Annals of Computer Science and Information Systems Volume 13

\title{
Communication Papers of the 2017 \\ Federated Conference on Computer Science and Information Systems
}

September 3-6, 2017. Prague, Czech Republic

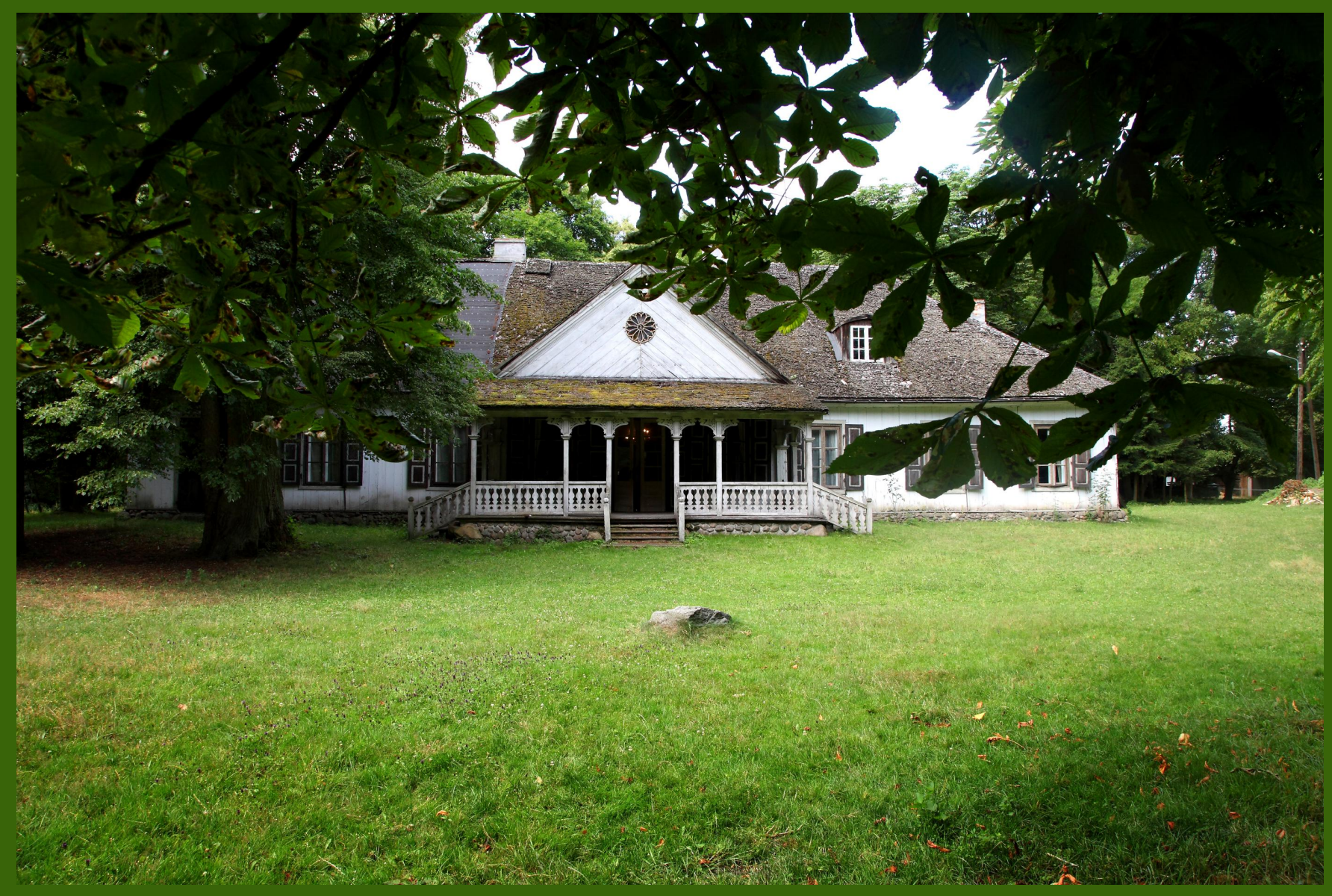

Maria Ganzha, Leszek Maciaszek, Marcin Paprzycki (eds.) 



\section{Annals of Computer Science and Information Systems, Volume 13}

\section{Series editors:}

Maria Ganzha,

Systems Research Institute Polish Academy of Sciences and Warsaw University of Technology, Poland

Leszek Maciaszek,

Wrockaw Universty of Economy, Poland and Macquarie University, Australia

Marcin Paprzycki,

Systems Research Institute Polish Academy of Sciences and Management Academy, Poland

\section{Senior Editorial Board:}

Wil van der Aalst,

Department of Mathematics \& Computer Science, Technische Universiteit Eindhoven (TU/e), Eindhoven, Netherlands

Frederik Ahlemann,

University of Duisburg-Essen, Germany

Marco Aiello,

Faculty of Mathematics and Natural Sciences, Distributed Systems, University of

Groningen, Groningen, Netherlands

Mohammed Atiquzzaman,

School of Computer Science, University of Oklahoma, Norman, USA

Barrett Bryant,

Department of Computer Science and Engineering, University of North Texas, Denton, USA

Ana Fred,

Department of Electrical and Computer Engineering, Instituto Superior Técnico

(IST-Technical University of Lisbon), Lisbon, Portugal

Janusz Górski,

Department of Software Engineering, Gdansk University of Technology, Gdansk, Poland

Mike Hinchey,

Lero-the Irish Software Engineering Research Centre, University of Limerick, Ireland

Janusz Kacprzyk,

Systems Research Institute, Polish Academy of Sciences, Warsaw, Poland

Irwin King,

The Chinese University of Hong Kong, Hong Kong

Juliusz L. Kulikowski,

Natecz Institute of Biocybernetics and Biomedical Engineering, Polish Academy of Sciences, Warsaw, Poland

Michael Luck,

Department of Informatics, King's College London, London, United Kingdom

Jan Madey,

Faculty of Mathematics, Informatics and Mechanics at the University of Warsaw, Poland Stan Matwin,

Dalhousie University, University of Ottawa, Canada and Institute of Computer Science, Polish Academy of Science, Poland

Michael Segal,

Ben-Gurion University of the Negev, Israel

Andrzej Skowron,

Faculty of Mathematics, Informatics and Mechanics at the University of Warsaw, Poland

John F. Sowa,

VivoMind Research, LLC, USA 


\section{Editorial Associate:}

Katarzyna Wasielewska, Systems Research Institute Polish Academy of Sciences, Poland Paweł Sitek,

Kielce University of Technology, Kielce, Poland

TEXnical editor: Aleksander Denisiuk,

University of Warmia and Mazury in Olsztyn, Poland 


\section{Communication Papers of the 2017 Federated Conference on Computer Science and Information Systems}

Maria Ganzha, Leszek Maciaszek, Marcin Paprzycki (eds.) 
Annals of Computer Science and Information Systems, Volume 13 Communication Papers of the 2017 Federated Conference on Computer Science and Information Systems

USB: ISBN 978-83-922646-3-7

WEB: ISBN 978-83-922646-2-0

ISSN 2300-5963

DOI 10.15439/978-83-922646-2-0

(C) 2017, Polskie Towarzystwo Informatyczne

Ul. Solec 38/103

00-394 Warsaw

Poland

Contact: secretariat@fedcsis.org

http://annals-csis.org/

Cover photo:

Iwona Burdecka, Elblag, Poland

Also in this series:

Volume 12: Position Papers of the 2017 Federated Conference on Computer Science and Information Systems, ISBN WEB: 978-83-922646-0-6, ISBN USB: 978-83-922646-1-3

Volume 11: Proceedings of the 2017 Federated Conference on Computer Science and Information Systems, ISBN WEB: 978-83-946253-7-5, ISBN USB: 978-83-946253-8-2, ISBN ART: 978-83-946253-9-9

Volume 10: Proceedings of the Second International Conference on Research in Intelligent and Computing in Engineering, ISBN WEB: 978-83-65750-05-1, ISBN USB: 978-83-65750-06-8

Volume 9: Position Papers of the 2016 Federated Conference on Computer Science and Information Systems, ISBN WEB: 978-83-60810-93-4, ISBN USB: 978-83-60810-94-1

Volume 8: Proceedings of the 2016 Federated Conference on Computer Science and Information Systems, ISBN WEB: 978-83-60810-90-3, ISBN USB: 978-83-60810-91-0, ISBN ART: 978-83-60910-92-7

Volume 7: Proceedings of the LQMR Workshop, ISBN WEB: 978-83-60810-78-1, ISBN USB: 978-83-60810-79-8

Volume 6: Position Papers of the 2015 Federated Conference on Computer Science and Information Systems, ISBN WEB: 978-83-60810-76-7, ISBN USB: 978-83-60810-77-4 Volume 5: Proceedings of the 2015 Federated Conference on Computer Science and Information Systems, ISBN WEB: 978-83-60810-66-8, ISBN USB: 978-83-60810-67-5 Volume 4: Proceedings of the E2LP Workshop, ISBN WEB: 978-83-60810-64-4, ISBN USB: 978-83-60810-63-7

Volume 3: Position Papers of the 2014 Federated Conference on Computer Science and Information Systems, ISBN WEB: 978-83-60810-60-6, ISBN USB: 978-83-60810-59-0 Volume 2: Proceedings of the 2014 Federated Conference on Computer Science and Information Systems, wEB: ISBN 978-83-60810-58-3, USB: ISBN 978-83-60810-57-6, ART: ISBN 978-83-60810-61-3

Volume 1: Position Papers of the 2013 Federated Conference on Computer Science and Information Systems (FedCSIS), ISBN WEB: 978-83-60810-55-2, ISBN USB: 978-83-60810-56-9 
$\mathrm{D}$ EAR Reader, it is our pleasure to present to you Communication Papers of the 2017 Federated Conference on Computer Science and Information Systems (FedCSIS), which took place, for the first time outside of Poland, in Prague, Czech Republic, on September 3-6, 2017.

This year, communication papers have been introduced as a separate category of contributions. They report on research topics worthy of immediate communication. They may be used to mark a hot new research territory or to describe work in progress in order to quickly present it to scientific community. They may also contain additional information omitted from the earlier papers or may present software tools and products in a research state.

FedCSIS 2017 was Chaired by prof. Pavel Tvrdik, while prof. Jan Janousek acted as the Chair of the Organizing Committee. This year, FedCSIS was organized by the Polish Information Processing Society (Mazovia Chapter), IEEE Poland Section Computer Society Chapter, Systems Research Institute Polish Academy of Sciences, Warsaw University of Technology, Wrocław University of Economics, and Czech Technical University in Prague.

FedCSIS 2017 was technically co-sponsored by: IEEE Region 8, IEEE Czechoslovakia Section, IEEE Poland Section, IEEE Computer Society, IEEE Computer Society Technical Committee on Intelligent Informatics, IEEE Czechoslovakia Section Computer Society Chapter, IEEE Poland Section Gdańsk Computer Society Chapter Poland, SMC Technical Committee on Computational Collective Intelligence, IEEE Poland Section Systems, Man, and Cybernetics Society Chapter, IEEE Poland Section Control System Society Chapter, IEEE Poland Section Computational Intelligence Society Chapter, ACM Special Interest Group on Applied Computing, Łódź ACM Chapter, International Federation for Information Processing, Committee of Computer Science of the Polish Academy of Sciences, Polish Operational and Systems Research Society, Mazovia Cluster ICT Poland, Polski Klaster Badań i Rozwoju Internetu Rzeczy, and Eastern Cluster ICT Poland. FedCSIS 2017 was sponsored by Intel, Profinit and Abra.

FedCSIS 2017 consisted of the following events (conferences, symposia, workshops, special sessions). These events were grouped into FedCSIS conference areas, of various degree of integration. Specifically, those listed without indication of the year 2017 signify "abstract areas" with no direct paper submissions to them (but with submissions to their enclosed events).

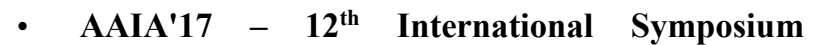
Advances in Artificial Intelligence and Applications

- AIMA'17 $-7^{\text {th }}$ International Workshop on Artificial Intelligence in Medical Applications

- AIRIM'17 - $2^{\text {nd }}$ International Workshop on AI aspects of Reasoning, Information, and Memory

- ASIR'17 - $7^{\text {th }}$ International Workshop on Advances in Semantic Information Retrieval

- JAWS'17 $-11^{\text {th }}$ Joint Agent-oriented Workshops in Synergy

- LTA'17 - $2^{\text {st }}$ International Workshop on Language Technologies and Applications
- WCO'17 - 10 $0^{\text {th }}$ International Workshop on Computational Optimization

- CSS - Computer Science \& Systems

- CANA'17 - $10^{\text {th }}$ Computer Aspects of Numerical Algorithms

- C\&SS'17 $-4^{\text {th }}$ International Conference on Cryptography and Security Systems

- CPORA'17 - $2^{\text {nd }}$ Workshop on Constraint Programming and Operation Research Applications

- MMAP'17 - 10 th $^{\text {th }}$ International Symposium on Multimedia Applications and Processing

- WAPL'17 $-6^{\text {th }}$ Workshop on Advances in Programming Languages

- WSC'17 $-9^{\text {th }}$ Workshop on Scalable Computing

- iNetSApp - International Conference on Innovative Network Systems and Applications

- INSERT'17 $-1^{\text {st }}$ International Conference on Security, Privacy, and Trust

- IoT-ECAW'17 - $1^{\text {st }}$ Workshop on Internet of Things - Enablers, Challenges and Applications

- WSN'17 $-6^{\text {th }}$ International Conference on Wireless Sensor Networks

- IT4MBS - Information Technology for Management, Business \& Society

- $A I T M^{\prime} 17-15^{\text {th }}$ Conference on Advanced Information Technologies for Management

- ISM'17 $-12^{\text {th }}$ Conference on Information Systems Management

- IT4L'17 $-5^{\text {th }}$ Workshop on Information Technologies for Logistics

- KAM'17 - 23 ${ }^{\text {nd }}$ Conference on Knowledge Acquisition and Management

- SSD\&A - Software Systems Development \& Applications

- IWCPS'17 $-4^{\text {th }}$ International Workshop on Cyber-Physical Systems

- LASD'17 - $1^{\text {st }}$ International Conference on Lean and Agile Software Development

- MIDI'17 $-4^{\text {th }}$ Conference on Multimedia, Interaction, Design and Innovation

- SEW-37 - The $37^{\text {th }}$ IEEE Software Engineering Workshop

- DS-RAIT'17 $-4^{\text {th }}$ Doctoral Symposium on Recent Advances in Information Technology

The 2017 edition of an AAIA'17 Data Mining Challenge, focused on "Helping AI to Play Hearthstone". Awards for the winners of the contest were sponsored by: Silver Bullet Solutions and the Mazovia Chapter of the Polish Information Processing Society.

Each paper, found in this volume, was refereed by at least two referees. 
The program of FedCSIS required a dedicated effort of many people. Each event constituting FedCSIS had its own Organizing and Program Committee. We would like to express our warmest gratitude to all Committee members for their hard work in attracting and later refereeing 504 submissions (regular and data mining).

We thank the authors of papers for their great contribution to research and practice in computing and information systems. We thank the invited speakers for sharing their knowledge and wisdom with the participants. Finally, we thank all those responsible for staging the conference in Prague. Organizing a conference of this scope and level could only be achieved by the collaborative effort of a highly capable team taking charge of such matters as conference registration system, finances, the venue, social events, catering, handling all sorts of individual requests from the authors, preparing the conference rooms, etc.
We hope you had an inspiring conference and an unforgettable stay in the beautiful city of Prague. We hope to meet you again for FedCSIS 2018 in Poznań, Poland.

\section{Co-Chairs of the FedCSIS Conference Series}

Maria Ganzha, Warsaw University of Technology, Poland and Systems Research Institute Polish Academy of Sciences, Warsaw, Poland

Leszek Maciaszek, Wroctaw University of Economics, Wrocław, Poland and Macquarie University, Sydney, Australia

Marcin Paprzycki, Systems Research Institute Polish Academy of Sciences, Warsaw Poland and Management Academy, Warsaw, Poland 
Annals of Computer Science and Information Systems, Volume 13

\title{
Communication Papers of the 2017 Federated Conference on Computer Science and Information Systems (FedCSIS)
}

\author{
September 3-6, 2017. Prague, Czech Republic
}

TABLE OF CONTENTS

$12^{\text {TH }}$ INTERNATIONAL SYMPOSIUM ADVANCES IN ARTIFICIAL

INTELLIGENCE AND APPLICATIONS

Call For Papers

Application of Pattern Recognition for a Welding Process Oto Haffner, Erik Kučera, Štefan Kozák, Erich Stark

Data Mining with Trusted Knowledge Viktor Nekvapil

Decision Making in Building Maintenance Using a Graph-Based Knowledge

Representation

Wojciech Palacz, Grażyna Ślusarczyk, Andrzej Łachwa, Barbara Strug, Anna Paszyńska, Ewa Grabska

Critical Success Factors in IT Projects-From the Practice of Polish Clients Bartosz Wachnik

A Decision Support System for Demand Forecasting based on Classifier Ensemble Irem Isslek, Sule Gunduz, Oguducu

\section{Aaia data Mining Challenge}

Call For Papers

Application of machine learning to help AI to play Hearthstone

Evgeny Patekha

$7^{\text {TH }}$ INTERNATIONAL WORKSHOP ON ARTIFICIAL INTELLIGENCE IN MEDICAL APPLICATIONS

Call For Papers

Analysys of the impact of disturbance on the arteriovenous fistula state classification 
$7^{\text {TH }}$ INTERNATIONAL WORKSHOP ON ADVANCES IN SEMANTIC

INFORMATION RETRIEVAL

Call For Papers

Generating AIML Rules from Twitter Conversations

Maxim Mozgovoy, Hiroshi Yamaguchi

\section{$11^{\text {TH }}$ JOINT AgENT-ORIENTED WORKSHOPS IN SYNERGY}

Call For Papers

Collision-Free Agent Migration in Spatial Simulation

Munehiro Fukuda, Christopher Bowzer, Benjamin Phan, Kasey Cohen

\section{$10^{\text {TH }}$ INTERNATIONAL WORKSHOP ON COMPUTATIONAL}

\section{OPTIMIZATION}

Call For Papers

InterCriteria Analysis of Multi-population Genetic Algorithms Performance Maria Angelova, Tania Pencheva

A new optimization-based approach for aircraft landing in the presence of windshear Pawet Drag, Krystyn Styczeń

Concepts Selection in Fuzzy Cognitive Map using Evolutionary Learning Algorithm based on Graph Theory Metrics Katarzyna Poczeta, Łukasz Kubus, Alexander Yastrebov

Binary Segmentation Methods for Identifying Boundaries of Spatial Domains Nishanthi Raveendran, Georgy Sofronov

The Revised Stochastic Simplex Bisection Algorithm and Particle Swarm Optimization Christer Samuelsson

\section{COMPUTER SCIENCE \& SYSTEMS}

Call For Papers

\section{$10^{\text {TH }}$ WORKSHOP ON COMPUTER ASPECTS OF NUMERICAL} Algorithms

Call For Papers

Laboratory tests of two-dimensional wavelet trigger in radio detection of cosmic rays Zbigniew Szadkowski, Anna Szadkowska

$4^{\text {TH }}$ INTERNATIONAL CONFERENCE ON CRYPTOGRAPHY AND SECURITY SYSTEMS

Call For Papers

Trustworthiness 5G Enabler

Rafat Artych, Krzysztof Bocianiak, Tomasz Ośko

$10^{\text {TH }}$ InTERNATIONAL SYMPOSIUM ON MULTIMEDiA A PPLiCATIONS AND Processing

Call For Papers

Utilization of Renewable Resources in Rehabilitation of Existent Micro-hydropower plants Victorita Radulescu

Extracting Acoustic Features of Japanese Speech to Classify Emotions Takashi Yamazaki, Minoru Nakayama 


\section{$6^{\text {TH }}$ Workshop on Advances in Programming Languages}

Call For Papers

Analysis of Include Dependencies in $\mathbf{C}++$ Source Code Bence Babati, Norbert Pataki

A QA System for learning Python

Parsing with Earley Virtual Machines

Audrius Šaik-unas

\section{$9^{\text {TH }}$ WORKSHOP ON SCAlable COMPUTING}

Call For Papers

Business Intelligence Platform for Big Data based on Scalable Distributed Two-Layer Data Store

Adam Krechowicz, Stanistaw Deniziak

The Algorithm for Sequential Analysis of Variants for Distribution of Virtual Machines in Data Center

Oleksandr Rolik, Maksym Bodaniuk, Valerii Kolesnik, Volodymyr Samotyy

INTERNATIONAL CONFERENCE ON INNOVATIVE NETWORK SYSTEMS AND APPLiCATIONS

Call For Papers

\section{$1^{\text {ST }}$ International Conference on Security, Privacy, AND}

\section{TRUST}

\section{Call For Papers}

Identification of Fingerprints using Circular String Approximation for Mobile Devices Oluwole I. Ajala, Costas S. Iliopoulos, Mujibur R. Khan Rodolfo Barriviera, Carlos Maziero

Multimodal Biometric System for Identity Verification Based on Hand Geometry and

Hand Palm's Veins Luiz Eduardo de Christo

Load-balanced Integrated Information Security Monitoring System Tomasz Klasa, Imed El Fray

A Robust Watermarking Technique for Image Content Authentication Obaid Ur-Rehman, Natasa Zivic

$1^{\text {ST }}$ WORKShOP ON INTERNET OF THINGS - ENABLERS, ChALlENGES AND APPLICATIONS

Call For Papers

Leveraging virtualization for scenario based IoT application testing Tomasz Szydlo, Joanna Sendorek

\section{INFORMATION TECHNOLOGY FOR MANAGEMENT, BUSINESS \&} SOCIETY 
$15^{\text {TH }}$ CONFERENCE ON ADVANCED INFORMATION TECHNOLOGIES FOR MANAGEMENT

Call For Papers

Commitment Modeling

Frantisek Hunka, Jaroslav Zacek, Jiri Matula

Eye Tracking Data Acquisition and Preprocessing for Modeling of Manager Knowledge in

Financial Report Analysis Adrian Kaźmierczak

An analysis of the use of the web-accessibility validator

Eukasz Krawiec

Towards an Architecture of Electronic Service Market System - requirements analysis and evaluation

Robert Kutera, Wieslawa Gryncewicz, Tomasz Skalniak

\section{$12^{\text {TH }}$ CONFERENCE ON INFORMATION SYSTEMS MANAGEMENT}

\section{Call For Papers}

WaterSocial.org as the social platform aimed to promote an efficient water use

Tomasz Jach, Andrea Capiluppi, Ewa Magiera, Zhenchen Wang

Task Execution Support in Research Activity using RAC System

Shigeki Ohira, Naoya Morita, Katashi Nagao

\section{$2^{\text {RD }}$ Conference on KnOWledge ACQuisition And Management}

Call For Papers

Overview of Verification Tools for Business Process Models

Anna Suchenia (Mroczek), Piotr Wiśniewski, Antoni Ligeza

Design Thinking and Cognitive Science: An Exploratory Approach to Create Intellectual

Capital with Decentralised Organisations

Salim Chujfi, Christoph Meinel

\section{SoftWARE Systems DeVelopMent \& APPlications}

Call For Papers

\section{$4^{\text {TH }}$ INTERNATIONAL WORKSHOP ON CYBER-PHYSICAL SYSTEMS}

Call For Papers

Virtual Laboratory Based on Node.js Technology and Visualized in Mixed Reality Using

Microsoft HoloLens

Erich Stark, Pavol Bisták, Erik Kučera, Oto Haffner, Štefan Kozák

BAGS: A Tool to Quantify Smart Grid Resilience

Yatin Wadhawan, Clifford Neuman

$1^{\text {ST }}$ International Conference on Lean and Agile Software DEVELOPMENT

Call For Papers

Distinguishing and defining the role of a technical leader in outsourced teams developing

IT solutions using Scrum

Jan Werewka, Marcelina Wietecha, Kamil Wotczyk 
$4^{\text {TH }}$ Conference on Multimedia, Interaction, Design AND INNOVATION

Call For Papers

Affective Design Patterns in Computer Games. Scrollrunner Case Study

Grzegorz J. Nalepa, Barbara Giżycka, Krzysztof Kutt, Jan K. Argasiński

Proposal of an efficient rank-ordering method based on subjectivity

Taiki Nishijima, Aoi Honda, Makoto Ohki

A Method for Converting Colors for Color-impaired People, Considering Saturation and Contrast Ratio

Junko Shirogane, Yuko Iwase, Hajime Iwata, Miho Saito, Yoshiaki Fukazawa

Magdalena Tkacz

\section{THE $37^{\text {TH }}$ IEEE SOFTWARE ENGINEERING WORKSHOP}

Call For Papers

Threefold Analysis of Distributed Systems: IMDS, Petri Net and Distributed Automata DA3

Wiktor Daszczuk

Jianyu Lu, Wanling Xie, Huibiao Zhu, Yuan Fei

Author Index 



\section{$12^{\text {th }}$ International Symposium Advances in Artificial Intelligence and Applications}

\begin{abstract}
A AIA'17 will bring scientists, developers, practitioners, and users to present their latest research, results, and ideas in all areas of Artificial Intelligence. We hope that successful applications presented at AAIA'17 will be of interest to researchers who want to know about both theoretical advances and latest applied developments in AI.
\end{abstract}

\section{TOPICS}

Papers related to theories, methodologies, and applications in science and technology in this theme are especially solicited. Topics covering industrial applications and academic research are included, but not limited to:

- Decision Support

- Machine Learning

- Fuzzy Sets and Soft Computing

- Rough Sets and Approximate Reasoning

- Data Mining and Knowledge Discovery

- Data Modeling and Feature Engineering

- Data Integration and Information Fusion

- Hybrid and Hierarchical Intelligent Systems

- Neural Networks and Deep Learning

- Bayesian Networks and Bayesian Reasoning

- Case-based Reasoning and Similarity

- Web Mining and Social Networks

- Business Intelligence and Online Analytics

- Robotics and Cyber-Physical Systems

- AI-centered Systems and Large-Scale Applications

We also encourage researchers interested in the following topics to submit papers directly to the corresponding workshops, which are integral parts of AAIA'17:

- AI in Computational Optimization (WCO'17 workshop)

- AI in Language Technologies (LTA'17 workshop)

- AI in Medical Applications (see AIMA'17 workshop)

- AI in Reasoning and Computational Foundations (AIRIM'17 workshop)

- AI in Information Retrieval (ASIR'17 workshop)

All papers accepted to the main track of AAIA'17 and to the above workshops will be treated equally in the conference programme and will be equally considered for the awards listed below.

\section{Professor Zdzislaw Pawlak Best Paper Awards}

We are proud to continue the tradition started during the AAIA'06 and award two "Professor Zdzislaw Pawlak Best Paper Awards" for contributions which are outstanding in their scientific quality. The two award categories are:
- Best Student Paper-papers qualifying for this award must be marked as "Student full paper" to be eligible.

- Best Paper Award.

In addition to a certificate, each award carries a prize of 300 EUR provided by the Mazowsze Chapter of the Polish Information Processing Society.

\section{ZdZislaw Pawlak Award CommitTeE}

- Kacprzyk, Janusz, Polish Academy of Sciences, Poland - Kwaśnicka, Halina, Wroclaw University of Technology, Poland

- Marek, Victor, University of Kentucky, United States

- Markowska-Kaczmar, Urszula, Wroclaw University of Technology, Poland

- Matwin, Stan, Dalhousie University, Canada

- Michalewicz, Zbigniew, University of Adelaide, Australia

- Skowron, Andrzej, University of Warsaw, Poland

- Śluzek, Andrzej, Khalifa University, United Arab Emirates

\section{SECTION EDITORS}

- Janusz, Andrzej, University of Warsaw, Poland

- Ślęzak, Dominik, University of Warsaw, Poland

\section{REVIEWERS}

- Bartkowiak, Anna, Wrocław University, Poland

- Bazan, Jan, University of Rzeszów, Poland

- Betlinski, Pawel, Security On Demand, Poland

- Borkowski, Janusz, Polish-Japanese Academy of Information Technology \& Security On Demand, Poland

- Błaszczyński, Jerzy, Poznań University of Technology, Poland

- Carrizosa, Emilio, Universidad de Sevilla, Spain

- Chakraverty, Shampa, Netaji Subhas Institute of Technology, India

- do Carmo Nicoletti, Maria, UFSCar \& FACCAMP, Brazil

- Duentsch, Ivo, Brock University, Canada

- Eklund, Patrik, Umeå University, Sweden

- Foresti, Gian Luca, University of Udine, Italy

- Froelich, Wojciech, University of Silesia, Poland

- Girardi, Rosario, Federal University of Maranhão, Brazil

- Jaromczyk, Jerzy, University of Kentucky, United States

- Jatowt, Adam, Kyoto University, Japan 
- Jin, Xiaolong, Institute of Computing Technology, Chinese Academy of Sciences, China

- Karhang, Maylor Leung, Universiti Tunku Abdul Rahman, Malaysia

- Kasprzak, Włodzimierz, Warsaw University of Technology, Poland

- Kayakutlu, Gulgun, Istanbul Technical University, Turkey

- Konikowska, Beata, Polish Academy of Sciences, Poland

- Korbicz, Józef, University of Zielona Góra, Poland

- Kostek, Bożena, Gdańsk University of Technology, Poland

- Kryszkiewicz, Marzena, Warsaw University of Technology, Poland

- Kulikowski, Juliusz, Institute of Biocybernetics and Biomedical Enginee, Poland

- Lopes, Lucelene, PUCRS, Brazil

- Madalińska-Bugaj, Ewa, University of Warsaw, Poland

- Matson, Eric T., Purdue University, United States

- Menasalvas, Ernestina, Universidad Politécnica de Madrid, Spain

- Miyamoto, Sadaaki, University of Tsukuba, Japan

- Moshkov, Mikhail, King Abdullah University of Science and Technology, Saudi Arabia

- Myszkowski, Paweł B., Wroclaw University of Technology, Poland

- Nourani, Cyrus F., Akdmkrd-DAI TU Berlin \& Munich Transmedia \& SFU Burnaby, Germany

- Nowostawski, Mariusz, Norwegian University of Technology and Science (NTNU), Norway

- Ogiela, Marek, AGH University of Science and Technology, Poland

- Ohsawa, Yukio, University of Tokyo, Japan

- Peters, Georg, Munich University of Applied Sciences, Germany

- Po, Laura, Università di Modena e Reggio Emilia, Italy

- Porta, Marco, University of Pavia, Italy

- Przybyła-Kasperek, Małgorzata, University of Silesia, Poland

- Raghavan, Vijay, University of Louisiana at Lafayette,
United States

- Rakus-Andersson, Elisabeth, Blekinge Institute of Technology, Sweden

- Ramanna, Sheela, University of Winnipeg, Canada

- Ras, Zbigniew, University of North Carolina at Charlotte, United States

- Rauch, Jan, University of Economics, Prague, Czech Republic

- Reformat, Marek, University of Alberta, Canada

- Ruta, Dymitr, EBTIC, Khalifa University of Science and Technology, United Arab Emirates

- Schaefer, Gerald, Loughborough University, United Kingdom

- Sikora, Marek, Silesian University of Technology, Poland

- Sikos, Leslie F., Flinders University, Australia

- Subbotin, Sergey, Zaporizhzhya National Technical University, Ukraine

- Sydow, Marcin, Polish Academy of Sciences \& PolishJapanese Academy of Information Technology, Poland

- Szczęch, Izabela, Poznań University of Technology, Poland

- Szczuka, Marcin, University of Warsaw, Poland

- Szpakowicz, Stan, University of Ottawa, Canada

- Szwed, Piotr, AGH University of Science and Technology, Poland

- Tomczyk, Arkadiusz, Lodz University of Technology, Poland

- Unland, Rainer, Universität Duisburg-Essen, Germany

- Unold, Olgierd, Wrocław University of Technology, Poland

- Velastin, Sergio A., Kingston University, United Kingdom

- Weber, Richard, Universidad de Chile, Chile

- Werghi, Naoufel, Khalifa University of Science and Technology, United Arab Emirates

- Zakrzewska, Danuta, Łódź University of Technology, Poland

- Zielosko, Beata, University of Silesia, Poland

- Ziółko, Bartosz, AGH University of Science and Technology, Poland 


\title{
Application of Pattern Recognition for a Welding Process
}

\author{
Oto Haffner, Erik Kučera, Štefan Kozák, Erich Stark \\ Faculty of Electrical Engineering and Information Technology \\ Slovak University of Technology in Bratislava \\ Bratislava, Slovakia \\ Email: oto.haffner@stuba.sk
}

\begin{abstract}
The paper deals with the development of a system for automatic weld recognition using new information technologies based on cloud computing and single-board computer in the context of Industry 4.0. The proposed system is based on a visual system for weld recognition, and a neural network based on cloud computing for real-time weld evaluation, both implemented on a single-board low-cost computer. The proposed system was successfully verified on welding samples which correspond to a real welding process in the car production process. The system considerably contributes to the welds diagnostics in industrial processes of small- and medium-sized enterprises.
\end{abstract}

\section{INTRODUCTION}

$\mathrm{T}$ HE quality of manufacturing process is one of the most important aspect of production. Welding processes are used in many manufacturing processes and automotive industry. This makes prerequisites for researching and developing new modern methods and system for welding quality evaluation.

Visual inspection of the weld is one of the weld quality diagnostic methods for weld quality diagnostic. This method enables to check weld joint and its various parameters. Visual inspection does not destruct the weld so it is notdestructive methods for weld joints and materials. This examination is done as the first examination and it detects various defects. There is no need to use another method after detecting a defect with this method, so it is cost and timesaving.

This work deals with application for evaluation algorithm and weld recognition. It is based on the single-board computer using the visual system. This method is based on the method for visual quality evaluation and modern computer vision and image processing methods. Industry production, as well as mobile robotics, will find the usage of these methods.

This work has been supported by the Cultural and Educational Grant Agency of the Ministry of Education, Science, Research and Sport of the Slovak Republic, KEGA 030STU-4/2015 and KEGA 030STU-4/2017, by the Scientific Grant Agency of the Ministry of Education, Science and by the Slovak Research and Development Agency APVV-0772-12.
Actual trend in the industry is Industry 4.0 also named fourth industrial revolution. It is collective name for current automatization, exchanging of data and manufacturing technologies. It can be defined as a collective name for technologies and concept for organising value chain, which unites Cyber-Physical Systems, Internet of Thing and Internet of Service [1]. Industry 4.0 understands manufacturing units as complex distributed systems made by "smart" partial integration of individual autonomous subsystems. The integration is provided by suitable communication of each other based on actual demand, activity coordination and coordination among autonomous subsystems. [8]

Authors [7] uses improved Beamlet Transformation for weld edge detection. Images they work with are loaded by noise. The aim of authors work is to detect edge borders of welds. Beamlet algorithm does the dynamic thresholding in one of the steps. The algorithm predicts the directional characteristic of the weld and it is possible to filtrate unsuitable edges. Using this method, it is capable of directly extracting weld seam edges from highly noisy welding images without any pre-processing or post-processing steps, thus is characterized by its high efficiency and its prominent anti-noising performance.

Authors [11] worked with weld images with very low contrast. The weld images come from the pipeline and are loaded with low contrast and noise which do problems to convent edge detectors. At first, the image is noise filtered using morphological operation of opening and closing. The next is improved algorithm of fuzzy edge detection. The algorithm is based on two steps: multi-level fuzzy image improvement based on interactive searching of optimal threshold level and based on multi-directional edge detector, which convolution kernel is $5 \times 5$ with 8 directions and is based on gradient searching. The result of the algorithm was compared with detectors as Sobel, canny FED and fast FED. Authors obtained whit their method better results.

Edge detection and histogram projection use authors [9] They compare histogram projections of tested welds with set similarity threshold. This threshold evaluates the quality of the weld. Loaded image of the pattern has the same 
specifications- width and position as the tested image. One vertical line is tested from the pattern and tested image. The correlation threshold is set. Line histogram od pattern image and the tested image is computed. The correlation degree of two histograms using Tukey HSD difference is computed. If the degree is lower than set threshold, this part of the image contains edge defects. The procedure is repeated over the entire width.

Authors [10] deals with weld evaluation on metal cans. On the weld can be a defect wich do not directly relate to weld (rest of glue, dust etc.). Because of this, authors use probability evaluation of two evaluation methods. The first, Column Gray-Level Accumulation Inspection represents in general histogram projection. The histogram projections of pattern and rested weld are compared. They compare also the first derivation for making better results. Defects of the wider surface can be detected by this method. DempsterShafer theory of evidence for overall evaluation is used.

Authors in other work [12] deal with edge detection based on pixel intensity difference of foreground and background. The background pixels intensity is occurred with maximal probability and the distribution of background pixels fits Gauss distribution.

\section{PROBLEM FORMULATION}

One of the most important production process for a wide range of products is also welding. Visual inspection of welds is one of the most effective and cheap unrestrictive methods for weld joints. This method of inspection is made by a manhuman vision. However, this can be loaded by human error because of fatigue, stress, inattention or lack of experience. In some manufactures, weld view is provided by the camera system. This image is shown on the monitor and the operator based on this image determine if the weld fits the set criteria. In the cases like this, it is possible to use automated method for weld evaluation by the visual system.

Incoming new modern trend Industry 4.0 and Internet of Things request new needs for all parts of manufacturing systems. It requests capability to communicate and share information for each part via cloud as a collector of data using computing intelligence.

The mentioned facts make the demand for research and development of new modern methods with are in line with the vision of Industry 4.0. One of these methods is also recognition and evaluation of welds [3].

The quality of weld joint must be evaluated during the whole welding process not only by testing of finished joint or construction. In terms of construction quality, weld joints are the most dangerous place in construction. This is the reason why the quality of weld joints is very important. [3]

The basic parts of the weld joint can be seen in Fig. 1:

1. Fundamental material

2. Boundary of weld

3. Weld reinforcement
4. Groove face

5. Root of weld

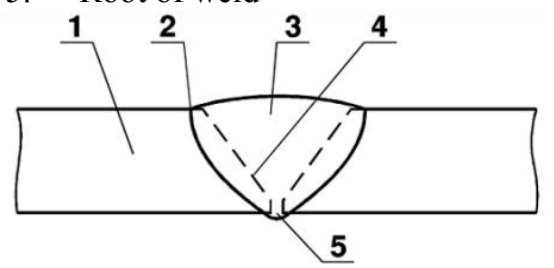

Fig. 1 Illustrated main terms of weld joint [3].

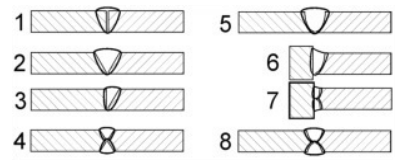

Fig. 2 Some butt weld types [3]

A. Selected weld types

Some types of butt welds are illustrated Fig. 2 , namely:
1. square
2. single- $\mathrm{V}$
3. single bevel
4. double-V
5. single-U
6. single-J
7. double $\mathrm{J}$
8. double side U-weld

\section{B. Defections of weld}

During all welding processes, there are many problems and most of them refer to non-integrity. The main quality of weld criteria is shape, integrity and required physical and chemical features. [3]

Butt weld defects are divided generally to internal and surface. To surface defects belongs: excess weld metal (Fig. 3), overlap, imperfect shape, root concavity, excessive meltthrough, undercuts (Fig. 4), poor restart (Fig. 3), cracks and spatter (Fig. 5) incomplete root penetration.

Testing using visual is non-destructive weld joints testing. Visual examination is performed at first before the others. Visual examination often reveals the most of the defects so the other time-consuming and expensive examinations are not done.

The visual examination focuses on the overall condition of the weld. The examination is performed by worker's eyes. The distance of examination should be $600 \mathrm{~mm}$ above surface and angle should not be lower than $30^{\circ}$. [3]

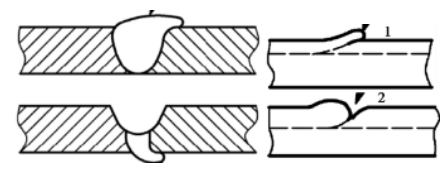

Fig. 3 Excess metal of weld (left) and poor restart (right) [2]

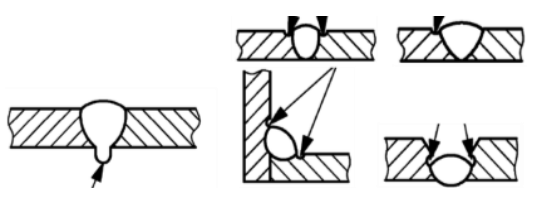

Fig. 4 Excessive melt-through (left) and undercut (right and middle) [2] 


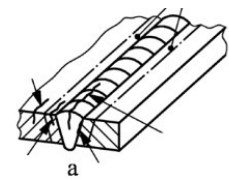

Fig. 5 Examples of cracks [2
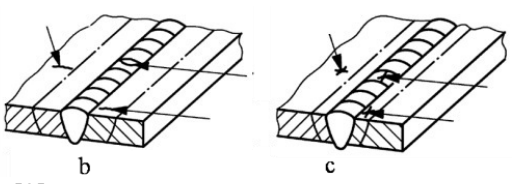

\section{CONCEPTION OF SYSTEM FOR WELD RECOGNITION AND DIAGNOSTIC}

Based on analysis of methods for weld evaluation, computer vision and industrial trends, technology there were proposed system for weld recognition and evaluation. In Fig. 6 is shown the scheme. Main parts of the system are singleboard computer Raspbery Pi 2 Model B, camera IDS uEye $\mathrm{XS}$ and web service Azure Machine Learning (Haffner, 2016).

Computing and communication kernel of this system is single-board computer Raspberry Pi 2 Model 2. Computer provides communication with camera which is mounted to laboratory preparation and takes images of weld samples. Obtained image data are processed and result is weld segmentation. Segmentation is used for computing of invariant image moment and then saved to text file. Text file is read by communication programme and sent to web service. Web service based on input data provide classification based on artificial computing intelligence and results are send back to communication programme.

Each parts of system are realised using low-cost components. Some of components are not suitable for industrial environment, however, for research and evaluation of proposed new modern method are. Using of low-cost components enables to utilize proposed system also for pedagogical purposes or modern ways for teaching of weld specialist.

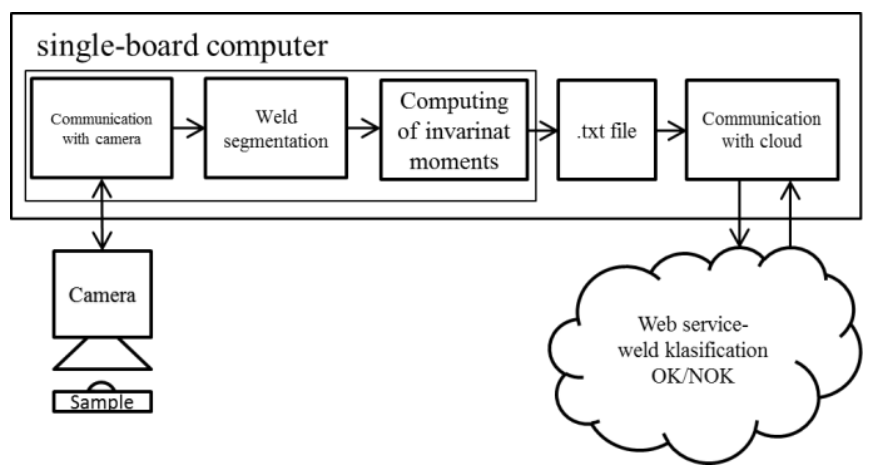

Fig. 6 Conception scheme for system for weld recognition and diagnostic

\section{COMPUTING TIME ANALYSIS}

Based on proposal method for weld recognition and evaluation, we made simple computing time comparison of chosen computer vision algorithms. As input image, we used image of laboratory made weld (Fig. 7 left). One tested algorithm is algorithm made in our work (Haffner \& Duchoň, 2014). This algorithm makes an environment map for mobile robot. In the next part of this section we will describe tested algorithms in detail.

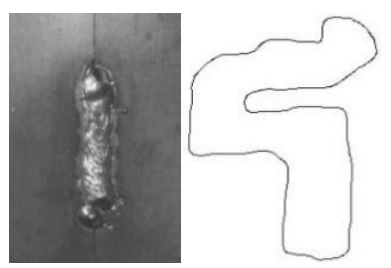

Fig. 7 Laboratory made weld (left) and result of algorithm for map making.

\section{A Tested algorithms}

All tested algorithms are realized in computer vision library OpenCV.

Used threshold algorithm uses Gauss method and block size for neighborhood 15 .

Opening algorithm is realized as erode and then dilate. The structuring element has ellipse shape and size $5 \times 5$.

Blur is realized as Gauss blur with kernel size $11 \times 11$.

Algorithm for map making will be described briefly. The laser scanner data are loaded from text file. Based on measured data the coordinates are computed and plotted in image. After plotting the map is thresholded. Then multiple time blur and threshold is used. After this Zhang-Suen skeletonizing is used. The result of this algorithm is in Fig. 7 right.

\section{B Computing time measurement}

Tested algorithms was compiled and measured on Raspberry Pi, Banana Pi, Raspberry PI2 and personal computer with CPU Intel Core i7 $9302.80 \mathrm{GHz}$. Result of simple time measurement we can see in Table 1. Based on result of the measurement, the Banana $\mathrm{Pi}$ is the fastest among embedded computers even when it's CPU has 2 cores and Raspberry Pi2 CPU has 4 cores. Better results from Raspberry PI2 can be expected using optimized algorithms for multi-core CPU.

Table 1

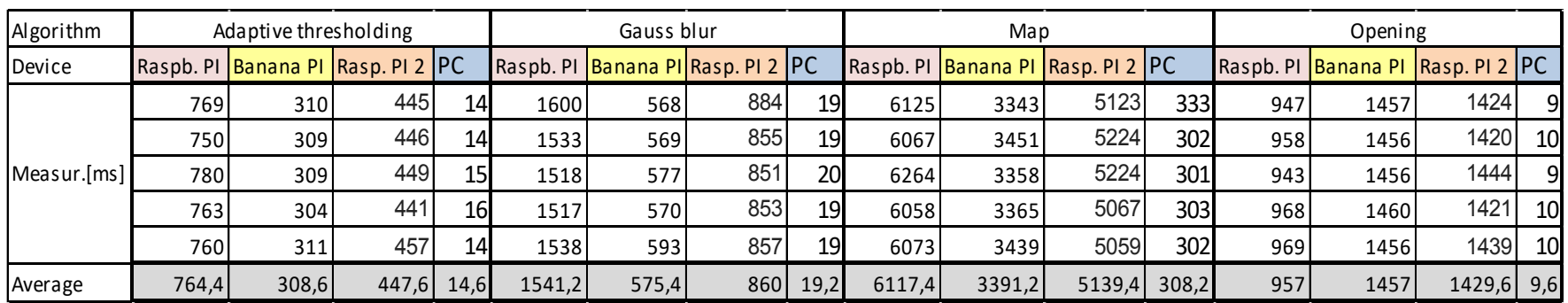




\section{VISUAL SYSTEM}

Industrial camera IDS uEye XS for image acquisition was used. Advantage of this camera are measurements, USB 2.0 connection, plenty of integrated functions and SDK for embedded operating systems and processors with ARMv7 Cortex.

\section{A Industrial camera IDS uEye XS}

Camera uEye XS is USB 2.0 industrial camera by company IDS Imaging Development Systems. It has 5.04 Mpix CMOS sensor by company ON Semiconductor with pixel size $1,4 \mu \mathrm{m}$. Supplied complex software package enables to integrate camera to application using standard interface as DirectShow, ActiveX, GenICam or directly by using uEye API.

\section{B Communication with Raspberry Pi 2}

For camera communication with single-board computer Raspberry Pi 2 was used SDK IDS Software Suite and operating system Raspbian based on Linux. SDK obtain libraries and header files and enable using function which operate with camera. The basic principle is shown in Fig. 9. In general, image from camera is loaded in a loop, until the key is pressed. To the image is plotted rectangle which represents region of interest in image (Fig. 8). The weld sample should be located to this area.

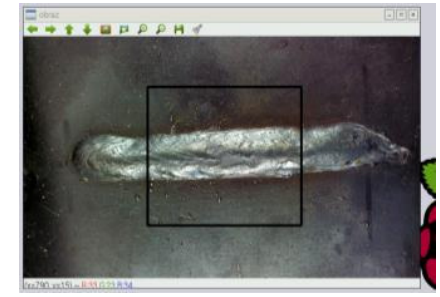

Fig. 8 Window with captured image of weld in ROI

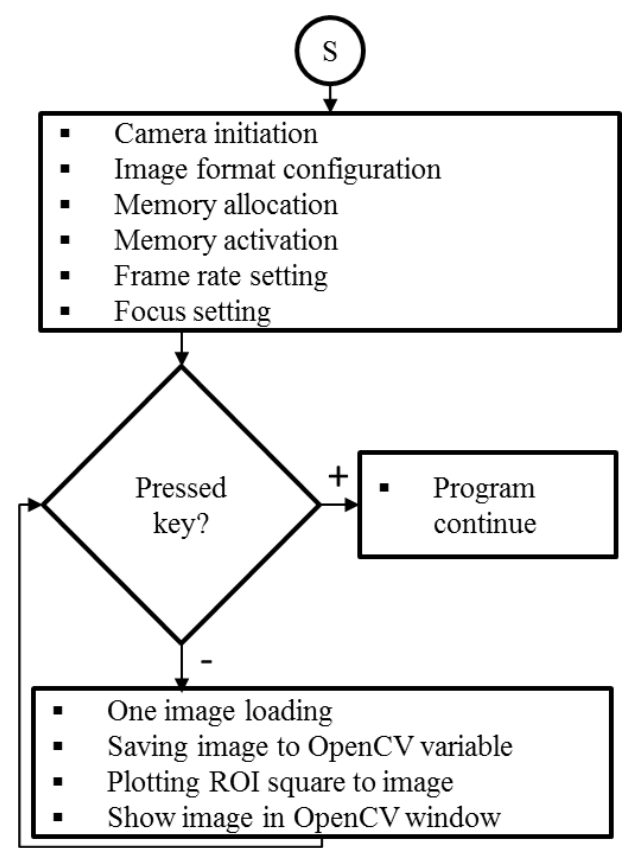

Fig. 9 Camera communication scheme

\section{Laboratory experiment}

For the tasks of image acquisition and processing there was needed to propose the preparation where will be camera mounted and take images of laboratory prepared weld samples under the artificial light. The experiment (Fig. 10) was constructed using low-cost components as building kit Merkur and ligh source (12V LED spot lights).

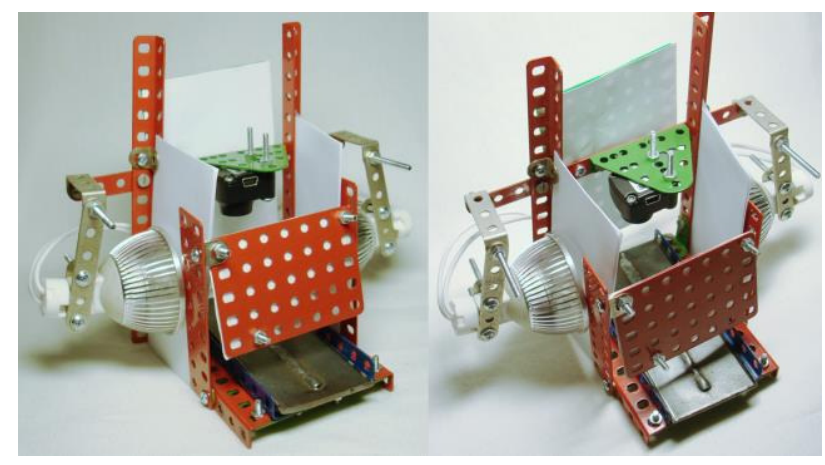

Fig. 10 Laboratory experiment

\section{WELD SEGMENTATION}

The weld segmentation method is based on namely local entropy of image which works with a statistical measure of randomness. Using this method comes from idea, that the surface around weld is flat and monotone however welded area has textured (random) surface.

\section{A Experiment}

We used Matlab Image processing toolbox and laboratory made weld samples for this experiment. The image (Fig.11 a) is filtered at first by median filter with 5-by5 neighborhood around the corresponding pixel. After median filtering the entropy filter is applied. The neighbourhood for filtering is 15-by-15. Resulting image (Fig.11 b) is threshelded with threshold 165 . The results of thresholding we can see in Fig. 11 c. As we can see, the thresholding did not give ideal results. Around weld mask are several blobs. Blobs are removed using the Matlab function bwareaopen. The basic steps of this algorithms are: determining the connected components, computing the area of each component and removing small objects. In our experiment we remove objects smaller than 2000 pixels. The result of removing small objects we can see in Fig. 11 d.

The mask contains unfilled holes (Fig12a). The wholes are filled using binary closing with disk structural element with size 10 (Fig.12b). In the Fig.12c we can see the result of weld segmentation and in the Fig.12d we can see corresponding edges of segmentation.

Also the others weld samples was tested with the same parameters. The results we can see in Fig. 13 and 14 as a) original image, b) entropy, c) mask, d) highlighted edges of segmented weld. 


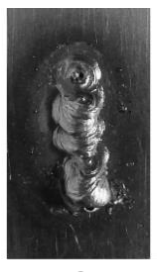

a

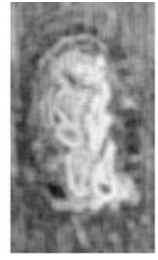

b

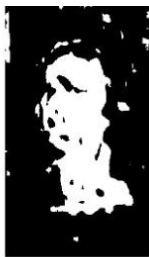

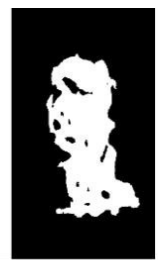

d
Fig. 11 a) original image of weld, b) entropy filtered image, c) thresholded entropy image, d) removed blobs

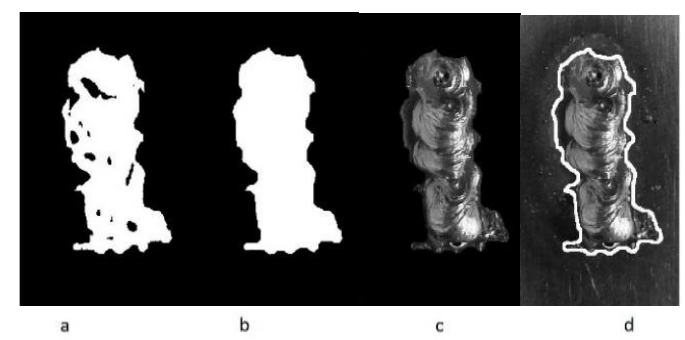

Fig. 12 a) original mask, b) filled mask, c) segmented weld, d) highlighted edges of segmented weld

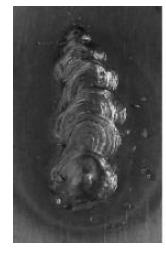

a

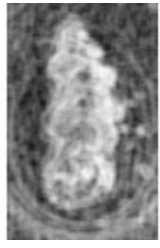

b

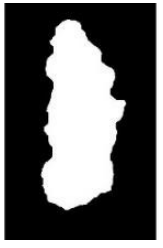

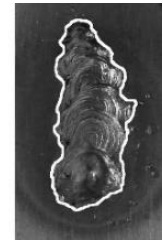

d
Fig. 13 Weld sample 1

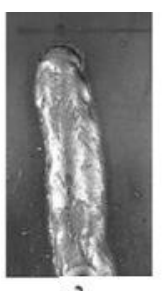

a

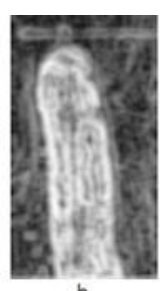

$2^{b}$

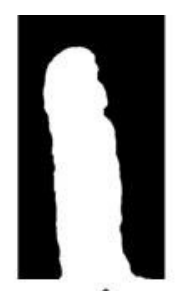

c

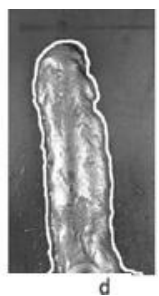

Fig. 14 Weld sample 2

\section{B Algorithm implementation}

Proposed algorithm for weld segmentation needs to be converted to $\mathrm{C}$ code, which is able to implement to singleboard computer Raspberry Pi 2. This can be done using MATALB Coder.

The proposed algorithm had to be adjusted. MATLAB Coder do not support al MATLAB functions and some function had to be replaced by functions from other toolbox. Function bwareaopen from Image Processing Toolbox had to be replaced by vision.BlobAnalysys from Computer Vision Toolbox. The function for entropy computing is not supported at all. This was the reason, why algorithm had to be adjusted.

Tasks for thresholding, blob removal and masking was moved to separate function. The input parameter is image entropy and output is segmented image. Computing of image entropy is not done using MATLAB but programme realization using OpenCV. The resulting image is transpositioned because of different data representation in
MATALB and OpenCV. After transpositin the image is converted to array and this array is input parameter for function generated from MATALB Coder. This proces is illustrated in Fig. 15.

\begin{tabular}{|c|c|c|c|c|c|}
\hline $\begin{array}{l}\text { grayscale } \\
\text { image }\end{array}$ & $\rightarrow \begin{array}{c}\text { entropy } \\
\text { computing- } \\
\text { OpenCV }\end{array}$ & transposition & $\begin{array}{c}\text { Conversion to } \\
\text { array }\end{array}$ & $\begin{array}{l}\text { segmentation- } \\
\text { code from } \\
\text { MATALB }\end{array}$ & $\begin{array}{c}\text { segmented } \\
\text { image }\end{array}$ \\
\hline
\end{tabular}

Fig. 15 Algorithm implementation scheme

Computing time of implemented code on Raspberry Pi2 (in table as RPi2) was compared with algorithm in MATLAB. Compared was the whole segmentation algorithm, generated function texture_seg2 and entropy computing. Measurement of MATLAB algorithm was done on computer Intel Core i7-3610QM 2.3GHz. in Table 2 we can see the time comparison. The computing time 2,2 seconds is for solved problem satisfying.

Table 2

\begin{tabular}{|c|r|r|r|r|r|r|}
\hline & \multicolumn{2}{|c|}{ segmentation } & \multicolumn{2}{c|}{ texture_seg2 } & \multicolumn{2}{c|}{ entropy } \\
\hline device & RPi 2 & MATLAB & RPi 2 & MATLAB & RPi 2 & MATLAB \\
\hline \multirow{4}{*}{ time[s] $]$} & 2,271 & 0,430 & 0,924 & 0,056 & 1,353 & 0,302 \\
\cline { 2 - 7 } & 2,259 & 0,432 & 0,912 & 0,057 & 1,319 & 0,303 \\
\cline { 2 - 7 } & 2,284 & 0,431 & 0,897 & 0,056 & 1,335 & 0,305 \\
\cline { 2 - 7 } & 2,243 & 0,432 & 0,899 & 0,055 & 1,324 & 0,302 \\
\cline { 2 - 7 } & 2,265 & 0,423 & 0,892 & 0,057 & 1,321 & 0,304 \\
\hline average[s] & 2,264 & 0,429 & 0,904 & 0,0562 & 1,330 & 0,303 \\
\hline
\end{tabular}

\section{Weld representation for classification}

The computing intelligence methods which will classify welds need its suitable representation. The features which will represent the weld were set as invariant moment characteristics. Computing of these characteristics are implemented in library OpenCV. The result is 7 numbers (moments), which are saved in text file. The average computing time of the moment on Raspberry Pi 2 is 5,59E-4 seconds.

\section{WELD CLASSIFICATION}

The feature representation of weld as invariant moments can be used for classification. For classification using computing intelligence method Microsoft Azure Machine Learning was chosen.

For training classifier, it is needed training data. It was unable to make thousands of weld samples. Instead of this, the training data was generated from real images of weld by moving weld through the ROI. 6428 training samples was generated from 50 laboratory samples.

Artificial neural network was used for classifying. There were done 6 experiment. For each experiment was set normalizer as Gauss normalizer. Structures of neural networks was default, i.e. one hidden layer, number of input nodes are the same as number of input features (7), nodes hidden layer is set by experimentator and output layer is set by number of classify classes (2). In Table 3 we can see the set parameters for individual layers. 
Table 3

\begin{tabular}{|l|r|r|r|r|r|r|r|}
\hline & $\begin{array}{c}\text { Hidd. } \\
\text { layers }\end{array}$ & $\begin{array}{c}\text { Learn. } \\
\text { tempo }\end{array}$ & $\begin{array}{c}\text { Numb. } \\
\text { of itter. }\end{array}$ & $\begin{array}{c}\text { Init. Learnig } \\
\text { weight }\end{array}$ & $\begin{array}{c}\text { mom- } \\
\text { entum }\end{array}$ & $\begin{array}{c}\text { Mean sqrd. } \\
\text { deviation }\end{array}$ & $\begin{array}{c}\text { Standard } \\
\text { deviation }\end{array}$ \\
\hline ANN 1 & 15 & 0,1 & 100 & 0,1 & 0 & $1,054 \mathrm{E}-04$ & $2,887 \mathrm{E}-03$ \\
\hline ANN 2 & 15 & 0,1 & 500 & 0,1 & 0 & $1,558 \mathrm{E}-06$ & $4,938 \mathrm{E}-05$ \\
\hline ANN 3 & 20 & 0,1 & 500 & 0,1 & 0 & $2,048 \mathrm{E}-06$ & $6,569 \mathrm{E}-05$ \\
\hline ANN 4 & 20 & 0,1 & 300 & 0,1 & 0 & $7,213 \mathrm{E}-06$ & $2,304 \mathrm{E}-04$ \\
\hline ANN 5 & 15 & 0,1 & 1500 & 0,1 & 0 & $1,159 \mathrm{E}-07$ & $3,401 \mathrm{E}-06$ \\
\hline ANN 6 & 20 & 0,1 & 1500 & 0,1 & 0 & $1,052 \mathrm{E}-07$ & $3,069 \mathrm{E}-06$ \\
\hline
\end{tabular}

Results for experiments ANN 2 to 6 can be characterized so, that all classifications were right in case of true positive result with probability range $0.9-1$ and true negative result with probability range $0,1-0$. In this case Metrics as Accuracy, Precision or Recall is 1 . To compare these neural networks, each other we used exact probabilities of each prediction, which can be exported from the Score module. Experiment with ANN 6 had the best results and was used as a classifier.

The ANN6 classifier was than tested with new dataset. Images for this dataset was generated from previous images, but the images were deformed in graphical software. Using this approach, we generated new dataset. The Mean squared deviation was 3,080E-07 and Standard deviation 6,581E-06. Results confirmed proposed method applicability for the problem of weld evaluation.

\section{VIII.VERIFICATION OF IMPLEMENTATION}

After integration of all parts of the system as communication with visual system, weld segmentation and communication with web service it was possible to verify the functionality of the system and computing time. Recognition provides one console application, which starts the camera image acquisition, image is saved after any key is pressed, with image are executed proposed algorithms, the feature representation is send to the web service and it send the results to the program and the results are showed to the console. Application was tested with set of 14 weld samples which was not used for training dataset. In Table 4 we can see the results.

Table 4

\begin{tabular}{|c|c|c|c|c|}
\hline Num. & classify & predicted & probability & time [s] \\
\hline 1 & OK & OK & $1,000000 \mathrm{E}+00$ & 5,31419 \\
\hline 2 & $\mathrm{OK}$ & OK & $9,999996 \mathrm{E}-01$ & 4,85362 \\
\hline 3 & NOK & NOK & 2,787349E-19 & 5,34659 \\
\hline 4 & NOK & NOK & $3,546546 \mathrm{E}-13$ & 5,16983 \\
\hline 5 & OK & OK & 9,999999E-01 & 4,87419 \\
\hline 6 & $\mathrm{OK}$ & $\mathrm{OK}$ & $1,000000 \mathrm{E}+00$ & 5,19455 \\
\hline 7 & OK & OK & $9,999998 \mathrm{E}-01$ & 4,86545 \\
\hline 8 & NOK & NOK & $8,053897 \mathrm{E}-11$ & 5,16845 \\
\hline 9 & NOK & NOK & $2,887203 \mathrm{E}-15$ & 4,96445 \\
\hline 10 & NOK & NOK & $4,755493 \mathrm{E}-32$ & 5,44418 \\
\hline 11 & NOK & NOK & $3,072803 \mathrm{E}-13$ & 5,34558 \\
\hline 12 & NOK & NOK & $2,193890 \mathrm{E}-16$ & 5,10893 \\
\hline 13 & NOK & NOK & $4,700284 \mathrm{E}-12$ & 5,18963 \\
\hline 14 & $\mathrm{NOK}$ & $\mathrm{NOK}$ & $3,459683 \mathrm{E}-09$ & 4,95562 \\
\hline & & & average & 5,128233 \\
\hline
\end{tabular}

\section{CONCLUSION}

The performed analysis of the current state in pattern recognition applications for welding has revealed that there has not been implemented and presented a solution based on low-cost single-board computer using cloud technologies yet. The proposed solution enables to recognize a weld in 5,2 seconds which is satisfactory for 0,5-1minute-long manufacturing tact used in automotive industry, and is suitable even for recognition of larger weld deformations. The used low-cost components are suitable for small and medium-sized companies. The proposed methodology can be effectively used especially in automotive industry.

\section{ACKNOWLEDGMENT}

This work has been supported by the Cultural and Educational Grant Agency of the Ministry of Education, Science, Research and Sport of the Slovak Republic, KEGA 030STU- 4/2015, KEGA 011STU-4/2015 and KEGA 030STU-4/2017, by the Scientific Grant Agency of the Ministry of Education, Science, Research and Sport of the Slovak Republic under the grant VEGA 1/0819/17.

\section{REFERENCES}

[1] Deng, S., LIpei, J., \& Long, X. (2008). Detecting linear features in weld seam images based on beamlet transform. 2008 9th International Conference on Signal Processing (s. 1145-1148). IEEE.

[2] Haffner, O. (2016). Contribution to modern methods. (in slovak). Ph.D. thesis: Slovak University of Technology in Bratislava.

[3] Haffner, O., \& Duchoň, F. (2014). Making a Map for Mobile Robot Using Laser Rangefinder. 23rd International Conference on Robotics in Alpe-Adria-Danube Region. Conference Proceedings. Bratislava: Publishing House of Slovak University of Technology.

[4] Haffner, O., Kučera, E., \& Kozák, Š. (2016). Weld Segmentation for Diagnostic and Evaluation Method. Levoča: 2016 Cybernetics \& Informatics (K\&I), IEEE. doi:10.1109/CYBERI.2016.7438605

[5] Hou, X., \& Liu, H. (2012). Welding Image Edge Detection and Identification Research Based on Canny. 2012 International Conference on Computer Science and Service System.

[6] Kagermann, H. (2013). Recommendations for implementing the strategic initiative INDUSTRIE 4.0. Dostupné na Internete: http://www.acatech.de/fileadmin/user upload/Baumstruktur nach W ebsite/Acatech/root/de/Material_fuer_Sonderseiten/Industrie_4.0/Fina 1_report_Industrie_4.0_accessible.pdf

[7] Liao, Z., \& Sun, J. (2013). Image segmentation in weld defect detection based on modified background subtraction. 2013 6th International Congress on Image and Signal Processing (CISP). IEEE.

[8] Marônek, M., \& Bárta, J. (2008). Multimediálny sprievodca technológiou zvárania (elektr. monografia). Trnava: AlumniPress.

[9] Mařík, V. (2015). National initiative Industry 4.0. Available on Internet:

http://download.mpo.cz/get/53723/62020/640376/priloha001.pdf

[10] Shen, Z., \& Sun, J. (2013). Welding seam defect detection for canisters based on computer vision. 2013 6th International Congress on Image and Signal Processing (CISP).

[11] Ulrich, K., Koleňák, R., \& Karvanská, S. (2006). Skúšanie zvarových spojov. Bratislava: STU v Bratislave.

[12] Xuming, Z., Zhouping, Y., \& Youlun, Y. (2007). Edge Detection of the Low Contrast Welded Joint Image Corrupted by Noise. 8th International Conference on Electronic Measurement and Instruments. Lab., Cambridge, MA Rep. ARCRL-66-234 (II), 1994, vol. 2. 


\title{
Data Mining with Trusted Knowledge
}

\author{
Viktor Nekvapil \\ University of Economics, Prague \\ nam. W. Churchilla 4 \\ 13067 Praha 3 \\ Email: viktor.nekvapil@vse.cz
}

\begin{abstract}
In this paper, a new concept of Trusted Knowledge (TK) is introduced. Trusted Knowledge are data from trusted organizations such as ministries, statistical offices and so on which can replace a domain expert in the evaluation phase of the data mining task. Two approaches to applying Trusted Knowledge are introduced. The first one called "Explanation system" offers additional information relevant to the resulting patterns which can help the user to better understand results of the task. The second one called "A/TKformulas" filters out the resulting patterns which are consequences of Trusted Knowledge and thus enables the user to concentrate on the interesting patterns. Conversely, the user can request to be shown only the resulting patterns which are consequences of TK to see which of them are in line with TK. Feasibility of the newly proposed framework is demonstrated in a case study.
\end{abstract}

\section{INTRODUCTION AND RELATED WORK}

A S STATED in the paper " 10 challenges of data mining research" [1], "there is a strong need to integrating data mining and knowledge inference". Although there have been some achievements since the paper has been published (see e.g. [2], [3]), data mining systems are still "unable to relate the results of mining to the real-world decisions they affect", as the authors claimed. Moreover, they stated that "Doing these inferences, and thus automating the whole data mining loop requires representing and using world knowledge within the system. One important application of the integration is to inject domain information and business knowledge into the knowledge discovery process".

The approach presented in my paper contributes to this challenge. It incorporates additional knowledge in the evaluation phase of data mining but avoids a lengthy and complex task of building a belief system of the user (see e.g. [4], [5], more recently in [6]). The idea is to enhance user's domain knowledge using available trusted sources of data that is, data from trusted organisations such as statistical offices, ministries and so on. I refer to this knowledge as trusted knowledge.

As domain experts are often not at disposal or are costly, the research aim is to replace to some extent the domain

The work described here has been supported by the internal grant agency of the University of Economics, Prague under project IGA 29/2016. expert with automate means - the system which will offer additional information to the user instead of the domain expert. Summarisingly, using knowledge and experience of himself/herself, the user evaluates whether the resulting pattern is interesting or not, or if he/she does not have enough knowledge in the particular domain, he/she uses the knowledge from the domain expert.

Further related work includes the approaches which use linked open data (LOD), which allow for publishing interlinked datasets employing machine interpretable semantics. For example, [7] developed an extension for Rapid Miner, which can extend a dataset with additional attributes drawn from the Linked open data cloud. Many approaches (e.g. [8], [9]) use DBPedia as a source of knowledge when evaluating resulting patterns; however, in my opinion, the nature of the data - it comes from community - is not trustworthy source. Reference [10] traverses Linked Data to find commonalities that form explanations for items of a cluster. However, the feature of LOD-based approaches is the fact that one has to map data to the ontology first; on contrary, the approach presented in this paper does not require lengthy setup.

The concept of Trusted Knowledge is inspired by FOFRADAR framework [3]. FOFRADAR is based on a logical calculus of association rules. The interpretation is based on mapping important items of knowledge to the sets of association rules which can be considered as their consequences. Important items of knowledge are expressed using a simple mutual influence among attributes. These are predefined relationships of attributes which are used to determine whether the association rule can be seen as a consequence of the item of knowledge or not. For example, the simple mutual influence (SI-formula) Income $\uparrow \uparrow$ Loan means: "if Income increases, then Loan increases as well". The set of atomic consequences of this SI-formula can be expressed by the following union: LowIncome $\times$ LowLoan $U$ MediumIncome $\times$ MediumLoan $\cup$ HighIncome $\times$ HighLoan, saying that "if Income is high, than Loan is high or if Income is medium then Loan is medium or if Income is high then Loan is high". Based on the levels in the union, it is possible to say whether the resulting rule is a consequence of the defined SI-formula or not. This feature is used in the 
proposed framework and further developed, as obvious in the following sections.

Theoretical concepts in this paper are demonstrated on a real data set from a financial institution. There are data concerning clients, who were given a loan, including geographical and demographical client data, data from the loan application, data concerning the agent who arranged the loan, and so on.

The rest of the paper is organised as follows. In section II, Trusted Knowledge is defined. Two possible ways of applying Trusted Knowledge are described in section III. In section IV, a case study is presented. In section V, conclusions and suggestions for the future work are included.

\section{TRUSTED KNOWLEDGE}

\section{A. Sources of Trusted Knowledge}

There are various data publicly available that can be used as Trusted Knowledge. Government institutions, EU institutions and statistical offices offer more and more data. This is boosted by the Open government data initiatives (see http://opengovernmentdata.org/), which offer a catalogue of publicly available data sets. In the Czech Republic, the Open data initiative (see http://www.opendata.cz/en) offers a catalogue of data using the linked data paradigm which refers to the Czech Republic. The data from those organisations are generally considered to be trusted sources.

I define Trusted Knowledge as follows: Trusted Knowledge (TK) is the data from trusted sources which can be connected to the results of a data mining task and are used in the evaluation phase of the data mining task to help with the understanding of the results. Trusted Knowledge can be seen as a special case of domain knowledge.

Trusted Knowledge is obtained from a trusted organisation. An example of such knowledge is the average and median income per district in the Czech Republic obtained from Czech Statistical Office [11].

\section{B. Items of Trusted Knowledge}

The following items of Trusted Knowledge are defined measures of TK, levels of measures of TK, explanations and a mutual influence of the attribute and measure of TK (A/TK-formula). The first two items are discussed in the following sections, the remaining items are described in section III.

\section{Measures of $T K$}

Measure of Trusted Knowledge (measure of TK) is a formalised piece of Trusted Knowledge. I formalise the measure of TK as follows:

a) Each measure has its name; b) is stored as per another dimension - in our case per geographical dimension; c) each value of dimension has its rank within the measure stored; d) each value of dimension has the absolute value of the measure stored.

An example of the measure of TK is depicted in Table III.
In Fig. 1, I outline the basic feature of the measure of TK its close connection to the results of a data mining task (resulting patterns). I use association rules as an example. Geographical dimension (locality) is used as a connecting element between the measure of TK and resulting patterns.

An average income in District $X$ as a measure of TK and The loan amount taken by a client in District $X$ as an attribute from analysed data can be examples of such a connection. If such a connection is done, it is assumed that the client is a member of the population which has an average income amounting to 20456, because he or she lives in the same district as the people whose income was collected by a trusted organisation (Czech Statistical Office in this case).

Of course, there are some challenges for this assumption, as for example, when the client lives in a particular region but works in a different one. Nonetheless, I believe that this situation is not occurring frequently and the given principle can be used in general.

To distinguish between data and Trusted Knowledge, I use the term attribute for the variables derived from the analysed data and the measure of TK for the variables used as Trusted Knowledge. Note that both the measure of TK and the attribute connected via a connecting element are ordinal.

\section{Levels of measures of TK}

The relationship depicted in Fig. 1 above does not bring much insight on its own. It is necessary to bring more context to the relationship. Levels of measures of TK enables to easily compare attributes and measures of TK. The way how domain experts evaluate the found patterns is commonly expressed by easily interpretable phrases saying for example "Income is low", "Amount is high" and so on. This simple approach is followed in FOFRADAR, as described in section I.

Recall the set of atomic consequences of SI-formula Income $\uparrow \uparrow$ Loan: LowIncome $\times$ LowLoan $\cup$ MediumIncome $\times$ $\times$ MediumLoan $\cup$ Highincome $\times$ HighLoan. Now we have to define what means, for example, "Income is low" (that is to define the level LowIncome).

\begin{tabular}{|c|c|c|}
\hline \multirow{2}{*}{$\begin{array}{c}\text { Trusted } \\
\text { knowledge }\end{array}$} & Connecting element & \multirow{2}{*}{$\begin{array}{c}\text { Resulting } \\
\text { pattern }\end{array}$} \\
\hline & & \\
\hline
\end{tabular}

\begin{tabular}{|c|c|}
$\begin{array}{c}\text { Measure } \\
\text { of TK }\end{array}$ & Locality \\
$\begin{array}{c}\text { Association } \\
\text { rule }\end{array}$ \\
\hline
\end{tabular}

\begin{tabular}{|c|c|c|}
\hline $\begin{array}{c}\text { Average } \\
\text { Income in } \\
\text { District X } \\
\text { (20456) }\end{array}$ & District X \\
\hline
\end{tabular}

Fig. 1: Relationship between measure of TK and resulting patterns different degrees of abstraction 
The task is to assign a set of values (called categories in FOFRADAR) $\alpha_{l}$ of a particular attribute $A$ to each level $\operatorname{Lev}(l)$. Levels have scales of various length - for example, if $1=3$, then levels $\operatorname{Lev}(1)=\{$ low, medium, high $\}$ or if $1=5$, then levels Lev(l) $=\{$ very low, low, medium, high, very high $\}$ and so on.

Now, I will present two approaches of defining levels, one of which is the newly proposed Rank-based approach.

Expert-based approach means that the domain expert decides which category is assigned to each level. For example, he/she can decide that Level=low for attribute Loan can be considered for the following categories: $<0 ; 100000),<100000 ; 150000)$. Formally written, LowLoan $=$ $\{<0 ; 100000),<100000 ; 150000)\}$. This approach is now used in the FOFRADAR framework and its feature is that it is necessary to define sets of categories with the help of the domain expert.

Rank-based approach is the newly proposed way of the automatic definition of levels. Categories of a particular attribute or measure of TK are sorted from the lowest to the highest. Then, we assign rank to each of the category according to the value of attribute or measure of TK. Last step comprises assigning Level( $l$ ) to each rank. For example, consider the categories of the attribute Loan_amount depicted in Table I. Based on the rankings of the categories, it is possible to assign respective categories to the levels.

This can be done by applying Assignment rules which state the principles of assigning categories to levels. Assignment rules are generic and not dependent on the analysed data. For example, assignment rules can look as depicted in Table II.

Table I: Levels for attribute Loan_amount

\begin{tabular}{|l|l|l|}
\hline \multicolumn{1}{|c|}{ Loan_amount category } & Rank & \multicolumn{1}{c|}{ Level } \\
\hline$<0 ; 100000)$ & 1 & Very low \\
\hline$<100000 ; 150000)$ & 2 & Very low \\
\hline$<150000 ; 200000)$ & 3 & Low \\
\hline$<200000 ; 270000)$ & 4 & Low \\
\hline$<270000 ; 300000)$ & 5 & Medium \\
\hline$<300000 ; 400000)$ & 6 & Medium \\
\hline$<400000 ; 500000)$ & 7 & High \\
\hline$<500000 ; 550000)$ & 8 & High \\
\hline$<550000 ; 650000)$ & 9 & Very high \\
\hline$<650000 ; 2600000>$ & 10 & Very high \\
\hline
\end{tabular}

Table II: Assignment rules

\begin{tabular}{|c|l|l|l|}
\hline$\#$ & $\begin{array}{c}\text { Number of } \\
\text { levels } \alpha\end{array}$ & $\begin{array}{c}\text { Number of } \\
\text { categories }\end{array}$ & Assignment rule \\
\hline 1 & 5 & 10 & 1 level per 2 categories \\
\hline 2 & 5 & 14 & $\begin{array}{l}\text { 1 level per 4 categories, (top and } \\
\text { bottom levels per 3 categories), } \\
\text { overlapping levels }\end{array}$ \\
\hline
\end{tabular}

Table III: Levels for measure of TK Income

\begin{tabular}{|l|l|l|l|}
\hline \multicolumn{1}{|c|}{ District } & Income & \multicolumn{1}{|c|}{$\begin{array}{c}\text { Income } \\
\text { rank }\end{array}$} & \multicolumn{1}{|c|}{ Level } \\
\hline Hlavni mesto Praha & 35115 & 1 & Very high \\
\hline Stredocesky kraj & 27345 & 2 & Very high \\
\hline Jihomoravsky kraj & 26116 & 3 & Very high/High \\
\hline Plzensky kraj & 26026 & 4 & High \\
\hline Moravskoslezsky kraj & 24877 & 5 & High \\
\hline Liberecky kraj & 24767 & 6 & High/Medium \\
\hline Kralovehradecky kraj & 24387 & 7 & Medium \\
\hline Ustecky kraj & 24336 & 8 & Medium \\
\hline Jihocesky kraj & 24321 & 9 & Medium/Low \\
\hline Kraj Vysocina & 24293 & 10 & Low \\
\hline Olomoucky kraj & 24175 & 11 & Low \\
\hline Pardubicky kraj & 24067 & 12 & Low/Very low \\
\hline Zlinsky kraj & 23873 & 13 & Very low \\
\hline Karlovarsky kraj & 22707 & 14 & Very low \\
\hline
\end{tabular}

First rule says that 2 consecutive categories are contained in 1 level. This assignment rule is applied in Table I. Second rule is example of overlapping levels, one category could be assigned to two levels. This behaviour is demonstrated in Table III on the measure of TK Income.

It is possible to prepare categories of attribute in such a way that it is easy to assign levels to each category. That means, if one desires 5 levels of an attribute, one creates 5 or 10 categories of the attribute, and similarly, this applies when 3 levels are considered, and so on.

Having the levels of attributes and measures of TK defined, we can compare levels and draw consequences based on values of the levels. This is further elaborated upon in section III.

\section{APPLYING TRUSTED KNOWLEDGE}

Here, I will introduce Trusted Knowledge Framework (TK Framework) - a framework which shows how TK is applied in the data mining process.

An important component of the framework is Trusted Knowledge Repository (TKR), a database where the items of $\mathrm{TK}$ are stored. Its feature is that it will be possible to share TKR among different projects in a similar way, as for example in [17]. The principles of sharing are left for the future work.

Now, I define two approaches of applying TK. The first approach is less demanding on the domain knowledge that has to be defined in advance but enables less automation in the evaluation phase. I call this approach Explanation system and elaborate upon this in section III.A. The second approach follows closely the FOFRADAR principles of automatic conclusions. It is called $A / T K$-formulas and is discussed in section III.B. 


\section{A. Explanation system}

Although the term explanation is broadly used in relation to expert systems (see e.g. [12]), I use it here in connection with a data mining system. Here, I perceive explanation as an item of TK that could help the user of data mining system to better understand results of a data mining task.

This is especially useful in situations when it is hard to obtain relevant knowledge from domain experts. In this case, a relevant explanation can be used as a support for the user and no knowledge from domain experts is needed.

Explanation is based on the measure of TK. As an example, I will mention the following explanation based on the measure of TK Income from Table III:

$$
\text { Zlinsky kraj => Income (very low), }
$$

meaning that in Zlinsky kraj district, Income is very low.

The TK Framework specified for the Explanation system is depicted in Fig. 2. As can be seen, after the results are obtained from the data mining system, TKR is queried for relevant explanations. If found, the relevant explanations are handed back to the user. Additionally, the user can request context of the explanation to better understand the explanation.

Now I will describe the experimental implementation of the proposed approach. It is a semi-automatic implementation based on LISp-Miner, SQLite and Python. The LISp-Miner System has been chosen for its ability to fine-tune the set of association rules which is mined; for more details see [13]. Further advantage is the possibility to automate the task through LMCL scripting language [14]. A simple database table is used as TKR. SQLite database is used for this purpose. Python is used as an engine that retrieves data from the TKR and presents it in the form of an explanation for the user.

I will continue using the following example. The $4 \mathrm{ft}-$ Miner procedure of the LISp-Miner is employed. I define the task so that attribute District is in the antecedent of the resulting rules, in consequent, Loan_amount is present.

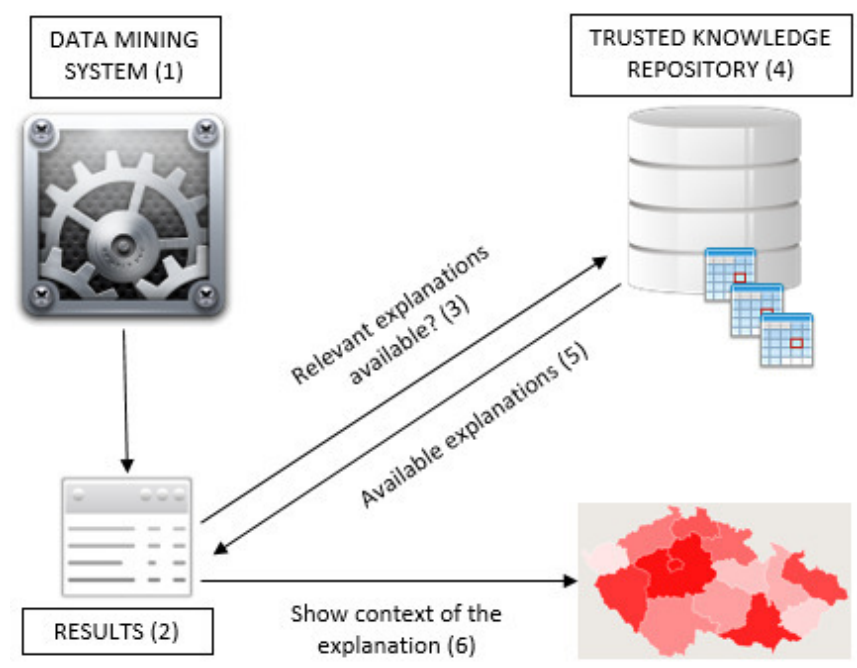

Fig. 2: TK framework of the Explanation system
In the TK Framework (see Fig. 2), this activity corresponds to number 1 (Data mining system and its usage). Three rules were found which are relevant to the task definition; see Fig. 3. This step corresponds to number 2 Results in the TK framework.

If we take, for example, rule (1), the user knows from the distribution of the attribute Loan (see Table I) that Loan $<100000 ; 150000$ ) is rather low (has the level very low). Are there some explanations for this fact? This question corresponds to the activity number 3 in the TK Framework. The TKR contains data from trusted organisation - Czech Statistical Office. The average income as presented in Table III above is contained in the TKR. Relevant explanations will be handed back to the user. The explanations which signal that a district presented in the resulting rule is outstanding (unusual) as regards the selected measure of TK, is considered to be relevant. The relevancy criterion in the experimental implementation is defined as the value of geo dimension having a very high or very low level according to the selected measure of TK. The example output of Explanation system for the first rule is presented below (see Fig. 4). First of all, basic information about the rule is summarised. Then, the explanations found are presented.

The user can conclude from the explanation that the average income in district 'Zlinsky kraj' is rather low (very low level). He/she can conclude that the clients' data he/she has at disposal in the data set are in line with his/her domain knowledge, because there is a direct proportion between the amount of loan and income (which is a part of the users' domain knowledge).

The reasoning used here is a combination of domain knowledge of the user and the measure of TK which brings the user to the conclusion that the rule is in line with his domain knowledge. The user knows that there is a direct proportion between the amount of loan and income, but does not know if the income in the district ('Zlinsky kraj') is rather low or high.

(1) District (Zlinsky kraj) -> Loan_amount <100000; 150000)

(2) District (Hlavni mesto Praha) -> Loan_amount <500000; 550000)

(3) District (Hlavni mesto Praha) -> Loan_amount <550000; 650000)

Fig. 3: Resulting rules

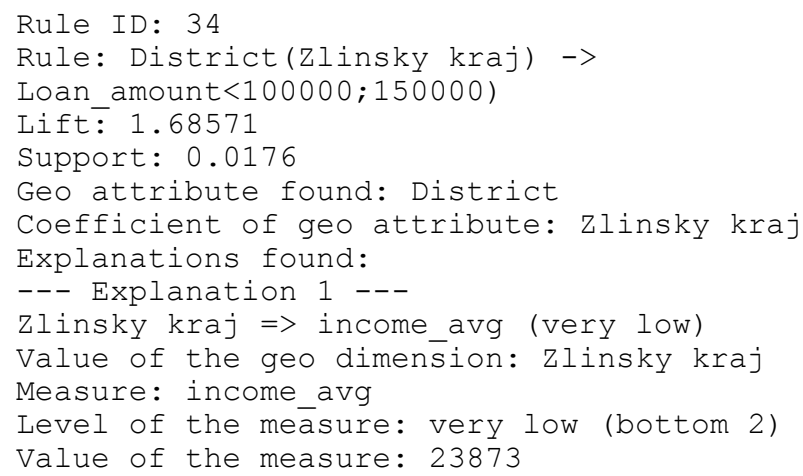

Fig. 4: Example output of the Explanation system 
This reasoning can be further automated as presented in section III.B. At this stage, the context of the explanation (as depicted in Fig. 2) can be obtained in a very simple manner. A table with districts, values of the measure, rank and level is retrieved. As a future work, a map with all the data mentioned above will be retrieved to enable to see values of neighbouring districts.

\section{B. Consequences of Trusted Knowledge (A/TK- formulas)}

One of the possible solutions of the automatic formulation of conclusions using domain knowledge is presented in the FOFRADAR framework, as described above. Using the measures of TK, it is possible to define mutual influence between an attribute and measure of TK. I call this mutual influence Attribute / Trusted Knowledge-formula (A/TKformula) and consider it an item of Trusted Knowledge. The principle of A/TK-formula is the same as in FOFRADAR, but instead of one of the attributes, the measure of TK is used in the mutual influence.

The TK Framework of A/TK-formulas is depicted in Fig. 5. After results are obtained, TKR is queried for A/TKformulas (3) which are available and relevant for the resulting patterns. The formulas are returned to (5) and their consequences are applied to the resulting patterns (6). Alternatively, the user can define a new A/TK-formula in advance using measures of TK that is available in TKR and then, consequences can be applied.

There are two ways how the consequences of $\mathrm{A} / \mathrm{TK}-$ formulas can be applied:

I. to obtain patterns which are consequences of A/TKformula - this way is useful when the user wants to know which resulting patterns are in line with the overall knowledge (trusted knowledge)

II. to filter out patterns which are consequences of

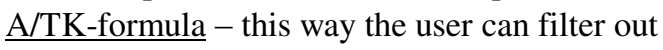
resulting patterns which are in line with trusted knowledge and concentrate on patterns which are not consequences of TK (they are either in contradiction to TK or have no TK available).

As an example, let us discuss the A/TK-formula Income $\uparrow \uparrow$ Loan. Income is a measure of TK. Using the rank-based approach, it is possible to assign values to respective levels as shown in Table III. The categories of the attribute Loan can be assigned to the levels, as depicted in Table I. Then the set of consequences of the A/TK-formula Income $\uparrow \uparrow$ Loan is defined by the following union:

Very low INCOME $\times$ Very low LOAN $_{U} \cup$ LOw $_{I N C O M E} \times$ Low $_{L O A N}$ $\cup$ Medium $_{I N C O M E} \times$ Medium $_{L O A N} \cup$ High $_{I N C O M E} \times$ High $_{L O A N} U$ Very high ${ }_{I N C O M E} \times$ Very high ${ }_{L O A N}$

Using the $4 \mathrm{ft}-$ Miner procedure, the following 8 rules are results of the task (see Fig. 6). An important feature of the $4 \mathrm{ft}-$ Miner procedure is that it is possible to define sequences of coefficients. In our example, Loan_amount was defined as a sequence of max. length 3 .

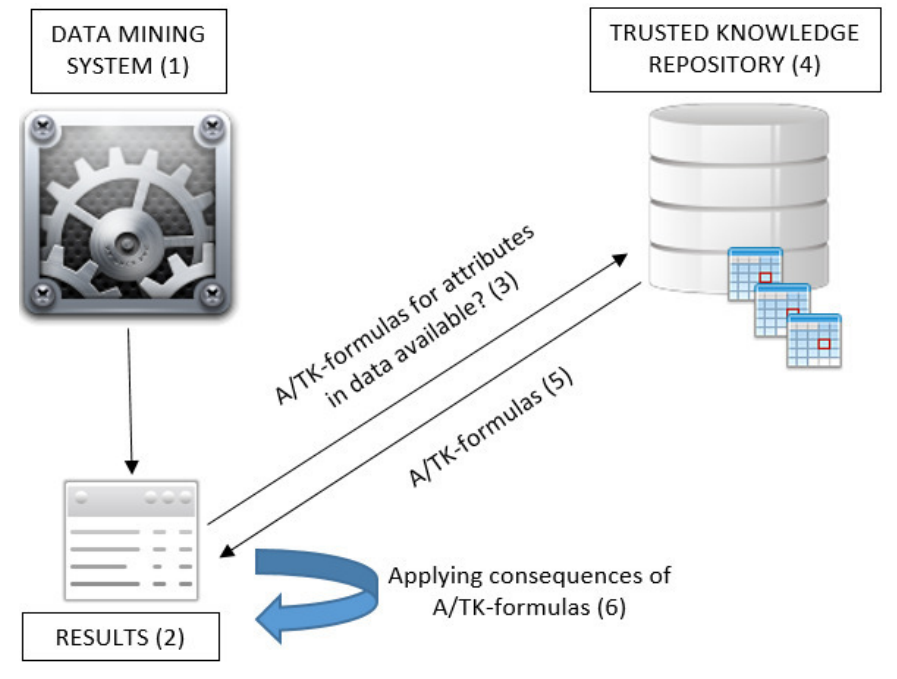

Fig. 5: TK framework of A/TK-formulas

This means that up to 3 consecutive categories can be chained together to increase the support of the rule; see [13] for details on sequences. This behaviour is performed onthe-fly when the $4 \mathrm{ft}$-Miner is running. Here, I do not state the measure of interestingness due to space reasons.

For rule (1), Loan has category <100000; 150000), so the assignment rule assigns the level 'very low' to the attribute. Now, the connecting element District is used to link the rule to the measures of TK., in our case, to the Income measure. The district value is Zlinsky kraj. If one looks at the level of the measure Income, it is very low according to the assignment rule in Table III. Note that in some rules, for example (4), the sequence option resulted in broader intervals. For example Loan_amount ( > = 500000) stands for the following categories: <500000; 550000), <550000; $650000),\langle 650000 ; 2600000\rangle$. It is possible to determine levels of the attribute District and the measure of TK Income for each rule, as can be seen in Table IV.

Then, we can apply consequences of the A/TK-formula Income $\uparrow \uparrow$ Loan. It is possible to filter out consequences of the formula, that is, rules 1 and 5 and reduce the number of displayed rules from 8 to 6 . Conversely, one can request all rules which are consequences of the A/TK-formula Income $\uparrow \uparrow$ Loan and display only rules 1 and 5. The idea is further elaborated upon in section 4 .

The novelty of the approach is that the A/TK-formula contains only one attribute of the data analysed, the second element is a measure of TK. Another important feature is that a connecting element is used to link up the measure of TK and the attribute in the resulting patterns.

(1) District (Zlinsky kraj) -> Loan_amount (<100000; 150000)

(2) District (Hlavni mesto Praha) -> Loan_amount (<500000; 550000)

(3) District (Hlavni mesto Praha) $->$ Loan_amount $(<500000 ; 650000)$

(4) District (Hlavni mesto Praha) - $>$ Loan_amount $(>=500000)$

(5) District (Zlinsky kraj) $->$ Loan_amount $(<150000)$

(6) District (Olomoucky kraj) -> Loan_amount (<270000; 400000)

(7) District (Zlinsky kraj) -> Loan_amount (<100000; 200000)

(8) District (Hlavni mesto Praha) -> Loan_amount (<400000; 550000) 
Table IV: Categories and corresponding levels for each rule

\begin{tabular}{|c|c|c|c|c|c|}
\hline \# & $\begin{array}{c}\text { Loan } \\
\text { category in } \\
\text { rule }\end{array}$ & $\begin{array}{l}\text { Loan } \\
\text { level }\end{array}$ & $\begin{array}{c}\text { Connecting } \\
\text { element } \\
\text { value }\end{array}$ & $\begin{array}{c}\text { Income } \\
\text { level }\end{array}$ & $\begin{array}{c}\text { Cons. of } \\
\text { Income } \uparrow \uparrow \\
\text { Loan }\end{array}$ \\
\hline 1 & $\begin{array}{l}<100000 ; \\
150000)\end{array}$ & Very low & Zlinsky kraj & Very low & Yes \\
\hline 2 & $\begin{array}{l}<500000 ; \\
550000)\end{array}$ & High & $\begin{array}{l}\text { Hlavni } \\
\text { mesto Praha }\end{array}$ & Very high & No \\
\hline 3 & $\begin{array}{l}<500000 ; \\
650000)\end{array}$ & $\begin{array}{l}\text { High } \\
\text { AND } \\
\text { Very high }\end{array}$ & $\begin{array}{l}\text { Hlavni } \\
\text { mesto Praha }\end{array}$ & Very high & No \\
\hline 4 & $\begin{array}{l}<500000 \text {; } \\
2600000)\end{array}$ & $\begin{array}{l}\text { High } \\
\text { AND } \\
\text { Very high }\end{array}$ & $\begin{array}{l}\text { Hlavni } \\
\text { mesto Praha }\end{array}$ & Very high & No \\
\hline 5 & $\begin{array}{l}<0 ; \\
150000)\end{array}$ & Very low & Zlinsky kraj & Very low & Yes \\
\hline 6 & $\begin{array}{l}<270000 ; \\
400000)\end{array}$ & Medium & $\begin{array}{l}\text { Olomoucky } \\
\text { kraj }\end{array}$ & Low & No \\
\hline 7 & $\begin{array}{l}<100000 ; \\
200000)\end{array}$ & $\begin{array}{l}\text { Very low } \\
\text { AND } \\
\text { Low }\end{array}$ & Zlinsky kraj & Very low & No \\
\hline 8 & $\begin{array}{l}<400000 \\
550000)\end{array}$ & High & $\begin{array}{l}\text { Hlavni } \\
\text { mesto Praha }\end{array}$ & Very high & No \\
\hline
\end{tabular}

\section{CASE STUDY}

The case study makes use of the same data that were used in the examples in the above sections. The goal of the case study is to show a more complex example of using the proposed framework. The combination of Explanation system and A/TK-formulas is shown as well as the relationship between the definition of categories of rational attributes (generally known as binning) and the assigning of levels to the categories of attributes.

After discussions with business experts, the task was specified as follows:

Are there any interesting combinations of client properties and indicators (including Loan_amount) on one side and locality on the other?

I am interested in filtering out rules which are consequences of A/TK-formulas contained in TKR. I also want to obtain explanations for the rules.

\section{A. Items of Trusted Knowledge in TKR}

In TKR, the following measures of TK are contained.

- Average income per district (see Table III)

- Average price of flat per square meter per district (see Table V) - source [15]

- Average amount of mortgage per district (Table VI) - source [16]

The following A/TK formulas are contained in TKR:

- Loan_amount $\uparrow \uparrow$ Price of flat - if the amount of a loan is high, then the price of a flat is also high

- Loan_amount $\uparrow \uparrow$ Income - If the amount of a loan increases, then the income increases as well.

The relevancy criterion for explanations is set to District 'very high' or 'very low'. The connecting element is the attribute / dimension District.
Table V: Levels for measure of TK Price of flat

\begin{tabular}{|l|l|l|l|}
\hline \multicolumn{1}{|c|}{ District } & $\begin{array}{c}\text { Price per } \\
\text { square } \\
\text { meter }\end{array}$ & Rank & \multicolumn{1}{c|}{ Level } \\
\hline Hlavni mesto Praha & 61500 & 1 & Very high \\
\hline Jihomoravsky kraj & 46800 & 2 & Very high \\
\hline Kralovehradecky kraj & 37100 & 3 & Very high/High \\
\hline$\ldots$ & $\ldots$ & $\ldots$ & $\ldots$ \\
\hline Karlovarsky kraj & 22100 & 12 & Low/Very low \\
\hline Moravskoslezsky kraj & 18400 & 13 & Very low \\
\hline Ustecky kraj & 10700 & 14 & Very low \\
\hline
\end{tabular}

Table VI: Levels for measure of TK Average amount of mortgage (mortgage_avg)

\begin{tabular}{|l|l|l|l|}
\hline \multicolumn{1}{|c|}{ District } & $\begin{array}{c}\text { Mortgage } \\
\text { amount (mil.) }\end{array}$ & Rank & \multicolumn{1}{c|}{ Level } \\
\hline Hlavni mesto Praha & 2.721 & 1 & Very high \\
\hline Jihomoravsky kraj & 1.933 & 2 & Very high \\
\hline Plzensky kraj & 1.806 & 3 & Very high/High \\
\hline$\ldots$ & $\ldots$ & $\ldots$ & $\ldots$ \\
\hline Zlinsky kraj & 1.59 & 12 & Low/Very low \\
\hline Kraj Vysocina & 1.542 & 13 & Very low \\
\hline Karlovarsky kraj & 1.467 & 14 & Very low \\
\hline
\end{tabular}

\section{B. Task definition in LISp-Miner}

The 'Clients' properties' group of attributes include the attributes Bonity, Collection, Age, Proposal_delivery and Sex. The 'Indicators' group of attributes include the attributes derived from the Loan_amount column in the analysed data. For the purpose of the case study, it is important to mention the type of the loan - it is a building savings loan. Note that in TKR, the mortgage loan amount is a measure of TK. Four variants of the Loan_amount are created to get the maximum chance to find interesting relationships. All four attributes are included in one class of the equivalence 'Loan'. It means that in one rule, only one of the four attributes can appear. The attributes differ in the number of categories which are created and the algorithm which is used to create them (equifrequent, equidistant). The Equifrequent option creates categories with the same number of objects (clients). The Equidistant option creates categories with the same length of intervals.

- Loan_ed5: Equidistant intervals, 5 categories, the class of equivalence 'Loan', levels - very high, high, medium, low, very low, for the respective category

- Loan_ef5: Equifrequent intervals, 5 categories, the class of equivalence 'Loan', levels - very high, high, medium, low, very low, for the respective category

- Loan_ef11: equifrequent intervals, 11 categories, levels (overlapping) as shown in Table VII, the class of equivalence 'Loan' 
- Loan_ed11: equidistant intervals, 11 categories, levels (overlapping) as shown in Table VII, the class of equivalence 'Loan'

Moreover, in the task definition, different coefficients are used. For the Loan_ed5 and Loan_ef5, a subset is used - that is, a usual attribute-value pair creation. For the Loan_ed11 and Loan_ef11, a sequence of minimal length 1 and maximal length 3 is used. The inclusion of the derived attributes in the 'Loan' class of equivalence ensures that only one attribute representing the column Loan from the data matrix will be present in the rule.

The task definition is as follows. In the antecedent, Client properties and the Indicators group of attributes is set. In the consequent, the attribute District is placed. A minimal support is set to 30 objects, minimal Lift=1.5.

\section{Results of the task}

After 3 seconds, 221 rules were found. To obtain less rules which are potentially interesting, it is possible to filter out the consequences of AT/K-formulas contained in TKR. Following the TK Framework of A/TK-formulas (see Fig. 5), TKR is queried to obtain relevant formulas. The Loan_amount attribute is present in the resulting rules and the connecting element District is present in both resulting rules and A/TK-formulas. This means that the A/TKformulas Loan_amount $\uparrow \uparrow$ Income and Loan_amount $\uparrow \uparrow$ Price of flat are relevant.

After filtering out the consequences of Loan_amount $\uparrow \uparrow$ Income, 100 rules remain. 121 rules are consequences of the Loan_amount $\uparrow \uparrow$ Income. 24 rules are consequences of the Loan_amount $\uparrow \uparrow$ Price of flat, 20 of them are also consequences of the Loan_amount $\uparrow \uparrow$ Income. This means that 4 additional rules are filtered out and 96 rules remain.

After the consequences of A/TK-formulas are filtered out, we query the TKR for explanations for the remaining 96 rules. 66 of them have a relevant explanation which is based on the measure of the TK Average amount of mortgage. It makes no sense to use explanations based on Income and Price of flat, because those measures of TK were already used in A/TK-formulas.

Table VII: Levels for Loan attributes with 11 categories

\begin{tabular}{|l|l|l|}
\hline $\begin{array}{c}\text { Loan category } \\
\text { (Loan_ef11, Loan_ed11) }\end{array}$ & Rank & \multicolumn{1}{c|}{ Levels } \\
\hline c1 & 1 & Very low \\
\hline c2 & 2 & Very low \\
\hline c3 & 3 & Very low / low \\
\hline$\ldots$ & $\ldots$ & $\ldots$ \\
\hline c9 & 9 & High / Very high \\
\hline c10 & 10 & Very high \\
\hline c11 & 11 & Very high \\
\hline
\end{tabular}

As for explanations, let us take following rule as an example: Agent(internal) \& Collection(No) \& Loan_ed11(ed_8..ed_10) $\Rightarrow$ (1.69) District(Hlavni mesto Praha)

The rule says that percentage of clients having internal agent, not having collections, having high loan and living in the district Hlavni mesto Praha is of 1.69 times higher than the percentage of clients living in the district Hlavni mesto Praha.

Found explanation for that rule is:

Hlavni mesto Praha $=>$ mortgage_avg(very high)

The explanation says that in the district Hlavni mesto Praha, average amount of mortgage is very high. This explanation could explain the high amount of the building savings loan (coefficient c8..c10, present in the resulting rule) because both loans (building savings loan and mortgage) are loans for housing purposes which behave very similarly. This knowledge (as stated in the last sentence) is a part of user's domain knowledge; the explanation supports and enhances user's domain knowledge.

Note that the framework of A/TK-formulas is not yet implemented. The features presented above were solved manually.

\section{CONCLUSIONS AND FUTURE WORK}

I have defined two approaches to applying Trusted Knowledge $-A / T K$-formulas and the Explanation system. As shown in section IV, using A/TK-formulas can significantly reduce the number of resulting patterns which are generated by the data mining system. This helps the user to concentrate on the rules which are interesting from the user's perspective (they are not the consequences of known Trusted Knowledge). Moreover, as introduced in section III.B, the explanations enhancing user's knowledge could help the user to better understand the results of the task.

Furthermore, I have defined a new way of assigning categories to the levels of attributes and measures of TK the rank-based approach based on assignment rules.

In Fig. 7, I summarise the three approaches introduced in the paper - SI-formulas, A/TK-formulas and the Explanation system. I distinguish three types of knowledge. Data knowledge is the knowledge obtained applying the data mining system on the data. Domain knowledge is the knowledge obtained from domain experts. Trusted Knowledge is the knowledge obtained from trusted organisations such as state ministries, statistical offices and so on. Based on this categorisation, it is possible to describe the three approaches mentioned above.

\section{A. FOFRADAR and SI-formulas}

Domain knowledge in the form of mutual influence of attributes from analysed data (SI-formulas) is used to draw consequences and obtain adjusted (filtered) results of the task. By now, the levels of each attribute were created in coordination with the domain expert. Additionally, a newly proposed rank-based approach was introduced to create 


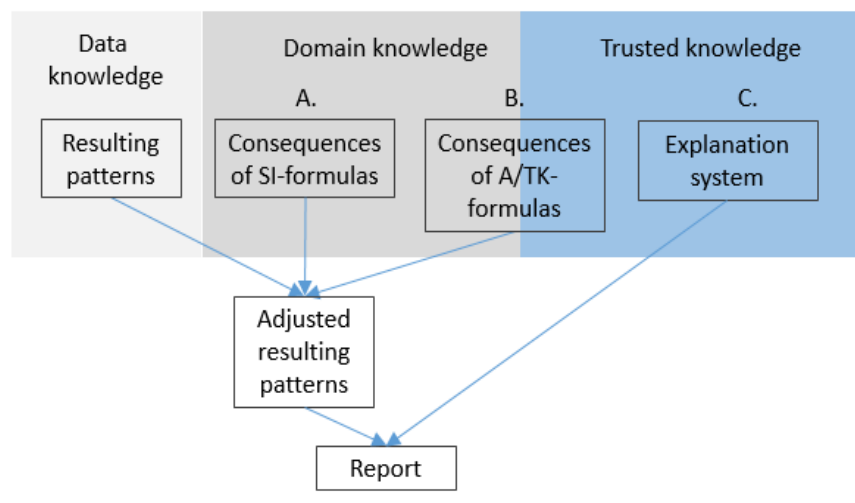

Fig. 7: Summary of used approaches

levels of attributes automatically in situations when the domain expert is not available.

\section{B. A/TK-formulas}

A/TK-formulas utilize the newly proposed concept of Trusted Knowledge in combination with the concept of mutual influence introduced in FOFRADAR. This combination of the domain knowledge and Trusted Knowledge is also highlighted in Fig. 7. In this case, it is a mutual influence between an attribute from analysed data and a measure of TK. The levels of the attribute derived from analysed data can be created either in coordination with the domain expert or with the use of the rank-based approach. The levels of the measure of TK are created using the rank-based approach. The consequences of the mutual influence are drawn automatically and again, adjusted results are obtained. A connecting element is used to connect the measure of TK and resulting patterns.

\section{Explanation system}

The third approach uses the concept of explanations. Trusted Knowledge in the form of the explanations which are relevant to the results of the task is offered to the user. The consequences of Trusted Knowledge are not drawn automatically. The reasoning is left to the user. A connecting element is used to connect an explanation and resulting patterns.

Implementation of $\mathrm{A} / \mathrm{TK}$-formulas is left for the future work. The framework also needs to be tested on more complex rules. To get even more benefits from the proposed framework, a sort of publically available sharing of TKR seems to be a next logical step, as for example in [17]. Moreover, a further automation of the task definition and drawing conclusions will be possible due to the defined LMCL scripting language. Another way how to elaborate upon the framework is to applicate it to the data mining with histograms [18].

\section{REFERENCES}

[1] Qiang, Y., Xindong, W., 2006. 10 Challenging Problems in Data Mining Research, International Journal of Information Technology \&
Decision Making, Vol. 5, No. 4, 2006, 597-604. DOI: $10.1142 / \mathrm{S} 0219622006002258$

[2] Mansingh, G., Osei-Bryson, K.-M., Reichgelt. H.: Using ontologies to facilitate post-processing of association rules by domain experts, Information Sciences, 181(3), 2011, 419-434. DOI: 10.1016/j.ins.2010.09.027

[3] Rauch, J., 2015. Formal Framework for Data Mining with Association Rules and Domain Knowledge - Overview of an Approach. Fundamenta Informaticae, 137 No 2, pp. 1-47. DOI: 10.3233/FI-2015-1175

[4] Silberschatz, A., Tuzhilin, A., 1995. On subjective measures of interestingness in knowledge discovery. In Proc. of the 1st ACM SIGKDD International Conference on Knowledge Discovery and Data Mining (KDD), pages 275-281, 1995. DOI: 10.1.1.88.146

[5] Padmanabhan, B., Tuzhilin, A., 1998. A belief-driven method for discovering unexpected patterns. In Proc. of the 4th ACM SIGKDD International Conference on Knowledge Discovery and Data Mining (KDD), pages 94-100, 1998. DOI: 10.1.1.28.728

[6] De Bie, T., 2013. Subjective interestingness in exploratory data mining. In Advances in Intelligent Data Analysis XII: 12th International Symposium, IDA 2013, London, UK, October 17-19, 2013. DOI: $10.1007 / 978-3-642-41398-8 \_3$

[7] Paulheim, H., Ristoski, P., Mitichkin, E., Bizer, C., 2014. Data Mining with Background Knowledge from the Web. RapidMiner World, At Boston, USA. August 2014

[8] Paulheim, H., 2012. Generating possible interpretations for statistics from linked open data, in: 9th Extended Semantic Web Conference, ESWC, 2012.

[9] Z. Huang, H. Chen, T. Yu, H. Sheng, Z. Luo, Y. Mao, 2009. Semantic text mining with linked data, in: INC, IMS and IDC, 2009. NCM'09. Fifth International Joint Conference on, 2009, pp. 338-343. DOI: $10.1109 / \mathrm{NCM} .2009 .131$

[10] Tiddi I., d'Aquin M., Motta E. 2014. Dedalo: Looking for Clusters Explanations in a Labyrinth of Linked Data. In: Presutti V., d'Amato C., Gandon F., d'Aquin M., Staab S., Tordai A. (eds) The Semantic Web: Trends and Challenges. ESWC 2014. Lecture Notes in Computer Science, vol 8465. Springer, pp. 333-348. DOI: 10.1007/978-3-319-07443-6_23

[11] Czech Statistical Office (CSO), 2015. Výsledky sčítání lidu, domů a bytů 2011 (Census 2011 _ in Czech) [online]. https://www.czso.cz/csu/czso/otevrena_data_pro_vysledky_scitani_li du_domu_a_bytu_2011_-sldb_2011- Last modified on 14 th April 2015.

[12] Buchanan, B. G., Smith, R. G., 1988. Fundamentals of expert systems. Annual review of computer science, 1988, 3.1: 23-58.

[13] Rauch, Jan. Observational Calculi and Association Rules [online]. 1. ed. Berlin : Springer-Verlag, 2013. ISBN 978-3-642-11736-7. Available at: http://link.springer.com/book/10.1007/978-3-64211737-4

[14] Šimůnek, Milan. 2014. LISp-Miner Control Language - description of scripting language implementation. Journal of Systems Integration [online], Vol 5, No 2 (2014), p. 28-44. ISSN 1804-2724. URL: http://www.si-journal.org/index.php/JSI/article/view/193 DOI: http://dx.doi.org/10.20470/jsi.v5i2.193

[15] Deloitte Real Index Q3 2016, (in Czech) [online]. Available at https://www2.deloitte.com/content/dam/

Deloitte/cz/Documents/realestate/Deloitte_Real_Index_Q3_2016_CZ.pdf

[16] Czech Ministry of Regional Development. Stav hypotečních úvěrů v krajích za leden až prosinec 2016 (in Czech). Available at http://www.mmr.cz/getmedia/a5bd12f0-2322-4037-80d4648163 c28e50/Stav-hypotecnich-uveru-v-krajich-za-leden-azprosinec-2016,-s-logem.pdf

[17] Vanschoren, J. 2012. The Experiment Database for Machine Learning (demo) [electronic document]. Workshop PlanLearn 2012. Available from http://datamining.liacs.nl/planlearnpapers/ planlearn2012_submission_7.pdf

[18] Rauch, Jan, Šimůnek, Milan. 2015. Data Mining with Histograms - A Case Study. In: Foundations of Intelligent Systems [online]. Lyon, 21.10.2015 - 23.10.2015. Cham : Springer International Publishing, 2015, s. 3-8. ISBN 978-3-319-25251-3. DOI: 10.1007/978-3-31925252-0. 


\title{
Decision Making in Building Maintenance Using a Graph-Based Knowledge Representation
}

\author{
Wojciech Palacz, Grażyna Ślusarczyk, Andrzej Łachwa, Barbara Strug, Anna Paszyńska, Ewa Grabska \\ The Faculty of Physics, Astronomy and Applied Computer Science \\ Jagiellonian University \\ ul. Łojasiewicza 11, 30-348 Kraków, Poland \\ Email: \{wojciech.palacz, grazyna.slusarczyk\}@uj.edu.pl
}

\begin{abstract}
This paper is an attempt to support effective decision making in building management by assisting maintenance processes. Knowledge about buildings is stored in a graph with many hierarchies. This representation allows us to express different types of hierarchical dependencies between building parts, like geometrical and functional ones, in one structure. Moreover, such a structure is useful to extract subgraphs containing information necessary for a given computational task, such as locating a desired place and the shortest path leading to it. As maintanance processes often require dynamic path target selection, modified indoor navigation methods are proposed. The paper presents the capability of the described knowledge model to cope with complex queries referring to different types of information. The considered examples show that the proposed approach can be used for various facility maintenance management applications.
\end{abstract}

\section{INTRODUCTION}

A COMPUTER-AIDED facility management system requires extensive information of multiple dimensions as it deals with problems of inspecting, repairing and substituting equipment in a cost-effective way [1]. As facility maintenance management directly affects the satisfaction of building users it should be able to identify problems and maintain the facilities in a time, cost and resource effective manner [2]. Simulation of equipment running conditions in a design phase of a building model can reveal the need of changes in facilities arrangement or even of the redesign of the equipment location spaces.

Good decisions for building maintenance require integration of various types of information and knowledge. Systems providing such an integration are actively researched [3]. Information about the geometrical and physical properties of a building is the most basic one. On top of this data, additional types of information can be added: which doors can be freely opened and which require a keycard, how rooms are numbered, which are used as office rooms and which as storage rooms, which employees are assigned to which rooms, etc. Some types of information may concern not the physical building, but be related to the building users, for example an organisational chart of the company which occupies the building, working hours for company departments, etc.

Having more types of information increases the breadth of knowledge available to a facility management system. This, in turn, makes the system more likely to be able to answer unusual and complex questions, which can enable innovative ways of managing the building. One of the problems of facility maintenance in modern buildings is finding information about equipment which can be turned off for some time to lower energy consumption. For example, in order to reduce the costs of building maintenance the current requirements for air conditioning of different building spaces should be considered.

Finding the useful information in a facility management system can greatly reduce costs of building maintenance. The effective maintenance of building facilities often needs navigation tools to locate a target place, like a storeroom of spare parts or a broken appliance, and the shortest path leading to it. It is also important to navigate the maintenance staff to equipment which should be operated manually by the shortest paths.

Navigation systems are, in general, concerned with determining the current location of their user, finding the optimal path to a target point, and then guiding the user along this path. Outdoor devices usually use satellite-based location services, but these services are unreliable inside buildings. Indoor systems use alternative approaches based on Wi-Fi [4], UWB, RFID [5], etc. Approaches based on matching images from smartphone cameras to the database of unique landmarks were also proposed [6]. In the last resort, the user can simply be asked to mark their current location on a map.

The optimal path is usually defined as the shortest one, and calculated by an appropriate graph algorithm. This implies that the system must have an alternative map in the form of a graph. This graph will usually have nodes representing doors and other important points in the building, and edges representing possible movements of a person walking from one point to another one [7].

The guidance part can be limited to showing a map with a drawn path, or can be provided as a list of commands ("go straight 100 meters, then turn right and walk another 50 meters"). Humans do not have an innate understanding of numeric distances and angles, therefore instructions using landmarks ("walk up the stairs, then turn towards the coffee machine") are easier to follow [6]. In the extreme case, when the navigation device does not use any location service and cannot update its own position in real-time, the guidance instructions may simply be a sequence of photos showing landmarks the user should walk by on their way from the starting to the target point [8].

Navigation strategy proposed in this paper is based on 
knowledge about the building layout stored in the hierarchical graph structure. Its multiple hierarchies allow for building models which represent not only the spatial hierarchy of rooms and floors, but also other functional and administrative dependencies. This additional knowledge stored in the graph can be used in the target selection phase.

Currently available navigation tools require users to specify a single point as a target, either by directly marking it on a map or by providing a description which can be translated by the device to point coordinates ("the room with the number 303", "the coffee machine closest to my current position"). The system based on the graph model proposed in this paper will be able to find targets described in a parametric way or specified by selected attributes (for example, the type of a scientific equipment shop). The hierarchy grouping graph nodes into larger entities makes it possible to accept requests for "the closest laboratory belonging to the Department of Organic Chemistry". What is even more important, specifying targets which resolve to a set of points will also be possible: "all office rooms belonging to the Department of Games" will make the navigation device guide its user from one room to the other.

The hierarchical nature of the graph model can also be useful for path searching algorithms. It provides the possibility of filtering the graph by hierarchy type and level, eliminating knowledge not related to the task of finding a path between two points in the chosen fragment of the building. The number of data to be analyzed is greatly reduced, thus speeding up the search.

\section{RELATED WORKS}

Building maintenance can be seen as the combination of administrative and technical actions to ensure the items of the building in an acceptable standard to perform its required functions [9]. The main objectives of building maintenance are: extending the useful life of the buildings, providing save and efficient working or living environment and maximization of economic and aesthetic values of the buildings. Building maintenance can be seen as an activity in facilities management [10] and considered as the part of the construction sector [11].

Building management requires taking many important decisions regarding the way of inspecting, maintaining, renewing or replacing existing facilities [1]. To support decision making at different stages of a building lifecycle the constant acquisition and analysis of data is needed [12]. Limited access to accureate data, knowledge and integrated of multiple domains makes it difficult to create user-friendly interfaces for decision support in operation and maintanance of building facilities [13].

Good decisions for building maintenance require integration of various types of information and knowledge. Current BIM applications are not able to fully exploit the capabilities of the knowledge systems. In [14] and [15] Radio Frequency Identification (RFID) is proposed to collect and share maintenance data with the most minimal manual data entry. The method is used in an integrated system proposed in [3] which captures both, information and knowledge. The system developed in [3] concerns rather capturing information and knowledge, while the efficiency of the system is improved by the automation of data capturing by means of technologies such as RFID. The system helps maintenance teams learn from previous experience and trace the history of a building elements on the base of Case-Based Reasoning system (CBR).

Majority of the current building maintenance systems mainly focus on capturing either information or knowledge. On the other hand many currently available building maintenance system capturing information and knowledge are concentrated on tracing the history of the building and learning from previous experience.

In our approach, we focus on another aspect of the effective maintenance of building facilities-navigation to locate a target place, like a storeroom of spare parts or a broken appliance, and the shortest path leading to it as well as finding information about equipment which can be turned off for some time to lower energy consumption. To allow for effective navigation and finding information about equipment, the hierarchical graphs with multiple hierarchies of various types are used as a representation of building models.

Graph structures are commonly used to model relations in many systems [16], [17]. They can be used in design systems to model topological relations between components, in databases to model logical relations between objects as well as to model data and control flow or specification and analysis of software systems. In many real applications, modelling systems by plain graphs (graphs where edges and nodes are sets without additional structure) is insufficient. The complex systems are very often hierarchical, thus graphs with hierarchy should be used as the models [18]-[20]. Graphs of this kind allow not only for modelling hierarchical relations between components of the system but also for hiding some details of "encapsulated" subgraphs. Hierarchical graphs used in conjunction with graph transformation rules can be used to manage changes in the state of big and complex systems like computing grids [21].

During the last years several models based on graphs with hierarchy have been defined [22]-[33]. They were used for modelling global computing systems, syntactical structure of agents, mobile systems, design systems, granular computing, etc. It turns out that existing hierarchical graphs are not sufficient to efficiently model objects considered in architectural design and engineering problems. The good representation should allow for expressing multi-argument relations between parts of different components, hierarchical dependencies between different parts of design, as well as many hierarchies of different types, where not all components must belong to the hierarchy. None of the mentioned models allows for modelling of all required features.

Therefore in this paper hierarchical graphs with multiple hierarchies of various types are proposed as representation of building models. The considered graphs are composed of nodes, which represent object components, and edges repre- 
senting relations between them. In given graph nodes other nodes and edges can be nested. Graph nodes with nested atoms are called hierarchical. Hierarchical nodes may be used to hide certain details of a designed object that are not needed in a given moment or to group objects having common features (geometrical or functional). Graph hierarchies enable us to group elements according to different objectives and consider the project at a chosen level of detail. Hierarchical graphs encode the knowledge about created designs and therefore enable to efficiently reason about the conformity of designs with specified design criteria [34].

In this paper, a multi-hierarchical graph model is used to search for possible routes in a given building. The most common graph algorithm for route calculation is the Dijkstra's algorithm. Other graph algorithms like A*, Floyd-Warshall or Bellman-Ford [35] are also used to determine the shortest path from a source to a destination. In [36] a reduced visibility graph is used to reflect possible route choices and the FastestPath Algorithm finds the fastest path for travelers [37]. In [38] metrical-topological models constructed from labelled floor plan geometry are used to describe the shape and connectivity of space, and constitute useful underlying tools for wayfinding. However, for almost all current navigation applications complex building interiors with large open spaces and vertical communication means present challenges.

\section{THE GRAPH-BASED REPRESENTATION OF BUILDING-RELATED KNOWLEDGE}

In this section the extended graph-based data structures, which better reflect the intrinsic nature of design objects, are described. They enable to express geometrical properties of objects together with their other attributes and different types of relations between object components.

Flat graphs are often used to model internal structures of objects. Nodes represent components of an object, and relations between them are represented by edges. These simple models are no longer convenient when components themselves need to be decomposed into sub-components without losing their identity. In such a case, the model has to contain nodes representing both the component and its parts.

Hierarchical graphs can represent the connection between the whole and its parts in a straightforward way, because nodes and edges can be nested inside other nodes. A graph node with nested atoms is called hierarchical and represents a component as a whole, while the children atoms represent this component's internal structure. Graphs considered in this paper also allow for expressing relationships between components located on different hierarchy levels, i.e., edges can connect nodes nested in different parents.

The considered graphs are multi-hierarchical, i.e., there is a possibility to define hierarchies of different types in one graph. Many hierarchies defined in one graph allow us to specify different types of hierarchical dependencies between graph elements. Such a representation makes it possible to filter the knowledge stored in the graph by selecting specific hierarchies. Moreover, there is a choice of different views of the project consisting of selected hierarchies including only relevant subsets of graph atoms.

The proposed multi-hierarchical graphs can represent a complex object at different levels of detail and different stages of the building lifecycle. They allow for hiding unnecessary at the moment low-level data and presenting only an outline of the object or showing a detailed view of the whole object (or of its part). The considered multi-hierarchical graphs are an extension of labelled and attributed graphs. Nodes and edges are labelled by names of components and relations, respectively. Semantic aspects of components and relations are encoded in sets of attributes assigned to nodes and edges.

Use of multiple graph hierarchies, instead of a single hierarchy, allows for additional knowledge to be encoded in the graph structure. Each hierarchy can correspond to different element grouping criteria. Reasoning about designs [34] can be more efficient thanks to the possibility of selecting both the hierarchy type, level, and the appropriate components of the object.

Let us introduce a formal description of the proposed multihierarchical graph model.

Let $\Sigma_{V}$ and $\Sigma_{E}$ be two disjoint alphabets of node and edge labels, respectively. Let $A$ be a nonempty, finite set of node and edge attributes. Let $D_{a}$ denote a fixed, nonempty set of admissible values for attribute $a \in A$, called the domain of $a$.

Let the set of nodes and edges of a graph $G$, known together as graph atoms, be denoted by $U$, i.e., $U=V \cup E$. It can be assumed without loss of generality that $\Sigma_{V}, \Sigma_{E}, A, V$ and $E$ are pairwise disjoint.

Some graph atoms may be completely outside the hierarchy, in the sense that concepts like "set of children" and "parent" are not applicable to them at all. Let $\perp$ be a fixed value, different from all graph atoms, which will be used to denote this case in the following.

Definition 1: A mapping ch : $V \rightarrow 2^{U} \cup\{\perp\}$ is a child nesting function if and only if:

- $\forall v, w \in V: w \in \operatorname{ch}(v) \Rightarrow \operatorname{ch}(w) \neq \perp$, i.e. if a node is a child of some other node, then it is a part of the hierarchy and thus must have its own set of children (which may be empty),

- $\forall x \in U \forall v, u \in V: x \in \operatorname{ch}(v) \wedge x \in \operatorname{ch}(u) \Rightarrow v=u$, i.e., an element cannot have two distinct parents,

- $\forall v \in V: v \notin c h^{+}(v)$, i.e., no element can be its own descendant.

For node $v \in V, \operatorname{ch}(v)$ is known as a set of its children, $c h^{+}(v)$ as a set of its descendants, and $c h^{*}(v)$ denotes a set containing both node $v$ and its descendants.

Definition 2: A multi-hierarchical graph over $\Sigma_{V}, \Sigma_{E}$ and $A$ with $n$ hierarchies (where $n$ is a fixed natural number) is a system $G=\left(V, E, s, t, c h_{1}, \ldots, c h_{n}, v l b, e l b, a t r, v a l\right)$, where:

- $V$ and $E$ are disjoint finite sets, with $V$ being non-empty, whose elements are called nodes and edges, respectively,

- $s: E \rightarrow V$ and $t: E \rightarrow V$ are mappings assigning to edges their source and target nodes, respectively, 


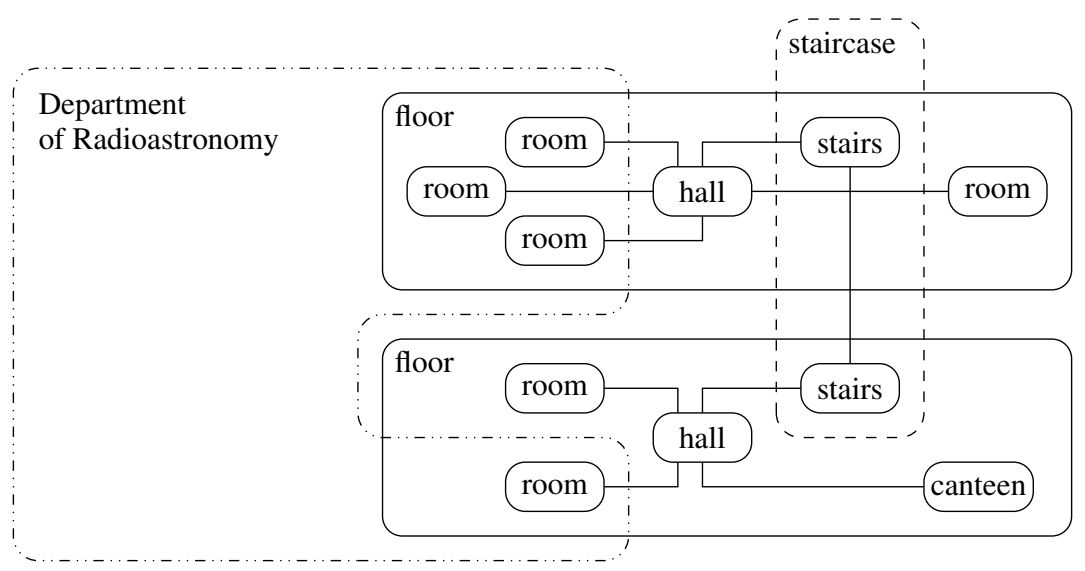

Fig. 1. An example graph model with three hierarchies

- $c h_{i}: V \rightarrow 2^{U} \cup\{\perp\}$, where $i=1, \ldots, n$ are child nesting functions,

- $v l b: V \rightarrow \Sigma_{V}$ and elb $: E \rightarrow \Sigma_{E}$ are node and edge labelling functions, respectively,

- atr : $U \rightarrow 2^{A}$ is a node and edge attributing function,

- val : $U \times A \rightarrow D$, with $D=\bigcup_{a \in A} D_{a}$, is a partial function such that for all $x \in U$ and $a \in A$ if $a \in \operatorname{atr}(x)$, then $\operatorname{val}(x, a) \in D_{a}$.

In Fig. 1 an example of a graph with three different hierarchies is presented. In the first hierarchy, nodes representing rooms and other spaces are nested in hierarchical nodes representing floors. Edges which connect nodes represent accessibility. All graph elements belonging to this hierarchy are drawn as solid ovals and lines.

The second hierarchy is used to group stair segments into staircases. While stair runs and their landings are contained in their specific floors, staircases considered as a whole do not belong to any floor. Therefore, the node in Fig. 1 which represents a staircase is not a part of the first hierarchy, and is drawn with a dashed line to underline this fact.

The third hierarchy stores knowledge about administrative room assignments. In this example there are four rooms assigned to the Department of Radioastronomy. Nodes representing these rooms are nested in the node representing the department.

It should be noted that a graph atom can belong to several hierarchies at the same time. For example, nodes representing stair segments are a part of the spatial and of the vertical spatial hierarchy; they are not a part of the administrative one.

Notions of a subgraph of a multi-hierarchical graph, an induced subgraph and a single view are useful tools for filtering the knowledge stored in a graph model.

Parts of an object represented by a multi-hierarchical graph correspond to its subgraphs.

Definition 3: A graph $G=\left(V_{G}, E_{G}, s_{G}, t_{G}, c h_{1 G}, \ldots\right.$, $\left.c h_{n}, v \operatorname{vlb}_{G}, e l b_{G}, a t r_{G}, v a l_{G}\right)$ is a subgraph of a graph $H=\left(V_{H}, E_{H}, s_{H}, t_{H}, c h_{1 H}, \ldots, c h_{n H}, v l b_{H}, e l b_{H}, a t r_{H}\right.$, $\left.v a l_{H}\right)$ if:

- $V_{G} \subseteq V_{H}$;
- $E_{G} \subseteq E_{H}$ and $\forall e \in E_{G}: s_{H}(e) \in V_{G} \wedge t_{H}(e) \in V_{G}$;

- $s_{G}=\left.s_{H}\right|_{E_{G}}, t_{G}=\left.t_{H}\right|_{E_{G}}$;

- $\forall x \in V_{G}: \operatorname{ch}_{i}(x)=\operatorname{ch}_{i H}(x) \cap\left(U_{G} \cup\{\perp\}\right)$ where $i=1, \ldots, n$,

- $v l b_{G}=\left.v l b_{H}\right|_{V_{G}}, e l b_{G}=\left.e l b_{H}\right|_{E_{G}}$;

- $\forall x \in U_{G}: \operatorname{atr}_{G}(x) \subseteq \operatorname{atr}_{H}(x)$,

- $\forall x \in U_{G}, a \in A: \operatorname{val}_{G}(x, a)=\operatorname{val}_{H}(x, a)$.

A subgraph in which all edges connecting selected nodes of a given graph are present is called an induced subgraph.

Definition 4: A graph $\boldsymbol{I n d}\left(\boldsymbol{V}_{\boldsymbol{I}}\right)=\left(V_{I}, E_{I}, s_{I}, t_{I}, c h_{1 I}\right.$, $\left.\ldots, c h_{n I}, v l b_{I}, e l b_{I}, a t r_{I}, v a l_{I}\right)$ is an induced subgraph of an graph $H=\left(V_{H}, E_{H}, s_{H}, t_{H}, c h_{1 H}, \ldots, c h_{n H}, \operatorname{atr}_{H}\right.$, val $\left._{H}\right)$ if $\operatorname{Ind}\left(V_{I}\right)$ is a subgraph of $H$, where $E_{I}=\left\{e \in E_{H}\right.$ : $\left.s_{H}(e) \in V_{I} \wedge t_{H}(e) \in V_{I}\right\}$.

Searching for specific information about the design, the designer often needs to take into consideration only one graph hierarchy. A subgraph which contains all nodes of the selected hierarchy and edges representing relations between them is called a single view.

Definition 5: Let $H=\left(V_{H}, E_{H}, s_{H}, t_{H}, c h_{1 H}, \ldots, c h_{n H}\right.$, $\left.v l b_{H}, e l b_{H}, a t r_{H}, v a l_{H}\right)$ be a graph and let $i$ be a fixed number $1 \leq i \leq n$. A graph $\boldsymbol{S}\left(\boldsymbol{c h}_{\boldsymbol{i}}\right)=\left(V_{S}, E_{S}, s_{S}, t_{S}\right.$, $\left.c h_{i S}, v l b_{S}, e l b_{S}, a t r_{S}, v a l_{S}\right)$ is a single view determined by the hierarchy $c h_{i H}$ if $S\left(c h_{i}\right)$ is a fragment of an induced subgraph of $H$ where $V_{S}=c h_{i H}^{*}\left(V_{H}\right) \cap V_{H}$ and $c h_{j}$ for $j \neq i$ are omitted.

\section{CASE Study}

A university building is used as an example in this section. Fig. 2 displays a part of its floor plan, which includes several rooms belonging to two departments, a student canteen and a staircase. A multi-hierarchical graph which models this building has four hierarchies: spatial, vertical spatial, functional and administrative. A graph with many hierarchies is difficult to arrange on a diagram in a clear way, therefore the following figures show different views of this graph.

The first hierarchy, which groups together nodes representing rooms belonging to the same floor and places them inside a node representing this floor, is the most obvious one. It also 


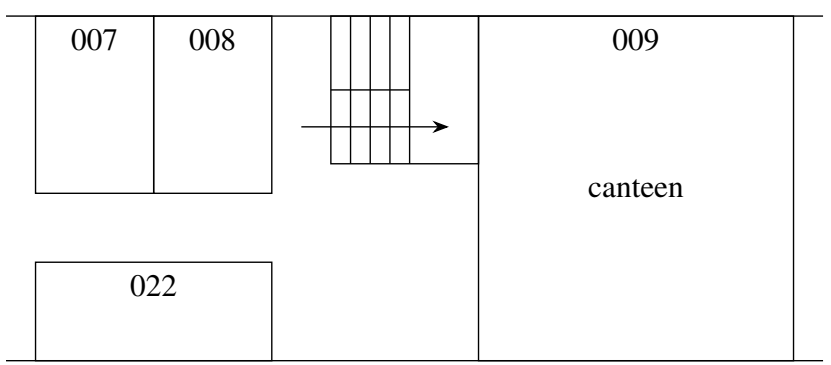

ground floor

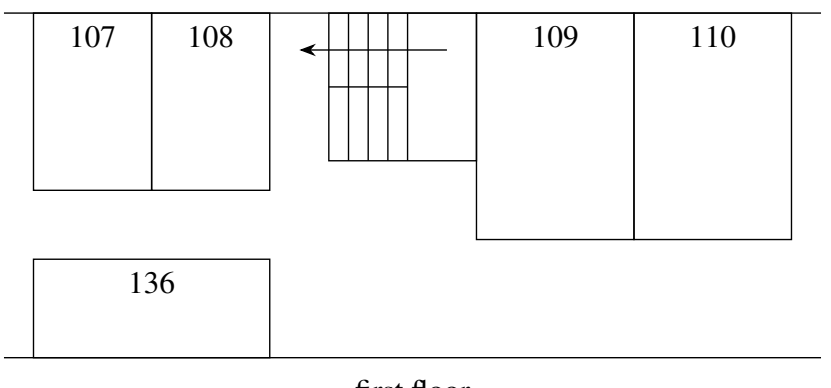

first floor

Fig. 2. A fragment of a university building floor plan

provides nodes representing entry/exit points which are used to move between adjacent spaces in the building. Most of them represent doors, some represent stair landings. Edges connecting these points denote accessibility and are attributed with numbers representing costs of traversing the edges (i.e., the time required to walk from one point to the other). A single view showing this spatial hierarchy is displayed in Fig. 3.

The second hierarchy is also based on physical containment, but it groups spaces in the vertical direction. It is named "vertical spatial" and provides hierarchical nodes representing staircases and lifts-that is, objects which cross floor boundaries.

The third hierarchy (see Fig. 4) divides the building into functional areas. All corridors and stairs are assigned as children of the "communication" node, personal rooms of university employees as children of the "staff offices" node, canteens and other places where food can be bought are assigned to the "gastronomy" node, and so on.

The fourth hierarchy (see Fig. 5) provides nodes corresponding to the administrative structure of a university (chairs, departments, etc.) and places rooms inside these nodes.

Usually, floor layouts like the one displayed in Fig. 2 show only the geometry of the building and room labels. A multi-hierarchical graph model is able to store additional information. This extra knowledge can be used to support specific facility management tasks.

\section{A. Case 1: HVAC system}

Modern buildings usually have HVAC (heating, ventilation, and air conditioning) systems which are centrally managed by a BMS (building management system). To reduce the energy consumption, the BMS often switches off the HVAC systems at the end of a day and turns them back on the next morning.

In a university building there are additional chances for saving energy, offered by irregular events. A special event can be accompanied by a university-wide cancellation of classes, which means that nobody will use lecture halls and student laboratories. On a smaller scale, a conference attended by a department can mean that the staff from that department will be absent for several days, and $\mathrm{AC}$ in their office rooms can be turned off for that period.

To take advantage of such events, a manual HVAC schedule adjustment must be made at a BMS computer terminal. Mak- ing adjustments room by room is time-consuming and errorprone. The BMS can make this procedure easier by letting the operator specify a group of rooms and then apply the adjustment to the whole group in a single step. The procedure gets even easier if the group can be automatically defined.

Integrating the BMS and the multi-hierarchical graph model makes it possible. The set of graph nodes representing office rooms belonging to a considered department is calculated on the basis of knowledge stored in the administrative (see Fig. 5) and functional (see Fig. 4) hierarchies. Alg. 1 shows the algorithm which implements this calculation. For example, when given the name "Department of Games" as an argument, function OfficeRooms returns a set of nodes representing rooms number 007 and 008 . Returned graph nodes are mapped to objects representing these rooms in the BMS.

\section{B. Case 2: navigation support}

Let us consider a case of guiding a maintenance technician to a correct shop. The technician was called in to check a non-working piece of scientific equipment, and he determined that a repair is possible but requires a spare part and/or use of specific tools. The required items can be obtained from workers in an equipment shop. The problem is, the building contains several such shops, and going to a wrong one is a waste of time. The shops are assigned to departments, and

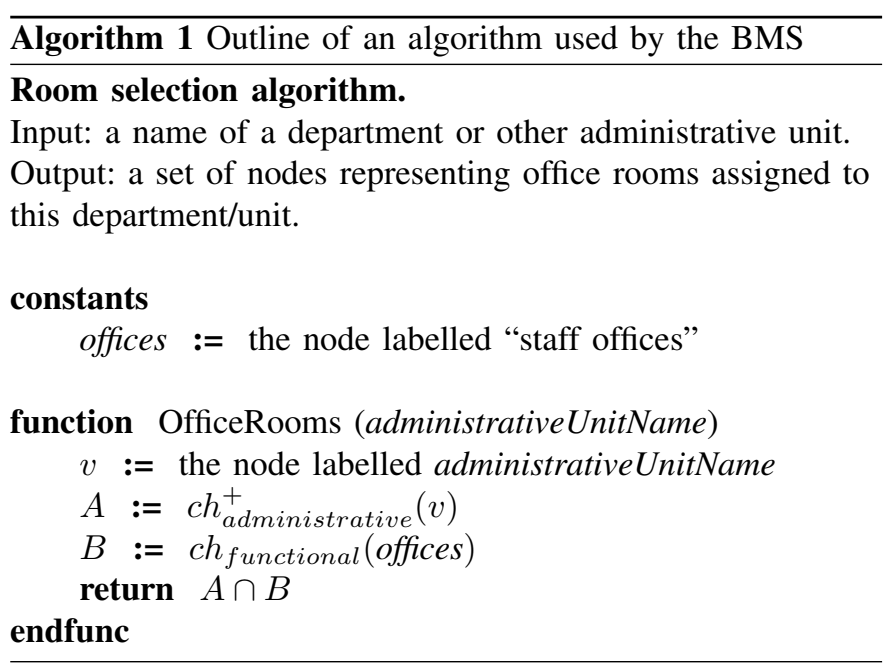




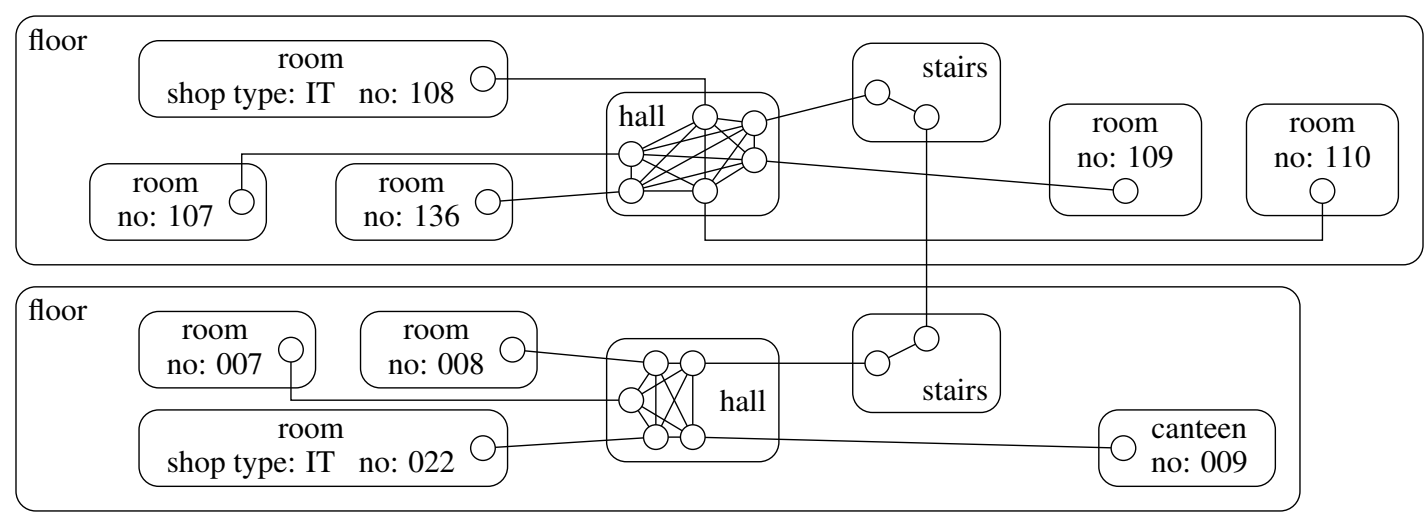

Fig. 3. A view showing the spatial hierarchy of the building model (edge costs omitted)
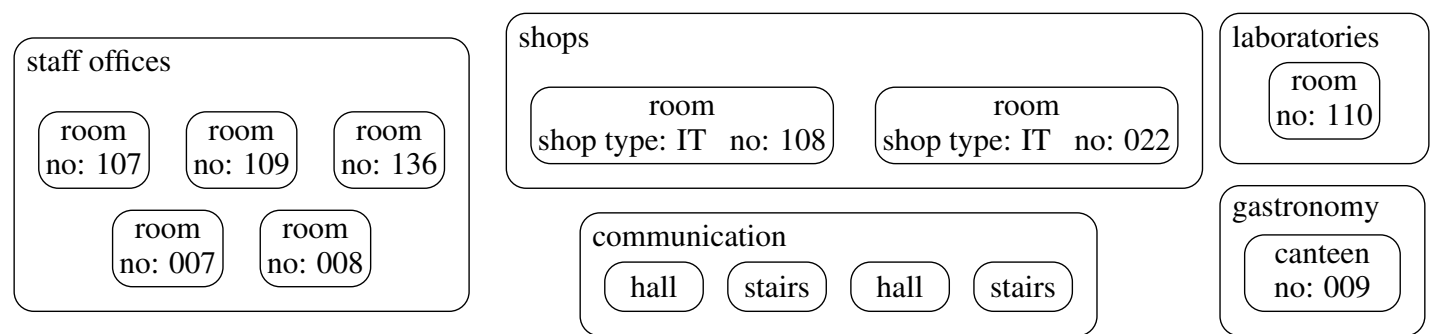

Fig. 4. A view showing the functional hierarchy of the building model
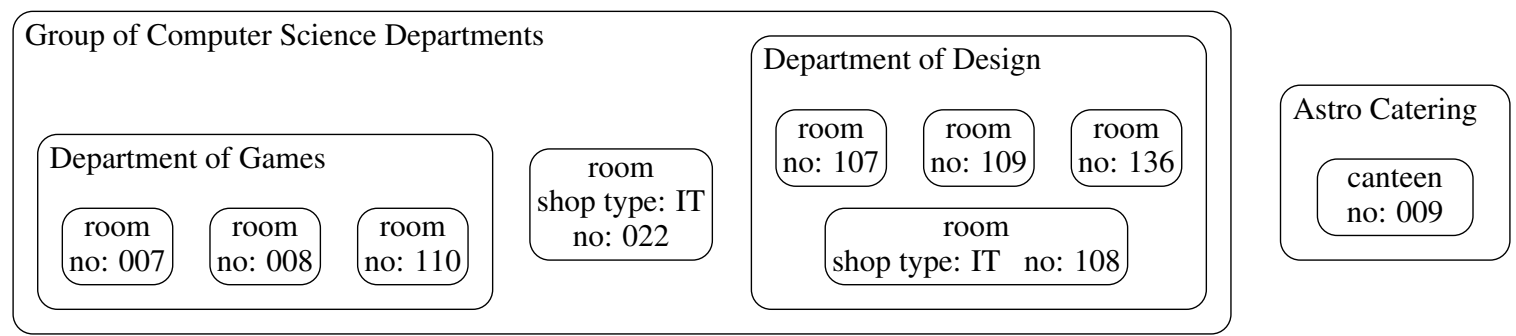

Fig. 5. A view showing the administrative hierarchy of the building model

because of university accounting rules, shop workers may not expend resources to fix equipment belonging to an unrelated department.

A navigation application with a dynamic target selection algorithm can help the technician to get to the correct shop. Pseudocode in Alg. 2 shows how to implement this guidance algorithm. We assume that the technician starts in the room with a broken piece of equipment. The SearchForShop procedure uses this assumption to determine which department this equipment belongs to. It can then take the set of nodes contained in the node representing this department (administrative hierarchy) and intersect it with the set of nodes nested in the "shops" node (functional hierarchy). This determines the room-or, if the department has more than one shop assigned, the set of rooms- to check in the first search phase.

Then, the application should direct the technician to the nearest room from this set. This is implemented in the TryNodes procedure. The code finds the shortest path from the node representing the room the technician is currently in to any of the nodes representing rooms to check (or rather, from a node representing an entry/exit point in the current room to any node representing a point in the destination rooms). To speed up the search the Dijkstra's algorithm is applied not to the complete graph model, but to its subgraph. Specifically, to a spatial hierarchy subgraph induced by start and destination nodes as well as by nodes which in the functional hierarchy are nested in the "communication" node.

If, when arriving at the destination point, the technician finds the shop closed or the replacement part unavailable, then they command the navigation application to direct them to the next shop. The application removes the node representing the visited shop from the set of destination nodes and repeats the procedure described in the previous paragraph.

If required parts can not be obtained from any shop in the department, the application checks the administrative hierarchy to find the parent unit of the department, and considers shops assigned directly to that unit. If necessary, it then proceeds to the grandparent unit, the great-grandparent unit, etc. 


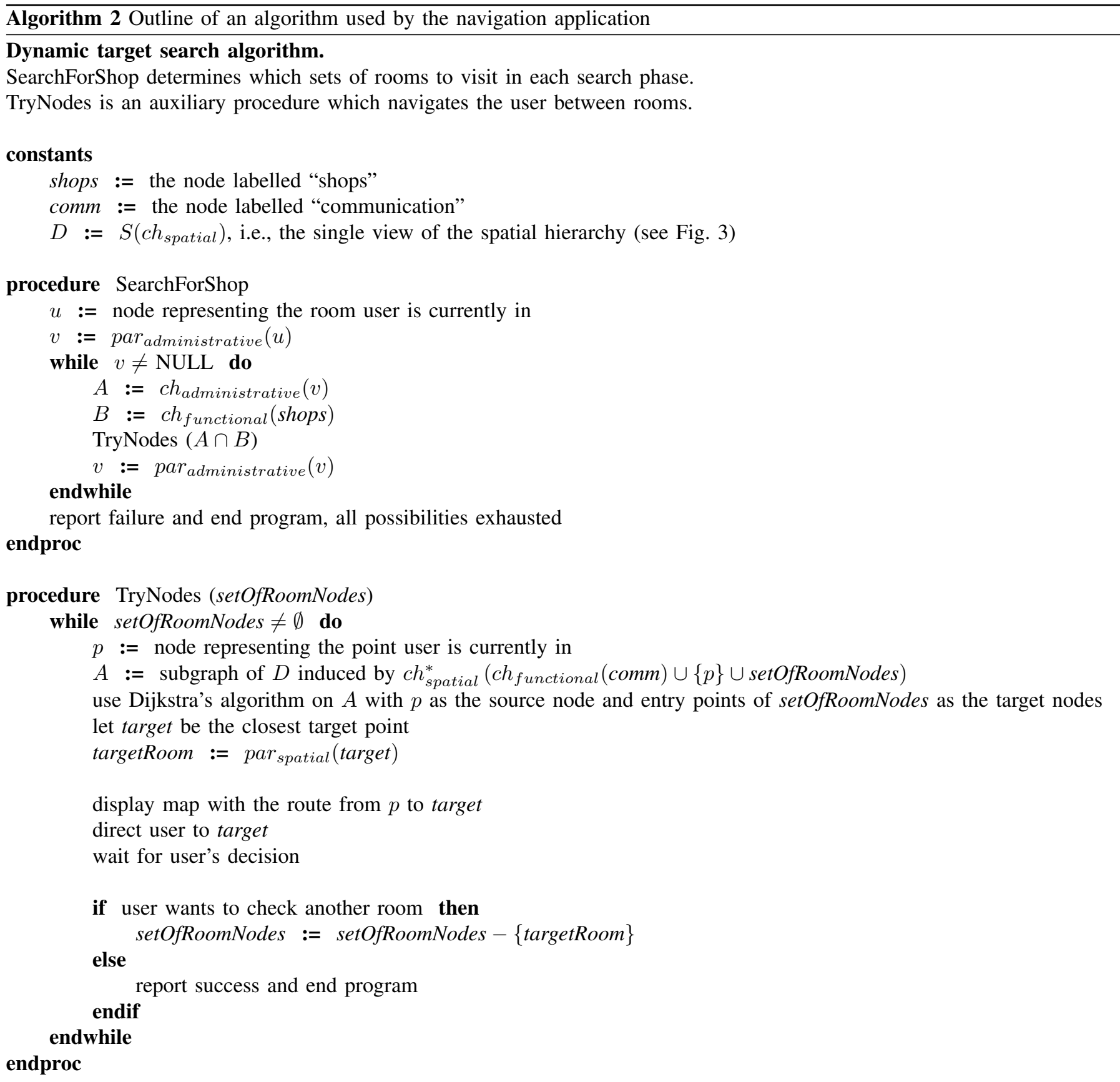

Fig. 6 illustrates the result of this process: the shop assigned to the Department of Design is closed, and the navigation application displays directions to the shop belonging to the Group of CS Departments. This path was calculated by the Dijkstra's algorithm invoked on a graph displayed in Fig. 7.

It should be noted that while SearchForShop implements a very specialised algorithm specific to this case, TryNodes is a general navigational procedure, able to direct people to successive rooms from the set given as its invocation argument. It should be possible to reuse TryNodes without modification in many other algorithms solving different navigation problems faced by university visitors, students and staff.

\section{Discussion}

As demonstrated by these case studies, the use of multiple hierarchies in a graph model allows that model to represent several different hierarchical relations in a natural way. Knowledge stored in these relations can be combined and used to extract subgraphs which contain information necessary for a given computational task. This reduction of data size speeds up processing.

Knowledge concerning properties of a single building component can be stored in attributes of the graph node corresponding to that component. In the case studies, types of shops are represented in this way. This additional information could 


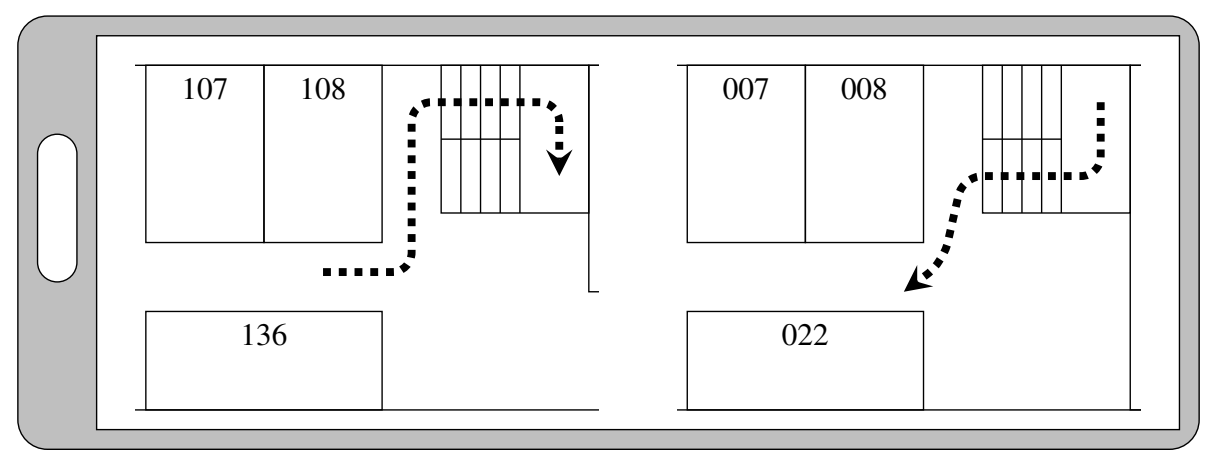

Fig. 6. A smartphone with the navigation application

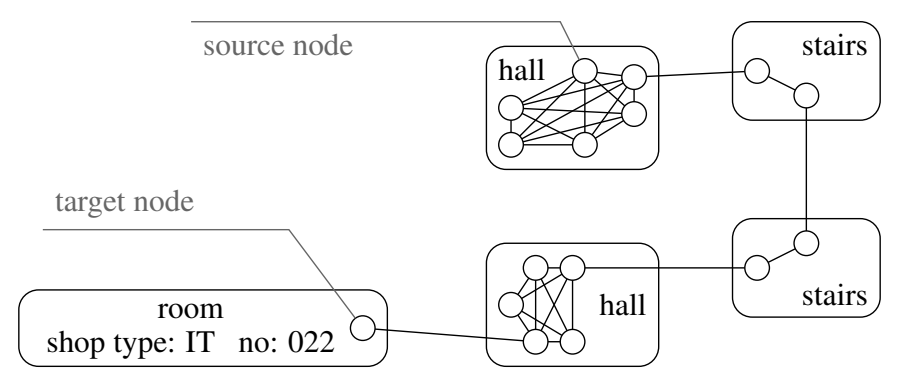

Fig. 7. One of temporary subgraphs used by the navigation algorithm (edge costs omitted)

be used by an enhanced navigation algorithm to narrow the set of rooms to be visited, but it would require the technician to specify the type of a shop he is looking for, which would consist an additional input parameter of the SearchForShop procedure.

In the presented approach, it is assumed that architectural building designs are created by means of CAD tools with the use of Building Information Modeling (BIM) technology. As the IFC (Industry Foundation Classes) format [39] is an interoperable BIM standard for CAD applications, a graph model of the considered building can be created by extracting the information about the topology of its spatial layout from the IFC file. On the basis of IFC entities and relations between them, the accessibility relations between building spaces are computed and stored in the graph structure. However grouping nodes into other hierarchies than the spatial one have to be done later by the person responsible for the maintenance of the building information (BMS operator).

Therefore the BMS operator has to take care of modifications in the building design structure or functionality during its lifecycle in order to maintain the consistency of multi-hierarchical graph representation of the building with its current status. The proposed formalism has an additional capability of supporting the preservation of information consistency and integrity. For example, when a room is divided into two new ones, the system can provide the additional level of securing correct assignment of information. It assigns to new rooms places in both functional and/or administrative hierarchies. In a similar situation of adding an extension to the building (new rooms) the formalism will also help to assure that all required information is provided.

However, there are some situations which require our formalism to be adjusted to cope with them. For example in case when two staff members work in the same room, and one of them changes the department in which he works, a problem arises as the same room can not be assigned to two different functional units, as an in graph terms it would mean that one node has two parents in the same hierarchy. In some cases this problem can be solved by assigning a room to a functional unit at the higher level of the hierarchy, in others it could be virtually divided into two parts.

\section{CONCLUSION}

This paper deals with supporting effective decision making in building management by assisting maintenance processes. As building maintenance require integration of various types of information and knowledge, the multi-hierarchical graph representation of buildings was proposed. This representation allows us to express different types of hierarchical dependencies between building parts, like geometrical and functional ones, in one structure. The additional knowledge concerning properties of building components is stored in attributes of the graph nodes corresponding to these components.

The described multi-hierarchical graph-based building model is useful in facility maintenance aspects. It provides the possibility of filtering the graph by the hierarchy type and level, eliminating knowledge not related to the given task. On the one hand, it helps in finding information constituting answers to complex queries referring to different types of information. On the other hand, it helps in finding a path between a starting point and a dynamically selected target 
point in the chosen fragment of the building, by enabling to consider only relevant subgraphs specified by selected hierarchies. In both cases the number of data to be analyzed is greatly reduced.

The considered problems were presented on examples set in the environment typical for the campus/educational establishments. The proposed solutions can be used with minor changes for BM systems of public buildings or other complex buildings where people have to be navigated. The proposed model can be extended in several ways. It was used in this paper as a knowledge base for the navigation within the building on the basis of the shortest path algorithm. It can also be used for creating tools which would support persons with disabilities helping the to find the easiest/most accessible route (not always the shortest).

\section{REFERENCES}

[1] D. J. Vanier, "Advanced asset management: Tools and techniques," Innovations in Urban Infrastructure Seminar of the APWA International Public Works Congress, pp. 39-56, 2000.

[2] J. C. P. Cheng, W. Chen, Y. Tan, and M. Wang, "A BIM-based decision support system framework for predictive maintenance management of building facilities," in Proceedings of the 16th International Conference on Computing in Civil and Building Engineering (ICCCBE2016), 2016.

[3] I. Motawa and A. Almarshad, "A knowledge-based BIM system for building maintenance," Automation in Construction, vol. 29, pp. 173182, 2013. doi: 10.1016/j.autcon.2012.09.008

[4] B. Köbben, A. H. van Bunningen, and K. Muthukrishnan, "Wireless campus LBS: Building campus-wide location based services based on WiFi technology," in Geographic Hypermedia: Concepts and Systems, E. Stefanakis, M. P. Peterson, C. Armenakis, and V. Delis, Eds. Springer, 2006, pp. 399-408. ISBN 978-3-540-34238-0

[5] H. M. Khoury and V. R. Kamat, "Evaluation of position tracking technologies for user localization in indoor construction environments," Automation in Construction, vol. 18, no. 4, pp. 444-457, 2009. doi: 10.1016/j.autcon.2008.10.011

[6] A. Basiri, P. Amirian, A. Winstanley, S. Marsh, T. Moore, and G. Gales, "Seamless pedestrian positioning and navigation using landmarks," The Journal of Navigation, vol. 69, no. 1, pp. 24-40, 2016. doi: 10.1017/S0373463315000442

[7] D. Büchel and P.-Y. Gilliéron, "Pedestrian navigation inside buildings," Géomatique Suisse, vol. 11/2004, pp. 664-668, 2004, (in French).

[8] C. Faure, P. Benci, A. Danzart, and E. Lecolinet, "Design of mobile services for students," in Proceedings of the UbiMob'06 Conference, 2006, (in French).

[9] Y. A. Wahab and A. S. H. Basari, "Building maintenance management preliminary finding of a case study in ICYM," Middle-East Journal of Scientific Research, vol. 17, no. 9, pp. 1260-1268, 2013.

[10] P. Barrett and D. Baldry, Facilities Management: Towards Best Practice, 2nd Edition. Blackwell Science, Oxford, 2003.

[11] N. Ali, M. Sun, G. Aouad, R. M. Mazlan, and F. D. Mustapa, "Understanding the business process of reactive maintenance projects," in Proceedings of the International Conference on Construction Industry, 2006, june 21-25, Padang, Sumatera Barat Indonesia.

[12] P. Teicholz, Ed., BIM for Facility Managers. Wiley, 2013. ISBN 9781-118-38281-3

[13] A. Akanmu, C. Anumba, and J. Messner, "Critical review of approaches to integrating virtual models and the physical construction," International Journal of Construction Management, vol. 14, no. 4, pp. 267-282, 2014. doi: 10.1080/15623599.2014.972021

[14] C. Legner and F. Thiesse, "RFID-based maintenance at Frankfurt airport," IEEE Pervasive Computing, vol. 5, no. 1, pp. 34-39, 2006. doi: 10.1109/MPRV.2006.14

[15] C.-H. Ko, "RFID-based building maintenance system," Automation in Construction, vol. 18, no. 3, pp. 275-284, 2009. doi: 10.1016/j.autcon.2008.09.001
[16] B. Strug, A. Paszyńska, M. Paszyński, and E. Grabska, "Using a graph grammar system in the finite element method," International Journal of Applied Mathematics and Computer Science, vol. 23, no. 4, pp. 839853, 2013.

[17] M. Minas, "Concepts and realization of a diagram editor generator based on hypergraph transformation," Science of Computer Programming, vol. 44, no. 2, pp. 157-180, 2002.

[18] E. Grabska, A. Łachwa, G. Ślusarczyk, K. Grzesiak-Kopeć, and J. Lembas, "Hierarchical layout hypergraph operations and diagrammatic reasoning," Machine GRAPHICS \& VISION, vol. 16, no. 1/2, pp. 23-38, 2007.

[19] E. Grabska, G. Ślusarczyk, and Sz. Gajek, "Knowledge representation for human-computer interaction in a system supporting conceptual design," Fundamenta Informaticae, vol. 124, pp. 91-110, 2013.

[20] B. Strug, E. Grabska, and G. Ślusarczyk, "Supporting the design process with hypergraph genetic operators," Advanced Engineering Informatics, vol. 28, pp. 11-27, 2014.

[21] E. Grabska, W. Palacz, B. Strug, and G. Ślusarczyk, "A graph-based generation of virtual grids," Lecture Notes in Computer Science, vol. 7203, pp. 451-460, 2012.

[22] R. Milner, "Bigraphs and their algebra," Electronic Notes in Theoretical Computer Science, vol. 209, pp. 5-19, 2008.

[23] G. Ferrari and U. Montanari, "Tile formats for located and mobile systems," Information and Computation, vol. 156, no. 1-2, pp. 173-235, 2000.

[24] R. Bruni, U. Montanari, G. Plotkin, and D. Terreni, "On hierarchical graphs: reconciling bigraphs, gs-monoidal theories and gs-graphs," in 13th Italian Conference on Theoretical Computer Science, 2012.

[25] H. Barendregt, M. C. J. D. van Eekelrn, J. R. W. Glauert, J. Kennaway, M. J. Plasmeijer, and M. R. Sleep, “Term graph rewriting," Lecture Notes in Computer Science, vol. 259, pp. 141-158, 1987.

[26] F. Drewes, B. Hoffmann, and D. Plump, "Hierarchical graph transformation," Journal of Computer and System Sciences, vol. 64, pp. 249-283, 2002.

[27] A. Poulovassilis and M. Levene, "A nested-graph model for the representation and manipulation of complex objects," ACM Transactions on Information Systems, vol. 12, pp. 35-68, 1994.

[28] J. F. Sowa, "Conceptual graphs," in Handbook of Knowledge Representation, F. van Harmelen, V. Lifschitz, and B. Porter, Eds. Elsevier, 2008, pp. 213-237.

[29] — "Conceptual graphs for a database interface," IBM Journal of Research and Development, vol. 20, no. 4, pp. 336-357, 1976.

[30] - Conceptual Structures: Information Processing in Mind and Machine. Addison-Wesley, 1984.

[31] D. Harel, "On visual formalisms," Communications of the ACM, vol. 31, pp. 514-530, 1988.

[32] G. Chen and N. Zhong, "Granular structures in graphs," Lecture Notes in Computer Science, vol. 6954, pp. 649-658, 2011.

[33] — , "Three granular structure models in graphs," Lecture Notes in Computer Science, vol. 7414, pp. 351-358, 2012.

[34] E. Grabska and G. Ślusarczyk, "Knowledge and reasoning in design systems," Automation in Construction, vol. 20, no. 7, pp. 927-934, 2011. doi: 10.1016/j.autcon.2011.03.009

[35] T. H. Cormen, C. E. Leiserson, R. L. Rivest, and C. Stein, Introduction to Algorithms (2nd ed.). MIT Press, 2001.

[36] A. Kneidl, A. Borrmann, and D. Hartmann, "Generating sparse navigation graphs for microscopic pedestrian simulation models," in 18th EG-ICE International Workshop, Twente, Netherlands, 2011.

[37] M. Höcker, V. Berkhahn, A. Kneidl, A. Borrmann, and W. Klein, "Graph-based approaches for simulating pedestrian dynamics in building models," in 8th European Conference on Product \& Process Modelling (ECPPM). Cork, Ireland: University College Cork, 2010.

[38] E. Whiting, J. Battat, and S. Teller, "Topology of urban environments: Graph construction from multi-building floor plan data," in ComputerAided Architectural Design Futures 2007: Proceedings of the 12th International CAAD Futures Conference. Dordrecht, Netherlands: Springer, 2007, pp. 115-128.

[39] "buildingSMART IFC," http://www.buildingsmart.org/standards/ifc, 2015, accessed on September 24, 2015. 



\title{
Critical Success Factors in IT Projects-From the Practice of Polish Clients
}

\author{
Bartosz Wachnik \\ Warsaw University of Technology, \\ Faculty of Production Engineering \\ Institute of Organization of Production Systems \\ ul. Narbutta 85, 02-524 Warszawa, Poland \\ Email: bartek@wachnik.eu
}

\begin{abstract}
The article presents research results concerning critical success factors in IT projects consisting in ERP, CRM, BI and DM system implementations, completed between 2013 and 2017. The research stems from the need to update knowledge concerning critical success factors in projects completed in commercial enterprises. The research was carried out in medium and large enterprises implementing outsourcing-based projects. The research was conducted on five types of IT projects: standard implementation, upgrade, re-implementation, roll-out and implementation of a standard system with an add-on. The article will help better understand the influence of critical success factors on the implementation of IT projects during the whole project life cycle.
\end{abstract}

\section{INTRODUCTION}

$\mathrm{T}$ HE article belongs to a cycle [1] [2] of publications concerning critical success factors in IT projects consisting in the implementations of ERP, CRM, BI and DM management information systems completed based on outsourcing, defined by Auksztol [3] as a contract with an external body or internal organizational unit, set up in order to obtain IT services along with the supporting HR management, resource management and processes. The article presents the most recent research results on Critical Success Factors (CFSs) in Poland in the group of ERP, CRM, BI and DMS implementations in enterprises, thus updating Soja's research conducted in the group of ERP [4] system implementations. During the research, two important assumptions were made, differentiating this study from others:

1. According to Kerzner [5], research and analyses of projects completed based on outsourcing should consider the relations between the client and the supplier. We need to indicate, following Lichtenstein [6], that there is a partial incompatibility of interest between the client and the supplier, manifesting itself in a clear contradiction be- tween the maximization of the supplier's profit and the client's wish to complete the project at the lowest possible cost. My research [2] has confirmed Lichtenstein's observations-the goals and interests of a client and a supplier implementing an IT project are partially contradictory. For this reason, in my opinion, it is important to analyze CSFs separately from two different perspectives: the client's and the supplier's. The presented research results refer to the client's perspective.

2. Studying IT projects with the use of institutional economics, and especially the agent theory and the contractual theory, we can state that information asymmetry between the supplier and the client can influence project completion [7]. In my research, I carried out a series of studies employing qualitative methods indicating that information asymmetry between the supplier and the client influences the completion of IT projects during the entire project life cycle, i.e. at the preparatory stage (defining business requirements, selecting a system and a supplier), project completion stage and system operation (service level agreement-SLA) [8] [9]. Qualitative research led me to quantitative research concerning CSFs, indicating that information asymmetry is one of many CSFs requiring analysis. It is noteworthy that this factor was not accounted for in earlier research concerning CSFs, which can be seen from research results concerning ERP system implementations [10].

Research on CSFs in IT projects consisting in ERP, CRM, BI and DMS implementations in Poland was prompted by the following phenomena:

1. We observe the paradox of IT outsourcing, manifesting through a dynamic development of this concept in literature with a simultaneously high failure rate of IT projects completed based on outsourcing [11].

2 . The increasing technological and business complexity of IT projects, which leads to changes in CSFs.

The presented article consists of four sections. The literature studies chapter, concerning CSFs, is a synthesis of the problem as discussed by researches over the last 10 years. The methodology chapter presents research questions, the methods of data collection, a characteristic of the respondents and the research method. The chapter dedicated to research results presents data describing 
CSFs in Poland. In the last chapter, data obtained from the study is interpreted, limitations of the research are presented and further challenges in conducting CSF studies are indicated.

\section{Critical success factors in IT projects}

Bullen and Rockart [12] have defined CSF as "the limited number of areas in which satisfactory results will ensure successful competitive performance for the individual, department, or organization. CSFs are the few critical areas where 'things must go right' for the business to flourish and for the manager's goals to be attained". The increasing significance and the relevance of issues linked to the effectiveness of IT project implementation have contributed to the proliferation of publications containing many diverse approaches-and different research perspectives-of crucial success factors in IT projects. A noteworthy example is the cognitive taxonomy map of the axis of analysis of critical success factors in management information systems, mainly ERP systems, designed by Shaul and Tauber [10].

\section{Strategic-tactical axis.}

It groups critical success factors according to the completion of strategic and tactical plans of the enterprise where the implementation project is completed [13]. Tactical success factors cover factors concerning the technical configuration of IT systems and activities linked to project management carried out by the medium level of company management. Strategic success factors cover factors linked to the strategy of IT project completion carried out by the higher-level company management.

\section{Organization-end user axis.}

Esteves, Pastor [14] and Al-Mashari [15] indicate crucial success factors linked to economic and non-economic benefits of the organization's investment in management information systems. Researchers group them separately for the organization and for the end users.

\section{Public institutions-companies axis.}

Chang [16] identifies crucial success factors for IT projects completed in public institutions and companies. Also, Dyczkowski [17] indicates critical success factors in IT implementation projects in the public sphere in Poland. The character and nature of the organization influences the key success factors.

\section{National-global axis.}

According to Zhang [18], a unique nature of organizational culture and national culture of the organization where an IT project is implemented influence the final result. This type of research was also completed by Soja [19] in Poland, Colmenares [20] in Venezuela, Wu and Wang [21] in Taiwan, He and Brown [22] in China and Kamhawi in Bahrain [23].

\section{Endogenic-exogenic axis.}

Researchers point out that in defining the crucial success factors, it is important to provide for factors such as the competitiveness of the enterprise, the character of the industry, the level of innovativeness and the goals and interests of the external group of stake-holders [24]. Markus and Tanis [25], as well as Soh [26] stress that culture and enterprise management methods, its financial situation, size and organizational structure influence the crucial success factors.

\section{Developing-developed countries axis.}

Livermore and Ragowski [27] indicate differences in the completion of MIS implementation projects in different cultures. Ngai identifies and discusses critical success factors in IT projects completed both in developed and developing countries [28]. The specific character of crucial success factors in developed and developing countries is analyzed by many researchers, including Soja [19] in Poland, Colmenares [20] in Venezuela, Wu and Wang [29] in Taiwan, as well as He and Brown [22] in China.

\section{Cultural-technological axis.}

Estevez and Pastor [30] stress that critical success factors should provide for technical aspects of information management systems. Plant and Willcocks [31] indicate that even though an MIS implementation in an enterprise is not a technological, but mainly organizational event, we need to consider specific technological characteristics of software, including systems requirements for servers. On the opposite side of the axis, we can find cultural factors influencing an IT implementation project.

\section{Project life cycle-individual project phase axis.}

Umble stresses that critical success factors should be designed for individual phases of project life cycle, considering all the technical and organizational conditioning [32]. 


\section{Corporations-SME axis.}

Researchers classify critical success factors based on the organization size. Buonanno [33] presents separate groups of critical success factors both for small and medium-size enterprises. Loh and Koh identified two groups of critical success factors for IT projects of ERP implementations in small and medium-sized enterprises in Great Britain [34]. Wu and Wang [21] have carried out a comparative analysis of IT project completions in SMEs and large corporations, and identified two CSF groups.

\section{RESEARCH METHODOLOGY}

The main research goal is to identify the factors which influence the effectiveness of completing ERP, CRM, BI and DMS implementation projects based on outsourcing from the client's perspective. The research was completed based on the formula of four project-research steps. The use of three research methods in four steps stemmed from the wish to complete data triangulation and verify individual conclusions in subsequent projects.

Step 1. Literature analysis.

Step 2. Case studies allowing us to form a research hypothesis based on qualitative research, which will then be verified in Step 3.

The research hypothesis is:

$$
\text { H1: }
$$

Do the factors-predictors presented in Table 1 really influence the effectiveness of MIS implementation projects from the client's perspective?

According to the classic definition of IT project success, it is understood as completing the project within the planned budget, schedule and achieving the planned business goals, i.e. implementing the functional range.

Step 3. Quantitative research using structural equation modeling. The research goal is verifying the research hypothesis posed in Step 2.

In Step 3, in the period between January 2016 and March 2017, 127 clients-participants, using a website, completed a survey consisting of the following questions for each of the factors-predictors presented in Table 1: P1-6, P2-7, P3-5, P4-6, P5-6, P6-5, P7-5, P8-6, P9-5.

The research covered 61 observations concerning ERP systems, 26-BI systems, 25-DMS and 15-CRM systems. The studied sample of 127 IT projects contained the following number and structure of implementation types: 32
Table 1. Identified project success factors and their interpretation from the client's perspective.

\begin{tabular}{|c|l|}
\hline Predictor code & \multicolumn{1}{|c|}{ Factor-predictor } \\
\hline P1 & $\begin{array}{l}\text { Effective process of establishing functional requirements } \\
\text { for the implemented system }\end{array}$ \\
\hline P2 & Effective IT project management \\
\hline P3 & $\begin{array}{l}\text { Credible estimation and agreement with the supplier } \\
\text { regarding project parameters, i. e. project scope, budget } \\
\text { and schedule }\end{array}$ \\
\hline P4 & Competence and engagement of the client project group \\
\hline P5 & Choice and use of an IT implementation method \\
\hline P6 & Motivation of the client project group \\
\hline P7 & $\begin{array}{l}\text { Added value brought by the supplier into the implemented } \\
\text { system, i. e. knowledge concerning the functioning of } \\
\text { processes vs. the implemented functionalities }\end{array}$ \\
\hline P8 & $\begin{array}{l}\text { Information asymmetry between the supplier and the } \\
\text { client }\end{array}$ \\
\hline P9 & $\begin{array}{l}\text { Sharing knowledge about functionalities and technology } \\
\text { of the implemented system }\end{array}$ \\
\hline
\end{tabular}

standard implementations, 19 roll-outs, 31 reimplementations, 27 upgrades and 18 vertical system implementations. The research was carried out in medium and large enterprises, as classified by the EU. The analyzed projects were completed in the following types of enterprises: 25 - enterprises specializing in internet sale of FMCG products; 28-enterprises specializing in the installation and servicing of industrial machinery; 37enterprises from the life sciences industry; 5-transport and shipping enterprises; 16-IT enterprises; 8-retail enterprises; 8-food processing enterprises. All the enterprises showed profits in the year when the studied IT project was completed. The geographic distribution of the enterprises was as follows: Mazovia-25\%, Lesser Poland39\%, Greater Poland-17\% and Pokarpacie-19\%.

In Step 3, quantitative methods were applied, i. e. structural equation modeling, which allow for capturing the structure of relations between phenomena where hidden variables occur As structural equation modeling allows for modeling and testing complex phenomena, it becomes the preferred method of confirming or rejecting theoretical models using quantitative methods [35].

Structural equation modeling (SEM) is used to test the suitability of a given model to data and theory, understood as hypothetical relations between conceptually defined variables [36]. In the study, in order to test the consistency of the theoretical model, a structural equation analysis with the Partial Least Squares method (PLS) was carried out. The analytical model was created according to the reflexive model concept of latent characteristics. Each of the 10 latent characteristics in the model was represented by the observed indicators (50 altogether). The study used WarpPLS, which allows users to implement the PLS model [37] according to its requirements. 
Step 4. In-depth analytical workshops with a selected group of 10 respondents from the group of projects researched in Step 3. The respondents included project managers, board members and enterprise owners. The analytical workshops consisted in completing structured interviews with the respondents. One session with each respondent was completed, lasting between 1 to 3 hours each.

\section{RESEARCH RESULTS}

Coefficient of determination, otherwise known as the co-efficient of definiteness, or $\mathrm{R}^{2}$, is the measure of proportion of the variance in the dependent (explained) variable, which in our case is the evaluation of success in IT project completion representing projects completed with full success, understood as completed on budget, on time, and with all the functional requirements implemented. Independent variables are the nine predictors presented.

An analysis of the coefficients of model-to-data fit has indicated that the created theoretical reflexive model had a very good model-to-data fit.

The Tennenhaus GoF (Goodness-of-Fit) index equaled 0.41 (ideally $>=0.36$ ), the SPR index equaled 0.89 (ideal$\mathrm{ly}=1$ ), the RSCR index equaled 0.98 (ideally $=1$ ), the SSR index equaled 0.89 (acceptable if $>=0.7$ ) and the NLBCDR index equaled 0.83 (acceptable if $>=0.7$ ). An analysis of the total collinearity index of the AFVIF predictors showed that the average collinearity equaled 1.17 (ideally $<=3.3$ ).

Fig. 1 presents the model layout. Structural equation analysis has revealed that the model was characterized by a very good model-to-data fit and its parameters were not affected by collinearity of variables. An analysis of coefficients of explained variable has shown that model 9 of latent variables accounted for $23 \%$ of variance in success factors (the adjusted index equaled 17\%). $77 \%$ were factors not considered in the study.

\section{Study results interpretation}

The presented results of both qualitative and quantitative research on CSF, which influence the effectiveness of outsourcing-based ERP, CRM, BI and DMS implementation projects from the client's perspective, allow us to formulate the following key conclusions.

In the group of analyzed factors-predictors, we can differentiate between two groups: the first group is directly correlated with project success, while the second group is negatively correlated with project success. Below, a group of seven factors positively correlated with project success, the so-called CSFs, is described.

First of all, the SEM analysis has shown that the strongest factor-predictor influencing project success was effective project management. In-depth analytical workshops have shown that clients understand CSF as their project manager's care to keep the deadlines, complete the project on or under budget and achieve planned goals reflecting the implemented functionality.

The second most important factor-predictor influencing project success is a low level of information asymmetry between the supplier and the client, both at the

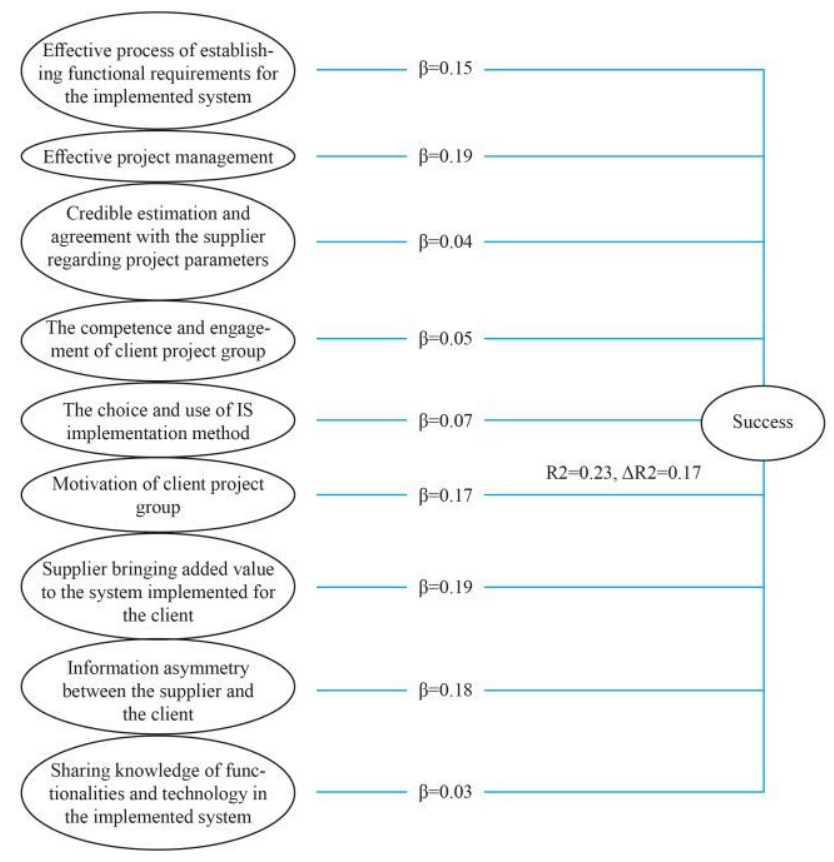

Figure 1. Model layout

preparatory stage and during implementation and operation. From a researcher's point of view, we need to stress that there are no precise methods of defining information asymmetry in IT projects and this parameter can only be estimated. Hence, the expression "low level of information asymmetry" declared by the respondents can be interpreted as client's relatively high level of knowledge concerning the implementation method, the methods of adapting systems, the knowledge of system operation, and the knowledge of how to minimize the TCO of the system, etc, in relation to the supplier, who naturally possesses more knowledge of the issues mentioned above.

We need to stress that the sub-chapter on literature research presented by Shaul and Tauber [10] does not mention any parameters reflecting the phenomenon of information asymmetry, understood as an information gap between the supplier and the client in an IT project. Analytical workshops have shown the client's concern that, as a consequence of the high level of information asym- 
metry and the phenomenon of negative selection, their project management costs will end up being higher than expected. To sum up, a significant novelty of the research results lay in identifying a high level of information asymmetry between the supplier and the client as a CSF.

The third most important factor-predictor influencing project success is the motivation of client's project group. In-depth analytical workshops have indicated that client's project group, i. e. project manager and key users, expected the enterprise management to provide a coherent and positive motivational system which would strengthen the project engagement. The respondents have pointed out that during project implementation they would spend the average of additional 2 to 4 hours in work, and thus expected the management to compensate them and motivate them to be engaged in project completion, mostly through economic benefits.

The fourth most important factor-predictor influencing project success is an effective process of establishing functional requirements for the implemented system, both at the preparatory stage aimed at selecting a system and a supplier, and the implementation stage, especially the functional analysis. As shown during analytical workshops, this factor-predictor depends mostly on client's organizational skill in obtaining and accumulating knowledge concerning the merits of the implemented systems.

The fifth most important factor-predictor influencing project success is the competence and engagement of client's project group. As demonstrated by in-depth analytical workshops, the competence of project group meant practical skills linked to project organization, the skill to pass on one's expectations for the system to the supplier in a precise manner, and skills linked to managing the project in critical situations. In-depth analytical workshops have indicated that the engagement of client's project group is understood as the engagement of key users on the client's side, who are responsible for designing business process modifications aimed at helping the company achieve a temporary competitive edge.

The sixth most important factor-predictor influencing project success was credibly evaluating and then agreeing on project parameters with the supplier. In-depth analytical workshops have indicated that respondents understood these parameters as: fixed implementation budget, license cost, detailed functional scope of the project and the TCO of the implemented system in the perspective of 3 to 6 years.

The seventh most important factor-predictor influencing project success is sharing the knowledge about functionalities and technologies in the implemented system. In-depth analytical workshops have shown that respond- ents understood this factor as a transfer of formal and informal knowledge, trainings and operating procedures.

Presented below is the group of factors negatively correlated with project success, the so-called critical failed factors.

The first most important factor-predictor negatively correlated with project success is the choice and use of system implementation method. In-depth analytical workshops have shown that the choice of implementation method from among Agile, Waterfall, Scrum and others did not positively influence project success.

The second most important factor-predictor negatively correlated with project success is bringing added value by the supplier to the system implemented for the client. During in-depth analytical workshops, respondents admitted that the knowledge and experience of supplier's consultants related to the implementation services was low. For this reason, they believed that, in practice, their supplier's consultants did not improve the functioning of their client's enterprise. The respondents admitted that these observations applied both to local and international companies. There are many CSFs critical to the success of an IT project. It is important that research concerning CSFs in IT projects is constantly updated due to the highpaced changes in technology and organization of IT projects, visible in the techniques, procedures and implementation methods. The changeability of implementation parameters may affect the structure of changing CSFs. There is not, however, a golden rule that would define the most important factors affecting project success. Successful projects do not necessarily include all the factors highlighted in this article. However, we should emphasize that the more CSFs there are, the higher the probability of success.

\section{CONCLUSIONS}

The research was completed between 2013 and 2017 on a sample of 971 projects in Poland, where the average percentage of ERP and CRM system implementation projects completed successfully in large enterprises equaled $49 \%$, and $48 \%$ in medium-sized enterprises, meaning that on average $50 \%$ of IT implementation projects ended in complete or partial failure [38]. In my opinion, the issues linked to analyzing failed projects from both the supplier's and the client's perspective are still noteworthy and it is important to analyze CSFs in outsourcing-based projects from both perspectives: the supplier's and the client's.

Only then will we be able to research what influences project success, not always understood equally by the client and the supplier. The CSFs diagnosed in the presented study are prevalently consistent with the axis of 
CSF analysis. It is noteworthy that quantitative research confirmed the hypothesis that a fuller picture of CSF in the selected group of IT projects completed through outsourcing should also consider the theories that belong to the new institutional economics, which describes business relations between suppliers and clients of the services provided. The phenomenon of information asymmetry between the supplier and the client constitutes a CSF influencing the success of an IT project. Despite the fact that it is impossible to measure the information gap between the supplier and the client in IT projects, the respondents indicated that a low level of information between the supplier and the client influences the success of project implementation.

The respondents understand low level of information asymmetry as knowledge, skills and experience possessed by the client concerning the implemented system, allowing for a more effective implementation of both the preparatory stage and project completion, which manifests itself through:

1. Defining precise functional requirements for an IT system.

2. Defining precise requirements for an IT system license.

3. Designing an implementation agreement and license purchase agreement that will ensure the completion of client's goals and interest.

4. Completing the project with the supplier so that it ends successfully.

5. Defining the total cost of ownership for the entire project life cycle.

6. Eliminating the phenomenon of moral hazard on the supplier's side.

7. Minimizing transaction costs on the client's side.

8. Partially eliminating the phenomenon of negative selection.

To sum up this synthetic presentation, I would like to stress that the research will be continued and the results will be published accordingly. I hope that by gathering and popularizing knowledge about CSFs of IT projects completed in commercial companies, it is possible to contribute directly to improving the effectiveness of completion process and management, as well as indirectly to the improving products and services that they are aimed at.

\section{REFERENCES}

[1] Wachnik B., 2015, "An Analysis of Effectiveness Factors in the Completion of it Projects-the supplier's Perspective," Scientific
Journals (Zeszyty Naukowe), Katowice: University of Economics in Katowice Publisher, vol. 234, pp. 1-12.

[2] Wachnik B., 2016, Wdrażanie Systemów Informatycznych Wspomagajacych Zarzadzanie, Warszawa: PWE.

[3] Auksztol J., 2008, Outsourcing Informatyczny w Teorii i Praktyce Zarządzania. Gdańsk: Wydawnictwo Uniwersytetu Gdańskiego.

[4] Soja P., 2006, "Success Factors in ERP Systems Implementations: Lessons from Practice," Journal of Enterprise Information Management, vol. 19, no. 6, pp. 646-661.

[5] Kerzner H., 2001, Strategic Planning for Project Management Using a Project Management Maturity Model. New York: John Wiley \& Sons..

[6] Lichtenstein Y., 2004, "Puzzles in Software Development Contracting," Communications of the ACM, vol. 47, no. 2, pp. 61-65, 2004, DOI: 10.1016/s0378-4754(00)00185-3.

[7] Dibbern J., T. Goles, R. Hirscheim, B. Jayatilaka, "Information Systems Outsourcing: A Survey and Analysis of the Literature," The Data Base for Advance in Information Systems, vol. 35, no. 4, pp. 6102, 2004 DOI: $10.1145 / 3051473.3051475$.

[8] Wachnik B., 2014, "Reducing Information Asymmetry in IT Projectsaction Research Results," Scientific Journals (Zeszyty Naukowe), Katowice: University of Economics in Katowice Publisher, vol. 188, pp. 237-249.

[9] Wachnik B., 2015, "Information Asymmetry in Four IT Projects: The Client's Perspective. A Multiple Case Study," Information Systems in Management, vol. 2, pp. 155-168, DOI: 10.1145/2379776.2379777.

[10] Shaul L., D. Tauber, 2012, "CSFs in ERP Systems: Review of the Last Decade," ACM Computing Surveys, vol. 45, no. 4, pp. 1-39.

[11] Wachnik B., 2017, "An Analysis of ERP and CRM System Implementations in Poland Between 2013 and 2016," Journal of Economics and Management, DOI: 10.1111/jems.12187.

[12] Bullen C., J. Rockart, Eds, 1986, "A Primer on Critical Success Factors," The Rise of Managerial Computing: The Best of the Center for Information System Research, Homewood, Illinois: Dow JonesIrwin, pp. 383-423.

[13] Holland C., B. Light, N. Gibson, 1999, "A Critical Success Factors Model for ERP Implementation," IEEE Software, vol. 16, no. 3, pp. 30-36, DOI: 10.1109/IPCC.1999.799146.

[14] Esteves J., V. Bohorquez, 2007, “An Updated ERP Systems Annotated Bibliography: 2001-2005," Communications of the Association for Information Systems, vol. 19, no. 1, pp. 1-59, DOI: 10.1016/j.lrp.2007.

[15] Al-Mashari M., 2001, "Process Orientation Through Enterprise Resource Planning (ERP): A Review of Critical Issues," Knowledge and Process Management, vol. 8, no. 3, pp. 175-185, DOI: $10.1002 / \mathrm{kpm} .128$

[16] Chan J. W. K., 2008, "Prioritization the Critical Success Factors for ERP Implementation Project: Production Postponement Perspective," Industrial Engineering Research, vol. 5, no. 2, pp. 11-23.

[17] Dyczkowski M., 2008, "Wiedza o krytycznych czynnikach sukcesu jako istotny element poprawy efektywności przedsięwzięć informatycznych w sferze zarządzania publicznego," in Technologie Wiedzy w Zarzadzaniu Publicznym '07, J. Gołuchowski, A. Frączkiewicz-Wronka, Eds. Katowice: Prace Naukowe Akademii Ekonomicznej w Katowicach, vol. 7, pp. 291-309.

[18] Zhang Z., M. K. Lee, P. Huang, L. Zhang, X. Huang, 2005, “A Framework of ERP Systems Implementation Success in China: An Empirical Study," International Journal of Production Economics, vol. 98 , no. 1 , pp. 63-64.

[19] Soja P., "Difficulties in Enterprise System Implementation in Emerging Economies. Insight from an Exploratory Study in Poland," 2008, Information Technology for Development, vol. 14, no. 1, pp. 3151, DOI: $10.1002 /$ itdj.20040.

[20] Colmenares L., 2004, "An Exploratory Study on the Critical Success Factors in the Implementation of Enterprise Resource Planning Systems in Venezuela," Journal of Information Systems and Technology Management, vol. 2, no. 2, pp. 167-187, DOI: 10.4301/10.4301\%2FS1807-17752007000100002.

[21] Wu J. H. , Y. M. Wang, 2003, "Enterprise Resource Planning Experience in Taiwan: An Empirical Study and Comparative Analysis," in Proc. of the 36th Hawaii International Conference on System Sciences, pp. 1-10. 
[22] He L., D. Brown, 2005, "The Adoption of ERP Applications in China," in Proc. of the 11th Americas Conference on Information Systems, pp. 284-292.

[23] Kamhawi E. M., "Critical Factors for Implementation Success of ERP Systems: An DEmpirical Investigation from Bahrain", International Journal of Enterprise Information Systems, DOI: 10.4018/IJEIS, vol. 3, no. 2, pp. 34-49, 2007.

[24] Bouchbout K., Z. Alimazighi, 2008, “A Framework for Identifying the Critical Factors Affecting the Decision to Adopt and Use Interorganizational Information Systems", World Academy of Science, Engineering and Technology, DOI: 10.1108/09504121011045809, vol. 43 , pp. $338-345$.

[25] Markus M. L., C. Tanis, 2000, "The Enterprise Systems Experiencefrom Adoption to Success," in Framing the Domains of IT Research: Glimpsing the Future Through the Past, Zmud R. W., Ed. Cincinnati: Pinnaflex Educational Resources Inc..

[26] Soh C., S. S. Kien, J. Tay-Yap, 2000, "Enterprise Resource Planning: Cultural Fits and Misfits: is ERP a Universal Solution?," Communications of the ACM, vol. 43, no. 4, pp. 47-51, DOI: 10.1016/s0378-4754(00)00185-3.

[27] Livermore C., A. Ragowsky, 2002, "ERP Systems Selection and Implementation: A Cross-cultural Approach," in Proc. of the 8th Americas Conference on Information Systems, pp. 1332-1339,.

[28] Ngai E. W., C. C. Law, F. K. Wat, 2008, "Examining the Critical Success Factors in the Adoption of Enterprise Resource Planning," Computers in Industry, vol. 59, no. 6, pp. 548-564.

[29] Wu J. H., Y. M. Wang, 2007, "Measuring ERP Success: the Criticalusers'Viewpoint of the ERP to Produce a Viable IS in the Organization," Computers in Human Behavior, vol. 23, no. 3, pp. 1582-1596.
[30] Esteves J., J. Pastor, 2000, "Towards the Unification of Critical Success Factors for ERP Implementation," in Proc. of the 10th Annual Business Information Technology (BIT) Conference, pp. 1-9.

[31] Plant R., L. Willcocks, 2007, "Critical Success Factors in International ERP Implementations: A Case Research Approach," Journal of Computer Information Systems, vol. 47, no. 3, pp. 60-70.

[32] Umble E. J., R. R. Haft, M. M. Umble, 2003, "Enterprise Resource Planning: Implementation Procedures and Critical Success Factors," European Journal of Operational Research, vol. 146, no. 2, pp. 241257.

[33] Buonanno G., P. Faverio, F. Pigni, A. Ravarini, D. Sciuto, M. Tagliavini, 2005, "Factors Affecting ERP System Adoption-A Comparative Analysis Between SMEs and Large Companies," Journal of Enterprise Information Management, vol. 18, no. 4, pp. 384-426, DOI: 10.1108/17410390810888697.

[34] Loh T., S. Koh, 2004, "Critical Elements for a Successful Enterprise Resource Planning Implementation in Small and Medium Sized Enterprises," International Journal of Production Research, vol. 2, no. 17, pp. 3433-3455, DOI: 10.1080/00207543.2017.1346318.

[35] Schumacher C. R., R.E. Lomaz, 2004, A Beginner's Guide to Structural Equation Modeling. Mahwah: Lawrence Erlbaum Associates, p. 7.

[36] Blunch N. J., 2008, Introduction to Structural Equation Modeling Using SPSS and AMOS. London: London Sage Publication.

[37] http://warppls.blogspot.co.uk/2009/12/welcome-to-warpplscommunity.html accessed: 20.04.2017.

[38] Wachnik B., 2017, "An Analysis of ERP and CRM System Implementations in Poland Between 2013 and 2016," Journal of Economics and Management, DOI: 10.1111/jems.12187. 



\section{A Decision Support System for Demand Forecasting based on Classifier Ensemble}

\author{
Irem Islek \\ Idea Teknoloji Cozumleri, \\ Istanbul, Turkey \\ Email: irem.islek@ideateknoloji.com.tr
}

\author{
Sule Gunduz Oguducu \\ Department of Computer Engineering, \\ Istanbul Technical University, \\ Istanbul, Turkey \\ Email:sgunduz@itu.edu.tr
}

\begin{abstract}
Demand forecasting is the process of constructing forecasting models to estimate the quantities of several products that customers will purchase in the future. When the warehouse and the number of products grow, forecasting the demand becomes dramatically hard. Most of the demand forecasting models rely on a single classifier or a simple combination of these models. In order to improve demand forecasting accuracy, we investigate several different classifiers such as MLP, Bayesian Network, Linear Regression and SVM analyzing their accuracy and performance. Moreover, we also studied some classifier combination techniques by approaching from demand forecasting perspective. In this paper, we propose a methodology to combine various forecasting models using neural networks rather for supporting demand forecasting. The proposed methodology is tested against single classifiers and classifier ensemble models using a real dataset. Experiments indicate that the proposed methodology outperforms all the other single classifiers tested in this study and their simple combinations.
\end{abstract}

\section{INTRODUCTION}

$\mathbf{S}$ UPPLY chain is defined as a set of entities directly involved in the activities associated with the upstream and downstream flows of products, services, finances, and/or information from a source to a customer [1]. Supply chains can be categorized into three groups such as Direct Supply Chain, Extended Supply Chain and Ultimate Supply Chain [2]. In our problem, we focus on Direct Supply Chain which contains some manufacturers, warehouses and customers. In this type of supply chain, products of manufacturer are transported to warehouses and customers reach these products through warehouses. Considering all given definitions above, supply chain management (SCM) can be thought as a process which deals with the total flow of materials from suppliers through end users [3]. There are various sub-processes of supply chain management which are quite complicated and challenging such as demand forecasting. Demand forecasting can be summarized as an estimation of a supply chain constituent's (such as warehouse, end sale point etc.) expected sales during a specified future period. Forecasting demand correctly for different constituents provides planning all processes of supply chain effectively. For instance, accurate demand forecasting prevents redundant shipping charges or storage costs. Thus, forecasting the demand of warehouses is an important task and it forms the motivation of our work. In this paper, we study forecasting the demand of warehouses with low error rate problem.
In our previous work, we tried to cluster warehouses according to their sale behaviors using bipartite graphs with the purpose of reducing error rate of demand forecasting. After that warehouse clusters are constructed, we set individual Bayesian Network models for every warehouse cluster. This approach provided improvement in forecasting performance. We defined using different machine learning models and combining them as our future work in an attempt to provide further improvement in forecasting performance [4].

Because of the fact that forecasting demand of warehouses is considerably hard, using a single model can be incapable to solve this problem. Thus, combining multiple models rather than using stand-alone models seems reasonable. Stacked Generalization [5] is a way of combining machine learning models. There are several studies which use Stacked Generalization for combining machine learning models in different domains such as predicting protein types [6], automatic music tagging [7], forecasting fraudulent financial statements [8], etc. In Stacked Generalization, there are two levels which are called as level0 and level-1. The forecasting outputs of level-0 algorithms are used as input to a level-1 generalizer. In other words, the output results of level- 0 are added as parameters to the level-1 generalizer. For example, if level-0 contains three different models, three input parameters must be added to level-1 generalizer. Essentially, level-1 generalizer learns how to map combined outputs to real class values. In this paper, we propose a novel method to combine our four different level-0 methods which are Multi Layer Perceptron (MLP), Bayesian Network, Linear Regression and Support Vector Machine (SVM) in level-1 generalizer. Instead of using only level-0 forecasting outputs as input parameters to level-1, we construct different instances using both level-0 model input parameters and level- 0 model forecasting outputs. Then, we use these instances in level-1 generalizer. In addition to that, we apply Stacked Generalization using only binary combinations of our level- 0 models in other trial. In other words, we take level-0 models' binary combinations separately to apply stacked generalization. After that, we apply same methodology using only triple combinations of level-0 models. In another trial, we apply regular stacked generalization methodology for comparison. All the results of mentioned trials can be seen in experiments section.

One of the contributions of this study is using level-0 input 
parameters and level-0 forecasting outputs together as input to level-1 generalizer differently from Stacked Generalization. In addition to that, there is any study that uses stacked generalization in order to forecast demand of warehouses.

The paper is organized as follows. Section II reviews the related literature. In Section III we describe the background of models which are used in this paper. Section IV presents the proposed approach. Section V describes the experiments and the results obtained. Finally, in Section VI we conclude and discuss some possible future works.

\section{RELATED WORKS}

Forecasting demand of warehouses is a problem of estimating the future-dated sales amounts of products for warehouses. There are some different methodologies which have been applied in the domain of warehouse demand forecasting problem. These techniques can be collected as two main types such as (1) stand-alone forecasting models and (2) hybrid forecasting models which use multiple models together. The stand-alone models can be collected two different types which are (1) statistical models and (2) machine learning models.

Moving average and Box-Jenkins are some examples of the basic and popular statistical models. There are some prior works which used these traditional models for demand forecasting [9], [10]. These methodologies were insufficient to solve demand forecasting problem which is quite complex and hard. Thus, artificial intelligence models were started to use. Especially, Neural Networks were used in a large number of works [11]-[16]. Some works compared Neural Networks with traditional statistical models and showed that Neural Networks provides better results than traditional statistical models. Because of the popularity of Neural Networks models for demand forecasting problem, some studies compared another models with Neural Networks. For instance, Efendigil et. al. compared Adaptive Neural Fuzzy Inference System (ANFIS) with Neural Networks. They claimed that ANFIS provides better results than Neural Networks in their study [17].

Some recent studies tried to combine different models in order to reduce the error rate of demand forecasting. In some studies, statistical models were combined with machine learning models. For instance, Aburto and Weber combined Autoregressive Integrated Moving Average (ARIMA) model with Neural Networks in their study [18]. On the other hand, Doganis et. al. used genetic algorithm with Neural Networks for demand forecasting [19].

Combining classifiers on the purpose of improving success rate is a popular approach in most domains. Stacked Generalization which is one of the classifier combining techniques combines more than one machine learning models using another machine learning model [5]. There are some studies which compared stacked generalization with standalone models [20]-[24]. According to these studies, stacked generalization provided better results than stand-alone models. In addition to that, some studies claimed that stacked generalization performs better than some other combining models. For instance, Ting and Witten compared Stacked Generalization with majority vote and provided lower error rate with Stacked Generalization [25]. This technique applied in a wide variety of domains likewise biomolecular event extraction [26], early diagnosis of Alzheimer disease [27], forecasting fraudulent financial statements [8], image classification [28], credit risk assessment [29], anti-spam filtering of e-mail [30], city traffic related geospatial data analysis [31], etc.

Conducted studies on demand forecasting handle different parts of the supply chain. Forecasting the demand of an end sale point is one of the most common type of demand forecasting studies. Moreover, most of the demand forecasting studies handle a limited number of warehouses and products. Because of the fact that our problem contains quite number of warehouses and products, one model cannot be sufficiently successful. Thus, approach of combining multiple models is used in this study.

\section{BACKGROUND}

In this section, we briefly describe the stacked generalization and the forecasting models which are adopted in our work.

\section{A. Stacked Generalization}

Ensemble of classifiers is defined as a concept of combining classifiers to improve performance of individual classifiers. Stacked generalization is one of the ensemble classifiers methodologies which is used for minimizing error rate of one or more classifiers [5]. This methodology proposed by David H. Wolpert in 1992.

In stack generalization methodology, there are two basic steps which are sequential. The first step which is called level0 contains independent machine learning models. These level0 models are combined in level-1 generalization step. In the level-1 step, outputs of each individual level- 0 models are used as an input parameter to generalizer model. Any machine learning model can be selected as generalizer according to suitability to the problem.

Level-0 models of stacked generalization are trained using a set of training data. Afterwards, another set of training data is created from prediction outputs of level- 0 models. This dataset is used for training level-1 generalizer. The key point of this operation is that, forecast results in this dataset are estimated from the instances which are not in the training dataset of level- 0 models. To evaluate the stacked generalization model, the output of every instance of a third dataset is predicted by level-0 models separately. Estimated forecasting results are used as input parameters in the level-1 generalizer. Finally, the forecasting output of level-1 generalizer is compared with the real output of every instance to obtain a final evaluation result. Scheme of stacked generalization can be seen at Fig. 1 .

\section{B. Bayesian Network Algorithm}

Bayesian Network is a simple, graphical representation for conditional independence assertions. In this graphical representation, every node of graph symbolize a random variable, where a random variable can take on possible values from a random experiment. In addition to that, every edge between 


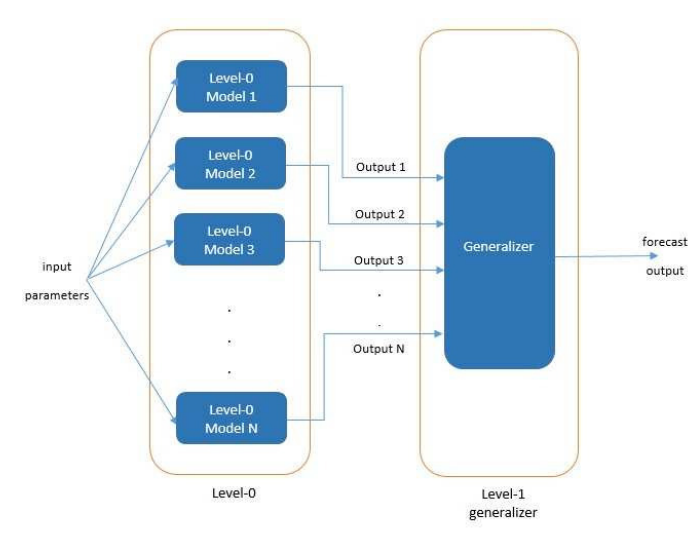

Fig. 1. Stacked Generalization

these nodes represents probabilistic dependencies among these random variables. If a Bayesian Network contains $n$ nodes (random variables), the joint probability distribution is represented as Eq. 1.

$$
p\left(x_{1}, x_{2}, x_{3}, \ldots, x_{n}\right)=\sum_{i=1}^{n} p\left(x_{i} \mid x_{P_{i}}\right)
$$

In Eq. 1, $p\left(x_{i} \mid x_{P_{i}}\right)$ represents the local conditional probability distribution for node $i$. $P_{i}$ corresponds to parent nodes indices of node $i$. Thanks to conditional independence relationship, joint probability distribution can be represented more conveniently for large networks.

Bayesian Networks can be used for numerous applications such as classification, regression, segmentation [32].

\section{Multi Layer Perceptron Algorithm}

Multilayer Perceptron (MLP) which maps a set of input onto a set of appropriate outputs is one of the feed-forward artificial neural network models. MLP can be thought as multiple layers which are fully connected to next layer. A multilayer perceptron contains an input layer, an output layer and also one or more hidden layers. Each node is accepted as a neuron and has a nonlinear activation function except for the input nodes. This nonlinear function can be seen in Eq. 2 .

$$
y=\varphi\left(\sum_{j=1}^{n} w_{j} x_{j}-\theta\right)
$$

In Eq. $2, x_{j}$ values are input signals and $w_{j}$ values are weights associated with the $j$ th input. $\theta$ corresponds to threshold value and $\varphi($.$) is a sigmoid activation function. Eq. 3$ shows this sigmoid activation function.

$$
\varphi(x)=\frac{1}{1+\exp (-x)}
$$

The weights are adjusted during training phase in order to obtain input-output mapping of the network. Weight updating phase continues until weights no longer change or error value reaches a threshold value. [33].

\section{Linear Regression Algorithm}

Linear regression is a technique which is used for modeling the relation between a scalar dependent variable $y$ and one or more independent variables. If there is only one independent variable, it is called simple linear regression. In other case, it is called multiple linear regression [34]. In multiple linear regression, the relation between the scalar dependent variable $y$ and independent variables is defined by Eq. 4 .

$$
y=\alpha+x_{1} \beta_{1}+x_{2} \beta_{2}+x_{3} \beta_{3}+\ldots+x_{n} \beta_{n}+\epsilon
$$

\section{E. Support Vector Machine Algorithm}

Support Vector Machine (SVM) which is a machine learning algorithm can be used for not only classification, but also regression problems. SVM regression is a nonparametric technique because it uses several kernel functions. The relation between input $\left(x_{i}\right)$ and output $\left(y_{i}\right)$ can be mapped by a regression function $f(x)$ which can be seen in Eq. 5 .

$$
f(x)=w x+b \quad w \in X, b \in \Re
$$

After that, following problem must be solved:

$$
\begin{gathered}
\text { minimize } \frac{1}{2}\|w\|^{2} \\
\text { subject to }\left\{\begin{array}{l}
y_{i}-w x_{i}-b \leq \epsilon \\
w x_{i}+b-y_{i} \leq \epsilon
\end{array}\right.
\end{gathered}
$$

There is a case where the constraints are infeasible. In this case which is called soft margin formulation, slack variables $\left(\xi_{i}, \xi_{i}^{*}\right)$ are used.

$$
\begin{gathered}
\text { minimize } \frac{1}{2}\|w\|^{2}+C \sum_{i=1}^{l} \xi_{i}+\xi_{i}^{*} \\
\text { subject to }\left\{\begin{array}{l}
y_{i}-w x_{i}-b \leq \epsilon+\xi_{i} \\
w x_{i}+b-y_{i} \leq \epsilon+\xi_{i}^{*} \\
\xi_{i}, \xi_{i}^{*} \geq 0
\end{array}\right.
\end{gathered}
$$

In Eq. 8, $C$ controls the penalty amount based on deviations which are larger than $\epsilon$. The linear $\epsilon$-insensitive loss function $\left(|\xi|_{\epsilon}\right)$ ignores errors that are within $\epsilon$ distance of the observed value by treating them as equal to zero. As can be seen in Eq. 10, the loss is measured based on the distance between observed value and the $\epsilon$ boundary.

$$
|\xi|_{\epsilon}= \begin{cases}0, & \text { if }|\xi| \leq \epsilon \\ |\xi|-\epsilon, & \text { if }|\xi| \succ \epsilon\end{cases}
$$

After applying Lagrangian multipliers, a model solution can be found in dual representation.

$$
\begin{aligned}
& f(x)=\sum_{i=1}^{l}\left(\alpha_{i}-\alpha_{i}^{*}\right) K\left(x_{i}, x\right)+b \\
& K\left(x_{i}, x_{j}\right)=\exp \left(-\gamma\left\|x_{i}-x_{j}\right\|^{2}\right)
\end{aligned}
$$


In Eq. $11, \alpha_{i}, \alpha_{i}^{*}$ are nonzero Lagrangian multipliers. $K\left(x_{i}, x\right)$ is the kernel function. Eq. 12 shows radial basis function (RBF) kernel and $\gamma$ corresponds to width parameter of RBF kernel [35].

\section{Details Of The Methodology}

In this paper, we study the problem of forecasting the sale amounts of products for main distribution warehouses. Because of the fact that there are a large number of warehouses and products in our case, problem is harder than regular demand forecasting problem. Thus, it is needed to use hybrid methodologies in order to solve given problem.

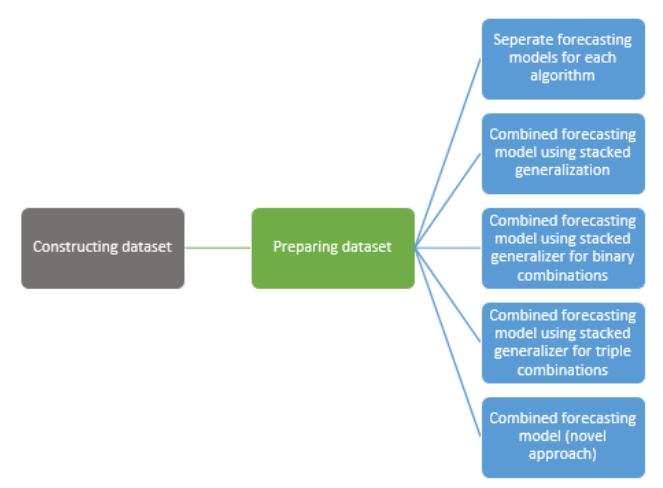

Fig. 2. Steps of the study

First step of our methodology is constructing a dataset which contains necessary information to forecast demand of warehouses. Our dataset is constructed from real sales transaction data of a national dried fruits and nuts company from Turkey. Sales transactions of 2011, 2012 and 2013 are used in this dataset. There are ninety eight warehouses and seventy different products in the dataset. Additionally, this dataset contains warehouse related attributes such as location, number of transportation vehicles, total amount of weekly selling product, selling area in square meter, number of employees and product related attributes such as selling amount, selling time, product category.

Next step of the proposed methodology is preparing the dataset for data mining operations. In this step, data is cleaned and prepared for further operations. Moreover, moving average value of product sale amounts are calculated using past three weeks. For a specific week $t$, the moving average calculation equation can be seen in Eq. 13.

$$
\text { Moving avg. }(t)=\frac{\sum_{i=1}^{3} \text { sale amount }(t-i)}{3}
$$

After these steps, forecasting model is constructed using machine learning algorithms. In this step, different methodologies are tried and compared in order to find the one with lowest error rate. Some of these methodologies use model combining techniques in order to provide better performance. In the first forecasting model construction trial of our study,

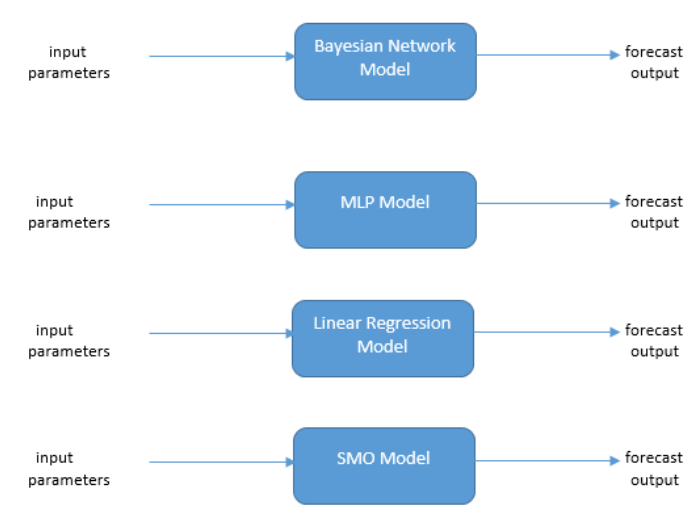

Fig. 3. Stand-alone models

four different algorithms are determined to forecast demand of warehouses. Selected algorithms are Bayesian Network, Multi Layer Perceptron, Support Vector Machine and Linear Regression. In order to make comparison with further combined forecasting models, selected algorithms are used separately for constructing a forecasting model as a baseline. Warehouse related attributes, product related attributes, time information and moving average values are used as input, selling amounts are used as output in all models. Detailed results and reviews about these four stand-alone models can be seen at Experiments and Results section.

Stacked Generalization methodology which is explained in Background section in detail, contains two different levels and combines selected models on the purpose of reducing the error rate. With the same purpose, stacked generalization methodology is used for combining four different machine learning models in our study. As can be seen in Figure 4, there are four separate forecasting models in level-0 step which will be used for estimating forecasting output in the next level. For every instance, forecast results are produced by individual level-0 models. After that, these forecast results are used as input parameters in level-1 generalization model. In our study, MLP machine learning algorithm is used for constructing level-1 generalizer. Level-1 generalizer maps input parameters into real sale amounts. In this approach, the output of the level-1 is the forecasting output of the total methodology.

With the aim of determining whether or not some of the selected machine learning algorithms are dominant to other ones, various combinations of the selected four machine learning algorithms are used in level-0, differently from the previous trial. Firstly, 2-combinations of the four algorithms are determined: MLP and Bayesian Network, MLP and Linear Regression, MLP and SVM, Bayesian Network and SVM, Bayesian Network and Linear Regression, SVM and Linear Regression. These six different combinations are used respectively as level-0 models. Instead of using four different models, two models which are from one of the 2-combination set are used as level- 0 models in this trial. Thus, two forecasting outputs are added as input parameters to the level- 1 generalizer in this trial. Constructing stacked generalization is repeated six 


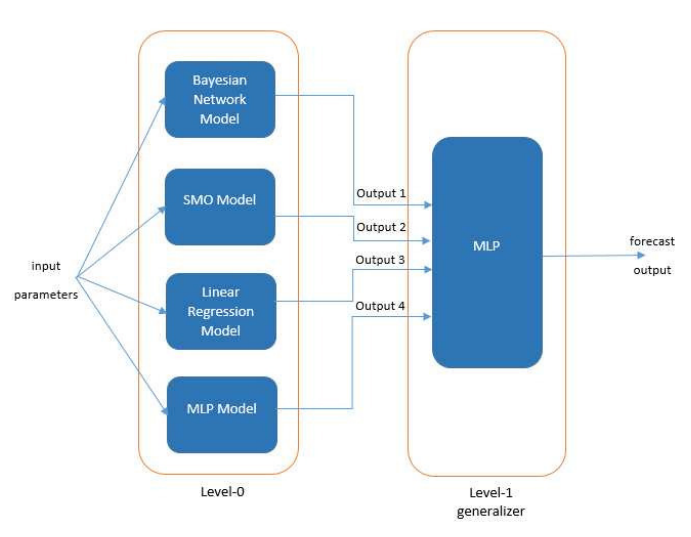

Fig. 4. Stacked Generalization for our problem

times because of that 2-combination set contains six element. Detailed figure of this trial can be seen at Figure 5.

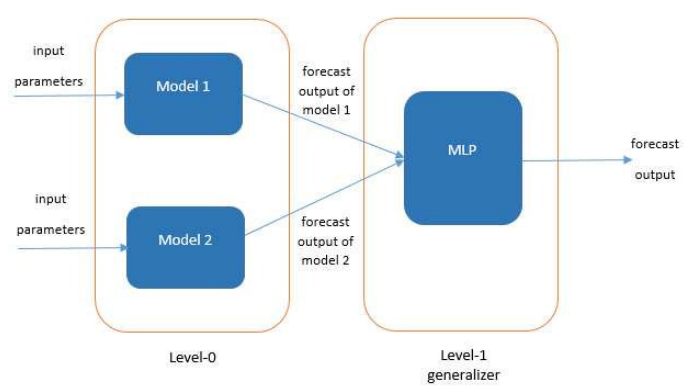

Fig. 5. Stacked Generalization using binary combinations

Same procedure is applied using 3-combinations in the next trial. 3-combination set contains MLP, Bayesian Network, SVM, MLP, Bayesian Network, Linear Regression, MLP, SVM, Linear Regression and Bayesian Network, SVM, Linear Regression. There are three different models in level-0 and three forecasting outputs are added to level-1 generalizer model as input parameters. As might be expected, stacked generalizer methodology is constructed four times in this trial. Relevant figure can be seen at Figure 6.

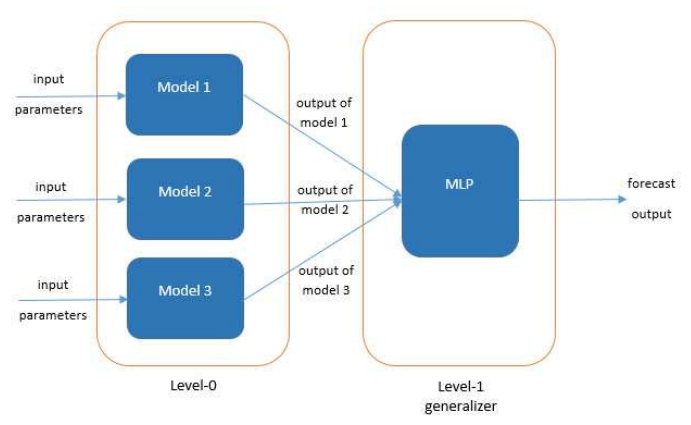

Fig. 6. Stacked Generalization using triple combinations

In the last trial, a novel approach which is similar to Stacked Generalization from some aspects is applied. This approach is also contains two levels. Especially, level-0 is completely similar to level-0 of Stacked Generalization. This methodology differs from Stacked Generalization in respect to construction input parameters of level-1. In Stacked Generalization, forecasting outputs of the level- 0 models are added to input parameters of level-1 generalizer. For instance, if there are four different models in level-0, four forecasting outputs will be added as new input parameters to the level-1 for every instance. Instead of using only forecasting outputs of level-0 in level-1, input parameters of level-0 and forecasting outputs of level- 0 are used together as input parameters of level-1.

As can be seen in Figure 7, level-0 models estimate forecasting outputs using $\mathrm{x}$ number of input parameters. Because of that there are four different models in level- 0 of our trial, four different forecasting outputs are estimated. There are four forecasting outputs after level-0. In the level-1, these forecasting outputs and input parameters of level-0 are used as inputs of level-1 to estimate the output of the total methodology. Using this new instance, a new forecasting output is estimated by level-1 neural network.

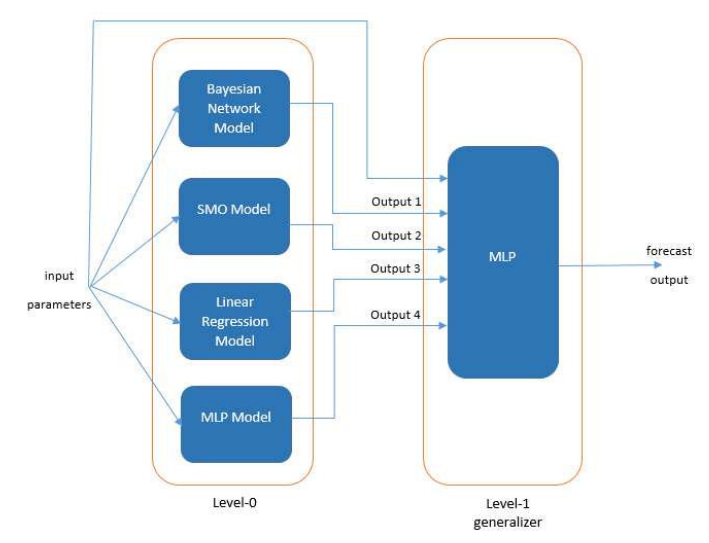

Fig. 7. Our approach for combining models

Comparison of all methodologies can be seen in the next section, Experiments and Results.

\section{EXPERIMENTS AND RESULTS}

In this section, we describe the sales data used in our experiments and present the results obtained by our approach for demand forecasting compared to other applied methodologies.

\section{A. Dataset}

Dataset is composed from the real sales data of a national dried fruits and nuts company from Turkey. This company gives service to all of the cities in Turkey. The warehouse and product counts are 98 and 70, respectively. Dataset contains 15317141 instances which are taken from real sales transaction data of 2011, 2012 and 2013 years. These instances are obtained from 93987868 sales transactions detail lines.

Attributes in the dataset can be collected two main groups: warehouse attributes and item attributes. Warehouse related attributes are location, number of sub-warehouses it has, number of employees, number of customers, number of vehicles, 
selling area in square meter and total amount of weekly selling products. Item related attributes are item category, selling time and moving average of previous 3 weeks. Selling amount of the item is selected as output of the model.

\section{B. Experimental Results}

First of all, the dataset is divided into 3 partitions in order to train and test the methodologies. The first, second and third datasets are composed from real sales transaction data of 2011, 2012 and 2013, respectively. Cause of dividing dataset into 3 partitions is that combined methodologies contain two levels of machine learning models and these sequential levels cannot be trained using the same training dataset. Thus, first dataset is used for training level-0 of combined methodology while second dataset is used for training level-1. Third dataset is used for testing the combined methodologies. Differently from the combined methodologies, methodologies which contain only one machine learning algorithm are trained using both first and second datasets. The third dataset is used for testing the standalone methodologies likewise the combined methodologies.

The error rate of the methodologies are estimated using Mean Average Percentage Error (MAPE). The calculation formula of MAPE can be seen in Eq. 14. In the given formula, $A_{t}$ means actual sales amount and $F_{t}$ means forecasting sales amount, for this study.

$$
M A P E=\frac{100}{n} \sum_{t=1}^{n} \frac{\left|A_{t}-F_{t}\right|}{\left|A_{t}\right|}
$$

Table 1 shows the error rate of stand-alone methodologies. As can be seen in this table, Bayesian Network gives better results than the other stand alone models for our problem.

TABLE I

ERROR RATES OF STAND-ALONE METHODOLOGIES

\begin{tabular}{|l|c|}
\hline \multicolumn{1}{|c|}{ Model } & MAPE Value \\
\hline Bayesian Network & $17.5 \%$ \\
\hline MLP & $21.2 \%$ \\
\hline Linear Regression & $19.8 \%$ \\
\hline SVM & $23 \%$ \\
\hline
\end{tabular}

With the motivation of reducing error rate, combining more than one model approach is applied. For combining models, stacked generalization is used at the beginning. Table 2 shows the result of stacked generalization error rate.

TABLE II

ERROR RATE OF STACKED GENERALIZATION

\begin{tabular}{|c|c|}
\hline Model & MAPE Value \\
\hline Stacked Generalization & $21.9 \%$ \\
\hline
\end{tabular}

After that, instead of combining all of the selected models (Bayesian Network, SVM, Linear Regression and MLP), binary combinations of these models are used as level-0 models. Reason of this trial is determining whether some models are dominant or not. Comparisons of these 2-combinations can be seen in Table 3.

TABLE III

ERROR RATE OF STACKED GENERALIZATION WITH 2-COMBINATIONS

\begin{tabular}{|l|c|}
\hline \multicolumn{1}{|c|}{ Model } & MAPE Value \\
\hline $\begin{array}{l}\text { Stacked Generalization with Bayesian } \\
\text { Network and SVM }\end{array}$ & $22 \%$ \\
\hline $\begin{array}{l}\text { Stacked Generalization with Bayesian } \\
\text { Network and MLP }\end{array}$ & $20.7 \%$ \\
\hline $\begin{array}{l}\text { Stacked Generalization with Bayesian } \\
\text { Network and Linear Regression }\end{array}$ & $20.5 \%$ \\
\hline $\begin{array}{l}\text { Stacked Generalization with SVM } \\
\text { and MLP }\end{array}$ & $23.8 \%$ \\
\hline $\begin{array}{l}\text { Stacked Generalization with SVM } \\
\text { and Linear Regression }\end{array}$ & $23 \%$ \\
\hline $\begin{array}{l}\text { Stacked Generalization with MLP } \\
\text { and Linear Regression }\end{array}$ & $22.8 \%$ \\
\hline
\end{tabular}

Table 4 shows the results of stacked generalization with 3combinations.

TABLE IV

ERROR RATE OF STACKED GENERALIZATION WITH 3-COMBINATIONS

\begin{tabular}{|l|c|}
\hline \multicolumn{1}{|c|}{ Model } & MAPE Value \\
\hline $\begin{array}{l}\text { Stacked Generalization with Bayesian } \\
\text { Network, SVM and MLP }\end{array}$ & $22.6 \%$ \\
\hline $\begin{array}{l}\text { Stacked Generalization with Bayesian } \\
\text { Network, SVM and Linear Regression }\end{array}$ & $21.4 \%$ \\
\hline $\begin{array}{l}\text { Stacked Generalization with Bayesian } \\
\text { Network, MLP and Linear Regression }\end{array}$ & $20.3 \%$ \\
\hline $\begin{array}{l}\text { Stacked Generalization with SVM, } \\
\text { MLP and Linear Regression }\end{array}$ & $23.4 \%$ \\
\hline
\end{tabular}

Finally, we try our methodology for the same dataset. In this methodology, we use both input parameters of level- 0 and forecasting outputs of level-0 as input of level-1.

TABLE V

COMPARISON OF BEST RESULTS OF ALL TRIALS

\begin{tabular}{|l|c|}
\hline \multicolumn{1}{|c|}{ Model } & MAPE Value \\
\hline Our methodology & $12.7 \%$ \\
\hline Stacked Generalization & $21.9 \%$ \\
\hline Bayesian Network & $17.5 \%$ \\
\hline $\begin{array}{l}\text { Stacked Generalization with Bayesian } \\
\text { Network and Linear Regression }\end{array}$ & $20.5 \%$ \\
\hline $\begin{array}{l}\text { Stacked Generalization with Bayesian } \\
\text { Network, MLP and Linear Regression }\end{array}$ & $20.3 \%$ \\
\hline
\end{tabular}

The results of warehouse demand forecasting indicate that our methodology which uses input parameters and forecasting outputs of level-0 together in level-1 has the overall best performance.

\section{CONCLUSION}

In this paper, we proposed an approach which combines multiple machine learning models in order to forecast demands 
of warehouses. Our methodology consists of two levels likewise Stacked Generalization. Differently from Stacked Generalization methodology that constructs level-1 generalizer with only the forecast outputs of level- 0 models, our methodology takes input parameters of level-0 and forecast outputs of level0 models together as input parameters into level-1 generalizer. In addition to that, we apply Stacked Generalization which has never been used before in warehouse demand forecasting, in order to compare results with our methodology. Experiments are performed on three-year real sales data of a national dried fruits and nuts company from Turkey.

The experimental results show that our approach achieves better results for forecasting demands of warehouses. In terms of MAPE, the proposed method provides nearly 5\% less error than best result of stand-alone models. Moreover, it drops error rate nearly $9 \%$ compared to Stacked Generalization.

\section{ACKNOWLEDGMENT}

This research was supported by Ministry of Science, Industry and Technology of Turkey SANTEZ project 0484.STZ.2013-2.

\section{REFERENCES}

[1] M. L. Christopher, Logistics and Supply Chain Management, Pitman Publishing, 1992.

[2] J. T. Mentzer, W. DeWitt, J.S. Keebler, S. Min, N. W. Nix, C. D. Smith, Z. G. Zacharia, "Defining Supply Chain Management," Journal of Business Logistics, vol. 22, no. 2, 2001.

[3] T. C. Jones, D. W. Riley, "Using Inventory for Competitive Advantage through Supply Chain Management,“ International Journal of Physical Distribution and Materials Management, vol. 15, no. 5, pp. 16-26, 1985.

[4] I. Islek, S. G. Oguducu, "A retail demand forecasting model based on data mining techniques," in 24th International Symposium on Industrial Electronics (ISIE), Buzios, Brasil, 2015, pp. 55-60.

[5] D. H. Wolpert, "Stacked Generalization," Neural Networks, vol. 5, pp. 241-259, 1992.

[6] S. Q. Wang, J. Yang, K. C. Chou, "Using stacked generalization to predict membrane protein types based on pseudo-amino acid composition," Journal of Theoretical Biology, vol. 242, no. 4, pp. 941-946, 2006.

[7] S. R. Ness, A. Theocharis, G. Tzanetakis, "Improving automatic music tag annotation using stacked generalization of probabilistic svm outputs,' in 17th ACM international conference on Multimedia, 2009, pp. 705-708.

[8] S. Kotsiantis, E. Koumanakos, D. Tezlepis, V. Tampakas, "Forecasting Fradulent Financial Statements Using Data Mining," International Journal of Computational Science, vol. 3, no. 2, pp. 104-110, 2006.

[9] L. M. Liu, S. Bhattacharyya, S. L. Sclove, R. Chen, W.J. Lattyak, "Data Mining on Time Series: An Illustration Using Fast-Food Restaurant Franchise Data," Computational Statistics \& Data Analysis, vol. 37, pp. 455-476, 2001.

[10] K. Gilbert, "An ARIMA supply chain model," Management Science, vol. 51, no. 2, pp. 305-310, 2005.

[11] P. C. Chang, Y. W. Wang, C. H. Liu, "The Development of a Weighted Evolving Fuzzy Neural Network for PCB Sales Forecasting," Expert Systems with Applications, vol. 32, pp. 86-96, 2007.

[12] Z. L. Sun, T. M. Choi, Y. Yu, "Sales Forecasting Using Extreme Learning Machine With Applications In Fashion Retailing," Decision Support Systems, vol. 46, pp. 411-419, 2008.
[13] Y. Yu, T. Choi, C. Hui, "An Intelligent Fast Sales Forecasting Model for Fashion Products," Expert Systems with Applications, vol. 38, pp. 73737379, 2011.

[14] S. H. Ling, Genetic Algorithm and Variable Neural Networks: Theory and Application, Lambert Academy Publishing, 2010.

[15] K. F. Au, T. M. Choi, Y. Yu, "Fashion Retail Forecasting by Evolutionary Neural Networks," International Journal of Production Economics, vol. 114, pp. 615-630, 2008.

[16] R. S. Gutierrez, A. Solis, S. Mukhopadhyay, "Lumpy Demand Forecasting Using Neural Networks," International Journal of Production Economics, vol. 111, pp. 409-420, 2008.

[17] T. Efendigil, S. Onut, C. Kahraman, "A Decision Support System for Demand Forecasting with Artificial Neural Networks and NeuroFuzzy Models: A Comparative Analysis," Expert Systems with Applications, vol. 36, no. 3, pp. 6697-6707, 2009.

[18] L. Aburto, R. Weber, "Improved Supply Chain Management Based On Hybrid Demand Forecasts," Applied Soft Computing, vol. 7, no. 1, pp. 136-144, 2007.

[19] P. Doganis, A. Alexandridis, P. Patrinos, H. Sarimveis, "Time Series Sales Forecasting For Short Shelf-Life Food Products Based On Artificial Neural Networks And Evolutionary Computing," Journal Of Food Engineering, vol. 75, pp. 196-204, 2006.

[20] A. Ghorbani, K. Owrangh, "Stacked Generalization In Neural Networks: Generalization On Statistically Neutral Problems," in IEEE International Joint Conference on Neural Networks, 2001, pp. 1715- 1720.

[21] D. W. Fan, P. K. Chan, S. J. Stolfo, "A comparative evaluation of combiner and stacked generalization," in AAAI-96 workshop on Integrating Multiple Learned Models, 1996, pp. 40-46.

[22] G. Sigletos, G. Paliouras, C. D. Spyropoulos, M. Hatzopoulos, "Combining information extraction systems using voting and stacked generalization," Journal of Machine Learning Research, vol. 6, pp. 1751-1782, 2005.

[23] L. Breiman, "Stacked regressions," Machine learning, vol. 24, no. 1, pp. 49-64, 1996.

[24] I. Guler, E. D. Ubeyli "ECG beat classifier designed by combined neural network model," Pattern recognition, vol. 38, no. 2, pp. 199-208, 2005.

[25] K. M. Ting, I. A. Witten "Issues in Stacked Generalization," Journal Of Artificial Intelligence Research, vol. 10, pp. 271-289, 1999.

[26] A. Majumder, A. Ekbal, S. K. Naskar, "Biomolecular Event Extraction using a Stacked Generalization based Classifier," in 13th International Conference on Natural Language Processing, 2016.

[27] H. Gandhi, D. Green, J. Kounios, C. M. Clark, R. Polikar, "Stacked Generalization for Early Diagnosis of AlzheimerâÁŹs Disease," in 28th IEEE EMBS Annual International Conference, 2006.

[28] C. F. Tsai, "Training Support Vector Machines Based On Stacked Generalization For Image Classification,” Neurocomputing, vol. 64, pp. 479-503, 2005.

[29] M. Doumpos, C. Zopounidis, "Model Combination For Credit Risk Assessment: A Stacked Generalization Approach," Annals of Operations Research, vol. 151, no. 1, pp. 289-306, 2007.

[30] G. Sakkis, I. Androutsopoulos, G. Paliouras, V. Karkaletsis, C. Spyropoulos, P. Stamatopoulos, "Stacking Classifiers for Anti-Spam Filtering of E-mail," in Empirical Methods in Natural Language Processing, pp. 44-50, 2001.

[31] X. Liu, L. Yu, P. Peng, F. Lu, "A Stacked Generalization Framework for City Traffic Related Geospatial Data Analysis," in Asia-Pacific Web Conference, 2016, pp. 265-276.

[32] N. Friedman, D. Geiger, M. Goldszmidt, "Bayesian Network Classifiers," Machine Learning, vol. 29, no. 2-3, pp. 131-163, 1997.

[33] S. Haykin, Neural Networks: A Comprehensive Foundation, Prentice Hall, 1998.

[34] D. A. Freedman, Statistical Models: Theory and Practice, Cambridge University Press, 2009.

[35] D. Basak, S. Pal, D. C. Patranabis, "Support vector regression," Neural Information Processing-Letters and Reviews, vol. 11(10), pp. 203-224, 2007. 



\section{AAIA'17 Data Mining Competition: Prediction model which would help AI to play the game of Hearthstone: Heroes of Warcraft}

\begin{abstract}
AAIA'17 Data Mining Challenge is the fourth data mining competition organized within the framework of International Symposium Advances in Artificial Intelligence and Applications. This time, the task is to come up with an efficient prediction model which would help AI to play the game of Hearthstone: Heroes of Warcraft. The competition is kindly sponsored by Silver Bullet Solutions and Polish Information Processing Society (PTI).
\end{abstract}

\section{Special Session}

As in previous years, a special session devoted to the competition will be held at the conference. We will invite authors of selected reports to extend them for publication in the conference proceedings (after reviews by Organizing Committee members) and presentation at the conference. The publications will be treated as short papers and will be indexed by IEEE Digital Library and Web of Science. The invited teams will be chosen based on their final rank, innovativeness of their approach and quality of the submitted report.

\section{AwARDS}

Authors of the top-ranked solutions (based on the final evaluation scores) will be awarded prizes funded by our sponsors (Silver Bullet Solutions and PTI):

- First Prize: 1000 USD + one free FedCSIS'17 conference registration

- Second Prize: 500 USD + one free FedCSIS'17 conference registration,

- Third Prize: one free FedCSIS'17 conference registration.

Contest Organizing Committee

- Andrzej Janusz, University of Warsaw

- Maciek Świechowski, Silver Bullet Solutions

- Damian Zieniewicz, Silver Bullet Solutions

- Krzysztof Stencel, University of Warsaw

- Jacek Puczniewski, Silver Bullet Solutions

- Jacek Mańdziuk, Warsaw University of Technology

- Dominik Ślęzak, University of Warsaw \& Infobright Inc 



\title{
Application of machine learning to help AI to play Hearthstone
}

\author{
Evgeny Patekha \\ Russia
}

Email: evgeny.patekha@gmail.com

\begin{abstract}
This paper presents a solution, which was developed as a part of the competition AAIA'17 Data Mining Challenge: Helping AI to Play Hearthstone. The goal of the competition was to predict the probability of AI player win in different intra-game states of Hearthstone game (online computer game with cards). This solution got the third place at the final leaderboard. The paper describes models and local validation approach, which was very useful for models development without overfitting.
\end{abstract}

\section{INTRODUCTION}

$\mathrm{H}$ EARTHSTONE: Heroes of Warcraft [1] is a free-toplay online video game developed and published by Blizzard Entertainment [2]. It is a turn-based collectible card game between two opponents, who use constructed decks of thirty cards along with a selected hero with a unique power. Players use mana points (money equivalent) to cast spells or summon minions (units for battle) to attack the opponent, with the goal to reduce the opponent's health to zero.

Author did not play this game before competition, but read the game rules and wiki [3] when developed this solution.

AAIA'17 Data Mining Challenge is the fourth data mining competition organized within the framework of International Symposium Advances in Artificial Intelligence and Applications [4].

For the purpose of this challenge, organizers simulated a large number of Hearthstone gameplays. The task for participants of this competition was to construct a prediction model that can learn how to evaluate accurately particular intra-game states. These models would help to improve AI to play the game of Hearthstone: Heroes of Warcraft.

The paper contains:

- the short description of a competition, data provided to the competitors and the evaluation method that was applied to submitted models

- the description of validation scheme which was used for the models development

- the description of models and main features

- the final results of competition

\section{COMPETITION TASK}

The ability to assess accurately a winning chance in different game states is substantial for designing efficient and challenging AI players in many games. In this data mining challenge, participants worked to develop a prediction model for a popular game Hearthstone: Heroes of Warcraft - a collectible card video game developed and published by Blizzard Entertainment.

The data for the competition was generated by the simulation of games between weak AI players. Ideas and models from this competition could be used to improve AI play.

The detailed information about the competition can be found in [5].

\section{A. Data}

The data for this competition were provided in two different formats: JSON and tabular. I worked with JSON files as they contained more information than tabular ones. Files with train data contained information about condition of each of the competing heroes, played minion cards, cards in the hand of the first player (it is assumed that the first player always starts the game) and other features.

The "decision" was a target variable to predict with values ' 1 ' if the first player won the game and ' 0 ' otherwise.

The test data is available in the same format as the training sets, however, there is no information about the "decisions".

It was allowed to use external knowledge bases about Hearthstone cards.

Initially, the training data contained descriptions of $2,000,000$ game states. During the competition test data were replaced by new ones, data from old test became available for training, and full training set appeared to be 3,250,000 records.

New test data contained 750,000 game states.

\section{B. Evaluation}

The participants of the competition were asked to submit likelihoods of winning by the first player. 
The submitted solutions were evaluated on-line and the preliminary results were published on the competition leaderboard (public LB). The preliminary score was computed on a subset of the test set, fixed for all participants. It corresponded to approximately $5 \%$ of the test data. The final evaluation was done after the completion of the competition having use the remaining part of the test data (final LB). Those results were also published on-line.

The assessment of solutions was done using the Area Under the ROC Curve (AUC) measure.

\section{SOLUTION}

The final solution was the mix of the Gradient Boosted Decision Trees and the Neural Net models. All models were developed in R with LightGBM [6] and MXNet [7] libraries. data.table library [8] was used for data processing before training.

\section{A. Validation}

During the competition organizers decided to replace test data, because of the information that different stages of one game could be both in the train and the test sets, and it could lead to inadequate and useless result of the competition.

However, the same problem was actual for local validation while model training. Training records with different stages of the same game led to model overfitting when Gradient Boosting Trees were used for training. To overcome this issue I decided to split data to different folds and tried to put all potential records from one game to one fold.

The only features that could be used for this goal were "hero_card_id" of a player and an opponent (each could have $\overline{9}$ different values). Therefore, I split data into 9 folds by the unique combination of "pl.hero_card_id" and "op.hero_card id" (total 81 combinations, 9 to each fold). I tried to achieve uniform distribution of data among the folds so that only one kind of "pl.hero_card_id" and one kind of "op.hero_card_id" were put in each fold.

This solution fixed the problem of overfitting and was very good for local evaluation of created models. The local results of cross-validation $(\mathrm{CV})$ had high correlation with public leaderboard. This kind of CV gave me excellent tool for fine-tuning of my models without overfitting (to choose right features and to find best parameters).

The score of my model dropped less between public and final leaderboards than the scores of other participants in top-10 (Table I). I suppose that was thanks to the good validation scheme.

\section{B. Features}

Based on the initial data many of new features were created and tested by cross-validation. Features were selected to be used in the final models if they improved score with local validation by more than 0.0001 , and scores were improved for most of validation folds.
The following features made a major contribution to improving the score (measured by cross-validation):

- Difference between cumulative "attack" (sum of "attack" of a player and his played cards) and cumulative "health" of an opponent (sum of "health" of an opponent and his played cards) and vice versa

- Difference of "health" of a player and an opponent divided by "health" of a player

- Sum of "health" of minions at player's hand

- Cumulative "attack" gain (compared to base levels) of played cards

- Number of played cards ready to attack

In addition, I used as features a number of cards (separately played and in hand) with specific IDs, specific costs. The most useful of IDs features were features with IDs of spell cards.

The full list of used features listed in the Appendix.

\section{External data}

The competition rules allowed to use external data. I used information about cards properties from hearthstonejson.com [9]. For each card, the database contains base information about cost, attack, health plus some additional features like card class, race, faction, collectible and others.

I tried to use additional features in my model. The most of those features did not improve my models. Only feature "number of neutral class cards" (number of cards with neutral class in player's hand) used in the main final model.

\section{D.Models}

The main model was the Gradient Boosting Decision Trees (GBDT) implemented by the LightGBM library. GBDT is a machine learning technique for regression and classification problems, which produces a prediction model in the form of an ensemble of weak prediction models of decision trees $[10,11]$.

The main model had 140 features. The individual score (AUC) of this model was 0.7987 at public LB.

The second model was the LightGBM too. The features were taken from the first model. There was an idea to review the main model features and create another model by adding them one by one from the beginning. As a result, I got another model with only 58 features with score similar to the main model ( 0.7983 at public LB). The predictions of these models were slightly different. The mix of these models produced a good gain (0.001) on CV and public LB.

The third model was the Neural Network by the MXNet library. I used the same features as in the first model. The model had 2 fully connected hidden layers of 192 units each, ReLu activation with 50\% dropout and softmax output [12]. This model had score 0.7980 at public LB and gave moderate improvement when combined with two LightGBM models. 
I used grid-search with cross-validation to find the best parameters for each models. The final parameters listed in Table I.

TABLE I

PARAMETERS OF THE MODELS

\begin{tabular}{|l|c|c|c|}
\hline \multicolumn{1}{|c|}{ Parameters } & LGBM 1 & LGBM 2 & NN \\
\hline learning_rate & 0.03 & 0.03 & 0.07 \\
\hline num_leaves & 12 & 12 & - \\
\hline max_depth & 4 & 4 & - \\
\hline feature_fraction & 0.8 & 0.8 & - \\
\hline bagging_fraction & 0.75 & 0.75 & - \\
\hline $\begin{array}{l}\text { averaged by CV } \\
\text { num_iterations }\end{array}$ & 3599 & 3547 & 46 \\
\hline batch_size & - & - & 32768 \\
\hline
\end{tabular}

\section{E. Additional model}

Based on analysis of the data, I discovered that for the first two turns the outcome of the game is mostly uncertain. A definite outcome is very rare at this stage. I decided to build another model only for first two turns. The idea was to restrict the model by exclusion of usage of some patterns from the next turns. The prediction of this model was scaled and inserted to the prediction of the main model. This approach helped to drop some of the false predictions with moderate improvements of the score.

\section{$F$. Other improvements}

After replacement of the test data during competition, some features of the new test set became very different from train set. The rules of the game were changed while new test data were collected. The changes affect features "turn", "op.deck_count" and "op.hand_count".

I tried to reduce those differences and changed train data:

- data with value of the feature "turn" more than 16 were not used in the models because maximum value of the feature "turn" in the new test data was 16 ;

- all cases where value of the feature "turn" was less than 11 and value of the feature "pl.crystals_all" was less than value of the feature "turn" were equate to "turn" value;

- for "turn" 1 and 2 values of the feature "op.deck_count" were increased by 1 ;

- for "turn" 1 and 2 values of the feature "op.hand_count" were decreased by 2 and 1 respectively;

These changes led to moderate improvement of the score.

\section{G.Other steps without success}

One of ideas that I tried without success was the idea to swap data between player and his opponent (with missing cards in player's hand which we did not have for opponent) to get additional data for training. I hope that some different game states would be good addition to the train data, but this approach did not help to improve the score. I suppose that the reason for this is that we already have enough data for training.

I tried other machine learning technologies, such as xgboost [13], as it was the best of gradient boosting implementation before LightGBM, K-nearest neighbor (KNN) with different number of neighbors and logistic regression, but all of them had worse score and did not improve the score of the main models when I tried ensemble.

I also tried to build second level model to stack different models, as it very popular in many competitions method to improve score, but did not find a way to validate second level model without overfitting. Prediction from stacked model did not improve score at public leaderboard while it was better on my CV.

\section{H.Training process and final prediction}

As we had big training dataset, predictions for the test data were made by each of iterations during 9-folds crossvalidation (at the point of the best validation score) and were averaged before submit. This approach had better scores than predictions from single model with full train set with approximation of number of training iteration.

For the final submission all models were trained with 3 different random seeds and the predictions were averaged. This approach is traditional way to increase stability of models.

The final prediction was a weighted mean of models with weights $30 \%$ for each of 2 LightGBM models and $40 \%$ for the MXNet model. This blend got score 0.8001 at public LB and 0.79895 at final LB (3 place).

\section{FINAL RESULTS}

There were submissions from 188 teams from 28 different countries. Top-10 scores are listed in Table II.

I think that the main contribution to my good result was made by a good local validation scheme. My CV allowed me to check many different ideas and to choose the best ones without overfitting the models.

TABLE II

FINAL RESULTS (TOP-10)

\begin{tabular}{|c|l|c|c|c|}
\hline Rank & Participants & $\begin{array}{c}\text { Public } \\
\text { lb }\end{array}$ & $\begin{array}{c}\text { Final } \\
\text { Ib }\end{array}$ & Drop \\
\hline 1 & iwannabetheverybest & 0,8041 & $\mathbf{0 , 8 0 1 8 5}$ & $-0,0022$ \\
\hline 2 & hieuvq & 0,8016 & $\mathbf{0 , 7 9 9 2 2}$ & $-0,0024$ \\
\hline $\mathbf{3}$ & johnpateha & 0,8001 & $\mathbf{0 , 7 9 8 9 5}$ & $-\mathbf{0 , 0 0 1 1}$ \\
\hline 4 & vz & 0,7997 & $\mathbf{0 , 7 9 7 3 3}$ & $-0,0024$ \\
\hline 5 & jj & 0,7997 & $\mathbf{0 , 7 9 7 0 7}$ & $-0,0026$ \\
\hline 6 & karek & 0,8000 & $\mathbf{0 , 7 9 6 8 5}$ & $-0,0032$ \\
\hline 7 & podludek & & $\mathbf{0 , 7 9 6 5 7}$ & \\
\hline 8 & akumpan & 0,7995 & $\mathbf{0 , 7 9 6 5 4}$ & $-0,0030$ \\
\hline 9 & iran-amin & & $\mathbf{0 , 7 9 6 3 7}$ & \\
\hline 10 & basakesin & 0,7988 & $\mathbf{0 , 7 9 6 1 7}$ & $-0,0026$ \\
\hline
\end{tabular}


ApPendix

At the first and the third models were used 140 features(for played IDs used only top important features):

- pl.hero_card_id, pl.crystals_all, pl.crystals_current,

- $\quad$ pl.hp, pl.armor, pl.attack, pl.special_skill_used,

- pl.weapon_durability,

- pl.deck_count,

- pl.hand_count, pl.played_minions_count

- op.hero_card_id, op.crystals_all, op.crystals_current,

- op.hp, op.armor, op.attack, op.special_skill_used,

- op.weapon_durability,

- op.deck_count,

- op.hand_count, op.played_minions_count

- pl.cum_attack, pl.cum_hp_cur, pl.cum_attack_gain,

- pl.cum_hp_loss, pl.num_taunt, pl.num_can_attack,

- pl.cum_crystals_cost

- op.cum_attack, op.cum_attack_gain, op.cum_hp_cur,

- op.cum_hp_loss,

- op.num_taunt,

- op.num_can_attack, op.cum_crystals_cost

- turn, pls.cum_attac_hp_dif, pls.cum_attac_hp_difl,

- pls.cum_attac_hp_dif2,

- pls.hp_dif,

- pls.hp_dif_to_hp,

- pl.ids_count,

- op.ids_count,

- pl.crystals_use, op.crystals_use, pl.cristal_turn_dif,

- op.cristal_turn_dif

- m_cum_attack,

- m_cum_hp,

- m_num_taunt,

- $\mathrm{m} \_$num_freezing, m_cum_crystals_cost, s_num,

- s_cum_crystals_cost,

- w_attack,

- W_num,

- W_cum_crystals_cost,

- m_ids (30), s_ids (44), pl_ids (top 3), op_ids (top 3)

At the second model were used 58 features:

- pl.hero_card_id, pl.crystals_all, pl.hp, pl.armor,

- pl.attack,

- pl.deck_count,

- pl.hand_count,

- pl.special_skill_used,

- pl.played_minions_count,

- pl.max_cost, pl.avg_hp_cur, pl.cum_attack_gain,

- pl.cum_hp_loss, pl.nocan_cum_attack
- op.hero_card_id, op.crystals_all, op.hp, op.armor,

- op.attack,

- op.deck_count,

- op.hand_count,

- op.special_skill_used, op.played_minions_count,

- op.max_cost, op.avg_hp_cur,op.cum_attack_gain,

- op.cum_hp_loss, “op.nocan_cum_attack

- m_cum_attack, m_cum_hp, m_num_neutral_class,

- s_cum_cost

- turn, pls.cum_attac_hp_dif, pls.cum_attac_hp_dif1,

- pls.cum_attac_hp_dif2,

- $\quad$ pls.hp_dif_to_hp,

- pls.cum_attac_hp_dif_by_turn,

- pl.crystals_use,

- op.crystals_use,

- $\quad$ l.cristal_turn_dif,

- op.cristal_turn_dif

- op.cost1, op.cost2, op.cost3, op.cost4, op.cost7,

- m_cost5, m_cost6, m_cost7, s_cost 1, s_cost 2 ,

- s_cost 3, s_cost 4, s_cost5, s_cost6, s_cost7, pl.cost7

Next prefixes were used in the features names:

- $\quad$ pl. - player played cards

- op. - opponent played cards

- $\mathrm{m}_{-}$- minions at player hand

- $\quad$ s_ - spell at player hand

- $\quad \mathrm{w}_{-}$- weapon at player hand

\section{REFERENCES}

[1] Hearthstone: Heroes of Warcraft. [Online]. Available: http://eu.battle.net/hearthstone/en/

[2] Blizzard Entertainment. [Online]. Available: http://www.blizzard.com

[3] Hearthstone wiki. [Online]. Available: http://hearthstone.gamepedia. com

[4] International Symposium Advances in Artificial Intelligence and Applications. [Online]. Available: https://fedcsis.org/2017/aaia

[5] AAIA'17 Data Mining Challenge. [Online]. Available: https://knowledgepit.fedcsis.org/contest/view.php?id=120

[6] Microsoft LightGBM lidrary. [Online]. Available: https://github.com/Microsoft/LightGBM

[7] MXNet library. [Online]. Available: http://mxnet.io

[8] data.table library. [Online]. Available: http://r-datatable.com

[9] hearthstonejson.com. [Online]. Available: https://api.hearthstonejson. com/v1/18792/enUS/cards.json

[10] Jerome H. Friedman, "Greedy function approximation: A gradient boosting machine." Ann. Statist. 29 (2001), no. 5, 1189-1232. doi:10.1214/aos/1013203451 [Online].Available: https://statweb. stanford.edu/ jhf/ftp/trebst.pdf

[11] Tianqi Chen, Carlos Guestrin, "XGBoost: A Scalable Tree Boosting System” KDD '16, August 13-17, 2016, San Francisco, CA, USA doi:10.1145/2939672.2939785 [Online]. Available: https://arxiv.org/abs/1603.02754

[12] N. Srivastava, G. E. Hinton, A. Krizhevsky, I. Sutskever, R. Salakhutdinov, "Dropout: A Simple Way to Prevent Neural Networks from Overfitting" Journal of Machine Learning Research 15 (1). 1929-1958 [Online]. Available: http://jmlr.org/papers/volume15/ srivastava14a/srivastava14a.pdf

[13] xgboost library. [Online]. Available: https://github.com/dmlc/xgboost 


\section{$7^{\text {th }}$ International Workshop on Artificial Intelligence in Medical Applications}

$\mathbf{T}$ HE workshop on Artificial Intelligence in Medical Applications - AIMA'2017-provides an interdisciplinary forum for researchers and developers to present and discuss latest advances in research work as well as prototyped or fielded systems of applications of Artificial Intelligence in the wide and heterogenious field of medicine, health care and surgery. The workshop covers the whole range of theoretical and practical aspects, technologies and systems based on Artificial Intelligence in the medical domain and aims to bring together specialists for exchanging ideas and promote fruitful discussions.

\section{TOPICS}

- Artificial Intelligence Techniques in Health Sciences

- Knowledge Management of Medical Data

- Data Mining and Knowledge Discovery in Medicine

- Health Care Information Systems

- Clinical Information Systems

- Agent Oriented Techniques in Medicine

- Medical Image Processing and Techniques

- Medical Expert Systems

- Diagnoses and Therapy Support Systems

- Biomedical Applications

- Applications of AI in Health Care and Surgery Systems

- Machine Learning-based Medical Systems

- Medical Data- and Knowledge Bases

- Neural Networks in Medicine

- Ontology and Medical Information

- Social Aspects of AI in Medicine

- Medical Signal and Image Processing and Techniques

- Ambient Intelligence and Pervasive Computing in Medicine and Health Care

\section{SECTION EDITORS}

- Lasek, Piotr, University of Rzeszow, Poland

- Paja, Wiesław, University of Rzeszów, Poland

- Pancerz, Krzysztof, University of Rzeszów, Poland

\section{REVIEWERS}

- Bamidis, Panagiotis, o Aristotle University of Thessaloniki, Greece

- Ciureanu, Adrian, University of Medicine and Pharmacy from Iasi, Romania

- Iantovics, Barna, Petru Maior University, Romania

- Jónsson, Björn Pór, IT University of Copenhagen, Denmark

- Komenda, Martin, Institute of Biostatistics and Analyses, Faculty of Medicine, Masaryk University, Brno, Czech Republic

- Komenda, Martin, Masaryk University, Czech Republic

- Kononowicz, Andrzej, Jagiellonian University Medical College, Poland

- Leniowska, Lucyna, University of Rzeszow, Poland

- Majernik, Jaroslav, Pavol Jozef Safarik University in Kosice, Slovakia

- Mapayi, Temitope, University of KwaZulu-Natal, Durban, South Africa, South Africa

- Olszewska, Joanna Isabelle, University of Gloucestershire, United Kingdom

- Papagelis, Manos, York University, Canada

- Perner, Petra, IBaI Leipzig, Germany

- Pokorná, Andrea, Institute of Biostatistics and Analyses, Faculty of Medicine, Masaryk University, Brno

- Rabl, Tilmann, Technische Universität, Berlin, Germany

- Schwarz, Daniel, Masaryk University, IBA, Czech Republic

- Stańczyk, Urszula, Silesian University of Technology, Poland

- Subbotin, Sergey, Zaporizhzhya National Technical University, Ukraine

- Víta, Martin, Faculty of Informatics, Masaryk university, Czech Republic

- Woodham, Luke, St George's University of London

- Yakovets, Nikolay, Eindhoven University of Technology, The Netherlands

- Zaitseva, Elena, University of Zilina, Slovakia

- Zary, Nabil, Nanyang Technological University 



\section{Analysys of the impact of disturbance on the arteriovenous fistula state classification}

\author{
Marcin Grochowina \\ University of Rzeszów \\ al. Rejtana 16, 35-310 Rzeszów, Poland \\ Email: gromar@ur.edu.pl
}

\author{
Lucyna Leniowska \\ University of Rzeszów \\ al. Rejtana 16, 35-310 Rzeszów, Poland \\ Email: lleniow@ur.edu.pl
}

\begin{abstract}
The quality of data sets used in the classification process has a significant impact on the outcome. The noise contained in the input data depending on the nature and intensity may have a different effect on the classification result. This paper presents the results of research on the quality and reliability of arterio-venous fistula classification based on the signal recorded under controlled disturbance conditions and in the model of artificial disturbations.

Typical environmental noise that may occur when the acoustic signal produced by the fistula was recorded and it is used as a disturbance. Its influence on the features extraction process and on the result of the fistula assessment was determined. Finally, a relationship between the intensity of the disturbances and the degree of shifting of the classification result to the pathological state of the fistula was demonstrated
\end{abstract}

\section{INTRODUCTION}

$\mathbf{T}$ HE problem of disturbations contained in the data sets in classification process is a well-known phenomenon. Each data recording is affected by external factors, and disturbations results in classification errors [7].

As it is usually the case in medical context, even slight errors might lead to grave consequences. Thus, the quality in decision support is of particularly high importance. Diagnosis based on deformed data can carry dangerous implications for the patient. Therefore, in the area of medical diagnostics, classifiers are built in such a way that, in case of uncertainty, they indicate a worse condition than it is in real. Such diagnosis can be then verified by other methods. Too optimistic diagnosis could lead to oversight threatening life and health symptoms.

This paper presents the testing results of sensitivity of arteriovenous fistula state classification for disturbations. Because our decision support system is based on the analysis of the acoustic signal produced by the blood flowing through the fistula, it is sensitive to sounds coming from the environment [1]. The level of ambient noise in locations like medical rooms or a private apartments is far from ideal, hence, we have to ascertain the system performance under such conditions.

As part of the study, a fistula acoustic signal was recorded in the anechoic chamber (in silence). Then, the artificially mixed disturbances were added into the signals previously recorded. In case of some patients the signal has also been registered under specified distortion conditions with known intensity. The data obtained was analyzed for the effect of disturbances on the fistula state estimation error.

\section{MATERIALS AND METHODS}

The research material was registered in the dialysis station of the Regional Clinical Hospital No.2 in Rzeszów. Collected sounds of radio-cephalic and brachio-cephalic fistulas were recorded from 38 patients. Registration was performed using specially developed recording head [4].

The teaching set was based on records of 31 patients. A total of 2645 points were obtained in the feature space, with each point mapping the signal fragment analysis for a single heartbeat.

The data from remaining 7 patients was used to build a test set uncorrelated with the training set. The test set contained a total of 10561 points in the feature space.

The feature space, originally 23 dimensional, was reduced to 7 dimensions by selection procedure [3]. Features taken into consideration in the classification process contain information about the amount of power contained in the frequency spectrum at intervals of the tertian center frequencies 63, 80, 100, $315,400,500$ and $630 \mathrm{~Hz}$.

The state of the fistula was assigned to one of the six classes named $\boldsymbol{a}-\boldsymbol{f}$, where class $\boldsymbol{a}$ was the best, and the class $\boldsymbol{f}$ was the worst condition.

The experiment was performed using Weka 3.7.13 package[13] using the $\mathrm{k}-\mathrm{NN}$ classifier with distance weighted voting and $k=10$ [2].

The recording process under natural conditions which takes into account the influence of external disturbance is shown in Fig.1.

However, two important issues emerged during the preparation of the research material:

1) natural sources of disturbances are not characterized by a single and fixed level of sound intensity,

2) there is no possibility of making recordings with all patients under controlled conditions of disturbance in the anechoic chamber.

Due to the inability to perform measurements under controlled conditions of a given level of disturbance, an artificial model of disturbance environment was used. For this purpose 


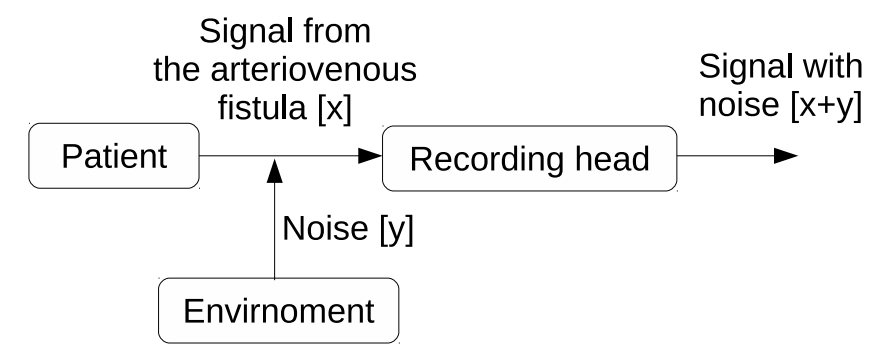

Fig. 1: The recording process under natural conditions

4 different sources were considered as potential disturbance signals (Tab.I):

- Urban noise - registered near the intersection of two busy streets,

- TV movie - sounds coming from a TV set that displays a movie,

- Conversation - lively discussion led by 3 people,

- Rock music - loudly played in the neighboring room hard rock music.

In addition, white noise as the fifth, control interfering signal was generated using software Audacity [14]. Registered and generated interfering signals were then used to create an artificial model of the noisy envirnoment.

TABLE I: Interference signals and their intensity

\begin{tabular}{l|c} 
Type of disturbance & Sound intensity during recording \\
\hline Urban noise & $70 \mathrm{~dB}$ \\
TV movie & $65 \mathrm{~dB}$ \\
Conversation & $60 \mathrm{~dB}$ \\
Rock music & $70 \mathrm{~dB}$
\end{tabular}

The impossibility of involving patients for multiple recording the fistulas sound under defined conditions was resolved by artificially mixing disturbances into the signals previously recorded in the dialysis station (Fig.2).

\section{Signal from}

the arteriovenous

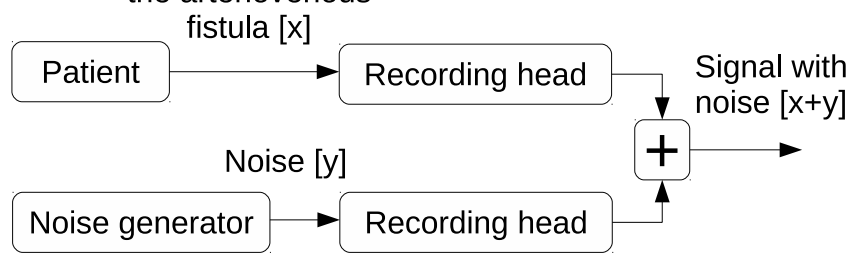

Fig. 2: The recording process with artificial disturbantion

For this purpose, two series of registrations were performed in the anechoic chamber, during which were recorded:

1) single patient fistula signal — during each recording, a previously recorded interfering signal with specific sound values of $55,60,65$ and $70 \mathrm{~dB}$ was played,

2) interfering signal - the recording header was applied to the patient's body at a distance from the fistula, and at the same time as the previous point, interfering sounds were played at the specified intensity.

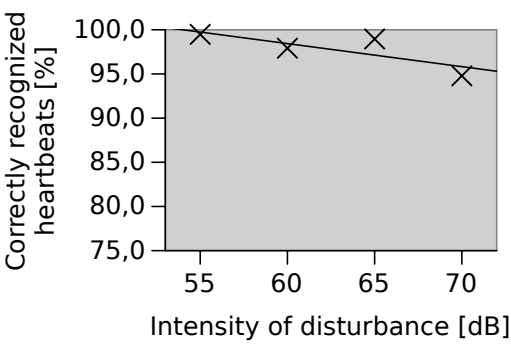

(a) White noise

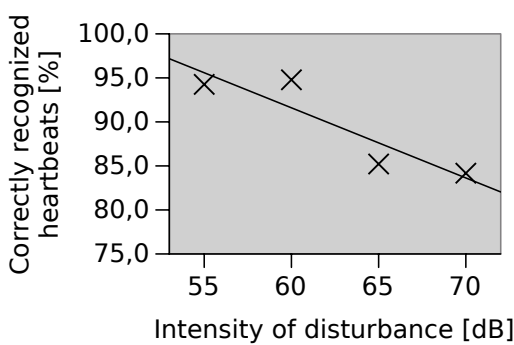

(b) Urban noise

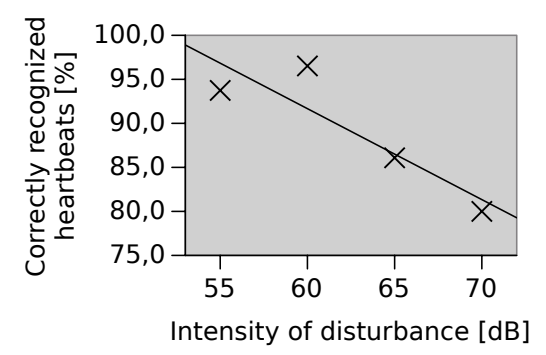

(c) TV movie

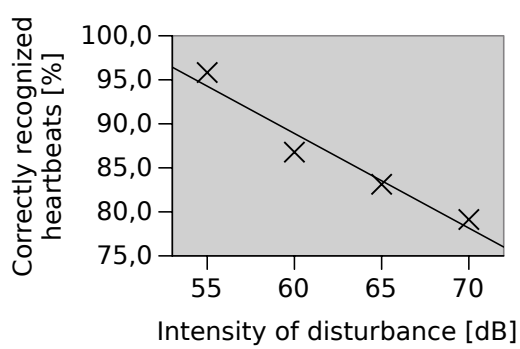

(d) Conversation

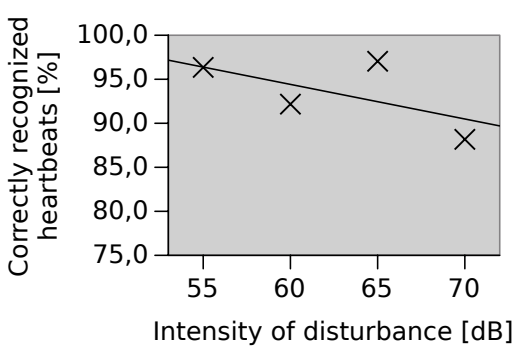

(e) Rock music

Fig. 3: Percentage of correctly recognized heartbeats in function of intensity of disturbance 
The volume value in both cases was measured with the Digital Sound Level Meter AZ8921 near the recording head [15].

Signals from patients registered in the dialysis station were added to the anechoic chamber recordings in Audacity. Each of the registered and artificially obtained signals was subjected to a classification process and the results were analyzed.

\section{RESULTS AND DISCUSSION}

A. The effect of disturbances on the operation of the feature extraction algorithm

First, after the registration of the research material, the effects of disturbances on the operation of the extraction algorithm were investigated [3].

For this purpose, the extraction algorithm was used on interrupted samples and on interfering samples. The disturbance was introduced into the signal in the artificial noising process.

The performance and robustness the feature extraction algorithm has no direct bearing on the quality of classification, however, increase the level of disturbance in a signal translates into a reduction of the number of points in feature space extracted from this signal.

The Tab.II shows the percentage of correctly extracted parts of signal corresponding to a single heartbeat. The number of heartbeats identified in the clean signal was assumed to be the baseline value.

TABLE II: Percentage of correctly recognized heartbeats

\begin{tabular}{c|ccccc}
\multirow{2}{*}{$\begin{array}{c}\text { Intensity of } \\
\text { disturbance }\end{array}$} & White & Urban & Type of disturbance \\
[dB] & noise & noise & movie & Conversation & Rock \\
\hline 55 & 99,5 & 94,3 & 93,7 & 95,8 & 96,3 \\
60 & 97,9 & 94,8 & 96,5 & 86,8 & 92,2 \\
65 & 99,0 & 85,2 & 86,1 & 83,1 & 97,0 \\
70 & 94,8 & 84,2 & 80,0 & 79,1 & 88,2
\end{tabular}

The data were presented graphically on Fig. 3. The charts were supplemented by trend lines.

In each of the cases studied, the increase in the intensity of the disturbances caused a decrease in the number of correctly interpreted heart rhythms.

The biggest impact on the number of correctly identified heartbeats had disturbances including sudden changes in sound intensity. Especially harmful were the conversation and containing a lot of dialogues television transmission of the TV movie.

White noise had the smallest effect on the extraction result. Because of its nature, it did not introduce sudden changes in sound intensity into the test signal, so it did not interfere with the process of detecting the extremes that indicate heartbeats.

Interestingly, rock music, as well as white noise, had little effect on the quality of the feature extraction algorithm, and apparently noise that is monotonous and devoid of sudden changes reduced the quality of extraction to $85 \%$.

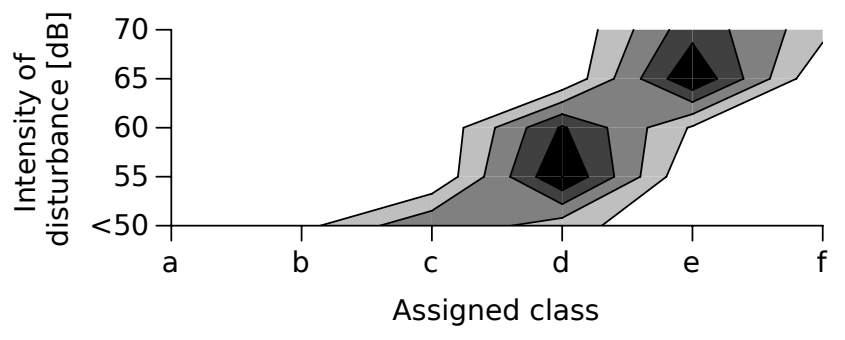

(a) White noise

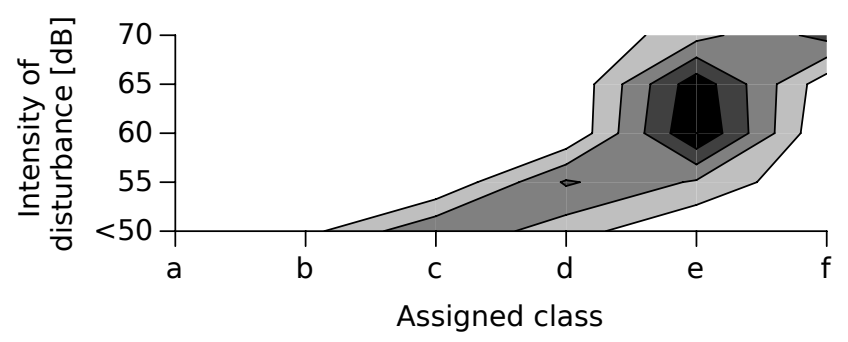

(b) Urban noise

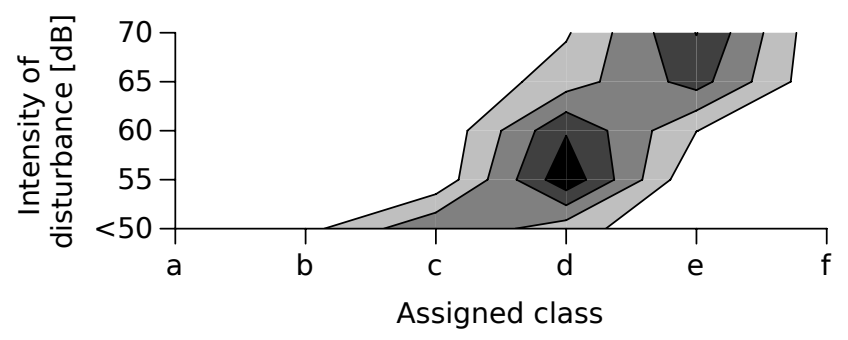

(c) TV movie

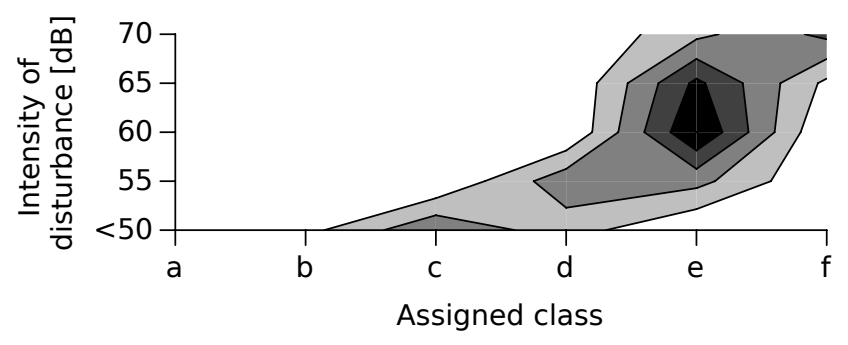

(d) Conversation

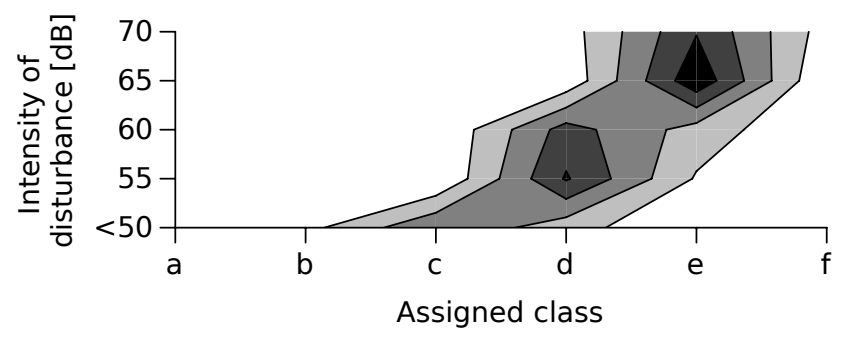

(e) Rock music

Fig. 4: Offset of the result of classification with increasing the disturbance intensity 


\section{B. Case study}

The next step was the analysis of a single case of a patient who agreed to register a fistula signal under controlled acoustic conditions of the anechoic chamber. During the registration of the fistula signal, the noise of a known intensity was played.

All the recorded signals were classified by the classifier built on the basis of 31 patients recorded with no disturbances. The results are shown in the Tab.III.

The first row of the table described "none" contains the result of analysis of 90 seconds of recording done in total silence. The results indicate that the condition of the examined patient's fistula was classified in class c. Leakage of results to neighboring classes $b$ and $d$ is the result of statistical uncertainty of classification and is a normal thing in the discussed problem of analysis of arteriovenous fistula state estimation.

TABLE III: Case 103 - complete result

\begin{tabular}{|c|c|c|c|c|c|c|c|}
\hline \multirow{2}{*}{$\begin{array}{l}\text { Type of } \\
\text { disturbance }\end{array}$} & \multirow{2}{*}{$\begin{array}{l}\text { intensity } \\
{[\mathrm{dB}]}\end{array}$} & \multicolumn{6}{|c|}{ Recognized class } \\
\hline & & $\mathrm{a}$ & $\mathrm{b}$ & c & $\mathrm{d}$ & $\mathrm{e}$ & $\mathrm{f}$ \\
\hline \multirow[t]{2}{*}{ none } & 0 & 0 & 11 & 46 & 23 & 0 & 0 \\
\hline & 55 & 0 & 0 & 0 & 80 & 0 & 0 \\
\hline White & 60 & 0 & 0 & 0 & 66 & 14 & 0 \\
\hline \multirow[t]{3}{*}{ noise } & 65 & 0 & 0 & 0 & 1 & 76 & 0 \\
\hline & 70 & 0 & 0 & 0 & 0 & 54 & 20 \\
\hline & 55 & 0 & 0 & 0 & 47 & 28 & 0 \\
\hline Urban & 60 & 0 & 0 & 0 & 0 & 74 & 0 \\
\hline \multirow{3}{*}{ noise } & 65 & 0 & 0 & 0 & 0 & 65 & 5 \\
\hline & 70 & 0 & 0 & 0 & 0 & 21 & 43 \\
\hline & 55 & 0 & 0 & 3 & 66 & 1 & 0 \\
\hline TV & 60 & 0 & 0 & 1 & 58 & 15 & 0 \\
\hline \multirow{2}{*}{ movie } & 65 & 0 & 0 & 0 & 19 & 43 & 1 \\
\hline & 70 & 0 & 0 & 0 & 11 & 50 & 1 \\
\hline & 55 & 0 & 0 & 0 & 40 & 35 & 0 \\
\hline \multirow[t]{3}{*}{ Conversation } & 60 & 0 & 0 & 0 & 0 & 76 & 0 \\
\hline & 65 & 0 & 0 & 0 & 0 & 61 & 11 \\
\hline & 70 & 0 & 0 & 0 & 0 & 23 & 43 \\
\hline \multirow{4}{*}{$\begin{array}{l}\text { Rock } \\
\text { music }\end{array}$} & 55 & 0 & 0 & 0 & 64 & 14 & 0 \\
\hline & 60 & 0 & 0 & 0 & 48 & 22 & 0 \\
\hline & 65 & 0 & 0 & 0 & 4 & 71 & 0 \\
\hline & 70 & 0 & 0 & 0 & 7 & 52 & 7 \\
\hline
\end{tabular}

The data in the table shows that even low intensity disturbances cause drastic changes in the assessment of the condition of the fistula. Each interfering signal tested has shifted the results of the classification towards the class indicating a condition that is worse than actual, with the amount of displacement increasing with the intensity of the disturbance.

Graphical visualization of results from the Tab.III was presented in Fig.4.

Areas marked with a darker color indicate the focus of classification hits on a given class. Gray areas indicate blurring results between classes.

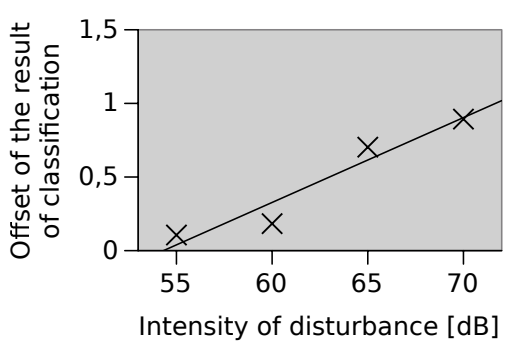

(a) White noise

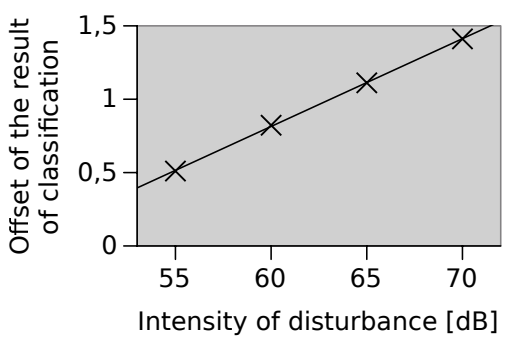

(b) Urban noise

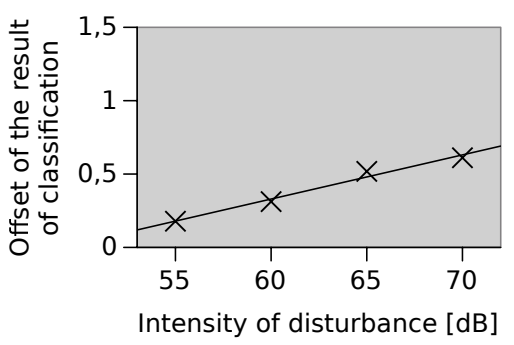

(c) TV movie

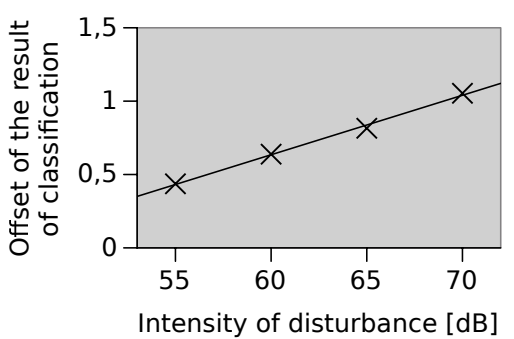

(d) Conversation

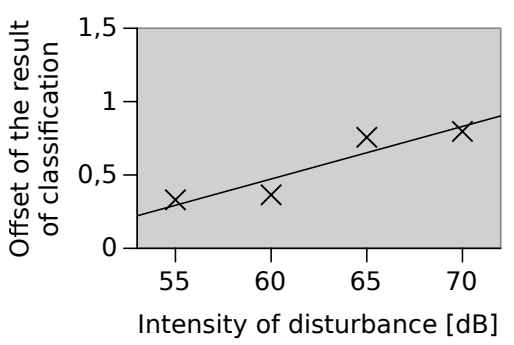

(e) Rock music

Fig. 5: Offset of the result of classification in function of intensity of disturbance 


\section{Analysis of all cases}

Assuming the case study as a model, an analysis of other fistulas sounds registered in dialysis station was performed. The only difference was that the disturbances were introduced artificially. The results obtained for each of the cases tested were consistent with the results obtained for the fistula registered in the anechoic chamber described in the previous section. It was assumed, therefore, that the model of artificial disturbance is correct and produces reliable results.

The results of all cases were averaged and shown in Tab.IV, and their graphical representation is shown in Fig.5. For the purposes of analysis, the letter labels $\boldsymbol{a}-\boldsymbol{f}$ were converted to numeric labels 1-6, which allowed for the calculation of the mean shift of the classification result relative to the base class.

TABLE IV: Offset of the result of classification as a result of disturbance

\begin{tabular}{c|ccccc}
\multirow{2}{*}{$\begin{array}{c}\text { Intensity of } \\
\text { disturbance }\end{array}$} & White & Urban & $\begin{array}{c}\text { Type of disturbance } \\
\text { TV }\end{array}$ & Conversation & Rock \\
[dB] & noise & noise & mivie & & music \\
\hline 55 & 0,11 & 0,51 & 0,18 & 0,44 & 0,33 \\
60 & 0,18 & 0,82 & 0,31 & 0,64 & 0,36 \\
65 & 0,70 & 1,11 & 0,52 & 0,82 & 0,76 \\
70 & 0,89 & 1,41 & 0,61 & 1,05 & 0,80
\end{tabular}

The increase in disturbance intensity in each of the cases studied led to an offset in the classification result, with the algorithm reporting worse condition of the fistula than what it actually was. It seems that the value of this offset can be approximated as linearly dependent on the intensity of the disturbance. If that is correct, extrapolating the trend line for smaller than tested noise intensities, it can be concluded that in order to obtain a sound quality sufficient for correct classification, the level of ambient noise should not exceed approximately $45 \mathrm{~dB}$.

\section{CONCLUSION}

The extraction algorithm proved to be quite resistant to disturbance - even loud noises at $70 \mathrm{~dB}$ did not prevent it from working, reducing only the amount of correctly recognized heart beats by $25 \%$. However, robustness of the extraction algorithm has not translated into maintaining the quality of the result of classification - noises of even slight intensity caused significant distortion of results.

A positive aspect of the study is the awareness that, if the recording is conducted under abusive acoustic conditions, the result will be unequivocally falsified in the pathology of the fistula. Its result will prompt the patient to verify rather than to optimistically accept misleading indication.
It is worth noting that only material recorded under low noise conditions allows for reasonable confidence in the results of classification process. While more complex denoising schemes (for example using a second microphone) seem possible, the complexity of implementing and testing such a solution makes it unfeasible.

\section{REFERENCES}

[1] Marcin Grochowina, Lucyna Leniowska and Piotr Dulkiewicz, "Application of Artificial Neural Networks for the Diagnosis of the Condition of the Arterio-venous Fistula on the Basis of Acoustic Signals," Brain Informatics and Health, Springer, 2014, pp. 400-411.

[2] Marcin Grochowina and Lucyna Leniowska, "Comparison of SVM and $\mathrm{k}-\mathrm{NN}$ classifiers in the estimation of the state of the arteriovenous fistula problem," Proceedings of the 2015 Federated Conference on Computer Science and Information Systems (FedCSIS), IEEE, 2015, pp. 249-254.

[3] Grochowina Marcin and Lucyna Leniowska, "The new method of the selection of features for the k-NN classifier in the arteriovenous fistula state estimation," Proceedings of the 2016 Computer Science and Information Systems (FedCSIS), IEEE, 2016, pp. 281-285.

[4] Grochowina Marcin and Lucyna Leniowska, "Analysis of the head prototype acoustic parameters for the acquisition of arteriovenous fistula signal," Mechanics in Medicine 12 (2014), University of Rzeszow, 2014, pp. 64-73. (in Polish)

[5] Mikkel Grama , Jens Tranholm Olesena , Hans Christian Riisa , Maiuri Selvaratnama and Michalina Urbaniaka, "Stenosis detection algorithm for screening of arteriovenous fistulae," 15th Nordic-Baltic Conference on Biomedical Engineering and Medical Physics (NBC 2011), Springer, 2011, pp. 241-244

[6] Duda Richard and Hart Peter and Stork David G "Pattern classification", John Wiley \& Sons 2012.

[7] Aha David and Kibler Dennis "Noise-Tolerant Instance-Based Learning Algorithms", IJCAI 1989, Citeseer, 1989, pp. 794-799,

[8] Jain Anil and Ross Arun "Learning user-specific parameters in a multibiometric system", Image Processing. 2002. Proceedings. 2002 International Conference on, IEEE, 2002, pp. I-I.

[9] Grochowina Marcin and Lucyna Leniowska, "The selection of features for the svm classifier in the arteriovenous fistula state estimation on the basis of acoustic signal", Acta Bio-Optica et Informatica Medica. Inżynieria Biomedyczna, vol.22(4), pp. 207-212. (in Polish)

[10] Domeniconi Carlotta and Peng Jing and Gunopulos Dimitrios "Locally adaptive metric nearest-neighbor classification", IEEE Transactions on Pattern Analysis and Machine Intelligence vol.1, IEEE, 2002, pp. 12811285.

[11] Aleksander Cisłak and Szymon Grabowski, "Experimental evaluation of selected tree structures for exact and approximate k-nearest neighbor classification," Proceedings of the 2014 Federated Conference on Computer Science and Information Systems, IEEE, 2014, pp. 93-100.

[12] Przemysław Wiktor Pardel and Jan G. Bazan and Jacek Zarychta and Stanisława Bazan-Socha, "A two-level classifier for automatic medical objects classification," Proceedings of the 2015 Federated Conference on Computer Science and Information Systems, IEEE, 2015, pp. 139-143.

[13] "WEKA documentation," http://www.cs.waikato.ac.nz/ml/weka/ documentation.html

[14] "Audacity manual," http://manual.audacityteam.org/\#tutorials

[15] "Digital Sound Level Meter AZ8921," http://www.az-instrument.com. tw/az-instrument/en/productsinfo/147.html 



\section{$7^{\text {th }}$ International Workshop on Advances in Semantic Information Retrieval}

$\mathbf{R}$ ECENT advances in semantic technologies form a solid basis for a variety of methods and instruments that support multimedia information retrieval, knowledge representation, discovery and analysis. They influence the way and form of representing documents in the memory of computers, approaches to analyze documents, techniques to mine and retrieve knowledge. The abundance of video, voice and speech data also raises new challenging problems to multimedia information retrieval systems.

We believe that our workshop will facilitate discussions of new research results in this area, and will serve as a meeting place for researchers from all over the world. Our aim is to create an atmosphere of friendship and cooperation for everyone, interested in computational linguistics and semantic information retrieval. The ASIR'17 workshop will continue to maintain high standards of quality and organization, set in the previous years. We welcome all the researchers, interested in semantic information retrieval, to join our event.

\section{TOPICS}

The workshop addresses semantic information retrieval theory and important matters, related to practical Web tools. The topics and areas include but not limited to:

- Domain-specific semantic applications.

- Evaluation methodologies for semantic search and retrieval.

- Models for document representation.

- Natural language semantic processing.

- Ontology for semantic information retrieval.

- Ontology alignment, mapping and merging.

- Query interfaces.

- Searching and ranking.

- Semantic multimedia retrieval.

- Visualization of retrieved results.

\section{SECTION EDITORS}

- Klyuev, Vitaly, University of Aizu, Japan

- Mozgovoy, Maxim, University of Aizu, Japan

\section{REVIEWERS}

- Carrara, Massimiliano, Universita di Padova, Italy

- Dobrynin, Vladimir, Saint Petersburg State University, Russia

- Goczyła, Krzysztof, Gdansk University of Technology, Poland

- Haralambous, Yannis, Institut Telecom - Telecom Bretagne, France

- Homenda, Wladyslaw, Warsaw University of Technology, Poland

- Jin, Qun, Waseda University, Japan

- Lai, Cristian, CRS4, Italy

- Leonelli, Sabina, University of Exeter, United Kingdom

- Nalepa, Grzegorz J., AGH University of Science and Technology, Poland

- Pyshkin, Evgeny, University of Aizu, Japan

- Shtykh, Roman, CyberAgent Inc., Japan

- Suárez-Figueroa, Mari Carmen, Ontology Engineering Group, Scool of Computer Science at Universidad Politécnica de Madrid, Spain

- Tadeusiewicz, Ryszard, AGH University of Science and Technology, Poland

- Vacura, Miroslav, University of Economics, Czech Republic

- Zadrożny, Sławomir, Systems Research Institute of Polish Academy of Sciences, Poland

- Kawrynowicz, Agnieszka, Poznan University of Technology, Poland 



\title{
Generating AIML Rules from Twitter Conversations
}

\author{
Hiroshi Yamaguchi, Maxim Mozgovoy \\ The University of Aizu \\ Tsuruga, Ikki-machi, Aizu-Wakamatsu, Fukushima, 965-8580 Japan \\ \{m5201115, mozgovoy\}@u-aizu.ac.jp
}

\begin{abstract}
A chat dialogue system or a conversational agent is a computer program designed to hold a conversation using natural language. Many popular chat dialogue systems are based on handcrafted rules, written in Artificial Intelligence Markup Language (AIML). However, manual design of rules requires significant efforts, so certain approaches for automating this process can be helpful. This paper presents some preliminary experiments to generate AIML knowledge automatically using conversation data acquired from Twitter. The experimental results show the possibility of obtaining natural-language conversation between the user and a dialogue system without the necessity of handcrafting its knowledgebase.
\end{abstract}

\section{INTRODUCTION}

A chat dialogue system or a conversational agent is a computer program designed to imitate a conversation with users [1]. In general, chat dialogue systems can be categorized into two types: task-oriented systems that are used to help the user to complete various tasks, and open-domain systems that aim to perform a natural conversation with the user [2].

A large number of dialog systems are rule-based, and one of the most popular mechanisms of representing rules is AIML (Artificial Intelligence Markup Language). AIML is a simple XML-based markup language that gained popularity after being used in a successful dialog system A.L.I.C.E. [3] that won the Loebner Prize three times. The main drawback of AIML-based systems lies in the large number of rules required to imitate a natural conversation, especially in case of opendomain systems. Therefore, AIML-based dialogue system require a lot of manual effort to describe its knowledgebase, leading to expensive and error-prone development process.

The goal of the proposed research is to demonstrate the possibility of generating AIML rules from Twitter conversations of real users. This method provides a potential possibility to greatly reduce the amount of manual effort needed to design an open-domain dialog system. We discuss the steps of this process, from gathering of the initial textual corpus to the operation of the resulting system, and provide example conversations.

Our ultimate aim is to develop a dialogue system supporting natural human-computer conversation, which can be useful in a variety of applications. The AIML-based approach is much simpler comparing to more advanced dialog system architectures [2]. Yet rule-base systems were still able to win the Loebner Prize, which proves their potential as human-like conversation agents. One of the main drawbacks of rule-based systems lies in the great number of rules that need to be handcrafted. We want to show that it is possible to reduce the amount of human effort by automating rule generation process, using a large dataset of real human conversations, such as Twitter dialogs.

\section{Elements OF AIML}

As mentioned in the previous section, AIML is based on XML, and thus consists of hierarchically organized elements. The following explanations will include fragments of Japanese-language conversations since we relied on a Japanese Twitter corpus in the experiments.

\section{A. AIML Categories}

Individual "units of knowledge" are known as categories in AIML. Each category should define at least two compulsory elements: a pattern that contains a sample input, and a template that contains the corresponding response of the bot [4]. There are three types of categories in AIML: atomic categories, default categories, and recursive categories [1].

Atomic categories contain patterns without wildcard symbols $\star,{ }^{\prime}, \ldots$, and \#. These categories describe exact responses for precisely defined user input:

$$
\begin{aligned}
& \text { <category> } \\
& \text { <pattern>おはよう</pattern> } \\
& \text { <template>おはようございます。</template> } \\
& \text { </ category> }
\end{aligned}
$$

Here if the user inputs おはよう (“morning”), the bot should reply おはようございます (“good morning”).

Default categories contain patterns with some wildcard symbols. These patterns can be matched against a family of input strings and thus can be used to describe more universal conversational elements. For example, the wildcard character * can be used to match one or more input words as follows:

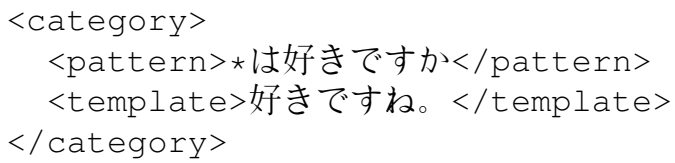

Here if the user input anything that ends with は好きですか (“do you like smth”), the bot should reply 好きですね。(“I like it.") The words matched with the wildcard characters can be programmed to appear in the bot response as well.

Recursive categories are used to handle certain patterns according to other rules already present in the system. In these categories, a $<$ srai $>$ tag is used to redirect the rule handler to another rule:

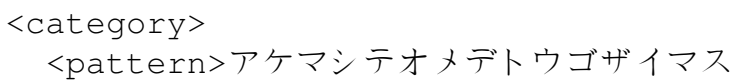




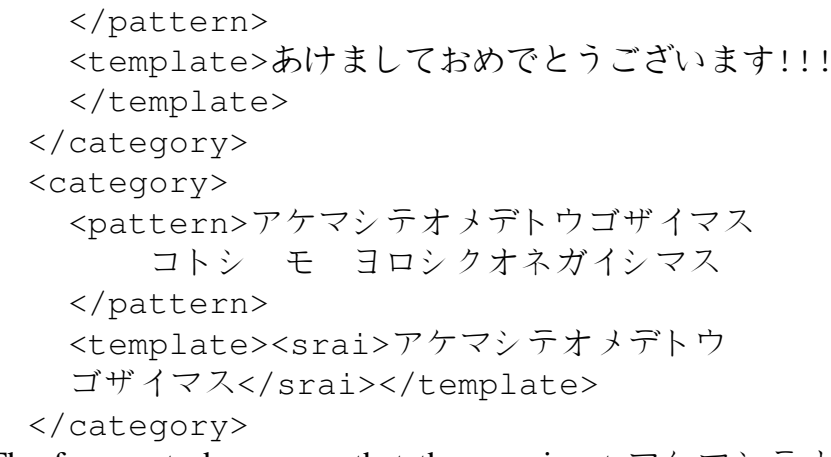

The fragment above says that the user input アケマシテオ メデトウゴザイマスコトシ モ ヨロシクオネガイシ マス ("Happy New Year") should be handled in the same way as アケマシテオメデトウゴザイマス (alternative way of wishing a happy New Year), thus the content of the tag <srai $>$ in the second category matches the content of the tag < pattern> in the first category. Recursive categories are typically used to handle synonyms and complex grammatical forms by splitting them into simpler parts.

\section{B. Interpreting AIML}

The set of AIML rules forming a chatbot's knowledgebase is processed with an AIML interpreter, responsible for actual dialogs with the user. There are several AIML interpreters available. We use Python-based pyAIML system, aimed to be fully compliant with AIML 1.0.1 standard [5].

AIML interpreter selects the rule that provides the longest match for the user input [1]. One can fine-tune rule prefernces by using wildcards in the rules, since different wildcards have different priorities.

\section{Generating AiML Rules}

\section{A. Twitter Streaming API}

The Internet contains numerous samples of conversation data. Perhaps, Twitter dialogs come relatively close to informal daily conversations, and there is an API available to retrieve individual tweets and tweet streams [6].

We used Twitter API to create the "Utterance Pairs" database, consisting of individual utterances and replies to them. The collected data corresponds to the tweets posted from October 2016 to April 2017. To build the database, more than 70 thousands utterance pairs were collected using Twitter Streaming API [7] that allows to extract tweets in real time. Individual tweets are tagged with a number of attributes, including the tweet language, timestamp, and in-reply-to field. We fetched Japanese tweets only, and extracted the content of each attribute.

\section{B. Converting Tweets to Rules}

The process of converting the raw tweet dataset into a set of AIML rules consists of the following steps.
1) Preprocessing: Raw tweet data contains numerous messages without any conversational meaning that have to be considered garbage for our purposes. These tweets typically consist of hyperlinks, hashtags and user names, or contain no Japanese characters. We remove such tweets from the source collection. We also remove very long messages, containing over 30 Japanese characters. In general, noise filtering in Twitter data is a well-researched problem [8], and we are planning to extend our filtering algorithm in the future.

2) Normalization: Each utterance pair consists of the input sentence and the reply to the input sentence. These pair elements will be mapped to the AIML tags <pattern> and <template>. Since the pattern has to be matched against the user input during the actual user-bot interaction, we increase the chances of a successful match by normalizing the patterns. A typical normalization procedure for English consists of stemming and capitalization of the pattern. For the tweets in Japanese, we tokenize the input into invididual morphemes using a morphological analyzer $\mathrm{MeCab}$ [9] convert them to katakana. We also use MeCab to perform a part-ofspeech tagging of the input text and eliminate non-significant morphemes, such as auxiliary verbs, postpositional particles, conjunctions, and pre-noun adjectivals.

3) Rule Generation: Unknown words that cannot be recognized by $\mathrm{MeCab}$ are substituted with wildcards, thus forming the patterns of default category rules. All other patterns are transformed into the rules of atomic category. As a result, we obtain the rules of the following kind:

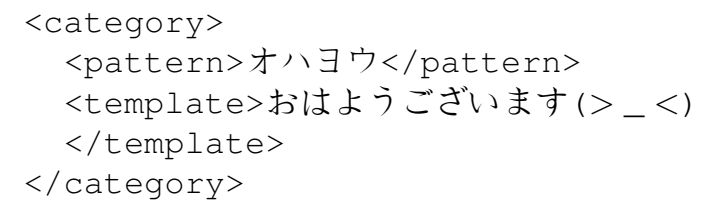

\section{RESUlts}

There are 71179 utterance pairs in the resulting dataset. After normalization and filtering, our program has generated 30991 atomic category rules and 39308 default category rules. By using the rules, our AIML bot is able to converse with the user, but the dialogs often contain unnatural responses, as the examples below show:

\section{Conversation 1: USER:こんにちは (Hello)}

BOT: こんにちは!!...君に会えるなんて今日はきっと良 い日だね (Hello, today is a good day to see you)

USER: それはよかった (It's good.)

BOT : 最高ですな。(It's really good.)

USER：何かいいことあった？(Any good news today?)

BOT: はるる (haruru)

USER：好きな人でもできたの？(Do you have a crush on someone?)

BOT: めちゃドン引きやん？ (笑) (Does something puts you off?)

USER：いや、別に引いてないけど（笑） (No, nothing) 
BOT: べつにWwww (Nothing.)

Conversation 2:

USER: おっはー (Good Morning)

BOT: おっはー！(Good Morning!)

USER: 今日めっちゃ暑くなるらしいよ (It seems that it

will be really hot today)

BOT : え- (Oh-)

USER：早く秋になってほしいね (I hope the season will change to fall early)

BOT: 仕方ない......ミラあったし...... (It can not be helped... There was Comic Market...)

USER: コミケとか行ったことないわ (I have never been to Comic Market)

Bот：はーい! コミケです！ (Yes, Comic Market!)

USER: コミケとか絶対行きたくないわ (I really don't want to go to Comic Market)

BOT: はーい！コミケです! (Yes, Comic Market!)

USER：アニ〉好き? (Do you like animation?)

BOT: 追加して (Please add it)

\section{Discussion AND CONCLUSION}

In this paper, we have presented a simple chatbot based on the AIML knowledge, extracted from Twitter streams. The bot is able to hold a conversation with the user, but many parts of the dialogs do not sound natural, and should be improved.

Probably, the largest shortcoming of the current implementation is related to the lack of context during conversations. The present bot implementation is based on the database of utterance pairs, while real dialogs include longer series of replies. AIML allows to incorporate context information in <that > tags, and we are planning to use this capability, and to substitute utterance pairs with longer dialog sequences.

Another difficulty is caused with the lack of approximate matching support in AIML. The only mechanism of approximate matching is wildcard characters, so the user-input strings are expected to be precisely specified, or, at least, to follow well-defined grammatical patterns. Without wildcards, even a relatively large dataset like ours can be insufficient for providing an exact match in many cases, and thus lead to unnatural conversations (we currently generate a random utterance from the knowledgebase if no match is found).

However, we are planning to address this problem in the next revision of the system. Our present goal was to show that Twitter data can be indeed used as a basis for automated AIML rules generation, and the current system demonstrates that this work can be accomplished, and the proposed approach has a potential to assist labor-intensive manual design of chatbot knowledge.

\section{REFERENCES}

[1] B. A. Shawar and E. Atwell, "ALICE chatbot: trials and outputs," Computación y Sistemas, vol. 19, no. 4, pp. 625-632, 2015.

[2] R. Higashinaka, K. Imamura, T. Meguro, C. Miyazaki, N. Kobayashi, H. Sugiyama, T. Hirano, T. Makino, and Y. Matsuo, "Towards an opendomain conversational system fully based on natural language processing.," in COLING, pp. 928-939, 2014.

[3] B. A. Shawar and E. Atwell, A comparison between ALICE and Elizabeth chatbot systems. University of Leeds, School of Computing research report 2002.19, 2002.

[4] R. Wallace, "The elements of AIML style," Alice AI Foundation, 2003.

[5] C. Stratton, "PyAIML: A Python AIML interpreter," https://github.com/andelf/PyAIML.

[6] F. Bessho, T. Harada, and Y. Kuniyoshi, "Dialog system using real-time crowdsourcing and twitter large-scale corpus," in Proceedings of the 13th Annual Meeting of the Special Interest Group on Discourse and Dialogue, pp. 227-231, Association for Computational Linguistics, 2012.

[7] Twitter, Inc., "Twitter Streaming API," https://dev.twitter.com/streaming/overview.

[8] R. Higashinaka, N. Kobayashi, T. Hirano, C. Miyazaki, T. Meguro, T. Makino, and Y. Matsuo, "Syntactic filtering and content-based retrieval of twitter sentences for the generation of system utterances in dialogue systems," in Situated Dialog in Speech-Based Human-Computer Interaction, pp. 15-26, Springer, 2016.

[9] T. Kudo, "Mecab: Yet another part-of-speech and morphological analyzer," http://mecab.sourceforge.net/, 2005. 



\section{$11^{\text {th }}$ Joint Agent-oriented Workshops in Synergy}

M ULTI-AGENT systems (MASs) provide powerful models for representing both real-world systems and applications with an appropriate degree of complexity and dynamics. Several research and industrial experiences have already shown that the use of MASs offers advantages in a wide range of application domains (e.g. financial, economic, social, logistic, chemical, engineering). When MASs represent software applications to be effectively delivered, they need to be validated and evaluated before their deployment and execution, thus methodologies that support validation and evaluation through simulation of the MAS under development are highly required. In other emerging areas (e.g. ACE, ACF), MASs are designed for representing systems at different levels of complexity through the use of autonomous, goal-driven and interacting entities organized into societies which exhibit emergent properties The agent-based model of a system can then be executed to simulate the behavior of the complete system so that knowledge of the behaviors of the entities (micro-level) produce an understanding of the overall outcome at the system-level (macro-level). In both cases (MASs as software applications and MASs as models for the analysis of complex systems), simulation plays a crucial role that needs to be further investigated.

\section{TOPICS}

JAWS'17 aims at providing a forum for discussing recent advances in Engineering Complex Systems by exploiting Agent-Based Modeling and Simulation. In particular, the areas of interest are the following (although this list should not be considered as exclusive):

- Agent-based simulation techniques and methodologies

- Discrete-event simulation of Multi-Agent Systems

- Simulation as validation tool for the development process of MAS

- Agent-oriented methodologies incorporating simulation tools

- MAS simulation driven by formal models

- MAS simulation toolkits and frameworks

- Testing vs. simulation of MAS

- Industrial case studies based on MAS and simulation/testing

- Agent-based Modeling and Simulation (ABMS)

- Agent Computational Economics (ACE)

- Agent Computational Finance (ACF)

- Agent-based simulation of networked systems

- Scalability in agent-based simulation

\section{Steering Committee}

- Cossentino, Massimo, ICAR-CNR, Italy

- Fortino, Giancarlo, Universita della Calabria, Italy

- Gleizes, Marie-Pierre, Universite Paul Sabatier, France

- Pavon, Juan, Universidad Complutense de Madrid, Spain

- Russo, Wilma, Universita della Calabria, Italy

\section{SECTION EdITORS}

- Fuentes-Fernández, Rubén, Research Group on Agentbased, Social \& Interdisciplinary Applications (GRASIA), University Complutense of Madrid (UCM), Spain

- Gravina, Raffaele, University of Calabria, Italy

- Niazi, Muaz, COSMOSE Research Group, COMSATS Institute of IT, Pakistan

- Seidita, Valeria, Università degli Studi di Palermo, Italy

\section{REVIEWERS}

- Antunes, Luis

- Azar, Ahmad Taher, Benha University, Egypt, Egypt

- Bernon, Carole, Universite Paul Sabatier, France

- Bremer, Joerg, University of Oldenburg

- Cipresso, Pietro, Applied Technology for NeuroPsychology Lab, Italy

- Davidsson, Paul, Malmö University, Sweden

- Derksen, Christian, University Duisburg-Essen, Germany

- Fortino, Giancarlo, Universita della Calabria, Italy

- Garro, Alfredo, University of Calabria, Italy

- Guerrieri, Antonio, University of Calabria, Italy

- Kowalczyk, Ryszard, Swinburne University of Technology, Melbourne, Victoria, Australia

- Linnenberg, Tobias

- Moench, Lars, FernUniversität Hagen, Germany

- Molesini, Ambra, Università di Bologna, Italy

- Özdemir, Serkan, University of Duisburg-Essen, Germany

- Petta, Paolo, OFAI, Austria

- Ribino, Patrizia, Istituto di Reti e Calcolo ad Alte Prestazioni - Consiglio Nazionale delle Ricerche, Italy

- Savaglio, Claudio, Universita della Calabria

- Sonnenschein, Michael, University of Oldenburg, Germany

- Sudeikat, Jan, Hamburg Energie GmbH, Germany

- Törsleff, Sebastian

- Unland, Rainer, Universität Duisburg-Essen, Germany

- Vizzari, Giuseppe, Università di Milano Bicocca, Italy

- Zia, Kashif, Sohar University, Oman, Pakistan 



\section{Collision-Free Agent Migration in Spatial Simulation}

Christopher Bowzer

\author{
Benjamin Phan
}

Computing and Software Systems

University of Washington Bothell

18115 NE Campus Way, Bothell, WA 98011

Email: \{cjbowzer, firstbbp, kcparker, mfukuda\}@uw.edu

\author{
Munehiro Fukuda
}

\begin{abstract}
Parallelization of agent-based models (ABMs) is one solution for scaling up their simulation size sufficiently covering more realistic problems. In order to break through memory limitation, some ABM simulators such as RepastHPC and FLAME enabled parallel simulation over a cluster system, (i.e. distributed memory). They visualize to agents remote processors' boundary data as ghost space or facilitate message broadcast among agents, so that agents can still share a full or partial view of their simulation space. Yet, ABMs encounter a parallelization problem where multiple agents may migrate to and thus collide with each other on the same logical coordinates, which should not occur in some applications, (e.g., traffic simulation where two vehicles cannot change to the same lane). Although such collision problems have been addressed algorithmically at a user level where an agent stops before or hops over another agent, moves faster or slower, ticks over time, or cuts coordinates finer, they yet require inter-agent synchronization such as serializing agent migration over all collision-inducing sub-spaces or cells, using a single thread. To facilitate collision-free agent migration more ef ficiently, we considered two migration algorithms named locationordered and direction-ordered migration, and implemented them over three ABM simulators: Multi Agent Spatial Simulation (MASS), RepastHPC, and FLAME. This paper discusses about programmability and execution performance among these three simulators in collision-free agent migration.
\end{abstract}

\section{INTRODUCTION}

$\mathbf{S}$ CALABILITY of simulation size is quite important for agent-based models (ABMs) including transport simulation [1], neural network simulation [2], ecological simulation [3], and immune system simulation [4], all requiring millions of agents to predict practical phenomena. Obviously, one solution is parallelization of underlying simulators or applications themselves. However, the biggest challenge is that most ABM applications have been based on a sharedmemory paradigm where agents interact with each other on a global simulation space. Although multithreading or even GPU computing has been applied to ABMs such as MATSim [1] and $\mathrm{TB}$ simulation [5] to reserve their share-memory-based implementations, some simulators such as RepastHPC [6], FLAME [7], and FluTE [3] enabled parallel simulation over a cluster system, (i.e., distributed memory) in support with MPI. Their implementation facilitates message broadcast among agents or visualizes to agents remote processors' boundary data as ghost space, so that agents can still share a full or partial view of their simulation space.
Yet, departed from a shared-memory paradigm, parallelized ABMs encounter another problem where multiple agents may migrate to and thus collide with each other on the same logical coordinates, which should not occur in some applications, (e.g. transport simulation where two vehicles cannot change to the same lane). In pedestrian simulation [8], each pedestrian agent avoids a collision by calculating its repulsive force with others, which results in $O\left(N^{2}\right)$ complexity. In traffic simulation [9], collision-inducing subspaces are serialized by a single thread, which requires careful space partitioning. Other simulators [10], [11] leave this collision problem on userlevel solutions where an agent stops before or hops over another agent, moves faster or slower, ticks over time, or cuts coordinates. These algorithms and implementations burden model designers with more programming complexity.

We particularly focus on agent migration ${ }^{1}$ over cellularbased or logical network space in transport simulation [12], [9] and artificial life [13], [14]. For this type of simulation, we consider three different algorithms that enforce collision-free agent migration, each named trial-and-error, location-ordered, and direction-ordered migration. Trial and error considers all such agent actions including a stop, a hop, or a faster/slower move upon encountering a collision. On the other hand, the location and direction-ordered algorithms move only agents at a time, which are located on the same group of coordinates, (e.g. those with their coordinates $[i, j]$ where $i$ and $j$ are divisible by three) or migrate to the same direction, (e.g. moving to the north). We implemented the location and directionoriented algorithms over our own ABM simulator named the MASS (Multi-Agent Spacial Simulation) library [15] as well as RepastHPC and FLAME as our benchmark platforms, and compared their programmability and execution performance.

The contribution of this paper is two-fold: (1) comparing three approaches to collision-free agent migration in parallel simulation and (2) demonstrating the programmability and performance superiority of the MASS library over RepastHPC and FLAME in collision-free migration. The rest of the paper is organized as follows: Section II surveys the conventional user-level and system-level collision-free migrations, and compares them with our location/direction-ordered algorithms in

\footnotetext{
${ }^{1}$ As mobile agents frequently use "migration", we use this terminology rather than "move".
} 


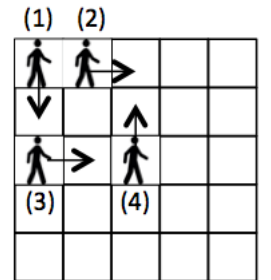

(a) Sequential migration

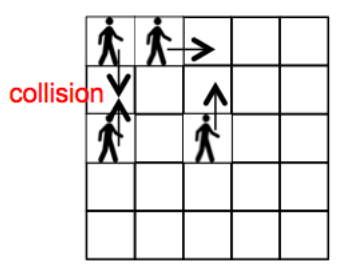

(b) Naïve parallel migration
Fig. 1. Agent migration over a meshed space

parallel simulation; Section III analyzes implementations of collision-free agent migration, each on MASS, RepastHPC, and FLAME; Section IV compares execution performance of these three simulation systems; and Section V summarizes the MASS-based collision-free agent migration.

\section{Agent Migration Algorithms}

Some ABM applications such as TRANSIMS [12], AIMSUN2 [9], Wa-Tor [14], and Sugarscape [13] simulate agent migration over a cellular-based or logical network space where at most one agent should reside at a given cell, node, or link, which however needs special cares in parallel simulation. This section gets started with a sequential execution of such agent migration over a space, thereafter raises an agent-collision problem in parallel execution, and examines three solutions to address this problem.

\section{A. Agent Migration in Spatial Simulation}

For simplicity, let us consider agent migration over a twodimensional (or 2D) cellular space as shown in Figure 1-(a). Sequential execution scans the space from upper left to lower right as moving an agent at each cell at a time. This gives a higher priority to an agent residing on an upper-left cell so that another at a lower-right cell can safely find its next destination cell without considering any potential collisions.

However, parallel execution removes such agent priorities that all agents in a space are allowed to migrate to their next destinations in any orders (see Figure 1-(b)), which causes an agent collision on a cell. Therefore, we need to consider parallel implementations to facilitate collision-free agent migration.

\section{B. Related Work}

Several collision avoidance algorithms have been proposed in both conceptual models and underlying parallel implementations. In conceptual models, Kirchner et al. [10] gave four resolutions for conflicts of pedestrians that move over a cellular automata model: (1) having an agent hop over or stop before another agent, (2) allowing an agent to move as far as possible, (3) dividing simulation time into sub-time steps, and (4) keeping a pedestrian from crossing the trajectory of another pedestrian that has already moved. Bandini et al. [11] gave another set of four methods to avoid agent collisions in pedestrian simulation: (1) changing their walking speeds, (2) modifying the space discretization towards a finer grain, (3) modifying the current time scale, and (4) combining methods 2 and 3 . However, these conceptual solutions still need careful implementation techniques in parallel simulation. If a simulation space is partitioned and mapped over a distributedmemory system, the simulator must facilitate so-called ghost space that visualizes a remote computing node's boundary data to the local node, so that each agent can observe others even on remote nodes. Furthermore, an agent must move into its destination cell exclusively no matter how finely simulation time and space are sliced. Otherwise "trial and error" will be repeated where multiple agents may end up moving to the same cell, in which case all except one that can stay there must follow one of the above resolutions to change their destination.

In parallel implementations, AIMSUN2 [9], a cellular-based transport simulator serializes agent migration over collisioninduing adjacent cells with a single thread, which handles these cells as a critical section and guarantees exclusive agent migration. However, users are burdened with grouping such collision-inducing cells into a non-interruptible block. In pedestrian simulation, Wagoum et al. [8] avoided a conflict of pedestrian agents by calculating each agent's repulsive force with others. Their simulator was parallelized with MPI where each rank maintains a neighborhood list of agents and computes their repulsive forces in parallel. However, the simulation results in $O\left(N^{2}\right)$ complexity yet within each MPI rank.

Our goal is to mitigate these user burdens incurred by conceptual models and to reduce complexity of parallel implementations and their computation.

\section{Parallel Algorithms}

We consider the following three collision-free migration algorithms: (1) trial-and-error migration, (2) location-ordered migration, and (3) direction-ordered migration.

1) Trial-and-Error Collision-Free Migration: As illustrated in Figure 2, this algorithm allows agents to collide with each other on the same destination cell. However, upon a collision, only one agent (with the highest identifier in most cases) can keep residing there while all the others must back off to their source cells and thereafter choose another available cell to move. This trial-and-error migration needs to be repeated until all agents find their next destination or conclude no more cells to go. It belongs to a so-called distributed-termination detection problem. If simulation wants to avoid solving this problem, it will end up examining every single direction of four or eight destinations in the von Neumann neighborhood, (i.e., north, east, south, and west) or the Moore neighborhood, (i.e., the former four directions plus north east, south east, south west, and north west). Unless parallel implementations guarantee serialization of agent migration as seen in AIMSUN2 [9], collision avoidance in conceptual models may result in trial and error.

2) Location-Ordered Collision-Free Migration: Let us consider a 2D space tiled with 3-by-3 cells, each numbered 0 through to 8. As shown in Figure 3, if we pick up only 


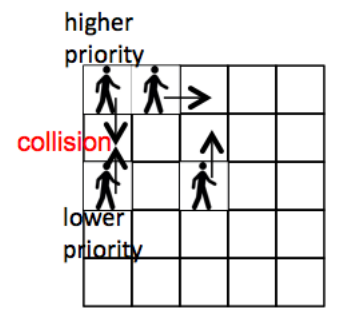

Step 1: collision detection and back off

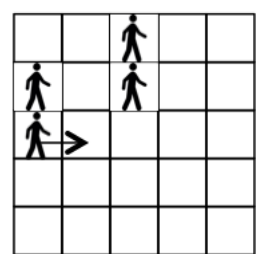

Step2: migration to another destination
Fig. 2. Trial-and-error collision-free migration

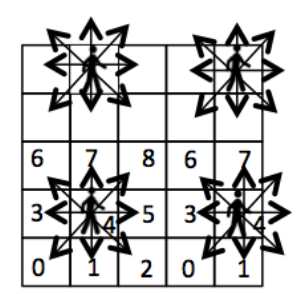

Moore neighborhood

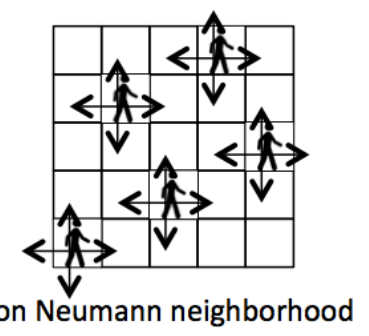

Fig. 3. Location-ordered collision-free migration agents residing on cells with the same number, (e.g., those with \#4), their migration based on the Moore neighborhood will cause no collision. Upon moving these agents, each cell must make its agent occupancy readable to its neighbors (as ghost space over distributed memory), so that the other agents will no longer choose the occupied cells. An iteration of agent migration and cell communication needs to be repeated nine times. The von Neumann neighborhood can reduce this repetition to five times. We call this algorithm location-ordered collision-free migration in the following discussions.

3) Direction-Ordered Collision-Free Migration: The third collision-free migration is direction-ordered. As shown in Figure 4, we will choose only agents that migrate toward the same direction. This ordered migration must be repeated eight times in the Moore and four times in the von Neumann neighborhood, (e.g., north first, east second, south third, and finally west in Figure 4). Note that, similar to the locationordered migration, all cells must inform their neighbors of their agent occupancy each turn of agent migration.

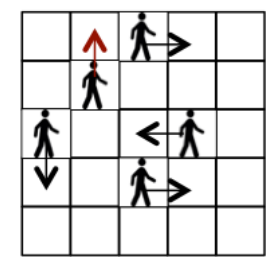

Turn 1: choose $\mathrm{N}$

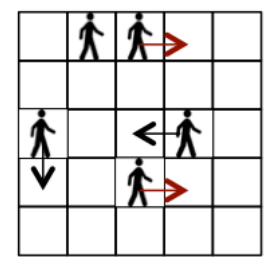

Turn 2: choose E

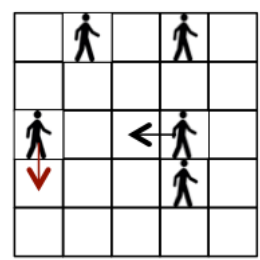

Turn 3: choose S
Fig. 4. Direction-ordered collision-free migration

\section{Pros and Cons}

Among the three collision-free algorithms, trial-and-error migration is potential to move agents in the least iterations, (e.g., $\leq 4$ in the von Neumann neighborhood). It prioritizes agents with a higher ID, which allows an agent with the highest ID to stay at a new destination and thus can reproduce a deterministic migration over multiple simulation runs. On the other hand, trial-and-error migration needs to have agents back off to their source cell upon a collision as well as to implement a distributed voting algorithm among all processes to see if they have to conduct the next trial. The vote needs three phases of collective communication: (1) a vote initiated from the master to all the slave processes, (2) ballots collected to the master, and (3) the result broadcast from the master. Although these three phases can be described in one MPI_Allreduce() function, the complexity of the underlying implementation doesn't change. Therefore, trial-and-error migration costs $p \times(5 c+a)$ where $p=\#$ trials, $5 c=$ agent sending, agent retracting, and three phases of communication needed for a distributed vote, and $a=$ agent synchronization overheads. We may avoid these distributed votes and therefore repeat four trials as default, in which case the cost will be $4(2 c+a)$.

Figure 5 shows how quickly the number of collisions grows and how many trial iterations are required as increasing the number of agents randomly distributed and migrating over a $1000 \times 1000$ simulation space. Even 10,000 agents, (i.e., only $1 \%$ population density) results in 103 collisions in average. This in turn means that trial-and-error migration in most cases can't complete in the first trial phase. With 50,000 agents, (i.e., $5 \%$ population density), the algorithm needs three trial phases. Finally it repeats four phases beyond 200,000 agents, (i.e., $20 \%$ population density). Considering practical applications that populate agents non-uniformly and move them toward the same direction, (e.g., toward an exit door in an evacuation), we may estimate $3(5 c+a)$ as the cost of trials and errors or end up $4(2 c+a)$ with four default trials.

The location-ordered migration is the easiest to implement at an application level by scheduling multiple events, (e.g., 9 in the Moore neighborhood), each moving agents that reside on cells with the same number: 0 through to 8 . However, unless an application regroups all agents into these nine events before migration, it must inevitably scan all agents at each event for the purpose of identifying which agent resides on which cell. Therefore, the location-ordered algorithm is the most expensive from the viewpoints of the number of iterations in migration and agent-scanning cost per iteration: $9(2 c+s)$ where $2 c=$ ghost-space updating and agent sending overheads and $s=$ memory access overheads for scanning agents.

The direction-ordered migration can complete less iterations of migration, (e.g., four in the von Neumann) than the locationordered algorithm although it has the same agent regrouping or scanning problem as the location-ordered migration. Therefore, the algorithm costs $4(2 c+s)$ where $2 c$ and $s$ are the same parameters as location-order migration. If this algorithm should be implemented at a system level, the system needs to 


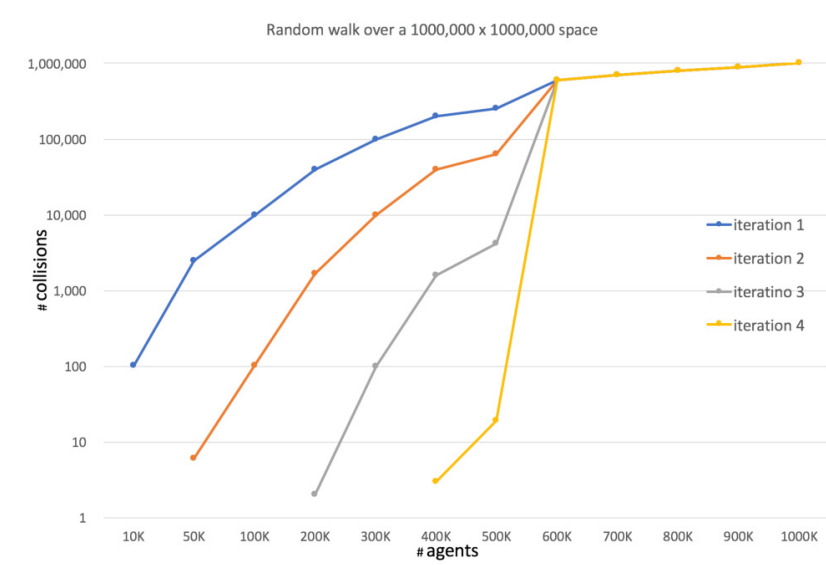

Fig. 5. \#Collisions in each trial phase when increasing agent population over a $1000 \times 1000$ space

directly examine each agent's data members to check its next destination.

In summary, the trial-and-error migration apparently burdens model designers with implementing inter-agent synchronization as well as a distributed voting algorithm, and it has little performance superiority as discussed above. Therefore, we will focus on location- and direction-ordered migration algorithms in our programmability analysis of three agentbased systems: our MASS library, RepastHPC, and FLAME.

\section{IMPLEMENTATIONS OF PARALlEL MigRATION}

This section compares MASS, RepastHPC, and FLAME systems in coding agents' random walk over a 2D space, (named RandomWalk). Our analysis covers location-ordered collision-free migration at an application level as well as direction-ordered migration at a system level.

\section{A. MASS}

We have developed the MASS (Multi-Agent Spatial Simulation) library in Java and C++. Places and Agents are keys to the MASS library. Places is a multi-dimensional array of elements that are dynamically allocated over a cluster of multi-core computing nodes. Each element is called a place, is pointed to by a set of network-independent array indices, and is capable of exchanging information with any other places. Agents are a set of execution instances that can reside on a place, migrate to any other places with array indices (thus as duplicating themselves), and indirectly interact with other agents through variables local to the current place.

Parallelization with the MASS library uses a set of multithreaded communicating processes that are forked over a cluster of multi-core computing nodes with JSCH in Java or libssh2 in $\mathrm{C}++$ and are connected to each other through TCP sockets. Multi-threads take charge of method call and information exchange among places and agents in parallel. A user designs a behavior of a place and an agent by extending the Place and Agent base classes respectively. They are populated through the Places and Agents classes. Actual computation is performed between MASS.init() and MASS.finish(), using the following major $\mathrm{C}++$ methods, each performed in parallel [15].

\section{Places Class}

- public Places(int handle, String className, int boundary, void *argument, int argSize int dim, int size[]) instantiates a shared array with size and a ghost space with boundary from className as passing an argument to the className constructor.

- public void* callAll(int functionId, void *arguments, int argSize) calls the method specified with functionId of all elements as passing arguments[i] to element[i], and receives a return value into (void $*$ ) [i].

- public void exchangeAll(int handle, int functionId, vector $<$ int* $>*$ destinations) calls from each element to a given method of all destination elements, each indexed with a vector element, and exchanges data among the elements.

- public void exchangeBoundary( ) exchanges boundary data with neighboring cluster nodes as a ghost space.

\section{Place Class}

- private vector $<$ int $>$ size, index maintain the size of the shared array that each element belongs to and the index of each array element.

- public void* callMethod(int functionId, void *arguments) is invoked from Places.callAll() or exchange-All() so as to call a function specified with functionId.

\section{Agents Class}

- public Agents(int handle, String className, void *arguments, int argSize, Places *places, int population) instantiates agents from className, passes arguments to their constructor, and populates them over a given Places, based on Agent.map().

- public void* callAll(int functionId, void *arguments, int argSize, int retSize) is the same as Places.callAll().

- public void manageAll() updates each agent's status, based on its latest calls of migrate(), spawn(), and kill(). These methods are invoked within callAll().

\section{Agent Class}

- migrate(int[] index...) allows a calling Agent to migrate or propagate itself to one or more Places specified with index upon Agents.manageAll().

- spawn(int nChildren, Object arguments) spawns children as passing arguments to them.

- $k i l l()$ terminates a calling Agent.

- public void* callMethod(int functionId, Object argument) is the same as Place.callMethod().

Figure 6 shows abstract $\mathrm{C}++$ code for parallelizing RandomWalk with MASS. We use location-ordered agent migration, focusing on the von Neumann neighborhood, (i.e., including four $\mathrm{N}, \mathrm{E}, \mathrm{S}$, and $\mathrm{W}$ neighbors) while numbering places from 0 to 8 and scheduling nine turns of migration as in the Moore neighborhood for simplicity. The main() function (lines 26-38) serves as a simulation scenario. It creates an $s z \times s z$ virtual land over which the $n$ number of nomad agents 


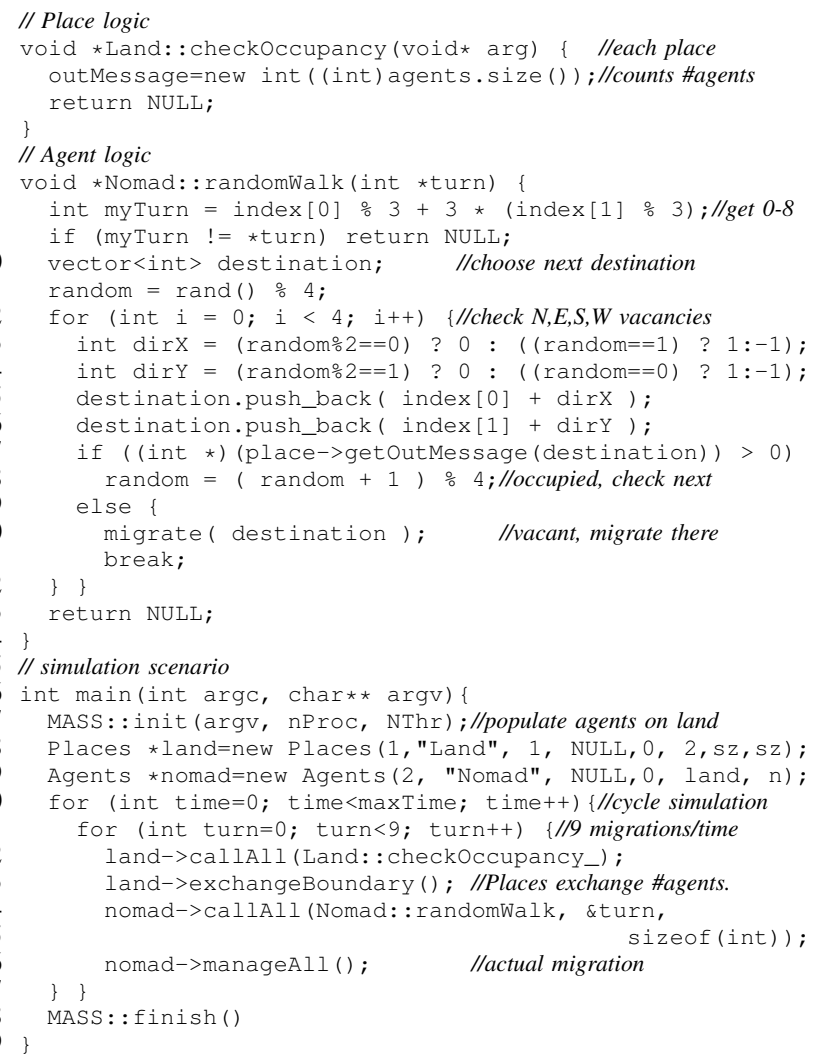

Fig. 6. Application-level agent migration in MASS

are populated (lines 28-29). Thereafter, a cycle simulation gets started with time $=0$ through to maxTime -1 (line 30). For each cycle, we schedule nine turns of agent migration (line 31). For each turn, we need to update each place's ghost space (stored in outMessage) (lines 32-33), for whose purpose each land updates its agent population in its outMessage (lines 2-5). Then, each agent invokes its randomWalk() function (lines 3435 and thereafter 7) that checks if it is this agent's turn (lines 89), randomly chooses one of its four neighboring places (lines 10-16), checks its occupancy (line 17), and decides to migrate there or to choose another place (line 18 or 20). The actual agent migration is committed at once by manageAll() (line 36).

Needless to say, model designers do not want to be aware of agent collisions that are not the essence of their simulation and thus should be supported by a system. Therefore, we have implemented direction-ordered collision-free migration in MASS. The implementation used the 2D von Neumann neighborhood only. As shown in Figure 7, RandomWalk can remove all the code related to collision avoidance and therefore focus on agents' randomWalk() function.

\section{B. RepastHPC}

RepastHPC is an MPI-supported parallel simulation platform for agent-based modeling, which was developed by the Argonne National Laboratory. In RepastHPC, Context is an

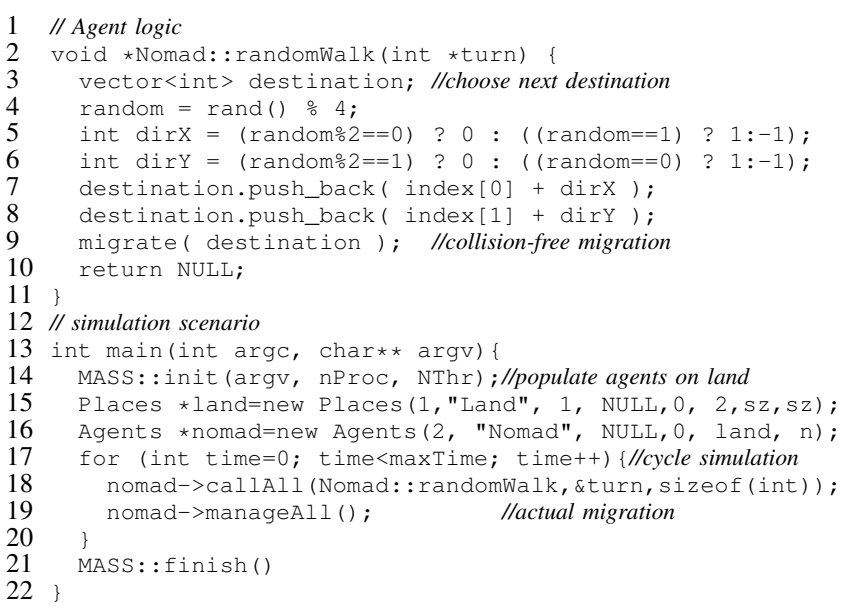

Fig. 7. System-supported agent migration in MASS

execution environment that populates agents over a given Projection instance such as a shared network, gird, and space. We can regard RepastHPC's contexts, agents, and projections as MASS processes, agents, and places respectively. RepastHPC has the following similarities to MASS:

1) Ghost space views adjacent MPI ranks' simulation boundary so that agents in each rank can see their neighborhoods' information including sub-space occupancy by other agents.

2) Agent migration are all committed at once by projection.balance() (similar to MASS agents.manageAll()) and are carried out physically by moving agents to a remote rank.

On the other hand, RepastHPC is different from MASS in the following aspects:

1) Simulation events are scheduled in a context separately from main(), whereas MASS schedules events as forloop iterations in main().

2) agent collisions are not supported at the system level unlike MASS

3) spatial operations are passively invoked from a context or each agent, whereas MASS allows each place to manipulate agents through its agents vector.

In a similar way to MASS, we parallelized RandomWalk with RepastHPC. Figure 8 shows the abstract code in $\mathrm{C}++$. The main() function initializes RepastHPC (line 35), instantiates a context (line 36), populates agents (line 38), schedules nine turns or events of agent migration (line 39), and starts a cycle simulation (line 40). In RepastHPC, Context schedules actual events and controls agents. Its schedule() function (lines 2-6) needs to declared these nine migration events separately, each calling the move() function (lines 7-16). This actually means that, upon each invocation, move() must scan all agents from the top in the context (lines 8-9) for examining each agent's current location (lines 10-11). It invokes Nomad::randomWalk() if a given agent resides on a cell with the current turn. The randomWalk() logic is the same as that 
of MASS: repeating a selection of the next destination up to four times until finding a vacant neighbor.

As compared to MASS, RepastHPC facilitates to its users more generic and various types of simulation space and gives them more freedom of agent management, which however tends to burden model designers with a much steeper learning curve and to increase their code size.

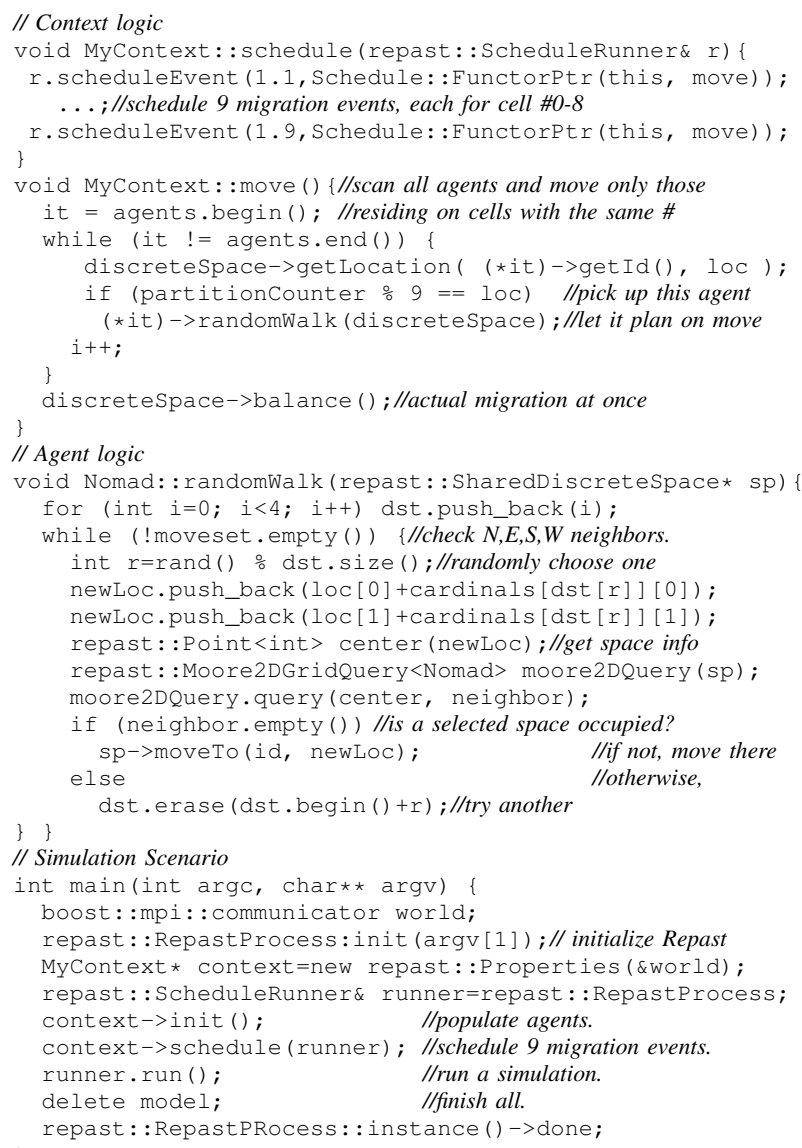

Fig. 8. Agent migration in RepastHPC

\section{FLAME}

FLAME is another MPI-based ABM system, originally developed by University of Sheffield, UK. Since FLAME users write their simulation in $\mathrm{C}$, for object-based programming purposes, they need to declare all agents, their data and method members, and environment variables in XML, in a similar way to $\mathrm{C}++$ header files. Although FLAME uses environment variable such as env_north_x, env_south_y, env_min_x, and env_max_y to shape a simulation space, it does not instantiate any actual space on memory beyond making these variables accessible to all agents. Instead, agents are capable of broadcasting their messages among one another through message boards, each launched at a different MPI rank. Contrary to MASS and RepastHPC, both moving agents over a distributed simulation space, FLAME is considered as a collection of communicating, state-transitting agents statically mapped over MPI ranks. FLAME's other notable differences include:

1) Simulation events are scheduled in the model XML separately from auto-generated main() and C-described agents.

2) agent collisions are impossible to support at the system that cannot keep track of agent locations except looking into their initial coordinates to uniformly map agents over the system.

3) spatial information is captured by and maintained inside each agent that informs the others of its migration through a broadcast message.

Figure 9 describes RandomWalk in FLAME. The source code consists of two files: (1) an XML file for declaring events and agent functions and (2) a C program for describing each agent's random-walk logics. More specifically, XML schedules 10 events for each simulation cycle (lines 2-6) where the first event at time 0 calls each nomad agent's new_turn function (lines 11-15) and the rest nine events, each at time 1-9 invoke random_walk() (lines 16-20). The new_turn() function computes each agent's current cell number: 0-8 (lines 24-26). Only agents whose MY_TURN equals the current time 1 to 9 can invoke random_walk() that allows the calling agent to examine four potential neighbors to migrate to (lines 29-46). Although FLAME's random_walk() is almost similar to the logics of MASS and Repast, the notable difference is that a FLAME agent needs to read all the others' current locations to examine a potential collision (lines 33-37).

As compared to MASS and RepastHPC, FLAME is purely agent-based. There is no actual implementation of simulation spaces. Functions including main() necessary to start and stop a simulation, to allocate and deallocate memory space, and to facilitate inter-agent communication are all automated, which allows model designers to focus on their own agent descriptions.

\section{Programmability Comparisons}

Given the RandomWalk code above in MASS, RepastHPC, and FLAME, we compare their programmability in collisionfree migration from the following four aspects.

1) Agent Handling and Description: For scheduling nine migration events, (each corresponding to moving agents on cells with the same number 0-8), MASS and FLAME can describe them in a for-loop or a simple XML statement, whereas RepastHPC needs to declare nine events separately. For each migration event, all these three systems must scan an entire list of agents whether or not they need to migrate at the current event. However, decision on moving a given agent can be made by each agent itself in MASS and FLAME while RepastHPC needs to describe such an agent selection separately in its Context logic, (i.e., in MyContext::move()), which implies that MASS and FLAME agents tend to be more self-descriptive. This is RepastHPC's inherent problem in using a separate Context class as an agent controller. Contrary to that, MASS decouples agents from places and FLAME is purely agent-based with no space concept. 


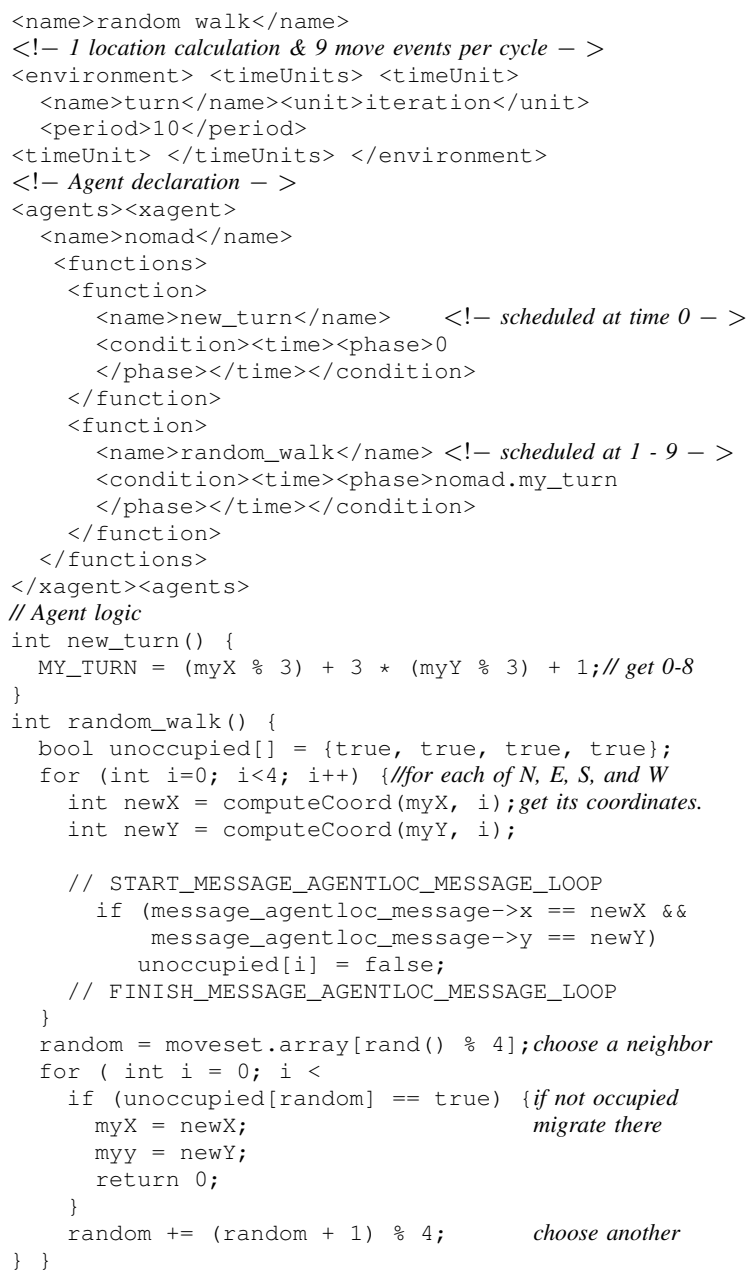

Fig. 9. Agent Migration in FLAME

2) Collision Detection: Agent migration needs a space concept to detect agent collisions on given coordinates. Both MASS and RepastHPC facilitate such a space as the Places or the SharedDescreteSpace class, both visualizing remote processor boundary as ghost space to local agents. For this purpose, logically neighboring processors have to exchange their ghost space with each other at each migration event. On the other hand, due to lack of space concepts, FLAME must have each agent broadcast its current location to all the others and compare its coordinates with others to avoid collisions. This results in $N \times N$ message exchanges and comparisons by each migration event.

3) System-level Collision-free Migration: System-level location-ordered migration is feasible as far as a space concept is supported by a system. From this standpoint, MASS and RepastHPC have potential to implement this migration algorithm. However, direction-ordered migration is more challenging unless a system can access each agent's next destination. RepastHPC can capture such agent information only when an agent calls projection.balance() to migrate over a space, which is too late for collision detection. Needless to say, FLAME cannot access each agent's private data. The reason why MASS was able to support direction-ordered migration at the system level is that MASS can forecast each agent's next destination by comparing a difference in coordinates between the agent and its current place, (i.e., Agent.index[] and Place.index[]).

4) Quantitative Measures: For each of MASS, RepastHPC, and FLAME, we measured the number of files and lines of code (LOC) necessary to implement RandomWalk and embed an application-level location-ordered migration algorithm in it.

\begin{tabular}{l|rr}
\hline Systems & \#Files & Lines of Code (LOC) \\
\hline \hline MASS & 5 & 727 \\
RepastHPC & 5 & 921 \\
FLAME & $5(16)$ & 783 \\
\hline
\end{tabular}

All of these three systems need five different files. Both MASS and RepastHPC requires (1) a simulation scenario in main.cpp, (2) a simulation space definition in Land.h, (3) its implementation in Land.cpp, (4) an agent definition in Nomad.h, and (5) its implementation in Nomad.cpp. On the other hand, FLAME needs one XML definition, three Cbased agent-controlling functions, and one more $\mathrm{C}$ program that describes simulation rules. Notable is that, since FLAME automatically generates $\mathrm{C}$ template and stub files from the XML definition, the nominal number of files is 16 . For the code size comparison, MASS was slightly the smallest. More specifically, it was $7 \%$ smaller than FLAME. Although we gave RepastHPC a few advantages by re-engineering the source code and subtracting space and comment lines from it, its LOC could not beat out neither MASS or FLAME. This was resulted from RepastHPC's coding style as discussed above: nine events must be declared independently and agent management need to be implemented in Context separately.

From these four observations, we feel that MASS facilitates collision-free migration more efficiently than the other two.

\section{Performance Evaluation}

We coded all the four RandomWalk programs with MASS both at the application and system levels, with RepastHPC, and with FLAME. They respectively correspond to Figures 6, 7, 8, and 9 in Section III. To compare their execution performance, we implemented system-level direction-ordered collision-free agent migration in MASS C++ and installed RepastHPC 2.1.0, and FLAME 2.1.3 on a cluster of $163.4 \mathrm{GHz}$ Intel Core i7 desktop machines, each equipped with 16GB memory and running Ubuntu 14.04. Figure 10 shows an execution of RandomWalk with the MASS library.

\section{A. Performance Comparison of MASS, Repast-HPC, and FLAME}

We first compared MASS at both application- and systemlevel implementations (called MASS App and MASS Lib respectively in the following discussions) with RepastHPC and FLAME for their parallel performance.

Figure 11 shows their performance with four computing nodes as increasing the number of threads from one to four. 

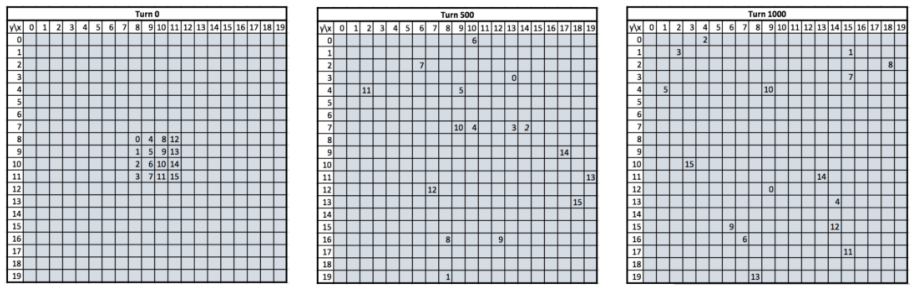

Fig. 10. A random walk execution with the MASS library

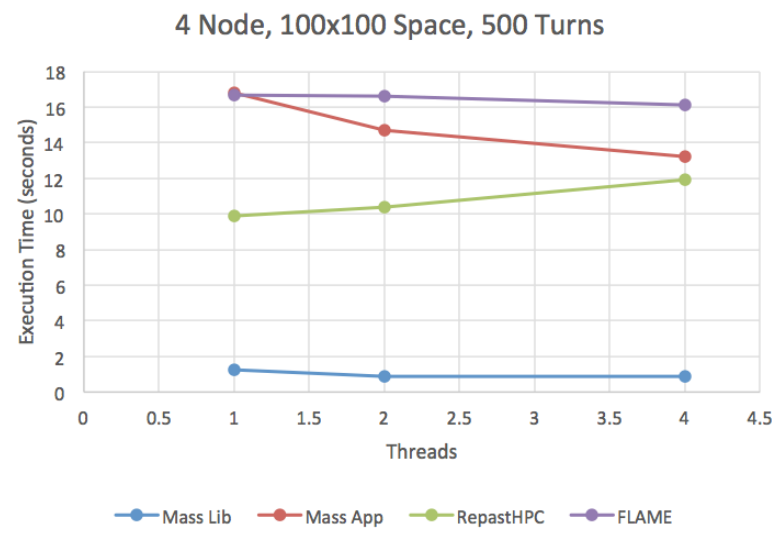

Fig. 11. Performance of MASS, RepastHPC, and FLAME running over 4 computing nodes

In this evaluation, 400 agents walked over a $100 \times 100$ space. With a single thread, RepastHPC performed 1.7 times better than MASS App and FLAME. This is because RepastHPC's Context object quickly scans a list of agents to invoke each agent's randomWalk function, whereas MASS needs two phases of operations: (1) updating ghost space among neighboring places and (2) calling each agent's randomWalk(); and FLAME needs $N \times N$ inter-agent communication over four computing nodes. However, using four threads per each node, RepastHPC slowed down due to its serialized accesses to the same agent list by multithreaded Context objects. FLAME could not improve its parallel performance because of its inter-agent communication overheads. On the other hand, MASS App improved 1.27 times faster than its singlethreaded execution. This is because MASS controls finegrained synchronizations among threads.

Notable is MASS Lib's performance that showed 7.9 to 13.6 times faster than RepastHPC. The biggest factor of this improvement is that MASS Lib groups agents into north, east, south, and west directions, thus scans the agent list only once for this grouping work, and completes migration in four turns. On the other hand, RepastHPC needs nine turns of migration where each turn must scan the entire agent list.

Figure 12 measures execution performance with 16 computing nodes. The evaluation was able to extend its simulation size to 3600 agents over a $300 \times 300$ space. The reason was that both RepastHPC and FLAME needed a large space of memory

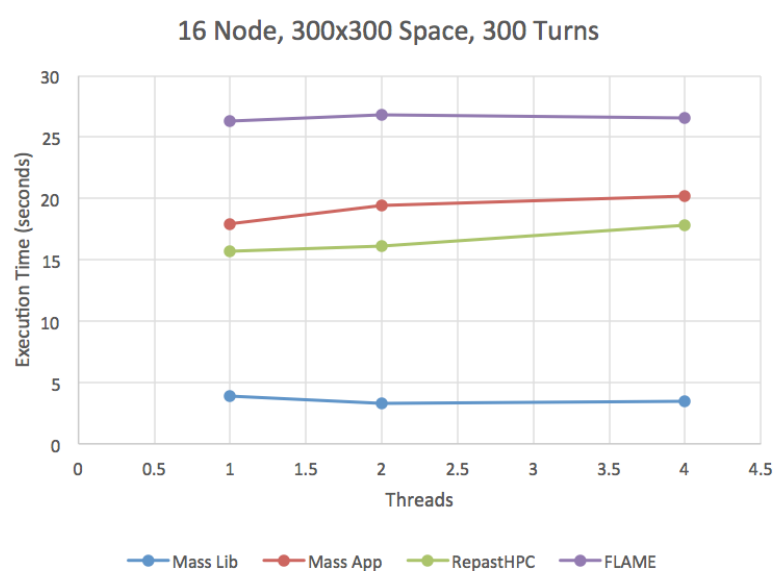

Fig. 12. Performance of MASS, RepastHPC, and FLAME running over 16 computing nodes

that couldn't fit a smaller number of computing nodes. Similar to the four-way parallelization, FLAME was the slowest and unable to improve its performance with multithreading due to its communication overheads. RepastHPC ran faster than MASS App whose performance loss was however mitigated to 14\%-21\% slow-down as we used a larger problem size. Again, MASS Lib performed fastest among the four test cases, more specifically 4.0 to 5.1 times better than RepastHPC.

\section{B. Performance of MASS Library}

We focused on the MASS library's CPU scalability for its collision-free agent migration. Our performance measurement walked 3,600 agents over a $300 \times 300$ space, (i.e., $4 \%$ population density) as increasing the number of nodes from one to 16 and the number of threads from one to four.

Figures 13 and 14 demonstrate MASS App's and Lib's CPU-scalable execution respectively. Despite RandomWalk's fine-grained pallalelization where each agent computes only the next destination to visit, MASS App showed that 16 singlethreaded computing nodes performed 2.2 times faster than a sequential execution. MASS Lib scaled up its parallelization to 8 ways, (i.e., four computing nodes, each with two threads) and ran 2.9 times faster than a sequential execution.

These results confirm three advantages of the MASS library: (1) both MASS App and Lib are CPU scalable; (2) MASS Lib is at least four times faster than the other ABM simulators; and (3) MASS saves memory space efficiently to run a larger simulation with a fewer computing nodes.

\section{CONCLUSIONS}

Needless to say, there are many applications that need no agent migration, which FLAME could benefit well with static agents that communicate with each other. On the other hand, a number of application domains need to observe agent migration over a space: vehicle flow in traffic simulation, pedestrian flow in emergency evacuation, and immune cells' attack to bacteria, etc. Model designers used to address such 
Mass Application Level Collision Avoidance (300x300 grid, 500 turns)

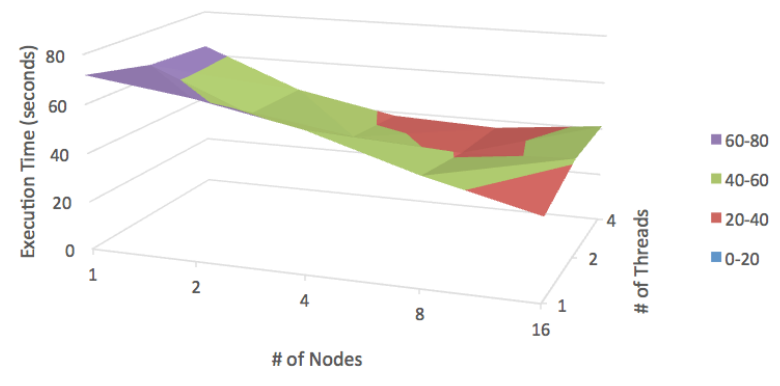

Fig. 13. Performance of MASS application-level collision-free migration

Mass Library Level Collision Avoidance (300x300 grid, 500 turns)

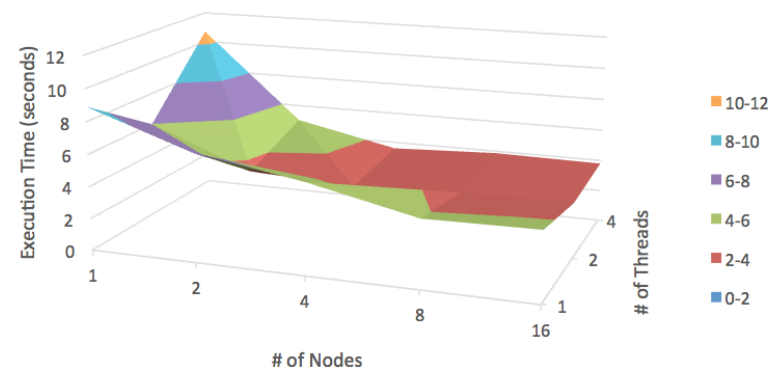

Fig. 14. Performance of MASS system-level collision-free migration

agent collisions in conceptual models [11], [10] by controlling agent migration or slicing simulation time and space finely or to implement collision avoidance mechanisms in their application-specific parallel execution [8], [9]. Therefore, from the programmability viewpoint, it is worthwhile supporting system-level collision-free agent migration in general-purpose simulators.

As shown in Figure 5, agent collisions increase in proportional to the growth of agents' population density until the growth reaches $50 \%$ of the entire simulation space. As observed in Figure 3, if agents are uniformly distributed to occupy less than only $1 / 9$ or $1 / 5$ of an entire simulation space in the Moore or the von Neumann neighborhood, the probability of agent collisions remains negligible (but does not result in zero even with the $1 \%$ population density). In such scenarios, the trial-and-error migration could most effectively reduce the turns of agent migration. However, the population of agents is dynamic in most cases, (e.g., in Sugarscape [13]), and agents may gather around (as observed in Wa-Tor [14]). Once a collision is detected, the trail-and-error algorithm may cost more than the other two migrations.

Therefore, we developed and focused on location and direction-ordered collision-free migration. Since the MASS library has access to each agent's index[] variable, (i.e., the current and the next locations), it was able to implement direction-ordered migration at the system level. This paper demonstrated its simple programmability in and faster execution of system-supported agent migration.

Our future plan is to continue our verification work on the MASS library's collision-free migration, using actual applications such as Sugarscape and Wa-Tor. We recently made the MASS library available to the public through:

http: : //depts.washington.edu/dslab/MASS.

\section{REFERENCES}

[1] MATSim Homepage, "http://www.matsim.org," 2012.

[2] F. Kawasaki, "Accelerating large-scale simulations of coortical neuronal network development," Master's thesis, Master of Science in Computing and Software Systems, University of Washington, 2012.

[3] D. L. Chao, M. E. Halloran, V. J. Obenchain, and I. M. Longini Jr, "FluTE, a Publicly Available Stochastic Influenza Epidemic Simulation Model," PLoS Computational Biology, vol. Vol.6, no. No.1, pp. 517527, January 2010.

[4] M. Oryani, "Applying agent-based modeling to studying emergent behaviros of the immune system cells," Master's thesis, KTH Electrical Engineering, Stockholm, Sweden, May 2014.

[5] R. M. D'Souza, S. Marino, and D. Kirschner, "Data-parallel algorithms for agent-based model simulations of tuberculosis on graphics processing units," in Proc. of Agent-Directed Symposium - ADSO9. San Diego, CA: SCS, March 2009.

[6] Argonne National Laboratory, "Repast for High Performance Computing, http://repast.gifhub.io/repast_hpc.html."

[7] C. Geenough and M. Holcombe, "FLAME Flexible Large-scale Agent Modeling Environment, http://www.flame.ac.uk."

[8] A. U. K. Wagoum, B. Stefen, A. Seyfried, and M. Chraibi, "Parallel real time computation of large scale pedestrian evacuations," Advances in Engineering Software, vol. Vol.60-61, pp. 98-103, 2013.

[9] J. Barceló, J. Ferrer, D. Garcća, and R. Grau, "Microscopic traffic simulation for att systems analysis. a parallel computing version," in 25th Anniversary of CRT, August 1998.

[10] A. Kirchner, H. Klüpfel, K. Nishinari, A. Schadschneider, and M. Schreckenberg, "Discretization effects and the influence of walking speed in cellular automata models for pedestrian dynamics," Journal of Statistical Mechanics: Theory and Experiment, vol. Vol.2004, no. No.10, p. P10011, October 2004.

[11] S. Bandini, L. Crociani, and G. Vizzari, "Towards a more comprehensive estimation of social costs in pedestrian facilities," in Proc. of the Workshop on The Challenge of Ageing Society: Technological Roles and Opportunities for Artificial Intelligence in conjunction with the 13th Conference of the Italian Association for Artificial Intelligence, (AI*IA 2013), Turin, Italy, December 2013, p. paper 7.

[12] C. L. Barrett et al., "TRANSSIMS(TRansportation ANalysis SIMulation System) Volume 0 - Overview," Los Alamos National Laboratory," LAUR-99-1658, May 281999.

[13] J. Epstein and R. Axtell, Growing artificial societies: social science from the bottom up. Brookings Institution Press, October 1996, p. 224.

[14] A. K. Dewdney, "Computer recreations sharks and fish wage an ecological war on the toroidal planet wa-tor," Scientific American, pp. 14-22, December 1984.

[15] M. Fukuda, "MASS: A Parallelizing Library for Multi-Agent Spatial Simulation, http://depts.washington.edu/dslab/MASS." 



\section{$10^{\text {th }}$ International Workshop on Computational Optimization}

$\mathbf{M}$ ANY real world problems arising in engineering, economics, medicine and other domains can be formulated as optimization tasks. These problems are frequently characterized by non-convex, non-differentiable, discontinuous, noisy or dynamic objective functions and constraints which ask for adequate computational methods.

The aim of this workshop is to stimulate the communication between researchers working on different fields of optimization and practitioners who need reliable and efficient computational optimization methods.

\section{TOPICS}

The list of topics includes, but is not limited to:

- combinatorial and continuous global optimization

- unconstrained and constrained optimization

- multiobjective and robust optimization

- optimization in dynamic and/or noisy environments

- optimization on graphs

- large-scale optimization, in parallel and distributed computational environments

- meta-heuristics for optimization, nature-inspired approaches and any other derivative-free methods

- exact/heuristic hybrid methods, involving natural computing techniques and other global and local optimization methods

The applications of interest are included in the list below, but are not limited to:

- classical operational research problems (knapsack, traveling salesman, etc)

- computational biology and distance geometry

- data mining and knowledge discovery

- human motion simulations; crowd simulations

- industrial applications

- optimization in statistics, econometrics, finance, physics, chemistry, biology, medicine, and engineering.

\section{Best PAPER Award}

The best WCO'17 paper will be awarded during the social dinner of FedCSIS 2017.

The best paper will be selected by WCO'17 co-Chairs by taking into consideration the scores suggested by the reviewers, as well as the quality of the given oral presentation.

\section{SECTION EDITORS}

- Fidanova, Stefka, Bulgarian Academy of Sciences, Bulgaria

- Mucherino, Antonio, INRIA, France

- Zaharie, Daniela, West University of Timisoara, Romania

\section{REVIEWERS}

- Bonates, Tibérius, Universidade Federal do Ceará, Brazil

- Breaban, Mihaela

- Chira, Camelia, Technical University of Cluj-Napoca, Romania

- Gonçalves, Douglas, Universidade Federal de Santa Catarina, Brazil

- Hosobe, Hiroshi, Hosei University, Japan

- Iiduka, Hideaki, Kyushu Institute of Technology, Japan

- Lavor, Carlile, IMECC-UNICAMP, Brazil

- Marinov, Pencho, Bulgarian Academy of Science, Bulgaria

- Micota, Flavia, West University of Timisora, Romania

- Muscalagiu, Ionel, Politehnica University Timisoara, Romania

- Parsopoulos, Konstantinos, University of Ioannina, Greece

- Pintea, Camelia, Tehnical University Cluj-Napoca, Romania

- Roeva, Olympia, Institute of Biophysics and Biomedical Engineering, Bulgaria

- Siarry, Patrick, Universite Paris XII Val de Marne, France

- Stefanov, Stefan, South-West University ""Neofit Rilski, Bulgaria

- Stoean, Ruxandra

- Stoean, Catalin

- Stuetzle, Thomas, Université Libre de Bruxelles (ULB), Belgium

- Tamir, Tami, The Interdisciplinary Center (IDC), Israel

- Zilinskas, Antanas, Vilnius University, Lithuania 



\title{
InterCriteria Analysis of Multi-population Genetic Algorithms Performance
}

\author{
Maria Angelova, Tania Pencheva \\ Institute of Biophysics and Biomedical Engineering \\ Bulgarian Academy of Sciences \\ 105 Acad. G. Bonchev Str., Sofia 1113, Bulgaria \\ Emails: maria.angelova@biomed.bas.bg, tania.pencheva@biomed.bas.bg
}

\begin{abstract}
InterCriteria Analysis approach is here applied for the assessment of promising genetic algorithms optimization techniques. Altogether six multi-population genetic algorithms are here considered, differing in the execution order of main genetic operators selection, crossover and mutation. InterCriteria Analysis approach, based on the apparatuses of index matrices and intuitionistic fuzzy sets, is implemented to assess the performance of multi-population genetic algorithms for the parameter identification of Saccharomyces cerevisiae fed-batch fermentation process. Degrees of "agreement" and "disagreement" between the algorithms outcomes convergence time and model accuracy, from one hand, and model parameters estimations, from the other hand, have been established. Outlined relations are going to lead to an additional exploring of the model, expected to be extraordinary valuable especially in the case of modelling of living systems, such as fermentation processes.
\end{abstract}

\section{INTRODUCTION}

$\mathbf{Y}$ EAST is widely used model organisms in contemporary biotechnology and genetic engineering due to its well known metabolic pathways [1], [15]. Specifically, $S$. cerevisiae has found significant application in the production of medicines, food and beverages. Meanwhile, the complex structure of fermentation processes, usually described by systems of non-linear differential equations with several specific growth rates, turns their modelling in a challenging and rather difficult task.

Genetic algorithms (GA) is a stochastic global optimization technique, proven in successful solving of a variety of challenging problems in the field of complex dynamic systems optimization [9], [10], [16], among them for parameter identification of various fermentation process models [1], [3], [14], [15], [16]. GA are one of the methods based on biological evolution, inspired by Darwins theory of survival of the fittest. Simple genetic algorithms, originally presented in [10], search a global optimal solution using three main genetic operators in a sequence selection, crossover and mutation over the individuals in one population. Meanwhile, multipopulation genetic algorithms (MpGA) is more similar to the nature since in it many populations, called subpopulations, evolve independently from each other. After a certain number of generations, a part of individuals migrates between the subpopulations.

The main purpose of this investigation is to be assessed the algorithms performance of MpGA modifications with different sequence of implementation of the main genetic operators, namely selection, crossover and mutation. InterCriteria Analysis ( ICrA), developed as an alternative to the traditional methods for the assessment of algorithms performance, is here implemented. So far, there are some successful ICrA application for parameter identification of fermentation process models [2], [12], [13], [17], where the approach applicability has been demonstrated for establishing correlations between model parameters and GA objective function value and convergence time, from one side, and model parameters themselves, from the other side. This investigation is focussed on the assessment of six MpGA modifications, consequently applied to parameter identification of a $S$. cerevisiae fed batch fermentation process.

\section{PROBLEM FORMULATION}

Parameter identification of a considered here $S$. cerevisiae fed-batch fermentation process model is performed using real data from on-line and off-line measurements, carried out in the Institute of Technical Chemistry, Hanover, Germany. The details about the process conditions and experimental data set could be found in [15].

According to the mass balance and considering mixed oxidative functional state [15], non-linear mathematical model of $S$. cerevisiae fed-batch fermentation process is commonly described as follows:

$$
\begin{gathered}
\frac{d X}{d t}=\left(\mu_{2 S} \frac{S}{S+k_{S}}+\mu_{2 E} \frac{E}{E+k_{E}}\right) X-\frac{F_{i n}}{V} X \\
\frac{d S}{d t}=-\frac{\mu_{2 S}}{Y_{S X}} \frac{S}{S+k_{S}} X+\frac{F_{i n}}{V}\left(S_{i n}-S\right) \\
\frac{d E}{d t}=-\frac{\mu_{2 E}}{Y_{E X}} \frac{E}{E+k_{E}} X+\frac{F_{i n}}{V} E \\
\frac{d V}{d t}=F_{\text {in }}
\end{gathered}
$$

where $X$ is the biomass concentration, $[\mathrm{g} / \mathrm{l}] ; S$ - substrate concentration, $[\mathrm{g} / \mathrm{l}] ; E$ - ethanol concentration, $[\mathrm{g} / \mathrm{l}] ; F_{\text {in }}$ - feeding rate, $[1 / \mathrm{h}] ; V$ - bioreactor volume, [1]; $S_{\text {in }}$ substrate concentration in the feeding solution, $[\mathrm{g} / \mathrm{l}] ; \mu_{2 S}, \mu_{2 E}$ - maximum values of the specific growth rates, $[1 / \mathrm{h}] ; k_{S}, k_{E}-$ saturation constants, [g/l]; $Y_{S X}, Y_{E X}$ - yield coefficients, [-]. All functions are continuous and differentiable and all model parameters fulfil the requirement for non-zero division. 
For the considered here model (1)-(4), the vector $p=$ $\left[\mu_{2 S}, \mu_{2 E}, k_{S}, k_{E}, Y_{S X}, Y_{E X}\right]$, including six model parameters should be identified.

Mean square deviation between the model output and the experimental data for the process variables biomass, substrate and ethanol, has been used as an optimization criterion:

$$
\begin{array}{r}
J=\sum_{i=1}^{m}\left(X_{\exp }(i)-X_{\text {mod }}(i)\right)^{2}+\sum_{i=1}^{n}\left(S_{\exp }(i)-S_{\text {mod }}(i)\right)^{2}+ \\
\sum_{i=1}^{l}\left(E_{\exp }(i)-E_{\text {mod }}(i)\right)^{2} \rightarrow \min
\end{array}
$$

where $m, n$ and $l$ are the dimensions of the experimental data; $X_{\text {exp }}, S_{\text {exp }}, E_{\text {exp }}, X_{\text {mod }}, S_{\text {mod }}$ and $E_{\text {mod }}$ are, respectively, experimental and model predicted data for biomass, substrate and ethanol.

\section{Multi-POPUlation Genetic AlgORIthMS FOR PARAMETER IDENTIFICATION OF $S$. cerevisiae FED-BATCH FERMENTATION PROCESS}

Genetic algorithms, firstly proposed by Holland [11] and later upgraded by Goldberg [10], are a stochastic searching tool inspired by processes of natural evolution. Frequently used as an alternative to the conventional optimization techniques, both simple and multi-population GA have been successfully applied for different problems solving [9], [10], [15], [16], but multi-population GA is more similar to nature than simple GA. Standard multi-population GA works with many populations of coded parameter sets, called subpopulations and searches a global optimal solution using the main genetic operators in a sequence selection, crossover and mutation. For brevity, this algorithm is here denoted as MpGA-SCM, coming from the operators execution order selection, crossover, mutation. MpGA-SCM starts with a creation of $k$ randomly generated subpopulations, each of them with $n$ chromosomes. After that each chromosome in the subpopulation is evaluated and assigned a fitness value. According to the fitness function, the most suitable solutions are selected. Then, crossover and mutation proceed to form a new offspring. After certain number of generations, called isolation time, individuals migrate between the subpopulations. For the purposes of this investigation, MpGA-SCM terminates when a certain number of generations is reached.

While the main idea of GA is to imitate the processes occurring in nature, one can assume that the probability crossover to come first and then mutation is comparable to that both processes to occur in a reverse order; or selection to be performed before or after crossover and mutation, no matter of their order. Following this idea, five modifications of MpGA-SCM, with different sequence of execution of main genetic operators, have been developed aiming to improve model accuracy and algorithms convergence time [1], [3], [14]. The modifications, namely MpGA-SMC (selection, mutation, crossover), MpGACMS (crossover, mutation, selection), MpGA-MCS (mutation, crossover, selection), MpGA-CSM (crossover, selection, mutation) and MpGA-MSC (mutation, selection, crossover) have been proposed and basically investigated for parameter identification of a fed-batch cultivation of $S$. cerevisiae in [1], [3], [14]. The performance of mentioned above altogether six MpGA, applied to the parameter identification of a $S$. cerevisiae fed-batch cultivation process, is going to be assessed by promising ICrA approach.

\section{INTERCRITERIA ANALYSIS}

The theoretical framework of the InterCriteria Analysis approach, based on the apparatuses of index matrices (IM) [5], [6], [7] and intuitionistic fuzzy sets (IFS) [4], is given in details in [8]. Here, ICrA is briefly presented for a completeness.

The initial IM $A$ in a form presented in (6) consists of the criteria $C_{p}$ (for rows), objects $O_{q}$ (for columns) and real number elements $a_{C_{p}, O_{q}}$ for every $p, q,(1 \leq p \leq m, 1 \leq q \leq n)$.

Further, an IM with index sets consisting of the criteria (for rows and for columns) with IF pair elements determining the degrees of "correspondence" between the respective criteria is constructed. A real number $a_{C_{p}, O_{q}}$ is comparable about relation $R$ with the other $a$-object, so that $R\left(a_{C_{k}, O_{i}}, a_{C_{k}, O_{j}}\right)$ is defined for each $i, j, k$. Let $\bar{R}$ be the dual relation of $R$ in the sense that if $R$ is satisfied, then $\bar{R}$ is not satisfied, and vice versa. For example, if " $R$ " is the relation "<", then $\bar{R}$ is the relation " $>$ ", and vice versa. If $S_{k, l}^{\mu}$ is the number of cases in which $R\left(a_{C_{k}, O_{i}}, a_{C_{k}, O_{j}}\right)$ and $R\left(a_{C_{l}, O_{i}}, a_{C_{l}, O_{j}}\right)$ are simultaneously satisfied, while $S_{k, l}^{\nu}$ is the number of cases is which $R\left(a_{C_{k}, O_{i}}, a_{C_{k}, O_{j}}\right)$ and $\bar{R}\left(a_{C_{l}, O_{i}}, a_{C_{l}, O_{j}}\right)$ are simultaneously satisfied, it is obvious, that

$$
S_{k, l}^{\mu}+S_{k, l}^{\nu} \leq \frac{n(n-1)}{2} .
$$

Further, for every $k, l$, satisfying $1 \leq k<l \leq m$, and for $n \geq 2$,

$$
\mu_{C_{k}, C_{l}}=2 \frac{S_{k, l}^{\mu}}{n(n-1)}, \quad \nu_{C_{k}, C_{l}}=2 \frac{S_{k, l}^{\nu}}{n(n-1)}
$$

are defined. Therefore, $\left\langle\mu_{C_{k}, C_{l}}, \nu_{C_{k}, C_{l}}\right\rangle$ is an intuitionistic fuzzy pair. Next, the following IM is constructed:

$$
\begin{array}{c|ccc} 
& C_{1} & \ldots & C_{m} \\
\hline C_{1} & \left\langle\mu_{C_{1}, C_{1}}, \nu_{C_{1}, C_{1}}\right\rangle & \ldots & \left\langle\mu_{C_{1}, C_{m}}, \nu_{C_{1}, C_{m}}\right\rangle \\
\vdots & \vdots & \ddots & \vdots \\
C_{m} & \left\langle\mu_{C_{m}, C_{1}}, \nu_{C_{m}, C_{1}}\right\rangle & \ldots & \left\langle\mu_{C_{m}, C_{m}}, \nu_{C_{m}, C_{m}}\right\rangle
\end{array},
$$

that determines the degrees of "correspondence" between criteria $C_{1}, \ldots, C_{m}$.

The sum $\mu_{C_{k}, C_{l}}+\nu_{C_{k}, C_{l}}$ is not always equal to 1 . The difference

$$
\pi_{C_{k}, C_{l}}=1-\mu_{C_{k}, C_{l}}-\nu_{C_{k}, C_{l}}
$$

is considered as a degree of "uncertainty".

The final step of ICrA is to classify the degrees of "correspondence" between criteria. Let $\alpha, \beta \in[0 ; 1]$ are the threshold values for comparison of $\mu_{C_{k}, C_{l}}$ and $\nu_{C_{k}, C_{l}}$. In general, the criteria $C_{k}$ and $C_{l}$ are respectively:

- in a positive consonance, if $\mu_{C_{k}, C_{l}}>\alpha$ and $\nu_{C_{k}, C_{l}}<\beta$; 


\begin{tabular}{c|ccccccc} 
& $O_{1}$ & $\ldots$ & $O_{k}$ & $\ldots$ & $O_{l}$ & $\ldots$ & $O_{n}$ \\
\hline$C_{1}$ & $a_{C_{1}, O_{1}}$ & $\ldots$ & $a_{C_{1}, O_{k}}$ & $\ldots$ & $a_{C_{1}, O_{l}}$ & $\ldots$ & $a_{C_{1}, O_{n}}$ \\
$\vdots$ & $\vdots$ & $\ddots$ & $\vdots$ & $\ddots$ & $\vdots$ & $\ddots$ & $\vdots$ \\
$C_{i}$ & $a_{C_{i}, O_{1}}$ & $\ldots$ & $a_{C_{i}, O_{k}}$ & $\ldots$ & $a_{C_{i}, O_{l}}$ & $\ldots$ & $a_{C_{i}, O_{n}}$ \\
$\vdots$ & $\vdots$ & $\ddots$ & $\vdots$ & $\ddots$ & $\vdots$ & $\ddots$ & $\vdots$ \\
$C_{j}$ & $a_{C_{j}, O_{1}}$ & $\ldots$ & $a_{C_{j}, O_{k}}$ & $\ldots$ & $a_{C_{j}, O_{l}}$ & $\ldots$ & $a_{C_{j}, O_{n}}$ \\
$\vdots$ & $\vdots$ & $\ddots$ & $\vdots$ & $\ddots$ & $\vdots$ & $\ddots$ & $\vdots$ \\
$C_{m}$ & $a_{C_{m}, O_{1}}$ & $\ldots$ & $a_{C_{m}, O_{k}}$ & $\ldots$ & $a_{C_{m}, O_{l}}$ & $\ldots$ & $a_{C_{m}, O_{n}}$
\end{tabular},

- in a negative consonance, if $\mu_{C_{k}, C_{l}}<\beta$ and $\nu_{C_{k}, C_{l}}>\alpha$; - in a dissonance, otherwise.

\section{NUMERICAL RESULTS AND DISCUSSION}

All identification procedures as well as InterCriteria Analysis implementation are performed on PC Intel Pentium 4 (2.4 $\mathrm{GHz}$ ) platform running Windows XP.

Six modifications of MpGA with different execution order of main genetic operators selection, crossover and mutation have been consequently applied to estimate the model parameters (vector $p$ ) of the considered model (1)-(4). Due to the stochastic nature of GA, 30 independent runs for each of the applied here six MpGA have been performed. MpGA operators and parameters are tuned according to [1].

In terms of ICrA, altogether eight criteria are taken into consideration: objective function value $J$ is considered as $C_{1}$; convergence time $T-$ as $C_{2}$; specific growth rates $\mu_{2 S}$ and $\mu_{2 E}$ - respectively as $C_{3}$ and $C_{4}$; saturation constants $k_{S}$ and $k_{E}$ - respectively as $C_{5}$ and $C_{6}$; yield coefficients $Y_{S X}$ and $Y_{E X}-$ respectively as $C_{7}$ and $C_{8}$. Six investigated here objects, referred to MpGA modifications, respectively are: $O_{1}$ corresponding to MpGA-CMS; $\mathrm{O}_{2}$ - to MpGA-CSM; $\mathrm{O}_{3}$ - to MpGA-MCS; $O_{4}$ - to MpGA-MSC; $O_{5}$ - to MpGASCM; and $O_{6}$ - to MpGA-SMC. For convenience, forenames of objective function, convergence time, fermentation process model parameters and MpGA modifications are further used instead of a criterion $C_{i}$ or an object $O_{j}$.

IMs (10)-(12) present, respectively, the average estimates (10), the best ones (11), and the worst ones (12) of the values of objective function $J$, the algorithm convergence time $T$, [s], as well as of all model parameters towards vector $p$. Three IMs list the objective function values rounded to the fifth digit after the decimal point, while the rest criteria - to the fourth digit after the decimal point. However, at the step of ICrA implementation, all parameter estimates are used as they have been obtained as a result from parameter identification procedures, in order to be distinguishable and the degrees of "uncertainty" to be decreased.

As seen from (10)-(12), obtained results show similar values for objective function $J$ after application of the considered here six MpGA for fermentation process model parameter identification. There is about $1 \%$ difference between the best among the best results $(J=0.02193$ for MpGA-SMC, (11)) and the worst among the worst results $(J=0.02218$ for
MpGA-CSM, (12)). On the other hand, the convergence time $T$ increases more than 2.5 times (347.3300 for MpGA-CMS, (12) towards 131.4000 for MpGA-SCM, (11)). Such a small deviation of $J$ proves all six considered here MpGA modifications as equally reliable and it is of user choice to make a compromise between the model accuracy and convergence time.

ICrA approach has been consequently implemented for each of the constructed IMs $A_{1}$ (average), $A_{2}$ (best) and $A_{3}$ (worst). After ICrA application, six IMs that determine the degrees of agreement and disagreement between investigated criteria have been obtained. IMs themselves are not shown here, but the results from the ICrA implementation for the cases of average, best and worst evaluations have been summarized in Table I. Obtained results are ranked by $\mu_{C_{k} ; C_{l}}$ values in the case of average evaluations. As could be seen from Table I, there are no pairs with a degree of "uncertainty" for the cases of average and worst evaluations, while such criteria pairs have been observed in the case of the best evaluations. The logical explanation of this fact is that even using a "row data" from parameter identification procedures there are some equal evaluations for some of the model parameters in different MpGA.

Aiming at better interpretation of the obtained results listed above, they are also graphically presented in Fig. 1.

Table II presents the scale of consonance and dissonance [17], on which basis each pair of criteria is going to be assessed.

Based on the presented scale, the following pair dependencies might be outlined for the case of average results of the examined criteria.

A positive and a weak positive consonance have been observed respectively for the pairs $\mu_{2 E}-Y_{E X}$ and $k_{S}-Y_{E X}$. There are three criteria pairs in a negative consonance - $J-k_{E}$, $Y_{S X}-Y_{E X}$ and $\mu_{2 E}-Y_{S X}$ and another one $-\mu_{2 S}-k_{S}$ in a weak negative consonance. The rest of the criteria pairs hit the intervals of a dissonance.

Some coincidences for the three investigated here case studies (of average, best and worst results) should be mentioned. The criteria pair $\mu_{2 E}-Y_{E X}$ is in a positive consonance for the cases of average and best results. The same criteria pair hits the upper boundary of the $\mu$-values in the case of worst results, as such showing a strong positive consonance. The criteria pair $\mu_{2 E}-Y_{E X}$ is the only one with the maximum value (i.e. $\mu=1$ ) for the degree of "agreement". A negative consonance for the pair $\mu_{2 E}-Y_{S X}$ has been observed for 


\begin{tabular}{c|c|c|c|c|c|c} 
& MpGA-CMS & MpGA-CSM & MpGA-MCS & MpGA-MSC & MpGA-SCM & MpGA-SMC \\
\hline$J$ & 0.02203 & 0.02210 & 0.02196 & 0.02203 & 0.02206 & 0.02195 \\
$T$ & 370.9408 & 317.6379 & 265.4929 & 214.0625 & 113.9127 & 164.3988 \\
$\mu_{2 S}$ & 0.9002 & 0.9063 & 0.9076 & 0.9023 & 0.9018 & 0.9004 \\
$A_{1}$ (average $)=\mu_{2 E}$ & 0.1494 & 0.1242 & 0.1485 & 0.1216 & 0.1389 & 0.1484 \\
$k_{S}$ & 0.1500 & 0.1496 & 0.1487 & 0.1466 & 0.1500 & 0.1500 \\
$k_{E}$ & 0.8000 & 0.8000 & 0.8339 & 0.8366 & 0.8000 & 0.8420 \\
$Y_{S X}$ & 0.3973 & 0.4090 & 0.3960 & 0.4126 & 0.4022 & 0.3993 \\
$Y_{E X}$ & 2.0193 & 1.6524 & 1.9939 & 1.6035 & 1.8630 & 2.0019
\end{tabular}

\begin{tabular}{c|c|c|c|c|c|c} 
& MpGA-CMS & MpGA-CSM & MpGA-MCS & MpGA-MSC & MpGA-SCM & MpGA-SMC \\
\hline$J$ & 0.02203 & 0.02204 & 0.02195 & 0.02199 & 0.02203 & 0.02193 \\
$T$ & 377.9200 & 343.0900 & 288.7900 & 226.7900 & 131.4000 & 177.1900 \\
$\mu_{2 S}$ & 0.9000 & 0.9004 & 0.9009 & 0.9024 & 0.9000 & 0.9004 \\
$A_{2}$ (best $)=\mu_{2 E}$ & 0.1500 & 0.1444 & 0.1496 & 0.1244 & 0.1500 & 0.1484 \\
$k_{S}$ & 0.1500 & 0.1500 & 0.1500 & 0.1465 & 0.1500 & 0.1500 \\
$k_{E}$ & 0.8000 & 0.8000 & 0.8363 & 0.8491 & 0.8000 & 0.8509 \\
$Y_{S X}$ & 0.3969 & 0.3998 & 0.3952 & 0.4120 & 0.3975 & 0.3993 \\
$Y_{E X}$ & 2.0297 & 1.9453 & 2.0009 & 1.6398 & 2.0280 & 2.0020
\end{tabular}

\begin{tabular}{c|c|c|c|c|c|c} 
& MpGA-CMS & MpGA-CSM & MpGA-MCS & MpGA-MSC & MpGA-SCM & MpGA-SMC \\
\hline$J$ & 0.02204 & 0.02218 & 0.02198 & 0.02205 & 0.02211 & 0.02201 \\
$T$ & 347.3300 & 295.4300 & 253.5500 & 195.9700 & 92.2960 & 142.9400 \\
$\mu_{2 S}$ & 0.9044 & 0.9176 & 0.9248 & 0.9024 & 0.9220 & 0.9014 \\
$A_{3}$ (worst $)=\mu_{2 E}$ & 0.1460 & 0.1003 & 0.1472 & 0.1148 & 0.1211 & 0.1480 \\
$k_{S}$ & 0.1498 & 0.1488 & 0.1455 & 0.1466 & 0.1495 & 0.1500 \\
$k_{E}$ & 0.8000 & 0.8000 & 0.8359 & 0.8413 & 0.8000 & 0.8095 \\
$Y_{S X}$ & 0.4006 & 0.4196 & 0.3959 & 0.4143 & 0.4103 & 0.3992 \\
$Y_{E X}$ & 1.9660 & 1.3112 & 1.9771 & 1.5232 & 1.6125 & 2.0002
\end{tabular}

the three considered case studies. A negative consonance has been outlined for $Y_{S X}-Y_{E X}$ in the cases of average and worst results, while in the case of the best results the pair is in a weak negative consonance.

Although mentioned above coincidences, there are several discrepancies in criteria dependences, caused mainly by the stochastic nature of the considered here six modifications of MpGA. The attention is drawn only for the pairs, showing consonance in some of the considered case studies. For example, according to the scale presented in Table II, a weak positive consonance has been observed for criteria pair $k_{S}-Y_{E X}$ in the cases of average and best results, while in the case of the worst results the pair is in a dissonance. A weak positive consonance for $\mu_{2 E}-k_{S}$ and a positive consonance for $J-Y_{S X}$ could be seen only in the cases of best and worst results, respectively. Another discrepancy is found in the results for the criteria pairs $\mu_{2 S}-k_{S}$ and $J-k_{E}$. Both pairs are in a negative consonance for the case studies of average and best results, while in the case of the worst results they fall in the interval of a weak dissonance.

Distribution of dependencies between criteria pairs in the cases of average, best and worst evaluations are listed in Table III. The criteria pairs are equally distributed in the cases of average and worst results. In the case of the best results, there are more criteria pairs in a positive or in a negative consonance and less in a dissonance, but this is the only one case with observed degree of "uncertainty" for some of the criteria pairs.

Taking into account the obtained ICrA estimations in the cases of average, best and worst results, and having in mind the stochastic nature of GA, it is more reasonable to rely with a higher credibility on the results in the case of average values than to results obtained in another two cases.

\section{CONCLUSion}

Promising ICrA approach has been here implemented to examine the performance of six modifications of multipopulation genetic algorithms, applied for the purposes of a fermentation process model parameter identification. All considered MpGA modifications demonstrate almost equal degree of accuracy with about $1 \%$ objective function value divergence, but on the account of the convergence time. Thus, it is of user choice to make a compromise between model accuracy and convergence time. After applying MpGA to a parameter identification of $S$. cerevisiae fed-batch cultivation process, three case studies have been examined - of average, 
TABLE I

CRiteria RELATIONS SORTED By $\mu_{C_{k}, C_{l}}$ VALUES IN THE CASE OF AVERAGE RESUlts

\begin{tabular}{c|c|c|c}
\hline \multirow{2}{*}{ Criteria relation } & Average results & Best results & Worst results \\
\cline { 2 - 4 } & $\mu / \nu / \pi$ & $\mu / \nu / \pi$ & $\mu / \nu / \pi$ \\
\hline$\mu_{2 E}-Y_{E X}$ & $0.93 / 0.07 / 0.00$ & $0.87 / 0.07 / 0.06$ & $1.00 / 0.00 / 0.00$ \\
\hline$k_{S}-Y_{E X}$ & $0.80 / 0.20 / 0.00$ & $0.80 / 0.00 / 0.20$ & $0.67 / 0.33 / 0.00$ \\
\hline$\mu_{2 E}-k_{S}$ & $0.73 / 0.27 / 0.00$ & $0.80 / 0.07 / 0.13$ & $0.67 / 0.33 / 0.00$ \\
\hline$J-Y_{S X}$ & $0.67 / 0.33 / 0.00$ & $0.60 / 0.40 / 0.00$ & $0.93 / 0.07 / 0.00$ \\
\hline$T-\mu_{2 E}$ & $0.67 / 0.33 / 0.00$ & $0.47 / 0.47 / 0.06$ & $0.40 / 0.60 / 0.00$ \\
\hline$J-T$ & $0.60 / 0.40 / 0.00$ & $0.60 / 0.40 / 0.00$ & $0.47 / 0.53 / 0.00$ \\
\hline$T-Y_{E X}$ & $0.60 / 0.40 / 0.00$ & $0.47 / 0.53 / 0.00$ & $0.40 / 0.60 / 0.00$ \\
\hline$\mu_{2 S}-Y_{S X}$ & $0.60 / 0.40 / 0.00$ & $0.73 / 0.27 / 0.00$ & $0.47 / 0.53 / 0.00$ \\
\hline$J-\mu_{2 S}$ & $0.53 / 0.47 / 0.00$ & $0.47 / 0.53 / 0.00$ & $0.53 / 0.47 / 0.00$ \\
\hline$T-\mu_{2 S}$ & $0.53 / 0.47 / 0.00$ & $0.47 / 0.53 / 0.00$ & $0.53 / 0.47 / 0.00$ \\
\hline$T-k_{S}$ & $0.53 / 0.47 / 0.00$ & $0.33 / 0.47 / 0.20$ & $0.47 / 0.53 / 0.00$ \\
\hline$k_{E}-Y_{E X}$ & $0.53 / 0.47 / 0.00$ & $0.40 / 0.53 / 0.07$ & $0.60 / 0.40 / 0.00$ \\
\hline$\mu_{2 S}-k_{E}$ & $0.47 / 0.53 / 0.00$ & $0.60 / 0.33 / 0.07$ & $0.47 / 0.53 / 0.00$ \\
\hline$\mu_{2 E}-k_{E}$ & $0.47 / 0.53 / 0.00$ & $0.40 / 0.60 / 0.00$ & $0.60 / 0.40 / 0.00$ \\
\hline$k_{S}-k_{E}$ & $0.47 / 0.53 / 0.00$ & $0.47 / 0.40 / 0.13$ & $0.40 / 0.60 / 0.00$ \\
\hline$k_{E}-Y_{S X}$ & $0.47 / 0.53 / 0.00$ & $0.47 / 0.47 / 0.06$ & $0.33 / 0.67 / 0.00$ \\
\hline$J-\mu_{2 E}$ & $0.40 / 0.60 / 0.00$ & $0.53 / 0.40 / 0.07$ & $0.13 / 0.87 / 0.00$ \\
\hline$J-k_{S}$ & $0.40 / 0.60 / 0.00$ & $0.33 / 0.47 / 0.20$ & $0.47 / 0.53 / 0.00$ \\
\hline$T-Y_{S X}$ & $0.40 / 0.60 / 0.00$ & $0.47 / 0.53 / 0.00$ & $0.53 / 0.47 / 0.00$ \\
\hline$J-Y_{E X}$ & $0.33 / 0.67 / 0.00$ & $0.47 / 0.53 / 0.00$ & $0.13 / 0.87 / 0.00$ \\
\hline$\mu_{2 S}-\mu_{2 E}$ & $0.33 / 0.67 / 0.00$ & $0.13 / 0.80 / 0.07$ & $0.47 / 0.53 / 0.00$ \\
\hline$k_{S}-Y_{S X}$ & $0.33 / 0.67 / 0.00$ & $0.20 / 0.60 / 0.20$ & $0.40 / 0.60 / 0.00$ \\
\hline$T-k_{E}$ & $0.27 / 0.73 / 0.00$ & $0.27 / 0.67 / 0.06$ & $0.40 / 0.60 / 0.00$ \\
\hline$\mu_{2 S}-Y_{E X}$ & $0.27 / 0.73 / 0.00$ & $0.07 / 0.93 / 0.00$ & $0.47 / 0.53 / 0.00$ \\
\hline$\mu_{2 S}-k_{S}$ & $0.20 / 0.80 / 0.00$ & $0.07 / 0.73 / 0.20$ & $0.27 / 0.73 / 0.00$ \\
\hline$J-k_{E}$ & $0.13 / 0.87 / 0.00$ & $0.07 / 0.87 / 0.06$ & $0.27 / 0.73 / 0.00$ \\
\hline$Y_{S X}-Y_{E X}$ & $0.13 / 0.87 / 0.00$ & $0.20 / 0.80 / 0.00$ & $0.07 / 0.93 / 0.00$ \\
\hline$\mu_{2 E}-Y_{S X}$ & $0.07 / 0.93 / 0.00$ & $0.13 / 0.80 / 0.07$ & $0.07 / 0.93 / 0.00$ \\
\hline & &
\end{tabular}

TABLE II

SCALE OF CONSONANCE AND DISSONANCE

\begin{tabular}{c|l}
\hline Interval of $\mu_{C_{k}, C_{l}}$ & Meaning \\
\hline$[0.00-0.05]$ & strong negative consonance \\
\hline$(0.05-0.15]$ & negative consonance \\
\hline$(0.15-0.25]$ & weak negative consonance \\
\hline$(0.25-0.33]$ & weak dissonance \\
\hline$(0.33-0.43]$ & dissonance \\
\hline$(0.43-0.57]$ & strong dissonance \\
\hline$(0.57-0.67]$ & dissonance \\
\hline$(0.67-0.75]$ & weak dissonance \\
\hline$(0.75-0.85]$ & weak positive consonance \\
\hline$(0.85-0.95]$ & positive consonance \\
\hline$(0.95-1.00]$ & strong positive consonance \\
\hline
\end{tabular}

best and worst results in regard to chosen criteria. ICrA approach has been implemented to assists in establishing of
TABLE III

DISTRIBUTION OF DEPENDENCES BETWEEN CRITERIA PAIRS

\begin{tabular}{c|c|c|c}
\hline Meaning & Average & Best & Worst \\
\hline Positive consonance & 2 & 3 & 2 \\
\hline Dissonance & 22 & 18 & 22 \\
\hline Negative consonance & 4 & 7 & 4 \\
\hline
\end{tabular}

existing relations between fermentation process model parameters and MpGA outcomes, such as objective function value and convergence time. Obtained additional knowledge for relations between model parameters and algorithms outcomes might be useful for improving the model accuracy and the performance of optimization algorithms in further parameter identification procedures.

\section{ACKNOWLEDGEMENT}

The work is partially supported by the National Science Fund of Bulgaria under the grant DFNI-I-02-5 "InterCriteria 

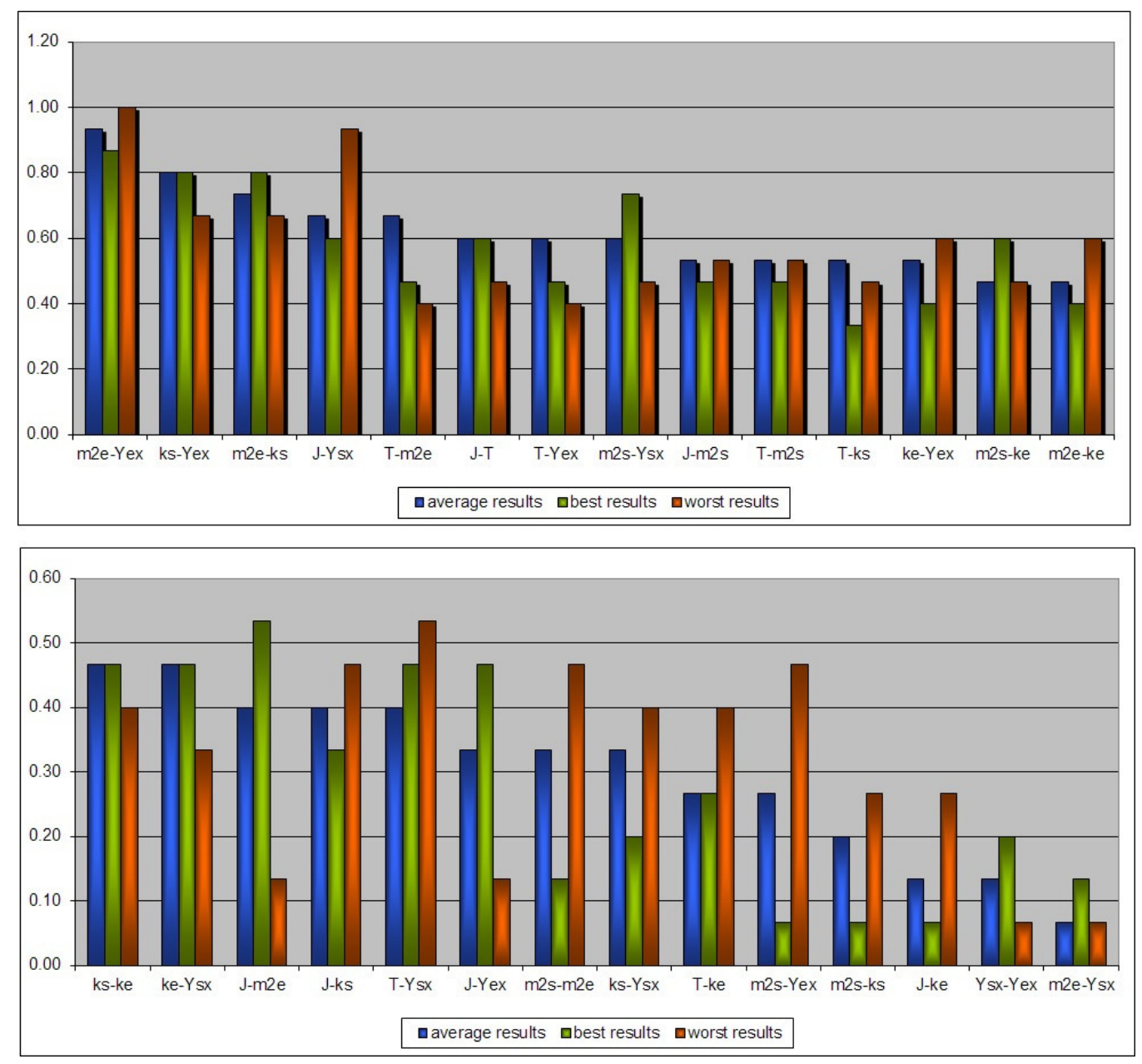

Fig. 1. Degrees of "agreement" $\left(\mu_{C_{k}, C_{l}}\right.$ values) for three considered cases

Analysis - A New Approach to Decision Making" and DM07/1 "Development of New Modified and Hybrid Metaheuristic Algorithms".

\section{REFERENCES}

[1] M. Angelova and T. Pencheva, "Genetic operators' significance assessment in multi-population genetic algorithms", Int. J. of Metaheuristics, vol. 3(2), 2014, pp. 162-173, DOI: 10.1504/IJMHEUR.2014.063146.

[2] M. Angelova, O. Roeva and T. Pencheva, "Intercriteria analysis of crossover and mutation rates relations in simple genetic algorithm", Ann. Comp. Sci. Info. Syst., vol. 5, 2015, pp. 419-424, DOI: 10.15439/2015F178.

[3] M. Angelova, S. Tzonkov and T. Pencheva,"Modified multi-population genetic algorithm for yeast fed-batch cultivation parameter identification", Int. J. Bioautomation, vol. 13(4), 2009, pp. 163-172.

[4] K. Atanassov, On intuitionistic fuzzy sets theory, Springer, Berlin, 2012, DOI: $10.1007 / 978-3-642-29127-2$.

[5] K. Atanassov, "Generalized index matrices", C. R. Acad. Bulg. Sci., vol. 40(11), 1987, pp. 15-18.

[6] K. Atanassov, "On index matrices, part 1: standard cases", Adv. Stud. Cont. Math., vol. 20(2), 2010, pp. 291-302.

[7] K. Atanassov, "On index matrices, part 2: intuitionistic fuzzy case", Proc. Jangjeon Math. Soc., vol. 13(2), 2010, pp. 121-126.

[8] K. Atanassov, D. Mavrov and V. Atanassova, "Intercriteria decision making: a new approach for multicriteria decision making, based on index matrices and intuitionistic fuzzy sets", Iss. Int. Fuz. Sets and Gen. Nets, vol. 11, 2014, pp. 1-8.
[9] A. Ghaheri, S. Shoar, M. Naderan and S. S. Hoseini, "The applications of genetic algorithms in medicine", Oman Med. J., vol. 30(6), 2015, pp. 406-416, DOI: $10.5001 / \mathrm{omj} .2015 .82$.

[10] D. E. Goldberg, Genetic algorithms in search, optimization and machine learning, Addison Wesley Longman, London, 2006.

[11] J. Holland, Adaptation in natural and artificial systems: an introductory analysis with application to biology, control and artificial intelligence, University of Michigan Press, 1975.

[12] T. Ilkova and M. Petrov, "Intercriteria analysis for identification of Escherichia coli fed-batch mathematical model", J. Int. Sci. Publ.: Mat., Meth. \& Techn., vol. 9, 2015, pp. 598-608.

[13] T. Pencheva and M. Angelova, "Intercriteria analysis of simple genetic algorithms performance", Advanced Computing in Industrial Mathematics, Vol. 681 of Studies in Computational Intelligence, 2017, 147-159. DOI: 10.1007/978-3-319-49544-6_13.

[14] T. Pencheva and M. Angelova, "Modified multi-population genetic algorithms for parameter identification of yeast fed-batch cultivation", Bulg. Chem. Comm., vol. 48(4), 2016, pp. 713-719.

[15] T. Pencheva, O. Roeva and I. Hristozov, Functional state approach to fermentation processes modelling, Prof. M. Drinov Acad. Publ. House, Sofia, 2006.

[16] O. Roeva (Ed.), Real-world application of genetic algorithms, InTech, 2012, DOI: $10.5772 / 2674$.

[17] O. Roeva, S. Fidanova, P. Vassilev and P. Gepner, "Intercriteria analysis of a model parameters identification using genetic algorithm", Ann. Comp. Sci. Inf. Syst., vol. 5, 2015, pp. 501-506, DOI: $10.15439 / 2015 \mathrm{~F} 223$. 


\section{A new optimization-based approach for aircraft landing in the presence of windshear}

\author{
Paweł Drąg \\ Department of Control Systems and Mechatronics \\ Wrocław University of Science and Technology \\ Wybrzeże Wyspiańskiego 27, 50-370, Wrocław, Poland \\ Email: pawel.drag@pwr.edu.pl
}

\author{
Krystyn Styczeń \\ Department of Control Systems and Mechatronics, \\ Wrocław University of Science and Technology \\ Wybrzeże Wyspiańskiego 27, 50-370, Wrocław, Poland \\ Email: krystyn.styczen@pwr.edu.pl
}

\begin{abstract}
In the article a new approach for aircraft landing with the presence of the windshear phenomena was presented. The differential-algebraic model with variability constraints was under considerations. To transform the optimal control problem into a nonlinear optimization task, a modified direct shooting method was used. Then, to solve the obtained largescale nonlinear optimization problem, a barrier method was applied. Moreover, in the proposed optimization-based approach, the variability constraints imposed on the state trajectory were considered directly.
\end{abstract}

Index Terms - variability constraints, DAE systems, aircraft landing, barrier method.

\section{INTRODUCTION}

$\mathbf{I}$ $\mathrm{N}$ THE past few decades a number of significant airplane accidents have resulted from windshear, which can be observed during takeoff or landing. Especially, such meteorological and physical phenomena as convective turbulences, gust fronts and terrain-influenced can be responsible for windshear. In general, windshear cannot constitute a danger to an airplane flying, nevertheless it may have a critical impact on flight safety at low altitude. Moreover, in takeoff and landing phases, the aircraft is low above the ground and it has no much time or space to maneuver [14].

It is worth to indicate, that low-level turbulence, below 1600 $\mathrm{ft}(500 \mathrm{~m})$, cannot be directly measured but only inferred from observational data. In their work, Hon and Chan [9] have analyzed such approaches, useful to observe a terraininduced low-level turbulence intensity. There are data obtained by the scanning Doppler lidar, the terminal Doppler weather radar (TDWR), a high-resolution anemometer, as well as the operational Windshear and Turbulence Warning System (WTWS).

Recently, Chan and Hon [5] have employed a high resolution numerical aviation model (AVM) to forecast the windshear occurrence and applied it at Hong Kong International Airport to predict the terrain-induced windshear. To improve the automatic landing procedure, Zhao et al. [22] proposed a linear model of the aircraft in longitudinal motion. The applied tracking error integral equations enables us to achieve a desirable tracking performance. The presented approach has been tested on the Boeing 747 airplane and the windshear model and showed the effectiveness and robustness of the proposed approach.
The aircraft landing problem in the presence of the windshear in the context of the feedback control has been extensively investigated by Patsko et al. [16]. It was assumed, that the bounds on the wind velocity deviations from some nominal values are known, while there is no information about the windshear location and wind velocity distribution in the windshear zone. Finally, to solve the presented problem, the methods of differential game theory have been employed

- pilot (the first player) need to choose the control parameters to minimize the payoff function,

- nature (the second player) need to design an appropriate wind disturbance to obtain an opposite interest.

The mathematical formulation of an aircraft abort landing problem has been presented by Bulirsch et al. [3], [4]. The flight maneuver can be described by a minimax optimal control problem with nonlinear differential-algebraic model of the aircraft dynamics. Moreover, a first-order state constraints, as well as a control variable constraint have been imposed on the model. The obtained optimal control problem has been solved numerically using the multiple shooting approach in connection with a homotopy strategy.

One of the first flight trajectories optimization procedures in the presence of windshear have been designed by the AeroAstronautics Group of Rice University [13]. In the mentioned research three situations have been deeply investigated: takeoff, abort landing, as well as penetration landing. Then, Pytlak and Vinter [17] have presented an optimization algorithm for optimal control problems with control, state, as well as terminal constraints. The designed algorithm has been successfully applied to solve the aircraft abort landing problem in the presence of windshear.

Recently, to solve the aircraft abort landing problem in a windshear downburst, $\mathrm{Li}$ et al. [10] designed and implemented a smooth approximate function method for solving a general class of minmax optimal control problems. Finally, the necessary and sufficient optimality conditions have been derived. To solve the obtained optimal control problem the control parameterization approach, as well as a time scaling transform have been applied.

The modern numerical control procedures are designed on the basis of efficient nonlinear optimization algorithms. Especially, new variants of a barrier method are treated as 
effective procedures to solve large-scale optimization problems. Baccari et al. [1] designed a new variant of the barrier function approach for constrained Model Predictive Control (MPC) problems. Moreover, the parallelization mechanism enabled to obtain the encouraging results without increasing the computation time. A Nonlinear Model Predictive Control (NMPC) method for the trajectory tracking problem of an autonomous underwater vehicle (AUV) has been investigated by Shen et al. [18]. Then, to handle the realistic constraints on the AUV thrusters, the authors have incorporated the log barrier functions into the cost function and modified the continuation/generalized minimal residual algorithm designed by Ohtsuka [15]. Finally, the effectiveness and efficiency of the new algorithm for the AUV tracking control problem has been demonstrated. Steinboeck et al. [20] designed a new NMPC algorithm for optimization complex systems described by hyperbolic partial differential equations. Then, the discretized constrained optimal control problem has been formulated in the form of barrier functions. The model predictive control approach for linear discrete-time systems subject to state constraints has been investigated in [8]. In the on-line optimization procedure a relaxed barrier function has been implemented. Finally, both the theoretical and numerical results have been presented.

The mentioned recently designed control procedures require efficient nonlinear optimization algorithms. Therefore, according to the presented advances in Nonlinear Model Predictive Control methods, in the next sections the new optimizationbased approach for aircraft landing in the presence of the windshear is presented.

The article is constructed as follows. In Section 2 a detailed description of the problem is presented. Then, in Section 3 , the new optimization-based solving procedure with the modified direct shooting method is discussed. The results of computational simulations are presented in Section 4. Finally, the presented considerations are concluded in Section 5 .

\section{THE PROBLEM DESCRIPTION}

During the aircraft landing maneuver, the situation presented on the Fig. 1 can be observed:

1) the plane lowers the flight,

2) the speed of the aircraft is reduced,

3) the downdraft zone,

4) the strong wind from the back of the plane.

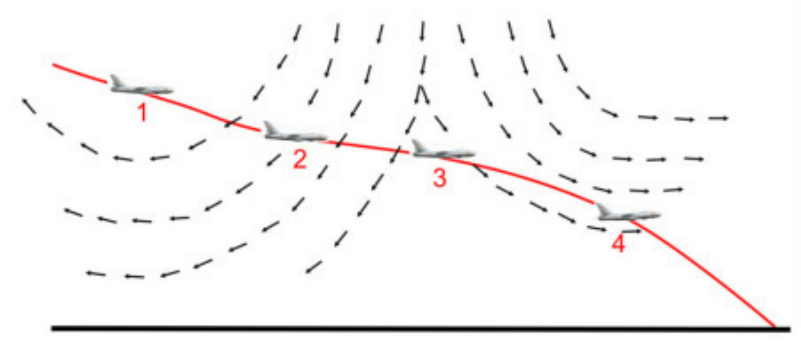

Fig. 1. The aircraft landing with the presence of the windshear [21].
The dynamical properties of the aircraft motion can be described by the following ordinary differential equations

$$
\begin{aligned}
& \dot{y}_{x}=y_{\nu} \cos y_{\gamma}+w_{x}, \\
& \dot{y}_{h}=y_{\nu} \sin y_{\gamma}+w_{h},
\end{aligned}
$$

$\dot{y}_{\nu}=\frac{1}{m}[T \cos (\alpha+\delta)-D]-g \sin y_{\gamma}-\left(\dot{w}_{x} \cos y_{\gamma}+\dot{w}_{h} \sin y_{\gamma}\right)$,

$\dot{y}_{\gamma}=\frac{1}{m y_{\nu}}[T \sin (\alpha+\delta)+L]-\frac{g}{y_{\nu}} \cos y_{\gamma}+\left(\dot{w}_{x} \sin y_{\gamma}-\dot{w}_{h} \cos y_{\gamma}\right)$,

where the state variables

$$
\mathbf{y}(t)=\left[\begin{array}{l}
y_{x}(t) \\
y_{h}(t) \\
y_{\nu}(t) \\
y_{\gamma}(t)
\end{array}\right]
$$

are the horizontal distance $y_{x}$, the altitude $y_{h}$, the relative velocity $y_{\nu}$ and the relative flight path angle $y_{\gamma}$. Moreover, the thrust and aerodynamic forces were defined

$$
\begin{aligned}
& T=\beta T_{\star}, \\
& T_{\star}=a_{0}+a_{1} y_{\nu}+a_{2} y_{\nu}^{2}, \\
& D=\frac{1}{2} C_{D} \rho S y_{\nu}^{2}, \\
& C_{D}(\alpha)=b_{0}+b_{1} \alpha+b_{2} \alpha^{2}, \\
& L=\frac{1}{2} C_{L} \rho S y_{\nu}^{2}, \\
& C_{L}(\alpha)=\left\{\begin{array}{r}
c_{0}+c_{1} \alpha, \text { for } \alpha \leq \alpha_{\star}, \\
c_{0}+c_{1} \alpha+c_{2}\left(\alpha-\alpha_{\star}\right)^{2} \\
\text { for } \alpha_{\star} \leq \alpha \leq \alpha_{\max },
\end{array}\right. \\
& \beta(t)= \begin{cases}\beta_{0}+\dot{\beta}_{1} t, & \text { for } 0 \leq t \leq t_{\beta} \\
1, & \text { for } t_{\beta} \leq t \leq t_{F}\end{cases}
\end{aligned}
$$

where the thrust, drag and lift were denoted as $T, D$ and $L$, respectively. Upon sensing a downdraft, the pilot increases power at a constant rate until reaching the maximum value at time

$$
t_{\beta}=\left(1-\beta_{0}\right) / \dot{\beta}_{0} .
$$

and holds it constant. The windshear is modeled as follows

$$
\begin{aligned}
& w_{x}=A\left(y_{x}\right), \\
& w_{h}=\frac{h}{h_{\star}} B\left(y_{x}\right),
\end{aligned}
$$

where

$$
A\left(y_{x}\right)=\left\{\begin{array}{c}
-50+a y_{x}^{3}+b y_{x}^{4}, \text { for } 0 \leq y_{x} \leq 500 \\
\frac{1}{40}\left(y_{x}-2300\right), \text { for } 500 \leq y_{x} \leq 4100 \\
50-a\left(4600-y_{x}\right)^{3}-b\left(4600-y_{x}\right)^{4} \\
50, \text { for } 4600 \leq y_{x}, \quad \text { for } 4100 \leq y_{x} \leq 4600
\end{array}\right.
$$


TABLE I

THE MODEL PARAMETERS OF A BOEING 727 AIRPLANE [2].

\begin{tabular}{|c|c|}
\hline$t_{F}$ & $40 \mathrm{sec}$ \\
\hline$u_{\max }$ & $3 \mathrm{deg} / \mathrm{sec}$ \\
\hline$\alpha_{\max }$ & $17,2 \mathrm{deg}$ \\
\hline$\rho$ & $0,2203 \times 10^{-2} \mathrm{lb} \mathrm{sec}^{2} \mathrm{ft}^{-4}$ \\
\hline$S$ & $0,1560 \times 10^{4} \mathrm{ft}^{2}$ \\
\hline$g$ & $3,2172 \times 10^{1} \mathrm{ft} \mathrm{sec}^{-2}$ \\
\hline$m g$ & $1,5 \times 10^{5} \mathrm{lb}$ \\
\hline$\delta$ & $2 \mathrm{deg}$ \\
\hline$a_{0}$ & $0,4456 \times 10^{5} \mathrm{lb}$ \\
\hline$a_{1}$ & $-0,2398 \times 10^{2} \mathrm{lb} \mathrm{sec} / \mathrm{ft}$ \\
\hline$a_{2}$ & $0,1442 \times 10^{-1} \mathrm{lb} \mathrm{sec}^{2} \mathrm{ft}^{-2}$ \\
\hline$\beta_{0}$ & 0,3825 \\
\hline$\dot{\beta}_{0}$ & $0,2 \mathrm{sec}^{-1}$ \\
\hline$b_{0}$ & 0,1552 \\
\hline$b_{1}$ & $0,12369 \mathrm{rad}^{-1}$ \\
\hline$b_{2}$ & $2,4203 \mathrm{rad}^{-2}$ \\
\hline$c_{0}$ & 0,7125 \\
\hline$c_{1}$ & $6,0877 \mathrm{rad}^{-1}$ \\
\hline$c_{2}$ & $-9,0277 \mathrm{rad}^{-2}$ \\
\hline$a_{\star}$ & $12 \mathrm{deg}$ \\
\hline$h_{\star}$ & $1000 \mathrm{ft}$ \\
\hline$a$ & $6 \times 10^{-8} \mathrm{sec}^{-1} \mathrm{ft}^{-2}$ \\
\hline$b$ & $-4 \times 10^{-11} \mathrm{sec}^{-1} \mathrm{ft}^{-3}$ \\
\hline$c$ & $-\ln (25 / 30,6) \times 10^{-12} \mathrm{ft}^{-4}$ \\
\hline$d$ & $-8,02881 \times 10^{-8} \mathrm{sec}^{-1} \mathrm{ft}^{-2}$ \\
\hline$e$ & $6,28083 \times 10^{-11} \mathrm{sec}^{-1} \mathrm{ft}-3$ \\
\hline$x_{0}$ & $0 \mathrm{ft}$ \\
\hline$\gamma_{0}$ & $-2,249 \mathrm{deg}$ \\
\hline$h_{0}$ & $600 \mathrm{ft}$ \\
\hline$\alpha_{0}$ & $7,353 \mathrm{deg}$ \\
\hline$\nu_{0}$ & $239,7 \mathrm{ft} / \mathrm{sec}$ \\
\hline$\gamma_{F}$ & $7,431 \mathrm{deg}$ \\
\hline
\end{tabular}

$$
B\left(y_{x}\right)=\left\{\begin{array}{c}
d y_{x}^{3}+e y_{x}^{4}, \text { for } 0 \leq y_{x} \leq 500, \\
-51 \exp \left[-c\left(y_{x}-2300\right)^{4}\right], \\
\quad \text { for } 500 \leq y_{x} \leq 4100, \\
d\left(4600-y_{x}\right)^{3}+e\left(4600-y_{x}\right)^{4}, \\
\quad \text { for } 4100 \leq y_{x} \leq 4600 \\
0, \text { for } 4600 \leq y_{x} .
\end{array}\right.
$$

The model parameters of a Boeing 727 airplane were summarized in the Table I.

The goal of this problem is to avoid having the airplane crash. To achieve this, two important conditions need to be satisfied. An unknown parameter $h_{\text {min }}$ represents the minimum altitude that occurs during the maneuver. Moreover, an additional inequality constraint

$$
y_{h}(t) \geq h_{\min }
$$

was introduced. Then, a new objective function can be constructed

$$
h_{\min } \rightarrow \max
$$

This representation of the optimization problem guarantees that aircraft will be as high above the ground as possible. In the considered task the angle of attack $\alpha(t)$ is treated as the control variable. Moreover, the control variable is subject to the two inequality constraints

$$
u(t)=\alpha(t) \leq \alpha_{\max }
$$

and

$$
|\dot{u}(t)|=|\dot{\alpha}(t)| \leq u_{\max }
$$

The eq. (14) has a character of a variability constraint.

In the presented task five flight phases can be distinguished. Each flight phase is characterized by the appropriate boundary conditions, as well as additional features.

Phase 1: $0=t_{0}^{1} \leq t \leq t_{F}^{1}$

$$
\begin{aligned}
y_{x}\left(t_{0}^{1}\right) & =y_{x_{0}}, \\
y_{\nu}\left(t_{0}^{1}\right) & =y_{\nu_{0}}, \\
y_{h}\left(t_{0}^{1}\right) & =y_{h_{0}}, \\
y_{\gamma}\left(t_{0}^{1}\right) & =y_{\gamma_{0}}, \\
u_{\alpha}\left(t_{0}^{1}\right) & =u_{\alpha_{0}}, \\
y_{x}\left(t_{F}^{1}\right) & =500, \\
A\left(y_{x}\right) & =-50+a y_{x}^{3}+b y_{x}^{4}, \\
B\left(y_{x}\right) & =d y_{x}^{3}+e y_{x}^{4}, \\
\beta(t) & =\beta_{0}+\dot{\beta}_{0} t .
\end{aligned}
$$

Phase 2: $t_{I}^{2} \leq t \leq t_{F}^{2}=t_{\beta}$

$$
\begin{aligned}
y_{x}\left(t_{0}^{2}\right) & =500 \\
A\left(y_{x}\right) & =-50+a y_{x}^{3}+b y_{x}^{4}, \\
B\left(y_{x}\right) & =d y_{x}^{3}+e y_{x}^{4}, \\
\beta(t) & =\beta_{0}+\dot{\beta}_{0} t .
\end{aligned}
$$

Phase 3: $t_{\beta}=t_{I}^{3} \leq t \leq t_{F}^{3}$

$$
\begin{aligned}
y_{x}\left(t_{F}^{3}\right) & =4100, \\
A\left(y_{x}\right) & =\frac{1}{40}\left(y_{x}-2300\right), \\
B\left(y_{x}\right) & =-51 \exp \left(-c\left(y_{x}-2300\right)^{4}\right), \\
\beta(t) & =1 .
\end{aligned}
$$

Phase 4: $t_{0}^{4} \leq t \leq t_{F}^{4}$

$$
\begin{aligned}
y_{x}\left(t_{0}^{4}\right) & =4100, \\
y_{x}\left(t_{F}^{4}\right) & =4600, \\
A\left(y_{x}\right) & =50-a\left(4600-y_{x}\right)^{3}-b\left(4600-y_{x}\right)^{4}, \\
B\left(y_{x}\right) & =d\left(4600-y_{x}\right)^{3}+e\left(4600-y_{x}\right)^{4}, \\
\beta(t) & =1 .
\end{aligned}
$$

Phase 5: $t_{0}^{5} \leq t \leq t_{F}^{5}$

$$
\begin{aligned}
y_{x}\left(t_{0}^{5}\right) & =4600, \\
y_{\gamma}\left(t_{F}^{5}\right) & =y_{\gamma_{F}}, \\
A\left(y_{x}\right) & =50, \\
B\left(y_{x}\right) & =0, \\
\beta(t) & =1 .
\end{aligned}
$$


As one can observe, each stage of the process is described by a system of differential-algebraic equations

$$
\begin{aligned}
\dot{\mathbf{y}}(t) & =F(\mathbf{y}(t), \mathbf{z}(t), \mathbf{u}(t), \mathbf{p}, t) \\
0 & =G(\mathbf{y}(t), \mathbf{z}(t), \mathbf{u}(t), \mathbf{p}, t)
\end{aligned}
$$

where $\mathbf{y}(t) \in \mathcal{R}^{n_{\mathbf{y}}}$ denotes a differential state trajectory, $\mathbf{z}(t) \in \mathcal{R}^{n_{\mathbf{z}}}$ is an algebraic state trajectory, $\mathbf{u}(t) \in \mathcal{R}^{n_{\mathbf{u}}}$ denotes a control function, $\mathbf{p} \in \mathcal{R}^{n_{\mathbf{p}}}$ is a vector of global parameters constant in the time. The indendent variable was time denoted by $t \in \mathcal{R}$. Moreover, two vector-valed functions are considered

$$
\begin{aligned}
& F: \mathcal{R}^{n_{\mathbf{y}}} \times \mathcal{R}^{n_{\mathbf{z}}} \times \mathcal{R}^{n_{\mathbf{u}}} \times \mathcal{R}^{n_{\mathbf{p}}} \times \mathcal{R} \rightarrow \mathcal{R}^{n_{\mathbf{y}}} \\
& G: \mathcal{R}^{n_{\mathbf{y}}} \times \mathcal{R}^{n_{\mathbf{z}}} \times \mathcal{R}^{n_{\mathbf{u}}} \times \mathcal{R}^{n_{\mathbf{p}}} \times \mathcal{R} \rightarrow \mathcal{R}^{n_{\mathbf{z}}}
\end{aligned}
$$

Assumption 2.1: The index of the DAEs system (20) is not greater than 1 .

To solve the flight optimization task presented by eq. (1)-(19), the new optimization-based solving procedure was designed.

\section{THE NEW OPTIMIZATION-BASED SOLVING PROCEDURE}

The optimization procedure is consisted on two main steps. The first one is based on the multiple shooting method and a control vector parameterization. In the second step - the parameterized model is optimized by a barrier algorithm.

Each phase of the flight was described by a system of differential-algebraic equations (1)-(19). Moreover, each phase was discretized using a modified direct shooting approach, where left-hand side of ODEs are treated also as additional decision variables [7]. Then, with 3 shooting points in each interval, in the considered process $3 \times 5=15$ subintervals were distinguished. Therefore, the time domain

$$
t \in\left[\begin{array}{ll}
t_{0} & t_{f}
\end{array}\right]
$$

was divided according to the indicated 15 subdomains in the following way

$$
t^{i} \in\left[\begin{array}{ll}
t_{0}^{i} & t_{f}^{i}
\end{array}\right], \quad i=1, \cdots, 15,
$$

where

$$
t_{0}=t_{0}^{1}<t_{f}^{1}<\cdots=t_{0}^{15}<t_{f}^{15}=t_{f}
$$

The considered DAE constraints were pointwise discretized as follows

$$
\begin{aligned}
\mathbf{x}_{\dot{\mathbf{y}}^{i}} & =\widetilde{F}^{i}\left(\mathbf{x}_{\mathbf{y}^{i}}, \mathbf{x}_{\mathbf{z}^{i}}, \mathbf{x}_{\mathbf{u}^{i}}, \mathbf{x}_{\mathbf{p}}, t^{i}\right) \\
0 & =\widetilde{G}^{i}\left(\mathbf{x}_{\mathbf{y}^{i}}, \mathbf{x}_{\mathbf{z}^{i}}, \mathbf{x}_{\mathbf{u}^{i}}, \mathbf{x}_{\mathbf{p}}, t^{i}\right)
\end{aligned}
$$

where

$$
\begin{aligned}
\mathbf{x}_{\dot{\mathbf{y}}^{i}} & =\dot{\mathbf{y}}\left(t_{0}^{i}\right) \\
\mathbf{x}_{\mathbf{y}^{i}} & =\mathbf{y}\left(t_{0}^{i}\right) \\
\mathbf{x}_{\mathbf{z}^{i}} & =\mathbf{z}\left(t_{0}^{i}\right) \\
\mathbf{x}_{\mathbf{u}^{i}} & =\mathbf{u}\left(t_{0}^{i}\right) \\
\mathbf{x}_{\mathbf{p}} & =\mathbf{p}
\end{aligned}
$$

and

$$
\begin{aligned}
& \widetilde{F}^{i}: \mathcal{R}^{n_{\mathbf{x}_{\mathbf{y}^{i}}}} \times \mathcal{R}^{n_{\mathbf{x}_{\mathbf{z}} i}} \times \mathcal{R}^{n_{\mathbf{x}_{\mathbf{u}} i}} \times \mathcal{R}^{n_{\mathbf{x}_{\mathbf{p}}}} \times \mathcal{R} \rightarrow \mathcal{R}^{n_{\mathbf{x}_{\mathbf{y}} i}} \\
& \widetilde{G}^{i}: \mathcal{R}^{n_{\mathbf{x}_{\mathbf{y}}}} \times \mathcal{R}^{n_{\mathbf{x}_{\mathbf{z}^{i}}}} \times \mathcal{R}^{n_{\mathbf{x}_{\mathbf{u}} i}} \times \mathcal{R}^{n_{\mathbf{x}_{\mathbf{p}}}} \times \mathcal{R} \rightarrow \mathcal{R}^{n_{\mathbf{x}_{\mathbf{z}^{i}}}}
\end{aligned}
$$

for $i=1, \ldots, 15$.

Moreover, if

$$
X=\left[\begin{array}{c}
\mathbf{x}_{\dot{\mathbf{y}}^{1}} \\
\vdots \\
\mathbf{x}_{\dot{\mathbf{y}}^{15}} \\
\mathbf{x}_{\mathbf{y}^{1}} \\
\vdots \\
\mathbf{x}_{\mathbf{y}^{15}} \\
\mathbf{x}_{\mathbf{z}^{1}} \\
\vdots \\
\mathbf{x}_{\mathbf{z}^{15}} \\
\mathbf{x}_{\mathbf{u}^{1}} \\
\vdots \\
\mathbf{x}_{\mathbf{u}^{15}}
\end{array}\right]
$$

and with differential-algebraic constraints in the following form

$$
\begin{aligned}
\widetilde{F}^{i}\left(\mathbf{x}_{\mathbf{y}^{i}}, \mathbf{x}_{\mathbf{z}^{i}}, \mathbf{x}_{\mathbf{u}^{i}}, \mathbf{x}_{\mathbf{p}}, t^{i}\right)-\mathbf{x}_{\dot{\mathbf{y}}^{i}} & =0 \\
\widetilde{G}^{i}\left(\mathbf{x}_{\mathbf{y}^{i}}, \mathbf{x}_{\mathbf{z}^{i}}, \mathbf{x}_{\mathbf{u}^{i}}, \mathbf{x}_{\mathbf{p}}, t^{i}\right) & =0
\end{aligned}
$$

for $i=1, \ldots, 15$. Then, the process constraints can be reduced to the general form

$$
\begin{aligned}
& \hat{F}(\mathbf{X})=0 \\
& \hat{G}(\mathbf{X})=0
\end{aligned}
$$


The presented modified direct shooting approach enables us to consider the variability constraints in the following form

$$
y^{L} \leq \dot{\mathbf{y}}(t) \leq y^{U}
$$

directly by

$$
\mathbf{x}_{\dot{y}}^{L} \leq \mathbf{x}_{\dot{y}} \leq \mathbf{x}_{\dot{y}}^{U},
$$

where $L$ and $U$ denote lower and upper bounds, respectively. After that, the appropriate nonlinear optimization problem with a pointwise-continuous differential-algebraic constraints can be considered

$$
\min _{\mathbf{X}} f(\mathbf{X})
$$

subject to

$$
\begin{aligned}
& \hat{F}(\mathbf{X})=0 \\
& \hat{G}(\mathbf{X})=0 \\
& \mathbf{X}_{\dot{\mathbf{y}}} \leq \mathcal{V}(\mathbf{X}) \\
& \mathbf{X}^{L} \leq \mathbf{X} \leq \mathbf{X}^{U}
\end{aligned}
$$

To solve the nonlinear optimization problem with the variability constraints, the barrier method implemented in function fmincon in MATLAB Optimization Toolbox was applied [11]. The obtained results were presented in the next section.

\section{RESULTS OF COMPUTATIONAL SIMULATIONS}

The aircraft state trajectories have been obtained after 1,101 iterations and 170,855 objective function evaluation. The final value of the objective function was equal to $429.6 \mathrm{~m}$. The results of the presented solving procedure were illustrated on the Fig. (2)-(6). The results and shape of the state trajectories are comparable with the solution obtained using Sparse Optimal Control Software - SOCS, developed by The Boeing Company [2].

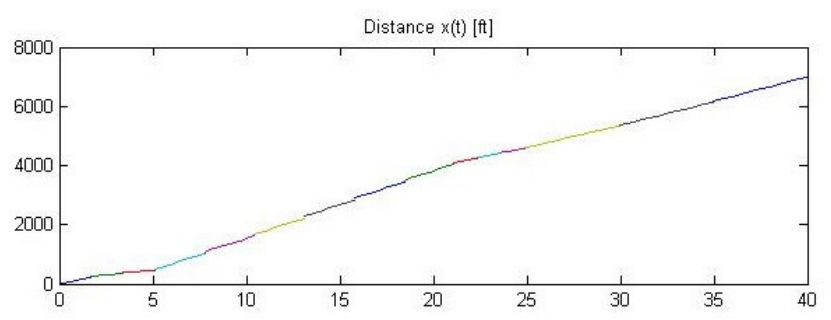

Fig. 2. The state trajectory $y_{x}(t)$.

\section{CONCLUSION}

In the article the modified direct shooting approach for the aircraft landing control problem in the presence of windshear was presented. In the discussed methodology, the values of the state trajectories, as well as left hand-side values of the ODEs, are treated as the additional decision variables. As the results of presented transformation, a large-scale nonlinear optimization problem with pointwise-continuous differential-algebraic

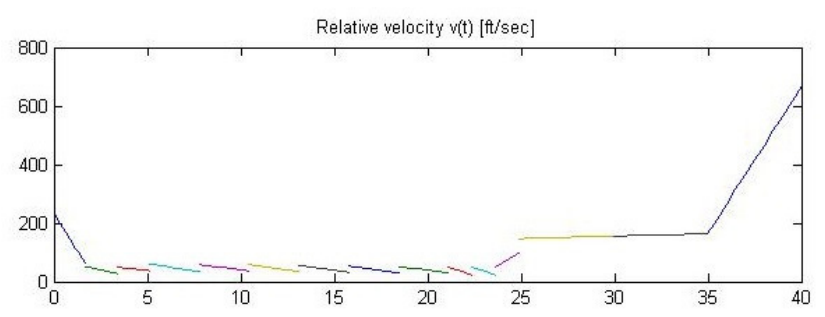

Fig. 3. The state trajectory $y_{v}(t)$.

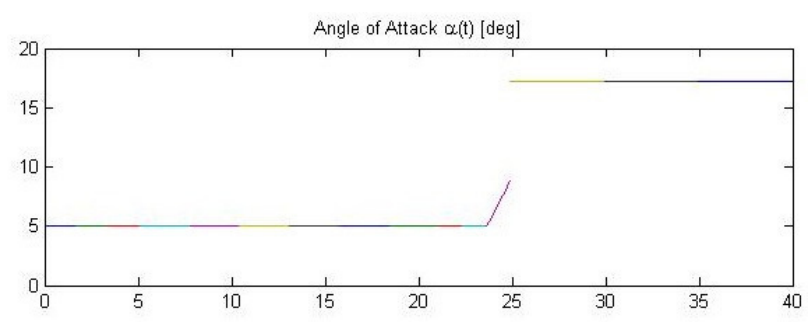

Fig. 4. The control trajectory $\alpha(t)$.

constraints was obtained. To solve the nonlinear optimization task, the barrier algorithm implemented in fmincon function in Matlab environment was used.

The future work should be concentrated on the NLP solver improvements in order to minimize number of the objective function evaluations. Moreover, the presented modifications can be applied in the autonomous takeoff and landing procedures [6], [12], [19].

\section{ACKNOWLEDGMENT}

The work of Paweł Drąg has been supported by the grant „Młoda Kadra”- „Young Staff” 0402/0228/16 at Wrocław University of Science and Technology. The work of Krystyn

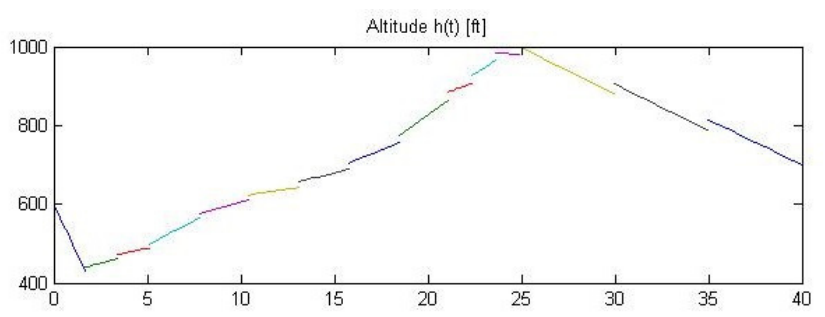

Fig. 5. The state trajectory $y_{h}(t)$.

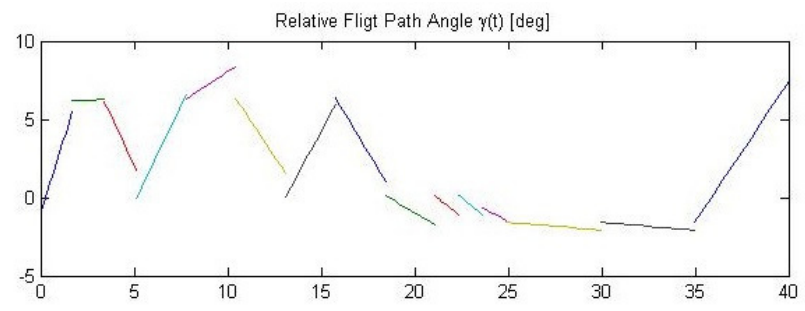

Fig. 6. The state trajectory $y_{\gamma}(t)$. 
Styczeń has been supported by the National Science Center under grant: DEC-2012/07/B/ST7/01216

\section{REFERENCES}

[1] S. Baccari, L. Iannelli, F. Vasca. 2012. A Parallel Algorithm for Implicit Model Predictive Control with Barrier Function. 2012 IEEE International Conference on Control Applications (CCA) Part of 2012 IEEE MultiConference on Systems and Control October 3-5, 2012. Dubrovnik, Croatia. 1405-1410, http://dx.doi.org/10.1109/CCA.2012.6402342

[2] J.T. Betts. 2010. Practical Methods for Optimal Control and Estimation Using Nonlinear Programming. SIAM, Philadelphia, http://dx.doi.org/10.1137/1.9780898718577

[3] R. Bulirsch, F. Montrone, H.J. Pesch. 1991. Abort landing in the presence of windshear as a minimax optimal control problem, part 1: Necessary conditions. Journal of Optimization Theory and Applications. 70:1-23, http://dx.doi.org/10.1007/BF00940502

[4] R. Bulirsch, F. Montrone, H.J. Pesch. 1991. Abort landing in the presence of windshear as a minimax optimal control problem, part 2: Multiple shooting and homotopy. Journal of Optimization Theory and Applications. 70:223-254, http://dx.doi.org/10.1007/BF00940625

[5] P.W. Chan, K.K. Hon. 2016. Observation and Numerical Simulation of Terrain-Induced Windshear at the Hong Kong International Airport in a Planetary Boundary Layer without Temperature Inversions. Advances in Meteorology. Article ID 1454513 , http://dx.doi.org/10.1155/2016/1454513

[6] L.H. De Godoy Patire, N.B.F Silva, K.R.L.J.C Branco. 2016. Data fusion techniques applied to takeoff and landing procedures - A VTOL case study. IEEE Latin America Transactions. 14:3962-3966. http://dx.doi.org/10.1109/TLA.2016.7785919

[7] P. Dra̧g. 2016. Algorytmy sterowania wielostadialnymi procesami deskryptorowymi. Warszawa, Akademicka Oficyna Wydawnicza EXIT, (in polish)

[8] C. Feller, C. Ebenbauer. 2017. A stabilizing iteration scheme for model predictive control based on relaxed barrier functions. Automatica. 80:328 339, https://doi.org/10.1016/j.automatica.2017.02.001

[9] K.K. Hon, P.W. Chan. 2014. Terrain-Induced Turbulence Intensity during Tropical Cyclone Passage as Determined from Airborne, Ground-Based, and Remote Sensing Sources. Journal of Atmospheric and Oceanic Technology. 31:2373-2391, http://dx.doi.org/10.1175/JTECH-D-14-00006.1

[10] B. Li, K.L. Teo, G.H. Zhao, G.R. Duan. 2009. An efficient computational approach to a class of minmax optimal con- trol problems with applications. ANZIAM Journal. 51:162-177, http://doi.org/10.1017/S1446181110000040

[11] MathWorks. 2017. Global Optimization Toolbox. User's Guide R2017a.

[12] F.L.L. Medeiros, V.C.F. Gomes, M.R.C. De Aquino, D. Geraldo, M.E.L. Honorato, L.H.M. Dias. 2015. Proceedings - 2015 Brazilian Conference on Intelligent Systems, BRACIS 2015, pp. 333-338, http://doi.org/10.1109/BRACIS.2015.53

[13] A. Miele. 1990. Optimal trajectories and guidance trajectories for aircraft flight through windshears. Proceedings of the 29th IEEE Conference on Decision and Control Part 6 (of 6); Honolulu, HI, USA; 5-7 December 1990. 2:737-746, http://dx.doi.org/10.1109/CDC.1990.203686

[14] C. Moscardini, F. Berizzi, M. Martorella, A. Capria. 2011. Signal spectral modelling for airborne radar in the presence of windshear phenomena. IET Radar, Sonar and Navigation. 5:796-805, http://dx.doi.org/10.1049/iet-rsn.2010.0234

[15] T. Ohtsuka. 2004. A continuation/GMRES method for fast computation of nonlinear receding horizon control. Automatica. 40:563-574, http://doi.org/10.1016/j.automatica.2003.11.005

[16] V.S. Patsko, N.D. Botkin, V.M. Kein, V.L. Turova, M.A. Zarkh. 1994. Control of an aircraft landing in windshear. Journal of Optimization Theory and Applications. 83:237-267, http://dx.doi.org/10.1007/BF02190056

[17] R. Pytlak, R.B. Vinter. 1999. Feasible Direction Algorithm for Optimal Control Problems with State and Control Constraints: Implementation. Journal of Optimization Theory and Applications. 101:623-649, http://dx.doi.org/10.1023/A:1021742204850

[18] C. Shen, B. Buckham, Y. Shi. 2016. Modified C/GMRES Algorithm for Fast Nonlinear Model Predictive Tracking Control of AUVs. IEEE Transactions on Control Systems Technology, http://dx.doi.org/10.1109/TCST.2016.2628803, (to appear)

[19] N.B.F. Silva, E.A. Marconato, K.R.L.J.C. Branco. 2015. AVALON: Definition and modeling of a vertical takeoff and landing UAV. Journal of Physics: Conference Series. Vol. 633, Article number 012125, http://dx.doi.org/10.1088/1742-6596/633/1/012125

[20] A. Steinboeck, M. Guay, A. Kugi. 2016. Real-Time Nonlinear Model Predictive Control of a Transport-Reaction System. Industrial and Engineering Chemistry Research. 55:7730-7741, http://dx.doi.org/10.1021/acs.iecr.6b00592

[21] Szefostwo Służby Hydrometeorologicznej Sił Zbrojnych RP. 2011. Meteorologia dla pilotów - poradnik. Warszawa, 2011, (in polish)

[22] L. Zhao, X. Yang, H. Gao, P. Shi. 2013. Automatic Landing System Design Using Multiobjective Robust Control. Journal of Aerospace Engineering. 26:603-617, http://dx.doi.org/10.1061/(ASCE)AS.19435525.0000174 


\title{
Concepts Selection in Fuzzy Cognitive Map using Evolutionary Learning Algorithm based on Graph Theory Metrics
}

\author{
Katarzyna Poczeta, Łukasz Kubuś, Alexander Yastrebov \\ Kielce University of Technology \\ al. Tysiąclecia Państwa Polskiego 7 \\ 25-314 Kielce, Poland \\ Email: \{k.piotrowska,lkubus,jastri\}@tu.kielce.pl
}

\begin{abstract}
Fuzzy cognitive map (FCM) allows to discover knowledge in the form of concepts significant for the analyzed problem and causal connections between them. The FCM model can be developed by experts or using learning algorithms and available data. The main aspect of building of the FCM model is concepts selection. It is usually based on the expert knowledge. The aim of this paper is to develop and analyze a new evolutionary algorithm for selection of key concepts and determining the weights of the connections between them on the basis of available data. The proposed approach allows to reduce concepts during learning process based on metrics from the area of graph theory: significance of each node and total influence of the concept. A simulation analysis of the developed algorithm was done with the use of real-life data.
\end{abstract}

\section{INTRODUCTION}

$\mathbf{F}$ UZZY cognitive map (FCM) is a directed weighted graph for representing knowledge [9]. It is an effective tool for modeling dynamic decision support systems [13], [25]. Fuzzy cognitive maps allow to visualize complex systems as a set of key concepts (nodes) and connections (links) between them. The FCM model can be built based on expert knowledge [3], [4]. Experts choose the most significant concepts and determine type and strength of the relationships between them (weights of the connections). Fuzzy cognitive map can be also initialized with the use of learning algorithms [16] and historical data. Standard supervised [8] and evolutionary algorithms [13], [23], [24] allow to determine the structure of the FCM model based on all available data. For each data attribute new concept is created. Next the weights of the connections are specified during learning process.

Fuzzy cognitive maps with the large number of concepts are difficult to analyze and interpret. Moreover, with the growth of the number of concepts, the number of connections between them that should be determined increases quadratically. Several researchers have attempted to develop methods of reduction of fuzzy cognitive map size. In [5] a new approach for reduction of the FCM model complexity by merging related or similar initial concepts into the same cluster of concepts is presented. These clusters can be used then as the real concepts in the reduced FCM model. Concepts clustering technique based on fuzzy tolerance relations was used in [17] for modeling of waste management system. The analysis of the decision making capabilities of the less complex FCM shows that proper concepts reductions make models easier to be used keeping their original dynamic behavior. Also cluster validity indexes were introduced to evaluate Fuzzy Cognitive Map design before training phase in [6]. The resulting FCM models are easy to interpret and properly perform the task of prediction. Homenda et al. [7] introduced a time series modeling framework based on simplified Fuzzy Cognitive Maps using a priori nodes rejection criteria. The obtained results confirmed that this approach for simplifying complex FCM models allows to achieve a reasonable balance between complexity and modeling accuracy. Selvin and Srinivasaraghavan proposed an application of the feature selection techniques to reduce the number of the input concepts of fuzzy cognitive map [21]. The feature selection methods were performed based on the significance of each concept to the output concept. However the influences of the connections between the concepts were not taken into consideration. In [18], [19] the structure optimization genetic algorithm for fuzzy cognitive maps learning was presented. It allows to select the most significant concepts and connections between them based on random generation of possible solutions and the error function that takes into account an additional penalty for highly complexity of FCM during learning process. The usefulness of the developed approach was shown on the example of the one-step ahead time series prediction.

The advantage of the FCM model is its graph-based representation that allows to use various methods and metrics from the area of graph theory to analyze the structure and behavior of the modeled system [26]. In this paper we propose to use two various metrics to reduce concepts of the FCM model during learning process. The first metric is the degree of a node. It denotes its significance based on the number of concepts it interacts with (is affected by and it affects) [4]. The second metric is one of the system performance indicators: the total (direct and indirect) influence of the concept [2], [22].

The aim of this paper is to develop the evolutionary learning algorithm that allows:

- to reduce the size of the FCM model by selecting the 
most significant concepts,

- to determine the weights of the connections between concepts,

- to approximate the real-life data [10], [15], [20].

The comparison of the developed approach with the standard one based on the all possible concepts and data error and the previously developed approach based on density and system performance indicators [12] was done. The learning process was performed using two effective techniques for FCMs learning: Elite Genetic Algorithm (EGA) [14] and Individually Directional Evolutionary Algorithm (IDEA) [11].

The outline of this paper is as follows. Section II briefly describes fuzzy cognitive maps. Section III presents the proposed approach for fuzzy cognitive map learning and concepts selection. In Section IV, the results of the simulation analysis based on real-life data are presented. Section V contains the conclusions and further work.

\section{Fuzzy Cognitive Maps}

Fuzzy cognitive map is a directed weighted graph for representing causal reasoning [9]:

$$
<X, W>
$$

where $X=\left[X_{1}, \ldots, X_{n}\right]^{T}$ is the set of the concepts, $n$ is the number of concepts determining the size of the FCM model, $W$ is the connection matrix, $w_{j, i}$ is the weight of the influence between the $j$-th concept and the $i$-th concept, taking on the values from the range $[-1,1] . w_{j, i}>0$ means $X_{j}$ causally increases $X_{i}, w_{j, i}<0$ means $X_{j}$ causally decreases $X_{i}$.

Fuzzy cognitive map allows to model behavior of dynamic decision support systems and can be used in a what-if analysis [1]. The values of the concepts determine the state of the FCM model and can be calculated according to the selected dynamic model. In the paper one of the most popular dynamic models was used [23]:

$$
X_{i}(t+1)=F\left(\sum_{j=1, j \neq i}^{n} w_{j, i} \cdot X_{j}(t)\right)
$$

where $X_{i}(t)$ is the value of the $i$-th concept at the $t$-th iteration, $i=1,2, \ldots, n, t$ is discreet time, $t=0,1,2, \ldots, T$. Transformation function $F(x)$ normalizes values of the concepts to a proper range. The most often used function is a logistic one, described as follows [23], [24]:

$$
F(x)=\frac{1}{1+e^{-c x}}
$$

where $c$ is a parameter, $c>0$.

\section{Proposed ApPROACH}

The aim of the proposed approach is automatic concepts selection in fuzzy cognitive map during learning process using metrics from the area of graph theory. This approach requires determination of the decision (output) concepts. Other concepts are input concepts. The obtained model consists only key input concepts that affect to the decision/output concept (or concepts). During learning process we evaluate the candidate FCMs based on data error calculated for decision concepts. The significance of the concept (the degree of the concept) and the total influence of the concept were taken into account in the process of the key concepts selection.

The proposed approach contains the following steps:

STEP 1. Initialize random population.

An initial population is generated before starting evolution loop. Each candidate FCM is described by the two vectors. The first vector (4) describes values of weights between concepts [23]:

$$
W^{\prime}=\left[w_{1,2}, \ldots, w_{1, n}, w_{2,1}, w_{2,3}, \ldots, w_{2, n}, \ldots, w_{n, n-1}\right]^{T}
$$

where $w_{j, i} \in[-1,1]$ is the weight of the connection between the $j$-th and the $i$-th concept, $, j=1,2, \ldots, n$ and $n$ is the number of concepts.

The second vector (5) describes the state of each concept:

$$
\begin{gathered}
C=\left[c_{1}, c_{2}, \ldots, c_{n},\right]^{T} \\
c_{i} \in\{A S, I A S, A A S\}
\end{gathered}
$$

where $c_{i}$ is the state of $i$-th concept and $n$ is the number of concepts.

Each concept can be in the one of the three states: active (AS), inactive (IAS) and always active (AAS). The decision concept is always active. This mean, that obtained model always contains decision concept (concepts). The concepts with AS state and the decision concepts creates the collection of key concepts.

During the first step, the elements of the $W^{\prime}$ vector are initialized with the random values form the interval $[-1,1]$. The state for every node is active for all individual in the initial population. For this reason, the elements of the $C$ vector are equal to AAS for the decision concept (concepts) and AS for the other concepts.

STEP 2. Evaluate population.

Each individual is evaluated based on the following fitness function:

$$
\text { fitness }(\text { Error })=- \text { Error }
$$

where Error is the objective function calculated on the basis of data error for the decision concepts:

$$
\text { Error }=\sum_{t=1}^{T} \sum_{i=1}^{n_{d}}\left|Z_{i}(t)-X_{i}(t)\right|
$$

where $X_{i}(t)$ is the value of the $i$ th decision concept at iteration $t$ of the candidate FCM, $Z_{i}(t)$ is the value of the $i$-th decision concept at iteration $t$ in the input data, $t=0,1,2, \ldots, T, T$ is the input data length, $i=1, \ldots, n_{d}$ and $n_{d}$ is the number of decision concepts.

STEP 3. Check stop condition.

If the number of iterations is greater than iteration max $_{\text {max }}$ then the learning process is stopped.

STEP 4. Select new population.

The temporary population is created from a current base population using roulette-wheel selection with dynamic linear scaling of the fitness function [14]. 
STEP 5. Select key concepts.

Process of selection of key concepts is carried out in 3 ways:

1) Key concepts are selected at random (SC_RND).

The state of each input concept for each individual may be changed with a certain probability. The value of state change probability is in the range $(0,1)$. The concept, whose state is AS may be removed from the key concepts collection by changing the state to IAS. The concept, whose state is IAS may be added to the key concepts collection by changing the state to AS. The values of $W^{\prime}$ vector are not modified.

2) Key concepts are selected based on the degree of the node (CS_DEG).

The degree of the node (8) denotes its significance based on the number of concepts it interacts with (is affected by and it affects) [4]:

$$
\begin{gathered}
\operatorname{deg}_{i}=\frac{\sum_{j=1, j \neq i}^{n} \theta\left(w_{i, j}\right)+\sum_{j=1, j \neq i}^{n} \theta\left(w_{j, i}\right)}{2 n-1}, \\
\theta\left(w_{i, j}\right)=\left\{\begin{array}{ll}
1 & , w_{i, j} \neq 0 \\
0 & ,
\end{array} w_{i, j}=0\right.
\end{gathered}
$$

where $n$ is the number of the concepts; $w_{j, i}$ is the weight of the connection between the $j$-th and the $i$-th concept; $i, j=1,2, \ldots, n$.

The state of some concept without the decision concept (concepts) for each individual may be changed with a certain probability. The value of state change probability is in the range $(0,1)$. The $i$-th concept with minimum value of $\operatorname{deg}_{i}$ (8) from the set of key concepts (concepts whose state is AS) will be removed from the key concepts collection. The value of state attribute of this concept will be changed to IAS. The $i$-th concept with maximum value of $\operatorname{deg}_{i}$ (8) from concepts whose does not belong to the key concepts collection (concepts whose state is IAS) will be added to the key concepts collection. The value of the state attribute of this concept will be changed to AS. The value of the state attribute change probability is equal to 0.5 .

3) Key concepts are selected based on total influence of each concept (CS_INF).

The total (direct and indirect) influence between concepts is described as follows [2], [22]:

$$
\text { inf } f_{i}=\frac{\sum_{j}^{n}\left(p_{i, j}+p_{j, i}\right)}{2 n}
$$

where $n$ is the number of the concepts, $p_{j, i}$ is the total (direct and indirect) influence between the $j$-th concept and the $i$-th concept calculated on the basis of the total causal effect path between nodes [12], $i, j=1,2, \ldots, n$. The state of input concept for each individual may be changed with a certain probability. The value of state change probability is in the range $(0,1)$. The $i$-th concept with minimum value of the total influence $i n f_{i}$ (9) will be removed from the key concepts collection. The value of the state attribute of this concept will be changed to IAS. The $i$-th concept with maximum value of the total influence $i n f_{i}$ (9) from concepts whose does not belong to the key concepts collection (concepts whose state is IAS) will be added to the key concepts collection. The value of the state attribute of this concept will be changed to AS. Also, in this case the value of the state attribute change probability is equal to 0.5 .

STEP 6. Apply genetic operators with the use of selected evolutionary algorithm.

In this paper Elite Genetic Algorithm [14] and Individually Directed Evolutionary Algorithm were used [11]. The genetic operators were applied only to the $W^{\prime}$ vector. The $C$ vector was processed by independent procedure described in STEP 5 .

STEP 7. Analyze population.

Evolution loop is extended by the process of the analysis of potential solution according to the previously developed approach [12]. The values of weights from $[-0.05,0.05]$ are rounded down to 0 as suggested in [23]. Next, the matrices with the total influence between concepts $p_{j, i}$ are calculated. If the value of $p_{j, i}$ is in the interval $[-0.1,0.1]$, the corresponding weight value $w_{j, i}$ is rounded down to 0 . Moreover, genetic operators implement density control method of potential solution for consistency of the algorithm. Go to STEP 2.

STEP 8. Choose the best individual and calculate evaluation criteria.

To evaluate performance of the proposed approach, we used two criteria that are commonly used in fuzzy cognitive map learning:

1) Initial error allowing calculation of similarity between the input learning data and the data generated by the FCM model for the same initial state vector:

$$
\text { initial }_{\text {error }}=\frac{1}{T \cdot n_{d}} \sum_{t=1}^{T} \sum_{i=1}^{n_{d}}\left|Z_{i}(t)-X_{i}(t)\right|
$$

where $X_{i}(t)$ is the value of the $i$-th decision concept at iteration $t$ of the candidate FCM, $Z_{i}(t)$ is the value of the $i$-th decision concept at iteration $t$ of the input model, $t=0,1,2, \ldots, T, T$ is the input data length, $i=1, \ldots, n_{d}$, $n_{d}$ is the number of decision concepts.

2) Behavior error allowing calculation of similarity between the input testing data and the data generated by the FCM model for the same initial state vectors:

behavior $_{\text {error }}=\frac{1}{P \cdot T \cdot n_{d}} \sum_{p=1}^{P} \sum_{t=1}^{T} \sum_{i=1}^{n_{d}}\left|Z_{i}^{p}(t)-X_{i}^{p}(t)\right|$

where $X_{i}^{p}(t)$ is the value of the $i$-th decision concept at iteration $t$ of the candidate FCM started from the $p$-th initial state vector, $Z_{i}^{p}(t)$ is the value of the $i$-th decision concept at iteration $t$ of the input model started form the $p$-th initial state vector, $i=1, \ldots, n_{d}, n_{d}$ is the number of decision concepts, $p=1,2, \ldots, P, P$ is the number of the initial testing state vectors. 


\section{EXPERIMENTS}

To analyze the performance of the developed evolutionary algorithm for concepts selection real-life data were used. The aim of the analysis is to select the most significant concepts, determine the influence between them and approximate the real-life data for the output concepts.

Standard approach for fuzzy cognitive maps learning (STD), the approaches: for random concepts selection (CS_RND), for selection based on the degree of the concept (CS_DEG), for selection based on the total influence of the concept (CS_INF) and two previously analyzed algorithms based on density (DEN) [12] and based on system performance indicators (SPI) [12] were compared.

\section{A. Dataset}

Real-life data were obtained based on the three FCMs reported in literature [10], [15], [20]. The first real-life model is a decision support system in radiotherapy [15]. It contains 16 concepts: the factor-concepts $\left(X_{1}-X_{5}\right)$, that represent the depth of tumor, the size of tumor, the shape of tumor, the type of the irradiation and the amount of patient thickness irradiated, the selector-concepts $\left(X_{6}-X_{13}\right)$, representing size of radiation field, multiple field arrangements, beam directions, dose distribution from each field, stationery vs. rotationisocentric beam therapy, field modification, patient immobilizing and use of $2 \mathrm{D}$ or $3 \mathrm{D}$ conformal technique, respectively and the three output-concepts $\left(X_{14}-X_{16}\right)$ : dose given to treatment volume, amount of irradiated volume of healthy tissues and amount of irradiated volume of sensitive organs. The second model is a notional FCM model for the evaluation of mining jurisdiction investment favorability [20]. It contains 11 input concepts: national gov. stability $\left(X_{1}\right)$, regional gob. stability $\left(X_{2}\right)$, support for mining industry $\left(X_{3}\right)$, workforce education $\left(X_{4}\right)$, workforce skills/experience $\left(X_{5}\right)$, infrastructure availability $\left(X_{6}\right)$, permitting delays $\left(X_{7}\right)$, gov. royalty rates $\left(X_{8}\right)$, tax rates $\left(X_{9}\right)$, environmental activism $\left(X_{10}\right)$, union activism $\left(X_{11}\right)$ and one output node: investment favorability $\left(X_{12}\right)$. The last fuzzy cognitive map for modeling the behavior of soldiers consists of 10 concepts: cluster $\left(X_{1}\right)$, proximity of enemy $\left(X_{2}\right)$, receive fire $\left(X_{3}\right)$, presence of authority $\left(X_{4}\right)$, fire weapons $\left(X_{5}\right)$, peer visibility $\left(X_{6}\right)$, spread out $\left(X_{7}\right)$, take cover $\left(X_{8}\right)$, advance $\left(X_{9}\right)$ and fatigue $\left(X_{10}\right)$ [10]. Concepts: $X_{5}, X_{8}$ and $X_{9}$ were selected as an output of the system.

The input data for the learning process were generated starting from the one random initial vector for every map. The resulting FCM models were tested on the basis of 10 testing state vectors $(P=10)$ and evaluated with the use of criteria (10)-(11) and the number of concepts $n$.

\section{B. Learning parameters}

The following parameters were used for the EGA algorithm:

- selection method: roulette wheel selection with linear scaling

- recombination method: uniform crossover,

- crossover probability: 0.75 ,

- mutation method: non-uniform mutation,
- mutation probability: 0.02 ,

- population size: 100 ,

- number of elite individuals: 2 ,

- maximum number of iterations: 100 ,

The following parameters were used for the IDEA algorithm:

- selection method: roulette wheel selection with linear scaling

- mutation method: directed non-uniform mutation,

- mutation probability: $\frac{1}{n^{2}-n}$

- population size: 100 ,

- maximum number of iterations: 100 ,

10 experiments were performed for every set of the learning parameters and the average values (Avg) and standard deviations (Std) were calculated.

\section{Results}

Table I summarizes the average results of the experiments with the real-life data: the number of the concepts for the resulted FCM models $n$, initial and behavior error.

TABLE I

AVERAGE RESULTS WITH REAL-LIFE DATA

\begin{tabular}{ccccc}
\hline \hline Approach & Method & $n$ & initial error & $\begin{array}{c}\text { behavior } \\
\text { Avr } \\
\text { Avg }\end{array}$ \\
& & Avg \pm Std & \\
\hline STD & & 16 & $0.013 \pm 0.002$ & $0.014 \pm 0.002$ \\
CS_RND & & 14 & $0.013 \pm 0.001$ & $0.013 \pm 0.001$ \\
DEN & IDEA & 16 & $0.011 \pm 0.002$ & $\mathbf{0 . 0 1 0} \pm 0.002$ \\
CS_DEG & Model 1 & 14 & $0.011 \pm 0.002$ & $\mathbf{0 . 0 1 0} \pm 0.001$ \\
SPI & & 16 & $\mathbf{0 . 0 1 0} \pm 0.001$ & $\mathbf{0 . 0 1 0} \pm 0.002$ \\
CS_INF & & 15 & $\mathbf{0 . 0 1 0} \pm 0.002$ & $\mathbf{0 . 0 1 0} \pm 0.002$ \\
\hline STD & & 16 & $0.014 \pm 0.002$ & $0.015 \pm 0.001$ \\
CS_RND & & 14 & $0.013 \pm 0.002$ & $0.013 \pm 0.001$ \\
DEN & EGA & 16 & $\mathbf{0 . 0 1 1} \pm 0.002$ & $\mathbf{0 . 0 1 1} \pm 0.001$ \\
CS_DEG & Model 1 & 14 & $0.012 \pm 0.002$ & $0.012 \pm 0.002$ \\
SPI & & 16 & $\mathbf{0 . 0 1 1} \pm 0.001$ & $0.012 \pm 0.002$ \\
CS_INF & & 14 & $\mathbf{0 . 0 1 1} \pm 0.002$ & $\mathbf{0 . 0 1 1} \pm 0.002$ \\
\hline STD & & 12 & $0.016 \pm 0.002$ & $0.016 \pm 0.003$ \\
CS_RND & & 11 & $0.012 \pm 0.003$ & $0.013 \pm 0.001$ \\
DEN & IDEA & 12 & $\mathbf{0 . 0 0 9} \pm 0.002$ & $0.010 \pm 0.004$ \\
CS_DEG & Model 2 & 11 & $\mathbf{0 . 0 0 9} \pm 0.001$ & $\mathbf{0 . 0 0 9} \pm 0.002$ \\
SPI & & 12 & $0.010 \pm 0.001$ & $\mathbf{0 . 0 0 9} \pm 0.003$ \\
CS_INF & & 11 & $\mathbf{0 . 0 0 9} \pm 0.002$ & $0.010 \pm 0.004$ \\
\hline STD & & 12 & $0.008 \pm 0.002$ & $0.011 \pm 0.002$ \\
CS_RND & & 8 & $\mathbf{0 . 0 0 6} \pm 0.001$ & $0.009 \pm 0.004$ \\
DEN & EGA & 12 & $\mathbf{0 . 0 0 6} \pm 0.001$ & $\mathbf{0 . 0 0 7} \pm 0.001$ \\
CS_DEG & Model 2 & 10 & $\mathbf{0 . 0 0 6} \pm 0.001$ & $0.008 \pm 0.002$ \\
SPI & & 12 & $\mathbf{0 . 0 0 6} \pm 0.001$ & $0.008 \pm 0.003$ \\
CS_INF & & 10 & $\mathbf{0 . 0 0 6} \pm 0.002$ & $0.008 \pm 0.002$ \\
\hline STD & & 10 & $0.019 \pm 0.003$ & $0.022 \pm 0.004$ \\
CS_RND & & 9 & $0.017 \pm 0.002$ & $0.023 \pm 0.002$ \\
DEN & IDEA & 10 & $\mathbf{0 . 0 1 3} \pm 0.001$ & $\mathbf{0 . 0 1 8} \pm 0.002$ \\
CS_DEG & Model 3 & 10 & $0.014 \pm 0.001$ & $0.019 \pm 0.002$ \\
SPI & & 10 & $\mathbf{0 . 0 1 3} \pm 0.002$ & $\mathbf{0 . 0 1 8} \pm 0.002$ \\
CS_INF & & 9 & $0.014 \pm 0.001$ & $0.019 \pm 0.002$ \\
\hline STD & & 10 & $0.018 \pm 0.002$ & $0.024 \pm 0.004$ \\
CS_RND & & 9 & $0.016 \pm 0.001$ & $0.022 \pm 0.004$ \\
DEN & EGA & 10 & $0.013 \pm 0.001$ & $\mathbf{0 . 0 1 8} \pm 0.002$ \\
CS_DEG & Model 3 & 7 & $0.013 \pm 0.001$ & $\mathbf{0 . 0 1 8} \pm 0.001$ \\
SPI & & 10 & $\mathbf{0 . 0 1 2} \pm 0.001$ & $\mathbf{0 . 0 1 8} \pm 0.001$ \\
CS_INF & & 9 & $0.013 \pm 0.001$ & $\mathbf{0 . 0 1 8} \pm 0.002$ \\
\hline \hline & & & & \\
\hline
\end{tabular}

Table II shows the best results of the experiments. The highlighted values in bold show the best values achieved for 
a)
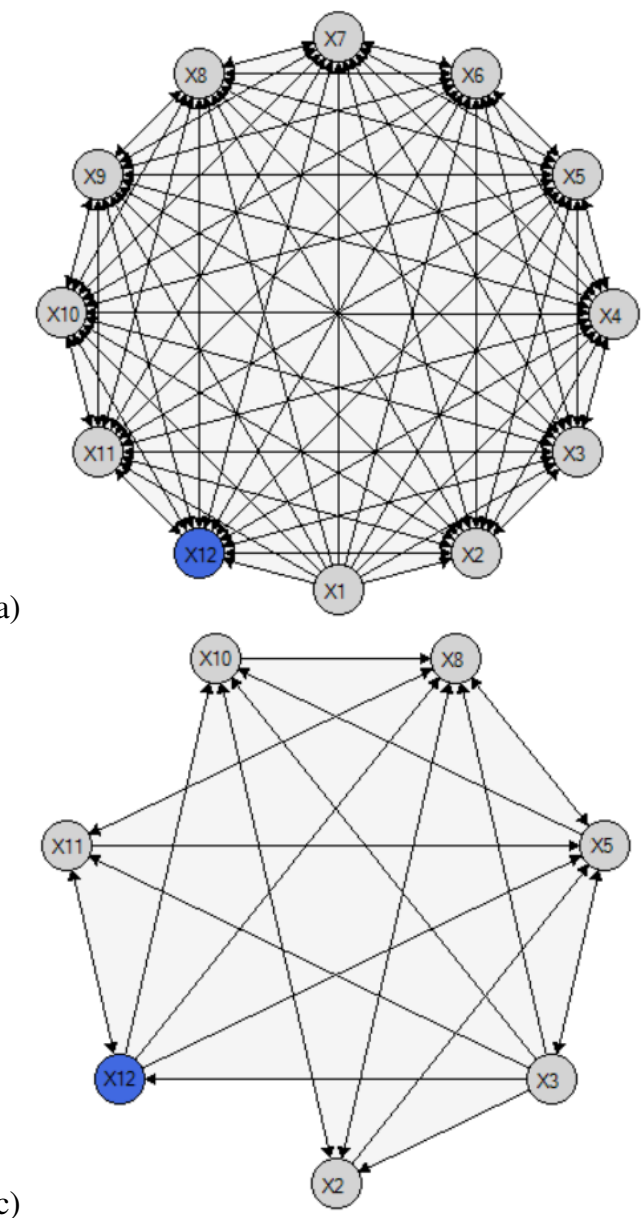

b)

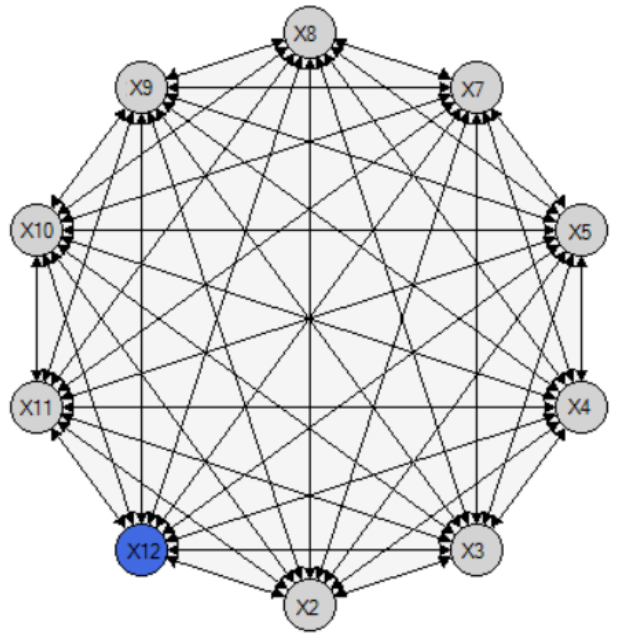

d)

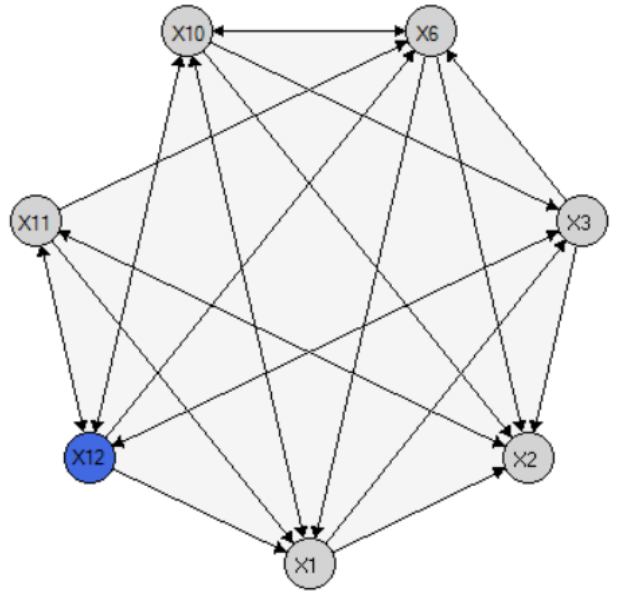

Fig. 1. Structures of the best FCM models for the analyzed approaches: a) STD, b) CS_RND, c) CS_DEG, d) CS_INF

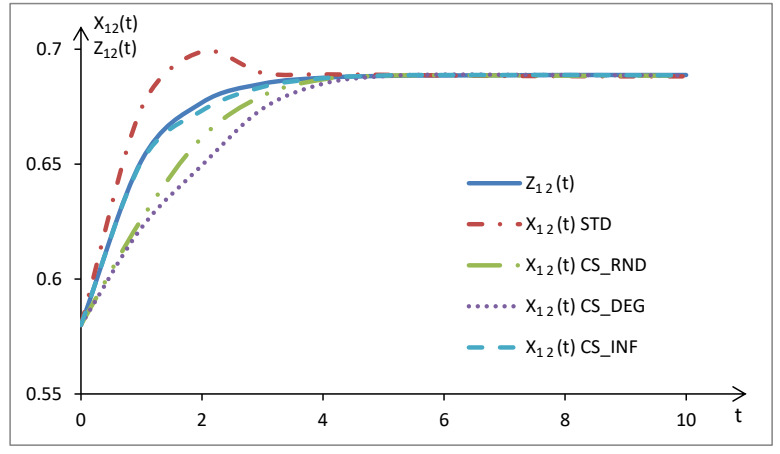

Fig. 2. Sample results of testing

initial and behavior error. Figure 1 presents the structures of the best models received using the standard method and the developed approaches for the second real-life model and EGA algorithm. Figure 2 shows the sample results of testing of the presented FCM models.

The obtained results show that the developed approach for fuzzy cognitive maps learning allows to approximate the real- life data with satisfactory accuracy comparable to the other approaches. It is observed that the proposed algorithm, in most of the cases, gives the lowest or very close to the lowest values of initial and behavior error. The advantage of the developed algorithm is the ability to reduce the size of the FCM models (the number of concepts $n$ ) by selecting the most significant concepts using graph theory metrics.

\section{CONCLUSION}

This paper introduces the evolutionary algorithm for selection of key concepts and determining the weights of the connections between them on the basis of real-life data. Graph theory metrics were used to reduce the number of concepts of fuzzy cognitive map during learning process. Effectiveness of the proposed approach was analyzed with the use of Elite Genetic Algorithm and Individually Directional Evolutionary Algorithm. The experiments confirmed that the developed approach allows to reduce the size of the FCM model by selecting key concepts and determine the weights of the connections between them keeping satisfactory level of error data. We are going to continue analysis of the developed technique for fuzzy cognitive maps learning with the use of 
TABLE II

THE BEST RESULTS OF THE EXPERIMENTS

\begin{tabular}{|c|c|c|c|c|}
\hline Approach & Method & $n$ & initial $_{\text {error }}$ & behavior $_{\text {error }}$ \\
\hline STD & & 16 & 0.011 & 0.011 \\
\hline CS_RND & & 15 & 0.011 & 0.013 \\
\hline$\overline{D E N}$ & IDEA & 16 & 0.008 & 0.013 \\
\hline CS_DEG & Model 1 & 13 & 0.008 & 0.010 \\
\hline SPI & & 16 & 0.008 & 0.007 \\
\hline CS_INF & & 12 & 0.009 & 0.010 \\
\hline STD & & 16 & 0.010 & 0.014 \\
\hline CS_RND & & 12 & 0.009 & 0.012 \\
\hline DEN & EGA & 16 & 0.008 & 0.010 \\
\hline CS_DEG & Model 1 & 16 & 0.009 & 0.010 \\
\hline SPI & & 16 & 0.008 & 0.009 \\
\hline CS_INF & & 12 & 0.009 & 0.008 \\
\hline STD & & 12 & 0.012 & 0.014 \\
\hline CS_RND & & 11 & 0.009 & 0.013 \\
\hline$\overline{\mathrm{DEN}}$ & IDEA & 12 & 0.007 & 0.007 \\
\hline CS_DEG & Model 2 & 10 & 0.007 & 0.008 \\
\hline SPI & & 12 & 0.008 & 0.007 \\
\hline CS_INF & & 8 & 0.005 & 0.006 \\
\hline STD & & 12 & 0.004 & 0.010 \\
\hline CS_RND & & 10 & 0.005 & 0.007 \\
\hline DEN & EGA & 12 & 0.004 & 0.010 \\
\hline CS_DEG & Model 2 & 7 & 0.004 & 0.012 \\
\hline SPI & & 12 & 0.004 & 0.014 \\
\hline CS_INF & & 7 & 0.003 & 0.006 \\
\hline STD & & 10 & 0.015 & 0.017 \\
\hline CS_RND & & 9 & 0.013 & 0.023 \\
\hline$\overline{\mathrm{DEN}}$ & IDEA & 10 & 0.012 & 0.017 \\
\hline CS_DEG & Model 3 & 9 & 0.011 & 0.017 \\
\hline SPI & & 10 & 0.011 & 0.020 \\
\hline CS_INF & & 7 & 0.012 & 0.015 \\
\hline STD & & 10 & 0.014 & 0.025 \\
\hline CS_RND & & 10 & 0.014 & 0.018 \\
\hline DEN & EGA & 10 & 0.011 & 0.017 \\
\hline CS_DEG & Model 3 & 8 & 0.011 & 0.018 \\
\hline$\overline{\text { SPI }}$ & & 10 & 0.011 & 0.016 \\
\hline CS_INF & & 8 & 0.011 & 0.015 \\
\hline
\end{tabular}

historical data. We plan also to extend our approach with other graph theory metrics.

\section{REFERENCES}

[1] J. Aguilar, “A Survey about Fuzzy Cognitive Maps Papers," International Journal of Computational Cognition,vol. 3 (2), pp. 27-33, 2005.

[2] V. V. Borisov, V. V. Kruglov, and A. C. Fedulov, Fuzzy Models and Networks, Publishing house Telekom, Moscow, 2004 (in Russian).

[3] A. Buruzs, M. F. Hatwágner, and L. T. Kóczy, "Expert-Based Method of Integrated Waste Management Systems for Developing Fuzzy Cognitive Map," Studies in Fuzziness and Soft Computing 2015, DOI: 10.1007/978-3-319-12883-2_4

[4] A. Christoforou, A. S. Andreou, "A framework for static and dynamic analysis of multi-layer fuzzy cognitive maps," Neurocomputing, vol. 232, pp. 133-145, 2017, http://dx.doi.org/10.1016/j.neucom.2016.09.115.

[5] M. F. Hatwagner, L. T. Koczy, "Parameterization and concept optimization of FCM models," Fuzzy Systems (FUZZ-IEEE), 2015 IEEE International Conference on, 2015, http://dx.doi.org/10.1109/FUZZIEEE.2015.7337888

[6] W. Homenda, and A. Jastrzebska, "Clustering techniques for Fuzzy Cognitive Map design for time series modeling," Neurocomputing vol 232, pp. 3-15, 2017, http://dx.doi.org/10.1016/j.neucom.2016.08.119

[7] W. Homenda, A. Jastrzebska, W. Pedrycz, "Nodes Selection Criteria for Fuzzy Cognitive Maps Designed to Model Time Series,"
In: Filev D. et al. (eds) Intelligent Systems'2014. Advances in Intelligent Systems and Computing, vol 323. Springer, Cham, 2015, http://dx.doi.org/10.1007/978-3-319-11310-4_75

[8] A. Jastriebow and K. Poczęta, "Analysis of multi-step algorithms for cognitive maps learning," BULLETIN of the POLISH ACADEMY of SCIENCES TECHNICAL SCIENCES, vol. 62, Issue 4, pp. 735-741, 2014, http://dx.doi.org/10.2478/bpasts-2014-0079.

[9] B. Kosko, "Fuzzy cognitive maps," International Journal of Man-Machine Studies, vol. 24, no.1, pp. 65-75, 1986 , http://dx.doi.org/10.1016/S0020-7373(86)80040-2.

[10] B. Kosko, Fuzzy Engineering, Prentice-Hall, Englewood Cliffs, NJ, 1997.

[11] Ł. Kubuś, "Individually Directional Evolutionary Algorithm for Solving Global Optimization Problems - Comparative Study," International Journal of Intelligent Systems and Applications (IJISA), Vol. 7, No. 9, 2015, str. 12-19.

[12] Ł. Kubuś, K. Poczęta, and A. Yastrebov, "A New Learning Approach for Fuzzy Cognitive Maps based on System Performance Indicators," 2016 IEEE International Conference on Fuzzy Systems, Vancouver, Canada, pp. 1-7, 2016.

[13] N. H. Mateou and A. S. Andreou, "A Framework for Developing Intelligent Decision Support Systems Using Evolutionary Fuzzy Cognitive Maps," Journal of Intelligent and Fuzzy Systems, Vol. 19, Number 2, pp. 171-150, 2008.

[14] Z. Michalewicz, Genetic algorithms + data structures = evolution programs, Springer-Verlag, New York, 1996.

[15] E. Papageorgiou, "A Novel Approach on Constructed Dynamic Fuzzy Cognitive Maps Using Fuzzified Decision Trees and KnowledgeExtraction Techniques," In: Michael Glykas (Ed.) Fuzzy Cognitive Maps: Advances in Theory, Methodologies, Tools and Applications, Springer, pp. 43-70, 2010.

[16] E. I. Papageorgiou, "Learning Algorithms for Fuzzy Cognitive Maps A Review Study." IEEE Transactions on Systems, Man, and Cybernetics - Part C: Applications and Reviews, vol. 42, no. 2, pp. 150-163, 2012, http://dx.doi.org/10.1109/TSMCC.2011.2138694.

[17] E I. Papageorgiou, M. F. Hatwágner, A. Buruzs, and L. T. Kóczy, "A concept reduction approach for fuzzy cognitive map models in decision making and management," Neurocomputing vol. 232, pp. 16-33, 2017.

[18] E. I. Papageorgiou and K. Poczeta, "A two-stage model for time series prediction based on fuzzy cognitive maps and neural networks," Neurocomputing, vol. 232, pp. 113-121, 2017 , http://dx.doi.org/10.1016/j.neucom.2016.10.072.

[19] K. Poczęta, A. Yastrebov, and E. I. Papageorgiou, "Learning Fuzzy Cognitive Maps using Structure Optimization Genetic Algorithm," 2015 Federated Conference on Computer Science and Information Systems (FedCSIS), Lodz, Poland, pp. 547-554, 2015, http://dx.doi.org/10.15439/2015F296.

[20] J. T. Rickard, J. Aisbett, and R. R. Yager, "A New Fuzzy Cognitive Map Structure Based on the Weighted Power Mean," IEEE TRANSACTIONS ON FUZZY SYSTEMS, VOL. 23, NO. 6, DECEMBER 2015.

[21] N. N. Selvin and A. Srinivasaraghavan, "Dimensionality Reduction of Inputs for a Fuzzy Cognitive Map for Obesity Problem," Inventive Computation Technologies (ICICT), International Conference on, 2016, https://dx.doi.org/10.1109/INVENTIVE.2016.7830187.

[22] V. B. Silov, Strategic decision-making in a fuzzy environment. Moscow: INPRO-RES, 1995 (in Russian).

[23] W. Stach, L. Kurgan, W. Pedrycz, and M. Reformat, "Genetic learning of fuzzy cognitive maps," Fuzzy Sets and Systems, vol. 153, no. 3, pp. 371-401, 2005, http://dx.doi.org/10.1016/j.fss.2005.01.009.

[24] W. Stach, W. Pedrycz, and L. A. Kurgan, "Learning of fuzzy cognitive maps using density estimate," IEEE Trans. on Systems, Man, and Cybernetics, Part B, vol. 42(3), pp. 900-912, 2012 http://dx.doi.org/10.1109/TSMCB.2011.2182646

[25] G. Słoń, "Application of Models of Relational Fuzzy Cognitive Maps for Prediction of Work of Complex Systems," Lecture Notes in Artificial Intelligence LNAI 8467, Springer-Verlag, pp. 307-318, 2014 http://dx.doi.org/10.1007/978-3-319-07173-2_27.

[26] R. J. Wilson, An Introduction to Graph Theory, Pearson Education, India, 1970. 


\section{Binary Segmentation Methods for Identifying Boundaries of Spatial Domains}

\author{
Nishanthi Raveendran \\ Department of Statistics \\ Faculty of Science and Engineering \\ Macquarie University, Sydney, Australia \\ Email: nishanthi.raveendran@students.mq.edu.au
}

\author{
Georgy Sofronov \\ Department of Statistics \\ Faculty of Science and Engineering \\ Macquarie University, Sydney, Australia \\ Email: georgy.sofronov@mq.edu.au
}

\begin{abstract}
Spatial clustering is an important component of spatial data analysis which aims in identifying the boundaries of domains and their number. It is commonly used in disease surveillance, spatial epidemiology, population genetics, landscape ecology, crime analysis and many other fields. In this paper, we focus on identifying homogeneous sub-regions in binary data, which indicate the presence or absence of a certain plant species which are observed over a two-dimensional lattice. To solve this clustering problem we propose to use the change-point methodology. We develop new methods based on a binary segmentation algorithm, which is a well-known multiple change-point detection method. The proposed algorithms are applied to artificially generated data to illustrate their usefulness. Our results show that the proposed methodologies are effective in identifying multiple domains and their boundaries in two dimensional spatial data.
\end{abstract}

\section{INTRODUCTION}

I DENTIFYING homogeneous domains is of particular interest in spatial statistics. It is often the case that spatial data have pre-defined subdivisions of interest. For example, data are often collected on non-overlapping administrative or census districts and these districts are often irregular in shape; see [1]. As a part of statistical modelling, spatial clustering is also an important component of spatial data analysis since spatial data may be heterogeneous and difficult to understand. However, if we cluster the data into homogeneous domains, then we can construct appropriate statistical models for each cluster. The problem of finding regional homogeneous domains is known as segmentation, partitioning or clustering. The two main problems in spatial clustering are identifying the number of domains, which is usually not known in advance, and estimating the boundaries of such domains.

Many clustering algorithms have been developed in the literature, ranging from hierarchical methods such as bottom-up (or agglomerative) methods top-down (or divisive) methods, to optimization methods such as the $k$-means algorithm [2]. The algorithms have numerous applications in pattern recognition, spatial data analysis, image processing, market research; see [3]. Spatial clustering covers enormous practical problems in many disciplines. For example, in epidemiological studies and public health research, it is known that the disease risk varies across space and it is important to identify regions of safety and regions of risk.
A model using Bayesian approach for spatial clustering was discussed in [4]. Recently, a two-stage Bayesian approach for estimating the spatial pattern in disease risk and identifying clusters which have high (or low) disease risks was proposed in [5].

The homogeneity changes in space is an important research subject in ecology. In a large area, the spatial distribution of plant or animal species is never homogeneous. Studying these kinds of changes is important in several ways. For example, detecting early changes in vegetation improves productivity. A class of Bayesian statistical models to identify thresholds and their locations in ecological data was introduced in [6]. A method to estimate the change-point distribution between two patches was presented in [7].

Studies of weather and climatic systems at a global scale have become a prime area of research for a number of reasons; one of these is the concern about global climatic change. Mann-Kendall trend test, Bayesian change point analysis and a hidden Markov model to find changes in the rainfall and temperature patterns over India are used in [8].

There has also been extensive literature on image recognition with some articles presenting statistical approaches to the boundary identification in statistical imaging. For example, [9] presented a Markov chain Monte Carlo (MCMC) method to identify closed object boundaries in gray-scale images. Change curve estimation problem is also referred as multidimensional detection problem or boundary estimation problem. A wavelet method to estimate jumps and sharp curves in the plane was proposed in [10].

Even though there is wide range of applications to spatial clustering, many statistical methods for detecting clusters have some limitations: either they detect the number of clusters and do not determine their locations, or they provide the inference with no clustering. In this study, we are interested in identifying the boundaries of domains and their number with applications to an ecological landscape. In general, these problems are typically challenging due to the multivariate nature of the data which leads to complex and highly 
parametrized likelihoods. We use binary data indicating the presence or absence of plant species, which are observed over a two-dimensional lattice. We consider our problem as a change-point detection problem, which is commonly used in analysing time series to detect changes and their locations. We develop new algorithms based on a binary segmentation algorithm, which is a well-known recursive partitioning tool in change-point literature and it leads to simple solutions for such problems and it has an advantage on simplicity and less computational cost compare to other methods.

Binary spatial data are commonly involved in various areas such as economics, social sciences, ecology, image analysis and epidemiology. Also, such data frequently occur in environmental and ecological research, for instance, when the data correspond to presence or absence of a certain invasive plant species at a location or, when the data happen to fall into one of two categories, say, two types of soil. The general overview of spatial data can be found in [11-13].

This study aims to develop effective procedures based on the binary segmentation method for estimating both the number of domains and their locations in spatial data. This paper is organized as follows. Section II describes the multiple change-point problem. We provide the mathematical model for our problem in section III. We explain both general binary segmentation and new algorithms in section IV and section $\mathrm{V}$, respectively, and provide the numerical results in section VI. Section VII gives a discussion. Section VIII concludes the paper with the future directions.

\section{Multiple Change-Point Problem}

Let us formulate the general multiple change-point problem in mathematical terms.

Let $y_{n}=\left(y_{1}, \ldots, y_{n}\right)$ be a sequence of observations of length $n, y_{1}, y_{2}, \ldots, y_{n}$ be independent random variables with the probability distribution functions $F_{1}, F_{2}, \ldots, F_{n}$. Let $\tau_{1}, \tau_{2}, \ldots, \tau_{m}$ be unknown positions of $m$ change-points, where $\tau_{1}<\tau_{2}<\cdots<\tau_{m}$. We define $\tau_{0}=0$ and $\tau_{m+1}=n$. The sequence of observations is divided into $m+1$ segments based on $m$ change-points. In general, the multiple changepoint problem involves the following null hypothesis,

$$
H_{0}: F_{1}=F_{2}=\cdots=F_{n}
$$

versus

$$
\begin{aligned}
& H_{1}: F_{1}=\cdots=F_{\tau_{1}} \neq F_{\tau_{1}+1}=\cdots=F_{\tau_{2}} \\
& \neq F_{\tau_{2}+1}=\cdots=F_{\tau_{m}} \neq F_{\tau_{m}+1}=\cdots=F_{n} .
\end{aligned}
$$

\section{Mathematical Model}

Let us assume that we have independent binary observations on an $n \times m$ lattice. We assume the observations at each cell are univariate. Let $M$ be the number of domains and $p=$ $\left(p_{1}, \ldots, p_{M}\right)$ be the parameters of Bernoulli distribution for the domains. The likelihood function is given by:

$$
L(X \mid p)=\prod_{j=1}^{M} p_{j}^{I_{D_{j}}}\left(1-p_{j}\right)^{O_{D_{j}}}, \quad j=1,2, \ldots, M,
$$

$X$ is the data (a matrix of zeroes and ones),

$M$ is the number of domains,

$D_{j}$ is the $j$-th (rectangular) domain,

$p=\left(p_{1}, \ldots, p_{M}\right)$ is the vector of probabilities,

$I_{D_{j}}$ is the number of ones in $D_{j}$,

$O_{D_{j}}$ is the number of zeroes in $D_{j}$.

In order to estimate the boundaries of domains and their number, we maximize the log-likelihood function

$$
l(X \mid p)=\sum_{j=1}^{M} I_{D_{j}} \log p_{j}+O_{D_{j}} \log \left(1-p_{j}\right) .
$$

\section{A. Maximum Likelihood Framework}

Let $X$ is an $n \times m$ matrix. A natural approach to split a domain into homogeneous sub-domains is to view it as the following hypothesis testing:

$$
H_{0} \text { : No sub-domains; Vs } H_{1} \text { : Two domains. }
$$

Under the null hypothesis, the log-likelihood function for the entire domain is given as

$$
l(X \mid \hat{p})
$$

where $\hat{p}$ is the maximum likelihood estimate of the $p$. Under the $H_{1}$, the log-likelihood function given a change-point (in our case, cut or boundary) $c$, which divides the domain into two homogeneous domains $D_{1}$ and $D_{2}$, is

$$
P(c)=l\left(D_{1} \mid \hat{p}_{1}\right)+l\left(D_{2} \mid \hat{p}_{2}\right)
$$

where $\hat{p}_{1}$ and $\hat{p}_{2}$ are the maximum likelihood estimates of the parameters for the first and the second domain, respectively. To estimate the location of the change-point, the log-likelihood function under $H_{1}$ is maximized.

Test statistic:

$$
\lambda(X)=2\left[\max _{c} P(c)-l(X \mid \hat{p})\right]
$$

where a threshold $\beta$ is chosen such that if $\lambda(X)>\beta$, the null hypothesis is rejected. The threshold could be based on the use of an information criterion: AIC, $\beta=2 k$, and SIC, $\beta=k \log n$, where $k$ is the number of extra parameters as a result of adding another domain.

The likelihood ratio test statistic can be extended to multiple change-point detection by summing the likelihood for the $m$ data segments. 
One way to detect multiple change-points is to minimize

$$
\sum_{i=1}^{m+1}\left[C\left(y_{\left(\tau_{i-1}+1\right): \tau_{i}}\right)\right]+\beta f(m),
$$

where $C$ is a cost function for a segment and $\beta f(m)$ is a penalty term in order to avoid overfitting.

\section{The Binary Segmentation Method}

The binary segmentation is a well-known multiple changepoint method and has been studied by various authors. It was first introduced in [14] in the context of cluster analysis. The concept of binary segmentation in detecting changes in mean was proposed in [15]. Later, this procedure was extended to detect the number of change-points in a multidimensional random process and proved the consistency of the estimates produced by binary segmentation under mild conditions, the first of which is based on the minimal distance between change-points; see [16]. Similar results when the changepoints are allowed to approach one another are achieved in [17]. Recent studies include many applications and it can be found in [24-32]. Thus, this method is now the most understood and widely cited search algorithm used within the multiple change-point literature.

Binary segmentation can be used to extend any single change-point method to multiple change-points. In the early works, binary segmentation was performed using a simple CUSUM test. It starts with applying the chosen single change-point detection method to the entire data set $y_{1: n}$, sequence of observations of length $n$. If no change-point is found, then the algorithm stops. If a change-point is detected, say, $\tau$, then the data set is split into two separate segments, $y_{1: \tau}$ and $y_{\tau+1: n}$. The single change-point method is applied to two segments and the procedure is repeated iteratively. Finally, we stop when no more change-points are detected. The generic binary segmentation algorithm [18] is given below.

One of the good features of the binary segmentation algorithm is that it detects the number of change-points and their locations simultaneously. It can be seen as an approach with $f(m)=m$ to minimize (1) by iteratively deciding whether a change-point should be added or not. It is a fast algorithm and saves lots of computational time and it can be implemented with the computational complexity $O(n)$.

There are some exact methods to minimize (1) but at a higher computational cost (for example, see [33]). It is clear that in many situations the number of change-points increases as we collect more data and the computational burden increases as well. Therefore, many authors are working on developing new algorithms which are fast and exact. Recently, a dynamic programming technique called PELT (Pruned Exact Linear Time) which is $O(n)$ under certain assumptions such as the number of true change-points being linear with the data

\begin{tabular}{cr}
\hline Algorithm & The Generic Binary Segmentation Algorithm \\
\hline Input: & A set of data of the form, $\left(y_{1}, y_{2}, \ldots, y_{n}\right)$. \\
& A test statistic $\lambda(\cdot)$ dependent on the data. \\
& An estimator of change-point position $\hat{\tau}(\cdot)$. \\
& A rejection threshold $\beta$.
\end{tabular}

Initialise: Let $C=\emptyset$, and $S=[1, n]$

Iterate: while $S \neq \emptyset$

1. Choose an element of $S$; denote this element as $[s, t]$.

2. If $\lambda\left(y_{s: t}\right)<\beta$, remove $[s, t]$.

3. If $\lambda\left(y_{s: t}\right) \geq \beta$ :
(a) remove $[s, t]$ from $S$;
(b) calculate $r=\hat{\tau}\left(y_{s: t}\right)+s-1$, and add $r$ to $c$;
(c) if $r \neq s$ add $[s, r]$ to $S$;
(d) if $r \neq t-1$ add $[r+1, t]$ to $S$.

Output: The set of change-points recorded $C$.

length was introduced in [19]. Still, this method has $O\left(n^{2}\right)$ complexity at the worst case.

\section{THE BINARY SEGMENTATION METHOD FOR SPATIAL CLUSTERING}

We would like to identify the number of homogeneous domains and their boundaries in binary lattice data. In this case, the change-point locations are the points which is used to draw a horizontal or vertical line to divide the domain into two homogeneous rectangular segments. Here we present three algorithms. Our proposed algorithms use maximum likelihood test as described in the previous section.

\section{A. Algorithm 1}

The algorithm searches every column and row to detect the change-point and selects the maximum test statistic for the optimum cut. If the test statistic is greater than a threshold value, it splits the domain according to the index (row or column) and stores the obtained domains. Otherwise, the algorithm stops. This procedure is repeated until a stopping criterion is met. In this study, we consider rectangle shaped domains.

We also propose two more algorithms with modifications. In general, our method can be summarized by a three-step iterative procedure (given in Algorithm 1).

\section{B. Algorithm 2}

We introduce a modification of Algorithm 1. It follows the similar structure but at each iteration it identifies two change-points and three domains. Here, all three segments have different means.

\section{Algorithm 3}

Algorithm 3 is a modified version of Algorithm 1. The main difference is that at each iteration it selects the bigger domain for next iteration assuming that bigger domain has a higher 


\begin{abstract}
Algorithm 1 Main Algorithm
Step 1: Given the data, search the change point column-wise and find the optimal cut which maximizes the test statistic. Repeat this procedure row-wise.

Step 2: Select the maximum of the two test statistics for the optimal column and row cuts and compare with the threshold value. If the test statistic is greater than the threshold value, then split the data in two domains.

Step 3: Repeat steps 1 and 2 for each domain until no new domains are identified.
\end{abstract}

chance to be split at the next iteration. Here, the "bigger" means the area of the rectangle. This algorithm performs well compare to Algorithm 1 and Algorithm 2. The great advantage of this algorithm is that it performs fast because at each iteration it selects only one domain to split. But in Algorithm 1 , at each iteration it considers two segments in parallel. This algorithm would be useful when we need to split the data into major domains (few number of domains).

\section{Model selection}

Our objective is to estimate both the number of domains and their boundaries. Thus, it can be formulated as a model selection problem, which is usually done by using a specific criterion. There are several popular model selection criteria that have been proposed in different contexts; see [34-36]. The model selection criteria are mainly used for two different purposes: first, to choose a model that well approximates the true model; second, to find the true model in a list of candidate models [20]. In this study, we use AIC [21], BIC [22] and mBIC (modified BIC, defined for change-point problems) [23]. The AIC, BIC and mBIC for our model can be described as below:

$$
\begin{aligned}
\operatorname{AIC}(k) & =-2 \log L\left(\hat{\Theta}_{k}\right)+2 k, \\
\operatorname{BIC}(k) & =-2 \log L\left(\hat{\Theta}_{k}\right)+k \log n, \\
\operatorname{mBIC}(k) & =-2 \log L\left(\hat{\Theta}_{k}\right)+2(k+1) \log n,
\end{aligned}
$$

where $L\left(\hat{\Theta}_{k}\right)$ is the maximum likelihood for the model with $k$ parameters, $k=1,2, \ldots, M$, and $n$ is the sample size. A model that minimizes a criterion (for example, AIC) is considered to be the most appropriate model.

\section{E. Stopping criteria}

In the binary segmentation, one has to define a stopping criterion to terminate the iterative procedure. We use one of two methods:

1) The algorithm is reiterated while we have significant cuts based on the results of a hypothesis testing. Let us define that number of cuts $c=C$, the process is stopped and the corresponding solution is considered as the optimal solution for the problem.

2) The decision to stop the algorithm is based on an information criterion.

\section{F. Likelihood test for spatial clustering}

In this study, we use the likelihood ratio test to check whether the domains obtained by the proposed algorithms are homogeneous or not. The null hypothesis for this model is given as:

\section{$H_{0}$ : Domain 1 and Domain 2 are homogeneous.}

The alternative hypothesis is

$H_{1}$ : Domain 1 and Domain 2 are not homogeneous.

The test statistic is:

$$
\text { LRT }=-2 \log \left(\frac{\text { likelihood for null model }}{\text { likelihood for alternative model }}\right) .
$$

After the algorithm finishes, we obtain several homogeneous domains. The next step is to perform a multiple comparison test for all combinations of the domains. Further, we consider the Bonferroni correction, which is used to control the familywise error rate when conducting multiple hypothesis tests. The Bonferroni correction adjusts $p$-values when several statistical tests are being performed simultaneously on a single data set. To perform the Bonferroni correction, divide the critical $p$ value $(\alpha)$ by the number of comparisons or the number of hypothesis being made. For example, if we have $M$ domains for our data set and have to perform $N$ comparisons, then the Bonferroni correction would test each individual hypothesis at $\alpha / N$. Here we do not need to perform all comparisons since we consider only rectangular domains in this study.

\section{RESULTS}

In this section we include all numerical results to illustrate and validate the proposed algorithms. A simulation study was carried out to demonstrate the properties of our algorithms and to analyse their segmentation capabilities. We present an example to illustrate the usefulness of our method. Finally, we compare our three algorithms using the Root Mean Square Error (RMSE) and information criteria. All proposed algorithms have been implemented using the statistical software R.

\section{A. Simulation study}

To perform simulation study, we generate artificial matrices using a Bernoulli distribution. We apply Algorithm 1, record the position of the optimal cut and estimate the parameter of the Bernoulli distribution for each domain. Each time we calculate the RMSE and plot a kernel density estimation curve to analyze the effectiveness of the algorithm. Hereinbelow we denote the number of obtained domains by $D$.

The RMSE is calculated as

$$
\mathrm{RMSE}=\sqrt{\frac{\sum_{i=1}^{n} \sum_{j=1}^{m}\left(e_{i j}-t_{i j}\right)^{2}}{N}},
$$

where $e_{i j}, t_{i j}$ denotes the estimated and the true values, respectively, for each cell of the matrix; $i, j$ indicate the corresponding rows and columns; $n \times m$ is the size of the 
TABLE I

THE NUMBER OF DOMAINS WITH FREQUENCIES

\begin{tabular}{ccccccc}
\hline $\boldsymbol{D}$ & 1 & 2 & 3 & 4 & 5 & $5+$ \\
\hline Frequency & 0 & 0 & 0 & 316 & 529 & 155 \\
\hline
\end{tabular}

matrix.

1) Case 1: Testing the performance of the binary segmentation algorithm: Our aim is to find the optimal number of domains for a particular data set. We generate artificial data with four domains and run our algorithm 1000 times. We record the number of domains identified by Algorithm 1.

Table I shows that the algorithm correctly found four domains in 316 simulations (out of 1000). However, the algorithm tends to overestimate the number of domains.

2) Case 2: Reporting the RMSE on the parameters of the domains: We analyse how the RMSE depends on the parameters of domains, that is, the probability of " 1 ". In this study, we generate artificial data with two domains, where $p_{1}$ and $p_{2}$ are the parameters of the Bernoulli distributions for the domains.

The algorithm performs well in identifying the correct position of the cut when the difference between $p_{1}$ and $p_{2}$ is rather large (for example, $p_{1}=0.8$ and $p_{2}=0.2$ ). Note that even if the difference is getting smaller, the algorithm works quite well; in this situation the RMSE is slightly higher compare to the case with the large difference of the probabilities. Figure 2 shows a kernel density estimation for three different cases. In the first row, we fix $p_{1}=0.8$ and we change $p_{2}$ from 0.1 to 0.9 . Likewise, in the second row, we fix $p_{1}$ as 0.5 and in the third row, $p_{1}=0.2$. It is clear that the effectiveness depends on the difference of the probabilities.

3) Case 3: Reporting the RMSE on the size of the data: In this section, we analyse how the RMSE changes depending on the size of the data. It is important to test our algorithms for different sizes. We generate matrices of different sizes $(50 \times 50,100 \times 100,200 \times 200)$ but with the same probabilities $p_{1}$ and $p_{2}$ for domain 1 and domain 2 , respectively. Here we restrict the number of domains to two.

Figure 1 shows the plot of kernel density estimation, which illustrates that the average value of the RMSE is not significantly influenced by the size of the data, whereas it is clear that the variability in the RMSE is getting smaller when the data size is becoming larger.

\section{B. Example}

We generate a $100 \times 100$ matrix using Bernoulli distributions with four domains (all are vertical cuts); the parameters are

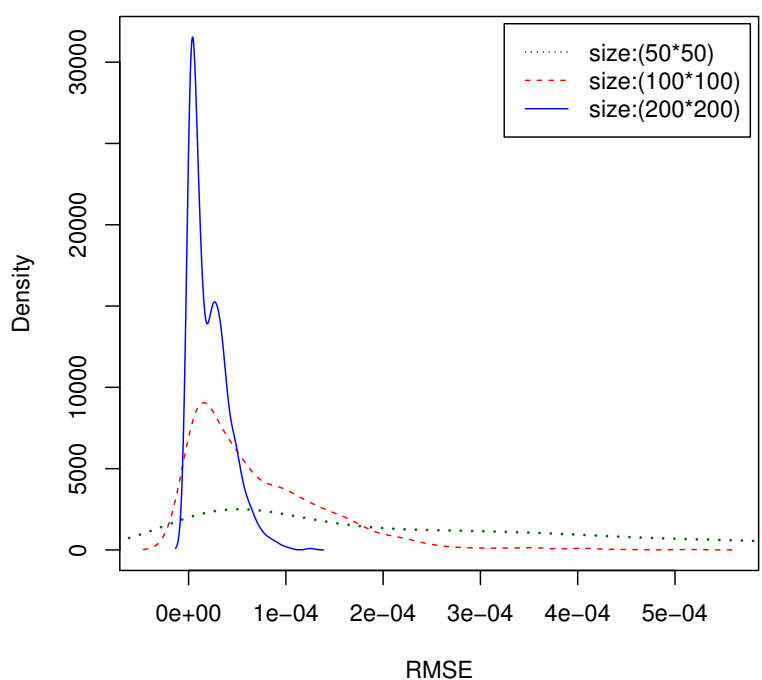

Fig. 1. Kernel density estimation of the RMSE for different sizes

TABLE II

THE PARAMETERS OF THE GENERATED DATA MATRIX

\begin{tabular}{ccc}
\hline Domains & Coordinates (top left to bottom right) & Probability $\left(p_{i}\right)$ \\
\hline Domain 1 & $(1,1)-(100,20)$ & $p_{1}=0.1$ \\
Domain 2 & $(1,20)-(100,60)$ & $p_{2}=0.5$ \\
Domain 3 & $(1,60)-(100,90)$ & $p_{3}=0.9$ \\
Domain 4 & $(1,90)-(100,100)$ & $p_{4}=0.2$ \\
\hline
\end{tabular}

given in Table II. We apply our binary segmentation algorithms, record the positions of the optimal cuts and estimate the parameters of the Bernoulli distributions at each iterations. Each time we calculate the RMSE, AIC, BIC and mBIC.

1) Result on Algorithm 1: We applied our binary segmentation algorithm to the data generated above (Table II). Table III shows that the algorithm run up to four iterations and at the end it identified seven domains. The RMSE value attains its minimum at the third iteration (Number of domains $=5$ ), which coincides with the results given by the information criteria AIC, BIC and $\mathrm{mBIC}$.

Figure 3 plots the values of the information criteria versus the number of domains; it shows that the minimal values for all three criteria correspond to five domains. Now we examine the obtained domains for their heterogeneity using the likelihood ratio test. Here, we consider only rectangle shaped domains so we do not need to check all possible comparisons. Therefore, in this example, we perform only four comparisons.

Table IV shows the obtained domains for this example. Table $\mathrm{V}$ illustrates the results of the likelihood ratio test. According to Table V, Domain 4 and Domain 5 can be considered as homogeneous. Thus, we combined those two 


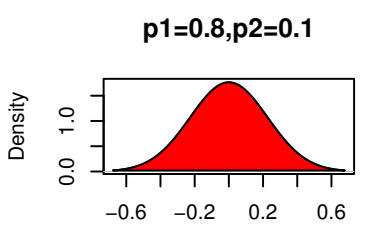

$\mathrm{N}=1000$ Bandwidth $=0.2261$

$\mathrm{p} 1=0.5, \mathrm{p} 2=0.1$

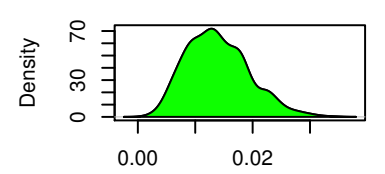

$\mathrm{N}=1000$ Bandwidth $=0.001233$

$\mathrm{p} 1=0.2, \mathrm{p} 2=0.1$

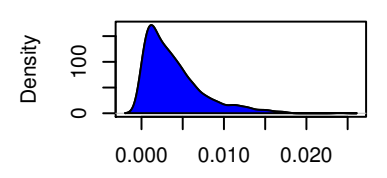

$\mathrm{N}=1000$ Bandwidth $=0.0006706$

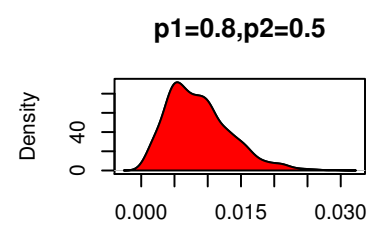

$\mathrm{N}=1000$ Bandwidth $=0.001051$

$\mathrm{p} 1=0.5, \mathrm{p} 2=0.5$

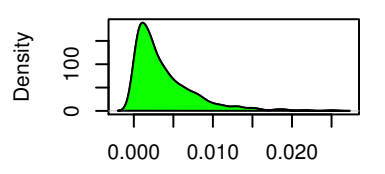

$\mathrm{N}=1000 \quad$ Bandwidth $=0.0006748$

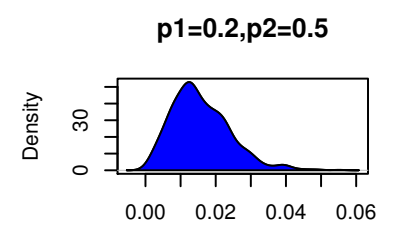

$\mathrm{N}=1000$ Bandwidth $=0.001853$

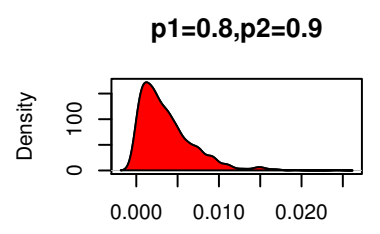

$\mathrm{N}=1000$ Bandwidth $=0.0006284$

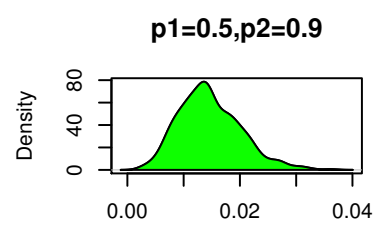

$\mathrm{N}=1000$ Bandwidth $=0.001195$

$\mathrm{p} 1=0.2, \mathrm{p} 2=0.9$

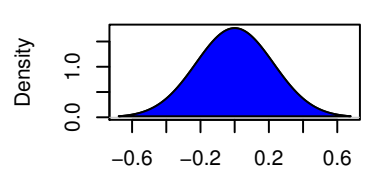

$\mathrm{N}=1000$ Bandwidth $=0.2261$

Fig. 2. Kernel density estimation of the RMSE

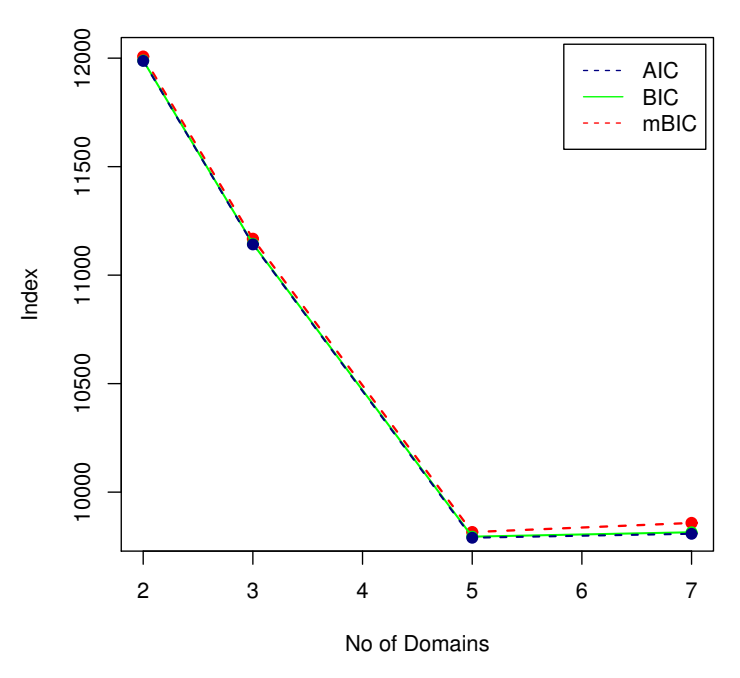

Fig. 3. The values of the AIC, BIC and mBIC

TABLE III

Results ON ALgORITHM 1

\begin{tabular}{ccrrrr}
\hline \# Iterations & $\boldsymbol{D}$ & RMSE & AIC & BIC & mBIC \\
\hline 1 & 2 & 0.217 & $11,987.2$ & $11,989.2$ & $12,007.2$ \\
2 & 3 & 0.164 & $11,141.5$ & $11,144.5$ & $11,167.5$ \\
3 & 5 & 0.007 & $9,789.8$ & $9,794.8$ & $9,815.8$ \\
4 & 7 & 0.019 & $9,807.9$ & $9,814.9$ & $9,857.9$ \\
\hline
\end{tabular}

TABLE IV

ObTaIned DOMAINS FOR ALgORITHM 1

\begin{tabular}{cc}
\hline Domains & Coordinates (top left to bottom right) \\
\hline D1 & $(1,1)-(100,20)$ \\
D2 & $(1,20)-(100,60)$ \\
D3 & $(1,60)-(100,90)$ \\
D4 & $(1,90)-(6,100)$ \\
D5 & $(6,90)-(100,100)$ \\
\hline
\end{tabular}

TABLE V

LIKELIHOOD RATIO TEST FOR ALGORITHM 1

\begin{tabular}{ccc}
\hline Domain combinations & $p$-value & Results \\
\hline D1 and D2 & $<0.00001$ & Significant \\
D2 and D3 & $<0.00001$ & Significant \\
D4 and D5 & 0.077242 & Not significant \\
\hline
\end{tabular}

domains into one domain. Finally, we obtained the same domains as in our generated data matrix (see Table II).

2) Results on Algorithm 2: We applied Algorithm 2 for the same example described in the previous section and, as before, we recorded the positions of cuts at each iterations. It

TABLE VI

Results ON ALgORITHM 2

\begin{tabular}{ccrrrr}
\hline \# Iterations & $\boldsymbol{D}$ & RMSE & AIC & BIC & mBIC \\
\hline 1 & 3 & 0.146 & $10,838.1$ & $10,859.7$ & $10,864.1$ \\
2 & 9 & 0.004 & $9,974.5$ & $10,039.4$ & $10,036.5$ \\
3 & 17 & 0.030 & $18,840.4$ & $18,857.4$ & $18,950.4$ \\
\hline
\end{tabular}


TABLE VII

RESULTS ON ALGORITHM 3

\begin{tabular}{ccrrrr}
\hline \# Iterations & $\boldsymbol{D}$ & RMSE & AIC & BIC & mBIC \\
\hline 1 & 2 & 0.217 & $11,987.2$ & $11,989.2$ & $12,007.2$ \\
2 & 3 & 0.164 & $11,141.5$ & $11,144.5$ & $11,167.5$ \\
3 & 4 & 0.000 & $9,790.9$ & $9,794.9$ & $9,822.9$ \\
4 & 5 & 0.017 & $9,880.5$ & $9,885.5$ & $9,918.5$ \\
\hline
\end{tabular}

is clear from Table VI that the RMSE, AIC, BIC and mBIC values are lowest for the case when the number of domains is equal to nine. Thus, Algorithm 2 identified nine domains for the same example illustrated above. Furthermore, all obtained domains identified by Algorithm 2 are significantly different.

3) Results on Algorithm 3: In this section, we applied Algorithm 3 for the same example described in above section. We recorded the positions of cuts at each iterations. Table VII shows that the algorithm found five domains in four iterations. The RMSE, AIC, BIC and mBIC values are lowest for the case when number of domains equals four. Thus, Algorithm 3 identified four domains (the same domains as we expected) in three iterations.

\section{Comparison of the Algorithms}

In this section, we compare our all algorithms. Final results of all three algorithms in the form of the RMSE, AIC, BIC and $\mathrm{mBIC}$ are given in Table VIII.

Our results show that the algorithms based on binary segmentation work well in identifying correct number of domains and their boundaries. Algorithm 2 finds more domains which are buried within larger domains. Algorithm 3 is fast and it is accurate in identifying major domains but overestimates the total number of domains.

\section{DISCUSSION}

There have been very few studies in the existing literature that focus on the development of statistical segmentation methods for spatial data. To address this issue, we have generalised the binary segmentation method for identifying the number of homogeneous domains and their boundaries in spatial data. In particular, we have applied the modified versions of the binary segmentation algorithms to binary spatial data indicating the presence or absence of a certain plant species, which are observed over a two dimensional lattice. The numerical results have illustrated that the algorithms work well under different scenarios; they accurately identify both the expected number of domains and their boundaries in few iterations.

Binary segmentation is described as "arguably the most widely used change-point search method" [19] and it is used for multidimensional data sequence. The benefits of binary segmentation include low computational complexity (typically of order $O(n)$ ), conceptual simplicity, the fact that it is usually easy to code, even in more complex models, and at each stage
TABLE VIII

COMPARISON OF ALL THREE ALGORITHMS (A1, A2, A3)

\begin{tabular}{rccrrrr}
\hline & \# Iterations & $\boldsymbol{D}$ & RMSE & AIC & BIC & mBIC \\
\hline A1 & 3 & 5 & 0.007 & $9,789.8$ & $9,794.8$ & $9,815.8$ \\
A2 & 2 & 9 & 0.004 & $9,974.5$ & $10,039.4$ & $10,036.5$ \\
A3 & 3 & 4 & 0.000 & $9,790.9$ & $9,794.9$ & $9,822.9$ \\
\hline
\end{tabular}

it involves one-dimensional rather than multi-dimensional optimization. On the other hand, the method is a "greedy" procedure in the sense that it is performed sequentially, with each stage depending on the previous ones, which are never revisited.

Analysing literature on binary segmentation, we have found out that it has been never discussed with respect to identifying both number of domains and boundaries in spatial data. To fill this gap, we develop effective procedures for estimating both the number of domains and their locations in spatial data by modifying the binary segmentation method. The applications of the proposed procedures are not limited to analysing ecological data. They can be easily extended and applied to other spatial data. For instance, it can be applied to epidemiological and economic data.

\section{FUTURE DIRECTIONS}

Over the last decades, spatial statistical models have been studied by many authors from different angles and the spatial clustering problem is one of main topics in spatial statistics. However, the problem has not been considered as a change-point detection problem. In this study, we have demonstrated how spatial clusters can be identified by using a new approach based on binary segmentation. At this stage, we have considered a simple model which assumes that observations are independent. However, statistical models that involve spatial dependence are more realistic. Extension to dependent data is considered as one of our future works. Moreover, we have only considered rectangular shaped domains and we plan to extend it to other more complex shapes in the future.

In this work, we have used univariate binary data. It is possible to consider multivariate case (for example, for several species) and other types of data such as count or continuous data as well. Furthermore, we have assumed that data is observed over a regular shaped lattice but it is also possible to consider a set of random points on a plane. The problem that we consider can be seen as a model selection problem and one of the major challenges is to determine the optimal number of domains. We have used well-known information criteria such as the AIC, BIC and modified BIC. The criteria may not work well for spatial cluster models because of irregularities in their likelihood functions. Our intention is to develop new modified information criteria particularly for specific spatial segmentation problems under different assumptions. 
In this study, we have focused on constructing binary segmentation methods because of their simplicity and low computation cost. We plan to develop new spatial segmentations algorithms bases on well-known statistical computational methods such as Cross Entropy (CE) [37], Markov chain Monte Carlo (MCMC) [39], [40] and Sequentially Importance Sampling (SIS) [38] methods.

\section{REFERENCES}

[1] T. Y. Yang and T.B Swartz, "Application of binary segmentation to the estimation of quantal response curves and spatial intensity," Biometrical journal, vol. 4, 2005, pp. 489-501. https://doi.org/10.1002/bimj.200310136

[2] S. S. Chen and P. S. Gopalakrishnan, "Clustering via the Bayesian information criterion with applications in speech recognition," In Acoustics, Speech and Signal Processing, 1998. Proceedings of the 1988 IEEE International Conference on, vol. 2, 1998, pp. 645-648. https://doi.org/10.1109/ICASSP.1998.675347

[3] A. K. Tung, J. Hou and J. Han, "Spatial clustering in the presence of obstacles," In Data Engineering, 2001. Proceedings. 17th International Conference on, IEEE, 2001, pp. 359-367. https://doi.org/10.1109/ICDE.2001.914848

[4] R. E. Gangnon and M.K Clayton, "Bayesian detection and modeling of spatial disease clustering," Biometrics, vol. 3, 2000, pp. 922-935. https://doi.org/10.1111/j.0006-341X.2000.00922.x

[5] C. Anderson, D. Lee and N. Dean, "Bayesian cluster detection via adjacency modelling." Spatial and spatio-temporal epidemiology, 2016, pp. 11-20. https://doi.org/10.1016/j.sste.2015.11.005

[6] B. Beckage, L. Joseph, P. Belisle, D. B. Wolfson and W. J. Platt, "Bayesian change-point analyses in ecology," New Phytologist, vol. 2, 2007, pp. 11-20. https://doi.org/10.1111/j.1469-8137.2007.01991.x

[7] I. López, M. Gámez, J. Garay, T. Standovár and Z. Varga, "Applications of change-point problem to the detection of plant patches," Acto biotheoretica, vol. 1, 2010, pp. 51-63. https://doi.org/10.1007/s10441 009-9093-x

[8] S. Tripathi and R. S. Govindaraju, "Change detection in rainfall and temperature patterns over India," In Proceedings of the Third International Workshop on Knowledge Discovery from Sensor Data, ACM, 2009, pp. 133-141. https://doi.org/10.1145/1601966.1601988

[9] J. D. Helterbrand, N. Cressie and J. L. Davidson, "A statistical approach to identifying closed object boundaries in images," Advances in applied probability, vol. 4, 1994, pp. 831-854. https://doi.org/10.1017/S0001867800026641

[10] Y. Wang, "Change curve estimation via wavelets," Journal of the American Statistical Association, vol. 441, 1998, pp. 163-172. http://dx.doi.org/10.1080/01621459.1998.10474098

[11] G. J. Upton and B. Fingleton, "Spatial data analysis by example. vol 1: Point pattern and quantitative data," Chichester: Wiley, vol. 1, 1985.

[12] A. D. Cliff and J. K. Ord, Spatial processes: models \& applications, Taylor \& Francis, 1981.

[13] N. Cressie, Statistics for spatial data, John Wiley and Sons, 2015

[14] A. J. Scott and M. Knott, "A cluster analysis method for grouping means in the analysis of variance," Biometrics, 1974, pp. 507-512. https://doi.org/10.2307/2529204

[15] A. Sen and M. S. Srivastava, "On tests for detecting change in mean," The Annals of statistics, vol. 1, 1975, pp. 98-108. https://doi.org/10.1214/aos/1176343001

[16] L. Vostrikova, "Detection of the disorder in multidimensional random point problems," Doklady Akademii Nauk SSSR, vol. 2, 1998, pp. 270274.

[17] I. A. Eckley, P. Fearnhead and R. Killick, "Analysis of changepoint models," Bayesian Time Series Models, 2011, pp. 205-224.

[18] E. S. Venkatraman, Consistency results in multiple change-point problems, $\mathrm{PhD}$ thesis, to the Department of Statistics. Stanford University. 1992.

[19] R. Killick, P. Fearnhead and I. A. Eckley "Optimal detection of change-points with a linear computational cost," Journal of the American Statistical Association, vol. 500, 2012, pp. 1590-1598. http://dx.doi.org/10.1080/01621459.2012.737745

[20] W. Li, "DNA segmentation as a model selection process," In proceedings of the fifth annual international conference on Computational biology, ACM, 2001, pp. 204-210. http://dx.doi.org/10.1145/369133.369202
[21] $\mathrm{H}$. Akaike, "A new look at the statistical model identification" IEEE transactions on automatic control, vol. 6, 1974, pp. 716-723. http://dx.doi.org/10.1109/TAC.1974.1100705

[22] G. Schwarz, "Estimating the dimension of a model," The annals of statistics, vol. 2, 1978, pp. 461-464 http://dx.doi.org/10.1214/aos/1176344136

[23] J. Chen, A. Gupta and J. Pan "Information criterion and change point problem for regular models," Sankhyā: The Indian Journal of Statistics, 2006, pp. 252-282.

[24] J. Chen and A. K. Gupta, "Testing and locating variance changepoints with application to stock prices," Journal of the American Statistical association, vol. 438, 1997, pp. 739-747. http://dx.doi.org/10.1080/01621459.1997.10474026

[25] T. Young Yang and L. Kuo, "Bayesian binary segmentation procedure for a poisson process with multiple changepoints," Journal of Computational and Graphical Statistics, vol. 4, 1998, pp. 772-785. http://dx.doi.org/10.1198/106186001317243449

[26] R. Killick, I. A. Eckley, P. Jonathan and U. Chester, "Efficient detection of multiple changepoints within an oceano-graphic time series," In Proceedings of the 58th World Science Congress of ISI, 2011.

[27] P. Fryzlewicz, "Wild binary segmentation for multiple change-point detection," The Annals of Statistics, vol. 6, 2014, pp. 2243-2281. http://dx.doi.org/10.1214/14-AOS1245

[28] W. J. R. M. Priyadarshana, The cross-entropy method and multiple change-point detection in genomic sequences, $\mathrm{PhD}$ thesis, Macquarie University, 2015.

[29] H. Cho and P. Fryzlewicz, "Multiple-change-point detection for high dimensional time series via sparsified binary segmentation," Journal of the Royal Statistical Society: series B (statistical methodology), vol. 2, 2015, pp. 475-507. http://dx.doi.org/10.1111/rssb.12079

[30] A. B. Olshen, E. Venkatraman, R. Lucito and M. Wigler,"Circular binary segmentation for the analysis of array-based DNA copy number data," Biostatistics, vol. 4, 2004, pp. 557-572. https://doi.org/10.1093/biostatistics/kxh008

[31] J. Chen and A. K. Gupta, Parametric statistical change point analysis. with applications to genetics, medicine, and finance, Springer Science \& Business Media, 2011.

[32] D. V. Hinkley and E. A. Hinkley, "Inference about the change-point in a sequence of binomial variables," Biometrika, vol. 3, 1970, pp. 477-488 https://doi.org/10.2307/2334766

[33] B. Jackson, J. D. Scargle, D. Barnes, S. Arabhi, A. Alt, P. Gioumousis, E. Gwin, P. Sangtrakulcharoen, L. Tan and T. T. Tsai,"An algorithm for optimal partitioning of data on an interval," IEEE Signal Processing Letters, vol. 12, 2005, pp. 105-108. https://doi.org/10.1109/LSP.2001.838216

[34] J. Pan and J. Chen, "Application of modified information criterion to multiple change point problems," Journal of multivariate analysis, vol. 10, 2006, pp. 2221-2241. https://doi.org/10.1016/j.jmva.2006.05.009

[35] A. N. Shiryaev, "On optimum methods in quickest detection problems," Theory of Probability \& Its Applications, vol. 1, 1963, pp. 22-46. https://doi.org/10.1137/1108002

[36] N. R. Zhang and D. O. Siegmund, "A modified Bayes information criterion with applications to the analysis of comparative genomic hybridization data," Biometrics, vol. 1, 2007, pp. 22-32. https://doi.org/10.1111/j.1541-0420.2006.00662.x

[37] T. Polushina and G. Sofronov, "Change-point detection in binary Marko DNA sequences by the Cross-Entropy method," In Computer Science and Information Systems (FedCSIS), 2014 Federated Conference on, IEEE, vol. 2, 2014, pp. 471-478. https://doi.org/10.15439/2014F88

[38] G. Yu Sofronov, G. E. Evans, J. M. Keith and D. P. Kroese, "Identifying change-points in biological sequences via sequential importance sampling," In Environmental Modeling \& Assessment, vol. 5, 2009, pp. $577-$ 584. https://doi.org/10.1007/s10666-008-9160-8

[39] G. Sofronov, "Change-Point Modelling in Biological Sequences via the Bayesian Adaptive Independent Sampler," In International Proceedings of Computer Science and Information Technology, vol. 5, 2011, pp. 122 126.

[40] M. Manuguerra, G. Sofronov, M. Tani and G. Heller, "Monte Carlo methods in spatio-temporal regression modeling of migration in the EU," In Computational Intelligence for Financial Engineering \& Economics (CIFEr), 2013 IEEE Conference on, IEEE, 2013, pp. 128-134. https://doi.org/10.1109/CIFEr.2013.6611708 


\title{
The Revised Stochastic Simplex Bisection Algorithm and Particle Swarm Optimization
}

\author{
Christer Samuelsson \\ $25 \mathrm{~A}$ rue des Sports \\ 57200 Sarreguemines, FRANCE \\ Email: csamuelsson42@yahoo.com
}

\begin{abstract}
The stochastic simplex bisection (SSB) algorithm is evaluated against particle swarm optimization (PSO) on a prominent test set. The original SSB algorithm performs on par with the PSO algorithm and a revised version of the SSB algorithm outperforms both of them. Detailed analysis of the performance on select objective functions brings to light key properties of the three algorithms. The core SSB algorithm is here viewed as a sampling tool for an outer loop that employs statistical pattern recognition. This opens the door for a host of other schemes.
\end{abstract}

\section{INTRODUCTION}

TOCHASTIC optimization [1] involves introducing some $\checkmark$ degree of randomness, when searching for optima. Particularly successful approaches include genetic algorithms [2], particle swarm optimization [3], and ant-colony optimization [4]. Such schemes are often applied to continuous optimization problems, especially when the gradient and Hessian of the objective functions are not readily available. In addition to this they have proven effective when optimizing highly multimodal functions, i.e., function with a large number of optima.

We here compare the performance of the stochastic simplex bisection (SSB) algorithm-first proposed in [5] and first evaluated against other optimization algorithms in [6] - with a particle swarm optimizer (PSO). The former employs a common stochastic optimization scheme, but unlike other stochastic approaches, it applies the scheme to search space regions, rather than to individual points. The latter is a wellknown global optimization scheme, which has spawned a number of related schemes, such as firefly optimization [7] and fish school search [8]. This is the first time the SSB algorithm is tested against another state-of-the-art global optimizer.

Two versions of the SSB algorithm were tested: the original one from [5], [6] and a new one, which uses the same core SSB algorithm and a modified outer loop that clusters a shrinking set of arguments with relatively low function values.

The rest of the article is organized as follows. Section II describes the new and old SSB algorithms. Section III details the PSO optimizer used. Section IV discusses the experimental setup, and reports and analyses the findings.

\section{The Stochastic Simplex Bisection Algorithm}

Consider the simple problem where we wish to minimize $f(x)$, which is strictly convex on $\mathbf{R}$. Assume further that we have found $x_{1}<x_{3}<x_{5}$, where $f\left(x_{1}\right) \geq f\left(x_{3}\right) \leq f\left(x_{5}\right)$, i.e., that we have found an interval that has an interior point $x_{3}$ with a smaller function value than its end points. ${ }^{1}$

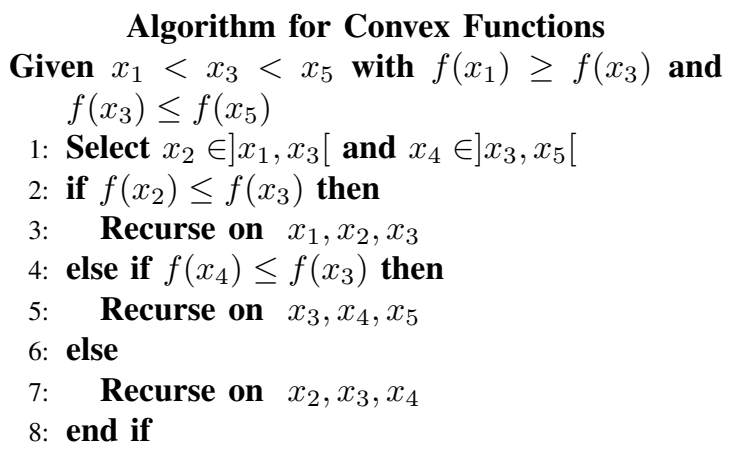

We thus recurse on the subinterval that has an interior point with a smaller function value than its end points. ${ }^{2}$

\section{A. The Core SSB Algorithm}

The SSB algorithm generalizes this to non-convex functions in $n$ dimensions. An interval is generalized to a simplex, rather than to a hyperbox, The latter has $2^{n}$ corners, and bisecting it requires computing the function value in $2^{n-1}$ new corners. The former has only $n+1$ corners, and bisecting it only requires calculating the function value in one new point.

\section{Core SSB Step}

Given a set $\left\{T_{k^{\prime}}\right\}$ of non-overlapping simplexes,

1: Select the next simplex $T_{k}$ to bisect at random with probability $\frac{s_{k}}{\sum_{k^{\prime}} s_{k^{\prime}}}$.

2: Select a bisection point at random roughly in the middle of the longest edge of $T_{k}$.

3: Bisect $T_{k}$, yielding two new simplexes.

4: Replace $T_{k}$ in $\left\{T_{k^{\prime}}\right\}$ with its two offspring.

The simplex score $s_{k}$, which is defined in Section II-A1 below, requires the function value in the simplex midpoint. Thus only three new function values need be calculated for each bisection: that of the bisection point and those of the midpoints of the two new simplexes, see Section II-A2.

The core SSB algorithm starts with a simplex $T_{0}$ and maintains a partition of it by repeatedly performing the core SSB

\footnotetext{
${ }^{1}$ By strict convexity, one of the two end point values must be strictly larger, i.e., either $f\left(x_{1}\right)>f\left(x_{3}\right)$ or $f\left(x_{3}\right)<f\left(x_{5}\right)$, or both.

${ }^{2}$ If $f\left(x_{2}\right)=f\left(x_{3}\right)$, the algorithm could be clever and instead recurse on $x_{2}, x_{23}, x_{3}$, with $\left.x_{23} \in\right] x_{2}, x_{3}\left[\right.$, where, by necessity $f\left(x_{2}\right)>f\left(x_{23}\right)<$ $f\left(x_{3}\right)$, due to strict convexity. Similarly for $f\left(x_{4}\right)=f\left(x_{3}\right)$.
} 
step, until some termination criterion is met. It then returns the best point found thus far. The algorithm is complete in the sense that no portion of the search space is ever discarded, and it avoids redundancy by using non-overlapping simplexes. These are two common pitfalls of stochastic optimization. The algorithm still reaps the benefits of stochastic search in exploring more promising regions earlier, in average, while granting also less promising regions a non-zero chance of being explored.

1) The Simplex Score: We define the simplex scores $s_{k}$ as follows. Let $\left\{T_{k}=\left\langle\mathbf{x}_{k}^{(1)}, \ldots, \mathbf{x}_{k}^{(n+1)}\right\rangle\right\}$ be a collection of $n$ dimensional simplexes with (dropping the index $k$ for clarity),

$$
\overline{\mathbf{x}}=\frac{1}{n+1} \sum_{i=1}^{n+1} \mathbf{x}^{(i)} \quad ; \quad \bar{f}=\frac{f(\overline{\mathbf{x}})+\sum_{i=1}^{n+1} f\left(\mathbf{x}^{(i)}\right)}{n+2}
$$

where $\overline{\mathbf{x}}$ is the midpoint and $\bar{f}$ is the average function value over the corners and the midpoint. We then set

$$
f^{-}=\min \left(f(\overline{\mathbf{x}}), \min _{i} f\left(\mathbf{x}^{(i)}\right)\right) \quad ; \quad \delta=\frac{\bar{f}-f^{-}}{4}
$$

and introduce

$$
f^{\star}=f^{-}-\delta
$$

which is a combined measure of the lowest function value $f^{-}$ and an estimate $\delta$ of how much it might potentially decrease, judging by the average function value $\bar{f}$ and this lowest value.

We next make $f^{\star}$ offset- and scale-invariant through minmax normalization, using the lowest function value $f_{\mathrm{vb}}$ in the very best point found this far, and some relatively high, fixed function value $f_{\mathrm{W}}$ found early in the search. ${ }^{3}$

$$
f^{\star} \leftarrow \frac{f^{\star}-f_{\mathrm{vb}}}{f_{\mathrm{W}}-f_{\mathrm{vb}}}
$$

Thus, all simplexes must be rescored whenever a new very best point is found. As this happens rather seldom, it incurs very little overhead in practice.

The simplex score $s$ is then defined as

$$
s=l \cdot \exp \left(-\lambda_{0} f^{\star}\right)
$$

where $l$ is the length of its longest edge:

$$
l=\max _{i j}\left|\mathbf{x}^{(i)}-\mathbf{x}^{(j)}\right|
$$

The parameter $\lambda_{0}$, which defaults to one, controls the tradeoff between exploration and exploitation. A small $\lambda_{0}$ will make the simplexes with different $f^{\star}$ more equiprobable, thus promoting exploration, a large one will do the opposite.

${ }^{3}$ In practice, we require that the $f^{\star}$ be non-negative and that the denominator $f_{\mathrm{W}}-f_{\mathrm{vb}}$ be at least 0.1 and at most one, yielding the expression:

$$
f^{\star} \leftarrow \frac{\max \left(0, f^{\star}-f_{\mathrm{vb}}\right)}{\max \left(0.1, \min \left(1, f_{\mathrm{w}}-f_{\mathrm{vb}}\right)\right)}
$$

2) Simplex Bisection: Each round only creates two new simplexes: the longest edge of the selected simplex is bisected. All corners remain the same, save one of the two connected by this edge. Let these corners be $\mathbf{x}^{(i)}$ and $\mathbf{x}^{(j)}$. The edge bisection point $\overline{\mathbf{x}}^{\prime}$ - the new corner-is also randomized to counter-act any symmetries of the objective function $f(\mathbf{x})$ in its argument $\mathbf{x}$ :

$\overline{\mathbf{x}}^{\prime}=(0.5+\theta) \mathbf{x}^{(i)}+(0.5-\theta) \mathbf{x}^{(j)} \quad$ for $\quad \theta \sim \mathrm{U}(-\alpha, \alpha)$

An empirically good choice is $\alpha=0.05$ ( $\max 10 \%$ randomness). $\overline{\mathbf{x}}^{\prime}$ replaces $\mathbf{x}^{(i)}$ in one offspring simplex and $\mathbf{x}^{(j)}$ in the other one. Thus only three new function values need be calculated for each bisection: $f\left(\overline{\mathbf{x}}^{\prime}\right)$ and the function values of the midpoints of the two new simplexes.

\section{B. Outer Loop: Maintaining a Hyperbox}

It is often desirable to start from a hyperbox. For example, constraints often take the form of bounds on the individual variables of each dimension. The tested SSB algorithms repeatedly restart an SSB algorithm from a hyperbox created in a previous iteration. They use an outer loop over epochs that maintains the hyperbox, and an inner loop over rounds, each of which performs the core SSB step. Note that although partitioning a hyperbox into a set of simplexes is trivial in two dimensions, it is a challenging and time-consuming task in higher ones, see [9] and [10].

The SSB algorithms thus consist of two nested loops. The outer loop over epochs maintains a hyperbox. Each epoch runs the inner loop over rounds, where each round contains one simplex bisection, which replaces one simplex with two new ones. Each epoch is divided into two phases, see below. In the first phase, the simplexes are processed as a first-in-first-out (FIFO) queue to create an initial grid. In the second phase, the next simplex to bisect is selected as in the core SSB step: at random, with probabilities proportional to the simplex scores.

\section{Inner Loop}

Given A hyperbox $H$.

1: Partition $H$ into a set $\left\{T_{k^{\prime}}\right\}$ of simplexes.

2: Initialize other variables as described below.

: Convert $\left\{T_{k^{\prime}}\right\}$ into FIFO queue.

4: while not End-of-epoch do

\section{if Phase 1 then}

Run Core SSB step, but select as $T_{k}$ the first simplex of the FIFO queue and add its offspring to the end of the queue.

7: $\quad$ else

8: $\quad$ Run Core SSB step. $\{$ Phase 2\}

\section{9: $\quad$ end if}

\{This yields three new objective function val-

ues $f\left(\mathbf{x}_{a}\right), f\left(\mathbf{x}_{c}\right), f\left(\mathbf{x}_{c}\right)$.

10: if $f\left(\mathbf{x}_{a / b / c}\right)<f_{\mathrm{vb}}$ then

11: $\quad f_{\mathrm{vb}} \leftarrow f\left(\mathbf{x}_{a / b / c}\right) ; \mathbf{x}_{\mathrm{vb}} \leftarrow \mathbf{x}_{a / b / c}$

12: $\quad$ Rescore all simplexes.

13: end if

14: Update other variables as described below.

15: end while 
For $f_{\mathrm{W}}$, we use the highest function value in a corner of the bounding box. The manner in which the hyperbox is modified after each epoch depends on the sequence of points sampled.

1) Old Scheme: The sequence order matters in the old scheme, and a single hyperbox is created. To this end, we need some terminology. A best point is any point found during the second phase of an epoch that is the best this far in that epoch. The best points thus start over each epoch. The very best point, on the other hand, is the globally best point found in any round of any epoch. Its objective function value is $f_{\mathrm{vb}}$. The first phase of each epoch consists of the first quarter of its rounds. The rest of its rounds constitute the second phase. Other choices than one quarter have been tested and found effective in other settings, see [5], [6].

\section{Initializing other variables}

1: Let $f_{b}$ be the best function value found when scoring the simplexes partitioning the hyperbox.

2: BestPoints $\leftarrow \emptyset$;

Updating other variables

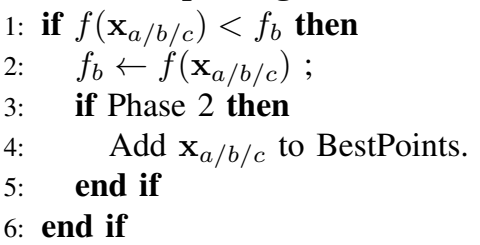

If the elapsed epoch had enough best points, the next hyperbox is one that contains all best points and the very best point as interior points. It turns out that the simple scheme of updating the lower and higher bounds of the hyperbox in each dimension, for each new best point, works well in practice. Otherwise, the previous hyperbox is increased in size and recentered around the current very best point. In the tested SSB algorithm, the box is padded by 100 percent of the interval length in each dimension, when there are enough best points, and the old interval length is quadrupled, when there aren't.

\section{Creating a new hyperbox}

1: Let $H$ be the smallest hyperbox that contains BestPoints $\cup\left\{\mathrm{x}_{\mathrm{vb}}\right\}$.

2: Retain the new midpoint of $H$.

3: if the set BestPoints is large enough then

4: Multiply the length of each side of $H$ by some fixed margin factor (e.g., 1.1).

5: else

6: Set the length of each side of $H$ to a fixed factor (e.g., 2) times that the old hyperbox.

7: end if

8: for all sides of the new hyperbox $H$ do

9: $\quad$ if the side length is zero then

10: $\quad$ Set that side length of $H$ to a fixed factor (e.g., 2) times that of the old hyperbox.

11: end if

12: end for

The old scheme loop may seem somewhat ad hoc. It captures the idea, that if there has been non-trivial local improvements, search should focus on these improvements, yet also consider the globally best point found. We note that any new best point must be a bisection point, or the midpoint of a simplex, with a lower function value than its corner points. In one dimension, these cases coincide. For convex functions, such an interval must contain the minimum, which our introductory algorithm exploits. For non-convex functions, or in several dimensions, the region surrounding such a point merits further investigation.

2) New Scheme: The next hyperbox is in the new scheme created by drawing inferences from the set of sample points using statistical pattern recognition techniques. In its current incarnation, it selects all sample points with a function value below a certain threshold. We call these low points. To generate more low points, they are clustered using k-means clustering, see, e.g., [11], pp. 424-430. Points far away from the cluster means are pruned from the clusters. A hyperbox is then created for each cluster and the core SSB algorithm is run on this hyperbox. This will add new sample points and thus presumably additional low points, and is done to search a region containing this cluster for an optimum. In addition, the midpoints between clusters are evaluated and added as sample points. This is done to encourage merging clusters that in fact belong to the same optimum. Since sample points are retained between epochs, their number increases steadily.

The number of rounds in each epoch, where each round requires three function evaluations, is constant and distributed between running the core SSB algorithm on a hyperbox derived from the entire set of low points and on the hyperboxes derived from individual clusters. We found that using half of the rounds for each purpose, and thus one half divided by the number of viable clusters for each of the latter, was appropriate. Thus, compared to the old scheme, fewer rounds are used for global exploration.

The final hyperbox used in the next epoch contains the set of low points. To gradually reduce it in size, the threshold is reduced from epoch to epoch to shrink the relative number of low points. In the first epoch, $n_{i}$ points, say all points, are included; in the last one, only $n_{f}$, say three points, are. For intermediate epochs we calculate the rank of the highest function value to be included as

$r(x)=\frac{n_{i}}{1+c \cdot x^{4}}$ with $c=\frac{n_{i}}{n_{f}}-1$ and $x=\frac{\text { epoch }}{\text { maxEpoch }}$

It is clear that $r(0)=n_{i}$ and that $r(1)=n_{f}$, independent of the power that $x$ is raised to; four was found empirically to be a good choice. This successively reduces the number of low points, and hopefully forces the algorithm to eventually commit to one cluster containing the global optimum. Thus, the algorithm initially performs global exploration, but gradually resorts to searching a shrinking number of low points and clusters, and eventually focuses on exploiting a small number of low points and very few-if not a single - clusters, to converge on a potential global optimum.

This is an example of using a level curve-aka a contour curve, called a level (hyper-)surface in higher dimensions - of the function. In this case, we select all points inside it. Current 
work includes applying statistical recognition of level surfaces to identify regions that warrant further investigation, as well as the use of more sophisticated clustering techniques.

\section{Related Work}

The SSB algorithm uses a typical stochastic optimization scheme. It maintains a set of elements, each with a positive score; randomly selects some elements based on the scores; uses these elements to explore the search space, often creating new elements in the process; and updates the set of elements and their scores according to the findings. The scheme is however here applied to regions of the search space, not to points in it, as in, e.g., PSO [12], shuffled complex evolution [13], covariance matrix adaptation evolution strategy [14], controlled random search [15], differential evolution [16], and firefly optimization [7].

Convergent optimization via most-promising-area stochastic search (COMPASS) [17] sounds similar, but it is a technique for discrete optimization via simulation, where "there is no explicit form of the objective function, and function evaluations are stochastic and computationally expensive." Predictably, it always samples the most promising area next, rather than select it probabilistically using scores, and stochastic search refers to uniform sampling within that area.

The simplex method [18], Chapter 9, doesn't actually use simplexes. To create new points, controlled random search [15] generates random simplexes. These may overlap and are not guaranteed to cover the search space. Nor are they subdivided. DIRECT [19] uses hyperboxes that partition the search space. It avoids the $2^{n}$ complexity by directional search from their midpoints, ignoring their corners. Each hyperbox potentially containing the global minimum is trisected, rather than bisected, along each dimension in turn. There is no randomized selection.

Whereas [20] uses stochastic optimization to improve kmeans clustering by avoiding local optima, the new SSB scheme applies k-means clustering to data generated by the core SSB algorithm to distinguish different optima and coordinate disparate data points corresponding to the same one.

\section{The PARTICLE SWARM OptIMIZER}

The employed PSO optimizer follows [1], p 121, with a few modifications.

The swarm consists of a number, typically 20-40, of boids - a word play on bots, birds, and droids - each possessing a position $\mathbf{x}$ and a velocity $\mathbf{v}$. The objective function value $f(\mathbf{x})$ is also recorded. In each iteration, the position and velocity of each boid are updated as follows.

$$
\begin{aligned}
& \mathbf{v} \leftarrow \theta \mathbf{v}+c_{1} \mathbf{u}_{1} \circ\left(\mathbf{x}_{1}^{b}-\mathbf{x}\right)+c_{2} \mathbf{u}_{2} \circ\left(\mathbf{x}_{2}^{b}-\mathbf{x}\right) \\
& \mathbf{x} \leftarrow \mathbf{x}+\mathbf{v}
\end{aligned}
$$

Here $\mathbf{x}_{1}^{b}$ is the best point historically of the boid in question, while $\mathbf{x}_{2}^{b}$ is the best current point of all boids. ${ }^{4}$ Thus, $\mathbf{x}_{i}^{b}-\mathbf{x}$ is the vector from the current point $\mathbf{x}$ to the best point $\mathbf{x}_{i}^{b}$.

${ }^{4}$ Best here means "with the lowest objective function value."
The idea is that the velocity $\mathbf{v}$ is attracted to both these two best points, with acceleration, or attraction coefficients, $c_{i} \mathbf{u}_{i}$. The first of these two terms of the velocity update is called the cognitive component, depending only on the history of the individual boid, and the second one is called the social component, depending on the swarm as a whole.

In [1], $\mathrm{p} 121, \mathbf{u}_{i}$ is in fact a random scalar $u_{i}$, drawn uniformly from $[0,1]$, and the multiplication is scalar multiplication of the difference vector. This guarantees that this acceleration term is along the line connecting $\mathbf{x}$ and $\mathbf{x}_{i}^{b}$. We instead use the Hadamard product, i.e., the component-wise product, denoted $\circ$, and let $\mathbf{u}_{1}$ and $\mathbf{u}_{2}$ be vectors where each element is independently uniform on $[0,1]$. This is the method used in [21], p 219. The acceleration term is then in a cone or pyramid centered around $\mathbf{x}_{i}^{b}-\mathbf{x}$. We set both the two scalars $c_{1}$ and $c_{2}$ to 2 , which is standard practice and which means that each component of $c_{i} \mathbf{u}_{i}$ has expectation 1 .

The first term, $\theta \mathbf{v}$, where $\theta$ is called the inertia weight, is simply the previous velocity for $\theta=1$. With $\theta>1$, this term accelerates the boid in the direction it is currently travelling, preferring exploration over exploitation. With $\theta<1$, it instead dampens the velocity, making it pay more heed to the cognitive and social acceleration components, thus preferring exploitation over exploration. It thus makes sense to start with an inertia weight above one, and then successively reduce it, ending the search with an inertia weight below one. To this end, we use dynamic inertia weighting to bring down $\theta$ geometrically from 1.4 in the first iteration to 0.3 in the last, cf. [1], pp 127-128.

As the velocities are initially self-accelerating, it is important to restrain them. Each velocity component is capped to have an absolute value that does not exceed a given maximum $v_{\max }$. This is another key parameter for balancing exploration and exploitation. The boids must stay within the feasibility domain, which is here a bounding hyperbox. Any boid that attempts to leave it has the offending $\mathbf{x}$ coordinate set to the boundary point. The corresponding $\mathbf{v}$ coordinate is set to the negative of its value. This leads to hard (elastic) reflection in the boundary, much like billiard balls.

\section{EXPERIMENTS}

\section{A. Experimental Setup}

We tested all two-dimensional objective functions of Figure 1 (penultimate page), most of which are from [22]. Function 0 comes from [5], Function 20 from [23], and Functions 21, 22, 24, and 25 are of our own design. All functions have unique global minima, except Function 0, due to symmetry in $x$ and $x+y$, and Functions 12, 14, and 17, which have four global minima, due to symmetry in $\pm x, \pm y$. Functions 16 and 17 were corrected using [24].

We tested the optimizers on the three domains:
1) $[-80,120] \times[-80,120]$,
2) $[-800,1200] \times[-800,1200]$, and
3) $[-8000,12000] \times[-8000,12000]$.

These are larger than those of the test set, which are typically $[-10,10] \times[-10,10]$ or even $[-5,5] \times[-5,5]$. The domains 
were made asymmetric in $x$ and $y$, since many test functions have their global minimum in $\mathbf{x}=\mathbf{0}$. In each trial, each optimizer was allowed 50000 function evaluations. Success was defined as finding any argument with a function value within $10^{-13}$ of the known global minimal value.

The optimal value of $v_{\max }$ was determined for the PSO in each test domain. It used 20 boids and 2500 iterations to achieve the limit of 50000 function evaluations. Fewer iterations result in failure to converge to within $10^{-13}$ of the minimum, whereas fewer boids fail to explore the search space effectively. Both SSB schemes used 40 epochs of 415 rounds each $(40 \times 415 \times 3=49800)$, which deviates from the 60 epochs and 276 rounds $(60 \times 276 \times 3=49680)$ used in [5]. This was done to accommodate the revised SSB scheme, where each epoch requires running the core SSB algorithm on a set of hyperboxes, one for each cluster, in addition to the hyperbox of the current epoch, see Section II-B, New Scheme.

\section{B. Experimental Results}

Table I shows their respective success rates in 1000 trials. We first and foremost note that the original SSB algorithm holds its own against the PSO algorithm and that the revised SSB scheme performs significantly better.

Ignoring ties, the revised SSB scheme is better than the PSO algorithm in 10 cases of 13 in the smallest domain and 10 of 14 cases in the other two domains. The pairwise sign test [25], which is not a very powerful test, yields p-values of 0.046 in the smallest domain, and 0.09 in the other two domains. Similarily, the revised SSB scheme is better than the original SSB scheme 18 times of 20 in the smallest domain and 15 times of 20 in two larger domains, yielding yields pvalues of 0.0002 and 0.021 , respectively. We used the sign test, rather than, say, the Wilcoxon signed-rank test [26], which is more powerful, since the objective functions vary greatly in difficulty. Thus, the difference pairs cannot be seen as drawn from the same distribution.

To further analyze these results, we need to consider the nature of each test objective function. These can be classified into:

- unimodal quadratic forms, Fcns 2, 6, 8;

- oligo-modal ${ }^{5}$ polynomials, Fens 3, 4, 5, 10, 18;

- multimodal damped trigonometrics, Fcns 0, 1, 12-17, 25;

- mixed trigonometrics and quadratics, Fcns 9, 11, 20, 24

- other (unimodal) functions, Fcns 7, 21, 22.

The first group is simple there to see that the optimizers are sane; any deviation from a perfect score signals a fundamental problem. Functions 9, 10, 16, and 21 behave similarly. For these functions, the PSO and the new SSB perform flawlessly, whereas the old SSB fails in a small number of the trials. Inspection reveals that the reason for this is that the old SSB prematurely commits to a hyperbox that does not contain the global optimum. Subsequent epochs tend to repair this by increasing the hyperbox size, but this does not happen fast

\footnotetext{
${ }^{5}$ Oligo-modal means "with a few modes." (Apologies for mixing Greek and Latin roots...)
}

enough to allow convergence to within $10^{-13}$ of the optimum value. Conversely, this behavior pays dividends in the largest domain and for the hardest functions-0,11, and 14-where the old SSB algorithm prevails.

Function 7 is very hard, and defeats all optimizers. It has a concave valley, kinks, and a very anisotropic variable coupling. The gradient is ill-defined and unbounded in the valley bottom, and especially ill-behaved in the optimum. It is included as a reminder that our successes are relative. Memento mori.

Functions 1,11,14 are essentially constant at some distance to the optimum. This makes it harder for the exploration aspect of the algorithms to locate the target optimum. As the domain size increases, performance drops from full score, for at least some optimizer, to essentially total failure for all of them.

Functions 12, 14, and 17 have multiple global optima due to symmetries. This profits the old SSB scheme and leaves the PSO scheme in the dust. In conjunction with the very localized variation of function 14, this defeats the new SSB scheme, as it does not have enough rounds for exploration.

The only functions for which the old SSB scheme does better than the new one are Functions 0, 4, 12, 14, and 17. We have just discussed the latter three, which have multiple global optima due to symmetries. Function 0 has very many local optima and the only real remedy is exploration. Function 4 is interesting in that it is a case where the clustering fails to some extent. Better clustering methods raise the success rate from 0.435 to 0.864 . This is however beyond the scope of the current article.

\section{Summary AND CONCLUSIONS}

We evaluated the stochastic simplex bisection (SSB) algorithm against a particle swarm optimizer (PSO) on a prominent test set. The former employs a common stochastic optimization scheme, but unlike other stochastic approaches, it applies the scheme to search space regions, rather than to individual points. The latter is a well-known workhorse for stochastic optimization. This is the first evaluation of the SSB algorithm against a state-of-the-art global optimizer.

The original SSB scheme holds its own against the PSO. The revised SSB scheme is better at exploitation than the old one, allowing it to significantly outperform both the PSO and old SSB schemes in all three domains.

The key difference between the new and original SSB schemes is that the new one applies statistical pattern recognition to the data points sampled using the core SSB algorithm. This opens the door for a host of other schemes that view stochastic optimization not as a random walk, but as statistical inference. Current work includes using more sophisticated statistical pattern recognition techniques to identify regions that warrant further investigation.

The PSO algorithm has been in extensive use since 1995, and it comes with a large body of experience and knowhow. The SSB algorithm was first published in 2015 and it is still very much evolving. It already outperforms the PSO algorithm. There is every reason to expect rapid progress in its performance. 
TABLE I

SUCCESS RATE IN 1000 TRIALS OF THE PSO THE SSB ALGORITHMS.

\begin{tabular}{|c|c|c|c|c|c|c|c|c|c|}
\hline \multirow{2}{*}{$\begin{array}{l}\text { Domain } \\
\text { Fcn }\end{array}$} & \multicolumn{3}{|c|}{$[-80,120]^{2}$} & \multicolumn{3}{|c|}{$[-800,1200]^{2}$} & \multicolumn{3}{|c|}{$[-8000,12000]^{2}$} \\
\hline & PSO & Old SSB & New SSB & PSO & Old SSB & New SSB & PSO & Old SSB & New SSB \\
\hline 0 & 0.025 & 0.124 & 0.066 & 0.002 & 0.107 & 0.036 & 0.000 & 0.086 & 0.023 \\
\hline 1 & 0.999 & 0.925 & 1.000 & 0.567 & 0.331 & 0.827 & 0.044 & 0.002 & 0.004 \\
\hline 2 & 1.000 & 0.999 & 1.000 & 1.000 & 0.999 & 1.000 & 1.000 & 0.999 & 1.000 \\
\hline 3 & 0.007 & 0.832 & 0.999 & 0.001 & 0.693 & 0.992 & 0.002 & 0.507 & 0.963 \\
\hline 4 & 0.997 & 0.986 & 1.000 & 0.971 & 0.978 & 0.939 & 0.852 & 0.905 & 0.435 \\
\hline 5 & 0.994 & 0.763 & 1.000 & 0.865 & 0.787 & 1.000 & 0.681 & 0.758 & 0.988 \\
\hline 6 & 1.000 & 0.998 & 1.000 & 1.000 & 0.987 & 1.000 & 1.000 & 0.929 & 1.000 \\
\hline 7 & 0.000 & 0.000 & 0.000 & 0.000 & 0.000 & 0.000 & 0.000 & 0.000 & 0.000 \\
\hline 8 & 1.000 & 1.000 & 1.000 & 1.000 & 0.993 & 1.000 & 1.000 & 0.954 & 1.000 \\
\hline 9 & 1.000 & 0.979 & 1.000 & 1.000 & 0.939 & 1.000 & 1.000 & 0.923 & 0.999 \\
\hline 10 & 1.000 & 0.930 & 1.000 & 1.000 & 0.877 & 1.000 & 1.000 & 0.897 & 1.000 \\
\hline 11 & 0.173 & 0.984 & 0.999 & 0.001 & 0.060 & 0.006 & 0.000 & 0.000 & 0.000 \\
\hline 12 & 0.953 & 0.975 & 0.997 & 0.255 & 0.966 & 0.981 & 0.003 & 0.861 & 0.593 \\
\hline 14 & 0.064 & 0.977 & 0.951 & 0.000 & 0.922 & 0.670 & 0.000 & 0.103 & 0.004 \\
\hline 16 & 1.000 & 0.920 & 1.000 & 1.000 & 0.874 & 1.000 & 0.998 & 0.860 & 1.000 \\
\hline 17 & 0.999 & 0.928 & 0.934 & 0.793 & 0.870 & 0.863 & 0.194 & 0.819 & 0.817 \\
\hline 18 & 0.917 & 0.999 & 1.000 & 0.992 & 1.000 & 1.000 & 1.000 & 0.994 & 1.000 \\
\hline 20 & 1.000 & 0.667 & 1.000 & 1.000 & 0.715 & 1.000 & 0.858 & 0.669 & 0.976 \\
\hline 21 & 1.000 & 0.848 & 1.000 & 1.000 & 0.813 & 0.999 & 1.000 & 0.749 & 1.000 \\
\hline 22 & 1.000 & 0.082 & 0.667 & 1.000 & 0.073 & 0.692 & 1.000 & 0.067 & 0.620 \\
\hline 24 & 0.998 & 0.365 & 0.966 & 0.960 & 0.337 & 0.854 & 0.169 & 0.312 & 0.752 \\
\hline 25 & 0.948 & 0.995 & 1.000 & 0.658 & 0.954 & 1.000 & 0.348 & 0.956 & 0.982 \\
\hline Ave & 0.776 & 0.785 & 0.890 & 0.685 & 0.694 & 0.812 & 0.552 & 0.607 & 0.689 \\
\hline
\end{tabular}

\section{ACKNOWLEDGMENT}

This work was funded by the BAULT network and the METALOGUE project. BAULT, building and using language technology, is a multidisciplinary research community at the University of Helsinki. METALOGUE is a Seventh Framework Programme collaborative project funded by the European Commission, grant agreement number 611073 (http://www.metalogue.eu).

\section{REFERENCES}

[1] M. Wahde, Biologically Inspired Optimization Algorithms. WIT Press, 2008.

[2] D. E. Goldberg and J. H. Holland, "Genetic algorithms and machine learning," Machine Learning, vol. 3, no. 2-3, pp. 95-99, 1988. doi: 10.1023/A:1022602019183

[3] J. Kennedy and R. Eberhart, "Particle swarm optimization," 1995. doi 10.1109/ICNN.1995.488968

[4] M. Dorigo, V. Maniezzo, and A. Colorni, "The ant system: Optimization by a colony of cooperating agents," IEEE Trans. on Systems, Man, and Cybernetics, vol. 26, no. 1, pp. 29-41, 1996. doi: 10.1109/3477.484436

[5] C. Samuelsson, "The stochastic simplex bisection algorithm," in Procs. 15th Int.'l Conf. Computational Science, ser. ICCS/15. Elsevier, 2015. doi: 10.1016/j.procs.2015.05.215 pp. 855-864. [Online]. Available: http://www.sciencedirect.com/science/article/pii/S1877050915010236

[6] - "Comparative evaluation of the stochastic simplex bisection algorithm and the scipy.optimize module," in Procs. 8th Workshop on Computational Optimization, ser. WCO/15, 2015, pp. 573-578.

[7] X.-S. Yang, "Firefly algorithms for multimodal optimization," in Procs. 5th Int.'l Conf. Stochastic Algorithms: Foundations and Applications, ser. SAGA'09. Springer-Verlag, 2009. doi: 10.1007/978-3-642-049446_14. ISBN 3-642-04943-5, 978-3-642-04943-9 pp. 169-178. [Online]. Available: http://dl.acm.org/citation.cfm?id=1814087.1814105
[8] C. J. A. B. Filho, F. B. de Lima Neto, A. J. da C. C. Lins, A. I. S. Nascimento, and M. P. Lima, "A novel search algorithm based on fish school behavior," in Proceedings of the IEEE International Conference on Systems, Man and Cybernetics, Singapore, 12-15 October 2008, 2008. doi: 10.1109/ICSMC.2008.4811695 pp. 2646-2651. [Online]. Available: http://dx.doi.org/10.1109/ICSMC.2008.4811695

[9] M. Haiman, "A simple and relatively efficient triangulation of the n-cube," Discrete \& Computational Geometry, vol. 6, no. 1 pp. 287-289, 1991. doi: 10.1007/BF02574690. [Online]. Available: http://dx.doi.org/10.1007/BF02574690

[10] R. B. Hughes and M. R. Anderson, "Simplexity of the cube," Discrete Mathematics, vol. 158, no. 1â̧̆̊3, pp. 99 - 150, 1996. doi: http://dx.doi.org/10.1016/0012-365X(95)00075-8. [Online]. Available: http://www.sciencedirect.com/science/article/pii/0012365X95000758

[11] C. M. Bishop, Machine Learning and Pattern Recognition. Springer Verlag, 2006.

[12] A. I. Vaz and L. N. Vicente, "A particle swarm pattern search method for bound constrained global optimization," J. of Global Optimization, vol. 39, no. 2, pp. 197-219, Oct. 2007. doi: 10.1007/s10898-007-9133-5. [Online]. Available: http://dx.doi.org/10.1007/s10898-007-9133-5

[13] Q. Duan, S. Sorooshian, and V. Gupta, "Effective and efficient global optimization for conceptual rainfall-runoff models," Water resources research, vol. 28, no. 4, pp. 1015-1031, 1992. doi: 10.1029/91WR02985

[14] N. Hansen and S. Kern, "Evaluating the CMA evolution strategy on multimodal test functions," in Parallel Problem Solving from Nature VIII, X. Yao et al., Eds. Springer, 2004. doi: 10.1007/978-3-540-302179_29 pp. 282-291.

[15] P. Kaelo and M. M. Ali, "Some variants of the controlled random search algorithm for global optimization," J. Optim. Theory Appl, vol. 130 , no. 2, pp. 253-264, 2006. doi: 10.1007/s10957-006-9101-0

[16] K. V. Price, R. M. Storn, and J. A. Lampinen, Differential Evolution A Practical Approach to Global Optimization, ser. Natural Computing. Springer-Verlag, 2006, iSBN 540209506. 


\section{Objective Functions}

Fcns 21, 22, 24, 25 are novel; Fen 0 from [5]; Fcn 20 from [23]; remainder from [22]. Fens 16 and 17 corrected using [24].

$$
\begin{aligned}
& f(\mathbf{x})=f\left(x_{1}, \ldots, x_{n}\right)=\prod_{k=1}^{n} f_{0}\left(\sum_{i=1}^{k} x_{i}\right) \quad \text { with } \\
& f_{0}(x)=\sin \left(\sqrt{1+x^{2}}\right) \cdot \cos (2 x(x+1)) \cdot \frac{\ln \left(1+x^{2}\right)}{\sqrt{1+x^{2}}} \\
& f(\mathbf{x})=20\left(1-\exp \left(-0.2 \sqrt{\frac{1}{n} \sum_{i=1}^{n} x_{i}^{2}}\right)\right)+e-\exp \left(\frac{1}{n} \sum_{i=1}^{n} \cos \left(2 \pi x_{i}\right)\right) \\
& f(\mathbf{x})=f\left(x_{1}, \ldots, x_{n}\right)=\sum_{i=1}^{n} x_{i}^{2} \\
& f(\mathbf{x})=f\left(x_{1}, \ldots, x_{n}\right)=\sum_{i=1}^{n-1}\left(100 \cdot\left(x_{i+1}-x_{i}^{2}\right)^{2}+\left(x_{i}-1\right)^{2}\right) \\
& f(x, y)=(1.5-x+x y)^{2}+\left(2.25-x+x y^{2}\right)^{2}+\left(2.625-x+x y^{3}\right)^{2} \\
& f(x, y)=\left(1+(x+y+1)^{2}\left(19-14 x+3 x^{2}-14 y+6 x y+3 y^{2}\right)\right) \text {. } \\
& \cdot\left(30+(2 x-3 y)^{2}\left(18-32 x+12 x^{2}+48 y-36 x y+27 y^{2}\right)\right) \\
& f(x, y)=(x+2 y-7)^{2}+(2 x+y-5)^{2} \\
& f(x, y)=100 \sqrt{\left|y-0.01 x^{2}\right|}+0.01|x+10| \\
& f(x, y)=0.26\left(x^{2}+y^{2}\right)-0.48 x y \\
& f(x, y)=\sin ^{2}(3 \pi x)+(x-1)^{2}\left(1+\sin ^{2}(3 \pi y)\right)+(y-1)^{2}\left(1+\sin ^{2}(2 \pi x)\right) \\
& f(x, y)=2 x^{2}-1.05 x^{4}+\frac{x^{6}}{6}+x y+y^{2} \\
& f(x, y)=-\cos (x) \cos (y) \exp \left(-(x-\pi)^{2}-(y-\pi)^{2}\right) \\
& f(x, y)=-0.0001\left(|\sin (x) \sin (y)| \exp \left(\left|100-\frac{\sqrt{x^{2}+y^{2}}}{\pi}\right|\right)+1\right)^{0.1} \\
& f(x, y)=-|\sin (x) \cos (y)| \exp \left(\left|1-\frac{\sqrt{x^{2}+y^{2}}}{\pi}\right|\right) \\
& f(x, y)=0.5+\frac{\sin ^{2}\left(x^{2}-y^{2}\right)-0.5}{1+0.001\left(x^{2}+y^{2}\right)^{2}} \\
& f(x, y)=0.5+\frac{\cos ^{2}\left(\sin \left(x^{2}-y^{2}\right)\right)-0.5}{1+0.001\left(x^{2}+y^{2}\right)^{2}} \\
& f(\mathbf{x})=f\left(x_{1}, \ldots, x_{n}\right)=\frac{1}{2} \sum_{i=1}^{n}\left(x_{i}^{4}-16 x_{i}^{2}+5 x_{i}\right) \\
& f(\mathbf{x})=f\left(x_{1}, \ldots, x_{n}\right)=\sum_{i=1}^{n}\left(x_{i}^{2}+10 \cdot\left(1-\cos \left(2 \pi x_{i}\right)\right)\right) \\
& f(\mathbf{x})=f\left(x_{1}, \ldots, x_{n}\right)=\sum_{i=1}^{n}\left|x_{i}\right| \\
& f(\mathbf{x})=f\left(x_{1}, \ldots, x_{n}\right)=\sum_{i=1}^{n} \sqrt{\left|x_{i}\right|} \\
& f(\mathbf{x})=f\left(x_{1}, \ldots, x_{n}\right)=\sum_{i=1}^{n}\left|x_{i}\right|+\left(10+x_{i}^{2}\right) \cdot\left(1-\cos \left(2 \pi x_{i}\right)\right) \\
& f(\mathbf{x})=f\left(x_{1}, \ldots, x_{n}\right)=\sum_{i=1}^{n} 1-\operatorname{sink}\left(x_{i}\right) \quad \text { with } \operatorname{sink}(x)=\frac{\sin (x)}{x}
\end{aligned}
$$

Fig. 1.

Fig. 1 List of objective functions 
[17] L. J. Hong and B. L. Nelson, "Discrete optimization via simulation using compass," Operations Research, vol. 54, no. 1, pp. 115-129, 2006.

[18] N. Andréassson, A. Egrafov, and M. Patriksson, An Introduction to Continuous Optimization. Studentlitteratur, 2005.

[19] D. R. Jones, C. D. Perttunen, and B. E. Stuckman, "Lipschitzian optimization without the Lipschitz constant," J. Optim. Theory Appl., vol. 79, no. 1, pp. 157-181, Oct. 1993. doi: 10.1007/BF00941892. [Online]. Available: http://dx.doi.org/10.1007/BF00941892

[20] R. Tang, S. Fong, X.-S. Yang, and S. Deb, "Integrating nature-inspired optimization algorithms to k-means clustering." in ICDIM, S. Fong, P. Pichappan, S. Mohammed, P. Hung, and S. Asghar, Eds. IEEE, 2012. ISBN 978-1-4673-2428-1 pp. 116-123.

[21] X.-S. Yang, Nature-Inspired Optimization Algorithms. Elsevier, 2014. ISBN 9780124167452, 9780124167438
[22] Wikipedia, "Test functions for optimization," http://en.wikipedia.org/ wiki/Test_functions_for_optimization.html, 2014, accessed: 2014-12-14.

[23] K. Mullen, D. Ardia, D. Gil, D. Windover, and J. Cline, "DEoptim: An R package for global optimization by differential evolution," $J$. of Stat. Software, vol. 40, no. 6, pp. 1-26, 2011. [Online]. Available: http://www.jstatsoft.org/v40/i06/

[24] A. Gavana, "Global optimization benchmarks and AMPGO," http:// infinity77.net/global_optimization, 2015, accessed: 2015-01-09.

[25] Wikipedia, "Sign test," https://en.wikipedia.org/wiki/Sign_test, 2017, accessed: 2017-06-19.

[26] - "Wilcoxon signed-rank test," https://en.wikipedia.org/wiki/Wilcoxon_signed-rank_test, 2017 , accessed: 2017-06-19. 


\section{Computer Science \& Systems}

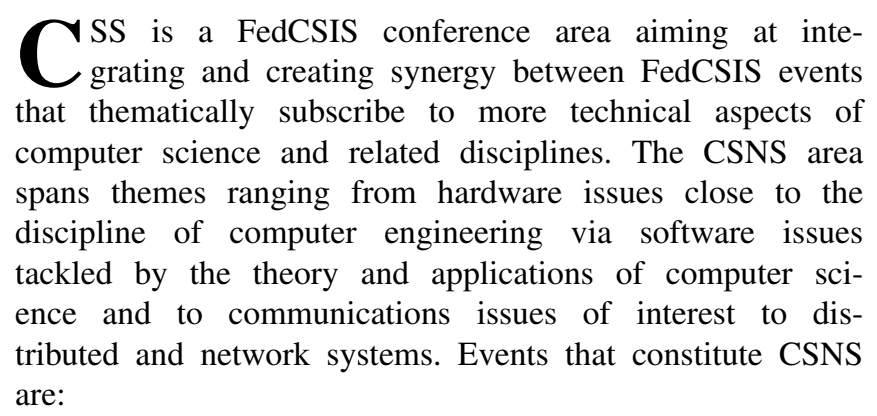

CSS is a FedCSIS conference area aiming at integrating and creating synergy between FedCSIS events that thematically subscribe to more technical aspects of computer science and related disciplines. The CSNS area clomes the discipline of computer engineering via software issues tackled by the theory and applications of computer scitributed and network systems. Events that constitute CSNS are:
- CANA'17-10 ${ }^{\text {th }}$ Computer Aspects of Numerical Algorithms

- C\&SS'17_th International Conference on Cryptography and Security Systems

- CPORA'17-2 $2^{\text {nd }}$ Workshop on Constraint Programming and Operation Research Applications

- MMAP'17-10 ${ }^{\text {th }}$ International Symposium on Multimedia Applications and Processing

- WAPL'17-6th Workshop on Advances in Programming Languages

- WSC'17-9th Workshop on Scalable Computing 



\section{$10^{\text {th }}$ Workshop on Computer Aspects of Numerical Algorithms}

$\mathbf{N}$ UMERICAL algorithms are widely used by scientists engaged in various areas. There is a special need of highly efficient and easy-to-use scalable tools for solving large scale problems. The workshop is devoted to numerical algorithms with the particular attention to the latest scientific trends in this area and to problems related to implementation of libraries of efficient numerical algorithms. The goal of the workshop is meeting of researchers from various institutes and exchanging of their experience, and integrations of scientific centers.

\section{TOPICS}

- Parallel numerical algorithms

- Novel data formats for dense and sparse matrices

- Libraries for numerical computations

- Numerical algorithms testing and benchmarking

- Analysis of rounding errors of numerical algorithms

- Languages, tools and environments for programming numerical algorithms

- Numerical algorithms on coprocesors (GPU, Intel Xeon Phi, etc.)

- Paradigms of programming numerical algorithms

- Contemporary computer architectures

- Heterogeneous numerical algorithms

- Applications of numerical algorithms in science and technology

\section{SECTION EDITORS}

- Bylina, Beata, Maria Curie-Sklodowska University, Poland

- Bylina, Jaroslaw, Maria Curie-Sklodowska University, Poland

- Stpiczyński, Przemysław, Maria Curie-Sklodowska University, Poland

\section{REVIEWERS}

- Amodio, Pierluigi, Università di Bari, Italy

- Anastassi, Zacharias, Qatar University, Qatar

- Banaś, Krzysztof, AGH University of Science and Technology, Poland

- Brugnano, Luigi, Universita' di Firenze, Italy

- Czachorski, Tadeusz, IITiS

- Dongarra, Jack
- Fialko, Sergiy, Tadeusz Kościuszko Cracow University of Technology

- Filote, Constantin

- Fourneau, Jean-Michel

- Gansterer, Wilfried, University of Vienna, Austria

- Georgiev, Krassimir, IICT - BAS, Bulgaria

- Gravvanis, George, Democritus University of Thrace, Greece

- Knottenbelt, William, Imperial College London, United Kingdom

- Kozielski, Stanislaw

- Księżopolski, Bogdan

- Kucaba-Pietal, Anna, Politechnika Rzeszowska, Poland

- Lirkov, Ivan, Institute of Information and Communication Technologies, Bulgarian Academy of Sciences, Bulgaria

- Luszczek, Piotr, University of Tennessee, United States

- Marowka, Ami, Bar-Ilan University, Israel

- Petcu, Dana, West University of Timisoara, Romania

- Satco, Bianca-Renata, Stefan cel Mare University of Suceava, Romania

- Sergeichuk, Vladimir, Institute of Mathematics of NAS of Ukraine, Ukraine

- Shishkina, Olga, Max Planck Institute for Dynamics and Self-Organization, Germany

- Srinivasan, Natesan, Indian Institute of Technology, India

- Szadkowski, Zbigniew, University of Lodz, Poland

- Szajowski, Krzysztof, Institute of Mathematics and Computer Science, Poland

- Telek, Miklos

- Tudruj, Marek, Inst. of Comp. Science Polish Academy of Sciences/Polish-Japanese Institute of Information Technology, Poland

- Tůma, Miroslav, Academy of Sciences of the Czech Republic, Czech Republic

- Ustimenko, Vasyl, Marie Curie-Sklodowska University, Poland

- Vajtersic, Marian

- Vazhenin, Alexander, University of Aizu, Japan

- Wyrzykowski, Roman, Czestochowa University of Technology, Poland 



\section{Laboratory tests of two-dimensional wavelet trigger in radio detection of cosmic rays}

\author{
Zbigniew Szadkowski \\ University of Łódź \\ Department of Physics and Applied Informatics \\ ul. Pomorska 149, 90-236 Łódź, Poland \\ Email: zszadkow@kfd2.phys.uni.lodz.pl
}

\author{
Anna Szadkowska \\ Łódź University of Technology \\ Center of Mathematics and Physics \\ ul. Żeromskiego 116, 90-024 Łódź, Poland \\ Email: aszadkow@p.lodz.pl
}

\begin{abstract}
The origin of ultrahigh-energy cosmic rays (UHE$\mathrm{CRs})\left(\mathrm{E} \geq 10^{17} \mathrm{eV}\right)$ is a fundamental question of astroparticle physics. The induced shower of secondary particles in the atmosphere of the Earth provides essential information on the cosmic ray itself: arrival direction, primary energy, and mass. In the air shower many electrons and positrons form a pancakeshaped particle front with a typical thickness less than $1 \mathrm{~m}$ close to the shower axis to more than $10 \mathrm{~m}$ far from the shower axis The geomagnetic field induces a drift velocity in these particles which is perpendicular to the direction of the initial cosmic ray. The generated current is a source of coherent emission of electromagnetic waves at wavelengths larger than the size of the dimension of the charge cloud i.e., for radio frequencies in the range of 30-300 $\mathrm{MHz}$.

The radio technique allows a detail study of the electromagnetic part of an air shower in the atmosphere and provide information complementary to that obtained by surface detectors water Cherenkov tanks, which are predominantly sensitive to the muonic content of an air shower at the ground. One of the promising attempts to observe UHECRs by the detection of their coherent radio emission is a wavelet trigger based on a FPGA.

The paper presents first laboratory results from the twodimensional wavelet trigger, implemented into the prototype Front-End Board developed for the Auger surface detector based on the Cyclone ${ }^{\circledR} V$ FPGA 5CEFA9F31I7. The wavelet trigger investigates a distribution of partial power contributions for two Fourier indices, simultaneously in time and frequency domains. Preliminary results are very promising and show that the wavelet trigger could improve a radio detection system
\end{abstract}

\section{INTRODUCTION}

$\mathbf{T}$ HE Pierre Auger Observatory is the ground based detector of the ultrahigh-energy cosmic rays (UHECR) [1] It contains from three types of detectors: fluorescence (FD), surface (SD) and radio (RD). The present challenges of the RD are to understand the emission mechanisms and the features of the radio signal, and to develop an adequate measuring instrument. Electron-positron pairs generated in the shower development are separated and deflected by the Earth magnetic field, hence introducing an electromagnetic emission [2]-[4]. During shower development, charged particles are concentrated in a shower disk a few meters thick. This results in a coherent radio emission up to about $100 \mathrm{MHz}$. Short but coherent radio pulses of duration from $10 \mathrm{~ns}$ up to several $100 \mathrm{~ns}$ are generated with an electric field strength increasing approximately linearly with the energy of the primary cosmic particle inducing the extended air showers (EAS), i.e. quadratic dependence of the radio pulse energy vs. primary particle energy [5]-[9]. In contrast to the fluorescence technique with a duty cycle of about $10 \%$ (fluorescence detectors can operate only during moonless nights [10]), the radio technique allows nearly fulltime measurements and long-range observations because of the high transparency of the air to radio signals in the investigated frequency range.

One of the currently developing techniques is radio signal power estimation based on wavelet transforms, which can be an alternative proposal to the algorithms currently in operation.

The radio signal is spread over time intervals in the order of 200 nanoseconds; most registered samples have a time interval below $150 \mathrm{~ns}$. The frequency window for the maximal antenna efficiency is $\sim 30-80 \mathrm{MHz}$ [11]. This range is additionally filtered by a band-pass filter. According to Nyquist's theorem the sampling frequency should be twice as high as the maximal frequency in an investigated spectrum. The anti-aliasing filter should have the cut-off frequency of $\sim 85 \mathrm{MHz}$. Taking into account the width of the transition range for the filter (from pass-band to stop-band) the final sampling frequency should not be lower than $170 \mathrm{MHz}(200 \mathrm{MHz}$ in our test setup and measurements).

\section{WAVELETS}

The contribution of non-stationary power at many different frequencies [12] in ADC traces (a time series X, with values of $x_{n}$, at time index $\mathrm{n}$ ) is analyzed by the wavelet transform. Each ADC sample is separated in time by a constant time interval $\Delta \mathrm{t}$. The wavelet transform $W_{n}(s)$ is a convolution of the wavelet function $\psi$ with our original time series:

$$
W_{n}(s)=\sum_{m=0}^{N-1} x_{m} \psi^{*}\left[\frac{(m-n) \Delta t}{s}\right]
$$

where $\mathrm{s}$ denotes the frequency scale and the asterisk (*) complex conjugate, $\mathrm{N}$ - number of ADC samples involved in a wavelet approximation, respectively.

A convolution in time domain corresponds to a simple product of Fourier coefficients in frequency domain. Fourier coefficients for reference wavelets are pre-calculated and are implemented into the FPGA code as constants. We need only to calculate on-line N-point Fourier coefficients of ADC 


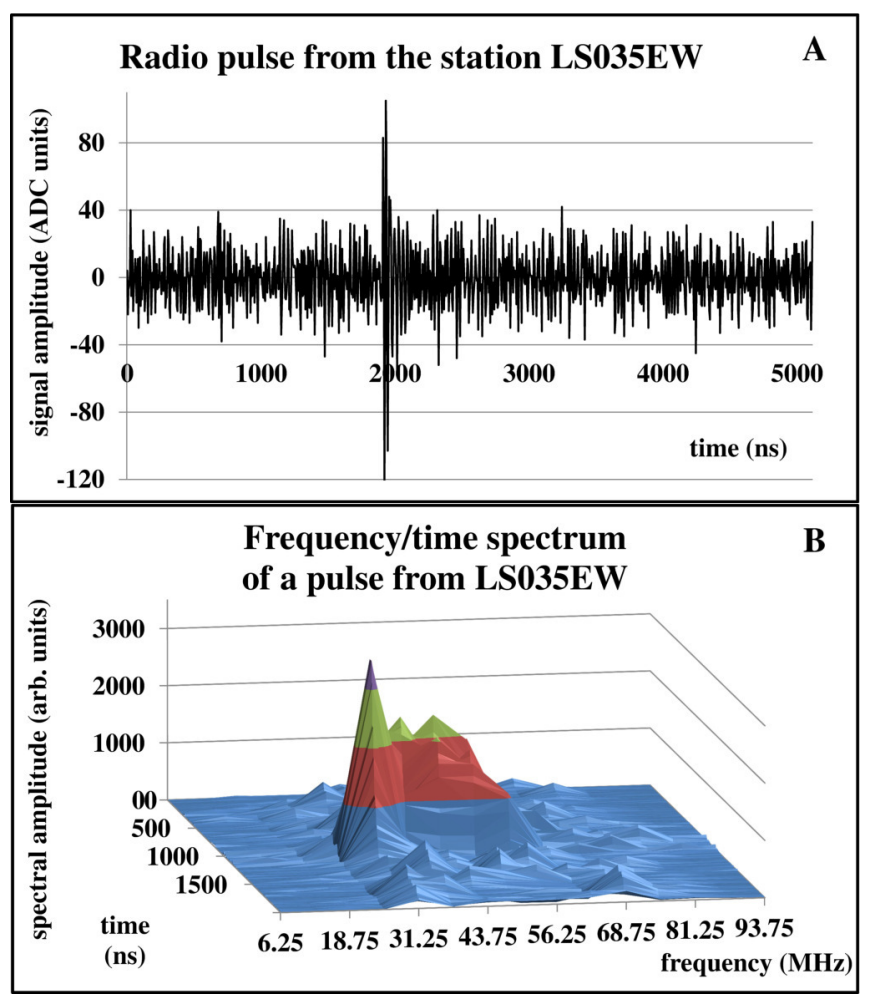

Fig. 1. Real AERA event for the radio station LS035EW

samples Selected $\mathrm{N}=32$. The FPGA routine of 32-point FFT (Fig. 1 in [13]) shows a sufficient speed for the FPGA 5CEFA9F31I7, the heart of the new Front-End Board for the prototype Auger surface detector, which contains 3 inputs for 3 PMTs from the water Cherenkov surface detector +2 inputs for the radio detector with two polarizations.

In order to get a 2-dim frequency/time distribution of a temporary signal power, the convolution (1) should be cal-

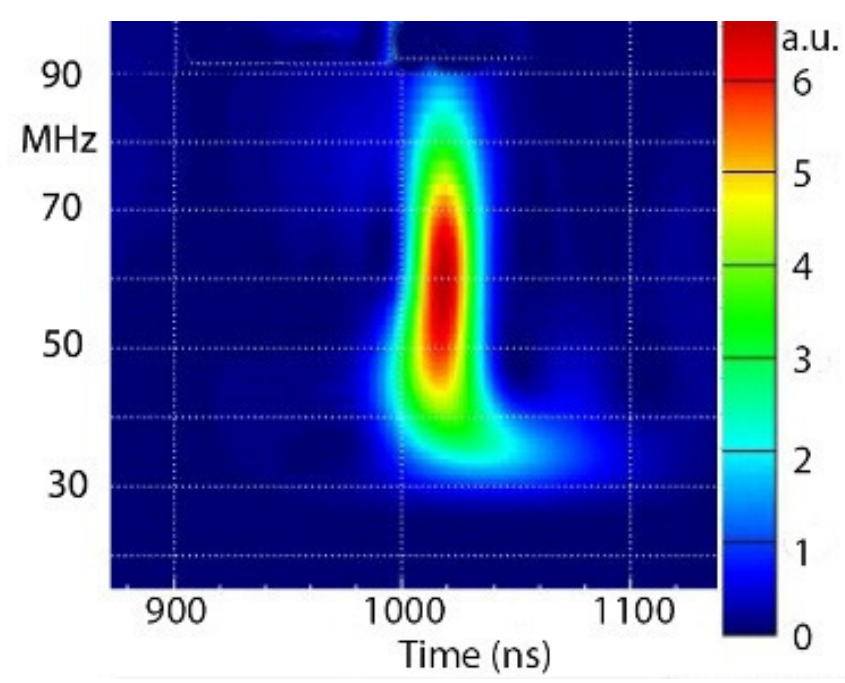

Fig. 2. High-resolution distribution of a power of a real cosmic ray event
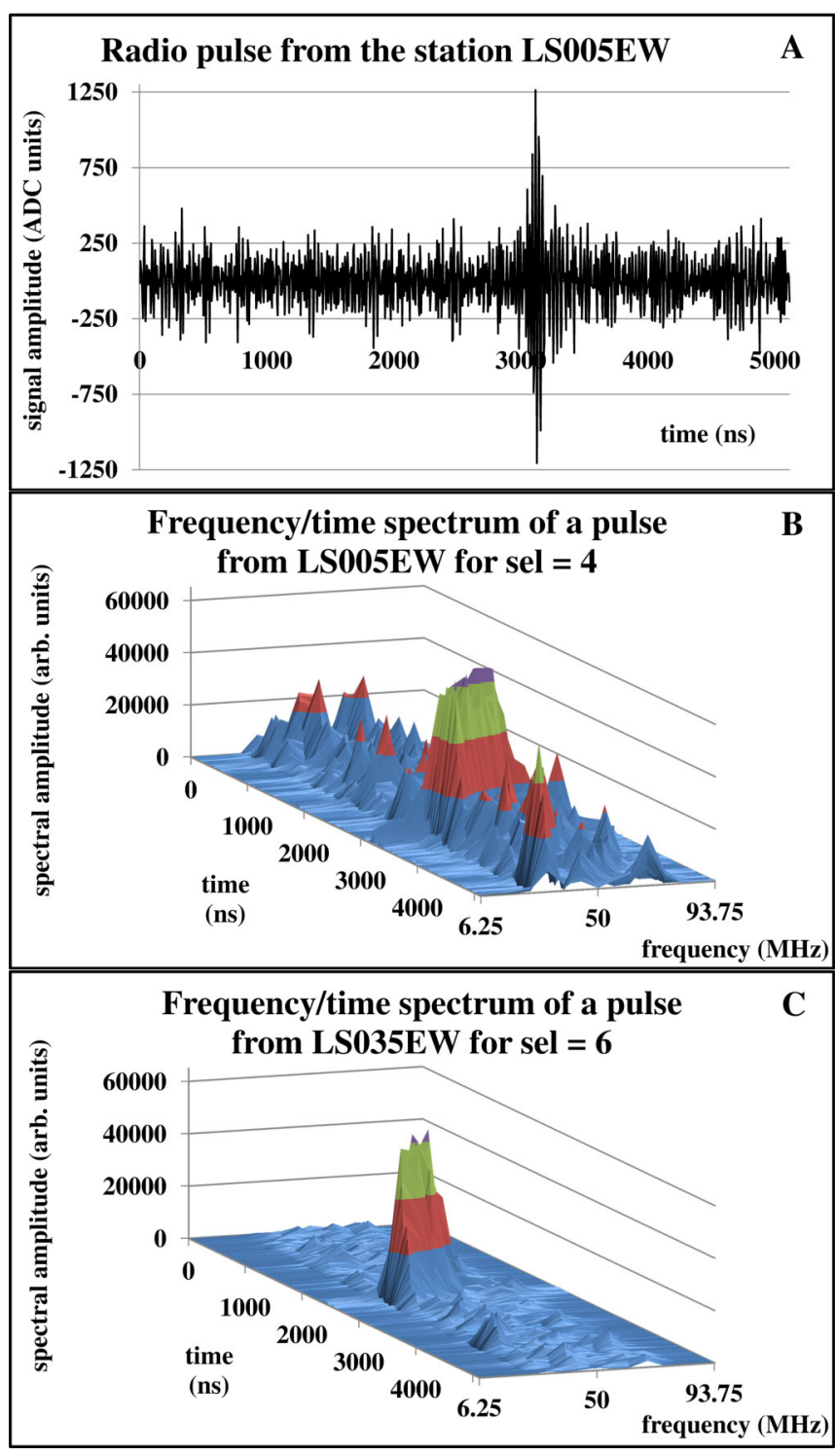

Fig. 3. Real AERA event for radio station LS005EW

culated $\mathrm{M}$ times for each scale s. M is a compromise between a frequency resolution and a resources utilization, which determines a registered performance of the FPGA implementation. The global wavelet power spectrum is defined as $\left|W_{n}(s)\right|^{2}[14]$ and the total signal power $\bar{W}^{2}$ can be expressed as follows:

$$
\sum_{j=0}^{M-1}\left|W_{j}\right|^{2}=\frac{1}{N} \sum_{j=0}^{M-1} \sum_{k=0}^{N-1}\left|\bar{X}_{k} \times \bar{\Psi}_{k, j}\right|^{2}
$$

The 32-point FFT routine gives only 16 independent FFT coefficients due to well known their symmetry. For $200 \mathrm{MHz}$ sampling, the grid is $6.25 \mathrm{MHz}$. Only 15 wavelet engines were implemented for 15 FFT real and imaginary coefficients from 6.25 to $93.75 \mathrm{MHz}$.

Several families of reference Morlet wavelets were tested for the scaling factor $s^{-1}=\alpha$, e.g. $0.04,0.01$ and 0.004 . 


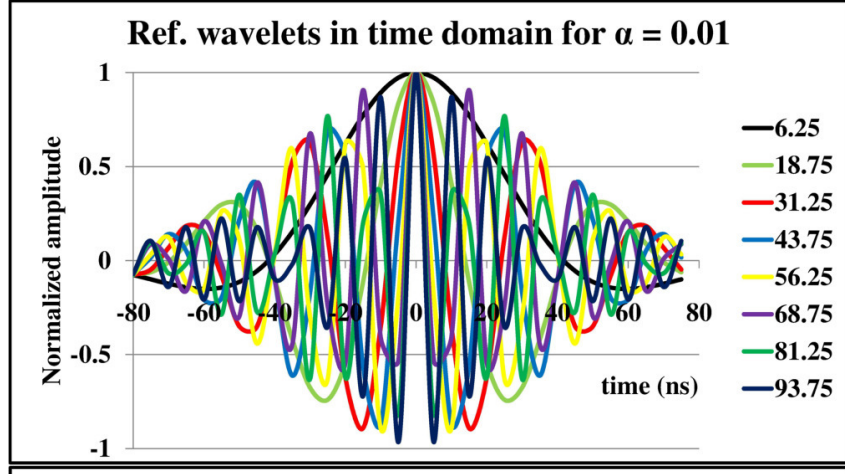

Ref. wavelets in time domain : $\alpha=0.001$

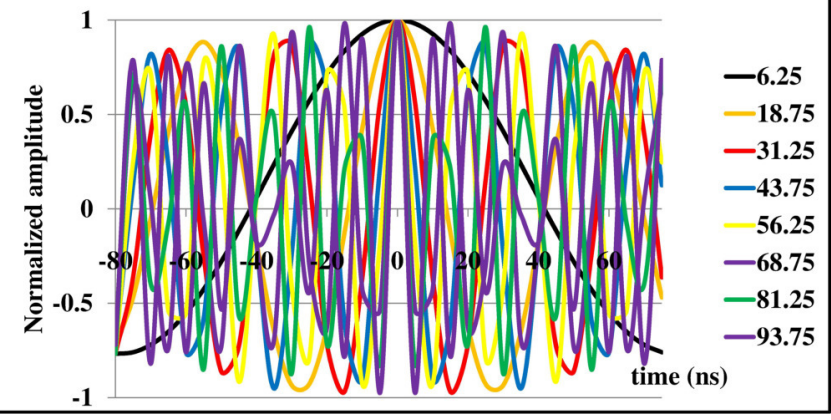

Fig. 4. The Morlet reference wavelets for 6.25-93.75 MHz investigating range for $\alpha=0.01$ and $\alpha=0.001$, respectively. Wavelets for $\alpha=0.01$ cover almost exactly the investigating time window. For $\alpha=0.001$ wavelets are far outside the time window. Practically, the time window is analyzed in an uniform way.

$$
f_{\alpha, \text { freq }}(k)=\cos \left(2 \pi k \frac{\text { freq }_{\text {wavelet }}}{\text { sampling }}\right) \exp \left(-\alpha \cdot k^{2}\right)
$$

where $-16 \leq k \leq 15$, sampling $=200(\mathrm{MHz})$.

Wavelets for $\alpha=0.04$ are squeezed to non-negligible values in a range of $-10 \leq k \leq 10$. For $\alpha=0.004$, wavelets are stretched to a larger range beyond $-16 \leq k \leq 15$. The factor $\alpha=0.01$ covers almost perfectly the time window of 32-points (Fig. 4A). The factor $\alpha=0.001$ covers the time window almost in an uniform way (Fig. 4B). I.e timing of signals does not correlate with reference wavelets, as e.g. for $\alpha=0.01$.

Fig. 5 shows a contribution to the power spectrum from neighboring wavelets due to a spectral leakage. For $\alpha=$ 0.001 the spectral leakage is almost negligible. Practically, the system will work on a base of pure Fourier coefficients. For the $\alpha=0.01$ at least two neighboring frequency bins (giving a significant contribution) have to be taken into considerations. This requires a usage of several multipliers. However, for $\alpha=$ 0.01 the spectral amplitudes of neighboring frequency bins are roughly twice lower, corresponding values are simply cropped by removing the LSB according to:

$$
W_{n}[13 . .0]=\bar{X}_{n}[13 . .0]+\bar{X}_{n-1}[13 . .1]+\bar{X}_{n+1}[13 . .1]
$$

where $W_{n}$ the power for the n-wavelet, $\bar{X}_{n}, \bar{X}_{n-1}$ and $\bar{X}_{n+1}$ modules of Fourier coefficients (corresponding to the contribution of power from wavelets: main with the index $n$

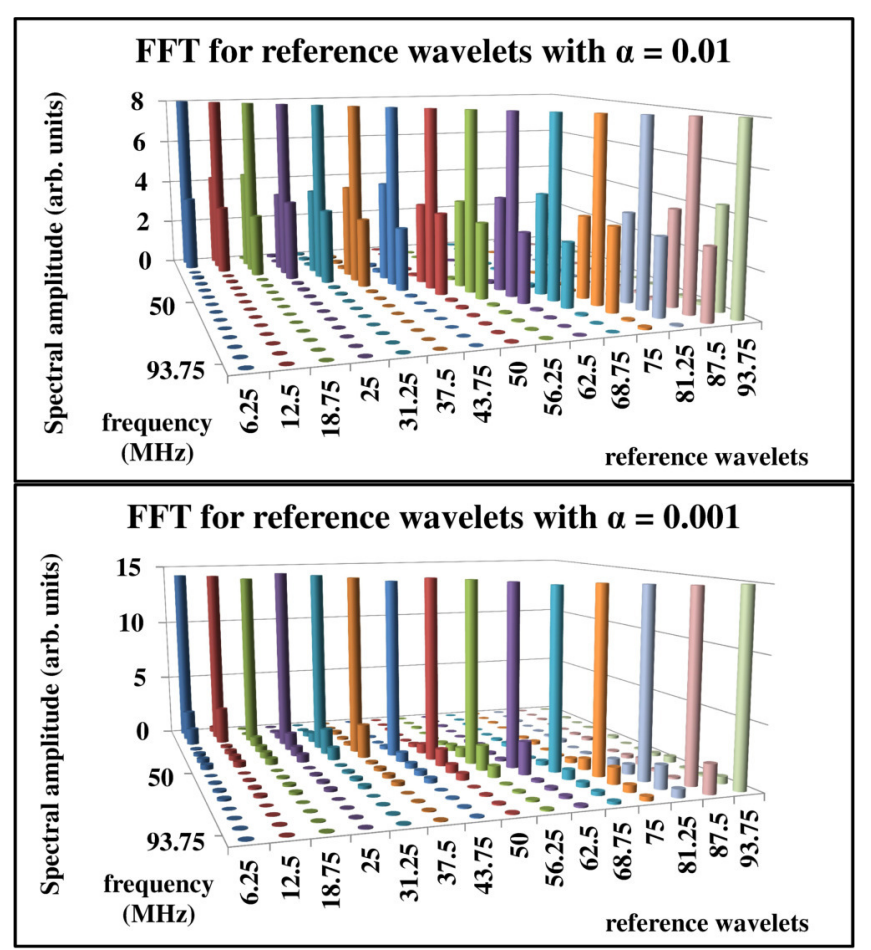

Fig. 5. The Fourier spectrum for 15 reference wavelets scaled for $\alpha=0.01$ and $\alpha=0.001$, respectively.

and neighboring with indices $n-1$ and $n+1$.). Multipliers can be replaced by the sum of the peak with two additional neighboring frequency bins. The FPGA sum routine is shown in Fig. 4. Outputs from 15 sum routines (wavelet engines) are inputs to the 2-dimensional array in Fig. 7.

\section{FPGA IMPLEMENTATION}

Modules:

$$
\left\|W_{n}\right\|=\bar{X}_{n, R e}^{2}+\bar{X}_{n, I m}^{2}
$$

are calculated by the Altera ${ }^{\circledR}$ IP routine ALTMULT_ADD. Altera ${ }^{\circledR}$ provides also a routine allowing a calculation of a square root ALTSQRT, however, the routine significantly slows down the global registered performance even for a large latency (additional pipeline stages for a speed improvement). We had to operate with $\left\|W_{n}\right\|$ modules to keep $200 \mathrm{MHz}$ global clock.

The algorithm of the wavelet trigger has been published in [13]. It has been implemented into Altera ${ }^{\circledR}$ Cyclone ${ }^{\circledR} \mathrm{V}$ E FPGA 5CEFA9F31I7, the heart of the new prototype FEB developed for the upgraded surface detector in AugerPrime [15][19]. After the conversion from the LVDS ADC outputs to a single-ended internal standard of the FPGA fabric signals are put on the wavelet engine.

15 outputs corresponding to 15 frequencies from 6.25 to 93.75 MHz with a $6.25 \mathrm{MHz}$ grid are pipelined in 15 chains of shift registers (Fig. 7). Outputs from this matrix drive comparators with fixed threshold set by the NIOS ${ }^{\circledR}$ processor $15 \times 32$ matrix of comparator outputs are next Ex-NORed with 


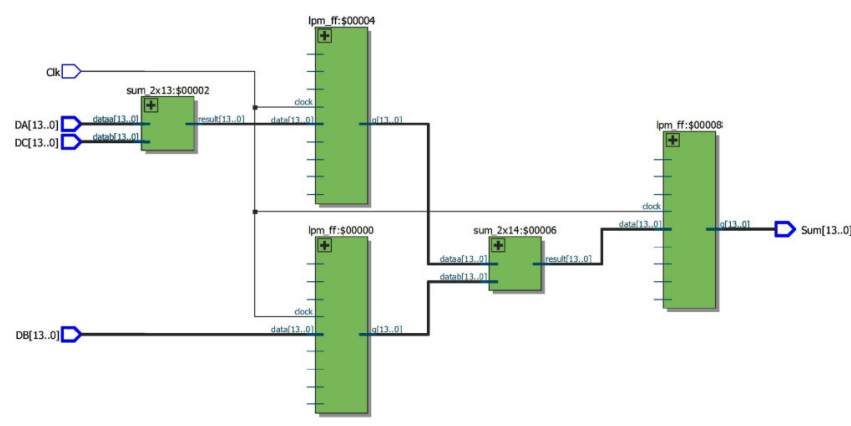

Fig. 6. The FPGA routine calculating wavelet contributions from neighboring frequency bins.

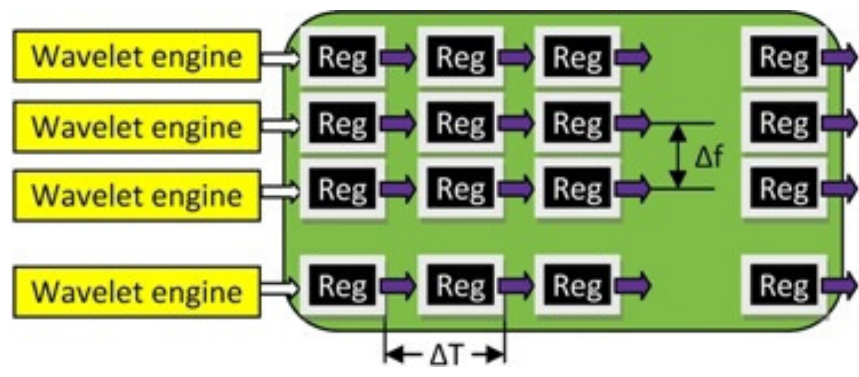

Fig. 7. The scheme of the 2-dimensional analysis. Partial power estimators calculated for reference wavelets spread in the frequency domain with the grid $\Delta \mathrm{f}$ are propagated in the registered pipelines to be available simultaneously for series time bins shifted by $\Delta \mathrm{T}$

the $15 \times 32$ matrix of patterns (Fig. 10) established by the NIOS $^{\circledR}$ processor. Positive recognized Ex-NOR conditions are summed and give 10-bit "occupancy" power estimator. A trigger is generated if the online calculated "occupancy" is above an arbitrary threshold, again set by the NIOS ${ }^{\circledR}$ processor. The same algorithm has been also implemented into the Cyclone ${ }^{\circledR}$ IV EP4CE75F29C6 FPGA. This FPGA is currently in use in the real radio stations. The resource occupancy of the wavelet engine for EP4CE75F29C6 is on the same level as for 5CEFA9F31I7, both for the "pure" wavelet engine and the trigger with the NIOS. For a full configuration the resource occupancy is relatively high, however, the register performance is still above $200 \mathrm{MHz}$ with a sufficient safety margin which allows an operation of the algorithm simultaneously with the standard code. The FPGA used in Dutch radio stations is

TABLE I

RESOURCES OCCUPATION OF WAVELET ENGINE FOR THE FPGA CURRENTLY IN USE : CYCLONE IV E EP4CE75F29C6 AND THE FPGA IN THE PROTOTYPE FRONT-END BOARD : 5CEFA9F31I7 FROM CYCLONE V E FAMILY

\begin{tabular}{|c||c|c|}
\hline FPFA & EP4CE75F29C6 & 5CEFA9F31I7 \\
\hline logic elements & $21737 / 75408(29 \%)$ & \\
\hline ALMs & & $11488 / 113560(10 \%)$ \\
\hline Total registers & 16195 & 20499 \\
\hline 9-bit multipliers & $162 / 400(42 \%)$ & $54 / 342(16 \%)$ \\
\hline DSP blocks & & $2541 \mathrm{k} / 12220 \mathrm{k}(21 \%)$ \\
\hline Memory bits & $2038 \mathrm{k} / 2745 \mathrm{k}(74 \%)$ & \\
\hline
\end{tabular}
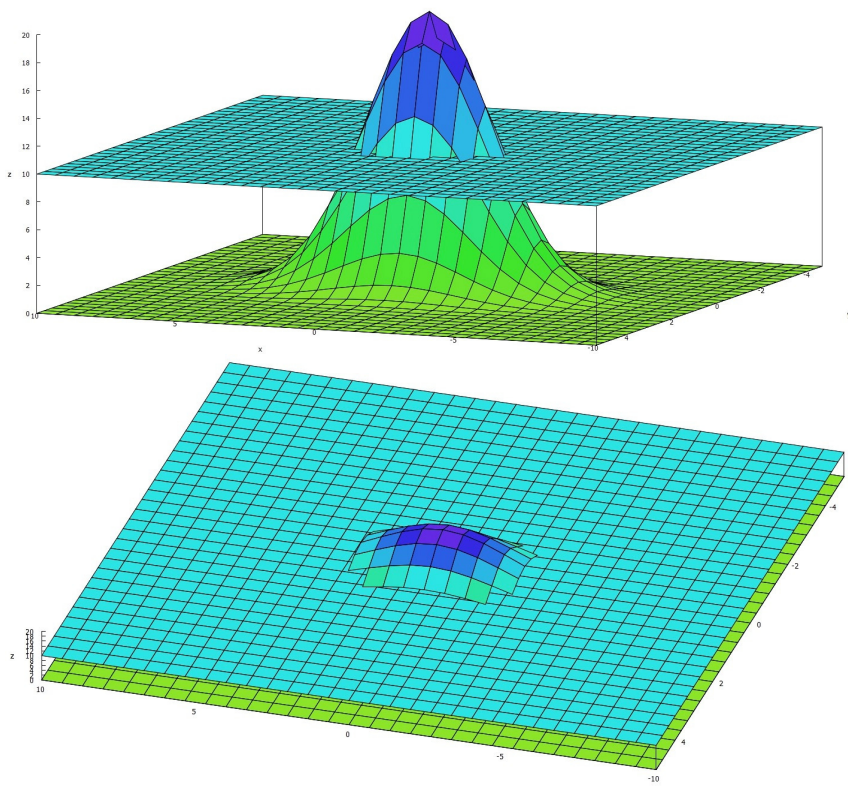

Fig. 8. Illustration of a calculation of power over the threshold. Here, the threshold $=10$ (arb. units) while signal power corresponds to the twodimensional "Gauss bell". The upper graph shows two-dimensional distribution of signal power with a surface cutting the "bell" on the threshold level The lower graph shows the part above the threshold interesting for the trigger.

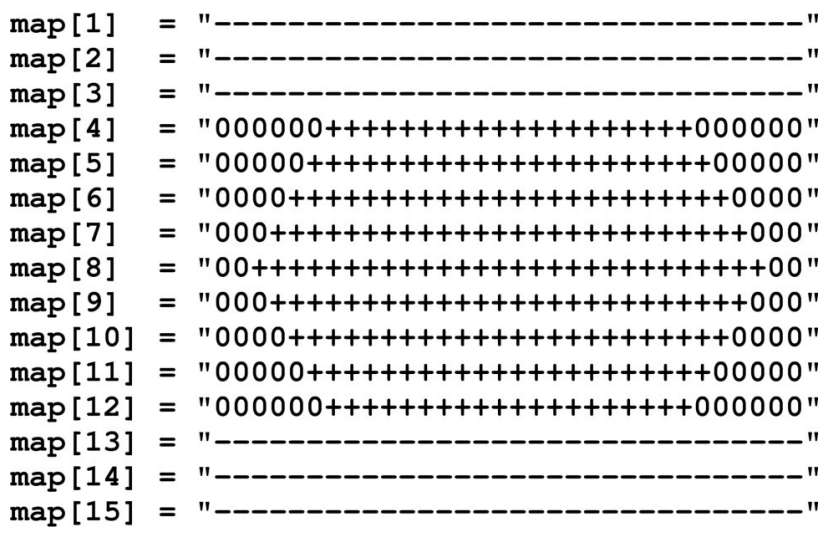

Fig. 10. An example of expected pattern. map[i] correspond to frequencies from 6.25 to $93.75 \mathrm{MHz}$ range $(\mathrm{i}=1, \ldots, 15)$ with $6.25 \mathrm{MHz}$ grid. The accepted frequency range for a signal detection is within $25-75 \mathrm{MHz}$ (map[4] $\operatorname{map}[12])$.

EP4C75F29C6 from Cyclone ${ }^{\circledR}$ IV E Altera family. This chip is large enough the wavelet trigger to be implemented and tested in real environment of Argentinean pampas.

\section{TWO DIMENSIONAL TRIGGER}

Wavelet power coefficients are propagated in the set of shift registers creating a two-dimensional matrix with a power distribution (Fig. 7). A set of comparators detect the active areas of power vs time and frequencies to estimate a probability of an existence of real signal. Fig. 8 shows a principle of a detection of an example signal. The two-dimensional set of registers (Fig. 7) creates a two-dimensional distribution of 

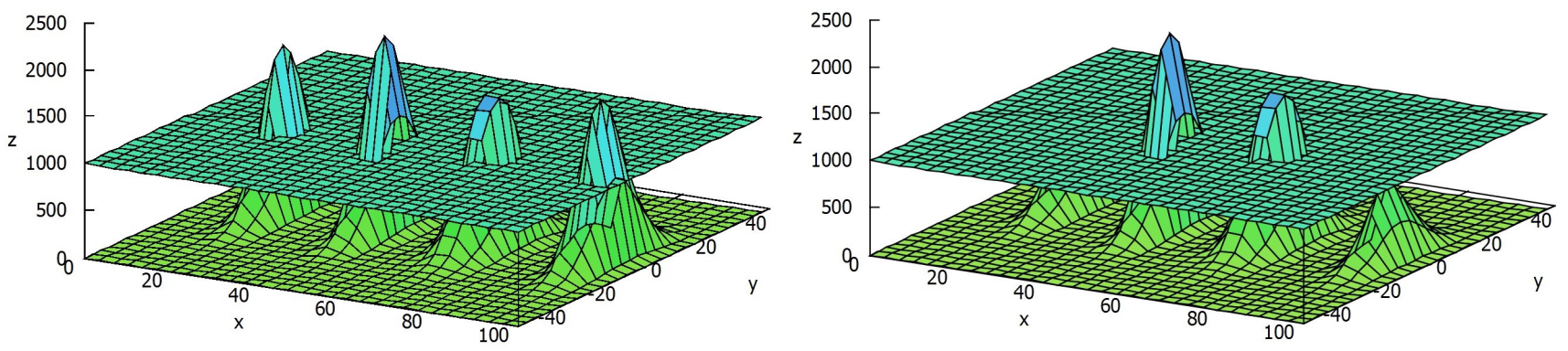

Fig. 9. 3D-plots generated by MAXIMA for arbitrarily assumed frequency spectrum of artificially generated pulse by iFFT with 4 peaks above the threshold (corresponding to $17.12,40.9,65.2$ and $90.7 \mathrm{MHz}$, respectively, left graph) and for with only 2 peaks (corresponding to 40.9 and $65.2 \mathrm{MHz}$, respectively, right graph). The axis $x$ corresponds to the frequency range from 0 to $100 \mathrm{MHz}$, the axis $y$ denotes time evolution, the axis $z$ is in arbitrarily units corresponding to $(6)$.

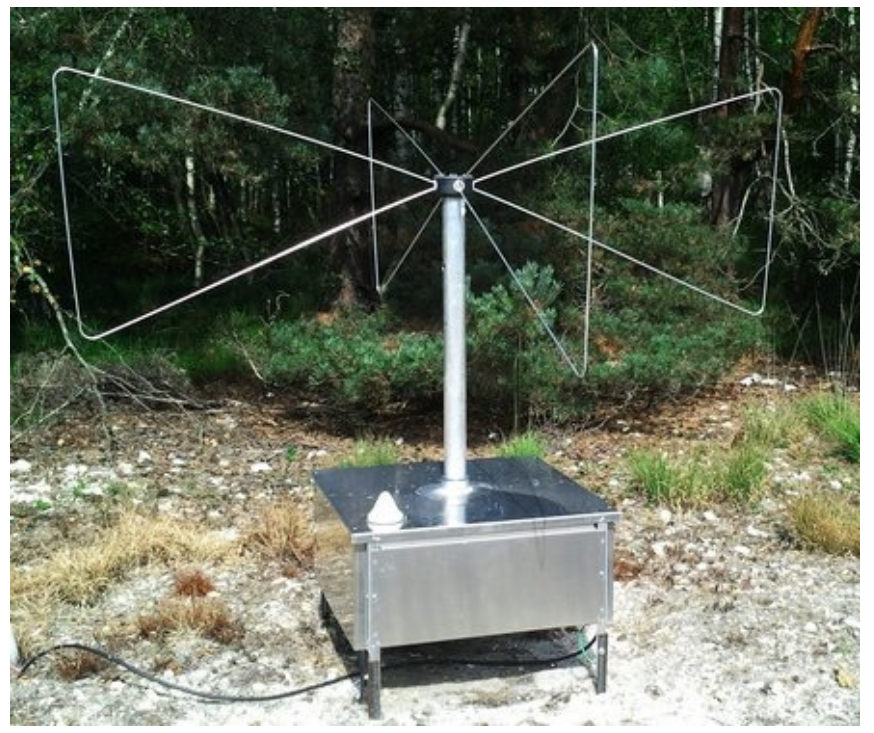

Fig. 11. The Pierre Auger radio station [18]

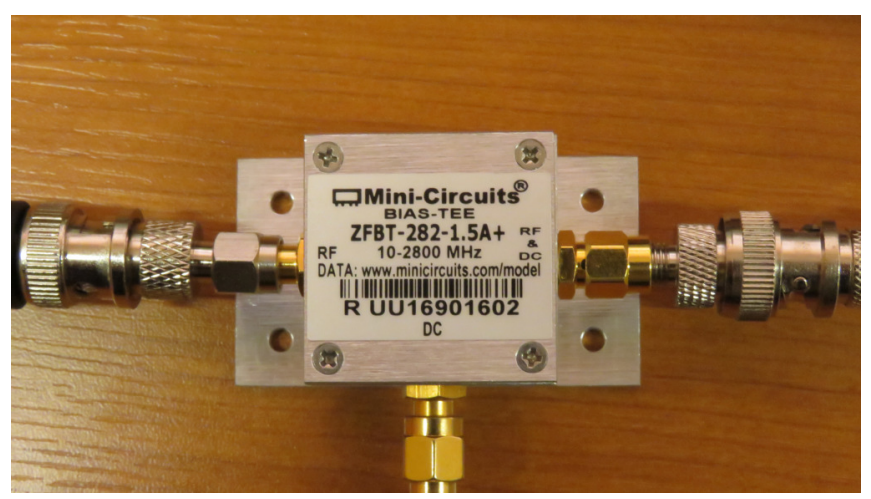

Fig. 12. Circuit which allows driving analog ASIC via antenna cable.

signal power simultaneously for time and frequency. The flat surface in Fig. 8 corresponds to a threshold, which cuts-off a part of distribution lying above the surface. These parts create flat distributions of active power regions.

The power distributions are next compared with an expected

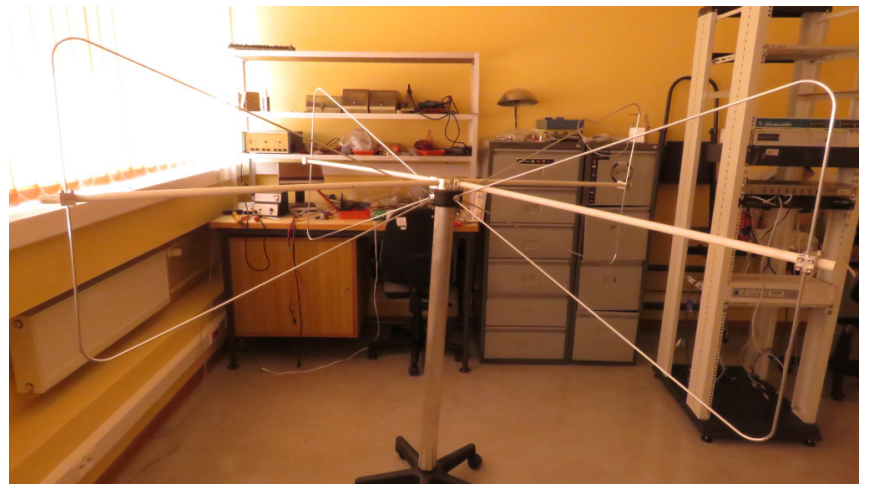

Fig. 13. AERA antenna in the Łódź laboratory for test of various RFI filters and wavelet trigger

patterns generated by the NIOS ${ }^{\circledR}$ processor, which transfers the pattern to the FPGA fabric to some dedicated registers. Comparator results are next Ex-NORed with the expected pattern (an example shown in Fig. 10). Ex-NORed 1-bit signals are next summed to get an "occupancy" in a power space. If the "occupancy" is greater than an arbitrary value (also established by the NIOS $^{\circledR}$ processor), the final trigger is generated. Internal frequencies (marked as "+") give a positive contribution, but peripheral ones (marked as "--") give negative contribution. Zeros limit the time range to 28 time bins.

\section{AdVANCED TESTS}

For tests, a pulse (Fig. 17A) with the following frequency spectrum (Fig. 9 - simulated by MAXIMA and Fig. 18B measured in the FEB) was generated

$$
f(t)=\sum_{k=0}^{3} A_{j} * \exp ^{-\frac{\left(\left(t-f_{k}\right)^{2}\right)}{\left(2 * \sigma_{k}^{2}\right)}}
$$

where amplitudes: $A_{0}=1700, A_{1}=2213, A_{2}=1500, A_{3}=$ 1800 (in ADC units), frequencies: $f_{0}=17.12, f_{1}=40.9, f_{2}$ $=65.2, f_{3}=90.7$ (in $\mathrm{MHz}$ ) and variances: $\sigma_{0}^{2}=862, \sigma_{1}^{2}=$ 662, $\sigma_{2}^{2}=1262, \sigma_{3}^{2}=762$, respectively (Fig. 9).

According to our expectation the spectrum contains two peaks for very low $(\sim 18.25 \mathrm{MHz})$ and very high $(\sim 93.75$ $\mathrm{MHz}$ ) frequencies, which are out of range for a standard 

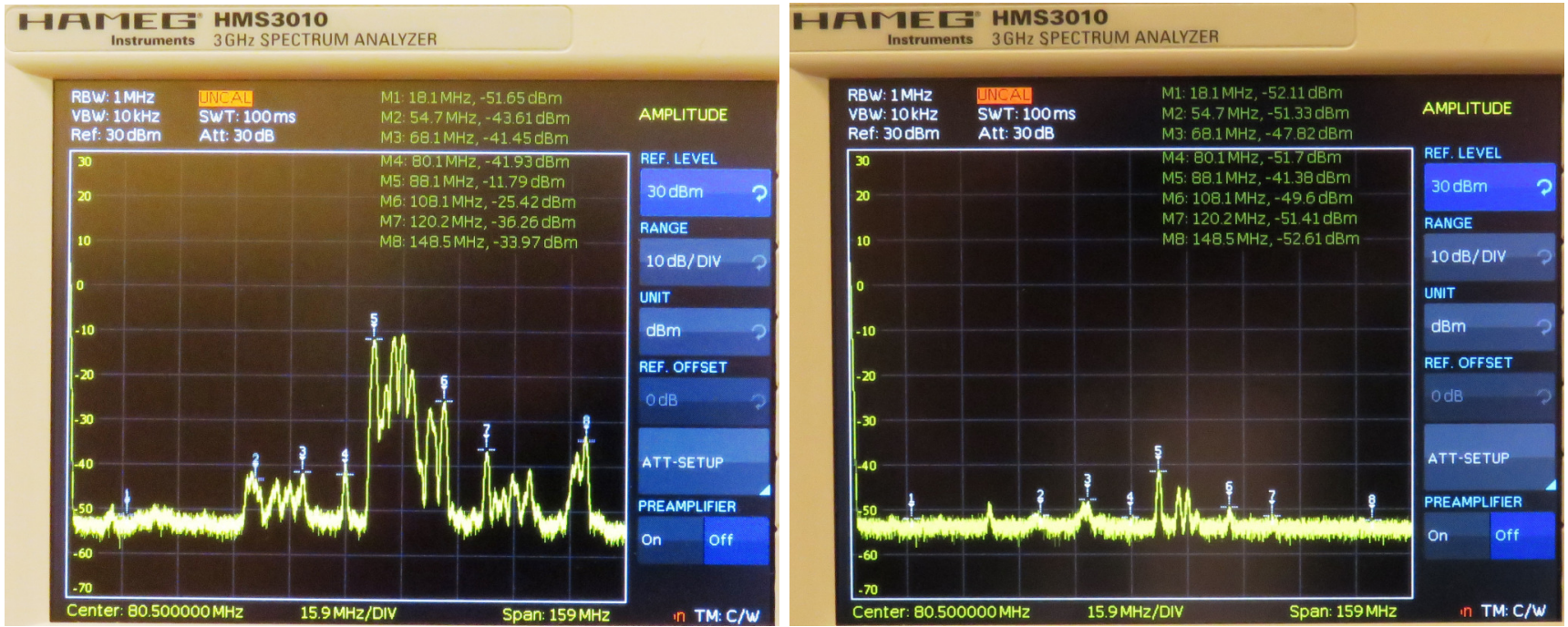

Fig. 14. Spectra of radio environment in Łódź direct from the antenna (left) and after low-pass Chebyshev analog filter (right)
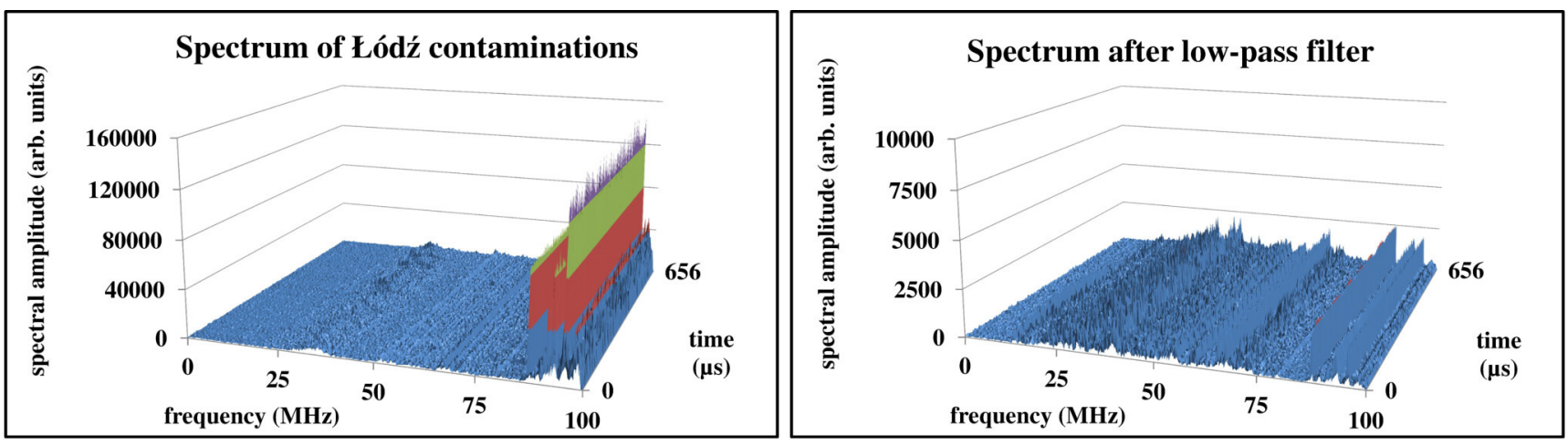

Fig. 15. Radio spectrum without (left) and with the filter (right) in the frequency range of 0-100 MHz. The sampling frequency equals $200 \mathrm{MHz}$.

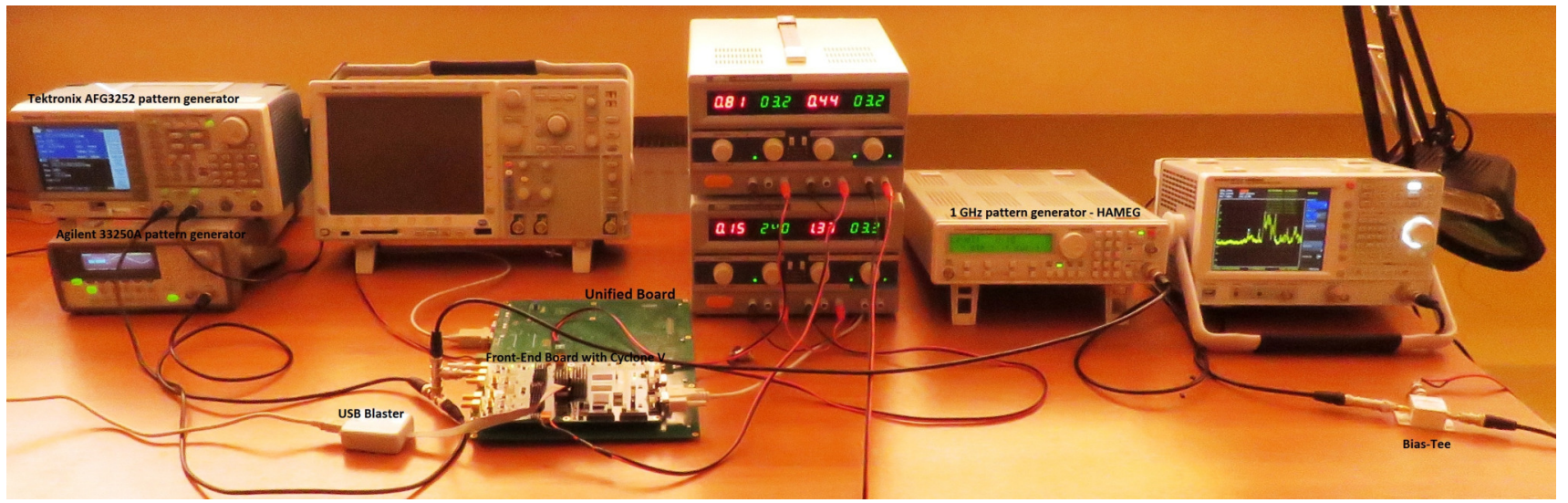

Fig. 16. Mesurement setup. Agilent 33250A, Tektronix AFG3252 and HAMEG pattern generators drive (as Wired-OR) the Front-End Board (FEB) with Cyclone ${ }^{\circledR}$ V FPGA 5CEFA9F32I7 as daughter board of the Unified Board (with micro-controller for a management of the Pierre Auger surface detector). The FEB is a prototype board for the surface detector but equipped with two radio channels with $200 \mathrm{MHz}$ sampling allowing a DAQ for AERA. The radio channel is driven either from from the pattern generators or from the AERA antenna via Bias-Tee.

AERA frequency characteristics due to the analog band-pass filter with 30-80 MHz cut-off. Fig. 17C shows that these peaks give a significant contribution to a total power. A task of Ex-NOR filtering is an elimination of pulses with spurious 

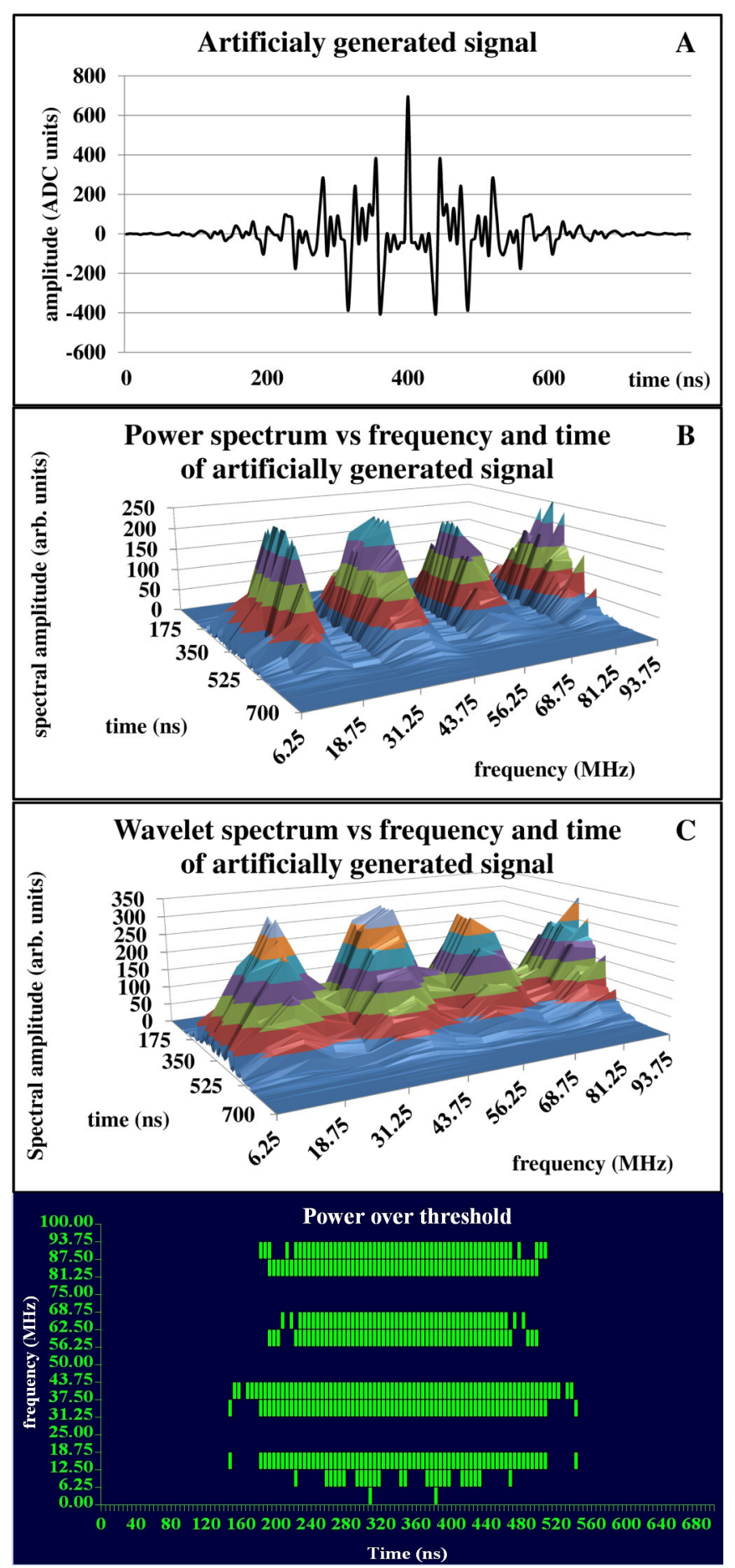

Fig. 17. Artificially generated pulse (A), its pure time/frequency spectrum without the wavelet scaling (B), the wavelet taking into account $(\mathrm{C})$ and power over threshold. Measured in the FPGA internal peaks $(B, C)$ and active regions (green regions in bottom graph) correspond to correct frequency range. Peripheral peaks correspond to spurious frequency leafs, which should not appear in real radio signals coming from cosmic rays (red regions). Amplitudes of peaks in graph B are reduced in comparison to Fig. 9. Taking into account wavelet coefficients to the power spectrum according to (4) peaks corresponding to the signals are soften with higher amplitude.

C
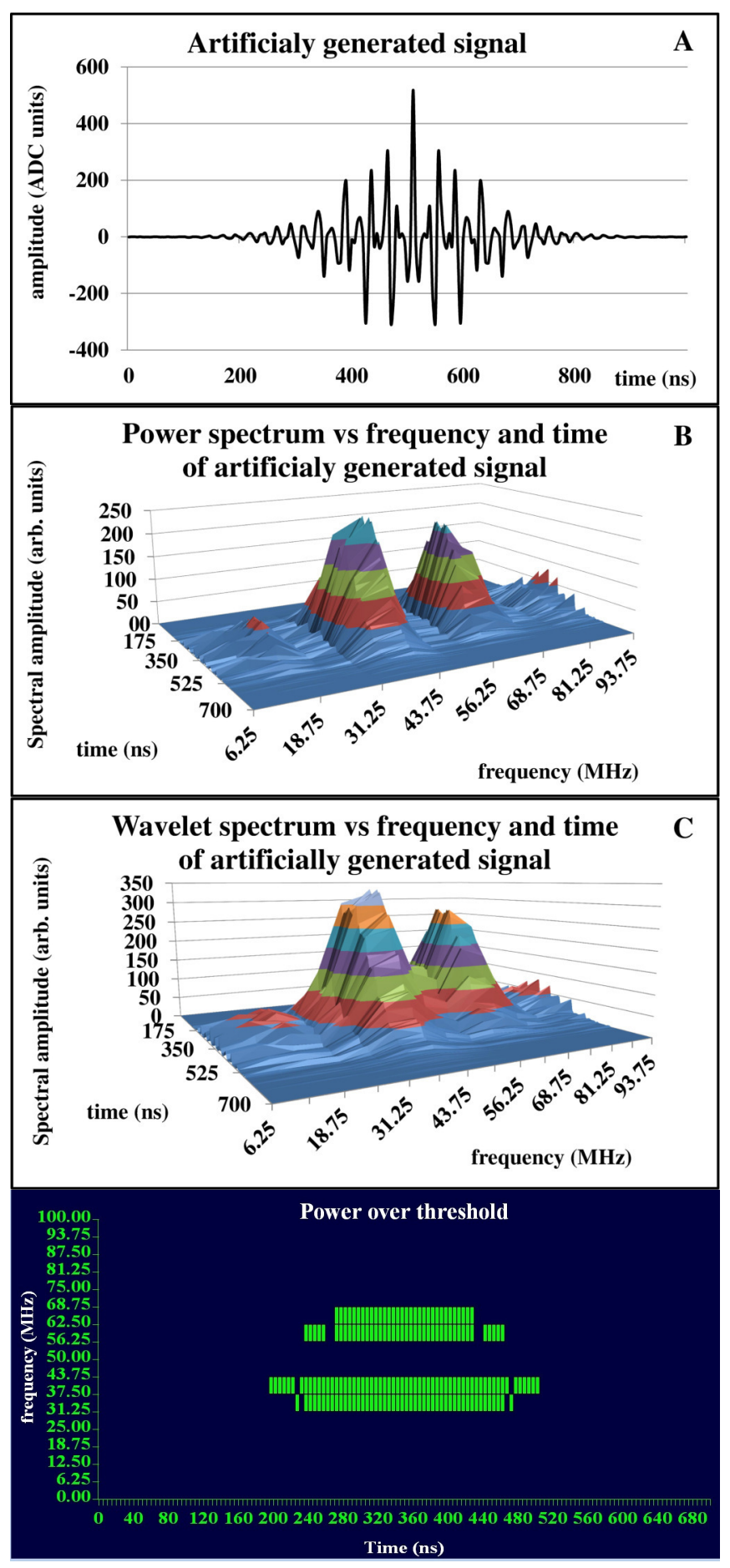

Fig. 18. Artificially generated pulse (A) with two peaks only. Description of the graph the same as for Fig. 17. Although the amplitudes $A_{0}$ and $A_{3}$ were reduced only twice (Fig. 9 - right graph), a selection of most significant bits by the NIOS almost eliminates peripheral contribution (graphs B and C). Only the green region appears in the bottom graph. The graph B shows the pure Fourier spectrum, the graph $\mathrm{C}$ with wavelet modification. The contribution of wavelets to the pure Fourier spectrum makes graphs soften, but peaks are amplified. 


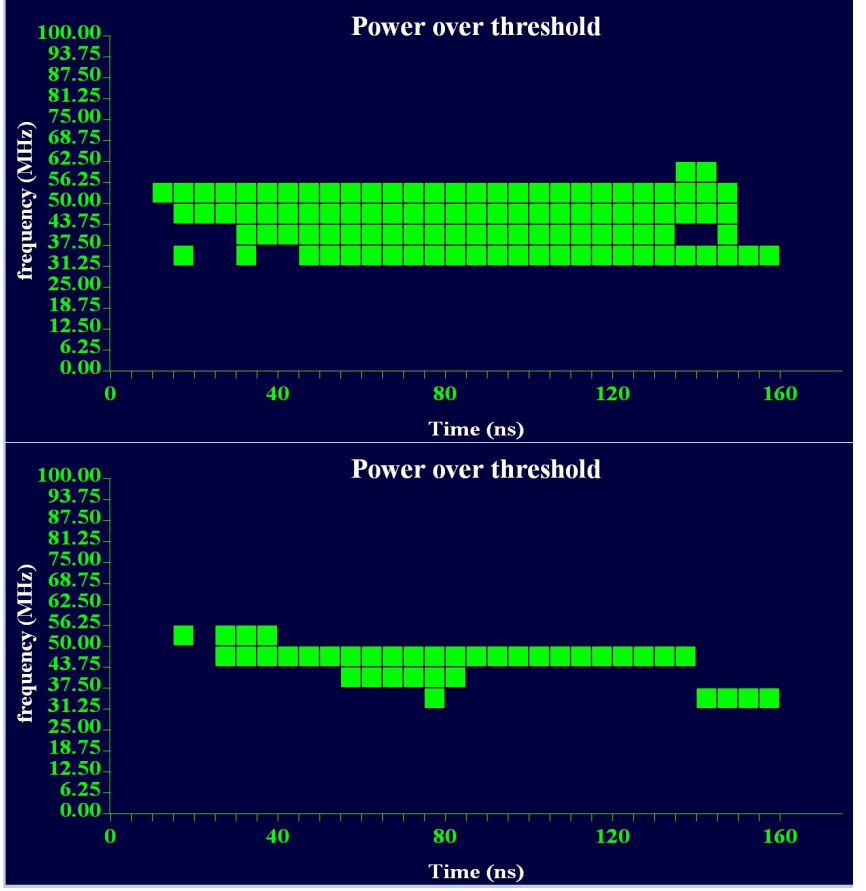

Fig. 19. Graphs presenting the power over threshold after the wavelet trigger Patterns from radio station LS035EW (upper) and LS005EW (lower) stored in the $256 \mathrm{kS}$ Agilent ROM are sent to the air via quarter-length dipole and received by the antenna.

frequency characteristics like in Fig. 17C.

Peripheral contributions are marked by the Ex-NOR filter as negative and they are subtracted from a positive contribution of internal peaks. Spurious regions are denoted in Fig. 17C by the red color. Because a contribution of peripheral (spurious) peaks is on the same level as internal one, the total power is negligible and this event is classified to be rejected.

However, if amplitudes $A_{0}=850$ and $A_{3}=900$ (in ADC units), respectively, the contribution of peripheral "bells" are much less and the peaks are below the threshold. Although there is some spurious contribution from peripherals, a final power is positive and such an event will be classified as potentially recognized cosmic-ray radio signal (Fig. 18).

\section{PRELIMINARY LABORATORY MEASUREMENTS}

Burst pulses from Tektronix AFG3252C arbitrary pattern generator have driven the radio channel in the prototype Front End Board (FEB) developed for the upgraded surface detector in the Pierre Auger Observatory [15][19]. The FEB is equipped with the biggest FPGA 5CEFA9F31I7 from the Cyclone ${ }^{\circledR} \mathrm{V}$ E family.

Signals from various radio stations may have significantly different amplitudes (compare Fig. 1A and 3A). The trigger algorithm has to take into account a wide amplitude range. $\left\|W_{n}\right\|$ modules outputs are put on a multiplexer dynamically selecting an optimal dynamic range. The multiplexer is controlled by the NIOS ${ }^{\circledR}$ processor. The $\bar{X}_{R e, I m}$ are 21-bit (seven pipeline stages for 32-point FFT) for 14-bit input ADC data $x_{n}$. To keep a maximal accuracy, intermediate pipeline results are not cropped. However, the product (5) for very wide inputs would be too wide and too sophisticated for an analysis. $\bar{X}_{R e, I m}$ are cropped to 16-bit range only (5 LSBs are neglected). The obtained $\left\|W_{n}\right\|$ is 32 bits. For an analysis, 16-bit window is selected by the NIOS ${ }^{\circledR}$ processor.

Fig. 3B shows a non-optimal selection (sel $=4$ as for LS005). Power peaks descended from noise are well visible. However, if an analysis window is shifted 2 bits higher, a frequency/time spectrum shows a pure peak descended from a real radio signal. The NIOS $^{\circledR}$ processor periodically analyses a level of ADC noise and signal amplitudes and adjusts the multiplexer address for an optimal dynamic range (not to crop a signal and not to leave unused bits).

\section{Measurements with the AERA antenna}

The wavelet trigger was tested in the Łódź laboratory. Preamplifier as analog ASIC provided by SUBATECH was supplied from the antenna cable, via Bias-Tee ZFBT-282-1.5A from Mini-Circuit (Fig. 12). The Łódź radio environment is significantly contaminated by the high-power FM transmitters in the frequency range of $88-108 \mathrm{MHz}$ (Fig. 14 left). We used the Chebyshev low-pass filter to suppress significantly the frequency range above the Nyquist cut-off limit. Both HAMEG spectrum analyzer (Fig. 14 right) and the data analyzed by the Fron-End Board (Fig. 15 right) show that a contamination level coming from FM transmitter is now at the acceptable low level.

The pulses from Agilent arbitrary pattern generator (33250A) were being sent to the air by the quarter-length dipole and received by the butterfly antenna.

At first we tested the system by the artificial pulses with several peaks in the frequency spectrum (Fig. 17 and Fig. 18). The artificial pulses are much longer than the cosmic ray ones. Tests with real AERA pulses stored at first as patterns in the Agilent arbitrary pattern generator and next sent to the air by the quarter-length dipol and received by the butterfly antenna gave exactly the same distributions (Fig. 1B and Fig. 3C) as by direct driving from the FPGA ROM.

\section{CONCLUSION}

Laboratory tests show that the wavelet engine with the NIOS $^{\circledR}$ processor and set of registers, comparators and adders still provides a sufficient registered performance higher than $200 \mathrm{MHz}$ for Cyclone ${ }^{\circledR}$ IV E (EP4CE75F29C6) and Cyclone ${ }^{\circledR}$ V E (5CEFA9F31I7) FPGAs. Trigger operates according to our expectations.

We realize that the wavelet trigger utilizes a significant percent of FPGAs resources. Nevertheless, it is worth testing the algorithm in real radio stations in Argentinean pampas [20] using the Front-End Boards equipped with the standard AERA radio FEBs with EP4CE75F29C6 as a potential improvement of the AERA trigger for the next generation electronics. 


\section{ACKNOWLEDGMENT}

This work is supported by the National Science Centre (Poland) under NCN Grant HARMONIA-4 No. 2013/08/M/ST9/00322 and NCN Grant HARMONIA-8 No. 2016/22/M/ST9/00198

The author would like to thank the Aachen University for providing the AERA antenna and SUBATECH for the lownoise amplifier.

\section{REFERENCES}

[1] Pierre Auger Collaboration, A. Aab et al., The Pierre Auger Cosmic Ray Observatory, Nucl. Instrum. Meth. A 798 (2015) 172, DOI: 10.1016/j.nima.2015.06.058

[2] H.R. Allan, Radio Emission From Extensive Air Showers, Progress in Elementary Particle and Cosmic Ray Physics, vol. 10 (1971) 171.

[3] H. Falcke, P.W. Gorham, Detecting radio emission from cosmic ray air showers and neutrinos with a digital radio telescope, Astropart. Phys. 19 (2003) 477, DOI: 10.1016/S0927-6505(02)00245-1

[4] Pierre Auger Collaboration, A. Aab et al., Muons in air showers at the Pierre Auger Observatory: Measurement of atmospheric production depth, Phys. Rev. D90 Issue 1 (2014) 012012, DOI: 10.1103/PhysRevD.90.012012

[5] Pierre Auger Collaboration, S. Acounis et al., Results of a self-triggered prototype system at the Pierre Auger Observatory for radio-detection of air showers induced by cosmic rays, JINST 7 (2012) P11023, DOI: 10.1088/1748-0221/7/11/P11023

[6] Pierre Auger Collaboration, A. Aab et al., Probing the radio emission from air showers with polarization measurements, Phys. Rev. D89 (2014) 052002, DOI: 10.1103/PhysRevD.89.052002

[7] S. Fliescher for the Pierre Auger Collaboration, Radio detection of cosmic ray induced air showers at the Pierre Auger Observatory, Nucl. Instrum. Meth. A662 (2012) S124, DOI: 10.1016/j.nima.2010.11.045

[8] R. Dallier for the Pierre Auger Collaboration, Measuring cosmic ray radio signals at the Pierre Auger Observatory, Nucl. Instrum. Meth. A630 (2011) 218, DOI: 10.1016/j.nima.2010.06.069
[9] T. Huege for the Pierre Auger Collaboration, Radio detection of cosmic rays in the Pierre Auger Observatory, Nucl. Instrum. Meth. A617 (2010) 484, DOI: 10.1016/j.nima.2009.10.012

[10] Pierre Auger Collaboration, J. Abraham et al., The fluorescence detector of the Pierre Auger Observatory, Nucl. Instrum. Meth. A620 (2010) 227, DOI: 10.1016/j.nima.2010.04.023

[11] Pierre Auger Collaboration, P. Abreu et al., Antennas for the Detection of Radio Emission Pulses from Cosmic-Ray induced Air Showers at the Pierre Auger Observatory, JINST 7 (2012) P11011, DOI: 10.1088/17480221/7/10/P10011

[12] I. Daubechies, The wavelet transform time-frequency localization and signal analysis, IEEE Trans. Inform. Theory 36 (1990) 961.

[13] Z. Szadkowski, An Optimization of the FPGA Based Wavelet Trigger in Radio Detection of Cosmic Rays, IEEE Trans. on Nucl. Science 62 Issue 3 (2015) 993, DOI: 10.1109/TNS.2015.2422713

[14] C. Torrence, G.P. Compo, A Practical Guide to Wavelet Analysis, Bulletin of the American Meteorological Society 79 (1998) 61.

[15] Z. Szadkowski, Front-End Board with Cyclone $V$ as a Test HighResolution Platform for the Auger Beyond 2015 Front End Electronics, IEEE Trans. on Nucl. Science 62 Issue 3 (2015) 985, DOI: 10.1109/TNS.2015.2426059

[16] Z. Szadkowski, A. Szadkowska, FPGA Based Wavelet Trigger in Radio Detection of Cosmic Rays, Braz. J Phys 44 (2014) 805, DOI: 10.1007/s13538-014-0243-5

[17] Z. Szadkowski, 16-point discrete Fourier transform based on the Radix-2 FFT algorithm implemented into cyclone FPGA as the UHECR trigger for horizontal air showers in the Pierre Auger Observatory, Nucl. Instrum. Meth. A 560, Issue 2 (2006) 309, DOI: 10.1016/j.nima.2006.01.045

[18] Pierre Auger Collaboration,, P. Abreu et al., Antennas for the detection of radio emission pulses from cosmic-ray induced air showers at the Pierre Auger Observatory, JINST 7 (2012) P10011.

[19] Z. Szadkowski, First results of High-Resolution Front End Electronics for Water Cherenkov Air Shower Detectors equipped with Cyclone V FPGA, IEEE Trans. on Nucl. Science 63 Issue 3 (2016) 1446, DOI: 10.1109/TNS.2016.2567320

[20] Pierre Auger Collaboration, J. Abraham et al., Atmospheric effects on extensive air showers observed with the surface detector of the Pierre Auger Observatory, Astropart. Phys. 32 (2009) 88, DOI: 10.1016/j.astropartphys.2009.06.004 



\section{$4^{\text {th }}$ International Conference on Cryptography and Security Systems}

C

RYPTOGRAPHY and security systems are two fields of security research that strongly interact and complement each other. The International Conference on Cryptography and Security Systems (CSS) is a forum of presentation of theoretical, applied research papers, case studies, implementation experiences as well as work-in-progress results in these two disciplines.

\section{TOPICS}

The main topics of interests include:

- network security

- cryptography and data protection

- peer-to-peer security

- security of wireless sensor networks

- security of cyber physical systems

- security of Internet of Things solutions

- heterogeneous networks security
- privacy-enhancing methods

- covert channels

- steganography and watermarking for security applications

- cryptographic protocols

- security as quality of service, quality of protection

- data and application security, software security

- security models, evaluation, and verification

- formal methods in security

- trust and reputation models

- reputation systems for security applications

- intrusion tolerance

- system surveillance and enhanced security

- cybercrime: threats and countermeasures

- 5G Security

- DDoS attacks: detection and mitigation

- Security of Smart Grid systems 



\title{
Trustworthiness 5G Enabler
}

\author{
Rafał Artych, Krzysztof Bocianiak, Tomasz Ośko \\ Orange Polska S.A. \\ R\&D Center \\ ul. Obrzeżna 7, 02-691 Warszawa, Poland \\ Email: $\{$ rafal.artych, krzysztof.bocianiak, tomasz.osko\}@orange.com
}

\begin{abstract}
This paper introduces security enabler for 5G networks that can be applied to improve network's own security and utilized by network users in their own service protection methods. Its main role is to process technical information present in the network in order to provide trustworthiness information that can facilitate necessary trust decisions. Network Provided Trustworthiness system matching 5G architecture is proposed to provide this functionality.
\end{abstract}

\section{INTRODUCTION}

$\sum^{\mathrm{G}}$ $\mathrm{G}$ is the proposed next generation of network technology envisaging a wide variety of actors and device types, more use of the cloud and virtualization techniques and built-in security and privacy protection [1][2][3]. In this paper the idea of using network information in managing trust relations within the $5 \mathrm{G}$ network and services offered over the network is discussed. This idea follows popular network assets exposition principle while retaining security and privacy protection requirements.

Among multiple novel technological approaches (needed for variety of services e.g. enhanced Mobile Broadband (eMBB), Ultra-Reliable Low Latency Communications (URLLC), massive Machine Type Communication (mMTC)) 5G envisages delegation of access control to 3rd party, new authentication methods and security enablers used on demand. The security enablers are expected to protect subscribers, devices and their communications but also the integrity of the network itself.

New security requirements arisen during ongoing process of specification and standardization of $5 \mathrm{G}$ networks include:

- $\quad$ proper tool support and automation in order to face growing system complexity that could go outside human control,

- $\quad$ exposition of information about each step of service delivery for the purpose of end to end security management and orchestration,

- $\quad$ awareness of all $5 \mathrm{G}$ system stakeholders of their technical security context based on evidences, facts, indicators and proofs collected at network infrastructure level,
- provision by $5 \mathrm{G}$ systems and components of functionality to mutually assess the trustworthiness before and during interactions.

Presented above requirements can be considerably fulfilled by trustworthiness assessment enabler discussed in this paper.

Software Defined Networking (SDN), Network Function Virtualization (NFV) and cloud computing technologies are expected to enhance the flexibility of network function provisioning and update, as well as reduce deployment and maintenance costs. However, security and trust become a crucial issue in practical deployment of these technologies in $5 \mathrm{G}$ due to lack of practical security and trust architecture that can support virtualized networks[4]. General approach that can cover trust assessment in access networks, virtualized core networks and user-network relation is proposed.

Between network users and external service providers (SP) trustworthiness assessment is close to IP reputation or anti-fraud solutions based on IP address geolocation. Industry-leading IP geolocation solutions provide multiple data points, including: connection type, time zone/language, proxies, ISP, connection speed, latitude/longitude, home/business, demographics, phone Area Code, industry codes, autonomous system number (ASN), etc. Having this information allows companies to adapt their policies of security, content presentation and encoding at a granular level. However, accuracy, reliability and scope of commercial IP geolocation services depend on the employed algorithms and methodologies. Since the providers are using proprietary methodologies the quality of delivered information can be regarded as questionable [5]. In contrary to external providers, network operator can provide information that is based on data directly available from $5 \mathrm{G}$ network. Moreover, network operators are legally obliged to collect and retain data needed for business operation and required by law enforcement agencies. These unique data can be used to gain added value from the network that differentiate operators from other players.

Novel 5G systems bring opportunity of developing new solutions for attack prevention, security and trust management [6]. Proposed approach can be integrated into 
currently considered $5 \mathrm{G}$ architectures and help to combine privacy protection requirement with the need for detailed security monitoring. It can be further extended into contextbased security by adding contextual information available directly from network core platforms and support systems (contextualization).

The remainder of this paper is structured as follows. Section 2 provides brief introduction to the notion of trustworthiness. In section 3 we describe Network Provided Trustworthiness system and section 4 gives more details about its operation in specific use cases. Finally in section 5 conclusions and future plans are presented.

\section{II.PROBLEM STATEMENT - TRUSTWORTHINESS}

There is currently no defined standard for $5 \mathrm{G}$ and technical standardization work has just begun. The security considerations for 5G cover new trust models, where infrastructures are shared by multiple virtual mobile network providers, but also take into account novel technological approaches such as multi-tenancy, network slicing, network virtualization and other novel technologies. It is required to control the exposure surface to new types of threat specific to $5 \mathrm{G}$ networks and to provide proactive mechanisms to protect against them. Trust based mechanisms can be among possible solutions [7]. Therefore, notions of trust and trustworthiness need to be introduced.

Trust is a belief and is related to risk. A trust decision about given entity is then a decision to accept the risk that the entity will not act as expected [8]. Trustworthiness is a property of being reliable that could be measured objectively. Information on the trustworthiness of other entities is crucial in order to make correct trust decisions. The optimal situation is when trust in an entity and the trustworthiness of that entity are in balance. If trust in an entity is lower than its trustworthiness, the trustor will take additional precautions or limit the scope of the relation - as a result he will increase his costs or decrease his profits. If trust is higher than the trustworthiness of the entity, the trustor will be exposed to more risk than he expects, consequently he can suffer a loss [9].

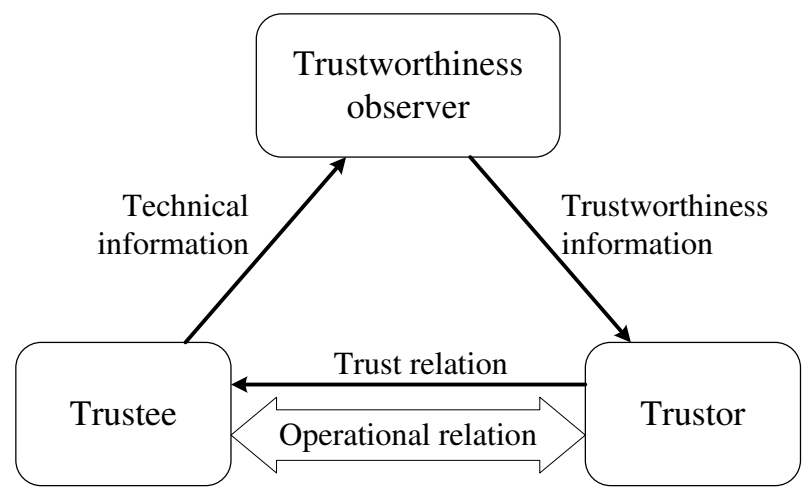

Fig. 1 Building trust relation using trustworthiness information delivered by Trustworthiness observer
Trustworthiness enabler that can be applied both to improve trust relation establishment within the network and to support external service providers in trust relation management with their customers is proposed in this paper.

While there are approaches to infer trust from comparison of observed metrics with expected values of given parameters, it is proposed to compute multi-dimensional trustworthiness level allowing direct interpretation. This computation is performed by dedicated entity having needed technical information from the network. The trustworthiness level is then used directly by trustor to build trust relation with trustee (cf. Fig.1). In this model the trustworthiness information is delivered to trustor while privacy of trustee is kept.

\section{ENABLER DESCRIPTION}

Proposed enabler should offer security context describing each connection that allows components of $5 \mathrm{G}$ system to take trust decisions. Moreover, it should support service providers in trust relation management with their customers. Referring to the model presented above the trustworthiness enabler plays the role of trustworthiness observer that gathers needed technical network information and exposes trustworthiness information.

In order to build the security context, relevant information needs to be collected at each segment of the network. When the connection is processed inside consecutive network segments the information included in the security context can be refined and extended. Finally it should be aggregated, correlated and propagated for future decisions regarding security, fraud prevention or characterization of risk.

In the 5G network architecture trustworthiness enabler functionality can be achieved with specialized subsystem distributed over the network. As depicted in Fig. 2 dedicated agents - Trustworthiness Watchers (TW) need to be placed in subsystems of $5 \mathrm{G}$ network, where information about connection is generated or processed. In the presented $5 \mathrm{G}$ ecosystem TW agents are located in network infrastructure (both physical and virtual), network functions and service layers both in access and core networks. Another set of information can be extracted from management systems and end to end (E2E) Orchestration layer.

Native network information received from TWs is aggregated and correlated by Network Provided Trustworthiness (NPT) system. The resultant trustworthiness information can be made available to network operator's service platforms or 3rd Party platforms offering services over the network in the form of security context. In considered here $5 \mathrm{G}$ architecture orchestrator can use security context of given connection to monitor required security level, but also to expose this information to 3rd parties.

Dynamic and programmable nature of $5 \mathrm{G}$ network allows not only computation of trustworthiness information but also negotiation and enforcement of required trustworthiness level. 


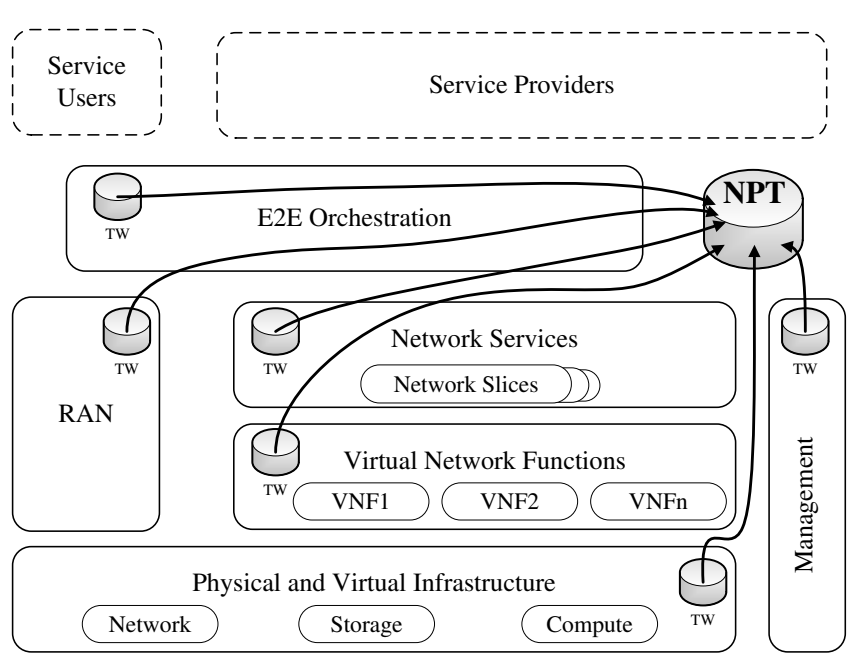

Fig. 2 NPT system within $5 \mathrm{G}$ ecosystem - logical view

The orchestrator can be seen as a key system of such solution where NPT system provides current security context of given connection. The user of that connection requires from the network to maintain current trustworthiness level or increase it according to needs. The orchestrator can then activate detailed monitoring of connection security metrics or change network configuration to fulfill user requirements.

In order to perform evaluation of given connection NPT system follows it inside the network, collecting metrics available in specified segment of the network. For example from Radio Access Network (RAN) the following information can be obtained: access type, location, location changes, radio link parameters, whereas the network Core segment can provide information on authentication scheme, service authorization, service parameters, associated resources. Finally, subscription parameters and its duration can be obtained from management systems.

The scope of security context in 5G network can include following areas:

- Customer's network context - encompassing the access network type, the equipment, the authentication scheme used, the device operating system and the equipment location;

- Infrastructure context - consisting of hardware and virtualization platforms' security parameters, attributes of VNFs (given function can be implemented using different software vendors - including open source software), network (physical and virtual) infrastructure security attributes including security policies, management capabilities and monitoring capabilities (telemetry, agents and probes);

- Customer relation context - covering parameters of contract and history of subscription.

The role of NPT system is to compute trustworthiness information from aggregated data (transform it into single value or vector of values) in order to facilitate trust decisions and hide details (customer privacy and network security principle). As trustworthiness is derived from technical context it does not cover all factors impacting the trust relation, nevertheless, this technical factor (beside human, social, formal and other factors) can play important role in trust establishment and risk management.

Service providers may be interested in more refined security context indicating directly risk of specific threats in this case technical information from the network needs to be transformed into warning about possibility of specific attack types, for example: Man in the middle, Eavesdropping, Denial of service, Rogue devices, Data manipulation, Content piracy, Spoofing, Impersonation, Unprotected endpoint used for network entry, Equipment cloning, etc. (cf. [10]). The proposed enabler can bring into operation such functionality by extending its analytics to be compatible with this new security context.

\section{USE CASES}

The usage of trustworthiness enabler can be illustrated by following examples.

\section{A. Trust in network access control}

Heterogeneity of access control to the network is one of considered 5G features [6] - it allows AAA (Authentication, Authorization and Accounting) function to be based on different authorization schemes and to be delivered by multiple providers. This approach implies the need to propagate the trustworthiness of the device accessing the network in the end to end relation. The NPT system can help to make the right trust decision to achieve required isolation and traffic segregation for devices using different access control configurations. Data about customer network context (access network type, equipment, authentication scheme and authentication provider) are discovered and transformed into trustworthiness information by NPT. Network service platforms can use this information in trust decisions about service level deciding about traffic priority and QoS (Quality of Service), fraud prevention monitoring, etc. For example specific security policy can be invoked for equipment authenticated within untrusted access network (indicated by low value trustworthiness information).

\section{B. Trusted network slicing}

Network slicing is often described as a technique that would help $5 \mathrm{G}$ network to implement variety of services with effective use of resources [11]. With NPT system the management of the slice with high-level security requirement can be made easier by indicating trustworthiness of each component from access and core network. Trustworthiness information computed by NPT reflects the security level of each component. The slice security policy can state that if this level is not high enough, the given (physical or real) component cannot be included in the slice. Using trustworthiness information the security level can be continuously monitored over the life time of the slice. In NFV based infrastructure NPT can be used to build E2E trust metrics that include information from a variety of monitoring and enforcement tools, including attestation, Intrusion Defense Systems (IDS), Network Domain Security (NDS) and software management of VNFs [12]. 


\section{Service provider risk management}

NPT system helps service providers using $5 \mathrm{G}$ network to manage the trust decisions and introduce proper risk management techniques for service requests received from the network. In this case the most helpful trustworthiness information would include possibility of specific threats within particular request coming from 5G network. Trustworthiness information used in service delivery should reflect the probability that the service request is initiated by the legitimate user in secure conditions in the rightful way. This probability is not static but corresponds to different parameters that can be easily identified by the network Operator.

Proposed here use case was demonstrated by the authors within the present convergent network ecosystem. In the proof of concept implementation so called Trust Level structured as shown in Table 1 was added to requests directed to exemplary service provider's website.

TABLE I.

EXAMPLE OF TRUSTWORTHINESS STRUCTURE

\begin{tabular}{|l|l|}
\hline $\begin{array}{l}\text { Trustworthiness } \\
\text { Dimension }\end{array}$ & Network information covered \\
\hline Access Type & $\begin{array}{l}\text { Origin of the request seen by the network } \\
\text { (access network, interconnected network, etc.) }\end{array}$ \\
\hline $\begin{array}{l}\text { Network } \\
\text { Authentication }\end{array}$ & $\begin{array}{l}\text { Strength and type of authentication used to } \\
\text { access network services }\end{array}$ \\
\hline Equipment & $\begin{array}{l}\text { Type of equipment originating the request on } \\
\text { network level including software version }\end{array}$ \\
\hline Contract & $\begin{array}{l}\text { Information about contract type } \\
\text { (postpaid/prepaid) and its duration seen by } \\
\text { CRM system }\end{array}$ \\
\hline
\end{tabular}

Trust Level information allowed SP to adapt its service logic and protection mechanisms to risk related with given service request (cf. [13]). For example after receiving tuple of trustworthiness values $\{\mathrm{v}, \mathrm{x}, \mathrm{y}, \mathrm{z}\}$ the risk of using stolen credentials in given request has been estimated by SP. According to this estimation the trust decision has been taken to offer the requesting user limited number of available actions on his account or to require additional authorization to perform sensitive operation. This way SP can improve protection of his electronic service accessed from multiple networks and provide fine-grained authorization.

Implementation of trustworthiness enabler as NPT system in $5 \mathrm{G}$ network can be based on more detailed information about the request and should offer service users (customers and service providers) the functionality to monitor and proactively modify connection parameters (access network, routing, protection methods) in order to achieve required trustworthiness level. On the other hand thanks to only numerical value delivered to service providers the privacy of end users is not disclosed.

\section{V.SUMMARY AND NEXT STEPS}

$5 \mathrm{G}$ is the proposed next generation of mobile wireless broadband technology. As with previous generations of mobile technology, security and privacy remain fundamental underlying requirements for mobile applications and services across devices that access wireless networks. Adoption of new paradigms like NFV and network slicing may further raise requirements aiming at properly securing such complex system. New trust models are one of responses to this demand. Introduction of trustworthiness enabler facilitates trust management on multiple layers of 5G architecture. Additionally, proposed enabler supports implementation of flexible security requirement: rather than enforcing user plane protection, the network may allow applications to select and dynamically adapt the way the user plane is protected [14].

Ongoing progress of $5 \mathrm{G}$ architecture shaping and standardization will enable more detailed design of NPT system compatible with this architecture. Beside definition how specific network information is used to calculate trustworthiness, further study of the concept should include exchange of trustworthiness information between $5 \mathrm{G}$ networks, cooperation with non-5G providers or other partners within network ecosystem. There is also a need for study of business model including liability of network operator to deliver trusted and qualified information to SP (security extension of Service Level Agreement between 5G network operator and vertical service providers).

\section{REFERENCES}

[1] Gupta, Akhil, and Rakesh Kumar Jha. "A survey of 5G network: Architecture and emerging technologies." IEEE access 3 (2015): 1206-1232. doi:10.1109/ACCESS.2015.2461602

[2] Next Generation Mobile Network Alliance, "5G White Paper", Version 1.0, Feb 17, 2015.

[3] 5G PPP Architecture Working Group, View on 5G Architecture Version 1.0, July 2016

[4] Yan, Zheng, Peng Zhang, and Athanasios V. Vasilakos. "A security and trust framework for virtualized networks and software-defined networking." Security and communication networks (2015). doi: $10.1002 /$ sec. 1243

[5] Koch, Robert, et al. "Using Geolocation for the Strategic Preincident Preparation of an IT Forensics Analysis." IEEE Systems Journal 10.4 (2016): 1338-1349. doi: 10.1109/JSYST.2015.2389518

[6] Selander, Goran, et al. "5G-ENSURE: D2. 1 use cases." (2016).

[7] Wang, Dongxia, et al. "Towards robust and effective trust management for security: A survey." Trust, Security and Privacy in Computing and Communications (TrustCom), 2014. doi:10.1109/TrustCom.2014.65

[8] Mohammadi, Nazila Gol, et al. "Trustworthiness attributes and metrics for engineering trusted internet-based software systems." International Conference on Cloud Computing and Services Science. 2013. doi: 10.1007/978-3-319-11561-0_2

[9] Phillips, Stephen, et al. "5G-ENSURE: D2.2 Trust model (draft)."

[10] 5G Security - Making the Right Choice to Match your Needs (A SIMalliance 5GWG technical white paper) http://simalliance.org/wpcontent/uploads/2016/02/5G-Security-\%E2\%80\%93-Making-theRight-Choice-to-Match-your-Needs.pdf

[11] Next Generation Mobile Network Alliance, "Description of Network Slicing Concept." NGMN 5 G P 1 (2016).

[12] Manzalini, A., et al., Towards 5G Software-Defined Ecosystems. Technical Challenges, Business Sustainability and Policy Issues, IEEE SDN White Paper, http://sdn.ieee.org/publications

[13] Li, Min, et al. "Privacy-aware access control with trust management in web service." World Wide Web 14.4 (2011): 407-430. doi:10.1007/s11280-011-0114-8

[14] Horn, Günther, and Peter Schneider. "Towards 5G Security." (2015). http://resources.alcatel-lucent.com/asset/200292 


\section{$10^{\text {th }}$ International Symposium on Multimedia Applications and Processing}

\section{BACKGROUND AND GOALS}

$\mathbf{M}$ ULTIMEDIA information has become ubiquitous on the web, creating new challenges for indexing, access, search and retrieval. Recent advances in pervasive computers, networks, telecommunications, and information technology, along with the proliferation of multimedia mobile devicessuch as laptops, iPods, personal digital assistants (PDA), and cellular telephones-have stimulated the development of intelligent pervasive multimedia applications. These key technologies are creating a multimedia revolution that will have significant impact across a wide spectrum of consumer, business, healthcare, educational and governmental domains. Yet many challenges remain, especially when it comes to efficiently indexing, mining, querying, searching, retrieving, displaying and interacting with multimedia data.

The Multimedia-Processing and Applications 2017 (MMAP 2017) Symposium addresses several themes related to theory and practice within multimedia domain. The enormous interest in multimedia from many activity areas (medicine, entertainment, education) led researchers and industry to make a continuous effort to create new, innovative multimedia algorithms and applications.

As a result the conference goal is to bring together researchers, engineers, developers and practitioners in order to communicate their newest and original contributions. The key objective of the MMAP conference is to gather results from academia and industry partners working in all subfields of multimedia: content design, development, authoring and evaluation, systems/tools oriented research and development. We are also interested in looking at service architectures, protocols, and standards for multimedia communicationsincluding middleware-along with the related security issues, such as secure multimedia information sharing. Finally, we encourage submissions describing work on novel applications that exploit the unique set of advantages offered by multimedia computing techniques, including home-networked entertainment and games. However, innovative contributions that don't exactly fit into these areas will also be considered because they might be of benefit to conference attendees.

\section{CALl FOR PAPERS}

MMAP 2017 is a major forum for researchers and practitioners from academia, industry, and government to present, discuss, and exchange ideas that address real-world problems with real-world solutions.

The MMAP 2016 Symposium welcomes submissions of original papers concerning all aspects of multimedia do- main ranging from concepts and theoretical developments to advanced technologies and innovative applications. MMAP 2016 invites original previously unpublished contributions that are not submitted concurrently to a journal or another conference. Papers acceptance and publication will be judged based on their relevance to the symposium theme, clarity of presentation, originality and accuracy of results and proposed solutions.

\section{TOPICS}

- Audio, Image and Video Processing

- Animation, Virtual Reality, 3D and Stereo Imaging

- Big Data Science and Multimedia Systems

- Cloud Computing and Multimedia Applications

- Machine Learning, Data Mining, Information Retrieval in Multimedia Applications

- Multimedia File Systems and Databases: Indexing, Recognition and Retrieval

- Multimedia in Internet and Web Based Systems

- E-Learning, E-Commerce and E-Society Applications

- Human Computer Interaction and Interfaces in Multimedia Applications

- Multimedia in Medical Applications

- Entertainment and games

- Security in Multimedia Applications: Authentication and Watermarking

- Distributed Multimedia Systems

- Network and Operating System Support for Multimedia

- Mobile Network Architecture

- Intelligent Multimedia Network Applications

- Future Trends in Computing System Technologies and Applications

\section{Best PAPER AWARD}

A best paper award will be made for work of high quality presented at the MMAP Symposium. The technical committee in conjunction with the organizing/steering committee will decide on the qualifying papers. Award comprises a certificate for the authors and will be announced on time of conference.

\section{Steering Committee}

- Amy Neustein, Boston University, USA, Editor of Speech Technology

- Lakhmi C. Jain, University of South Australia and University of Canberra, Australia

- Ioannis Pitas, University of Thessaloniki5, Greece

- Costin Badica, University of Craiova, Romania 
- Borko Furht, Florida Atlantic University, USA

- Harald Kosch, University of Passau, Germany

- Vladimir Uskov, Bradley University, USA

- Thomas M. Deserno, Aachen University, Germany

\section{SECTION EDITOR}

- Dumitru Dan Burdescu, University of Craiova, Romania

\section{General Co-Chairs}

- Adriana Schiopoiu Burlea, University of Craiova, Romania

- Marius Brezovan, University of Craiova, Romania

\section{PUBLICITY CHAIR}

- Amelia Badica, University of Craiova, Romania

- Adriana Schiopoiu Burlea, University of Craiova, Romania

\section{ORGANIZING COMMITTEE}

- Dumitru Dan Burdescu, University of Craiova, Romania

- Costin Badica, University of Craiova, Romania

- Marius Brezovan, University of Craiova, Romania

- Adriana Schiopoiu Burlea, University of Craiova, Romania

- Liana Stanescu, University of Craiova, Romania

- Cristian Marian Mihaescu, University of Craiova, Romania

\section{REVIEWERS}

- Azevedo, Ana, CEOS.PP-ISCAP/IPP, Portugal

- Badica, Amelia, University of Craiova, Romania

- Böszörmenyi, Laszlo, Klagenfurt University, Austria

- Botez, Ruxandra, University of Quebec

- Burlea Schiopoiu, Adriana, University of Craiova, Romania

- Camacho, David, Universidad Autonoma de Madrid, Spain

- Cano, Alberto, Virginia Commonwealth University

- Cordeiro, Jose, EST Setúbal/I.P.S.

- Cretu, Vladimir, Politehnica University of Timisoara, Romania

- Debono, Carl James, University of Malta, Malta

- Fabijańska, Anna, Lodz University of Technology, Poland - Institute of Applied Computer Science, Poland

- Fomichov, Vladimir, National Research University Higher School of Economics, Moscow, Russia., Russia

- Giurca, Adrian, Brandenburg University of Technology, Germany

- Grosu, Daniel, Wayne State University, United States

- Groza, Voicu, University of Ottawa, Canada

- Kabranov, Ognian, Cisco Systems, United States
- Kannan, Rajkumar, Bishop Heber College Autonomous, India

- Keswani, Dr. Bright, Suresh Gyan Vihar University, Mahal, Jagatpura, Jaipur

- Korzhik, Valery, State University of Telecommunications, Russia

- Kotenko, Igor, St. Petersburg Institute for Informatics and Automation of the Russian Academy of Science, Russia

- Kriksciuniene, Dalia, Vilnius University, Lithuania

- Lau, Rynson, City University of Hong Kong, Hong Kong S.A.R., China

- Lloret, Jaime, Polytechnic University of Valencia, Spain

- Logofatu, Bogdan, University of Bucharest, Romania

- Mangioni, Giuseppe, DIEEI - University of Catania, Italy

- Mannens, Erik, Ghent University

- Marghitu, Daniela, Auburn University

- Mihaescu, Cristian, University of Craiova, Reunion

- Mocanu, Mihai, University of Craiova, Romania

- Morales-Luna, Guillermo, Centro de Investigación y de Estudios Avanzados del Instituto Politécnico Nacional, Mexico

- Ohzeki, Kazuo, Professor Emeritus at Shibaura Institute of Technology, Japan

- Popescu, Dan, CSIRO, Sydney, Australia, Australia

- Querini, Marco, Department of Civil Engineering and Computer Science Engineering

- Radulescu, Florin, University "Politehnica" of Bucharest

- RUTKAUSKIENE, Danguole, Kaunas University of Technology

- Salem, Abdel-Badeeh M., Ain Shams University, Egypt

- Sari, Riri Fitri, University of Indonesia, Indonesia

- Sousa Pinto, Agostinho, Instituto Politécnico do Porto

- Stanescu, Liana, University of Craiova, Romania

- Stoicu-Tivadar, Vasile, University Politehnica Timisoara

- Tejera, Mario Hernández, University of Las Palmas de Gran Canaria, Spain

- Trausan-Matu, Stefan, Politehnica University of Bucharest, Romania

- Trzcielinski, Stefan, Poznan University of Technology, Poland

- Tsihrintzis, George, University of Piraeus, Greece

- Tudoroiu, Nicolae, John Abbott College, Canada

- Vega-Rodríguez, Miguel A., University of Extremadura, Spain

- Velastin, Sergio, Kingston University, United Kingdom

- Virvou, Maria, University of Piraeus, Greece

- Watanabe, Toyohide, University of Nagoya

- Wotawa, Franz, Technische Universitaet Graz, Austria

- Zurada, Jacek, University of Louisville, United States 


\title{
Utilization of Renewable Resources in Rehabilitation of Existent Micro-Hydropower Plants
}

\author{
Victorita Radulescu \\ University Politehnica of Bucharest, \\ Department of Hydraulics, Hydraulic \\ machinery and Environmental engineering, \\ Splaiul Independentei 313, Sector 6 \\ Bucharest, Romania \\ Email:vradul4@gmail.com
}

\begin{abstract}
The document presents a prototype Pilot station, realized near a Small hydro power plant, decommissioned in 1998, one between others 400 in Romania. In its structure, there are inter-connected a wind power plant equipped with a rotor of own production (a Romanian brevet under license), a photovoltaic power plant and the micro hydro power plant, needed to be rehabilitated. The wind turbine has a rotor able to start at low values of the wind speed, around $1,4 \mathrm{~m} / \mathrm{s}$. There are analyzed the environmental and geographic parameters, the economic necessity as to establish the optimum position for the pilot station. The theoretical modeling, the physical realization, the main steps in the project, the conditions needed to be satisfied for coupling these resources are mentioned. Consequently, are presented some obtained results during the first 6 months after implementation, some observations, and recommendation for further development as to improve its functioning for other conditions.
\end{abstract}

\section{INTRODUCTION}

In the actual context, the European Union decides to reduce during the period 2010-2020, the pollutant emission with $8 \%$ compared with the year 2010. Accordingly, some specific documents concerning the principal solutions are mentioned in the Green Cart, White Cart, the Directive concerning the increase of the utilization of the renewable resources from $14 \%$ to $22 \%$ until 2020 into EU countries.

In Romania [1], during 1980-1990 were built over 600 Micro-Hydro power plants, having as principal purpose the electrical power supply for the isolated areas or the soil irrigation. After 25 years, less than half of them are still functional, because there are no investments concerning their maintenance and rehabilitation Moreover, 150 of them are technically old, with parameters far outdone from their initial values. Consequently, in the present context concerning utilization of renewable resources in Romania, starting with 2005 there was held a constant campaign for monitoring the environmental data, in order to locate the economically favorable zones as to implement a wind power plant or a photovoltaic, connected to an existent small hydro power plant, needed to be rehabilitated.
Nowadays, in the RES structure, in Romania, the hydro resource still has the largest share. The energy provided by SHP-s has the installed capacity $\leq 10 \mathrm{MW}$. The potential possible to be produced is equivalent to almost $80 \%$ of the energy produced by the Iron Gates I power plant, except that they are distributed in the entire country, so their construction would lead to an economic growth in all the regions. Notwithstanding, the EU Directive 2009/28/EC, says that hydro, wind, solar, geothermal, aero-thermal, hydro-thermal, considered as renewable energy would avoid large emissions of carbon dioxide, as it happens in the case of burning the fossil fuels. SHP-s (small hydro power plants) can also solve some local problems, such as new jobs, the water alimentation, the irrigation, the electricity in various isolated villages, the decrease in imports of the primary energy resources, the elimination of the voltage drop in the far electrical networks, reduction of energy losses in transmission lines.

Accordingly, to the new attempts as to improve the efficiency of the SHP-s they must begin with some initial steps as mandatory works and technological operations:

- The cleaning of the vegetation from the dam and the adjacent channels,

- Repair works to eliminate all the cracks from the concrete structures, the surfaces with eroded areas and lack of the material,

- Consolidation works upstream/downstream of the dam with the construction of reinforced concrete piers,

- Construction of a fish ladder, required by the actual EU standards,

- Rehabilitation, adaptation, and modernization of all metal constructions of the dam,

- Sandblasting, cleaning, and restoration by welding and polishing of the metal damaged surfaces,

- Changing of all the fasteners and fastening,

- Realization of the corrosion protection

In Fig. 1 is presented the share of the energy based on primary resources, source ANRE, the Romanian Energy 
Regulatory Authority, [1], the report made in 2016 compared with the year 2012 .
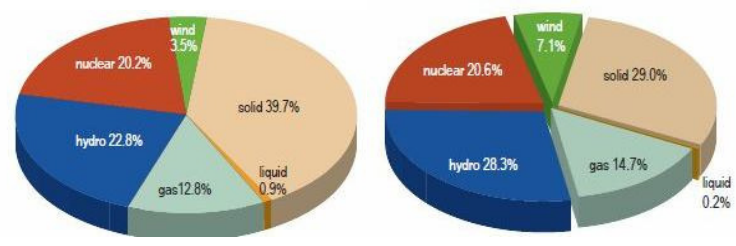

Fig 1. Components of the energetic balance 2012 and 2016

In present in Romania, the energy produced from renewable resources represents around $35 \%$, but more than $28 \%$ due to the hydro resources. In Table I are presented the new capacities, and the total investments estimated to be spent in 2010-2020. There are mentioned the realized investments during 2011-2015. The abbreviations refer at S-T solar- thermal; S-E solar electric; W-wind; H-hydro, B-T biomass-thermal; B-E biomass-electric; Ggeothermal; NI-New investment; TI-Total Investment and NC- New Capacities.

TABLE I.

ESTIMATION OF RR UTILIZATION IN ROMANIA

\begin{tabular}{|l|l|l|l|l|}
\hline \multirow{2}{*}{ RR } & \multicolumn{2}{|c|}{$\mathbf{2 0 1 0 - 2 0 2 0}$} & \multicolumn{2}{c|}{$\mathbf{2 0 1 1 ~ - 2 0 1 5}$} \\
\cline { 2 - 5 } & \multicolumn{1}{|c|}{$\mathbf{N I}$} & $\begin{array}{c}\text { TI } \\
\mathbf{1 0}^{6} \text { Euro }\end{array}$ & NC & $\begin{array}{c}\text { TI } \\
\mathbf{1 0}^{6} \text { Euro }\end{array}$ \\
\hline S-T $\left[10^{3}\right.$ tep] & 37,3 & 175,0 & 16,0 & 93,0 \\
\hline S-E $[\mathrm{MW}]$ & 11,5 & 87,5 & 9,5 & 48,0 \\
\hline W $[\mathrm{MW}]$ & 520 & 420,0 & 280 & 280,0 \\
\hline H $\leq 10 \mathrm{MW}$ & 320 & 350,0 & 120 & 120,0 \\
\hline B-T $[\mathrm{MW}]$ & 5250 & 240,0 & 3488 & 200,0 \\
\hline B-E $[\mathrm{MW}]$ & 590 & 480,0 & 380 & 400,0 \\
\hline G $\left[10^{3}\right.$ tep $]$ & 27,5 & 15,0 & 23,9 & 12,0 \\
\hline Total & 932 & 1887,5 & 789 & 1153,0 \\
\hline
\end{tabular}

To estimate the opportunity of implementation of the pilot station into the selected area, there were monitored in the beginning the environmental data, for a period of two years. There were used independent weather stations, with electric supply from the solar panels, equipped with a system of on-line registration, data storage and the transmission via the internet of the collected data.

\section{II.ENVIRONMENTAL DATA ACQUISITION}

It was selected the Station Weather Hawk Series Signature 500, able to measure, store, and transmit by GSM: the direction and intensity of the wind, air temperature, relative humidity of the air, barometric pressure, solar radiation, the quantity of rainfall and dew point. The system stores and calculates a value for the ET (vapor transpiration). In November 2014, the weather station was installed consequently the first data acquisitions were made. The measurements were set and stored at each hour. As a supplementary option, was set the registration of the minimum and the maximum of certain parameters, considered essentials for further applications, as in Table II (as an example, part of 03.04.2015).

TABLE II.

MEASUREMENTS REGISTERED DATA

\begin{tabular}{|l|l|l|l|l|l|}
\hline Parameter & Max & Max T & Min & Min T & Av. \\
\hline $\begin{array}{l}\text { Wind } \\
\text { Direction }\end{array}$ & 304 & $5: 27 \mathrm{am}$ & 267 & $1: 00 \mathrm{pm}$ & 292 \\
\hline Wind Speed & 11 & $9: 16 \mathrm{am}$ & 0 & $8: 00 \mathrm{am}$ & 6 \\
\hline Wind Gust & 11 & $10: 00 \mathrm{am}$ & 0 & $8: 00 \mathrm{am}$ & 8 \\
\hline In Humidity & 0 & $5: 24 \mathrm{am}$ & 0 & $5: 24 \mathrm{pm}$ & 0 \\
\hline Humidity & 86 & $8: 00 \mathrm{pm}$ & 44 & $8: 00 \mathrm{am}$ & 71 \\
\hline In Temp & 17.8 & $5: 24 \mathrm{am}$ & -18 & $5: 24 \mathrm{am}$ & 14.2 \\
\hline Out Temp & 20.5 & $8: 00 \mathrm{am}$ & 7.7 & $6: 48 \mathrm{pm}$ & 20 \\
\hline Raw Bar & 748 & $10: 00 \mathrm{am}$ & 732 & $8: 00 \mathrm{am}$ & 602 \\
\hline Total Rain & 0.00 & $5: 24 \mathrm{am}$ & 0.0 & $5: 24 \mathrm{am}$ & 0.0 \\
\hline Ch 1 Temp & 0.0 & $5: 24 \mathrm{am}$ & 0.0 & $5: 24 \mathrm{am}$ & 0.0 \\
\hline Ch 1 Hum & 0 & $5: 24 \mathrm{am}$ & 0 & $5: 24 \mathrm{am}$ & 0 \\
\hline Ch 2 Temp & 0.0 & $5: 24 \mathrm{am}$ & 0.0 & $5: 24 \mathrm{am}$ & 0.0 \\
\hline Ch2 Hum & 0 & $5: 24 \mathrm{am}$ & 0 & $5: 24 \mathrm{am}$ & 0 \\
\hline Ch 3 Temp & 0.0 & $5: 24 \mathrm{am}$ & 0.0 & $5: 24 \mathrm{am}$ & 0.0 \\
\hline Ch 3 Hum & 0 & $5: 24 \mathrm{am}$ & 0 & $5: 24 \mathrm{am}$ & 0 \\
\hline Ev.transp & 0.99 & $11: 00 \mathrm{pm}$ & 0.0 & $5: 24 \mathrm{am}$ & 0.5 \\
\hline Battery V & 14 & $11: 00 \mathrm{pm}$ & 12 & $5: 00 \mathrm{pm}$ & 13 \\
\hline Solar Rad. & 94 & $10: 00 \mathrm{pm}$ & 0 & $2: 00 \mathrm{pm}$ & 21 \\
\hline Wind Chill & 20.5 & $5: 24 \mathrm{am}$ & -17 & $8: 00 \mathrm{am}$ & 11 \\
\hline
\end{tabular}

Consequently, after the data stored for an entire month, the average values are calculated. Ordinarily are stored, in special files, the direction, and intensity of the wind, the solar radiation, and the humidity, important for future developments. Separately is measured the flow rate and the quantity of rain. The internal storage of recorded data, since they are measured more than twenty parameters at every minute and the minimum and the maximum daily values were a challenge. Moreover, a dynamic database was created, structured monthly and annually.

\section{THE SOLAR PHOTO-VOLTAIC PART OF THE PILOT STATION}

\section{A. Analysis of the zone opportunities}

Ordinarily, were analyzed the local conditions where the pilot station must be implemented. The produced energy [2] is directly dependent on the solar radiation, the geographic position, season, time of the day, etc. Consequently, after some months of monitoring of all the mentioned parameters and supplementary of the solar radiation and temperature, it was selected a specific area from the south of Moldavia, Romania. By using the Homer software [3], for numerical modeling was estimated the efficiency and established to be realized ten modules of $1.4 \mathrm{KW}$ each, meaning a pilot station of $14 \mathrm{KW}$.

For Romania, the solar radiation is between 1200-1400 $\mathrm{h} /$ year at maximum installed power. Making a compari- 
son with other areas we may estimate that the solar potential is at a medium level, and the average produced energy is somewhere between $2.75-4.8 \mathrm{kWh} / \mathrm{m}^{2}$. As medium estimated, the value for the selected area is $3.4 \mathrm{kWh} / \mathrm{m}^{2}$, with variation of $12 \%$.

Consequently, from the principal offers from the photovoltaic market were selected the first 12 producers (they assure in 2014 more than $90 \%$ of the whole production). It must be mentioned that today, from the market more than $25 \%$ of the total production is represented by the Japanese market, Europe 23\%, China 37\% and the rest by small firms, around $15 \%$.

\section{B. Numerical modeling of the PV system}

Moreover, some tests are realized at different angles from the horizontal:

1. Firstly, panels realized at different angles: between $30^{\circ}-35^{\circ}$, between $35^{\circ}-40^{\circ}$ and from $40^{\circ}-45^{\circ}$. The measurements and the estimation assume the pilot station realized at constant angle, for one of these values.

2. Secondly, measurements and numerical modeling take into account the realization of a pilot station having rows realized at different angles.

3. The third estimation considers the modules able to be oriented after the sun position.

Accordingly, to the measurements and the numerical modeling using specific software RET and Homer, some steps from the design are presented in Table III.

TABLE III.

RESULTS BY NUMERICAL MODELLING

\begin{tabular}{|l|l|l|l|}
\hline \multicolumn{4}{|c|}{ System in Grid Connected Operation } \\
\hline Location: & Racaciuni & PV Output: & $101.3 \mathrm{~kW}$ \\
\hline $\begin{array}{l}\text { Climate Data } \\
\text { Record: }\end{array}$ & Racaciuni & $\begin{array}{l}\text { Gross/Active Solar } \\
\text { PV Surface Area: }\end{array}$ & $\begin{array}{l}731.1 \mathrm{~m}^{2} / \\
725.5 \mathrm{~m}^{2}\end{array}$ \\
\hline Output: & $33.76 \mathrm{~kW}$ & Ground Reflection: & $20.0 \%$ \\
\hline $\begin{array}{l}\text { Gross/Active } \\
\text { Solar Area }\end{array}$ & $\begin{array}{l}243.7 \mathrm{~m}^{2} / \\
241.8 \mathrm{~m}^{2}\end{array}$ & Output Losses & - \\
\hline PV Module & $152 \mathrm{x}$ & Deviation AM 1.5: & $2.0 \%$ \\
\hline Manufacturer: & Polycrystalline & Deviation & $3.0 \%$ \\
\hline Type: & PV-210AE-A & Diodes: & $0.5 \%$ \\
\hline Power Rating: & $210 \mathrm{~W}$ & Due to Pollution: & $0.0 \%$ \\
\hline P. Deviation: & $0 \%$ & Inverter & $6 \mathrm{x}$ \\
\hline $\begin{array}{l}\text { Irradiation } \\
\text { Horizontal: }\end{array}$ & $979,414 \mathrm{kWh}$ & Own Use: & $161.9 \mathrm{kWh}$ \\
\hline $\begin{array}{l}\text { PV Array } \\
\text { Irradiation: }\end{array}$ & $980,171 \mathrm{kWh}$ & $\begin{array}{l}\text { Energy Produced by } \\
\text { PV Array: }\end{array}$ & $\begin{array}{l}112,551 \\
\mathrm{kWh}\end{array}$ \\
\hline $\begin{array}{l}\text { Irradiation - } \\
\text { Reflection: }\end{array}$ & $931,141 \mathrm{kWh}$ & System Efficiency: & $10.8 \%$ \\
\hline $\begin{array}{l}\text { Energy from } \\
\text { Inverter (AC): }\end{array}$ & $105,716 \mathrm{kWh}$ & Performance Ratio: & $94.1 \%$ \\
\hline $\begin{array}{l}\text { Consumption } \\
\text { Requirement: }\end{array}$ & $0 \mathrm{kWh}$ & Final Yield: & $2.9 \mathrm{~h} / \mathrm{d}$ \\
\hline Energy Grid: & $161 \mathrm{kWh}$ & Spec. Annual Yield: & $1,042 \mathrm{kWh}$ \\
\hline
\end{tabular}

Some general remarks must be mentioned after 6 months since the implementation:

- The intensity of global solar radiation, daily value is between 3.8-4.2 KWh $/ \mathrm{m}^{2}$

- The territorial dispersion of values for the average global solar intensity, measured on horizontal is $14-20 \%$ from the maximum value

- For the energetic purposes, the average value is 1390 $\mathrm{KWh} / \mathrm{m}^{2}$

The selected solution, adopted is with solar modules oriented towards the south with variable angles between summer and winter; in Fig 2. are presented some results.
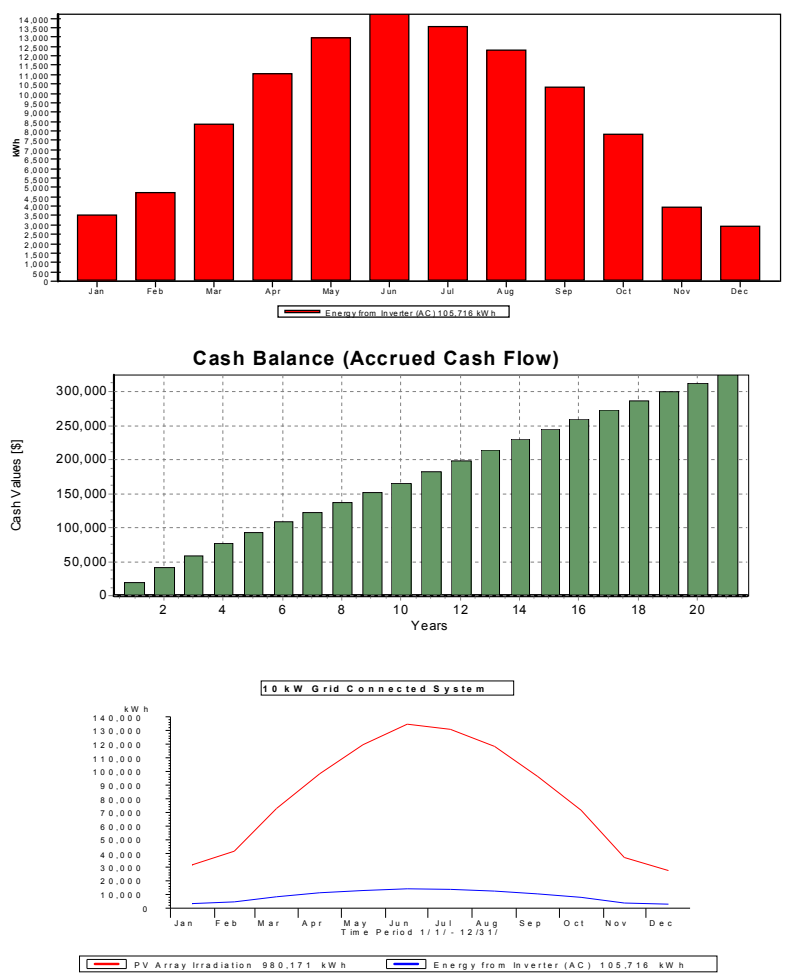

Fig 2. Components of the energetic balance 2012 and 2016

\section{Implementation of the $P V$}

Ordinarily, from offers of Sharp, Q-Cells, Kyocera, Sanyo, Mitsubishi, Schott Solar, BP Solar, Suntech, Motech, Shell Solar, Isofotón and Deutsche Cell was selected the variant Kyocera. The system is formed by panels Off Grid $1.4 \mathrm{~kW}$ PV with energy storage in batteries, recommended in areas where electricity is lacking. Each package contains two panels $700 \mathrm{~W}, 1$ inverter 24V/UPS system 1400 with 1 Controller 60A 12-24V EP Solar, etc. Reliable modules are PV panels with great efficiency and high yield. Even with a reduced incidence of light, the module achieves a good performance due to excellent low light behavior. Before and after lamination, each module undergoes an electroluminescence test. Performance guarantee: up to 10 years: $90 \%$ of nominal output; Effi- 
ciency to 25 years: $80 \%$ of nominal power; the product warranty is 11 years for the Photovoltaic panel Polycrystalline 700W. Technical data: Maximum Power Pmax $(\mathrm{W})=700 \mathrm{~W}$, Voltage at $\operatorname{Pmax}(\mathrm{V})=30,75 \mathrm{~V}$, Current at $\operatorname{Pmax}(\mathrm{A})=8.34 \mathrm{~A}$, tensions empty $(\mathrm{V})=38,32 \mathrm{~V}$, short circuit current $(\mathrm{a})=8.83=1000 \mathrm{~V}$ maximum voltage $\mathrm{Nr}$. $=60$ pieces of photovoltaic cells. The size of $156 \mathrm{~mm} \mathrm{x}$ $156 \mathrm{~mm}$ size cell panel $=1.640 \times 991, \mathrm{X}=38 \mathrm{~mm}$ Weight $=$ $18.0 \mathrm{Kg}, 1000 \mathrm{~W}$ inverter $24 \mathrm{~V}$.

The transformation of alternating current to direct one is provided through a robust integrated into the inverter of the transformer. For inverter is assured a management and a control with a microcontroller with amplification, in combination with the "MOS-FET" technology.

\section{THE Wind COMPONENT OF THE PILOT Station}

\section{A. Wind resources of the area}

In Romania are five zones of wind potential, considering the geographic and environmental conditions. The measurements are made at an altitude of $80 \mathrm{~m}$, estimated as the height of the wind turbine. From the European reports, Romania has important wind resources, especially in coastal areas and in some other three favorable zones: south and north in Moldavia and in the high mountains.

Some preliminary investigation proved that in the area of the Black Sea coast the intensity of the wind allows obtaining more than $4000 \mathrm{GWh}$ /year. Some other Bulgarian institutes confirmed the results. Consequently, after the implementation of the weather station, measurement, and registration of the intensity and of the wind direction, the initial estimation is confirmed. The environmental data are still stored in a database.

\section{B. Numerical modeling of the wind system}

For numerical modeling there were tested more than twenty possibilities considering the power station equipment, referring to the power and number of the wind turbines. As numerical modeling was considered and tested six variants:

1. A turbine model DEWIND power $1.25 \mathrm{MW}, 60 \mathrm{~m}$ high, Variant 1-one turbine, Variant 2-two turbines,

2. Model Nordex, power $1.3 \mathrm{MW}, 70 \mathrm{~m}$ high, Variant 3-1 turbine, Variant 4-two turbines,

3. Model Siemens $1 \mathrm{MW}, 60 \mathrm{~m}$ high, Variant 5- 3 turbines and finally Variant 6 with 4 turbines.

In Table IV, are mentioned some calculations taking into account, the Net average GHG reduction $\left(t_{\mathrm{CO} 2} / \mathrm{yr}\right)$ : 3.161, the Renewable energy delivered (MWh/yr): 6.430 and the Year-to-positive cash flow: more than 25 years.

The estimation of the wind system in the Pilot Station is for turbines with a capacity of 3 MW. In Fig. 3 are presented the economic efficiency of the wind power component of the pilot station.
TABLE IV.

ESTIMATED RESULTS

\begin{tabular}{|l|l|l|}
\hline Wind plant capacity & $\mathrm{kW}$ & 2.300 \\
\hline Unadjusted energy production & $\mathrm{MWh}$ & 7.412 \\
\hline Pressure adjustment coefficient & - & 1,00 \\
\hline Temp. adjustment coefficient & - & 0,96 \\
\hline Gross energy production & $\mathrm{MWh}$ & 7.116 \\
\hline Losses coefficient & - & 0,90 \\
\hline Specific yield & $\mathrm{kWh} / \mathrm{m}^{2}$ & 1.011 \\
\hline Wind plant capacity factor & $\%$ & $32 \%$ \\
\hline Renewable energy delivered & $\mathrm{MWh}$ & 6.430 \\
\hline
\end{tabular}
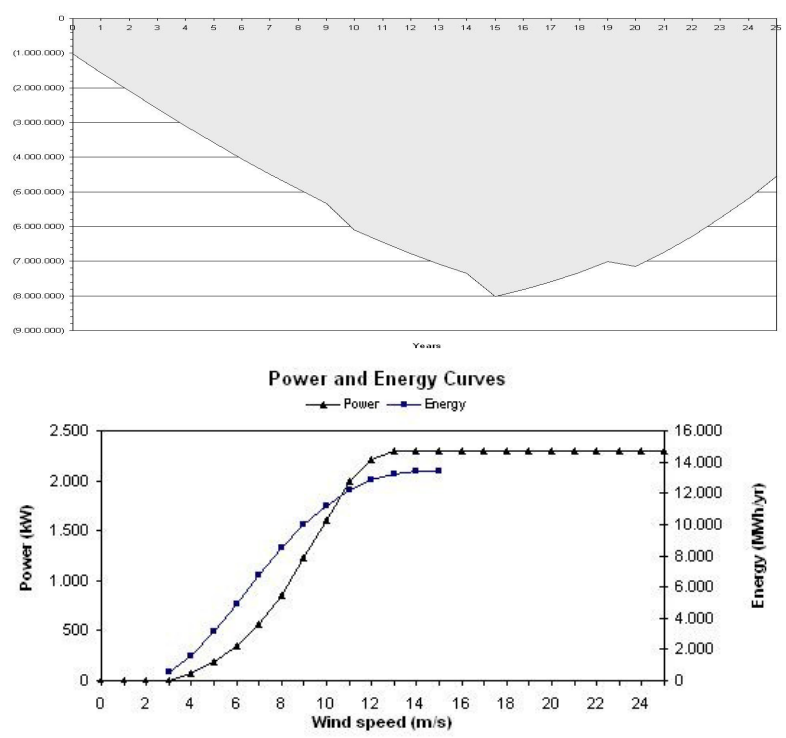

Fig 3. Cumulative Cash Flows and Dependence between the produced energy and wind speed

Nowadays there is implemented a wind turbine, equipped with an original rotor, REVIR able to start at low intensity of the wind velocity, around $0.4 \mathrm{~m} / \mathrm{s}$. It represents a Romanian brevet, under licence. A mixed collective of engineers from the ICPE Institute and University Politehnica of Bucharest creates it. Accordingly, to the numerical modeling and of the theoretical calculation a wind rotor with two blades with variable thickness was realized; such a wind profile is presented in Fig.4.

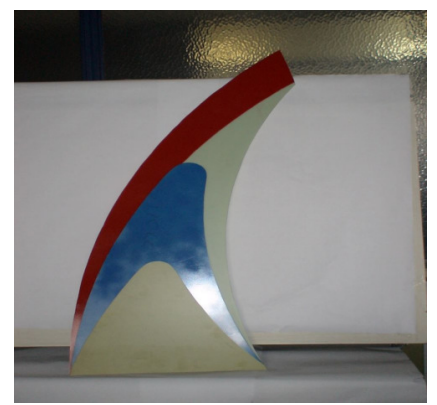

Fig 4. The REVIR wind blade 


\section{Experimental data for the new type of rotor, the REVIR}

There are presented some experimental data obtained with the original rotor, variant with two rotor blades, at a low wind velocity, between $0,5-4,5 \mathrm{~m} / \mathrm{s}$, medium between 4,6-10 $\mathrm{m} / \mathrm{s}$ and high values of wind velocities between $10,1-24 \mathrm{~m} / \mathrm{s}$, Fig.5. The rotor, nowadays tested, into the pilot station has three REVIR blades.
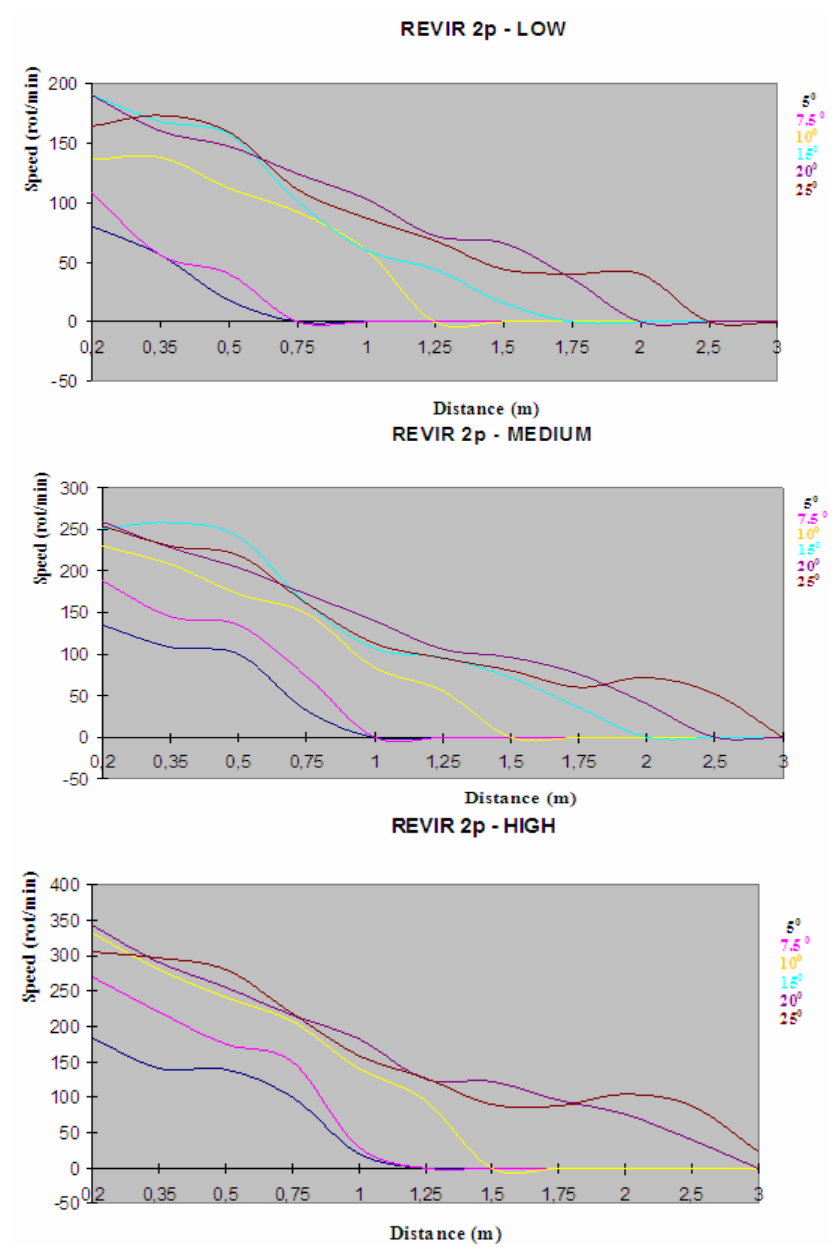

Fin 5. Experimental results with REVIR wind rotor

The fundamental advantage of this type of rotor is the fact that it starts to rotate at low intensity of the wind, at values between $1.3-1.5 \mathrm{~m} / \mathrm{s}$, compared with the classical type of rotors who start in the best case at $3.5-4 \mathrm{~m} / \mathrm{s}$. The rotor represents a Romanian Patent. Supplementary, the REVIR rotor has received the Gold Medal at International Salon of Inventions from Geneva, 2007.

This type of turbine may be implemented in areas with a medium intensity of the wind due to the small velocity necessary to start rotates. In the solution with the mobile rotor blades at a high value of wind velocity, the rotor stays out of the load [9].

As a further development, we intend extending the mechanical part, as to obtain a constant rotation at different values of the wind intensity, meaning an extension of zones with a rapid variation of the wind intensity.
Consequently, taking into account the number of the rotor rotations may be evaluated the produced energy [11]. New models of rotors with three and four blades are realized in University laboratory.

Moreover, must be mentioned some observations between the classic wind rotor and the REVIR wind rotor:

- the classic rotor, at values of the wind intensity under $3 \mathrm{~m} / \mathrm{s}$ does not start; at a velocity over $3.3 \mathrm{~m} / \mathrm{s}$ the velocity at the rotor axis is around $550 \mathrm{rot} / \mathrm{min}$, but only for the high value of the incidence angle, between $10^{\circ}-15^{\circ}$. Ordinarily, it is recommended for such applications somewhere between $5^{\circ}-9^{\circ}$. The rotation decreases quickly with decreasing of the wind intensity.

- the REVIR rotor starts to rotate at low values of wind velocity, in some conditions even under $1 \mathrm{~m} / \mathrm{s}$, but at incidence angles of $20^{\circ}-30^{\circ}$. For a velocity of the wind between $3,5-4,2 \mathrm{~m} / \mathrm{s}$ the rotor rotation at axis remains almost constant, due to the blades configuration, even when the wind velocity decreases. The decreasing of the rotation is smaller, compared with the classic rotors.

\section{V.THE SMALL HYDRO-POWER SYSTEM}

The energy produced by the hydropower systems represents the principal utilization of the renewable resources. In Romania in present are more than 600 small hydro-power plants (SHP); more than 300 belong to SC Hidroelectrica SA, 200 to the National Authority Romanian Waters and the rest of the local administration [4].

Accordingly, the geographical advantages of the selected area for the solar and wind developments, the implementation of the pilot station was selected near an existent SHP Racaciuni, in the south part of Moldavia.

\section{A. Energetic efficient management}

Firstly, it is necessary to assure a management of the local energy demand DSM-Demand - Side Management. DMS assumes to identify and implement initiatives that improve utilization of the production capacity of the provider by altering with the characteristics of the energy demand. Modern tracking in real time of the behavior of the electricity network, with automatic SHP may be realized with software, providing an energy management. They allow the optimal leadership of the local/regional energy systems with higher energetic efficiency by ensuring the demands of the power quality electricity, supplied to consumers [5].

Intelligent monitoring systems ensures

- Remote reading of energy meters

- Centralized load control consumer by changing the load curve of the system

- Control of the disturbances introduced by the consumer network

- Centralized control for changing tariffs / prices

- Automated billing 
TABLE V.

ADVANTAGES OF THE ENERGETIC MANAGEMENT

\begin{tabular}{|l|l|}
\hline \multicolumn{1}{|c|}{ Producing } & \multicolumn{1}{c|}{ Consuming } \\
\hline $\begin{array}{l}\text { Ensuring adequate energy pro- } \\
\text { duction }\end{array}$ & $\begin{array}{l}\text { Quantities accepted } \\
\text { Control required }\end{array}$ \\
\hline $\begin{array}{l}\text { Flexibility of the offer in plan- } \\
\text { ning/production in the medium } \\
\text { and long term }\end{array}$ & $\begin{array}{l}\text { Knowledge / estimation of the } \\
\text { consumption trends }\end{array}$ \\
\hline $\begin{array}{l}\text { Optimizing the production } \\
\text { planning and supply }\end{array}$ & $\begin{array}{l}\text { - demand forecast - daily load } \\
\text { curves, week timetables } \\
\text {-flattening, filling gaps, transfer } \\
\text { the load from peak hours in the } \\
\text { hours of low demand }\end{array}$ \\
\hline $\begin{array}{l}\text { Compliance with quality indi- } \\
\text { cators correlated with consumer } \\
\text { input frequency disturbances, } \\
\text { temporary and transient surges, } \\
\text { supply disruptions }\end{array}$ & $\begin{array}{l}\text { Secondary indicators - rapid } \\
\text { fluctuations of voltage unbal- } \\
\text { ance, non-sinusoidal regimes }\end{array}$ \\
\hline $\begin{array}{l}\text { Dispatching and common } \\
\text { commercial offer }\end{array}$ & \begin{tabular}{l} 
monitoring of the tender request \\
\hline
\end{tabular} \\
\hline
\end{tabular}

To determine the annual average flow $\mathrm{Q}$ in the natural conditions, are used for correlating, at least three methods

\section{B. Energetic efficient management}

\section{B1. Utilization of the graph curves}

The method assures a connection between the multilayer average flow $(\mathrm{mm})$ and the average altitude. It is determined from the statistic data, from the catchments area $\mathrm{F}\left(\mathrm{Km}^{2}\right)$ connected with the average elevation of the basin $\mathrm{H}_{\mathrm{m}}(\mathrm{m})$. From the local map, it is determined the watercourses. Then, the multi-annual average of flow, calculated, where $\mathrm{T}$ is the time reference(s), is:

$$
Q_{m}\left(\mathrm{~m}^{3} / \mathrm{s}\right)=\frac{F\left(\mathrm{~km}^{2}\right) \times H_{m}(\mathrm{~mm}) \times 10^{-3}}{T(\mathrm{~s})}
$$

\section{B2. The method of the water balance}

Notations: $Y_{0}$ - the Annual average of river leakage, $S_{0}$ the surface Leakage, $\mathrm{U}_{0}$ - the Groundwater flow, $\mathrm{W}_{0}$ - the Wetting annual average of soil, $\mathrm{K}_{\mathrm{u} 0}$ - the Feed rate underground river.

$$
\begin{aligned}
& K_{u 0}=\frac{U_{0}(1)}{W_{0}} \Rightarrow Y_{0}=S_{0}+K_{u 0} \times W_{0} \\
& Y_{0}=S_{0}+U_{0}
\end{aligned}
$$

By using of the map of the territorial distribution of the component of the hydraulic balance, are obtained the corresponding values of the geographical position of the surface stream. As it follows:

- From the full surface runoff layer, it was extracted the value $\mathrm{S}_{0}(\mathrm{~mm})$.

- From the map of the geographical spread of the total soil, the moisture, it is extracted the value $\mathrm{W}_{0}(\mathrm{~mm})$.

- From the map of the power, the underground distribution coefficient is extracted the $\mathrm{K}_{\mathrm{u} 0}$ value.

By substituting, the values extracted in the above point $\mathrm{Y}_{0}$ may be estimated the average annual average flow.

\section{B2. The method of hydrologic balance}

Notations: $\mathrm{X}_{0}$ - average annual rainfall, $\mathrm{Y}_{0}$ - average annual flow, $Z_{0}$ - the annual average evaporation from land, surface evaporate-transpiration

$$
X_{0}=Y_{0}+Z_{0}(1) \Rightarrow Y_{0}=X_{0}-Z_{0}
$$

From the precipitations map is extracted the value $\mathrm{X}_{0}$. From the map distribution, of the total average of evaporation-transpiration, it is extracted the value $Z_{0}$. By introducing the extracted values in (3) is obtained the average annual runoff value $Y_{0}(\mathrm{~mm})$ and determined the annual average flow rate.

\section{Numerical modeling}

For the numerical modeling, was selected a part of the Siret river basin. Here it was applied the software Vapidro-ASTE, which generates a hydro-graphic network of the area of interest. The model uses the local terrain, implemented as input data, based on the existent geographic and hydrologic maps of the field.

The software can locate the best position of the water supply, for the realization of the hydropower plant, optimized technically and economic. The selected area is the Siret basin, in the energetic sector Bistrita and Trotus, between cities Bacau and Adjud.

Consequently, the necessary data are introduced for the financial analysis: the equipment costs, the maintenance costs, the costs of execution, as to obtain the cost-benefit analysis. The water volume flowing along the riverbed is determined by numerical modeling, taking into account the soil roughness, the soil incidence, rainfall intensity, temperature, etc. Moreover, it is analyzed the existing situation for the local, realized SHP.

By numerical modeling, it is also estimated the installed capacity for further possible SHP, the total annual energy able to be produced, the total costs for realization, the benefits, the cost-benefit analysis and finally the amortization period. All the economical optimizations are performed and the results are transposed into a graphic form. Technical and economic optimization results consist of the most favorable locations for positioning of the hydroelectric power plant and of the water intakes for the new SHP, as mentioned in Fig.6.
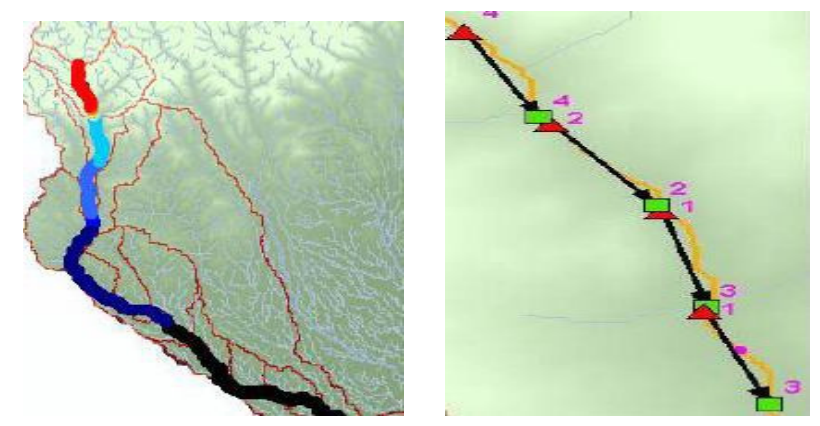

Fig 6. The modeled utile fall and the possible developments 
Note: with green are noted the possible future hydropower plant and with red the water intakes.

From the Siret river basin, they are selected three rivers for modeling; the initial database are presented in Table VI and the obtained results in Table VII [6].

TABLE VI.

INITIAL PARAMETERS

\begin{tabular}{|c|c|c|c|c|c|}
\hline $\mathrm{Nr}$ & Data parameters & Method & R1 & R2 & R3 \\
\hline 1 & Area $\mathrm{F}_{\mathrm{BH}}\left[\mathrm{km}^{2}\right]$ & Plane map & 10 & 16 & 18 \\
\hline 2 & $\begin{array}{l}\text { Average Altitude } \\
\mathrm{H}_{\mathrm{m}}[\mathrm{m}]\end{array}$ & Direct read & 1000 & 120 & 150 \\
\hline 3 & $\begin{array}{l}\text { Average Length } \\
\mathrm{L}_{\mathrm{m}}[\mathrm{m}]\end{array}$ & $\begin{array}{l}\text { Direct meas- } \\
\text { urement }\end{array}$ & 5100 & 380 & 440 \\
\hline 4 & $\begin{array}{l}\text { Cote upstream } \\
\mathrm{C}_{\mathrm{am}}[\mathrm{mdM}]\end{array}$ & Direct read & 810 & 850 & 800 \\
\hline 5 & $\begin{array}{l}\text { Cote upstream } \\
\mathrm{C}_{\mathrm{av}}[\mathrm{mdM}]\end{array}$ & Direct read & 608 & 700 & 600 \\
\hline 6 & Crude fall $\mathrm{H}_{\mathrm{br}}[\mathrm{m}]$ & Calculation & - & - & - \\
\hline 7 & $\begin{array}{l}\text { Annual average } \\
\text { flow } Y_{0}[\mathrm{~mm}]\end{array}$ & Direct read $^{1}$ & 800 & 840 & 820 \\
\hline 8 & $\begin{array}{l}\text { Surface leakage } \\
\mathrm{S}_{0}[\mathrm{~mm}]\end{array}$ & Direct read ${ }^{2}$ & $\begin{array}{l}400 \\
500\end{array}$ & $\begin{array}{l}440 \\
510\end{array}$ & $\begin{array}{l}420 \\
500\end{array}$ \\
\hline \multirow[t]{2}{*}{9} & Evaporation tran- & & 280 & 280 & 280 \\
\hline & spiration $Z_{0}[\mathrm{~mm}]$ & Direct read ${ }^{3}$ & 340 & 300 & 320 \\
\hline \multirow[t]{2}{*}{10} & The total wet soil & & 710 & 700 & 700 \\
\hline & $\mathrm{W}_{0}[\mathrm{~mm}]$ & Direct read ${ }^{4}$ & 700 & 700 & 700 \\
\hline \multirow[t]{2}{*}{11} & Underground & & 0,36 & 0,4 & 0,4 \\
\hline & $\begin{array}{l}\text { alimentation co- } \\
\text { efficient } \mathrm{K}_{\mathrm{u} 0}[\mathrm{~mm}]\end{array}$ & Direct read ${ }^{5}$ & 0,4 & 0,4 & 0,4 \\
\hline 12 & $\begin{array}{l}\text { Average annual } \\
\text { rainfall } \mathrm{X}_{0}[\mathrm{~mm}]\end{array}$ & Direct resd & 1000 & 110 & 120 \\
\hline 13 & $\begin{array}{l}\text { Min. leakage } \mathrm{q}_{\min } \\
{\left[1 / \mathrm{sxkm}^{2}\right]}\end{array}$ & Direct read ${ }^{5}$ & 4,8 & 5,5 & 4,2 \\
\hline 14 & $\begin{array}{l}\text { Minimum } \\
\mathrm{Q}_{\min }\left[\mathrm{m}^{3} / \mathrm{s}\right]\end{array}$ & Calculation & 0,04 & 0,08 & 0,08 \\
\hline
\end{tabular}

Where: ${ }^{1}=\mathrm{Y}_{0}=\mathrm{f}\left(\mathrm{H}_{\mathrm{m}}\right),^{2}=\mathrm{S}_{0}=\mathrm{f}\left(\mathrm{H}_{\mathrm{m}}\right),{ }^{3}=\mathrm{Z}_{0}=\mathrm{f}\left(\mathrm{H}_{\mathrm{m}}\right),{ }^{3}=\mathrm{W}_{0}=\mathrm{f}\left(\mathrm{H}_{\mathrm{m}}\right)$ ${ }^{4}=\mathrm{K}_{\mathrm{u} 0}=\mathrm{f}\left(\mathrm{H}_{\mathrm{m}}\right),{ }^{5}=\mathrm{q}_{\min }=\mathrm{f}\left(\mathrm{H}_{\mathrm{m}}\right)$

TABLE VII.

OBTAINED RESULTS

\begin{tabular}{|c|c|c|c|c|c|}
\hline $\mathrm{Nr}$ & \begin{tabular}{|c|} 
Data \\
parameters
\end{tabular} & R1 & $\mathbf{R 2}$ & R3 & Formula \\
\hline 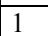 & $\mathrm{F}_{\mathrm{BH}}\left[\mathrm{km}^{2}\right]$ & 10 & 16 & 18 & Yes, by statistic data \\
\hline 2 & $\mathrm{H}_{\text {med }}[\mathrm{m}]$ & 1300 & 1400 & 1350 & Yes, by statistic data \\
\hline 3 & $\mathrm{~L}_{\mathrm{m}}[\mathrm{m}]$ & 4700 & 3500 & 400 & $\begin{array}{l}\text { Correlated with decreas- } \\
\text { ing altitude }\end{array}$ \\
\hline 4 & $\mathrm{C}_{\mathrm{am}}[\mathrm{mdM}]$ & 790 & 820 & 802 & Confirmed GPS \\
\hline 5 & $\mathrm{C}_{\mathrm{av}}[\mathrm{mdM}]$ & 580 & 670 & 610 & Confirmed GPS \\
\hline 6 & $\mathrm{H}_{\mathrm{br}}[\mathrm{m}]$ & 210 & 150 & 192 & $\mathrm{H}_{\mathrm{br}}=\mathrm{C}_{\mathrm{am}}-\mathrm{C}_{\mathrm{av}}$ \\
\hline 7 & $\mathrm{Y}_{0}[\mathrm{~mm}]$ & 800 & 780 & 790 & Adopted minimum value \\
\hline 8 & $\mathrm{~S}_{0}[\mathrm{~mm}]$ & 450 & 470 & 450 & Adopted average value \\
\hline 9 & $\mathrm{Z}_{0}[\mathrm{~mm}]$ & 290 & 280 & 280 & Adopted average value \\
\hline 10 & $\mathrm{~W}_{0}[\mathrm{~mm}]$ & 700 & 704 & 706 & Confirmed value \\
\hline 11 & $\mathrm{~K}_{\mathrm{u} 0}[\mathrm{~mm}]$ & 0,38 & 0,4 & 0,39 & Adopted average value \\
\hline 12 & $\mathrm{X}_{0}[\mathrm{~mm}]$ & 1100 & 1050 & 1100 & Consider arithmetic mean \\
\hline \multirow[t]{3}{*}{13} & \multirow{3}{*}{$\begin{array}{l}\text { Annual aver- } \\
\text { age flow } \\
\mathrm{Q}_{\mathrm{m}}\left[\mathrm{m}^{3} / \mathrm{s}\right]\end{array}$} & \begin{tabular}{l|l}
$\left.{ }^{1}\right)$ & 0,253 \\
\end{tabular} & 0,405 & 0,456 & 1) \\
\hline & & 2) 0,220 & 0,380 & 0,418 & 2 ) \\
\hline & & 3) 0,256 & 0,410 & 0,462 & 3 \\
\hline 14 & $\mathrm{Q}_{\min }\left[\mathrm{m}^{3} / \mathrm{s}\right]$ & 0,049 & 0,088 & 0,0756 & Calculation \\
\hline 15 & $\begin{array}{l}\text { Max. spe- } \\
\text { cific flow } \\
\text { rate insur- } \\
\text { ance of } 1 \%\end{array}$ & 71 & 113 & 27 & $\begin{array}{l}\text { Extrapolation } \\
\mathrm{Q}_{\max }=71001 / \mathrm{s} \mathrm{x} \mathrm{km}{ }^{2}\end{array}$ \\
\hline
\end{tabular}

$\left.{ }^{1}\right)$ Direct Method

$$
\begin{aligned}
& \text { Ts }=(8760 \times 3600)[\mathrm{s}] \\
& \frac{F\left(\mathrm{~km}^{2}\right) \times Y_{0}(\mathrm{~mm}) \times 10^{3}}{T_{s}(\mathrm{~s})}
\end{aligned}
$$

$\left.{ }^{2}\right)$ Water balance method-Variant I

$$
\frac{F\left(\mathrm{~km}^{2}\right) \times\left(S_{0}+K_{u 0} \times W_{0}\right) \times 10^{3}}{T_{s}(s)}
$$

$\left.{ }^{3}\right)$ Water balance method-Variant II

$$
\begin{aligned}
& \frac{F\left(\mathrm{~km}^{2}\right) \times\left(X_{0}-Z_{0}\right) \times 10^{3}}{T_{s}(s)} \\
& q_{\text {min }}\left(l / s \times \mathrm{km}^{2}\right) \times F\left(\mathrm{~km}^{2}\right) \times 10^{-3}
\end{aligned}
$$

Where $\mathrm{S}_{0}$ - Surface leakage $\mathrm{S}_{0}[\mathrm{~mm}], \mathrm{F}$ - the hydrographic basin $F[\mathrm{~km} 2], T s(\mathrm{~s})$ - significant time value, Evaporation -transpiration $Z_{0}[\mathrm{~mm}]$, Average annual rainfall $\mathrm{X}_{0}[\mathrm{~mm}]$, Minimum leakage $\mathrm{q}_{\min }\left[1 / \mathrm{sxkm}^{2}\right], \mathrm{Y}_{0}[\mathrm{~mm}]-$ the Annual average flow layer, $\mathrm{W}_{0}[\mathrm{~mm}]-$ The total wet soil, Underground alimentation coefficient $\mathrm{K}_{\mathrm{u} 0}[\mathrm{~mm}]$, [7].

In Figs.7-9 are presented some obtained results [8].

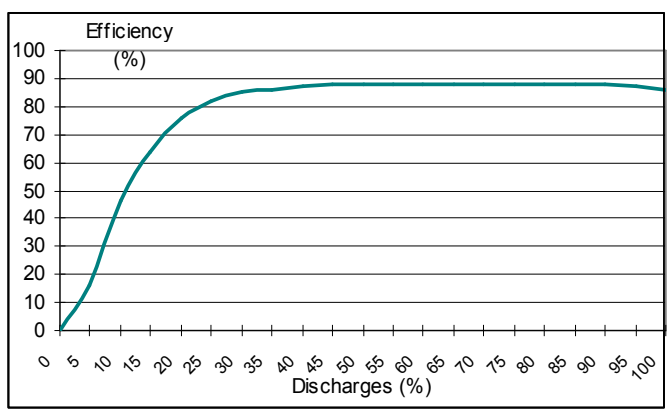

Fig 7. The efficiency curve

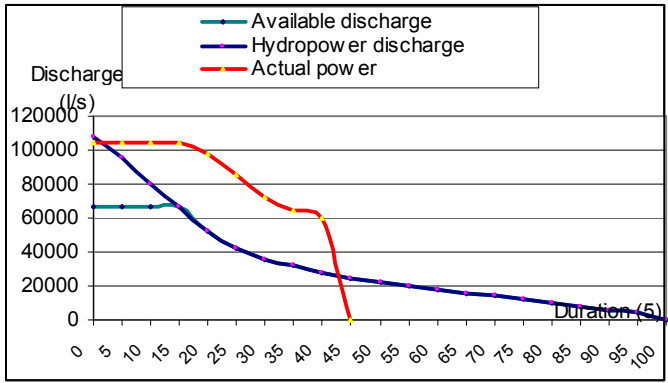

Fig 8 . The flow and the Duration curves

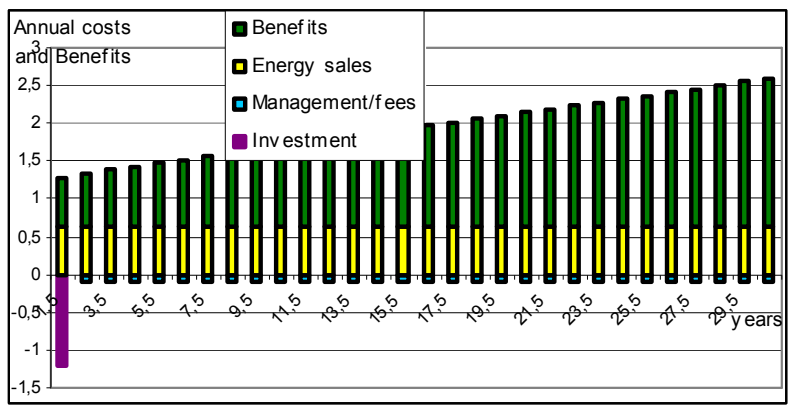

Fig 9. The Benefits and the Costs breakdown 


\section{CONCLUSION}

SHP represents a distinct category, both in terms of technical and financial as to use the relatively small hydraulic energy resources. They are ideal for promoting the interests of the local communities and industry, for small and medium entrepreneurs. It is a solution to recovery of a body of water and has many advantages:

- Small and medium unit $<10$ MW that can be controlled, constructed, and operated in less than two years, with the immediate production of the income

- Reduce the environmental impact

- Suited to the industrial, commercial, and residential area of the network

- The investment project can be developed modular as to cover the increasing of the consumption

- The Complexity and simple maintenance

- The cost of a unit of electricity can be determined with certain accuracy.

- Is ideal for application of the control technology systems, smart meters at customers premises, points of connection in the main nodes, to determine the flow rate

- The real-time charging and billing

By modeling with this software, Valpidro-Aste it can be achieved [10]:

- Identify the optimal locations as to determine the number possible SHP to be built

- The Yearly analysis of the cost-benefits

- The Model is adaptive at local topography

- Estimate the technical and economic feasibility

The wind rotor mentioned in this article, presented, and aerodynamic tested, in concrete natural conditions is a Romanian patent, awarded also and at the Inventors Fair in Geneva. The rotor has a great advantage by starting to rotate at low wind speeds. It may be used in areas where the wind intensity is lower.

For the implementation of the Pilot Station, it was selected an area, with an existent SHP Racaciuni, in the south part of Moldavia. The area is also favorable for utilization of solar and wind renewable resources. To test is realized a Solar Power Plant made by 10 panels, each one of $1.4 \mathrm{KW}$, meaning $14 \mathrm{KW}$, capable of delivering electric energy if necessary. Regarding the wind potential energy, the Pilot plant is placed into the Siret basin. The wind measurements made into the selected area confirm values of wind velocity constant between $6.8-18.5 \mathrm{~m} / \mathrm{s}$, at $60 \mathrm{~m}$ above the ground. Even in the hot days of summer, the wind is blowing. Another advantage of the selected area is represented by the relative constancy of the wind direction: 6 months it blows from N-E to S-W and 6 months from S-W to N-E. In present, there is also tested the prototype REVIR rotor. For the Pilot Station was implemented a wind turbine model EWT DW61, of $1 \mathrm{MW}$. A real advantage of the position of the Pilot station con- sists of the existent electric line of transport, near the Pilot Stations, due to the existing SHP [12].

Finally, in Fig. 10 are presented images with the Racaciuni SHP, before, and after rehabilitation.
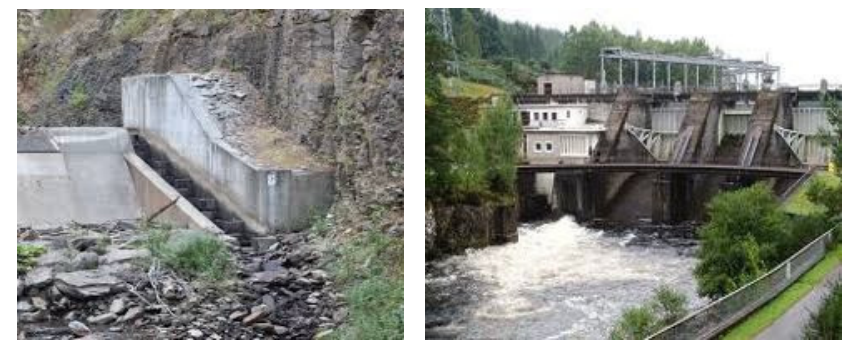

Fig 10. The SHP Racaciuni before, and after rehabilitation

\section{ACKNOWLEDGMENT}

The author wishes to thank colleagues from SC Hidroelectrica SA who funded the measurements and the elaboration of the efficiency study of the wind farm, and finally the realization of the feasibility study and realization of the Pilot Station. They trusted to let our collective rehabilitate the SHP Racaciuni.

I also thank Prof. PhD. Eng. Ion Seteanu, my first professor in Numerical modeling, who showed me fifteen years ago, the importance of the renewable resources, and he helped me in my early research in this area.

\section{REFERENCES}

[1] B. Popa, "Hidroenergetica, Microhidrocentrale", Hydroenergy, Small hydropower plants, University Politehnica of Bucharest, 2009.

[2] A. Osinowo, E. C. Okogbue, all, "Analysis of Global Irradiance over Climatic Zones in Nigeria for solar Energy Applications", Journal of Solar Energy, Vol. 2015, ID 819307.

[3] V. Khare, S. Nema, P. Baredar, "Optimization of the hybrid renewable energy system by HOMER, PSO and CPSO for the study area", Int. Journal of Sustainable Energy, vol. 36, Issue 4, pp. 326-343, 2015, http://dx.doi.org./10.1080/144786451.2015.1017500.

[4] P. G. Oguntunde, A. Babatunde, "A numerical modeling study of the hydro-climatology of the Niger River basin, West Africa" Hydrological Sciences Journal, vol. 61, 2016 Issue 1, doi: 10.1080/02626667.2014.980260.

[5]. M. Cruz-Zambrano, C. Corchero, "The impact of commercial quality on electricity consumer satisfaction", Congreso Asociación Española para la Economia Energética", València: 2013, p. 1-12., https:/upc.edu/bitstream/handle/2117/19561/047.pdf.

[6] R. J. Stockdale, S. J. McLelland, R. Middleton, T.J. Coulthard, "Measuring river velocities using GPS River Flow Tracers (GRiFTers)", Wiley Online Library, 2007, DOI: 10.1002/esp.1614.

[7] W-C. Chu, Yi-P. Chen, T-H. Lin, "The Competitive Model Based on the Demand Response in the Off-Peak Period for the Taipower System", IEEE Transactions on Industry, Vol.44, Issue: 4, 2008, doi: 10.1109/TIA.2008.926063.

[8] A. K. Verma, V. Vijay Venu, "A modeling paradigm for extending wellbeing analysis to a composite bilateral contract market", PSCE, 2009, IEEE, doi: 10.1109/PSCE.2009.4839959.

[9] M. Juresko, M. Pawlak, A. Mezyk, "Optimisation of wind turbine blades”, Journal of Materials Processing Technology 167 463-471, 2005, Elsevier .

[10] P. Garcia-Navarro, E. Playan, Numerical Modeling of Hydrodynamics of Water Resources", International Workshop on Numerical Modelling of Hydrodynamics foe Water resources, Zaragosa, Spain, ISBN 13:978-415-44056-1 (hbk).

[11] S. Lee, "Numerical modeling of wind turbine aerodynamic noise in the time domain", Journal of Acoustic Society, 2013, Doi: $10.1121 / 1.4774072$. 


\section{Extracting Acoustic Features of Japanese Speech to Classify Emotions}

\author{
Takashi Yamazaki \\ Human System Science \\ Tokyo Institute of Technolgy \\ Tokyo 152-8552, Japan \\ Email: yamazaki@nk.ict.e.titech.ac.jp
}

\author{
Minoru Nakayama \\ Information and Communications Engineering \\ Tokyo Institute of Technolgy \\ Tokyo 152-8552, Japan \\ Email: nakayama@ict.e.titech.ac.jp
}

\begin{abstract}
An emotional detection technique which extracts acoustic features from audio recordings of speech was developed. Though the formant frequency of individual voices may contribute to emotional variations in speech, the differences between vowels have an influence on feature extraction. To reduce the influence, a simple procedure was developed to extract relative features of vowels for every mora. The estimation performance of this emotional detection technique was improved by $11 \%$ using relative formant frequencies instead of formant frequencies. The strengths of some emotional expressions were also reflected in some features. The effectiveness of using acoustic features to estimate the category of emotionally inflected speech was confirmed.
\end{abstract}

\section{INTRODUCTION}

$\mathbf{E}$ MOTIONAL presentation is a key factor for sharing information in human communications. Its importance is not only for human-human communications, but for humanrobot communications and human computer communications as well. Possible resources for estimating the emotional state of people using natural verbal communication habits while speaking is verbal information (context of speech), acoustic feature of voices (speaking tone), and also visual features (facial expressions, behavioral actions).

Uemura [1] compared the degree of ability to detect emotional inflections using these resources, and suggested the order of intensity of presenting emotions by level of importance was voice audio $>$ facial expressions $>$ verbal contents. Therefore, any systems which include communication robots may be able to recognize the emotional states of speakers and may well play an important role as a communication partner once features of emotional expressions can be extracted and utilized. Although some robots have already been designed to recognize or present emotions during communications with humans using features of language and behavior, more specific features are required to improve their performance. As voice audio is the effective source of providing emotional inflections, acoustic feature of voices should be used for the communication aids. The appropriate technique to extract significant features from voice audio and their applications are required. When these techniques were developed, robots may be possible to detect emotional states of a human speaker as well as synthesizing an appropriate speech in response to human's emotional conditions. These fundamental techniques, in particular, emotional recognition and presentation in voice audio, should be developed.

The emotional information processing for speech as voice audio is still tough work in comparing with speech recognition and speech synthesis for even a specific speaker. As the problems are caused by phonological issue of language, additional lexical information are also used for predicting emotional state [2]. Previous phonological studies suggest that phonological components can represent factors of emotions [3]. The formant frequency is one of the features of phonological information of speech. When the formant frequency was used to predict emotion, the accuracy was $90 \%$ when the spoken words were identical [3]. Therefore, the formant frequency is confirmed as being one of the significant features which can be used to detect the emotional state of the speaker. However, performance for mixed sentences which contain several vowels decreases 15-20\% [3]. Since formant frequencies for each vowel are different, phonological information depends on the combinations of vowels in sentences.

As mixed sentences are naturally used in our communications, formant frequency should be employed as a feature of speech when considering vowels in mixed sentences. Therefore, an appropriate procedure for processing phonological information from mixed sentences is required in order to improve the performance of predicting emotional states, and this technique may also be capable of providing features of phonological information for use with the synthesis of voices containing emotions [4].

This paper will address the development of a procedure for the extraction of formant frquencies as phonological information of speech from spoken words which contain multiple vowels, and the effectiveness of these features is evaluated in order to predict the emotional state of mixed sentences. The details are as follows:

1) To develop a procedure for extracting acoustic features using the formant frequencies of every vowel, and to also develop a procedure for specifying the emotional state (emotional category) of speech using the extracted features.

2) The contributions of each acoustic feature are compared in order to determine the key information necessary for 
TABLE I

MEAN F1S [Hz] AND EXAMPLES OF RELATIVE RATIOS

\begin{tabular}{|l||c|c|c|c|}
\hline Emotions & $\mathrm{Ka}$ & $\mathrm{Ze}$ & $\mathrm{Bu}$ & $\mathrm{Ki}$ \\
\hline \hline Neutral & 450.5 & 488.8 & 384.3 & 299.5 \\
Surprised (Strength=1) & 521.0 & 509.3 & 398.1 & 409.7 \\
\hline Relative ratio & 1.16 & 1.04 & 1.04 & 1.37 \\
\hline
\end{tabular}

TABLE II

RESULTS OF F-TEST OF VOWELS (EXP. VALUES)

\begin{tabular}{|l|cccc|}
\hline Features & df & MSE & F & $p$ \\
\hline Fundamental Freq. & $(4,96)$ & 4198.97 & 2.10 & n.s. \\
Power & $(4,96)$ & 28.34 & 4.25 & $<0.01$ \\
F1 & $(4,96)$ & 17272.32 & 17.48 & $<0.01$ \\
F2 & $(4,96)$ & 134190.75 & 20.69 & $<0.01$ \\
F3 & $(4,96)$ & 116989.82 & 1.24 & n.s. \\
\hline
\end{tabular}

prediction of emotional states and strength levels of an emotion.

The results of these analyses will provide basic information to be used to produce emotionally inflected speech using speech synthesis engineering technologies.

\section{METHOD}

\section{A. Source of speech sound}

An audio processing procedure was developed using a set of voice audio corpus for short speech phrases (a voice audio corpus which consists of short phrases spoken by voice actors/actresses). This corpus was originally developed to record emotionally inflected voice audios (voice audio spoken by voice actors/actresses portraying emotions) in online gaming using voice chats which are used to compose an audio library [6]. Features of acoustic characteristics used to estimate emotional states are comparable when extracted from phrases or whole sentences [5]. In addition, two male and female voice actors recorded emotionally inflected voice audios of some phrases from chats from online games. The categories of emotions are based on Ekman's 8 basic emotions [7]. All phrases are presented and recorded in a neutral tone and 3 strength levels of every emotion (for example, very happy, moderately happy, and slightly happy). Though the extracted phrases have no meanings as independent terms, the emotional impressions of the phrases can be easily recognized.

\section{B. Analysis of audio data}

Three phrases were randomly selected from the corpus and used as a set of test data for four emotions: "happy", "angry", "sad" and "surprised". These were the same emotions that were used in a previous study [4], because the emotional features may be present in those categories of emotions to a significant degree. The overall number of test phrases is 48 , which consists 3 phrases $\times 4$ emotions $\times 4$ levels of emotional strength: neutral, and strength levels $1 \sim 3$.

Audio data was analyzed using software known as "Praat" [8]. Seven acoustic features, such as fundamental frequency,

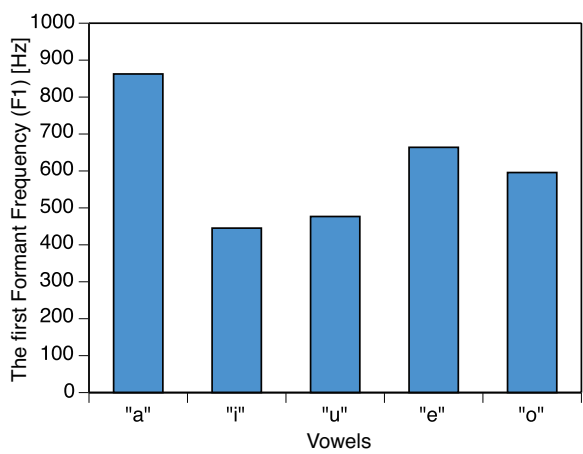

Fig. 1. Comparison of mean F1s between vowels

TABLE III

RESULTS OF F-TEST OF VOWELS (RELATIVE RATIOS)

\begin{tabular}{|l|cccc|}
\hline Features & df & MSE & F & $p$ \\
\hline Fundamental Freq. & $(4,96)$ & 0.06 & 1.92 & n.s. \\
Power & $(4,96)$ & 0.01 & 0.05 & n.s. \\
F1 & $(4,96)$ & 0.06 & 5.04 & $<0.01$ \\
F2 & $(4,96)$ & 0.15 & 3.46 & n.s. \\
F3 & $(4,96)$ & 0.01 & 0.45 & n.s. \\
\hline
\end{tabular}

power, duration, speech speed, and 3 formant frequencies (F1F3: the first, second and third frequencies as the formant frequency) were extracted manually.

\section{Acoustic Features}

\section{A. Features between vowels}

To compare the lexical features of phrases, the "mora" unit was introduced. The mora is a unit of lexical sound and corresponds with a piece of speech that includes a vowel. Therefore, for every mora the acoustic features mentioned above were extracted, except for duration and speed of speech. Exceptions were evaluated phrase by phrase.

Though the mora can be a useful unit for acoustic features, means of features of mora are used for evaluation, as the duration of a mora is too short. Therefore, an appropriate procedure that compensates for this is required in order to consider combinations of vowels.

Here, an example of analysis of the phrase "KaZeBuKi", which consists of 4 moras, is shown in Table I. Table I shows F1 frequencies for neutral and surprised speech (strength level=1) for every mora. In comparing the frequencies of the two speech conditions, the differences show the contributions of emotionally inflected speech.

In order to examine the factors for 5 categories of vowels using acoustic features, one-way ANOVA as F-test was conducted. This analysis was conducted using features of every mora, and the number of samples for each category differed. The strength of emotions was ignored during this analysis.

The $\mathrm{F}$ values for the main effects of variations, ratios of two variances, and their degrees of freedom (df) are summarized in Table II, and the effects are significant for power, F1 and F2 $(p<0.01)$. The results confirm the effects of acoustic 


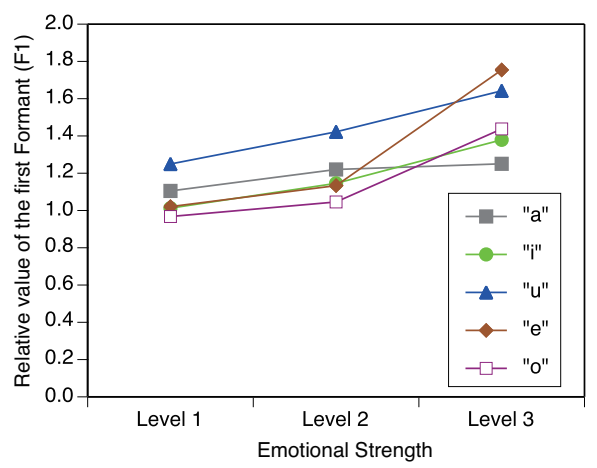

Fig. 2. Comparison of F1 relative ratios between vowels

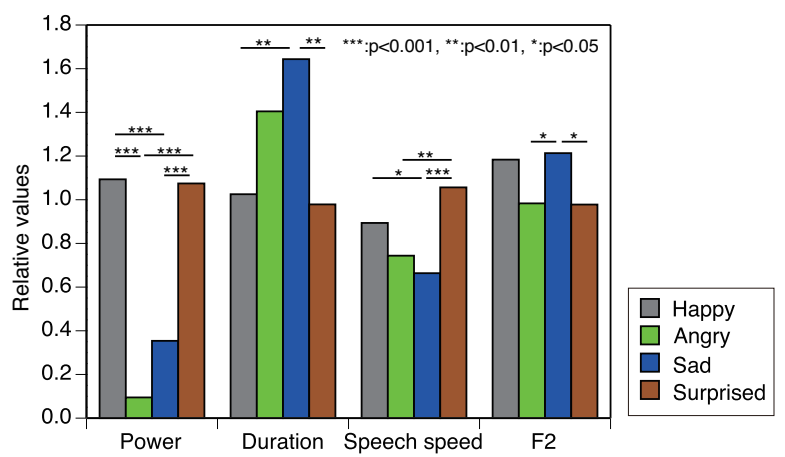

Fig. 3. Comparison of feature values between vowels

features between vowels. Figure 1 shows mean frequencies of F1 between vowels, and the results of sub-effect tests are presented. There are some significant differences between vowels, and the tests have confirmed that the fequency of the vowel " $\mathrm{a}$ " is the highest.

\section{B. Relative features}

Even with neutral emotion, the acoustic features between vowel categories are different. The relative values of features of emotional speech features which use neutral expression were introduced. The relative values as "relative ratio" are displayed in the third line in Table I.

The same analysis was applied to relative values. The results are shown in Table III. The Table shows that the factor for vowels is significant for F1 $(p<0.01)$, however. Mean relative values of $\mathrm{F} 1$ between levels of emotional strength are summarized in Figure 2. Some changes are observed at the level 3 of emotional strength. For the further analysis, multiple comparison between vowels was conducted as the sub-effect tests. The results show that there are no significant differences between vowel categories. Therefore, the factor of vowels with acoustic features decreased when the relative values were introduced.

\section{Differences in features between emotional categories}

The overall features of phrases can be generated using relative features. To extract the differences between the types
TABLE IV

RESULTS OF DISCRIMINANT ANALYSIS WITHOUT FORMANT FREQUENCIES

\begin{tabular}{|l||cccc|c|}
\hline Source emotions & HAP & ANG & SAD & SUP & Error (\%) \\
\hline Happy & 5 & 0 & 0 & 4 & 44.44 \\
Angry & 0 & 7 & 2 & 0 & 22.22 \\
Sad & 1 & 3 & 4 & 1 & 55.56 \\
Surprised & 1 & 0 & 1 & 7 & 22.22 \\
\hline Error rates & & & & & 36.11 \\
\hline
\end{tabular}

TABLE V

RESULTS OF DISCRIMINANT ANALYSIS WITH FORMANT FREQUENCIES

\begin{tabular}{|l||cccc|c|}
\hline Source emotions & HAP & ANG & SAD & SUP & Error (\%) \\
\hline Happy & 7 & 0 & 0 & 2 & 22.22 \\
Angry & 0 & 7 & 2 & 0 & 22.22 \\
Sad & 1 & 1 & 7 & 0 & 22.22 \\
Surprised & 3 & 0 & 0 & 6 & 33.33 \\
\hline Error rates & & & & \multicolumn{4}{|c|}{25.00} \\
\hline
\end{tabular}

of emotional presentations, one-way ANOVA was conducted on each acoustic feature. In the results, the effectiveness of the features of some types of emotional presentations, such as power, duration, speech speed and F2, is significant. The effectiveness is illustrated in Figure 3, which shows the results of sub-effect tests of the four emotions with significant levels.

These results show that emotional presentation changes some of the means of acoustic features.

\section{DISCRIMINANT ANALYSIS OF EMOTIONS USING ACOUSTIC FEATURES}

\section{A. Procedure and performance}

As some acoustic features reflect emotionally inflected speech, the possibility of predicting the type of emotional presentation using discriminant analysis exists. The amount of data was limited, and the randomForest technique was used for classification. The strengths of emotions were ignored in this analysis.

As mentioned in the introduction, the effectiveness of formant frequency should be tested using discriminant analysis. An analysis was therefore conducted with and without using formant frequencies.

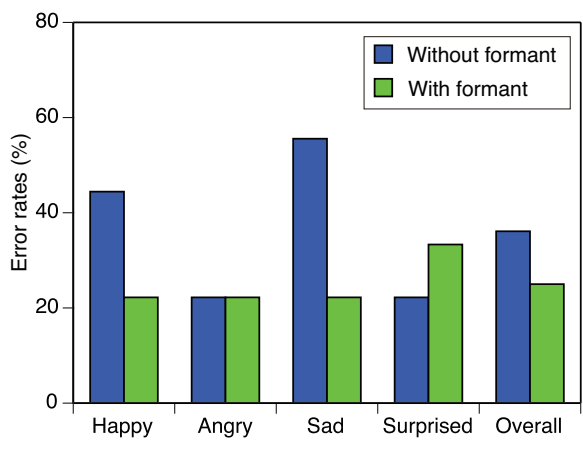

Fig. 4. Error rates of Emotions 


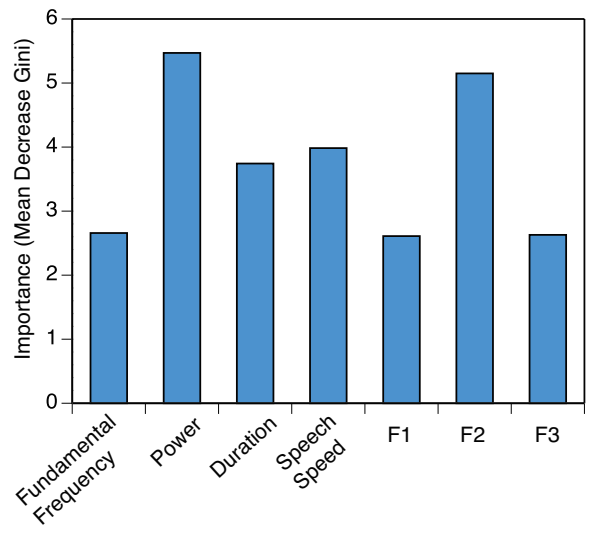

Fig. 5. Importance of acoustic features

TABLE VI

RESULTS OF F-TESTS OF THE STRENGTHS OF EMOTIONAL VOICES

\begin{tabular}{|l|cccc|}
\hline Features & df & MSE & F & $p$ \\
\hline Fundamental Freq. & $(2,102)$ & 0.05 & 13.35 & $<0.01$ \\
F1 & $(2,102)$ & 0.08 & 7.21 & $<0.01$ \\
\hline
\end{tabular}

Table IV shows the results of the discriminant performance without formant frequencies, and Table $\mathrm{V}$ shows the performance with formant frequencies. In both tables, the vertical cells represent emotional categories of speech, and the horizontal cells represent the discriminant results. The orthogonal components represent correct discriminations. The percentages of misclassification frequencies are calculated for each emotion, and they are summarized as error rates in the far right columns. In sum, the overall error rate decreased $11 \%$ when features of formant frequency were used. The error rates for the four emotions are summarized in Figure 4. The figure shows that formant frequency contributes to discriminant performance for "happy" and "sad" emotions, but the improvements are not present for all emotions.

B. Importance of acoustic features for classifying emotional states

The degree of importance of features, which means the contributions of classification of emotional state discrimination, are summarized in Figure 5. The most prominent features are power and the 2nd formant frequency (F2), which is related to the position of the speaker's tongue. The factor of emotionally inflected speech may be a concern when the tongue is moving.

\section{Relationship between acoustic features and emotional strengths}

The factor of emotional strengths for the three voice levels (since voices are presented using three levels of emotional strength) was tested for the acoustic features which were extracted using one-way ANOVA. In the results shown in Table VI, the main factor is significant for fundamental frequency and for 1st formant frequency (F1). Changes in the values for emotional strengths are summarized in Figure 6. Sub-effect

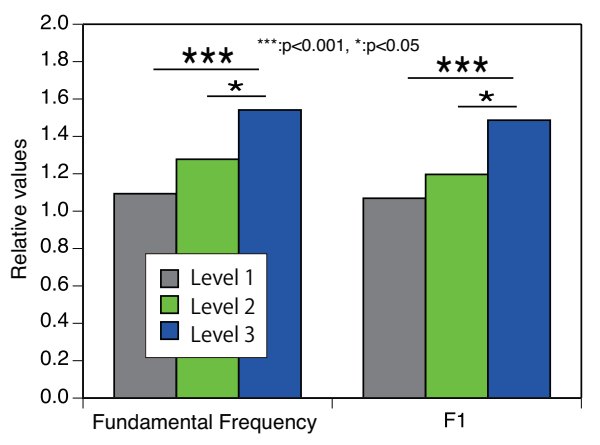

Fig. 6. Results of multiple comparisons of the strengths of emotionally inflected voices

tests show that there are some significant differences between the strengths of emotionally inflected speech, and the results are illustrated in Figure 6. These two features may respond to the level of the emotional presentation, and a detailed analysis of this will be the subject of our further study.

\section{SUMMARY}

The relationships between emotionally infected speech and its acoustic features were analyzed. In particular, a signal processing procedure for use with formant frequencies between vowels was developed to examine their contributions to detecting emotional factors. During the procedure acoustic features, including formant frequencies, were evaluated using relative values for emotional speech which were compared to relative values for natural speech for every mora unit. The features of phrases were evaluated as means of features between moras.

This technique improves discriminant performance between the four types of emotional speech by comparing formant frequencies such as F2. Performance was improved $11 \%$ for speech without formant frequencies. Power and the 1 st formant frequency are the two acoustic features which influence the strength of emotional speech the most.

The development of practical application procedures including adaptations for phonological features of other languages system such as English will be the topics of further study.

\section{REFERENCES}

[1] J.Uemura, K.Mera, Y.Kurosawa, T.Takezawa, "Analysis of Inconsistency among Emotions Estimated from Linguistics, Acoustic, and Facial Expression Features and A Proposal of the Inconsistency Detecting Method," Proc. of 78th annual meetings of IPSJ, 6Y-04, 4, 321-322, 2016.

[2] T. Matsui, M. Hagiwara, "A Dialogue System with Emotion Estimation and Knowledge Acquisition Functions," Trans. of Japan Society of Kansei Engineering, 16(1), 35-42, 2017. doi: $10.5057 /$ jjske.TJSKE-D-16-00058

[3] M. Shigenaga, "Features of Emotionally Uttered Speech Revealed by Discriminant Analysis," IEICE Trans., Vol.J83-A, No.6, 726-735, 2000.

[4] M. Shigenaga, "Characteristic Features of Emotionally uttered Speech Revealed by Discriminant Analysis (III): Discrimination of both Mixed Sentences and Test Data," IEICE Technical Report, SP, 97(396), 65-72, 1997-11-21, 1997. 
[5] M. Shigenaga, "Characteristic Features of Emotionally uttered Speech Revealed by Discriminant Analysis (VI)," Proc. of Acoustic Society of Japan, 3-3-12, 1999.

[6] NII Speech Resources Consortium, "Online gaming voice chat corpus with emotional label (OGVC)," URL http://research.nii.ac.jp/src/OGVC.html
[7] P. Ekman, W.V. Friesen, Unmasking the face, Prentice-Hall, Inc., NJ, USA, 1975.

[8] Paul Boersma, David Weenink, http://www.fon.hum.uva.nl/praat/ (accessed 27th Jan., 2017)

[9] H. Jouo, NIHONGO ONSEI KAGAKU, Badai Music Entertainment, Tokyo, Japan, 1998. 



\section{$6^{\text {th }}$ Workshop on Advances in Programming Languages}

$\mathbf{P}$ ROGRAMMING languages are programmers' most basic tools. With appropriate programming languages one can drastically reduce the cost of building new applications as well as maintaining existing ones. In the last decades there have been many advances in programming languages technology in traditional programming paradigms such as functional, logic, and object-oriented programming, as well as the development of new paradigms such as aspect-oriented programming. The main driving force was and will be to better express programmers' ideas. Therefore, research in programming languages is an endless activity and the core of computer science. New language features, new programming paradigms, and better compile-time and run-time mechanisms can be foreseen in the future.

The aims of this event is to provide a forum for exchange of ideas and experience in topics concerned with programming languages and systems. Original papers and implementation reports are invited in all areas of programming languages.

\section{TOPICS}

- Automata theory and applications

- Compiling techniques

- Context-oriented programming languages to specify the behavior of software systems and dynamic adaptations

- Domain-specific languages

- Formal semantics and syntax

- Generative and generic programming

- Grammarware and grammar based systems

- Knowledge engineering languages, integration of knowledge engineering and software engineering

- Languages and tools for trustworthy computing

- Language theory and applications

- Language concepts, design and implementation

- Markup languages (XML)

- Metamodeling and modeling languages

- Model-driven engineering languages and systems

- Practical experiences with programming languages

- Program analysis, optimization and verification

- Program generation and transformation

- Programming paradigms (aspect-oriented, functional, logic, object-oriented, etc.)

- Programming tools and environments

- Proof theory for programs

- Specification languages

- Type systems

- Virtual machines and just-in-time compilation

- Visual programming languages

\section{Best PAPER AwARD}

To celebrate WAPL's 10 years old, the $1^{\text {st }}$ edition was in 2007, a Best Paper award will be offered to distinguish a work of high quality presented in the workshop. Award comprises a certificate for the authors and will be announced during the conference dinner.

\section{KEYNOTE SPEAKERS}

- Marjan Mernik from University of Maribor (Slovenia) and University of Alabama at Birmingham (USA)

- Jan Vitek from the Programming Research Laboratory, CCIS at Northeastern University, Boston (USA)

\section{STEERING COMMITTEE}

- Janousek, Jan, Czech Technical University, Czech Republic

- Luković, Ivan, University of Novi Sad, Serbia

- Mernik, Marjan, University of Maribor, Slovenia

- Slivnik, Bo\&scaron;tjan, University of Ljubljana, Slovenia

\section{SECTION EDITORS}

- Rangel Henriques, Pedro, Universidade do Minho, Portugal

\section{REVIEWERS}

- Barisic, Ankica, Universidade Nova de Lisboa, Portugal

- Horvath, Zoltan, Eotvos Lorand University, Hungary

- Janousek, Jan, Czech Technical University, Czech Republic

- Kardaş, Geylani, Ege University International Computer Institute, Turkey

- Kern, Heiko, University of Leipzig, Germany

- Kollár, Ján, Technical University of Kosice, Slovakia

- Kosar, Tomaž, University of Maribor, Slovenia

- Lopes Gançarski, Alda, TELECOM SudParis, Evry, France

- Luković, Ivan, University of Novi Sad, Serbia

- Mandreoli, Federica, University of Modena, Italy

- Martínez López, Pablo E. "Fidel", Universidad Nacional de Quilmes, Argentina

- Mernik, Marjan, University of Maribor, Slovenia

- Milašinović, Boris, University of Zagreb Faculty of Electrical Engineering and Computing, Croatia

- Milewicz, Reed, University of Alabama at Birmingham, United States 
- Moessenboeck, Hanspeter, Johannes Kepler Universitat Linz, Austria

- Pai, Rekha, National Institute of Technology Calicut, India

- Papaspyrou, Nikolaos, National Technical University of Athens, Greece

- Porubän, Jaroslav, Technical University of Kosice, Slovakia

- Saraiva, João, Universidade do Minho, Portugal
- Sierra Rodríguez, José Luis, Universidad Complutense de Madrid, Spain

- Slivnik, Boštjan, University of Ljubljana, Slovenia

- Splawski, Zdzislaw, Wroclaw University of Science and Technology, Poland

- Van Wyk, Eric, University of Minnesota, United States

- Varanda Pereira, Maria João, Instituto Politecnico de Braganca, Portugal

- Watson, Bruce, Stellenbosch University, South Africa 


\title{
Analysis of Include Dependencies in C++ Source Code
}

\author{
Bence Babati, Norbert Pataki \\ Department of Programming Languages and Compilers, \\ Eötvös Loránd University \\ Pázmány Péter sétány 1/C H-1117 Budapest, Hungary \\ Email: \{babati, patakino\}@caesar.elte.hu
}

\begin{abstract}
The C++ Standard Template Library (STL) is the flagship example for libraries based on the generic programming paradigm. The usage of this library is intended to minimize classical $\mathbf{C} / \mathrm{C}++$ errors, but does not warrant bug-free programs. Furthermore, many new kinds of errors may arise from the inaccurate use of the generic programming paradigm, like dereferencing invalid iterators or misunderstanding remove-like algorithms.

Unfortunately, the C++ Standard does not define which standard header includes another standard headers. It is easy to write code that works perfectly on an implementation but fails to compile with another implementation of STL. These unportable codes should be result in compilation error with every STL implementation. However, in this case the compiler does not warn us that this code is erroneous.

In this paper we present our tool that is based on the Clang. This tool is able to detect the missing include directives that are patched by the STL implementation's internal structure. It also reports the unnecessary include directives to avoid extra compilation time. The background of our tool is discovered and we briefly present the underlying data structures and algorithms. We analyse how these problems occur in open source libraries and programs. Which environment proves oneself to be lazy or strict? How the developers take advantage of this portability issue?
\end{abstract}

\section{INTRODUCTION}

$\mathbf{N}$ OWADAYS, the C++ language is very popular in educational and industrial environments as well. It provides a wide range of programming language elements, from the low level bit manipulation and pointer usage, which mainly come from $\mathrm{C}$ language to the high level, modern programming paradigms, such as function overloading, exceptions and templates. Runtime efficiency is important in $\mathrm{C}++$, with the guiding principle being "do not pay for what you do not use". Efficiency can be very important in industrial environment.

On the other hand, the $\mathrm{C}++$ language is continuously evolving, new standards come with a bunch of new features, for instance the variadic templates which appeared in $\mathrm{C}++11$. These new features keep $\mathrm{C}++$ popular. Actually, there are millions of lines $\mathrm{C}++$ code and new lines are created day by day, that someone has to maintain later. Thus, the detection of bugs has become very important. The earlier the bug is found, the lesser the cost. There are analysis tools on the market that can catch bugs in the source code. Some of them find

Supported by the NKP-17-4 New National Excellence Program of the Ministry of Human Capacities problematic code snippets during the compilation stage, others can do it at runtime.

In this paper we describe an issue related to $\mathrm{C}++$ Standard Template Library (STL). This issue may cause portability problems because of the underlying STL implementation's undefined include dependency. We present our tool that can analyse the source code and find these kind of bugs. The tool is based on Clang compiler infrastructure [1].

This paper is structured as follows. The paper begins with an introduction to STL and static analysis in section II. We present the related problems in details in section III. After we describe our tool that is based on Clang in section IV. Our tool has been evaluated on open source projects, so the collected result can be seen in section V. Finally, this paper concludes.

\section{PREQuisites}

\section{A. Standard Template Library}

The Standard Template Library (STL), is a standard C++ library of container classes, algorithms, iterators, and functors [2]. STL provides many of the basic algorithms and data structures of computer science. The STL is a generic library, meaning that its components are heavily parametrized: almost every component in the STL is a template that cannot be compiled in advance. Therefore all definitions of the STL components should be written in the header files [3].

The STL includes container classes: classes whose purpose is to contain other objects. The library includes the classes vector, list, deque, set, multiset, map, multimap. Each of these structures is a template, and can be instantiated to contain many types of object.

The STL also includes a large collection of algorithms that manipulate the data stored in containers (for instance, for_each, copy, find_if, etc.). Algorithms are global function templates, not member functions. Every algorithms operate on a range of elements, rather than on a container. Algorithms are decoupled from the STL container classes. This means that algorithms can be used with vectors, but also work with lists, and even with elements of $\mathrm{C}$ arrays, etc.

Iterators make connection between the algorithms and containers, which are generalization of pointers. Their public interface originates from pointer-arithmetic. Iterator is the mechanism that makes it possible to decouple algorithms from containers: algorithms are templates, and are parametrized by 
the type of iterator, therefore they are not restricted to a single type of container.

Finally, the STL includes a large collection of function objects, also known as functors. Just as iterators are generalization of pointers, function objects are generalization of functions: a function object is anything that you can call using the ordinary function call syntax supported by operator(). Function objects are an important part of generic programming because they allow abstraction not only over the types of objects, but also over the operations that are being performed.

The complexity of the library is greatly reduced because of this layout. As a result of this layout we can extend the library with new containers and algorithms simultaneously. This is a very important feature because object-oriented libraries do not support this kind of extension [4].

The STL that is specified in the $\mathrm{C}++$ standard does not belong to a specific implementation. Many STL implementations are available and these are not the same. Extensions and different approaches appear in the STL implementations.

The usage of $\mathrm{C}++$ STL highly reduces the classical programming errors, like memory leaks and invalid pointer dereferences but misunderstanding generic programming paradigm can lead to new kinds of errors, for example, iterator invalidation or improper use of algorithms (unique, remove) [5]. The include dependencies in the STL are not well-defined and many dependencies belong to the implementation of STL that can be a main reason of a subtly portability issue [6].

\section{B. Static analysis}

Static analysis is a kind of software analysis. Its idea is analyzing the software without execution, it can target the source code or byte code as well. The main advantage of this method is that the code is not executed. In general, finding bugs in an earlier stage of software development highly could reduce the cost of the software [7]. The tools are not perfect, they could not find all bugs and sometimes go wrong. However, these tools are beneficial ones [8]. Using these kind of tools during the development can be effective in many respects, for example these tools can provide a kind of automatic checking of the source code.

Of course, the static analysis technique used to affects what kinds of problems can be detected. However, the language also affects the efficiency of the analysis. There are many analysis methods to detect issues from simple ones, like regular expression based searches to very complex algorithms. Nowadays, the market leading static analysis tools use very advanced algorithms to detect issues, for example symbolic execution[9]. Symbolic execution have become more and more important in the modern software development [10]. The complexity of the analysed language also can influence the algorithms, for example the analysis of a language which has pointers could be harder than others which do not have [11].

Clang is an open source compiler for $\mathrm{C} / \mathrm{C}++/ O b j e c t i v e-\mathrm{C}$. It is built on the top of the LLVM compiler infrastructure. It is a rapidly evolving project which is supported by Apple and Google. Clang is getting more and more popular.
One of the advantages of Clang is its modular architecture. One can use the parser as a library to build tools. The popularity of this compiler also implies that it is well tested. Clang has been applied in many different static analysis tasks: finding semantic mistakes in the usage of STL [5], searching for semantic differences in source code according to different $\mathrm{C}++$ standards [12], improving static analysis with function summaries [13], finding move semantics related bugs [14], detecting uses of uninitialized memory in $\mathrm{C}$ and C++ [15], customizable style validation [16], speed-up special operations [17] etc.

\section{THEORETICAL ISSUES}

The following theoretical issues will be addressed in this paper. To get a deeper view on these, the next subsections will summarize the problems. All of them are related to include hierarchy and the usage of header files, but each of them is different.

\section{A. Internal STL hierarchy}

1) Motivation: $\mathrm{C}++$ is a standardized language [3]. The first official standard released in 1998. Three improved C++ standards was released later and the next one comes in 2017. The language is continuously evolving to meet the expectations. The $\mathrm{C}++$ Standard is a very long and formal paper and defines the element of the $\mathrm{C}++$ language and the Standard Template Library provided features, but there are subtle opportunities for the compiler and library implementations.

Also, there are some weaker points in the Standard. In C++, to modularize our program, the header inclusion can be used which comes from the $\mathrm{C}$ language. Header inclusion happens during the preprocessing stage of the compilation. The include preprocessor directive provides header inclusion. When the preprocessor reaches out an include directive, it opens the given file and copies its content to the place of directive. Also, this process can be recursive. At the end of preprocessing of a translation unit, the preprocessed source file is ready that contains all data which is necessary for the compiler. For example, if there is an include for vector header, preprocessor will copy the content of vector header to the place of include [18]. This mechanism increases the build time intensively [19].

Standard Template Library uses header inclusion to separate headers into different files. The Standard defines for each STL header file what it has to provide, for example vector standard header has to provide a data structure called vector. The developers can include these files, if they would like to use their features. Some standard headers need to use a symbol from another standard header file. It is possible, but this mechanism results that, the features of included header file are available by including the other one.

It would be okay, the different modules have to be connected in some cases, for example map uses features of utility. However, it could lead issues later.

The main problem is that the $\mathrm{C}++$ Standard does not define exactly which standard headers must include the other ones. It 
depends on the developers of current STL library implementation because they create the hierarchy of the library. Thus, the hierarchy of STL implementations could be different. It is not a problem when the standard headers are used correctly, but the compilers do not check it comprehensively. Therefore it can be the root cause of portability problems.

Easy to write code that compiles with an implementation but fails with another. These unportable codes should result in compilation error with every STL implementation, but the compiler does not know that is wrong. It finds the used definitions and does not check how they can be reached.

2) Examples: This problem appears when the developers try to take advantages of hierarchy, that is not well defined. In the next examples, the problem will be highlighted more obviously and how easy to commit this kind of mistake.

In this case, the compiler have to be upgraded, that requires to upgrade the used STL implementation also. We used g++ 5.3 with libstdc++ STL implementation. Here is a simple program that compiles and works fine. For example, see Listing 1 , there is an include directive for algorithm header, but in the main function a variable with type std: :vector<int> is constructed.

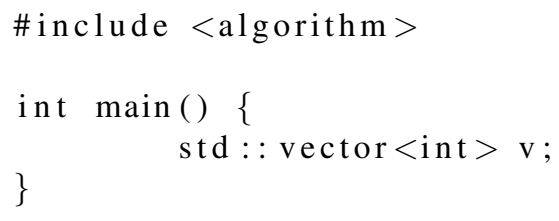

\section{Listing 1: Vector from algorithm}

Next step is upgrading our compiler to g++ 6.1, the STL implementation is also updated. The new compiler fails to compile the code because it does not find vector in std namespace.

error: 'vector' is not a member of 'std'

Listing 2: Compiler error

How this situation could happens? The hierarchy of libstdc++ STL implementation has been changed. Till this version algorithm included random, that included vector, so if one includes algorithm, the vector header also can be used.

In the next example, compiler is switched with another and this also results in switching one STL implementation with another. At the beginning, Clang compiler is used with libc++ STL implementation. Another interesting example can be seen in Listing 3, which is very simple, it just includes algorithm header and uses std: : shared_ptr through this header.

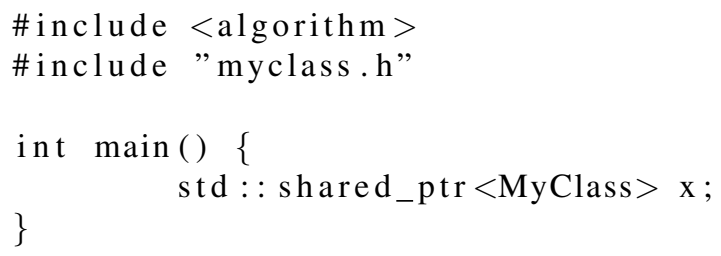

Listing 3: Header memory from header algorithm
It compiles and works with Clang using libc++ STL, but we change the compiler to g++ 6.1 with libstdc++ STL. It results a compilation error, because std: : shared_ptr class template cannot be found.

error: 'shared_ptr' is not a member of 'std' Listing 4: Compiler error

The root cause of this error has been that we have changed the used STL implementation to another one. In libc++, algorithm header includes memory because some implemented algorithms use smart pointers from memory header. In the libstdc++ implementation, the algorithms do not use smart pointers so algorithm does not include memory header necessarily.

3) Clarification: As a short summary for the problem, the include hierarchy in STL implementations could be different and it can be changed by time. It could be annoying, if the code that compiles with a specific compiler, does not compile with a different compiler that uses another STL implementation. To avoid this problem no one should take advantage of the include dependencies of the used STL implementation.

\section{B. Legacy style $C$ header include}

For backward compatibility with $\mathrm{C}$ language, the $\mathrm{C}++$ has to provide features of $\mathrm{C}$ standard library. It means, almost every $\mathrm{C}$ standard header file is available through their appropriate $\mathrm{C}++$ version, for example, features of stdlib.h is available by cstdlib. The main difference between these header files is that the $\mathrm{C}++$ version brings functions into the st $d$ namespace.

On the other hand, the original $\mathrm{C}$ header files can be included, but the $\mathrm{C}++$ standard formulates them as deprecated. It does not require, that these headers have to be available by definition at all times. The $\mathrm{C}$ headers are accessible only, if the build environment contains a $\mathrm{C}$ compiler beside the $\mathrm{C}++$ compiler, for example gcc and $\mathrm{g}++$. It is possible, the $\mathrm{C}++$ compiler is only a $\mathrm{C}++$ compiler and does not care about $\mathrm{C}$, that implies the $\mathrm{C}$ standard header files are not available certainly. Since the $\mathrm{C}++$ only requires the $\mathrm{C}++$ version of $\mathrm{C}$ standard header files, a short example can be seen in Listing 5 .

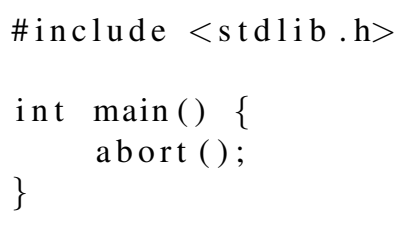

Listing 5: $\mathrm{C}$ header usage

\section{Unused header files}

The unused header files are not really an issue because they do not cause any compilation error. By definition a needless include does not contain any declaration or definition, that is used somewhere in the source code. That means, these kind of includes safely can be removed without affecting the build procedure [20]. 
However, the deletion of unnecessary header files is a build process optimization because unused include files can highly increase the build time [21]. Just for clarification, in the next sample in Listing 6 depicts how an unused header looks like.

a.h :

void foobar ();

main.cpp :

\#include "a.h"

int main () \{

\}

Listing 6: Unused header

These problems often occur in big $\mathrm{C} / \mathrm{C}++$ projects. Meyers devoted an item for the STL-related header files but no tool was mentioned to detect the misusage [6]. We have analysed many open-source projects and all of them contains at least one of mentioned problems, so we have developed a tool.

\section{THE TOOL}

\section{A. Technical background}

The previously described problems can appear from time to time, and it often results in a compiler error that must be fixed. To detect these kind of problems, a new tool was created to statically analyse the source code. The targeted problems are related to each translation unit and its include hierarchy, so translation units can be processed separately from each other.

The tool is based on Clang library and takes advantage of its libraries, for example. use its abstract syntax tree, which is created from the analyzed source. The main advantage of using Clang is the proper $\mathrm{C}++$ parser that follows the $\mathrm{C}++$ standard.

\section{B. Conceptual problems}

We have faced some interesting problems, which require to solve them in order to create a usable, handy tool.

a) Mandatory includes: Almost every standard header uses features from another ones, but it is not well documented. Among the used includes, there are many which are required to be included. That means, it must include them to implement functionality defined by the standard. This mechanism can help us to filter out a lot of false positive results because a header file cannot be implemented without include another one. This kind of required relation is transitive such as the header inclusion. For example, map has to include utility, because std: :map's insert member function returns a pair of iterator and bool, that means, the insertion succeeded if first member of std: :pair is an iterator to the newly inserted element and the second member's value is true. If it is not, the first contains an iterator to an element with equal key and the second is false.

pair $<$ iterator, bool $>$ insert ( const value \&); Listing 7: std::map::pair b) Private headers: To determine the exact location in terms of header of a given class or function template definition may be very hard. In theory, the expectation is that the standard header files contain the definition for data structures, functions and other programming units but in practice they do not. The $\mathrm{C}++$ Standard specifies that the vector header has to provide definition for vector type, but it does not specify that the definition of vector has to be placed in vector header. As previous examples depict that the header inclusion mechanism is transitive, so if $a . h$ includes $b . h$ and $b . h$ includes $c . h$, then the $a . h$ also includes $c . h$.

However, the definitions may be separated into different header files and these headers are included in the standard header. This mechanism fulfills the requirements of $\mathrm{C}++$ Standard, because if one includes vector in the source file and vector includes vector. $h$ that contains the definition for vector class template, then vector class can be accessed through vector header.

In some STL implementations header inclusion is used. That makes hard to determine the symbols real place because during the analyses the tool searches for a symbol, that it comes from $a . h$, but we need that information which Standard header functionality is implemented in this header. For example, in libstdc++ the implementations mainly are in other "internal" header files, those are included by standard headers, e.g: algorithm includes stl_algo.h and stl_algobase.h, which contain the implementation of algorithms. So, need to track these includes, to find their belonging standard header.

The tool can catch these internal headers, because they are not defined by user, so they come from the system and are not standard header files. In the dependency graph, the belonging relation between the header file is marked with a different kind of edge. For more precise result the belonging relation in the graph should be corrected in analysing stage that requires deep knowledge from the $\mathrm{C}++$ Standard. This mechanism can be used to determine which standard header file's functionality implemented in a system header. For example, vector header has to provide std: : vector, but it is implemented in vector_impl.h file, then the tool has to know that the vector_impl.h file implements a part of functionality of vector header, so it belongs to the vector header.

c) Unused header indirect usage: The deletion of needless includes can cause problems indirectly, because they do not contain any used declaration, but it can include other header file that contains a declaration, which is used. In this case, the tool has to detect dependencies between include files and before a header file is suggested to be removed, it should check this header file's include dependencies and add the used ones, if is not included by another header file.

d) Knowledge from $\mathrm{C}++$ standard: The tool requires information from the $\mathrm{C}++$ Standard. Some of them are not must have dependencies, but they can help to improve the process. Many kinds of knowledge are required from standard, to make analysis more precise.

- Header files: the tool has to determine whether a header file is $\mathrm{C}$ or $\mathrm{C}++$ standard file or it is not. The sys- 
tem_header pragma usually helps to determine, that is user defined or not, but more precise decision is necessary. To be able to select standard header files, some knowledge is required from the $\mathrm{C}++$ Standard. Those nodes which are standard header files should be marked in the graph.

- Mandatory includes, this is a list of headers which have to be included for each standard header file.

- Symbols: Symbols that are provided by standard header files. This is a very large data collection and it has collisions if we just check for names, for example in case of function overloading. These symbols are used during the visiting of AST to correct edges of these internal headers in the graph. This is not mandatory but it results in a more precise analysis.

\section{High-level overview}

In this part, a high-level overview will be depicted, how the tool works and solves any issues. The tool analyses the source code, thus execution of the code is not required. Also, it needs to known the exact compiler arguments for the analysis, for instance macro definitions. The build procedure usually has many different parts, such as compilation, linking etc. To follow this convention, the analysis can be separated into three stages.

a) Preprocessing: First of them is preprocessing, when the tool collects information about the include hierarchy. The preprocessor is used for this task. In Clang, the compilation process is very customizable, so someone can attach to the preprocessor with callbacks. When the preprocessor catches an include directive, the tool receives a callback to this event. The header inclusion uses deep first search, so all information about the include hierarchy of the processed translation unit can be collected.

During the preprocessing a dependency graph is building up that can be mapped to the origininal include hierarchy. The nodes of the graph are the files and the directed edges mean include relation between the two endpoints, the source node includes the destination node. Also the nodes contains additional information, that may be needed later, for example. user defined header or not, part of Standard Template Library or not, etc. In this graph, the relations between headers are clear, we know for each file which other files includes and which other files are included it.

Another important data is collected in this stage, macro usage only can be gathered right here. With the macro usage information the proper nodes are extended in the graph. At the end of the preprocessing, this graph can be considered as definitive, no one will extend the graph with any new node or directed edge, but extra information can be added to nodes in the next stage.

b) Analyzing: The second stage is the analyses of AST and extract the necessary information from. After the preprocessing, the dependency graph is done for a while, but it may change in this stage. Right now, the preprocessed source code has to be analyzed. The Clang will do every parsing related task, for example tokenization, parsing, etc and it builds up the abstract syntax tree from the source code. Through Clang libraries, we just take advantage of its architecture by creating visitor for abstract syntax tree. The visitors support the walking on AST. Thus, using visitors, all kind of declaration, expression or etc can be catched in the code. After AST visiting, the graph is done and is not be changed later. An example graph is depicted in Figure 1. For reference, the circle nodes are STL related headers and the rectangles are user defined or other system headers in the graph.

c) Visitors: Two different visitors were used for different purposes. One of them is for catching every usage place in the user files of every possible function, class, typedef, macro etc. A few number of properties is used from used symbols, but the most important one is the location to know where the symbol is and where it comes from. For each usage, the corresponding node in the graph will be extended with what symbol is used and where. Catching all symbols is necessary to produce feasible output.

Another visitor is capturing definitions only from system headers. This functionality is necessary to properly detect private headers in the STL implementation. It watches definitions of function, class, typedef etc. and if they are not in standard header file, but their file is included by standard headers, then corrections should be made in the graph. In case private files will behave as standard header in the graph, because they really implements features of standard header. This kind of private header mapping and validation can be important because popular STL implementations use private headers to modularize their structure.

d) Graph analyzers: The last stage is the graph analysis. The dependency graph is complete right now, the different analysers can start their work to walk over the graph. The graph analysers no not depend on each other, just visiting the given graph. Every previously described issues are implemented as a graph analyser, because they just seek after erroneous parts in the graph independently from each other. The Figure 2 depicts how the architecture looks like.

Different analyzers use different graph walking algorithms to detect the targeted issue. STL internal hierarchy analyzer walks over nodes which belongs to user defined files, checks the used symbols and searches for routes between their headers and the usage place. If there is no valid route, it creates a report for that header file where the definition is placed. At the end, it aggregates the results and removes duplicates.

In details, during the walking on nodes, the nodes are marked as valid or invalid flag, this flag indicates that using symbols from this file would be okay or not. The user-defined files always are valid, because we try to detect issues related to Standard Template Library. We can determine the routes from usage location to the location of definition of the symbol. If only routes are available which do not contain non standard header file, then the usage of this symbol could be marked an invalid.

For example: in main.cpp, std: : vector is used in the 


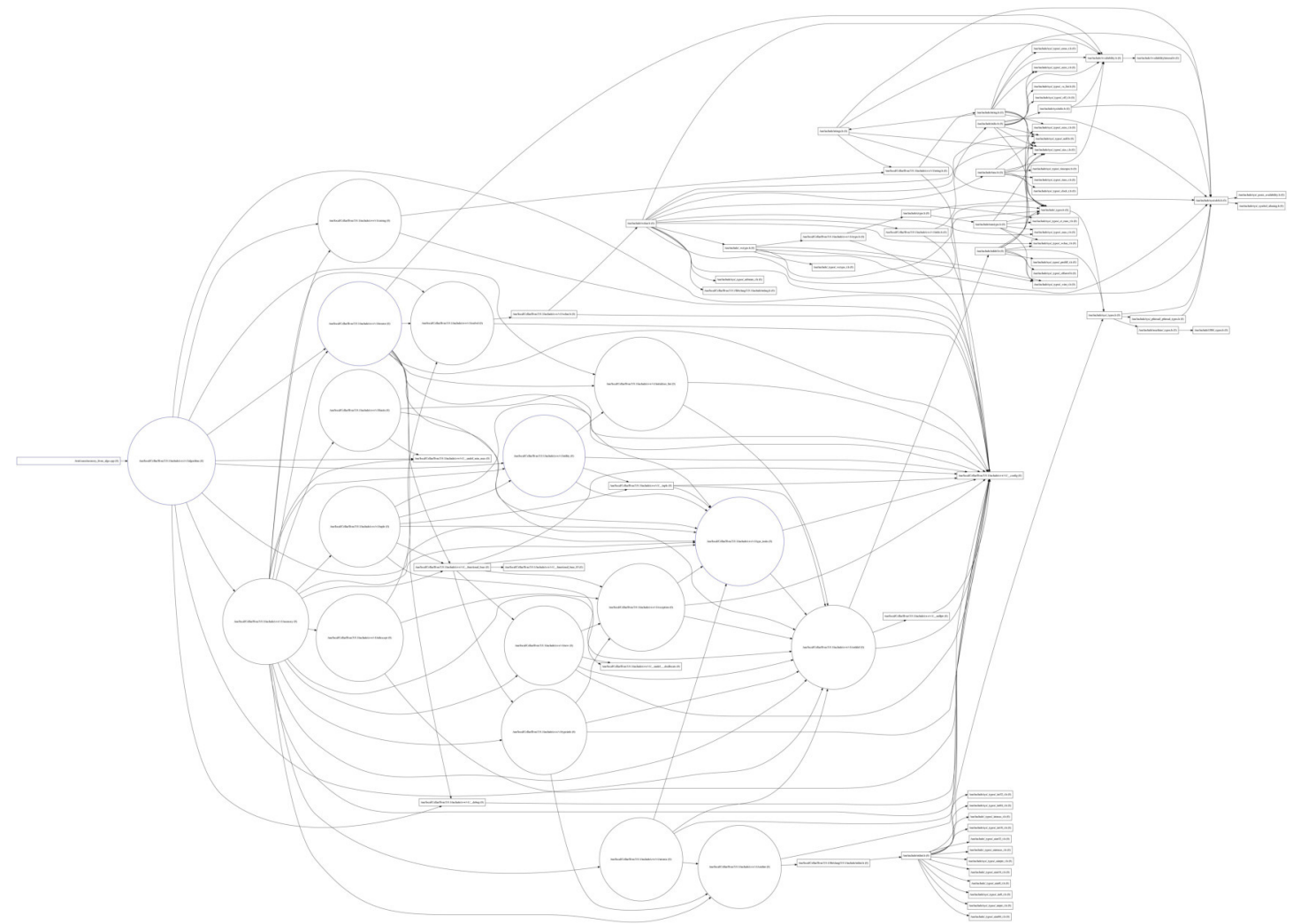

Fig. 1: Dependency graph

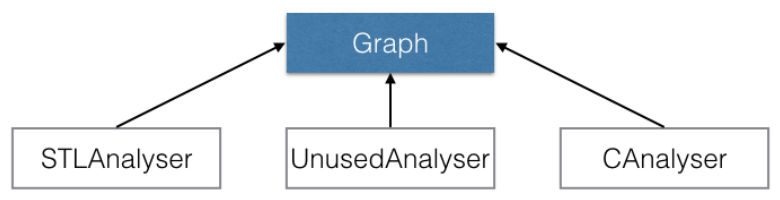

Fig. 2: Graph analyzers

declaration of function foo and the std: : vector definition comes from vector header, so the routes from main.cpp to vector header are searched from in the include hierarchy graph. This seems to be reasonable because main.cpp includes directly vector header.

main . cpp :

\#include $<$ vector $>$

void foo (const std: : vector $<$ int $>\&$ v);

Listing 8: Vector usage sample

There may be multiple include directive paths to a file. In this case, we have to aggregate them to set the flag correctly. The used rule for aggregation is that, if there is a flow from a file that is included by user-defined file or it is a user-defined file then this inclusion is valid, otherwise it is not. In the next example in Listing 9, the problem is explained.

main.cpp : \#include $<$ map $>$

\#include "myheader.h"

$\ldots$

std : : pair $<$ int, int $>$ p;

...

myheader.h:

\#include $<$ utility $>$

$\cdots$

Listing 9: Multiple path inclusion

In this example, there are two include directives for map and myheader.h in main.cpp file, but somewhere in the file the std: :pair is used as type of variable. In myheader.h file, there is only one include directive for utility. Just for reference std: :pair is defined in utility. However, map and myheader.h file include utility too. Two paths exist in the graph from main.cpp to utility that contain the std: :pair definition. In this example the node of utility should be marked as valid because it is included through an user header too.

\section{Output format}

The combination of functionalities results in a powerful tool that can be used to analyse source codes for detecting portability issues and unnecessary includes, which increase the compilation time. The tool at the end of the analysis need to aggregate the results of three kind of analysis. The program 
has many filenames with used symbol names and usage place, header files that are not used. The result of the analysis is three lists, which contain header files:

- header files to be added as include and a file for each, where you should be add

- header files which proposed to be removed and a file for each, where they are right now

- C style standard header files with their current location, e.g. stdlib.h

For example, check Listing 10 for sample code and Listing 11 for the output.

main . cpp :

0 \#include $<$ stdlib.h $>$

1 \#include $<$ algorithm $>$

2

3 int main() \{

$4 / /$ works with g++ 5.3

5 std:: vector $<$ int $>$ v;

6 abort ();

$7\}$

Listing 10: Sample

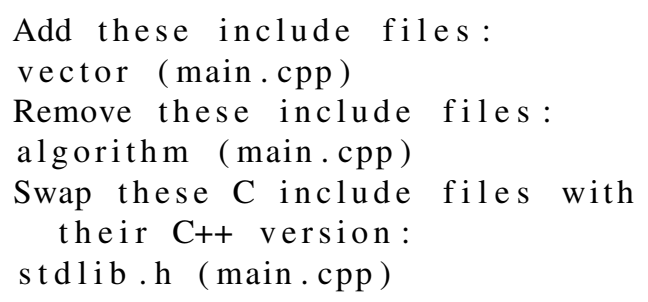

Listing 11: Output of analysis

\section{RESULTS}

We have developed the described tool and the comprehensive testing has been started. The tool finds all mistakes that are presented in this paper as examples. All mentioned features are implemented and the tool is able to detect portability issues and needless includes.

During the development the tool was tested on open-source projects to verify the prototype of software. Quite popular open source projects were used for this testing. Different characteristics projects were selected for testing purposes, some of them are library and some of them are standalone application that provides solution for a given problem. Their programming style differs from each other, that makes the testing more complete. However, the size of projects is not so large, about many ten or hundred thousand lines of code, but enough to analyse them with our tool. Every found bug required to check manually to verify if it is really a bug that process takes much time. The complete list of the analysed projects:

- Tinyxml2, https://github.com/leethomason/tinyxml2

- Json for Modern C++, https://github.com/nlohmann/json

- Bloaty, https://github.com/google/bloaty

- Guetzli, https://github.com/google/guetzli
- Yaml-cpp, https://github.com/jbeder/yaml-cpp

- Flatbuffers, https://github.com/google/flatbuffers

- Orc, https://github.com/apache/orc

To analyze projects, the tool requires exact compiler invocations for each translation unit. These are stored in a json formatted file called compilation database ${ }^{1}$. Compilation database contains a section for each translation unit that describes how it was originally compiled. The sections have three fields:

- directory: directory of file

- command: the exact build command

- file: name of the compiled file

For instance, see Listing 12.

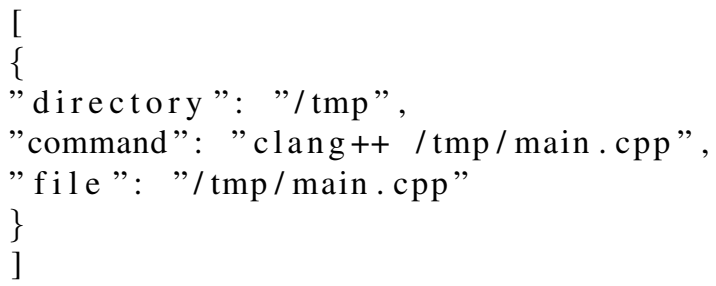

\section{Listing 12: Compilation database}

These can be produced by capturing the build execution of project or can be generated by some build systems. There are a few software products that can capture build flow and some build systems can generate it by default, e.g. CMake can generate compilation database if you pass CMAKE_EXPORT_COMPILE_COMMANDS parameter.

For testing purposes, to make this process easier, an executor script has been developed that parses compilation database json files and analyses every source file that with our analyzer tool. It analyses the source files with given compiler options and writes the output of tool into files on the disk. It makes analysing of projects and evaluating the results easier.

The previously described issues were searched for, portabality problem caused by taking advantage of internal STL hierarchy, unused header files, legacy $\mathrm{C}$ header file usage. In this the test phase, several open-source projects were analysed by our tool. The libc $++^{2}$ STL implementation was used to analyse projects. The results of analyses are depicted on Table I. The first column means the STL internal hierarchy usages, second means unused header count, and the third one means the $\mathrm{C}$ header usage in legacy style.

TABLE I: Results

\begin{tabular}{|l|r|r|r|}
\hline & STL & Unused & C \\
\hline Tinyxml2 & 0 & 0 & 1 \\
\hline Json for Modern C++ & 0 & 3 & 0 \\
\hline Bloaty & 3 & 9 & 14 \\
\hline Guetzli & 2 & 4 & 34 \\
\hline Yaml-cpp & 1 & 16 & 2 \\
\hline Flatbuffers & 0 & 3 & 7 \\
\hline Orc & 4 & 8 & 20 \\
\hline
\end{tabular}

${ }^{1}$ https://clang.llvm.org/docs/JSONCompilationDatabase.html ${ }^{2}$ https://libcxx.llvm.org/ 
As it can be seen, the hits by different issues are not same. The STL internal hierarchy usage issue were found in a few number of cases. Unused headers and $\mathrm{C}$ header usage have been found in a large number. The distribution of hits is not equal for each projects, some of them has many unused header and some of them has any other issues. To conclude this section, real issues were found in real open source projects, that proves that the tool can catch them. However, there were some false positive reports, but they have been fixed in the software and not counted in results.

\section{RELATED WORK}

The analysis of $\mathrm{C}++$ programs is quite popular these days, thus this is not the first tool which target to analyse the include dependency in any kind of way.

One of them is Include-what-you-use (IWYU) [22] tool from Google that is an open source project ${ }^{3}$. It analyses the include hierarchy as well, but it is different in regards of purpose of the tool compared to our. It generally suggests to include header file which provides the symbols that one uses in the source files. It aims to detect places where one does not include the header file which provides the given symbol. The similarity with our tool is both of them can detect unused headers but it comes from behaviour of the tools.

However, our tool tries to find several kind of portability issues along with unused headers in the include dependency.

\section{CONCLUSION}

In this paper we have introduced subtle issues in the $\mathrm{C}++$ Standard Template Library that cause portability issues. To overcome this kind of portability problem, we have created a tool to detect portability issues in the include hierarchy of Standard Template Library. Deep knowledge is required from the C++ Standard about the STL header files to detect this kind of mistakes, mainly how they include each other and what they need to provide by definition.

This tool is based on Clang library that is the most appropriate technique to create new kind of analysis tools for $\mathrm{C}$ and $\mathrm{C}++$ nowadays. It is a command line tool, so it analyzes the source code and prints out the result, where one can fix and improve the implementation. Also, it is able to determine whether a header file is not used in a translation unit. It is implemented as an extra feature with minimal cost, but it is very useful feature of this tool. The tool has some impressive results, it is tested on open source projects.

\section{REFERENCES}

[1] C. Lattner, "LLVM and Clang: Next generation compiler technology," 2008, lecture at BSD Conference 2008.

[2] M. H. Austern, Generic Programming and the STL: Using and Extending the C++ Standard Template Library. Addison-Wesley, 1999.

[3] B. Stroustrup, The C++ Programming Language (special edition). Addison-Wesley, 2000

[4] N. Pataki, "C++ Standard Template Library by safe functors," in Proc. of 8th Joint Conference on Mathematics and Computer Science, MaCS 2010, Selected Paper, H. F. Pop and A. Bege, Eds. Komárno: Novadat Ltd., 2011, pp. 363-374.
[5] G. Horváth and N. Pataki, "Clang matchers for verified usage of the C++ Standard Template Library," Annales Mathematicae et Informaticae, vol. 44, pp. 99-109, 2015. [Online]. Available: http://ami.ektf.hu/uploads/papers/finalpdf/AMI_44_from99to109.pdf

[6] S. Meyers, Effective STL. Addison-Wesley, 2001.

[7] N. Nagappan and T. Ball, "Static analysis tools as early indicators of pre-release defect density," in Proceedings of the 27th International Conference on Software Engineering, ser. ICSE '05. New York, NY, USA: ACM, 2005, pp. 580-586. [Online]. Available: http: //doi.acm.org/10.1145/1062455.1062558

[8] B. Johnson, Y. Song, E. Murphy-Hill, and R. Bowdidge, "Why don't software developers use static analysis tools to find bugs?" in Proceedings of the 2013 International Conference on Software Engineering, ser. ICSE '13. Piscataway, NJ, USA: IEEE Press, 2013 , pp. 672-681. [Online]. Available: http://dx.doi.org/10.1109/ICSE.2013. 6606613

[9] C. King., "Symbolic execution and program testing," Communication of the ACM, vol. 19, pp. 385-394, 1976. [Online]. Available: http://dl.acm.org/citation.cfm?id=360252

[10] C. Szabó, M. Kotul, and R. Petruš, "A closer look at software refactoring using symbolic execution," in The 9th International Conference on Applied Informatics - Volume 2, 2014, pp. 309-316. [Online]. Available: http://dx.doi.org/10.14794/ICAI.9.2014.2.309

[11] Z. Xu, T. Kremenek, and J. Zhang, "A memory model for static analysis of C programs," in ISoLA'10 Proceedings of the 4th international conference on Leveraging applications of formal methods, verification, and validation - Volume Part I. Heidelberg: Springer-Verlag Berlin, 2010, pp. 535-548.

[12] T. Brunner, N. Pataki, and Z. Porkoláb, "Backward compatibility violations and their detection in $\mathrm{C}++$ legacy code using static analysis," Acta Electrotechnica et Informatica, vol. 16, no. 2, pp. 12-19, 2016. [Online]. Available: http://dx.doi.org/10.15546/aeei-2016-0009

[13] G. Horváth and N. Pataki, "Source language representation of function summaries in static analysis," in Proceedings of the 11th Workshop on Implementation, Compilation, Optimization of Object-Oriented Languages, Programs and Systems, ser. ICOOOLPS '16. New York, NY, USA: ACM, 2016, pp. 6:1-6:9. [Online]. Available: http://doi.acm.org/10.1145/3012408.3012414

[14] A. Baráth and Z. Porkoláb, "Automatic checking of the usage of the C++11 move semantics," Acta Cybernetica, vol. 22, no. 1, pp. 5-20, 2015. [Online]. Available: http://dx.doi.org/10.14232/actacyb.22.1.2015. 2

[15] E. Stepanov and K. Serebryany, "Memorysanitizer: Fast detector of uninitialized memory use in C++," in Proceedings of the 13th Annual IEEE/ACM International Symposium on Code Generation and Optimization, ser. CGO '15. Washington, DC, USA: IEEE Computer Society, 2015, pp. 46-55. [Online]. Available: http: //dx.doi.org/10.1109/CGO.2015.7054186

[16] N. Pataki, T. Cséri, and Z. Szúgyi, "Task-specific style verification," AIP Conference Proceedings, vol. 1479, no. 1, pp. 490-493, 2012 [Online]. Available: http://dx.doi.org/10.1063/1.4756173

[17] V. Májer and N. Pataki, "Concurrent object construction in modern object-oriented programming languages," in The 9th International Conference on Applied Informatics - Volume 2, 2014, pp. 293-300. [Online]. Available: http://dx.doi.org/10.14794/ICAI.9.2014.2.293

[18] B. Babati, N. Pataki, and Z. Porkoláb, "C/C++ preprocessing with modern data storage devices," in 2015 IEEE 13th International Scientific Conference on Informatics, Nov 2015, pp. 36-40. [Online]. Available: http://dx.doi.org/10.1109/Informatics.2015.7377804

[19] J. Mihalicza, "Compile C++ systems in quarter time," in Proceedings of 10th International Scientific Conference on Informatics, 2009, pp. 136 141. [Online]. Available: http://dx.doi.org/10.14794/ICAI.9.2014.2.309

[20] Y. Yu, H. Dayani-Fard, and J. Mylopoulos, "Removing false code dependencies to speedup software build processes," in Proceedings of the 2003 Conference of the Centre for Advanced Studies on Collaborative Research, ser. CASCON '03. IBM Press, 2003, pp. 343-352. [Online]. Available: http://dl.acm.org/citation.cfm?id=961322.961375

[21] J. Mihalicza, "How \#includes affect build time in large systems," in Proceedings of the 8th international conference on applied informatics (ICAI 2010), 2010, pp. 343-350.

[22] C. Silverstein, "Implementing Include-what-you-use using clang," 2010 , 2010 LLVM Developers' Meeting Talk. 


\section{A QA System for learning Python}

\author{
Marcos Oliveira Ramos \\ Dpt. Informática, Centro Algoritmi \\ Universidade do Minho \\ Braga, Portugal \\ pg28503@alunos.uminho.pt
}

\author{
Maria João Varanda Pereira \\ Dpt. Informática e Comunicações, IPB \\ Centro Algoritmi, Universidade do Minho \\ Bragança, Portugal \\ mjoao@ipb.pt
}

\author{
Pedro Rangel Henriques \\ Dpt. Informática, Centro Algoritmi \\ Universidade do Minho \\ Braga, Portugal \\ pedrorangelhenriques@gmail.com
}

\begin{abstract}
This article proposes a Question Answering System that can automatically answer to questions presented in a natural language about the Python programming language. A system of this kind aims at the interaction with a human. Since it is natural for a human to communicate in a natural language, such as Portuguese or English, there is a need for systems that can respond to the user in the same language. When restricted to a closed or specific knowledge domain, these systems can offer satisfiable answers to the posed questions. So, it is expected that the proposed QA System can present reasonable answers to questions about Python. After surveying this emergent working area, that is growing every day, we will present the design and implementation of a Python QA system in order to prove that it is possible to adopt a systematic approach to construct this kind of systems.
\end{abstract}

\section{INTRODUCTION}

W ITH increasingly demanding users, look for answers on the WWW using standard search engines is no longer desirable having become a complicated and inefficient task.

The main goal of Question Answering (QA) Systems is to provide a new way of searching information based on natural language questions. At first, this can turn easier the user interaction and open the possibility to be used by people in general. At second, the QA System gives a concrete short answer to the user rather than a list of possible related documents where the desired answer is mixed with other kind of informations as happens in a common search engine.

A QA System can be open or closed domain but in a closed knowledge domain, it can be tuned to give more accurate answers. The system should be prepared with a set of databases resources and with a mechanism to analyse the input question. Techniques of information extraction are then used to construct the answer. Each question should be analysed based on its syntatic structure and on a set of keywords and it should be translated into a repository query. Complex data structures should be prepared to receive the query and avoid redundant, incomplete or wrong answers. At the end the given concrete answer can be complemented with a set of related documents.

PythonQAS, the QA System presented in this paper, is restricted to a closed knowledge domain: Python Programming Language. It is expected to be useful to students or to professionals that want to know more about this language. Moreover Python doesn't belong to the Informatics Engineering Degree Curricula and all the material and tools will be very useful people who wants to learn this language.
A survey of methods and tools to build Question \& Answering is presented in Section 2. Our proposal to construct a closed domain QA System for Python programming language is sketched in Section 3; a block diagram to depict the system's architecture is shown, and each component is described in detail. Section 4 is dedicated to discuss the implementation based on a first glance at the questions of the FAQ for Python. In section 5 the new web interface for Pyhton QA system will be presented. Section 6 closes the paper with some conclusions and directions for future work.

\section{RELATED WORK}

QA Systems are mostly separated by closed and open domain. Closed-domain systems answer questions within a specific knowledge domain or to only a certain type of questions. They target precision, rather than coverage. Dealing with a restricted scope of knowledge allows QA systems to resort to smaller amounts of information, usually structured data such as ontologies. With such limited, formalized and structured data, systems try to take great advantages of natural language processing techniques in order to be the most accurate possible in their answers. Open-domain systems answer questions about almost everything. These system rely on much larger amounts of data than closed-domain QA systems, using mostly unstructured data and general ontologies. Their primarily goal is to provide factoid answers to questions about world knowledge. While closed-domain systems aim at accuracy, open-domain systems intend to cover the greater scope of information that is possible. The ambition is to offer more than a conventional web search engine by answering the user's questions, rather than presenting a simple list of documents/web pages that match the search's query.

Concerning techniques and approaches involved, QA systems will be classified according to three different perspectives: techniques for question analysis; techniques for retrieving answers from knowledge repositories; and techniques for composing the final answer.

For question analysis, some QA systems are based on methods of natural language processing, i.e. methods that try to derive meaning from natural language input. Natural Language Processing is itself a very prominent field of computer science and artificial intelligence that involves techniques like parsing and machine learning. The process consists in converting the user's question into a database query written in a formal 
language such as SQL and SPARQL. The output of the query will usually be given as an answer. BASEBALL [4] and LUNAR are closed domain QA systems and they fit perfectly into this category. START ${ }^{1}$ is another example of a linguistic approach but it is an open domain.

Another technique for question analysis is Pattern Matching anf Tagging. The QA system analyses a question and labels it in order to find a pattern. If the pattern corresponds to the expected pattern for a certain answer, then this answer should be the right one. For example, when posed with the question, "Who is the President of Portugal?" the system interprets the question as " $<$ Person Name $>$ is the $<$ President of Portugal $>$ ?" and expects the answer to be the name of a person who is president of Portugal. QACID [3] an ontologybased QA system, applies tagging algorithms in order to extract a query pattern, i.e. a query in natural language labeled with morphological information and ontological concepts. To overcome tagging difficulties, due to the complexity of natural languages and human error, QA systems might rely on, for example, synonyms and algorithms for removing stem words, stop words and vowels. The AQUA system [11], among its various steps for processing a question, divides the sentence into subject, verb, propositional phrases, adjectives and objectives. Similar to QACID, it produces a semantic representation of the query that is used by search algorithms when trying to find an answer in the knowledge base. [10] proposes a method that combines patterns with machine learning techniques in an open domain QA system. They use the machine learning technique of bootstrapping to build a tagged corpus from some examples of hand crafted question-answer pairs. These examples are passed to a search engine and from the results of the search, the system extracts patterns. The precision of each pattern is calculated for each type of question. The patterns are then employed in finding answers for new questions.

Concerning Answer Retrieval, some systems rely on structured knowledge sources and ontologies about a specific domain and they are mostly closed-domain QA systems. The idea is to make queries over a database that contains structured information about the system domain. Meaning that the information was produced before the questions were asked and that, if the knowledge source works as it should, the effort lays mainly in understanding the question rather than finding the best answer. This is only feasible when the scope of the domain is well defined and restricted, and the knowledge source is relatively small, very well defined and structured. QACID [3] is a good example of this. In this case the information, from which the system derives answers to the user's questions, is stored in textual documents written in natural language. Ontologies are mostly used to define a language in which documents and questions can be represented and exploited [9]. The most interesting feature of these systems, and what makes them perfect for working with an open-domain, is the capacity of taking advantage of the ever increasing amount of textual information, available throughout

${ }^{1}$ http://start.csail.mit.edu the Internet. Web search engines like Google can be used to find and retrieve these knowledge sources from the Internet. Nonetheless, not all QA systems rely on this technique, since most of the time, there is no guarantee of the correctness of the retrieved information. The system described in [2] and MULDER [7] are examples of this.

Concerning Answer formulation, a QA system can give as result a set of fragments of texts or text highlighting or just a succinct answer. Some QA systems are text based, meaning that the answer to the question posed by the user, will be a fragment of a text. By using this approach, a system becomes intricately related to other information access techniques such as "document retrieval", in which entire documents are retrieved, and "passage retrieval" in which chunks of text are returned [8] as answers. Even though, this does not offer much more than a web search engine such as Google, Yahoo, etc. it might be enough to answer a question, since it relies on the intelligence of the user to extract the exact meaning from the text. In [1], PiQASso(Pisa Question Answering System) is an example of this and in [5] is explained how QuALIM [6] answers can be supplemented with paragraphs from the Wikipedia. Contrary to the last approach, some other systems give a succinct answer that directly satisfies the user information needs. Besides giving a direct answer in a natural language, some of these systems will also provide additional informations related to the topic. Evi ${ }^{2}$, formerly known as True Knowledge is an open-domain QA platform that translates the user's questions into a language independent query that is executed using the knowledge base and an inference system. The result of the question is a direct answer to the user's question.

\section{Python QA SYSTEM: OUR PROPOSED SOLUTION}

A closed-domain system is more adequate when wanting to built something different from what is available today. Since it deals with a smaller scope of information, it is possible to analyze the problem in greater detail, to create a system that could solve a real problem of a certain domain. Nonetheless, most QA system do not necessarily intend to replace the conventional search engines that we have become familiar with, but to complement them or to present a viable alternative.

\section{A. Chosen domain: Python}

Python has been capturing attention in recent years. Its popularity among both beginners and experienced programmers is rapidly increasing. Many people learn Python by themselves, resorting on large amounts of scattered information available throughout the Internet. The QA system being proposed could help programmers by reducing the effort necessary to find useful data among all this information.

Python is a powerful and very popular language. Nonetheless, other languages such as Java, C++ or Ruby have some or all of the same functionalities and could as well serve as the domain of the proposed system. The system should be capable

\footnotetext{
${ }^{2}$ https://www.evi.com
} 


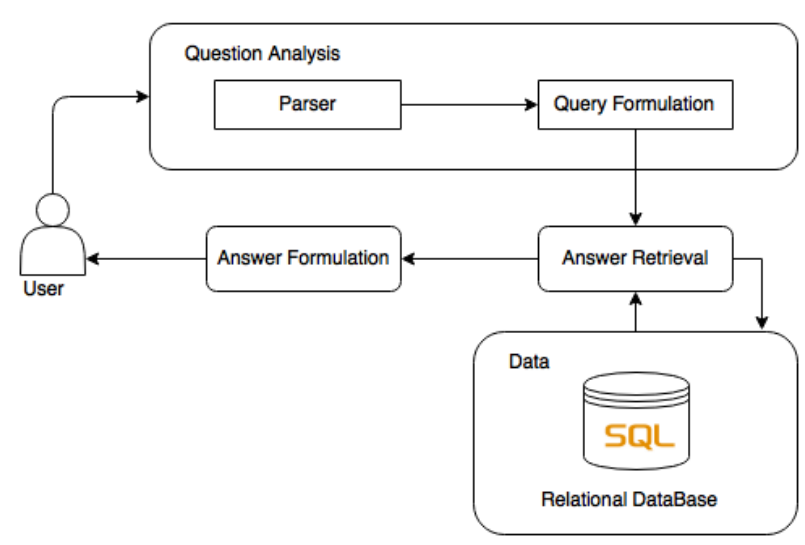

Fig. 1. Proposed System's Architecture

of being used in the domain of another programming language without the need for structural changes.

On a first phase, the Python Frequently Asked Questions $(\mathrm{FAQ})^{3}$, will be used as a knowledge source. Since the goal is to create a system capable of answering question in natural language about Python, the fastest way to create a functional prototype is to populate its knowledge base with answers and questions that the Python Software Foundation classifies as "frequently asked". On a second phase, more data should be added to the database in order to enhance the system capabilities.

\section{B. System's Architecture}

Figure 1 depicts the proposed system's architecture. The system starts by accepting a question from the user. Then it parses the question in order to produce a query that will be used to retrieve information from the database. This information will then be analyzed and presented to the user.

Every QA system starts by receiving a user's question. Understanding the meaning of the question is vital for the process of retrieving the correct information.

PythonQAS parses the question in order to identify certain words. Any other words are discarded leaving only the desired words and preserving the order that they had in the user's question. This 'meta-question' is then used to construct a query in order to retrieve data from a relational database. The knowledge data should be stored in a way that offers stability, safety and fast access to data. We decided to adopt a structured knowledge base such as a relational database.

When providing an answer to a user's question, we are usually confronted with a dilemma. Should the answer contain just enough information to answer the question succinctly or should the system present additional information about the context of the answer? We approach the problem by establishing a middle term between two methodologies. If a user's question makes sense within the domain of the system, then a succinct answer should be presented to the user. At the same time extracts of text or web links containing information about

${ }^{3}$ https://docs.python.org/faq/ the topics of the question are also shown as a complement. When the system can not find a satisfiable answer, a message notifying the user of this fact appears instead of the desired result.

\section{SySTEM IMPLEMENTATION}

The proposed system was built as a web application. This section describes the system functionalities, the used tools and the implementation approach to build the application.

\section{A. System's Overview}

To start implementing the QA system the Python FAQ (PyFAQ) was used as a source of questions and answers on this domain. That PyFAQ will be the seed to build the basic knowledge repository (KR) but in the near future it can and must be enriched with other sources. On one hand, it will support the processing of simple and literal questions; on the other hand, it will allow to provide direct answers.

First and foremost it is necessary to find a method to analyze questions and subsequently apply this method to build the knowledge base and find the respective answers.

We propose the creation of pairs of question-skeleton mapped to $(\mapsto)$ the respective answer.

A question-skeleton is a template, or pattern, that describes the intention of a question. For instance, the question "How to print out the value of a variable in Python?" can be abstracted by the following skeleton that defines the question intention: "HOW(print, [variable])". So, our KR will store the information as quadruples (or four-tuples), Question Type $\rightarrow$ Action $\rightarrow$ Keywords $\rightarrow$ Answer.

Looking at the PyFAQ we concluded that the majority of the questions are posed using a small, limited kind of words, mostly adverbs, that give meaning to the phrase. We can then join questions into groups of Question Types.

In Table I there are some examples of how questions in the PyFAQ could be classified by the proposed Question Types.

TABLE I

QUESTION TYPES

\begin{tabular}{ll} 
Question Type & Question \\
\hline \multirow{2}{*}{ Why } & Why are Python strings immutable? \\
& Why is there no goto? \\
\multirow{2}{*}{ How } & How are lists implemented? \\
& How do I convert a string to a number? \\
\hline Where & How do I modify a string in place? \\
\hline \multirow{2}{*}{ What } & Where is the math.py source file? \\
\hline When & What is a method? \\
\hline
\end{tabular}

Usually, a question fits only one Question Type. The answers can be reused by different (but similar) questions.

Answers will be given a confidence rate. That way even if a question does not satisfy the user's needs, others with a lower confidence rate might solve the problem.

To illustrate the idea, observe the following entry in the PyFAQ:

Q3.23: Why is there no goto? 
(A3.23:) You can use exceptions to provide a "structured goto" that even works across function calls. Many feel that exceptions can conveniently emulate all reasonable uses of the "go" or "goto" constructs of C, Fortran, and other languages.

This answer could also satisfy the question "How can I use a goto statement?". Even though the two questions are different, they could be both mapped to the same answer. But, for example the question "What is a goto statement?" can not be clarified by the answer. The Question Type is important to identify possible answers, but this does not imply that a question of some type can not be also responded by the answer to a question of a different type. It just means that the confidence of the system in the assertiveness of the answer is not as great as it would be in a different situation.

As said above, each entry in the $\mathrm{KR}$ (a pair < questionskeleton $\mapsto$ answer $>$ stored in our central relational database contains the Question Type and also an Action (verb) and a set of Keywords (mostly nouns) that abstract the question intention. This triple (Type, Action, Keywords) composes the so called question-skeleton.

Table II illustrates this principle with examples taken again from PyFAQ.

TABLE II

KEYWORDS

\begin{tabular}{lll}
\hline Question & Action & Keywords \\
\hline Why is there no goto? & not(exist) & goto \\
\hline How are lists implemented? & implement & list \\
\hline How do I convert a string to a number? & convert & string + number \\
\hline How do I modify a string in place? & modify & string + place \\
\hline What is a method? & define & method \\
\hline What is a class? & define & class \\
\hline When can I call a method? & call & method \\
$\begin{array}{l}\text { Is it possible to allocate memory } \\
\text { for strings in creation time? }\end{array}$ & allocate & $\begin{array}{c}\text { memory + string } \\
\text { creation time }\end{array}$ \\
\hline
\end{tabular}

Notice that the Action and Keywords that represent a question do not need to be precisely the same words that are found in the question sentence. We resort to an English dictionary or thesaurus to lemmatize verbs and find synonyms.

This approach introduces generality in the knowledge base and it is a way to be flexible in the question interpretation. For instance, the question "Why does not exist goto in Python ?" will be replied with the same answer (A3.23) because the sentence will be recognized and represented by the same skeleton of (Q3.23).

To sum up, the (Q\&A3.23) shown above is represented in our KR (contained in the central database) as a four-tuple ${ }^{4}$ illustrated by the entry in Table III.

If the user types the question "Why does not exist a jump statement in Python?", the system processes the sentence with NLP tools complemented by a NL Thesaurus and a PL Ontology (to convert nouns to the singular form, verbs to infinitive form, and find synonyms, etc.), in order to identify the Question Type, Action and the Keywords that characterize

\footnotetext{
${ }^{4}$ Actually three elements that describe the question-skeleton plus the answer.
}

TABLE III

REPRESENTATION OF AN ENTRY IN THE DATABASE

\begin{tabular}{lcc}
\hline Q Type Action & Kw & Answer \\
\hline & $\begin{array}{c}\text { You can use exceptions to provide a "structured } \\
\text { goto" that even works across function calls. } \\
\text { Many feel that exceptions can conveniently } \\
\text { emulate all reasonable uses of the "go" or "goto" } \\
\end{array}$ \\
Why not(exist) goto & constructs of C, Fortran, and other languages.
\end{tabular}

the question skeleton. By extracting that information, our system will generate a query to send to the database aiming at retrieve the associated answer.

To be more precise, after analyzing the input sentence our system tries to find an entry with the same Question Type (Why in this case), and with at least one Keyword and Action. If our database contained only the entries with the set of keywords shown in Table II, the system would not find any match. But the system resorts to synonyms and similar concepts, thus it would not only look for the keyword jump statement but also for goto. A simple scoring function is then used to rank the candidate answer(s) based on the frequency of the Question Type, Action and Keywords. The answer with the highest score is then presented to the user followed by the ones above a certain score. If there is no satisfiable answer, the system returns a simple message notifying the user of this fact and advises him to rewrite the question.

\section{B. Technology used}

Since the domain of the system is a programming language (Python), we decided to use the same language for the development of the application. Python is a very popular language among the natural language processing community, thus, there are many free and good tools, packages and libraries for the language, such as NLTK.

Django was chosen as a framework to construct the web site. Using a framework such as Django allow us to construct a web site without needing to build everything from scratch. Django is free and open-source, it is written in Python and promises to be fast, secure and scalable. It officially supports various relational databases which facilitates the building of data-driven websites and comes with an integrated web server that helps to test the application faster and almost effortless. It also offers an administration application and many capabilities that intend to reduce effort and increase safety like forms, and models to build and connect with the database.

PostgreSQL was chosen as the database engine for the project. It is a free and open source object relational database that runs on most modern operative systems and can be both used for small single-machine applications and larger data warehousing.

\section{Implementation approach}

This section presents all the main steps necessary to build PythonQAS, along with explanations regarding the different methods adopted, the reasons behind all major decisions and the most critical obstacles that were faced. 


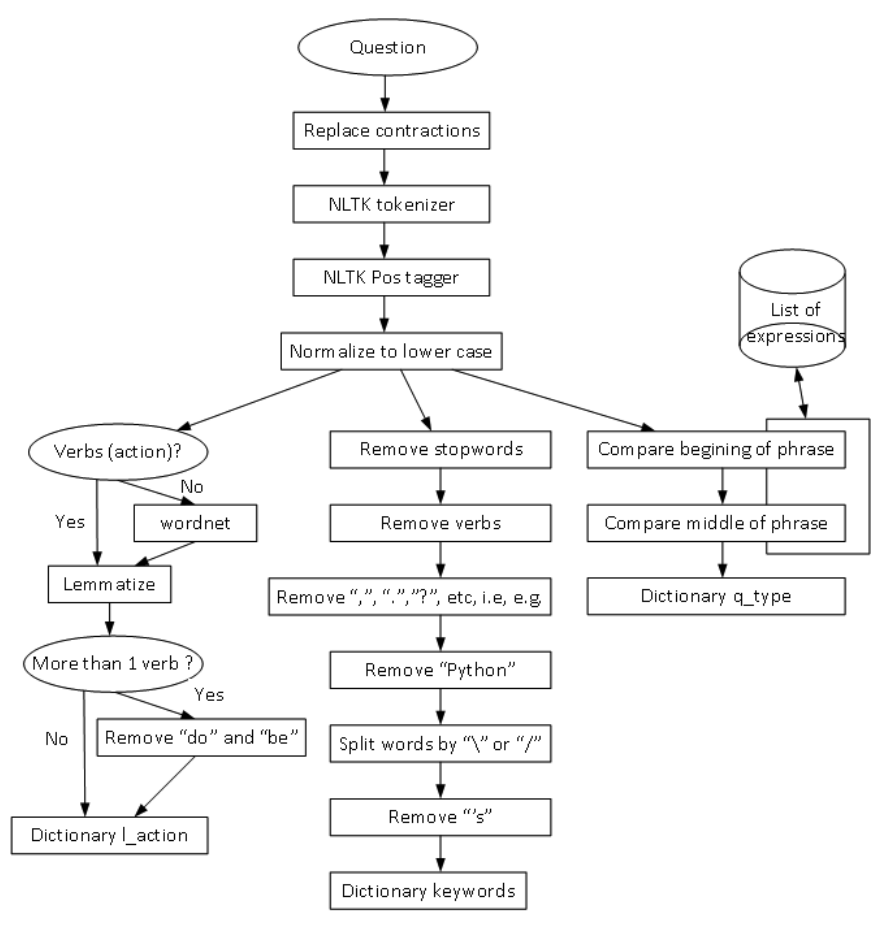

Fig. 2. Phrase Analysis

1) Phrase Analysis: A phrase or a question is always composed of many components such as adverbs, nouns, verbs, etc... To understand the meaning of a phrase it is necessary to split it into these components. Even if not in a conscientious way we, human beings, also do it. Take the example of when we are trying to learn a new language and we do not know all the vocabulary that a native uses in its daily life. Even if we do not know the word we are sometimes able to infer it's meaning by its position on the phrase. To analyze a question, the proposed QA system does a similar job by dividing a phrase into multiple components and trying to identify three different types: action, keywords and a question type.

Concerning the Action, usually, while using natural languages, human beings use a word that describes an action, an occurrence or a state of being. This word is obviously called a verb. But finding the right action or the main action of a question is not always an easy task. A question could have more than one verb, verbs have tenses (past, present, future), verbs change according to the personal pronoun and a verb might, for example, be no different from a noun, except for its position on the phrase. Even a human being could be mistaken about the right action of a question if not given more context or information about the it.

Figure 2 depicts the major techniques used by the system for analyzing a phrase.

In the application an Action is an object that starts by receiving a question as a simple string. Before doing anything to the question it is best to find any contractions that may exist in the phrase and if possible convert them to their full form. This may lead to ambiguous situations [12]. After some tests with different contractions it was concluded that the advantage of trying to disambiguate would not offer any significant advantages if taken to account the work that would be needed. Thus, the PythonQAS only converts contractions that are not ambiguous.

Next step, the phrase is divided into multiple substrings using the NLTK Tokenizer Package, in order to be used by the Pos (Part-of-speech) Tagger from the NLTK Tagging Package. Right after using the Pos Tagger, the words are converted to their lower form to avoid problems when comparing words. This could not be done before because it could decrease the efficacy of the Post Tagger. For example, the word "I" in a phrase has a complete different meaning from the letter "i".

The process continues by identifying all the verbs in the question. These will be saved to a dictionary where the key is a word (verb) and the object is a number from 0 to 1 . This number represents the trust in the assertiveness of the verb being the right action. It will be very useful to find an answer in the database and distinguish the different results of the database queries. This dictionary will be called $l$ _action along this document. If one or more verbs were found, these will be converted to their infinitive mode using the NLTK WordNetLemmatizer.

If at the beginning, no verbs were found, then a different path should be followed in order to find at least one verb. The process works by looking at the words of the phrase and trying to find any that could be a verb. But, since a word could be used both as a verb or an adjective, this process is not very reliable. So, the Trust of the verbs will be reduced.

Next, the Stopwords are removed. Stopwords are usually the most common used words in a certain language and do not contain any significant importance to the meaning of a phrase. Thus, its removal is very common when processing natural languages and usually do not compromise the success of the analysis.

The next step is to find which words could be verbs by using WordNet, a lexical database. It groups words into synonyms called synsets and provides many data about these words like number of relations, definitions and examples of usage. It might be seen as a mixture of a thesaurus and a dictionary. Next, PythonQAS resorts once again to the dictionary containing the results of the Pos Tagging process to verify if the remaining words are nouns or adjectives. If so, and if the words are positively identified by WordNet as verbs, the words are added to dictionary "1_action".

If more than one verb was found, regardless of the way the verbs were obtained, the tool will try to exclude false positive findings. First the PythonQAS removes all stopwords and words that contain characters which are not letters.

Concerning the Keywords, most phrases in a natural language contain a set of keywords that give the phrase meaning and context. These words are not the most frequent words used in a speech, but it would be impossible to express anything more than basic replies without them. For example, the question "How can I populate a dictionary?" has one keyword "dictionary". The question "How can I populate a dictionary 
with lists?" has a set of two keywords, "dictionary" and "lists". The first question relates to the context "dictionary" and the second to a context contained in the first one. Thus, the more keywords, the smaller the domain of the phrase will be.

In the Action process we knew that actions were verbs, but keywords are not as restrictive as that. So, the strategy here passes more by excluding unwanted information(keywords). PythonQAS starts by removing all stopwords and words that were identified by the Pos Tagging process as being verbs.

Next, the keywords are saved to a dictionary ("keywords"), similar to the "l_action". The keywords are the keys of the dictionary and their objects are numbers between 0 and 1, representing the trust in the assertiveness of the classification.

It was observed in some questions that similar concepts/keywords were separated by a slash(")" or "/"). Neither the NLTK Tokenizer or the Pos Tagger were able to separate them and they were considered to be a single word. To solve the problem, PythonQAS tries to identify any slashes or other separators inside every keywords. After the system splits the keywords using a list of separators, if the individual words contain more than one alpha character, the old word is erased from the dictionary and the new ones are added.

Concerning the Question Type, it was noticed that the questions of the Python FAQ are almost always formulated in similar ways. By grouping the questions by their similarity it was possible to find a set of question types. Question types are expressions such as "How", "When", "Where", etc.. These types are not directly related to the keywords or even the action of the question, but its use could change the entire meaning of a question. For example the questions "How should I use a dictionary?" and "Where should I use a dictionary?" are almost the same except for the use of "How" or "Where". The answers to both questions are also similar and we could actually find the answer to one of them in an indirect way in the answer of the other. Nonetheless, PythonQAS should be the most accurate possible and for that it tries to distinguish from the different question types.

A Question Type is, like the Keywords and Action, an object. The initial part of the procedure is shared with the Keywords and the Action classes.

After rhis, the system uses a list of lists of expressions to try to find the question type of the phrase. The list does not contain words but lists of words. Each list contains expressions that are similar. That way when searching for an answer, not only the question type of the users question can be used but also the similar expressions in the list.

The most important information produced by the object's different parts is contained within a dictionary, called "q_type", similar to the ones used for the Action and Keywords, whose keys are strings (question types) and the objects are numbers between 0 and 1 .

To identify the expressions in the phrase the system starts by trying to match the different question types with the beginning of the question. The question type is usually at the beginning of the phrase.
The next step is to find out if the question was formulated using two phrases. The system splits the text into multiple phrases and tries to identify question types at the beginning of each phrase and then tries to identify it in the middle.

If no question types were found, the system adopts a new way to find a satisfiable question type. When comparing the expressions, or words that constitute the question type of the phrase, with the results of the NLTK PosTagger a relation was discovered between the tags and the question types. The question type, or part of it, is usually tagged with one of the following tags: "WRB", "WP", "MD", "WDT" or "EX". But even though they are usually tagged with those tags, not all words tagged in the same way constitute question types. For example, Wh-adverbs (WRB) are adverbs that start by "wh", sometimes called interrogative words. "how" or "however" are such examples. Nonetheless "how" is a very common question type contrary to "however".

2) Information Storage: The Python FAQ is used as the initial knowledge base of the PythonQAS. The initial database is very important because it allow us to test the system and adjust it according to the results. All the different web pages containing the questions/answers were downloaded comprising not only the text, but also their structure and HTML annotations. That way, things like colored text and web links can keep being part of the answer.

The Django Framework offers many capabilities, such as Models, that try to decrease the complexity and work associated with the building of a web application. Models allow us to simply create python classes that contain and represent information. These classes are used by Django to create all the database structure. This not only saves time by building the database, but also, because all objects in the database are treated as python classes in the application, it allows the programmer to save, delete, update and retrieve data from the database without the need to ever use SQL code. The Answer Model contains five variables: question, question type, action, a set of keywords and an answer.

3) Information Retrieval: After receiving a question from the user, the system creates an object(AnswersRetrieval) that aggregates everything necessary to process the question and search for answers. When the object is created it starts by defining variables used to store relevant information about the execution of the different methods contained by the object. These variables indicate different weights and measures to calculate the probability of an answer being right, the NLTK Stemmer that is going to be used, answers found in the database, results of analyzing the question, etc.. It also creates three objects, explained before: Question Type, Action and Keywords.

The next step will be to call three auxiliary functions to retrieve answers from the database. Each of these correspond to the components extracted from the phrase. The different methods used to retrieve the answers and the analysis of the question are used to calculate a probability value.

If the system found any actions in the phrase, then for each action it will try to find all Answer objects with that action. 
First, a variable is created holding the Trust in the action that is being analyzed. This value is provided by the Action object. Second, the Answer objects that contain this action are obtained from the database using a direct match.

Then the word (action) is saved to a dictionary containing pairs as objects. This dictionary, called 'visited", saves all the words that were already used in the search process to avoid duplicated results. The keys are the words being used in the search. The first element of the pair is the a string "word" and the second is the actual word used to search the Answers.

Last, a dictionary "action_answ" is built containing all the answers. The key of the dictionary is a tuple where the first element is the Answer object and the second is the action that was used in the search process. The object appointed by the key is a value between 0 and 1 containing the trust in the assertiveness of the answer.

In the next step, instead of using the action to search for Answer objects, the system uses a stem word of that action using one of the Stemmers provided by the NLTK. After obtaining the stem word, the system searches for Answers with an action that starts with it. Even though a stem word does not always correspond to the beginning of the word, in most cases it does. The best option would be to apply the Stemmer to the action contained by each individual Answer, but by doing so the system would have to read and compare each Answer using only Python (we do execute any SQL code but of course Django still does). In a very small database this would not be a problem but in a medium sized to large database the time and resources necessary to do this would be very unsatisfactory. Then, the action is saved to the "visited" dictionary. Now, the key used in the dictionary is the stem word and not the action as before. This way the system saves what it really used in the search without discarding the action. This is necessary because two different words might have the same stem word and saving the action instead of the stem word could cause duplicate values. Lastly, the results obtained by search are saved to the "action_answ" dictionary.

The next step is to use synonyms by resorting to the WordNetLemmatizer. Note that only synonyms that are verbs will be used. The process used to find Answer objects using synonyms is not very different from what it was done before with the original word. Synonyms are received and for each of them a search for Answers will be performed followed by the use of Stem words.

In the next step, the system tries to find Answer objects using the keywords extracted from the phrase. The procedure is similar to the one used for finding answers with an action. First, the system declares a dictionary with the visited elements. For each keyword in the list of keywords contained by the object Keyword, a direct match will be tried .

After the first search there is a slight difference. Since the verbs/action were already in their infinitive form, nothing was done regarding this. Thus it is necessary to lemmatize the keywords using the WordNetLemmatizer. A dictionary "keywords_answ" will contain the Answers and the Trust in these same Answers, similarly to the "action_answ". Next, the stem word of the current keyword is used followed by the use of synonyms and their stem words.

For any question types found by the questions analysis, the system searches the database for matches. Because of the nature of question types and the methods used to extract them, the search for answers based on the question type is simpler from the Action and Keywords methods. Nonetheless, the idea is still very similar.

A list is created, temporarily storing the expressions that were already visited by the search process. For each question type in the Question Type object, the PythonQAS queries the database in order to get any entries whose question type corresponds to the current expression. Any Answers found will be stored in a dictionary called "type_answ", where the keys are tuples containing the Answer and the expression used to retrieve that answer, and the objects are values between 0 and 1 expressing the trust in that answer being the right one to the users question.

After creating the three dictionaries "action_answ", "keywords_answ" and "type_answ" containing all the answer extracted with the actions, keywords and question types, the goal is to merge these into a new dictionary "answ_prob". The keys of the dictionary are Answers and the objects are dictionaries containing the trust/probability of the assertiveness of the Answer regarding the action, keywords and question type of each Answer.

Regarding the Answers found with the question type, the dictionary "type_answ" will be treated in the same way as the "action_answ". The same strategy cannot be applied regarding the keywords because an Answer can have more than one keyword. Instead of simply copying the trust, the system increments the value of the keyword of the dictionary with that trust value. This way a previous value is never lost but summed. Now, the system will divide this number by the number of keywords of the Answer Object resulting in a trust value made of the values obtained by using all keywords found in the question.

The main goal of an object of type Answer is to provide answers, thus, after everything that was explained above, the system has the dictionary "answ_prob" containing all possible answers. Still there is not yet a way from where to choose the best possible answer. The system will iterate all the Answers in the dictionary and it will calculate its probability based on the trust value of the Keywords, Action and Question Type of the respective Answer.

After, the system returns a list containing tuples (answer, probability), sorted by the probability value.

A good example would be to ask PythonQAS, "What are the rules for local and global variables in Python?" and thus obtain a list of possible answers sorted by the probability value. This example can be consulted at http://pythonqas. epl.di.uminho.pt/qaSystem/answer/?question_text=What+are+ the+rules+for+local+and+global+variables+in+Python $\% 3 \mathrm{~F}$. 


\section{WEB APPLICATION}

PythonQAS uses a web interface to communicate with both users and system administrators. The website can be consulted at http://pythonqas.epl.di.uminho.pt.

Regarding the normal user's interface, the system provides a way to ask questions, view results and some information regarding the usage of PythonQAS, information about the authors and the developing methods used in its construction. When a user asks a question the system creates an AnswerRetrieval object using the question. If the object produces any answers and if these answers have a probability/trust value greater than a certain value defined in the system's programming, the answers will be presented to the user, sorted by their trust value. Only the most probable answer is shown to the user, the other answers are hidden inside collapsed divs showing only their order on the list and their trust value. Nonetheless, the user only has to click on the div to see its content.

Regarding the administrator's interface, PythonQAS provides, through the Django Administration web application a way to list all answers and keywords in the database, edit, delete, update and insert new entries, and add new users (administrators).

Even though Django offers a very good administration interface, it lacks in some functionality particular to the PythonQAS. Thus, the system was complemented in order to be able to: receive a text file containing pairs Question $\rightarrow$ Answer, parse this file, show the results to the user along with suggestions about the different components needed to insert the information into the database and a way to insert it after it is reviewed; easily insert an individual entry to the database; receive a question and creates three objects of the classes Action, Keywords and Question Type and return the results to the interface to test the system capabilities.

\section{Vi. CONCLUSION}

The design and implementation of a computer based system capable of understanding an Human question, about a knowledge domain, delivering an appropriate answer is a major dream that is stimulating, since a long time ago, the artificial intelligence, computer science and linguistic communities to moving them up to a deeper research. In that context of Q\&A (question and answering) Systems, this paper contributed, on one hand, with a survey on the work already done aimed at the classification of existing approaches and tools, and, on the other hand, as a proof of concept with the design and implementation of PythonQAS, a web-based system to answer questions set up by programmer about the language Python. Even returning satisfactory answers, the system still lacks the access to other information sources to be able to derive more answers (the more the system grows in terms of information, the better it will be able to provide the accurate answers). Another project contribution, also discussed in the paper, was the choice of Python information sources and the development of a back-end system to collect information from them and automatically populate PythonQAS knowledge repository. A first (and simple) test and evaluation of PythonQAS was performed to draw conclusions about the system outcomes (the lessons learned were pointed out) in order to understand the directions for future work (possible in a PHD context):

1) Increase the number of answers in the database, i.e. more reliable data should be added to the KR;

2) Adjust the different measures used to calculate the probability value of an answer;

3) Integrate the PythonQAS into a Question and Answer Web Site like Stackoverflow;

4) Apply this approach and technology to another Knowledge Domain, for instance Java, Perl or C\# programming languages, to test its validity;

5) Apply this approach in a different Natural Language environment, for instance to Portuguese.

\section{ACKNOWLEDGMENT}

This work has been supported by COMPETE: POCI-010145-FEDER-007043 and FCT - Fundação para a Ciência e Tecnologia within the Project Scope: UID/CEC/00319/2013.

\section{REFERENCES}

11] Giuseppe Attardi, Antonio Cisternino, Francesco Formica, Maria Simi, Alessandro Tommasi, and Cesare Zavattari. Piqasso: Pisa question answering system. In TREC, 2001.

[2] Susan Dumais, Michele Banko, Eric Brill, Jimmy Lin, and Andrew Ng. Web question answering: Is more always better? In Proceedings of the 25th annual international ACM SIGIR conference on Research and development in information retrieval, pages 291-298. ACM, 2002.

[3] Oscar Ferrández, Rubén Izquierdo, Sergio Ferrández, and José Luis Vicedo. Addressing ontology-based question answering with collections of user queries. Information Processing \& Management, 45(2):175-188, 2009.

[4] Bert F Green Jr, Alice K Wolf, Carol Chomsky, and Kenneth Laughery. Baseball: an automatic question-answerer. In Papers presented at the May 9-11, 1961, western joint IRE-AIEE-ACM computer conference, pages 219-224. ACM, 1961.

[5] Michael Kaisser. The qualim question answering demo: Supplementing answers with paragraphs drawn from wikipedia. In Proceedings of the 46th Annual Meeting of the Association for Computational Linguistics on Human Language Technologies: Demo Session, pages 32-35. Association for Computational Linguistics, 2008.

[6] Michael Kaisser and Tilman Becker. Question answering by searching large corpora with linguistic methods. In TREC, 2004

[7] Cody Kwok, Oren Etzioni, and Daniel S Weld. Scaling question answering to the web. ACM Transactions on Information Systems (TOIS), 19(3):242-262, 2001.

[8] Jimmy Lin, Dennis Quan, Vineet Sinha, Karun Bakshi, David Huynh, Boris Katz, and David R Karger. What makes a good answer? the role of context in question answering. In Proceedings of the Ninth IFIP TC13 International Conference on Human-Computer Interaction (INTERACT 2003), pages 25-32, 2003.

[9] Diego Mollá and José Luis Vicedo. Question answering in restricted domains: An overview. Computational Linguistics, 33(1):41-61, 2007.

[10] Deepak Ravichandran and Eduard Hovy. Learning surface text patterns for a question answering system. In Proceedings of the 40th Annual Meeting on Association for Computational Linguistics, pages 41-47. Association for Computational Linguistics, 2002.

[11] Maria Vargas-Vera and Miltiadis D Lytras. Aqua: A closed-domain question answering system. Information Systems Management, 27(3):217$225,2010$.

[12] Martin Volk and Rico Sennrich. Disambiguation of english contractions for machine translation of tv subtitles. 2011. 


\title{
Parsing with Earley Virtual Machines
}

\author{
Audrius Šaikūnas \\ Institute of Mathematics and Informatics \\ Akademijos 4, LT-08663 Vilnius, Lithuania \\ Email: tuxmarkv@gmail.com
}

\begin{abstract}
Earley parser is a well-known parsing method used to analyse context-free grammars. While being less efficient in practical contexts than other generalized context-free parsing algorithms, such as GLR, it is also more general. As such it can be used as a foundation to build more complex parsing algorithms.

We present a new, virtual machine based approach to parsing, heavily based on the original Earley parser. We present several variations of the Earley Virtual Machine, each with increasing feature set. The final version of the Earley Virtual Machine is capable of parsing context-free grammars with data-dependant constraints and support grammars with regular right hand sides and regular lookahead.

We show how to translate grammars into virtual machine instruction sequences that are then used by the parsing algorithm. Additionally, we present two methods for constructing shared packed parse forests based on the parse input.
\end{abstract}

\section{INTRODUCTION}

Parsing is one of the oldest problems in computer science. Pretty much every compiler ever written has a parser within it. Even in applications, not directly related to computer science or software development, parsers are a common occurrence. Date formats, URL addresses, e-mail addresses, file paths are just a few examples of everyday character strings that have to be parsed before any meaningful computation can be done with them. It is probably harder to come up with everyday application example that doesn't make use of parsing in some way rather than list the ones that do.

Because of the widespread usage of parsers, it no surprise that there are numerous parsing algorithms available. Many consider the parsing problem to be solved, but the reality couldn't be farther from the truth. Most of the existing parsing algorithms have severe limitations, that restrict the use cases of these algorithms. One of the newest $\mathrm{C}++$ programming language compiler implementations, the CLang, doesn't make use of any formal parsing or syntax definition methods and instead use a hand-crafted recursive descent parser. The HTML5 is arguably one of the most important modern standards, as it defines the shape of the internet. Yet the syntax of HTML5 documents is defined by using custom abstract state machines, as none of the more traditional parsing methods are capable of matching closing and opening XML/HTML tags.

It is clear that more flexible and general parsing methods are needed that are capable of parsing more than only context-free grammars.

As such, we present a new approach to parsing: the Earley Virtual Machine, or EVM for short. It is a virtual machine based parser, heavily based on the original Earley parser.
EVM is capable of parsing context-free languages with datadependant constraints. The core idea behind of EVM is to have two different grammar representations: one is userfriendly and used to define grammars, while the other is used internally during parsing. Therefore, we end up with grammars that specify the languages in a user-friendly way, which are then compiled into grammar programs that are executed or interpreted by the EVM to match various input sequences.

We present several iterations of EVM:

- $\mathrm{EVM}_{0}$ that is equivalent to the original Earley parser in it's capabilities.

- $\mathrm{EVM}_{1}$ is an extension of $\mathrm{EVM}_{0}$ that enables the use of regular operators within grammar rule definitions, thus easing the development of new grammars.

- $\mathrm{EVM}_{2}$ further extends $\mathrm{EVM}_{1}$ by allowing the use of regular-lookahead operator.

- Finally, $\mathrm{EVM}_{3}$ is an extension of $\mathrm{EVM}_{2}$ that enables general purpose computation during parsing, and as such allows to conditionally control the parsing process based on the results of previously parsed data. Therefore, $\mathrm{EVM}_{3}$ can be used to recognize data-dependant language constructs that cannot be parsed by more traditional parsing methods.

In section III we present two separate methods for constructing the abstract syntax trees (or more precisely, shared packed parse forests) based on the input data. Automatic AST construction enables automatic construction of shared packed parse forests, without any changes to the grammar. On the other hand, manual AST construction requires explicit instructions from the user to control the AST construction.

\section{EARLEy ViRTUAL MACHINE}

\section{A. EVM structure}

For every terminal input symbol input $_{i}$ EVM creates a state $S_{i}$. A state $S_{i}$ is a tuple $\left\langle\right.$ input $\left._{i}, F, F S\right\rangle$.

Each state $S_{i}$ contains a set of fibers $F$. Fibers in EVM loosely correspond to items in Earley parser. Each fiber is a tuple $\langle i p$,origin $\rangle$. ip is the instruction pointer of the current fiber and origin is the origin state number. The origin value indicates the input offset where the currently parsed rule has begun. In a away, the origin value may be considered as the return "address" for the rule.

Additionally, each state has a set of suspended fibers FS. Fibers are suspended when they invoke different rules/nonterminal symbols. A fiber remains suspended until the appropriate non-terminal symbol is matched, at which point the fiber 
is resumed by copying it to the set of running fibers $F$ in the current state.

An $\mathrm{EVM}_{0}$ grammar is a set of productions in form sym $\rightarrow$ body, where sym is a non-terminal symbol and body is a grammar expression.

An $\mathrm{EVM}_{0}$ grammar expression is defined recursively as:

- $a$ is a terminal grammar expression, where $a$ is a terminal symbol.

- $A$ is a non-terminal grammar expression, where $A$ is a non-terminal symbol.

- $\epsilon$ is an epsilon grammar expression.

- $(e)$ is a brace grammar expression, where $e$ is a grammar expression.

- $e_{1} e_{2}$ is a sequence grammar expression, where $e_{1}$ and $e_{2}$ are grammar expressions.

Every EVM grammar program is a tuple $\langle$ instrs, rule_map $\rangle$. instrs is the sequence of instructions that represents the compiled grammar. rule_map is mapping from non-terminal symbols to locations in the instruction sequence, which represents entry points for the grammar program. It is used to determine the start locations of compiled rules for specific non-terminal symbols.

Every instruction in EVM completes with one of the following results:

- r_continue. This result is used to indicate that the current fiber should continue executing instructions in order. That means that after executing an instruction ip of current fiber should be increased by 1 .

- r_continue_to $i p_{n e w}$. This behaves exactly like r_continue, however $i p$ of current fiber is set to $i p_{n e w}$ after completing execution of an instruction.

- r_discard. This result is used to indicate that the current fiber needs to be terminated.

- r_suspend. This result is used to indicate that the current fiber needs to be suspended.

To correctly represent all Earley parser grammars, the following instructions are required:

- i_call_dyn sym. This instruction is used to begin parsing of non-terminal symbol sym. It dynamically invokes all rules with product sym. This constitutes creating new fibers in state $S_{c u r r}$ with $i p$ pointing to beginning of every rule with product sym and origin curr. The instruction completes with result $r_{-}$cont inue. Because the fibers of every state are stored in a set, multiple or recursive invocations of the same product have no effect.

- i_match_sym $\operatorname{sym}_{1} \rightarrow i p_{1}, \ldots$, sym $_{n} \rightarrow i p_{n}$. This instruction is used to detect a successful reduction of one or more non-terminal symbols $s y m_{1}, \ldots, s y m_{n}$. When $s m_{i}$ is reduced, a new fiber is created with ip pointing to corresponding $i p_{i}$. The instruction operands essentially form a jumptable. Whenever instruction i_match_sym is executed, the current fiber is suspended and moved to the the set of suspended fibers in the current state (it completes with result r_suspend).
TABLE I

EVM $_{0}$ GRAMMAR COMPILATION RULES

\begin{tabular}{|c|c|}
\hline Grammar element & Instruction sequence \\
\hline $\begin{array}{l}\text { Grammar: } \\
\mathrm{G}=\left\{P_{1}, \ldots, P_{n}\right\}\end{array}$ & $\begin{array}{l}\text { i_call_dyn main } \\
\text { i_match_sym main } \rightarrow l_{\text {accept }} \\
l_{\text {accept }}: \\
\text { i_accept } \\
\text { i_stop } \\
\text { code }\left(P_{1}\right) \\
\ldots \\
\text { code }\left(P_{n}\right)\end{array}$ \\
\hline $\begin{array}{l}\text { Production rule: } \\
P \rightarrow e\end{array}$ & $\begin{array}{l}\text { code }(e) \\
\text { i_reduce } P \\
\text { i_stop }\end{array}$ \\
\hline $\begin{array}{l}\text { Terminal grammar expression: } \\
a\end{array}$ & i_match_char $a \rightarrow i p_{\text {next }}$ \\
\hline $\begin{array}{l}\text { Non-terminal grammar expression } \\
\text { (dynamic): } \\
A\end{array}$ & $\begin{array}{l}\text { i_call_dyn } A \\
\text { i_match_sym } A \rightarrow i p_{\text {next }}\end{array}$ \\
\hline \multicolumn{2}{|l|}{$\begin{array}{l}\text { Epsilon grammar expression: } \\
\epsilon\end{array}$} \\
\hline $\begin{array}{l}\text { Brace grammar expression: } \\
(e)\end{array}$ & $\operatorname{code}(e)$ \\
\hline $\begin{array}{l}\text { Sequence grammar expression: } \\
e_{1} e_{2}\end{array}$ & $\begin{array}{l}\operatorname{code}\left(e_{1}\right) \\
\operatorname{code}\left(e_{2}\right)\end{array}$ \\
\hline
\end{tabular}

- i_match_char $\operatorname{char}_{1} \rightarrow i p_{1}, \ldots, \operatorname{char}_{n} \rightarrow i p_{n}$. This instruction is used to match terminal symbols. If the current input symbol $i n_{\text {curr }}$ matches one of the instruction operands $\operatorname{char}_{i}$ then a new fiber $\left\langle i p_{i}\right.$, origin $\rangle$ in state $S_{\text {curr }+1}$ is created. After executing this instruction, the current fiber is discarded (it completes with result r_discard)

- i_reduce sym. This instruction performs the reduction of non-terminal symbol sym. It is used to indicate that a grammar rule with product sym has matched successfully. The instruction finds all suspended fibers in state $S_{\text {origin }}$ that have been previously suspended with instruction i_match_sym and resumes them in state $S_{c u r r}$. Only those fibers are resumed, which have sym among their operands. The fibers are resumed by creating a copy of suspended fiber in the current state with updated instruction pointer. The instruction completes with result r_continue.

- i_stop. This instruction stops and discards the current fiber. It is used to destroy the current fiber when a parse rule is matched successfully, usually immediately after executing a i_reduce instruction. The instruction completes with r_discard.

- i_accept. This instruction is used to indicate the parse input is valid and can be accepted.

$\mathrm{EVM}_{0}$ grammar compilation rules are shown in table I. Productions of a grammar are compiled in sequence. $\operatorname{code}(E)$ represents instruction sequence for grammar element $E$, where $E$ may be a grammar, a production rule or a grammar expression. $i p_{\text {next }}$ is instruction pointer of the next instruction.

An example $\mathrm{EVM}_{0}$ grammar and the corresponding grammar program are shown in table II. 


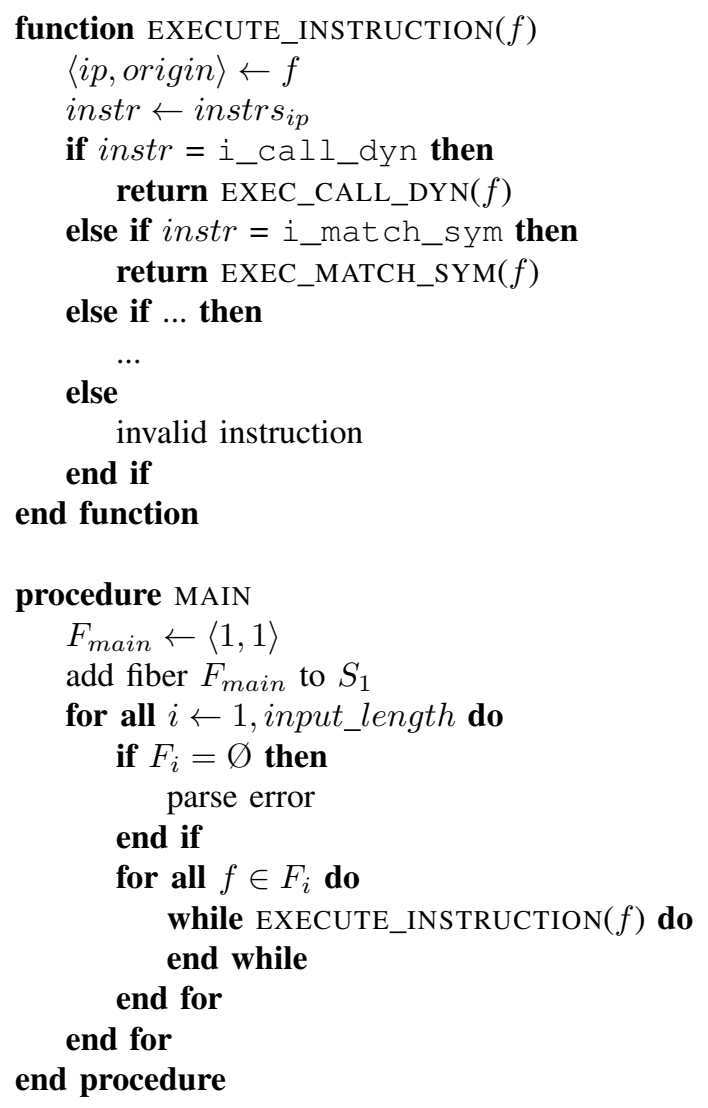

Fig. 1. $\mathrm{EVM}_{0}$ parser algorithm

TABLE II

EVM $_{0}$ GRAMMAR COMPILATION EXAMPLE

\begin{tabular}{|c|c|}
\hline Grammar & Instruction sequence \\
\hline $\begin{array}{l}I \rightarrow a \\
E->I \\
E \rightarrow D+I\end{array}$ & 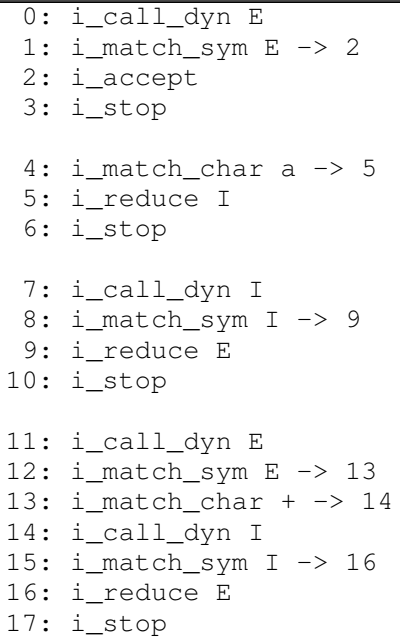 \\
\hline
\end{tabular}

\section{B. Extending EVM to support regular right-hand sides}

To support regular right-hand sides in production rules, our definition of a grammar needs to be extended.

$\mathrm{An} \mathrm{EVM}_{1}$ grammar is a set of productions in form sym $\rightarrow$ body, where sym is a non-terminal symbol and body is an
TABLE III

EVM $_{1}$ GRAMMAR COMPILATION RULES

\begin{tabular}{|c|c|}
\hline Grammar element & Instruction sequence \\
\hline $\begin{array}{l}\text { One-or-more grammar expression: } \\
e+\end{array}$ & 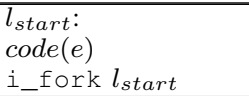 \\
\hline $\begin{array}{l}\text { Kleene star grammar expression: } \\
\text { e* }\end{array}$ & $\begin{array}{l}l_{\text {start }}: \\
\text { i_fork } l_{\text {end }} \\
\text { code }(e) \\
\text { i_br } l_{\text {start }} \\
l_{\text {end }} \text { : }\end{array}$ \\
\hline $\begin{array}{l}\text { Optional grammar expression: } \\
e ?\end{array}$ & $\begin{array}{l}\text { i_fork } l_{\text {end }} \\
\text { code }(e) \\
l_{\text {end }}:\end{array}$ \\
\hline $\begin{array}{l}\text { Alternative grammar expression: } \\
e_{1} \mid e_{2}\end{array}$ & $\begin{array}{l}\text { i_fork } l_{\text {other }} \\
\text { code }\left(e_{1}\right) \\
{\text { i_br } l_{\text {end }}} \\
l_{\text {other }}: \\
\text { code }\left(e_{2}\right) \\
l_{\text {end }}:\end{array}$ \\
\hline
\end{tabular}

$\mathrm{EVM}_{1}$ grammar expression.

An $\mathrm{EVM}_{1}$ grammar expression is defined as follows:

- If $e$ is an $\mathrm{EVM}_{0}$ grammar expression, it is also an $\mathrm{EVM}_{1}$ grammar expression.

- $e+$ is a one-more-more grammar expression, where $e$ is a grammar expression.

- $e^{*}$ is a klenne star grammar expression, where $e$ is a grammar expression.

- $e$ ? is an optional grammar expression, where $e$ is a grammar expression.

- $e_{1} \mid e_{2}$ is an alternative grammar expression, where $e_{1}$ and $e_{2}$ are grammar expressions.

To implement these new grammar constructs additional virtual machine instructions are required:

- i_br $i p_{\text {new }}$. This instruction is used to perform an unconditional branch to the given instruction pointer $i p_{n e w}$. This instruction completes with result $r_{\text {_continue_to }}$ $i p_{n e w}$.

- i_fork $i p_{n e w}$. This instruction is used to fork the current fiber. After forking, the instruction pointer of the new fiber is set to $i p_{n e w}$. This instruction completes with result $r$ _continue.

$\mathrm{EVM}_{1}$ grammar compilation rules are shown in table III. $l_{\text {start }}, l_{\text {end }}$ and $l_{\text {other }}$ are code labels. During grammar compilation these labels are replaced with concrete instruction pointer values.

An example grammar rule that uses regular operator + and its corresponding instruction sequence are shown in table IV.

\section{Regular lookahead in EVM}

In addition to having regular right-hand sides, sometimes it is helpful to perform regular lookahead during parsing. The $\mathrm{EVM}_{1}$ may be further augmented to support this feature.

An $\mathrm{EVM}_{2}$ grammar expression is defined as:

- $e$, where $e$ is $\mathrm{EVM}_{1}$ grammar expression.

- $e_{1}>e_{2}$ is a positive lookahead grammar expression, where $e_{1}$ and $e_{2}$ are grammar expressions. 
TABLE IV

EVM $_{1}$ GRAMMAR COMPILATION EXAMPLE

\begin{tabular}{|l||l|}
\hline Grammar rule & Instruction sequence \\
\hline \multirow{5}{*}{ B $\rightarrow$ (A a $)+$} & $20:$ i_call_dyn A \\
& $21:$ i_match_sym A $->22$ \\
& $22:$ i_match_char a $->23$ \\
& $23:$ i_fork 20 \\
& $24:$ i_reduce B \\
& $25:$ i_stop \\
& $\ldots$ \\
&
\end{tabular}

Grammar expression $A>B$ means that $A$ should only match if it is immediately followed by $B$.

There are two ways of implementing regular lookahead in the current model of EVM:

- When parsing $A(B>C) D$, EVM can first parse $A$, then $B$, then both $C$ and $D$ in parallel. Should it appear during parsing that $C$ fails to match, then the corresponding parse should be rejected.

- When parsing $A(B>C) D$, EVM can first parse $A$, then $B$, after which parsing of $D$ is delayed until $C$ matches successfully. Once $C$ matches successfully, parsing of $D$ is resumed.

Both of these approaches have advantages and disadvantages. The first approach is simpler and doesn't require any fundamental changes to the way EVM processes input: one terminal symbol at a time without an ability to backtrack and reparse certain parts of the input. However it also may require storing additional information about fiber relationships and complex logic for discarding fibers resulting of invalid parses. More importantly, this approach starts parsing $D$ preemptively even when it is not known whether or not the lookahead will succeed. As a result, such lookahead implementation may be less efficient.

The second approach requires changes to EVM to allow suspending fibers that depend on lookahead expressions and later resume then in backtracked position. This approach should be more efficient when the lookahead grammar expression fails to match often. We select the latter approach for implementing lookahead in EVM.

Right now, $\mathrm{EVM}_{1}$ is built under assumption that i_match_sym is always executed before corresponding i_reduce. However with grammars that contain lookahead this may no longer be true in all cases. Consider grammar expression $(A>B) B$. Initially, non-terminal symbol $A$ is matched, after which the instruction sequence for the lookahead sub-expression $B$ will be executed. It will cause matching of non-terminal symbol $B$. If $B$ parses successfully, then the whole sub-expression $(A>B)$ will be matched and the original fiber for parsing $(A>B) B$ will be resumed It will try to parse $B$ again by invoking symbol $B$ with instruction i_call_dyn, which will cause no new fibers to be created, as $B$ was already parsed in the lookahead sub-expression. That means that the original thread will be permanently suspended with instruction i_match_sym and the expression $(A>B) B$ will always fail to parse.

To avoid the previously described issue and to support parsing lookahead expressions, EVM can no longer rely on the strict ordering of i_match_sym and i_reduce instructions. As a result, the following changes to EVM are required:

- $\mathrm{EVM}_{2}$ in every state has to keep track of reductions that happened and their respective lengths.

- Instruction i_reduce has to store in origin state $S_{\text {origin }}$ reduction symbol and it's final state index curr.

- Instruction i_match_sym has to check all of it's operands for the reductions that may have already happened and to resume fibers in final states of the reductions. This may only occur if the matching symbol was already parsed in a lookahead sub-expression.

- Every fiber has to have a priority value, as lookahead sub-expressions have to be executed first.

An $\mathrm{EVM}_{2}$ state $S_{i}$ is a tuple $\langle i, R, T, F S\rangle$, where $i$ is the state index, $R$ is the reduction map, $T$ is execution trace set, $F S$ is the suspended fiber set. Because the running fiber set is longer stored stored within the state, it is necessary to ensure that there are no duplicate fibers. This is achieved by using execution trace set $T$, which stores instruction pointer and origin state index pairs. Whenever a new fiber is to be created, the EVM first checks whether or not such fiber already exists in target state. If it does, then no new fiber is created. This eliminates infinite left-recursion and having to reparse the same input with the same grammar rule multiple times.

An $\mathrm{EVM}_{2}$ fiber $F$ is a tuple $\langle$ sid,prio, ip, origin $\rangle$, where sid is state index, prio is priority value, $i p$ is instruction pointer and origin is state index of origin (caller/return) state.

An $\mathrm{EVM}_{2}$ parser is a tuple $\langle$ input, $P, Q, S\rangle$, where input is the input terminal symbol sequence, $P$ is the grammar program, $Q$ is the fiber queue and $S$ is the state sequence. During parsing, fibers are executed according to their priority. Fibers with lower priority are removed from the fiber queue first. If there multiple fibers with the same priority, then the fiber with lowest state index is removed first. Otherwise, the order of execution is unspecified.

To support regular lookahead, additional two instructions are required:

- i_lookahead $i p_{a h e a d}$. This instruction is used to begin parsing of a lookahead sub-expression at instruction pointer $i p_{a h e a d}$. It creates a new fiber $\langle c u r r, p r i o-$ $\left.1, i p_{\text {ahead }}, c u r r\right\rangle$, where curr is the index of the current state, prio is the priority of the current fiber. The instruction completes with result $r$ _continue.

- i_lookahead_ret sym. This instruction is used to finish parsing lookahead sub-expression. It is identical to i_reduce with one key difference: fibers are resumed not in current state, but in origin state. The instruction completes with r_discard.

EVM $_{2}$ grammar compilation rules are provided in table V. usym is an unique non-terminal symbol created for each lookahead expression. 
TABLE V

EVM $_{2}$ GRAMMAR COMPILATION RULES

\begin{tabular}{|l||l|}
\hline Grammar element & Instruction sequence \\
\hline \multirow{4}{*}{ Lookahead grammar expression: } & code $\left(e_{1}\right)$ \\
& i_lookahead $l_{\text {ahead }}$ \\
$e_{1}>e_{2}$ & i_match_sym usym $\rightarrow l_{\text {end }}$ \\
& $l_{\text {ahead }}:$ \\
& code $\left(e_{2}\right)$ \\
& i_lookahead_ret $u s y m$ \\
& $l_{\text {end }}:$ \\
\hline
\end{tabular}

\section{Eliminating dynamic rule resolution}

In the current model of EVM, rule resolution is performed dynamically during runtime by the i_call_dyn instruction. It is possible to eliminate this indirection by replacing every i_call_dyn prod instruction with a sequence of i_call $i p$ instructions, where every i_call instruction invokes a different production rule for the same product prod. This also means that the compiled grammar programs are no longer required to keep track of the rule_map variable, that was previously used by the i_call_dyn instruction.

\section{E. Parsing with data-dependant constraints}

It is well known that many languages used in practise cannot be represented purely by using context-free grammars. For example, in order to parse XML sources, an additional automata is needed to match opening and closing XML tags. Other languages have fixed-width fields of width $n$, where $n$ is an integer value preceded before the field. Specifying such languages with context-free grammars is impossible as well, as the parser has to semantically recognize the meaning behind the length field and to use that value to continue parsing.

In order for parser like $\mathrm{EVM}_{2}$ to parse XML, it needs somehow to "remember" the opening XML tags and later match the closing tag only against the remembered string. This can be achieved by further augmenting $\mathrm{EVM}_{2}$ into $\mathrm{EVM}_{3}$ by applying the following changes:

- Fibers have to be extended to contain stacks. A fiber's stack may be used to perform general-purpose computation during parsing.

- Execution trace set has to be extended to include fiber's stack. This enables $\mathrm{EVM}_{3}$ to simultaneously execute multiple fibers with the same instruction pointer and the same origin, but with different data-constraints, which will be stored in the stack

- i_call instruction has to be extended with an operand to contain the number of parameters to copy from the stack of the current fiber to the stack of target fiber.

- The following new categories of instructions are needed: stack instructions, conditional control transfer instructions, data processing instructions.

To support general-purpose computation, $\mathrm{EVM}_{3}$ grammars need to be extended to include statements and expressions (not to be confused with grammar expressions). In $\mathrm{EVM}_{3}$ grammar rule definitions look akin to function definitions or subroutines that are found in general-purpose programming languages: grammar rule definitions are composed out of 0 or more procedural statements. These statements enable to adjust the control flow of the grammar rule.

Expressions, just like in more traditional programming languages, enable manipulation of variable values. Both variable values and intermediate expression values are stored in the stack of the current fiber.

To separate "traditional" value-based expressions from the grammar expressions that are used to specify matching rules, a special parse statement is introduced to the grammar language. The parse statement may be mixed in with the the other grammar statements to more accurately control and constrain the parse process. This enables to direct the parsing process based on the variable values that may have been derived from the parse input.

To support these new features, new EVM instructions are required. Non-exhaustive list of new instructions in $\mathrm{EVM}_{3}$ :

- i_push_int $v$. Pushes an integer value $v$ to the stack of the current fiber.

- i_pop $n$. Removes the top $n$ values from the stack.

- i_peek $i$. Pushes a copy of stack value with index $i$ to the top of the stack.

- i_poke $i$. Removes the top value of the stack and sets the stack value with index $i$ to that value.

- i_bz $i p_{n e w}$. Conditional control transfer to $i p_{n e w}$. Removes the top stack value and performs jump to $i p_{n e w}$ if the value is 0 .

- i_match_char_dyn. Used for data-dependent matching of terminal symbols. Matches the top value of the stack with input $t_{c u r r}$. If the symbols match, it creates a new fiber $\langle$ curr +1 , prio, ip +1 , origin $\rangle$. The instruction completes with result $r$ _discard. An optimized version of the instruction may move the fiber from the $S_{\text {curr }}$ state to $S_{c u r r+1}$ with updated $i p$ value.

- i_foreign_call $n i d x$. Calls a foreign function with index $i d x$ and $n$ arguments. The call is performed by popping $n$ arguments from the stack and passing them to the foreign callee. The result of the callee is pushed back to the stack. The instruction may be used to implement various data processing operations without adding additional data-processing instructions.

- i_add_int. Pops two top elements from the stack and pushes their sum to the top of the stack.

- ...

Most of the newly added instructions complete with result r_continue, unless stated otherwise. New data processing (integer, string, list handling) instructions may be added as needed. The same operations may be implemented by using i_foreign_call instruction to call external functions in the environment that implements EVM.

$\mathrm{EVM}_{3}$ grammar to grammar program compilation rules are provided in table VI. The table lists only the core elements of the grammar language to illustrate the overall grammar compilation process. New statements and expressions may be added as needed. Additionally, the syntax of the grammar 
TABLE VI

EVM $_{3}$ GRAMMAR COMPILATION RULES

\begin{tabular}{|c|c|}
\hline Grammar element & Instruction sequence \\
\hline $\begin{array}{l}\text { EVM }_{3} \text { grammar rule: } \\
\text { rule } \operatorname{sym}\left(\arg _{1}, \ldots, \arg _{n}\right) \\
\quad \text { stmt }_{1} \\
\quad \ldots \\
\quad \text { stmt }_{n} \\
\text { end }\end{array}$ & $\begin{array}{l}\operatorname{code}\left(\operatorname{stm}_{1}\right) \\
\ldots \\
\text { code }\left(\text { stmt }_{n}\right) \\
\text { i_reduce sym } \\
\text { i_stop }\end{array}$ \\
\hline $\begin{array}{l}\text { If statement: } \\
\text { if cond } \\
\quad \text { body } \\
\text { end }\end{array}$ & $\begin{array}{l}\operatorname{code}(\text { cond }) \\
\text { i_bz } l_{\text {end }} \\
\text { code }(\text { body }) \\
l_{\text {end }}\end{array}$ \\
\hline $\begin{array}{l}\text { Parse statement: } \\
\text { parse grammar_expr }\end{array}$ & code $($ grammar_expr $)$ \\
\hline $\begin{array}{l}\text { While statement: } \\
\text { while cond } \\
\quad \text { body } \\
\text { end }\end{array}$ & $\begin{array}{l}l_{\text {start }}: \\
\text { code }(\text { cond }) \\
\text { i_bz } l_{\text {end }} \\
\text { code }(\text { body }) \\
\text { i_br } l_{\text {start }} \\
l_{\text {end }}:\end{array}$ \\
\hline $\begin{array}{l}\text { Variable declaration statement: } \\
\text { var } v=\operatorname{expr}\end{array}$ & code $($ expr $)$ \\
\hline $\begin{array}{l}\text { Integer constant expression: } \\
\text { value }\end{array}$ & i_push_int value \\
\hline $\begin{array}{l}\text { Variable read expression: } \\
v\end{array}$ & i_peek stack_slot $v$ \\
\hline $\begin{array}{l}\text { Variable write expression: } \\
v=e\end{array}$ & $\begin{array}{l}\text { code }(e) \\
\text { i_poke stack_slot }\end{array}$ \\
\hline $\begin{array}{l}\text { Dynamic terminal match } \\
\text { grammar expression: } \\
\text { @expr }\end{array}$ & $\begin{array}{l}\text { code }(\text { expr }) \\
\text { i_match_char_dyn }\end{array}$ \\
\hline $\begin{array}{l}\text { Parameterized non-terminal } \\
\text { grammar expression: } \\
A\left(a r g_{1}, \arg _{2}, \ldots, a r g_{n}\right)\end{array}$ & $\begin{array}{l}\operatorname{code}\left(\arg g_{1}\right) \\
\operatorname{code}\left(\arg g_{2}\right) \\
\ldots \\
\operatorname{code}\left(\arg g_{n}\right) \\
\text { i_call } n, i p_{1} \\
\ldots \\
\text { i_call } n, i p_{m} \\
\text { i_pop } n \\
\text { i_match_sym } A \rightarrow l_{\text {end }} \\
l_{\text {end }}:\end{array}$ \\
\hline
\end{tabular}

language may be changed to more closely suit the environment in which EVM is being implemented.

An example $\mathrm{EVM}_{3}$ grammar rule that imperatively matches $n$ of 'a' characters and the compiled instruction sequence are shown in table VII. This compiled instruction sequence may be later invoked with instruction i_call 1, 10, because this grammar rule has 1 parameter and the instruction sequence implementing the grammar rule starts at offset 10 .

\section{F. Garbage collection of suspended fibers}

The current version of EVM creates a state for every input terminal symbol. In case of a successful parse, every state needs to contain at least one fiber. If EVM is used in a scannerless setting, this means that the total amount of memory required for EVM will be significantly higher than that of the input string. As such, to support parsing longer strings, memory footprint of the EVM needs to be lowered.

There are several important observations to make:

- Most states and fibers after suspension will be never needed during parse again. As such, some states that are
TABLE VII

EVM $_{3}$ GRAMMAR COMPILATION EXAMPLE

\begin{tabular}{|l||l|}
\hline Grammar rule & Instruction sequence \\
\hline & $10:$ i_peek 0 \\
& $11:$ i_push_int 0 \\
$12:$ i_int_more \\
rule field $(\mathrm{n})$ & $13:$ i_bz 20 \\
while $\mathrm{n} 0$ & $14:$ i_match_char a -> 15 \\
parse a & $15:$ i_peek 0 \\
$\mathrm{n}=\mathrm{n}-1$ & $16:$ i_push_int 1 \\
end & $17:$ i_int_sub \\
end & $18:$ i_poke 0 \\
& $19:$ i_br 10 \\
& $20:$ i_reduce "field" \\
& $21:$ i_stop \\
\hline
\end{tabular}

unnecessary, together with the fibers they contain, may be discarded before the parsing process completes.

- The only instructions that access variables from previous states are i_reduce and i_lookahead_ret.

- State index sid of a fiber is always equal or higher to the lowest value sid in the fiber queue. In other words, new fibers are always created with monotonically increasing state indices.

Based on these observations, the following optimizations can be made:

- Execution trace sets may be discarded from states with indices from interval $\left[1, s i d_{\min }\right)$, where $s i d_{\min }$ is the lowest state index in fiber queue $Q$. These sets are only needed in states, where new fibers may be created. Because new fibers are created with monotonically increasing state indices, the sets are no longer needed.

- Unreachable states with indices $\left[2\right.$, sid $\left._{\min }\right)$ may be discarded completely.

A state with index sid is reachable if there exists a fiber (either running or suspended) with origin state index origin equal to sid. As such, mark-and-sweep garbage collector may be employed to identify reachable and unreachable states.

The described garbage collector will discard all states with the fibers they contain that are not part of any parse rule/active reduction that can be traced back to the starting non-terminal symbol. As a result, it will have a significant impact on overall memory usage, especially when EVM is used without a dedicated scanner. To reduce the garbage collector's performance impact to the parsing process, the garbage collector may be run every $n$ parsed terminal symbols.

\section{Constructing The ABStRACT SyntAX TREE}

So far, the last EVM version is only capable of recognizing the input. However recognizers have only limited practical applicability. As such, for EVM to truly be useful in practice, there needs to be a way to construct the abstract syntax tree from the terminal input symbol sequence.

Normally, extension of a recognizer into a parser is a fairly trivial task. However in case of EVM, constructing the AST is not as simple, because EVM supports parsing ambiguous inputs that may result in a parse forest, which represents 
multiple valid parse trees for the same input. In case of highly ambiguous parses, naive approach of storing a complete copy of a parse tree for each valid parse path is not viable, as it may lead to an exponential parse forest growth. As a result, parsers that support parsing ambiguous inputs use special data structures to represent the parse trees, called shared packed parse forests, or SPPFs for short.

SPPFs look and behave similarly to regular parse trees. However, to represent ambiguity within a parse tree, special packed nodes are used. Children of a packed node represent different parses for the same input fragment. Furthermore, matching subtrees of packed nodes may be shared to reduce space requirements for storing the SPPF. In case of $\epsilon$ grammars, SPPFs may contain cycles.

There are two methods available for constructing SPPFs within $\mathrm{EVM}_{3}$ : automatic and manual AST construction.

\section{A. Manual AST construction}

As the name implies, ASTs in $\mathrm{EVM}_{3}$ may be constructed manually by relying on $\mathrm{EVM}_{3}$ 's imperative capabilities. The virtual machine may be extended with additional instructions that allow the creation and management of tagged AST nodes.

Specifically, the following new instructions are required:

- i_new_node num, sym. This instruction pops num AST node indices from the stack and creates an AST node with num popped children and label sym. Additionally, this instruction stores the interval [origin, curr] within the newly created node to represent the source interval of the node. Upon node creation, i_new_node pushes the reference of the resulting node to the stack. The newly created node is considered to be detached.

- i_reduce_rsym. Used to reduce a non-terminal symbol with a node-value. Works similarly to i_reduce, however upon execution it additionally pops a reference to an AST node from the stack and attaches it to the AST. The node attachment process works by assigning the node an unique index and storing that index and the node reference pair within the AST node list. Additionally, the node's unique index is stored within the reduction map of the origin state. If a reduction with the same non-terminal symbol and length already exists in the origin state, this indicates existence of ambiguity, as now there are two candidate non-terminal reductions within the same source interval. As such, a packed node is created in place of the previously created node. The old node gets assigned a new index and the two newly assigned indices are added as the children of the newly created packed node. If the previous node index already points to a packed node, then the node that is being attached is added to the packed node's children list.

- i_match_sym_r sym $m_{1} \rightarrow i p_{1}, \ldots$, sym $_{n} \rightarrow i p_{n}$. Just like the original i_match_sym, this instruction is used to match a non-terminal symbol. Upon successful resumption of a fiber previously suspended by i_match_sym_r, the instruction also pushes an index of AST node that represents the recently matched nonterminal symbol. The node referenced by the returned node index may be mutated later on by i_reduce_r instruction, as new ambiguous reductions are performed.

By applying this strategy of AST construction, AST nodes are created with the i_new_node instruction. Leaf nodes have no children and only contain the source range of input they represent. Nodes are then "returned" from grammar rules by using i_reduce_r instruction, which associates every AST node with a tuple $\langle$ sym, start, end $\rangle$, where start and end represent the source range of the node. In the event that more than one reduction is being associated with the same tuple, then a packed node is created with children that represent the alternative parses of non-terminal symbol sym in the source range $[$ start, end]. The returned node index is retrieved with the help of i_match_sym_r instruction, which pushes the index of the node into the stack of the callee. This enables the grammar program to use that index in future i_new_node instruction calls to construct non-leaf AST nodes. The node index that is returned from the start rule represents the root node.

It is important to note the importance of using node indices to represent the nodes rather than node references (or pointers): at the time of any given reduction (the call to i_match_sym_r) there is no way to know if there will be a matching future reduction that will cause the original reduction to become ambiguous. As such, node indices exist as a form of indirection, which allows swapping of a regular non-ambiguous node into an ambiguous packed node, when it is determined that there is more than one way to parse a given source range with the same non-terminal symbol.

To make use of these instructions, additional changes are required to the grammar language:

- A new AST node construction expression is needed to allow construction of AST nodes.

- A new return statement is needed that allows returning a previously constructed AST node.

- A new assignment grammar expression is needed to allow assignment of non-terminal return values (which store AST node indices) to previously declared variables.

An example grammar rule that manually constructs AST and the corresponding instruction sequence are provided in table VIII.

Even though manual AST construction requires new instructions and additional changes to the way grammars are specified and compiled, it also enables us to fine-tune and precisely control the AST construction process. Nodes, which are not meant to be included in the final AST may not be included as arguments for the i_new_node instruction, which effectively excludes such nodes from the final AST. Some grammar rules, such as the ones for parsing whitespace may not include any calls to i_new_node at all and may use the old i_reduce instruction, which should decrease the total amount of unnecessary AST nodes constructed and increase the overall performance of $\mathrm{EVM}_{3}$ parser. 
TABLE VIII

ManUAL AST CONSTRUCTION EXAMPLE

\begin{tabular}{|l||l|}
\hline Grammar rule & Instruction sequence \\
\hline \multirow{4}{*}{ rule E } & $30:$ i_call_dyn E \\
parse I:E + r:I & $31:$ i_match_sym_r E $->32$ \\
$32:$ i_match_char $+->33$ \\
return node("plus", & $34:$ i_call_dyn I \\
l, r) & $35:$ i_peek O \\
end & $36:$ i_peek 1 \\
& $37:$ i_new_node 2, "plus" \\
& $38:$ i_reduce_r e \\
& $39:$ i_stop \\
& $\ldots$ \\
\hline
\end{tabular}

\section{B. Automatic AST construction}

In some cases it may be desirable to be able to construct ASTs without any additional changes to the parser grammars. In such event, automatic EVM AST construction may be used instead.

To support automatic AST node construction, the following changes to $\mathrm{EVM}_{3}$ are required:

- Every fiber has to be extended to contain a list (or a stack) of child node indices that will be used during reduction to construct the AST node.

- i_reduce sym instruction has to be extended: 1) it has to construct an AST node with label sym and children from the child stack of the current fiber; 2) it has to include the node packing logic of the i_reduce_r instruction.

- i_match_sym instruction has to be extended: upon resuming a previously suspended fiber by i_match_sym, the instruction has to push the corresponding node index of the matched non-terminal symbol to the children stack.

Essentially, automatic AST construction works by merging instructions i_reduce_r and i_new_node into i_reduce; i_match_sym_rinto i_match_sym and using a separate array/stack in each fiber instead of the general purpose stack to store the children node indices for future reductions.

While this approach is easier to implement, it is also requires more memory during parsing, as there is no way to exclude unnecessary nodes from the final abstract syntax tree. Both of these AST construction approaches create SPPFs.

\section{RELATED WORKS}

The original Earley parser was first described in [1]. Originally it was created for parsing natural languages and saw limited use for parsing computer languages. Back then, the Earley parser was too inefficient to parse computer languages, as the computer resources were limited and computer languages used in practise were designed to be parsable by simpler and more efficient parsing algorithms, such as LR.

In recent years, as the computer performance rose, newer variations of the Earley parser appeared specifically designed for parsing non-natural languages. An Efficient Earley Parser
[2] modifies the original Earley parser by moving away from raw productions for internal grammar representation. Instead, it uses Earley graphs to represent the grammars internally. The move also enables the use of regular operators within the grammars. By applying a variation subset construction to the generated Earley graphs, the authors of [2] achieve additional performance gains. Finally, a futher variation of Efficient Earley parser called Yakker [3] enables the use of data-dependant constraints within the grammars.

An Earley parser variation described in [4] moves in a different direction: the authors of [4] propose a version of Earley parser that is suitable for parsing reflective (more often referred as adaptable) grammars, that can be modified during parsing to augment the input language. A separate paper [5], describes how to translate Earley grammars into $\mathrm{C}$ programming language, thus eliminating many of dynamic elements of the original parser and improving the overall parsing performance.

It is also important to note that the original Earley parser is not a true parser, as it does not provide the means to construct the ASTs for the parse input. As such, paper by E. Scott proposes a method that enables construction of SPPFs during parsing in [6]. Because the way the garbage collection works in EVM, this method is not directly applicable to EVM.

Earley parser is not the only parser suitable for analysing context-free languages. The primary contender for that purpose is the GLR family of parsers. One of the more modern GLR variations is RNGLR parser [7]. However, much like like the original GLR, RNGLR is a table based parser and as such, while being very efficient, it is also fairly rigid and difficult to extend. Despite that, there have been attempts to augment various existing parsing methods, including Earley and GLR, to enable parsing of context-dependant constraints in [8]. A variation of RNGLR parser suitable for scanerless parsing is described in [9].

A recent alternative to context-free grammars for specifying languages is Parsing Expression Grammars, or PEGs for short [10] [11]. PEGs are often implemented by a Packrat parser, which is a memoizing recursive descent parser and as a result, the overall structure of the parser ends up being very simple. PEGs, just like EVM, support regular operators in rule definitions. Unfortunately, PEGs also inherit all the restrictions of recursive decent parsers, such as no support for parsing ambiguous grammars and no left-recursion. A virtual-machine based implementation for PEGs exists [12].

The use of virtual machines for parsing is not a new concept. One of the first descriptions of such parser was described by Donald E. Knuth in [13]. However, the inspiration for EVM came from [14], where a virtual machine for parsing regular expressions is proposed.

\section{FUTURE WORK}

There are several potential future research directions for EVM: 
- Better parser error handling. To ease the use of EVM, there needs to be a way to automatically generate de scriptive error messages in the event of a parse error.

- The performance of EVM needs to be evaluated. EVM is currently implemented as a prototype in Ruby programming language, which is used to parse a Ruby-like language, whose grammar consists of 500 lines of code. However, the Ruby implementation makes any performance comparisons to real-world parsers and parser generators, such as bison, invalid. Comparing performance to traditional parsers is further hampered by the fact that significant performance gains may be achieved by the use of regular operators to specify the repeated patterns in the parse input. As such, separate test grammars are required for EVM to maximize it's performance.

- Translation of EVM grammar programs to LLVM IR would enable compiling EVM grammars into native machine code, which should increase the overall performance of the parser even further.

- Additional optimizations may be applied to compiled grammars programs to further increase the parser performance. Specifically, a variation of subset construction may be applied to reduce nondeterminism and the number of fibers required for parsing.

- Negative lookahead, boolean operators and rule precedence specifiers would additionally simplify the development of new grammars.

\section{Conclusion}

We have presented a new, virtual machine based approach to parsing context-free grammars, which was heavily inspired by the classic Earley parser. We have shown several versions of the Earley Virtual Machine with increasing complexity and expanding feature sets. The final version of EVM, the EVM3 is capable of recognizing context-free grammars with data-dependant constraints. Furthermore, EVM3 grammars support regular expression operators and regular lookahead in right hand sides of production rules, which should simplify development of new grammars. Finally, we have shown two modifications of EVM3, which enable construction of shared packed parse forests during parsing.

\section{ACKNOWLEDGment}

Thanks to Institute of Mathematics and Informatics for financing this research.

\section{REFERENCES}

[1] J. Earley, "An efficient context-free parsing algorithm," Commun $A C M$, vol. 13 , no. 2, pp. 94-102, 1970

[2] T. Jim and Y. Mandelbaum, "Efficient earley parsing with regular right-hand sides," Electronic Notes in Theoretical Computer Science, vol. 253 , no. 7 , pp. $135-148,2010$.

[3] T. Jim, Y. Mandelbaum, and D. Walker, "Semantics and algorithms for data-dependent grammars," in Proceedings of the 37th Annual ACM SIGPLAN-SIGACT Symposium on Principles of Programming Languages, ser. POPL '10. New York, United States: ACM, 2010, pp. 417-430.

[4] P. Stansifer and M. Wand, "Parsing reflective grammars," in Proceedings of the Eleventh Workshop on Language Descriptions, Tools and Applications, ser. LDTA '11. New York, United States: ACM, 2011, pp. 10:1-10:7.

[5] J. Aycock and N. Horspool, Directly-Executable Earley Parsing Berlin, Heidelberg: Springer Berlin Heidelberg, 2001, pp. 229-243.

[6] E. Scott, "Sppf-style parsing from earley recognisers," Electron. Notes Theor. Comput. Sci., vol. 203, no. 2, pp. 53-67, Apr. 2008.

[7] E. Scott and A. Johnstone, "Right nulled glr parsers," ACM Trans. Program. Lang. Syst., vol. 28, no. 4, pp. 577-618, Jul. 2006.

[8] [8] T. Jim and Y. Mandelbaum, "A new method for dependent parsing," in Proceedings of the 20th European Conference on Programming Languages and Systems: Part of the Joint European Conferences on Theory and Practice of Software, ser. ESOP'11/ETAPS'11. Berlin, Heidelberg: Springer-Verlag, 2011, pp. 378-397.

[9] G. Economopoulos, P. Klint, and J. Vinju, "Faster scannerless glr parsing," in In Proceedings of the 18th International Conference on Compiler Construction (CC. Springer-Verlag, 2009.

[10] B. Ford, "Packrat parsing: a practical linear-time algorithm with backtracking," Master's thesis, Massachusetts Institute of Technology, 2002.

[11] — , "Parsing expression grammars: A recognition-based syntactic foundation," SIGPLAN Not., vol. 39, no. 1, pp. 111-122, 2004.

[12] S. Medeiros and R. Ierusalimschy, "A parsing machine for pegs," in Proceedings of the 2008 Symposium on Dynamic Languages, ser. DLS'08. New York, NY, USA: ACM, 2008, pp. 2:1-2:12.

[13] D. E. Knuth, "Top-down syntax analysis," Acta Inf., vol. 1, no. 2, pp. 79-110, Jun. 1971.

[14] R. Cox. (2009) Regular expression matching: the virtual machine approach. [Online]. Available: https://swtch.com/ rsc/regexp/ regexp2.html 



\section{$9^{\text {th }}$ Workshop on Scalable Computing}

T HE Workshop on Scale Computing (WSC) is a result of evolution in the world of computing. It originated (as Workshop on Large Scale Computing in Grids; LaSCoG) in 2005. Next, cloud computing became popular and, in response to this new trend, Workshop on Scalable Computing in Distributed Systems (SCoDiS) emerged. The two workshops (under a joint name LaSCoG-SCoDiS) have been organized till 2014 (information about past events can be found here). However, the world of large-scale computing continuously evolves. In particular, data-intensive computations (known as "Big Data") brough a completely new set of issues that have to be solved (in addition to those that exist since late 1990th and that still deserve our attention). Therefore we have decided to refresh the name of the event (to better represent the scope of interest). This is how the Workshop on Scalable Computing (WSC) came to being.

\section{TOPICS}

- General issues in scalable computing

- Algorithms and programming models for large-scale applications, simulations and systems

- Large-scale symbolic, numeric, data-intensive, graph, distributed computations

- Architectures for large-scale computations (GPUs, accelerators, quantum systems, federated systems, etc.)

- Data models for large-scale applications, simulations and systems

- Large-scale distributed databases

- Security issues for large-scale applications and systems

- Load-balancing / intelligent resource management in large-scale applications, simulations and systems

- Performance analysis, evaluation and prediction

- Portals, workflows, services and collaborative research

- Data visualization

- On-demand computing

- Virtualization supporting computations

- Self-adaptive computational / storage systems

- Volunteer computing

- Scaling applications from small-scale to exa-scale (and back)

- Computing for Big Data

- Business applications

- Grid / Cloud computing

- Cloud / Grid computing architectures, models, algorithms and applications
- Cloud / Grid security, privacy, confidentiality and compliance

- Mobile Cloud computing

- High performance Cloud computing

- Green Cloud computing

- Performance, capacity management and monitoring of Cloud / Grid configuration

- Cloud / Grid interoperability and portability

- Cloud / Grid application scalability and availability

- Economic, business and ROI models for Cloud / Grid computing

- Big Data cloud services

\section{SECTION EDITORS}

- Ganzha, Maria, University of Gdańsk and Systems Research Institute Polish Academy of Sciences, Poland

- Gusev, Marjan, University Sts Cyril and Methodius, Macedonia

- Paprzycki, Marcin, Systems Research Institute Polish Academy of Sciences, Poland

- Petcu, Dana, West University of Timisoara, Romania

- Ristov, Sashko, University of Innsbruck, Austria

\section{REVIEWERS}

- Barbosa, Jorge, University of Porto, Portugal

- Camacho, David, Universidad Autonoma de Madrid, Spain

- Carretero, Jesus

- D'Ambra, Pasqua, IAC-CNR, Italy

- Gordon, Minor, Software development consultant, United States

- Gravvanis, George, Democritus University of Thrace, Greece

- Grosu, Daniel, Wayne State University, United States

- Holmes, Violeta, The University of Huddersfield, United Kingdom

- Kalinov, Alexey, Cadence Design Systems, Russia

- Kecskemeti, Gabor, Liverpool John Moores University, United Kingdom

- Kitowski, Jacek, AGH University of Science and Technology, Department of Computer Science, Poland

- Knepper, Richard, Indiana University, United States

- Lang, Tran Van, Vietnam Academy of Science and Technology, Vietnam

- Lastovetsky, Alexey, University College Dublin, Ireland

- Margaritis, Konstantinos G., University of Macedonia, Greece

- Morrison, John, University College Cork, Ireland 
- Nosovic, Novica, Faculty of Electrical Engineering, University of Sarajevo, Bosnia and Herzegovina

- Prodan, Radu, University of Innsbruck

- Schikuta, Erich, University of Vienna, Austria

- Schreiner, Wolfgang, Johannes Kepler University Linz, Austria

- Shen, Hong, University of Adelaide, Australia

- Telegin, Pavel, JSCC RAS, Russia

- Tudruj, Marek, Inst. of Comp. Science Polish Academy of Sciences/Polish-Japanese Institute of Information Technology, Poland

- Vazhenin, Alexander, University of Aizu, Japan

- Wei, Wei, School of Computer science and engineering, Xi'an University of Technology, China

- Wyrzykowski, Roman, Czestochowa University of Technology, Poland

- Zavoral, Filip, Charles University, Czech Republic 


\section{Business Intelligence Platform for Big Data based on Scalable Distributed Two-Layer Data Store}

\author{
Adam Krechowicz \\ Kielce University of Technology, Poland \\ Email: a.krechowicz@tu.kielce.pl
}

\author{
Stanisław Deniziak \\ Kielce University of Technology Poland \\ Email: s.deniziak@tu.kielce.pl
}

\begin{abstract}
Data mining is one of the main business intelligence technique. The volume of Big Data expands in such a way that the classical Business Intelligence methods need to be redefined. First, the organization of large data sets requires new distributed database architectures. Second, it is necessary to develop distributed data processing models that provide a high degree of scalability.In this paper we introduce fully scalable BI platform that is suitable for the most common data processing issues. The platform is based on our scalable distributed two-layer data store, which is competitive to existing NoSQL distributed data base systems. We show examples and experimental results showing advantages of our approach.
\end{abstract}

\section{INTRODUCTION}

B USINESS Intelligence (BI) is very important branch of modern analytic in many companies. Analysing gathered data (like information about company's clients) can be a huge source of essential knowledge. Because of that companies can offer their services on higher level and can multiply their profits. There are many existing BI tools available to use out of the box. Some of them, which offers basic functionality, are even available free of charge. The prices of others, which offer advanced techniques of data processing, can be very high.

From the basic principles of statistics we know that to achieve more accurate data analysis, the set of analysed data should be really big. Unfortunately processing that huge data sets is still a great challenge. The popularity of Big Data nowadays is a reflection of this trend. There is still a wide pressure on developing efficient algorithms that are suitable for processing huge data sets.

Scalable Distributed Two-Layer Data Store (SD2DS) [1] is a very powerful data store that was developed in our research team. The ease of its scalability gave us the opportunity to develop an efficient BI platform that can process data distributed on nodes in the cluster. In this paper we present the prototype of such a system.

This paper is organized as follows. Firstly, we present the existing BI platforms for data analysis. Then, we discuss the demand of creating this novel platform. In the next section we introduce the basic concept of SD2DS. Next, we present key architectural elements of our platform which is evaluated in the next section. The paper ends with conclusions.

\section{RELATED WORK}

The Business Intelligence have a long history. For many years the basic tools were developed as stand alone systems that run on a standalone computer. For many people the first choice to create some kind of data analysis is to use Microsoft Excel or its open-source equivalent like Libre Office or Open Office. Among many other tools the QlikView [2] is worth highlighting. It allows to create advanced and responsive data analysis. That kind of programs are suitable for most typical applications.

On the other hand, there are many commercial platforms provided by the companies such as Oracle [3], SAP [4] or IBM [5]. They offer BI tools as a part of their whole enterprise management systems. In the vast majority they are oriented as client-server systems. In most cases they offer custom designed solution for specific needs.

Contemporary BI tools use advanced data mining techniques, expert systems and computational intelligence methods, but still simple methods for analysing large data sets are needed. Distributed storage and processing is required to ensure the required performance of Big Data analysis.

The truly distributed data analysis can be developed based on the framework such as Hadoop [6] which is the open source implementation of a very popular Google MapReduce [7] system. On the contrary to the previously presented tools, it can really process big data. There are many existing scenarios for BI based on Hadoop solutions [8], [9], [10]. The Hive [11] processing engine can be considered as one of the most recognizable examples of such systems.

\section{Motivation}

All of the systems presented in the previous section have their limitations. The stand alone systems are obviously not suitable for huge data sets. For example Libre Office allows to load only a finite number of data. In the case of the QlikView system, authors of this paper suffered themselves the "Out of Memory" error while processing huge data sets many times.

Commercial products are, in vast majority, based on the typical relational data bases which have their serious limitations. Those limitations are well known and are widely discussed [12], [13]. They were one of the main reason for expansion of NoSQL data storages.

The MapReduce framework tends to eliminate these drawbacks. However, it does not provide a complete tool to work with. It rather provide environment for designing custom solutions [7]. Their efficiency is strongly correlated with the programmers skills due to the fact that designing such 
distributed algorithms is not intuitive in comparison with programming single process programs.

All described above problems concerning processing big data were the driving force behind designing distributed and efficient tool for Business Intelligence. As a base of our platform we have chosen Scalable Distributed 2-Layer Data Store because it is a very effective architecture for storing really huge data.

\section{Scalable Distributed Two-Layer Data Store}

The Scalable Distributed Two-Layer Data Store (SD2DS) is an efficient NoSQL key-value database, which has been designed by the authors for the last couple of years. Its main goal is to store huge data sets that are too big to be efficiently stored by typical relational databases. It allows to run in distributed environment and is capable to scale its storage capacity almost infinitely.

On the base of the SD2DS the very mature standard of Distributed Linear Hashing $\left(L H^{*}\right)$ [14] was utilized. It organizes the data in so called buckets which are located on nodes in a cluster. Each bucket is responsible for storing some set of data portions (components). All of the buckets are distributed on a nodes in a cluster. The main advantage of the $L H^{*}$ is the ease of addressing the components without any central directory while preserving full data scalability. Each component consists of two parts: headers and bodies. The component body is the data itself while the component header consists of metadata that allows to manage the bodies. The $L H^{*}$ mechanism is responsible for managing the first layer of the store which is responsible for storing headers. The bodies are stored in the second layer of the store. That layer division was firstly introduced to eliminate the main drawback of the single layer store, namely the need of constantly reorganizing the whole structure [15].

The example architecture of SD2DS is presented in Fig. 1. The first layer consists of the set of headers while the second layer consists of the set of bodies. If a client wants to access specific component (component number 2 in the figure) it first needs to access specific header in the first layer. The appropriate first layer bucket, that contains desired header, is found based on the $h(k e y)$ function according to the $L H^{*}$ scheme. The locator inside header is used then to access the corresponding body. When a client wants to insert data item into SD2DS it first needs to insert appropriate data header. The first layer is responsible for allocating the space for the body in the second layer. This indirect access gave us opportunity to introduce additional functionality like throughput scalability [16] or fault tolerance.

The Fig. 2 and Fig. 3 present the time of accessing the data in SD2DS in comparison to the most recognizable representatives of the NoSQL systems: MongoDB [17] and MemCached [18]. The overall evaluation was presented in [1] and [19]. The MongoDB and MemCached was chosen because they have many similarities with SD2DS. First of all, all of those systems were developed using $\mathrm{C}++$ language and allowed to store the data in distributed environment. Secondly, they all allow to extensively use main memory of machines to speed up the all operations. Both figures 2 and 3 present the results of getting the data portions in the relation to the number of clients that simultaneously operate on the system. Figure 2 presents the results of getting data portions of $5 \mathrm{MiB}$ while figure 3 presents the results of getting $10 \mathrm{MiB}$ data portions.

In all of these two cases the best results were achieved for SD2DS. The times for MongoDB are strongly correlated to the number of the mongos elements. The mongos elements are responsible for properly addressing the data portions in MongoDB system. Because the clients does not know the exact location of the data it has to direct their queries to the central element that is aware of the current configuration of the system [17]. The SD2DS is free of that kind of drawback. Our SD2DS proved to have better performance even in comparison with the MemCached system. The MemCached is optimised mostly for the small data portions. It does not do best with the data portions that are greater that $1 \mathrm{MiB}$. It also does not work well under the heavy clients load when it just rejects clients after specified time [20].

In its basic form SD2DS stores the data in raw format which does not use any schema at all. Because of that its basic application was storing multimedia data [21], [22], [23] like photos or videos. We also successfully utilized our data store for gathering data from advanced Internet of Things system [24] and used it as a base for anonymous storing the data in the Cloud environment [25], [26].

\section{ARCHITECTURE OF SD2DS-BASED BI PLATFORM}

In this section we presented the essential parts of the architecture of our BI platform. The main goal to face with was to develop an efficient processing model that can process the data effectively in the distributed environment. Due to the fact that in our previous work we mostly used raw portion of the data we also needed to develop a simple yet effective data model to begin with.

\section{A. Data model}

In the original conception of SD2DS bodies were just a block of data that have no structural form at all. Hence, in our previous work, we used it mostly for large data portions like high resolution photos and videos. To efficiently store structural data we needed to develop a method of storing a set of structured data into a single body. We decided to introduce simple data schema in which the whole set of data is divided into records. Each record was then divided into dimensions. Because of the nature of the most data and also simplicity we assumed that all records had the same dimensions. Additionally we assumed that the number of dimensions $(n)$ was constant to all records. In that form all of the bodies could consist of a separable subset of all records (so called block). The Table I illustrate this concept.

This data model gave us opportunity to fast access value of any dimension $(i)$ in record $(j)$ within specified block $(b)$ simply by utilizing the following equation: 


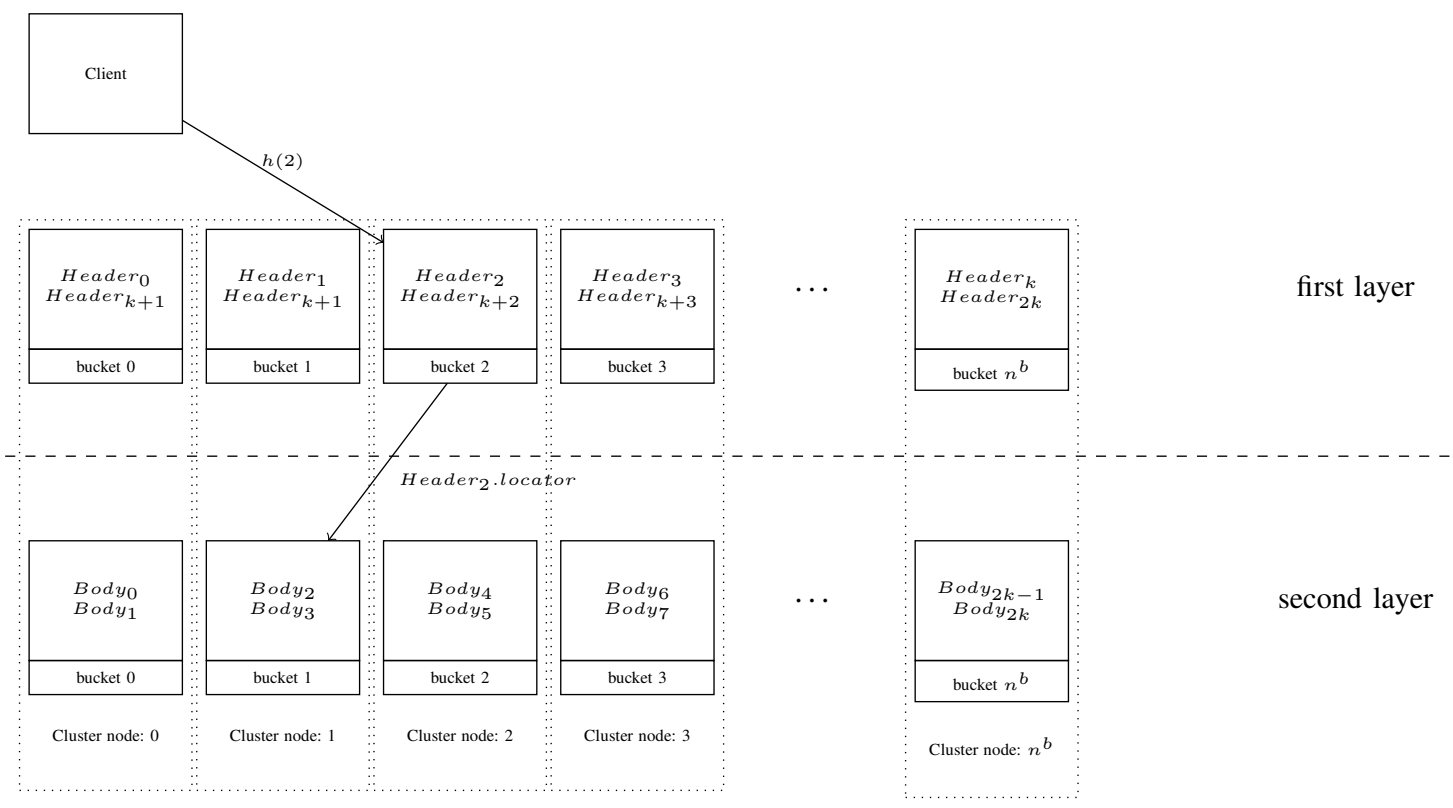

Fig. 1. Architecture of SD2DS

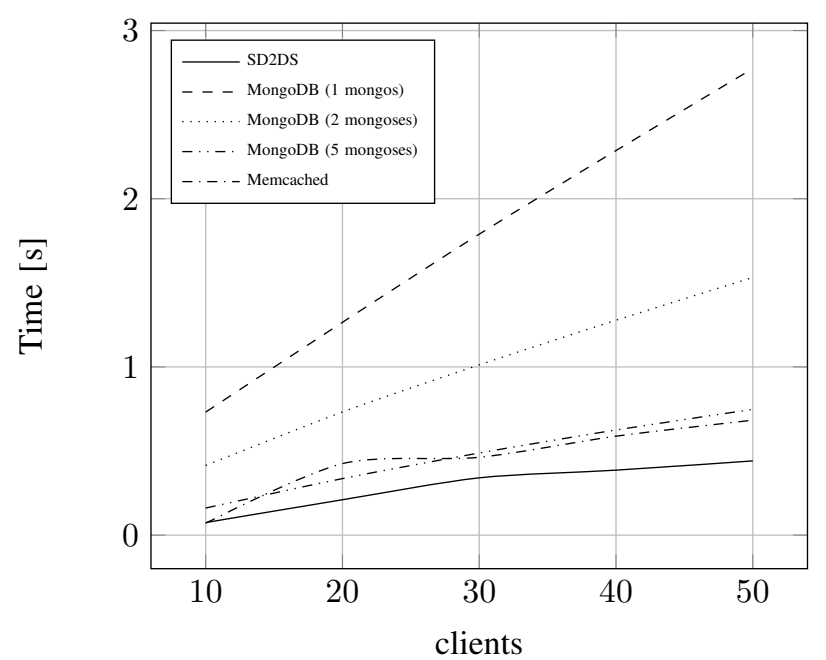

Fig. 2. Time comparison of getting components of fixed (5MiB) size [19]

TABLE I

DATA MODEL IN SD2DS

\begin{tabular}{|c|c|c|c|}
\hline dimension $_{1}$ & dimension $_{2}$ & $\ldots$ & dimension $_{n}$ \\
\hline value $_{1,1}$ & value $_{2,1}$ & $\ldots$ & value $_{n, 1}$ \\
\hline value $_{1,2}$ & value $_{2,2}$ & $\ldots$ & value $_{n, 2}$ \\
\hline$\ldots$ & $\ldots$ & $\ldots$ & $\ldots$ \\
\hline value $_{1, m}$ & value $_{2, m}$ & $\ldots$ & value $_{n, m}$ \\
\hline
\end{tabular}

$$
\operatorname{value}(i, j, b)=\operatorname{block}_{b}[j * n+i]
$$

where:

- $b$ - block number

- $j$ - record number in $b$-th block

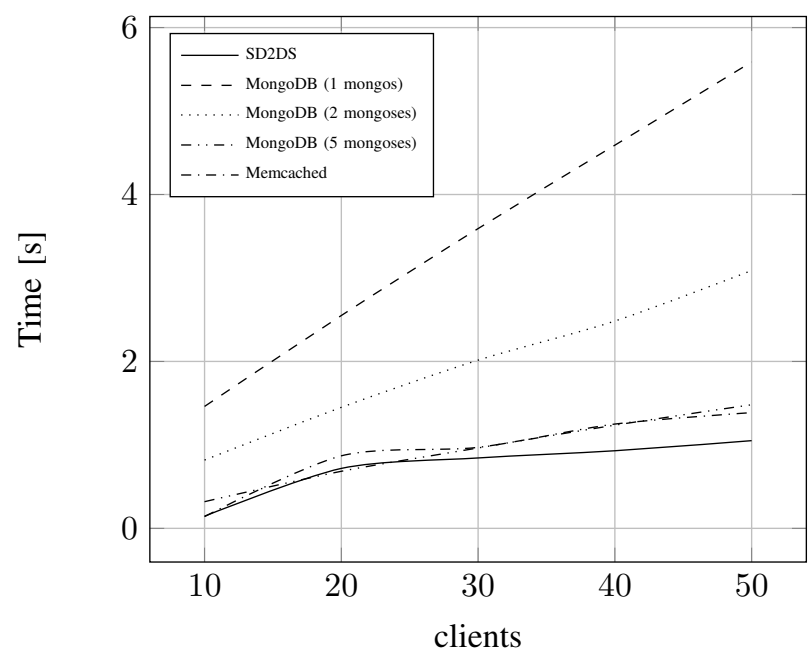

Fig. 3. Time comparison of getting components of fixed (10MiB) size [19]

- $i$ - dimension number in $j$-th record in $b$-th block

The main advantage of this model is that it can access each value within the block in constant time regardless of the number of dimensions and the number of records within block.

\section{B. Processing model}

The natural distribution of blocks within buckets creates a great opportunity to distribute the processing on all buckets within SD2DS. All buckets are responsible for processing blocks that are stored within. Because all bodies are accessible through the first layer there are used to aggregate the results given from the second layer. The partially aggregated results 
from the first layer are then fully aggregated by the client This creates the processing model which requires to define two functions:

- $f_{b}(b, \ldots)-$ Block function

- $f_{a}(x, y)$ - Aggregate function

The $f_{b}(b, \ldots)$ function is responsible for processing specified block of data $(b)$. Depending of the nature of the processing it may require additional parameters. The results of all $f_{b}(b, \ldots)$ functions are then used as a parameters of the $f_{a}(x, y)$ functions which aggregates the partial results. The $f_{b}(b, \ldots)$ function is executed on the second layer buckets while $f_{a}(x, y)$ is executed both on the first layer buckets and by clients. This creates a processing model similar to one introduced in MapReduce framework [7].

The results of both $f_{b}(b, \ldots)$ and $f_{a}(x, y)$ has to produce the result in the same form. Additionally aggregate function should work with only one parameter in such a way that:

$$
f_{a}\left(f_{b}\left(b_{1}\right), n u l l\right)=f_{a}\left(\text { null }, f_{b}\left(b_{1}\right)\right)=f_{b}\left(b_{1}\right)
$$

Additionally, because results of different blocks can be executed in different order the following two constraints should be satisfied:

$$
\begin{gathered}
f_{a}\left(f_{b}\left(b_{1}\right), f_{b}\left(b_{2}\right)\right)=f_{a}\left(f_{b}\left(b_{2}\right), f_{b}\left(b_{1}\right)\right) \\
f_{a}\left(f_{a}\left(f_{b}\left(b_{1}\right), f_{b}\left(b_{2}\right)\right), f_{b}\left(b_{3}\right)\right)= \\
=f_{a}\left(f_{a}\left(f_{b}\left(b_{2}\right), f_{b}\left(b_{3}\right)\right), f_{b}\left(b_{1}\right)\right)= \\
=f_{a}\left(f_{a}\left(f_{b}\left(b_{3}\right), f_{b}\left(b_{1}\right)\right), f_{b}\left(b_{2}\right)\right)
\end{gathered}
$$

The sample architecture and the execution model of defined functions are presented in Figure 4. In this example SD2DS consists of three first layer buckets and three second layer buckets. Each second layer bucket consists of three blocks (bodies). First second layer bucket consists of blocks $b_{1}$, $b_{4}$ and $b_{7}$, second bucket consists of blocks $b_{2}, b_{5}$ and $b_{8}$ while the third bucket consists of blocks $b_{3}, b_{6}$ and $b_{9}$. To accomplish processing, each second layer bucket executes $f_{b}(b, \ldots)$ function on all blocks for which it is responsible. Then the results of block functions are passed to appropriate first layer bucket which executes the $f_{a}(x, y)$ to aggregate the results from second layer buckets. Next, the results from all first layer buckets are transferred to the client which executes again $f_{a}(x, y)$ functions for each intermediate results.

\section{Example: Finding Maximum Value}

For better understanding of the role of the $f_{b}(b, \ldots)$ and $f_{a}(x, y)$ functions we present an example calculation of the maximum value of the i-th dimension in all records. The algorithm 1 presents the block function for finding max value while algorithm 2 presents the aggregate function.

The function $f_{b}^{\max }(b, i)$, like all other block function requires the $b$ parameter which indicates the block that operates on. Additional parameter $i$ determines the dimension on which the maximum value is searched. In its basic form it searches on all
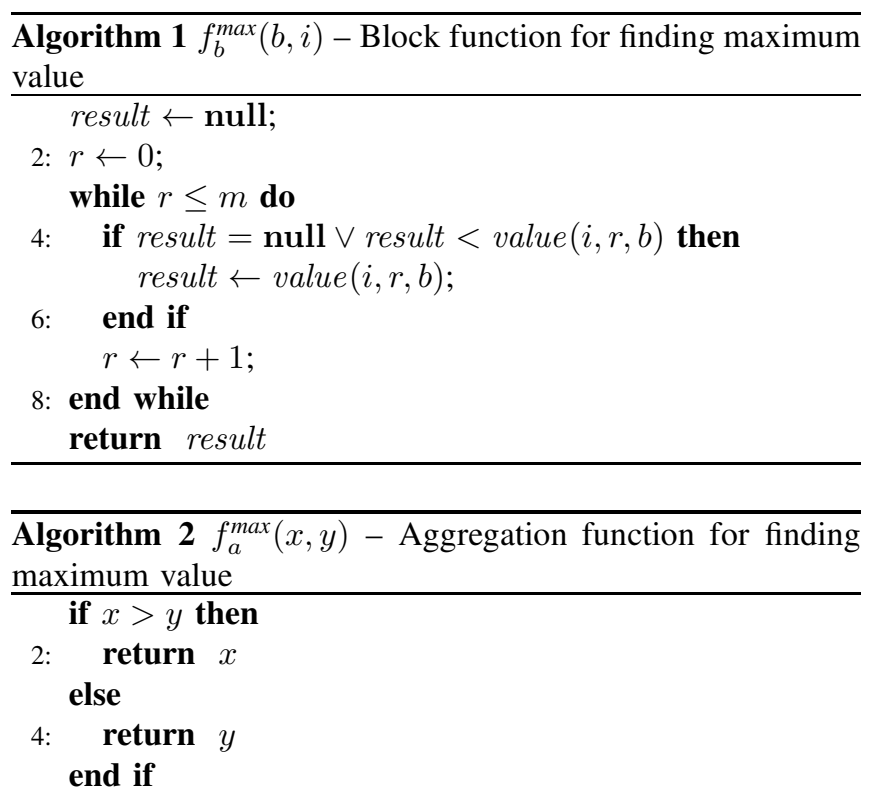

records within the blocks but can easily be modified to process only specific records that follow user defined constraints.

The $f_{a}^{\max }(x, y)$ function determine the maximum value from the two output of block functions, from other aggregate functions or from one block and one aggregate function. It simply determines the maximum value from two intermediate values. Its simplicity is very important because it is also executed by the client for every intermediate results obtained by the all first layer buckets. It is also important to not change the first layer buckets which are responsible for serving other clients requests.

\section{USE CASE}

To evaluate our platform we designed a special implementation that gave us an opportunity to visualise the exchange rates of different currencies in relation to the "polish złoty" (PLN). The data about the exchange rates was taken from the [27]. Although the data set was relatively small (approx. 250 records per year) it allowed us to evaluate the correctness of the calculation by comparing it with different standalone tools. The data consisted of 38 dimensions. Each record represented the exchange rates of 35 different currencies for the specified day. The 3 additional dimension was used to represent the date (one dimension per year, month and day). The user interface of the prepared tool is presented in figure 5. It allowed to create visualization data aggregated in SD2DS environment.

The figures 6-8 present the results of executing different queries of the different subsets of the whole data set. The figure 6 shows the average exchange rate of United State Dollar in the months of the year 2014. The figure 7 presents the maximal exchange rate of Great British Pound that was obtained in the evaluated years. At last figure 8 presents the average exchange rate of Euro of specified days in each month of the year 2012. 


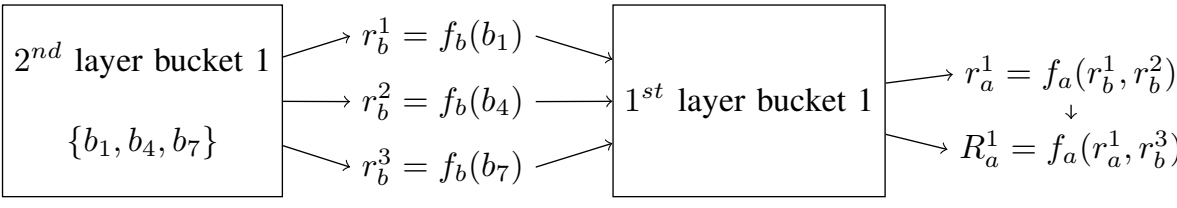

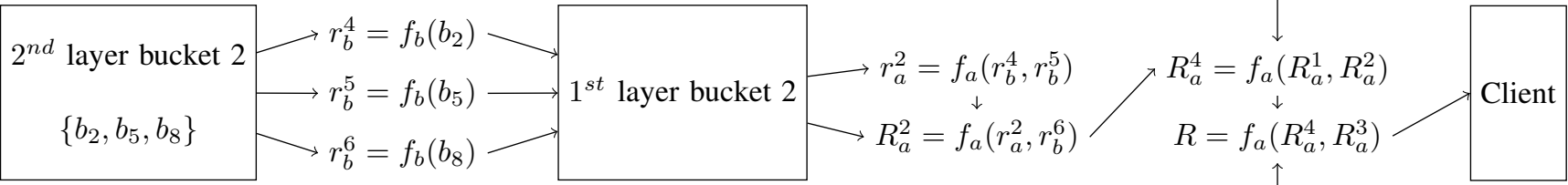

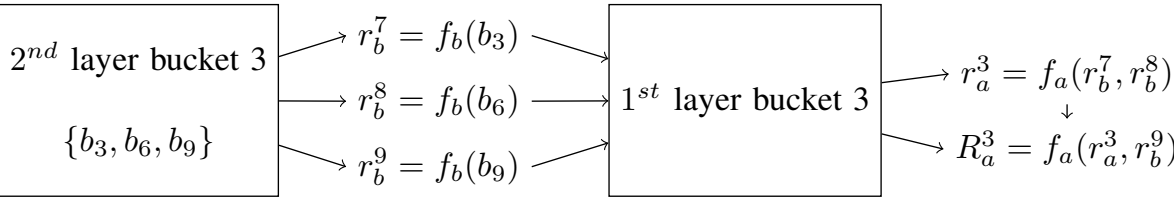

Fig. 4. Architecture of tool

Fig. 5. User interface

Fig. 6. Average value of United States Dollar per month in the year 2014

Fig. 7. Maximum value of Great British Pound per year

\section{EXPERIMENTAL RESULTS}

The exchange rate use case presented in section VI did not allow to evaluate the efficiency of the platform in relation to the size of the overall dataset. To determine the efficiency in that matter we used synthetic dataset that contained of the same value in all dimensions in all records. We use 16 nodes of cluster for the SD2DS buckets. Each node consists of one first layer bucket and one second layer bucket. Additional node was used for client application. In all cases each block consisted of 1,000 records. The time given in all experiments was the average value of 1,000 tries.

In our first evaluation we measured the processing time of 16 blocks with different number of dimensions. The results are presented in figure 9 . We measured the processing time for blocks that contained 1 to 100 dimensions. The obtained results of processing time were independent of the number of data dimensions. It oscillated from 0.008 to approximately 0.0083 second for all cases regardless on the dimensions number.

The goal of the next experiment was to evaluate the pro-

Fig. 8. Average value of Euro in days of the month in the year 2012

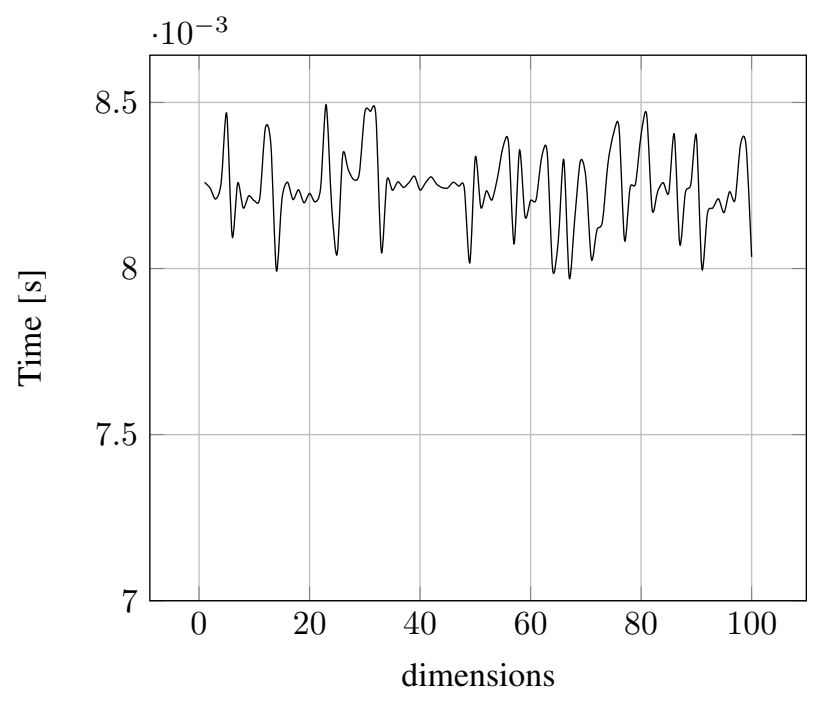

Fig. 9. Processing time in relation to the number of data dimensions

cessing time in relation to the number of data records. We evaluated our tool with the records number from 1,000 to 128,000 . The results are presented in figure 10. The overall figure can be divided into three sections. The first section (from 1,000 to 16,000 records) presents the situation where the processing time drastically increased. It was caused by the fact that the processing was not distributed on the whole set of buckets. Because of that the the client waits for reduced number of buckets to response and the number of execution of aggregate function is also reduced. The second section (from 


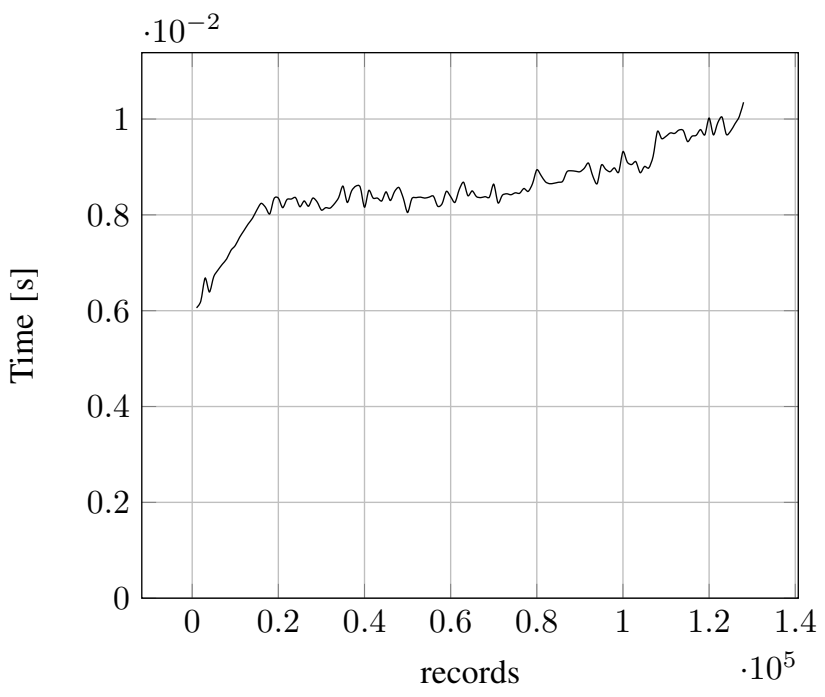

Fig. 10. Processing time in relation to the number of data records

16,000 to approx. 70,000) presents the situation where the processing time was very similar in each case. It represents the situation where all of the data were properly distributed on all buckets within the cluster. In the third section (from approx. 70,000 and above) the processing time started to increase slightly. It represents the situation when the buckets became heavy loaded and additional portions of data increased the overall processing time. This situation indicated the need to introduce additional buckets to the structure to ensure scalability.

\section{CONCLUSIONS}

In this paper we introduced our SD2DS based BI platform that is suitable for processing Big Data sets. We chose our Scalable Distributed Two-Layer Data Store for this purpose because of its efficiency that had been established in our previous work. We introduced a special data model that gave us the opportunity to take advantage of its high performance to achieve our goal. We also proposed a special processing model which allowed to distribute the computation on the nodes in the cluster on similar way than MapReduce model. The preliminary experiments, carried out in this paper, are very promising and we are planning to develop truly scalable BI platform as our future work.

\section{REFERENCES}

[1] A. Krechowicz, S. Deniziak, M. Bedla, A. Chrobot, and G. Łukawski, "Scalable distributed two-layer block based datastore," in International Conference on Parallel Processing and Applied Mathematics. Springer International Publishing, 2015, pp. 302-311.

[2] M. García and B. Harmsen, Qlikview 11 for developers. Packt Publishing Ltd, 2012

[3] Oracle, “Oracle business intelligence 12c," https://www.oracle.com/ solutions/business-analytics/business-intelligence/index.html, 2017, accessed 3rd April 2017.

[4] SAP, "Business intelligence (bi) tools \& software," https://www.sap.com/ solution/platform-technology/analytics/business-intelligence-bi.html, 2017, accessed 3rd April 2017.
[5] IBM "Business intelligence", https://www.ibm.com/ business-intelligence, 2017, accessed 3rd April 2017.

[6] P. Zikopoulos, C. Eaton et al., Understanding big data: Analytics for enterprise class hadoop and streaming data. McGraw-Hill Osborne Media, 2011.

[7] J. Dean and S. Ghemawat, "MapReduce: simplified data processing on large clusters," Communications of the ACM, vol. 51, no. 1, pp. 107-113, 2008

[8] D. Borthakur, J. Gray, J. S. Sarma, K. Muthukkaruppan, N. Spiegelberg, H. Kuang, K. Ranganathan, D. Molkov, A. Menon, S. Rash et al., "Apache hadoop goes realtime at facebook," in Proceedings of the 2011 ACM SIGMOD International Conference on Management of data. ACM, 2011, pp. 1071-1080.

[9] S. Chen, "Cheetah: a high performance, custom data warehouse on top of mapreduce," Proceedings of the VLDB Endowment, vol. 3, no. 1-2, pp. 1459-1468, 2010.

[10] J. Dittrich and J.-A. Quiané-Ruiz, "Efficient big data processing in hadoop mapreduce," Proceedings of the VLDB Endowment, vol. 5, no. 12, pp. 2014-2015, 2012

[11] A. Thusoo, J. S. Sarma, N. Jain, Z. Shao, P. Chakka, S. Anthony, H. Liu, P. Wyckoff, and R. Murthy, "Hive: a warehousing solution over a mapreduce framework," Proceedings of the VLDB Endowment, vol. 2, no. 2, pp. 1626-1629, 2009

[12] A. Moniruzzaman and S. A. Hossain, "Nosql database: New era of databases for big data analytics-classification, characteristics and comparison," arXiv preprint arXiv:1307.0191, 2013.

[13] C. J. Tauro, S. Aravindh, and A. Shreeharsha, "Comparative study of the new generation, agile, scalable, high performance nosql databases," International Journal of Computer Applications, vol. 48, no. 20, pp. $1-4,2012$.

[14] W. Litwin, M.-A. Neimat, and D. A. Schneider, LH*: Linear Hashing for distributed files. ACM, 1993, vol. 22, no. 2.

[15] K. Sapiecha and G. Lukawski, "Scalable distributed two-layer data structures (SD2DS)," International Journal of Distributed Systems and Technologies (IJDST), vol. 4, no. 2, pp. 15-30, 2013.

[16] K. Sapiecha, G. Łukawski, and A. Krechowicz, "Enhancing throughput of scalable distributed two-layer data structures," in Parallel and Distributed Computing (ISPDC), 2014 IEEE 13th International Symposium on. IEEE, 2014, pp. 103-110.

[17] E. Plugge, T. Hawkins, and P. Membrey, The Definitive Guide to MongoDB: The NoSQL Database for Cloud and Desktop Computing, 1st ed. Berkely, CA, USA: Apress, 2010. ISBN 1430230517, 9781430230519

[18] J. Petrovic, "Using memcached for data distribution in industrial environment," in Systems, 2008. ICONS 08. Third International Conference on. IEEE, 2008, pp. 368-372.

[19] A. Krechowicz, A. Chrobot, S. Deniziak, and G. Łukawski, "SD2DS based datastore for large files," in Federated Conference on Software Development and Object Technologies. Springer, 2015, pp. 150-168.

[20] Memcached, "Memcached - A Distributed Memory Object Caching System," http://memcached.org, accessed 3rd April 2017.

[21] S. Deniziak, T. Michno, and A. Krechowicz, "The scalable distributed two-layer content based image retrieval data store," in Computer Science and Information Systems (FedCSIS), 2015 Federated Conference on. IEEE, 2015, pp. 827-832.

[22] _ _Content based image retrieval using modified scalable distributed two-layer data structure." International Journal of Computer Science \& Applications, vol. 13, no. 2, 2016.

[23] T. Michno and A. Krechowicz, "SD2DS database in the direction of image retrieval," Applications of information technologies - theory and practice, vol. 11, 2015

[24] S. Deniziak, G. Łukawski, M. Bedla, and A. Krechowicz, "A scalable distributed 2-layered data store (SD2DS) for internet of things (IoT) systems," Measurement Automation Monitoring, vol. 61, no. 7, pp. 382 384, 2015.

[25] A. Krechowicz, "Scalable distributed two-layer datastore providing data anonymity," in International Conference: Beyond Databases, Architectures and Structures. Springer, 2015, pp. 262-271.

[26] A. Krechowicz and S. Deniziak, "SD2DS-based anonymous datastore for IoT solutions," DEStech Transactions on Computer Science and Engineering, no. wcne, 2016.

[27] Narodowy Bank Polski, "Statistics and reporting," http://www.nbp.pl/ home.aspx?f=/statystyka/kursy.html, accessed 3rd April 2017. 


\section{The Algorithm for Sequential Analysis of Variants for Distribution of Virtual Machines in Data Center}

\author{
Oleksandr Rolik, Maksym Bodaniuk, Valerii Kolesnik \\ Department of Automation and Control in Technical \\ Systems \\ National Technical University of Ukraine "Igor Sikorsky \\ Kyiv Polytechnic Institute" \\ Kyiv, Ukraine \\ e-mail: o.rolik@kpi.ua,max.bodaniuk@gmail.com, \\ kolesnik.valerii@gmail.com
}

\author{
Volodymyr Samotyy \\ Department of Automation and Information Technologies \\ Faculty of Electrical and Computer Engineering, \\ Cracow University of Technology, Cracow, Poland \\ Lviv State University of Life Safety. Lviv, Ukraine \\ e-mail: vsamotyy@pk.edu.pl
}

\begin{abstract}
- this work proposes an algorithm of sequential analysis of variants (SAV) to solve the distributional problem of allocation of virtual machines to physical servers in a data center. The set of tests and rules of the SAV algorithm is defined. The experimental results for problems of different dimensions are given. The comparison of the proposed algorithm with heuristic and genetic algorithms is accomplished. The time of finding solution required by the SAV algorithm depending on the dimension of the problem is evaluated. The recommendations for using the SAV algorithm are given. For tasks requiring high precision distribution it is better to use the SAV algorithm as it finds the optimal solution, whereas heuristic and evolutionary algorithms can quickly get an effective solution. The speed of the heuristic and evolutionary algorithms is not significantly dependent on the problem's size, but the quality of their solutions is worse than equivalent solution received with the SAV algorithm.
\end{abstract}

Keywords-VM allocation; data center; resource allocation; sequential analysis of variance, virtual machine distribution

\section{INTRODUCTION}

Virtualization of computational resources remains an essential attribute of modern data centers (DC). Maintaining of IT infrastructure in a DC requires significant financial expenses, which makes a problem of efficient resource allocation relevant. A maximal density of virtual machines (VM) allocation among physical servers (PS) allows operational expenses to be reduced and more users to be served with DC's server farm. That is why current paper is dedicated to solve the problem of optimal VMs distribution among physical servers, which is a challenging task.

\section{ANALYSIS OF LATTER WORKS}

Nowadays, increasing interest can be seen in the area of algorithms, which work according to the most important natural law, namely evolution. One of the well-known algorithms in this area are genetic algorithms, evolutional strategies and programming. These algorithms simulate the principle of evolution (survival of the fittest) [1].
Such algorithms maintain a population of potential solutions (individuals) through repeated application of some "evolutionary" operators. They yield individuals with successfully improved fitness, and converge, hopefully, to the fittest individuals representing optimal solutions [2].

In [3] the authors have considered the problem of managing the data center by a cloud service provider. The cloud service provider makes available a variety of services for the customers. Some services may be provided to other users by implementing multi-tenant data centers which represent a very complicated networking environment. Between the cloud service providers there are agreements on providing the services using different data centers. There are also the corresponding access policies to ensure the possibility of providing resources on demand.

The cloud service provider issues the following services: virtual machines, middle-boxes, services, service workflows. A separate virtual machine is allocated to provide a service.

A problem of VMs distribution among physical servers can be solved with classical genetic algorithm (CGA) [4-6]. Papers [4-6] show that usage of CGA can improve quality of problem solving and can control course of a solution on each iteration of algorithm. Heuristic algorithms can also be used for allocating VMs. Also, it should be mentioned that there are a few objective functions developed for VM distribution problem, including the energy consumption minimization [7], minimization of PS amount [8], network traffic minimization [9], availability maximization [10], resource utilization maximization [7] and other.

Additionally, those papers contain proof of relation between efficiency of heuristic algorithms and algorithm structure and parameters of distributed VMs. However, those publications do not propose an algorithm, which obtains optimal solution at VMs allocation problem. 


\section{GOAL OF THE RESEARCH}

The goal of current research is to develop and define feasibility of application of proposed algorithm for sequential analysis of variants (SAV) which is used to solve problem of VMs distribution on physical servers of a DC.

\section{MATHEMATICAL MODEL}

A data center contains an ordered set of physical servers $N=\left\{N_{1}, \ldots, N_{n}\right\}$, where $n$ is the number of servers; an ordered set of virtual machines $K=\left\{K_{1}, \ldots, K_{m}\right\}$, where $m$ is the number of VMs, which are subjected to distribution.

Each server $N_{i}, i=\overline{1, n}$, has the following parameters: $\Omega_{i}$ stands for processor performance or CPU load; $\Gamma_{i}$ stands for RAM capacity of the server $N_{i}$.

Each $\mathrm{VM} K_{j}, j=\overline{1, m}$, requests $\omega_{j}$ of CPU time and $\gamma_{j}$ of RAM resource;

Matrix $R=\left\|r_{j i}\right\|$ with dimensions $m \times n$ defines a distribution of VMs among servers, moreover

$$
r_{j i}=\left\{\begin{array}{l}
1, \text { if } \mathrm{VM} K_{j} \text { is located on server } N_{i}, \\
0, \text { otherwise. }
\end{array}\right.
$$

And matrix $R$ is a solution of the problem and it defines a distribution of set of VMs $K$ over set of physical servers $N$.

\section{PROBLEM STATEMENT}

In case where all servers from set $N$ have identical technical characteristics, it can be considered that $\Omega_{i}=1$ and $\Gamma_{i}=1$, $i=\overline{1, n}$, i.e.

$$
\left.\left\{\Omega_{i}, \Gamma_{i}\right\}\right|_{N_{i}}=\{1,1\}, \quad i=\overline{1, n}
$$

We can see the transition from resource measurement on servers in absolute units where RAM is measured in megabytes (MB) and CPU frequency in megahertz $(\mathrm{MHz})$, to relative units. Then, needs in resources of VMs from set $K$ are defined as a normalized part of resources capacities of a server.

In the paper the task of clustering is not considered, that is why requirements to resources of each VM from set $K$ cannot exceed capacities of a server

$$
\omega_{j} \leq 1 \text { and } \gamma_{j} \leq 1, i=\overline{1, n}
$$

For solving the problem of VMs distribution, all servers from set $\mathrm{N}$ must satisfy the following resource constraint:

$$
\sum_{j=1}^{m} r_{j i} \omega_{j} \leq 1 \text { and } \sum_{j=1}^{m} r_{j i} \gamma_{j} \leq 1, i=\overline{1, n}
$$

After that, next vector will be introduced $\bar{y}=\left\langle y_{i}\right\rangle, i=\overline{1, n}$, where

$$
y_{i}=\left\{\begin{array}{l}
1, \text { if server } N_{i} \text { contains at least one VM, } \\
0, \text { otherwise }
\end{array}\right.
$$

Then, optimum criteria for solving the problem of VMs distribution on physical servers will be:

$$
\min \sum_{i=1}^{n} y_{i}
$$

In other words, virtual machines should allocate physical server in a way that the minimal amount of physical servers was involved.

Such approach allows to minimize total costs $S$ for maintenance and power supply of DC servers. Objective function can be represented thus:

$$
S=s \sum_{i=1}^{n} y_{i}
$$

where $s$ is the cost of maintenance and power supply for one server.

Then problem statement can be represented with next interpretation: physical servers in DC should allocate resources for VM in a way that allows minimum value in expression (5) to be achieved.

\section{ALGORITHM OF SEQUENTIAL ANALYSIS OF VARIANTS FOR DISTRIBUTION OF VIRTUAL MACHINES}

The algorithm of SAV relates to combinatorial methods of problems solving. The main idea of combinatorial methods is in the use of finiteness of a feasible set and substitution of its complete enumeration with shortened one (directional enumeration). General scheme for sequential analysis of variants is developed by Myhalevich V.S. [11] based on ideas of theory of sequential solutions and dynamic programming. Methods of SAV are based on systematic solutions construction, or in other words: incremental specification of component values of the solution, and elimination of solutions which cannot be considered as a part of the optimal one.

Let us consider a minimization problem:

$$
\min f\left(x_{1}, \ldots, x_{m}\right)=\min s \sum_{i=1}^{n} y_{i}
$$

With constraints in the following representation:

$$
\left(\sum_{j=1}^{s}\left(r_{j 1} \omega_{j}\right) y_{1}, \ldots, \sum_{j=1}^{s}\left(r_{j i} \omega_{j}\right) y_{n}\right) \leq 1, j=\overline{1, m}, \quad i=\overline{1, n},
$$




$$
\begin{gathered}
\left(\sum_{j=1}^{s}\left(r_{j 1} \gamma_{j}\right) y_{1}, \ldots, \sum_{j=1}^{s}\left(r_{j i} \gamma_{j}\right) y_{n}\right) \leq 1, j=\overline{1, m}, \quad i=\overline{1, n}, \\
x_{j} \in Q, j=\overline{1, m}
\end{gathered}
$$

where $Q=\left\{q_{1_{1} j}, \ldots, q_{\alpha_{m} j}\right\}$ are given final sets [12]. Each variable $x=(j ; i)$ for all $j=\overline{1, m}$ expresses a location of $j$-th virtual machine on $i$-th server.

Vector $\bar{x}_{(m)}=\left(x_{1}, \ldots, x_{m}\right)$ is a solution if its components $x_{j} \in Q, \quad j=\overline{1, m}$. Each element of set $q_{\alpha_{m} i}$ represents one of possible components of the vector $\bar{x}_{(m)}$.

A set of all solutions is defined as $Z$. A solution is acceptable if it satisfies inequalities (7) and (8). A set of all acceptable solutions will be defined with $Z_{f}$. Vector $\bar{x}_{(p)}=\left(x_{1}, \ldots, x_{p}\right), \quad p<m$ is called partial solution [11] in case if $\bar{x}_{(p)} \in Q$. If under these conditions vector $\bar{x}_{(p)}$ can be constructed to the acceptable solution $\left(x_{1}, \ldots, x_{p}, x_{p+1}, \ldots, x_{m}\right)$, $\bar{x}_{(m)} \in Z_{f}$, then this vector is named an acceptable partial solution.

A set of all solutions represents a tree where path from root node to top nodes corresponds to partial solutions and path from the node $x_{0}$ to the node $x_{m}$ corresponds to complete solutions (see Fig. 1).

For the problem when all servers can provide the same amount of resources it is advisable to except mirrored solutions from consideration. For example if there are two identical servers then the number of possible solutions of VMs allocation can be reduced twice because transfer of all VMs from the first server to the second server will not influence the amount of used resources. For servers with identical configuration, the relation between their location and location of others is important, but not an absolute location (see Fig. 2). With increasing number of servers with similar configuration the number of mirrored combination significantly grows from $(m !-1)$ for the case of VMs allocation on one server, to $(m \times n-1)$ ! for the case of VMs allocation on a different servers.

For algorithm development it is necessary to define a set of rules for selecting solutions which will be improving each on each step. Rules are set in a way of eliminating tests, which define strategy of development of partial solutions. The definition of elimination tests set $\xi=\left\{\xi_{l}\right\}$ is necessary for selection of partial solutions, which cannot be improved either up to acceptable or to optimal solutions [12].

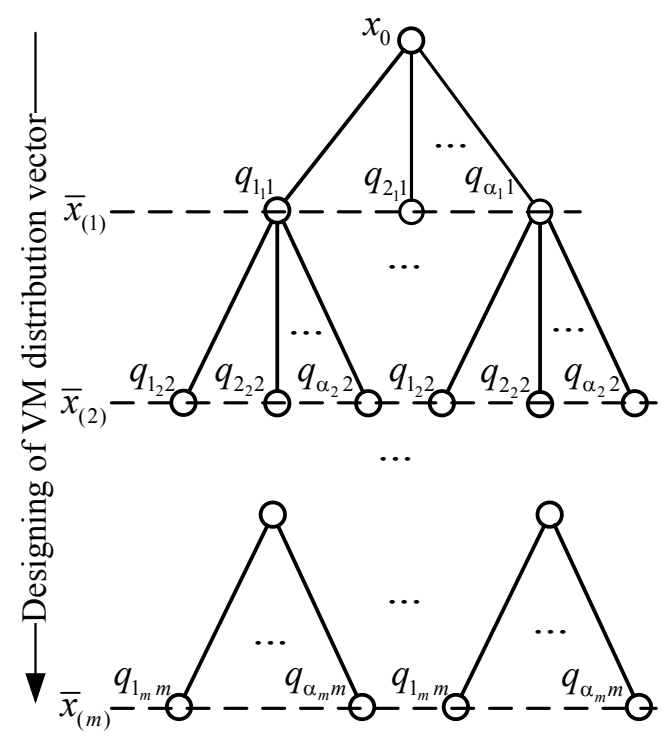

Fig. 1. Tree of solutions for algorithm SAV

Let $h$ be some set of partial solutions. Then in ordered set $\sigma(h)=\left\{\xi_{0}(h), \xi_{1}(h), \ldots, \xi_{l}(h)\right\}$ the variable $\xi_{l}(h)$ denotes a set of partial solutions, which are excepted with test $\xi_{l}$.

Let us next introduce a set of elimination tests:

$\xi_{0}$ - analysis of solutions admissibility;

$\xi_{1}$ - comparison of acceptable solutions by a value of objective function;

$\xi_{2}$ - evaluation of optimal value $\gamma_{f}$ of objective function on each of the sets (calculation of lower bound).

The test $\xi_{0}$ allows to exclude unacceptable solutions from consideration. The set of unacceptable solutions contains all solutions for which an amount of allocated VMs on server exceeds the server's capacity. The test $\xi_{1}$ is designed to search and find optimal distribution of VMs and exclude only not optimal but acceptable solutions from consideration..

The test $\xi_{2}$ and the use of rule for excluding of mirrored solutions allow to avoid a complete enumeration of acceptable solutions. 


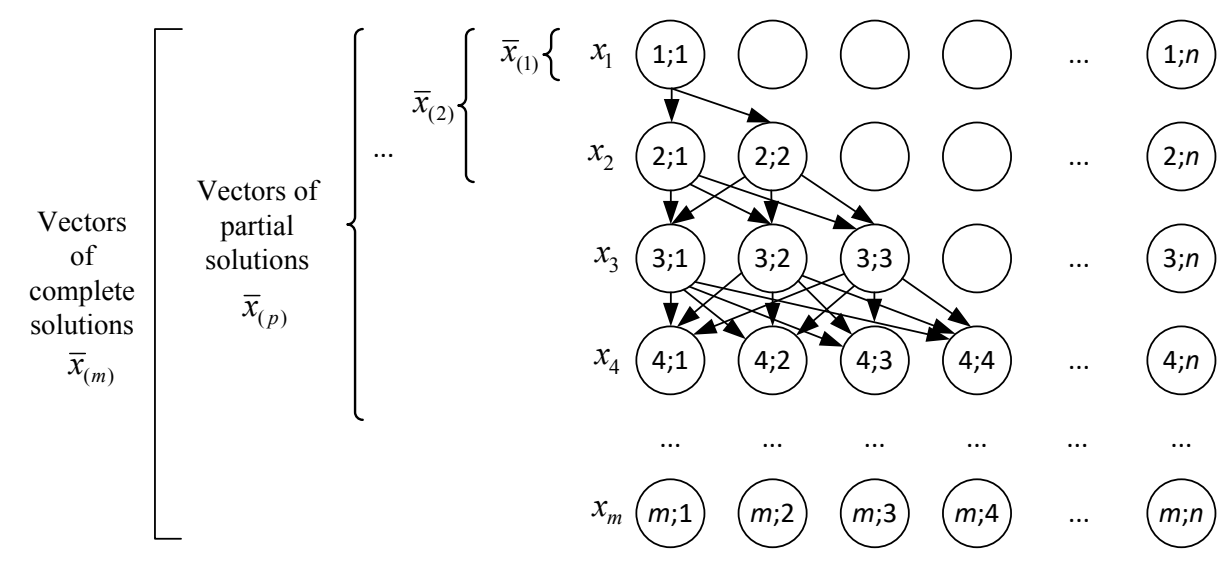

Fig. 2. Allocation of VMs on servers with the use of the SAV algorithm

Definition of elimination tests $\xi_{0}, \xi_{1}, \xi_{2}$ is represented below:

$$
\begin{gathered}
\xi_{0}(h)=\left\{\bar{x}_{(p)} \mid \bar{x}_{(p)} \in h, \sum_{j=1}^{p} x_{j i} \omega_{j}>1, \sum_{j=1}^{p} x_{j i} \gamma_{j}>1, i=\overline{1, n}\right\} \\
\xi_{1}(h)=\left\{\bar{x}_{(m)} \mid \bar{x}_{(m)} \in Z_{f}, f(x) \leq f\left(\bar{x}_{(m)}\right)\right\} \\
\xi_{2}(h)=\left\{\bar{x}_{(p)} \mid \bar{x}_{(p)} \in h, \gamma_{f}\left(\bar{x}_{(p)}\right)>f^{*}\right\}
\end{gathered}
$$

where $f^{*}$ is the upper bound for problem minimum (6). The test $\xi_{2}$ calculates lower bound for optimal value of objective function $f\left(\bar{x}_{(p)}^{0}\right)$ on each of sets $\mathrm{H}\left(\bar{x}_{(p)}^{0}\right)$ where elements are solutions, which extend partial solution $\bar{x}_{(p)}^{0}$.

The order of application of eliminating tests and rules of selection of partial solutions $U=\left(u_{1}, \ldots, u_{q}\right)$ has impact on results of SAV. In SAV algorithm for distribution problem the set of rules $U$ is defined by the following rules: $u_{1}, u_{2}, u_{3}$.

The rule $u_{1}$ is defined as follows: on each step of algorithm, all partial solutions are developed. These solutions are defined on previous step beside the solutions, which are mirrors of previously considered solutions.

The rule $u_{2}$ describes next condition: on each step of the algorithm from all previously received partial solutions, only those are selected, which allow to achieve a minimum of objective function.

The rule $u_{3}$ is defined as follows: selection of candidate on each step of the scheme is performed from set $h_{d}=h_{k}-h_{k-1}$ of partial solutions, which are obtained on previous step and only in case when $h_{d}$ does not contain a partial solution, it is necessary to look up whole list of solutions.
The set of rules $U=\left(u_{1}, u_{2}, u_{3}\right)$ and tests $\xi=\left\{\xi_{0}, \xi_{1}, \xi_{2}\right\}$ define a strategy of solutions enumeration. An order of rules and eliminating tests application corresponds to indexes. In the given case, rules and tests are ordered by ascending complexity.

\section{RESULTS OF EXPERIMENTAL RESEARCHES}

\section{A. Specifics of experiments}

Requests for allocation of the set of VMs in DC are incoming sequentially. Amount of requested resources for each $\mathrm{VM}$ is chosen randomly. Dependency of required RAM and CPU is distributed uniformly.

During experimental researches a given amount of sets of VMs was generated. Needs in computational resources such as RAM or CPU for VMs are also generated randomly within bounds of $0,05 \ldots 0,6$ from server's capacity.

\section{B. Results of experiments}

Efficiency of SAV algorithm performance was evaluated by the parameter of time required for searching for optimal solution, depending on problem complexity $m$. Results are shown on Figure 3. For comparison, figure depicts time of manageable genetic algorithm (MGA) and heuristic algorithms "T" and "M", proposed in [4].

Figure 3 shows that SAV shows acceptable results of allocation time on problems with low complexity, when number of VMs does not exceed range $4 \ldots 16$.

With further increase of VMs amount, the time for distribution obtained by SAV algorithm grows within exponential dependence, whereas heuristic and genetic algorithms demonstrate linear growth of time.

The huge difference in work time of SAV algorithm and heuristic algorithms is explained by their nature. SAV is combinatorial algorithm when heuristic algorithms are just modification of sort algorithms.

In addition, an analysis of errors during work of algorithms was performed as proposed in [4]. A value of objective function ST, SM, SMGA, obtained as a result of heuristic algorithms work "T", "M" and manageable genetic algorithm 
(MGA) respectively was compared to optimal solution provided by SAV. Figure 4 shows error of distribution of VMs plotted along ordinate axis, which shows how much more servers are used with distribution of VMs obtained by algorithms "T", "M" and MGA, comparing to optimal allocation. The number of VMs requested to distribution on servers in DC is plotted along abscissa axis. Values of error are averaged from the results of 10 experiments.

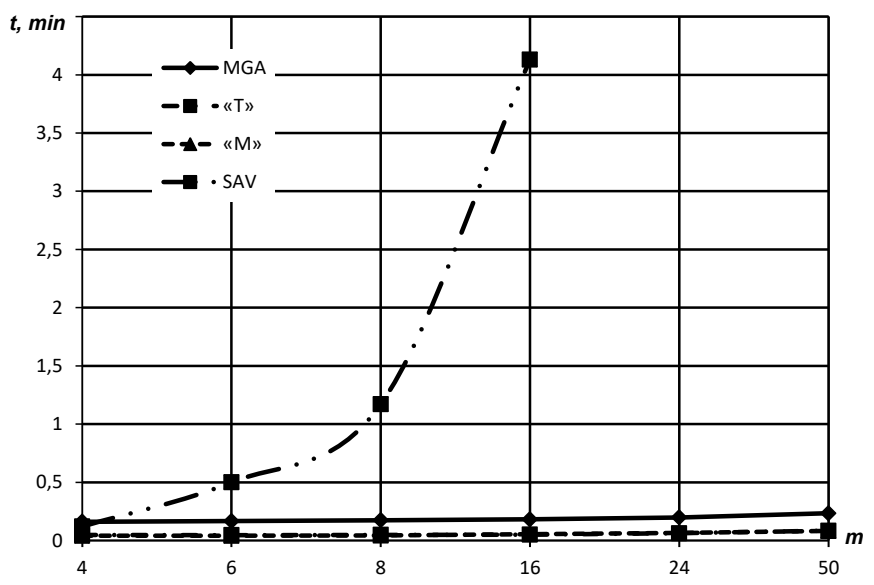

Fig. 3. Dependence of algorithms work time on problem complexity

The SAV algorithm gives slight gain in accuracy even on tasks with low complexity. With the increase in complexity, the error from heuristic and genetic algorithms grows, what indicates a dependence of error on the task complexity.

It should be noticed that for successful appliance of the SAV algorithm, it is necessary to have some amount of servers $N_{\text {min }}$, for which it is certainly known that they can allocate all VMs from set $K_{j}, j=\overline{1, m}$. A value $N_{\min }$ can be obtained with expert's help or with the use of one of heuristic algorithms.

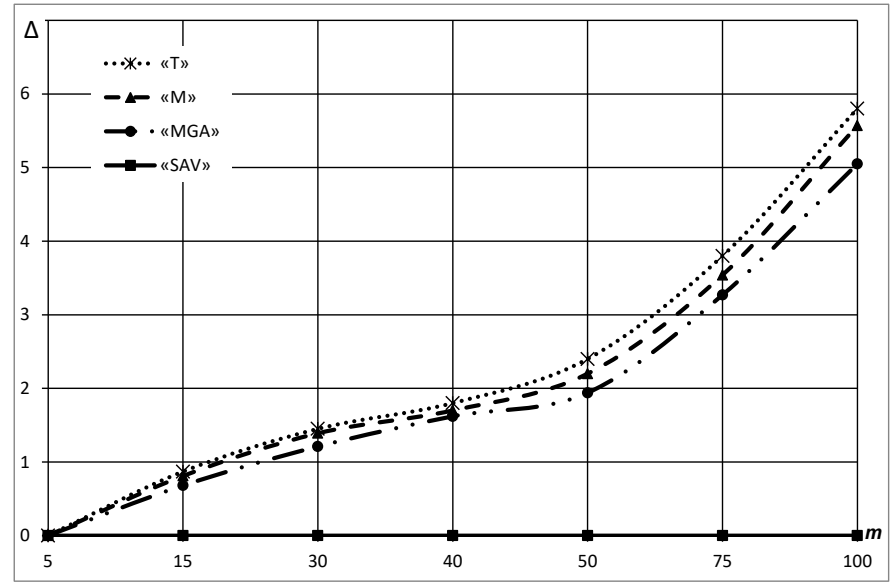

Fig. 4. Dependence of error of VMs distribution $\Delta$ relative to optimal solution depending on problem complexity

Researches have allowed to make the following recommendations for choosing an algorithm for VMs distribution on physical servers. For tasks where high accuracy with small amount of VMs is the main priority, it is necessary to use the SAV algorithm because it finds optimal solution. The SAV can also be used in case where time for obtaining the result is not critical.

Heuristic and genetic algorithms make it possible to get a fast solution. Speed of work of such algorithms loosely depends on complexity of a task but the quality of solution is not on the same level as the solution of the SAV algorithm.

\section{CONCLUSIONS}

The result of researches made in this paper is an algorithm of discrete optimization based on SAV scheme for solving the problem of VMs allocation on physical servers of a data center. During development of algorithm, evaluations of time expenses of SAV algorithm were made, which depend on problem size. Comparative analysis of efficiency of different algorithms was done. It was proved that heuristic algorithms allow to obtain a solution really fast. With the use of such algorithms it is possible to reduce expenses for maintaining servers. The SAV algorithm requires significant time expenses but makes it possible to find the best solutions.

\section{REFERENCES}

[1] J. H. Holland "Complex Adaptive Systems," in Daedalus, vol. 121, no. 1, pp. 17-30; Winter 1992.

[2] K. S. Leung, Q. H. Duan, Z. B. Xu and C. K. Wong, "A new model of simulated evolutionary computation: convergence analysis and specifications," IEEE Trans. on Evolutionary Computation, vol. 5, no. 1, pp. 3-16, 2001.

[3] S. Telenyk, E. Zharikov and O. Rolik, "Architecture and Conceptual Bases of Cloud IT Infrastructure Management," in Advances in Intelligent Systems and Computing, Springer, 2017, pp. 41-62.

[4] S. Telenyk., O. Rolik, P.S. Savchenko and M. E. Bodaniuk, "Manageable genetic algorithm in tasks of distribution of virtual machines in data centres," Visnyk of Cherkasy State Technological University), vol. 2, pp. 104-113, 2011.

[5] S. F. Telenik, A. I. Rolik, M. M. Bukasov and S. A. Androsov, "Genetic algorithms of decision of tasks of management resources and loading of centers of processing of data" Automatic. Automation. Electrical engineering complexes and systems, no. 1 (25), pp. 106-120, 2010.

[6] S. Singh and I. Chana, "A Survey on Resource Scheduling in Cloud Computing: Issues and Challenges," Journal of Grid Computing, pp. 148, 2016.

[7] Z. Cao and S. Dong, "An energy-aware heuristic framework for virtual machine consolidation in cloud computing," The Journal of Supercomputing, pp. 1-23, 2014.

[8] M. Sun, W. Gu, X. Zhang, H. Shi, and W. Zhang, "A matrix transformation algorithm for virtual machine placement in cloud," in Trust, Security and Privacy in Computing and Communications (TrustCom), 2013 12th IEEE Int. Conf. on. IEEE, 2013, pp. 1778-1783.

[9] F. Lopez Pires and B. Baran, "Multi-objective virtual machine placement with service level agreement: A memetic algorithm approach," in Proc. of the 2013 IEEE/ACM 6th Int. Conf. on Utility and Cloud Computing. IEEE Computer Society, 2013, pp. 203-210.

[10] W. Wang, H. Chen, and X. Chen, "An availability-aware virtual machine placement approach for dynamic scaling of cloud applications," in Ubiquitous Intelligence \& Computing and 9th Int. Conf. on Autonomic \& Trusted Computing (UIC/ATC), 2012, pp. 509-516.

[11] V. S. Mikhalevich, "Consecutive optimization algorithms and their application. I. II," Cybernetics, no.1, pp. 45-55, no 2. pp. 85-88, 1965.

[12] E. N. Sipko, "The method for sequential analysis of variants to solve a scheduling problem,” Iskusstvenny intellekt, no. 1, pp. 243-250, 2011. 



\section{International Conference on Innovative Network Systems and Applications}

M

ODERN network systems encompass a wide range of solutions and technologies, including wireless and wired networks, network systems, services and applications. This results in numerous active research areas oriented towards various technical, scientific and social aspects of network systems and applications. The primary objective of Innovative Network Systems and Applications (iNetSApp) conference is to group network-related events and promote synergy between different fields of network-related research. To stimulate the cooperation between commercial research community and academia, the conference is co-organised by Research and Development Centre Orange Labs Poland and leading universities from Poland, Slovak Republic and United Arab Emirates.

The conference continues the experience of Frontiers in Network Applications and Network Systems (FINANS), International Conference on Wireless Sensor Networks (WSN), and International Symposium on Web Services (WSS). As in the previous years, not only research papers, but also papers summarising the development of innovative network systems and applications are welcome.

iNetSApp currently consists of tracks:

- CAP-NGNCS' $17-1^{\text {st }}$ International Workshop on Communications Architectures and Protocols for the New Generation of Networks and Computing Systems

- INSERT'17 - $1^{\text {st }}$ International Conference on Security, Privacy, and Trust

- IoT-ECAW'17-1 ${ }^{\text {st }}$ Workshop on Internet of ThingsEnablers, Challenges and Applications

- SoFast-WS'17-6 $6^{\text {th }}$ International Symposium on Frontiers in Network Applications, Network Systems and Web Services

- WSN'17 - $6^{\text {th }}$ International Conference on Wireless Sensor Networks 



\section{$1^{\text {st }}$ International Conference on Security, Privacy, and Trust}

\begin{abstract}
A DMITTEDLY, information security works as a backbone for protecting both user data and electronic transactions. Protecting communications and data infrastructures of an increasingly inter-connected world have become vital nowadays. Security has emerged as an important scientific discipline whose many multifaceted complexities deserve the attention and synergy of the computer science, engineering, and information systems communities. Information security has some well-founded technical research directions which encompass access level (user authentication and authorization), protocol security, software security, and data cryptography. Moreover, some other emerging topics related to organizational security aspects have appeared beyond the long-standing research directions.

The $1^{\text {st }}$ International Conference on Security, Privacy, and Trust (INSERT'17) focuses on the diversity of the information security developments and deployments in order to highlight the most recent challenges and report the most recent researches. The conference is an umbrella for all information security technical aspects, user privacy techniques, and trust. In addition, it goes beyond the technicalities and covers some emerging topics like social and organizational security research directions. INSERT'17 is intended to attract researchers and practitioners from academia and industry, and provides an international discussion forum in order to share their experiences and their ideas concerning emerging aspects in information security met in different application domains. This opens doors for highlighting unknown research directions and tackling modern research challenges. The objectives of the INSERT' 17 can be summarized as follows:

- To review and conclude researches in information security and other security domains, focused on the protection of different kinds of assets and processes, and to identify approaches that may be useful in the application domains of information security

- To find synergy between different approaches, allowing elaborating integrated security solutions, e.g. integrate different risk-based management system.

- To exchange security-related knowledge and experience between experts to improve existing methods and tools and adopt them to new application areas
\end{abstract}

\section{TOPICS}

Topics of interest include but are not limited to:

- Biometric technologies

- Human factor in security

- Cryptography and cryptanalysis
- Critical infrastructure protection

- Hardware-oriented information security

- Social theories in information security

- Organization- related information security

- Pedagogical approaches for information security

- Social engineering and human aspects in security

- Individuals identification and privacy protection methods

- Information security and business continuity management

- Decision support systems for information security

- Digital right management and data protection

- Cyber and physical security infrastructures

- Risk assessment and risk management

- Tools supporting security management and development

- Trust in emerging technologies and applications

- Ethical trends in user privacy and trust

- Digital forensics and crime science

- Security knowledge management

- Privacy Enhancing Technologies

- Misuse and intrusion detection

- Data hide and watermarking

- Cloud and big data security

- Computer network security

- Security and safety

- Assurance methods

- Security statistics

\section{SECTION EDITORS}

- Awad, Ali Ismail, Luleå University of Technology, Sweden

- Bialas, Andrzej, Institute of Innovative Technologies EMAG, Poland

\section{REVIEWERS}

- Banach, Richard, University of Manchester, United Kingdom

- Bun, Rostyslav, Lviv Polytechnic National University, Ukraine

- Clarke, Nathan, Plymouth University, United Kingdom

- Cyra, Lukasz, DM/OICT/RMS (UN)

- Dworzecki, Jacek, Police Academy in Szczytno

- Furnell, Steven, Plymouth University, United Kingdom

- Furtak, Janusz, Military University of Technology, Poland

- Geiger, Gebhard, Technical University of Munich, Faculty of Economics

- Grzenda, Maciej, Orange Labs Poland and Warsaw University of Technology, Poland 
- Hämmerli, Bernhard M., Hochschule für Technik+Architektur (HTA), Switzerland

- Hasssaballah, M., South Valley University, Egypt

- Kapczynski, Adrian, Silesian University of Technology, Poland

- Kosmowski, Kazimierz, Gdansk University of Technology

- Krendelev, Sergey, Novosibirsk State University,JetBrains research, Russia

- Misztal, Michal, Military University of Technology, Poland

- Pańkowska, Małgorzata, University of Economics in Katowice, Poland
- Rot, Artur, Wroclaw University of Economics, Poland

- Soria-Rodriguez, Pedro, Atos Research \& Innovation

- Stokłosa, Janusz, WSB University in Poznan, Poland

- Suski, Zbigniew, Military University of Technology, Poland

- Szmit, Maciej, IBM, Poland

- Thapa, Devinder, Luleå University of Technology

- Wahid, Khan Ferdous, Airbus, Germany

- Yen, Neil, The University of Aizu, Japan

- Zamojski, Wojciech, Wrocław University of Technology

- Zieliński, Zbigniew, Military University of Technology, Poland 


\title{
Identification of Fingerprints using Circular String Approximation for Mobile Devices
}

\author{
Oluwole I. Ajala, Costas S. Iliopoulos, Mujibur R. Khan \\ Faculty of Natural \\ and Mathematical Sciences \\ Department of Informatics \\ King's College London \\ London, UK WC2R 2LS \\ Email:\{oluwole.ajala, costas.iliopoulos, md.khan\}@kcl.ac.uk
}

\begin{abstract}
Lately, Biometrics has employed the use of fingerprints to identify individuals. Out of the commonly used techniques, fingerprint authentication till date remains the most reliable. The bulk of research that has focused on fingerprint authentication, has however, neglected the issues that arise with fingerprints resulting to incorrect orientation identification. This is because it is assumed and often times wrongly, that the direction of the fingerprint will align with the stored fingerprint image. This singular issue poses tension in fingerprint matching, which only a negligible number of techniques have considered. As computers and mobile devices adopt fingerprint recognition as a way to authenticate the user, this apparent tension gains more popularity, becoming an integral research area which must be addressed.

Responding to this issue, this report proposes a novel pattern matching technique that caters for orientation differences in fingerprints, by implementing a pre-matching stage called the orientation identification stage and then match the fingerprint image with stored images using algorithms for approximate circular string matching. This fingerprint string information is now matched against a database of stored images using approximate string matching techniques.
\end{abstract}

Keywords-Biometrics, Rotation,Fingerprint, Circular String Matching.

\section{INTRODUCTION}

The complication of security increases when information is disseminated over a wide area network or larger number of devices and systems being shared by unrelated users. As more information on individuals and companies are placed in the 'cloud', user privacy protection becomes a fundamental requirement. Issues on integrity, confidentiality and user authorisation pose a major concern. Thus, the core principles of information security are compromised.

Biometric resolutions proffer confidential and authentic transactions and also personal data privacy. Its usage transcends the commercial applications and law enforcement bodies to all government parastatals at all levels.

Fingerprints have provided an impeccable means of user authentication and personal identification for a long time, possibly dating back to the 19th century, when the records of fingerprint details of criminals in Argentina were released [20]. It has long since been adopted not just for law enforcement purposes (forensics and police) but also for commercial purposes like financial transactions and most recently, it is used as an authentication method in mobile devices and computers. Fingerprints are made up of minutiae, which are basically ridges and furrows in parallelism with each other. These minutiae form a complicated pattern that when impressed on a fingerprint scanner, leaves a print. These prints are matched to stored images on a database for either verification, authentication or both purposes [18].

\section{ROAD MAP}

The organisation of the rest of this paper is as follows. In section III, we present a very brief literature review, followed by section IV where we summarize our contribution to this problem. We present our approach in details in subsection IV-D. The experiment and the result analysis will be presented in section V. Finally, we briefly conclude and state the future work in section VI.

\section{LITERATURE REVIEW}

As earlier mentioned, there have been extensive yet unsatisfactory algorithmic attempts at accurately processing and interpreting fingerprint patterns. Some of the hurdles encountered during the study of this overly researched area include but are not limited to application in comparing biological systems, signal processing, recovering information and correlating data from various files.

According to the Merriam-Webster dictionary, an algorithm is a procedure for solving a mathematical problem (as of finding the greatest common divisor) in a finite number of steps that frequently involves repetition of an operation; a step-bystep procedure for solving a problem or accomplishing some end especially by a computer [15].

A string is processed by an algorithm to search for a text pattern within a larger body of text i.e. finding all the occurrences of a string/pattern (x) within a larger string (y.)

Generally, the algorithms probe the text through a 'window' starting by fastening at the left end of both text and window. They then compare the pattern to the text within the window space for either a match or a mismatch. This is called an attempt. They move to the next segment of the text and recheck for a match or mismatch. This process is repeated until 
the whole text is read to the right end; a progression called sliding window mechanism [7].

Important algorithms in relation to this paper are the Needleman-Wunsch and Landau and Vishkin algorithms [14]. Approximate String Matching led to (i) Dynamic programming algorithm by Sellers and (ii) Landau and Vishkin algorithm [14]. The drawback of this algorithm was that it was expensive, space-consuming and inappropriate for long strings. Due to the complexities of matching strings across the grid and modification of edit distances, the exactness of the alignment within record time was challenging. Therefore, accuracy and speed became mitigating factors present in this algorithm.

Subsequently, a more operative algorithm that also took into consideration the no gap penalty was introduced individually by David Sankoff in 1972, T. K. Vintsyuk in 1968, Robert A. Wagner and Michael J. Fischer in 1974.

It was in an attempt to solve string matching more accurately in record time and use less space at a minimal cost that the Landau and Vishkin algorithm was presented [14]. The approach was to use suffix trees to reach more space over a minimal time frame. It takes into consideration that there could be errors or mismatches from unnecessary characters in both texts and patterns, insertions and deletions ( $\mathrm{k}$ differences).

The algorithm had two stages: (i.) Pre-processing phase and (ii.) Iteration phase. Both text and pattern are processed in the pre-processing phase to construct a generalised suffix tree and to get the longest common extension (LCE). The suffix trees are used to compute the dynamic programming table in an accelerated time.

In the iteration phase, the algorithm probes each of the diagonals of the programming grid $\mathrm{k}$ times as far as it can go searching for all the pattern matches - permitting a minimum number of $\mathrm{k}$ differences.

'Diagonal' refers to all the cells with the same k differences. A simple stretch of a diagonal path defines an accurate match. [14]

\section{OUR CONTRIBUTION}

This paper focuses on the identification stage by proposing a fast novel pattern matching technique for fingerprintbased authentication on mobile devices that attempts to match fingerprints via classification. The fingerprint is intercepted with a series of scan circles and the minutiae information is derived. This information will then be translated into a string. This fingerprint string information is now matched against a database of stored images using an efficient, error tolerant, pattern matching algorithm. With this approach, identification of fingerprints can be done in linear time, with respect to the total length of all strings to be searched.

We devised a comprehensive method to identify fingerprints using the circular string matching technique. Because it exploits the power of circular string matching, the rotation of the input image is no longer a problem here. And the impact due to the distortion of the input image is kept at a minimal level by using approximation instead of exact matching. This is more like a proof of concept where we demonstrate that a comprehensive strategy could be devised by exploiting the power of circular string matching.
Few new things have been introduced that are used as underlying building blocks of our algorithm. They are presented in the following three subsections - IV-A, IV-B, and IV-C. Finally, the algorithm is presented in the subsection IV-D.

\section{A. Angle of Rotation}

Let $x=x[0,1, . ., m-1]$ be any circular string of length $\mathrm{m}$. We know, $x^{i}=x[i, i+1, . ., m, 0,1, . . i-1]$ is the $i$ th rotation of $x$. The angle of rotation $a$ of $x^{i}$ in degrees is given by the following formulae

$$
a=\frac{i}{m} \times 360^{\circ}
$$

Hence,

$$
i=\left\lceil\frac{a}{360} \times m\right\rceil
$$

For example, if $x=A B C D E F G H$, then $x^{1}=$ $B C D E F G H A$ and $x^{1}$,s angle of rotation, $a$ is $45^{\circ}$. Similarly, if $y=0123456789 A B$ then the $45^{\circ}$ rotation of $y$ will be $y^{2}$, i.e. $23456789 A B 01$.

\section{B. Alignment of Concentric Circular Strings}

Let us consider figure IV-B that shows the concentric circular strings of three different fingerprints.
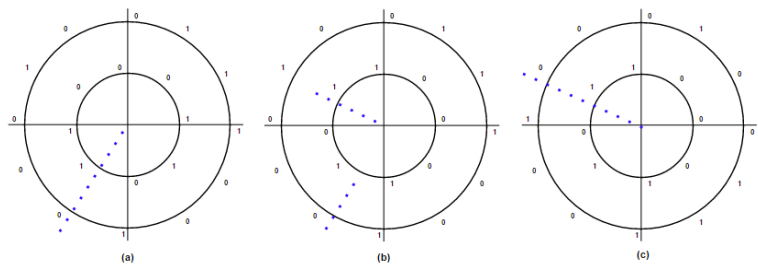

Fig. 1. Two concentric circles and the corresponding binary strings of three arbitrary images.

Only two circles are shown here for simplicity. The outer circle of figure IV-B(a) matches a rotation of the outer circle of figure IV-B(b) and the inner circle of figure IV-B(a) matches another rotation of the inner circle of figure IV-B(b). But the rotations of outer and inner circles are not aligned (as shown by the dotted lines). Hence, figure IV-B(b) is not a match of figure IV-B(a). On the other hand, the circular strings of figure $\mathrm{IV}-\mathrm{B}(\mathrm{c})$ matches the ones in figure IV-B(a) and also the outer and inner circles are both having the same amount angle of rotation (say, $a^{\circ}$ ) i.e. they are aligned. Hence, figure IV-B(c) is the $a^{\circ}$ th rotation of figure IV-B(a).

Therefore, in order to identify an input image as a rotation of a given fingerprint, all the circular strings of the input must match with a certain angular rotation of the corresponding circular string in the fingerprint.

In our algorithm, we take the longest circular strings from the input and the saved fingerprint. We check if any rotation of the circular string from the input matches the one from the saved fingerprint (or vice-versa) using asmf/acdmf. Suppose, the $a^{\circ}$ th rotation of the input is found to be a good match. We then take the $a^{\circ}$ th rotation of each of the remaining circular strings of the input image and match them with the 
corresponding circular string from the saved image using the approximation algorithm of Needleman-Wunsch. We do not need to perform circular string matching for these strings.

Due to the rotation and distortion of the input image, it is less likely that the circular strings will match exactly. Hence, using the approximate matching is more likely to lead us towards a valid result.

\section{Novel Minutiae Extraction 2}

Minutiae extractions are repeatedly used in our process. Hence, the speed of minutiae extraction has a significant impact on the overall speed of our process. That's why we have devised Novel_Minutiae_Extraction2 witch is found to be extremely faster than the Novel_Minutiae_Extraction presented in Wole's MSc project paper [2]. Outline of Novel_Minutiae_Extraction2 is presented below:

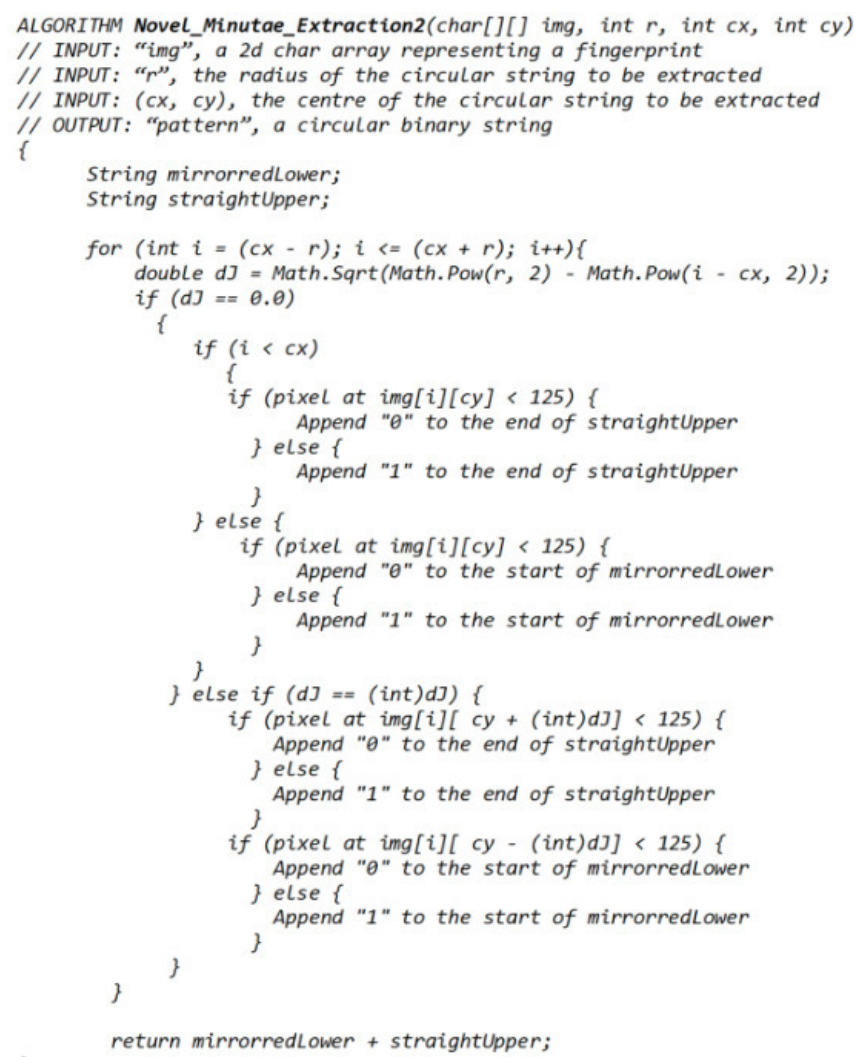

\section{Outline of the Algorithm}

We have outlined the complete process of Fingerprint matching as below:

1) Construct the database of circular strings taking the centre of the image as the centre. Draw concentric circles and then convert them into strings of 0 's and 1's (using the Novel_Minitiae_Extraction2 as outlined in IV-C). Say $X_{i r}$ be the circular string for the $i$ th image with a radius $r$.

2) Save all rotations of each of the circular strings in the database. Let $D R_{i r}$ be the collection of rotated

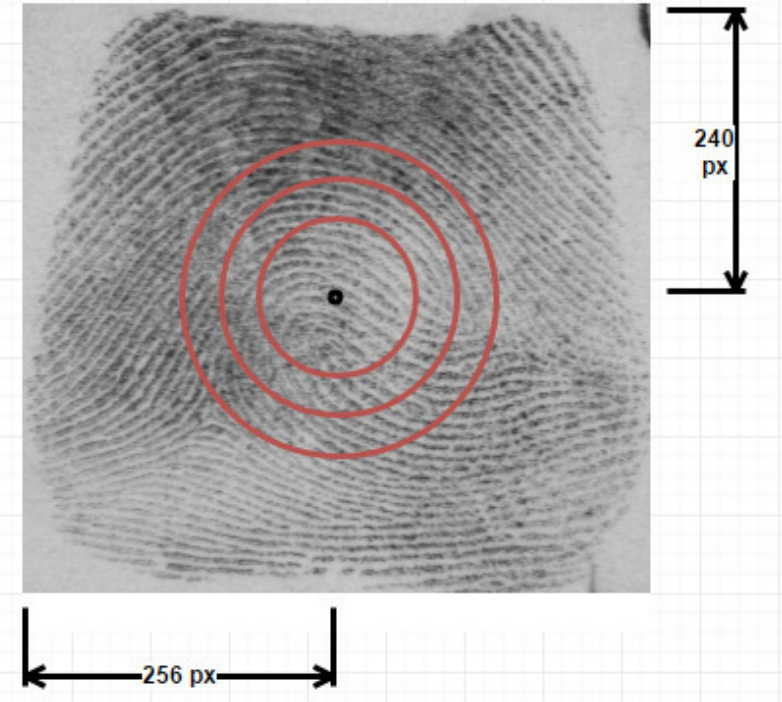

Fig. 2. A sample image from the database. (H:480px, W:512px)

strings from $X_{i r}$ e.g. if $X_{i r}=$ "1011" then $D R_{i r}$ will be "1011","0111","1110","1101"

3) For the input fingerprint, we shall identify the boundary of the input. Let $G v$ be the larger among the stretches in the height and width of the image. (See figure 3)

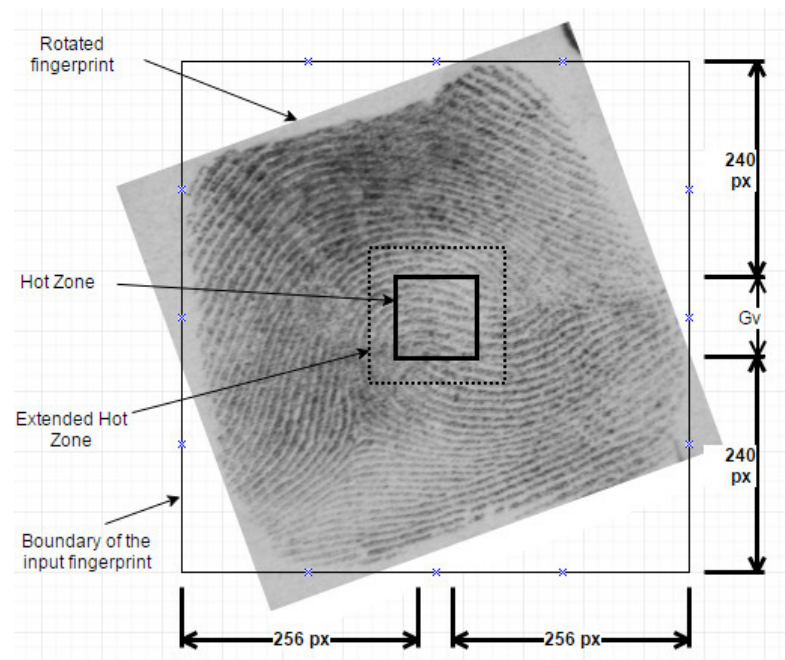

Fig. 3. Image boundary of a rotated input image

4) Let's draw a square with sides of length $G v$ at the centre of the boundary, as shown in the image (alternatively, we could draw a circle with radius $G v$ from the centre of the identified boundary). It is most likely that the centre of the original image lies somewhere within this hot zone.

5) We could add an arbitrary amount of tolerance $(d T)$ to $G v$ and get the extended hot zone. The centre of the original image is further likely to fall somewhere within the extended hot zone.

6) Now, start at the centre of the identified boundary and 
take it as the prospective centre (in the subsequent iterations, we shall spiral outwards from this point until the boundary of the extended hot zone). (See figure 4)

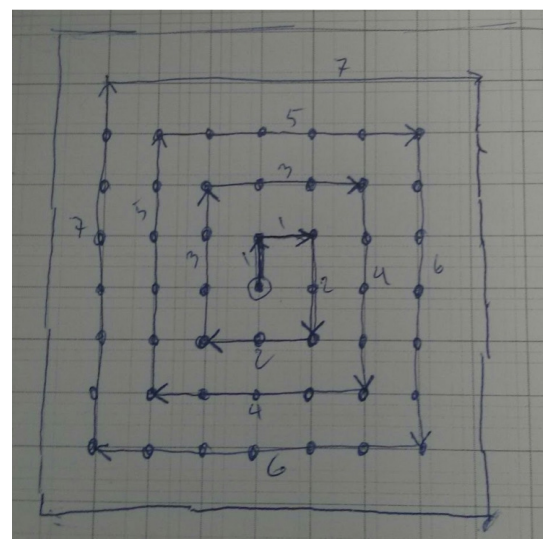

Fig. 4. Spiral outward from the centre

7) Draw concentric circles of various radius from this prospective centre. Then construct circular binary strings for each of the circle (using the Novel_Minitiae_Extraction2 as outlined in subsection IV-C). Make a sorted list $\mathbf{C s R}$ of circular string and radius pairs, $<C S t r$, Radius $>$, in descending order of the length of CStr.

8) a) Apply asmf/acdmf method to compare all the rotations of CsR[0].CStr (i.e. the first circular strings obtained in step 7) with the one in $D R_{i r}$ where $r=C s R[0]$.Radius. Let's assume the $q$ th rotation of $C s R[0] . C S t r$ has the highest match. Calculate the angle a for the $q$ th rotation using eq. 1 .

b) For each CsR[p].CStr where $1 \leq p<\operatorname{len}(C s R)$, find the $q$ th rotation of CsR[p].CStr, where $q$ is calculated for angle a using eq. 2. Then apply NeedlemanWunsch method to compare the $q$ th rotations of CsR[p].CStr with the one in $D R_{i r}$ where $r=C s R[p]$.Radius.

9) Find the average of matching obtained in steps $8 \mathrm{a}$ and $8 \mathrm{~b}$.

10) a) If the percentage of matched strings is higher than a given threshold (tS), then save the sum of percentage of match and mark the $i$ th image as a candidate and select the next $((i+1)$ th $)$ image. Proceed to step 11 .

b) Otherwise, repeat from step 6 by choosing a different centre point.

c) Repeat it until all the points within the extended hot zone are tried.

11) Repeat step 6 to step 10 until all the images in the database are exhausted.

12) Return the top ranked images.

\section{EXPERIMENTAL RESULTS}

We coded the program in C\# using Microsoft Visual Studio 2013. Intel Xeon processor with $3.5 \mathrm{GHz}$, the Operating system was 64 bit Windows 7 .
Figure $\mathrm{V}$ is an input panel with $\mathrm{H}: 1024$ and $\mathrm{W}: 2048$ pixels that simulates the input screen of an arbitrary mobile device. The original fingerprint is a $512 \times 512$ pixel bitmap. Which is rotated and placed at a random location inside the input panel. Then the boundary of the input image is identified.

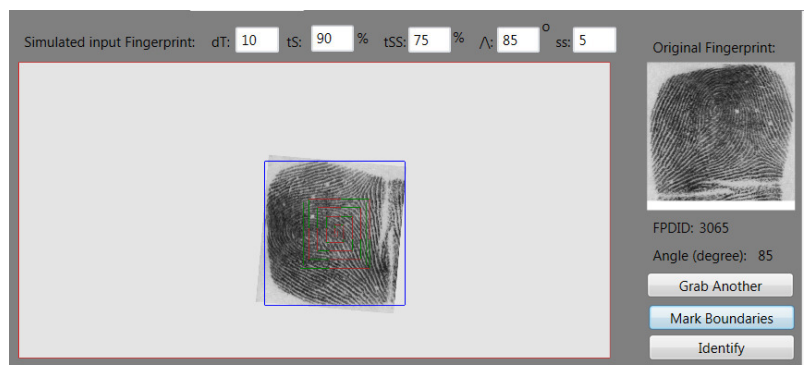

Fig. 5. Input panel with a simulated input and its identified image-boundary.

The performance and accuracy of the proposed algorithm vastly depends on the size of the database as well as on the number of probable centre points on the input image. This number varies depending on the rotation of the input image. We have collected data for this against the degree of rotation which is presented in Table I.

TABLE I: The average number of points considered as potential centre of the rotated image (rounded to nearest 100), against the angle of rotation of the input image.

\begin{tabular}{|c|c|}
\hline $\begin{array}{c}\text { Angle of rotation of } \\
\text { the input image } \\
\text { (in degree) }\end{array}$ & $\begin{array}{c}\text { Average number of } \\
\text { prospective centres } \\
\text { (dT=10) }\end{array}$ \\
\hline $0,90,180,270$ & 1500 \\
\hline $15,105,195,285$ & 15000 \\
\hline $30,120,210,300$ & 30000 \\
\hline $45,135,225,315$ & 40000 \\
\hline $60,150,240,330$ & 30000 \\
\hline $75,165,255,345$ & 15000 \\
\hline
\end{tabular}

Table II shows the result against three different database sizes. For every input, we captured the top 5 ranked images from the database. The second column shows the percentage of times when the correct fingerprint is returned as the top ranked image. The third column shows the percentage of times when the correct image is not the top ranked but is withing the top 5 .

TABLE II: Summery of findings (dT=10, $\mathrm{tS}=90, \mathrm{tSS}=75)$.

\begin{tabular}{|c|c|c|c|c|}
\hline $\begin{array}{c}\text { Db } \\
\text { Size }\end{array}$ & $\begin{array}{c}\text { \% of cases } \\
\text { where } \\
\text { the } \\
\text { correct } \\
\text { fingerprint } \\
\text { is top } \\
\text { ranked }\end{array}$ & $\begin{array}{c}\text { \% of cases } \\
\text { where } \\
\text { the } \\
\text { correct } \\
\text { fingerprint } \\
\text { is in } \\
\text { top 5 }\end{array}$ & $\begin{array}{c}\text { \% of cases } \\
\text { where } \\
\text { the } \\
\text { correct } \\
\text { fingerprint } \\
\text { is Not } \\
\text { in top 5 }\end{array}$ & $\begin{array}{c}\text { Average } \\
\text { time } \\
\text { (Seconds) }\end{array}$ \\
\hline 50 & $72.72 \%$ & $09.09 \%$ & $18.18 \%$ & 881.30 \\
\hline 100 & $47.62 \%$ & $23.81 \%$ & $28.57 \%$ & 3276.93 \\
\hline 500 & $14.29 \%$ & $28.57 \%$ & $57.14 \%$ & 63644.37 \\
\hline
\end{tabular}


It is clearly visible that the performance degrades significantly as the database size increases. That indicates the huge potential for the future scope to improve the algorithm.

\section{Conclusion}

This report establishes yet another example of an interdisciplinary effort where mathematical algorithms have been translated into codes in computer science that are used to solve problems extending beyond bioinformatics.

As more systems and devices adopt fingerprint recognition as biometric authentication, the need for effective pattern matching cannot be over emphasised. To this end, a plethora of algorithms and string matching techniques have erupted. This paper addressed the ever rising issue with fingerprint authentication- orientation. With the correct orientation figured out, matching becomes much easier and faster thus speed and accuracy is maximised.

\section{REFERENCES}

[1] Ajala, O., Aljamea, M., Alzamel, M., Iliopoulos, C. S., Fast Fingerprint Rotation Recognition Technique Using Circular Strings in Lexicographical Order. SAI Intelligent Systems Conference 2016, September 21-22, 2016, London, UK

[2] Ajala, O., Aljamea, M., Alzamel, M., Iliopoulos, C. S., Strigini, Y., Fast Fingerprint Recognition Using Circular String Pattern Matching Techniques. PATTERNS 2016 : The Eighth International Conferences on Pervasive Patterns and Applications

[3] Al-Jamea, M et al, 2015. A Novel Pattern Matching Approach for Fingerprint-based Authentication. PATTERNS 2015: The Seventh International Conferences on Pervasive Patterns and Applications, [Online]. 978-1-61208-393-3, 45-49. Available at: https://www.thinkmind.org/download.php? articleid=patterns 2015 2 2 40 70032 [Accessed 12 March 2016].

[4] Angle, S et al, 2005. Biometrics: A Further Echelon of Security. The Impact of Technology on Plagiarism Prevention and Detection: Research Process Automation, a New Approach for Preven tion, 3, 3-9.

[5] Barnes, J. G, 2014. The Fingerprint Sourcebook. 1st ed. UK: Create Space Independent Publishing Platform.

[6] Barton, Iliopoulos, Pissis, C., C. S., S. P., 2014. Fast algorithms for approximate circular string matching. Algorithms for Molecular Biology, 9:9, 10.
[7] Christian, C., Lecroq, T., Handbook of exact string matching algorithms. London, UK:: Kings College Publications, 2004

[8] Colussi, L., 1991. Correctness and efficiency of pattern matching algorithms. Information and Computation, 95, 225251. https://doi.org/10.1016/0890-5401(91)90046-5

[9] Cormen, Leiserson and Rivest, T. H., C. E., and R. L. , 1990. Introduction to Algorithms. 1st ed. Cambridge, Massachusetts: MIT Press.

[10] Dongjae, D.L, 2008. Systems, Man, and Cybernetics, Part B: Cybernetics, IEEE Transactions on Year: 2008, Volume: 38, Issue: 1. Recognizable-Image Selection for Fingerprint Recognition With a Mobile-Device Camera, 38, Issue 1, 233243.

[11] Donida, L.R, 2013. Computational Intelligence and Virtual Environments for Measurement Systems and Applications (CIVEMSA), 2013 IEEE International Conference on Year: 2013. Accurate 3D fingerprint virtual environment for biometric technology evaluations and experiment design, DOI: 10.1109/CIVEMSA. 2013.6617393, 43-48.

[12] Galton, F., 1892. Finger Prints. 1st ed. London, New York: Macmillan and Co. http://www.explainthatstuff.com/. 2004. Biometric fingerprint scanners. [ONLINE] Available at: http://www.explainthatstuff.com/ fingerprintscanners.html. [Accessed 31 August 16].

[13] Gao, Q, 2014. A preliminary study of fake fingerprints. I.J Computer Network and Information Security, 12, 1-8.

[14] Landau, Vishkin, G.M., U., 1998. Fast String Matching with k Differences* .Journal of Computer and System Sciences, 37, 63-78. https://doi.org/10.1016/0022-0000(88)90045-1

[15] Merriam-Webster. 2016. Algorithm. [ONLINE] Available at: http://www.merriam-webster.com/dictionary/algorithm. [Accessed 09 September 16].

[16] Miranda, R. C., Ayala-Rinc on, M., Solon, L, 2005. Advances in Bioinformatics and Computational Biology.. 1st ed. Sao Leopoldo, Brazil: Springer Berlin Heidelberg.

[17] Moses, K.R, 2014. The finger print source book. 1st ed. UK: CreateSpace Independent Publishing Platform

[18] Sebastian, S., Literature survey on automated person identification techniques, International Journal of Computer Science and Mobile Computing, vol. 2, no. 5, pp. 232237, 2013

[19] Sheik, S. S., Aggarwal, S. K., Poddar, A., Balakrishnan, N., Sekar, K., 2004. A FAST Pattern Matching Algorithm. J. Chem. Inf. Comput. Sci, 44, 1251-1256. https://doi.org/10.1021/ci030463z

[20] Unar, J., Seng, W. C., and Abbasi, A., A review of biometric technology along with trends and prospects, Pattern Recognition, vol. 47, no. 8, pp. 26732688, 2014 https://doi.org/10.1016/j.patcog. 2014.01.016 



\section{A model for context-sensitive usage control in healthcare information systems}

\author{
Rodolfo Barriviera \\ Federal Institute of Paraná State, \\ Londrina PR - Brazil \\ Email: rodolfo.barriviera@ifpr.edu.br
}

\author{
Carlos Maziero \\ Federal University of Paraná State, \\ Curitiba PR - Brazil \\ Email: maziero@inf.ufpr.br
}

\begin{abstract}
The secure management of access to patient information in medical and hospital environments is a relevant and widely debated problem. Although the electronic patient record is envisaged in the current legislation, it is still a promise to the Brazilian health reality. The aim of this work is to define a continuous access control model for patient's medical information, which considers contextual information and that can be implemented in large scale environments, such as computational clouds. A bibliographic study was conducted related to the computational area of access control, electronic and paper medical records, and on the current health legislation in Brazil. Subsequently, a qualitative research was conducted in the BHUs of the city of Londrina - Paraná (Brazil). The results presented a great lack of computerization, the use of paper charts, and the need for a computer model of access control that considers the aspects of a real medical-hospital environment. From the results obtained in this research, it was possible to elaborate a computational model of access control to the patient's electronic medical record for health information systems using the UCONabc access control model and the XACML architecture. A prototype of the proposed model was implemented. The experiments conducted and their results demonstrated the proposal's feasibility.
\end{abstract}

\section{INTRODUCTION}

C OMPUTATIONAL advancement allows known technologies to be enhanced and is used in different ways in computing environments, especially in cloud computing. However, cloud computing brings new concerns, and new paradigms of data management, as well as worries about users that require improvement related to the data access.

Since the 1990s, health information systems have been developed to provide medical records in distributed environments and cloud computing. Nevertheless, due to concerns to the fact that only authorized people should have access to the system, certain security requirements were required, such as authenticity, non-repudiation, integrity, confidentiality, availability, and especially access control.

Research into health information systems in the cloud has been intensified in the pursuit of technologies that can consider not only the attributes of those involved in the information access but also the characteristics of the environmental context of a specific system.

According to Abowd [1], context is any information that can be used to characterize an entity. The entity can be the representation of a person, place, object or physical state that can be considered relevant in the interaction between the user and the system.
In general, health studies are based on the information contained in the current legislation and do not consider real health environments as Basic Healthcare Units - BHUs and Hospitals. Thus, there is a gap between proposals for health information systems and the current reality.

In this work, a qualitative research approach was used to investigate the real health environment and, under the circumstances, to serve as a basis for the development of a conceptual model that meets the demands of the server's access to the patient's medical records on the health environment. However, since the objective of this article is not to show a qualitative approach, these results can be appreciated in another opportunity.

The objective of this work is to propose a computational model of continuous access control for health information systems, which considers contextual information and can be introduced in large scale environments.

This article is structured in 5 sections. Section 2 presents the concepts of health information systems; Section 3 describes the access control model proposed in this work, who is based on the results obtained through bibliographical and qualitative research; Section 4 encompass the evaluation and results of the access control model proposed in this paper; And, finally, Section 5 concludes this work.

\section{HoSPITAL INFORMATION SYSTEMS}

According to [2], the advancement of technology and its increasingly presence in the patient care process has increased the demand for collaborative sharing of patients' clinical data among health professionals. In 1997, the Institute of Medicine defined the Electronic Medical Record (EMR) as

\section{An electronic record that resides in a system specif- ically designed to support users through availabil- ity of complete and accurate data, practitioner re- minders and alerts, clinical decision support sys- tems, links to bodies of medical knowledge, and other aids.}

The world-renowned architecture of a healthcare and hospital environments includes the concepts of Personal Health Record - PHR, Electronic Medical Record - EMR, and Electronic Health Record - EHR and Picture Archiving and Communication Systems - PACS. The most widespread architectures worldwide are user centric. Thus, all access must have 
authorization from the owner user. In the Healthcare Cloud [3] software the user has autonomy to control the accesses to its electronic medical record through the mechanisms of control of access.

In Brazil, there is no system pattern, only a guideline of the Conselho Federal de Medicina (CFM, Portuguese for "Federal Council of Medicine"). The CFM Resolution No. 1,331/89, Ordinances No. 1,638/2002 and No. 1,639 / 2002, informs how the temporality, custody, and handling of the medical records have to be processed, as well as how the computerized systems have to be used. These Brazilian details allow the exploration and research in the health area aiming the computer vision of the access control area.

\section{A USAGE CONTROL MODEL FOR HOSPITAL INFORMATION}

The objective of this proposal is to elaborate a model that allows the continuous access control to medical information and considers external information (such as data of the entities context and the identities of the subjects and objects involved) besides considering the results of the qualitative research.

Studies starting from the reality of the subject through a previous qualitative research, involving the concepts of continuous control of access, using the contextual information of the environment, and applying this information to the processing of rules policies were not found in the area of access control and cloud computing. This aspect shows the relevance of this research not only in the conceptual aspect but also in the real applicability of the model.

In the next sections, the necessary requirements for the development of the proposed model will be discussed as well as the related works, the technologies involved, and the architecture of the model and its interaction form.

\section{A. Requirements analysis}

The objective of the qualitative research was to understand how the Basic Healthcare Units - BHUs control the access to patients' medical records, to verify the occurrence of the use of the medical record in paper and in digital format and to compare the reality practiced with the current legislation. Thus, through its results, serve as a basis for the development of a computer model of access control related to the real aspects of the health environments.

According to the results of the qualitative research, contextsensitive health information, such as patient address, blood pressure, and location of the care service, is essential for the proposed model. Interview reports, such as "Medical records are taken from the storage room. Particularly in medical visits", emphasize the importance of conducting qualitative research and the application of contextual information in the development of the model.

The contextual information identified by the qualitative research is processed along with other information through the rules policies in the proposed model. For example, if a patient does not reside in the BHU coverage area he will not have home care, the patient will have to request the service from the BHU of its area.

The health environments present several characteristics identified through the qualitative research that can be translated to the proposed model, for example: Subject and its attributes - the one that requests access to the available resource in the system; Object and its attributes - the resource available in the system in this model represented as the patient's medical record; Rights - the actions that the subject can execute on the object; Obligations - characterizes what the subject must do; Conditions - defines the characteristics to be respected related to the system environment; And authorizations - the policies that represent the rules that will be processed to determine whether or not the subject can access the object.

Because of the results of the qualitative research, it was verified that during the process of the patient care and access to its medical records, there was a continuous behavior: The patient's medical record was constantly used by health servers and, in some cases, shared with others workers to clarify cases.

From this result, the concept of continuity and delegation was applied in the proposed model. Thus, the proposed model was able to consider the continuous accesses to patients' medical records and also to provide the possibility of having several health professionals acting in the same clinical case through the delegation of access.

Continuous accesses of patients' medical records were associated with a constant change in patient characteristics (defined as contextual information) and should also be considered in the proposed model. The contextual information covers, for example, blood pressure, service location and health status.

To encompass the behavior identified by the results of the qualitative research, the concept of mutability was applied. Hence, the changes occurred during the patient care can be considered in the proposed model.

As an example, one of the situations considered in the model tests was the authorization request to access the patient medical records within a BHU. This request is referred to the moment the patient and the nurse are in the anamnesis room. The nursing auxiliary needs the access to the patient's medical record for a period of time to take the pertinent notes, so the auxiliary requests authorization.

The model considers for this authorization request: the subject and its attributes, the object and its attributes, rights, obligations, conditions, contextual information, and authorization request. Combining all this information, the model does the processing and issues the access authorization response.

\section{B. Technologies involved}

After a bibliographic study, the technologies considered essential for the success of the proposal were selected for the development of the suggested model. The technologies chosen are described in the following sections.

1) Usage Control - UCONabc: In the proposed model the concepts of continuous access control to medical information are essential because it is necessary to consider the dynamicity 
of real health environments. The UCONabc technology application has in its specification this behavior and has proved to be efficient.

The UCONabc is a Usage Control Model proposed in [4] that has as objective the definition of rigorous theoretical concepts for Usage Control in which it considers the dynamicity of the actions occurred during the use of the system resources. This model contains the concepts relevant to current computational applications and introduces in its architecture the concepts of Subject (S), Subject Attributes (ATT(S)), Objects $(\mathrm{O})$ and Object Attributes (ATT(O)), Authorizations (A), Rights (R), Obligations (B), Conditions (C), as well as the concepts of mutability and continuity.

The application of UCONabc allows the proposed model to use information related to the subject and object in a wider and more dynamic way. In addition, it allows the model to apply obligations and conditions to the subjects in the environments and control them from their rights and authorizations. Health environments are active, shared and in constant change, therefore it is important to consider the changes that are occurring in this environment as well as ensuring its continuity and delegation.

2) Context Aware Computing: During qualitative research, health environments presented quality contextual information, such as current location and blood pressure. Due to the relevance of this information they were applied in the model. Using the Context-Aware Computing technology it is possible to apply this information to the model.

The development of context-aware information systems brings new challenges. According to [5], the context architecture is the basis for the abstraction of a context application. In information system that uses context, it is necessary to obtain relevant environment information that has effects on the interaction of the user with the system. Contextual information is constantly obtained from the environment while using the system.

Context-Aware Computing is an essential factor in the proposed model. There are significant contextual characteristics in the UBS environment that were identified in the qualitative research, such as physical status of the patient and physician's current location. This type of information is very relevant and can be used to control access.

3) Extensible Access Control Markup Language - XACML: $\mathrm{XACML[6]} \mathrm{is} \mathrm{a} \mathrm{markup} \mathrm{language,} \mathrm{an} \mathrm{XML} \mathrm{extension} \mathrm{(eX-}$ tensible Markup Language) that allows the modeling, storage, and distribution of descriptive Usage Control policies across entire systems, specific resources, proprietary or public environments. In this language, the policies are defined to provide forms of requests and responses to Usage Control and authorization. Because of this standard, it is possible to control access and authorize the use of certain system contents.

According to [6], the XACML architecture is composed of: Access Requester, Policy Enforcement Point - PEP, Obligations Service, Context Handler, Policy Decision Point - PDP, Policy Administration Point - PAP, Policy Information Point - PIP e Environment. XACML is an XML-based request- response language that, after processing requests and rules, evaluates it to "permit", "deny", "indeterminate" or "notapplicable".

\section{Related work}

The proposed model presented in this paper was developed based on theoretical studies and from the results of a qualitative research in order to follow the reality of the healthcare environment. The related works described in this section are linked to this paper because they partially apply the technologies used here.

Anastasi in his work [7] presents a federated cloud access control framework based on the UCONabc model and on the XACML language. The framework extends the XACML technology and also proposes a way to manage the authorization session thus creating a control of continuity or revocation of access to the federated cloud environment.

In his study, Almutairi[8] applies the concepts of the $\mathrm{UCONabc}$ model and the contextual information to provide continuity of access to changes in the environment. The suggested model called CA-UCON uses contextual information in conjunction with UCONabc concepts to identify changes that occur during access.

The related works presented the development of models from the UCONabc model, contextual information, and processing with the XACML language. However, in the references cited and in the literature, it was not identified works that have proposed continuous access control models for health information systems that applied the concepts of the UCONabc model, obtained contextual information of the environment in a constant way and made the access session management. Also, no work found applied to real health environments on a large scale, considered the results of the qualitative research, or that was applied through of XACML.

\section{Architecture}

The proposed model applies the concepts of the UCONabc Usage Control Model mentioned in section III-B1 as well as the concepts of continuity or revocation of access mentioned in the work of Anastasi [7]. In addition, it applies the concept of Context-Aware Computing discussed in section III-B2 and the application of the results of the qualitative research. The model uses the XACML markup language, cited in III-B3, as a policy processing language. Thus, the attributes, the characteristics of the environment, the context information of the health environment, the attributes mutability, the continuity and the delegation of access are considered.

The model considers that the subjects requesting access to the object are already authenticated in the system. The architecture is concerned about the authorization process.

The model architecture, as can be seen in figure 1 , is structured through several components with specific actions. There are nine components: (i) Subjects and Attributes of the subjects: The subjects (user and/or system) are the entities that want to have access to the objects. The attributes represent the characteristics of the subjects. Examples of 


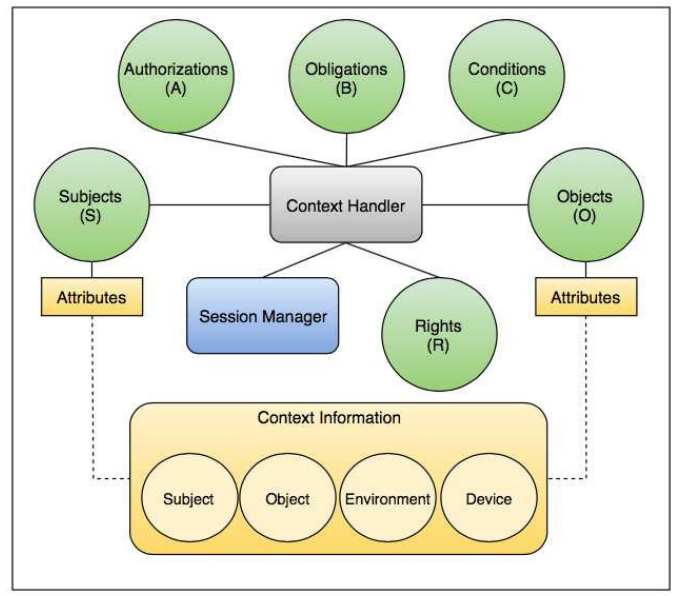

Fig. 1. Architecture of the model.

this component are name, address, and location; (ii) Objects and Attributes of objects: The objects (patient's electronic medical record) are the entities that the subjects want to access for a certain period of time. Attributes represent the characteristics of objects. Some examples are: ID, date, and location; (iii) Rights: Rights are the entities that represent the privileges that a certain subject can obtain during access to the object in a certain environment. Some examples are: the patient can be vaccinated in the health environment; (iv) Authorizations: Authorizations are the entities that represent the location of all the policies that will be processed are stored. The policy processing (containing the rules) results in the access authorization; (v) Obligations: Obligations are the entities that represent the obligations and/or requirements that must be fulfilled before or during the use of the authorization; (vi) Conditions: The conditions are the entities that represent the factors related to the environment and that are relevant to the authorization process; (vii) Contextual information: Contextual information is the entities that represent the attainment and updating of the relevant contextual information used in the authorization process. Any contextual information applied to the model is obtained and updated by that entity. The contextual information used is obtained and updated by this entity in parallel to the policy processing; (viii) Context Handler: Module responsible for integrating the entire model. It interacts with each architecture module in order to manage the operation of requests and responses; (ix) Session manager: The session manager is the entity that represents the control of the access period of the subject to the object. With this entity, it is possible to define the subject access time and the renewal (continuity) of its access or revocation. The proposed model reflects the application of the defined concepts and also the real environment of the UBSs.

\section{E. Interaction Model}

Based on the UCONabc model, a context-sensitive access control software was developed in health information systems for the computational environment.

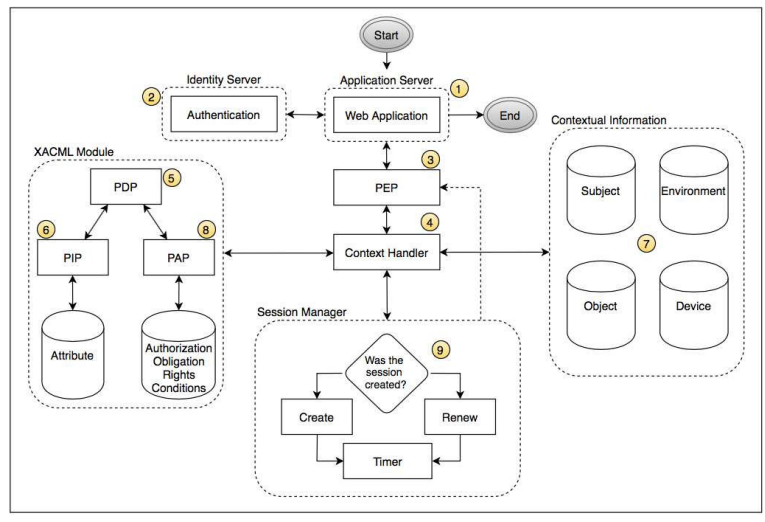

Fig. 2. Interaction of the modules.

As described in the figure 2, the prototype was implemented with the following modules: Application Server (1), Identity Server (2), PEP (3), Context Handler (4), PDP (5), PIP (6), Context Information (7), PAP (8) and Session Manager (9).

The interaction between the modules follows the order: Step 1. The web application receives the authentication request of the subject (containing login and password) through the web application and forwards the obtained data to the authentication module; Step 2. The authentication module checks the credentials of the subject and sends the authentication process response to the web application. If there is any problem with the authentication process, the user is refused in the web application and there will be no authorization request for access to the patient's electronic medical record; Step 3. After successful authentication, the Policy Enforcement Point - PEP module intercepts the subject's authorization request in its native language and converts it to XACML language. After the conversion, the PEP sends a request to the Context Handler; Step 4. The Context Handler receives a request in XACML language sent by the PEP. It is required to provide a response to the PEP. The response can be: "permit", "deny", "not applicable" or "indeterminate". To obtain a response, the Context Handler needs to request the processing of the policies that are associated with the request received. Thus, the Context Handler sends to the Policy Decision Point - PDP the XACML language request to be processed; Step 5. The Policy Decision Point - PDP receives the request processing of the Context Handler request. Then, the PDP requests the Policy Information Point - PIP the attributes related to the request received. The attributes refer to the subject, object, environment, and device; Step 6. PIP searches the stored attributes on its basis. The attributes not found are requested for the Context Information module, through the Context Handler. After receiving the requested attributes, PIP sends them to the PDP; Step 7. Contextual Information represents a module that acts in parallel with the model. This module is constantly obtaining the relevant contextual information through the environment sensors. A recording is performed in the corresponding database from the contextual information 
obtained; Step 8. After all necessary attributes were received from the PIP, the PDP requests to the Policy Administration Point - PAP the policies regarding the software environment. Then, the PAP searches the pertinent policies (authorizations, obligations, rights, and conditions) to the current requisition and sends them to the PDP to process. The PDP receives the policies of the PAP and does the processing of the policies. The result is sent to the Context Handler in XACML format; Step 9. The Context Handler receives the response from the PDP and checks it. If the response is "permit", it prompts to the Session Manager to create or renew the session for the last request. The Session Manager receives the request from the Context Handler to create or renew the session of the subject. Initially, it is checked if the session requested by the Context Handler has already been created (in the cases of continuity of access). If there is no session regarding the subject of the active request, then a session is created and its timer activated. If the session is active, it is refreshed and the timer restarts. When the subject's use time expires, the Session Manager sends a renewal request to the PEP (continuity process). From this request, all procedures for authorization requests are executed again; Final step. After the steps described above, the Context Handler receives the message from the Session Manager that the section was created. Next, the Context Handler sends to the PEP the response of the authorization request in XACML language. PEP converts the XACML language authorization result received from the Context Handler into native language and sends the result to the web application. Consequently to "permit" response, the web application authorizes the subject to access the electronic medical record of the patient.

The delegating access process is done by a user who is using the system. In other words, its access request has already been authorized. This user sends an access request to the system and as a result, a certain employee can access the medical record that the first user is accessing at that moment. This request follows the same steps described above.

\section{EVALUATION OF THE PROPOSED MODEL}

The objective of this section is to describe the implemented prototype that represents the model proposed in this work as well as its characteristics and results obtained.

\section{A. Prototype implemented}

For the evaluation of the model, a MacOS Sierra 10.12.3 (3.4 GHz Intel Core i5 processor, memory module holding 16GB RAM and $395 \mathrm{~Gb}$ Hard Drive capacity) was set as the server machine. The programming language used was Java SE 1.8.0 version and the free software framework WSO2 Application Server version 5.3.0 (WSO2 AS 5.3.0) as a web application server. The free software framework WSo2 Identity Server 5.3.0 was utilized as the authentication server. And, finally, MySQL 5.6.2 version 14.14 was used as a database and the Apache Axis2 module was chosen as SOAP message interceptor.

The proposed model was developed through Java SE language oriented to object with the Model View Controller -
MVC structure integrated with Java Server Pages - JSP, a total of 1,960 lines of code. The free software framework WSO2 Application Server is used to integrate web technologies such as Apache TomCat, Web Services (Apache Axis2), RESTful, cluster and log extensions. The framework makes it possible to host and manage web services and the integration with WSO2 frameworks. It was responsible for hosting the developed prototype as a web service.

The WSO2 Identity Server software framework is an identity manager. The LDAP is used as a native module to authenticate users. It also has the XACML engine for versions 1.0, 2.0 and 3.0 in order to process the rules. This framework was responsible for authenticating users and processing the rules defined in the proposed model. The Apache Axis2 module was used as the request interceptor. This module acts as the PEP in the XACML structure.

The MySQL database was used to store all the contextual information obtained from the environment. It has been integrated into the WSO2 Identity Server as an external data source. Therefore, the developed prototype was hosted on the WSO2 Application Server which was integrated with the WSO2 Identity Server to authenticate users and process the rules.

\section{B. Conducted Experiments}

The conducted experiments using the developed prototype were a load test. This test aims to overload the web service to observe its behavior from a certain volume of data. The volume measured in the prototype is from the access requisitions. For each test, all the services involved (the web server and the XACML authentication and processing server) were restarted. The restart of services avoids server caching and performance problems that occurred after long periods.

For the simultaneous execution of access requests, the free software Apache JMeter version 3.1 was used. The software was configured for each test with the variation of users between $8,16,32,64,128$ and 256 , and variation of connections per second between $8,16,26,41,55$ and 64 . The rules configuration was defined on the WSO2 Identity Server with XACML rule processing. The combinatorial algorithm used to execute the rules was deny-unless-permit. This rule forces the processing of all active rules in the system to check the permit or deny condition. The rules were varied between 8,16 and 32 users. The attributes were configured in the external database MySQL. The attributes varied between 8, 16 and 32 users as well. Initially, for configuration and adjustment of the tool, 50,000 tests were generated to verify the system stability.

Thus, for each test, we defined an attribute value, rules value and the variation of users with the variation of connections per second. For example, the first test was performed with 8 attributes, 8 rules, with the variation of $8,16,32,64,128$ and 256 users combined with the variation of connections per second of $8,16,26,41,55$ and 64. Each user value corresponded to a value of connections per second, such as: 8 users with 8 requests per second, 16 users with 16 requests per second, 32 users with 26 requests per second, 64 users with 41 
requests per second, 128 users with 55 requests per second, and 256 users with 64 requests per second. The next test was done with 8 attributes, 16 rules, and the same variation of users and requisitions per second. The tests were then successively conducted up to 32 attributes and 32 rules. In total, 4,536 executions were conducted.

\section{Results and discussion}

The results obtained with the configuration of the environment and experiments performed show the viability of the proposed model. These results had the objective of evaluating the prototype performance.

The figure 3 (a) shows the response time as a function of the requisition rate per second. It is verified that the lowest response value for the requests occurs when there are 8 attributes and 8 rules, whereas, when there are 8 attributes and 32 rules the response time is higher. This result shows that the variation of rules directly impacts the results. The same behavior is indicated in tests with 32 attributes and 8 rules compared to 32 attributes and 32 rules.

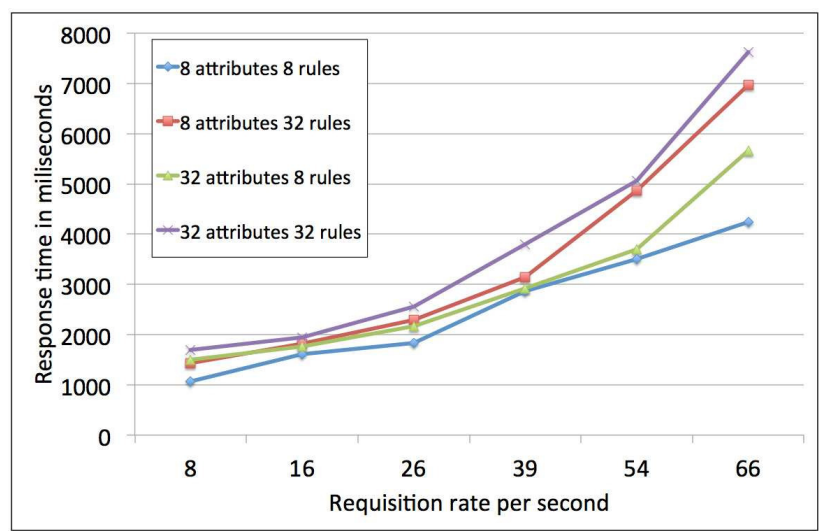

(a) Response time according to the requisition rate per second.

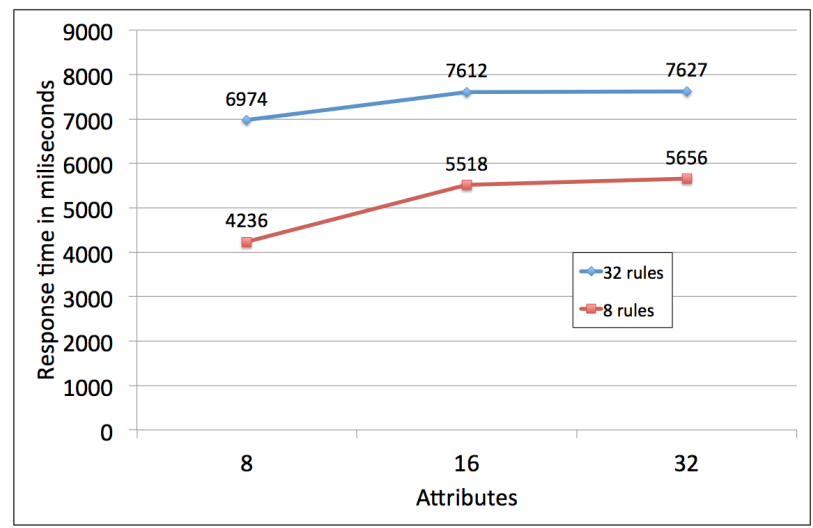

(b) Response time according to the number of attributes.

Fig. 3. Response time.

When a better case, the smallest number of attributes (8) and the lower number of rules (32), is observed and compared with the worst case, the highest number of attributes (32) and the biggest number of rules (32), there is also an increase in response time for requests.

This behavior was expected as a prototype performance because the prototype needs to process a larger number of rules and more requisitions per second, leading to an increase in the response time for each request.

The figure 3 (b) shows the performance of the response time in function of the variable attributes and rules. As the attributes increase, the response time also gradually increases. However, after 16 attributes, it turns out that the response time increases more slowly, representing a better performance in the search time in the database, since the attributes need to be collected in the external MySQL database.

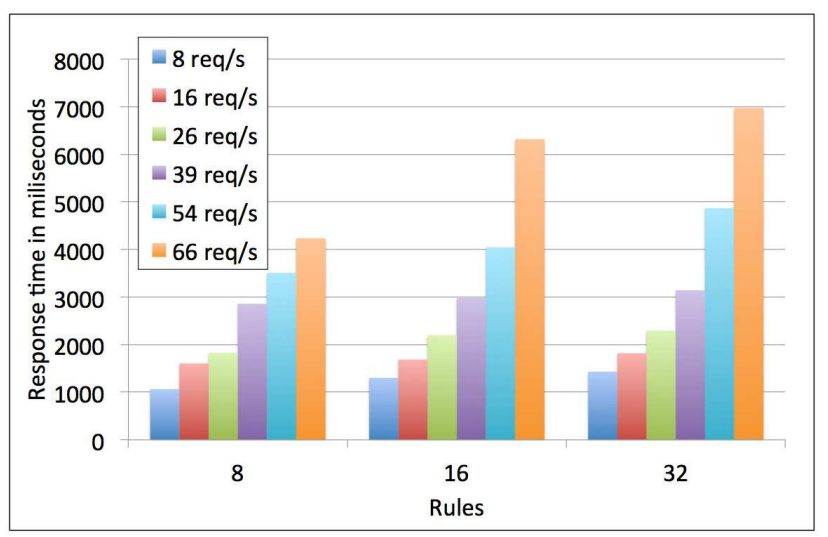

(a) Relation between rules and requisitions per second - 8 attributes.

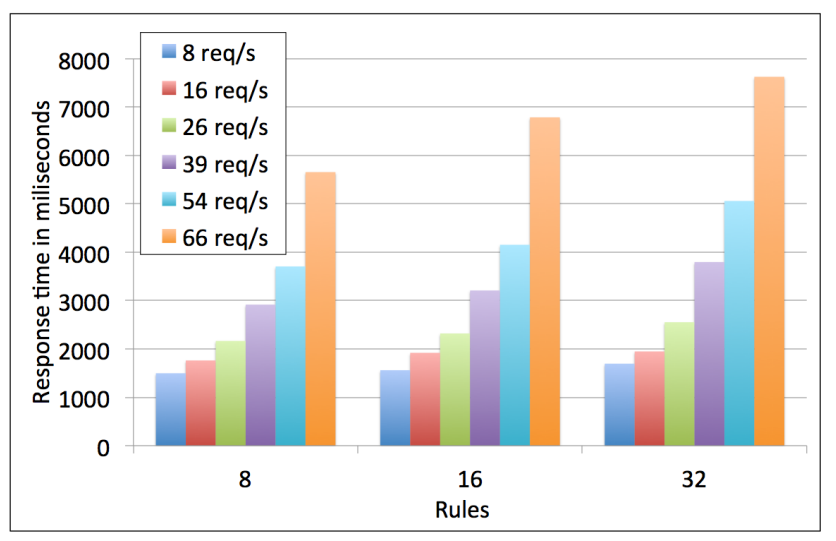

(b) Relation between rules and requisitions per second - 32 attributes.

Fig. 4. Rules in relation to 8 and 32 attributes.

Figures 4 (a) and (b) represent the response time with the relationship between the increase in the number of rules and the rates of requests per second. The chart confirms the expected prototype behavior. As more rule processing and requisitions per second are required, there is an increase in response time for each request.

\section{CONCLUSION}

The qualitative research allowed the proposal to be closer to the reality of the user. The UCONabc model granted through its concepts the development of a strict control of 
continuous access to the health environments that have several characteristics. The use of contextual information was essential to obtain from the environment the relevant data considered in decision making. The results obtained demonstrated good performance when simulating situations close to reality with several requisitions per second. We have not found any work in the literature with a proposal equivalent to the one presented in this paper. Future research opportunities include studying the impact of the model on higher requests rates per second and evaluating the prototype with more complex rules in a more realistic scenario.

\section{REFERENCES}

[1] G. D. Abowd, A. K. Dey, P. J. Brown, N. Davies, M. Smith and P. Steggles, "Towards a better understanding of context and context-awareness," in Proceedings of the 1st International Symposium on Handheld and Ubiquitous Computing, ser. HUC '99. London, UK, UK: Springer-Verlag, 1999, pp. 304-307. [Online]. Available: http://dl.acm.org/citation.cfm?id=647985.743843

[2] J. a. Filho, M. Figueiredo, D. Santos, D. A. Pizzol, L. C. D. Medeiros, A. Fernanda, F. Bezerra, G. Henrique, and M. Bezerra, "Infraestrutura de segurança para comunicação , autenticação e autorização transparentes em hospitais federados (in Portuguese), Journal of Health Informatics, vol. 3, no. 2, pp. 58-63, 2011

[3] R. Zhang and L. Liu, "Security models and requirements for healthcare application clouds," Proceedings - 2010 IEEE 3rd International Conference on Cloud Computing, CLOUD 2010, pp. 268-275, 2010.

[4] J. Park and R. Sandhu, "The UCON ABC Usage Control Model," ACM Trans. Inf. Syst. Secur, vol. 7, no. 1, pp. 128-174, Feb. 2004. [Online]. Available: http://doi.acm.org/10.1145/984334.984339

[5] A. Dey, G. Abowd, and D. Salber, "A Conceptual Framework and a Toolkit for Supporting the Rapid Prototyping of Context-Aware Applications," Human-Computer Interaction, vol. 16, no. 2, pp. 97-166, 2001.

[6] D. Ferraiolo, R. Chandramouli, R. Kuhn, and V. Hu, "Extensible access control markup language (xacml) and next generation access control (ngac)," in Proceedings of the 2016 ACM International Workshop on Attribute Based Access Control, ser. ABAC '16. New York, NY, USA: ACM, 2016, pp. 13-24. [Online]. Available: http://doi.acm.org/10.1145/2875491.2875496

[7] G. F. Anastasi, E. Carlini, M. Coppola, P. Dazzi, A. Lazouski, F. Martinelli, G. Mancini, and P. Mori, "Usage Control in Cloud Federations," 2014 IEEE International Conference on Cloud Engineering, pp. 141-146, Mar. 2014. [Online]. Available: http://ieeexplore.ieee.org/ lpdocs/epic03/wrapper.htm?arnumber $=6903467$

[8] A. Almutairi and F. Siewe, "Ca-ucon: A context-aware usage control model," in Proceedings of the 5th ACM International Workshop on Context-Awareness for Self-Managing Systems, ser. CASEMANS '11. New York, NY, USA: ACM, 2011, pp. 38-43. [Online]. Available: http://doi.acm.org/10.1145/2036146.2036153 



\section{MULTIMODAL BIOMETRIC SYSTEM FOR IDENTITY VERIFICATION BASED ON HAND GEOMETRY AND HAND PALM'S VEINS}

\author{
Luiz Eduardo de Christo \\ Universidade Federal do Paraná \\ Centro Politécnico, Av Cel Francisco H dos \\ Santos 81530-900 Curitiba, Brasil \\ Email: dudu.christo@gmail.com
}

\author{
Alessandro Zimmer \\ Universidade Federal do Paraná \\ Centro Politécnico, Av Cel Francisco H dos \\ Santos 81530-900 Curitiba, Brasil \\ Email: alessandro.zimmer@gmail.com
}

\begin{abstract}
This project was developed with the aim to implement a multibiometric system capable of handling hand palm images acquired using a touchless approach. This considerably increases the difficulty of the image processing task due to the fact that the images from the same person may vary significantly depending on the relative position of the hand regarding the sensor. A modular software tool was developed, providing the user a method for each of these steps: initial image preparation, the feature extraction, processing and fusion, ending with the classification, thus making the researcher's task much easier and faster. The biometric features used for identification include hand geometry features as well as palm vein textures. For the hand geometry data, an algorithm for determining finger tips and hand valleys was proposed and from there was possible to extract a handful of other features related to the geometry of the hand. The handpalm veins' texture features were extracted from a rectangle generated based on the hand's center of mass. The texture descriptor chosen was the Histogram of Gradients. In possession with all the biometric data, the fusion was done on feature level. Support Vector Machine technique was used for the classification. The database chosen for the development of this project was the CASIA Multi-Spectral Palmprint Image Database V1.0. The images used corresponds to the $940 \mathrm{~nm}$ spectrum due to allowing the visualization of the hand palm's veins. The achieved result for the hand geometry was an EER of $4.77 \%$, for the palm veins an EER of $3.11 \%$ and changing the threshold value a FAR of $0.50 \%$ and a FRR of $4.82 \%$ were achieved. For the fusion of both biometric systems the final result was an EER of $2.33 \%$ with a FAR of $1.30 \%$ and a FRR of $4.27 \%$.

Keywords: biometry, hand geometry, palm veins, multimodal biometry, biometric system, support vector machines, histogram of gradients.
\end{abstract}

\section{Introduction}

The development of technologies to store and handle data in the digital world created the necessity for new mechanisms responsible for ensuring the safety and access controls for such operations. The most common mechanisms are the use of passwords and tokens.
However, these mechanisms have proven insufficient to ensure the integrity of the user authentication process, either because the user doesn't follow a good security policy or because of the evolution of the hacking techniques. This raises the need for more secure authentication systems to protect the access to the data or the environment that contains it. It is possible to increase the security of the authentication process using biometry. Biometry is the science of establishing the identity of a person based on his physical, chemical or behavioral attributes [1]. A biometric system is defined as a pattern recognition system that operates acquiring biometric data of a person, extracting a set of characteristics and comparing them with a model in a database [1]. As these data are unique and inherent to the person, the task of hacking a system with biometric security proves itself difficult.

Some of the most recent work in hands multimodal biometric systems makes use of geometric attributes combined with tridimensional hand attributes as proposed by KANHANGAD[25] and the work of PARK and KIM [26] based on geometric attributes plus hand veins patterns.

\section{Database}

The image database CASIA-MS-Palmprint V1.0 [2] provided by the Institute of Automation, Chinese Academy of Sciences (CASIA). The database contains 7.200 images, acquired with a multispectral device. The resolution of the images is $72 \mathrm{dpi}$ with 8 bits of grayscale levels and in JPEG format. Each one of the samples of this database has 6 images for both hands, for each one of the following electromagnetic spectrum: $460 \mathrm{~nm}, 630 \mathrm{~nm}, 700 \mathrm{~nm}, 850 \mathrm{~nm}, 940 \mathrm{~nm}$ and white light. Fact that makes possible the extraction of one set of characteristics used in this project: the hand palm's veins patterns, acquired with the $940 \mathrm{~nm}$ spectrum of the right hand only.

Working with touchless hand image acquisition devices is more comfortable for the user but by the other hand considerably increases the difficulty to extract the features compared to a hand fixed (such as pins) device. This is due to the fact that the variations for the same person can be significant as we may have distance, inclination and light variations, thus making it difficult to normalize the data and even leading to problems before this such as not being able to apply filters like 
image segmentation. Figure 1 depicts examples of bad positioned images.
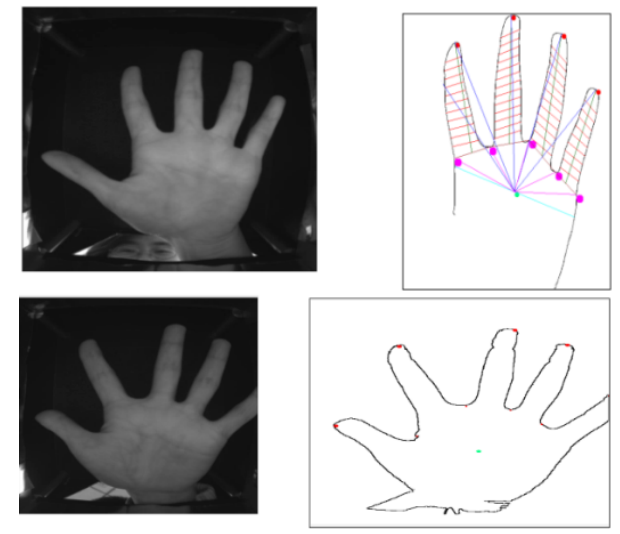

Fig. 1 - Challenges on processing images acquired through touchless devices.

Distance variations can be corrected applying a normalization method (like dividing all the attributes for the hand area or hand width). But in extreme cases (as the ones mentioned and illustrated on Figure 1) the image simply can't be further processed and its characteristics extracted. The algorithm implemented for the geometric feature extraction tries to extract the maximum numbers of features possible. If this number is less than an established limit the image is discarded. Four images were discarded in this work.

\section{Extraction of geometry characteristics set}

The very first filter applied in the image was the thresholding. It is a widely used segmentation technique due to its simplicity, intuitive properties and low computational costs [3]. The Otsu [4] algorithm was selected due to the light variations on the images and it was the algorithm that handled this variety in a better way. For the border detection task, the Canny algorithm [5] was the one with best results, hence the one applied. Finally to extract the hand contour a blob filter provided by the AForge.NET image processing framework [6] was used.

After all the proper filters were applied and the contour extracted the next step was to detect the finger tips and the hand valleys. This was achieved with a proposed method that aims to divide the hand contour into 5 distinct regions. Each of these regions is defined by a pair of curves surrounding each one of the fingers. The points related to these curves were stored on a dictionary data structure which the key is a point representing the coordinate of the pixel and the value is the Euclidian distance between this point and the hand's center of mass. The dictionary of each one of the valley regions was ordered in a descending way so that the smallest distance is the center point of the valley and for the finger tips the very opposite logic was used, the points were ordered according to the longest distance from the hand's center of mass. These regions are shown on Figure 2.

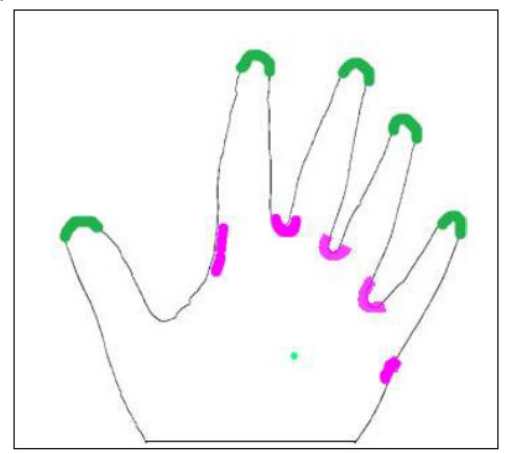

Fig. 2 - Algorithm implemented for the detection of hand valleys and finger tips. Finger tips regions on green color and valleys on pink color.

A total of 80 characteristics were extracted. They include: hand perimeter, hand palm width, distances between the fingers (base lines), Euclidian distance between the hand's center of mass and the finger's tips, as well each of the finger's valley. And for all the fingers except the thumb: 15 widths (maximum) and finger length. The Figure 3 depicts all these characteristics after the extraction algorithms were processed.

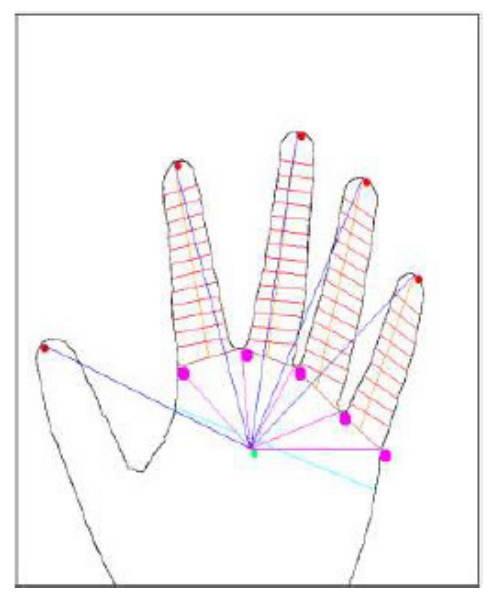

Fig. 3 - All geometric characteristics extracted.

\section{Hand palm's veins}

In order to be possible to process texture, the definition of a Region of Interest (ROI) is necessary. There are plenty of methods for ROI extraction [7] including dynamic ones [8]. In the developed project, the ROI was extracted growing a rectangle from the hand palm's mass center until the maximum possible area could be extracted without extrapolating the hand palm area of all users. The resulting image was a 160x120 pixels rectangle that represents the hand palm's veins pattern as shown in Figure 4. 


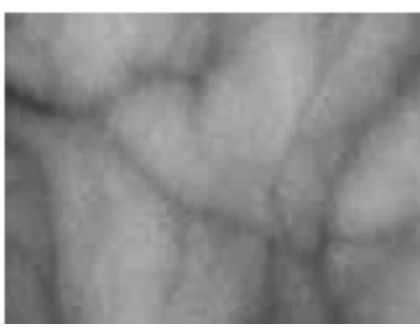

Fig. 4 - ROI of hand palm with its veins pattern.

Then the texture descriptor used was the Histogram of Oriented Gradients (HOG) introduced by DALAL and TRIGGS [9] and has been used for people detection with very good results. The HOG parameters used were $4 \mathrm{x} 4$ cells per block and 2x2 pixels per cell. The blocks and cells are depicted in Figure 5. The total amount of attributes generated for each hand palm image is an array of 3.780 float values for each window.

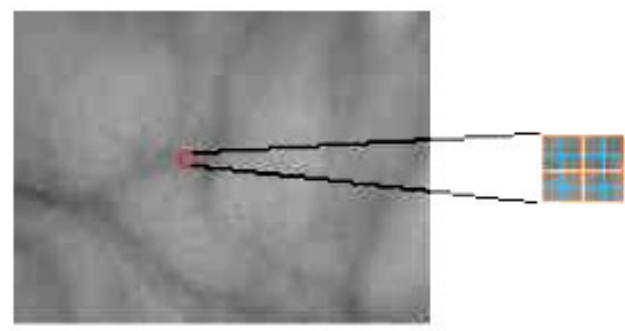

Fig. 5 - Representation of cells and blocks used in HOG texture descriptor algorithm.

On Figure 6 the representation of the veins can be visualized after a HOG texture descriptor is applied.
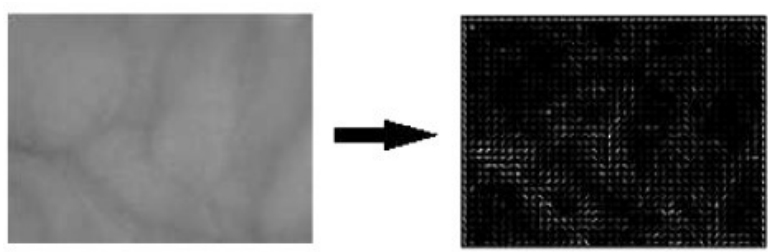

Fig. 6 - HOG representation of veins textures.

\section{Fusion}

The fusion process was done using the attribute level fusion method. As the geometric characteristics diverge in nature and scale from the characteristics acquired from the HOG descriptor before the fusion a normalization technique was applied. Two methods were tested: Z-Score and MinMax. The later was chosen because it achieved better results for machine learning data normalization [10]. The equation of this normalization is described below.
After the data was normalized the fusion process is simple, just concatenate the two output arrays (from geometric attributes and the HOG descriptor output array) into one. For each one of the images a fused array was generated.

\section{Classification}

The Supporting Vectors Machine (SVM) technique was chosen for the task of classification due to its good results regarding biometric patterns recognition. In order to achieve this, the library libSVM [11] was used together with Matlab.

Two distinct groups were created. One for training which had 500 images of $940 \mathrm{~nm}$ from the right hand and the other group used for testing which had 100 images from the total of 600 . This way for each user 5 images were used for training and 1 was used for testing.

Besides the recommended parameters from [11] the $c$ parameter (that defines the size of the margin separating hiperplanes i.e. how much we want to avoid misclassification, the smaller the c parameter value the bigger the separating margin) was changed to 0.001 . Also the kernel used for the current SVM implementation was the Radial Basis Function (RBF) kernel.

\section{Tests}

While all the image processing and attributes fusion were done in the developed system for this project the classification was done solely in Matlab. There were 3 testing groups: geometry, veins and the fusion of both. For each one of them a Receiver Operating Characteristic (ROC) as well as False Acceptance Rate (FAR) x False Rejection Rate (FRR) curves were generated and important quality indicators of biometric systems like Equal Error Rate (EER) were calculated.

\section{BiometricsLAB}

A software was developed with the intention to concentrate all the tasks pertinent to image processing, feature extraction and data fusion in only one place. This will allow future researchers to save time by being able to do all these operations in only one software and to avoid doing those operations in different places. Also, BiometricsLAB was designed keeping in mind its extensibility and reusability so others can add to it new biometrics and new functions. It is reusable because the user can choose to use only parts of it or some of its libraries instead of use the system in its totality. The Figure 7 represents the main screen of BiometricsLAB available at [27].

$$
x_{n o r m}=\frac{X-X_{\min }}{X_{\max }-X_{\min }}
$$




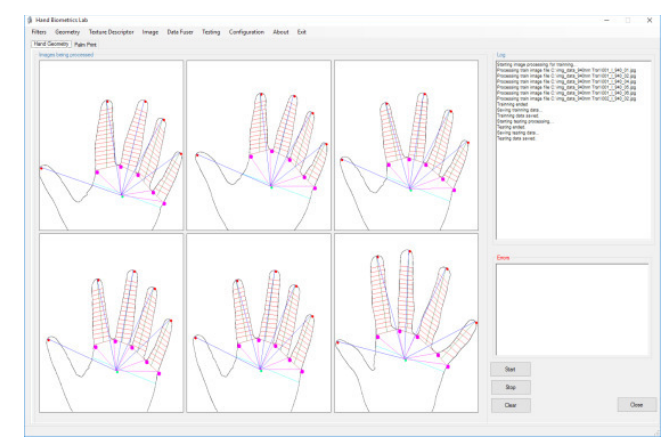

Fig. 7 - The main screen of the developed software: BiometricsLAB showing the processing of geometry hand biometrics.

\section{Results}

The results for the hand geometry experiment can be visualized in Figure 7 and Figure 8.

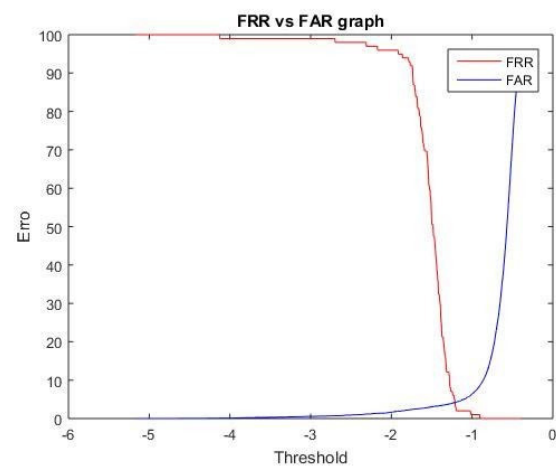

Fig. 8 - FAR x FRR curves for hand geometry biometric system.

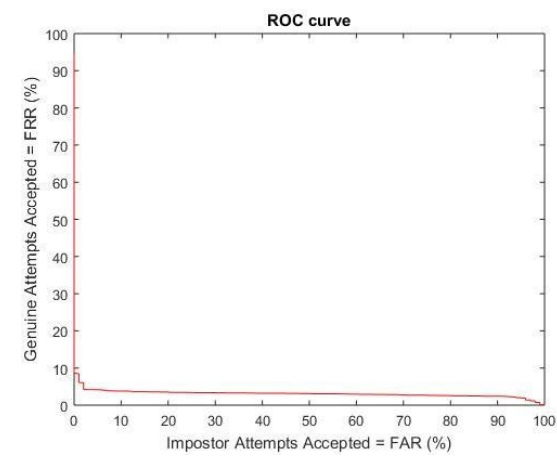

Fig. 8 - ROC curve for hand geometry biometric system.

The EER rate achieved was $4.77 \%$. It's easy to notice that a slight variation on the threshold value it changes the system's performance significantly, thus making it unfeasible to choose security at the expense of a higher reliability.

The second experiment shows a little improvement compared to the first one. The Figures 9 and 10 depicts the performance of the hand palm's vein biometric system.

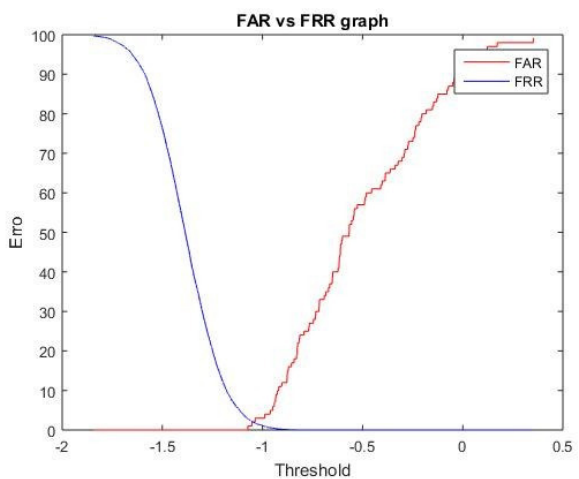

Fig. 9 - FAR x FRR curves for hand palm's veins biometric system.

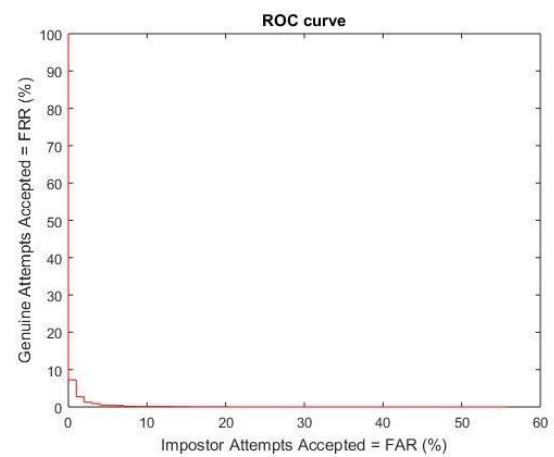

Fig. 10 - ROC curve for hand palm's veins biometric system.

For this experiment an EER rate of $3.11 \%$ was achieved which is clearly an improvement compared to the first one.

In the final experiment the multimodal biometric system proved itself more reliable and secure than both of the options that implemented only one biometry. After the fusion, the consolidated data that was generated and processed by the SVM produced even better results. These results are shown in Figure 11 and Figure 12.

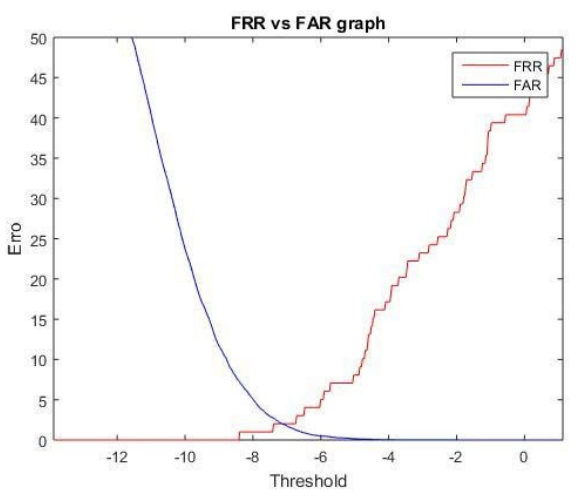


Fig. 11 - FAR x FRR curves for the multimodal biometric system (Geometry + veins).

An EER rate of $2.33 \%$ was reached and can be improved by changing the threshold obtaining this way rates for FAR and FRR of $1.30 \%$ and $4.27 \%$ respectively.

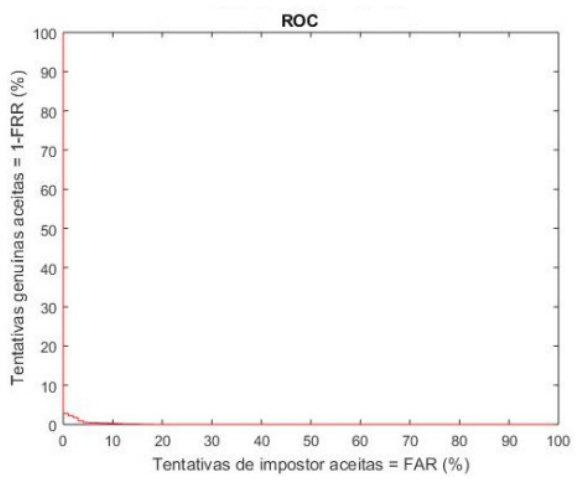

Fig. 12 - ROC curve for the multimodal biometric system (Geometry + veins).

\section{Discussion}

Comparing the obtained results with recent works presented in Table 1, it is possible to notice that there are better results especially regarding the hand geometry.

Here it is important to mention the differences of how the images were acquired. CASIA [2] database makes use of a touchless method for data acquisition which makes it harder to process the feature extraction due to the high level of variance in the images of a same person (distance, angles, light, etc) while systems that make use of a support for this task do not have to deal with these problems.

Table 1: Recent works results

\begin{tabular}{ccc}
\hline Author & Biometry & Succesrate(\%) \\
\hline $\begin{array}{c}\text { Xin et al. } \\
(2011) \text { [12] }\end{array}$ & Geometry & 97.84 \\
\hline $\begin{array}{c}\text { Guo et al. } \\
(2012) \text { [13] }\end{array}$ & Geometry & 96.23 \\
\hline $\begin{array}{c}\text { Lee } \\
\text { (2012) [14] }\end{array}$ & Veins & EER=0.44 \\
\hline $\begin{array}{c}\text { Gangopadhyay } \\
\text { et al. } \\
\text { (2013) [15] }\end{array}$ & Geometry & 96.67 \\
\hline $\begin{array}{c}\text { Singh et al. } \\
\text { (2014) [16] }\end{array}$ & Geometry & 95.84 \\
\hline $\begin{array}{c}\text { Abbas e } \\
\text { George } \\
(2014) \text { [17] }\end{array}$ & Veins & EER $=0.24$ \\
\hline $\begin{array}{c}\text { Wang et al. } \\
(2014) \text { [18] }\end{array}$ & Veins & 98.88 \\
\hline
\end{tabular}

\begin{tabular}{ccc}
\hline $\begin{array}{c}\text { Elnasir e } \\
\text { Shamsuddin } \\
(2014) \text { [19] }\end{array}$ & Veins & EER $=0$ \\
\hline $\begin{array}{c}\text { Kang et al. } \\
(2014) \text { [20] }\end{array}$ & Veins & $\begin{array}{c}\text { EER }=0.996 \text { e } \\
\text { EER }=3.11\end{array}$ \\
\hline $\begin{array}{c}\text { Yan et al. } \\
(2015)[21]\end{array}$ & Veins & EER $=0.16$ \\
\hline
\end{tabular}

Although this work has reached good results there are some points to improve especially regarding the analysis of other types of ROI

\section{Conclusion}

Looking to reduce the possibility of fraud in a system, the use of a biometric system is a viable alternative. By extracting the attributes from more than one source, the system becomes safer and less susceptible to successful hacking attempts, besides other benefits like enabling the inclusion of persons with physical limitations.

One innovation that can be stressed is the proposed method for extracting the finger tips and hand valleys. The algorithm produced very good results for the CASIA database, with $99 \%$ of successful extractions. Not only did it prove itself to be reliable and strong, but it also showed a good performance and low computational cost.

Another point, still regarding the hand geometry biometry, was the fail tolerant algorithm implemented in the feature extraction that allows obtaining the maximum number of features even when the image could not be processed completely. If the quality of the image is too low (due to noise factors) to the point that affects the extraction of canon features (like palm width, finger lengths, finger tips, etc) the algorithm will then discard the image.

The hand palm's veins biometric system was implemented with a different method from the ones used so far, the Histogram of Oriented Gradients, which has been successfully used for people recognition [12]. The HOG texture descriptor obtained good results when used for the veins textures as the results obtained proved.

\section{References}

[1] JAIN, et. al.. Handbook of Biometrics. 1st Edition. Springer, New York, 2008.

[2] CASIA-MS-Palmprint V1. Availableat: http://biometrics.idealtest.org.

[3] GONZALEZ , R. C.; WOODS, R. E. Digital Image Processing, 3rd edition, Prentice-Hall, 2008.

[4] OTSU N. A threshold selection method from graylevel histograms. IEEE Trans. Sys., Man., Cyber, 1979.

[5] CANNY, John. “A Computational Approach to Edge Detection”, IEEE Transaction onPattern Analysis and Machine Intelligence, Vol. 8, pp. 679-98, 1986. 
[6] AForge.NET framework V2.2.5. Available at: http://www.aforgenet com

[7] Kekre H, Sarode T, Vig R. An effectual method for extraction of ROI of palmprints. In: Communication, Information Computing Technology (ICCICT), International Conference on . 2012. P. 1-5.

[8] Kalluri, H. K.; Prasad, M. V. N. K.;Agarwal, A. 2012. Dynamic ROI extraction algorithm for palmprints. In Proceedings of the Third international conference on Advances in Swarm Intelligence - Volume Part II (ICSI'12), Ying Tan, Yuhui Shi, and Zhen Ji (Eds.), Vol. Part II. Springer-Verlag, Berlin, Heidelberg, 217-227.

[9] Triggs B, Dalal N. Histogram of Oriented Gradients for Human Detection. Proceedings of the 2005 IEEE Computer Society Conference on Computer Vision and Pattern Recognition, 2005.

[10] Aksoy, S.; Haralick, R. "Feature normalization and likelihood-based similarity measures for image retrieval," Pattern Recognit. Lett., Special Issue on Image and Video Retrieval, 2000.

[11] Chang, C.-C.; Lin, C.-J. Libsvm: A library for support vector machines. ACM Transactions on Intelligent Systems and Technology, v. 2, p. 27:1-27:27, 2011. Software available at http://www.csie.ntu. edu.tw/ cjlin/libsvm

[12] Xin, Cui; Wu, Xiangqian; Qiushi, Zhao; Youbao, Tang. "A Contactless HandShape Identification System", 3rd International Conference on Advanced Computer Control, pp. 561-565, 2011

[13] Guo, Jing-Ming; Hsia, Chih-Hsein; Liu, Yun-Fu; Yu, Jie-Cyun; Chu, Mei-Hui; Le, Thanh-Nam "Contact-free hand geometry-based identification system”, Expert System with Applications, Vol. 39, No. 14, pp. 11728-11736, 2012

[14] Lee, Jen-Chun. "A novel biometric system based on palm vein image", Pattern Recognition Letters, Vol. 33, No. 12, pp. 1520-1528, 2012.

[15] Gangopadhyay, Ahana; Chatterjee, Oindrila; Chatterjee, Amitava. "Hand shape based biometric authentication system using radon transform and collaborative representation based classification", IEEE Second International Conference on Image Information Processing, pp. 635-639, 2013.

[16] Singh, Aditya P.; Thakur, Ranjan K.; Kumar, Arabind; Baksh, Ram. "User Authentication Using Hand Images", International Journal of Science and Research, Vol. 3, No. 3, pp. 317-322, 2014
[17] Abbas, Asmaa M. J.; George, Loay E. "Palm Vein Recognition and Verification System Using Local Average of Vein Direction", International Journal of Scientific \& Engineering Research, Vol. 5, No. 4, pp. 1026-1033, 2014

[18] Wang, Ran; Wang, Guoyou; Chen, Zhong; Zeng, Zhigang; Wang, Yong. "A palm vein identification system based on Gabor wavelet features", Neural Computing and Applications, Vol. 24, pp. 161-168, 2014.

[19] Elnasir, Selma; Shamsuddin, Siti Mariyam." Palm Vein Recognition based on 2D-Discrete Wavelet Transform and Linear Discrimination Analysis", International Journal of Advances in Soft Computing and its Application, Vol. 6, No. 3, pp. 43-59, 2014

[20] Kang, Wenxiong; LIU, Yang; WU, Qiuxia; YUE, Xishun. "ContactFree Palm-Vein Recognition Based on Local Invariant Features", PLoS ONE, Vol. 9, No. 5, pp. 1- 12, 2014.

[21] Yan, Xuekui; Kang, Wenxiong; Deng, Feiqi; Wu, Qiuxia. "Palm vein recognition based on multi-sampling and feature-level fusion", Neurocomputing, Vol. 151, pp. 798-807, 2015.

[22] Said, Y; Atri M.; Tourki, R. Human Detection Based on Integral Histograms of Oriented Gradients and SVM.Conference: International Conference on Communications, Computing and Control Applications, 2011

[23] Candès, E.; Donoho, D. "Curvelets - a surprisingly effective nonadaptive representation for objects with edges." In: A. Cohen, C. Rabut and L. Schumaker, Editors, Curves and Surface Fitting: Saint-Malo 1999, Vanderbilt University Press, Nashville (2000), pp. 105-120.

[24] Rafiee, J. et al. Feature extraction of forearm EMG signals for prosthetics, Expert Systems with Applications 38, 2011.

[25] Park, G.; Kim, S.; Hand Biometric Recognition Based on Fused Hand Geometry and Vascular Patterns. Sensors, vol. 13, n. 3, pp. 2895-2910, 2013

[26] Park, G.; Kim, S.; Hand Biometric RecognitionBased on Fused Hand Geometry and Vascular Patterns. Sensors, vol. 13, n. 3, pp. 2895-2910, 2013.

[27] BiometricsLab v2 Available at: https://github.com/dudu84/ BiometricsLab-v2 


\section{Load-balanced Integrated Information Security Monitoring System}

\author{
Tomasz Klasa \\ TK Systems Security \\ Email: tklasa@tksystemsecurity.pl \\ West Pomeranian Business School, \\ Szczecin, Poland \\ Email: tklasa@zpsb.pl
}

\author{
Imed El Fray \\ Warsaw University of Life Sciences, Faculty of Applied \\ Informatics and Mathematics, Warsaw, Poland, \\ West Pomeranian University of Technology, \\ Faculty of Computer Science, Szczecin, Poland \\ Email: imed_el_fray@sggw.pl,ielfray@wi.zut.edu.pl
}

\begin{abstract}
Monitoring is the last step of the information security management process. It is intended to evaluate not the state of security itself, but rather the accuracy and quality of prior security evaluation and risk treatment applied. In other words, it is supposed to provide the answer, whether chosen countermeasures and all other decisions based on the security assessment and evaluation results were accurate, proper and sufficient. If during this phase of the security management process, any significant anomaly is found within the system, it means that either one of the accepted 'as is' risks occurred, or that the applied countermeasures did not provide assumed protection in some point of the system. In such a case it is necessary to identify all the areas that require security audit repeat. As information systems grow in complexity, an integrated solution for security monitoring that will prevent system overload caused by monitoring is proposed in this paper.
\end{abstract}

\section{INTRODUCTION}

$\mathrm{O}$ $\mathrm{NE}$ of the final results of the information security audit (or risk analysis) is a list of vulnerabilities that were identified, but not covered sufficiently, causing a necessity for further monitoring of a chosen part of the information system. As a consequence, a list of parameters to follow shall be determined and proper control actions taken. This, however, does not mean that applied monitoring solutions eliminated any risks remaining. This action, or rather plan of activities, will not prevent threat from happening. The most important goal for all those actions it to detect any such anomalies and evaluate their influence on the system, as this says, whether the prior risk evaluation was correct. If a severe anomaly is detected by the monitoring mechanisms, it means that some of the countermeasures applied were unsuccessful in preventing it from happening or at least limiting its scale to some acceptable level. As a consequence, parts of the system, that were touched by that detected anomaly, should be reevaluated, e.g. in a form of the audit repeat.

The problem is, while adding precision to security monitoring, one has to accept quickly rising costs of data acquisition and processing. The more frequently the status of specific elements of the system is to be verified or the more parameters of a similar nature are added to allow more complex and multilevel analysis, the bigger quantity of data must be collected, exchanged and processed within the information system. Because company's assets are always limited, the more of them are assigned to the information security monitoring process, the less are left to provide everyday key services of the organization. This may lead even to limiting basic activity of the organization, due to insufficient efficiency of the system as a whole.

To avoid that, it is necessary to determine, for a given set of monitoring parameters, how to control each of them and how frequently to refresh the information about the state of each of the monitored components of the system. In other words, it is necessary to decide which parameters are more important than others as their status has more significant impact of the state of security of the whole information system, not one of its elements alone. To solve the problem, a model of information security monitoring plan adjustment to known technical and organizational limits was proposed.

\section{RELATED WORK}

Research projects related to security monitoring can be divided into two major directions. One of them focuses on anomaly detection, especially increasing its accuracy, in some chosen security domain or part of the system. As an example, there are works on improvement of IDS/IPS [24], [26] or anomaly detection on the basis of network traffic analysis, e.g. [5], [12], [15]. Even if activity of users and individual system components is analyzed, it is done only on the basis of data gathered from networking appliances and the rest of the infrastructure [21]. As can be easily noted, each of them stands for a part of the image only - they provide the answer whether in some chosen part of the system an anomaly occurred, or not. Because of that, each of them can be seen as an improvement of the quality of the source data gathered from individual system components, but not as a verification of the applied set of risk treatment.

A second major direction of research aims at a definition or a model of a secure system. Often this takes a form of a dedicated security framework used to enforce specific 
scheme of security monitoring, like in [18] or a specific architecture of the whole system as in [8]. A different solution is to use existing classification of threats and vulnerabilities, e.g. for critical infrastructure systems a partition into four groups was proposed, including dedicated risk treatment techniques [13]. One of other attempts is project FORISK, based on a three-step process: once formal requirements and knowledge about the company are collected (phase 1), a formalized risk assessment is undergone (phase 2), and on that basis an automatic or semiautomatic choice of further treatment is performed (phase 3 ). The whole solution was designed to be easy to apply even for a not fully trained management [7]. Division into steps or layers is a common approach. For instance, in [16] a smartgrid system security was modelled with the help of three layers (social, software and hardware) and a set of allowed interactions between them. Another example of multi-layered approach, relies on game theory and impact assessment based on AHP [10]. The model of a secure system can be created also with the help of a specific descriptive notation or language, like SecBPMN [4], which defines expected behavior of the system and policies that can be used in monitoring. The common feature of security frameworks is that they introduce description of the expected form of the system (either its structure, activity, or both), so it can be easily said if there are any deviations from that state. This leads to quite simple anomaly detection and assumption, that if the system is not compliant with the framework definition, it is not secure. This, however, is still insufficient to verify applied set of countermeasures properly as usually there is no reference to the results of the security audit, which should be a basis for all further decisions and applied risk treatment. Thus, it is not clear whether introduced description of the system (framework) is adjusted to requirements that emerged from the results of the security audit, or if it was proposed regardless of risk analysis.

Modelling expected structure and behavior of system components means that a security framework usually does not apply to all security domains or is adjusted to a specific type of the organization. As a consequence, it is necessary to combine various techniques and solutions to address technical and organizational or immaterial layers of the information system at the same time. This causes further difficulties connected with dependencies between solutions (the same asset, e.g. computer network, may be defined in a different way, depending on chosen framework) resulting in unexpected and unnecessary data redundancy or even inconsistency.

Issues with efficiency caused by quantity of gathered and transmitted data are also typical, however this problem is not unique to information security monitoring. A similar issue was a subject of research e.g. in the area of securing supply chain with numerous dependencies between network elements, where system performance was controlled after implementation of treatment perceived as not influencing performance [20]. Amount of transmitted data was an issue also in the case of research under a smart-grid system, where calculations show that petabytes of data must be gathered to analyze the system of a whole country. When compared the number of data sources in that case with a number of individual data sources in a more sophisticated organization, it can be seen that they are quite comparable. Because the size of data transmission depends mostly on the number of unique messages, and it is impossible to limit the number of data sources without decreasing analysis quality, it is necessary to focus on the sampling frequency [1].

Generally, current solutions either focus on some chosen parts of the information system or attempt to model an enforced, secure state of the system. However, little is done to standardize and process the reverse feedback from the system, to identify weaknesses in applied security plan and improve it appropriately. The monitoring plan is usually used to detect problems and counter them instantly with the help of one of the predefined actions, not to induce long-term improvement of the plan itself, e.g. by the replacement of insufficient countermeasures. Current solutions focus on the best accuracy, it comes at a price of high load of the information system, caused by gathering and processing loads of data intended to maximize the level of details. This approach, however, cannot be implemented in many types of organizations, including virtual organizations, as excessive system load may even block basic operation of such an organization, due to limited resources. At the same time, little attention is paid to solutions that adapt to the requirements and limits of the examined organization in terms of operational costs, that may cover multiple functional areas of the organization at the same time.

\section{BACKGROUND}

There are two major elements of the whole integrated approach to security monitoring. First of all, the initial evaluation of information system's security is the basis for all further decisions and choice of countermeasures. Because of that, this step shall be done in a standardized way. Based on the analysis of the issues in widely adapted risk assessment methods, a more formalized, however still flexible, approach was proposed [6].

Another very important prerequisite is data description unification. The whole information system consists of multiple elements that, from the perspective of monitoring, can be treated as individual data sources. As they are incompatible with each other and cannot provide output data in a single, chosen arbitrarily, format, it necessary to convert their output into a chosen scheme, as is usually done in numerous research projects aimed at some specific part or type of the system, e.g. [3], [9], [25], [27]. To address that problem, a general solution of data scheme conversion, based on the meaning of individual data fields, was proposed in [14]. 


\section{INTEGRATED APPROACH TO INFORMATION SECURITY MONITORING}

Verification of adequacy of countermeasures taken on the basis of the results of security audit, that were supposed to guarantee some expected security level of the information system is complex. That evaluation is influenced not only by the fact of occurrence (or not) of some local anomalies on the level of individual system components, but most importantly the influence of such events on proper operation of the organization and its ability to proceed required business processes.

The main source of data for parametrization of the security monitoring system is the security audit report, which includes references to identified business processes and utilized assets, defines known threats and specifies for which of them there are uncovered vulnerabilities of used assets. This document explains therefore, which areas or components of the system should be monitored, to assure assumed security objectives. Elements of the system selected during the planning phase are supposed to periodically provide reports describing recent operational history. Among the data sources there are systems like SIEM, firewalls, other network devices, hardware and software sensors responsible for physical and environmental security, software telemetrics and other organizational solutions allowing governance of the implemented procedures.

The list of the data sources presented above shows clearly that they do not form a unified, common group. Also the way of storing and processing data by individual sources is different. Because of that it is reasonable to divide the system into a number of logical layers (ref. fig.1). The first of them is data source layer, gathering individual system components, the state or activity of which is to be monitored. Each element of this layer can be questioned - as a response to such a response it should reply with a status report.

Because of the diversity of data sources, it is necessary to convert messages, or at least convert metadata describing the data. It can be done before the actual communication with the central repository or after the message is received by the repository. Unification of data format before wrapping them into the message structure provides an opportunity for a more detailed control mechanisms and data verification on repository input.

Once the data description format is converted, and if necessary also the data format itself, the data is ready to be wrapped into the structure of the message, which is done at the level of the communication layer.

Received messages go to the data verification layer, which is responsible for the control of data structure, integrity and source. Positively verified data is then sent to the data archive layer, which is responsible for the repository update.

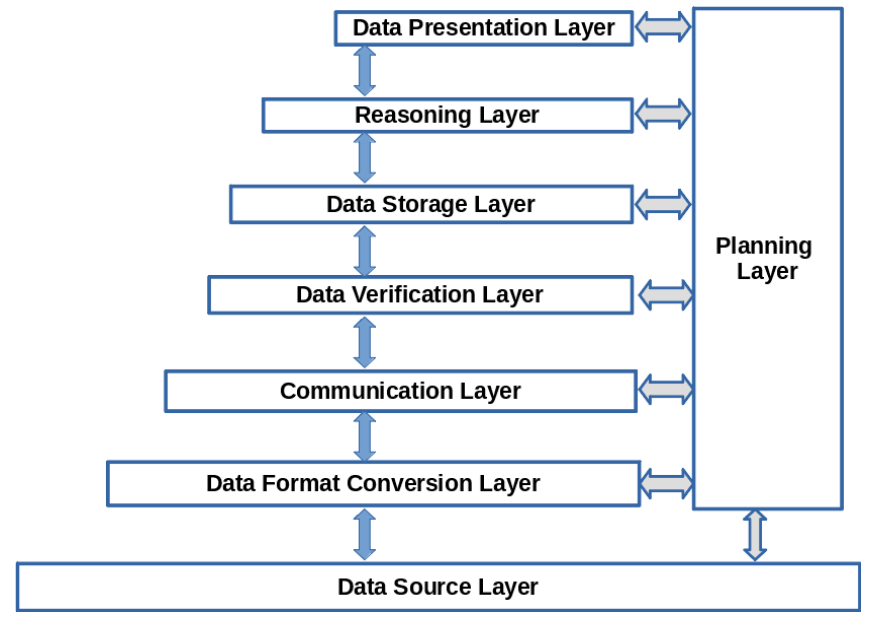

Fig. 1 Multi-layer model of monitoring data integration.

Gathered data are processed and analyzed by the algorithms forming reasoning layer. The result of all operations performed at this stage is an evaluation of effectiveness of implemented countermeasures. the last layer is responsible for mechanisms of results presentation and providing communication between the system and the user.

The only layer which allows communication with any other layer of the system is the planning layer. With its help a functional parametrization is done, as well as technical setup of the components responsible for tasks of the individual layers (e.g. it includes available definitions of data description formats and mappings necessary for their conversion).

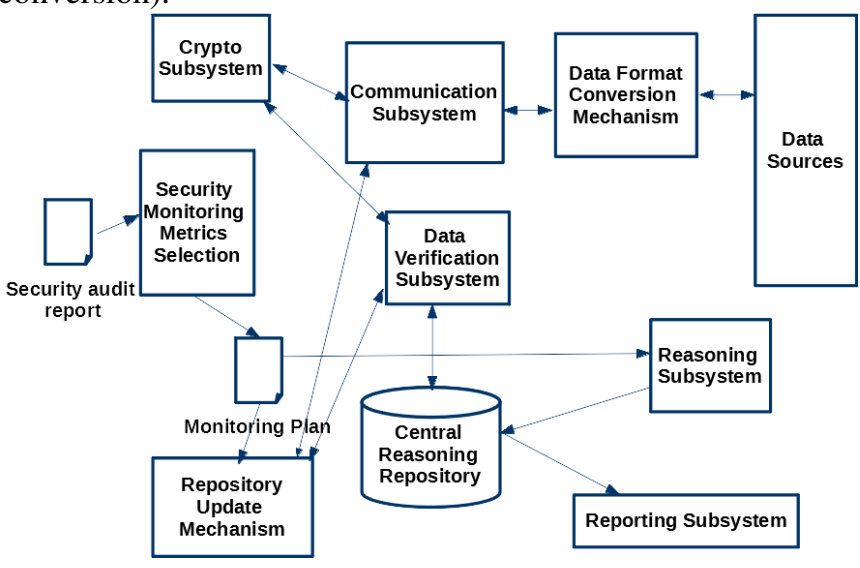

Fig. 2 Security Monitoring Metrics Selection.

This element is a part of the planning layer. Selection of monitoring scope, which means metrics of system components state and means of controlling them, is done on the basis of the security audit results. This provides a Plan of Monitoring, describing which metrics (parameters) should be followed for each of the assets, with the help of which mechanisms and at which frequency their status should be updated at the central repository.

The process consists of two steps. At first, a list of metrics is determined for each of the assets, on the basis of a set of relations described by matrixes, similar to those used by method FoMRA [6]. The results can be shown as an 
assignment matrix $\boldsymbol{R P} \boldsymbol{A}_{(\boldsymbol{M x N})}$ of parameters $\boldsymbol{p}_{i} \in \boldsymbol{P}\left\{\boldsymbol{p}_{1}, \boldsymbol{p}_{2}, \ldots, \boldsymbol{p}_{\boldsymbol{m}}\right\}$ to assets $\boldsymbol{a}_{i} \in A\left\{\boldsymbol{a}_{1}, \boldsymbol{a}_{2}, \ldots, \boldsymbol{a}_{\boldsymbol{n}}\right\}$, where $\boldsymbol{M}$ stands for the number of parameters, and $N$ number of assets.

$$
R_{P A}=\left[\begin{array}{cccc}
a_{1} p_{1} & a_{1} p_{2} & \cdots & a_{1} p_{m} \\
a_{2} p_{1} & a_{2} p_{2} & \cdots & a_{2} p_{m} \\
\vdots & \vdots & \ddots & \vdots \\
a_{n} p_{1} & a_{n} p_{2} & \cdots & a_{n} p_{m}
\end{array}\right]
$$

That scope cannot be modified, if the monitoring process is supposed to cover all assumed security objectives. The only possible way to avoid system overload caused by the monitoring process is to adjust the means of control assigned to each of those parameters in such a way, that the overall cost does not exceed some predefined, acceptable level. Then, for each of those parameters an appropriate way of control is selected, with attention to the overall system load that it will cause. This includes the choice of the right data description format to be used for communication with the data source, which is based on the Triage procedure [23], followed by a comparative analysis of the overhead generated by each of the remaining options (e.g. based on ROI).

Then, the frequency, at which the status of each of the monitored parameters is updated in the central repository is calculated. This is intended to provide as small data granularity as possible, processing and gathering of which will not cause a system load higher than assumed boundary values. Theoretically, the best scenario is to monitor everything in near real-time. Unfortunately, as it was proved by earlier research in various fields, implementation of such an approach is practically near to impossible, e.g. due to the amount of generated network traffic which may interfere with normal, daily operation of the organization. To avoid generating a serious risk of business discontinuity, it is necessary to define a total, acceptable (and safe) system load that can be caused by security monitoring, without significant negative impact on other business processes. Then, the plan of monitoring should be adjusted in such a way, that its implementation does not cause higher load than the one defined as a limit, which may be called a budget B.

A number of weights are used to adapt sampling frequency of various information system elements. All analyzed business processes, assets and parameters are given weights.

Each of the parameters that are subject of monitoring can be connected with only one security objective, but multiple assets and business processes, so they shall not be treated as equal. There are numerous methods for business process assessment, that can be used to evaluate all business processes of the organization, e.g. RAPID RE [17]. Obtained values, after normalization, provide business processes weights $\boldsymbol{W B P} \boldsymbol{P}_{\boldsymbol{i}}$
All assets that are combined with a given parameter can be treated as equal, so for $\boldsymbol{a}$ assets connected with parameter $\boldsymbol{k}$ an asset weight can be calculated as:

$$
W A_{j}=\frac{1}{a}, W A_{j} \in(0 ; 1\rangle
$$

As the parameter can be assigned to many assets and business processes, to calculate parameter's weight it is necessary apply proper aggregation. Due to the fact, that relations between business processes, assets and parameters form a tree structure, parameters' weights can be calculated similarly to FTA elements [11], [19], like a total probability of events forming a stochastic tree [2]:

$$
W P a r_{k}=\sum_{j=1}^{z}\left(W A_{j} * \sum_{i=1}^{p} W B P_{i}\right), W P a r_{k} \in\langle 0 ; 1\rangle
$$

Then, the cost of monitoring plan implementation (which is the system load caused by it) for $\mathrm{R}$ parameters and a standardized time period, can be calculated with the help of an adapted formula from process cost calculus [22]:

$$
M P C=\sum_{k=1}^{R}\left(C u_{k} * A_{k} * f_{k}\right)
$$

Where:

- $M P C$ - monitoring plan cost (additional system load),

- $A_{k}$ - number of assets connected with parameter $k$,

- $C u_{k}$ - load caused by a single update of parameter $k$ status (unit cost),

- $f_{k}$ - parameter $\mathrm{k}$ status update frequency.

Then, a following generalization can be assumed:

$$
f_{k}=\mathrm{WPar}_{k} * f
$$

Where:

- $W \operatorname{Par}_{k}$ - weight of parameter $k$,

- $f$ - unknown base frequency.

As a result, the following formula is obtained:

$$
M P C=\sum_{k=1}^{R}\left(C u_{k} * A_{k} * \mathrm{WPar}_{k} * f\right)
$$

As the maximum acceptable additional load to the system caused by security monitoring was defined as a budget $\boldsymbol{B}$, the problem got limited to calculation of a maximum base frequency $f$ for which equation $M P C \leq B$ holds. That boundary can be defined for a number of criteria, the list of which can differ depending on organizational structure and culture. They may include IT infrastructure load and workload of employees.

A set of four criteria was chosen during research:

- calculation power consumed,

- memory consumed,

- quantity of data transferred,

- human workload.

For each of those criteria a separate budget equation should be formed: 


$$
B_{a}=\sum_{k=1}^{R}\left(C u_{k} * A_{k} * \mathrm{WPar}_{k} * f\right)
$$

Base frequency $f$ must fulfill all the criteria of system workload, which means:

$$
f=\min \left\{f_{1}, \cdots, f_{c-1,} f_{c}\right\}
$$

Calculated frequency $f$ is used to determine the frequency of each parameter's update, with attention to its weight WPar, calculated earlier:

$$
f_{k}=\mathrm{WPar}{ }_{k} * f
$$

As a consequence, the final monitoring plan $\boldsymbol{P M}$, providing monitoring technique $\boldsymbol{M T}$ and status update frequency $\boldsymbol{f}$ for each of the parameters, takes a form of:

$$
P M=\left\{\left(\operatorname{Par}_{i}, M T_{\left(\operatorname{Par}_{i}\right)}, f_{\left(\operatorname{Par}_{i}\right)}\right), \cdots\right\}
$$

As it was stated at the beginning, a list of parameters was taken as an input and is a result of earlier steps of the security management process. Monitoring techniques were chosen with attention to various requirements and measurable criteria. Finally, update frequency for each of the parameters was established with attention to parameters' significance and within predefined load limit (budget). Changing a set of load criteria will influence the final result in such a way that the update frequencies may be different, but the overall load of the system will always be below predefined limit. Although that limit must be determined separately for every single organization, it is a far simpler task when compared to assessment of all possible monitoring plan combinations.

\section{A. Data Sources}

There are many different data sources that may provide data to the system, including SIEM solutions, firewalls and other networking devices, hardware and software sensors for physical and environmental security, software telemetrics mechanisms or other organizational solutions of applied procedures governance.

\section{B. Central Reasoning Repository}

It is the main element of the data gathering layer. It collects all status data from individual components of the system, as well as necessary system parametrization data. Depending on the scale of the information system that is subject of monitoring, it may be necessary to implement database architecture designed for the big data environment.

\section{Repository Update Mechanism}

Another key element of the data gathering layer. It is responsible for periodical overwrite of the oldest records with current, successfully verified valued received from data sources. It is based on the cyclic queue mechanism and makes a use of a typical logistic approach to managing performer operation.

\section{D.Data Verification Subsystem}

It is responsible for initial verification of integrity, source and structure of data. Verification of data source and integrity is done with the help of cryptography, implemented in the Crypto module. Verification of data structure is based on the data description format definition that is expected in the message from the agent that is responsible for processing this specific order on data.

\section{E. Data Format Conversion Mechanism}

The conversion model relies on assumption that there is some general set of description format fields, from which specific languages are constructed by selection of proper subsets of fields. Such assumption is correct as long as each field that exists in one description format forms a unique element of a set. This means that for instance in the case of field date that appears in $\mathrm{n}$ languages to meet that assumption a set of fields LF should contain up to $\mathrm{n}$ unique elements that corresponds to date. The mechanism was described in [14].

\section{F. Reasoning Subsystem}

To reduce time required to build or adjust the model and to make it easier, classification of local anomalies based on rough sets and linguistic knowledge base was used. It was combined with aggregation on the level of assets, business processes and security objectives.

A number of factors influencing changes in the state of security were chosen based on the widely used methods and standards (ISO2700x and ISO15408-x). They were used to define a model of linguistic base decomposition defined as $\mathrm{WL}=\mathrm{f}(\mathrm{x} 1, \mathrm{x} 2, \mathrm{x} 3, \mathrm{x} 4, \mathrm{x} 5, \mathrm{x} 6, \mathrm{x} 7)$, where:

$\mathrm{x} 1$ - business processes significance,

$\mathrm{x} 2$ - number of corresponding business processes,

$\mathrm{x} 3$ - asset recovery time,

$\mathrm{x} 4$ - dynamics of changes of asset's parameters state,

$\mathrm{x} 5$ - scale of changes of assets parameters,

$\mathrm{x} 6$ - available time,

$\mathrm{x} 7$ - environment influence.

Then, an aggregation based on a tree structure was done, where level 0 is a tree root that represents security of the whole information system, and nodes on level $\mathrm{n}$ described by the $W L$ value. The aggregation characteristics are as follows:

- At least one of the child nodes on level $\boldsymbol{n}+\boldsymbol{l}$ has value equal to $\mathrm{WL}$,

- None of the child nodes on level $\boldsymbol{n + 1}$ has value higher than WL,

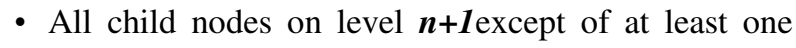
have value lower than $\mathrm{WL}$,

- Value on the level $\boldsymbol{n}$-1 must not be lower than WL.

Finally, starting with the deepest level of the tree, nodes with the highest value of influence WL are identified and that value is propagated up the tree, carrying the information about the status of the system according to approach "worst case first". Proposed aggregation model is in this way a 
combination of analytic side of the FTA and synthetic side of the ETA. In some way it can be perceived as a tree representation of the FMEA model. Because the attention of the administrator or the management is focused on those anomalies that caused the biggest abbreviation from the state perceived as safe, it is easy to prioritize anomaly handling and in this way correct detected weaknesses of the implemented security management system.

\section{V.CASE STUDY}

Proposed new approach was verified on the basis of data gathered during real life operation of an IT knowledge contest Tik?-Tak! in two different time periods. Tik?-Tak! was a national (Polish) contest organized by the Polish Information Processing Society, addressing primary, secondary and high school students. First set of data comes from its second edition in year 2012, and second set of data was gathered in year 2015, after the contest system was significantly reconstructed. The contest was divided into three phases: school level, regional level and finals. The first two of them were done online, with the help of mentioned contest system. Data used in case study were gathered during the first phase of the contest, in which over ten thousand students attended each of the editions. The contest system was located on a virtual machine provided by Cloudia (in 2015 rebranded to Atende Business Cloud). Users used their own computers to $\log$ in to the system.

The following case study presents key steps through the proposed integrated monitoring system. An entry point was a list parameters that came as a result of risk assessment process:
1) Contest system server,
2) Users database,
3) LAN network,
4) Internet connection,
5) Questions database,
6) Answers database,
7) Administrator.

Those assets are used in three main business processes:

- Solving tests,

- Calculating rank lists,

- User and school registration.

Five of the assets used were assessed as secure enough and because of that were not assigned to any monitoring parameter. For the remaining two there were known vulnerabilities that were not covered fully in other way, which caused them to require monitoring. In total, three parameters were defined in this case:

- Answer saved (t),

- Par 65 - Unsuccessful use of the user identification mechanism, including the user identity provided,

- Par 66 - Successful use of the user identification mechanism, including the user identity provided.

Those three parameters, mapped on assets, were taken as input to case study in this paper. The $\mathrm{R}_{\mathrm{PA}}$ matrix looks like:

$$
R_{P A}=\left[\begin{array}{lll}
1 & 1 & 1 \\
0 & 0 & 0 \\
0 & 0 & 0 \\
0 & 0 & 0 \\
0 & 0 & 0 \\
1 & 0 & 0 \\
0 & 0 & 0
\end{array}\right]
$$

For each of the parameters, a unit cost of monitoring was estimated, separately for each of the four conditions: caused system load in seconds, human workload in hours, data transmission in $\mathrm{kB}$ and quantity of data stored in $\mathrm{kB}$.

TABLE I.

BUDGET DEFINITION - LOAD ALLOWANCE FOR EACH OF THE TEST SCENARIOS FOR TIK?-TAK! 2012. SOURCE: OWN CONTRIBUTION.

\begin{tabular}{|c|c|l|l|c|}
\hline $\begin{array}{c}\text { test } \\
\text { no. }\end{array}$ & $\begin{array}{c}\text { data saved } \\
\text { increase }\end{array}$ & $\begin{array}{c}\text { system } \\
\text { load }\end{array}$ & $\begin{array}{c}\text { human } \\
\text { workload }\end{array}$ & $\begin{array}{c}\text { data } \\
\text { transmitted }\end{array}$ \\
\hline test 1 & $1 \mathrm{~GB}$ & $10000 \mathrm{~s}$ & $40 \mathrm{~h}$ & $5 \mathrm{~GB}$ \\
\hline test 2 & $2 \mathrm{~GB}$ & $1000 \mathrm{~s}$ & $40 \mathrm{~h}$ & $5 \mathrm{~GB}$ \\
\hline test 3 & $1 \mathrm{~GB}$ & $10000 \mathrm{~s}$ & $5 \mathrm{~h}$ & $5 \mathrm{~GB}$ \\
\hline test 4 & $1 \mathrm{~GB}$ & $1000 \mathrm{~s}$ & $20 \mathrm{~h}$ & $5 \mathrm{~GB}$ \\
\hline test 5 & $1 \mathrm{~GB}$ & $2000 \mathrm{~s}$ & $8 \mathrm{~h}$ & $5 \mathrm{~GB}$ \\
\hline
\end{tabular}

Frequencies of parameter status update were calculated for each of the test scenarios defined by budget limits shown in table1. The results are shown in Tab II.

TABLE II.

TEST RESUlTS FOR TIK?-TAK! 2012. SOURCE: OWN CONTRIBUTION.

\begin{tabular}{|c|c|c|c|}
\hline test no. & $\begin{array}{c}\text { unsuccessful } \\
\text { user } \\
\text { identification }\end{array}$ & $\begin{array}{c}\text { successful } \\
\text { user } \\
\text { identification }\end{array}$ & $\begin{array}{c}\text { answer saved } \\
\left(\mathrm{t}_{1}-\mathrm{t}_{0}\right)\end{array}$ \\
\hline test 1 & 103 & 103 & 96 \\
\hline test 2 & 30 & 30 & 28 \\
\hline test 3 & 12 & 12 & 12 \\
\hline test 4 & 30 & 30 & 28 \\
\hline test 5 & 20 & 20 & 19 \\
\hline
\end{tabular}

TABLE IIIII.

BUDGET LIMIT CONSUMPTION IN TEST SCENARIOS. OWN CONTRIBUTION.

\begin{tabular}{|l|l|l|l|l|}
\hline $\begin{array}{l}\text { test } \\
\text { no. }\end{array}$ & $\begin{array}{l}\text { data saved } \\
\text { increase }\end{array}$ & $\begin{array}{l}\text { system } \\
\text { load }\end{array}$ & $\begin{array}{l}\text { human } \\
\text { workload }\end{array}$ & $\begin{array}{l}\text { data } \\
\text { transmitted }\end{array}$ \\
\hline test 1 & $0,010 \mathrm{MB}$ & $3348,149 \mathrm{~s}$ & $39,999 \mathrm{~h}$ & $0,013 \mathrm{MB}$ \\
\hline test 2 & $0,003 \mathrm{MB}$ & $999,999 \mathrm{~s}$ & $11,946 \mathrm{~h}$ & $0,004 \mathrm{MB}$ \\
\hline test 3 & $0,001 \mathrm{MB}$ & $418,519 \mathrm{~s}$ & $4,999 \mathrm{~h}$ & $0,002 \mathrm{MB}$ \\
\hline test 4 & $0,003 \mathrm{MB}$ & $999,999 \mathrm{~s}$ & $11,946 \mathrm{~h}$ & $0,004 \mathrm{MB}$ \\
\hline test 5 & $0,002 \mathrm{MB}$ & $669,630 \mathrm{~s}$ & $8,000 \mathrm{~h}$ & $0,003 \mathrm{MB}$ \\
\hline
\end{tabular}

Parameter status update frequency is limited by the most restrictive cost criteria. As can be seen in table 3, in the first, third and fifth test scenario a maximum allowed human 
workload was reached. In the second and fourth, the limit was caused by the system load allowance.

For each of the parameters, a unit cost of monitoring was estimated, separately for each of the four conditions: caused system load in seconds, human workload in hours, data transmission in $\mathrm{kB}$ and quantity of data stored in $\mathrm{kB}$. Then, frequencies of their status update were determined, according to formula 7 and 9, for a number of test scenarios. To provide comparison with results obtained for the 2012 contest system, at first the same test cases were defined (based on the same budget limits).

TABLE IV.

BUDGET DEFINITION - LOAD ALLOWANCE FOR EACH OF THE TEST SCENARIOS FOR TIK?-TAK! 2015. SOURCE: OWN CONTRIBUTION.

\begin{tabular}{|c|c|c|c|c|}
\hline $\begin{array}{c}\text { test } \\
\text { no. }\end{array}$ & $\begin{array}{c}\text { data } \\
\text { saved } \\
\text { increase }\end{array}$ & $\begin{array}{c}\text { system } \\
\text { load }\end{array}$ & $\begin{array}{c}\text { human } \\
\text { workload }\end{array}$ & $\begin{array}{c}\text { data } \\
\text { transmitted }\end{array}$ \\
\hline test 1 & $1 \mathrm{~GB}$ & $10000 \mathrm{~s}$ & $40 \mathrm{~h}$ & $5 \mathrm{~GB}$ \\
\hline test 2 & $2 \mathrm{~GB}$ & $1000 \mathrm{~s}$ & $40 \mathrm{~h}$ & $5 \mathrm{~GB}$ \\
\hline test 3 & $1 \mathrm{~GB}$ & $10000 \mathrm{~s}$ & $5 \mathrm{~h}$ & $5 \mathrm{~GB}$ \\
\hline test 4 & $1 \mathrm{~GB}$ & $1000 \mathrm{~s}$ & $20 \mathrm{~h}$ & $5 \mathrm{~GB}$ \\
\hline test 5 & $1 \mathrm{~GB}$ & $2000 \mathrm{~s}$ & $8 \mathrm{~h}$ & $5 \mathrm{~GB}$ \\
\hline
\end{tabular}

Frequency of parameter status update was calculated again for each of the test scenarios defined by budget limits shown in table 4 , while table 5 contains results obtained for the Tik?-Tak! 2015 contest, with the following set of pareameters:

Par1: unsuccessful user identification

Par2: successful user identification

Par3: SSH attack

Par4: Fail2Ban bans

Par5: PHP errors nginx

Par6: PHP sock-fail

Par7: answer saved $\left(\mathrm{t}_{1}-\mathrm{t}_{0}\right)$

TABLE V.

TEST RESUlTS FOR TIK?-TAK! 2015. SOURCE: OWN CONTRIBUTION.

\begin{tabular}{|c|c|c|c|c|c|c|c|}
\hline $\begin{array}{c}\text { test } \\
\text { no. }\end{array}$ & Par1 & Par2 & Par3 & Par4 & Par5 & Par6 & Par7 \\
\hline test 1 & 33 & 33 & 33 & 33 & 33 & 33 & 33 \\
\hline test 2 & 7 & 7 & 7 & 7 & 7 & 7 & 7 \\
\hline test 3 & 4 & 4 & 4 & 4 & 4 & 4 & 4 \\
\hline test 4 & 7 & 7 & 7 & 7 & 7 & 7 & 7 \\
\hline test 5 & 6 & 6 & 6 & 6 & 6 & 6 & 6 \\
\hline
\end{tabular}

Similarly to tests for Tik?-Tak! 2012 contest, the first, third and fifth test scenario generated

a maximum allowed human workload value (refer to table $6)$. The second and fourth were limited by system load criterion. It can be seen as the increase of the parameters quantity, at an unchanged limit of allowed load, caused by monitoring implementation, reduces frequencies of parameters' status update.
TABLE VI.

BUDGET LIMIT CONSUMPTION IN TEST SCENARIOS. OWN CONTRIBUTION.

\begin{tabular}{|c|c|c|c|c|}
\hline $\begin{array}{c}\text { test } \\
\text { no. }\end{array}$ & $\begin{array}{c}\text { data saved } \\
\text { increase }\end{array}$ & system load & $\begin{array}{c}\text { human } \\
\text { workload }\end{array}$ & $\begin{array}{c}\text { data } \\
\text { transmitted }\end{array}$ \\
\hline test 1 & $0,008 \mathrm{MB}$ & $4333,334 \mathrm{~s}$ & $40,000 \mathrm{~h}$ & $0,014 \mathrm{MB}$ \\
\hline test 2 & $0,002 \mathrm{MB}$ & $999,999 \mathrm{~s}$ & $9,228 \mathrm{~h}$ & $0,003 \mathrm{MB}$ \\
\hline test 3 & $0,001 \mathrm{MB}$ & $541,666 \mathrm{~s}$ & $4,999 \mathrm{~h}$ & $0,002 \mathrm{MB}$ \\
\hline test 4 & $0,002 \mathrm{MB}$ & $999,999 \mathrm{~s}$ & $9,228 \mathrm{~h}$ & $0,003 \mathrm{MB}$ \\
\hline test 5 & $0,002 \mathrm{MB}$ & $866,666 \mathrm{~s}$ & $7,999 \mathrm{~h}$ & $0,003 \mathrm{MB}$ \\
\hline
\end{tabular}

Two additional test scenarios were added with increased budget limits. The idea was to show the difference in load of the given four criteria, generated by the increased scope of monitoring (when compared to Tik?-Tak! 2012), with the frequencies on comparable level. Table 7 presents budget limits for the additional test scenarios.

TABLE VII.

BUDGET DEFINITION - LOAD ALLOWANCE FOR ADDITIONAL TEST SCENARIOS FOR TIK?-TAK! 2015. OWN CONTRIBUTION.

\begin{tabular}{|l|c|l|l|l|}
\hline $\begin{array}{l}\text { test } \\
\text { no. }\end{array}$ & $\begin{array}{l}\text { data saved } \\
\text { increase }\end{array}$ & $\begin{array}{l}\text { system } \\
\text { load }\end{array}$ & $\begin{array}{l}\text { human } \\
\text { workload }\end{array}$ & $\begin{array}{l}\text { data } \\
\text { transmitted }\end{array}$ \\
\hline test 6 & $1 \mathrm{~GB}$ & $10000 \mathrm{~s}$ & $200 \mathrm{~h}$ & $5 \mathrm{~GB}$ \\
\hline test 7 & $1 \mathrm{~GB}$ & $20000 \mathrm{~s}$ & $200 \mathrm{~h}$ & $5 \mathrm{~GB}$ \\
\hline
\end{tabular}

Once again, frequencies were calculated. Results obtained for the new test scenarios were shown in table 8 .

TABLE VIII.

RESULTS FOR ADDITIONAL TEST SCENARIOS FOR TIK?-TAK! 2015. OWN CONTRIBUTION.

\begin{tabular}{|l|l|l|l|l|l|l|l|}
\hline $\begin{array}{l}\text { test } \\
\text { no. }\end{array}$ & Par1 & Par2 & Par3 & Par4 & Par5 & Par6 & Par7 \\
\hline test 6 & 76 & 76 & 76 & 76 & 76 & 76 & 76 \\
\hline test 7 & 153 & 153 & 153 & 153 & 153 & 153 & 153 \\
\hline
\end{tabular}

TABLE IX.

BUDGET LIMIT CONSUMPTION IN TEST SCENARIOS 6 AND 7. OWN CONTRIBUTION.

\begin{tabular}{|l|l|l|l|l|}
\hline $\begin{array}{l}\text { test } \\
\text { no. }\end{array}$ & $\begin{array}{l}\text { data saved } \\
\text { increase }\end{array}$ & $\begin{array}{l}\text { system } \\
\text { load }\end{array}$ & $\begin{array}{l}\text { human } \\
\text { workload }\end{array}$ & $\begin{array}{l}\text { data } \\
\text { transmitted }\end{array}$ \\
\hline test 6 & $0,019 \mathrm{MB}$ & $10000 \mathrm{~s}$ & $92,309 \mathrm{~h}$ & $0,031 \mathrm{MB}$ \\
\hline test 7 & $0,039 \mathrm{MB}$ & $20000 \mathrm{~s}$ & $184,615 \mathrm{~h}$ & $0,062 \mathrm{MB}$ \\
\hline
\end{tabular}

In both cases it was the system load that limited the frequency of parameters' status update. This can be clearly seen in table 9.

As can be seen, the monitoring plan is adjusted according to changing load allowance (limit) of the four budget criteria. The final parameters' status update frequencies are calculated in such a way, that it is impossible to exceed a predefined limit, which prevents from information system overload caused by the implementation of security monitoring procedures. 


\section{DISCUSSION}

It should be noted that data does not necessarily have to be updated in the form of readings from specific points in time. The described mechanism can be applied as well if the data source returns periodically a summary of status changes in time or some aggregated value of status readings from some time period. As a result, this mechanism is connected at least with some latency in status update or even change of data granularity.

\section{system response time [ms]}

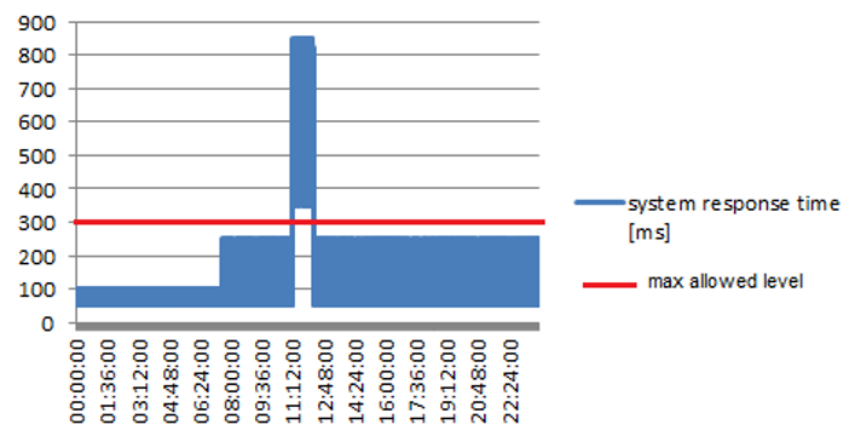

Fig. 3 Value in time of one of the Tik?-Tak! parameters gathered at $f=1$ s. Own contribution.

system response time [ms]

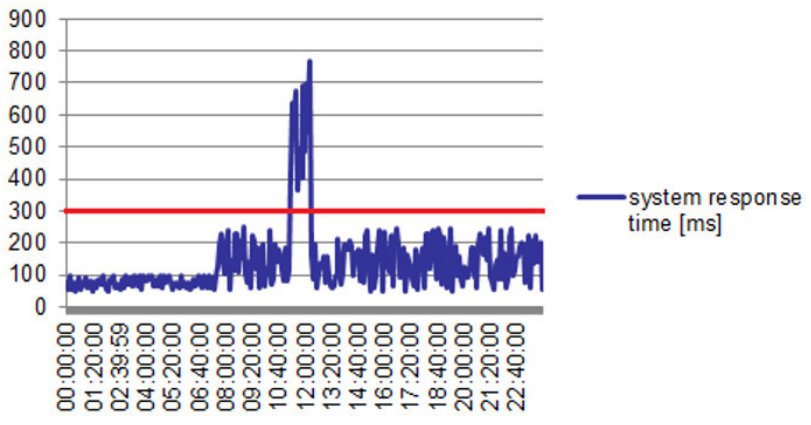

Fig. 4 Values in time of one of Tik?-Tak! parameters gathered with frequency $f=5 \mathrm{~min}$. Own contribution.

In this paper it was assumed that budget criteria values were evaluated according to some rules or methodology, which is irrelevant from the perspective of the proposed mechanism. However, those limits may have impact on the accuracy of further reasoning on the basis of gathered data (due to data granularity and latency in their receive). The method for calculation of those budget limits is not a part of this paper, however. To show, that the mechanism proposed in this paper, if it is not used with too strict budget limits, shall not reduce detectability of security incidents, data for one of the parameters chosen for Tik?-Tak! contest was compared in two scenarios. Figure 5 shows values gathered with a maximum possible frequency $(f=1 \mathrm{~s})$ within a $24 \mathrm{~h}$ time period. As the system designer defined the maximum comfortable system latency as $300 \mathrm{~ms}$, that value was defined as a limit - all incidents when this is exceeded should be treated as a significant system slow down. In analyzed time period such an anomaly occurred around 11:20, and lasted for about 15 minutes.

Figure 6 presents values of the same parameter, but updated/gathered during the same time period at a lower frequency $f=5 \mathrm{~min}$. as can be seen, although figures differ, the overall characteristics remained, so it is still possible to detect this anomaly, however with some latency. The reduction of reasoning precision (latency) caused by data granularity was paired with significant reduction of monitoring costs. As a result, application of a mechanism proposed in this paper should always be combined with proper evaluation of allowed load limits. Standardization of that evaluation is the most significant field of future work. The proposed solution, however, takes budget limits as input arguments, without considering how they were calculated.

\section{CONCLUSION}

The proposed approach to security monitoring, is a complex solution for all steps of the process, starting with planning and finishing with reasoning about the state of security of the whole system. Thanks to many adaptation mechanisms it can be adjusted to requirements and implemented in different organizations. What is important, the selection of monitoring plan is done in a way that is supposed to prevent system overload caused by the monitoring activity itself. Construction of the reasoning process, including knowledge aggregation, was prepared in such a way that it provides a clear answer which areas or elements of the system were affected by significant security incidents, which means that in those areas already implemented countermeasures are probably insufficient. Then, on the basis of such an information, it becomes easy to plan an audit repeat, if required.

\section{REFERENCES}

[1] Aiello, M. and Pagani, G.A. 2014. The Smart Grid's Data Generating Potentials. Annals of Computer Science and Information Systems. 2014, Vol. 2, pp. 9-16., DOI: 10.15439/2014F509

[2] Bean, Michael, A. Probability: The Science of Uncertainty with Applications to Investments, Insurance, and Engineering. Providence : American Mathematical Society, 2001.

[3] Brdys, M.A. 2014. Integrated monitoring, control and security of Critical Infrastructure Systems. Annual Reviews in Control. 2014, 38, pp. 47-70., DOI: 10.1016/j.arcontrol.2014.03.006

[4] Dalpiaz, F., Giorgini, P., Salnitri, M., Designing secure business processes with SecBPMN. Enterprise, Business-Process and Information Systems Modeling, pp.200-214, 2015, DOI: 10.1007/s10270-015-0499-4

[5] Deraison R., Gula R., Ranum M., Unified Security Monitoring (USM), Real-Time Situational Awareness of Network Vulnerabilities, Events and Configurations, Tenable Network Security, 2009

[6] El Fray, I. A Comparative Study of Risk Assessment Methods, MEHARI \& CRAMM with a New Formal Model of Risk Assessment (FoMRA). Information Systems. Computer Information Systems and Industrial Management. 2012, Vol. 7564 of the series Lecture Notes in Computer Science., DOI: 10.1007/978-3-642-33260-9_37

[7] Fenz, S., et al. 2013. FORISK: Formalising information security risk and compliance management. 2013 43rd Annual IEEE/IFIP Conference on Dependable Systems and Networks Workshop (DSNW). 2013., DOI: 10.1109/DSNW.2013.6615533 
[8] Fernandez E., Monge R., Building a security reference architecture for cloud systems, Requirements Eng (2016) 21:225-249,

DOI: $10.1007 / \mathrm{s} 00766-014-0218-7$

[9] Han, S., et al. 2014. Intrusion Detection in Cyber-Physical Systems: Techniques and Challenges. IEEE SYSTEMS JOURNAL. 2014, Vol. 8, 4., DOI: 10.1109/JSYST.2013.2257594

[10] Huang S., Zhou C., Yang S., Quin Y., Cyber-physical System Security for Networked Industrial Processes, International Journal of Automation and Computing, 12(6), December 2015, 567-578, DOI: $10.1007 / \mathrm{s} 11633-015-0923-9$

[11] IEC. Fault Tree Analysis (FTA), International Technical Commission, IEC Standard, Publication 1025. 1990.

[12] Jung H., Hwang I., Moon J., Park H., A security monitoring method for malicious P2P event detection, Peer-to-Peer Netw. Appl. (2016) 9:498-507, DOI: 10.1007/s12083-015-0369-4

[13] Karabacak, B. and Tatar, U. Strategies to Counter Cyberattacks: Cyberthreats and Critical Infrastructure Protection. NATO Science for Peace and Security Series - E: Human and Societal Dynamics. 2012, Vol. Volume 116: Critical Infrastructure Protection.,

[14] Klasa, T. and El Fray, I. Data Scheme Conversion Proposal for Information Security Monitoring Systems. [book auth.] S. Kobayashi, et al., et al. Hard and Soft Computing for Artificial Intelligence, Multimedia and Security. s.1. : Springer International Publishing, 2017., DOI: 10.1007/978-3-319-48429-7_15

[15] Kondakci, S., A causal model for information security risk assessment, 2010 Sixth International Conference on Information Assurance and Security, Atlanta, GA, 2010, pp. 143-148., DOI: 10.1109/ISIAS.2010.5604039

[16] Li, T., Horkoff, J. \& Mylopoulos, J. Softw Syst Model (2016). doi:10.1007/s10270-016-0560-y

[17] Manganelli, R. L., Klein, M. M. The reengineering handbook: a stepby-step guide to business transformation. New York: AMACOM, 1994.,
[18] Martinelli, F. and Matteucci, I. A framework for automatic generation of security controller. Software Testing Verification \& Reliability. 2008, pp. 563-582., DOI: 10.1002/stvr.441

[19] NASA. Fault Tree Handbook with Aerospace Applications', Version 1.1, NASA Publication. 2002.

[20] Pero, M. and Sudy, I. 2014. Increasing security and efficiency in supply chains: a five-step approach. International Journal of Shipping and Transport Logistics. 2014, Vol. 6, 3, pp. 257-279., DOI: 10.1504/IJSTL.2014.060785

[21] Shashanka M., Shen M., Wang J., User and Entity Behavior Analytics for Enterprise Security. BigData, 2016. DOI: 10.1109/BigData.2016.7840805

[22] Shim, J. K., Siegel J.G., Modern Cost Management and Analysis, Barron's Business Library, New York, 2009

[23] Stoppler, Melissa, Conrad, MD. 2014. MedicineNet.com. [Online] 12 $1,2014$. http://www.medicinenet.com/script/main/art.asp?articlekey=79529.

[24] Thakore U., Weaver G., Sanders W., A Quantitative Methodology for Security Monitor Deployment, 2016 46th Annual IEEE/IFIP International Conference on Dependable Systems and Networks, IEEE, 2016, DOI: 10.13140/RG.2.2.17533.56808

[25] Van Tan, V. and Yi, M.J. 2010. Design Issues and Approach to Internet-Based Monitoring and Control Systems. Trends in Applied Intelligent Systems, Pt1, Proceedings, Lecture Notes in Artificial Intelligence. 2010, Vol. 6096, pp. 478-488., DOI: 10.1007/978-3-64213022-9 48

[26] Vaarandi, R., Real-time Classification of IDS Alerts with Data Mining Techniques, Proceedings of the 2009 IEEE MILCOM Conference, IEEE 2009

[27] Wu, M.Z., et al. 2012. Development and Validation on Integrated Dynamic Security Monitoring Platform. 2012 Sixth International Conference on Genetic and Evolutionary Computing. 2012., DOI: $10.1109 /$ ICGEC.2012.80 



\title{
A Robust Watermarking Technique for Image Content Authentication
}

\author{
Obaid Ur-Rehman and Natasa Zivic \\ Chair for Data Communications Systems \\ University of Siegen \\ Hoelderlinstrasse 3, 57076 Siegen, Germany \\ email: \{obaid.ur-rehman, natasa.zivic\}@uni-siegen.de
}

\begin{abstract}
A robust watermarking technique for image content authentication is proposed. The proposed technique is robust to minor modifications resulting from legitimate image processing operations. At the same time, the modifications affecting image content are classified as forgery attacks by the proposed scheme. Simulation results are given for intentional modifications (forgery attacks) as well as unintentional modifications (channel noise). Different levels of noisy environments are assumed including the coded orthogonal frequency division multiplexing which is used in 5G networks, to prove the effectiveness of the proposed scheme. A security analysis of the proposed watermarking scheme is given in the end.
\end{abstract}

Keywords- Robust Watermarking; Content based Authentication; COFDM; Error Tolerance; Feature Extraction.

\section{INTRODUCTION}

$\mathrm{W}$ ITH the widespread availability of multimedia editing tools, image content can be easily edited for forgery. As a consequence, there is a strong need for image integrity verification and content authentication algorithms. A digital watermark is a signature of the image and typically inserted inside the image to prove its authenticity or ownership at a later stage. The watermark might be visible or invisible. Unlike digital signatures, digital watermarking [1] does not require extra transmission bandwidth. Watermarking methods are designed to be prone to minor modifications in the image data. On the other hand, digital signatures are very sensitive to modifications in the image data. Due to a wellknown phenomenon, called the Avalanche Effect [2], data authentication fails even in the presence of a single bit error.

A new class of authentication mechanisms have emerged recently, called noise tolerant or soft authentication mechanisms [3]. These mechanisms are different from the standard authentication mechanisms as they are designed to be tolerant to minor changes in the data. Simply put, the authentication succeeds despite the data protected by these mechanisms being a little different (decided based on a threshold) than the data on which the authentication tag was computed.

The watermarking technique proposed in this paper is based on generating the authentication tag using the approximate message authentication code (AMAC) [4] and then using it as a watermark. AMAC is tolerant to minor changes in data as opposed to standard message authentication code (MAC) $[5,6]$ which does not tolerate data modification. The proposed method is analyzed for security. Simulation results are given for different noisy environments and forgery attacks.

This paper is organized as follows. Related work is presented in Section II. The proposed watermarking technique is presented in Section III, including the improvements on the existing methods. Simulation results are given in Section IV. A security analysis of the proposed scheme is presented in Section V. The paper is concluded in Section VI.

\section{RELATED WORK}

Noise tolerant data authentication methods proposed in literature include approximate message authentication code (AMAC) [4], noise tolerant message authentication code (NTMAC) [7] and joint channel coding and cryptography [8], just to name a few. AMAC is considered in this work. It is based on the usage of majority logic for generation of the authentication tag. The tag is generated by grouping the data into rows and columns of a chosen dimension and XORing it with pseudo random bit stream. The final tag is obtained using the majority logic.

Another noise tolerant authentication technique is NTMAC, where the data is split into multiple blocks by calculating standard MAC on each block and then retaining only a portion of the MAC (called sub-MAC) for each block, which is then used to detect changes in the block. For tolerance to minor modifications, the concept of partitions is introduced. A variation on NTMAC was proposed by the authors as weighted noise tolerant message authentication code (WNTMAC) [9]. WNTMAC introduces the concept of weights to differentiate the important parts of data from the relatively lesser important parts. The authors also proposed error correcting (EC)-WNTMAC in the same work [9] as an extension of WNTMAC. In EC-WNTMAC, an error location and correction capability was introduced.

The above approaches are not directly suitable for image content authentication, rather only for (image) data authentication. They can be extended to image content authentication by using image features rather than the data. The use of error correction codes further extends the tolerance capability of these techniques. 


\section{WATERMARKING TECHNIQUE}

\section{A. Watermark based on Modified AMAC}

Discrete Wavelet Transform (DWT) is computed on the source image, splitting the image into four sub bands, i.e., Low-Low (LL), Low-High (LH), High-Low (HL) and HighHigh $(\mathrm{HH})$. An AMAC tag is computed on the LL sub band. The computation of AMAC tag is different from the standard AMAC tag [4] which was used in [10]. The modified and strengthened AMAC algorithm uses an extra pseudorandom bit for AMAC tag computation. This avoids the security attack proposed in [11]. The original AMAC uses one pseudorandom bit $\left(\mathrm{x}_{\mathrm{i}}\right)$ which is XORed with the message bit $\left(\mathrm{m}_{\mathrm{i}}\right)$. The modified AMAC uses two pseudorandom bits, $\mathrm{w}_{\mathrm{i}}$ and $x_{i}$ and calculates the AMAC tag bit $z_{i}$ using (1).

$$
z_{i}=m_{i} \cdot w_{i} \oplus x_{i}
$$

The AMAC tag is used as a watermark of the image. If there are minor changes in the image, the LL sub band will not change significantly and therefore the corresponding AMAC tag will be the same as for the original image. For changes beyond a chosen threshold, such as in the case of a forgery attack, such as object insertion or object removal, the LL sub band will change significantly resulting in a changed AMAC. The threshold can be chosen as desired using the approach presented in [4]. The watermark generation process for the "Lena" image is shown in Fig. 1.

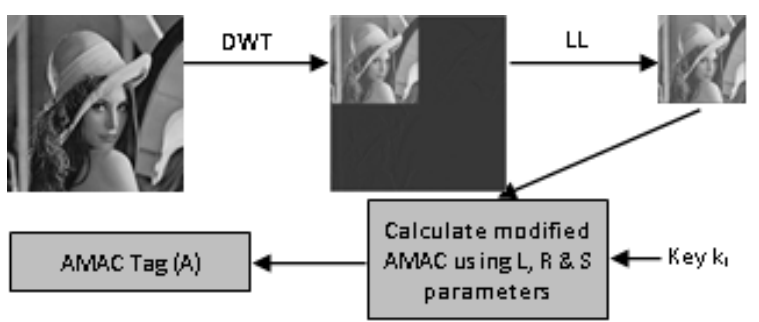

Figure 1. Watermark generation

\section{B. Watermark Embedding}

The watermark is self-embedded in the source image in this work. The source image is split into $8 \times 8$ pixel non overlapping blocks. The length of the AMAC tag is chosen to be 256 bits. The AMAC tag is split into 32 sub-AMACs of 8 bits each. One sub-AMAC is taken at a time and inserted into the Least Significant Bits (LSBs) of every $8^{\text {th }}$ pixel value inside the next image block. Since a block is chosen to be 8 $\times 8$ pixels, the block size is 64 pixels. To embed 8 bits, a total of 8 pixel values are modified. Instead of using the first 8 pixel positions, as in [10], every $8^{\text {th }}$ position is chosen. This enhancement spreads the watermark bits uniformly in the source image block and therefore the quality of the source image is not degraded considerably. The next image block for embedding a sub-AMAC is chosen randomly using a secret permutation, with the help of a secret key, $k_{2}$, as seed. This secret permutation makes it very hard for someone (an attacker), without the knowledge of the secret key $k_{2}$, to extract and replace the watermark. The watermarked image is the output of the watermark embedding process. It is assumed that the secret keys $\mathrm{k}_{1}$ and $\mathrm{k}_{2}$ are pre-shared using standard key exchange mechanisms.

\section{Watermark Extraction}

In order to prove the authenticity of the image, the watermark is extracted using the procedure as follows. The watermarked image is split into $8 \times 8$ pixel non overlapping blocks. The LSBs of every $8^{\text {th }}$ pixel's value of the next block (chosen randomly using the same seed $\mathrm{k}_{2}$ as used at the transmitter) is taken. These bits are appended together to obtain the modified-AMAC tag. This extracted watermark is used for image authentication.

\section{Image Authentication}

The image verification takes places by comparing the extracted watermark with the watermark recomputed on the image presented for authenticated. Since the watermark is embedded in the spatial domain, it distorts a minor part of the cover image. Authentication based on the standard mechanism will fail in this case. However, since AMAC is tolerant to modifications below a (chosen) threshold, the authentication succeeds in the presence of minor modifications.

\section{Simulation Results}

The simulations are performed using the following parameters. The dimension of source images used in the simulations is $256 \times 256$ pixels. The source image is converted into a grayscale image before any other processing. Single level DWT transform is performed on the resultant grayscale image giving four sub-bands, out of which the LL band is used for AMAC tag calculation. The length of modified-AMAC is chosen to be 256 bits and the length of sub-AMACs is chosen to be 8 bits. The watermark is generated using the strengthened AMAC tag as proposed in [11].

Simulation results for the following cases are presented.

\section{A. COFDM Transmission}

Orthogonal Frequency Division Multiplex (OFDM) is a crucial technique for modern and future mobile communications (4G, 5G and beyond). Together with Multiple-Input Multiple-Output (MIMO) systems, OFDM is a basis for high speed services to achieve a high Quality of Service (QoS), which is the main advantage of present over previous mobile generations. OFDM is interesting from application point of view as it is used in numerous applications like Digital Audio Broadcasting (DAB, DAB+), Digital Video Broadcasting - Terrestrial (DVB-T [2]), Worldwide Interoperability for Microwave Access (WiMAX), mobile communications 4G and 4.5G - Long Term Evolution (LTE) / LTE-A etc. Coded OFDM (COFDM) is used in case of extreme selective fading channels, e.g., in case that every subcarrier suffers from a different amount of noise measured with different signal-tonoise ratio (SNR). 
Results obtained in the presence of COFDM transmission are shown in Fig. 2. COFDM transmission is considered in the presence of regular LDPC codes of rate $1 / 2$ as the forward error correction codes. As all, or most, of the errors are corrected by the LDPC decoder, the image is declared authentic by the proposed algorithm.

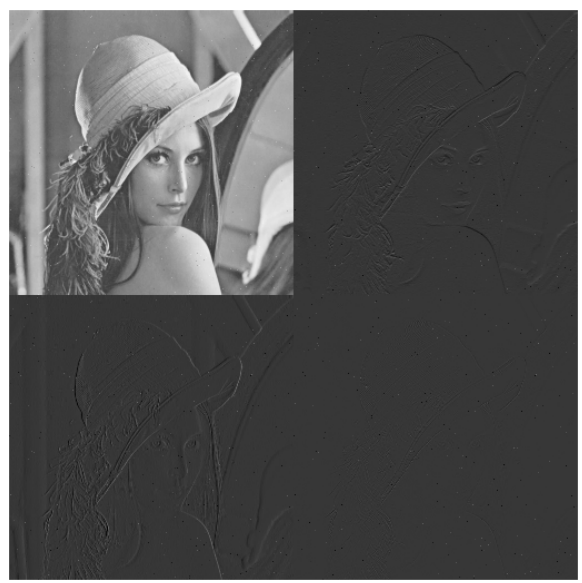

Figure 2. Lena image authentication in the presence of COFDM with rate $1 / 2$ LDPC codes

\section{B. Forgery Attack}

The authentication for a forged Lena image is shown in Fig. 3 (note the extra hair on the forehead). The LL band of the forged image has a Hamming distance of 17235 to the LL band of the original Lena image of similar dimension. This results in a failed authentication for the forged image.

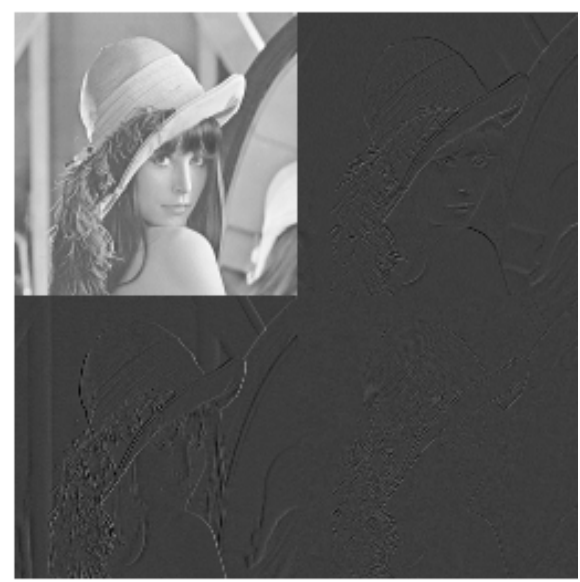

Figure 3. Lena image authentication in the presence of forgery attack (with extra hair on the forehead)

\section{SECURITY ANALYSIS}

In this section, the security analysis of the proposed scheme is given. The analysis of AMAC is given in [11], where it is proven that if Hamming distance is used for distance measurement then the scheme might not be secure for large messages, such as high resolution images. The security analysis of the used improved and strengthened AMAC is given in [11]-[12].

The security analysis of the proposed authentication technique is made by considering two major attacks as follows.

\section{A. Key Recovery Attack}

Two different keys are used in the proposed authentication technique. The first key $\left(\mathrm{k}_{1}\right)$ is used in watermark generation and the other $\left(\mathrm{k}_{2}\right)$ is used in watermark insertion. The attacker job is made difficult as he must recover two secret keys, e.g., through brute force attack. In AMAC, the reshaped matrix of size $\mathrm{R} \times \mathrm{L} \times \mathrm{S}$ bits is used, which means the attack complexity is about $2^{\mathrm{R} \times \mathrm{L} \times \mathrm{S}}$ function (tag generation and verification) operations, which is a very high complexity. For even the smaller image dimension used in the simulations, i.e., $256 \times 256$ pixels, this attack is computationally infeasible with the current technology.

\section{B. Substitution Attack}

A substitution attack can be executed in two steps, i.e., a forgery attack on the strengthened AMAC followed by an attack on the watermark embedding process. The possibility for an attacker to pass the first step can be calculated as follows. Let $T$ be the threshold value below which the difference between the AMAC tags is acceptable and let $t$ indicates the threshold for difference between DWT's LL sub-band tolerance. The probability $\left(\mathrm{P}_{\mathrm{t}}\right)$ of changes in the "majority" selection round of the AMAC is calculated in [16], as follows,

$$
P_{t}=\frac{1}{2^{L} \sum_{i=0}^{t}\left(\begin{array}{l}
L \\
i
\end{array}\right)} \sum_{i=0}^{t} \sum_{j=\left\{\frac{t+1-2 i}{2}\right\rfloor}^{\left\lfloor\frac{t-1}{2}\right\rfloor} \sum_{k=0}^{\left.\frac{2 i-2 j-t-1}{4}\right\rfloor}\left(\begin{array}{l}
t \\
j
\end{array}\right)\left(\begin{array}{l}
j \\
k
\end{array}\right)\left(\begin{array}{l}
t-j \\
i-k
\end{array}\right)
$$

Based on $\mathrm{P}_{t}$, the probability of deceiving the attacker $\left(\mathrm{P}_{\mathrm{D}}\right)$ by an image whose AMAC tag is close enough to the reference tag is calculated as,

$$
P_{D}=\sum_{i=0}^{T}\left(\begin{array}{l}
L \\
i
\end{array}\right) P_{t}^{i}\left(1-P_{t}\right)^{L-i}
$$

It can be observed from (3) that $\mathrm{P}_{\mathrm{D}}$ can be decreased by increasing the length of AMAC tag.

\section{CONCLUSION}

A robust watermarking technique for content based image authentication is proposed in the paper. The proposed technique consists of extracting image features using discrete wavelet transform, generating the watermark based on the image features and by protecting it using the noise tolerant 
AMAC algorithm. Simulation results are given to show the effectiveness of the proposed algorithm. A security analysis is provided in the end to analyze the security strength of the proposed scheme. In future, a complete mathematical framework, similar to the standard authentication mechanisms, is planned to be proposed for the robust authentication algorithms.

\section{REFERENCES}

[1] I. Cox, M. Miller, J. Bloom, J. Fridrich and T. Kalker, "Digital Watermarking and Steganography", Morgan Kaufmann, 2007.

[2] H. Fiestel, "Cryptography and Computer Privacy", Scientific American, May 1973, vol. 228, no. 5, pp. 15-23.

[3] O. Ur-Rehman, "Applications of iterative soft decision decoding". Aachen: Shaker Verlag; 2013. ISBN:978-3-8440-1641-3.

[4] R. Graveman and K. Fu, "Approximate message authentication codes," in Proceedings of $3^{\text {rd }}$ Fed. lab Symposium on Advanced Telecommuni-cations/Information Distribution, vol. 1, College Park, MD, Feb. 1999.

[5] ISO/IEC 9797-1:2011, "Information technology -- Security techniques -- Message Authentication Codes (MACs) -- Part 1: Mechanisms using a block cipher".
[6] ISO/IEC 9797-2:2011, "Information technology -- Security techniques -- Message Authentication Codes (MACs) -- Part 2: Mechanisms using a dedicated hash-function".

[7] C. Boncelet, "The NTMAC for authentication of noisy messages", IEEE Transactions on Information Forensics and Security, vol. 1, no. 1, pp. 35-42, Mar. 2006.

[8] Joint Channel Coding and Cryptography, Shaker Verlag, Aachen, 2008, ISBN 978-3-8322-7180-0.

[9] O. Ur-Rehman, N. Zivic, S.A.H.A.E. Tabatabaei, C. Ruland, "Error Correcting and Weighted Noise Tolerant Message Authentication Codes," $5^{\text {th }}$ International Conference on Signal Processing and Communication Systems (ICSPCS), pp. 1-8, December 12-14, 2011.

[10] O. Ur-Rehman, N. Živic, "Discrete Wavelet Transform based Watermarking for Image Content Authentication", $6^{\text {th }}$ International Conference on Patterns Recognition Applications and Methods, Feb 24-26, 2017, Porto, Portugal.

[11] D. Onien, R. Safavi-Naini, and P. Nickolas, "Breaking and repairing an approximate message authentication scheme," Discrete Mathematics, Algorithms and Applications, World Scientific Publishing Company, 2011.

[12] G. Di Crescenzo, R. F. Graveman, G. Arce and R. Ge, A Formal Security Analysis of Approximate Message Authentication Codes, Proc. of the 2003 CTA Annual Symposium, a US Dept. of Defense publication. 


\section{$1^{\text {st }}$ Workshop on Internet of Things-Enablers, Challenges and Applications}

T HE Internet of Things is a technology which is rapidly emerging the world. IoT applications include: smart city initiatives, wearable devices aimed to real-time health monitoring, smart homes and buildings, smart vehicles, environment monitoring, intelligent border protection, logistics support. The Internet of Things is a paradigm that assumes a pervasive presence in the environment of many smart things, including sensors, actuators, embedded systems and other similar devices. Widespread connectivity, getting cheaper smart devices and a great demand for data, testify to that the IoT will continue to grow by leaps and bounds. The business models of various industries are being redesigned on basis of the IoT paradigm. But the successful deployment of the IoT is conditioned by the progress in solving many problems. These issues are as the following:

- The integration of heterogeneous sensors and systems with different technologies taking account environmental constraints, and data confidentiality levels;

- Big challenges on information management for the applications of IoT in different fields (trustworthiness, provenance, privacy);

- Security challenges related to co-existence and interconnection of many IoT networks;

- Challenges related to reliability and dependability, especially when the IoT becomes the mission critical component;

- Zero-configuration or other convenient approaches to simplify the deployment and configuration of IoT and self-healing of IoT networks;

- Knowledge discovery, especially semantic and syntactical discovering of the information from data provided by IoT;

The IoT conference is seeking original, high quality research papers related to such topics. The conference will also solicit papers about current implementation efforts, research results, as well as position statements from industry and academia regarding applications of IoT. The focus areas will be, but not limited to, the challenges on networking and information management, security and ensuring privacy, logistics, situation awareness, and medical care.

\section{TOPICS}

The IoT conference is seeking original, high quality research papers related to following topics:

- Future communication technologies (Future Internet; Wireless Sensor Networks; Web-services, 5G, 4G, LTE, LTE-Advanced; WLAN, WPAN; Small cell Networks...) for IoT,
- Intelligent Internet Communication,

- IoT Standards,

- Networking Technologies for IoT,

- Protocols and Algorithms for IoT,

- Self-Organization and Self-Healing of IoT Networks,

- Trust, Identity Management and Object Recognition,

- Object Naming, Security and Privacy in the IoT Environment,

- Security Issues of IoT,

- Integration of Heterogeneous Networks, Sensors and Systems,

- Context Modeling, Reasoning and Context-aware Computing,

- Fault-Tolerant Networking for Content Dissemination,

- Architecture Design, Interoperability and Technologies,

- Data or Power Management for IoT,

- Fog-Cloud Interactions and Enabling Protocols,

- Reliability and Dependability of mission critical IoT,

- Unmanned-Aerial-Vehicles (UAV) Platforms, Swarms and Networking,

- Data Analytics for IoT,

- Artificial Intelligence and IoT,

- Applications of IoT (Healthcare, Military, Logistics, Supply Chains, Agriculture, ...),

- E-commerce and IoT.

The conference will also solicit papers about current implementation efforts, research results, as well as position statements from industry and academia regarding applications of IoT. Focus areas will be, but not limited to above mentioned topics.

\section{SECTION EDITORS}

- Cao, Ning, College of Information Engineering, Qingdao Binhai University

- Furtak, Janusz, Military University of Technology, Poland

- Zieliński, Zbigniew, Military University of Technology, Poland

\section{REVIEWERS}

- Amanowicz, Marek, Military University of Technology

- Antkiewicz, Ryszard, Military University of Technology, Poland

- Chudzikiewicz, Jan, Military University of Technology in Warsaw, Poland

- Cui, Huanqing, Shandong University of Science and Technology, China 
- Ding, Jianrui, Harbin Institute of Technology, China

- Fouchal, Hacene, University of Reims ChampagneArdenne, France

- Fuchs, Christoph, Fraunhofer Institute for Communication, Information Processing and Ergonomics FKIE, Germany

- Ghamri-Doudane, Yacine, Université La Rochelle

- Gluhak, Alexander, Intel Labs Europe

- Higgs, Russell, University College Dublin, Ireland

- Hodoň, Michal, University of Žilina, Slovakia

- Johnsen, Frank Trethan, Norwegian Defence Research Establishment (FFI), Norway

- Krco, Srdjan, DunavNET

- Lenk, Peter, NATO Communications and Information Agency, Other

- Li, Guofu, University of Shanghai for Science and Technology, China

- Ma, Fumin, Nanjing University of Finance and Economics, China

- Marks, Michat, NASK - Research and Academic Computer Network, Poland

- Murawski, Krzysztof, Military University of Technol- ogy, Poland

- Niewiadomska-Szynkiewicz, Ewa, Research and Academic Computer Network (NASK), Institute of Control and Computation Engineering, Warsaw University of Technology

- Paprzycki, Marcin, Systems Research Institute Polish Academy of Sciences, Poland

- Sabir, Essaid, Hassan II University of Casablanca

- Sikora, Andrzej, Research and Academic Computer Network (NASK)

- Skarmeta, Antonio, University of Murcia

- Suri, Niranjan, Institute of Human and Machine Cognition

- Wrona, Konrad, NATO Communications and Information Agency

- Xu, Jian, Northeastern University, China

- Xu, Lina, University College Dublin, Ireland

- Zhang, Tengfei, Nanjing University of Post and Telecommunication, China

- Zhao, Yongbin, Shijiazhuang Tiedao University, China

- Zheng, Lijuan, Shijiazhuang Tiedao University, China

- Zhou, Wei, Qingdao Technological University, China 


\section{Leveraging virtualization for scenario based IoT application testing}

\author{
Tomasz Szydlo \\ AGH University of Science and Technology, \\ Department of Computer Science, Krakow, Poland \\ Email: tszydlo@agh.edu.pl
}

\author{
Joanna Sendorek \\ AGH University of Science and Technology, \\ Department of Computer Science, Krakow, Poland \\ Email: send.joanna@gmail.com
}

\begin{abstract}
From day to day we can observe an increasing adoption of Internet of Things in the world. Number of devices connected to the Internet is constantly growing. At the same time, the applications are getting more and more complex and its development becomes more difficult and requires testing. Access to the IoT testbed and possibility to execute the subsequent version of application in the same environmental conditions is desirable. In the paper, we present the concept of virtual testing environment which enables a possibility to create on demand Linux virtual devices which have access to virtual sensors and effectors deployed in the emulated environment driven by the user defined scenarios.
\end{abstract}

\section{INTRODUCTION}

$\mathbf{T}$ HE Internet of Things settles rapidly in the world. Not only in the Smart City initiatives [1], smart homes, agriculture [2], but also enterprise adoption of the Internet of Things (IoT) [3] is starting to gain momentum. The concept of Industry 4.0 where machines will communicate directly with each other providing more flexible production setup and ability to react to changing conditions, increases the data variety, volume and the necessity of data processing at the network edge [4].

IoT applications are getting more and more complex which causes their implementation very challenging and error prone thus the application testing is necessary. There are several tools simplifying testing process. For example, in cloud platforms [5] such as Samsung Artik or IBM IoT there are the device simulation modules which are able to generate randomized data in order to verify the processing in the clouds. At the same time, WSN testbeds [6] are designed to verify low level interactions between devices. Such testbeds constitutes of several, sometimes even hundreds or thousands, of nodes deployed in the particular place. Additional infrastructure enables a possibility for node programming and debugging. There are also projects aimed at testing networking in the virtual environments. The ns-3 project [7] is a discrete event network simulator developed for networking research using the network and protocols models. In contrast, the CORE [8] emulator is a tool for emulating networks on one or more machines using lightweight Linux virtualization. Unfortunately, these solutions does not allow testing the whole IoT stack for datadriven application where the processing depends on the data gathered by the sensors. This is especially important in the Fog Computing concept [9], [10] where computations from the cloud are moved closer to the sensors in order to decrease the processing latency and data throughput from the sensors to the cloud through the Internet [4].

In the paper, we propose the concept of virtualized platform for testing IoT applications which enables a possibility to create virtual devices which along with real embedded devices could be put in the emulated environment driven by the scenarios. Devices instead of reading data from real sensors will be using virtual ones located in the virtual environment. This enables a possibility to repetitively execute the testing scenarios and observing how the new versions of tested application behave facing the same readings from the sensors. Moreover, such virtual environment composed of the virtual devices can be set up on almost any computer simplifying the development process of the IoT applications. In the paper we are focusing on the Linux based IoT devices but the presented concept can be further extended to cover other types of the devices.

The scientific contributions of the paper are: (i) introduction of Linux based embedded devices virtualization concept (ii) introduction of sensors virtualization concept (iii) event-driven environment emulation framework (iv) use case showing the functionalities of the framework.

The rest of the paper is organized as follows. Section II describes the virtualization of linux based IoT devices. In section III the scenario based testing and the environment emulation is discussed, while in section IV case study is presented. The last section summarizes the paper.

\section{ViRTUALIZATION OF LINUX BASED IOT DEVICES}

Operating systems based on the Linux kernel are used in embedded systems such as consumer electronics, personal video recorders, networking devices, industrial automation and IoT. They differs in kernel versions, available modules and standard libraries. Nevertheless, due to the fact that they are all based on Linux kernels, most of the software can be used on any of these platforms.

In the Linux OS for embedded systems, access to the device peripherals is usually achieved by the files in the / sys folder which are there exposed by the kernel modules. Pin numbers and their functionalities varies between embedded boards, so the applications have to be customized for particular hardware. 
In order to simplify the application development, Intel implemented the two libraries - MRAA ${ }^{1}$ and $\mathrm{UPM}^{2}$. First one is for low speed IO communication in $\mathrm{C}$ with bindings for $\mathrm{C}++$, Python, Node.js and Java. It supports generic platforms, as well as Intel Edison, Intel Joule and Raspberry Pi. It provides access, among others, to the GPIO (General Purpose Input/Output), ADC (analog-to-digital converter), $\mathrm{I}^{2} \mathrm{C}$ (Inter-Integrated Circuit) and UART (universal asynchronous receiver/transmitter) interfaces. The second one - UPM - is a high level repository that provides software drivers for a wide variety of commonly used sensors and effectors. It contains routines for accessing real sensors such as temperature sensors e.g. Texas Instruments LM $35^{3}$, humidity e.g. Sensirion SHT $31^{4}$ and others. These software drivers interact with the underlying hardware platform through calls to MRAA APIs. The idea is presented in Fig.1a.

For the purpose of device virtualization, it was necessary to virtualize the device and the hardware interfaces for connecting external peripherals and sensors. We have decided to virtualize the device using Docker containers, while for hardware interfaces virtualization, two solutions were considered:

- implementation of Linux kernel modules emulating the real hardware and placing particular files in / sys folder,

- modification of the system libraries that covers nuances of linux distributions and provides unified API for the users - in that case the aforementioned Intel MRAA which is open sourced and used in several projects.

We have decided to implement interface virtualization by modifying MRAA library. The decision was dictated by the fact, that the library covers several hardware platforms and enables possibility to use UPM library. Communication between the modified v-MRAA library on the Linux based device (real or Docker based) and the emulated environment is message driven. It is based on MQTT which is a much used light-weight publish/subscribe communication protocol. Currently, the GPIO and ADC peripherals are supported. Realization of the I2C and UART protocols is in progress and is out of scope of this paper. Fig.1b depicts the situation where embedded device is connected to virtual sensors through emulated interface. In order to use UPM library for reading values from virtual sensors using routines for real ones, their internal logic must be implemented in environment emulation framework, which is discussed in the next section.

Fig.1c depicts the situation, where the real embedded device instead of real sensors is connected to sensor emulation module. The concept is further elaborated in the previous work [11].

\section{SCENARIO BASED TESTING}

One of the biggest issues behind complex system testing of IoT solutions, which are based on sensors and real data

\footnotetext{
${ }^{1}$ http://mraa.io

${ }^{2} \mathrm{https}: / /$ github.com/intel-iot-devkit/upm

${ }^{3}$ www.ti.com

${ }^{4}$ https://www.sensirion.com
}

processing, is lying in the unpredictability of surrounding world and its changing conditions being the input for the system. While the separate components could be mocked for testing purposes, the necessity for being able to reproduce particular changes of the whole conditions, often dependent on each other, seems to be gaining in importance with more emerging IoT applications based on sensors measurements. The concept of applying the scenario-based testing to simulate behavior of external conditions, its influence on devices and interactions between them, is presented in this paper. We have made assumption that not only should person writing a scenario be able to specify exactly what is happening at the given time or under certain conditions, but also be able to apply potentially non deterministic mathematical model as the simulation base. This approach enables testing of the whole application including data analysis models and real data processing. In the least complex variant, the mathematical model could be reduced to replaying already recorded measurements.

\section{A. Environment emulation}

Emulation model presented in the paper consists of two main entities: (i) the world representing all parameters which can be measured and which are changing along with a particular model and (ii) observers which are able to read specified values of world's parameters. World itself has space mapped to measurement vectors $\mathrm{V}=\left(v_{0}, v_{1}, v_{2}, \ldots\right)$ where $v_{i}$ is the specific property of world able to be measured by sensors in the real world and to be observed by observers in the emulation model e.g. temperature, humidity etc. Main idea behind the proposed environment construction is the separation of the world which is responsible for simulating physical mechanisms from observers which main task is to read world's state and convey it further to the actual device (real or virtual) and sensors. Hence, observers have the role of data providers to the devices and their components and world acts as the source of this data. Observers are reading data from the world in the moments meeting requirements defined in the scenario while the world is transforming itself starting from the initial state.

One of the challenges coming with the construction of scenario and its execution have been the ability to make observers responsive to certain situations occurring during world transformation. In certain cases, it could be satisfactory to make observers read world state at specified moments in time domain. However, while this approach is closest to the real-world behavior of sensors, it does not address few important issues. First of all, running any transformation based on mathematical computations on computer gives no guarantee as to the length of intervals occurring between iterations of the simulation. Because complexity of model have impact on this length, this could lead to the point when observer reads data from the world while part of the vectors are still at previous state while the others are already in the next one. Another problem could arise when it would be necessary to synchronize few observers or make particular observations consecutive and dependent on each other. Finally, the nondeterministic 

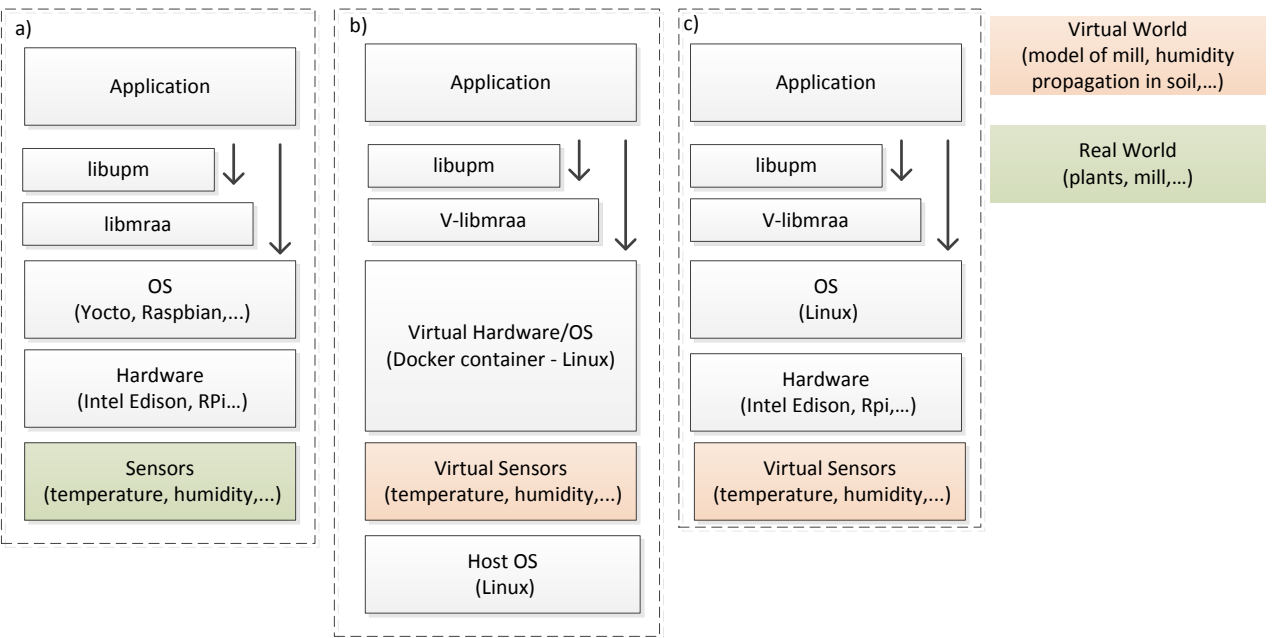

Fig. 1. Access to the sensors on Linux based devices

nature of applied mathematical model may cause some specific circumstances occur in unpredictable moments in time.

All of the mentioned issues decide on insufficiency of the time-based procedural scenario execution. Solution proposed in the paper is inspired by the reactive programming paradigm gaining lately on popularity. This lead to implementation of event-based scenario framework in Python, which enables both time based and reactive observing. In order to inform observers that world's state has already changed, special events can be produced after each iteration cycle which world needs to transform its measurement vectors from state $x_{k}$ to state $x_{k}+1$. In the proposed solution, scenario consist of steps - function declarations written in Python which can be decorated using predefined decorators in order to decide on moment of their execution. Decorators provided by framework for the time of writing are:

Time-based:

- @every (start=..., seconds=...) - periodic execution

- eafter ( $\operatorname{seconds=...)~-~one-time~execution~at~}$ given moment

Event-based:

- @every_event (event=...) - execution on the each occurrence of event

- eon_event (event=...) - one-off execution on occurrence of event

Example 3.1 (Python): Example scenario written in Python framework

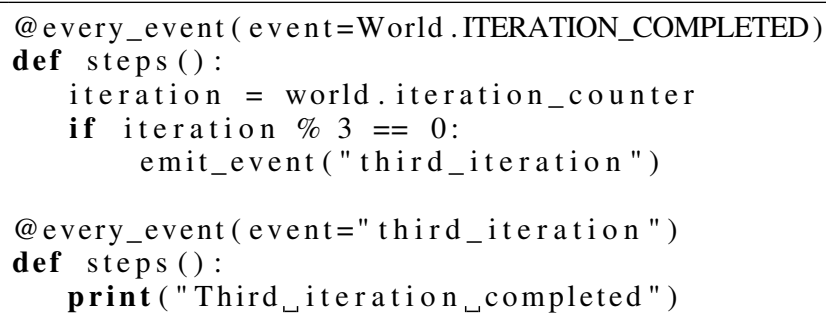

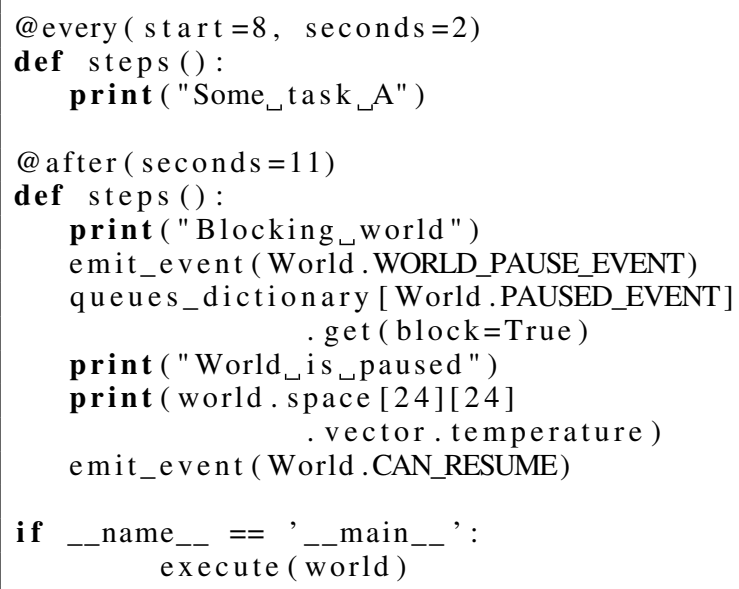

Example 3.1 presents scenario which consists of four function declarations, each representing different behavior of observer corresponding with specific event or time moment, declared in the decorator for function. Each event occurring during scenario execution has to be correlated with at most one observer function declaration, however it may be also generated or reproduced during function execution as presented in first declaration.

The idea of observer functions being able to withhold execution of world transformation stems from the necessity for confirming that world state read by observer after one completed iteration has not yet been modified by the following one. Implementation of world being withholdable on WORLD_PAUSED_EVENT should be the responsibility of programmer implementing world thus making it responsive to any events in the more complex example. Described mechanism is also enabling few observers to act during the same interval between particular two iterations.

As the possible development direction for framework, we consider adding effector functions which would have the 
ability not only to read world state at particular moment and pause world transformation, but also to influence actual way of transformation e.g. modifying underlying mathematical model responsible for data generation.

Underlying implementation of framework is based on blocking queues stored in the common dictionary, which is mapping event name to corresponding queue. When particular observing function declaration in scenario is decorated, the decorator handles execution of this function based on events appearing in the queue. When new event name is being detected during scenario execution, appropriate queue is added to the queues dictionary.

\section{B. Sensor virtualization}

During the scenario, observers read the particular values from the virtual world as physical values e.g. temperature. In order to read that value by the virtual device using v-MRAA and UPM libraries, the decision should be made which type of hardware sensor should be virtualized - for example DS18B20 by Maxim or LM35 by Texas Instruments.

In the case of LM35 temperature sensor, the voltage provided by the sensor is linearly related to the temperature $(0 \mathrm{mV}$ $\left.+10.0 \mathrm{mV} /{ }^{\prime} \mathrm{C}\right)$. The voltage can be measured by the ADC input on the virtual device. The python class LM35_V in the emulator framework has the method update_temp ( $t e m p$ ) which computes the voltage that would be generated by the real sensor measuring particular temperature $t$. The voltage is then converted to the 10bit $\mathrm{ADC}$ value $f_{A D C}(t)$ which is sent to the v-MRAA library on the device using MQTT protocol. The maximum voltage measured by the ADC is $5 \mathrm{~V}$. The equation is as follows:

$$
f_{A D C}(t)=t \frac{10 m V}{1^{\circ} C} \frac{2^{10}-1}{5 V}=\frac{t * 1023}{500}
$$

On the virtual device, the application that uses UPM library routine for LM35 sensor will read the already computed voltage value and will convert it to the proper temperature. When the same application would be executed on the real embedded device with LM35 temperature sensor, the value would be read from the real environment.

\section{Automation OF ENVIRONMENT SETUP}

Building emulation environment described in this paper is done with enclosing each virtual device in one Docker container thus isolating them from platform they would be run on. Usage of Docker containers resolves many problems including running each virtual device independently of the others or ensuring that software required by devices is provided. However, one still has to have Docker platform installed on the machine which will be hosting containers thus making whole environment not fully independent from platform it is build on. What is more, when running multiple virtual devices, one has to repeat the same process of running each container from the same image and setting environment variables for it. In the solution presented in the paper, we aim at environment portable, easy to maintain and as automated as possible. Fig. 2 depicts the idea of using automation tools for setting up virtualized devices. In the proposed implementation, we are using Ansible, which enables defining all platform setup e.g. installation of required software in YAML files called playbooks. Ansible also has build-in support for docker orchestration which enables creating images from Dockerfiles and running them in the containers.

While usage of Ansible and in general, automation tools, resolves inconvenience stemming from executing repetitive tasks for each instance of virtual device, it does not address issues coming from platform-dependence of installed software. In particular, Linux-oriented playbook will not work when run on Windows operating system and vice versa. Therefore, we have provided additional virtual machine as a docker host and made use of Vagrant tool, which enables building virtual environments which are portable and platform-independent. Vagrant has also support for Ansible provisioning thus making all three tools working together well and providing means for easy to build environment.

Fig 2 depicts the tools stack used to set environment up and automate the process.

\section{USE CASE AND VERIFICATION}

In order to present the idea of virtual testing environment we have constructed example cloud based IoT application whose purpose was to read temperature from the environment by the two devices and send that data to the cloud platform. As the cloud platform handling devices data, we used the Samsung Artik Cloud ${ }^{5}$ platform and applied simple case of plotting data. For temperature reading We decided to use the aforementioned LM35 sensors.

In order to test the whole layers of the application during development, some data had to be provided so we constructed two virtual devices. Data was generated by simulation framework and then conveyed to devices as the emulated temperature measurements. Whole test was executed due to scenario constructed in the framework described in section III.

After building emulation environment as described in the previous paragraph, we executed the example scenario. Code for this scenario, composed with framework characterized previously in the paper, is presented by example 5.1.

Example 5.1 (Python): Use-case scenario

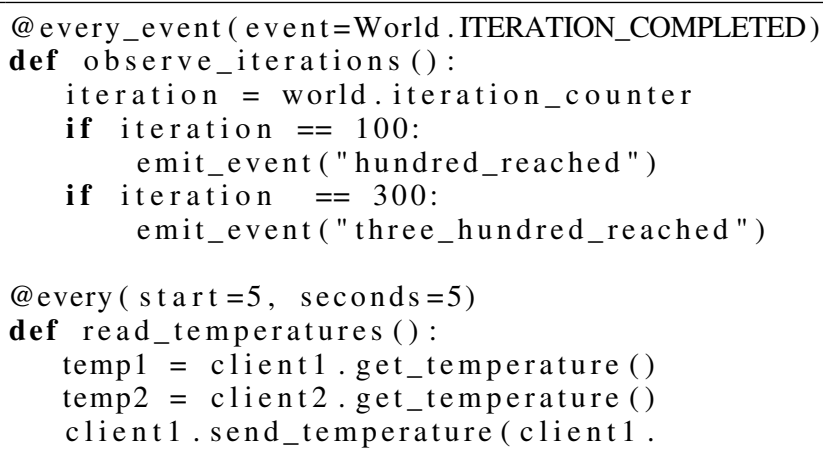

${ }^{5} \mathrm{https} / / /$ artik.cloud 


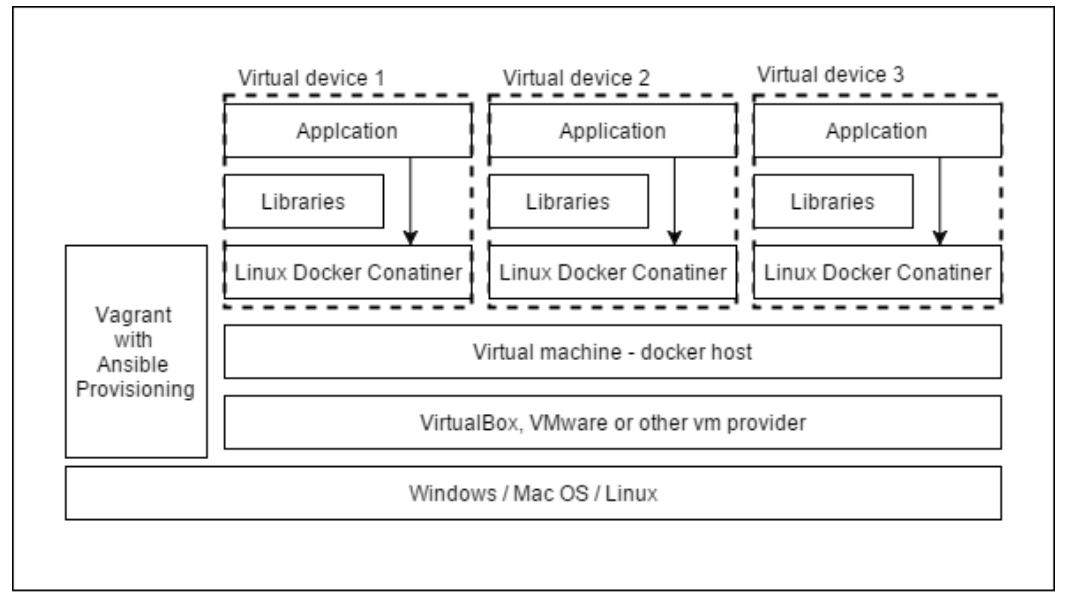

Fig. 2. Virtualization tools applied to building environment

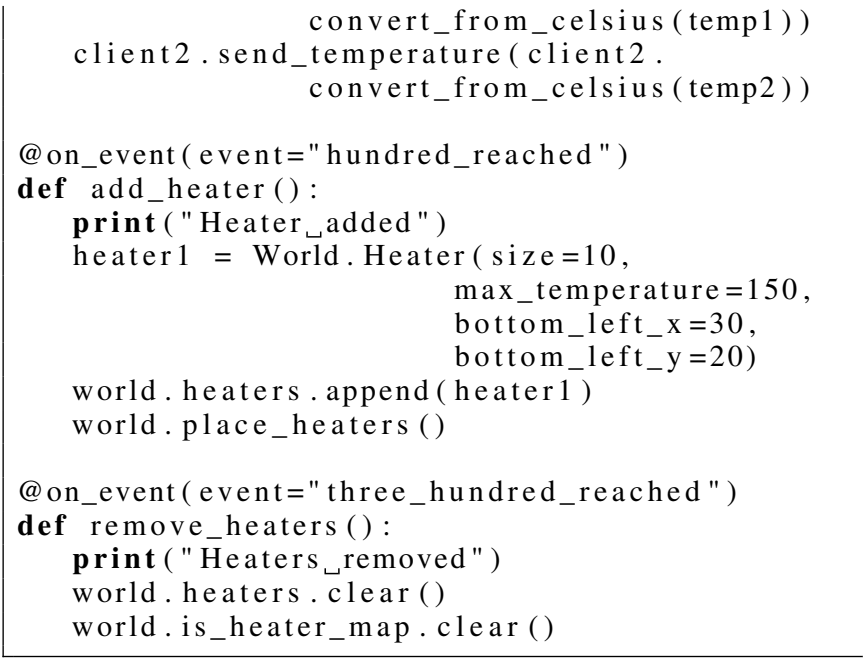

Simulation is based on the simplified heat diffusion process in the two-dimensional space with sensors being able to read temperature at the given points in space. Presented scenario consists of four observer functions. Three of them are event-based and are executed on the occurrence of events connected with iterations. The remaining function is being executed periodically in the time domain, at each five seconds starting from fifth one. Function observe_iterations is responsible for emiting events, based on the value of world's iteration counter that are handled by the other functions. Function read_temperatures which is working in the time domain, is responsible for providing data to the MQTT clients, which are then sending messages to the devices in simulation environment. Example 5.2 is presenting the initialization code for both clients.

Example 5.2 (Python): Initialization of MQTT clients

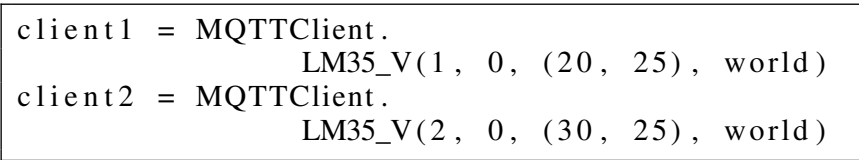

The clients have the role of virtualized temperature sensors LM35 and are instances of class LM35_V, which is taking as constructor arguments: device id, pin id, position of the sensor in the simulation space and the world which is main simulation entity, described in the previous paragraphs. First two parameters are determining MQTT topics, to which the data will be send.

World is starting with two dimensional space of size 50 by 50 units. Space is discretely sampled and each point of the space is mapped to the measurement vector, holding information on temperature value. One heating device with temperature of 150 Celsius is placed at $(15,25)$ and with each iteration, heat is diffusing on the space with simple mathematical model, reduced to replacing temperature value for the point with average of temperature values of its neighbors. Second heater will be placed in the world during scenario execution. Frequency of world's iterations is $1 \mathrm{sec}$.

Functions add_heater and remove_heaters are de facto more effectors than observer functions, because they influence the behavior of the world by placing additional heater and removing all heaters respectively. However, they do not use any synchronization mechanism described in the previous paragraphs and therefore behave in a manner similar to this of observer functions. The example is presenting different possibilities coming with current state of simulation framework, but the aspects of synchronization and separating observer functions from effector functions remain open.

In order to provide some verification for readings made by read_temperatures function, we implemented simple plotter that use matplotlib library for world simulation. Therefore, each fifty iteration, the plot of world state was generated. Fig. 4 shows plots of heatwave generated after $50,150,300$ and 350 iterations that corresponds to 50,150, 300 and 350 seconds since the beginning of the testing scenario, while Fig. 3 depicts graphs of data received by both devices. "Usecase_temp3" corresponds to device with virtualized sensor placed at coordinate $(20,25)$ - device A on figure and "Usecase_temp4" corresponds to device with 


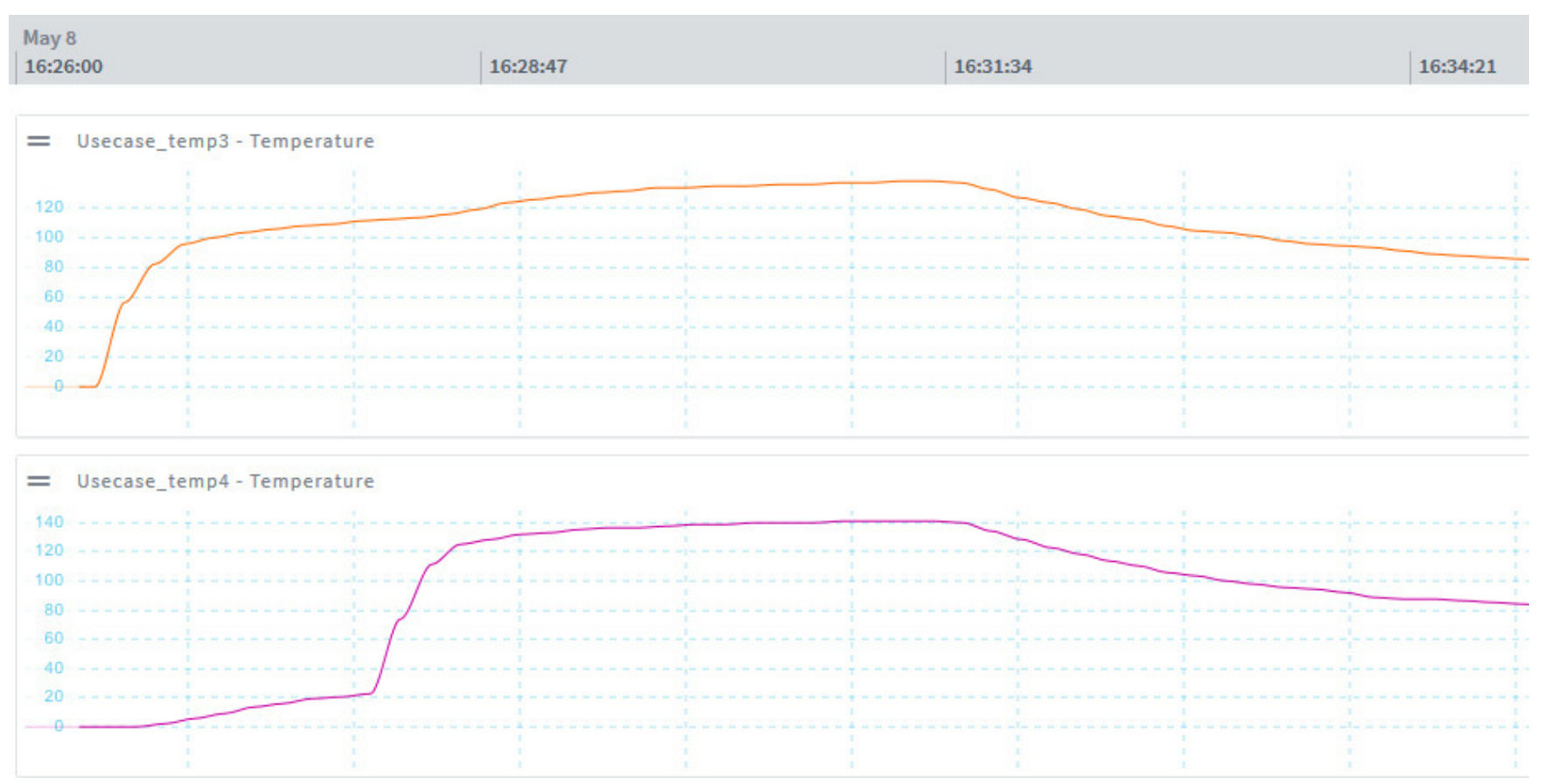

Fig. 3. Plots of data received by device in Samsung Artik Cloud
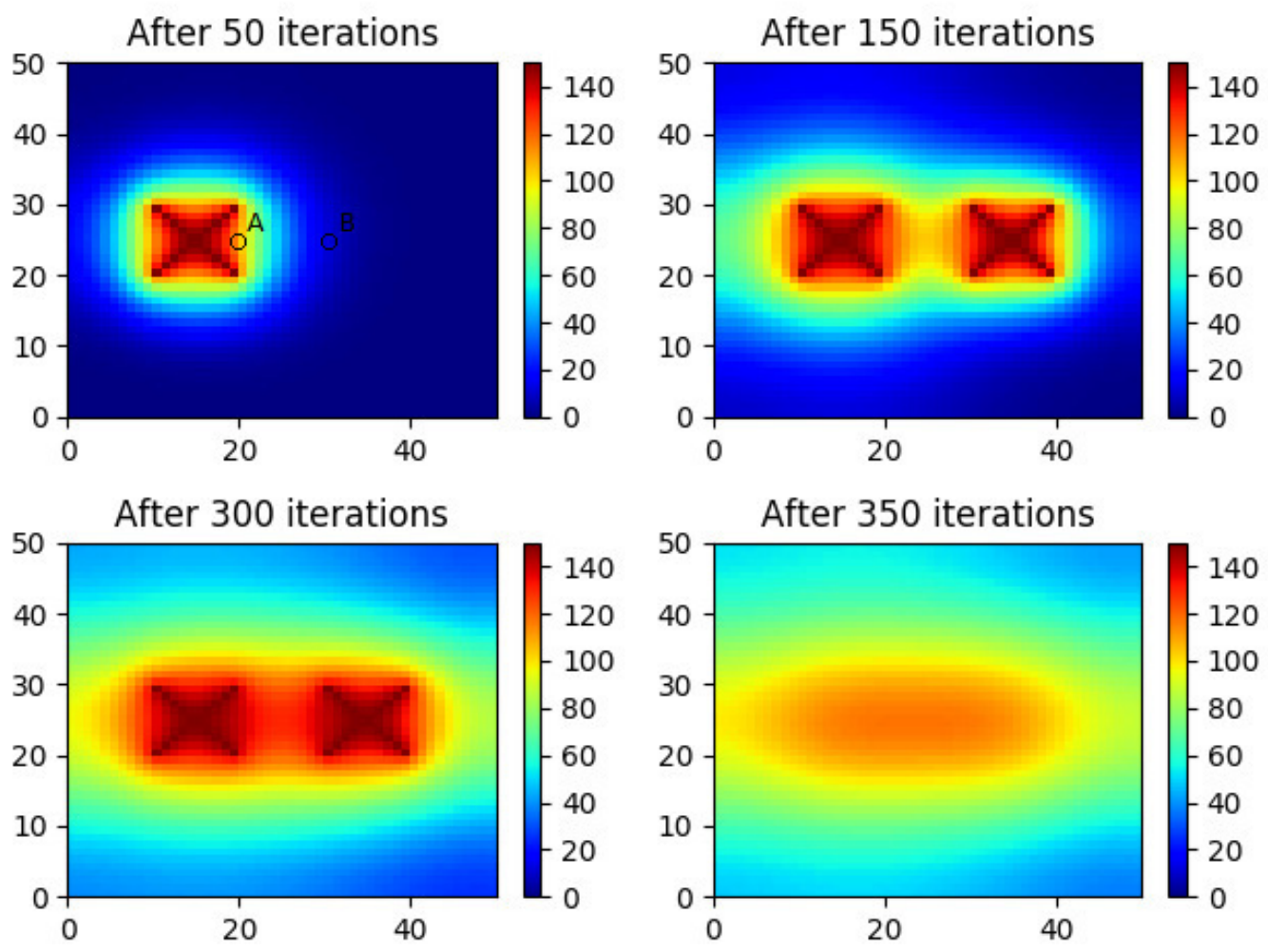

Fig. 4. Plots of world's state corresponding with data received by device 
virtualized sensor placed at $(30,25)$ - device B. There can be observed how firstly, only one sensor was noticing significant changes of temperature, then after 100 seconds the second one started and finally after about 300 seconds, both sensors started noticing declining temperatures.

The presented use case shows how the virtualized testing platform can be used for testing data-driven IoT applications. The virtual machine with docker containers representing virtual devices where executed on i5 notebook with 8GB of RAM, while the emulation environment was executed on i3 desktop computer with 4GB of RAM. During the experiments, the MQTT broker at iot.eclipse.org has been used. It proves that the framework can be executed even on development computers simplifying process of IoT applications development.

\section{SUMMARY}

In the paper we have presented the concept of virtualized testbed for scenario based IoT application testing. The concept has been verified by the use case where the virtual environment was used for testing application that measures the temperature using IoT devices and analyzes it in the cloud platform.

As a future work, concept could be extended by adding protocols for $\mathrm{I}^{2} \mathrm{C}$ and UART interfaces in v-MRAA library. It would extend the list of possible sensors that could be used by applications. Additionally, the external sensor emulation board would be useful for testing real devices - in that case instead of real sensors, such emulation boards could be connected and driven by the scenarios. Finally, the environment emulation framework can be extended by synchronization concepts simplifying implementation of effectors wanting to change the virtual worlds.

\section{ACKNOWLEDGMENT}

The research presented in this paper was partially supported by the National Centre for Research and De- velopment (NCBiR) under Grant No. LIDER/15/0144/L7/15/NCBR/2016.

\section{REFERENCES}

[1] A. Zanella, N. Bui, A. Castellani, L. Vangelista, and M. Zorzi, "Internet of Things for Smart Cities," IEEE Internet of Things Journal, vol. 1, no. 1, pp. 22-32, Feb 2014.

2] J. Shenoy and Y. Pingle, "IOT in agriculture," in 2016 3rd International Conference on Computing for Sustainable Global Development (INDIACom), March 2016, pp. 1456-1458.

[3] L. D. Xu, W. He, and S. Li, "Internet of Things in Industries: A Survey," IEEE Transactions on Industrial Informatics, vol. 10, no. 4, pp. 22332243, Nov 2014.

[4] R. Brzoza-Woch, M. Konieczny, P. Nawrocki, T. Szydlo, and K. Zielinski, "Embedded systems in the application of fog computing - levee monitoring use case," in 11th IEEE Symposium on Industrial Embedded Systems, SIES 2016, Krakow, Poland, May 23-25, 2016. IEEE, 2016, pp. 238-243. [Online]. Available: http://dx.doi.org/10.1109/SIES.2016.7509437

[5] P. P. Ray, "A survey of IoT cloud platforms," Future Computing and Informatics Journal, pp. -, 2017.

[6] A. Gluhak, S. Krco, M. Nati, D. Pfisterer, N. Mitton, and T. Razafindralambo, "A survey on facilities for experimental Internet of Things research,” IEEE Communications Magazine, vol. 49, no. 11, pp. 58-67, November 2011.

[7] G. F. Riley and T. R. Henderson, "The ns-3 Network Simulator." in Modeling and Tools for Network Simulation, K. Wehrle, M. GÃijnes, and J. Gross, Eds. Springer, 2010, pp. 15-34.

[8] J. Ahrenholz, C. Danilov, T. R. Henderson, and J. H. Kim, "CORE: A real-time network emulator," in MILCOM 2008 - 2008 IEEE Military Communications Conference, Nov 2008, pp. 1-7.

[9] F. Bonomi, R. Milito, J. Zhu, and S. Addepalli, "Fog computing and its role in the Internet of Things," in Proceedings of the first edition of the MCC workshop on Mobile cloud computing. ACM, 2012, pp. 13-16.

[10] S. Yi, C. Li, and Q. Li, "A survey of fog computing: concepts, applications and issues," in Proceedings of the 2015 Workshop on Mobile Big Data. ACM, 2015, pp. 37-42.

[11] R. Kaploniak, L. Kwiatkowski, and T. Szydlo, "Environment emulation for WSN testbed," Computer Science (AGH), vol. 13, no. 3, pp. 101-112, 2012. [Online]. Available: http://dx.doi.org/10.7494/csci.2012.13.3.101 



\section{Information Technology for Management, Business $\&$ Society}

T4MBS is a FedCSIS conference area aiming at integrating and creating synergy between FedCSIS events that thematically subscribe to the disciplines of information technology and information systems. The IT4BMS area emphasizes the issues relevant to information technology and necessary for practical, everyday needs of business, other organizations and society at large. This area takes a sociotechnical view on information systems and relates also to ethical, social and political issues raised by information systems. Events that constitute IT4BMS are:

- AITM' $17-15^{\text {th }}$ Conference on Advanced Information Technologies for Management

- ISM'17 - $12^{\text {th }}$ Conference on Information Systems Management

- IT4L'17-5 $5^{\text {th }}$ Workshop on Information Technologies for Logistics

- KAM'17-23 $3^{\text {rd }}$ Conference on Knowledge Acquisition and Management 



\section{$15^{\text {th }}$ Conference on Advanced Information Technologies for Management}

W E are pleased to invite you to participate in the $14^{\text {th }}$ edition of Conference on "Advanced Information Technologies for Management AITM'17'. The main purpose of the conference is to provide a forum for researchers and practitioners to present and discuss the current issues of IT in business applications. There will be also the opportunity to demonstrate by the software houses and firms their solutions as well as achievements in management information systems.

\section{TOPICS}

- Concepts and methods of business informatics

- Business Process Management and Management Systems (BPM and BPMS)

- Management Information Systems (MIS)

- Enterprise information systems (ERP, CRM, SCM, etc.)

- Business Intelligence methods and tools

- Strategies and methodologies of IT implementation

- IT projects \& IT projects management

- IT governance, efficiency and effectiveness

- Decision Support Systems and data mining

- Intelligence and mobile IT

- Cloud computing, SOA, Web services

- Agent-based systems

- Business-oriented ontologies, topic maps

- Knowledge-based and intelligent systems in management

\section{SECTION EDITORS}

- Andres, Frederic, National Institute of Informatics, Tokyo, Japan

- Dudycz, Helena, Wrocław University of Economics, Poland

- Dyczkowski, Mirosław, Wrocław University of Economics, Poland

- Hunka, Frantisek, University of Ostrava, Czech Republic

- Korczak, Jerzy, Wrocław University of Economics, Poland

\section{REVIEWERS}

- Abramowicz, Witold, Poznan University of Economics, Poland

- Ahlemann, Frederik, University of Duisburg-Essen, Germany

- Atemezing, Ghislain, Mondeca, Paris, France

- Brown, Kenneth, Communigram SA, France

- Cortesi, Agostino, Università Ca' Foscari, Venezia, Italy

- Czarnacka-Chrobot, Beata, Warsaw School of Economics, Poland
- De, Suparna, University of Surrey, Guildford, United Kingdom

- Dufourd, Jean-François, University of Strasbourg, France

- Franczyk, Bogdan, University of Leipzig, Germany

- Januszewski, Arkadiusz, UTP University of Science and Technology in Bydgoszcz, Poland

- Kannan, Rajkumar, Bishop Heber College (Autonomous), Tiruchirappalli, India

- Kersten, Grzegorz, Concordia University, Montreal, Poland

- Kowalczyk, Ryszard, Swinburne University of Technology, Melbourne, Victoria, Australia

- Kozak, Karol, TUD, Germany

- Leyh, Christian, Technische Universität Dresden, Chair of Information Systems, esp. IS in Manufacturing and Commerce, Germany

- Ligezza, Antoni, AGH University of Science and Technology, Poland

- Ludwig, André, University of Leipzig, Germany

- Magoni, Damien, University of Bordeaux - LaBRI, France

- Michalak, Krzysztof, Wroclaw University of Economics, Poland

- Owoc, Mieczyslaw, Wroclaw University of Economics, Poland

- Pankowska, Malgorzata, University of Economics in Katowice, Poland

- Pinto dos Santos, Jose Miguel, AESE Business School Lisboa

- Rot, Artur, Wroclaw University of Economics, Poland

- Stanek, Stanislaw, General Tadeusz Kosciuszko Military Academy of Land Forces in Wroclaw, Poland

- Surma, Jerzy, Warsaw School of Economics, Poland and University of Massachusetts Lowell, United States

- Teufel, Stephanie, University of Fribourg, Switzerland

- Tsang, Edward, University of Essex, United Kingdom

- Wątróbski, Jarosław, West Pomeranian University of Technology in Szczecin, Poland

- Wendler, Tilo, Hochschule fur Technik und Wirtschaft Berlin

- Wolski, Waldemar, University of Szczecin, Poland

- Zanni-Merk, Cecilia, INSA de Rouen, France

- Ziemba, Ewa, University of Economics in Katowice, Poland 



\section{Commitment Modeling}

\author{
Frantisek Hunka \\ University of Ostrava, Faculty of \\ Science \\ Dvorakova 7, 70103 Ostrava 1 \\ Czech Republic \\ Email: frantisek.hunka@osu.cz
}

\author{
Jaroslav Zacek \\ University of Ostrava, Faculty of \\ Science \\ Dvorakova 7, 70103 Ostrava 1, \\ Czech Republic \\ Email: jaroslav.zacek@osu.cz
}

\author{
Jiri Matula \\ University of Ostrava, Faculty of \\ Science \\ Dvorakova 7, 70103 Ostrava 1, \\ Czech Republic \\ Email: jiri.matula@osu.cz
}

\begin{abstract}
The notion of commitment is a principal notion in human communication in social systems. It can be also viewed as an elementary contract in which human beings enter into and comply with to achieve their business goals. However, different methodologies use slightly different paradigm to model this notion. The paper firstly analyzes the commitment usage in economic transactions in general, and then investigates the DEMO Enterprise Ontology (Design \& Engineering Methodology for Organizations) and Resource Event Agent (REA) ontology and their capabilities for commitment modeling. Possibilities of these modeling approaches are shown in an example of a practical application. The main asset of the paper is to empirically explore the DEMO's prescriptive and REA's descriptive abilities for enterprise information system design and to reveal their strong and weak points.
\end{abstract}

\section{INTRODUCTION}

$\mathrm{T}$ HE notion of commitment belongs to fundamental notions in the social systems, in which human beings are the core participants, according to [4]. In general, human beings plan and achieve their goals through conversation (coordination). Human beings who are involved in conversation concerning commitment are marked as performer (anyone who expose intention) and addressee (somebody, to which the intention is addressed). We can distinguish an individual commitment, in which the performer exposes intention towards the addressee and paired (coupled) commitments, in which one commitment is in consideration of another 'paired' commitment. Individual commitments are easier elaborated and structured because there is only one commitment over which human beings have their roles. In simple case 'paired commitments' are represented by two commitments in which one commitment is in consideration of the other or it may be represented by two groups of commitments in which one group of commitments is in consideration of the other group.

According to $[1,6]$, an economic commitment is a type of obligation by one human being to transfer economic resources to another human being at some specified point of time usually in future. Commitments can be a part of contract or may stay alone, in which case they are often re- garded as uncontracted commitment. Commitments are also regarded as atomic contracts, according to [5]. In the simplest form of commitment (contract) two contract parties (human beings) commits to an exchange of resources such as goods for money or service for money, i.e. to a pair of transactions of resources from one party to the other. The important thing about it is that one transaction is in consideration of the other.

There are two methodologies, we focus on, dealing with business process modeling and working with the notion of commitment. The DEMO enterprise ontology [3] and the REA value modeling ontology $[2,5,6]$. The DEMO methodology has its foundation in the DEMO enterprise ontology and provides a strong theoretical foundation for business process modeling. On the base of the transaction axiom DEMO has 'integrated' commitment in its transaction pattern. Universal transaction pattern is a very powerful concept, which among others enables working actions in current and in the future in the uniform way. DEMO as a generic methodology works primarily with individual commitment, which is structured in the form of transaction in a tree structures.

The REA value modeling ontology originates from accountancy systems and provides a domain specific platform for value modeling business processes. The term value modeling means that REA approach keeps track of primary and raw data about economic resource values. REA model is composed of at least two transactions of economic resources, where one transaction is in consideration of the other. REA considers service to be an economic resource. Rather than focusing on debits and credits which by design omit important data about economic event, REA proposes capturing the detail about each resource under the organization's control, the events that change the amount of each resource, and the agents who participate in these events, according to $[6,7,9]$. Both the REA and DEMO methodologies utilize the notion of transaction in which commitment is included. Commitment itself is a principal notion in human communication. The other methodologies do not provide such concepts and such ways for modeling. Our motivation is to empirically explore the DEMO's 
prescriptive and REA's descriptive abilities for enterprise information systems design, utilizing commitment modeling, and to reveal their strong and weak points.

The remainder of the paper is organized as follows: Section 2 deals with commitment modeling in DEMO. REA commitment modeling is depicted in section 3. Practical examples of commitment usage are stated in section 4 . The resulting findings are discussed in section 5. The final section contains conclusions and a summary of future work.

\section{DEMO COMmitment Modeling}

According to the DEMO methodology, human beings, of which an organization is composed, perform two kinds of acts: production acts and coordination acts. By performing coordination acts human beings enter into and comply with commitments towards each other regarding the performance of production acts $[3,4]$. Commitments in DEMO are included in the standard transaction pattern as its 'integral' part. The standard transaction is depicted in Fig. 1. Each act is followed by a corresponding fact representing a concrete state. In general, an elementary state of affairs in a world is a fact.

Actually, transaction pattern expresses coordination acts and facts that enclose the production act and fact. When studying the transaction pattern in detail one soon finds out that the proposition phase of the transaction pattern represents a commitment. In this phase the initiator exposes his or her request concerning the production fact and if the executor promises the request, both actors enter into a commitment. Execution phase of the transaction pattern concerns production act and fact. The result phase of the transaction pattern represents the fulfilling of the commitment in case of 'happy path'. However in reality, it often happens that the proposition phase and the execution phase do not follow immediately one after another. Usually, there is a time lag between both phases. The case in which there is a time lag between proposition and execution phases is more frequent and therefore more important for studying than the case when the proposition phase fluently continues into the execution phase and the result phase. As can be seen from Fig. 1, the DEMO methodology puts all phases into one transaction pattern. The time lag between phases makes the transaction pattern closer to reality. The transaction pattern is able to capture activities in which the execution phase and result phase fluently follows after the proposition phase and the case in which there is a time lag between the proposition phase and the execution and result phases. In practice it means that the case when a customer rents a car immediately is the same as the case when a customer makes a reservation of renting a car for the following weekend. Both these seemingly different cases are modelled according to the same transaction pattern.

In DEMO, a business process is formed by transaction(s) structured in a tree arrangement (parent-child relation). It frequently happens that one business process has transactions which the execution and result phases will happen in future and which will affect the other business process. These

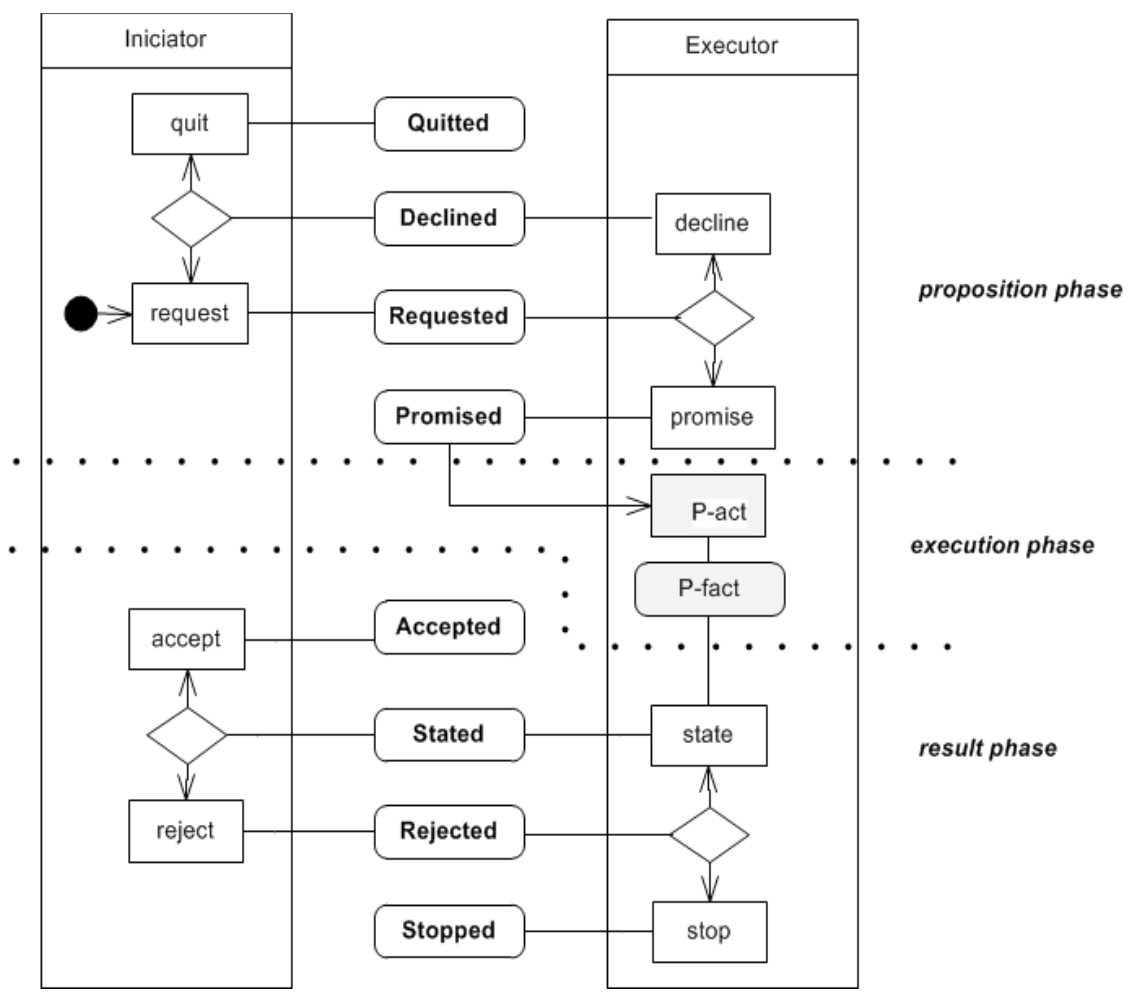

Fig. 1. The Standard Transaction Pattern Source: [3] 
processes have to be loosely coupled through information links.

Figure 1 contains two 'swimlanes', one for the initiator and the other for the executor. Inside these swimlanes are indicated coordination acts which may happen. In the middle, there are shown coordination facts, which are incurred by the acts.

The executor swimlane also contains the production act (P-act) and the production fact (P-fact). The production act and fact are placed in the executor's swimlane because production is exclusively executor's activity. This figure also represents the DEMO state machine in which transitions are drawn within the swimlanes and the states are situated in the middle between the swimlanes. As the DEMO methodology is a generic methodology for business process modeling the DEMO transaction pattern can be considered to be an individual commitment of the corresponding production fact.

It may be concluded that the DEMO methodology enables to model transactions with a time lag between the proposition and the result phases of transaction according to unique transaction pattern. In addition, the DEMO Enterprise Ontology and its models provide prescriptive information system for the enterprise. This information system enforces model compliance. In short, it enforces all actors to comply with the allowed state transitions. This is done by the Transaction axiom one of the four axioms on which the DEMO Enterprise Ontology is based.

\section{REA COMMITMENT Modeling}

Commitment entities and their relationships with other entities are shown in Fig. 2. The commitment entity copies to a considerable extent the structure of the event entity, by which we mean the existence of an increment and decrement commitment and the exchange reciprocity relationship. The exchange reciprocity relationship between the increment and decrement commitments identifies which resources are promised to be exchange for which others $[6,7,8]$.

Each commitment is related to an economic resource by a reservation relationship which specifies which resources will be needed or expected by future economic events. The reservation relationship between the resource and commitment represents the features of the resource and rights associated with the resource that will be changed or transferred by a future economic event. As a matter of fact, each commitment must be related by committed provide and committed receive relationships to economic agents.

The other crucial relationship is the fulfillment relationship which relates a corresponding commitment entity to an event entity. The purpose of this relationship is to validate whether the economic events fulfill corresponding commitments. A REA model represents one business process. REA transactions are paired.

Contrary to the DEMO methodology, REA ontology is a domain specific methodology, which focuses on all information systems where the values of resources are traced. There are three basic differences between the DEMO methodology and the REA modeling approach in terms of

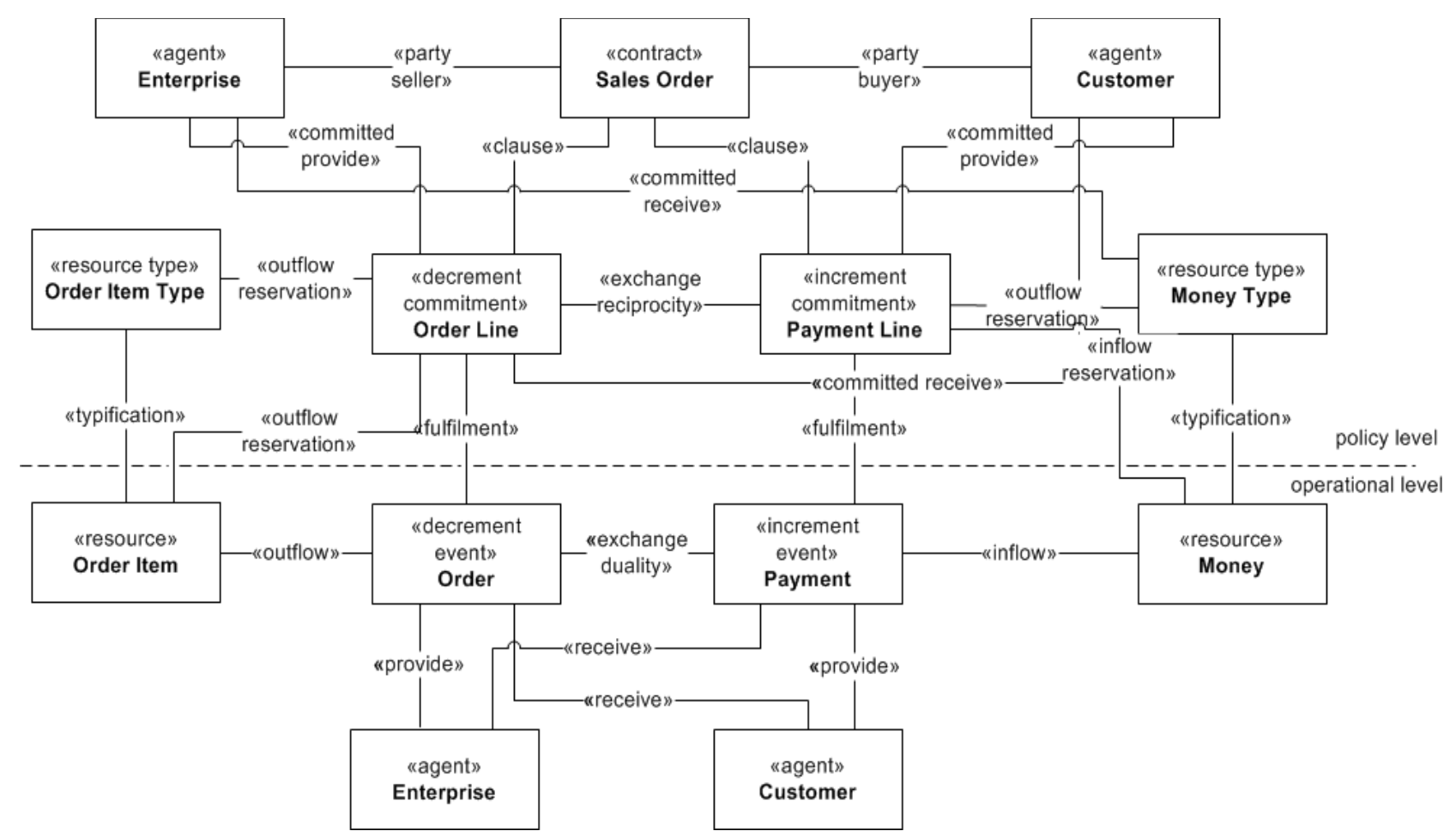

Fig. 2 REA model of sales order Adapted from[6] 
commitment modeling. At first, REA as other business process modeling methodologies works only with production activities and production results. It does not work with communication (coordination). At second, the REA modeling approach distinguishes between current and past economic events and future economic events which are denoted in REA as commitment entities. An economic event itself captures changing of property rights to economic resources or captures consuming, usage, and production of economic resources.

A commitment entity addresses the issue of modeling promises of future economic events and the issue of reservation of resources. The reason for this solution has its origin in accountancy systems from which REA ontology has developed into full-fledged tools for information systems modeling. Economic events specify according REA ontology only actual increment or decrement of resources, not the future increment or decrement of resources. Finally, REA distinguishes two principal transaction kinds, increment transaction, decrement transaction, and these transactions are inherently arranged as paired. The exchange reciprocity relates increment commitments to decrement commitments and the duality relationship relates increment economic events to decrement economic events. The REA modeling approach results in descriptive information systems that are based on exchange, consumption, usage and production of economic resources.

\section{Practical Example of Commitment Usage}

This practical example can elucidate the differences and similarities in a commitment modeling in the context of the above mentioned modeling approaches. The rent-a-car example covers both current and future events and on the other hand, it is not too complex to comprehend [4]. To introduce the problem a short narrative description follows. Rent-a-car is a service which is provided either to walk-in customers or customers who make a rental reservation by telephone, fax or email. A car may be rented on the same day or may be reserved for a specific term in the future after a contract between an employee of the rental company and a customer has been signed. The company which rents out cars has many branches around the country. So, the rented car may be picked up and dropped off at different branches. The rental payment depends directly on the number of days of rental and kind of rented car. Among other things, the signed contrast states the period of the rental and the name of the branch where the car will be dropped off. If the period of rental or/and the drop off branch do not coincide with the conditions in the signed contract, the customer is liable for a penalty payment. The rental payment must be made by the starting day of the rental at the latest. Additional penalty payments must be made at the drop off point.

\section{A. DEMO Modeling Approach}

From the short narrative actor roles and transaction kinds can be identified. There are four actor roles: CA01 - renter, CA02 - driver, A01 - rental starter and A02 - rental ender. The renter actor role and the driver actor role may represent the same or different person. In this example, we can identify six transactions which represent two business processes. The first business process is composed of the following transactions: T01 rental start, T02 rental payment, T03 car pick-up, and T04 car drop-off. The second business process

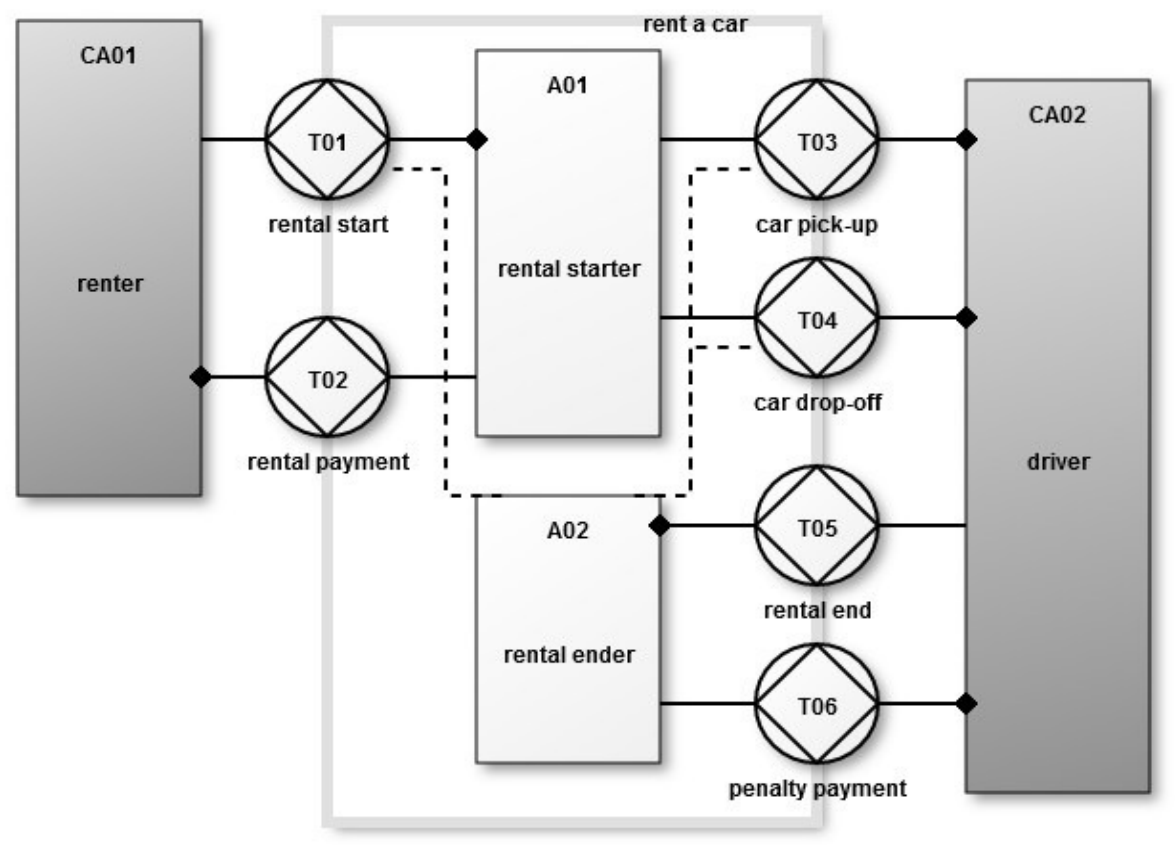

Fig. 3 The DEMO Construction Model 
is constituted of the following transactions: T05 rental end, and T06 penalty payment. For the commitment modeling, the first business process is more illustrative than the second one. The structure of the first business process is as follows. T01 - rental start is a parent transaction and all other three transactions are child transactions (T02, T03, and T04). Conversely, T02 rental payment, T03 car pick-up and T04 car drop-off transactions are enclosed in T01 rental start transaction. Conclusion of T01 rental start transaction also means that the contract between a renter and a rental starter has been signed. The DEMO construction model capturing actor roles and corresponding transactions is depicted in Fig.3.

The renter - CA01 and the driver - CA02 are composite actor roles colored dark-grey. The rental starter - A01 and the rental ender actor roles are elementary actor roles colored white. All six transactions are placed at the interface of the rent-a-car system and environment. The solid lines among a transaction and two different actor roles (each transaction is connected by solid lines exactly with two actor roles) represent initiator and executor. The executor role is indicated by the solid dot at the actor's role.

T01 transaction encloses T02, T03, and T04 transactions. When T01 transaction is completed it means the rental payment transaction (T02) must be paid (completed) and the car pick-up transaction (T03) and the car drop-off transaction (T04) must be promised. It means that when T01 rental start is completed the driver can either pick-up a corresponding car type now or pick-up a corresponding car at some future point in time. More formally, there may/ may not be a time lag between the promise transaction step and the accept transaction step in T03 transaction. In case of T04 transaction, we suppose that there will be a time lag between the promise transaction step and the accept transaction step in the duration of the rental.

However, information about rental start, car pick-up and car drop-off are necessary for the second business process, which is formed by T05 and T06 transactions. According to the above mentioned information, T05 transaction decides whether the driver must pay a penalty or not. The dashed lined in the DEMO construction model between a transaction and an actor role indicates the flow of information. Detailed view on the inside of an individual business process provides the Process Structure Diagram. This Process Structure Diagram of rent-a-car for the first business process is depicted in Fig. 4.

The solid lines in the figure represent causal relations between individual transaction steps. A small box at the edge of the transaction stands for an action (transition) whereas a small circle indicates a fact (state). On the request of the renter for the T01 rental start transaction the rental starter (A01) promises rental start and requests three next transaction kinds, namely, T03 car pick-up transaction, T02 rental payment transaction, and T04 car drop-off transaction. T03 and T04 transactions are functionally similar and the rental starter is satisfied with the promise transaction step in both cases. This means in practice that the driver or delegated renter makes an agreement with the rental starter about the particulars related to the car pick-up and the car drop-off activities. The further view on this state says that

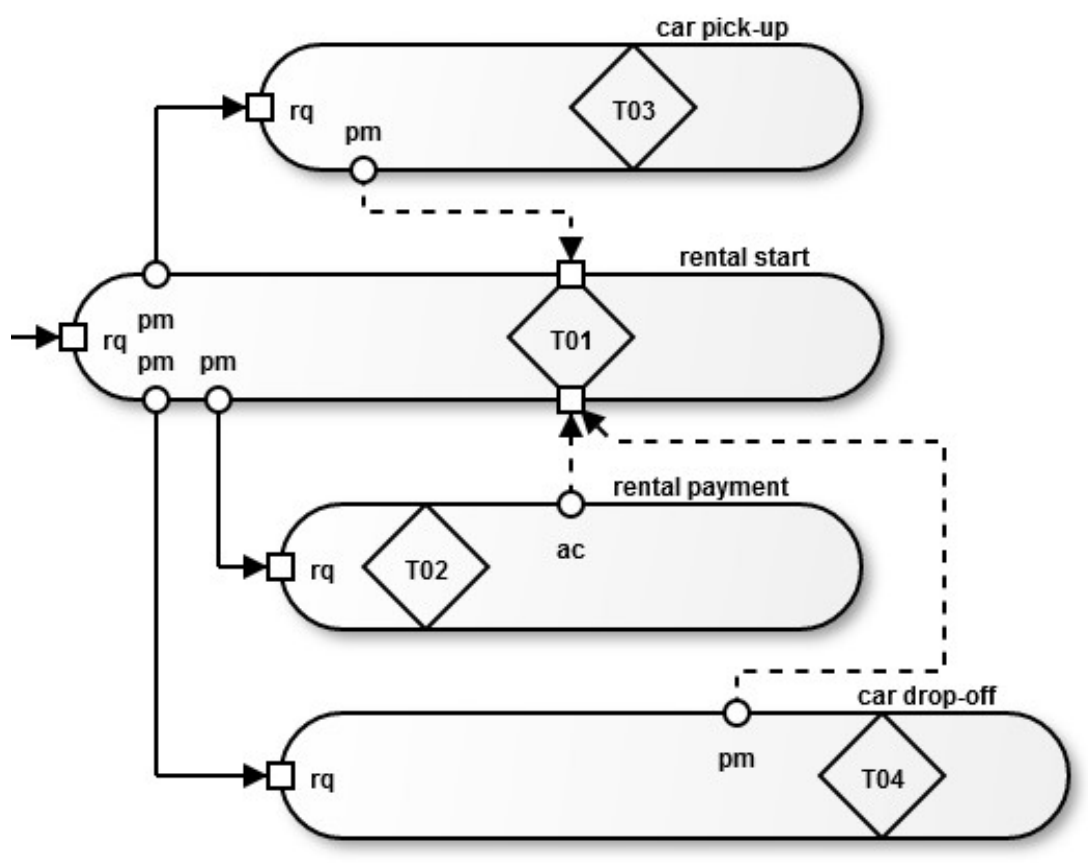

Fig. 4 The DEMO Process Structure Diagram 
this is the only possible way how to solve this issue in which there is evidently a time lag between the promise transaction step and the time when the driver really will pick-up a car at a given branch of the car rent company. The same also holds for the car drop-off transaction. T02 rental payment transaction needed to be completed entirely which means that the rental starter has to accept amount of the rental payment. In terms of time when the rental payment is made, it is essential for the car rental to be paid before a car is being picked up. Figure 4 makes it clear because in case the rental payment is not concluded it implicates that the rental start can't be concluded either. The details of the DEMO models concerning sequence of transaction steps within a given business process are captured in the process structure diagrams.

\section{B. REA MODELING APPROACH}

REA modeling approach represents rather descriptive than prescriptive model. The whole REA exchange model is formed in one business process. Transactions in a REA model are implicitly paired. Those transactions which increase the value of resources in a REA model are paired with transactions that decrease the value of given resources. Assigning the notions of increment and decrement is dependent on the view the REA model is seen. In the practical example, the following commitments and events are identified: Rent a Car commitment, Rent a Car event, Rental Payment commitment, Rental Payment event, Penalty Payment commitment, and Penalty Payment event. As the REA model introduces 'mirroring' each commitment is reflected in a corresponding event. In addition, each commitment is related to corresponding event by the fulfillment relationship.

The commitment entity in its essence represents promise of the future event and is separated from the corresponding event entity. Event entity itself represents an actual exchange or conversion of economic resources. As can be seen, commitment and event entities do not form an integral unit as it is in the case of the DEMO methodology. On the other hand, this separation is a source of many limitations and deficiencies of the REA modeling approach. A crucial role in a REA model plays a contract entity. The contract entity relates to commitment entities and parties which are involved in the REA model.

\section{Discussion}

One of the basic building blocks of the DEMO methodology is a transaction pattern that provides a detail and truthful generic template in which communication between human beings takes place. This pattern is composed

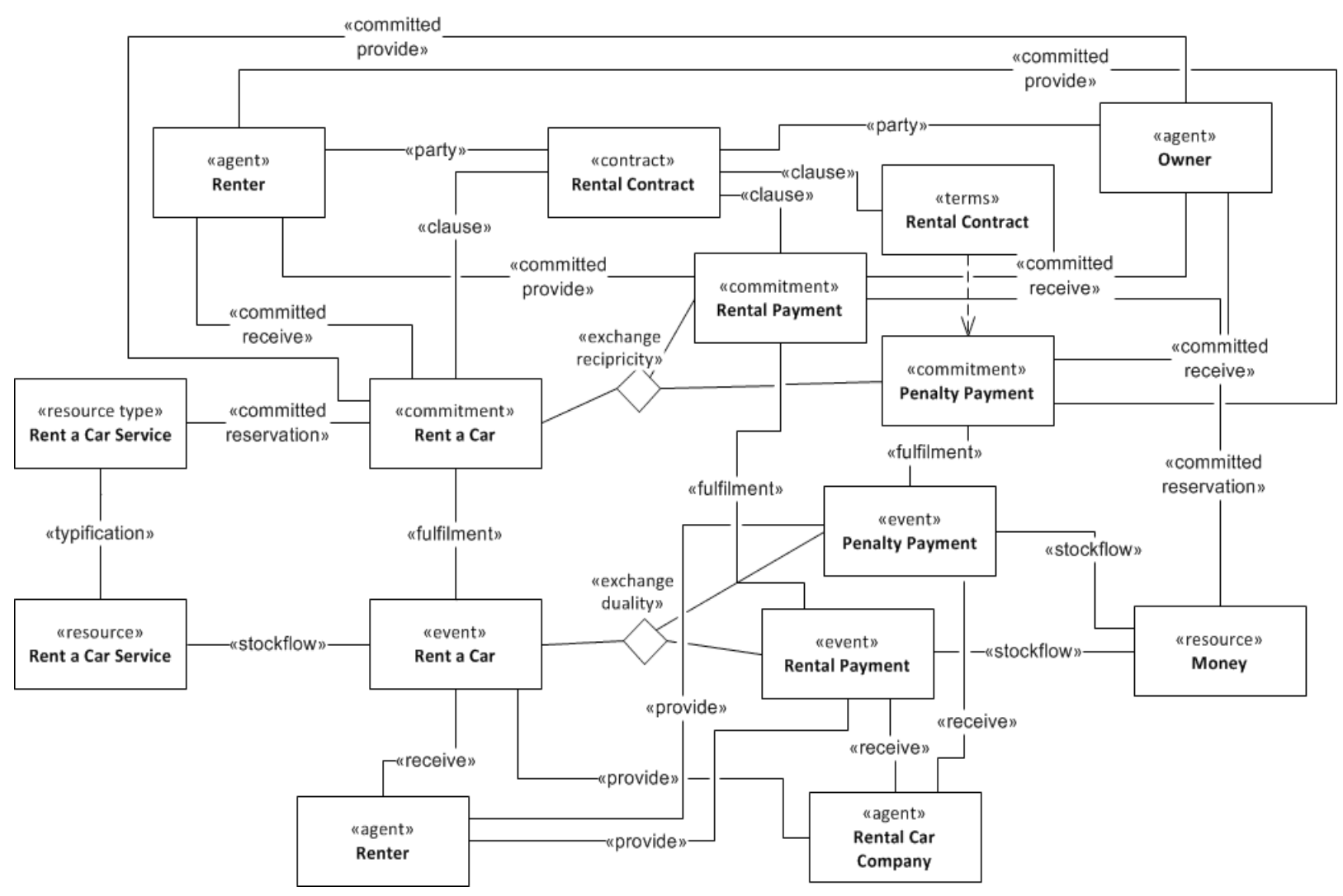

Fig. 5 REA model Source: [7] 
of three phases: the proposition phase, the execution phase and the result phase. The proposition phase represents this part of communication (coordination), in which human beings enter into an agreement concerning the production fact. In short, the proposition phase of the transaction pattern represents a commitment. It can be said that commitment is "integrated 'in this pattern.

The DEMO composition axiom enables DEMO transactions to be arranged in a tree structure. This structure provides a parent-child relationship between transactions. Child transactions are enclosed in the corresponding parent transaction and together form a business process. Business processes may be related to each other by information links (interstriction relationships). In this way, the DEMO methodology is able to capture all the things that happen in reality with great empirical evidence. However, the current DEMO methodology doesn't provide any mechanism supporting the pair transaction arrangement. The parent-child relationship, in which the DEMO transactions are arranged doesn't support this arrangement.

A commitment in REA ontology is modelled as an independent entity that deals with the future economic events and has a relation to economic event(s) that addresses the current and past activities incurred by change of property rights to economic resources or incurred by use, consume, or produce an economic resource.

The REA modeling approach is entirely focused on production activities which are indicated by the corresponding economic events. REA doesn't provide state machine in the sense of the DEMO methodology. Commitment and corresponding economic event are related to each other by the fulfillment relationship which simply indicates whereas the commitment was fulfilled, or not. The core of the REA transaction is formed by a commitment and a corresponding economic event. Two different kinds of the REA transactions are arranged into paired transactions in which one kind of transaction is plan/done in consideration of the other kind of transaction. At the commitment level the paired transactions are related to each other by the reciprocity relationship. At the economic event level the paired transactions are related to each other by the duality relationship. The reciprocity and the duality relationships are crucial relationships in the REA model.

In the practical example, the DEMO construction diagram is formed by six transactions which constitute two business processes. The REA solution represents three mutually paired transactions arranged in one business process (REA model).

\section{Conclusion and Future Research}

The paper presents utilization of the DEMO enterprise ontology and the REA ontology for commitment modeling. The DEMO approach is much precise and consistent. The commitment is inseparable part of the DEMO's transaction pattern. From the practical example and description it follows that the DEMO 'commitment' meets all requirements that are required in reality. However, the current DEMO Enterprise Ontology is not perfectly suitable for capturing an exchange process in which partaking transactions are mutually independent and are not related to each other by the parent-child relationship. This is given by the fact that the DEMO methodology represents a generic ontology in which transactions are arranged in a tree structures. The REA ontology has its origin in accountancy systems and is based on the mechanism of paired transactions. The REA commitment is an independent entity related to other corresponding entities in the REA model. The REA modeling approach is able to work with different transactions as mutually independent transactions with only reciprocity and duality relationships between them which corresponds to the descriptive abilities of REA ontology. Signing and fulfilling a contract is only expressed by the reciprocity and the duality relationships. REA modeling approach doesn't provide the DEMO consistency within its transaction which brings about limited utilization of this methodology.

\section{AcKNOWLEDGEMENT}

The paper was supported by the grant provided by Ministry of Education, Youth and Sports Czech Republic, reference no. SGS15/PRF/2017.

\section{REFERENCES}

[1] G.L. Geerts and W.E. McCarthy, "The Ontological Foundations of REA Enterprise Information Systems". Paper presented at the Annual Meeting of the American Accounting Association, Philadelphia, PA. (2000)

[2] G.L. Geerts and W.E. McCarthy, "Policy-Level Specification in REA Enterprise Information Systems". Journal of Information Systems. Vol 20, No. 2 pp. 37-63. (2006)

[3] J.L.G Dietz, "Enterprise Ontology - Theory and Methodology". Springer-Verlang (2006)

[4] J.L.G. Dietz, "The Essence of Organization. An Introduction to Enterprise Engineering". Sapio bv, 2012.

[5] C.L. Dunn, O.J. Cherrington and A.S. Hollander, "Enterprise Information Systems: A Pattern Based Approach". New York: McGraw-Hill/Irwin (2004)

[6] P. Hruby, "Model-Driven Design Using Business Patterns". SpringerVerlag Berlin Heidelberg (2006)

[7] F. Hunka and R. Belunek, "Transaction Based Business Process Modeling", in Proceedings of the 2015 Federated Conference on Computer Science and Information Systems (FedCSIS). Lodz, 2015, pp. 1397-1402. http://dx.doi.org/10.15439/2015F149.

[8] J. Korczak, M. Hernes and M. Bac, "Fundamental analysis in the multi-agent trading system", in Proceedings of the 2016 Federated Conference on Computer Science and Information Systems (FedCSIS). Gdansk, 2016, pp. 1169--1174. http://dx.doi.org/10.15439/2016F238

[9] P. Sitek, "A declarative decision support framework for supply chain problems", in Proceedings of the 2016 Federated Conference on Computer Science and Information Systems (FedCSIS). Gdansk, 2016, pp. 1215-1222. http://dx.doi.org/10.15439/2016F39 



\title{
Eye Tracking Data Acquisition and Preprocessing for Modeling of Manager Knowledge in Financial Report Analysis
}

\author{
Adrian Kaźmierczak \\ Wrocław University of Economics, Komandorska Str. 118/120, 53-345 Wroclaw, Poland \\ Email: adrian.kazmierczak@ue.wroc.pl
}

\begin{abstract}
The article presents process of data acquisition and detection of pattern in the way of perceiving economic data. For this purpose, results of eye tracking study were used to obtain information on how data are analyzed by SME managers in accordance with their level of economic and financial knowledge. As a result, information was derived on viewing patterns allowing for generation of sequence patterns. Research results will help design a mechanism for intelligent interpretation of economic information in Business Intelligence systems.
\end{abstract}

\section{INTRODUCTION}

A NALYSIS of a manager's eye movements during the time of reading financial reports constitutes one of the new areas of research. The objective is to identify perception processes, both overt and covert, for a participant's consciousness. Making eye movements in analysis and decision systems, as studies have shown [1], can play an active role in decision-making [2]. Eye tracking allows for very precise time measurement of perceiving data, for carrying out an operation and reaching a decision, which would not have been possible with other traditional methods.

Studies show that a significant proportion of managers of small and medium-sized enterprises (SMEs) have no background knowledge and qualification in economics making it difficult for them, on the one hand, to conduct correct data analysis and financial reports and, on the other, to make complete use of the many features and services available in decision support systems [3]. Universal, "madeto-measure" solutions prevent adaptation of system interface to the level of knowledge, skills and qualifications of a SMEs manager. Effectiveness of decision-making is impaired by lack of financial analysts in SMEs as well as lack of sufficient funds to cover the high costs of continuous business consultation. Their decisions, managers in SMEs need systemic support based on data from transactional systems. By processing these data, applying financial analysis tools help in the preparation of decision support projects. Advancement in information technology has provided an opportunity for managers to access diversified information stored in various databases and Internet resources. Excessive amounts of report data usually generated by transactional and dedicated decision support systems, among others, constitute a problem. When it comes to business processes, information overload significantly reduces one's ability to reach the right decision, in particular in the case of SMEs.

The aim of the article is to show the possibility of preparing data for patterns of sequence of eye-glance, or patterns of manager's analytical thinking using eye tracking system. Due to inability to automate process of generating data, it was necessary to find a solution that would make it possible to prepare them beforehand. The article will describe method of data processing, in the context of conducted research. These data could be assisted built ontologies relevant to managers in the knowledge areas of economic and financial, understood as formal conceptual layer specifications [2].

The structure of the article is as follows. The next section of the article describes the study that led to the research data. Another point is devoted to the possibility of generating a sequence of looking at financial reports. Taking into account, in point 4, the description of how to obtain sequence patterns achieved using data received. The article concludes with a summary description of experiments, showing the scope of planned research.

\section{ANALYSIS OF FINANCIAL STATEMENT}

Research has been conducted on interpretation of financial statements with the aim of modeling of a manager's knowledge. The purpose of the experiment was to examine perceptions of participants of different levels of knowledge in the field of financial reporting. Experienced managers as well as beginner analysts - economics students, took part in the study. During the experiment, attempt was made to acquire information on perception of report through the eyes of participants, as well as expert's and novice's patterns of observations. The purpose of this study was to use this information in modeling a manager's knowledge profile, specifying sequences and relationships in how appearance of presented reports is perceived.

The study, assumed that a manager uses business-oriented software (such as DSS/ERP/BI) supported by financial ontology, analytical models, and appropriate expertise.

In the experiment, participants were asked to evaluate an enterprise's economic and financial situation. Case study consisted of financial reports and adequate ontology prepared and made available to the participants.

The experiment started with an analysis of an internal managerial report containing widely used indicators of assessing financial situation, namely: liquidity and 
profitability, debt, and turnover ratios. The most important factor directly observed by participants in the experiment was a significant increase in profitability in an analyzed period.

An ontological approach for conceptualization of knowledge domain models was adopted in the study. Development of ontology for selected areas of economic and financial knowledge important to SME manager constitutes an important element. Ontologies are created by experts in the area, with the consideration of acquisition of hidden knowledge as a result of research using eye tracking, for example in order to correctly explain profitability ratio, it was necessary to make use of ontology describing the concept of profitability, as presented in Figure 1.

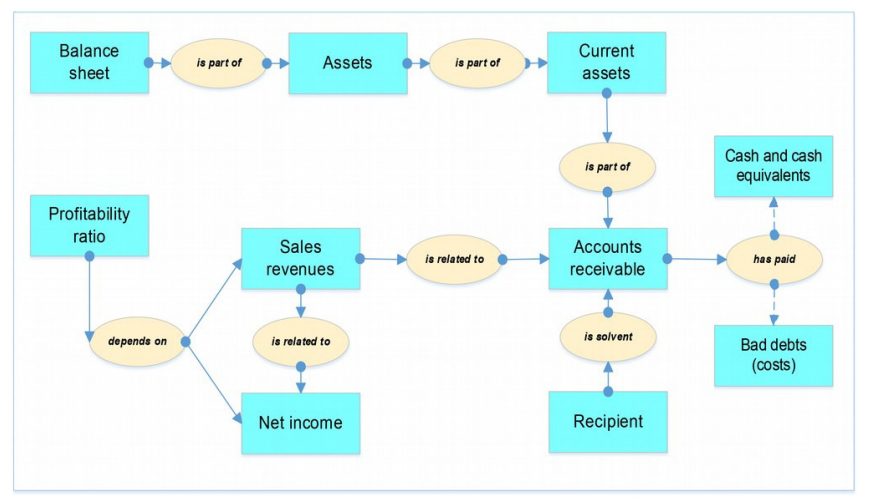

Fig. 1. Ontology: view of profitability

Many research projects show that an ontology of economic and financial indicators aid in decision making [5]. It is important to note that there is no single universal system of economic indicators that could be applied in all organizations. Besides, a lot of companies use varying assessment models of business based on analysis of various indicators. Design of a new intelligent interface, the ontological approach, was augmented by eye tracking methods. A visual presentation of knowledge and related data available in DSS allows for quick access to economic situation and for taking appropriate action [1]. The concepts and analytical operations obtained in study were matched with the financial ontology available in the system.

A balance sheet and profit and loss account of a real company were submitted for use in the research study. The financial statement included information indicating seemingly positive performance of the case company. However, in the periods analyzed, serious problems emerged as a result of debts. These problems should have been picked up by an analyst as threatening the loss of rationale behind going concern's basic assumption, i.e. the ability to function without threat of liquidation in the foreseeable future.

The experiment was divided into two phases. The first phase was to assess the overall situation of the company, based on core measures and indicators. After analyzing reports, each participant had to synthetically rate the state of company's assets and financial situation as very good, satisfactory, or poor. In the second phase, participants had to answer questions regarding financial indicators observed as positive and negative, with a higher level of detail. Detailed questions covered the key factors contributing to the development of the company, helping maintain its ability to continue its operations. Additionally, the purpose of this phase was to test ability to monitor adverse financial warnings which in many cases could have been concealed by the positive results and other financial indicators. Finally, participants had to comment on positive and negative aspects.

Data from experiments in the form of a log of operations along with ontology were analyzed in order to explore and produce an additional, often subconscious, knowledge on the manager. To analyze the data, algorithms of data mining and discovery sequential patterns were applied [7], however, it was necessary to prepare data beforehand.

\section{Generate SEQUENCE Using Eye Tracking}

Two concepts are important in order to analyze eye movement, namely:

- fixations, that is, focus of eye vision on a particular item;

- saccades, which are sequences of rapid eye movements between successive fixations.

The points a manager focuses on can be identified based on the duration and number of fixations. In general, the shorter the time of eye fixation upon a specific item, the lesser significant the item is. The longer the time of fixation on an analyzed element, the more significant the element is in the process of perception [8].

Eye tracker and Gazepoint programming software were used for the experiment. They offer high-performance, affordable eye trackers to clients in all fields of applications from academic researchers to video game developers and more. This allowed for recording test footage, showing subsequent fixations and saccades for a defined research problem. Exemplary report in the eye tracker software of the case study is illustrated in Figure 2. This program offers us an opportunity to obtain different types of results on the visual activity of the participant. A heat map and an inverted heat map showing aggregate information on focusing of attention by the participant are some of these results that can be distinguished.

Numerous eye movements may be analyzed and processed to produce a variety of reports. Logs of the user operations provide detailed information on how information is perceived on the screen, on the order in which the content is viewed, and the length of time the eye is fixed on a given point. After transformation of the data, information on how a document was analyzed can be obtained. 
(2) Gazepoint Analysis Standard Edition (v3.5.0) x64

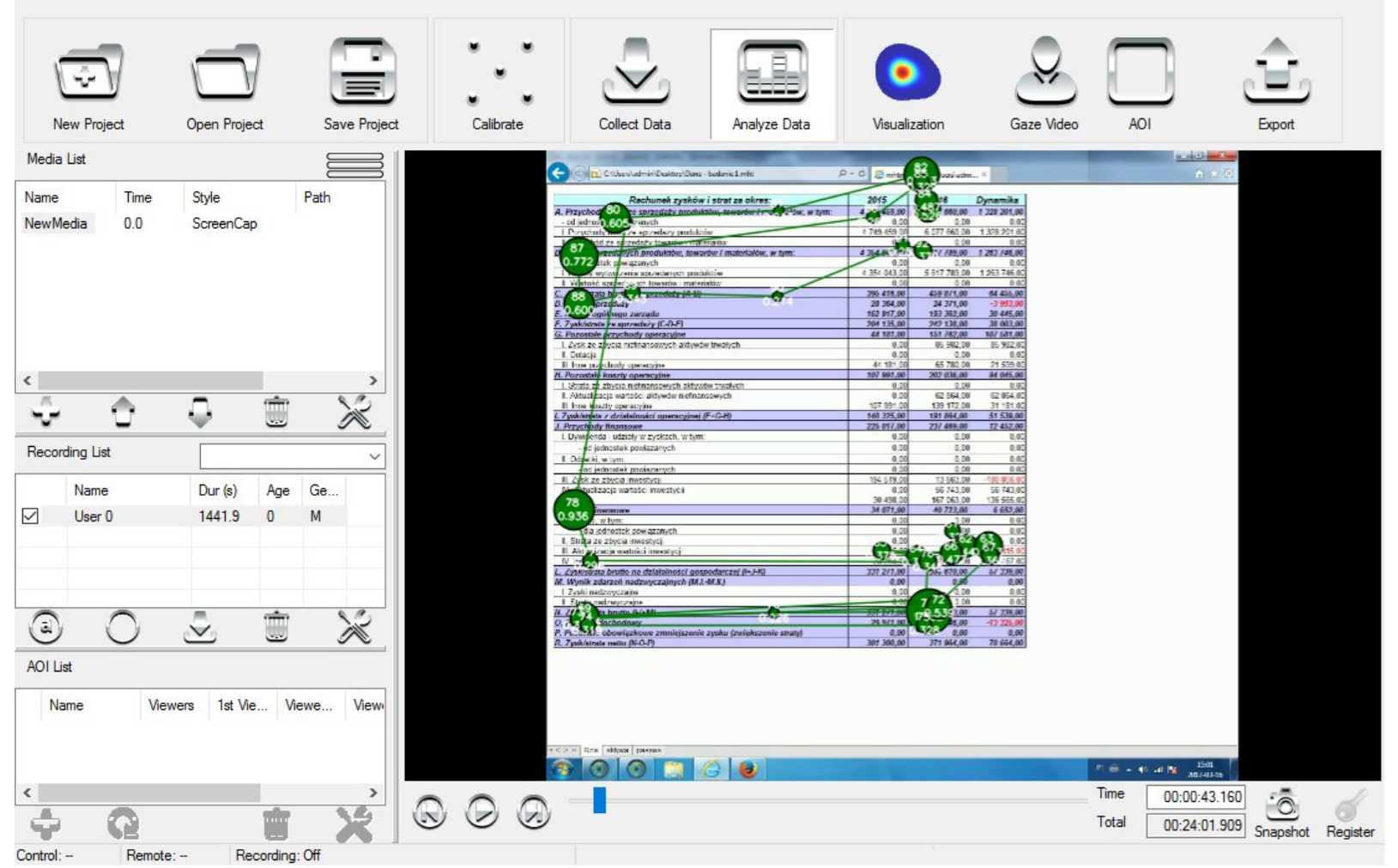

Fig. 2. Path of analysis financial statements

\begin{tabular}{|c|c|c|c|c|}
\hline & 1 & 2 & 3 & 4 \\
\hline $101 \ldots$ & Profit and loss account & 2015 & 2016 & Dynamics \\
\hline $102 \ldots$ & A. Net revenues from sales of products, goods and materials, including: & 4749459,00 & 6077660,00 & 1328201,00 \\
\hline $103 \ldots$ & - from related parties & 0,00 & 0,00 & 0,00 \\
\hline $104 \ldots$ & I. net revenues from sales of products & 4749459,00 & 6077660,00 & 1328201,00 \\
\hline $105 \ldots$ & II. net revenues from sales of goods and materials & 0,00 & 0,00 & 0,00 \\
\hline $106 \ldots$ & B. Cost of products, goods and materials sold, including: & 4354043,00 & 5617789,00 & 1263746,00 \\
\hline $107 \ldots$ & - to related parties & 0,00 & 0,00 & 0,00 \\
\hline $108 \ldots$ & I. Manufacturing cost of products sold & 4354043,00 & 5617789,00 & 1263746,00 \\
\hline $109 \ldots$ & II. Value of goods and materials sold & 0,00 & 0,00 & 0,00 \\
\hline $110 \ldots$ & C. Gross profit (loss) on sales (A-B) & 395416,00 & 459871,00 & 64455,00 \\
\hline $111 \ldots$ & D. Selling costs & 28364,00 & 24371,00 & $-3993,00$ \\
\hline $112 \ldots$ & E. General and administrative costs & 162917,00 & 193362,00 & 30445,00 \\
\hline $113 \ldots$ & F. Profit (loss) on sales (C-D-E) & 204135,00 & 242138,00 & 38003,00 \\
\hline $114 \ldots$ & G. Other operating revenues & 44181,00 & 151762,00 & 107581,00 \\
\hline $115 \ldots$ & 1. Gain on disposal of non-financial fixed assets & 0,00 & 85982,00 & 85982,00 \\
\hline $116 \ldots$ & II. Subsidies & 0,00 & 0,00 & 0,00 \\
\hline $117 \ldots$ & III. Other operating revenues & 44181,00 & 65780,00 & 21599,00 \\
\hline $118 \ldots$ & H. Other operating expenses & 107991,00 & 202036,00 & 94045,00 \\
\hline $119 \ldots$ & 1. Loss on disposal of non-financial assets & 0,00 & 0,00 & 0,00 \\
\hline $120 \ldots$ & II. Revaluation of non-financial fixed assets & 0,00 & 62864,00 & 62864,00 \\
\hline $121 \ldots$ & III. Other operating expenses & 107991,00 & 139172,00 & 31181,00 \\
\hline $122 \ldots$ & 1. Profit (loss) on operating activities ( $F+G-H)$ & 140325,00 & 191864,00 & 51539,00 \\
\hline $123 \ldots$ & J. Financial revenues & 225017,00 & 237469,00 & 12452,00 \\
\hline $124 \ldots$ & I. Dividend and profit sharing, including: & 0,00 & 0,00 & 0,00 \\
\hline $125 \ldots$ & - from related parties & 0,00 & 0,00 & 0,00 \\
\hline $126 \ldots$ & II. Interest, including: & 0,00 & 0,00 & 0,00 \\
\hline $127 \ldots$ & - from related parties & 0,00 & 0,00 & 0,00 \\
\hline $128 \ldots$ & III. Gain on disposal of investments & 194519,00 & 13663,00 & $-180856,00$ \\
\hline $129 \ldots$ & IV. Revaluation of investments & 0,00 & 56743,00 & 56743,00 \\
\hline $130 \ldots$ & V. Other & 30498,00 & 167063,00 & 136565,00 \\
\hline $131 \ldots$ & K. Financial expenses & 34071,00 & 40723,00 & 6652,00 \\
\hline $132 \ldots$ & I. Interest, including: & 0,00 & 0,00 & 0,00 \\
\hline $133 \ldots$ & - for related parties & 0,00 & 0,00 & 0,00 \\
\hline $134 \ldots$ & II. Loss on disposal of investments & 0,00 & 0,00 & 0,00 \\
\hline $135 \ldots$ & III. Revaluation of investments & 5815,00 & 0,00 & $-5815,00$ \\
\hline $136 \ldots$ & IV. Other & 28256,00 & 40723,00 & 12467,00 \\
\hline $137 \ldots$ & L. Profit (loss) on business activities (1+J-K) & 331271,00 & 388610,00 & 57339,00 \\
\hline $138 \ldots$ & M. Result on extraordinary events (M.I. - M.III.) & 0,00 & 0,00 & 0,00 \\
\hline $139 \ldots$ & I. Extraordinary gains & 0,00 & 0,00 & 0,00 \\
\hline $140 \ldots$ & II. Extraordinary losses & 0,00 & 0,00 & 0,00 \\
\hline $141 \ldots$ & N. Gross profit (loss) (L+/-M) & 331271,00 & 388610,00 & 57339,00 \\
\hline $142 \ldots$ & O. Income tax & 29971,00 & 16646,00 & $-13325,00$ \\
\hline $143 \ldots$ & P. Other statutory reductions in profit (increases in loss) & 0,00 & 0,00 & 0,00 \\
\hline $144 \ldots$ & R. Net profit (loss) (N-O-P) & 301300,00 & 371964,00 & 70664,00 \\
\hline
\end{tabular}

Fig. 3. Split report into areas 
Sequences, presentation of a scheme of analysis of financial statements played a key role in our study. As there was no easy way to acquire expected information from the system level, processing of data obtained was, arriving at specific sequences with accuracy of reports fields.

Details of data obtained are presented in Figure 3. Each report contains a given number of rows and columns. As a result of combination of these data, together with user scanning path, a sequence was obtained. The sequence provides information on method of analysis of SMEs reports.

The software provided by the eye tracker manufacturer allows for recording data on the log, with details on the recorded data. Figure 4 shows sample data that can be saved as a file. This has been described in same way in software documentation.

Among all the data, one of the most important in the attempt at defining areas of interest (AOI) is the information related to visual focus point, in relation to the $\mathrm{X}$ and $\mathrm{Y}$ axes. As these data are written on a scale of 0 to 1 showing the drawing, it is necessary to reference these values to resolution of the screen on which the study was conducted. In our case, it was $1680 \times 1050$ pixels for the $\mathrm{X}$ and $\mathrm{Y}$ axes, respectively. As a result, for example, if a participant looked closely at the center of the monitor, the data for both axes was 0.5 , that is 840 and 525 .

This information was used to define individual report fields as interest areas, where for each of them, on a proportional basis, a range of 0 to 1 was calculated for both axes. With the use of Excel and its functions IF, for each position, formulas were created to return information on whether a fixation described by $\log$ values is within defined AOI. Taking into account fixation number, duration and whether or not, in the area in which it occurred, a clear statement with a view of financial statement called the viewing sequence has been made.

Figure 5 shows, for example, generated viewing sequences for 5 different people. Numbers were assigned to relevant item in the financial statements, giving unequivocal information using numerical value:

- $\quad$ first - about the name of the report being analyzed;

- $\quad$ second and third - about the report line;

- fourth - about the report column.

Where there is 0 in the sequence, it means that a participant was not looking at a given fixation on the report, and his eyesight went beyond it. This may have been the result of either searching for data not on the report, such as system time or other software, or of fatigue, stress or dissociation. From the viewing sequence, it is hard to categorically pinpoint the cause, but testimony and recorded facial expression are helpful here. A detailed analysis of recordings will allow for more accurate identification of the reasons of the eyes straying outside the reports.

\section{SEQUENTIAL PATTERNS}

Given a set of sequences where each sequence consists of a list of elements and each element consists of a set of items, and given a user-specified min support threshold, sequential pattern mining consists of finding all of the frequent subsequences, i.e. subsequences whose occurrence frequency in the set of sequences is no less than min support [6].

Identification of sequential patterns of operations requires analysis of a database containing information about events that occurred within a specified period of time. Many methods used to detect sequential patterns have been applied in various fields, such as analysis of shopping cart, telecommunications, medicine, insurance banking, and Web pages [7].

Pattern recognition of analytical thinking may be performed using different algorithms. The choice depends on computational complexity as well as size of database. For the purposes of the study, only the most useful algorithms for detecting frequent sequential patterns will be examined.

The idea of an algorithm for discovering sequential patterns of is well-known [9]. First, minimum support for the occurrence of sequences in the database has to be defined. As a result, the algorithm produces in the first iteration frequent 1-element sequences for a given minimum support. In subsequent iterations, generating a candidate sequence uses the sequence discovered from a previous iteration. If the support of the discovered sequence is greater than or equal to predefined level of occurrences, the algorithm again generates a candidate sequence using the sequences detected in the previous iteration. Search algorithm ends when there are no more candidates or no common sequences.

Numerous modifications of algorithms to discover patterns of sequences resulted in elimination of identified difficulties and improved the efficiency of data mining. This is an example of data patterns conversion in the sequence shown in Figure 6. Conversion is a modification of the eye tracker logs arranged for fixation at a certain time. This made it possible to define occurrences at each AOI, which were then used to detect sequential patterns.

By comparing a manager's operations with the patterns of expertise and the domain ontology, the system is in a position to not only adapt the system interface to the level of the manager's knowledge, but also to identify "gaps" in his or her knowledge and propose an appropriate manner of economic analysis [10]. This will be possible using algorithms that identify dependencies and correlations in the way financial statements are analyzed. As a result, a detailed analysis of conclusions arrived at will allow for patterns generation and identify exceptions as well as their causes in the way reports are perceived. Pilot studies were also intended to provide information on opportunities to apply eye tracking tools to draw conclusions on the basis of sequential of perception of reports, and to define the rules and the process of realizing economic and financial expertise. 


\begin{tabular}{|c|c|c|c|}
\hline \multicolumn{3}{|l|}{ Dialog } & $\times$ \\
\hline $\mathrm{E} \ldots$ & CSVID & Description & $\hat{\imath}$ \\
\hline$\square$ & MEDIA_ID & A unique numeric identifier associated with the media item in the media list. & \\
\hline$\square$ & MEDIA_NAME & The user defined name of a media item. & \\
\hline$\square$ & WEB_ID & A unique numeric identifier associated with the web page recorded. & \\
\hline$\square$ & WEB_TITLE & The TITLE of the webpage from the webpage source (HTML). & \\
\hline$\square$ & WEB_URL & The URL of the webpage. & \\
\hline$\square$ & CNT & The COUNT increments once for each data record generated by Control. & \\
\hline$\square$ & TIME & Time elapsed in seconds since the last system initialization. & \\
\hline$\square$ & TIMETICK & CPU ticks recorded at time as TIME, can be used to syncronize data with other applications. & \\
\hline$\square$ & FPOGX & The X-coordinate of the fixation POG, as a percentage of the screen width $(0$ to 1$)$. & \\
\hline$\square$ & FPOGY & The $Y$-coordinate of the fixation POG, as a percentage of the screen height ( $\left(\begin{array}{ll}0 & 1\end{array}\right)$. & \\
\hline$\square$ & FPOGS & The starting time of the fixation POG in seconds since the system initialization or calibration. & \\
\hline$\square$ & FPOGD & The duration of the fixation POG in seconds. & \\
\hline $\bar{\square}$ & FPOGID & The fixation POG ID number. & \\
\hline$\square$ & FPOGV & The FPOG valid flag is 1 if the fixation data is valid and 0 if it is not. & \\
\hline$\square$ & BPOGX & The X-coordinate of the unfiltered POG (left\&right average), as a percentage of the screen wid... & \\
\hline$\square$ & BPOGY & The Y-coordinate of the unfiltered POG (left\&right average), as a percentage of the screen hei... & \\
\hline$\square$ & BPOGV & The valid flag with value of 1 if the data is valid, and 0 if it is not. & \\
\hline$\square$ & $\mathrm{CX}$ & The X-coordinate of the mouse cursor position, as a percentage of the screen width ( 0 to 1$)$. & \\
\hline$\square$ & $\mathrm{CY}$ & The Y-coordinate of the mouse cursor position, as a percentage of the screen height ( 0 to 1$)$. & \\
\hline$\square$ & CS & Mouse cursor state, 0 for steady state, 1 for left down, 2 for right down, 3 left up, 4 right up. & \\
\hline$\square$ & USER & A custom data field that may be set by the user via the API or Remote. & \\
\hline$\square$ & LPCX & The X-coordinate of the left eye pupil in the camera image, as a percentage of width ( $(0$ to 1$)$. & \\
\hline$\square$ & LPCY & The Y-coordinate of the left eye pupil in the camera image, as a percentage of height ( $(0$ to 1$)$. & \\
\hline$\square$ & LPD & The diameter of the left eye pupil in pixels. & \\
\hline$\square$ & LPS & The scale factor of the left eye pupil, normalized to 1 at the head depth at calibration. & \\
\hline$\square$ & LPV & The left pupil valid flag with value of 1 if the data is valid, and 0 if it is not & \\
\hline$\square$ & RPCX & The $\mathrm{X}$-coordinate of the right eye pupil in the camera image, as a percentage of width ( 0 to 1$)$. & \\
\hline$\square$ & $\mathrm{RPCY}$ & The $Y$-coordinate of the right eye pupil in the camera image, as a percentage of height ( 0 to 1$)$. & \\
\hline 可 & RPח & The diameter of the rinht eve nu unil in nivel & $\checkmark$ \\
\hline & & OK & \\
\hline
\end{tabular}

Fig. 4. Data from recordings

\begin{tabular}{|c|r|r|r|r|r|r|r|r|r|r|r|r|r|r|r|r|r|r|r|}
\hline Person & \multicolumn{10}{|c|}{ Sequence } \\
\hline 1 & 0 & 1352 & 1291 & 0 & 0 & 0 & 0 & 0 & 0 & 0 & 0 & 0 & 0 & 0 & 0 & 0 & 0 & 0 & 1011 \\
\hline 2 & 0 & 0 & 0 & 1442 & 1421 & 1393 & 1394 & 1402 & 1411 & 1421 & 1414 & 0 & 0 & 0 & 1031 & 1041 & 1091 & 1141 & 1161 \\
\hline 3 & 0 & 1321 & 1121 & 0 & 1012 & 1022 & 0 & 1091 & 1361 & 1041 & 1061 & 1081 & 1031 & 1041 & 1021 & 0 & 0 & 0 & 0 \\
\hline 4 & 1292 & 1301 & 1341 & 1411 & 1361 & 1381 & 1371 & 1342 & 1353 & 1354 & 1343 & 1351 & 1361 & 1321 & 1311 & 1291 & 1302 & 1431 & 0 \\
\hline 5 & 0 & 1331 & 1051 & 1031 & 1013 & 1044 & 0 & 1041 & 1081 & 1171 & 1151 & 1081 & 1051 & 1053 & 1044 & 1052 & 1012 & 1043 & 1084 \\
\hline
\end{tabular}




\begin{tabular}{|c|c|c|c|c|c|c|}
\hline ParticipantName & LocalTimeStamp & EyeTrackerTimestamp & GazeEventType & GazeEventDuration & FixationPointX (MCSpx) & FixationPointY (MCSpx) \\
\hline P02 & $09: 49: 10.627$ & 1427787312203270 & Fixation & 267 & 143 \\
\hline P02 & $09: 49: 10.644$ & 1427787312219940 & Fixation & 267 & 237 \\
\hline P02 & $09: 49: 10.661$ & 1427787312236610 & Fixation & 267 & 143 \\
\hline P02 & $09: 49: 10.677$ & 1427787312253270 & Fixation & 267 & 143 \\
\hline P02 & $09: 49: 10.694$ & 1427787312269930 & Fixation & 267 & 143 \\
\hline
\end{tabular}

\begin{tabular}{|c|c|c|c|c|c|c|c|c|c|}
\hline ParticipantName & LocalTimeStamp & Number of fixations & Fixation1 & Fixation2 Fixation3 Fixation4 Fixation5 Fixation6 Fixation7 Fixation8 \\
\hline P02 & $09: 49: 10.627$ & 5 & $R$ & $R$ & $R$ & $R$ & $R$ \\
\hline P02 & $09: 49: 13.798$ & 1 & $R$ & & & & \\
\hline P02 & $09: 49: 13.988$ & 8 & R & P & R & R & R \\
\hline P02 & $09: 49: 16.690$ & 3 & R & R & R & & \\
\hline P02 & $09: 49: 18.190$ & 4 & P & P & P & P & \\
\hline
\end{tabular}

Fig. 6. Converting a point on the screen

\section{V.CONCLUSION}

In this project, a new part, referred to as Patterns of Expertise, is created on the basis of data provided by an eye tracker that records a manager's perception and his manner of document analysis. The collected quantitative data are used to identify sequential patterns of his perception of information. A built-in algorithm explores the eye tracker $\operatorname{logs}$ and creates a model of managerial operational knowledge.

Arriving at these data is make possible with the help of appropriately prepared data. As the eye tracking software doesn't have advanced data analysis functions, it was necessary to obtain a self-tracking tool. Interpretation of the data generated by the software, due the brief documentation, also constituted another problem. As a result, it was necessary to separately prepare the data. Their manual processing, as described, yielded the expected results. As a result, a database containing sequences was prepared. This will be used to apply pattern search algorithms, which will eventually be used to build intelligent system interfaces.

\section{ACKNOWLEDGMENT}

Special thanks goes to the IT and financial experts, in particular to J. Korczak, B. Nita, P, Oleksyk and students of the Faculty of Management, Informatics and Finance of Wrocław University of Economics. The author would also like thank to Jaroslaw Jankowski for loaning eye tracker and for the support in experiment.

\section{REFERENCES}

[1] Korczak J., Dudycz H., Nita B., Oleksyk P., Kaźmieczak A., Attempt to extend knowledge of Decision Support Systems for small and medium-sized enterprises, Computer Science and Information Systems (FedCSIS), 2016, nr 8, pp. 1263-1271

[2] Korczak J., Kaźmierczak A., Discovery of Analytical Thinking Patterns of Managers using Eye Tracking, Intelligent Systems Conference (IntelliSys), 2017 [in print]

[3] Nita B., Korczak J., Dudycz H., Oleksyk P., Kaźmierczak A. (2015), W kierunku inteligentnych systemów analizy finansowej w zarządzaniu małym i średnim przedsiębiorstwem, Stowarzyszenie Księgowych w Polsce, Katowice, pp. 79-101

[4] Gruber T. (1995), Toward Principles for the Design of Ontologies Used for Knowledge Sharing, Academic Press, Duluth, pp. 907-928

[5] Dudycz, H.: The Topic Map as a Visual Representation of Economic Knowledge (in Polish). Wrocław University of Economics, Wrocław (2013)

[6] Agrawal R., Srikant R. (1995), Mining sequential patterns. Conf. Data Engineering (ICDE'95), Taipei, pp. 3-14

[7] Masseglia F., Teisseire M., Poncelet P. (2005), Sequential Pattern Mining: A Survey on Issues and Approaches, Institute of Electrical and Electronics Engineers, Gwalior, pp. 3-29

[8] Kamińska J. (2013), Okulografia w ocenie ergonomicznej stanowisk pracy - przykłady, Centralny Instytut Ochrony Pracy - Państwowy Instytut Badawczy. Warszawa, pp. 13-15

[9] Agrawal R., Srikant R. (1994), Fast Algorithms for Mining Association Rules, VLDB '94 Proceedings of the 20th International Conference on Very Large Data Bases, San Francisco, pp. 487-499

[10] Korczak J., Dudycz H., Nita B., Oleksyk P., Kaźmierczak A. (2015), Próba rozszerzenia wiedzy $w$ systemach wspomagania decyzji menedżerskich w małych i średnich przedsiębiorstwach, Wydawnictwo Uniwersytetu Ekonomicznego w Katowicach, Katowice, pp. 227-241 


\title{
An analysis of the use of the web-accessibility validator
}

\author{
Łukasz Krawiec \\ Wrocław University of Economics \\ ul. Komandorska 118-120, 53-345 Wrocław, Poland \\ Email: lukasz.krawiec@ue.wroc.pl
}

\begin{abstract}
A very important aspect of websites nowadays is their accessibility. Thanks to modern, constantly evolving technologies, it is possible to create friendly services for each user, regardless of his state of health. Web sites accessibility may be considered in aspects of their functionality and readability. One of methods for exploring this issue is the use of validators, i.e. automated tests to check the syntax of the documents posted on the Internet. The purpose of this paper is to analyze of the use one of the selected tools. The structure is as follows. First part of the article explained the importance of the accessibility of the web sites and present the most important accessibility standard. Then there is briefly characterized proposal of validators evaluation criteria for studying web-accessibility and present a list of selected tools in this way. In the next step is presented the results of analyzes carried out using the most promising tool. Finally, there are conclusions from the study. The paper is a continuation of the study of the website validation [1].
\end{abstract}

\section{INTERPRETATION OF WEBPAGE ACCESSIBILITY VALIDATION RESULTS}

$\mathbf{J}_{\mathrm{v}}^{\mathrm{u}}$ UST as any other type of common good, the World Wide Web network, with its ubiquitous nature, multitude of functionalities, and a broad range of benefits it offers to the modern information society seems to require proper care and maintenance in support of its continued development. This responsibility lies largely in the hands of web designers and those involved in the administration of individual pages and sites as fundamental units of the entire virtual reality of the WWW system. It is therefore advisable for any designer to not only emphasise the high quality of the distributed content, but also its proper presentation and its adjustment to the technical standards of the profession.

Webpage is a term so commonly used in modern contexts that it needs no detailed definition. However, from this study's perspective, it may seem useful to approach it in contrast to the term 'website', although both terms are often perceived as synonymous. A webpage is a document formatted in a HTML standard, containing multimedia elements arranged in an orderly fashion, and links (hyperlinks) to other webpages or to specific sections of the document itself [2]. In contrast to the above, a website is a term used to represent entire sets of cross-linked documents [3].

The application of best standards and practices in web design may be seen as a warrant to the quality of content presentation, functionality, and integration with the entire WWW system. Professional benefits offered by this approach require proper execution of the most fundamental qualitative features of WWW presentation, such as: web visibility, web benefits, web usability, and web accessibility [4]. The latter parameter is of special significance since, regardless of the original intentions of the designer, the ultimate objective here is to ensure effective presentation of the content and efficient propagation of information to the largest possible audience. Hence, the most obvious line of approach is to make sure that both the content and the rage of functionalities offered on the website can easily be accessed by all users, regardless of any disabilities or other conditions that may impair the reception of WWW content [5].

Webpage is a type of computer interface, so its features should be analysed in the area of human-machine interactions also. In this context, webpage accessibility represents a sum of user interface (UI) qualities designed to provide functionalities to all users, regardless of their preferred platform or client software, or any disabilities or impairments that may affect standard reception of the content [5]. In other words, webpages should be designed in accordance with the established standards of presentation to make them accessible from various platforms (e.g. both stationary and mobile), software profiles (operating systems and browsers), and configurations (e.g. with or without additional accessories or pointing devices, compatibility with audio readers). This requires a great deal of informed preparation and creative prognostication of possible scenarios, both at website design phase, and during code implementation. The large amount and the complexity of elements to be taken into account in the task of designing a well-accessible website may prove quite challenging to even the most experienced web designers.

\section{STANDARDS OF WeB ACCESSIBILITY}

'Best practices' is a term of a fairly general character, particularly when used in the complex context of web accessibility. Luckily, designers have easy access to a number of formal repositories of such standards, such as those formulated by the World Wide Web Consortium (W3C) in cooperation with the Web Accessibility Initiative (WAI). From the viewpoint of website designers, the most important segment of the WAI guidelines is the Web Content Accessibility Guidelines (WCAG). This document represents the most fundamental and the most widely propagated global standard of web content accessibility [6]. In addition to the above, researchers and analysts use a number of other standards and guidelines, as dictated by the 
need to generalise the findings or to present them in a specific legal context at regional or national level. A good example of such a supplementary set is the US set of laws and regulations on accessibility provided in Section 508 of the Rehabilitation Act of 1973 [7]. Standard supplementation is a justifiable approach, since no existing standards can warrant complete and exhaustive solution to the problems at hand. However, the WCAG - due to its specificity and the expertise of the authoring organisation (W3C) - may be considered as a fundament and a starting point for analyses and validations of web content accessibility.

Table 1 presents the structure and the organisation of WCAG guidelines in its current 2.0 version. Here, the four main principles are used as basis for the arrangement of 10 guidelines, and each guideline is evaluated on the basis of testable success criteria, producing a total of 61 elements, grouped into three levels of conformance with the guidelines of web accessibility: A - the lowest, offering basic accessibility; AA - medium, representing adequate accessibility of web content; and AAA - the highest standard, which (for design reasons) may not readily be available to all sites. To satisfy a certain level of accessibility, the page under examination should meet the whole set of success criteria for this level and the level(s) below it. In other words, to meet the AA level standards, a page should meet all criteria required at level AA and A, while the AAA conformance level would require the satisfaction of standards in all three levels from A to AAA [8].

To help users translate the WCAG success criteria into specific design choices, the standard is supplemented by a number of clarification documents and supporting materials. 'Techniques for WCAG 2.0' - one of the most important supplementary documents in this context - is a repository of techniques, common design errors, technical descriptions, practical examples, source code solutions, and conformity tests [9]. The document is available at https://www.w3.org/TR/WCAG20-TECHS.

TABLE 1.

THE STRUCTURE OF WCAG 2.0

\begin{tabular}{|l|l|lc|}
\hline \multicolumn{3}{|c|}{ WCAG 2.0 structure } \\
\hline Principles & Guidelines & Level A & $\begin{array}{c}\text { Success criteria } \\
\text { Level AA Level AAA }\end{array}$ \\
\hline
\end{tabular}

\begin{tabular}{|l|l|l|l|l|}
\hline 1. Perceivable & 1.1. Text alternatives & 1.1 .1 & \multicolumn{2}{|c|}{} \\
\cline { 3 - 5 } & 1.2. Time-based media & $1.2 .1-1.2 .3$ & $1.2 .4-1.2 .5$ & $1.2 .6-1.2 .9$ \\
\cline { 3 - 5 } & 1.3. Adaptable & $1.3 .1-1.3 .3$ & \multicolumn{3}{|c|}{} \\
\cline { 3 - 5 } & 1.4. Distinguishable & $1.4 .1-1.4 .2$ & $1.4 .3-1.4 .5$ & $1.4 .6-1.4 .9$ \\
\hline
\end{tabular}

\begin{tabular}{|l|l|l|l|l|}
\hline 2. Operable & 2.1. Keyboard accessible & $2.1 .1-2.1 .2$ & & 2.1 .3 \\
& 2.2. Enough time & $2.2 .1-2.2 .2$ & & $2.2 .3-2.2 .5$ \\
& 2.3. Seizures & 2.3 .1 & 2.3 .2 \\
\cline { 5 - 5 } & 2.4. Navigable & $2.4 .1-2.4 .4$ & $2.4 .5-2.4 .7$ & $2.4 .8-2.4 .10$ \\
\hline
\end{tabular}

\begin{tabular}{|l|l|l|l|l|}
\hline 3. Understandable & 3.1. Readable & 3.1 .1 & 3.1 .2 & $3.1 .3-3.1 .6$ \\
\cline { 2 - 5 } & 3.2. Predictable & $3.2 .1-3.2 .2$ & $3.2 .3-3.2 .4$ & 3.2 .5 \\
\cline { 2 - 5 } & 3.3. Input assistance & $3.3 .1-3.3 .2$ & $3.3 .3-3.3 .4$ & $3.3 .5-3.3 .6$ \\
\hline
\end{tabular}

\begin{tabular}{|l|l|l|}
\hline 4. Robust & 4.1. Compatible & 4.1.1 - 4.1.2 \\
\hline
\end{tabular}

Source: Michael Gaigg: Über UI/UX Design, Web Content Accessiblity Guidelines (WCAG) 2.0: Overview and Structure, http://www.michaelgaigg.com/blog/2008/12/19/web-content-accessiblity-guidelines-wcag-20-overview-and-structure

(25.01.2017) (see also: [1]).

\section{VALIDATORS AND THEIR USE IN WEB ACCESSIBILITY TESTS}

There are many approaches in the verification of webpage conformance with web accessibility guidelines. Since some of the methods, such as expert evaluation, source code analyses and panel group observations, may prove fairly laborious, it may be advisable to reach for automated procedures offered by validators. These are sets of tools and instruments designed to perform automated verifications of technical conformance with WWW standards of web design. Reports generated by validators are produced based on analyses, valuations and assessments of the various webpage elements in relation to the adopted standard of evaluation [10]. 
Automated validators of webpage accessibility come in many forms and utilise a multitude of methods. Some common elements can be found between them, but there are also fairly large discrepancies on many levels, some of them of key significance to the final result, depending on the objectives adopted for testing procedures. Oftentimes, the same web page may be found to generate a different number of errors when tested in different validators, even when they use the same sets of accessibility criteria. Thus, before deciding on the use of validators for accessibility testing, it may be advisable to select the most appropriate variant, since this type of instruments is generally used in the early stages of analytical examination and, as such, they may greatly affect the course of subsequent activities [10].

Validators of online services have become increasingly popular over the last few years. Choosing the right one is not, therefore, easy. Specific tools vary greatly, by form of access, by the number of web pages they can verify at a time, by their interface, etc. When analysing various tools for automated tests and the accessibility requirements for websites, I have distinguished the following criteria [10], which can be used to compare and grade validator programs:

1. Verification according to WCAG 2.0 standard. This standard was created by the World Wide Web Consortium, an organisation that aims to create standards in the virtual reality of the Internet. The history of $\mathrm{W} 3 \mathrm{C}$, its importance for the development of the Internet, and the fact that hundreds of companies, institutions and schools belong to it, it can be assumed that their WCAG standard is the most complex and precise. Therefore, conformity to WCAG 2.0 standard should be a requirement for validator programs. A tool that can verify a website according to this standard should be preferred.

2. Verification according to other standards and additional test options. Validator programs are very useful in all tests of websites and in evaluating the quality of a website - company's website, for instance. In both these cases, any additional remarks can be very useful. A tool that allows testing according to other standards than the WCAG (such as the US Section 508), or testing for other criteria (such as spelling errors) should be preferred.

3. Number of web pages tested at a time. Free validator tools often test only one page at a time, while in most cases, the user wants to grade their whole website, not just a part of it. A tool that can verify several pages simultaneously should be preferred.

4. Way of presenting results. Accessibility evaluation is a complex subject and cannot be simplified into one final grade. A tool that generates an in-depth, precise and clear report of its analysis should be preferred.

5. Access form. This describes the way of using the automated tests: locally or online. Online access allows to test anywhere (anywhere with an Internet access, that is). A tool that offers both these forms of access should be preferred.

6. User interface. A graphical interface makes use easier, while a text interface (from the console, for instance) allows for better effectiveness or automatisation. Interface quality should also be taken into account. A tool offering a clear graphical interface with additional option of command console access should be preferred.

7. Software updates. These allow for stable program use, and when choosing a validator, one should check whether it is being updated, as well as whether or not it underwent a testing phase, or how long is it present on the market. A tool that is being supported, developed and suited to current standards should be preferred.

8. Cost. There are three main groups of validator software: commercial products, open source for non-commercial use, and open source. From the point of view of financial advantage only, free open source tool should be preferred.

Based on these criteria and prior research [5], the author produced a list of the most functional and practical instruments of this type:

- Cynthia Says - http://cynthiasays.com (10.02.2017),

- Functional Accessibility Evaluator https://fae.disability.illinois.edu (10.02.2017),

- Wave - http://wave.webaim.org (10.02.2017),

- HTML Validator - http://validator.w3.org (10.02.2017),

- $\quad$ aChecker - http://achecker.ca (10.02.2017),

- Utilitia - https://validator.utilitia.pl (10.02.2017),

- TAW Web Accessibility Test http://www.tawdis.net. (10.02.2017).

Each of the above validators uses the WCAG 2.0 standard as the main platform of reference. Regardless of the above, and due to the aforementioned discrepancies, results produced by those agents can differ to a large extent. Due to its features and comprehensive operation, the first tool - Cynthia Says was selected for further analysis.

\section{ANALYSIS OF THE VALIDATION PROCESS}

Accessibility analysis was submitted to the nonpublic (under construction) website, which is a virtual showcase of the small company, consisting of 6 subpages (Pages 1-6: Start, Company, Offer A, Offer B, Projects, Contact).

Results of validation processes are presented in four tables. Tab. 2 presents detailed results for a single WCAG 2.0 criterion within the main page of the website (Page 1). For each of the 61 success criteria, the following results may be obtained:

- Violation, representing lack of conformance with the required standard, 
- Warning, representing a potential violation of the standard, and requiring manual validation (designer or coder),

- Passed, representing the only positive outcome of the validation process,

- N/A - this result is returned when no elements in the code are found to correspond with the criterion under study; rows containing empty criteria are deleted.

Each of the above results is described by three elements:

- The WCAG technique, with code reference (e.g. H37) as a link to the detailed description of the problem provided in 'Techniques for WCAG 2.0' (see section 2 above for address),

- Meaning,

- Element - the corresponding part of content or code.

Using the formula provided at the bottom of Tab. 2, scores are calculated in the range of 0 to 100 for each subsequent criterion. The score represents the ratio of results in the 'passed' category to the total number of results generated.

Tab. 3 presents an overview of all success criteria generated for Page 1. Detailed descriptions of validation results were truncated and simplified to make them fit in a single row. Initial figures represent the number of occurrences in a given category, and codes in parentheses are references to specific sections of the 'Techniques for WCAG 2.0' document, to help users find detailed information on the problem and respond to it in an effective manner. The last column presents scores calculated for each of the studied criteria. Scores are also calculated for each WCAG level. Due to the characteristics of the A - AA AAA progression, these are presented in the form of cumulative sums (the last three rows of Tab. 3).

The above tables serve to illustrate and explain the approach in the calculation of validation scores based on the example of Page 1 content. Due to editorial constraints, the large volume of results obtained for the remaining pages of the website are presented in a collated form in Tab. 4. Since the results were found to be similar across the entire website, the final results for the site under examination were presented as average, and in separation into all three levels of the WCAG 2.0 compliance.

In summary, Tab. 4 (all scores summaries for the whole website) is a generalization of Tab. 3 (all scores for the first page only), which in turn is a generalization of Tab. 2 (descriptions for first exemplary guideline). To better illustrate the connections between them, Figure 1 was prepared.

Apart from the report of accessibility tests representing the site's compliance with the WCAG guidelines, the paper presents all violations registered in the process, i.e. all of the errors, omissions and other problems that seriously affect the website accessibility. Those errors are typically replicated across many pages, making them suitable for grouping into categories in Tab. 5.

TABLE 2.

DETAILED DESCRIPTION OF VALIDATION PROCEDURES FOR THE FIRST CRITERION, AS APPLIED TO PAGE 1

\begin{tabular}{|c|c|c|}
\hline \multicolumn{3}{|c|}{ WCAG 2.0 checklist - Page 1 - Guideline 1.1.1 score (example of success criteria with description) } \\
\hline Violations & Warnings & Passed \\
\hline $\begin{array}{l}\text { Technique: H37: Using alt attributes on } \\
\text { img elements } \\
\text { Meaning: IMG element contains no ALT } \\
\text { attribute } \\
\text { Element: Line 476, column 6,img } \\
\text { element, SRC = } \\
\text { "http://i.imgur.com/xdyD1J2.png?1" }\end{array}$ & $\begin{array}{l}\text { Technique: F3: Failure of Success Criterion } \\
\text { 1.1.1 due to using CSS to include images } \\
\text { that convey important information) } \\
\text { Meaning: Ensure that image used in } \\
\text { background-url for element is not conveying } \\
\text { information } \\
\text { Element: Line 398, 407, 416, column } 6 \text {, } \\
\text { DIV elements } \\
\text { Technique: G144: Ensuring that the Web } \\
\text { Page contains another CAPTCHA serving } \\
\text { the same purpose using a different modality } \\
\text { Meaning: CAPTCHA found, please verify } \\
\text { that the information is conveyed through } \\
\text { audio as well as visual } \\
\text { Element: Line 520, } 521 \text { (...) column 9, } \\
\text { \#document element, \#content = "captcha" }\end{array}$ & $\begin{array}{l}\text { Technique: F30: Failure of } \\
\text { Success Criterion 1.1.1 and 1.2.1 } \\
\text { due to using text alternatives that } \\
\text { are not alternatives (e.g., } \\
\text { filenames or placeholder text) } \\
\text { Meaning: Image has descriptive } \\
\text { alt text }\end{array}$ \\
\hline \multicolumn{3}{|c|}{ Issues count } \\
\hline 1 & 2 & 2 \\
\hline
\end{tabular}


Score of 1.1.1 (percent passed) $=$ Passed / ( Violations

+ Warnings + Passed $) * 100=2 /(1+2+2) * 100=40$

Source: own research based on results obtained using Cynthia Says validator (see also: [1]).

TABLE 3.

DETAILED SUMMARY OF VALIDATION RESULTS FOR ALL CRITERIA, AS APPLIED TO PAGE 1

\begin{tabular}{|c|c|c|c|c|c|}
\hline & \multicolumn{5}{|c|}{ WCAG 2.0 checklist - Page 1 - Summary (extended example) } \\
\hline & \multirow{2}{*}{$\begin{array}{c}\text { Guide- } \\
\text { lines }\end{array}$} & \multicolumn{3}{|c|}{ Success criteria } & \multirow{2}{*}{ Score } \\
\hline & & Violations & Warnings & Passed & \\
\hline & \multicolumn{5}{|c|}{ Level A } \\
\hline \multirow{39}{*}{ Table $2>$} & 1.1 .1 & $1(\mathrm{H} 37)$ & $2(\mathrm{~F} 3, \mathrm{G} 144)$ & $2(\mathrm{~F} 30, \mathrm{H} 2)$ & 40 \\
\hline & 1.3 .1 & 0 & 0 & $2(\mathrm{H} 42, \mathrm{H} 97)$ & 100 \\
\hline & 1.3 .3 & 0 & $1(\mathrm{~F} 14)$ & 0 & 0 \\
\hline & 1.4 .1 & 0 & $1(\mathrm{G} 14)$ & 0 & 0 \\
\hline & 2.1 .1 & 0 & 0 & $1(\mathrm{SCR} 2)$ & 100 \\
\hline & 2.2 .1 & 0 & 1 (SCR1) & 0 & 0 \\
\hline & 2.3 .1 & 0 & 1 (G19) & 0 & 0 \\
\hline & 2.4 .2 & 0 & 0 & $1(\mathrm{H} 25)$ & 100 \\
\hline & 2.4 .3 & 0 & $1(\mathrm{H} 4)$ & 0 & 0 \\
\hline & 2.4 .4 & $1(\mathrm{H} 30)$ & 0 & 0 & 0 \\
\hline & 3.1 .1 & 1 (H57) & 0 & 0 & 0 \\
\hline & 4.1 .1 & 0 & $1(\mathrm{G} 134)$ & 0 & 0 \\
\hline & \multicolumn{5}{|c|}{ Level AA } \\
\hline & 1.4 .3 & $1(\mathrm{~F} 24)$ & $2(\mathrm{G} 145, \mathrm{G} 18)$ & 0 & 0 \\
\hline & 1.4 .4 & $1(\mathrm{C} 12)$ & $1(\mathrm{C} 17)$ & 0 & 0 \\
\hline & 1.4 .5 & 0 & $2(\mathrm{C} 30, \mathrm{HS} 2)$ & 0 & 0 \\
\hline & 2.4 .6 & 0 & $1(\mathrm{G} 130)$ & 0 & 0 \\
\hline & 2.4 .7 & 0 & 1 (G149) & 0 & 0 \\
\hline & 3.1 .2 & 0 & $1(\mathrm{H} 58)$ & 0 & 0 \\
\hline & 3.2 .3 & 0 & $1(\mathrm{~F} 66)$ & 0 & 0 \\
\hline & 3.2 .4 & 0 & 1 (G197) & 0 & 0 \\
\hline & \multicolumn{5}{|c|}{ Level AAA } \\
\hline & 1.4 .6 & $1(\mathrm{~F} 24)$ & $3(\mathrm{G} 145, \mathrm{G} 17, \mathrm{G} 18)$ & 0 & 0 \\
\hline & 1.4 .8 & $1(\mathrm{~F} 24)$ & $1(\mathrm{C} 17)$ & 0 & 0 \\
\hline & 3.2 .5 & 1 (H83) & 1 (SCR24) & 0 & 0 \\
\hline & 3.3 .5 & $1(\mathrm{G} 71)$ & 0 & 0 & 0 \\
\hline & 1.4 .9 & 0 & $1(\mathrm{C} 30)$ & 0 & 0 \\
\hline & 2.1 .3 & 0 & 1 (HS5) & 0 & 0 \\
\hline & 2.2 .3 & 0 & 1 (G5) & 0 & 0 \\
\hline & 2.2 .4 & 0 & $1(\mathrm{G} 75)$ & 0 & 0 \\
\hline & 2.3 .2 & 0 & 1 (G19) & 0 & 0 \\
\hline & 3.1 .3 & 0 & $1(\mathrm{H} 60)$ & 0 & 0 \\
\hline & 3.1 .4 & 0 & $1(\mathrm{H} 60)$ & 0 & 0 \\
\hline & 3.1 .5 & 0 & 1 (G79) & 0 & 0 \\
\hline & 2.4 .9 & 0 & 0 & $1(\mathrm{H} 2)$ & 100 \\
\hline & 2.4 .10 & 0 & 0 & 1 (G141) & 100 \\
\hline & Sum A & 3 & 8 & 6 & 35,3 \\
\hline & Sum AA & 5 & 18 & 6 & 20,7 \\
\hline & Sum AAA & 9 & 31 & 8 & 16,7 \\
\hline
\end{tabular}

Source: own research based on results obtained using Cynthia Says validator (see also: [1]). 
TABLE 4.

SUMMARY OF VALIDATION RESULTS FOR ALL PAGES (PAGES 1-6) OF TESTED WEBSITE

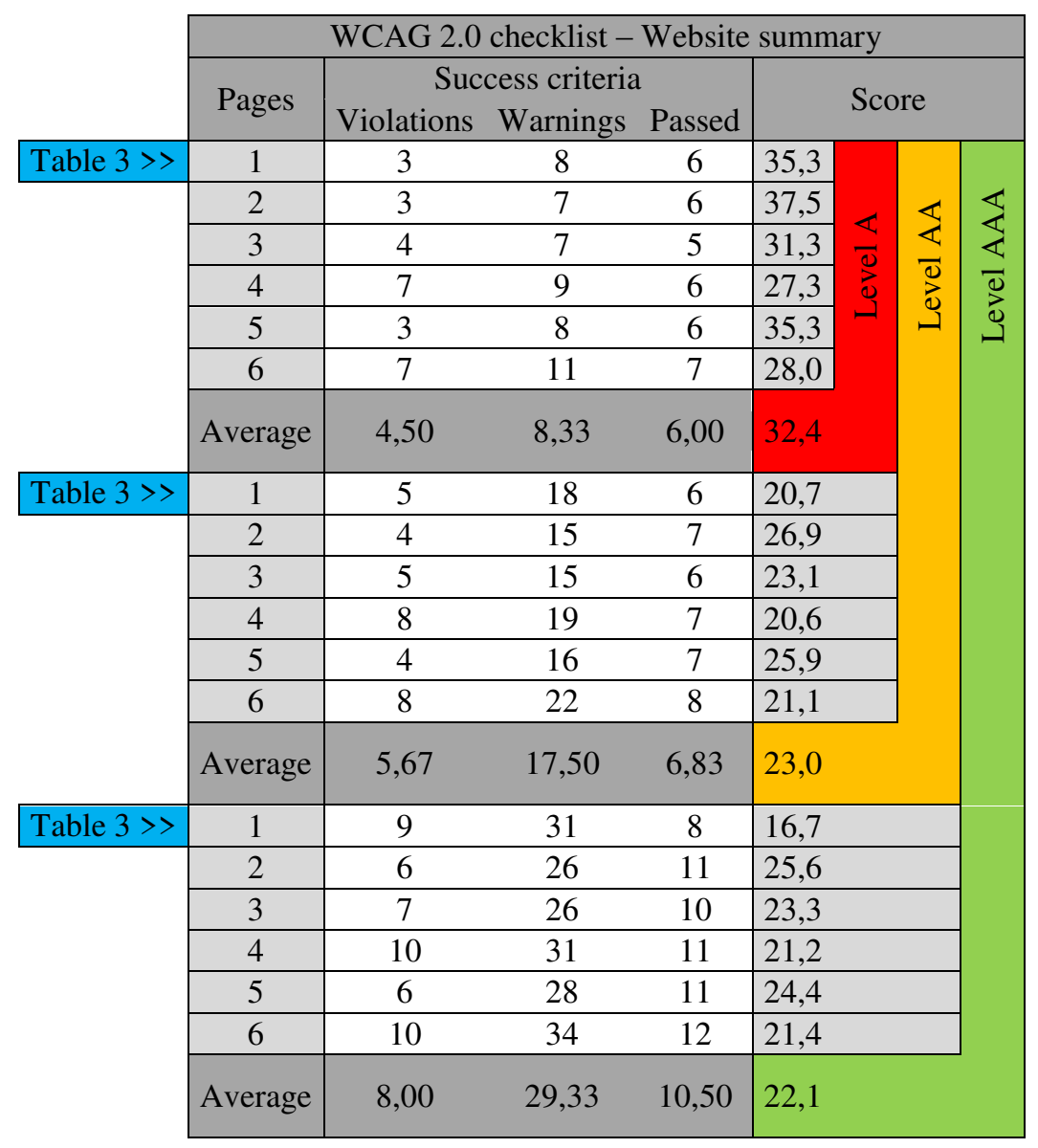

Source: own research based on results obtained using Cynthia Says validator (see also: [1]).

TABLE 5.

DETAILED REVIEW OF VIOLATIONS OF THE WCAG 2.0 STANDARD, AS REGISTERED AT THE TESTED WEBSITE

\begin{tabular}{|c|c|c|}
\hline & & WCAG 2.0 checklist - All pages - Violations (full description) \\
\hline & & $\mathrm{G}=$ Guideline, $\mathrm{T}=$ Technique, $\mathrm{M}=$ Meaning, $\mathrm{E}=$ Element \\
\hline & & Level A \\
\hline 1 & G & 1.1.1 [Non-text Content] \\
\hline & $\mathrm{T}$ & H37 Use alt attributes on img elements \\
\hline & M & IMG element contains no ALT attribute \\
\hline & $\mathrm{E}$ & All pages: img element, SRC = "http://i.imgur.com/xdyD1J2.png?1" \\
\hline & & Page 5: line 135, column 7, img element, SRC = "http://i.imgur.com/X8TwNrx.jpg" \\
\hline 2 & G & 2.4.4 [Link Purpose (In Context)] \\
\hline & $\mathrm{T}$ & H30 Providing link text that describes the purpose of a link for anchor elements \\
\hline & M & Anchor element does not have alternative text which describes purpose of the link \\
\hline & $\mathrm{E}$ & Page 1: a elements, HREF = "..." (news pages links \& social media links) \\
\hline & & Pages 2-6: a elements, HREF = "..." (social media links) \\
\hline 3 & $\mathrm{G}$ & 3.1.1 [Language of Page] \\
\hline & $\mathrm{T}$ & H57 Use language attributes on the html element \\
\hline & M & Page must specify LANG and/or XML:LANG attribute on the html element. \\
\hline & $\mathrm{E}$ & All pages: general \\
\hline 4 & $\mathrm{G}$ & 1.1.1 [Non-text Content] \\
\hline
\end{tabular}




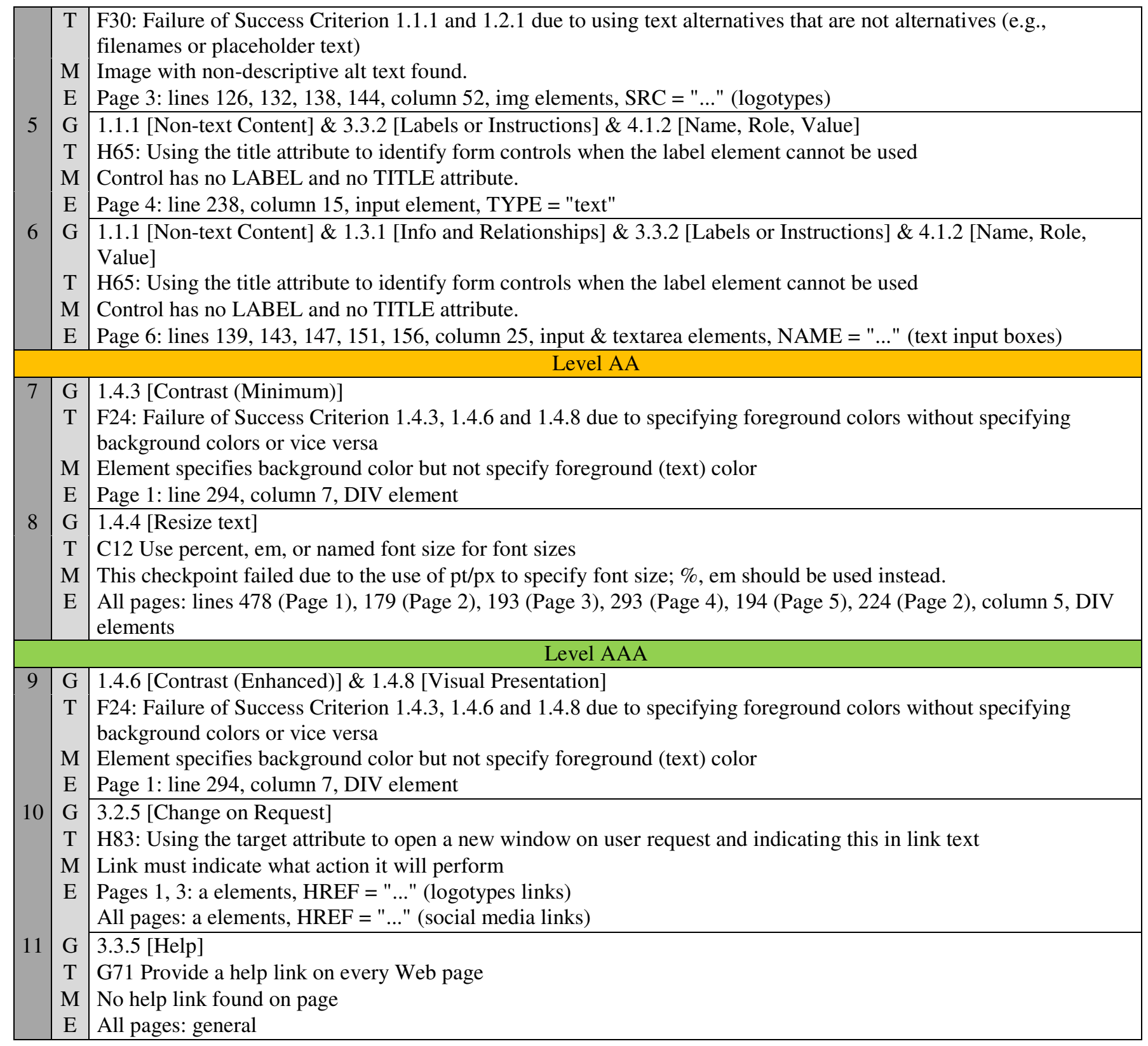

Source: own research based on results obtained generated by Cynthia Says validator (see also: [1]). 


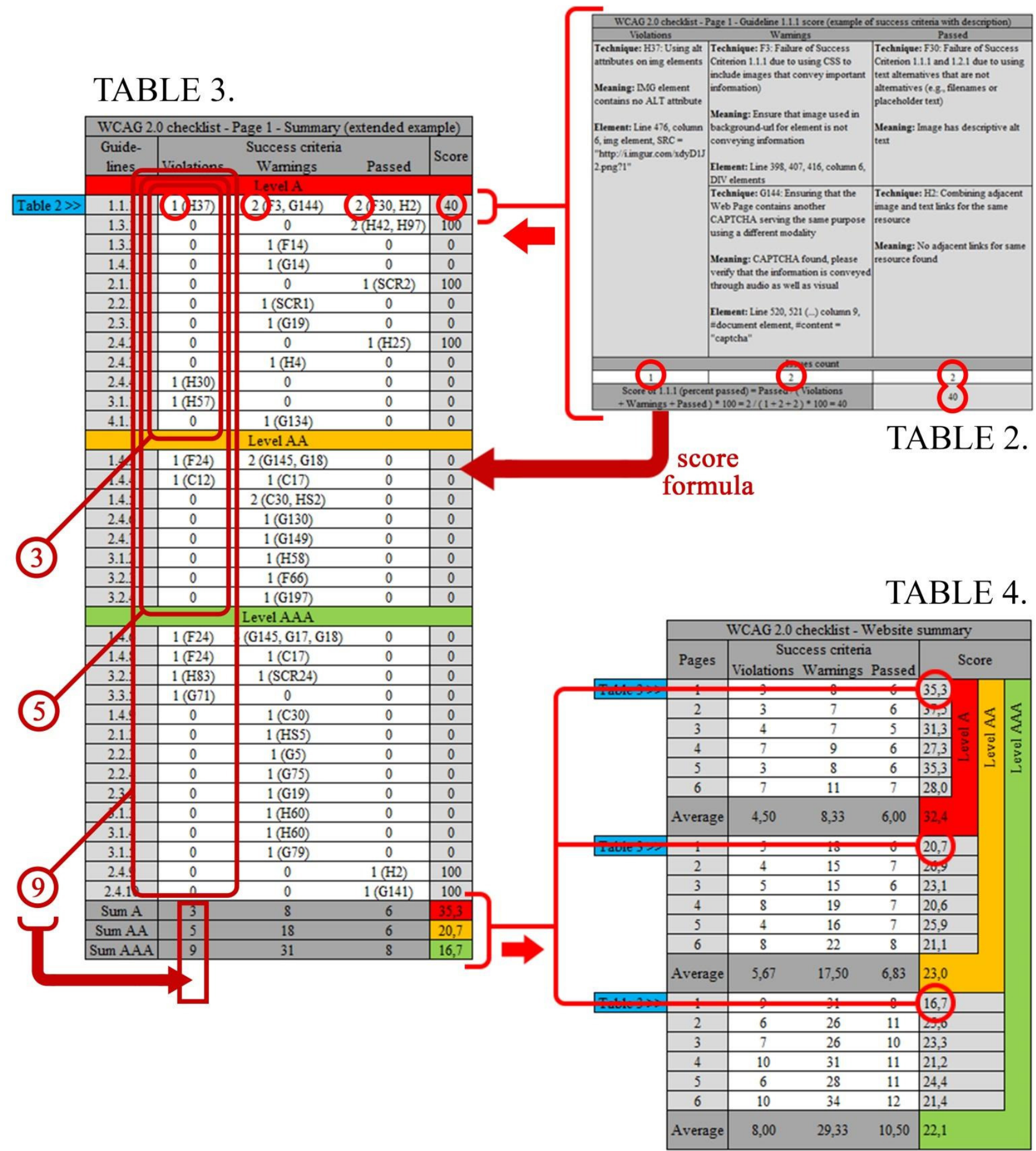

Fig. 1 Information flow between Tables 2-4

Source: own research. 


\section{RECAPITULATION AND CONCLUSIONS}

Web accessibility validation of the analyzed website was conducted in accordance with the WCAG 2.0 standard, for all three levels of compliance. The final report, consisting of numerical scores and detailed error reports, was generated on the basis of results produced by the Cynthia Says validator. Generated reports are fairly large, with most items presented in a nestled format to render the consecutive levels of detail in their presentation. Each item can also be opened in a new window. Due to editorial constraints and largely technical character of the results, this paper presents only a selection of those, to serve as examples in Tabs. 2. and 3.

Evaluating the effects of validation processes, one should bear in mind that the tested website is still under construction. The R\&D phase of the design is also a good time to conduct accessibility evaluations and to address any errors and violations of the WCAG 2.0 standard; for the website under examination, these are detailed in Tab. 5. Based on results presented in Tab. 4, the final score for the analyzed website is very low. Regardless of the adopted level of compliance, the overall ratio of 'passed' occurrences to all occurrences registered under the WCAG 2.0 success criteria for the site is very low, falling short of $40 \%$. Let us reiterate here: for a site to be judged as compliant at the very level of A, it must infallibly reach the $100 \%$ ratio within this level.

However, one can suggest at least two factors to justify such low scores obtained for the site under study. Firstly, for each of the three WCAG 2.0 compliance levels, the sum of warnings was found to be in excess of both the number of violations and 'passed' scores. Warnings represent elements which cannot be validated using the automatic methods; hence, they may not necessarily refer to errors in the website design but rather those of the tool itself. Such a dilemma can only be solved by the designer. However, the process is time-consuming and requires good knowledge of the site's source code. It is possible that the final score for the site under study would be much more favourable were we to apply this type of analytical reasoning. Secondly, it seems that the very notion of web accessibility is still lacking adequate propagation. If web designers fail to perceive web accessibility as the fundamental objective of their designs, there is small chance for it to satisfy the required standards to even a minimal extent, even when the quality of coding is otherwise flawless.

Cynthia Says is a well-designed tool with a convenient and intuitive interface. Most importantly, it provides useful and complex reports. But unfortunately they are not fully complete. The second problem is the items that the validator could not check automatically.

Previous research has shown that Cynthia Says is one of the best free license walidators, but also that none of them is perfect. Other tools were also used during the tests. For example, results were found to be largely similar to those produced by the Functional Accessibility Evaluator. One the other hand, additional or supplementary information could be obtained from other validators, such as Wave, HTML Validator, and aChecker. Rudimentary Web Developer Tools, of the type provided with most of the modern browsers for source code examination purposes, would be also very useful in the process. Therefore, prior to the initiation of testing procedures, and depending on webpage construction and reporting standard preferences, it may be advisable to utilise at least three mutually supplementing instruments.

\section{REFERENCES}

[1] Krawiec Ł., Nycz M., Pólkowski Z., Validation of website accessibility, a case study of DIMBI project, IEEE Xplore Digital Library, ECAI-2017 VOLUMES, Vol. 9-2017, ISSN-1843-2115, IN PRINT.

[2] Benicewicz-Miazga A., e-Business w Internecie i multimediach, Wydawnictwo Mikom, Warsaw, Poland, 2003, p. 24.

[3] Ziemba E., Metodologia budowy serwisów internetowych dla zastosowań gospodarczych, Wydawnictwo Akademii Ekonomicznej w Katowicach, Katowice, Poland, 2005, p. 27.

[4] Zawiła-Niedźwiecki J., Rostek K., Gąsiorkiewicz A. (red.), Informatyka gospodarcza, C. H. BECK, Warsaw, Poland, 2010, p. 227.

[5] Dudycz H., Krawiec Ł., The approach to the assessment of validators for studying web-accessibility - Orłowski A., Łukasiewicz P., Ząbkowski T., Gaj K., Szczesny W. (red.), Information Systems in Management, SGGW w Warszawie, Warsaw, Poland, 2017, IN PRINT.

[6] World Wide Web Consortium, Getting Started with Web Accessibility, https://www.w3.org/WAI/gettingstarted (20.01.2017).

[7] PowerMapper Website Testing and Site Mapping Tools, Government accessibility standards and WCAG 2, https:/www.powermapper.com/blog/governmentaccessibility-standards (20.01.2017).

[8] World Wide Web Consortium, Web Content Accessibility Guidelines (WCAG) 2.0, https://www.w3.org/TR/WCAG20 (25.01.2017).

[9] World Wide Web Consortium, Introduction to Techniques for WCAG 2.0, https://www.w3.org/TR/WCAG20-TECHS/intro.html (25.01.2017).

[10] Dudycz H., Krawiec Ł., Kryteria oceny walidatorów do badania dostępności stron WWW - Brzozowska A., Pawełoszek I., Turek T. (red.), Wiedza i technologie informacyjne $\mathrm{w}$ zarządzaniu procesami biznesowymi, Wydawnictwo Wydziału Zarządzania Politechniki Częstochowskiej, Częstochowa, Poland, 2016, p. 117. 



\section{Towards an Architecture of Electronic Service Market System - requirements analysis and evaluation}

\author{
Robert Kutera, Wiesława Gryncewicz \\ Wrocław University of Economics \\ ul. Komandorska 118-120, 53-345 Wrocław, Poland \\ Email: \{robert.kutera, wieslawa.gryncewicz\}@ue.wroc.pl
}

\author{
Tomasz Skalniak \\ Leipzig University \\ Ritterstraße 26 \\ 04109 Leipzig, Germany \\ Email: skalniak@wifa.uni-leipzig.de
}

\begin{abstract}
The last decade was a time of a very successful growth of Electronic Markets (EMs). EM is an electronicallysupported system which is rather focused on selling physical goods. Electronic Service Market (ESM) is a special case of EM, focused on selling services. The raising business of running ESM seems to be the next possible evolution of the classic EM, and in last few years, several ESM projects were started by various organizations. This paper presents the essential Functional and Architectural Requirements needed in ESM system. The way of their transformation into the architecture of real-life application based on STCMBER meta-architecture was presented. The proposed architecture was validated in the series of pilot implementations performed in compliance with Consortium Research method.
\end{abstract}

\section{INTRODUCTION}

\section{A. Structure of the Paper}

T HIS paper is organized as follows: this section describes the basic concepts of ESM and introduces the basic terms used in this publication. In addition, this section describes the origins of this paper and the used research method. The Section II contains the fundamental, functional (FR) and architectural (AR) requirements of ESM. The Section III introduces the meta-architecture used by the authors in various ESM projects, as well a concrete ESM architecture based on it. The Section IV describes how the architecture presented in the previous section was used in different projects and settings. What is more, this section shows how the used architecture is supporting the requirements described in the Section II. Section V contains the summary and proposes the potential next steps.

\section{B. Basics Concepts and Notation}

ESM is a raising business of selling services using the new Information Technologies (IT) [1]. ESM is a type of system which can be compared with the classic EM. In EM the main actors are: consumers, sellers and intermediaries. In ESM context, similar actors can be defined, but the sellers sell services, so we could name them also as "service providers". Service provider is an owner of the business which offers services. The person who is performing the ordered service is called a "service performer" (usually it is an employee of the service provider). Service performer is a new, additional actor, whose presence is distinctive in case of ESM - this role doesn't appear in classic EM. To sell the service, the service consumer (buyer) and the service performer (seller) have to meet. The meeting of the two sides of the transaction can be called simply as "service appointment". The process of coordinating the appointment of the stakeholders of the transaction, and the time needed to execute the service are the distinctive factors of ESM (when compared to the classic EM). Other fundamental, distinctive and unique feature of ESM is the m:n model of the offered service types. There are many electronic stores of the food delivery services, the hotel booking services, the transportation services, the home cleaning services, and other types of services. All similar solutions, offering a specific type of services, are based on the 1:n model - 1 type of services is available for many people. The n:m model means that in one system, different types of services are available to the same group of people. In this context, ESM can be understood as a business innovation: all the possible services available using one account, one password, and the same user interface. In the context of this paper, the "service" term means a "Bricks-and-Mortar" service: a service offered usually by SME and rather addressing the needs of individuals, instead of the needs of other companies [1]. The Bricks-andMortar services require to make an appointment between the service performer and service consumer, such a service can't be offered electronically.

What seems to be important to underline, is that the business of ESM means not only running a web store of services. ESM can also be used as an internal tool for enterprise companies or big corporations, or in the context of offering and coordinating public services to public consumers. What's more, in some contexts of use, the transactions done using ESM don't have to be money-based. Sometimes the services can be bought using the internal, artificial currencies, or even can be offered for free, or in the barter-based process. Similar characteristic of the various potential contexts of use can be observed in the classic EM. Described fundamental concepts of the ESM show that the ESM is an evolution of the classic EM. However ESM has some distinctive characteristics which together with some 
classic EM characteristics create a new class of enterprise systems which requires own architectural models and a new definition of crucial requirements.

This paper describes both functional and architectural requirements of ESM, so the authors discuss both the businessoriented (functional requirements) and technical details (architectural requirements) of ESM. Architectural requirements are the technical requirements which come from the functional requirements. The architectural requirements are the requirements which are analysed in the process of creating the software architecture (SA) [2], [3], [4]. Based on the architectural requirements, the design decisions are made, and a complete set of taken design decisions form a concrete SA. That's why, to propose the model of SA dedicated for ESM, there is a need of defining the crucial architectural requirements of such a system. On the classic electronic markets different types of requirements are already known and analysed. Also some requirements were defined for ESM [5]. This paper's research input is the analysis of the functional and architectural requirements of ESM more deeply, their synthesis and the indication of how ESM architecture is realizing them in real-life use cases.

The next section will introduce the research method used for identification and evaluation of the developed research artefacts.

\section{Origins and the Research Method}

The reason behind the idea of gathering the most important ESM requirements, and proposing the concept of ESM architecture was to do the next step in the process of building the theory about the ESM. In addition, one of the key theoretical aspects of this paper, was to emphasize the role of the appointment coordination as a key process in the age of service economy [6]. The requirements for the ESM system were researched by using a Consortium Research method [7], [8] which is a design-oriented research method based on the "design science" approach [9], [10]. The main goal and reason of why to use the design-oriented methods is to engage in the research process different stakeholder groups with different points of views and to analyze the research object from different perspectives. This approach is often used in the Information Systems (IS) research projects. The case-oriented research methods (including design-oriented) often produce "solution proposals" as the reserch results. Solution proposals are valid research artefacts of the Consortium Research, and are very often used while researching SA models [11]. It is important to note that the design-science approach is not based on the deductive reasoning, but rather on the natural language deduction by taking into consideration existing models and theories [9]. That is why the process of designoriented reaserch is always iterative. The iterative nature of the design science enables to validate the research artefact in different contexts and different environments, making it better and better with every iteration. The design science process consists of Analysis, Design, Evaluation and Diffusion (for a detailed explanation please see [7], [8]). This process used in a specific context and under concrete conditions can be named as the Consortium Research method. In the Consortium Research method the research team consists of practitioners and researchers, university workers as well as people from real businesses. They work closely together to find a solution (as a research artefact) which solves a real-life problem. The minimum research duration is 2 years. To ensure that the artefact will be usable in real-life context, the research has to be partly finance by the partner companies (practitioners). The results of the research have to be disseminated in both the researchers and practitioners communities, and the research results have to accessible in a public domain. The research artefacts have to be tested in a business context of the partner companies. The research artefacts don't have to be theoretical - the practical solution is acceptable. [8] Only in rare cases the constructed and validated reasearch artefacts can be formally verified. Rather the artefacts are rejected or accepted by experts, who can judge if the verification was correct or not [9]. In the case of this paper, the research artefact (ESM architecture model) was tested, judged and verified by the partner companies and social organizations who was engaged in the research process, and who used the designed ESM architecture model in real-life cases.

The ESM system built during the research, its requirements and architecture were researched by the Consortium Research team. The research was started few years ago, and the partial results were tested in different contexts and projects in the last 10 years. The developed artefacts, including the discovered appointment coordination (AC) processes, ESM SA, software delivery concepts, social impact of ESM and related-platforms, UI-specific issues related with cross-platform applications, and other topics, were researched thanks to the long and deep engagement of different researchers and practitioners [12], [13], [14], [15], [1], [16], [17], [18], [19]. Right now, for more than 2 years, the team includes 3 universities from 3 different countries (Switzerland, Germany and Poland), 2 different companies (Switzerland), 3 social organisations (one from Switzerland and two from Germany) and more than 20 researchers and practitioners engaged.

In the next section the requirements gathered during many performed iterations will be presented in a synthetic form and marked with proper symbols (FRx, ARx, where $\mathrm{x}$ is the subsequent number) for their better identification within the paper.

\section{REQUIREMENTS ANALYSIS}

\section{A. Functional Requirements}

On electronic service markets, both parties must agree to conclude a contract. There are many requirements that are significant to them. The most important ones are described below. Some of them (e.g. FR1, FR4, FR5 - see below) are inherited from the classic EM systems, and even if they are not unique for the ESM, they play a significant role, and can't be omitted.

First of all, while surfing the Internet people are using different tools: desktop computers (PCs), laptops, tablets, 
smartphones and other mobile devices. That is why applications for electronic services have to work on various platforms (FR1), not only web platforms, but also mobile ones. Users expect to have access to the system from diverse places and with different devices. The interface should ensure the similar positive user experience on each platform (similar navigation and content logic, easiness of use). Also the process of coordinating the place, time and choosing the actors needed to realize a given bought service is crucial (FR2). Selling a service means, that the service consumer and the service performer need to meet at some point. In addition, in some cases to fully utilize the ordered service, the service has to be served few times, e.g. some massages might need to be repeated few times. The place of the actual execution of the service has to be coordinated too. For example the gardener might offer his services at the client location, at the same time the hairdresser might offer own services only in the hairdressing salon.

The service performers, providers and the service consumers should be able to manage their calendars with the view of all the past and upcoming appointments. Such a view is very important when the negotiation of the date and place of a concrete appointment takes place. What is worth to note, is that some parts of the different AC processes are the same. For example the process triggered by the service consumer, or started by the service provider, can achieve a state of a date and time of the appointment already agreed by both sides of the transaction. From such a state it doesn't matter who started the process, so both the consumer-triggered and provider-triggered coordination processes can have shared, common parts.

Of course managing all the appointments means not only having them all on one view, but also being able to filter them based on different conditions. For example, some appointments which are not yet coordinated should be possible to be filtered. Also, some upcoming appointments might require the action of a specific actor in the coordination process. For example the data proposed by the service performer might not be suitable for the service consumer. So the service consumer should be able to propose an alternative date. Then, the service performer should be able to accept the data, or to propose a different one (or a set of alternative dates). Such a state of the appointment in the AC process requires the performer's action - the process of coordination will not proceed until he/she does the action. To handle the appointments, both sides of AC process should be able to filter the appointments which require their actions to proceed.

What is more, it is very important for both sides to have the possibility of changing the time of an appointment. Nowadays people are very busy, have a lot of additional jobs and duties which can cause unexpected changes in plans. Both, the service provider (and his performers) and the service consumer must be flexible, they must enable changes of previously established appointments.

Very important functional requirement, directly connected with previously mentioned one, is the support for manual and automatic dispatching of appointments to proper performers
(FR3). It is very important for providers to have as many automated processes as possible in each possible case. Such an automation of dispatching process can spare their time spent for performing simple repeatable day-to-day operations of looking for free time slots of particular perfomers at the particular time. In both scenarios of automatic and manual decision making, the calendar with already agreed appointments and the free timeslots is needed. In addition, the service provider or the manager responsible for manual dispatching the services requested by the consumers, might see all the companies past and up-coming appointments in one calendar view. Such a functionality might be needed in different use cases, e.g.: while preparing the internal company event (when all the employees have to be available). Dispatching the performer might be done automatically, based on the personal calendars of the performers, or even directly by the performers - the one, who wants to perform the requested service, performs it. The processes of matching the service consumers and providers (needed services) might be very complex. For example, in some cases, the service consumer might want to just select the needed type of the service he is looking for. After selecting the desired type of service, the service provider (and his specific service) could be automatically matched with the consumer (using different algorithms) or could be chosen using different method, e.g. "First-come First-served" (FCFS) method.

Another important requirement is the mutual confirmation of service delivery (FR4). The customer should have the opportunity to express an opinion about the service he has used and about the provider. Also the service provider (or performer) should be able to rate his customers. In that way both sides of the transaction become less anonymous and the overall service market - more transparent. When buying a service, customers don't want to rely only on its description and expect to get familiar with the opinions of other customers. They can give credibility to the service provider. On the other hand, the service performer will gladly perform a service for the customer that has positive opinions. For this purpose, a system of stars or points can be used.

In times of social media expansion an important requirement is also the recommendation possibility (FR5). Satisfied customers could be the best ambassadors of the service and could share the information about particular offer among people in their closest environment. An interesting information may even become part of an advertising campaign for a particular service provider. This phenomenon is described in the literature as viral marketing [20]. An intriguing idea can inspire more and more new audiences to further spread the information and create opinions. In social media it is also very popular to comment and assign "Likes" to interesting offers. These are examples of market stimulation by those who have not used the service, but whose opinion may be of interest to potential buyers.

In addition, some of the users of ESM would like to use a different calendar system then the internal calendar view offered by ESM. There is a big variety of the electronic calendars on the market, many users already do use some of them. 
Switching to a new electronic calendar, or using few calendars to maintain all the appointments is very uncomfortable. That's why one of the most important functional requirements is the need of being able to see ESM appointments in different calendars (FR6). Calendars integration is a difficult problem, because there are different implementations on the market, and different implementations use different standards of live synchronization of the content. On the other hand, the need of calendar synchronization is one of the most important issues when it comes to lowering ESM entry barriers.

Service providers, who deliver services on electronic markets, should adopt flexible pricing policy (FR7). They should offer different discounts, promotions and special offers for particular user groups. The possibility of price negotiation is also very appreciated (e.g. I have a large house, so I expect lower window cleaning rates). In addition, services can be reckon up on the basis of duration (e.g. lawn mowing time), number of completed units of service (e.g. number of massages) or level of complexity (eg type of hydraulic failure). Moreover, service settlements should be available in different currencies. Due to the high involvement of people in all kinds of non-profit organizations, the possibility of providing services free of charge should also be considered.

$\mathrm{AC}$ processes consist of many possible stages and require a cooperation of both sides in determining the place and time of the appointment. That is why both sides have to be informed about any changes in the process to stay up-to-date all the time. If any action was performed, there is always a side of the transaction, which has to wait until the second side will react. That's why, it's important in ESM, to offer the functionality of notifications about changing the state of the appointment to shorten the waiting time as much as possible (FR8). In case of any action required, such notifications should motivate users to act immediately.

The list of crucial functional requirements described in this section is basic but they seem to be universal for many electronic service markets. That is why this list was chosen as a basis for determining the list of architectural requirements for univeral ESM system.

\section{B. Architectural Requirements}

The analysis of the functional requirements of ESM, described in the last section, leads to discover a set of very concrete architectural requirements. The first architectural requirement can be defined as multi-platform character of end-user applications with a strict separation of its layers (AR1) and is related directly to the requirement FR1. Support of different end-user applications (on various hardware platforms) and using/extending the native platform elements (e.g. native platform calendars), leads to the separation of the application logic code and the business logic code. In a result the SA must consist of separate layers with a very detailed inter-layer communication protocol. It defines basic rules of how the layers are dependent on each other, and what kind of communication is allowed in which direction. For example, the communication between the layers, and modules written in different technologies (e.g. Android/iOS application and responsive web site developed in PHP or Python) might be done using the REST architectural style [21]. What is more, using layer-based approach forces the strong authorization policy for ensuring an access to particular layers only for allowed requestors to avoid any security breaches and unauthorized data leaks. For that purpose, OAuth protocol could be used [22].

Another very important requirement is to have the specialized AC services, with the very different AC processes available inside (AR2). It is based on a set of functional requirements: FR2, FR3, FR4. ESM should be usable and understandable for users so that the coordination processes should be similar to each other with unified interfaces and common set of possible actions. The processes should be adapted to the functional requirements, including the manual or automatic appointment dispatching (FR3), flexible pricing policy (FR7) and post-sale phase supporting with two-sided confirmation of performed service (FR4). The processes require automation to some extent so the respective business logic should allow for performing tasks not only on user request (action pinned to the specific button) but also catching some application events and handle them automatically with taking into account the dedicated algorithm. An example could be the already mentioned feature of auto-dispatching appointments to performers who defined their availability in theirs individual calendars (FR3). It also requires to design complex data structures and business entities for appointment, service or user in a way that allows for handling all process tasks. In order to do that it is necessary to equip the SA of ESM with the Business Process Management (BPM) system. The possibility of defining new sub-processes based on the existing processes, having one set of the available actions between the processes, changing the flow of the process without the need of a deep code refactoring and re-engineering the backend parts of the system, seems to be the most crucial part of ESM system. On the basis of AR2 different design decisions can be made: different BPM engines can be used, different technologies might be needed to address this functionality and to fit into the overall SA. For example, if JAVA technologies are used, maybe the good idea would be to use the Activiti framework [23] as the BPM engine.

The next architectural requirement for ESM is the need of synchronization of different user calendars for the use within $\mathrm{AC}$ processes as well as within company management ones (AR3). It seems to be the natural consquence of the FR6 requirement. In order to do that, some native implementations and extensions to the commonly used calendar systems should be made. Consequently, the SA of ESM might contain modules dedicated to different end-user devices (some popular calendars are only available on a very specific hardware platforms).

Functional requirement of flexible pricing policy (FR7) leads to another significant architectural requirement of having the user groups entity which allows for addressing offers to specific public or private groups of users (AR4). What is more, many organizations on the electronic service market would 
expect to have the multitenancy-ready ESM which allows for not only addressing specific offers, but also providing the tenant-specific interface of the application. For this purpose, new processes for managment of those entities should be designed as well as those entities should be taken into account in main processes of ACS, as it has been mentioned above.

The functional requirement of a fast and convenient notification system (FR8) leads to another architectural requirement. There should be the dedicated component to handle the notifications (AR5), not only for catching events and sending proper messages, but also queuing and postponing the notifications if they are being tried to send at the inappropriate time (e.g. at night). Depending on the specific context, sometimes the notifications engine can work only using the Internet - the notifications can be implemented on the end-user devices using the native platform notifications system (e.g. Android native notifications system), sometimes the SMS engine might be required.

By taking an advantage of efficient notification system the recommendation tool (AR6) could be also designed for satisfying the requirement FR5. The special notification, which will be sent on user request to another person, even if he/she is not the actual ESM user. What is more, the data structures should be designed in a way to allow different kinds of promoting the services within the ESM catalog.

This Section introduced main architectural requirements, and potential design decisions which would have to be taken in real-life use cases. Designing ESM SA is a very complex process of gathering a lot of functional requirements, and based on the aggregated set of such requirements, the architectural requirements have to be defined. Then, based on the defined architectural requirements, the design decisions have to be made. What is more, the strict non-functional requirements should be defined to enable quick, safe and convenient performance of ESM. The whole process of software design is very complex, and it is not a goal of this publication, to address all the problems of such a process.

In the next section the Authors will present the concept of the meta-architecture developed by the Consortium which allows to build on top of it concrete ESMs architectures, based on the modern enterprise design patterns.

\section{ESM ARCHITECTURE}

\section{A. STCBMER Meta-Architecture}

The meta-architecture is an abstract software model which can be used to build on top of it a concrete SA [24]. STCBMER (Smart Client-Template-Controller-BeanMediator-Entity-Resource) is such a component-based [25], [11] meta-architecture [14] designed to support building modern, layered enterprise software systems. STCBMER was comparatively evaluated with other component-based, layered meta-architectures in previous works of the authors [12], [13]. STCBMER is following various architectural principles [13] which are simple pieces of practical architectural knowledge [4], captured by SA researchers and practitioners, and used to build different kinds of software models. Due to the limitations of the paper it is not possible to describe all of them [13], but just as an example: the DDP (Downward Dependency Principle) principle states that the architecture model is topdown, and objects of higher layers depend on objects in lower layers. Based on the principles, STCBMER constructs a component-model which is a graph of several main nodes attached to three layers:

1) Business Logic Layer
a) Resource
b) Entity
c) Mediator

2) Application Logic Layer
a) Bean
b) Controller
c) Template

3) Smart-Client Logic Layer

The base component is Resource - it's main goal is to communicate with the data sources. Entity is component which includes all the data definitions. The Mediator is a main Business Logic component, it contains all the business logic. Mediator manipulates on the Entity objects but is also responsible for storing them in the data sources and caching. What's more, Mediator prepares the REST API available for higher layer - Application Logic. Bean is a component from the Appication Logic layer, it is similar to the Entity, but is never stored in a database - Bean classes rather live in the memory. Controller is a heart of the Application Logic - it consists of server-side application functionalities. Controller has various internal sub-elements responsible for: consuming the Business Layer API, defining the application logic pieces (application processes), defining the REST API for the higher layers. To define the application logic, Controller has to manipulate on data - Bean objects. Both Controller and Bean object are used by the Template to prepare the results of the REST web services of the Application Logic layer. Depending on the needs, Template can prepare UI elements (e.g. HTML, JavaScript, CSS), data (e.g. XML or JSON) and other types of results. The Smart-Client Layer is a layer which contains elements needed to prepare the web application run by the web browser or other end-point environment. It's internal components are usually based on one of classic MVC variants and are usually implemented in the same technology, usually JavaScript. Smart-Client connects to the Application Logic via REST API and complements the logic delivered by the Application Logic layer. Thanks to the Smart-Client Layer, the application is faster and more responsive, because some parts of the processing are performed directly on the end users machines. Smart-Client Layer enables also to cache some parts of the application in the end users devices what allows for using the system in the off-line mode.

\section{B. STCBMER Concrete Implementation}

The ESM system (Amiona) prepared by the Consortium (see Section I-C) researching in the field of ESM, was based on the STCBMER meta-architecture. The presented ESM 


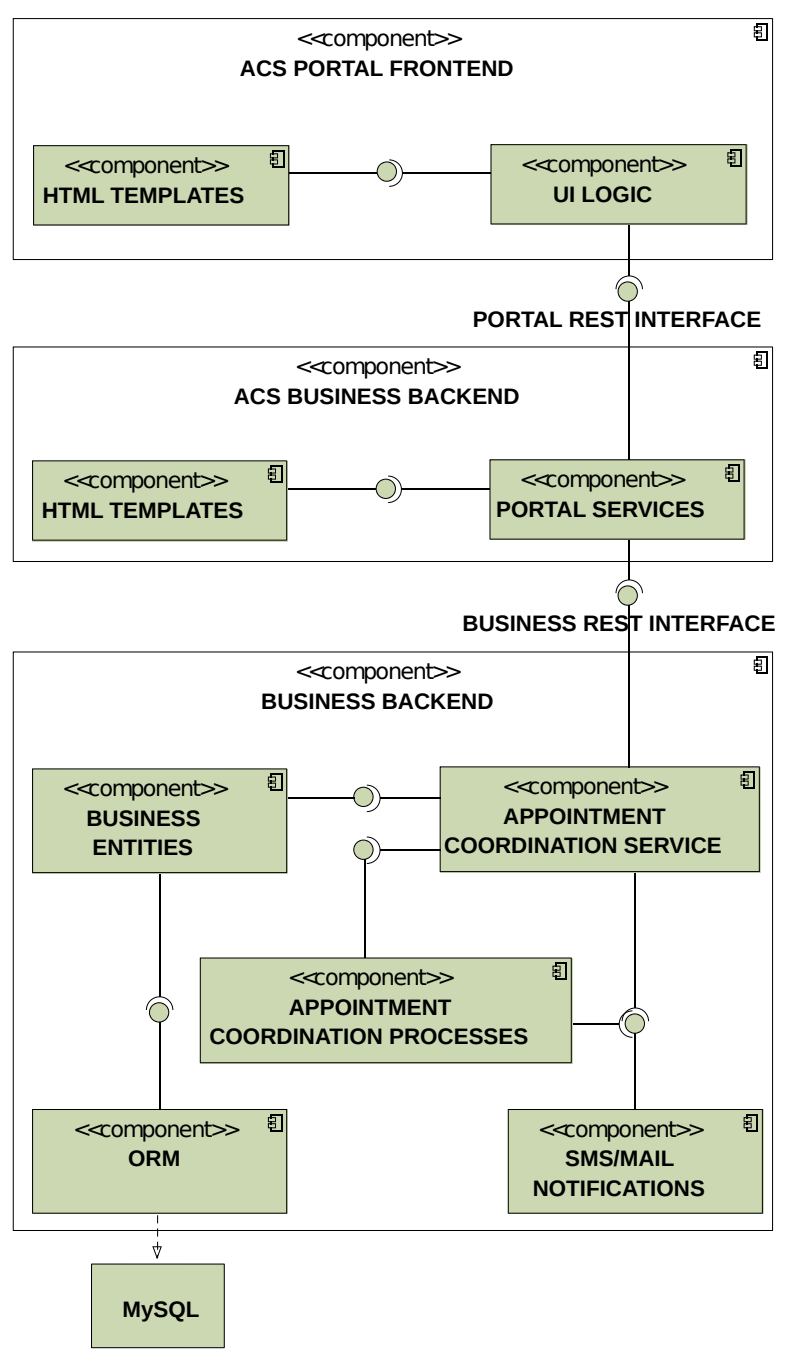

Fig. 1. ESM architecture

architecture (see Fig. 1) consists of 4 layers:

1) Business Backend

2) ACS Business Backend

3) ACS Portal Frontend

The Business Backend is based on the STCBMER Business Logic Layer. The Business Entities and ORM can be mapped as the Entity sub-elements, The Appointment Coordination Service can be mapped as the Mediator. Appointment Coordination Service contains all the crucial ESM elements: implementations of various $\mathrm{AC}$ processes as well as the notification engine. In the presented ESM architecture the Resource responsibilities are delivered by the ORM subcomponent (because of the concrete framework used in the implementation).

The Portal Services can be mapped as the Controller, while the HTML Templates can be understood as Template.

The ACS Portal Frontend is based on the Smart-Client Layer concept. UI Logic is containing all the client-side logic (including data definitions), while the HTML Templates produce the pieces of HTML to build the UI.

It is worth noticing that both Appointment Coordination Service and Portal Services are both equipped with the REST interfaces, and could be consumed by other, third-party system as well. The data source used in the presented ESM architecture is MySQL, the basic frameworks and libraries used in this architecture implementation are: SQL-Alchemy (ORM, Entity), Pyramid (Appointment Coordination Service, Appointment Coordination Processes, Portal Services), Mako (ACS Business Backend: HTML Templates), Angular.js (ACS Portal Frontend), Bootstrap (HTML Templates).

To read more about how frameworks can fit into STCBMER, and other software models, see [14].

The web application for ESM, implemented on the basis of this architecture, was used and deployed for various market conditions. Those use cases gradually delivered new requirements in compliance with the principles of chosen research method. Thanks to it the application was evaluated by real customers and the current state of the application architecture is the joint effect of gathering feedback from various use cases. The next section will provide information how the proposed architecture meets the special requirements in different community settings (rural, small town and urban).

\section{Evaluation}

\section{A. ESM in small town setting}

The Consortium (details in Section I-C) organized three pilot implementations of Amiona ESM in three different cities in Northern Germany (urban setting), Southern Germany (rural setting) and Switzerland (small town setting). These 3 pilots addressed different target groups, focused on different occupational modules, and had to deal with different local systems. Thereby, the final solution is flexible enough to adapt to varying situations, and, consequently, better suitable for a roll-out to different regions. The ESM architecture model was positively evaluated by the experts (see Section I-C) on the basis on their experiences, required business knowledge, and test results. Due to the limitations of the paper, it is not possible to show the details of the pilot-specific systems in a graphical form. Therefore, the pilot descriptions contain only the most important remarks, and some references to the presented requirements (see Section II).

This pilot implementation (Sankt Gallen, Switzerland) is the main foundation for the shape of proposed ESM. The general objective of the pilot implementation is to improve the quality of life of elderly people from small town by facilitating their access to all kinds of brick and mortar services. Their independence is associated with self-attaining extremely broad spectrum of everyday life activities. Consequently, they have an increasing need for services of specialists in specific fields. The main purpose of the ESM in that case is to eliminate the existing problems related to service orders, like fragmentation of suppliers, the complicated process of ordering and negotiating the terms of performance and to reduct to the maximum of the complexity of these processes[26]. The requirements 
taken into account in this case are: FR1, FR2, FR6, FR8 (full covarage), FR3, FR4, FR5, FR7 (partial coverage). They affect all architectural components of ESM.

The proposed architecture meets these requirements well. First of all, the AC processes are very intuitive (on the basis of AR2) and supported by simple and concrete notifications at each stage (on the basis of AR5). They consist of small tasks which are organized in a specific way for each separate process, like a standard process (consumer-triggered), a provider-triggered process, a direct booking process (request on specific date with automatic acceptance of appointment) or an ad hoc process (when at least one side of appointment isn't identified in the system). All those processes are supported by Appointment Coordination Processes component and respective web services from Appointment Coordination Service component. However the processes are different in general, they use the same tasks in a different order and the user is navigated through the process by the system itself. Information about user appointments is gathered in one common view and all operations on them are accessible from one place.

ESM supports also service providers in day-to-day operations of company management by providing them with tools for managing: company profile, its services, its performers and all company appointments (FR3). In addition, it offers a wide range of price configuration, with choice of the currency, price units and so on (FR7). Discounts could be also defined, e.g. for specific dates offered in direct booking process (which allows for offering discounts to specific time slots, e.g. less popular hours of using a specific service). Users have an access to the calendar which could be synchronized with external calendars with the iCalendar (.ics) file format (AR3) to have a better control of his appointments schedule (FR2, FR3, FR6).

The proposed architecture, thanks to the Smart-Client Layer concept, and using REST as communication channel allows to offer the application simultaneously as the web application (meeting the responsive web design standards) as well as the mobile application for Android devices (AR1/FR1). The backend of the application could be also easily used for providing business logic for other devices, like Smart TV or wereables. Thanks to that user can have an access to his appointments from multiple devices and operate on them easily. Recommendation tool (AR6/FR5) is only partially implemented and relies on the intermediary-triggered approach where intermediary can recommend a particular service offer, which is marked with special formatting on public catalog views. In general this marketplace is using most of the features provided by the ESM and all of them have a high rate of acceptance within the local community of users as well as service providers, who have got the another profitable channel of distribution of their services. The ESM is also continously maintained and improved on the basis of user feedback and experiences from another field trials.

\section{B. ESM in rural setting}

The next setting, rural, is focused on the voluntary transportation services (SeniorenMobil) for senior citizens (Weil der Stadt, Southern Germany), supervised by one of the Consortium's end user organisation. The existing process works as follows:

1) The elderly citizen calls in to make a request for a drive.

2) The order is taken by the intermediary via their InternetPlatform or phone.

3) The service provider is informed about the request and looks for a possible driver, most likely to be the scheduled driver for the requested date.

4) The drive is conducted.

In this process, the drivers at first have to schedule the dates on which they are free to drive for the transportation service. They have to be available on the scheduled day via e-Mail or telephone. If they get a request for the scheduled day, they make contact with the customer to confirm the request. Then they pick up the customer and carry out the drive. One of the most important requirements from the end user organization is to support AC by an automatic matchmaking of appointment requests with driver's schedules at the initial stage of the process. It is an important part of the FR3 requirement. ESM, by satisfying the AR2 requirement, provides necessary enhacements to Business Entities component (especially to Performer and Appointment entities) as well as in Appointment Coordination Processes component (improved manual dispatching logic and added the handling of the algorithm). The frontend part, UI Logic and HTML Templates components contain the templates and web services for handling the operations on new calendar view asynchronously. As a result, service providers get the dedicated calendar view for managing theirs performers and theirs availability schedules. The service providers have access to company management tools, and among them the "Availability schedule". The tool is responsible for managing schedules of all performers drivers in the test case. The provider has permissions to modify schedules of all his performers. In parallel, performers (drivers) are able to see all the schedules in their personal profiles, but they can only modify their own schedule by entering or editing availability slots. As already mentioned, in the particular AC process, a special algorithm of automatic dispatching was included. This algorithm takes into account drivers' availability, and the appointment is dispatched to the first available driver, whose availability slot is long enough to fit the current service duration. For that purpose each appointment should have identified the duration parameter, which depends on the service specificity. The driver, within the AC process, could accept the appointment or refuse it, what triggers the next iteration of the algorithm, when the appointment is proposed to the next driver (all based on normal AC proccess, FR2). If none of appointments is accepted, the intermediary is asked for manual dispatching.

The proposed ESM architecture was able to handle the case-specific requirements and make the whole process very clear and efficient. Users were trained for using the ESM and now the service is offered to the end users. First user feedback shows they like the flexibility of the service, it 
spares time of both sides, drivers as well as intermediary. The information on availability of drivers is clear and usable on calendar view (as a Gantt diagram) and works efficiently because of using Smart-Client layer concept and processing data asynchronously, mostly on user device.

\section{ESM in urban setting}

Third pilot implementation takes place in Hamburg, the second largest city in Germany and is performed for social organisation that supervises several neighborhoods and aims at helping elderly people in their inclusion into social life. The first neighborhood, which is using the ESM, is Rungehaus that consists of 73 fully accessible apartments and offers shared space of 220 square meters. Its quarter-related approach of living with secured maintenance and without a lump sum for care is a peculiarity of this model. By integration into the existing housing quarter and the neighborhood of the Rungehaus, living for people who do and do not need assistance, or are old is enabled. Similarly to the Rungehaus, tenants from the extended neighborhood ( 3 housing complexes that consist of 1,027 apartments owned by the same owner as Rungehaus) have also access to common rooms. The rooms are open to use for the whole neighborhood, and there is a special location for professional service providers. The service providers of the quarter office enable a broad range of services which is available at all times. In general, assistance and supporting services are available for all tenants. The range of services is ensured by a mixed financing of ambulant care service providers and integration assistance.

From the architectural point of view, the most important requirements concern the necessity of modification of the AC process (AR2, AR5). End users expect to have information about engagement opportunities, about different possibilities to become involved as a volunteer, and about searching and offering neighbourly help in the immediate vicinity. Both - provide support and receive assistance - should be possible. Any typical ESM system is able to handle supplyside processes of publishing offers of services. One of the ESM innovative approaches is that the user can define his expecations and create a demand which is propagated to all formal and informal providers (some of them, potientially interested are also directly informed by the notification). The process is being continued by gathering offers for that particular demand, choosing of offer(s), and creating the respective appointment(s) on that basis. Next steps are the same as in standard process. The presence of a new entity in Business Entities component requires to introduce new data structures. This new entity still can share many of these structures with the core entity - Service (e.g. Address or Category data structure).

Another important issue is to support intermediaries in the mediation of volunteers as well as searching and finding volunteers (AR2, FR3). Intermediaries have the possibility to act on behalf of other users and enter a demand for another person if he/she is not able to do that because of some kind of disability. What is more, intermediaries and service providers can create specific user groups and manage them on the separate view. The users gathered in user groups can get special offers with discounts or other benefits depending on business context (AR4). That is why the core AC processes and searching/browsing offers had to be updated (AR2) to be able to handle the specificity of user groups.

The Portal Frontend component is able to meet the specific requirements of having the web application developed in compliance with responsive web design paradigm to be available on such devices as PCs, laptops, tablets and smartphones. All features, including consumer and service providers tools, are available to use on mobile platforms. The implementation in the urban area of Hamburg is only a part of a larger, integrated solution, however this topic goes beyond the scope of the paper. More about the integrated project in: [27], [17], [19]. The first user feedback in test implementation of the integrated solution, including the Amiona ESM instance per neighborhood, provides a good feedback and shows an interest of potential users.

\section{SUMMARY AND FUTURE WORK}

The digitalization of the consumer world brings a significant shift for companies. Electronic markets are growing and more and more consumers are looking for convenient ways of satysfying their needs in order to improve their quality of life. The consequence of this shift could be a discipline of life engineering which together with business engineering have to continue to ensure efficiency in the satisfaction of needs [28]. ESM plays a special role in providing access to brick and mortar services to users via the electronic channel in easy, quick and secure way. Such circumstances require a strong software foundation for ESM system. The SA needs to be flexible and allows for its customization for specific cases.

In order to find the universal ESM architecture Authors gathered a set of functional requirements from many reallife use cases and synthesised them into 8 main ones. On that basis the architectural requirements were developed and the final shape of the ESM architecture proposed. The ESM system implemented with the new architecture principles was evaluated in many use cases, however three of them are presented in the paper.

The results of evaluation show that the architecture is flexible enough to handle specific requirements of different territorial settings and business context (from commercial services to voluntary help). Still, the new opportunities emerge and for sure the proposed architecture will evolve over time. However, the taken approach of using Service-oriented architectural principles and modern design patterns can assure the evolution won't turn into a revolution in the next few years.

Not all the described requirements (see Section II) were covered by the Evaluation phase (see Section IV). The twosided confirmation requirement (part of the AR2) was never implemented in any of the Evaluation iterations. The AC process confirmation was implemented only as a one-sided (the service provider confirms that the service is done, not confirmation on the consumers side is done). The second 
requirement not yet implemented and validated is the recommendations tool (AR6). In this case, there are no notifications assigned to the recomendation process yet, as well as so far consumers cannot recommend any offer to each other. Both requirements are thought to be a part of the solution, and will be fully implemented in the future iterations.

\section{REFERENCES}

[1] P. Schenkel, P. Osl, and H. Österle. Towards an electronic maketplace for bricks-and-mortar services. In 24th Australasian Conference on Information Systems, pages 1-11, Melbourne, 2013. ACIS 2013.

[2] J. Bosch. Software architecture: The next step. In F. Oquendo, B. C. Warboys, and R. Morrison, editors, Software Architecture, pages 194-199. Springer, Berlin, Heidelberg, 2004. [Online]. Available: http://dx.doi.org/10.1007/978-3-540-24769-2_14

[3] M. Babar. A web-based system for managing software architectural knowledge. In J. Yao, editor, Web-based Support Systems, pages 305-332. Springer, London, 2009. [Online]. Available: http://dx.doi.org/10.1007/978-1-84882-628-1_14

[4] M. Babar. Supporting the software architecture process with knowledge management. In M. Babar, T. Dingsyr, P. Lago, and H. van Vliet, editors, Software Architecture Knowledge Management, pages 69-86. Springer, Heiderberg, 2009. [Online]. Available: http://dx.doi.org/10.1007/978-3642-02374-3_5

[5] A. Schmidt, B. Otto, and H. Österle. Integrating information systems: case studies on current challenges. Electronic Markets, 20(2):161-174, 2010. [Online]. Available: http://dx.doi.org/10.1007/s12525-010-0037-8

[6] Z. Zhou. The Development of Service Economy : A General Trend of the Changing Economy in China. Springer Singapore, 2016. [Online]. Available: http://dx.doi.org/10.1007/978-981-287-901-1

[7] B. Otto and H. Österle. Relevance through consortium research? findings from an expert interview study. In R. Winter, J. L. Zhao, and S. Aier editors, Global Perspectives on Design Science Research, pages 1630, Berlin, Heidelberg, 2010. Springer Berlin Heidelberg. [Online] Available: http://dx.doi.org/10.1007/978-3-642-13335-0_2

[8] H. Österle and B. Otto. Consortium research. Business \& Information Systems Engineering, 2(5):283-293, 2010.

[9] H. Österle, J. Becker, U. Frank, T. Hess, D. Karagiannis, H. Krcmar, P. Loos, P. Martens, A. Oberweis, and E. Sinz. Memorandum on designoriented information systems research. European Journal on Information Systems, 20:7-10, 2011.

[10] J. R. Venable. The role of theory and theorising in design science research. In in: Design Science Research in Information Systems and Technology, 2006.

[11] T. Vale, I. Crnkovic, E. S. de Almeida, P. A. da Mota Silveira Neto, Y. C. Cavalcanti, and S. R. de Lemos Meira. Twentyeight years of component-based software engineering. Journal of Systems and Software, 111:128 - 148, 2016. [Online]. Available: http://dx.doi.org/10.1016/j.jss.2015.09.019

[12] L. Maciaszek, T. Skalniak, and G. Biziel. A meta-architecture for service-oriented systems and applications. In Fourth International Symposium on Business Modeling and Software Design, pages 20-28. ScitePress, 2014. [Online]. Available: http://dx.doi.org/10.5220/0005423900200028

[13] L. Maciaszek, T. Skalniak, and G. Biziel. Architectural principles for service cloud applications. In B. Shiskov, editor, Business Modeling and System Design, volume 220, pages 1-21. Springer International Publishing, 2015. [Online]. Available: http://dx.doi.org/10.1007/978-3319-20052-1
[14] L. Maciaszek and T. Skalniak. Confluent factors, complexity and resultant architectures in modern software engineering: A case of service cloud applications. In Fifth International Symposium on Business Modeling and Software Design, pages 37-46. ScitePress, 2015. [Online]. Available: http://dx.doi.org/10.5220/0005885300370045

[15] G. Biziel, A. Pyka, T. Skalniak, J. Słowik. Platforma zarzadzania usługami jako narzędzie wspierające życie osób starszych (Service Management Platform as an Independent Living Supporting Tool for Senior Citizens). Informatyka Ekonimiczna (Business Informatics). Wydawnictwo Uniwersytetu Ekonimicznego we Wrocławiu, 2(32):33-40, 2014.

[16] Ł. Łysik, R. Kutera, P. Machura. Social Collaboration Solutions as a Catalyst of Consumer Trust in Service Management Platforms - A Research Study. In W. Abramowicz, editor, Business Information Systems: 18th International Conference, pages 220-232, 2015. Springer International Publishing. [Online]. Available: http://dx.doi.org/10.1007/978-3-31919027-3_18

[17] L. Maciaszek, W. Gryncewicz, R. Kutera. Integrated Service EMarketplace for Independent and Assisted Living - Business and Software Engineering Challenges. In B. Shishkov, editor, Business Modeling and Software Design: 6th International Symposium, pages 221-231, 2017. Springer International Publishing. [Online]. Available: http://dx.doi.org/10.1007/978-3-319-57222-2_11

[18] W. Gryncewicz, R. Kutera, M. Leszczyńska, B. Butryn. The model of delivering an IT product the designed to activate and support senior citizens in Poland. In M. Ganzha, L. Maciaszek, M. Paprzycki, editors, Position Papers of the 2016 Federated Conference on Computer Science and Information Systems, Annals of Computer Science and Information Systems, pages 195-202, 2016. Polskie Towarzystwo Informatyczne. [Online]. Available: http://dx.doi.org/10.15439/2016F209

[19] A. Rot, R. Kutera, W. Gryncewicz. Design and Assessment of User Interface Optimized for Elderly People. A Case Study of Actgo-Gate Platform. In C. Rocker, J. O'Donoghue, M. Ziefle, L. Maciaszek, W. Molloy, editors, Proceedings of the 3rd International Conference on Information and Communication Technologies for Ageing Well and e-Health. (ICT4AWE 2017), Annals of Computer Science and Information Systems, pages 157-163, 2017. ScitePress. [Online]. Available: http://dx.doi.org/10.5220/0006320001570163

[20] J. Berger. Contagious: Why Things Catch On Paperback. Simon \& Schuster, 2016

[21] R. T. Fielding. Architectural Styles and the Design of Network-based Software Architectures. $\mathrm{PhD}$ thesis, University of California, Irvine, 2000.

[22] I. Microsoft. The OAuth 2.0 Authorization Framework https://tools.ietf. org/html/rfc6749 Accessed: 09-05-2017.

[23] I. Alfresco Software. Activiti BPM Platform http://activiti.org/ Accessed: 09-05-2017.

[24] A. Yadin. Computer Systems Architecture. Chapman \& Hall/CRC Textbooks in Computing. Taylor \& Francis, 2016.

[25] C. Szyperski. Component Software: Beyond Object-Oriented Programming. Addison-Wesley Professional, 2nd edition, 2002.

[26] Department of Information Systems, Wrocław University of Economics. IT in Service Economy Competence Center, Independent Living http: //itse.ue.wroc.pl/index.php/independent-living/ Accessed: 09-05-2017.

[27] R. Kutera and W. Gryncewicz. Web Oriented Architectural Styles for Integrating Service E-marketplace Systems. In Proceedings of the 7th International Symphosium on Business Modeling and Software Design BMSDâĂŹ17, pages 72-80. ScitePres, 2017.

[28] H. Österle. Business oder Life Engineering? HMD Praxis der Wirtschaftsinformatik, 51(6):744-761, 2014. [Online]. Available: http://dx.doi.org/10.1365/s40702-014-0097-x 



\section{$12^{\text {th }}$ Conference on Information Systems Management}

T HIS event constitutes a forum for the exchange of ideas for practitioners and theorists working in the broad area of information systems management in organizations. The conference invites papers coming from two complimentary directions: management of information systems in an organization, and uses of information systems to empower managers. The conference is interested in all aspects of planning, organizing, resourcing, coordinating, controlling and leading the management function to ensure a smooth operation of information systems in an organization. Moreover, the papers that discuss the uses of information systems and information technology to automate or otherwise facilitate the management function are specifically welcome.

\section{TOPICS}

- Management of Information Systems in an Organization:

- Modern IT project management methods

- User-oriented project management methods

- Business Process Management in project management

- Managing global systems

- Influence of Enterprise Architecture on management

- Effectiveness of information systems

- Efficiency of information systems

- Security of information systems

- Privacy consideration of information systems

- Mobile digital platforms for information systems management

- Cloud computing for information systems management

- Uses of Information Systems to Empower Managers

- Achieving alignment of business and information technology

- Assessing business value of information systems

- Risk factors in information systems projects

- IT governance

- Sourcing, selecting and delivering information systems

- Planning and organizing information systems

- Staffing information systems

- Coordinating information systems

- Controlling and monitoring information systems

- Formation of business policies for information systems

- Portfolio management,

- CIO and information systems management roles

\section{SECTION EDITORS}

- Arogyaswami, Bernard, Le Moyne University, USA

- Chmielarz, Witold, University of Warsaw, Poland

- Karagiannis, Dimitris, University of Vienna, Austria

- Kisielnicki, Jerzy, University of Warsaw, Poland

- Ziemba, Ewa, University of Economics in Katowice, Poland

\section{REVIEWERS}

- Ahmad T., Al-Taani, Yarmouk University, Jordan

- Alghamdi, Saleh, University of Sussex, United Kingdom

- András, Nemeslaki, National University of Public Service, Budapest, Hungary

- Antlová, Klára, Technical University of Liberec, Czech Republic

- Bialas, Andrzej, Institute of Innovative Technologies EMAG, Poland

- Bicevska, Zane, DIVI Grupa Ltd, Latvia

- Bontchev, Boyan, Sofia University St Kliment Ohridski

- Carreño, Alberto Mora, Universitat Oberta de Catalunya, Spain

- Chatzoudes, Dimitrios, Greece

- Chmielewski, Mariusz, Military University of Technology

- Csikosova, Adriana, The Technical University of Košice, Slovakia

- Czarnacka-Chrobot, Beata, Warsaw School of Economics, Poland

- Damaševičius, Robertas, Kaunas University of Technology, Lithuania

- Dragomirescu, Horatiu, Bucharest University of Economic Studies, Romania

- Duan, Yanqing, University of Bedfordshire, United Kingdom

- Dudycz, Helena, Wrocław University of Economics, Poland

- El Emary, Ibrahim, King Abdulaziz Univetrsity, Saudi Arabia

- Espinosa, Susana de Juana, University of Alicante, Spain

- Gallego Duran, Francisco Jose, Universidad de Alicante, Spain

- Gawel, Aleksandra, Poznan University of Economics and Business

- Geri, Nitza, The Open University of Israel, Israel

- Glassman, Aaron M., Embry-Riddle Aeronautical University, United States 
- Halawi, Leila, Embry-Riddle Aeronautical University, United States

- Hamari, Juho, University of Tampere

- Jankowski, Jarosław, West Pomeranian University of Technology in Szczecin, Poland

- Jelonek, Dorota, Czestochowa University of Technology, Poland

- Kobyliński, Andrzej, Warsaw School of Economics, Poland

- Leal, José Paulo, University of Porto

- Leyh, Christian, Technische Universität Dresden, Chair of Information Systems, esp. IS in Manufacturing and Commerce, Germany

- Michalik, Krzysztof, University of Economics in Katowice, Poland

- Mullins, Roisin, University of Wales Trinity Saint David, United Kingdom

- Muszyńska, Karolina, University of Szczecin, Poland

- Nicklas, Daniela, University of Bamberg, Germany

- Nuninger, Walter, Polytech'Lille, Université de Lille, France

- Ohira, Shigeki, Nagoya University, Japan

- Ozkan, Necmettin, Turkiye Finans Participation Bank, Turkey

- Paar, Alexander, TWT GmbH Science \& Innovation

- Pastuszak, Zbigniew, Maria Curie-SKlodowska University, Poland

- Popescu, Elvira, University of Craiova

- Queirós, Ricardo, Escola Superior de Media Artes e Design, Politécnico do Porto, Portugal
- Ranjan, Jayanthi, Institute of Management Technology in Ghaziabad, India

- Rizun, NIna, Alfred Nobel University, Dnipropetrovs'k, Ukraine

- Rozevskis, Uldis, University of Latvia, Latvia

- Schroeder, Marcin Jan, Akita International University, Japan

- Sillaots, Martin, Tallinn University, Estonia

- Simões, Alberto, Instituto Politécnico do Cávado e do Ave

- Sobczak, Andrzej, Warsaw School of Economics, Poland

- Sobolewski, Piotr, Wroclaw University of Science and Technology

- Šušol, Jaroslav, Comenius University in Bratislava, Slovakia

- Swacha, Jakub, Institute of Information Technology in Management, Faculty of Economics and Management, University of Szczecin, Poland

- Symeonidis, Symeon, Democritus University of Thrace, Greece

- Temperini, Marco, Sapienza University in Rome, Italy

- Travica, Bob, University of Manitoba, Canada

- Tupia, Manuel, Pontificia Universidad Catolica del Peru, Peru

- Wątróbski, Jarosław, West Pomeranian University of Technology in Szczecin, Poland

- Wielki, Janusz, Opole University of Technology, Poland

- Wolski, Waldemar, University of Szczecin, Poland

- Žemlička, Michal, Charles University in Prague, Czech Republic 


\section{WaterSocial.org as the social platform aimed to promote an efficient water use}

\author{
Tomasz Jach \\ Institute of Computer Science, \\ University of Silesia, \\ 41-200 Sosnowiec, Bedzinska 39, Poland \\ Email: tomasz.jach@us.edu.pl \\ Ewa Magiera \\ Institute of Computer Science, \\ University of Silesia, \\ 41-200 Sosnowiec, Bedzinska 39, Poland \\ Email: ewa.magiera@us.edu.pl
}

\author{
Andrea Capiluppi \\ Department of Computer Science, \\ Brunel University London, UK \\ Email: Andrea.Capiluppi@brunel.ac.uk \\ Zhenchen Wang \\ Medicines \& Healthcare \\ products Regulatory Agency \\ London, UK \\ Email: zhenchen.wang@mhra.gov.uk
}

\begin{abstract}
ISS-EWATUS is a EU founded project which aims to increase the effectiveness of water saving awareness. One of its key components is the social media platform, along with connectors and information exchanging throughout whole system. In this paper, the brief description of this platform is stated, as well as the dissemination results of a whole ISS-EWATUS project.
\end{abstract}

\section{INTRODUCTION}

W ATERSOCIAL.ORG is an online social media platform (SMP) that was specially designed for promoting an efficient water use. This platform was designed and developed as a part of the project named Integrated Support System for Efficient Water Usage and Resources Management (ISSEWATUS) [1], [2]. By using this website, ISS-EWATUS has established a supportive community of users who are willing to take on the role of a "local leader" in changing the water behaviour of a local community[3]. This includes its impact on local, national and international levels across Europe and its target audiences of water stakeholders at different levels of individuals, households, green NPOs, water managers, researchers and policy makers. The SMP aims to ease the communication and creation of relationships between stakeholders and to produce a sustainable impact for the communities involved. Apart from supporting mainstream social networking activities such as sharing, communications, being friends, asking and answering, discussion, the SMP allows users to share water tips and photos under different environmental scenes, and the shared content can be pinned on a global map. Gamification enables the entire SMP to be used as a platform with gaming elements, which involve game tasks, competitions and rewards. The game tasks can be any user tasks on the social networks or any water use related offline activities such as recording water use activities. Each of these user tasks can be rewarded upon accomplishment. The rewards system has paid off in terms of acquiring users and convincing them to change their behaviour. Watersocial.org is unique in its vision to harness gamification and social media to reinforce water saving behaviours. It incorporates a set of gamification designs into the online social networking activities in which the users are able to get rewarded upon each accomplishment of a task or even are able to compete with each other through the WaterSocial.org leaderboards.

\section{GAMIFICATION IN WATERSOCIAL.ORG}

Gamification refers to the use of game design elements in non-game contexts such as gaining rewards from competition or participation. It can be used to engage communities of interest in changing behaviours. In the WaterSocial.org platform, the gamification mechanism is driven by three motivational affordances including Aqoins, Titles and Leaderboards. Aqoins and Titles can be won by participating to a variety of social networking activities; while the leaderboards allow users to compete with each other.

\section{A. Aqoins and titles}

Aqoins are points that used to reward user activities on the platform. In order to encourage more user activities, most of the activities can be used to earn Aqoins. Table 1 shows the tariffs of each activity: after completing any of the activities illustrated, users will be rewarded with the corresponding aqoins.

When a user earns certain amount of Aqoins, they can obtain titles (see Figure 1).

\section{B. Leaderboard}

The leaderboard aims to trigger more "social comparison" so that more user engagement can be achieved. There have been implemented three types of leaderboards, namely the weekly leaderboard within friend circle, monthly leaderboard within the whole community and the all-time leaderboard which includes all users throughout all time. 
TABLE I

USER ACTIVITIES THAT CAN EARN AQOINS

\begin{tabular}{|l|c|}
\hline User Activities & Aqoins \\
\hline submit data to water diary & 30 \\
\hline ask a new question & 30 \\
\hline answer a question & 10 \\
\hline reply an item from social stream & 10 \\
\hline like a topic & 10 \\
\hline vote a question/answer & 10 \\
\hline upload a photo from mobile app & 80 \\
\hline upload a photo on map & 60 \\
\hline post texts including tips on map & 30 \\
\hline reply a topic discussion & 20 \\
\hline
\end{tabular}

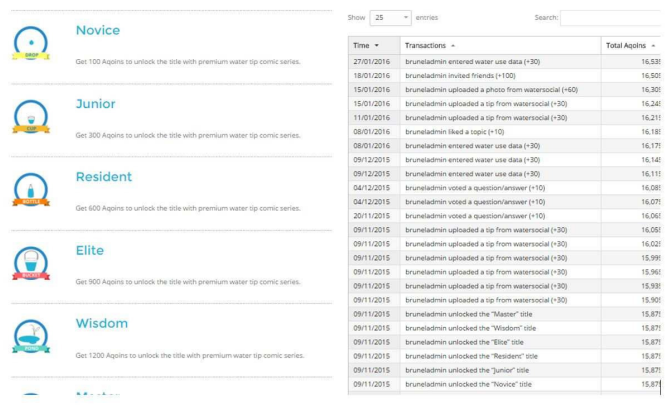

Fig. 1. My aqoins page

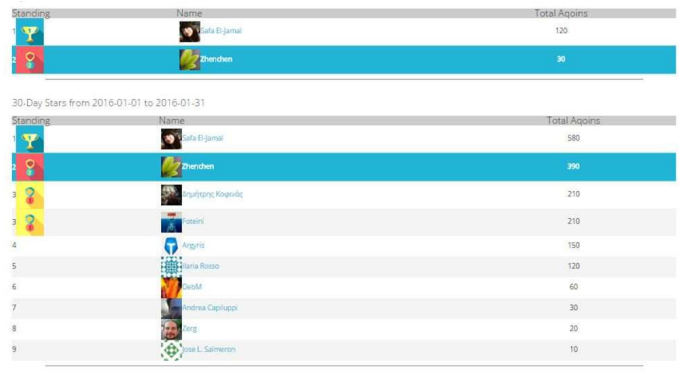

Fig. 2. Leader board page

\section{Social-MEdia PLATFORMS ENGENDERING SOCIAL ENGAGEMENT AND BEHAVIOUR CHANGE}

According to a report from Pew Internet Project3, as of early $201474 \%$ of the online adults use social networking sites. A qualitative study conducted in the UK by Binsted \& Hutchins [5] mapped individuals' usage patterns of social networks, namely: keeping in touch with contacts, sharing photos, playing games, following celebrities, organising social events, getting recommendations, as well as gaining and sharing information.

In addition to the general social network sites (e.g. Facebook, Twitter, and Google+), there are many other ones focused on specific topics. Some of them are linked to sports and life-style apps, such as Endomondo, RunKeeper, and WeightWatchers. These applications provide users with communities to exchange information about performances, to keep each other up-to-date, and to link their account with other networks. In the water conservation field the use of social networks are also emerging. For instance, Twitter has often been used as a media to share news and tips about water uses (Water-Use It Wisely).

Additionally, Facebook has been used to post water use related news to rise people's awareness of water conservation such as Water - Use It Wisely4. Since social networks are an emerging trend and their applications are growing very rapidly, it has a high potential to persuade people to practice water conservation.

\section{A. Social Engagement}

Self-gratification plays an important role in explaining why people tend to voluntarily take part in the social networks. It includes the gratification received from entertainment, information seeking, and social interaction [6], [7], [8]. Getting to know new people with common interest in order to share ideas seems to be one of the main reasons for people to use a social-media platform [9]. Furthermore according to the same research, people are interested in getting updates from their social network connections as this makes them better informed about other people's activities and opinions. In [10], self-gratification is further defined as one's attachment, sense of duty and good way to meet people, in the political context, which in turn are argued to play a major role towards influencing citizens to help curb social problems.

In addition to psychological factors, there are also other objective factors or incentives to cause people to join social networks. For example point systems (such as scores, stars, reputations and badges) are a common practice in many game applications. In some social forums, question and answer Websites, there is also an increasing number of computer and smartphone games that use point system to motivate players to keep playing the games. Reasons of why such a reward system is successful can perhaps be explained by using behavioural model of [11]. He argues that an activity that is easy to do (such as playing games) will require only low motivation for a person to finally do it. Thus, triggers, such as points, may be enough for making him/her play a game. However, when an activity is hard to do, it will require much higher motivation. Therefore, different kind of triggers may be needed to boost motivation.

\section{B. Social Behaviour Change}

Behaviour can be influenced through social norms. People are more likely to participate in an activity if those around are participating in the activity. Previous studies including [12] have also indicated that social media/networks can be used to motivate or to give certain pressure to people to behave in certain ways. One study performed in [13] showed that water conservation could be influenced by the presence of people modelling the promoted water saving action of turning off the shower while soaping. It was found that before the study $6 \%$ of shower users carried out the action while $93 \%$ were aware of the sign suggesting the action. Following the presence of 
one role model, $49 \%$ carried out the action. The presence of two role models resulted in $67 \%$ participation.

Behaviours can also be influenced via designed intervention. [14] defines a concept called CBSM (Community Based Social Marketing) referring to intervention programs that attempt to apply a structured approach and the insights of social psychology when influencing community behaviour. CBSM approach has been found to be effective in changing routine behaviour and the design of CBSM has been further developed in [15]. There four steps to design a CBSM, the first step involves recognising the behaviours relevant to a particular environmental goal, for example, installing a lowflow showerhead. CBSM starts with identifying the barriers which prevent the action from being taken, and then selecting a specific barrier to promote based on its potential impact on the behaviour campaign, the relevance to the behavioural change and the resources available to overcome the barrier. The chosen barrier varies depending on whether the desired behaviour change is a one off action, or involves changing a particular habit or routine. The study in [15] cognises that habitual or routine change is much more difficult to influence compared to changing a one-off behaviour. The second step is to design a programme to overcome the selected barrier to achieve the behavioural change. The important aspect of the design stage is to target interventions very specifically towards the identified barriers, drawing on social-psychological devices such as commitments, prompts, or signals, to promote the desired behaviours. The third and final steps in the CBSM approach are piloting and testing the programme and evaluating its effectiveness before it is applied on a wider scale.

\section{DESIGN AND IMPLEMENTATION OF WATERSOCIAL.ORG}

This chapter details the development aspects of WaterSocial.org. The system architecture, the components and their connectors are described, together with the process model and the data model of each component. The process model is needed to show the degree of interactions of the component with other components; the data model is needed to show the data structures and information flowing from one component to the other, as part of their interaction.

In the next few sections, each of the modules is presented in terms of process model, data model and their current deployment status in the system.

\section{A. Software components}

The software components are shown in Figure 3 (with the indication of which of the project tasks are implemented in). These components seek to fulfil the scenarios and the associated use cases defined in the gathered requirements via the following modules:

1) ISS-EWATUS Web Portal (ISNP): This is the landing Web page for the whole system.

2) ISNP Data Sensing (IDS): It detects social networking activities including sharing and inter-user communication and individual user water use input to the system.
IDS is cross-platform which means it also can receive data from multiple devices such as mobile phones and laptops. In addition, it also stores and dispenses the pre-processed data flows to other expected SMP components.

3) Water Use Visualizer (WUV): It performs the visualisation features which enable the system to present the water use patterns in different media forms such as charts, histograms from different dimensions such as temporal and activity.

4) Water Use Pattern Visualization (WUPV): This is the front end component that handles the user input from WUV.

\section{INTER-COMPONENT INTERACTIONS}

Interactions connect modules within components. Below we report, for each interaction, their process and data models, and the API specifications to implement these interactions. Also, the modules that are connected by each interaction are described. The modules are associated to three types of intercomponent interactions, as defined below:

1) Social Network Activity and Water Use Visualization

2) Gamification

3) Evaluation

Interactions connect modules within components. Below we report, for each interaction, their process and data models, and the API specifications to implement these interactions. Also, the modules that are connected by each interaction are described.

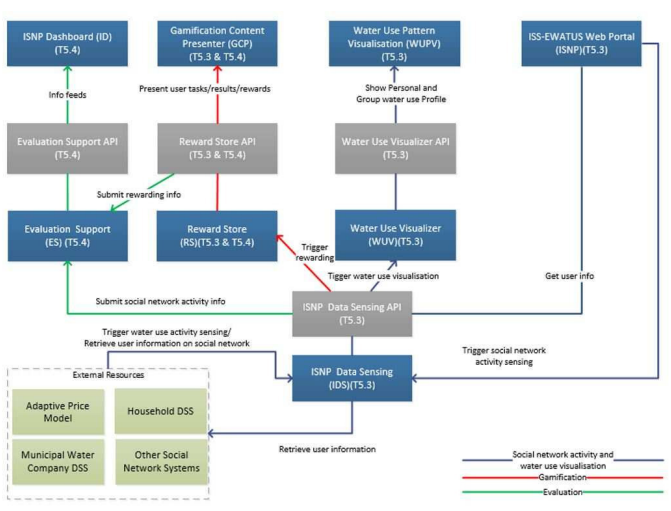

Fig. 3. ISS-EWATUS SMP Components

\section{A. Inter-component Interactions: Social Network Activity and Water Use Visualization}

This inter-component interaction has the purpose of handling, for each user account, the user account management; the social network activity management; and the water use visualization. The interactions that are allowed are two-fold:

1) internally to the SMP, its components interact through this layer at the user-level, 
2) externally, the interactions also extend to components outside the SMP, such as the household DSS (within the ISS-EWATUS scope) and the other social network systems (outside the ISS-EWATUS scope) to allow them to retrieve information provided by the platform.

1) Process model: Below we display the process model of the cluster of interactions named "Social Network Activity and Water Use Visualization". It is presented in a UML Sequence Diagram, where the main components are shown in their box, and the interactions are the arrows that travel between them.

At current implementation, we have enable the new user registration, post/reply and water use diagram request processes.

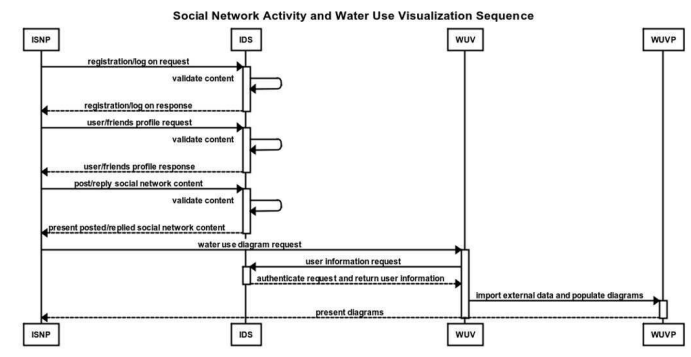

Fig. 4. Process model for social network activity and water use visualisation

2) Data Model: The data model used in the platform customises and extends the data structure supplied in the WordPress database framework. There are mainly three types of data types used in this module.

1) user: contains user information that comprises the use profile,

2) posts: defined as a base data structure used to be extended and implemented to define user input and other component data input to SMP,

3) postmeta: used to define the additional data fields in forms of key-value pairs of an extended posts type.

Figure 5 shows the user data structure, the full details of data field definition can be viewed on http://watersocial.org/ extensions-json/rest/api/users/schema.

Figures 6 and 7 show the base posts type and post meta data type data structures. In the platform we particularly extended the base posts type in terms of the post_type data field which allow the platform to handle different social networking activities input (see Table II). We also implemented three platform original data types used to handle user input of water use, water use tips and water use images, namely the tips object (see Figure 8), media object (see Figure 9). The descriptions of data fields of these two objects can also be found at http://watersocial.org/extensions-json/rest/api/tips/schema and http://watersocial.org/extensions-json/rest/api/media/schema.

As the platform is a Web-based application system, we developed a set of Web APIs (application programming interface) which allow the platform data can be accessed and managed within and outside the platform. The Web API here is defined as an API for either a Web server or a Web browser.

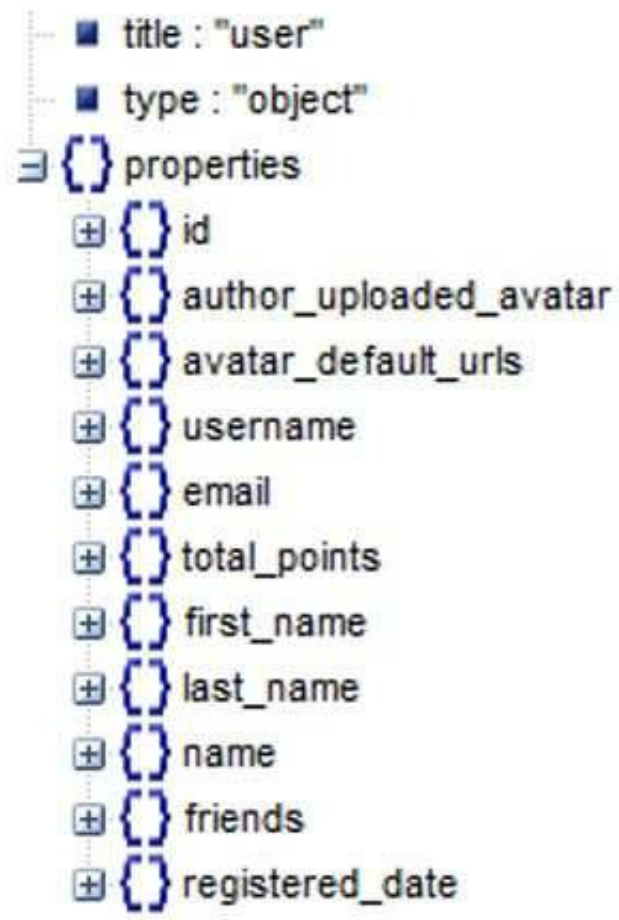

Fig. 5. User type data structure

TABLE II

CUSTOMIZED POST TYPE USED THE PLATFORM

\begin{tabular}{|l|l|}
\hline $\begin{array}{l}\text { Post Type Used in the } \\
\text { Platform }\end{array}$ & Use \\
\hline achievement-type & $\begin{array}{l}\text { To store different types of the gamification } \\
\text { tasks }\end{array}$ \\
\hline answer & $\begin{array}{l}\text { To store users answers in the Questions } \\
\text { and Answers activities }\end{array}$ \\
\hline attachment & $\begin{array}{l}\text { To store user uploaded content such as } \\
\text { images }\end{array}$ \\
\hline badge-log-entry & To log users'rewarding transactions \\
\hline bgmp & $\begin{array}{l}\text { To store geo-related content such as tips } \\
\text { and photos }\end{array}$ \\
\hline faq & To store FAQs of website \\
\hline forum & To store different types of forums \\
\hline mobilechallenge & $\begin{array}{l}\text { One of the achievement-type defined for } \\
\text { mobile users }\end{array}$ \\
\hline question & $\begin{array}{l}\text { To store users' questions in the Questions } \\
\text { and Answers activities }\end{array}$ \\
\hline reply & To store users' replies for a forum topic \\
\hline title & $\begin{array}{l}\text { One of the achievement-type defined for } \\
\text { all users }\end{array}$ \\
\hline topic & To store users' topics in a forum \\
\hline watersocial_survey & To store survey answers \\
\hline wateruse & $\begin{array}{l}\text { To store water use diary input from } \\
\text { individual users }\end{array}$ \\
\hline contact_form & $\begin{array}{l}\text { To log messages sent to users from } \\
\text { manager }\end{array}$ \\
\hline
\end{tabular}

The INSP and IDS components expose a set of Web APIs by using Restful Web services. This allows the SMP to be flexibly integrated with heterogeneous systems including Android and/or iOS apps. The APIs are well documented 


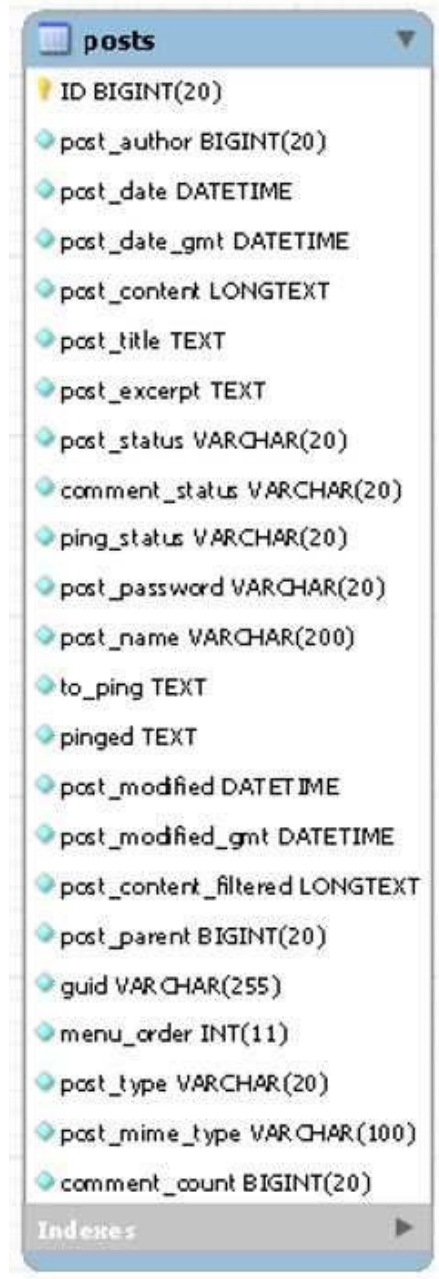

Fig. 6. Posts type data structure

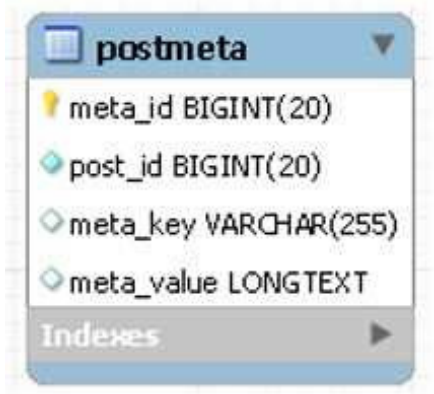

Fig. 7. Post meta data structure

in thehttp://watersocial.org/extensions-json/rest/api where IDS and WUV APIs are included and general descriptions of these APIs are given below (see Table III and Table IV).

\section{B. Inter-component Interactions: Gamification}

The gamification enables the whole SMP to be used as a platform with gaming elements, which involve game task,

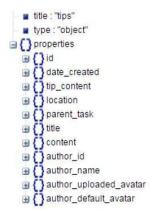

Fig. 8. Tips object, the full schema definition can be found at http: //watersocial.org/extensions-json/rest/api/tips/schema

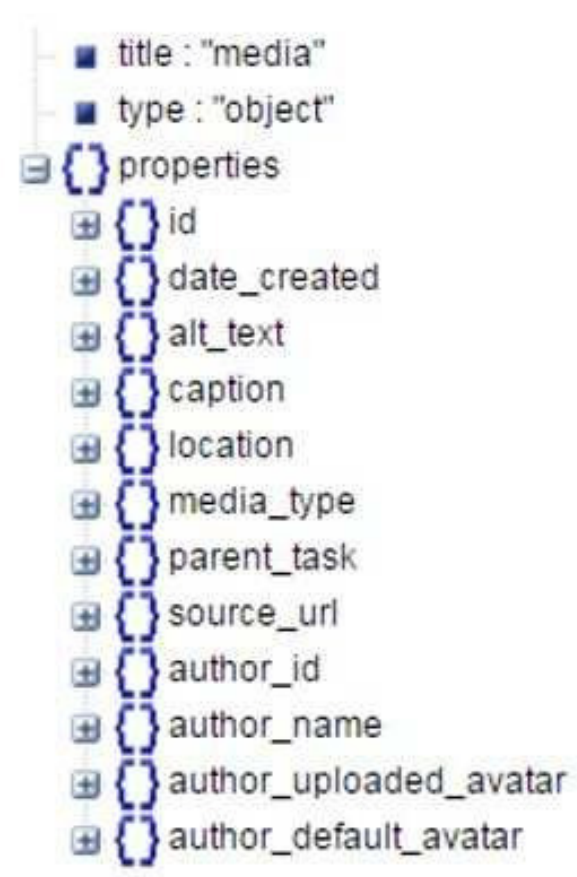

Fig. 9. Media object, the full schema definition can found at http://watersocial. org/extensions-json/rest/api/media/schema

competition and rewarding. The game tasks can be any user tasks on the social networks or any water use related offline activities such as recording down water use activities. Each of these user tasks can be rewarded upon its accomplishment. The gamification mechanism provided here allows managers to design user tasks and to reward points/badges as the user task progresses. The components involved include:

- Reward Store (RS) plays a pivotal role in incentivising users participating in the whole system. The features of RS include calculation of rewards such as points, experience level, ranking etc. for each gamified social network activity. RS outputs the rewarding results in the forms of leaderboard of users and notification of rewards on an individual basis.

- Gamification Content Presenter (GCP) is a front end component which handles information from RS.

1) Process Model: The gamification process involves user task design, task status check and rewarding procedures (see Figure 10). 
TABLE III

IDS APIS

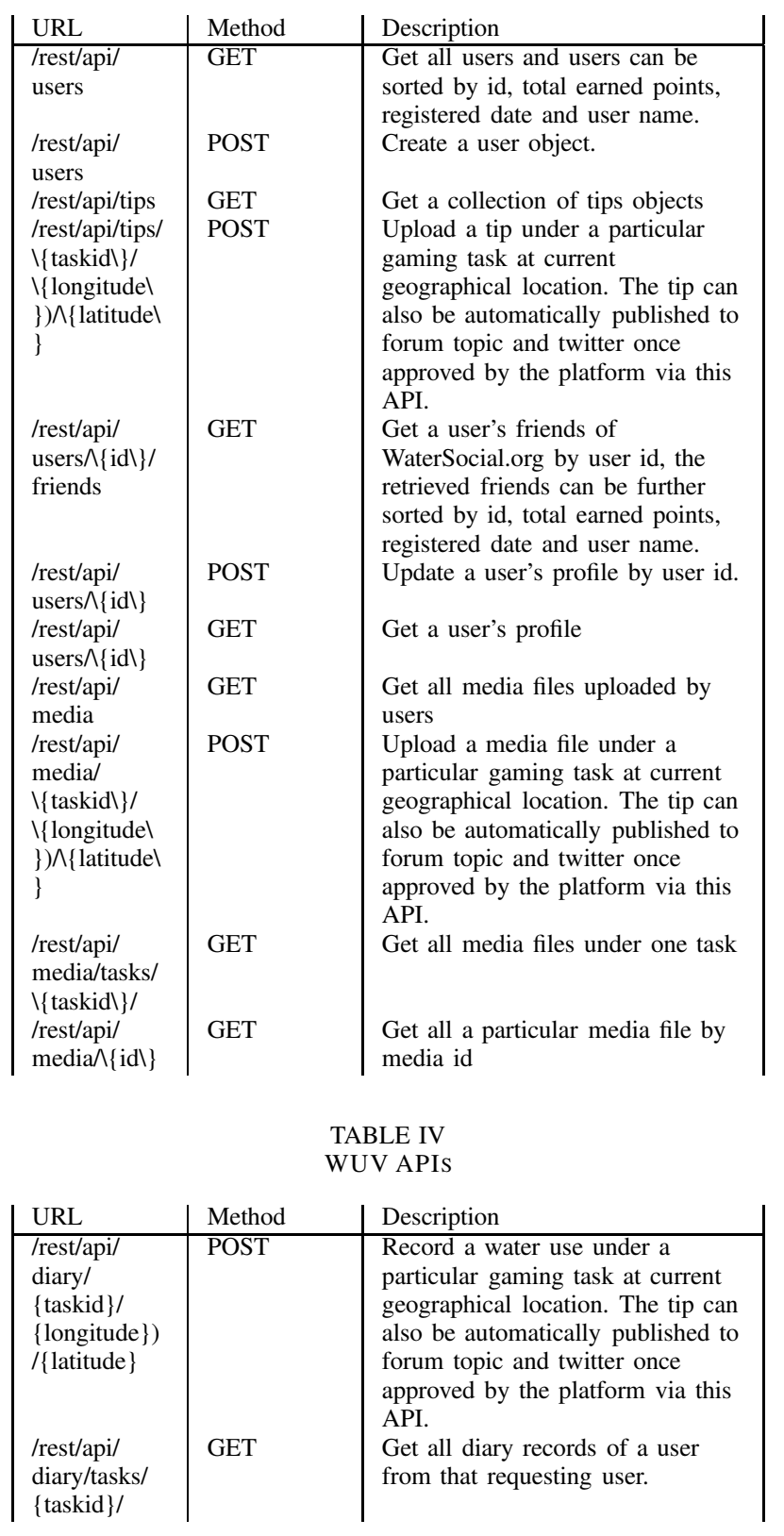

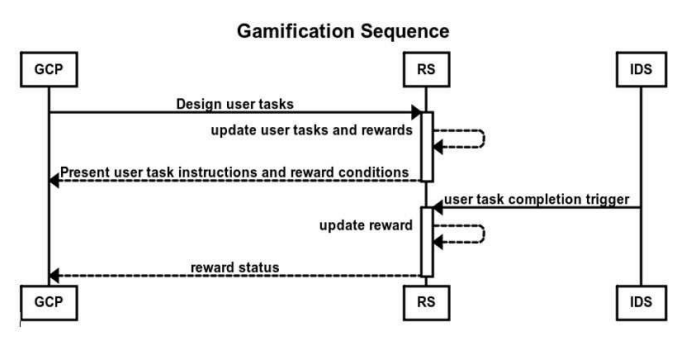

Fig. 10. Process model for gamification
2) Data Model: There are two data objects defined in this module namely the task object and reward object. The specifications of two objects are defined online. The tasks object is defined in http://watersocial.org/extensions-json/rest/api/tasks/ schema (see Figure 11) and the reward object is defined in http: //watersocial.org/extensions-json/rest/api/rewards/schema (see Figure 12).

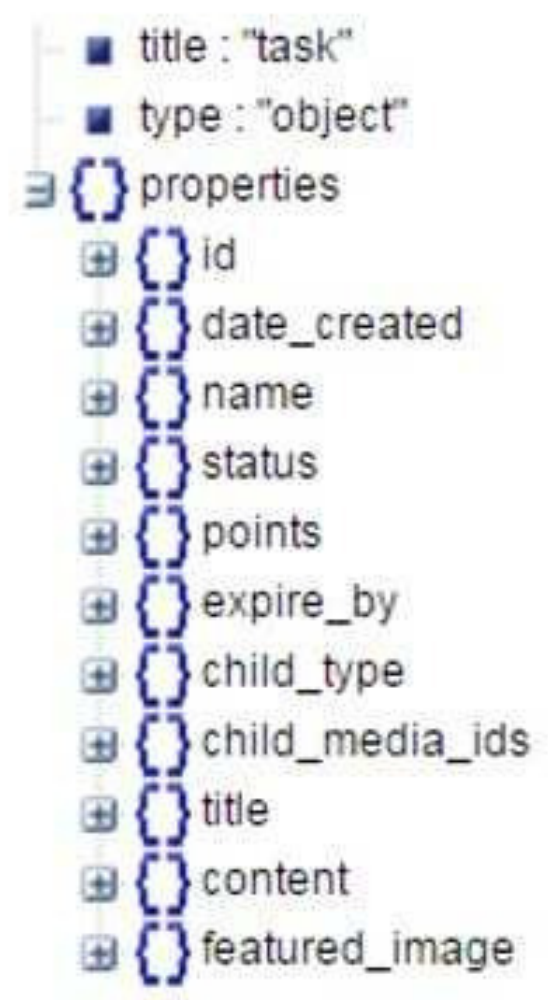

Fig. 11. Task object, the full schema definition can be found at http: //watersocial.org/extensions-json/rest/api/tasks/schema

The Web APIs for RS include those used to retrieve the points from the platform and those from the external game service providers (see Table V).

TABLE V
RS APIS

\begin{tabular}{|l|l|l|} 
URL & Method & Description \\
\hline $\begin{array}{l}\text { /rest/api/ } \\
\text { rewards/ } \\
\text { /userid }\} \\
\text { /rest/api/ } \\
\text { rewards/ } \\
\text { game }\end{array}$ & GET & Get a rewards of a user. \\
$\begin{array}{l}\text { /rest/api/ } \\
\text { rewards/ } \\
\text { game/ } \\
\text { decrypt/ } \\
\text { fcode }\end{array}$ & GET & $\begin{array}{l}\text { Get point redemption code from } \\
\text { watersocial, this API is used to } \\
\text { integrate the games from outside } \\
\text { the platform. } \\
\text { Get points value from a rewarding } \\
\text { code. This code is generated from } \\
\text { WaterSocial.org for external game } \\
\text { service provider. }\end{array}$ \\
\hline
\end{tabular}

In addition to the Web APIs, there are also APIs that used 


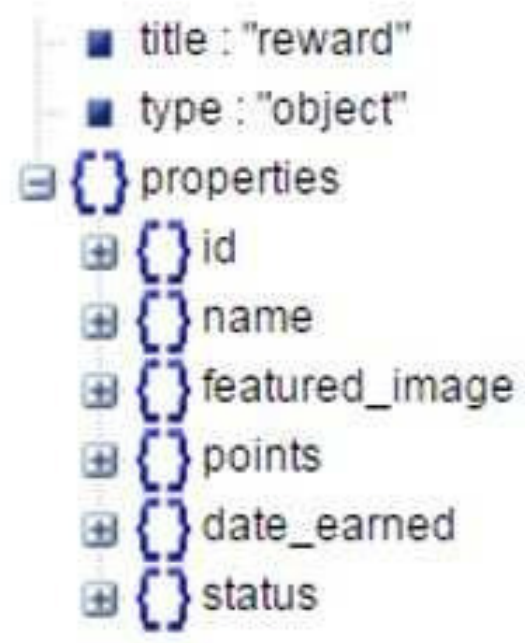

Fig. 12. Reward object, the full schema definition can be found at http //watersocial.org/extensions-json/rest/api/rewards/schema

to specifically target the game tasks. In the platform, the following task actions APIs are developed to support gamified social networking tasks and water use tasks. The use of these APIs can follow the pattern add_action(ACTION_NAME, CUSTOMISED_FUNCTION) in a WordPress plugin.

TABLE VI

GAME TASK ACTIONS APIS

\begin{tabular}{|l|l|} 
Action Name & Description \\
\hline publish_wateruse & $\begin{array}{l}\text { It can be used if a user uploads a } \\
\text { water use diary } \\
\text { It can be used if a user posts a } \\
\text { new question in Q\&A feature. } \\
\text { It can be used if a user posts an } \\
\text { answer in Q\&A feature. }\end{array}$ \\
bp_activity_comment__ & $\begin{array}{l}\text { It can be used if a user } \\
\text { commented in social activity } \\
\text { stream. }\end{array}$ \\
posted & $\begin{array}{l}\text { It can be used if a user unlike or } \\
\text { like a topic. } \\
\text { wp_ulike_mycred_like } \\
\text { ap_vote_casted }\end{array}$ \\
publish_bgmp & $\begin{array}{l}\text { answer/question in Q\&A feature. } \\
\text { It can be used if a user posts } \\
\text { some information on the map, e.g. } \\
\text { a photo or a tip. } \\
\text { It can be used if a user replies a } \\
\text { topic. } \\
\text { It can be used if a user sends } \\
\text { invitation of join water social on } \\
\text { other social networks. }\end{array}$ \\
send_friend_invitation &
\end{tabular}

\section{Inter-component Interactions: Evaluation}

Evaluation allows managers and researchers to continuously monitor and get the statistics of users, social network activities and rewards information. There are two components designed to handle these tasks, namely:
- Evaluation support (ES) is the component serves dashboard, it collects data from RS and IDS so that it can produces summative results of rewarding and all other social network activities occurred. ES performs different statistical features to produce the meaningful results.

- ISNP Dashboard (ID) This is a Web based application that presents the results generated from ES based upon a user's privilege.

The detailed description of the evaluation is beyond of this paper.

\section{DisSEMINATION OF WATERSOCIAL.ORG}

A number of dissemination activities were carried out between 1st January 2016 and 30th December 2016. To disseminate the WaterSocial.org the following types of activities were undertaken: 1. Online activities: a) Social media: the use of social media platforms to share water saving messages. b)Publications: newsletters and blogs that detailed the WaterSocial platform. 2. Offline activities: a) Competitions: to enhance the gamification layer. b) School visits: pupil engagement that showcased the WaterSocial platform. These activities are related as they all publicised the WaterSocial platform by promoting water saving messages [4]. Their monitoring and analysis help in evaluating whether a measurable user uptake has been observed and the types of messages used and the degree of relevance to users. The results of the portal dissemination have been very satisfying, for example 102 new users were registered to WaterSocial.org, 127 friendships and 145 topics were created.

\section{ACKNOWLEDGMENT}

The research was undertaken as part of Integrated Support System for Efficient Water Usage and Resources Management (ISS-EWATUS) project, funded by European Union's Seventh Framework Programme for research, technological development and demonstration under grant agreement No. [619228].

\section{REFERENCES}

[1] http://issewatus.eu

[2] Magiera, E., Froelich, W.: Integrated support system for efficient water usage and resources management (iss-ewatus). Procedia Engineering 89, 1066/1072 (2014)

[3] Capiluppi a., Wang Z.: A Social-Centred Gamification Approach to Improve Household Water Use Efficiency, 7th International Conference on Games and Virtual Worlds for Serious Applications (VS-Games), DOI: 10.1109/VS-GAMES.2015.7295761, 2015

[4] Safa El-Jamal, A.C., Wang, Z.: A holistic dissemination strategy to deliver water conservation messages through gamication and social networks. In: Conference Water Efficency Network (WATEF). pp. 1/10 (2016)

[5] Binsted, A., Hutchins, R., Transport Research Laboratory (Great Britain). The role of social networking sites in changing travel behaviours. http://www.trl.co.uk/online_store/reports_publications/trl_ reports/cat_traffic_and_the_environment/report_the_role_of_social_ networking_sites_in_changing_travel_behaviours.htm, accessed 22/09/2014.

[6] Raacke, J., Bonds, J. MySpace and Facebook: Applying the uses and gratifications theory to exploring friend-networking sites. CyberPsychology \& Behavior, 11(2), pp. 169âĂŞ174, 2008.

[7] Jung, T., Youn, H., Mcclung, S. Motivations and self-presentation strategies on Korean-based âĂIJCyworldâĂİ weblog format personal homepages. CyberPsychology \& Behavior, 10(1), pp. 24âĂŞ31, 2007. 
[8] Dunne, ÃĄ., Lawlor, M. -A., Rowley, J. Young people's use of online social networking sites âĂ $\breve{T}$ A uses and gratifications perspective. Journal of Research in Interactive Marketing, 4(1), pp. 46âĂŞ58, 2010

[9] Brandtzaeg P. and Heim J. Why People Use Social Networking Sites. In Proceedings of the $3 \mathrm{~d}$ International Conference on Online Communities and Social Computing: Held as Part of HCI International 2009 (OCSC '09), A. Ant Ozok and Panayiotis Zaphiris (Eds.). Springer-Verlag, Berlin, Heidelberg, pp. 143-152, 2009.

[10] Pattie, C., Seyd, P., Whiteley, P. Citizenship and civic engagement: Attitudes and behaviour in Britain. Political Studies, 51(3), pp. 443âĂŞ468, 2003.

[11] Fogg. BJ A behavior model for persuasive design. In Proceedings of the 4th International Conference on Persuasive Technology (Persuasive
'09). ACM, New York, NY, USA, Article 40, 7 pages, 2009.

[12] Barry W., Are personal communities local? A Dumptarian reconsideration, Social Networks, 18(4), pp. 347-354, 1996.

[13] McKenzie-Mohr, D. Fostering Sustainable Behavior: An Introduction to Community-Based Social Marketing. New York: New Society Publishers, 2011.

[14] Rolls, J. M. A review of Strategies Promoting Energy Related Behaviour Change. International Solar Energy Society Solar World Congress. Adelaide, South Australia 25th November-2nd December, 2001.

[15] Jackson, T. Motivating Sustainable Consumption: A review of evidence on consumer behaviour and behaviour change. Report to the Sustainable Development Research Network. Centre of Environmental Strategy, University of Surrey. Guilford, Surrey, 2005. 


\section{Task Execution Support in Research Activity using RAC System}

\author{
Shigeki Ohira \\ Nagoya University \\ Furo-cho, Chikusa-ku, Nagoya, Japan \\ Email: ohira@nagoya-u.jp
}

\author{
Naoya Morita, Katashi Nagao \\ Nagoya University \\ Furo-cho, Chikusa-ku, Nagoya, Japan \\ Email: \{morita,nagao\}@ nagao.nuie.nagoya-u.ac.jp
}

\begin{abstract}
Research activities carried out daily in research laboratories, include conducting investigations, giving seminars on progress, performing experiments, analyzing results, and writing papers, but all of these research activities involve steady, methodical work that does not produce immediate, visible results. For this reason, a mechanism to maintain motivation when research is not going well or to help students get on track with research when they have just been assigned to a laboratory could be useful. Students that have just begun their research may not yet understand how to proceed. We previously developed a research activity concierge (RAC) system, which is a platform to encompass general research activities, and applied gamification to this system to keep user motivation high. However, even with the RAC, non-research-savvy students have difficulty handling challenges and executing tasks. In this research, we focused on discussions in seminars and introduced a mechanism to support task execution in students' research activities by implementing automatic extraction of task statements into the RAC.
\end{abstract}

\section{INTRODUCTION}

$\mathbf{R}$ ESEARCH consists of various activities. It begins with surveying related research and includes giving seminars on progress, discussing future plans, programming or constructing equipment, performing experiments, analyzing results, and writing papers. However, by looking carefully at these activities, it can be seen that the everyday activities are very mundane; sometimes they go well, and sometimes they do not. Scientists must often carry on without immediate visible results.

Motivation to research increases when everything is on track, and the everyday tasks become more interesting. However, when research does not go as expected or when students have just been assigned to a laboratory and do not know which way to turn, a high level of motivation can be difficult to maintain.

On the other hand, gamification - the use of game design elements in non-game contexts [1][2]—has attracted enormous interest across a range of different areas, including education [3][4]. Bodnar and Clark put game-based learning into practice in undergraduate engineering classrooms and obtained very positive results [5]. Ohira et al. incorporated gamification into seminars and found that students' motivation to speak better and debate was markedly improved [6]. González et al. presented an intelligent tutoring system including gamification

This work was supported by JSPS KAKENHI Grant Number 15K01066. elements, which improved student achievement and enhanced learning [7].

We have developed a comprehensive gamification framework for general research activities [8][9]. Specifically, it provides a research activity concierge (RAC) system, which is a platform for recording and organizing everyday challenges and tasks arising in discussions and for visualizing the results when they are applied to real actions.

However, it is difficult for non-research-savvy students to accurately handle day-to-day challenges and completely execute day-to-day tasks. In this research, we focused on the discussions in seminars and introduced a mechanism to support task execution in students' research activities by implementing automatic extraction of task statements into RAC. The paper will report the results of supporting task execution and the improvement of the RAC system.

\section{RESEARCH ACtivity}

The IDC (interest driven creator) theory [10] has recently been attracting much attention, and learning and teaching methods have been changing from "examination-driven" to "interest-driven," that is, students study what interests them. Also, to fully engage in research activities, researches must be interested in them. However, students often do not know what kind of activity to carry out because they have not been shown a global image of research. Therefore, it is thought that research activities can be more smoothly executed by preparing guidelines of research activities for such students. We have classified all research activities into 11 main activities and 100 sub-activities [8]. We call this the research activity map and express it in the mind map format.

- Gaining expertise

- Deciding research themes

- Implementation

- Deciding evaluation methods

- Experimentation

- Project meetings

- Seminar presentation

- Discussion

- Conference participation

- Conference presentation

- Writing papers 


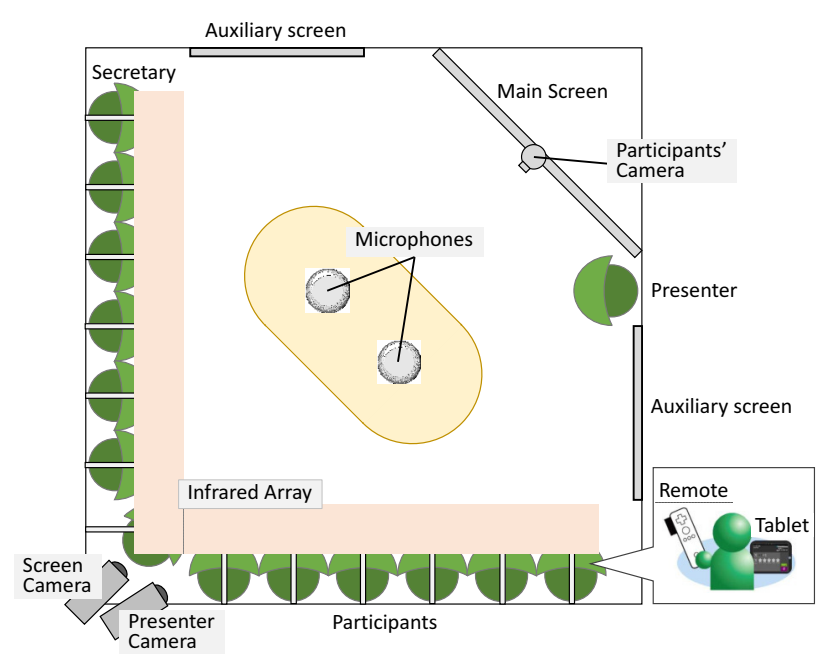

Fig. 1. Discussion room.

The main activities focus on the activities performed in every area of research. Moreover, the main activities cover activities from when a student is assigned to a laboratory until he/she graduates. We arranged activity nodes in a clock pattern on the research activity map in such a way as to be able to carry out activities in sequence. Each activity is subdivided into simple and practicable actions in accordance with the level of difficulty. In this research, we subdivide the main and sub-activities into about 500 actions. Thus, by seeing the specific actions to achieve a goal that should be performed in research activities, users can choose actions without omitting any necessary one. Furthermore, we prepared about 240 activity results that can acquire the achievement status of a target action automatically and can perform a statistical assessment.

Challenges and tasks also need to be clear for research activities to be smoothly executed. In particular, students just starting their research have difficulty setting appropriate challenges and tasks on their own, and discussion within the research group is extremely important in resolving this.

Our laboratory has been developing and operating a system to record seminar content [11]. Generally, proceedings of a seminar consist of an abstract and hand-outs, but seminar content can also include a summarized transcript, audio or video recordings of the seminar, or the slides used in the presentation. We have held seminars in the environment illustrated in Figure 1. After a seminar presentation, students use a Web application called Discussion Browser (Figure 2) for searching for and viewing seminar content to review and organize what was discussed.

The discussion during a seminar usually contains many useful opinions and much useful advice, so it is a good starting point in deciding future research directions. Thus, students should thoroughly review the seminar content as soon as possible after the seminar. However, reviewing a long seminar from beginning to end is not always practical. Discussions

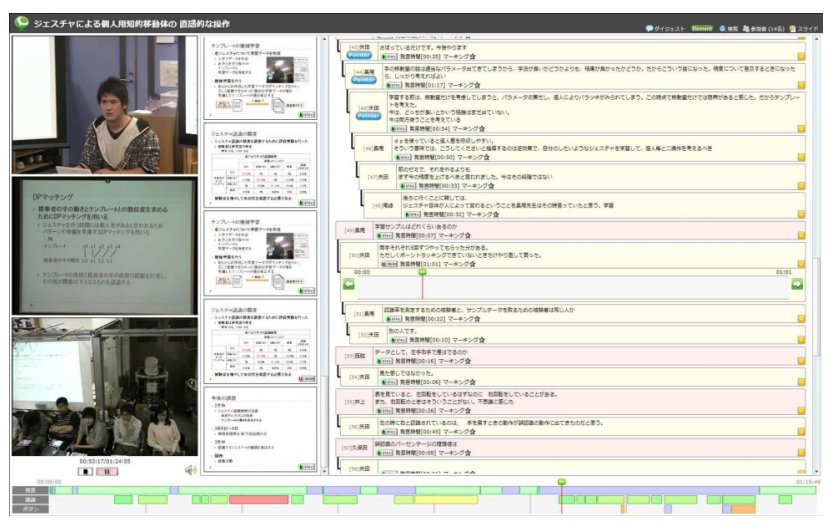

Fig. 2. Discussion Browser.

contain statements that will not affect future research activities, so statements must be divided into those that are needed and those that are not when reviewing. Further, a transcript recorded by a secretary is presumed to be an incomplete summary that does not record all content, so it may be important to listen to the audio again to decide which parts are important. Later, after time passes, the discussion may be difficult to recall correctly, even if you remembered the content at some point. Thus, to avoid having to review the content over and over, summarizing notes should be left.

Reviewing seminar content in this way takes effort and is a significant hurdle for students, so a mechanism is needed for focusing on salutary opinions and advice for subsequent research activities and actively promoting the recording and organizing of challenges and tasks.

\section{RESEARCH ACTIVITy CONCIERGE}

Systems developed to support research activity include, Papits[12] for sharing information about "know-who" in a university laboratory, MIRASS[13] for improving usability of scientific search engines for medical informatics through data mashup, and systems for managing repositories of resources accumulated through research activities in a laboratory [14][15].

The research activity concierge (RAC) [8][9] is a comprehensive support system for general research activities that introduces a gamification framework for organizing challenges and tasks and visualizing research activity.

As shown in Figure 3, the research activity concierge system consists of three basic tools: the research activity organizer (RAO), the research activity visualizer (RAV), and the research activity watch-dog (RAW). RAO organizes challenges and tasks on the basis of seminar content, and RAV visualizes research activity on the basis of the performance and tasks. RAW is a tool that constantly monitors the information input and output from RAO and RAV, describes the current status of activity to the user, and recommends actions. RAO and RAV are applications that run on a web browser. In this research, since we carried out experiments and analysis using enhanced RAO and RAV, we will explain them both briefly. 


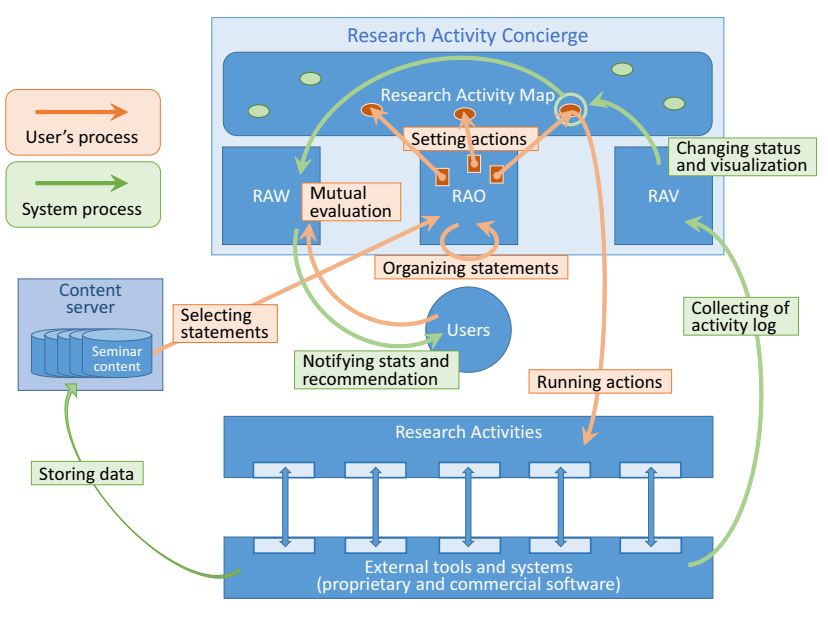

Fig. 3. Research Activity Concierge system overview.

\section{A. Research Activity Organizer (RAO)}

A screen shot of RAO is shown in Figure 4. The right side is the statement view for browsing the content of a discussion in a seminar, and the left side is the note view for organizing challenges and tasks. Users organize the statements in note view while viewing them in statement view. Users can create notes for various research activities (such as surveys, implementations, or paper writing) and record detailed information in memos. Users can also create a note on a research activity map displayed by RAV, which we will describe later. When users choose an activity on a research activity map and click on the icon of "creating a note," a screen on the browser transitions to RAO, and the created note is displayed.

\section{B. Research Activity Visualizer (RAV)}

RAV can visualize challenges, tasks, and relationships that result from related research activities organized by RAO on a research activity map (Figure 5). RAV can also show the state of processing for such items quantitatively.

For this research, we used external tools and systems as much as possible for recording digital data so that the results for practically dealing with challenges and tasks could be handled quantitatively. In our laboratory, we use both commercial and independently developed software. These software packages enable the recording of the number of statements in discussions, times users view seminar content, items committed to in repositories, and words users input when writing papers, together with their dates and times. An application programming interface (API) or plug-in is used to retrieve information from each external tool or system, and when the user performs a research activity on the basis of a challenge or task, an outline of the action is retrieved by RAV.

\section{TASK EXECUTION SUPPORT}

\section{A. Goal Setting based on Challenges and Tasks}

Although looking again at seminar content including useful discussions for research activities is important, it also requires time and effort. Thus, task statements have been automatically extracted by using a machine learning model that has metadata in a seminar content and linguistic information of the utterance as features [16][17]. The extracted task statement list is shown in the statement view of RAO (Figure 4, 6).

A user judges whether or not the statement extracted automatically is really a task statement and clicks the appropriate button on a statement list. The fixed task statement information is fed back as a teacher signal that a system uses for learning a task statement extraction model and is used for active learning [18] that updates a model. After judging a task statement, a user quotes the fixed task statement to a note view and creates a memo. Four types of progress tags can be attached to a created memo: not-started, in-progress, completed, or pending. Moreover, target actions can also be selected from elements included in a research activity map and attached to memos in the form of tags (Figure 7). Thus, created memos can record the research activity to which they are related.

A student with little experience of research activities often feels uncertain about how long to spend executing one of several tasks. Therefore, the time spent on each task should be managed. In this research, we introduced a scheduler for task execution as a function of RAV (Figure 8). In the upper part of the screen, graphs and a calendar are arranged, and users can see the approximate ratio and achievement status of tasks. In the lower part of the screen, users can schedule task executions on a timetable.

Four types of tasks can be added to the task scheduler.

- Event for the whole laboratory

(ex. seminar).

- Research task (ex. computer experiment).

- Tasks other than research (ex. instruction to a lower-grade student).

- Other than these (ex. classes).

Each task on a timetable is colored in accordance with its type, and the ratio for all types of tasks is displayed as a pie chart. The free time in a pie chart is calculated by subtracting the scheduled time of all the tasks from the maximum activity time set beforehand. Users can plan a reasonable task execution schedule by checking their free time.

Since RAV sets a rule-of-thumb achievement time of a target action on the basis of results of a questionnaire to students, the simple automatic scheduling function is also implemented in the task scheduler.

\section{B. Recording and Evaluation of Task Achievements}

After task execution, users record details, such as the contents of execution, on the memo that quotes the task statement and change the progress tag of a memo to "completed." Furthermore, users evaluate the contents of task execution. The present RAC already has self-assessment and mutual evaluation functions. However, since the memos about research activities were private, we had not previously touched upon the assessment of the contents. In this research, we enabled the RAC to unfold the memo about a task that was clarified at 


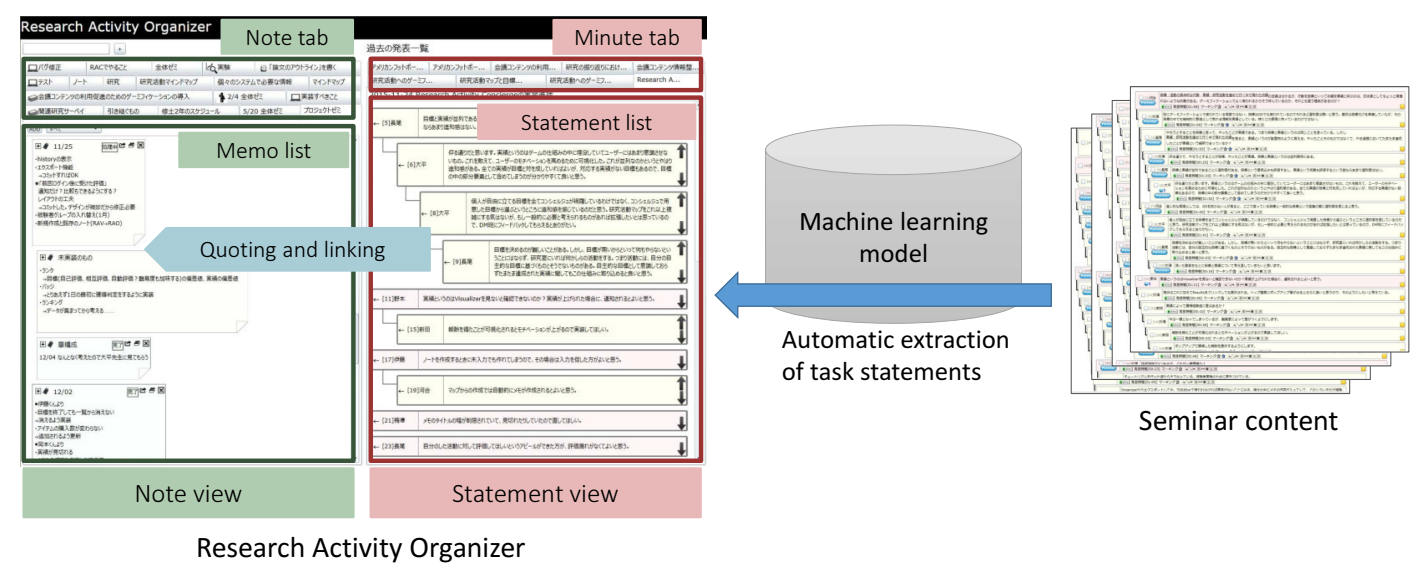

Fig. 4. Process of task statement extraction and Research Activity Organizer.

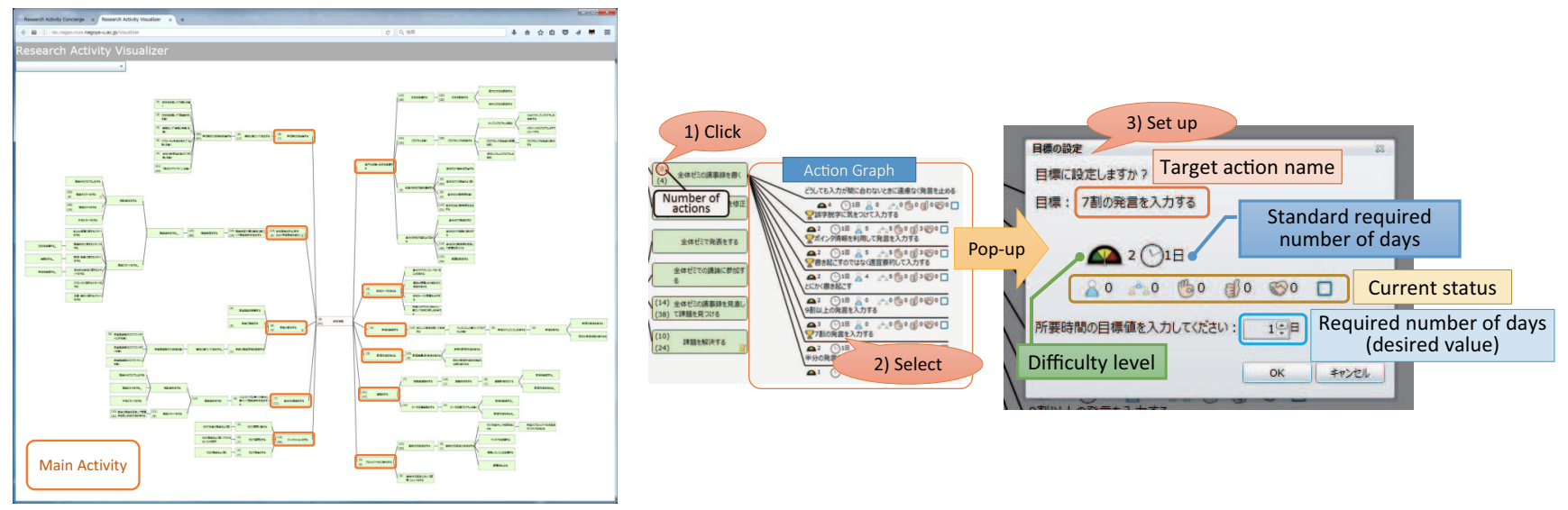

Fig. 5. Research activity map and goal setting on RAV.

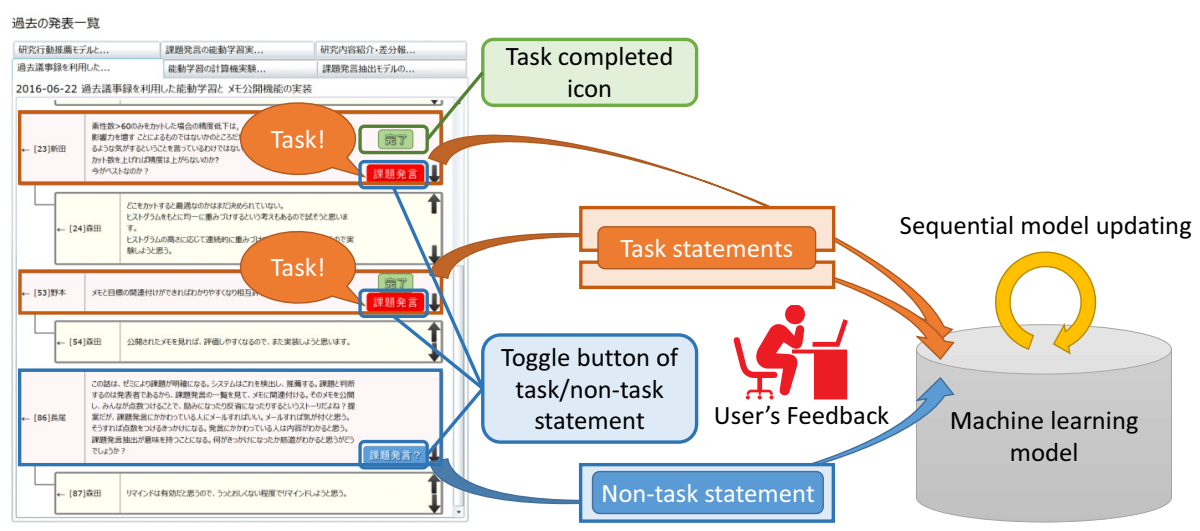

Fig. 6. Task statement list in statement view of RAO and extraction model updating. 


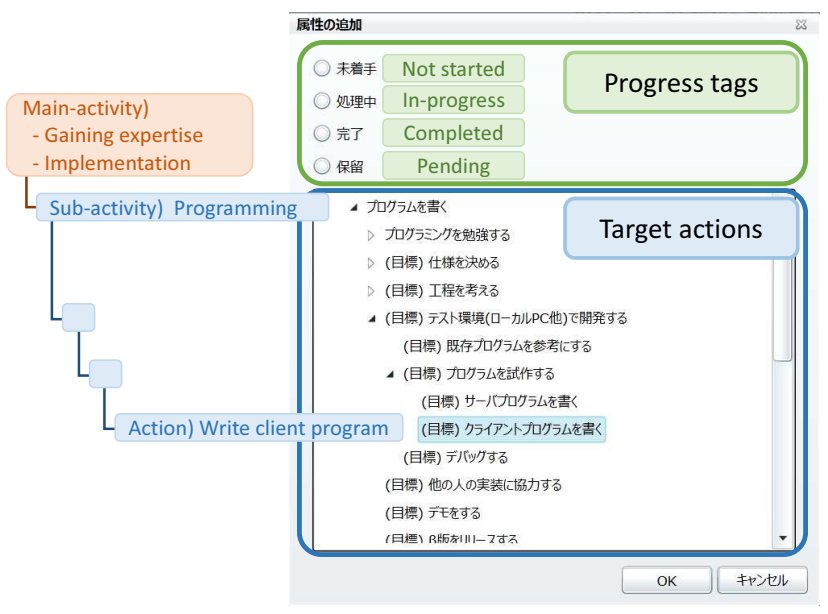

Fig. 7. Attachment of progress tags and target actions to memo.

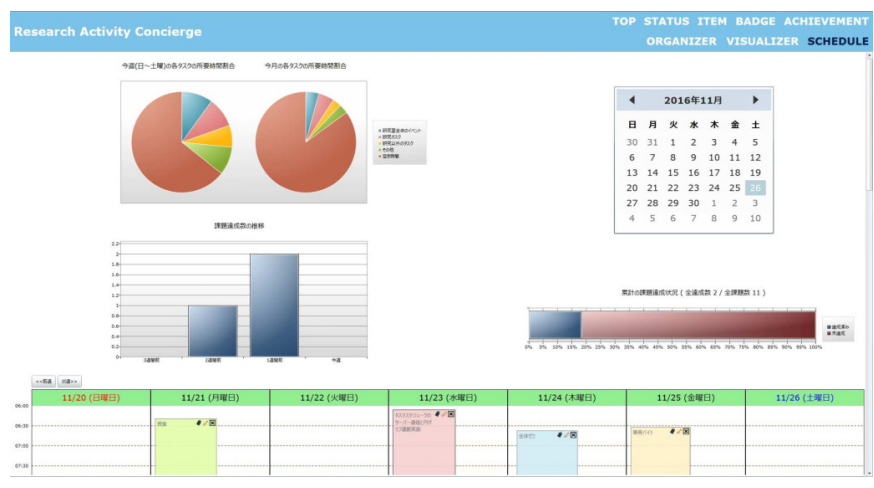

Fig. 8. Task scheduler.

a seminar before laboratory members. Then, we enabled it to receive a sensitive assessment in accordance with the contents of execution of the task.

After completing a task, users can associate the memo about their own activity with the target action and can perform self-assessment in a self-assessment dialog (Figure 9). In self-assessment, the user evaluates his/her own actions on a five-point scale. When mutual evaluation from other users is received, the memos associated with the target action during self-assessment are displayed on the right-hand side of a mutual evaluation dialog (Figure 10), and users can use them as judgment material for evaluating the contents of execution of a task. In mutual evaluation, other users evaluate a user's action. Mutual evaluation consists of two indicators. One is a stamp assessment. This is concerned with evaluating not the content of the activity but the activity itself. There are three types of stamp: "Like!", "Great!", and "Thanks!" The other indicator is a five-point scale assessment. The stamp assessment evaluates the activity itself, and a five-point scale assessment is used to evaluate the content of the activity.

\section{EXPERIMENTS AND ANALYSIS}

We conducted experiments to quantitatively evaluate the effect on research activities of the proposed RAC and a

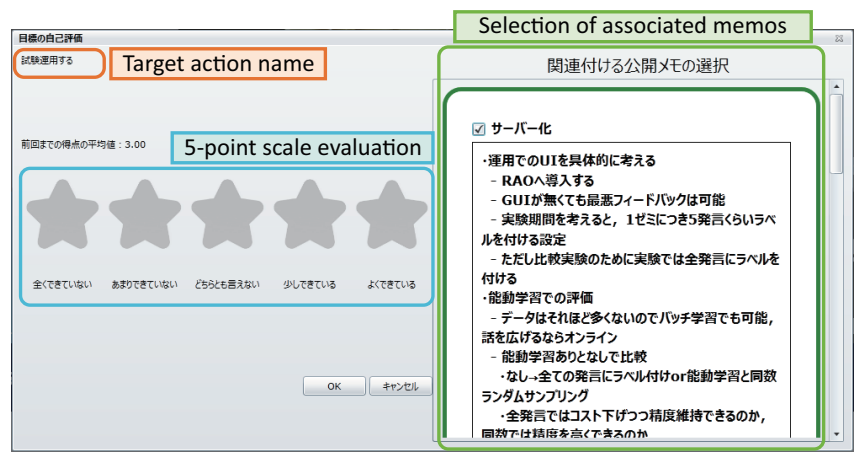

Fig. 9. Self-assessment dialog.

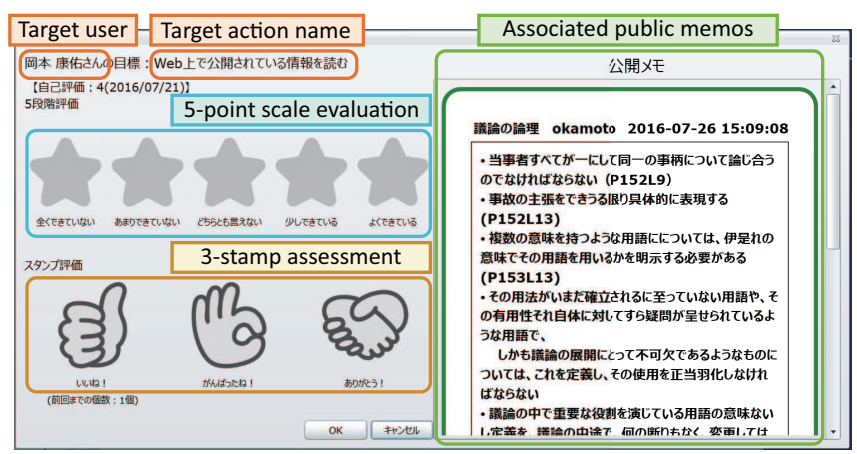

Fig. 10. Mutual evaluation dialog.

qualitative assessment by a questionnaire. The subjects that participated in the experiments ranged from undergraduate seniors (B4) to second-year graduate students (M2). There were eight students (B4: three, M1: three, and M2: two) in our laboratory. We randomly divided each grade into the intervention group (proposed system) and the control group (conventional system) and carried out crossover comparison tests in the first semester (April to July) and the second semester (October to December) of fiscal year 2016.

\section{A. Quantitative Evaluation of Effect on Research Activities}

The evaluation criteria of the system in quantitative evaluation are as follows.

- Task awareness rate

- Goal attainment level

- Self-assessment and mutual evaluation score

The task awareness rate is the proportion of task statements collected while using the RAC from the true task statements selected by reviewing all the seminar contents at the end of a semester. The goal attainment level expresses the number of goals achieved per day. The comparison results for the proposed system (P) and the conventional system (C) are shown in Table I.

As a result of performing a t-test of the task awareness rates between the proposed and conventional RACs, a difference was found that had the significance level of .05 ( $p$-value = 0.0481). Moreover, when two students used the conventional 
TABLE I

EVAluATION OF PROPOSED (P) AND CONVENTIONAL (C) RACS.

\begin{tabular}{|l|c|c|c|c|c|}
\hline Evaluation criteria & System & Ave. & SD & \multirow{2}{*}{ S.D. } & $\begin{array}{c}\text { Effective \# } \\
\text { of students }\end{array}$ \\
\hline \hline \multirow{2}{*}{$\begin{array}{l}\text { Task awareness rate } \\
(\%)\end{array}$} & $\mathrm{P}$ & 61.4 & 23.3 & \multirow{2}{*}{$*$} & 6 \\
\cline { 2 - 4 } \cline { 3 - 4 } & $\mathrm{C}$ & 36.2 & 32.1 & & 2 \\
\hline Goal attainment level & $\mathrm{P}$ & 0.23 & 0.10 & \multirow{2}{*}{ n.s. } & 5 \\
\cline { 2 - 4 } & $\mathrm{C}$ & 0.27 & 0.33 & & 3 \\
\hline \multirow{2}{*}{$\begin{array}{l}\text { Self-assessment score } \\
\text { (max. 5) }\end{array}$} & $\mathrm{P}$ & 4.24 & 0.47 & \multirow{2}{*}{ n.s. } & 5 \\
\cline { 2 - 4 } $\begin{array}{l}\text { Mutual evaluation score } \\
\text { (max. 5) }\end{array}$ & $\mathrm{C}$ & 4.09 & 0.58 & & 3 \\
\cline { 2 - 4 } & $\mathrm{P}$ & 4.76 & 0.18 & \multirow{2}{*}{ n.s. } & 2 \\
\cline { 2 - 3 } & $\mathrm{C}$ & 4.79 & 0.27 & & 4 \\
\hline
\end{tabular}

n.s. : not significant

system, their task awareness rates became high, but the difference between systems was several percent and thus not very large. Thus, the function for automatically extracting the task statement in the proposed system effectively distinguishes between tasks.

The goal attainment level, self-assessment score, and mutual evaluation score do not significantly differ between systems as shown in Table I. However, the goal attainment level and the self-assessment score showed higher values when five out of eight students used the proposed RAC. Thus, this suggests that we need to increase the amount of data after this and to analyze them carefully. Since the quality of an execution result also changes a lot depending on the difficulty of a task, raising the proposed RAC's sensitivity to tasks and the goal attainment level or assessment scores may not necessarily be related.

\section{B. Qualitative Assessment using Questionnaire}

The results of a questionnaire given to users after they had used the proposed RAC are shown in Figure 11.

Seven out of eight students answered "strongly agree" to the statement "Tasks I forgot were extracted." Moreover, seven out of eight students responded positively to "RAC positively affected research activities," "I was more motivated to organize tasks," and "RAC is useful." In their free descriptions, users who answered "undecided" to these three statements said they had "not acquired the habit of using the system," so we can consider using push messages to urge use of the system.

On the other hand, only four students agreed that the proposed "RAC is easy to use." Since half the users did not think it was easy-to-use, the challenge is preparing an interface that raises use volition.

The task execution using RAC system mainly contains five behaviors: 1) selection of task statements, 2) organizing of the content of tasks, 3) goal setting based on tasks, 4) scheduling of tasks, and 5) recording of task execution results. To assess the usability of RAC about each behavior, we conducted a questionnaire using System Usability Scale (SUS) [19]. The score of SUS and the related scales [20][21], and use/nonuse of RAO/RAV in each behavior are shown in Table II. The result shows that it is necessary to greatly improve the usability of RAV.

As mentioned above, although the proposed RAC was useful in many users' research activities on the whole and was especially useful for arranging tasks, problems such as usability and routinization of use are left to be solved.

\section{CONCLUSIONS}

In this research, to smoothly promote a student's research activities in a university laboratory, we added a task execution support function to a research activity concierge (RAC), which is our present research-activities support system. As a result of conducting a practical use experiment of the system for students engaged in undergraduate and postgraduate research, we found that the proposed RAC was able to grasp the existence of tasks more correctly than the present RAC.

In the present RAC, the user him/herself needs to set goals. However, since all research activities are large scale and many target actions are prepared by the RAC, it is difficult for the students unfamiliar with research activities to select target actions. Therefore, it is thought that an RAC that recommends a target action in accordance with a student's progress will be effective. For a recommendation system, many algorithms of user-based collaborative filtering will need to be adopted. By using collaborative filtering for a user's activity log obtained by long-term practical use of the proposed RAC, more suitable target actions for students should be able to be recommended and more advanced task execution support achieved.

\section{REFERENCES}

[1] S. Deterding, D. Dixon, R. Khaled, and L. Nacke, "From Game Design Elements to Gamefulness: Defining "Gamification"," in 15th International Academic MindTrek Conference: Envisioning Future Media Environments, Tampere, Finland, 2011, pp. 9-15, https://doi.org/10.1145/2181037.2181040

[2] K. Werbach and D. Hunter, For the Win: How Game Thinking Can Revolutionize Your Business, Wharton Digital Press, 2012.

[3] R. Cronk, "Using Non-interactive Games to Increase Student Engagement and Participation in Class Discussion," in World Conference on Educational Multimedia, Hypermedia and Telecommunications, Denver, CO, 2012, pp. 311-315.

[4] I. Kotini and S. Tzelepi, A Gamification-Based Framework for Developing Learning Activities of Computational Thinking, Gamification in Education and Business, Springer International Publishing, 2015, pp. 219-252, DOI: 10.1007/978-3-319-10208-5

[5] C. A. Bodnar and R. M. Clark, "Exploring the Impact Gamebased Learning Has on Classroom Environment and Student Engagement Within an Engineering Product Design Class," in Second International Conference on Technological Ecosystems for Enhancing Multiculturality, Salamanca, Spain, 2014, pp. 191-196, https://doi.org/10.1145/2669711.2669899

[6] S. Ohira, K. Kawanishi, and K. Nagao, "Assessing Motivation and Capacity to Argue in a Gamified Seminar Setting," in Second International Conference on Technological Ecosystems for Enhancing Multiculturality, Salamanca, Spain, 2014, pp. 197-204, https://doi.org/10.1145/2669711.2669900

[7] C. González, A. Mora, and P. Toledo, "Gamification in Intelligent Tutoring Systems," in Second International Conference on Technological Ecosystems for Enhancing Multiculturality, Salamanca, Spain, 2014 pp. 221-225, https://doi.org/10.1145/2669711.2669903 
Tasks I forgot were extracted. RAC positively affected research activities.

I was more motivated to organize tasks.

RAC is useful.

RAC is easy to use.

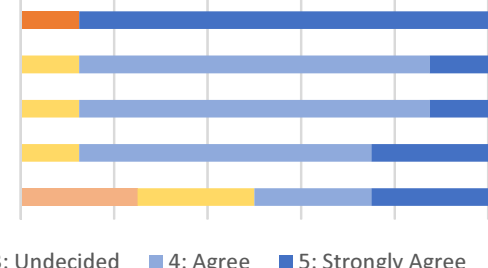

1: Strongly Disagree $\quad 2$ : Disagree $\quad 3$ : Undecided $\quad 4$ : Agree $\quad 5$ : Strongly Agree

Fig. 11. Results of questionnaire.

TABLE II

SUS SCORE AND RELATED SCALES OF TASK EXECUTION WITH RAC.

\begin{tabular}{|l|c|c|c|c|c|c|}
\hline & RAO & RAV & SUS score [19] & Acceptability ranges [20] & Adjective ratings [20] & Grade [21] \\
\hline \hline 1) Selection of task statements & + & - & 86.9 & Acceptable & Excellent & A+ \\
\hline 2) Organizing the content of tasks & + & - & 77.5 & Acceptable & Good & B+ \\
\hline 3) Goal setting based on tasks & - & + & 51.3 & Marginal low & OK & F \\
\hline 4) Scheduling of tasks & - & + & 57.5 & Marginal low & OK & OK \\
\hline 5) Recording of task execution results & + & + & 62.5 & Marginal high & D \\
\hline
\end{tabular}

[8] S. Ohira, S. Sugiura, and K. Nagao, "Proposed Framework for Gamifying Research Activities," in Third International Conference on Technological Ecosystems for Enhancing Multiculturality, Porto, Portugal, 2015, pp. 245-250, https://doi.org/10.1145/2808580.2808617

[9] S. Ohira, S. Sugiura, and K. Nagao, "Gamifying Research Activity Support System, " in Fourth International Conference on Technological Ecosystems for Enhancing Multiculturality, Salamanca, Spain, 2016, pp. 739-745, https://doi.org/10.1145/3012430.3012601

[10] L.-H. Wong, T.-W. Chan, Z.-H. Chen, R. B. King, and S. L. Wong, "The IDC theory: interest and the interest loop," in 23rd International Conference on Computers in Education, Hangzhou, China, 2015, pp. 804-813.

[11] K. Nagao, K. Kaji, D. Yamamoto, and H. Tomobe, "Discussion Mining: Annotation-Based Knowledge Discovery from Real World Activities," in Fifth PacificRim Conference on Multimedia, Tokyo, Japan, 2004, pp. 522-531.

[12] T. Ozono and T. Sintani, "P2P based Information Retrieval on Research Support System Papits," in IASTED International Conference on Artificial and Computational Intelligence, Bologna, Italy, 2002, pp. 49-50, https://doi.org/10.1145/544741.544755

[13] M. L. M. Kiah, B. B. Zaidan, A. A. Zaidan, M. Nabi, and R. Ibraheem, "MIRASS: medical informatics research activity support system using information mashup network," Journal of medical systems, vol. 38, no. 4 2014, pp. 1-15, https://doi.org/10.1007/s10916-014-0037-x

[14] Y. Miyadera, S. Nakamura, and T. Nanashima, "LabChart: A Support System for Collaborative Research Activities in University Labora- tories and its Practical Evaluations," in 12th International Conference on Information Visualisation, London, UK, 2008, pp. 169-178, https://doi.org/10.1109/IV.2008.15

[15] O. Koyama and Y. Katsuyama, "Design and performance analysis of unified education and research activity support systems over WWW," Journal of Innovative Computing, Information and Control, vol. 2, no. 4, 2006, pp. 807-818.

[16] K. Nagao, K. Inoue, N. Morita and S. Matsubara, "Automatic Extraction of Task Statements from Structured Meeting Content," in 7th International Conference on Knowledge Discovery and Information Retrieval, Lisbon, Portugal, 2015, pp. 307-315, http://dx.doi.org/10.5220/0005609703070315

[17] K. Nagao, N. Morita, and S. Ohira, "Evidence-Based Education: Case Study of Educational Data Acquisition and Reuse," in 8th International Conference on Education, Training and Informatics, Orlando, FL, 2017.

[18] B. Settles, Active Learning Literature Survey, Computer Sciences Technical Report 1648, University of Wisconsin-Madison, 2010.

[19] J. Brooke, "SUS - A quick and dirty usability scale," Journal of Usability

[20] A. Bation in industry, vol. 189, no. 194, London, UK, 1996, pp. 4-7. SUS Scores Mean: Adding an Adjective Rating Scale," Journal of Usability Studies, vol. 4, no. 3, 2009, pp. 114-123.

[21] J. Sauro, A Practical Guide to the System Usability Scale, Measuring Usability LLC, 2011. 



\section{$23^{\text {rd }}$ Conference on Knowledge Acquisition and Management}

$\mathbf{K}$ NOWLEDGE management is a large multidisciplinary field having its roots in Management and Artificial Intelligence. Activity of an extended organization should be supported by an organized and optimized flow of knowledge to effectively help all participants in their work.

We have the pleasure to invite you to contribute to and to participate in the conference "Knowledge Acquisition and Management". The predecessor of the KAM conference has been organized for the first time in 1992, as a venue for scientists and practitioners to address different aspects of usage of advanced information technologies in management, with focus on intelligent techniques and knowledge management. In 2003 the conference changed somewhat its focus and was organized for the first under its current name. Furthermore, the KAM conference became an international event, with participants from around the world. In 2012 we've joined to Federated Conference on Computer Science and Systems becoming one of the oldest event.

The aim of this event is to create possibility of presenting and discussing approaches, techniques and tools in the knowledge acquisition and other knowledge management areas with focus on contribution of artificial intelligence for improvement of human-machine intelligence and face the challenges of this century. We expect that the conference\&workshop will enable exchange of information and experiences, and delve into current trends of methodological, technological and implementation aspects of knowledge management processes.

\section{TOPICS}

- Knowledge discovery from databases and data warehouses

- Methods and tools for knowledge acquisition

- New emerging technologies for management

- Organizing the knowledge centers and knowledge distribution

- Knowledge creation and validation

- Knowledge dynamics and machine learning

- Distance learning and knowledge sharing

- Knowledge representation models

- Management of enterprise knowledge versus personal knowledge

- Knowledge managers and workers

- Knowledge coaching and diffusion

- Knowledge engineering and software engineering

- Managerial knowledge evolution with focus on managing of best practice and cooperative activities

- Knowledge grid and social networks
- Knowledge management for design, innovation and ecoinnovation process

- Business Intelligence environment for supporting knowledge management

- Knowledge management in virtual advisors and training

- Management of the innovation and eco-innovation process

- Human-machine interfaces and knowledge visualization

\section{SECTION EDITORS}

- Hauke, Krzysztof, Wroclaw University of Economics, Poland

- Nycz, Malgorzata, Wroclaw University of Economics, Poland

- Owoc, Mieczyslaw, Wroclaw University of Economics, Poland

- Pondel, Maciej, Wroclaw University of Economics, Poland

\section{REVIEWERS}

- Abramowicz, Witold, Poznan University of Economics, Poland

- Andres, Frederic, National Institute of Informatics, Tokyo, Japan

- Badica, Amelia, University of Craiova, Romania

- Berio, Giuseppe, Universite de Bretagne Sud, France

- Bodyanskiy, Yevgeniy, Kharkiv National University of Radio Electronics, Ukraine

- Chmielarz, Witold, Warsaw University, Poland

- Christozov, Dimitar, American University in Bulgaria, Bulgaria

- Christozov, Dimitar, American University in Bulgaria, Bulgaria

- Grabowski, Mariusz, Krakow University of Economics, Poland

- Helfert, Markus, Dublin City University, Ireland

- Hussain, Fehmida, School of Science and Technology, Dubai

- Jan, Vanthienen, Katholike Universiteit Leuven, Belgium

- Jelonek, Dorota, Faculty og Management of Czestochowa University of Technology

- Kania, Krzysztof, Ue Katowice

- Kayakutlu, Gulgun, Istanbul Technical University, Turkey

- Khachidze, Manana, Tbilisi State University, Georgia

- Kisielnicki, Jerzy, University of Warsaw, Poland 
- Konikowska, Beata, Institute of Computer Science, Poland

- Korwin-Pawlowski, Michael L., Universite du Quebec en Outaouais, Canada

- Kulczycki, Piotr, Systems Research Institute, Polish Academy of Sciences, Poland

- Ligęza, Antoni, AGH University of Science and Technology, Poland

- Mach-Król, Maria, University of Economics in Katowice, Poland

- Mercier-Laurent, Eunika, University Jean Moulin Lyon3, France

- Michalik, Krzysztof, University of Economics in Katowice, Poland

- Milewski, Robert, Medical University of Bialystok, Department of Statistics and Medical Informatics, Poland

- Nalepa, Grzegorz J., AGH University of Science and Technology, Poland

- Olszak, Celina M., University of Economics in Katowice, Poland

- Opiła, Janusz, AGH University of Science and Technology, Poland

- Paliński, Andrzej, AGH University of Science and Technology, Poland

- Petryshyn, Lubomyr, AGH University of Science and Technology, Poland

- Pełech-Pilichowski, Tomasz, AGH University of Science and Technology, Poland

- Prasad, T. V., Godavari Institute of Engineering and Technology, India

- Pulvermueller, Elke, University Osnabrueck, Germany

- Reimer, Ulrich, University of Applied Sciences St. Gallen, Switzerland

- Rossi, Gustavo, National University of La Plata, Argentina
- Salem, Abdel-Badeeh M., Ain Shams University, Egypt

- Sankowski, Dominik, University of Technology in Łódź, Poland

- Sauer, Jurgen, University of Oldenburg, Germany

- Schroeder, Marcin Jan, Akita International University, Japan

- Skalna, Iwona, AGH University of Science and Technology, Faculty of Management, Poland

- Sobińska, Małgorzata, Wroclaw University of Economics, Poland

- Soja, Piotr, Cracow University of Economics, Poland

- Stawowy, Adam, AGH University of Science and Technology, Faculty of Management, Poland

- Surma, Jerzy, Warsaw School of Economics, Poland and University of Massachusetts Lowell, United States

- Szpyrka, Marcin, AGH University of Science and Technology, Poland

- Teufel, Stephanie, University of Fribourg, Switzerland

- Tvrdikova, Milena, VŠB Technological University of Ostrava, Faculty of Economics, Czech Republic

- Vasiliev, Julian, University of Economics in Varna, Bulgaria

- Wielki, Janusz, Opole University of Technology, Poland

- Zaliwski, Andrew, University of Auckland

- Zhelezko, Boris, Belorussian State Economic University, Belarus

- Zhu, Yungang, College of Computer Science and Technology, Jilin University, China

- Zurada, Jozef, College of Business University of Louisville, United States

\section{ORGANIZING COMMITTEE}

- Hołowińska, Katarzyna

- Przysucha, Lukasz, Wroclaw University of Economics 


\section{Overview of Verification Tools for Business Process Models}

\author{
Anna Suchenia (Mroczek) \\ Cracow University of Technology \\ ul. Warszawska 24, 31-155 Kraków, Poland \\ Email: asuchenia@pk.edu.pl
}

\author{
Piotr Wiśniewski, Antoni Ligęza \\ AGH University of Science and Technology \\ al. A. Mickiewicza 30, 30-059 Krakow, Poland \\ E-mail: \{wpiotr,ligeza\}@agh.edu.pl
}

\begin{abstract}
Formal verification of process models is an important issue in Business Process Management. Such a verification provides the information about the correctness of a process model, can be also used for checking business compliance or as a preliminary step to simulation. In this paper, we provide an overview of the existing tools for such a verification.

Index Terms-process models verification, process models anomalies, verification tools, business process verification
\end{abstract}

\section{INTRODUCTION}

D ESIGNING process models is currently a broad term. Considering designing processes, one can think about models designed manually by business analysts, as well as models generated from other representations such as: natural text description [1], structured text [2], spreadsheets [3], other representations like UML [4], or mining such models from event logs [5]. All such models can suffer from various anomalies [6]-[8]. Some of such anomalies can be avoided by validation models [9], [10] or verification [11] of models.

Business Process Model and Notation (BPMN) [12] is the most common notation for representing process models. BPMN supports process documentation, communication and visualization using clear graphical representation understandable even for non-technical business people. Although it provides a standardized understanding of BPMN elements and constructs, no formal semantics is provided within this specification. This can lead to misinterpretations or errors in models. Thus, there is a field for studies focusing on formal representation and verification of such process models.

Such a verification of process models can provide the information about the correctness of a process model in terms of syntactic and structural anomalies of models. The main focus of this paper is to present an overview of the verification tools for process models which can detect some anomalies in business processes. This paper provides an overview of several existing tools. Their capabilities and properties were analyzed.

The rest of the paper is organized as follows. Section II covers the presentation of the BPMN notation. Section III and IV give the literature overview on process anomalies, especially concerning anomalies in BPMN models. Section V provides the description and comparison of various verification tools used for verifying business process models. The final section concludes the paper.

The paper is supported by the AGH UST grant.

\section{Business Process Model and Notation}

Process models in BPMN are represented as diagrams modeled using a limited set of graphical elements. There are four main groups of elements, namely: flow objects, connecting objects, swimlanes and artifacts.

Flow objects are the key elements describing the process. The set of flow objects consist of three core element types: events, activities and gateways (see Fig. 1):

1) activities - represented by rounded-corner rectangles, describe the work that has to be completed within a process,

2) events - represented by circles, describe something that happen during the time of the process,

3) gateways - represented by diamond shapes, control the flow of the token between flow objects.
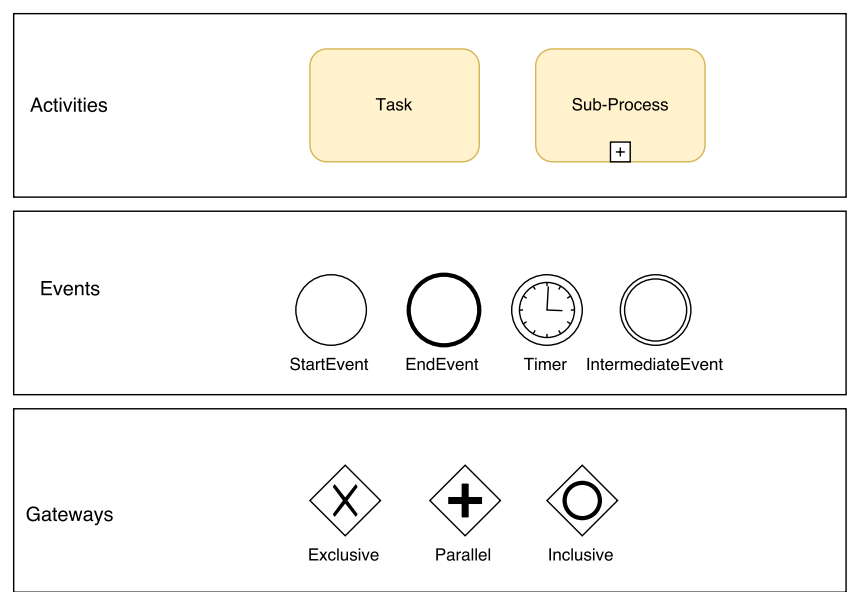

Fig. 1. BPMN flow objects of internal Business Process Model

In the case of activities, there are two kinds of them: tasks and sub-processes. A task represents a single unit of work should not be divided. A sub-process is used for complex work which can be divided into smaller units and specified in the lower level as a separated process.

There are three types of events: the start, intermediate and the end event. The start event works like a trigger to a process and shows its beginning (and under what conditions the process begins). The end event indicates where the business process ends. The intermediate event represents what happens between start and end events. 
Gateways constitute the mechanism of controlling the way in which business process is executed. The most typical types of gateways are as follows:

- data-based exclusive gateway - used for controlling the process flow based on given process data (see Fig. 2),

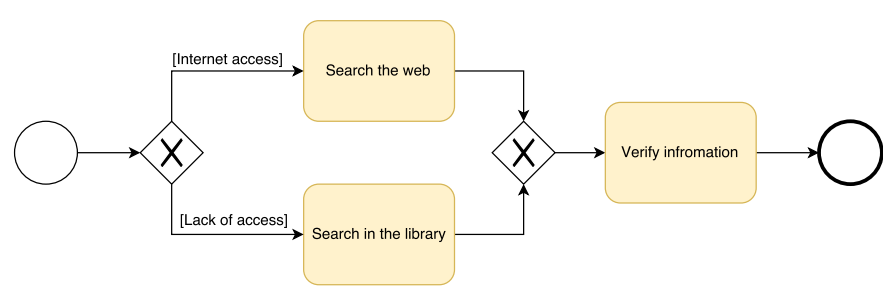

Fig. 2. Data-Based Exclusive Gateway

- inclusive gateway - used for creating potentially parallel paths based on the conditions of all outgoing flows (see Fig. 3),

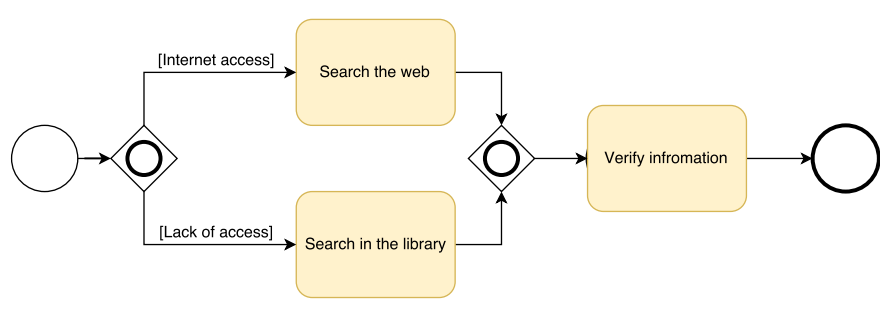

Fig. 3. Inclusive gateway

- parallel gateway - used for paralleling the flows without the need of checking any conditions (see Fig. 4).

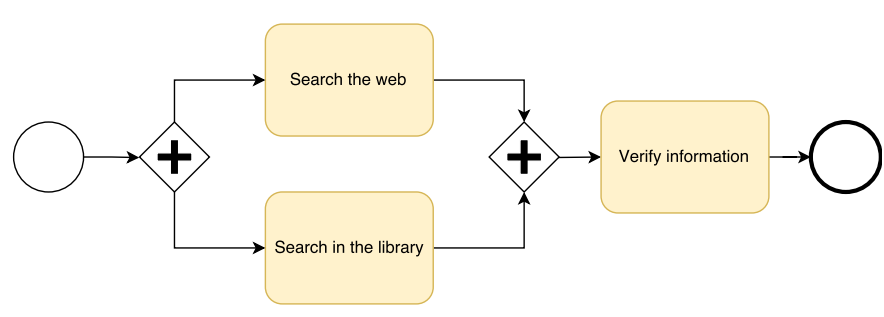

Fig. 4. Parallel gateway

- event-based gateway - used for modeling alternative paths that are based on events (Fig. 5).

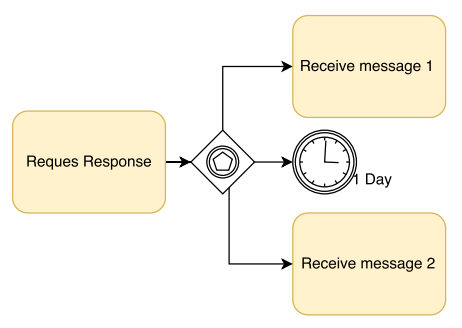

Fig. 5. Event-based gateway
Flow objects are connected using connecting objects, which are of three types: sequences, messages, and associations. Sequence flow shows the order in which particular activities are performed in a process. Message flow shows the flow of messages between two process participants. Association links some artifacts to activities, events, gateways or flows.

A simple auction process, presenting the basic BPMN flow objects, is depicted in Fig. 6.

For demonstrating what business function a particular flow object is connected with or by which part of system it is executed, BPMN usually uses the concept of swimlanes. Participants are represented as pools, which can be divided into sub-partitions called lanes. These can be used for representing specific objects or roles in a process. Artifacts, in turn, are diagram elements which show additional pieces of information. BPMN naively supports several kinds of artifacts like data objects and data stores, groups and annotations.

Additionally, many different extensions of BPMN have been proposed for capturing various aspects of business processes [13]-[16]. Thus, in such an advanced and complex notation, it is hard to avoid model anomalies. Moreover, it is possible to mistake a correct model with incorrect one, especially because two BPMN models with different structure, but behaviorally equivalent, can be both correct and unambiguous [17]. It is because the BPMN notation allows for expressing the same semantics using various syntactic structures. However, it is possible to transform such equivalent structures to the equivalent ones [18]. Another way is to use some kind of a guide which provides help in modeling. This can be done using some recommendation technique during process modeling [19]-[21] or the environment capable to detect some semantic issues [22]-[25].

\section{AppliCATIONS OF THE VERIFICATION IN THE AREA OF ANOMALIES IN PROCESS MODELS}

There are many possibilities of defining incoherent business logic specification and its interpretation. Even in basic process models, some anomalies can be observed [26]. An improvement is required in the mechanism which provides cohesion in detecting anomalies in business processes [27]. Anomalies have been defined in numerous papers [28], yet a unified definition was presented in IEEE standard classification for Software Anomalies [29]: Each condition different from the expected is an anomaly. In a business logic, an anomaly can be considered as every negative influence on modeling and models. There is a special kind of anomaly - a defect, which blocks the correct and efficient flow of objects completely.

Some anomalies can be found by searching the BPMN models for some patterns. In [30], several anti-patterns are found using a query language for BPMN. In [31], typical gateway constellations leading to problematic situations in the flow work diagram are presented. A similar solution was used in [32], where an 'anomaly pattern' are detected using antipatterns in the data flow. Patterns as well as anti-patterns can be represented as LTL formulae and be used in formal 


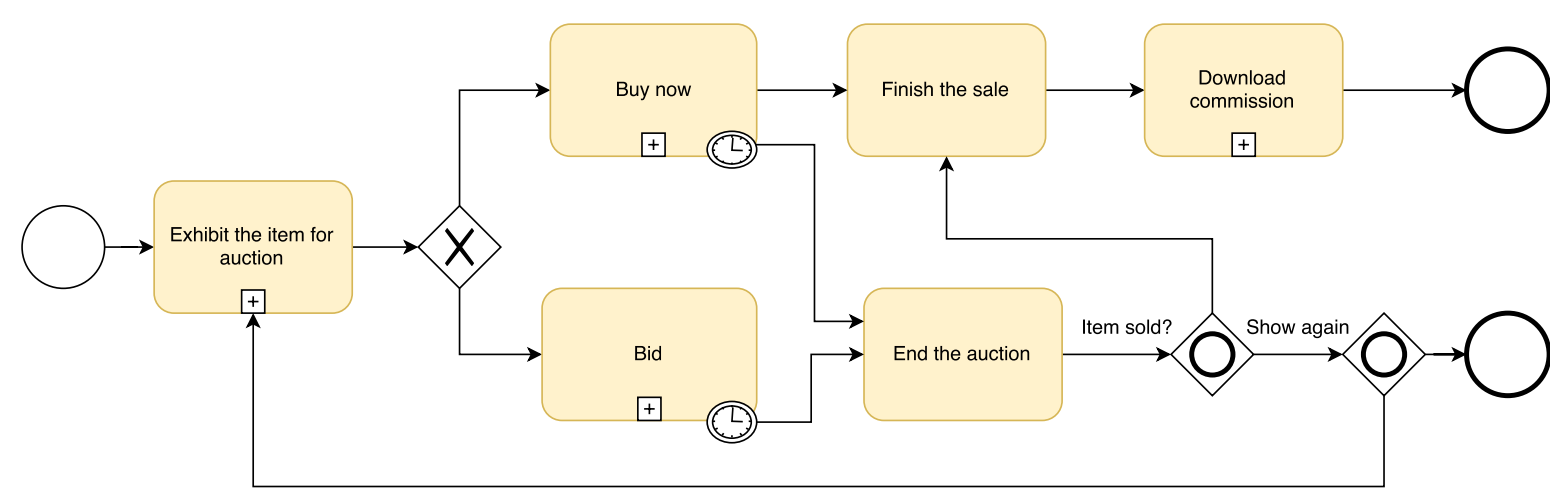

Fig. 6. An example of a process model in BPMN

verification of a business process model transformed to a highlevel Petri Net [33]. The approach of Lam [34] transforms BPMN models to the New Symbolic Model Verifier (NuSMV) language in order to do a model-checking analysis. This approach has formal foundations and addresses the correctness issue of the transformation. It requires to encode properties of a model using Computation Tree Logic (CTL) formulas. Another approach, which stems from formalism or inadequacy of the tools, is presented in [35].

Control flow anomalies concern problems connected with flow control and gateways conditions [36]. In [37], a problem of control over many semantically identical connections between two workflow elements was presented. This multiplicity complicates changes in the workflow, which is not desired. Roy et al. [38] described the method of control flow error detection based on Petri Net analysis and evaluated it on a set of industrial examples. An extensive survey of process model verification techniques regarding flow correctness and variability was presented in [39].

Formal verification of workflow-oriented software models based on the semantic tableaux method that uses the deductive approach was proposed in [40].

Ou-Yang and Lin proposed a Petri-net-based approach [41], which evaluates a feasibility of a BPMN model, e.g. to reveal deadlocks and infinite loops. This approach consists in manually translating of the BPMN model to the Modified BPEL4WS representation, and then to Colored Petri-net XML (CPNXML). The CPNXML representation can be then verified using CPN Tools. The approach has some major restrictions, such as limited assessment criteria, and lack of support of the multiple merge and split conditions.

Gateways can also caused some anomalies. XOR-gateways with undefined gateway conditions can cause practical problems or even be a reason of an error. A similar thing occur when XOR-gateways conditions do not exclude each other and partially or fully overlap. What happens in flow control in a case of a lack of synchronization is multiple flow execution. For example, branches and some loop instruction cause such an anomaly [42].

A flow deadlock is a situation, in which the workflow is stopped in the current position of the path and cannot be accomplished. Another lock of flow is known as livelock. In
[30] it is called an 'infinite loop'. Flow livelock keeps the operating work flow system in an infinite loop. The reasons are bad modeling conditions, which prevent leaving the loop. An approach based on business process event logs, where both deadlocks and livelocks can be detected as well as tasks which can never be executed, was presented in [43].

Badica et al. consider formalized models using Role Activity Diagrams for BP business process verification [44] as well as including logic-based ones in similar multi-agent approaches [45]. Other formalized approaches in modeling complex heterogeneous information systems include [46].

Another class of anomalies in processes are anomalies in the rules used in a process model. Such rule-based anomalies are described in a number of papers [47]-[51]. These involve several problems connected with rules, such as: rule-base consistency (concerning the coherence of rules), rule-base livelock, also called 'circular rules' [48] or rule-base deadlock. This type of anomaly suggests that rule-base does not encompass the basic context in which it is used. Coverage anomalies concern the rules in which conditions can be fulfilled by the context but conclusions are modeled in such a way that no effect will ever be seen. A formal verification of business rules violations using Business Rule Language (BRL) and DepthFirst Search algorithm was conducted in [52].

Business process related anomalies may also refer to the violation of temporal constraints and dependencies added to the process model as well as can be used for validation of time related processes [53]-[59]. In [59], a BPMN model is transformed to a set of Timed Automata (TA) and is verified using Clocked Computation Tree Logic (CCTL). A similar method is described in [57], where BPMN models are verified using TA-networks with respect to business performance indicators. Temporal properties of a business process can be also verified using a framework based on the declarative specification of a process model and the Answer Set Programming (ASP) technique [58]. Dynamic detection of temporal violations and providing possible solutions to a specific problem was proposed in [55]. In [56], in turn, the authors proposed a solution where an extended BPMN model is mapped onto timed automata and then verified using UPPAAL model checker. 


\section{ANOMALIES IN BUSINESS PROCESSES}

There is a number of business process anomalies which can occur during modeling business processes. A common classification distinguishes: syntactic and structural anomalies.

\section{A. Syntactic Anomalies in Business Process}

Syntactic anomalies constitute the problem in improper usage of modeling elements. Such anomalies can be divided into four groups:

- Incorrect usage of activities - this anomaly results from improper use of start or end event. The BPMN specification defines the start and end events as optional. However, if there is a start event used in a diagram, each activity - that do not have an end event on its path - can be considered as incorrect.

- Invalid use of gateway - this anomaly is especially connected with the data-based XOR gateway and eventbased XOR gateway. The data-based gateway has to use a data to determine the token flow, so using event-based objects for data-based gateway is incorrect. In the case of the event-based gateway, it cannot be used as a merge gateway, but can only be used as a decision type gateway.

- Incorrect usage of connecting object - this anomaly is concerned with such situations as using a message flow within the pool or using conditional flow from the event type source.

- Incorrect usage of swimlanes - such an anomaly can occur when a model uses multiple pools as a single process where message flows indicate sequence of activities. This can lead to situation, where activities in a pool are not connected with sequence flows (see Fig. 7). On the other hand, there are also situations of improper use of lane as a pool.

\section{B. Structural Anomalies}

Structural anomalies are broadly described in the literature [60]-[64]; such anomalies correspond to a wrong dynamic behavior and can be divided into four types:

- Deadlock - a situation, in which the flow cannot continue because a requirement of the model is not satisfied. There are two types of deadlocks: deterministic deadlock, when concurrent flow are connected by the AND-join (parallel) gateway (see Fig. 8) and non-deterministic one, if they are connected by an OR-join (inclusive) or complex gateway.

- Lack of synchronization - a situation where there is more than one token on some sequence flow that it should be. It occurs when concurrent paths (starting with an OR-split or an AND-split) are joined by an XOR-join (see Fig. 9). If the paths were split by the AND gateway, this problem is deterministic. It is non-deterministic, if decision about splitting is made at the execution time.

- Dead Activity - a situation, in which there is an activity which cannot be executed, because there is no path leading from the start event to this activity (see Fig. 10).

- Infinite Loop - also called the closed loop - is a cycle in the process, in which token is looped and can not escape the loop. This can be caused by improper use of gateways (see Fig. 11). If such gateway is of the OR type, the loop is non-deterministic; if it is the AND type, then the loop is deterministic.

\section{VERIFICATION TOOLS}

There are several automatic or interactive tools for process model verification such as: Signavio (BpStruct, LoLA) [65], UPPAAL [66], SPIN, Wolfan, NuSMV, nuXmv, and Alvis. This section provides the analysis of several of such tools.

\section{A. Signavio}

Signavio [65] uses colored Petri nets for process verification. These can handle unreachable states (such as unreachable activities) as well as deadlocks (caused by wrong usage of gateways). Signavio also uses two additional tools BPStruct and LoLA [65].

As it comes to the BPMN syntax, Signavio can check the usage of the elements from the defined BPMN subset, as well as mandatory attributes, definition of required dictionary links and consistency with attributes of the linked dictionary item, uniqueness of element names, etc. In the process structure, usage of different elements in various contexts can be checked, e.g. usage of activities before or-splits, consistent usage of signals correct usage of boundary events, message flows, etc. Additionally, absence of loops, deadlocks, multi merges, subprocess relation cycles, multiple incoming sequence flows can be checked.

There are many properties checked by Signavio's tool, for example:

- Lack of the flow source or flow target.

- Source and target of the sequence flow are not part of the same process.

- Start-event without outgoing flows.

- Event-based gateway with less than two outputs.

BPStruct supports transforming unstructured process models into well-structured ones. A model can be called wellstructured, if for every node with multiple outgoing arcs (a split), there is a corresponding node with multiple incoming arcs (a join), and the other way round. Such the fragment of the model between the split and the join should form a singleentry-single-exit (SESE) component.

LoLA (Low Level Petri Net Analyzer) is a tool used to verify a model in Signavio. The LoLA can load the model as a Petri net and the provided properties specified in CTL*. These properties are analyzed and in the case a property is not fulfilled, it can provide a counter-example.

\section{B. UPPAAL}

UPPAAL [66], [67] is an integrated tool for modeling, simulation and verification of real-time systems. It consists of two main parts: a graphical user interface and a model-checker engine. Its model-checker is based on symbolic processing, which reduces the problem of verification to constraint programming. It is an appropriate tool for systems which can be modeled as a set of non-deterministic processes with finite 


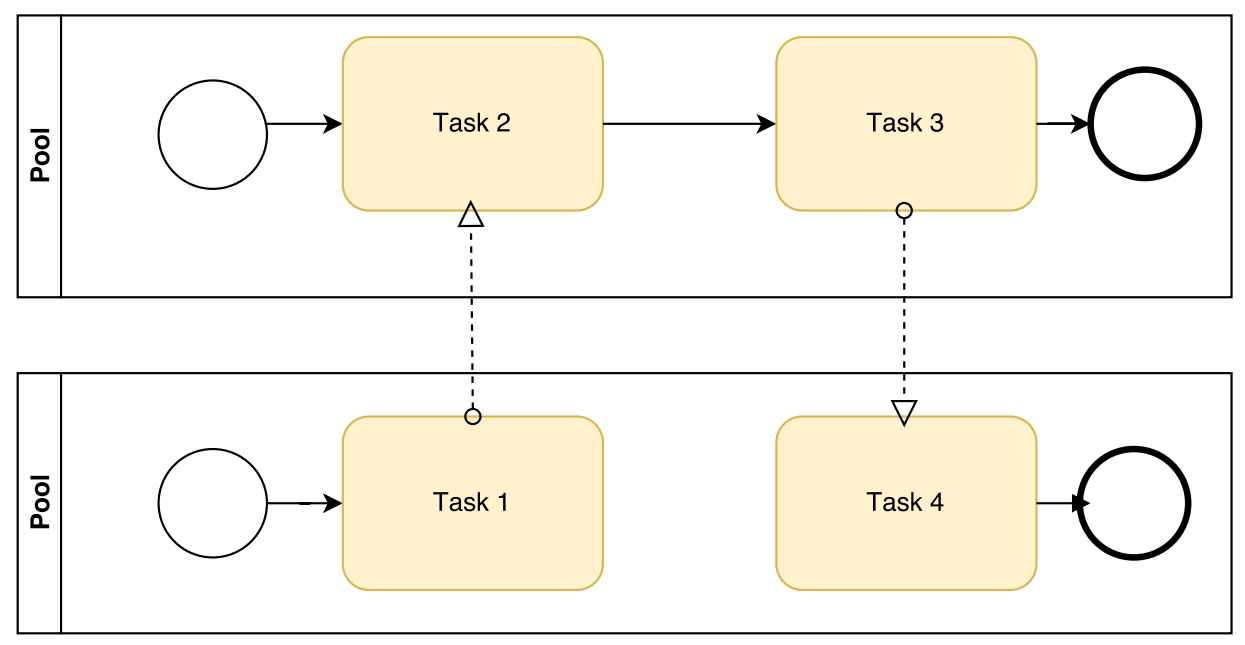

Fig. 7. Missing sequence flow

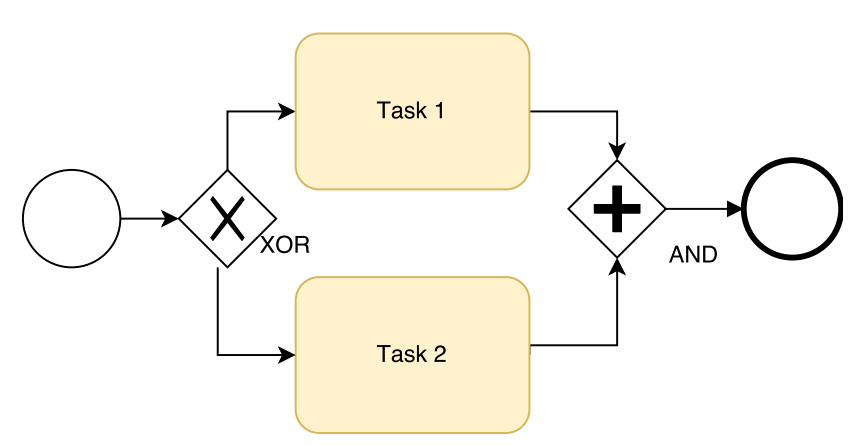

Fig. 8. Deterministic deadlock

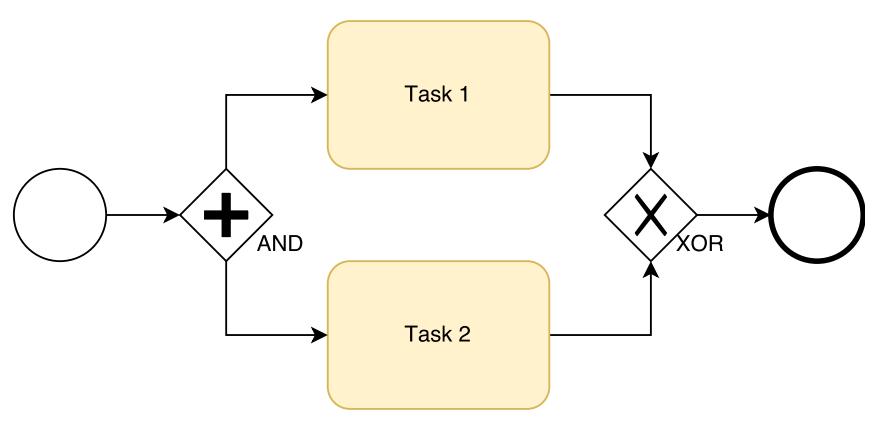

Fig. 9. Lack of synchronization

control structure and real-valued clocks that communicates using channels or shared variables [68].

UPPAAL provides a graphical editor and a graphical simulator as well as path generator for system verification and visualization.

\section{SPIN}

SPIN model checker [69] is an open-source verification tool which can be used to verification of multithreaded applications. SPIN is also a general tool for verifying the correctness

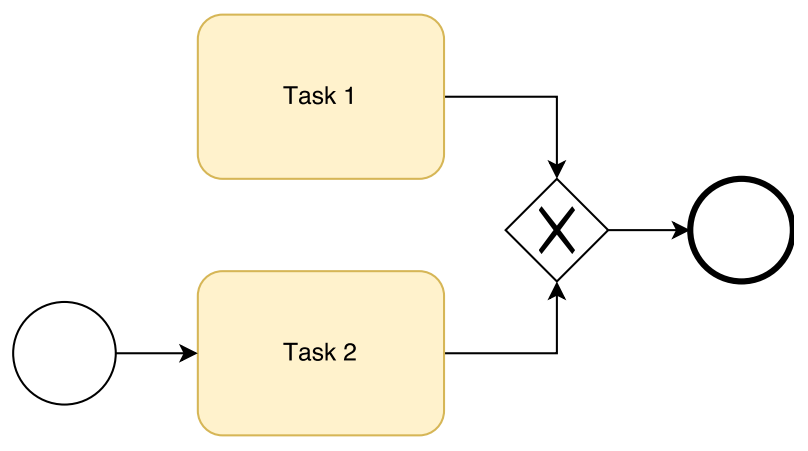

Fig. 10. Dead Activities

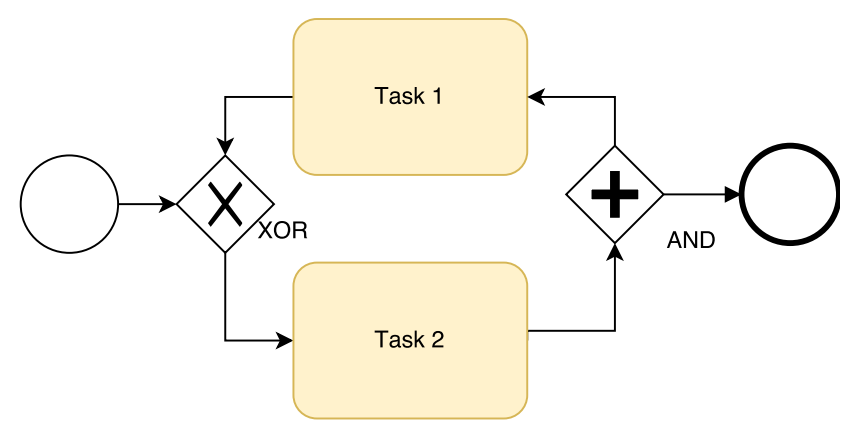

Fig. 11. Infinite Loop

of models in an automated fashion. It is oriented on checking the interactions of processes.

The tool provides 'on-the-fly' verification (the full state graph is not required, so much larger models can be verified) as well as it supports native $\mathrm{C}$ code, what allows code based verification.

\section{Woflan}

Woflan (WOrkFLow ANalyser) [70], [71] is a workflow analysis tool, which checks if a Petri net conforms to specified 
restrictions for workflows [72]. Woflan can help in detecting errors made at design-time Woflan also helps in providing the diagnostics why the process is wrong and how it can be repaired. For that reason, Woflan generates high-quality diagnostic information, which can guide the designer towards the error. The advantage of the tool is that it transforms the model into the coverage graph, which often causes a significant reduction in state space.

\section{E. NuSMV}

NuSMV [73], [74] is a symbolic model checker for temporal logic. In this approach, the process is modeled as a finite state transition system and specifications are given as formulae, which can be expressed in LTL or CTL logic. This tool is used to verify if the model satisfies the provided specifications. The input file with a model definition in SMV language can be generated based on a Petri Net [75] or behavioral elements extracted from a business process specification [76].

\section{F. $n u X m v$}

Another symbolic model checker is nuXmv [77], an extension of the NuSMV tool, which can be used for both finite and infinite state transition systems. It offers more functionalities such as boundedness and liveness verification. It also allows a user to generate an explicit state representation in a form of an XMI file which can be then visualized as a UML diagram. Process model verification using nuXmv can be executed by generating model definition from the coverability graph of a real-time coloured Petri Net [78].

\section{G. Alvis}

Alvis is a formal modelling language, tool set, and framework created to verify and model check distributed concurrent systems [79]-[82]. The advantage of Alvis modelling framework are readable graphical and a code layers of specification of modelled system behaviour. The graphical layer presents data exchange channels of communicating distributed units and enables hierachical specification that hides complexity of huge models [83]. The code layer specifies behaviour of particular distributed units using mixture of domain specific language (called Alvis language) and Haskell. The possibility of exchanging Haskell with other standard programming languages was studied in [84].

Alvis was used in BPMN model verification [85], [86]. One can verify time constraints of concurrent system with time version of Alvis framework [87]. The Alvis tool set may be used not only to verify a model of concurrent system but to simulate a provided model together with computation of time dependent statistics of simulation results [88], [89].

\section{CONCLUSION}

The relatively broad spectrum of tools for a process model verification is discussed in this paper. We have given the literature overview on process anomalies, especially concerning anomalies in BPMN models and provided the description of various verification tools used for verifying process models.
The research presented in this paper is a proposal for further studies related to verification issues of BPMN process models. Our future work will focus on practical assessment of process models especially with the existing tools [90]-[93].

\section{REFERENCES}

[1] F. Friedrich, J. Mendling, and F. Puhlmann, "Process model generation from natural language text," in Advanced Information Systems Engineering, ser. Lecture Notes in Computer Science, H. Mouratidis and C. Rolland, Eds. Springer Berlin Heidelberg, 2011, vol. 6741, pp. $482-496$.

[2] K. Kluza and K. Honkisz, "From SBVR to BPMN and DMN models. proposal of translation from rules to process and decision models," in $\mathrm{Ar}$ tificial Intelligence and Soft Computing: 15th International Conference, ICAISC 2016, Zakopane, Poland, June 12-16, 2016, Proceedings, Part II, ser. Lecture Notes in Computer Science, L. Rutkowski, M. Korytkowski, R. Scherer, R. Tadeusiewicz, L. A. Zadeh, and J. M. Zurada, Eds. Springer International Publishing, 2016, vol. 9693, pp. 453-462.

[3] K. Kluza and P. Wiśniewski, "Spreadsheet-based business process modeling," in Computer Science and Information Systems (FedCSIS), 2016 Federated Conference on. IEEE, 2016, pp. 1355-1358.

[4] J. R. Nawrocki, T. Nedza, M. Ochodek, and L. Olek, "Describing business processes with use cases," in BIS, 2006, pp. 13-27.

[5] A. A. Kalenkova, M. de Leoni, and W. M. van der Aalst, "Discovering, analyzing and enhancing BPMN models using ProM?" in Business Process Management-12th International Conference, BPM, 2014, pp. $7-11$.

[6] A. Ligeza, K. Kluza, and T. Potempa, "AI approach to formal analysis of BPMN models. towards a logical model for BPMN diagrams," in Proceedings of the Federated Conference on Computer Science and Information Systems - FedCSIS 2012, Wroclaw, Poland, 9-12 September 2012, M. Ganzha, L. A. Maciaszek, and M. Paprzycki, Eds., 2012, pp. 931-934.

[7] A. Suchenia and A. Ligęza, "Event anomalies in modeling with BPMN," International Journal of Computer Technology \& Applications, vol. 6, no. 5, pp. 789-797, 2015.

[8] A. Mroczek and A. Ligeza, "A note on BPMN analysis. Towards a taxonomy of selected potential anomalies," in Computer Science and Information Systems (FedCSIS), 2014 Federated Conference on. IEEE, 2014, pp. 1097-1102.

[9] M. A. Mach and M. L. Owoc, "Validation as the integral part of a knowledge management process," in Proceeding of Informing Science Conference, 2001.

[10] M. Mach-Król and K. Michalik, "Validation and verification of temporal knowledge as an important aspect of implementing a temporal knowledge base system supporting organizational creativity," in Computer Science and Information Systems (FedCSIS), 2015 Federated Conference on. IEEE, 2015, pp. 1315-1320.

[11] M. Mach-Król and K. Michalik, "Verification of temporal knowledge bases as an important aspect of knowledge management processes in organization," in Advances in Business ICT: New Ideas from Ongoing Research. Springer, 2017, pp. 1-15.

[12] OMG, "Business Process Model and Notation (BPMN): Version 2.0 specification," Object Management Group, Tech. Rep. formal/2011-0103, January 2011.

[13] A. Yousfi, C. Bauer, R. Saidi, and A. K. Dey, "ubpmn: A bpmn extension for modeling ubiquitous business processes," Information and Software Technology, vol. 74, pp. 55-68, 2016.

[14] R. Martinho, D. Domingos, and J. Varajão, "Cf4bpmn: a bpmn extension for controlled flexibility in business processes," Procedia Computer Science, vol. 64, pp. 1232-1239, 2015.

[15] R. M. Pillat, T. C. Oliveira, P. S. Alencar, and D. D. Cowan, "Bpmnt: A bpmn extension for specifying software process tailoring," Information and Software Technology, vol. 57, pp. 95-115, 2015.

[16] K. Kluza, K. Jobczyk, P. Wiśniewski, and A. Ligęza, "Overview of time issues with temporal logics for business process models," in Computer Science and Information Systems (FedCSIS), 2016 Federated Conference on. IEEE, 2016, pp. 1115-1123.

[17] K. Kluza and K. Kaczor, "Overview of BPMN model equivalences: towards normalization of BPMN diagrams," in 8th Workshop on Knowledge Engineering and Software Engineering (KESE2012) at the at the biennial European Conference on Artificial Intelligence 
(ECAI 2012): August 28, 2012, Montpellier, France, J. Canadas, G. J. Nalepa, and J. Baumeister, Eds., 2012, pp. 38-45. [Online]. Available: http://ceur-ws.org/Vol-949/

[18] V. S. W. Lam, "Equivalences of BPMN processes," Service Oriented Computing and Applications, vol. 3, no. 3, pp. 189-204, 2009.

[19] S. Bobek, G. J. Nalepa, and O. Grodzki, "Integration of activity modeller with bayesian network based recommender for business processes," in Proceedings of 10th Workshop on Knowledge Engineering and Software Engineering (KESE10) co-located with 21st European Conference on Artificial Intelligence (ECAI 2014), Prague, Czech Republic, August 19 2014, ser. CEUR Workshop Proceedings, G. J. Nalepa and J. Baumeister, Eds., vol. 1289, 2014. [Online]. Available: http://ceur-ws.org/Vol-1289/kese10-05_submission_10.pdf

[20] S. Bobek, M. Baran, K. Kluza, and G. J. Nalepa, "Application of bayesian networks to recommendations in business process modeling," in Proceedings of the Workshop AI Meets Business Processes 2013 co-located with the 13th Conference of the Italian Association for Artificial Intelligence (AI*IA 2013), Turin, Italy, December 6, 2013, L. Giordano, S. Montani, and D. T. Dupre, Eds., 2013. [Online]. Available: http://ceur-ws.org/Vol-1101/

[21] K. Kluza, M. Baran, S. Bobek, and G. J. Nalepa, "Overview of recommendation techniques in business process modeling," in Proceedings of 9th Workshop on Knowledge Engineering and Software Engineering (KESE9) co-located with the 36th German Conference on Artificial Intelligence (KI2013), Koblenz, Germany, September 17, 2013, G. J. Nalepa and J. Baumeister, Eds., 2013. [Online]. Available: http://ceur-ws.org/Vol-1070/

[22] G. J. Nalepa, K. Kluza, and U. Ciaputa, "Proposal of automation of the collaborative modeling and evaluation of business processes using a semantic wiki," in Proceedings of the 17th IEEE International Conference on Emerging Technologies and Factory Automation ETFA 2012, Kraków, Poland, 28 September 2012, 2012.

[23] K. Kluza, K. Kaczor, G. Nalepa, and M. Slazynski, "Opportunities for business process semantization in open-source process execution environments," in Computer Science and Information Systems (FedCSIS), 2015 Federated Conference on, Sept 2015, pp. 1307-1314.

[24] K. Kluza, G. J. Nalepa, M. Ślażyński, K. Kutt, E. Kucharska, K. Kaczor, and A. Łuszpaj, "Overview of selected business process semantization techniques," in Advances in Business ICT: New Ideas from Ongoing Research. Springer, 2017, pp. 45-64.

[25] W. T. Adrian, S. Bobek, G. J. Nalepa, K. Kaczor, and K. Kluza, "How to reason by HeaRT in a semantic knowledge-based wiki," in Proceedings of the 23rd IEEE International Conference on Tools with Artificial Intelligence, ICTAI 2011, Boca Raton, Florida, USA, November 2011, pp. 438-441. [Online]. Available: http: //ieeexplore.ieee.org/xpls/abs_all.jsp?arnumber=6103361\&tag=1

[26] J. Mendling, H. Verbeek, B. F. van Dongen, W. M. van der Aalst, and G. Neumann, "Detection and prediction of errors in epcs of the sap reference model," Data \& Knowledge Engineering, vol. 64, no. 1, pp 312-329, 2008

[27] A. Hallerbach, T. Bauer, and M. Reichert, "Capturing variability in business process models: the provop approach," Journal of Software Maintenance and Evolution: Research and Practice, vol. 22, no. 6-7, pp. 519-546, 2010.

[28] A. Suchenia, T. Potempa, A. Ligęza, K. Jobczyk, and K. Kluza, "Selected approaches towards taxonomy of business process anomalies," in Advances in Business ICT: New Ideas from Ongoing Research. Springer, 2017, pp. 65-85.

[29] I. Group et al., "1044-2009-ieee standard classification for software anomalies," IEEE, New York, 2010. [Online]. Available: https: //standards.ieee.org/findstds/standard/1044-2009.html

[30] R. Laue and A. Awad, "Visualization of business process modeling anti patterns," Electronic Communications of the EASST, vol. 25, 2009.

[31] S. Kühne, H. Kern, V. Gruhn, and R. Laue, "Business process modeling with continuous validation," Journal of Software Maintenance and Evolution: Research and Practice, vol. 22, no. 6-7, pp. 547-566, 2010.

[32] N. Trčka, W. M. Van der Aalst, and N. Sidorova, "Data-flow antipatterns: Discovering data-flow errors in workflows," in Advanced In formation Systems Engineering. Springer, 2009, pp. 425-439.

[33] A. Kheldoun, K. Barkaoui, and M. Ioualalen, "Formal verification of complex business processes based on high-level petri nets," Information Sciences, vol. 385-386, pp. $39-54,2017$.
[34] V. S. W. Lam, "Formal analysis of BPMN models: a NuSMV-based approach," International Journal of Software Engineering and Knowledge Engineering, vol. 20, no. 7, pp. 987-1023, 2010.

[35] N. Lohmann and K. Wolf, "How to implement a theory of correctness in the area of business processes and services," in Business Process Management. Springer, 2010, pp. 61-77.

[36] S. A. White, "Process modeling notations and workflow patterns," Workflow handbook, vol. 2004, pp. 265-294, 2004.

[37] L. Olkhovich, "Semi-automatic business process performance optimization based on redundant control flow detection," in Telecommunications, 2006. AICT-ICIW'06. International Conference on Internet and Web Applications and Services/Advanced International Conference on. IEEE, 2006, pp. 146-146.

[38] S. Roy and A. S. M. Sajeev, "A formal framework for diagnostic analysis for errors of business processes," in Transactions on Petri Nets and Other Models of Concurrency XI, M. Koutny, J. Desel, and J. Kleijn, Eds. Berlin, Heidelberg: Springer Berlin Heidelberg, 2016, pp. 226-261.

[39] H. Groefsema and D. Bucur, "A survey of formal business process verification: From soundness to variability," in Proceedings of International Symposium on Business Modeling and Software Design. SciTePress, 2013, pp. 198âĂŞ-203.

[40] R. Klimek, "A system for deduction-based formal verification of workflow-oriented software models," International Journal of Applied Mathematics and Computer Science, vol. 24, no. 4, pp. 941-956, 2014.

[41] C. Ou-Yang and Y. D. Lin, "BPMN-based business process model feasibility analysis: a petri net approach," International Journal of Production Research, vol. 46, no. 14, pp. 3763-3781, 2008.

[42] R. Liu and A. Kumar, "An analysis and taxonomy of unstructured workflows," in Business Process Management. Springer, 2005, pp. 268-284.

[43] O. Allani and S. A. Ghannouchi, "Verification of BPMN 2.0 process models: An event log-based approach," Procedia Computer Science, vol. 100, pp. 1064 - 1070, 2016.

[44] A. Badica and C. Badica, "Formal verification of business processes represented as role activity diagrams," in Federated Conference on Computer Science and Information Systems - FedCSIS 2011, Szczecin Poland, 18-21 September 2011, Proceedings, M. Ganzha, L. A. Maciaszek, and M. Paprzycki, Eds., 2011, pp. 277-280.

[45] A. Badica and C. Badica, "Fsp and fltl framework for specification and verification of middle-agents," Applied Mathematics and Computer Science, vol. 21, no. 1, pp. 9-25, 2011.

[46] J. Stepaniuk, J. G. Bazan, and A. Skowron, "Modelling complex pattern by information systems," Fundam. Inform., vol. 67, no. 1-3, pp. 203$217,2005$.

[47] D. Xu, K. Xia, D. Zhang, and H. Zhang, "Model checking the inconsistency and circularity in rule-based expert systems," Computer and Information Science, vol. 2, no. 1, p. 12, 2009.

[48] A. K. Zaidi and A. H. Levis, "Validation and verification of decision making rules," Automatica, vol. 33, no. 2, pp. 155-169, 1997.

[49] M. Dohring and S. Heublein, "Anomalies in rule-adapted workflowsa taxonomy and solutions for vbpmn," in Software Maintenance and Reengineering (CSMR), 2012 16th European Conference on. IEEE, 2012, pp. 117-126.

[50] A. Ligęza and G. J. Nalepa, "A study of methodological issues in design and development of rule-based systems: proposal of a new approach," Wiley Interdisciplinary Reviews: Data Mining and Knowledge Discovery, vol. 1, no. 2, pp. 117-137, 2011.

[51] M. A. Mach and P. J. Kalczynski, "Technique for reducing the number of rules in a temporal knowledge base." in BIS, 2006, pp. 442-454.

[52] A. Rachdi, A. En-Nouaary, and M. Dahchour, "Verification of common business rules in bpmn process models," in Networked Systems: 4th International Conference, NETYS 2016, Marrakech, Morocco, May 1820, 2016, Revised Selected Papers, P. A. Abdulla and C. Delporte-Gallet, Eds. Cham: Springer International Publishing, 2016, pp. 334-339.

[53] S. Cheikhrouhou, S. Kallel, N. Guermouche, and M. Jmaiel, "The Temporal Perspective in Business Process Modeling : An Evaluative Survey and Research Challenges," Service Oriented Computing and Applications, vol. 9, no. 1, pp. 75-85, 2015.

[54] R. Klimek and P. Szwed, "Verification of archimate process specifications based on deductive temporal reasoning," in Computer Science and Information Systems (FedCSIS), 2013 Federated Conference on. IEEE, 2013, pp. 1109-1116.

[55] Y. Du, P. Xiong, Y. Fan, and X. Li, "Dynamic checking and solution to temporal violations in concurrent workflow processes," IEEE Transac- 
tions on Systems, Man, and Cybernetics - Part A: Systems and Humans, vol. 41, no. 6, pp. 1166-1181, 2011.

[56] K Watahiki, F Ishikawa, and K Hiraishi, "Formal verification of business processes with temporal and resource constraints," in Systems, Man, and Cybernetics (SMC), 2011 IEEE International Conference on, 2011, pp. 1173-1180.

[57] L. E. Mendoza Morales, C. Monsalve, and M. Villavicencio, "Application of formal methods to verify business processes," in Formal Methods. Foundations and Applications: 19th Brazilian Symposium, SBMF 2016 , Natal, Brazil, November 23-25, 2016, Proceedings, L. Ribeiro and T. Lecomte, Eds. Cham: Springer International Publishing, 2016, pp. 41-58.

[58] L. Giordano, A. Martelli, M. Spiotta, and D. T. Dupre, "Business process verification with constraint temporal answer set programming," Theory and Practice of Logic Programming, vol. 13, no. 4-5, pp. 641-655, 2013.

[59] L. E. Mendoza Morales, "Business process verification: The application of model checking and timed automata," CLEI Electronic Journal, vol. 17 , no. $2,2014$.

[60] W. M. van der Aalst, A. Hirnschall, and H. Verbeek, "An alternative way to analyze workflow graphs," in Advanced Information Systems Engineering. Springer, 2002, pp. 535-552.

[61] L. Hong and Z. J. Bo, "Research on workflow process structure verification," in e-Business Engineering, 2005. ICEBE 2005. IEEE International Conference on. IEEE, 2005, pp. 158-165.

[62] H. Lin, Z. Zhao, H. Li, and Z. Chen, "A novel graph reduction algorithm to identify structural conflicts," in System Sciences, 2002. HICSS Proceedings of the 35th Annual Hawaii International Conference on. IEEE, 2002, pp. 10-pp.

[63] G.-W. Kim, J. H. Lee, and J. H. Son, "Classification and analyses of business process anomalies," in Communication Software and Networks, 2009. ICCSN'09. International Conference on. IEEE, 2009, pp. 433 437.

[64] E. Börger, O. Sörensen, and B. Thalheim, "On defining the behavior of or-joins in business process models." Journal of Universal Computer Science, vol. 15, no. 1, pp. 3-32, 2009.

[65] Signavio, "Following academic signavio," http://www.signavio.com/ bpm-academic-initiative/, 2009, accessed: 2017-05-01.

[66] C. Pan, J. Guo, L. Zhu, J. Shi, H. Zhu, and X. Zhou, "Modeling and verification of can bus with application layer using uppaal," Electronic Notes in Theoretical Computer Science, vol. 309, pp. 31-49, 2014.

[67] Basic Research in Computer Science at Aalborg University in Denmark and the Department of Information Technology at Uppsala University in Sweden, "Uppaal," http://www.uppaal.org/, 1999, accessed: 2017-05-01.

[68] G. Rodriguez-Navas, J. Proenza, and H. Hansson, "An uppaal model for formal verification of master/slave clock synchronization over the controller area network," in Proc. of the 6th IEEE International Workshop on Factory Communication Systems, Torino, Italy, IEEE Computer Society Press, Los Alamitos, 2006.

[69] Bell Labs, "Spin," http://spinroot.com/spin/whatispin.html, 1990, accessed: 2017-05-01.

[70] W. M. Van Der Aalst, "Woflan: a petri-net-based workflow analyzer," Systems Analysis Modelling Simulation, vol. 35, no. 3, pp. 345-358, 1999.

[71] H. M. Verbeek, T. Basten, and W. M. van der Aalst, "Diagnosing workflow processes using woflan," The computer journal, vol. 44, no. 4, pp. 246-279, 2001

[72] Eindhoven University of Technology, "Woflan - the workflow analyser," http://www.win.tue.nl/woflan/, 1998, accessed: 2017-05-01.

[73] A. Cimatti, E. Clarke, F. Giunchiglia, and M. Roveri, "Nusmv: A new symbolic model verifier," in International conference on computer aided verification. Springer, 1999, pp. 495-499.

[74] A. Cimatti, E. Clarke, F. Giunchiglia, and M. Roveri, "Nusmv: a new symbolic model checker," International Journal on Software Tools for Technology Transfer, vol. 2, no. 4, pp. 410-425, 2000

[75] M. Szpyrka, A. Biernacka, and J. Biernacki, "Methods of translation of petri nets to nusmv language." in $C S \& P, 2014$, pp. 245-256.

[76] P. Szwed, "Evaluating efficiency of archimate business processes verification with nusmv," in Information Technology for Management. Springer, 2016, pp. 179-196.

[77] R. Cavada, A. Cimatti, M. Dorigatti, A. Griggio, A. Mariotti, A. Micheli, S. Mover, M. Roveri, and S. Tonetta, "The nuxmv symbolic model checker," in International Conference on Computer Aided Verification. Springer, 2014, pp. 334-342.
[78] A. Biernacka, J. Biernacki, M. Szpyrka, T. E. Simos, Z. Kalogiratou, and T. Monovasilis, "State-based verification of rtcp-nets with nuxmv," in AIP Conference Proceedings, vol. 1702, no. 1. AIP Publishing, 2015, p. 100011.

[79] M. Szpyrka, P. Matyasik, and R. Mrówka, "Alvis - modelling language for concurrent systems," in Intelligent Decision Systems in Large-Scale Distributed Environments, ser. Studies in Computational Intelligence, P. Bouvry, H. Gonzalez-Velez, and J. KoÅĆodziej, Eds. SpringerVerlag, 2011, vol. 362 , ch. 15, pp. 315-341.

[80] M. Szpyrka, P. Matyasik, and M. Wypych, "Generation of labelled transition systems for alvis models using haskell model representation,' in Proceedings of the 22nd International Workshop on Concurrency, Specification and Programming (CS\&P 2013), vol. 1032. Warsaw, Poland: CEUR Workshop Proceedings, 2013, pp. 409-420.

[81] M. Szpyrka, P. Matyasik, R. Mrówka, and L. Kotulski, "Formal description of Alvis language with $\alpha^{0}$ system layer," Fundamenta Informaticae, vol. 129 , no. $1-2$, pp. 161-176, 2014

[82] T. Szmuc and M. Szpyrka, "Formal methods - support or scientific decoration in software development," in Proc. of Mixdes 2015, the 22nd International Conference Mixed Design of Integrated Circuits and Systems, Torun, Poland, June 25-27 2015, pp. 24-31.

[83] M. Szpyrka, P. Matyasik, J. Biernacki, A. Biernacka, M. Wypych, and L. Kotulski, "Hierarchical communication diagrams," Computing and Informatics, vol. 35, no. 1, pp. 55-83, 2016.

[84] M. Wypych, M. Szpyrka, and P. Matyasik, "Extension of Alvis compiler front-end," in International Conference of Computational Methods in Sciences and Engineering (ICCMSE 2015), ser. AIP Conference Proceedings, vol. 1702. Athens, Greece: AIP Publishing, March 20-23 2015, pp. 100 015-1-100015-4.

[85] M. Szpyrka, G. J. Nalepa, A. Ligęza, and K. Kluza, "Proposal of formal verification of selected BPMN models with Alvis modeling language," in Intelligent Distributed Computing V. Proceedings of the 5th International Symposium on Intelligent Distributed Computing - IDC 2011, Delft, the Netherlands - October 2011, ser. Studies in Computational Intelligence, F. M. Brazier, K. Nieuwenhuis, G. Pavlin, M. Warnier, and C. Badica, Eds. Springer-Verlag, 2011, vol. 382, pp. 249-255. [Online]. Available: http://www.springerlink.com/content/m181144037q67271/

[86] K. Kluza, G. J. Nalepa, M. Szpyrka, and A. Ligęza, "Proposal of a hierarchical approach to formal verification of BPMN models using Alvis and XTT2 methods," in 7th Workshop on Knowledge Engineering and Software Engineering (KESE2011) at the Conference of the Spanish Association for Artificial Intelligence (CAEPIA 2011): November 10, 2011, La Laguna (Tenerife), Spain, J. Canadas, G. J. Nalepa, and J. Baumeister, Eds., 2011, pp. 15-23. [Online]. Available: http://ceur-ws.org/Vol-805/

[87] M. Szpyrka, P. Matyasik, and M. Wypych, "Alvis language with time dependence," in Proceedings of the Federated Conference on Computer Science and Information Systems, Krakow, Poland, 2013, pp. $1607-$ 1612

[88] P. Matyasik, "Alvis virtual machine," in Proceedings of the Federated Conference on Computer Science and Information Systems, 2014, pp. 1639-1645.

[89] M. Szpyrka, P. Matyasik, L. Podolski, and M. Wypych, Simulation of Multi-agent Systems with Alvis Toolkit. Zakopane, Poland: Springer International Publishing, 2017, pp. 599-608. [Online]. Available: http://dx.doi.org/10.1007/978-3-319-59060-8 54

[90] M. Mach-Król, "Tools for building a temporal knowledge base system supporting organizational creativity," Procedia Computer Science, vol. 65, pp. 1031-1037, 2015.

[91] E. Kucharska, K. Grobler-Dębska, J. Gracel, and M. Jagodziński, "Idea of impact of erp-aps-mes systems integration on the effectiveness of decision making process in manufacturing companies," in International Conference: Beyond Databases, Architectures and Structures. Springer, 2015, pp. 551-564.

[92] E. Dudek-Dyduch, E. Kucharska, L. Dutkiewicz, and K. Raczka, "Almm solver-a tool for optimization problems," in International Conference on Artificial Intelligence and Soft Computing. Springer, 2014, pp. 328338.

[93] E. Kucharska, K. Grobler-Dębska, and K. Rączka, "Almm-based methods for optimization makespan flow-shop problem with defects," in Information Systems Architecture and Technology: Proceedings of 37th International Conference on Information Systems Architecture and Technology-ISAT 2016-Part I. Springer, 2017, pp. 41-53. 


\section{Design Thinking and Cognitive Science: An Exploratory Approach to Create Intellectual Capital with Decentralised Organisations}

\author{
Salim Chujfi \\ Hasso Plattner Institut \\ Potsdam, Germany \\ salim.chujfi@hpi.de
}

\author{
Christoph Meinel \\ Hasso Plattner Institut \\ Potsdam, Germany \\ christoph.meinel@hpi.de
}

\begin{abstract}
The significance of managing intellectual capital within organisations so as to be competitive in the digital age, combined with the distributed or discontinuous essence of geographically decentralised organisational forms, places enormous challenges on today's business leaders who want to retain control of the knowledge created dispersedly. This analysis explores a cyber-humanistic approach to improve the effectiveness of interaction in distributed settings, based on individual cognitive preferences, addressing the management of intangible tacit knowledge to develop intellectual capital. We aligned elements of the Japanese Style of global organisational knowledge creation and Design Thinking to develop a context to identify cognitive values of a descentralized organisation style. It provides individuals and teams with the grounds to effectively function in order to produce quality work that can be assessed as capital. The improved interaction scheme aims to create intellectual capital, aligning staff personalities that are cognitively sympathetic improving information gathering and creating business value.
\end{abstract}

\section{INTRODUCTION}

$\mathrm{T}$ HE idea that we are capable of producing extraordinary quantities of data is not new. The quantity of digital data is growing exponentially and is astronomically vast, however, having more data does not necessarily mean that we are processing it entirely, nor making proper use of it transforming it in something valuable for individuals or organizations.

The opportunities of having more data enable organisations to do things differently and indeed to profit from this. The transformation of data into knowledge, and its further refinement of transforming knowledge into intelligence, needs to be addressed independently, as suggested in Figure 1, which is an adaptation of the DIKW Pyramid of Ackoff [1]. Firstly, to transform data into information, basic categorisations and sorting are necessary to segment information according to any given parameter. Secondly, to transform information into knowledge, it is possible to apply Big-Data algorithms to convert defined signals, records and other different sources of classified data to improve efficiency, detect patterns, resolve problems and provide new perspectives on the world around us. Nevertheless, the final step, the transformation of knowledge into intelligence, requires, to a certain degree, creativity and human values to take decisions, innovate and thus create intellectual capital.

This research focuses specifically in how to cognitively improve the last step of the chain, transforming knowledge into intelligence and increase the generation of intellectual capital. In order to achieve that, a crucial prerequisite is to understand that the digital literacy of organizations means much more than just a number of technological skills their employees can master. Rather, it is synonymous with a new Cyber Cognition that encompasses a variety of motor, emotional, cognitive and sociological behavioural adaptations: competencies that enable people to function in digital environments. Cyber Cognition is a paradigm that shifts interaction from an exclusively technology-based context to consider in addition individual cognitive preferences and digital literacy.

Intellectual capital in the digital world relies on the ability of individuals or groups to share knowledge online with others, whereas the interaction involves identifying with others and distinguishing ourselves from others. Considering differences and similarities between individuals, their interests and expertise, the use of cognitive and behavioural instruments online is also necessary to allow the monitoring and guiding characteristics of team members to identify preferred and compatible modes and styles of interaction. It allows better communication that produces valuable outputs that organizations can materialize and retain in new ideas or products.

Another important factor to consider is that the creator of the intellectual capital in the digital world needs an online identification (the online self), which, in our context, is viewed by others. It develops, interacts, takes form, and works with other online users. Each individual is unique in the digital world, and his/her online self is simply a projection of the cognitive capabilities that allow him/her to interact. 


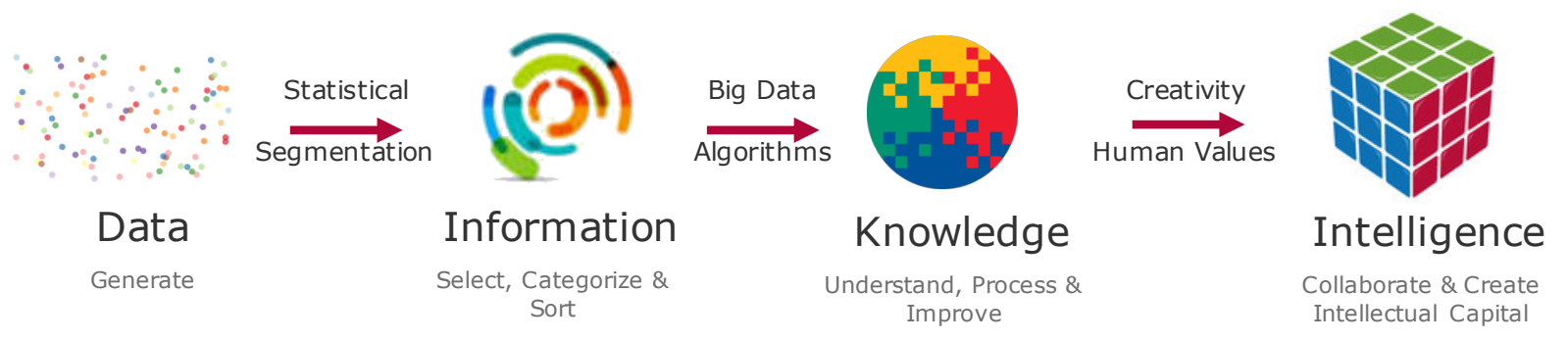

Fig. 1 Extended Data Chain Transformation

To provide a sympathetically grounded interaction to a group of individuals online, this study explores Design Thinking as a context of interaction to be used in the last step of the chain described in Figure 1. Design Thinking is brought into place as a synergy, considering the human as the intellectual (cognitive) generator and multiplicator of intellectual capital. Figure 2 presents the schema proposed for multidisciplinary teams supporting the concept of radical collaboration, enhancing interaction and developing more human contact and trust-based relationships within virtual teams considering cognitive capabilities.

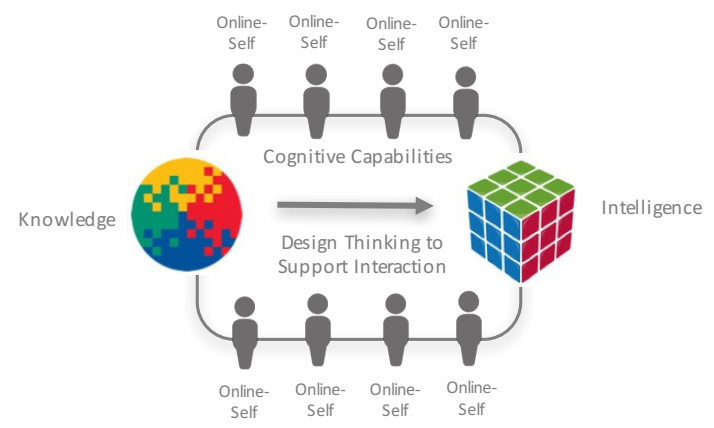

Fig. 2 Cognitive Schema of Virtual Interaction

\section{RELATED WORK}

Several researchers have identified the challenges that decentralised settings engender [2]. The processing of knowledge in the online community requires a methodology to guide the interaction and to support the cyclical cognitivecentred process as a trustable source of sustained competitive advantage. For instance, the influence of trust and the difficulty of building it in decentralised settings has received particular attention [3]. In response, researchers have proposed different approaches. Some of these examine the operational network structure of virtual organisations [4]; and some others investigate the interactions and communication among people within decentralised organisations [5].

One of the most widely recognised problems in knowledge management is that tacit knowledge is difficult to transfer [6][7]. From a communication perspective, this can be explained by the stance that lean (non-face-to-face) communication channels in virtual settings do not provide sufficient support for the necessary transfer of rich information to assist the recipient to acquire tacit knowledge
[8]. This can certainly be considered as an issue related to the use of digital technologies, however it also implies a communication problem that needs to be individually attended [9]. Nevertheless, creating and transferring knowledge are more likely to improve if the communication is supported by a structured method that supports the interaction and data exchange in virtual settings [10].

Researchers have also obtained significant results, indicating that there is evidence for a collective intelligence factor in the performance of human groups [11]. They have established that groups can exhibit a collective intelligence (or c factor) and also develop an analogous "collective" intelligence that could regulate how efficaciously they can address these cognitive assignments. This is a relevant finding to consider, because organizational supervision, and some other kinds of jobs are progressively performed by groups — working both face-to-face and virtually [9]. In order to optimise collective performance, it is therefore required to comprehend what defines a group more intelligent. This factor is not related to cohesion, motivation or satisfaction, but to the average social sensibility [9]. This has given us a new path by which to examine cognitive dimensions more intensively.

Interaction plays an important role as well since it helps developing communities, and the feeling of engagement for the individuals and the group, leading to the development of the social knowledge fabric [9]. The feeling of belonging and experiencing social networks can stimulate people sense of trust and tolerance. The concept of social capitalism is presented as the formal medium to collect any current or potential knowledge which is related to ownership of a robust network of more or less established relationships with mutual awareness and recognition [12].

Despite several social and rational dimensions of knowledge management having been analysed by researchers, considering the learning processes of an organisation, how knowledge is represented and how IT systems manage their transformation, the cognitive dimensions of individuals and their values are still not substantially considered, and very little has been studied in regard to the human-centred discussion considered in geographically decentralised teams. 


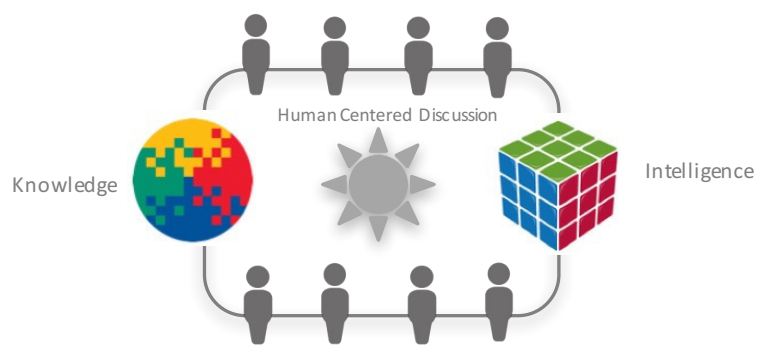

Fig. 3 Knowledge Transformation Schema

\section{ORGANIZATIONAL INTELLECTUAL CAPITAL}

Considering the creation of intellectual capital with particular attention to the relation between human-centered discussions and collective thinking, we started studying knowledge management approaches that are radically different, and which take place on a global scale. We reviewed and summarized in Table 1, the Japanese Style Knowledge management and compared it to the Western approaches, following the premises suggested by Nonaka \& Takeuchi [13]. The target was to identify the different core elements of both styles that could be particularly cognitively relevant for geographically distributed communities where individuals are challenged to have a particular digital environment of interaction. Considering how multidisciplinary geographically decentralised teams are built, we validated particularly three aspects which need to support the cognitive dimensions of interaction between the actors thanks to the human-centred discussion it drives.

\section{A. The Interaction between Tacit and Explicit Knowledge}

In the West, interaction between tacit and explicit knowledge takes place at the individual level. Concepts and ideas tend to be created through externalisation efforts coming from top managers, or through the creation of innovative products, which are then combined organisationally into new admirable personalities or archetypes of new products. In Japan, however, the interaction between tacit and explicit knowledge usually takes place at the group level. Knowledge-creation project teams are led by middle managers, who play a key role in sharing tacit knowledge among the team, considering all the relevant information, such as advanced concepts coming from top managers, or from front-line members who directly interact with the world offering services or products. The intensive human interaction produces a wide range of novel concepts for developing target products, services and business systems. This is an analogous approach to what Design Thinking defines as an "iterative process".

\section{B. Thinking Skills (externalisation and socialisation)}

Analytical thinking skills and concrete forms of oral and visual presentation, such as manuals, documents and databases, are commonly used by Western business organisations. Western leaders' strengths are mostly based on externalisation and combination, which could riskily trap them into the so-called "paralysis by analysis" syndrome. The state of over-analysing or over-thinking a situation, so that decisions or actions are never taken, in effect paralyse the outcome. Japanese, on the other hand, tend to rely heavily on tacit knowledge. They use intuition, figurative and sometimes ambiguous - language and are relatively weak in analytical skills. To compensate for this, they seek interaction and discussion among people (socialisation) as much as they can. The Japanese Style of knowledge creation is internationalisation. As soon as an archetype is created, high-quality tacit knowledge is quickly accumulated at individual and organisational levels by mass-producing or implementing an archetype. The style of Japanese knowledge creation can lead to what is called "collective thinking". This approach is what Design Thinking proposes as "radical collaboration".

\section{Organisational Intentions and Ambiguities}

Western-style intellectual capital creation relies on clear organisational intentions, a low redundancy of information and tasks, low fluctuation from top management and high autonomy at the individual level. In contrast, Japanese-style intellectual capital creation is characterised by relatively ambiguous organisational intention, frequent fluctuation from top management and high autonomy at the group level. The dynamic that Design Thinking offers mimics the Japanese Style, as it does motivate constant re-thinking of new contrasting approaches, and also relies on the autonomy of the cluster groups commonly defined in each of the sessions.

Additional to the previous three aspects on how the knowledge-creation process differ between Japanese and Western, we proceeded to summarise in Table 1 some relevant aspects particularly considering the individual and his interaction in the environment within the organization.

TABLE 1:

DifFERENCE BETWEEN JAPANESE AND WESTERN KNOWLEDGECREATION PRACTICES [13]

\begin{tabular}{l|l}
\hline \multicolumn{1}{c|}{ Japanese Organisation } & \multicolumn{1}{c}{ Western Organisation } \\
\hline Group-based & Individual-based \\
\hline Tacit knowledge-oriented & Explicit knowledge-oriented \\
\hline $\begin{array}{l}\text { Strong socialisation and } \\
\text { internationalisation }\end{array}$ & $\begin{array}{l}\text { Strong externalisation and } \\
\text { combination }\end{array}$ \\
\hline Emphasis on experience & Emphasis on analysis \\
\hline Danger of group thinking & $\begin{array}{l}\text { Danger of "paralysis by } \\
\text { analysis" }\end{array}$ \\
\hline $\begin{array}{l}\text { Ambiguous organisational } \\
\text { intention }\end{array}$ & Clear organisational intention \\
\hline Group autonomy & Individual autonomy \\
\hline
\end{tabular}




\begin{tabular}{l|l}
\hline $\begin{array}{l}\text { Creative chaos through } \\
\text { overlapping tasks }\end{array}$ & $\begin{array}{l}\text { Creative chaos through } \\
\text { individual differences }\end{array}$ \\
\hline $\begin{array}{l}\text { Frequent fluctuation from top } \\
\text { management }\end{array}$ & $\begin{array}{l}\text { Less fluctuation from top } \\
\text { management }\end{array}$ \\
\hline Redundancy of information & Less redundancy of information \\
\hline $\begin{array}{l}\text { Requisite variety through cross- } \\
\text { functional teams }\end{array}$ & $\begin{array}{l}\text { Requisite variety through } \\
\text { individual differences }\end{array}$ \\
\hline
\end{tabular}

We identify in Table 1 how some fundamental concepts from the Japanese Style that can be supported online with the application of Design Thinking. For instance, strong socialization through human-centred discussion, creative chaos through the culture of prototyping and crossfunctional teams through radical collaboration. As represented in Figure 4, we take the effectiveness human centered discussion of the Japanese Style as reference to build up the interaction framework supported in Design Thinking, which we proceed now to model as an intellectual capital driver in virtual teams.

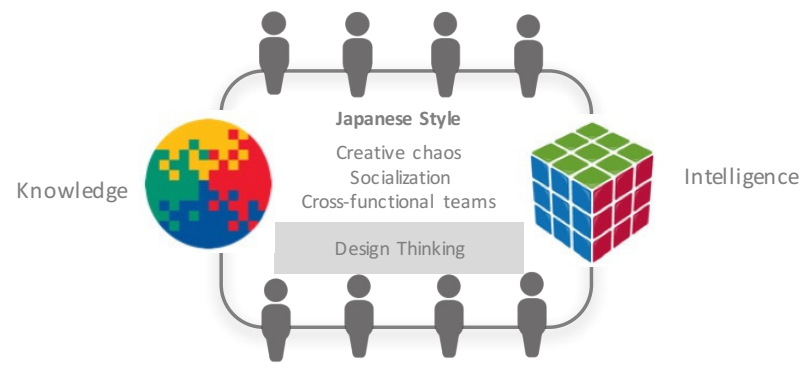

Figure. 4 Knowledge and Interaction with Design Thinking

\section{DESIGN THINKING}

The Japanese Style of intellectual capital creation and its effectiveness could be materialised in geographically decentralised organisations with the aid of Design Thinking, as it promotes "collective thinking" over "individual thinking", stimulates multidisciplinary human interaction, constantly reflects the creation of archetypes, while ideating prototyping and testing. Thus, the creation of intellectual capital is a shared action of human values between several individuals and is not the product of individual minds working independently. This plays a key role when considering that tacit and multidisciplinary knowledge needs to be transformed into intelligence, while unlocking the creative potential of individuals and including the human values that each participant, independent of its speciality, brings with him/her, as shown in Figure 5.

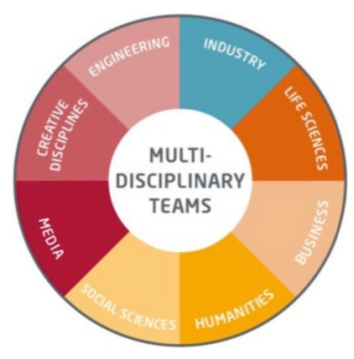

Figure. 5 Interaction of Multidisciplinary Teams [14]
To develop teamwork, it is also necessary for the right combination of leadership and grassroots activism to achieve innovation to scale. Intellectual capital creation in organisations is seldom a solo activity. For the interaction required in each of the Design Thinking stages shown in Figure 5, we propose a systematic approach to cognitively manage the use of information in order to provide a continuous flow of knowledge to the right people at the right time, thus enabling efficient and effective decision-making processes to create business value.

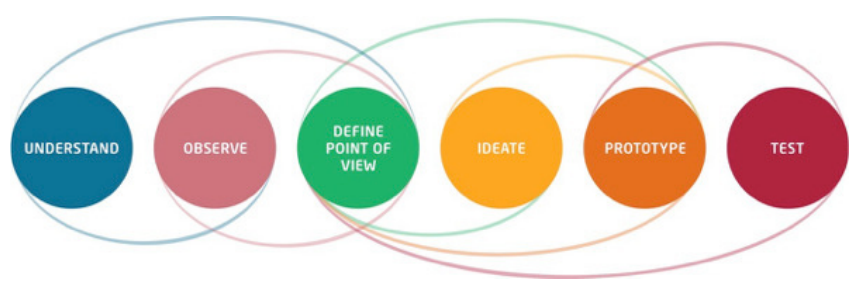

Figure. 5 Design Thinking Stages [14]

To consider Design Thinking as an intellectual capital driver, we base our analysis on the nature of knowledge suggested by Polany's Theory [15], considering that "the knower" requires to actively participate and become a "knower-dependent". Knowledge is a process of individual assembly of data and its elaboration is closely related to the cognitive preferences each individual has [9]. Whenever we express ourselves to communicate what we know, we are able to do it using different channels in different forms. However, messages transmitted can only be interpreted by a knowing mind that can decode and understand them adapting them to its own knowledge structures [16]. Independently of how we process knowledge, it has very little relevance until it is used for some purpose. The main purpose is to realize how individuals' cognitive styles can impact the sharing of knowledge in descentralized and collaborative environments [9].

This means developing properly aligned conditions between human cognition calls for cognitive models dynamic and adaptable computational representations of the cognitive structures and mechanisms.

\section{V.COGNITIVE SCIENCE}

De Bono [17] considered that cognitive styles are required to facilitate a knowledge management system in terms of helping the individual to identify appropriate information and to be able to transfer it at the accurate location.

\section{A. Cognitive Preferences}

Previous researches [9] show that according to Sternberg's Cognitive Theory, people can be understood in terms of the functions, forms, levels, scopes and leanings of governance. However, only three of these: scopes, leanings and levels were identified as relevant to the nature of remote work and virtual teams [18]. 
Levels: Because local cognitive styles prefer concrete detail in their work, whereas global cognitive styles prefer abstract thinking, and remote work elevates ambiguity, people with global cognitive styles will have greater commitment to working with tacit knowledge than people with local cognitive styles. In addition, because the nature of virtual collaboration involves heightened ambiguity from a traditional office setting, these differences are surmised to disproportionately increase the cognitive costs of information exchange for locals during virtual collaboration [19][20]. Thus, people with global cognitive styles will have greater commitment to manage tacit knowledge than people with local cognitive styles.

Scopes: As individuals with internal cognitive styles are more likely to choose to work alone instead of in a group, this would indicate that individuals with those styles would better empathize with environments where less interaction is required, and consequently would this perhaps be related with high levels of commitment to work in virtual environment. [9]. On the other hand, individuals seeking out interaction with their virtual team members have been shown to increase identification with and commitment to coworkers [21]. Thus, people with external cognitive styles will have greater commitment to group interaction than people with internal cognitive styles.

Leanings: As the remote work function has limited capability to support observational modelling, and because it has increased discretion and reduced feedback compared to traditional office settings [22][23][24], working remotely is an activity that is less externally structured. Because similarity between a person's cognitive style and a person's task environment is an important condition for building commitment [25][26], liberals should be more committed to the less structured environment of remote work than conservatives [27]. Thus, people with liberal cognitive styles will have greater commitment to conceptualising and modelling than conservative people.

\section{Table 2: Cognitive Styles [9]}

\begin{tabular}{l|l}
\hline \multicolumn{1}{c}{ LEVELS } \\
\hline Global & $\begin{array}{l}\text { Individual prefer to work with big ideas, however it } \\
\text { may lose perception of the details (can process } \\
\text { abstract information) }\end{array}$ \\
\hline Local & $\begin{array}{l}\text { Individual prefer work that demands keeping track } \\
\text { of details and concentrating on specific (requires } \\
\text { concrete details) }\end{array}$ \\
\hline
\end{tabular}

\begin{tabular}{l|l}
\hline \multicolumn{1}{c}{ SCOPES } \\
\hline Internal & $\begin{array}{l}\text { Individual is usually introvert and does not feel } \\
\text { comfortable in groups (prefers working } \\
\text { independently) }\end{array}$ \\
\hline External & $\begin{array}{l}\text { Individual avoids working and being alone (prefers } \\
\text { working with more people) }\end{array}$ \\
\hline
\end{tabular}

\begin{tabular}{l|l}
\hline \multicolumn{2}{c}{ LEANINGS } \\
\hline Liberal & $\begin{array}{l}\text { Individual may prefer change simply for the sake of } \\
\text { change, even when it is not ideal (goes beyond } \\
\text { boundaries) }\end{array}$ \\
\hline Conservative & $\begin{array}{l}\text { Individual likes to minimise change and avoid } \\
\text { ambiguity (prefers conventions and structures) }\end{array}$ \\
\hline
\end{tabular}

Considering the dimensions presented in Table 2, we classify them against the Design Thinking stages to find the empathy in each stage within the context of team collaboration. Table 3 is developed using ' - ' and '+' indicating lower and higher affinity of the respective styles to each of the stages of the Design Thinking Model. It tabularly represents the cognitive profiles that represent lower and higher empathy and best match the requirements of each stage.

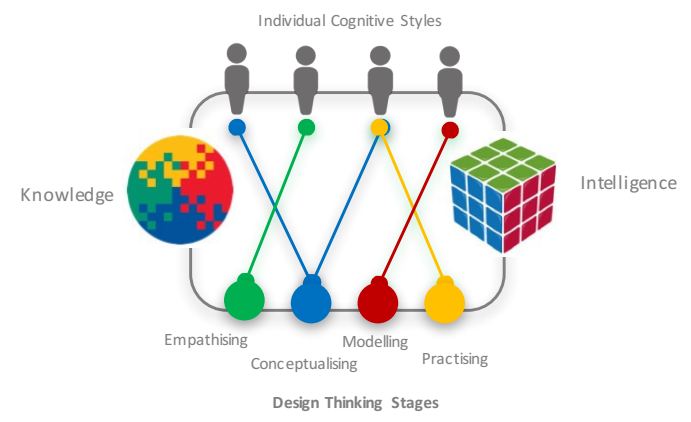

Figure. 6 Model of Cognitive Affinity with Design Thinking

TABLE 3: EMPAThy Foundation BETwEen Design ThinKING AND Cognitive Styles [9]

\begin{tabular}{|c|c|c|c|c|}
\cline { 1 - 4 } $\begin{array}{c}\text { Design } \\
\text { Thinking } \\
\text { Stages }\end{array}$ & Empathising & $\begin{array}{c}\text { Concep- } \\
\text { tualising }\end{array}$ & $\begin{array}{c}\text { Model- } \\
\text { ling }\end{array}$ & Practising \\
\cline { 1 - 4 } $\begin{array}{c}\text { Individual } \\
\text { Styles }\end{array}$ & + & + & - & + \\
\cline { 1 - 4 } Global & - & + & + & + \\
\hline Local & + & - & - & + \\
\hline Internal & + & + & + & - \\
\hline External & - & & & \\
\hline & - & + & + & - \\
\hline Liberal & + & - & - & + \\
\hline Conservative & + & & & \\
\hline
\end{tabular}

\section{Design Thinking and Cognitive Empathy}

In order to create intellectual capital, the collective knowledge of individuals in an organisation needs to produce wealth, multiply output of physical assets, gain competitive advantage, and/or enhance the value of other types of capital. Using the cognitive dimension of Design Thinking in decentralised workforces, the target is to develop creatively confident groups that are able to trigger 
competitive value to transform multidisciplinary knowledge into valuable intellectual capital for organisations.

Design Thinking as a cognitive and intellectual process that balances the rational and emotional, when applied, harmonises with other modes of thinking and closes knowledge and information gaps, creating order and refining meaning. Because Design Thinking is a dynamic, constructive process that is iterative in nature, developing ideas requires ongoing definition, redefinition, representation and assessment. To achieve those tangible outcomes, it is necessary to open the process to multiple participants and to socialise the process and outcomes. Visualisation is a very critical part of the process, not an outcome [28].

Empathy provides a foundation for guiding our behaviours towards others [29]. Emotional empathy, also called affective empathy or primitive empathy, is the subjective state resulting from emotional contagion. Cognitive empathy is principally responsible for precisely recognise and comprehend another's expressive and emotional state. This kind of empathy is called sometimes "perspective taking."'[9]

Table 3 presents an individual cognitive foundation to empathise Design Thinking in a new decentralised dimension, considering:

- A human-centred discussion: to derive a point of view POV- (conceptualising).

- Fomenting a culture of prototyping: trying different models and ideas (modelling).

- Bringing theories to a real context: showing and not just telling (practising).

- Fomenting radical collaboration: listening to other people (empathising).

- Bias towards action: moving forward when knowing where to go (conceptualising).

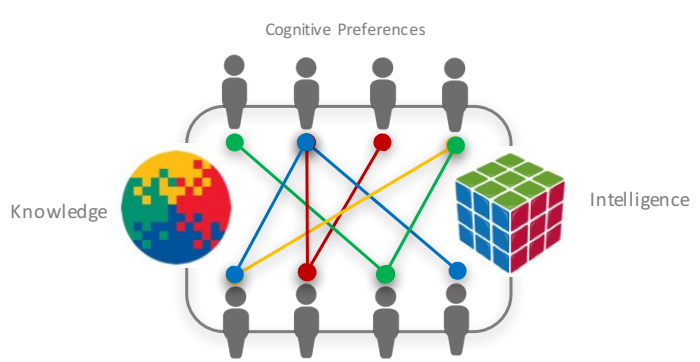

Figure. 7 Model of Individual Cognitive Empathy

\section{CYBER-COGNITIVE CONDITIONS}

A complementary and crucial, prerequisite to implement a collective intellectual capital model with decentralised workforces is the realisation that digital literacy means far more than just a number of technological skills. Rather, it is synonymous with a new cyber-humanism, which encompasses a variety of cognitive, motor, emotional, and sociological behavioural adaptations: competencies that enable people to function in digital environments.

The core is a complex mix of six basic components suggested by Eshet-Alkalai [30] and supported by empirical research.

- Photo-Visual Literacy

- Reproduction Literacy

- Branching Literacy Information

- Information Literacy

- Socio-Emotional Literacy

- Real-Time Thinking

These meta-competences are indispensable in an organisation that is constantly moving on several levels. They go hand in hand with a cyber-humanist paradigm shift: from technology to pedagogy. To this day, these six facets form the basic model for the understanding, creating and processing of digital content [31].

\section{A. Photo-Visual Literacy}

Digital communication now offers us the chance to reverse this "cognitive load", which is a way of going backwards. Because, in the digital age, image-improved communication significantly reduces the time required for interfacing with interfaces. Due to the low cognitive load, image processing functions almost automatically. Communicatively, it goes back to the cave.

\section{B. Reproduction Literacy}

Digitisation also allows a fundamental "persistence" of content. "Reproduction Literacy" describes the ability to create meaningful, authentic and creative works or interpretations that incorporate the existing: the art of creative recycling of existing material. The prerequisite for this is multidimensional synthetic thinking.

\section{Branching Literacy}

Knowledge must increasingly be constructed from large quantities of independent, disordered pieces of information. This requires the competence to be able to think in associative, branched and non-linear ways.

The intellectual source of this ability is the "Cognitive Flexibility Theory" [32]. In essence, it describes how individuals manage to spontaneously restructure their own knowledge and understand complexity meaningfully - a kind of hypermedia skill. Branched thinking is based on a sense of spatial multidimensional orientation. It prevents people from losing sight of reality when they move around in the "virtual" world. It also requires metaphorical thinking: the ability to create mental models and other representations [33].

\section{D.Information Literacy}

"Information Literacy" functions like a filter: it helps to identify erroneous, irrelevant or pre-existing information. It 
is the proverbial step on the perceptual brake. It prevents people from succumbing to the temptation to accept information as given, even if it appears "binding" and valid. It therefore concerns critical thinking and a permanent scepticism about the quality of information.

The transfer of these findings into the working world is as obvious as it is relevant: the diversity of perspectives and diversity of sources require a critical analysis of information, especially in the face of a perceived overload of information. What is missing is not necessarily the mental resources. Rather, it takes a kind of filter training to transfer the critical thinking into the world of information.

\section{E. Socio-Emotional Literacy}

The spread of digital communication platforms has opened up a multitude of new opportunities for collaborative learning and sharing of information. But with these new opportunities come new problems which were simply unknown before the digital era.

Outsourcing, Ideology, Self-awareness and Power are some examples of known problems. Competent users may know how to avoid these traps, and to focus on the benefits of digital communications. They practise a relatively new method of digital literacy: "Socio-Emotional Literacy". To acquire this, users must be critically, analytically mature and equipped with a high degree of Information Literacy and Branching Literacy. Investing in this ability will be crucial for business in the future.

\section{F. Real-Time Thinking}

"Real-Time Digital" sounds promising. And at the same time, it is the semantic cornucopia for the parallel bombardment of sound, text and image in the digital age. But it is our everyday life, and accordingly, the demand for the ability to process these different stimuli in parallel is commonplace.

The transition from digital literacy to cyber-humanism is much more than just dealing with digital technology. Really forward-looking is an all-embracing cyber-humanism which is at the same time a digital optimism, by learning to acquire digital competences.

Digital literacy becomes not only necessary for the effective transformation of knowledge, but also a consequence that supports sustainable creation of intellectual capital.

Figure 8 shows the proposed process of transformation (KI - Knowledge - Intelligence) presenting the sequence of patterns, models and resources necessary to accomplish the effective creation of intelligence as expression of intellectual capital.

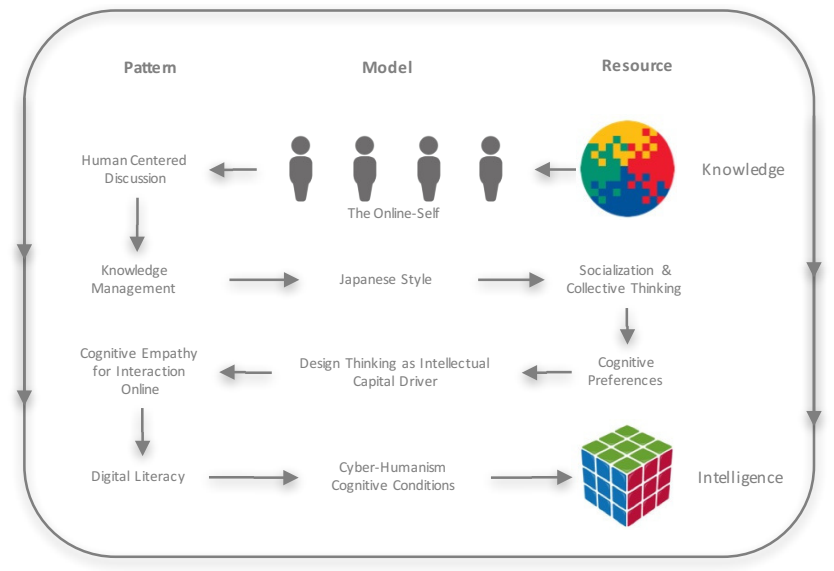

Figure. 8 Transformation Process Knowledge Intelligence

\section{CONCLUSION}

This study suggested how to combine models, methods, conditions and relevant cognitive aspects necessary to transform knowledge into intelligence considering the conditions of descentralized organizations.

Creation of intelligence as expression of intellectual capital in the digital age cannot be achieved merely through knowledge collection. A proper model is necessary to promote the exchange of human values and participation within collective-thinking environments. This is particularly important for organisations working with geographically distributed teams that are "missing" face-to-face interaction, and that are at high risk of having isolated members. They should be guided towards the implementation of working models, supported by methods like Design Thinking, not just for innovation and to create new products or services, but to embed them as a working philosophy, taking into consideration the relevance of the cognitive preferences of each individual. Creating intellectual capital should not be a project, but the continuous creative working environment for all members of the organisation that should become a habit.

The management of knowledge nowadays can be very complex and treat individuals unfairly, resulting in their isolation. However, digital literacy should be aligned not just to collect distributed knowledge but to build a cyberhumanistic cosmos, creating it as a collection, bearing in mind the wide spectrum of individuals' locations and their cognitive preferences.

\section{REFERENCES}

[1] Ackoff, Russell (1989). "From Data to Wisdom". Journal of Applied Systems Analysis. 16: 3-9.

[2] Mark, G. (2001) Meeting current challenges for virtually collocated teams: participation, culture, and integration. In Our Virtual World: the transformation of work, play and life via technology (Eds., Chidambaram, L. and Zigurs, I.) pp.74-93. Idea group publishing, Hershey USA.

[3] Jarvenpaa, S. L. \& Leidner, D. E. (1999) Communication and trust in global virtual teams. Organization Science, 10, 6, 791-815. 
[4] Ahuja, M. K., Galletta, D. F. \& Carley, K. M. (2003) Individua centrality and performance in virtual $R \& D$ groups: An empirical study. Management Science, 49, 1, 21-38.

[5] Paul, D. L. \& McDaniel Jr, R. R. (2004) A field study of the effect of interpersonal trust on virtual collaborative relationship performance. MIS Quarterly, 28, 2, 183.

[6] Alavi, M. \& Leidner, D.E. (2001) Review: knowledge management and knowledge management systems: conceptual foundations and research issues. MIS Quarterly, 25, 107-136.

[7] Allport, F. H. Lepkin , M. (1943), Building war morale with news headlines. Public Opinion Quarterly, 7, 211-221

[8] Daft, R.L. \& Lengel, R.H. (1986) Organizational information requirements, media richness and structural design. Management Science, 32, 554-571.

[9] Chujfi, S. Meinel, C. (2015), Patterns to explore cognitive preferences and potential collective intelligence empathy for processing knowledge in virtual settings. Springer Open. Journal of Interaction Science 2015 3:5.

[10] Ko, D.-G., Kirsch, L.J. \& King, W.R. (2005) Antecedents of knowledge transfer from consultants to clients in enterprise system implementations. MIS Quarterly, 29, 59-85.

[11] A. W Woolley, "Evidence for a Collective Intelligence Factor in the Performance of Human Groups" Science 330, 686 (2010);

[12] Bourdieu, Pierre. 1984. Distinction: A Social Critique of the Judgment of Taste. Cambridge MA: Harvard University Press. pp. 170, 466.

[13] Nonaka, I. Takeuchi H. The Knowledge-Creating Company. Oxford University Press. 1995. 197-199.

[14] Plattner, H, Meinel C., Weinberg U. (2009) 'Design Thinking', FinanzBuch Verlag München.

[15] Smith, M. K. (2003) 'Michael Polany and tacit knowledge', the encyclopedia of informal education, http://infed.org/mobi/michaelpolanyi-and-tacit-knowledge/. Retrieved: 13. Nov 2016

[16] Wilson, T. (2002) "The nonsense of "knowledge management", Information Research, vol. 5, paper no. 144

[17] De Bono, E. (2000). Thinking Course, Great Britain: BBC Book.

[18] Dunn, Kenneth, and Rita Dunn, "Dispelling Outmoded Beliefs aboutStudent Learning" Educational Leadership, March, 1987.

[19] Stout, R. J., Cannon-Bowers, J. A., Salas, E., \& Milanovich, D. M (1999). Planning, shared mental models, and coordinatedperformance: An empirical link is established. Human Factors, 41, 61-71.

[20] Volkma, R. J., \& Gorman, R. H. (1998). The influence of cognitivebased group composition on decision-making process and outcome. Journal of Management Studies, 35, 105-121.
[21] Heald, M. R., Contractor, N. S., Koehly, L. M., \& Wasserman, S. (1998). Formal and emergent predictors of coworkers perceptual congruence on an organizations social structure. Human Communication Research, 24, 536-563.

[22] Baruch, Y., \& Nicholson, N. (1997). Home, sweet work: Requirements for effective home working. Journal of General Management, 23, 15-31.

[23] Hill, E. J., Miller, B. C., Weiner, S. P., \& Colihan, J. (1998). Influences of the virtual office on aspects of work and work/life balance. Personnel Psychology, 51, 667-683.

[24] Wieland, R. (1999). Mental workload in VDU-assisted office work: Consequences for the design of telework. Zeitschrift Fur Arbeits-Und Organisationaspsychologie, 43, 153-158.

[25] Ferrari, M., \& Sternberg, R. J. (1998). The development of mental abilities and styles. In W. Damon, D. Kuhn, \& R. S. Siegler (Eds.), Handbook of child psychology: Vol. 2 (5th ed., pp. 899-946). NY: Wiley.

[26] Jehng, J. J. (1997). The psycho-social processes and cognitive effects of peer-based collaborative interactions with computers. Journal of Educational Computing Research, 17, 19-46.

[27] Workman, M., Kahnweiler, W., \& Bommer, W. H. (2003). The effects of cognitive style and technology media on commitment to telework and virtual teams, Journal of Vocational Behavior, 63, 199-21.

[28] Mootee, Idris. (2012) 'Design Thinking Is A Cognitive And Intellectual Process That Balances The Rational And Emotional', $\mathrm{http}$ ://idr.is/design-thinking-is-a-cognitive-and-intellectual-processthat-balances-the-rational-and-emotional-not/. Retrieved: 13. Nov 2016

[29] Rogers, Kimberley, Isabel Dziobek, Jason Hassenstab, Oliver T. Wolf, and Antonio Convit. "Who cares? Revisiting empathy in Asperger syndrome." Journal of Autism and Developmental Disorders 37, no. 4 (2007): 709-715.

[30] Eshet-Alkalai, Yoram (2004): Digital Literacy: A Conceptual Framework for Survival Skills in the Digital Era. Journal of Educational Multimedia and Hypermedia (2004) 13 (1), 93-106

[31] Naughton, C. (2016): Digitale Erleuchtung: "Cyber-Humanismus: Digitale Ermächtigung". Zukunftsinstitut GmbH, 58-65.

[32] Spiro, R. J., Feltovich, P. J., Jacobson, M. J., \& Coulson, R. L. (1992). Cognitive flexibility, constructivism and hypertext: Random access instruction for advanced knowledge acquisition in ill-structured domains. In T. Duffy \& D. Jonassen (Eds.), Constructivism and the Technology of Instruction. Hillsdale, NJ: Erlbaum.

[33] Eshet-Alkalai, Yoram \& Amichai-Hamburger Yair (2004). Experiments in Digital Literacy. CyberPsychology \& Behaviour, 7(4), 425-434. 


\section{Software Systems Development \& Applications}

SD\&A is a FedCSIS conference area aiming at integrating $\checkmark$ and creating synergy between FedCSIS events that thematically subscribe to the discipline of software engineering. The SSD\&A area emphasizes the issues relevant to developing and maintaining software systems that behave reliably, efficiently and effectively. This area investigates both established traditional approaches and modern emerging approaches to large software production and evolution. Events that constitute SSD\&A are:
- IoTM'17 - $1^{\text {st }}$ Workshop on Internet of Things, Process Modelling and Microservices

- IWCPS' 17 - $4^{\text {th }}$ International Workshop on CyberPhysical Systems

- LASD'17 - $1^{\text {st }}$ International Conference on Lean and Agile Software Development

- MIDI'17- $4^{\text {th }}$ Conference on Multimedia, Interaction, Design and Innovation

- SEW-37 - The $37^{\text {th }}$ IEEE Software Engineering Workshop 



\section{$4^{\text {th }}$ International Workshop on Cyber-Physical Systems}

$\mathbf{P}$ ROLIFERATION of computers in everyday life requires cautious investigation of approaches related to the specification, design, implementation, testing, and use of modern computer systems interfacing with real world and controlling their surroundings. Cyber-Physical Systems (CPS) are physical and engineering systems closely integrated with their typically networked environment. Modern airplanes, automobiles, or medical devices are practically networks of computers. Sensors, robots, and intelligent devices are abundant. Human life depends on them. Cyber-physical systems transform how people interact with the physical world just like the Internet transformed how people interact with one another.

The event is a continuation and extension of 2006-2010 Real-Time Software FedCSIS workshops and 2013, 2015, 2016 IWCPSs. The objective of the workshop is to serve the community with main interest in CPS.

The workshop will accept papers in the following areas:

- Control Systems

- real-time/embedded/networked

- wireless sensing/actuation

- process control \& cloud computing

- Internet of Things

- system organization/implementation

- device security

- impact on business

- Scalability/Complexity

- modularity

- design methodologies

- legacy systems

- tools

- Interoperability

- concurrency

- models of computation

- networking

- heterogeneity

- Validation and Verification

- safety assurance \& certification

- simulation

- Cyber-security

- intrusion detection

- resilience

- privacy

- attack vectors

- Applications of CPS
- intelligent measurements in medicine, environment, etc.

- robotics, manufacturing

- intelligent/autonomous cars

- transportation, ITS

- power systems including smart grids

- smart cities

- military

- smart consumer devices

- CPS Education

- curriculum development

- on-line and virtual laboratories

- academic courses

- pedagogy issues

\section{SECTION EDITORS}

- Grega, Wojciech, AGH University of Science and Technology, Poland

- Kornecki, Andrew J., Embry Riddle Aeronautical University, United States

- Szmuc, Tomasz, AGH University of Science and Technology, Poland

- Zalewski, Janusz, Florida Gulf Coast University, United States

\section{REVIEWERS}

- Babiceanu, Radu, Embry Riddle Aeronautical University, United States

- Bianchini, Devis, Università degli Studi di Brescia

- Čaplinskas, Albertas, Vilnius University, Lithuania

- Černohorský, Jindřich, VSB Technical University of Ostrava, Czech Republic

- Cicirelli, Franco, Universita della Calabria, Italy

- Cosulschi, Mirel, University of Craiova, Romania

- Ehrenberger, Wolfgang, University of Applied Science Fulda, Germany

- Friesel, Anna, Technical University of Dennmark, Denmark

- Furht, Borko, Florida Atlantic University, United States

- Giurca, Adrian, Brandenburg University of Technology, Germany

- Golatowski, Frank, University of Rostock, Germany

- Gomes, Luis, Universidade Nova de Lisboa, Portugal

- Greitans, Modris, Institute of Electronics and Computer Science, Latvia

- Grosu, Radu, Technische Universität Wien, Austria 
- Gumzej, Roman, Faculty of Logistics, University of Maribor, Slovenia

- Haverkort, Boudewijn R., University of Twente, The Netherlands

- Laplante, Phillip A., PennState University, United States

- Letia, Tiberiu, Technical University of Cluj-Napoca, Romania

- Majstorovic, Vidosav D., University of Belgrade, Serbia

- Marwedel, Peter, Technische Universität Dortmund, Germany

- Monostori, László, Hungarian Academy of Sciences, Hungary

- Motus, Leo, Tallinn University of Technology, Estonia

- Nalepa, Grzegorz J., AGH University of Science and Technology, Poland

- Obermaisser, Roman, Universität Siegen, Germany

- Roman, Dumitru, SINTEF / University of Oslo, Norway

- Rozenblit, Jerzy W., University of Arizona, United States

- Rysavy, Ondrej, Brno University of Technology, Czech Republic

- Sachenko, Anatoly, Ternopil National Economic University, Ukraine
- Saglietti, Francesca, University of Erlangen-Nuremberg, Germany

- Sanden, Bo, Colorado Technical University, United States

- Sanz, Ricardo, Universidad Politecnica de Madrid, Spain

- Schagaev, Igor, London Metropolitan University, United Kingdom

- Selic, Bran, Simula Research Lab, Norway

- Sojka, Michal, Czech Technical University, Czech Republic

- Sveda, Miroslav, Brno University of Technology, Czech Republic

- Trybus, Leszek, Rzeszow University of Technology, Poland

- van Katwijk, Jan, Delft University of Technology, The Netherlands

- van Lier, Ben, Rotterdam University of Applied Sciences, The Netherlands

- Vardanega, Tullio, University of Padova, Italy

- Veža, Ivica, University of Split, Croatia

- Villa, Tiziano, Università di Verona, Italy

- Waeselynck, Hélène, LAAS-CNRS Toulouse, France

- Zlatogor, Minchev, Bulgarian Academy of Sciences

- Zoebel, Dieter, University Koblenz-Landau, Germany 


\title{
Virtual Laboratory Based on Node.js Technology and Visualized in Mixed Reality Using Microsoft HoloLens
}

\author{
Erich Stark, Pavol Bisták, Erik Kučera, Oto Haffner and Štefan Kozák \\ Faculty of Electrical Engineering and Information Technology \\ Slovak University of Technology in Bratislava \\ Bratislava, Slovakia \\ Email: erich.stark@stuba.sk, erik.kucera@stuba.sk
}

\begin{abstract}
The paper demonstrates remote control of test experiment in the virtual laboratory. This is a common problem, but another way can always be used to solve it. The paper compares several existing virtual laboratories and their possible issues at present. To develop such a new solution JavaScript technology was used on both client and server side using Node.js runtime. The modern approach is a visualization of received data in mixed reality using Microsoft HoloLens or another compatible device with Windows Mixed Reality platform.
\end{abstract}

\section{INTRODUCTION}

$\mathbf{P}$ RACTICAL exercises in the laboratory are an important part of the process of training people with technical background in general. Ancient Chinese philosopher Confucius once said: "Tell me, and I will forget. Show me, and I may remember. Involve me, and I will understand" [1]. We know from experience that man can learn in the fastest way when he tries things several times, and after that, he understands how it works. Unfortunately, you cannot always provide direct access to real devices to perform the experiment for researchers or students. There may be several issues: the higher price of laboratory equipment, workplace safety (depending on the experiment), or lack of qualified assistants.

In recent years, the development of virtual machines has increased mainly due to the technological evolution of software engineering. The progress of modern technology gives us the better approach to solve new challenges while creating whether the virtual systems for online teaching or specific virtual laboratories where physical processes can be simulated. In experiments conducted in a virtual environment, it is possible to share resources of this environment for more connected users who want to perform the same experiment, which would not be possible on our computers. This makes virtual laboratory a good complement to study whether research, where you can try different variations of the experiment without risk to health or destruction of the device. Later, experiments can be tested on real devices, if necessary.

\section{VIRTUAL LABORATORIES}

At the time when the Internet was not yet widespread in use, the experiments were done in real laboratories. It was

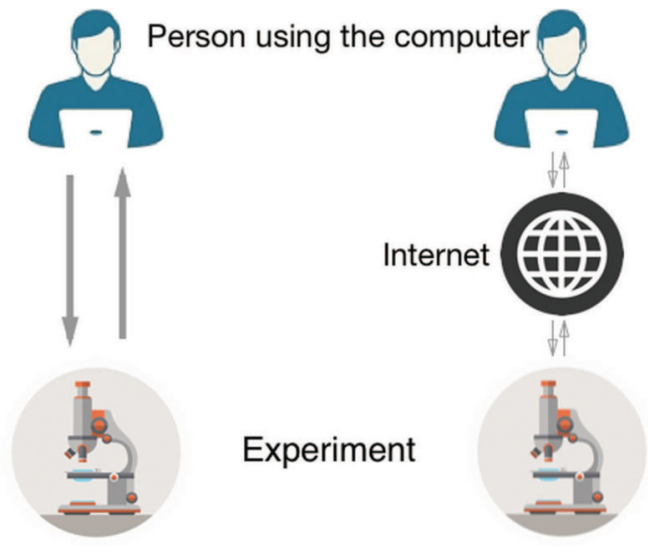

Fig. 1. The difference between a face-to-face and remotely controlled experiments

important to keep on with different safety regulations to the possibility of personal injury or damage to equipment.

Distance and lack of financial resources make real experiments difficult to perform, especially in cases where it is necessary to have some advanced and sophisticated tools. Another encountered problem is the lack of good teachers. Although at present there are already online courses that provide instructional videos, but it solves the problem only partially. Thanks to internet experiments can be structured for visualization and control remotely. Nowadays, a lot of equipment already provides an interface to connect computer and process data from it. Experimenting over the internet allows the use of resources, knowledge, software and data when physical experiments cannot [2].

In this paper, we discuss the creation of virtual laboratory (VL). Before we describe the list of technologies to create VL, we must explain what we consider under VL. Generally, we can say that VL is a computer program, where students interact with the experiment by the computer via the Internet as it is depicted in the Fig. 1.

A typical example is the simulation experiment, where the student interacts with the web/app interface. Another 
TABLE I

COMPARISON OF REAL, VIRTUAL AND REMOTE LABORATORIES [3]

\begin{tabular}{|l|l|l|}
\hline Laboratory Type & Advantages & Disadvantages \\
\hline Real & $\begin{array}{l}\text { real data, interaction } \\
\text { with real experiment, } \\
\text { collaborative work, in- } \\
\text { teraction with supervi- } \\
\text { sor }\end{array}$ & $\begin{array}{l}\text { time and place restric- } \\
\text { tions, requires schedul- } \\
\text { ing, expensive, supervi- } \\
\text { sion required }\end{array}$ \\
\hline Virtual & $\begin{array}{l}\text { good for concept ex- } \\
\text { planation, no time and } \\
\text { place restrictions, in- } \\
\text { teractive medium, low } \\
\text { cost }\end{array}$ & $\begin{array}{l}\text { idealized data, lack of } \\
\text { collaboration, no inter- } \\
\text { action with real equip- } \\
\text { ment }\end{array}$ \\
\hline Remote & $\begin{array}{l}\text { interaction with real } \\
\text { equipment, calibration, } \\
\text { realistic data, no time } \\
\text { and place restrictions, } \\
\text { medium cost }\end{array}$ & $\begin{array}{l}\text { only "virtual presence" } \\
\text { in the lab }\end{array}$ \\
\hline
\end{tabular}

possibility is a remote-controlled experiment where the student interacts with the real device via the computer interface, although he can be far away. This is the case when a virtual laboratory turns into a remote laboratory.

When the web excludes the second option, so we have the following definition: "We call it a virtual laboratory where the student interacts with the experiment, which is physically at distant from him or her and not to demand any physical reality".

After explaining what is VL, look at the benefits they can bring. They are described in the Table I.

People often think that the main benefit of a virtual laboratory is to replace the real one. But it is not. You cannot replace the experience of the real work with the VL. Although VL is better than no experience. VL should not be seen as providing the maximum possible interaction experience.

\section{A. Existing solutions}

There are currently many different virtual and remote laboratories, which are used by foreign universities for teaching or research. This paper briefly reviews often used laboratories that are accessible over the Internet. A comparison of functionality and the use of technology can be seen in the Table II, where different virtual laboratories created in the world are summarized.

There are also some from our Faculty of Electrical Engineering and Information technology, Slovak University of Technology in Bratislava in the Table III.

\section{B. Disadvantaged of existing solutions}

At the beginning of the design of a virtual laboratory, it was appropriate to examine the possibilities of existing solutions. Avoiding various design issues is important. Alternatively, technologies that have been used are already outdated. Nowadays, the development of new technologies is incredibly fast. Such an analysis of existing solutions we have done in the previous section. Our aim was to create a crossplatform solution using one programming language on client and server side, which cannot be done with WCF or COM technology as in the previous solutions. JMI is only suitable for
TABLE II

COMPARISON OF VIRTUAL LABORATORIES CREATED OUTSIDE OF FEI STU [4]

\begin{tabular}{|l|l|l|l|}
\hline Name of VL & $\begin{array}{l}\text { Client technol- } \\
\text { ogy }\end{array}$ & $\begin{array}{l}\text { Server technol- } \\
\text { ogy }\end{array}$ & $\begin{array}{l}\text { Simulation } \\
\text { software }\end{array}$ \\
\hline $\begin{array}{l}\text { Weblab- } \\
\text { DEUSTO }\end{array}$ & $\begin{array}{l}\text { AJAX, Flash, } \\
\text { Java applets, } \\
\text { LabVIEW, } \\
\text { Remote panel }\end{array}$ & $\begin{array}{l}\text { Web services, } \\
\text { Python, } \\
\text { LabVIEW, } \\
\text { Java, NET, C, } \\
\text { C++ }\end{array}$ & $\begin{array}{l}\text { Xilinx- } \\
\text { VHDL, } \\
\text { LabVIEW }\end{array}$ \\
\hline NCSLab & AJAX, Flash & PHP & $\begin{array}{l}\text { Matlab, } \\
\text { Simulink }\end{array}$ \\
\hline ACT & $\begin{array}{l}\text { HTML, Java ap- } \\
\text { plets }\end{array}$ & PHP & $\begin{array}{l}\text { Matlab, } \\
\text { Simulink }\end{array}$ \\
\hline $\begin{array}{l}\text { LabShare } \\
\text { Sahara }\end{array}$ & $\begin{array}{l}\text { AJAX, Java ap- } \\
\text { plets }\end{array}$ & $\begin{array}{l}\text { Web services, } \\
\text { Java }\end{array}$ & Java \\
\hline iLab & $\begin{array}{l}\text { HTML, Active } \\
\text { X, Java applets }\end{array}$ & $\begin{array}{l}\text { Webservices, } \\
\text { NET }\end{array}$ & LabVIEW \\
\hline RECOLAB & HTML PHP & $\begin{array}{l}\text { Matlab, } \\
\text { Simulink }\end{array}$ \\
\hline SLD & AJAX, HTML & $\begin{array}{l}\text { Web services, } \\
\text { PHP }\end{array}$ & $\begin{array}{l}\text { Matlab, } \\
\text { Simulink }\end{array}$ \\
\hline
\end{tabular}

TABLE III

COMPARISON OF VIRTUAL LABORATORIES CREATED AT FEI STU [5]

\begin{tabular}{|c|c|c|c|c|c|}
\hline Year & Author & $\begin{array}{l}\text { Simulation } \\
\text { software }\end{array}$ & Data flow & $\begin{array}{l}\text { Client } \\
\text { technol- } \\
\text { ogy }\end{array}$ & $\begin{array}{l}\text { Server } \\
\text { technol- } \\
\text { ogy }\end{array}$ \\
\hline 2011 & $\begin{array}{l}\text { R. } \\
\text { Farkas }\end{array}$ & $\begin{array}{l}\text { Matlab, } \\
\text { Simulink, } \\
\text { Real } \\
\text { device }\end{array}$ & $\begin{array}{l}\text { JMI Sock- } \\
\text { ets }\end{array}$ & Java & Java \\
\hline 2012 & $\begin{array}{l}\mathrm{T} . \\
\text { Borka }\end{array}$ & $\begin{array}{l}\text { Matlab, } \\
\text { Simulink, } \\
\text { Real } \\
\text { device }\end{array}$ & WCF & $\begin{array}{l}\text {.NET, } \\
\text { WPF }\end{array}$ & .NET \\
\hline 2014 & $\begin{array}{l}\text { M. } \\
\text { Kun- } \\
\text { drat }\end{array}$ & $\begin{array}{l}\text { Matlab, } \\
\text { Simulink }\end{array}$ & $\begin{array}{l}\text { JMI, } \\
\text { SOAP }\end{array}$ & HTML, JS & $\begin{array}{l}\text { Tomcat, } \\
\text { Java JSF, } \\
\text { EJB3, } \\
\text { MySQL }\end{array}$ \\
\hline 2014 & $\begin{array}{l}\text { T. Cer- } \\
\text { veny }\end{array}$ & $\begin{array}{l}\text { Matlab, } \\
\text { Simulink }\end{array}$ & $\begin{array}{l}\text { JMI, } \\
\text { HTTP }\end{array}$ & HTML, JS & Jetty, Java \\
\hline 2015 & $\begin{array}{l}\text { S. } \\
\text { Varga }\end{array}$ & $\begin{array}{l}\text { Matlab, } \\
\text { Simulink }\end{array}$ & $\begin{array}{l}\text { COM, } \\
\text { HTTP }\end{array}$ & HTML, JS & $\begin{array}{l}\text {.NET, } \\
\text { PHP }\end{array}$ \\
\hline
\end{tabular}

solutions where Java platform is used. The server cannot also be used with LabVIEW technology or .NET (multi-platform version - .NET core is already under development). Client solutions such as Flash, ActiveX and Java applets are no longer supported in browsers, so their use is not appropriate.

\section{Components of virtual laboratory}

There are plenty of existing laboratories, but usually, it is not possible to guarantee compatibility between them, because there is not a solid standard. Anyway, it is always possible to identify the basic components that virtual laboratories can use. Some of them can be even used more times.

Components:

- The experiment itself

- The device with possibility to control and acquiring data

- Laboratory server, which provides control, monitoring and data processing of the experiment 


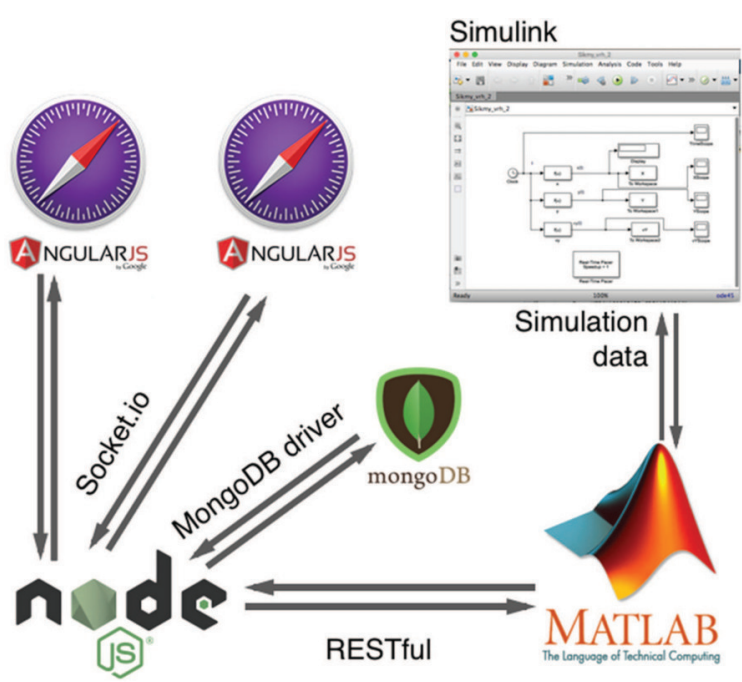

Fig. 2. Design of communication between components

- Server providing connection between remote users and laboratory server, usually via the internet

- Web camera connected to a server, which can be used for remote user as a visual and audible feedback on the actual status of the experiment

- Tools enabling multi-user audio, video and chat communication

- Client software controlling and representing data of the experiment [6]

It is important to realize which of these components could be used, because for a creation of a virtual laboratory it is not necessary to have them all. Alternatively, others that are perfectly suited for a role can also be used. Sometimes it is used e.g. database server if experiments will be stored and processed later. It is also important to realize what type of VL we want to create. Certainly, differences will be in the design of single-user as opposed to multi-user VL, even with multiple experiments simultaneously. It should bear in mind as properly solve the scalability, potential safety issues, multi-user access and other possible issues.

\section{ARChitecture Propos AL}

As the main component, Node.js was selected. It is the server which handles communication between components of VL. The parts of architecture will be explained based on Fig. 2. The data are fetched periodically from Simulink into Matlab workspace. In the beginning, it was not sure whether it would be possible to achieve to run multiplatform soft real-time Simulink based simulations. Because only Windows based solution was found directly from MathWorks. For our solution Real-Time Pacer [7] was used that allows us to run simulations in soft real-time even under MacOS or Linux. It is used to slow down the simulation to the soft real-time.

To communicate with RESTful web service Matlab R2015a uses the built-in rarely used function webread and webwrite [8]. Firstly, the simulation must be run through the web browser, after that data will be transferred over socket.io library channel. These data will be shown in the graph of the web browser, and it is possible to save them to MongoDB database for later processing (Fig. 2).

\section{A. Reference simulation model}

For a development purpose, we used the simulation of the dynamic system called projectile motion implemented in Simulink that runs through the web interface. This simulation needs to be run with two files. The purpose of the first is the initialization of variables needed to calculate the coordinates of the point. This experiment has three parameters. The first and second parameter are initial values for simulation. The last parameter userFromWeb is not necessary for simulation itself, but it is important to identify the user who runs the simulation. This makes it possible to assign the simulation results in later processing from the database.

\section{B. Experiment handler}

The second Matlab file is a handler code sending the data to Node.js. Because of its length of implementation, it is not possible to display the whole source code, so we describe only the key part.

During initialization, the URL path is set for Express.js REST API where Matlab will send the data.

The model is preloaded using the Matlab function load_system('projectile_motion'). This function searches in the current folder for projectile_motion.mdl file and sets it as the top-level model. After this initial settings, simulation must be run using the command set_param(model, 'SimulationCommand', 'Start').

In the next block of the Matlab code, it is running an infinite while loop that makes possible to collect data from the simulation to the state until it is complete. Inside of the while loop the function set_param(model, 'SimulationCommand', 'WriteDataLogs') is called, which is looking for the current top-level simulation. In the soft realtime the calculated data are written to the Matlab workspace. Without that function, data would be written only after the simulation ends.

Meanwhile, it is necessary to prepare required format of data for the web service. Thus, before sending them to the REST API, it is suitable to wrap data to the JSON structure. We used the Matlab library JSONlab v1.2 [9].

A sequence of these two commands is required to create the desired JSON format and send it to Express.js API. Create JSON with the command json = savejson('result', struct('user', userFromWeb, 'status', 'Running', 'data', struct('time', timeFinal, 'you', vyFinal, 'y', yFinal, ' $x$ ' $x$ fine))) and transfer it to the service with response $=$ webwrite (URL, JSON, options).

The command get_param(model, 'SimulationStatus') is used to check current status of the simulation. If the simulation is still running the status is "running". As soon as the status is "stopped", the loop needs to be stopped using the break keyword and we know that all data is transferred to Node.js. 


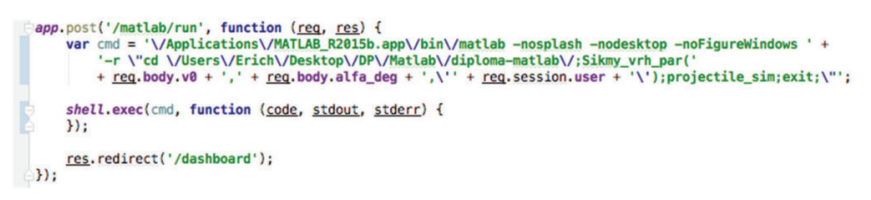

Fig. 3. Start Matlab in command line using shell.js library

\section{Communication between components}

One of the aspects of the individual components of the laboratory is communication. Although in each component communication works differently, it is still based on the HTTP protocol.

The sequence diagram in the Fig. 6 shows that communication starts from the web browser. The user inserts the parameters of simulation, which are sent to StarkLab via the REST web service. This service starts Matlab on the current operating system with the necessary files and simulation parameters. Meanwhile, the user waits until Matlab starts in the background. Simulation is immediately initialized and starts sending data to StarkLab, which sends them directly to the web client from where the simulation has been originated. All the received data will be reflected in the chart, animation, and table in the web browser. This sequence is repeated until the condition contains SimulationStatus $==$ "running". After stopping the simulation, the client sends a request to save data through StarkLab directly into the document database MongoDB.

\section{Run Matlab from command line}

In the beginning, it was not clear how to run the simulation. It was necessary to determine whether Node.js allows to carry out the commands of the operating system, respectively run programs. The simulation was working in such a way that the Matlab was opened manually and we put there all the necessary initialization files, then the simulation itself. But this solution is not sufficient in terms of automation and autonomy.

It has been found that Node.js can launch any software that can run through the terminal. To simplify this workflow the shell.js library [10] was used which provides such an option.

The sample of code in the Fig. 3 shows how Matlab is started via Node.js route http://localhost/matlab/run. This route is called immediately after the form was sent with initial parameters of the experiment from the web browser.

\section{REMOTE CONTROL OF EXPERIMENT}

\section{A. Web client created with Angular framework}

Client application was created with the JavaScript framework Angular [11] (version 1.5.5). The role of the web client was to verify the functionality of the server that sends simulated data. The functionality has been verified, and screens will be described specifically.

Fig. 4 shows login page for web client application. It is authenticated against LDAP server of Slovak University of Technology.

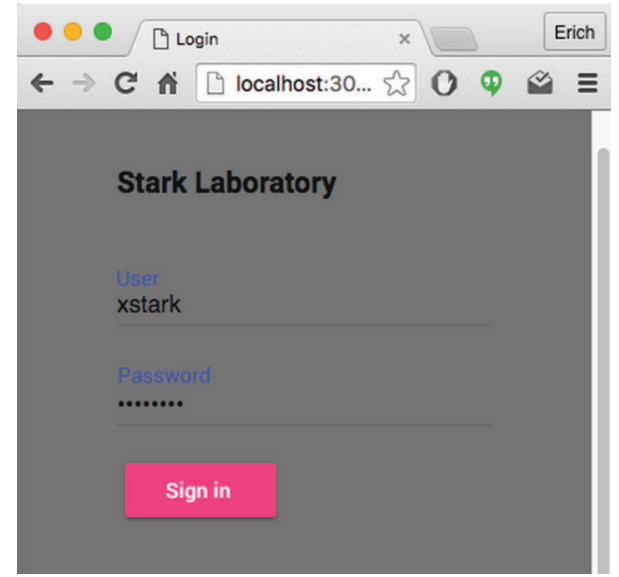

Fig. 4. Login to web application

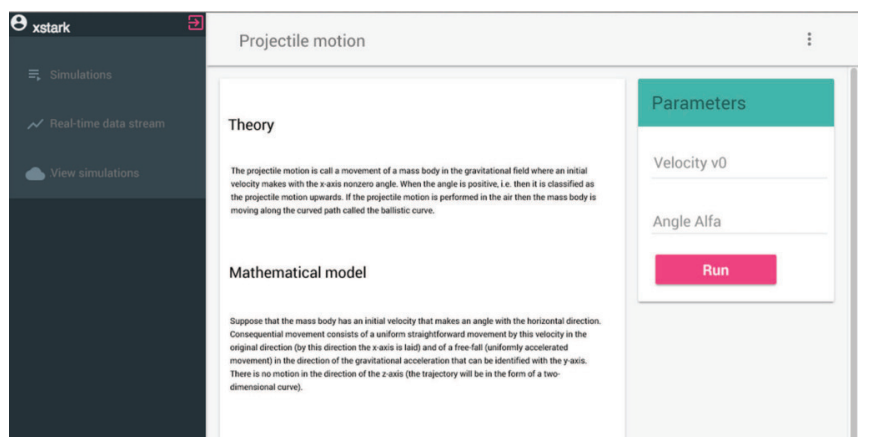

Fig. 5. Parameters of simulation / initial velocity and angle in degrees

The details of the login process via LDAP is not interesting for this part of the paper. After successful login, the dedicated page for the tested experiment is showed. Our experiment was projectile motion. It takes two parameters to run a simulation. On Fig. 5 it can be seen the form that takes two parameters to run a simulation. The page is redirected to http://localhost/matlab route, where the user is waiting to see the data from Node.js REST API.

It redirects to the dashboard page, and the user has to wait until the start of Matlab simulation. When it starts, the user will see new data coming to graph, animation, and table in his web browser. This part could be accelerated by a powerful server running with Matlab.

Visualization of the received data is done by Chart.js library on Fig. 7. Our implementation of chart was created using Angular directive with name $<$ ui-graph $><$ ui-graph $>$. Because of this approach (the usage of Angular components), it can be used multiple times with the same codebase.

In the beginning, it is necessary to get an element from DOM (Document Object Model) tree. Next step is to obtain canvas context and create the object with initial data.

The plotted data at the bottom of the picture is identical to the data in the graph. The difference is in the way of implementation as animation. This animation was created using HTML Canvas technology. 


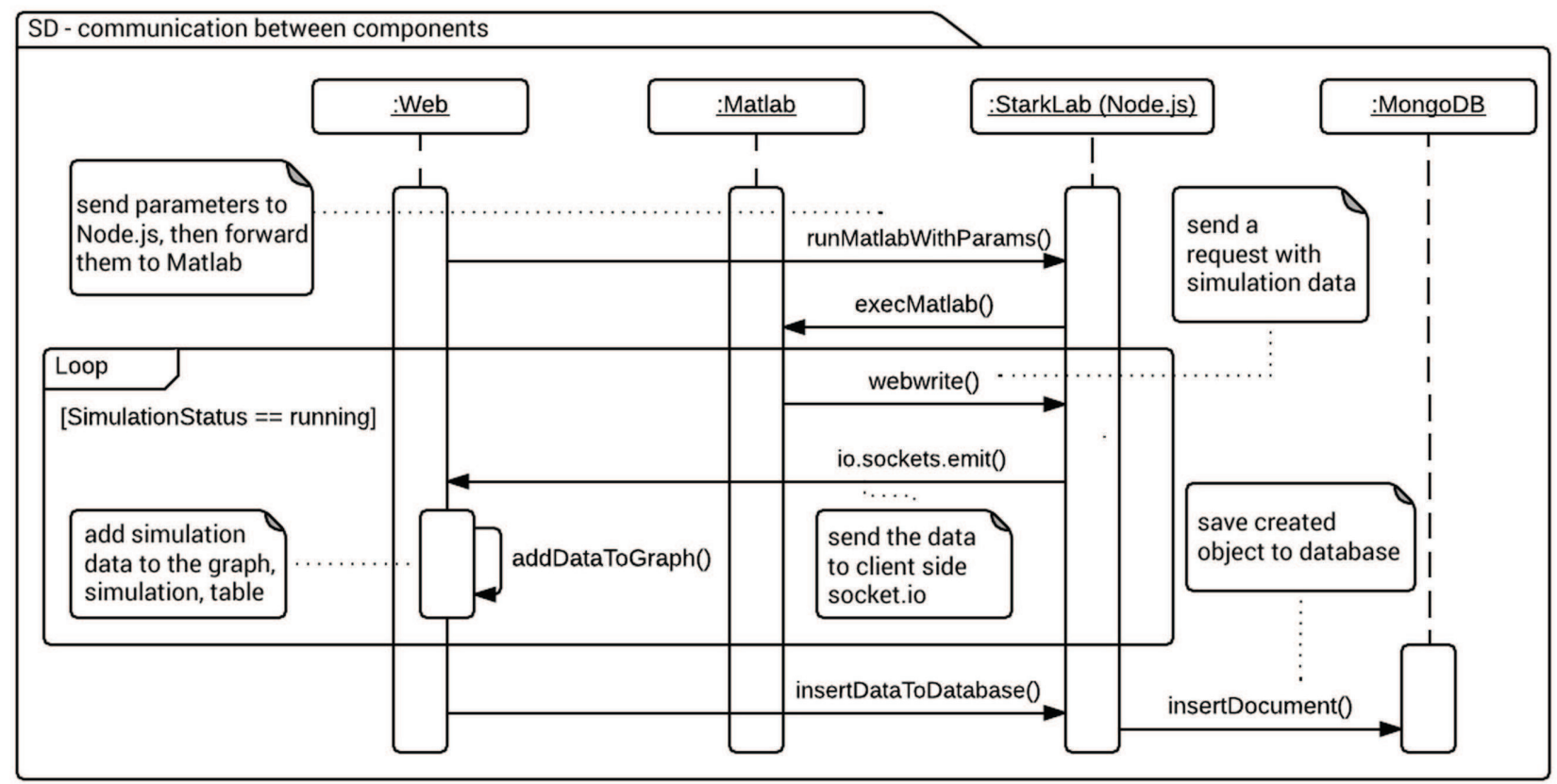

Fig. 6. Communication between components

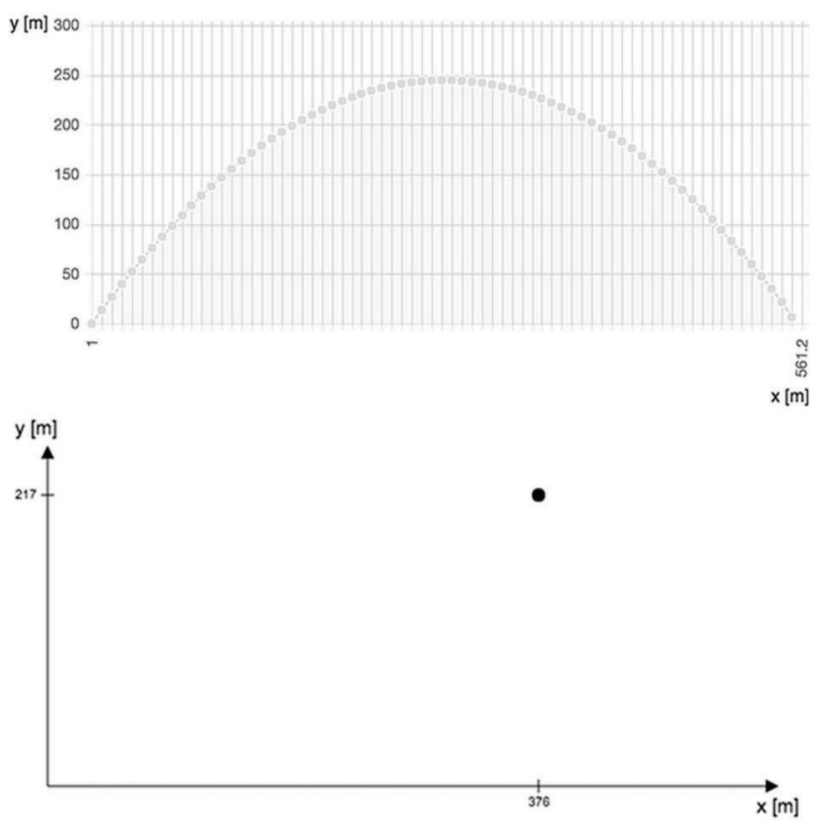

Fig. 7. Graph and Animation of projectile motion in $[\mathrm{x}, \mathrm{y}]$ position

The last section, where the data can be seen is a table where data were added over time as well as chart and animation before. In this table Angular databinding [11] is used to set received object as one row with their properties. As simulation runs, the Angular adds new rows to table dynamically.

This system is not only about the real-time rendering of

\begin{tabular}{|c|c|c|c|}
\hline \multicolumn{3}{|c|}{ Simulation data } & (8) \\
\hline Time [s] & Axis $\mathrm{x}[\mathrm{m}]$ & Axis y [m] & Velocity $[\mathrm{m} / \mathrm{s}]$ \\
\hline 0 & 0 & 0 & 38.57 \\
\hline 0.01 & 0.46 & 0.39 & 38.47 \\
\hline 0.02 & 0.92 & 0.77 & 38.37 \\
\hline 0.03 & 1.38 & 1.15 & 38.27 \\
\hline 0.04 & 1.84 & 1.53 & 38.17 \\
\hline 0.05 & 2.3 & 1.92 & 38.08 \\
\hline 0.06 & 2.76 & 2.3 & 37.98 \\
\hline 0.07 & 3.22 & 2.68 & 37.88 \\
\hline 0.08 & 3.68 & 3.05 & 37.78 \\
\hline 0.09 & 4.14 & 3.43 & 37.68 \\
\hline 0.1 & 4.6 & 3.81 & 37.59 \\
\hline 0.11 & 5.06 & 4.18 & 37.49 \\
\hline 0.12 & 5.52 & 4.56 & 37.39 \\
\hline 0.13 & 5.98 & 4.93 & 37.29 \\
\hline 0.14 & 6.43 & 5.3 & 37.19 \\
\hline 0.15 & 6.89 & 5.67 & 37.1 \\
\hline 0.16 & 7.35 & 6.05 & 37 \\
\hline
\end{tabular}

Fig. 8. Table data / time, $x, y$, vy values of projectile motion experiment 


\section{List of simulations}

\begin{tabular}{|c|c|c|c|c|}
\hline User & Date & Model & View & Delete \\
\hline xstark & 09.04.2016 11:39 & projectile & 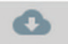 & 士 \\
\hline xstark & 09.04.2016 11:42 & projectile & 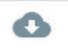 & $\overline{0}$ \\
\hline xstark & 13.04.2016 09:22 & projectile & A & $\bar{\square}$ \\
\hline xstark & $13.04 .201609: 23$ & projectile & $A$ & \\
\hline xstark & $13.04 .201609: 23$ & projectile & $A$ & \\
\hline xstark & $14.04 .201602: 00$ & projectile & 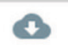 & \\
\hline xstark & $14.04 .201602: 00$ & projectile & \& & \\
\hline xstark & $14.04 .201602: 00$ & projectile & 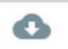 & $\Rightarrow$ \\
\hline xstark & $14.04 .201611: 41$ & projectile & A & \\
\hline xstark & $14.04 .201611: 46$ & projectile & $A$ & 量 \\
\hline xstark & 14.04.2016 11:52 & projectile & $A$ & 厂 \\
\hline xstark & $14.04 .201611: 53$ & projectile & 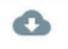 & D \\
\hline xstark & $14.04 .201611: 54$ & projectile & A & - \\
\hline
\end{tabular}

Fig. 9. Table of saved simulation for currently logged user

data, but also for later viewing and processing of them. On the site of simulations, we can see all the entries for the currently logged in user - Fig. 10. The list is obtained from MongoDB using Angular \$http.get (url, callback) function from web client to our Node.js server, which can have access to database.

When the one of the results is opened, the output looks the same as in Fig. 7, but it is possible to set data sampling and time of simulation. The second option is about time rendering. There are two options: to see data output immediately or soft real-time as it was firstly run.

\section{Summary OF EXPERIENCE WITH THE CREATION OF MATLAB-NODE.JS VIRTUAL LABORATORY}

After the experience with this kind of development, we assess that the creation of virtual laboratory platform on Node.js development was easier thanks to the use of JavaScript on the server and client side. We thought that due to the single thread loop of Node.js would handle more clients and simulations than the similar solution on a different platform. The problem was not in many of registered users, but only when we run multiple simulations in Matlab. In our test computer - MacBook Pro there was already a problem with two parallel simulations. It can be improved using a powerful server for Matlab calculations.

The work is not over yet and StarkLab can be extended with another interesting functionality such as the creation of the unified protocol for data interchange. Suitable would also be interfaces for other calculation and simulation software. Matlab deployment on a separate server with an available domain would help to availability. Another interesting functionality would be uploading simulation and calculation scripts through a web interface.
The current solution is not possible to deploy into production without certain modifications and integrations, but it might serve as a solid basis for adding new features. There are many ways to improve this solution.

All code is open source and available at https://github.com/erichstark/.

\section{VISUALISATION OF VIRTUAL LABORATORY IN MIXED REALITY}

Modern forms of education are now realized on the basis of the development of new ICT technologies (e.g. interactive applications made in 3D engine [12], virtual reality or mixed reality). Visualisation of process modelling, identification and control of complex mechatronic systems, elements and drives using virtual and mixed reality allows students to get a much better and quicker understanding of the studied subject compared to conventional teaching methods.

\section{A. Introduction to mixed reality}

Nowadays, there is a trend of using interactive 3D applications and virtual reality in many prestigious universities.

Very interesting project is a virtual clinic [13]. This project is supported by the University of Miami or Charles R. Drew University of Medicine and Science in Los Angeles. This interactive application offers an insight into the actual functioning of a larger clinic, and they can also try to diagnose patients. Students are thus trained through a real experience with the health system, but this complex system is modelled and simulated in virtual reality.

There are also interactive applications from Animech Technologies. This company offers many education modules like Virtual Car, Virtual Truck or Virtual Gearbox [14]. Using these applications students can understand the functioning of mentioned devices and they can look into their interior and detach their individual components in detail.

An absolute novelty is Microsoft HoloLens [15], the arrival of which has led to the emergence of a completely new segment of mixed reality. Mixed reality has unquestionable advantages over virtual reality, as the user perceives a real world and also a virtual world in the same time. The use of this feature is in practice undisputed and it is assumed that mixed reality will become a new standard in many areas such as education, marketing, modeling of complex mechatronic systems, etc.

For Microsoft HoloLens there are more education applications.

Application HoloTour [17] provides 360-degree spatial video of historical places like Rome or Peru. The application complements 3D models of important landmarks that have not been retained or supplementary holographic information about elements in the scene.

Application HoloAnatomy [18] allows interactive education of anatomy of the human body. The advantage is that if the application is used by more users in the same time, everyone sees the same model of a part of the human body. This allows 


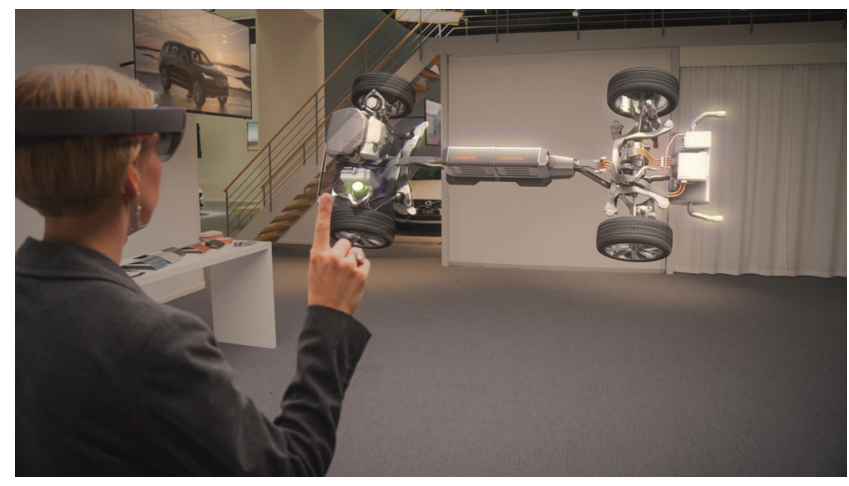

Fig. 10. Microsoft HoloLens - mixed reality application (Volvo) [16]

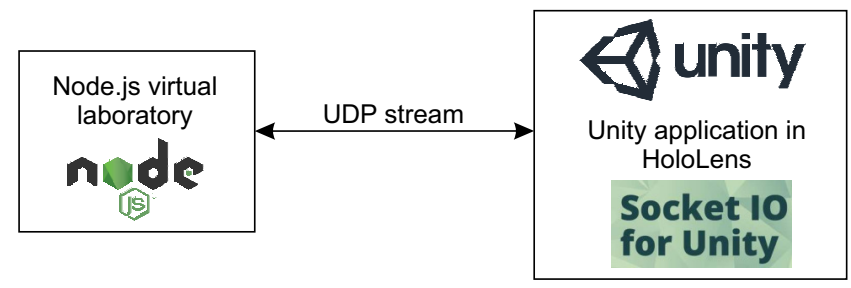

Fig. 11. Scheme - visualisation of virtual laboratory in mixed reality developed in Unity

an interaction between students that results in a significant multiplier education effect.

From technical fields there is an application called HoloEngine [19]. This application allows understanding of the complex 3D mechanical structures of the combustion engine. The application allows you to see the engine in the air, start it and even look inside it and closely monitor the mutual interaction of the mechanical parts.

\section{B. Virtual laboratory in mixed reality}

For development Unity engine was used. Proposed Unity application for Microsoft HoloLens brings a visualisation of results from described Node.js virtual laboratory in mixed reality. By this application, students get better insight into the results of the experiment.

It was needed to connect Unity application with Node.js laboratory. There is a free library for Unity called Socket.IO for Unity [20] which was used in the proposed application.

In Fig. 12, it is possible to see the results from the virtual laboratory in Unity engine. The application was deployed on Microsoft HoloLens. The results in mixed reality you can see in Fig. 13.

\section{CONCLUSION}

After the experience with this kind of development, we assess that the creation of virtual laboratory platform on Node.js development was easier thanks to the use of JavaScript on the server and client side. We thought that due to the single thread loop of Node.js would handle more clients and simulations than the similar solution on a different platform. The problem was not in many of registered users, but only

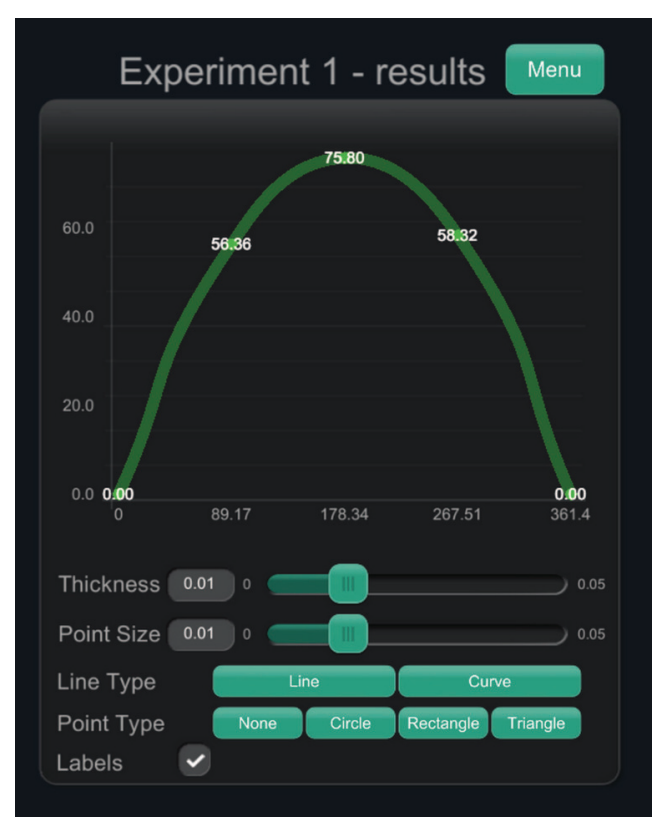

Fig. 12. Results from virtual laboratory in Unity engine

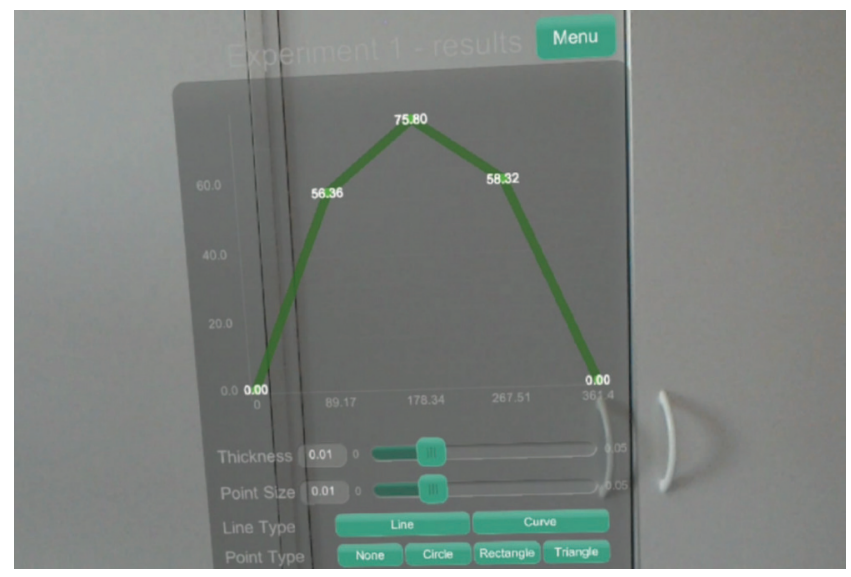

Fig. 13. Results from virtual laboratory in mixed reality (Microsoft HoloLens)

when we run multiple simulations in Matlab. In our test computer - MacBook Pro there was already a problem with two parallel simulations. It can be improved using a powerful server for Matlab calculations.

The work is not over yet and StarkLab can be extended with another interesting functionality such as the creation of a unified protocol for data interchange. Suitable would be also interfaces for other calculation and simulation software. Matlab deployment on a separate server with an available domain would help to availability. Another interesting functionality would be uploading simulation and calculation scripts through a web interface.

The future work will also focus on additional development for Windows Mixed Reality platform and Microsoft HoloLens headset. 
The source code of the virtual laboratory is available as open source at https://github.com/erichstark/.

\section{ACKNOWLEDGMENT}

This work has been supported by the Cultural and Educational Grant Agency of the Ministry of Education, Science, Research and Sport of the Slovak Republic, KEGA 030STU4/2015 and KEGA 030STU-4/2017, by the Scientific Grant Agency of the Ministry of Education, Science, Research and Sport of the Slovak Republic under the grant VEGA 1/0937/14 and VEGA 1/0819/17 and by the Tatra banka Foundation within the grant programme Quality of Education, project No. 2016vs046 (Support of education in mechatronics through virtual reality).

\section{REFERENCES}

[1] R. R. Wright, "Using 3 dimensional simulation in nursing education," in 43rd Biennial Convention (07 November-11 November 2015). STTI, 2015.

[2] V. team. (2016) The philosophy of virtual laboratories. [Online]. Available: http://vlab.co.in

[3] Z. Nedic, J. Machotka, and A. Nafalski, Remote laboratories versus virtual and real laboratories. IEEE, 2003, vol. 1.

[4] I. Santana, M. Ferre, E. Izaguirre, R. Aracil, and L. Hernandez, "Remote laboratories for education and research purposes in automatic control systems," IEEE transactions on industrial informatics, vol. 9, no. 1, pp. 547-556, 2013.

[5] E. Stark, "Virtual laboratory using javascript on the server side (in slovak)," Master's thesis, Slovak University of Technology in Bratislava, 2016.
[6] L. Gomes and S. Bogosyan, "Current trends in remote laboratories," IEEE Transactions on industrial electronics, vol. 56, no. 12, pp. 4744 4756,2009

[7] G. Vallabha, "Real-time pacer for simulink," The MathWorks, Inc, vol. $21,2010$.

[8] M. team. (2017) Web access. [Online]. Available: https://www. mathworks.com/help/matlab/internet-file-access.html

[9] Q. Fang. (2016) Jsonlab: a toolbox to encode/decode json files. [Online]. Available: https://www.mathworks.com/matlabcentral/ fileexchange/33381-jsonlab--a-toolbox-to-encode-decode-json-files

[10] Contributors. (2017) shell.js. [Online]. Available: https://github.com/ shelljs/shelljs

[11] M. Hevery and team. (2016) Angular framework. [Online]. Available: http://angularjs.org

[12] Triseum. (2017) Variant: Limits. [Online]. Available: https://triseum. com/calculus/variant/

[13] D. Parvati, W. L. Heinrichs, and Y. Patricia, "Clinispace: a multiperson $3 \mathrm{~d}$ online immersive training environment accessible through a browser," Medicine Meets Virtual Reality 18: NextMed, vol. 163, p. 173, 2011.

[14] A. Technologies. (2014) Virtual gearbox. [Online]. Available: http //www.animechtechnologies.com/showcase/virtual-gearbox/

[15] P. A. Rauschnabel, A. Brem, and Y. Ro, "Augmented reality smart glasses: definition, conceptual insights, and managerial importance," Working paper, The University of Michigan-Dearborn, Tech. Rep., 2015.

[16] E. Uhlemann, "Connected-vehicles applications are emerging [connected vehicles]," IEEE Vehicular Technology Magazine, vol. 11, no. 1, pp. 25 96, 2016.

[17] M. Corporation. (2017) Holotour. [Online]. Available: https://www. microsoft.com/en-us/hololens/apps/holotour

[18] S. Prajapati, E. Madrigal, and M. T. Friedman, "Acquisition, visualization and potential applications of 3d data in anatomic pathology," 2016 doi: $10.15190 /$ d.2016.15

[19] 360world Europe Kft. (2016) Holoengine. [Online]. Available: https://www.microsoft.com/en-us/store/p/holoengine/9nblggh4wkh9

[20] F. Panettieri. (2014) Socket.io for unity. [Online]. Available: https: //www.assetstore.unity3d.com/en/\#!/content/21721 


\section{BAGS: A Tool to Quantify Smart Grid Resilience}

\author{
Yatin Wadhawan \\ Department of Computer Science \\ University of Southern California \\ Los Angeles, USA \\ ywadhawa@usc.edu
}

\author{
Clifford Neuman \\ Information Science Institute \\ University of Southern California \\ Los Angeles, USA \\ bcn@isi.edu
}

\begin{abstract}
In this paper, we present the Bayesian Attack Graph for Smart Grid (BAGS) tool to quantify smart grid resilience in the presence of multiple cyber-physical attacks. BAGS takes system functions, network architecture, applications and a vulnerability report as input and generates three Bayesian Networks at three different levels of hierarchy. The top level network is called Functional Bayesian Network that defines how smart grid functions are connected. System engineers can select a particular function on a dashboard and view the Network Bayesian Network of that function at the second level. They can also choose a particular network component to see the list of vulnerabilities and the probability of associated compromise at the third level. System engineers can incorporate this functionality into their system and analyze the impact of any compromised component of the smart grid system on its resilience. Furthermore, BAGS helps to identify the failure paths in advance from one power grid function to another so that they can devise secure strategies and deploy resources effectively and efficiently.
\end{abstract}

Index Terms-Smart Grid; Bayesian Network; Cyber Security; Cyber-Physical Security; Resilience; Industrial Control System

\section{NOMENCLATURE}

S1: Quality of Smart Meter and Electric Vehicle Reads

S2: Quality of Smart Sync Head

S3: Billing System Performance

S4: Performance of Outage Management System

S6: Quality of Data captured by Vendor

S7: Performance of Electricity Energy Control Center

S8: Meter Data Management

R: Resilience

\section{INTRODUCTION}

$\mathrm{C}$ YBER-PHYSICAL Systems (CPS) [16] refer to a new generation of systems where physical processes are controlled and monitored from the cyber domain through advanced computation and communication technologies including humans in the loop. Utilization of advanced Information and Communication Technologies (ICT) in the CPS provides the ability for system engineers to control and monitor the physical processes in real-time. The growing interdependence between the cyber and physical world has opened the doors for various Cyber-Physical Threats (CPT) thus imposes extraordinary challenges on the security of CPS such as Smart Grid (SG). A Stuxnet-style attack on US SG could cost \$1 trillion to US government [15]. According to the report, such high-profile attacks on CPS would be used to infect the electricity generation control rooms in some parts of the northern US by exploiting vulnerabilities in network components with a motive to control power generation. Recent multiple attacks on the Ukraine power grid [13] [14] indicate that cyber attacks on such critical infrastructures will be frequent. Hence, identifying, understanding and modeling CPTs and defining system's resilience in the presence of CPTs is now a necessity.

Researchers have focused on describing the resilience of the SG system by analyzing different types of attacks on one of its components/functions [5-9]. Although such efforts provide relevant insights about the security of the $\mathrm{SG}$, they are incomplete in the sense that they do not consider the effect of one compromised function onto the other functions and ultimately on the system's resilience. Furthermore, the current work does not consider the dynamic nature of the vulnerabilities [7-9] and associated attack vectors. The likelihood of system component compromise is changing based on the dynamic nature of the vulnerabilities associated with the components and actions taken by the system engineers. For instance, we know the initial probability of compromising a server and that probability might change if we say that system engineers have applied security controls (patched) to that server. So we revise our belief based on current information, and this belief would be changed when there exists a zero-day attack for that server. Another challenge is that it is misleading to consider the vulnerabilities of the individual components [7]. It is possible that the vulnerability score of the single component is lower than the combination of multiple components, which provide the same functionality [4]. It is necessary to include the causal relationship between various functions, and components during risk analysis. It is insufficient to quantify and analyze Smart Grid Resilience (SGR) based on the static analysis of the system. We have to consider the dynamic nature of the vulnerabilities which is not yet considered by the previous approaches [7] [5]. They have failed to define a metric that can be used in real time to assess the SGR.

In this paper, we propose the Bayesian Attack Graph for Smart Grid (BAGS) tool to quantify the SGR in the presence of multiple CPAs in real time. BAGS takes functions, network architecture, applications and a vulnerability report as input and generates three Bayesian Networks (BN). The top level network is called Functional Bayesian Network (FBN) that defines how SG functions are connected to each other, their probability of failure and connection with the resilience variables. The possibility of a function compromise is the joint 
probability distribution of its network components that is based on the vulnerabilities of each component. FBN can be expanded to the second level as Network Bayesian Network (NBN) that can be further expanded to the third level as Vulnerability Bayesian Network (VBN). BAGS provides ease to the system engineers to perform an in-depth study of one of the functions of the SG and evaluates its effect on the overall system resilience. The system engineers can incorporate this functionality into their system, and they can see the impact of any compromised component of the SG on its resilience. The tool enables them to analyze how a failure of a network component controlling a particular power grid functionality propagates from the cyber to the physical domain and its impact on the SGR. It also helps them to identify the failure paths in advance from one SG function to another so that they can devise appropriate secure strategies and deploy resources effectively and efficiently.

The structure of the paper is as follows: Section II describes the related work. Section III discusses the challenges faced during SG risk analysis. Section IV and V describe the Resilience and Bayesian Network respectively. Section VI describes the SG architecture. Section VII explains the design of the BAGS tool. Section VIII describes the tool prototype and Section IX discusses the conclusion and future work.

\section{RELATED WORK}

Traditionally, researchers have focused on modeling and analyzing the impact of CPTs on various functions and components of the SG system [5-9] [18-19]. Sanjab et al. [10] defined threats targeting the SG infrastructure, challenges involved in the understanding of CPAs and defensive strategies against such attacks. Neuman and Tan [8] described different types of CPAs and how they propagate from cyber to the physical domain and vice versa. Srikantha and Deepa [5] formulated a differential game that describes stealthy strategies for attackers to disrupt transient stability by leveraging control over Distributed Energy Resources (DERs). Researchers in [6] demonstrated an optimal attack scenario using false data injection attacks on Automatic Generation Control (AGC) functionality of the SG system. In [7], the authors defined the systematic approach to quantify the resilience of the SG system through PowerWorld simulation. The authors performed load drop attack by sending remote disconnect commands to smart meters at various locations and analyze its impact on the power system. Although such methods clearly demonstrate how power system gets affected because of changes in one of the functions of the SG due to the cyber attacks, they have failed to capture the dynamic behavior of attacks based on the vulnerabilities associated with the system components.

Findrik et al. [11] present a framework for the development and evaluation of secure and resilient SG control applications. The coupling between the communication system simulator $\mathrm{OMNeT}++$ to the power system simulator PowerFactory using flexible middleware is used to develop the proposed tool. After describing the development of the tool, the paper modeled the cyber attacks in OMNeT++ simulator and analyzed the impact of those attacks on the power voltage control system. One of the limitations of this approach is that it does not include the real time vulnerabilities of the SG system into consideration.
Suppose a zero-day vulnerability is discovered or vulnerability is patched in a network component. How this tool would incorporate this information in the modeling and how SGR is affected by that change? A risk assessment approach for power system considering the reliability of the information system is presented in [17]. Through simulations, authors demonstrated that the failure of the information system brings more risk to the power system operation. The line overload and risk of bus voltage out of range are defined and calculated as risk indices. Both software and hardware components are considered to quantify the indices. The paper provides insights into the impact of the information system failure on the SG system which was not performed earlier in the literature.

The concept of defining resilience using $\mathrm{BN}$ has frequently been used in securing engineered systems such as in [1-4] [1819]. Li. et.al [1] described a three-layer framework that assesses the potential risks introduced by the mobile apps within the Android mobile system. The authors formulated three layers called as static, dynamic and behavioral network where risks are identified, and their propagation through each layer is modeled as Bayesian Risk Graph. Similarly, researchers in [4] modeled IT infrastructure using $\mathrm{BN}$ that enables system engineers to quantify the chances of network compromise. In [3], researchers defined a $\mathrm{BN}$ framework with a motive to compute the resilience of inland waterway network. Nita and Pingfeng [2] outlined a framework using $\mathrm{BN}$ to measure the resilience of engineered systems quantitatively. According to them, resilience is the combination of reliability and restoration. And it is similar to what is described in [3] that is absorptive, adaptive and restorative capacity.

We are motivated by the definition of resilience in [3] [4]. We have formulated a BN for the SG with a motive to quantify SGR in the presence of multiple CPAs in real time. Our research is different from current approaches (for assessing SGR) in a way that we have modeled the SG system as a dynamic BN where the probability to compromise system gets updated whenever there is a change in the vulnerabilities of the system. There is a need to develop a tool like BAGS so that system engineers not just can monitor the SG system components, functions and take decisions but can also control and compute the probability to compromise a particular function and analyze its effect on the SGR in real time. Furthermore, they should have the ability to perform an indepth study from functional level to vulnerability level of any function of the SG system.

Note, this tool is not a replacement for intrusion detection and prevention systems or SCADA system, rather it works along with such systems. This tool provides better attack predicting capability and also enables system engineers to perform containment of compromised components to stop the propagation of attacks to other parts of the system.

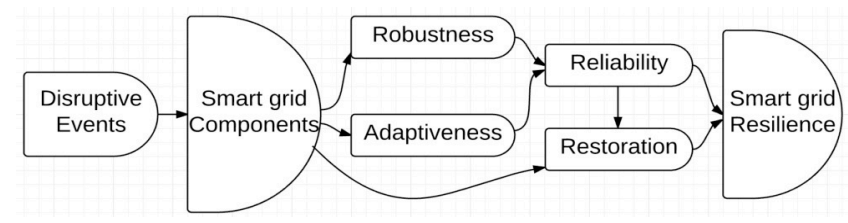

Fig. 1. Smart Grid: Resilience Modeling 


\section{CHALLENGES IN SMART GRID RISK ANALYSIS}

$\mathrm{SG}$ is a federated system with multiple complex components. These components are interconnected to each other, and they focus on different functionalities of the SG system. Each federated system consists of various network components that have different attack vectors to compromise. Due to the presence of large number of attack vectors in different SG components and platforms, the risk assessment is challenging. The main challenges faced by the system engineers to perform risk assessment are as follows:

1. Legacy System: Traditionally, SG systems were developed not keeping security in mind. This makes them the hub of security vulnerabilities that can be exploited remotely through the internet. Furthermore, it is impossible to patch vulnerabilities altogether since it requires to shutdown the system during the patching process. Thus, it is hard to track and patch all the potential risks present in such federated systems.

2. Complex Attack Vectors: Due to large surface area of attack, there are large number of attack vectors present. The cyber attackers can perform multiple attacks by combining different attack vectors. For instance, they combine remote code execution at smart meters or buffer overflow in smart meter head server followed by a DDOS attack to prevent legitimate signals reaching physical units and finally, they send control signals remotely to smart meters to manipulate power supply. To avoid such attacks, it is important to develop a tool that can track vulnerabilities of all the system components in real time and alert in case of emergency.

3. Risk Monitoring: The wide variety of vulnerabilities are associated with the SG network components. These vulnerabilities are changing in real time. Thus, it is hard for the system engineers to perform risk monitoring. Each function in the SG consists of network components, which further comprise of various vulnerabilities. There is a need of a tool that combines the risks associated with the network components of different functions into one model and quantifies it.

\section{RESILIENCE}

Resilience is the ability of a given system to avoid failures of its functions and components in the presence of the disruptive events and to quickly recover from those failures to an acceptable state without affecting function delivery. Fig. 1 shows the general concept of SGR modeling. To model the resilience of complex engineering systems different variables are defined in the literature [2-4]. We define those variables in the context of the SG system:

1. Robustness: It is the ability of the SG to withstand disruptive events. For instance, if there is a shortage of power during the natural disaster such as earthquake or Tsunami, the power grid continues to meet electricity demand by using power storage units. To provide robustness against CPAs, the system must have following capabilities in place: generation units, power storage, fault protection system, peaker power plant, gas storage, backup of the control system and critical workstations on standby mode. Such set of capabilities provides robustness to the infrastructure with a motive to meet power demand even in the presence of attacks.

2. Adaptiveness: It is the ability of the SG to adapt in the presence of changing environment so that to overcome disruptive events. For instance, if some generation units in the grid stop producing power, the power capacity is redistributed to other working generation units within the same area and neighboring areas to meet the demand. To provide the adaptive capacity to the SG, the system must have following capabilities: automation feeder and switching control, automatic generation control, automatic voltage control, phase angle regulator, real time load management such as demand response, and real-time load transfer. All such functionalities regulate power generation, transmission, and delivery even in the presence of attacks.

3. Reliability: It is the ability of the SG to continue to perform its functions normally. It depends on the Robustness and Adaptive capacity of the system which further depend on the system characteristics and disruptive events that take place on the system components.

4. Restorative: It is the ability of the SG to restore/recover from the disruptive events to an acceptable state where system is reliable to deliver its function. The restoration capacity depends on the reliability of the system and system characteristics in the presence of disruptive events. To provide the restorative capacity, the system must have following capabilities: automatic islanding and reconnection, containment of system components, fault current limiting, automatic software patching and hidden networks to operate. All such features enhance resilience of the system against various contingencies.

\section{BAYESIAN NETWORK}

In this section, we provide brief description of the $\mathrm{BN}$ and then, we describe how it is applied to the SGR modeling.

\section{A. Bayesian Belief Network (BBN)}

BBN is a probabilistic graphical model based on Bayes' theorem. It represents the conditional dependency between a set of random variables in a form of Directed Acyclic Graph (DAG) $G=(V, E) . V=\left\{V_{l}, V_{2}, \ldots \ldots, V_{N}\right\}$ is a set of nodes or variables of the system and $E$ is the set of edges representing the dependencies among variables. A link $E_{i, j}$ from $V_{i}$ to $V_{j}$ represents the causal dependency between these two nodes. Here, $\mathrm{V}_{\mathrm{j}}$ depends on the value of $\mathrm{V}_{\mathrm{i}}$ and $\mathrm{V}_{\mathrm{i}}$ is called parent (pa) of $\mathrm{V}_{\mathrm{j}}$. The relationship between variables of the $\mathrm{BBN}$ is measured using the conditional probability distribution (CPD). The joint probability distribution of $\mathrm{N}$ variables is:

$$
\mathrm{P}\left(\mathrm{V}_{1}, \ldots, \mathrm{V}_{\mathrm{N}}\right)=\Pi_{\mathrm{i}}^{\mathrm{N}} \mathrm{P}\left(\mathrm{V}_{\mathrm{i}} \mid \mathrm{V}_{\mathrm{i}+1} \ldots, \mathrm{V}_{\mathrm{N}}\right)
$$




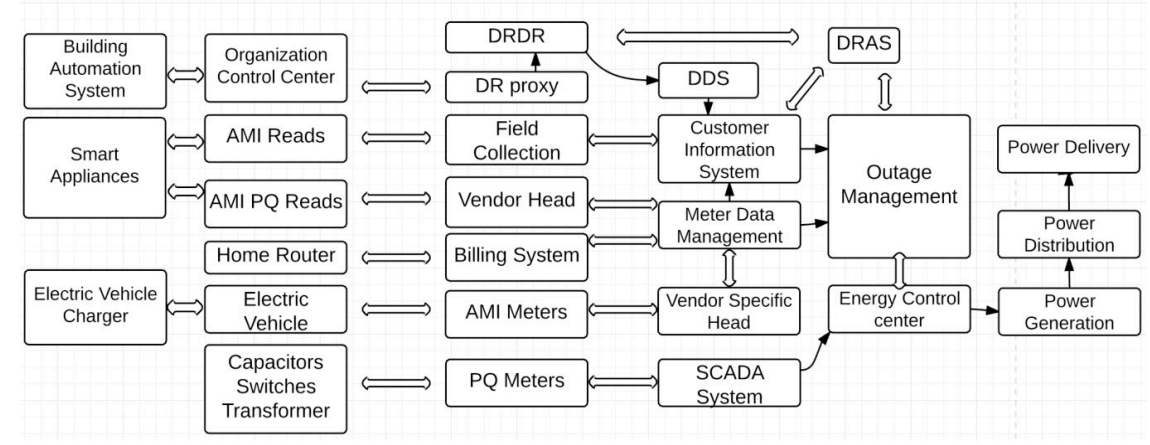

Fig. 2. Smart Grid Architecture

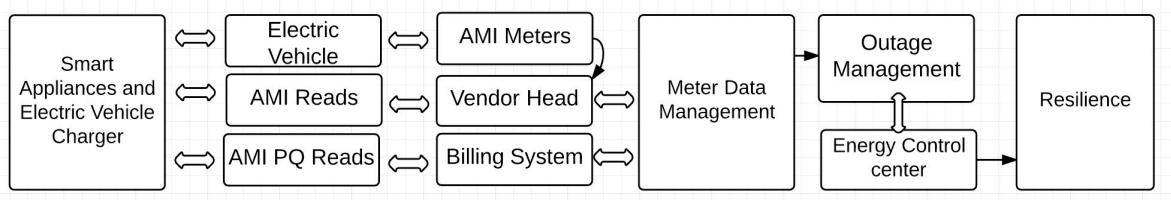

Fig. 3. Test Network

$$
\mathrm{P}\left(\mathrm{V}_{1}, \ldots, \mathrm{V}_{\mathrm{N}}\right)=\Pi_{\mathrm{i}}^{\mathrm{N}} \mathrm{P}\left(\mathrm{V}_{\mathrm{i}} \mid \text { Parents }\left(\mathrm{V}_{\mathrm{i}}\right)\right)
$$

To calculate the joint probability distribution, the individual distributions and conditional distributions among parent and children must be predetermined. Such expectations are measured from data analysis, expert knowledge or the combination of both and by performing the simulation. The major advantage of using $\mathrm{BBN}$ is to compute posterior probabilities of an event when certain events are observed in real time. This is called belief propagation. For instance, the likelihood of the SG to be in the resilient state is updated when certain disruptive events are observed on some of its components. Now we discuss how this concept is applied to quantify resilience.

\section{B. BBN Applied to Resilience Modeling}

The probability of system resilience [2] is expressed regarding the probability of reliability and restorative capacity of the system. The likelihood of restoration depends on the likelihood of reliability and system characteristics when disruptive events are observed. The probability of reliability depends on robustness and adaptiveness of the system. The disruptive events can happen on different components of the SG. It depends on how system components are connected and how an event propagates from one part to other parts of the system. The joint probability distribution of the system according to Fig. 1 is defined as:

$\mathbf{P}($ Resilience $)=\mathrm{P}($ Disruptive Events $) *$

P (Smart grid Components State | Disruptive Events) *

P (Robustness | Smart grid Components State) *

P (Adaptiveness | Smart grid Components State) *

P (Reliability | Robustness, Adaptiveness) *

P (Restoration | Reliability, Smart grid Components State) *

P (R | Reliability, Restoration)

\section{SMART GRID ARCHITECTURE AND TEST NETWORK}

In this section, we provide brief description about the SG architecture and then, we discuss the test network used for experiments.

\section{A. Smart Grid Architecture (SGA)}

SGA (see Fig. 2) provides a full vision of the proposed system and ensures that minimum qualification of system requirements such as security management, network deployment, and policy implementation. It also identifies the key domain areas, functions, and their weaknesses. Thus, it is important to understand the SGA so that the system engineers can establish and implement security policies effectively.

Starting from the left-hand side, building automation system, smart appliances, and electric vehicles are the endpoints that consume power from the grid. Building automation system manages the power consumption of the smart buildings and interacts with the central control system which controls other parts of the power network; smart appliances refer to power consumption devices at homes that connect to smart phones, desktop or laptops and provide you more control and information remotely. All such components send readings about the use of power by each appliance and electricity quality delivered to endpoints to AMI meters. Further, AMI meters send the collected data to the field collection system, vendor head end, and billing system. At these places, data is processed and stored in relevant databases. The data will then be sent to Meter Data Management (MDM) system where the data is used for various functions such as power prediction, customer load profile management, power outages, power quality at different places, and billing customers. MDM connects to Customer Information System (CIS) which maintains all the customer's database. All the power quality readings are transferred to the SCADA where it monitors the overall power grid functionality. 


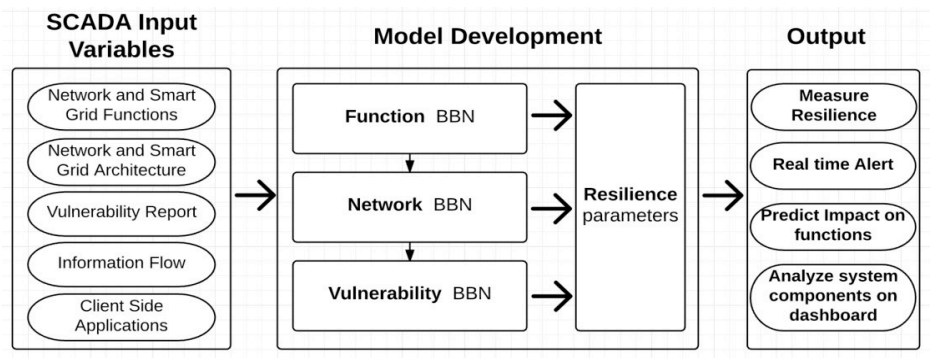

Fig. 4. Tool Design

Energy control center further manages power generation, distribution, and delivery. The data stored in the CIS and MDM, Outage Management System (OMS) predicts power outages and send information to the control center to manage power generation. Demand Response (DR) functionality is controlled through DR proxy. Many independent organizations are connected to DR proxy. DR proxy is further connected to DR Data Repository (DRDR). DRDR connects to DR Decision Support (DDS) and DR Automation Server (DRAS) that takes decision whether to perform DR functions in a given organization. DRAS interact with the OMS to check whether $\mathrm{DR}$ is required in response to reduced power generation. By understanding SGA, we know various power grid functions and components and how they are connected to each other. This understanding forms the basis of developing FBN in our model development of system design, which is explained in the Section VII-B.

\section{B. Test Smart Grid Architecture}

To develop a mock-up of our proposed design, we consider a test network in Fig. 3 which is a part of the SGA. The system consists of smart appliances and electric vehicle attached to the grid. The power consumption and electricity quality readings from these components are collected by the field collection systems, vendor specific heads, and billing system. Further, this information is communicated to MDM, and via MDM it is transferred to OMS to keep track of reserved power and power outages that might happen shortly. It also manages how much amount of power should be stored to meet the demand during contingencies. OMS interacts with the ECC to take decisions regarding power generation, DR, power delivery, etc. We develop the FBN, NBN, and VBN for this test graph and show how our proposed model is useful in quantifying resilience of the system. We explain the BN of the test network in the next section.

\section{TOOL DESIGN}

In this section, we discuss the input variables given to the tool, model development and output of the tool (see Fig. 4).

\section{A. Input Variables}

1. Network and $S G$ functions: It represents the list of higher level network and SG funcitons such as energy management system, outage management system, power generation, demand response, smart meters, billing function and vendor head end.
2. Network and SG Architecture: The network architecture of the system that supports all the functions of the SG system. For instance, MDM consists of servers, workstations, database, and communication network. It also represents the interconnection between all these components.

3. Applications: The list of client side and server side applications is also given as input to the system. It also includes vendor side applications which integrate to the SGA.

4. Vulnerability Report: To compute the likelihood of a particular system compromise, we must have a list of vulnerabilities that exist in system's components and applications. For instance, billing server has Cross Site Scripting (XSS), local file inclusion vulnerabilities, which can be exploited to gain control over the billing server. We will use Common Vulnerability Scoring System (CVSS) [12] scores to compute likelihood of compromise.

Once we have these inputs to the system, it generates three $\mathrm{BN}$ at three different levels of hierarchy, which we describe in the next subsections. The output of the model is displayed on an interactive dashboard where system engineers can select a particular function and can view its system architecture, components, and information flow. Furthermore, they can select a particular component and can view its vulnerability report and likelihood of its compromise. These features give power to the system engineers to perform real-time monitoring and predict the impact of a compromise on different components of the system and finaly, quantify the resilience of the system.

\section{B. Function Bayesian Network (FBN)}

FBN represents the causal interconnection between different functions of the SG. Nodes in the FBN describes the functions of the SG and edges represent the information flow from one function to another. It also describes how the impact of a system compromise travels across different functions. We use test network (in Fig. 3) to provide a better understanding of the FBN. Fig. 5 depicts the FBN of the test network. Smart meter function (S1) sends messages to the SG head function (S2) which further send messages to the billing system (S3). Similarly, electric vehicle charging data (consumption and power quality) is captured by the function (S5) and given to the Vendor particular head end (S6). 


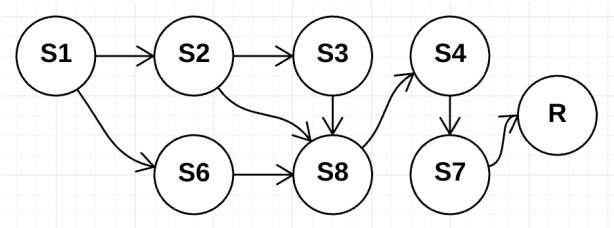

Fig. 5. Test Network: Function Bayesian Network

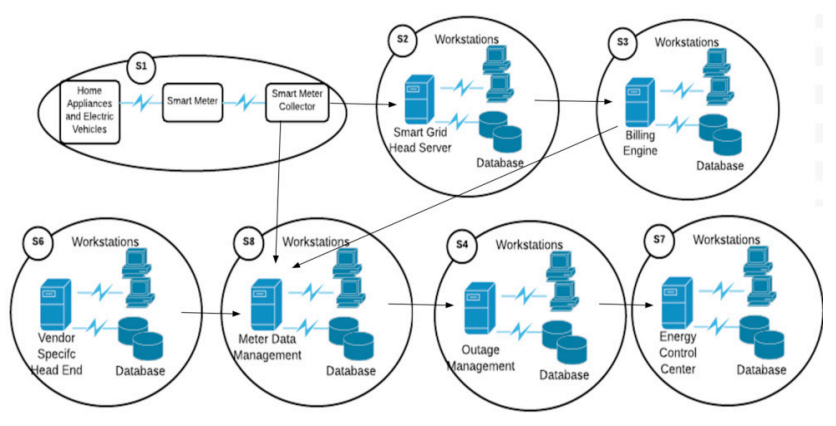

Fig. 6. Test Network: Network Bayesian Network

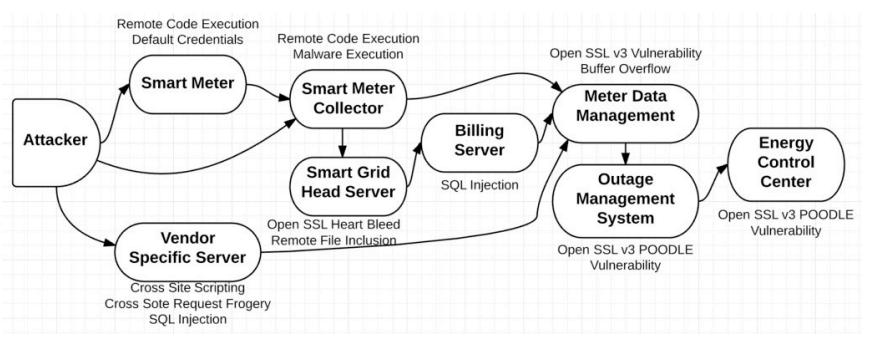

Fig. 7. Test Network: Vulnerability Bayesian Network

The data set from the billing system, head end and vendor specific end is provided to the MDM (S8). MDM connects with the OMS (S4), which is further connected to the ECC (S7). Finally, ECC is connected to Resilience function (R). Suppose an adversary compromises a smart meter by injecting a malware, he sends compromised messages about the power consumption all the way to the billing center and also exploits the vulnerability to gain control over the component. If he gains control over the billing system, he escalates privileges by exploiting vulnerabilities of other systems. FBN provides understanding to the system engineers that how information and control flow works in the SGA. They can also identify various attack paths an adversary may take if a certain function is compromised. In our model, they can choose any function on FBN and view its network components, which we describe in the next subsection.

\section{Network Bayesian Network (NBN)}

NBN represents various network components supporting a particular function. The system engineers can select a specific function at the FBN level on the dashboard and can see its network components and analyze its functional status and information flow. Fig. 6 describes the NBN of the FBN illustrated in Fig. 5. To illustrate with an example, the smart meter functionality (S1) has components: home appliances, electric vehicles, smart meters and smart meter collector server. The communication between these components is mostly wireless. Smart Meter collector server collects data from all the smart meters defined in its zone and sends that information to the smart grid head end (S2) over the wireless mesh network. Further, S2 consists of workstations, database servers and smart grid head server which collects data from various S1 systems. The significance of such modeling is to understand how data flows from one system to another, in other sense, whether there is any vulnerable path from one system to another. By providing such an interface, it provides the ability to analyze every system component and its impact on the overall system. In the next subsection, we describe that how an adversary compromises system vulnerabilities to move forward in the network. We have not shown firewalls and routers in this network diagram. We have displayed components that provide functionality to the SG and have vulnerabilities in their implementation. But we can extend this model to show other network components.

\section{Vulnerability Bayesian Network (VBN)}

The system engineers can select a particular network component from the NBN and view the list of its vulnerabilities by vulnerability report submitted as an input to the system. It also provides the information about the likelihood of network component compromise based on the CVSS score. The possibility of the components combined to calculate the compromise probability of the network component and ultimately of the function to which network components belong to. Also, if there is a change in the vulnerability, the system automatically updates the belief of system compromise and propagate to other parts of the network.

Attack Graph: Fig. 7. describes the attack graph from remote attacker's point of view according to our test case. A remote attacker performs variety of attacks to gain access to the SG functions. According to our test case, the attacker exploits XSS, CSRF or SQL Injection vulnerability to gain access to the Vendor specific server or performs remote code execution on smart meters or smart meter collector. Once the attacker has access to the smart meter collector, he exploits open SSL heart bleed to gain access to the smart grid head server from where he targets the billing engine server. The SQL Injection vulnerability can be exploited to gain remote access through username and password from the database. Once he gains access to the billing engine, he performs the port scan over the network range and identify the MDM server.

Once MDM server is identified, he exploits the buffer overflow vulnerability present in the server operating system. Then, the attacker gains access to the MDM directly without going through billing engine. Once he gains access to the MDM server, he performs the variety of attacks such as integrity attack by changing meter readings, but in this case, he is interested in having access to the energy control center. So he further performs scanning and identifies the OMS server which is connected to the MDM server. He exploits the open SSL v3 POODLE vulnerability and gets root access. Once he has access to the OMS server, he identifies and attacks the ECC by exploiting open heart bleed vulnerability. Table I indicates all the vulnerabilities corresponding to different servers in our test network. 
CVSS: Our motive is to compute the local probability distribution of system compromise based on the vulnerabilities of the system. For that, first, we need to calculate the likelihood of success for an attacker to exploit the known vulnerability. We use the process described in CVSS [12] to calculate this probability. CVSS score consists of three different scores: Base, Temporal and Environmental. The base score includes the essential properties of the vulnerability such as Attack Vector (AV), Attack Complexity (AC), User Interaction (UI) required or not, Privileged Required (PR) or not, affecting Confidentiality, Integrity, and Availability (CIA). The temporal score indicates the characteristics of the vulnerability that evolve over its lifetime. It includes variables like exploit code maturity, remediation level, and report confidence. And finally, the environmental score includes characteristics that are dependent on the implementation and environment of the organization. It includes variables such as the requirement of CIA and modified scope. We compute the probability of exploiting vulnerability by considering exploitability score as described in CVSS specification [12]:

$$
\mathrm{P}\left(\mathrm{V}_{\mathrm{i}}\right)=(8.22 * \mathrm{AV} * \mathrm{AC} * \mathrm{UI} * \mathrm{PR}) / 10
$$

Table II represents the CVSS probabilities of compromise corresponding to each vulnerability. The local conditional probability distribution (LCPD) is computed when many exploits are involved. It depends on whether each vulnerability exploitation is a distinct event or not. If it is a distinct event, we compute LCPD using product rule (see eq. 5) otherwise we calculate joint probability for OR case (see eq. 6). Suppose an adversary compromises a known vulnerability of the system $(\mathrm{X})$ and earns a user privilege $(\mathrm{Y})$ to that system. It forms a causal relationship as $\mathrm{X}->\mathrm{Y}$.

TABLE I. TEST SGA VULNERABILITIES

\begin{tabular}{|c|c|}
\hline $\begin{array}{l}\text { System } \\
\text { Name } \\
\end{array}$ & Vulnerabilities \\
\hline Smart Meters & $\begin{array}{l}\text { Default Password } \\
\text { Remote Code Execution }\end{array}$ \\
\hline Smart Meter Collector Server & Remote Code Execution \\
\hline $\begin{array}{l}\text { Smart Grid Head End } \\
\text { (Windows Server 2008) }\end{array}$ & Open SSL Heart Bleed \\
\hline $\begin{array}{l}\text { Billing Engine Server } \\
\text { (Windows Server 2008) }\end{array}$ & SQL Injection \\
\hline $\begin{array}{l}\text { Meter Data Management } \\
\text { Server (Windows Server } \\
\text { 2012) }\end{array}$ & $\begin{array}{l}\text { Open SSL v3 Vulnerability } \\
\text { XSS client end }\end{array}$ \\
\hline $\begin{array}{l}\text { Outage Management Server } \\
\text { (Windows Server 2012) }\end{array}$ & Open SSL POODLE Vulnerability \\
\hline $\begin{array}{l}\text { Energy Control Center Server } \\
\text { (Windows Server 2012) }\end{array}$ & Open SSL POODLE Vulnerability. \\
\hline $\begin{array}{l}\text { Vendor Specific Server } \\
\text { (Windows Server 2012) }\end{array}$ & $\begin{array}{l}\text { Cross Site Request Frogery } \\
\text { Cross Site Scripting (XSS) } \\
\text { SQL Injection }\end{array}$ \\
\hline Workstations & Buffer Overflow \\
\hline MS SQL Server & $\begin{array}{l}\text { SQL Injection } \\
\text { MS SQL Buffer Overflow }\end{array}$ \\
\hline
\end{tabular}

TABLE II. EXPLOITABLE Probabilities BASED ON CVSS

\begin{tabular}{|l|l|}
\hline \multicolumn{1}{|c|}{ Vulnerabilities } & \multicolumn{1}{c|}{$\begin{array}{c}\text { CVSS } \\
\text { Probability }\end{array}$} \\
\hline Remote code execution & 0.84 \\
\hline Buffer Overflow & 0.78 \\
\hline Denial of Service & 0.74 \\
\hline SQL Injection MS SQL Server & 0.72 \\
\hline Open SSL Heart Bleed & 0.75 \\
\hline Open SSL POODLE & 0.31 \\
\hline Cross Site Scripting & 0.61 \\
\hline Cross Site Request Frogery & 0.88 \\
\hline
\end{tabular}

$$
\begin{aligned}
& \mathrm{P}(\mathrm{Y} \mid \operatorname{Parents}(\mathrm{Y}))=\Pi_{\mathrm{i}}{ }^{\mathrm{N}} \mathrm{P}\left(\text { Exploit }_{\mathrm{i}}\right) \\
& \mathrm{P}(\mathrm{Y} \mid \operatorname{Parents}(\mathrm{Y}))=1-\Pi_{\mathrm{i}}{ }^{\mathrm{N}}\left(1-\mathrm{P}\left(\text { Exploit }_{\mathrm{i}}\right)\right)
\end{aligned}
$$

The probability of compromise of $\mathrm{Y}$ depends on the vulnerabilities that $\mathrm{Y}$ has and the likelihood of $\mathrm{X}$ to get compromised. $\mathrm{X}$ is the parent of $\mathrm{Y}$. In case of multiple exploits that need to be exploited, the probability is computed via product rule. We compute the product of all the probabilities of exploits that are present in the parent of Y. LCPD is used to calculate the unconditional probability distribution corresponding to each vulnerability, by merging the marginal cases at each node. For instance, the unconditional probability of compromising billing server depends on all the nodes that influence it (smart meter, smart meter collector and smart grid head server). Similarly, the unconditional probability of the ECC server depends on all the nodes shown in the graph Fig. 7. The probability whether remote attacker attacks the SG system components is provided by the system engineers based on their experience and they revise their belief over a period. In our tool, the system engineers can change this probability to see the impact on the resilience of the system.

Posterior Probabilities: The probability to compromise a function changes over a period depending on the vulnerabilities of its network component's and other factors. The posterior probabilities of system components are useful to evaluate the risk in the dynamic environment. For example, if we know that OMS is compromised, we can calculate the likelihood of MDM compromise using Bayes rule (eq. 7):

$$
\begin{aligned}
\mathrm{P}(\mathrm{MDM} / \mathrm{OMS}) & =\mathrm{P}(\mathrm{OMS} / \mathrm{MDM}) \mathrm{P}(\mathrm{MDM}) / \mathrm{P}(\mathrm{OMS}) \\
& =0.99
\end{aligned}
$$

We already know the value of $\mathrm{P}(\mathrm{MDM})=0.4786$, $\mathrm{P}(\mathrm{OMS})=0.1484$ (see fig. 12.) and $\mathrm{P}(\mathrm{OMS} / \mathrm{MDM})=0.31$. The unconditional probability of MDM getting compromised was 0.4786. But once we know that an attack incident at OMS, the posterior probability becomes 0.99 . Similarly, the system engineers can calculate probabilities of successors of MDM and other nodes in response to an attack incident on OMS. Such technique allows the system engineers to see how the effect of an attack propagates to other parts of the system. For 
instance, when the individual system gets outdated and has more vulnerabilities exposed to the outer world, the attacker has larger surface area to compromise the system, and that will affect the probability of compromise of other nodes as well. Similarly, the system engineers can also evaluate how attack surface area is reduced when a particular security control is placed on the node or vulnerabilities are reduced by updating the software. They can develop resource allocation algorithm to minimize the risk using posterior probabilities.

Another advantage of this approach is to partition the network by compromised probabilities. Once the likelihood of a component crosses a threshold, that component is either fixed immediately or removed from the network to reduce its effect on other parts of the system. The tool provides the ability to calculate posterior probabilities and show them on the dashboard so that system engineers can monitor the status of each network component.

\section{E. Tool Output}

The main motive of this tool is to provide following functionalities:

1. Measure Resilience: The primary motive is to measure the resilience of the system in real time. By considering the vulnerabilities of the system components, the likelihood of their compromise is calculated. Using BN, we connect different system components and see how an attack propagates from one system to another. The resilience is computed based on the probability of likelihood of ECC compromise, which controls the power generation and distribution. If the probability of ECC compromise is high, the system is not resilient, and power delivery will be affected in case of an attack. As described in section IV, resilience is dependent on reliability and restorative measures. Due to lack of SG data, we only consider reliability. The probability of the ECC compromise works as an identifier of the resilience of the system.

2. Alert Mechanism: The alert mechanism helps system administrator to put check points on the probabilities of the system component compromise. Based on their knowledge of the system, they assign probability thresholds to each component described in the test network. If the probability of the system component compromise crosses threshold, an alarm is raised. This enables system engineers to identify most of the vulnerable components so that they can assign appropriate security controls and perform vulnerability assessment and penetration testing.

3. Predict Impact: The system engineers evaluate and predict the impact of a system component compromise on another components by computing posterior probabilities.

4. View System Architecture: The system engineers view the whole system on an interactive dashboard. The interactive dashboard provides a view of 1) FBN where all the system functions are logically connected, 2) NBN which is a detailed description of the function components and 3) VBN which describes the list of vulnerabilities associated with the components and probabilities of their compromise (unconditional probabilities) and attack graph. This enables system engineers to analyze the status of the SG system remotely, and they can perform impact analysis by simulating different attack scenarios on different components of the system.

\section{TOOL PROTOTYPE}

In this section, we describe the simulation setup and the mock-up of the tool.

\section{A. Tool Development}

We have developed the User Interface (UI) of the tool in Java language using regular window toolkit class. It represents the framework that is visible to the system engineers on the dashboard. We maintain the static files database of the network components, functions and vulnerabilities (described in Section VII-A) as input to the tool. The tool parses the file and generates the FBN (see fig. 8.). In this mock-up, the lines are not directed. But in the real tool, the lines will be directed and marked by the type of information they represent. Note, here, our motive is to demonstrate a mock-up/UI of the tool that shows how powerful it is.

FBN represents the acyclic graph of the connected components according to the test network (see fig. 3 and 5). When a user clicks on a node of FBN, the function's network components are represented and how they are connected (see fig. 9.). When the user clicks on a particular network component, the list of vulnerabilities associated with that component is generated (see fig. 10.). It also contains the details of the vulnerabilities, CVSS score and the probability of compromise. The system engineers can change the system configuration in the database consistently. The same changes are reflected in the tool on refreshing it. The system engineers can add or remove any component, discover or patch any vulnerabilities, and disconnect any component.

In this mock-up, we use the Bayes.jar to represent the unconditional probabilities of the functions. We create nodes corresponding to each functions and link them according to the test network (see fig. 11). Then, we provide the probability of compromise to each component. It gives the unconditional probabilities of compromise of each function as output. The system engineers view the expectations by selecting any function on the dashboard (see fig. 12.).

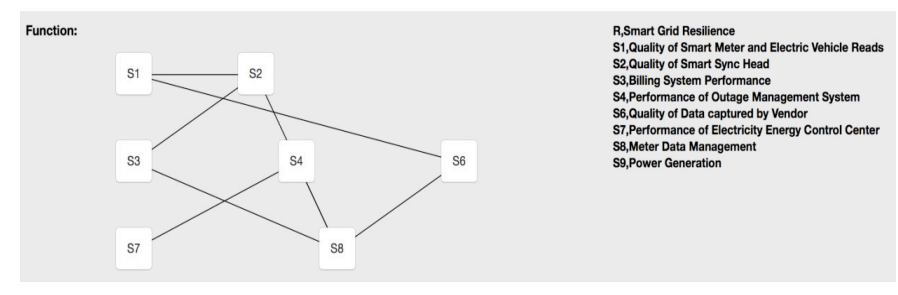

Fig. 8. Tool prototype: FBN. 


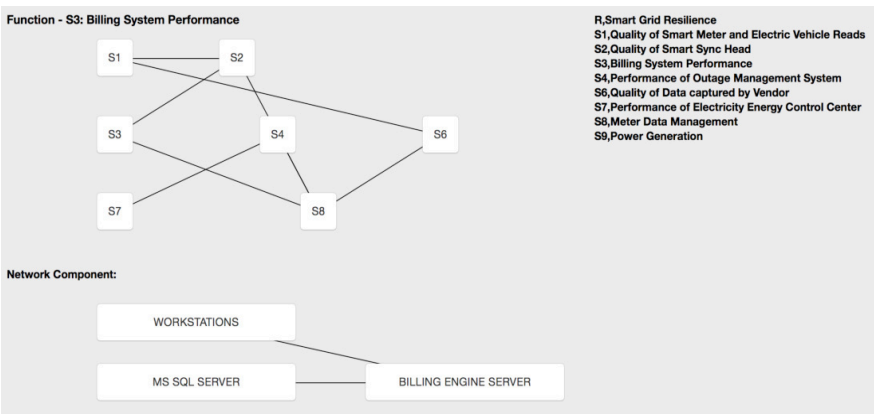

Fig. 9. Tool Prototype: NBN

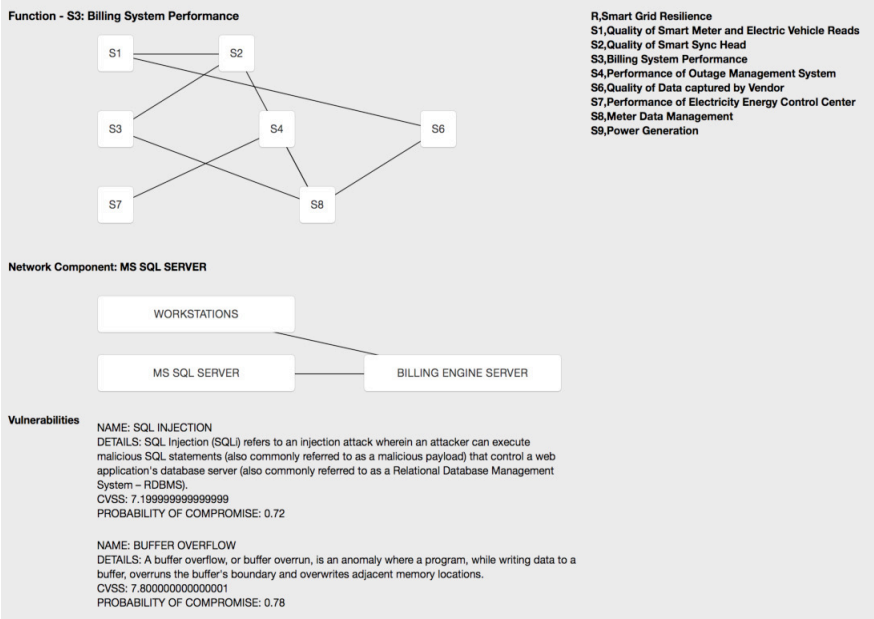

Fig. 10. Tool Prototype: VBN

In the future, we will implement the Bayesian algorithm in our tool. Over here our motive is to show the features of the BAGS tool available to the system engineers. In fig. $8, \mathrm{~S} 7$ stands for the performance of ECC. This is the main component of the SG system. If ECC gets compromised, the power system may get shutdown. If this system has high probability of compromise, the overall system is at high risk. The cyber attackers can easily exploit the vulnerabilities and compromise S7. Functions that fall under the category of reliability, restoration, and adoption will be considered in our future work. Due to the limitation of space and the absence of real world dataset, it is difficult to demonstrate how they are modeled.

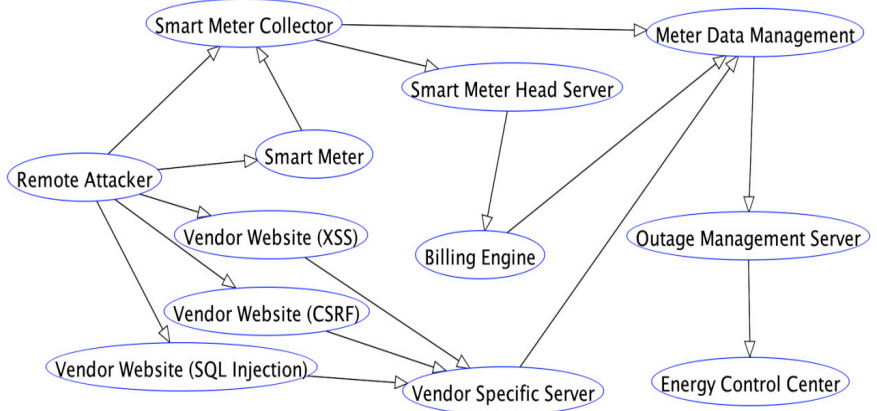

Fig. 11. Bayes.jar tool Function Nodes.

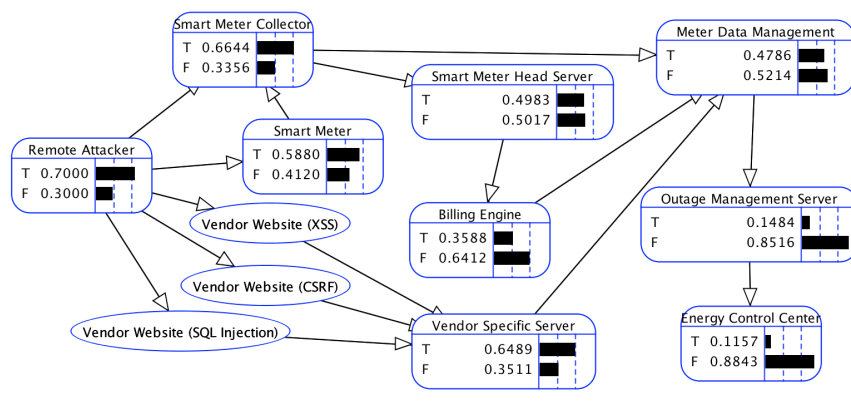

Fig. 12. Bayes.jar Unconditional Probability Distributions when Probability of remote attacker to attack is 0.70 .

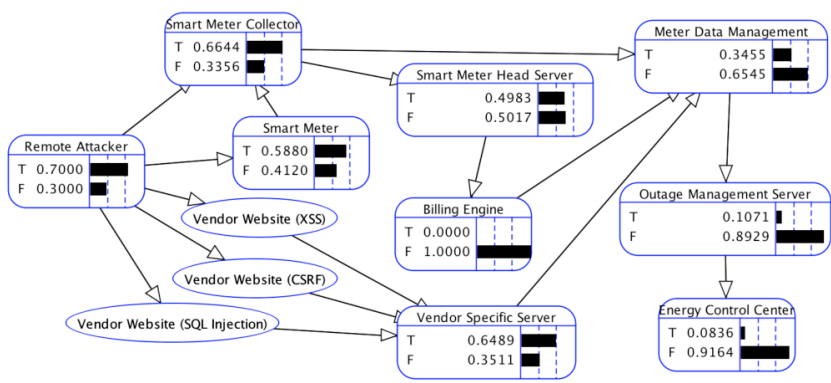

Fig. 13. Bayes.jar Unconditional Probability Distributions when Billing Engine's SQL Injection vulnerability is patched. The effect of such change is propagated to other components.

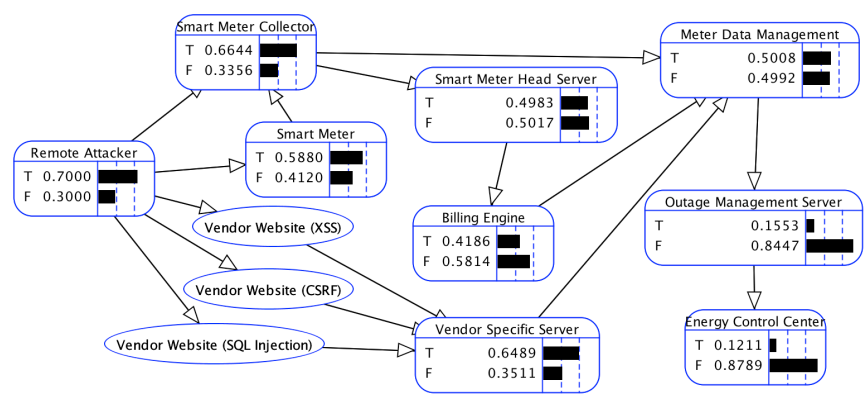

Fig. 14. Bayes.jar: Unconditional Probability Distributions when remote Code Execution is vulnerability is discovered in Billing Engine server. The effect of such change is propagated to other components.

\section{B. Results}

Fig. 12. represents the unconditional probabilities of all the functions of the test network. Each function is a Bernoulli variable. True $(\mathrm{T})$ variable represents the likelihood of compromise, and False $(\mathrm{F})$ represents the probability of not compromise. They are computed by Bayes rule described in equation 2. Also, they are used to calculate the posterior probabilities of the components to get compromised. The system engineers can easily monitor the status of the components regarding compromise probabilities by analyzing the graph in fig. 5. If any vulnerability is discovered in a component or if any vulnerability is patched, the unconditional probabilities will automatically get change.

Fig. 13. represents the case when system engineers have patched the billing engine server, and its probability of 
compromise becomes zero. There is a drastic change in the likelihood of compromise of other components which are children of billing engine server. Similarly, fig. 14 represents when the zero-day vulnerability is discovered in the billing engine server and how the probabilities to compromise of its children change. This is how BAGS enables engineers to evaluate the risk associated with every component and how risk propagates from one component to another in such interdependent network. Based on the experience of the system engineers, they set a threshold on the unconditional probability of any function and create an alert mechanism. This feature will also be implemented as part of the future work.

\section{CONClusion ANd Future WORK}

In this research paper, we presented the Bayesian Attack Graph for Smart Grid tool to quantify the resilience of a given smart grid system in the presence of multiple cyber-physical attacks in real time. BAGS takes system functions, network architecture, applications and vulnerability report as input and generates $\mathrm{BNs}$ at three different levels of hierarchy: FBN, NBN, and VBN. We have implemented a mock-up/UI of this tool by maintaining a static database of network architecture. The system engineers can incorporate this functionality into their system, and they can see the impact of any compromised component of the smart grid system on its resilience. BAGS enables system engineers to analyze how a failure of a cyber network component controlling a particular power grid functionality propagates from the cyber to the physical domain and its impact on SGR. It also helps them to identify the failure paths in advance from a smart grid function to another so that they can devise relevant secure strategies and deploy resources effectively and efficiently. BAGS works along with the intrusion detection and prevention systems or SCADA system. It provides better attack predicting capability and ability to perform containment of compromised components to stop the propagation of attacks to other parts of the system. The system engineers can feed this input to their dashboard of Security Operations Center to expedite the process of security risk assessment. Note, one can develop this tool to quantify the resilience of other CPSs such as oil and gas systems, nuclear plants, water treatment plants, etc. For that, they need to understand and incorporate the system's cyber and physical infrastructure. Our future work will be to develop this tool using the real world dataset and deploy it in a SCADA system. We are in the process of discussion with companies that may provide SGA data to us. We will also implement Bayesian Algorithm and add features such as alert mechanisms and posterior probabilities as a part of our tool. Furthermore, we will develop an algorithm to allocate security controls to maximize the resilience of the system using game theoretic approaches.

\section{ACKNOWLEDGEMENT}

This work was conducted with partial funding by Northrop Grumman Information Systems through the Cyber Security Research Consortium, and with support from Schlumberger.

\section{REFERENCES}

[1] Li, S., Tryfonas, T., Russell, G., \& Andriotis, P. (2016). Risk assessment for mobile systems through a multilayered hierarchical Bayesian network. IEEE transactions on cybernetics, 46(8), 1749-1759.

[2] Yodo, N., \& Wang, P. (2016). Resilience modeling and quantification for engineered systems using Bayesian networks. Journal of Mechanical Design, 138(3), 031404.

[3] Hosseini, S., \& Barker, K. (2016). Modeling infrastructure resilience using Bayesian networks: a case study of inland waterway ports. Computers \& Industrial Engineering, 93, 252-266.

[4] Poolsappasit, N., Dewri, R., \& Ray, I. (2012). Dynamic security risk management using bayesian attack graphs. IEEE Transactions on Dependable and Secure Computing, 9(1), 61-74.

[5] Srikantha, Pirathayini, and Deepa Kundur. "A DER Attack-Mitigation Differential Game for Smart Grid Security Analysis." IEEE Transactions on Smart Grid 7, no. 3 (2016): 1476-1485.

[6] Tan, Rui, Hoang Hai Nguyen, Eddy YS Foo, Xinshu Dong, David KY Yau, Zbigniew Kalbarczyk, Ravishankar K. Iyer, and Hoay Beng Gooi. "Optimal False Data Injection Attack against Automatic Generation Control in Power Grids." In 2016 ACM/IEEE 7th International Conference on Cyber-Physical Systems (ICCPS), pp. 1-10. IEEE, 2016.

[7] AlMajali, A., Rice, E., Viswanathan, A., Tan, K., \& Neuman, C. A systems approach to analysing cyber-physical threats in the Smart Grid. In 2013 IEEE International Conference on Smart Grid Communications (SmartGridComm).

[8] Neuman, C., and Tan, K.: Mediating cyber and physical threat propagation in secure smart grid architectures. In Smart Grid Communications (SmartGridComm), IEEE International Conference on (pp. 238-243). (2011)

[9] Yi, Ping, Ting Zhu, Qingquan Zhang, Yue Wu, and Li Pan. "Puppet attack: A denial of service attack in advanced metering infrastructure network." Journal of Network and Computer Applications 59 (2016): 325-332.

[10] Sanjab, A., Saad, W., Guvenc, I., Sarwat, A., \& Biswas, S. (2016). Smart Grid Security: Threats, Challenges, and Solutions. arXiv preprint arXiv:1606.06992.

[11] Findrik, M., Smith, P., Kazmi, J. H., Faschang, M., \& Kupzog, F. (2016, Novemher). Towards secure and resilient networked nower distrihution grids: Process and tool adontion. In Smart Grid Communications (SmartGridComm), 2016 IEEE International Conference on (pp. 435440). IEEE.

[12] CVSS Score. https://www.first.org/cvss/specification-document

[13] Ukraine's Power outage was a cyber attack: Ukrenergo, 2017. http://www.reuters.com/article/us-ukraine-cyber-attack-energyidUSKBN1521BA

[14] Analysis of the Cyber Attack on the Ukrainian Power Grid, March 2016. http://www.nerc.com/pa/CI/ESISAC/Documents/EISAC_SANS_Ukraine_DUC_18Mar2016.pdf

[15] Stuxnet style attack on US Smart Grid could cost governemnt \$1 trillion. https://www.scmagazineuk.com/stuxnet-style-attack-on-us-smart-gridcould-cost-government-1-trillion/article/535452/

[16] Baheti. Radhakisan. and Helen Gill. "Cvher-nhysical systems." The impact of control technology 12 (2011): 161-166.

[17] Lu, D., Liu, Y., \& Zeng, Y. (2016, November). Risk assessment of power grid considering the reliability of the information system. In Smart Grid Communications (SmartGridComm), 2016 IEEE International Conference on (pp. 723-728). IEEE.

[18] J. Zalewski, S. Drager, W. McKeever, A. Kornecki, B. Czeido, Modeling Resiliency and Its Essential Components for Cyberphysical Systems, Annals of Computer Science and Information Systems (Proc. FedCSIS'2015), Vol. 6, 107-114 (2015)

[19] A. Kornecki, N Subramanian, J. Zalewski, Studying Interrelationships of Safety and Security for Software Assurance in CyberPhysical Systems: Approach Based on Bayesian Belief Networks, Proceedings of the 2013 FedCSIS Conference, pp. 1381-1387. 


\section{$1^{\text {st }}$ International Conference on Lean and Agile Software Development}

T HE evolution of software development life cycles is driven by the perennial quest on how to organize projects for better productivity and better quality. The traditional software development projects, which followed well-defined plans and detailed documentations, were unable to meet the dynamism, unpredictability and changing conditions that characterize rapidly changing business environment. Agile methods overcame these limits by considering that requirements are not static but dynamic, while customers are unable to definitively state their needs up front. However, the advent of agile methods divided the software engineering community into opposing camps of traditionalists and agilists. After more than a decade of debate and experimental studies a majority consensus has emerged that each method has its strengths as well as limitations, and is appropriate for specific types of projects, while numerous organizations have evolved toward the best balance of agile and plan-driven methods that fits their situation.

In more recent years, the software industry has started to look at lean software development as a new approach that could complement agile methods. Lean development further expands agile software development by adopting practices from lean manufacturing. Lean emphasizes waste elimination by removing all nonvalue-adding activities.

\section{TOPICS}

The objective of LASD' 17 is to extend the state-of-the-art in lean and agile software development by providing a platform at which industry practitioners and academic researchers can meet and learn from each other. We are interested in high quality submissions from both industry and academia on all topics related to lean and agile software development. These include, but are not limited to:

- Combining lean and agile methods for software development

- Lean and agile requirements engineering

- Scaling agile methods

- Distributed agile software development

- Challenges of migrating to lean and agile methods

- Balancing agility and discipline

- Agile development for safety systems

- Lean and agility at the enterprise level

- Conflicts in agile teams

- Lean and agile project management

- Collaborative games in software processes

- Lean and agile coaching

- Managing knowledge for agility and collaboration
- Tools and techniques for lean and agile development

- Measurement and metrics for agile projects, agile processes, and agile teams

- Innovation and creativity in software engineering

- Variability across the software life cycle

- Industrial experiments, case studies, and experience reports related to all of the above topics

\section{SECTION EDITORS}

- Przybyłek, Adam, Gdansk University of Technology, Poland

\section{REVIEWERS}

- Akman, Ibrahim, Atilim University, Turkey

- Alshayeb, Mohammad, King Fahd University of Petroleum and Minerals, Saudi Arabia

- Angelov, Samuil, Fontys University of Applied Sciences, The Netherlands

- Bagnato, Alessandra, SOFTEAM R\&D Department, France

- Bauer, Veronika, Technische Universität München, Germany

- Belle, Alvine Boaye, École de Technologie Supérieure, Canada

- Bhadauria, Vikram, Texas A\&M International University, United States

- Binti Abdullah, Nik Nailah, Monash University Malaysia, Malaysia

- Biró, Miklós, Software Competence Center Hagenberg and Johannes Kepler University Linz, Austria

- Blech, Jan Olaf, RMIT University, Australia

- Borg, Markus, SICS Swedish ICT AB, Sweden

- Buglione, Luigi, Engineering Ingegneria Informatica SpA, Italy

- Carreira, Paulo, Instituto Superior Técnico, Portugal

- Chatzigeorgiou, Alexandros, University of Macedonia, Greece

- Cruzes, Daniela, SINTEF ICT, Norway

- Dejanović, Igor, Faculty of Technical Sciences, Novi Sad

- Diebold, Philipp, Fraunhofer IESE, Germany

- DUTTA, ARPITA, NIT ROURKELA, India

- GODBOLEY, SANGHARATNA, NIT ROURKELA, India

- Gonzalez Huerta, Javier, Blekinge Institute of Technology, Sweden

- Górski, Janusz, Gdańsk University of Technology, Poland 
- Gregory, Peggy, University of Central Lancashire, United Kingdom

- Hohenstein, Uwe, Siemens AG, Germany

- Janes, Andrea, Free University of Bolzano, Italy

- Janousek, Jan, Czech Technical University, Czech Republic

- Järvinen, Janne, F-Secure Corporation, Finland

- Jarzębowicz, Aleksander, Gdańsk University of Technology, Poland

- Jovanović, Miloš, University of Novi Sad, Serbia

- Kaloyanova, Kalinka, Sofia University, Bulgaria

- Kapitsaki, Georgia, University of Cyprus, Cyprus

- Kassab, Mohamad, Innopolis University, Russia

- Katić, Marija, School of Computing, Engineering and Physical Sciences, United Kingdom

- Knodel, Jens, Fraunhofer IESE, Germany

- Kuciapski, Michał, University of Gdansk, Poland

- Landowska, Agnieszka, Gdansk University of Technology, Poland

- Lehtinen, Timo O. A., Aalto University, Finland

- Luković, Ivan, University of Novi Sad, Serbia

- Lunesu, Ilaria, Università degli Studi di Cagliari, Italy

- Mangalaraj, George, Western Illinois University, United States

- Marcinkowski, Bartosz, Department of Business Informatics, University of Gdansk, Poland

- Mazzara, Manuel, Innopolis University, Russia

- Mesquida Calafat, Antoni-Lluís, University of the Balearic Islands, Spain

- Miler, Jakub, Faculty of Electronics, Telecommunications And Informatics, Gdansk University of Technology, Poland

- Misra, Sanjay, Covenant University, Nigeria

- Mohapatra, Durga Prasad, NIT ROURKELA, India

- Morales Trujillo, Miguel Ehecatl, National Autonomous University of Mexico, Mexico

- Mordinyi, Richard, Vienna University of Technology, Austria
- Norta, Alex, Tallinn University of Technology, Estonia

- Noyer, Arne, University of Osnabrueck and Willert Software Tools GmbH, Germany

- Oktaba, Hanna, National Autonomous University of Mexico, Mexico

- Ortu, Marco, University of Cagliari, Italy

- Özkan, Necmettin, Turkiye Finans Participation Bank, Turkey

- P, Adam

- Panda, Subhrakanta, Birla Institute of Technology and Science, Pilani, India

- Pereira, Rui Humberto R., Instituto Politecnico do Porto - ISCAP, Portugal

- Przybyłek, Michał, Polish-Japanese Academy of Information Technology, Poland

- Ramsin, Raman, Sharif University of Technology, Iran

- Ristić, Sonja, University of Novi Sad, Faculty of Technical Sciences, Serbia

- Salah, Dina, Sadat Academy, Egypt

- Salnitri, Mattia, University of Trento, Italy

- Sedeno, Jorge, University of Seville, Spain

- Śmiałek, Michał, Politechnika Warszawska, Poland

- Soares, Michel, Federal University of Sergipe, Brazil

- Spichkova, Maria, RMIT University, Australia

- Tarhan, Ayca, Hacettepe University Computer Engineering Department, Turkey

- Thomaschewski, Jörg, University of Applied Sciences Emden/Leer, Germany

- Torrecilla Salinas, Carlos, University of Seville, Spain

- Weichbroth, Pawel, Gdansk University of Technology, Poland

- Wróbel, Michat, Gdańsk University of Technology, Poland

- Yilmaz, Murat, Çankaya University, Turkey

- Zarour, Nacer Eddine, University Constantine2, Algeria

- Lukasiewicz, Katarzyna, Gdańsk University of Technology, Poland 


\section{Distinguishing and de ning the role of a technical leader in outsourced teams developing IT solutions using Scrum}

\author{
Jan Werewka \\ Department of Applied Com- \\ puter Science \\ AGH University of Science and \\ Technology \\ al. Mickiewicza 30, 30-059 \\ Kraków, Poland \\ Email: werewka@agh.edu.pl
}

\author{
Marcelina Wietecha \\ Faculty of Management \\ AGH University of Science and \\ Technology \\ al. Mickiewicza 30, 30-059 \\ Kraków, Poland \\ Email:marcelina.wietecha@gmail. \\ com
}

\author{
Kamil Wołczyk \\ Department of Applied Com- \\ puter Science \\ AGH University of Science and \\ Technology \\ al. Mickiewicza 30, 30-059 \\ Kraków, Poland
}

\begin{abstract}
Outsourcing software development is gaining popularity and importance. The value of an outsourced team is much higher when this team can be represented by a technical leader. Many outsourced teams use various agile methodologies, Scrum being the most popular among them. Distinguishing specialized roles in teams, such as e.g. a role of a technical leader, does not conform to some agile principles. When building an IT system, it is important for the team to have a technical leader who can communicate with internal and external stakeholders. In this paper the aspects of the communication abilities of a technical leader are considered in more detail based on conducted surveys. Developing successful technical leadership is not an easy task for a company, and that is why career paths of software engineers are an important issue regarding $H R$ competencies and leveraging costs. The paper offers some remarks concerning useful competences of technical leaders based on an example related to databases.
\end{abstract}

\section{INTRODUCTION}

$\mathrm{I}$ N IT companies many employees are software developers. An important issue for businesses and the whole IT market is their proper competence development. Nowadays, many development teams work using agile methodologies, out of which Scrum is the most popular. Having a technical leader in a team can significantly increase the value of the team, e.g. when the team is outsourced. According to Scrum principles, specialization of team members is not a desirable feature. However, outsourced teams can greatly benefit from defining the role of a leader who will perform certain functions. If teams are not outsourced, sometimes it is better to have a separate company division dealing with technical leadership.

Our hypothesis states that it is advantageous to create the role of a technical leader in Scrum teams because it results in increased efficiency and quality. It is also possible to define the requirements for a technical leader and his role in the team. On the other hand, building proper leadership in teams is a long-lasting and costly process.

The problem is that Scrum defines a team as a group of collaborators that consists of people with assigned roles: a Scrum master, a product owner, and a team member (developer). The team is inherently self-organized and no one outside the group should affect their work. Developers are responsible for creating a product. All team members contribute to product development, therefore, they are not only programmers, but also testers, analysts, etc.. In [1] some informal, implicit, transient, and spontaneous roles are identified in self-organizing teams. The selected roles of a Mentor, Coordinator, Translator, Champion, Promoter, and Terminator help the team to become more efficient. In our study we investigate the role of a technical leader in a broader context of IT company needs.

To have a basis for proposed solutions, a series of surveys were conducted in Kraków, Poland. There are many software development outsourcing projects in this region, which is the biggest outsourcing center in Europe (it occupies the ninth place in the world, according to Tholons [2]). Outsourcing causes a division of teams into these which work in the office (on-site), and these which work remotely (offshore). The issue of outsourcing IT teams has been addressed in various publications.

However, few papers have investigated applying agile methodologies and outsourcing solutions in distributed software development. In [3] adapting the Scrum methodology to work in Network Organization is proposed. The Scrum core roles are extended, and changes in Scrum artifacts are proposed; changes and high competition being the cornerstone of the approach. In [4] the problem of an effective use of Scrum practices in Global Software Development is investigated. The paper reports a multi-case study that investigates the impact of key contextual project factors on the use of Scrum practices in GSD. A similar study [5] proposes a SO-SPRM (Software Outsourcing Service Provider Relationship Model) model concerning the cooperation of both teams in the customer's office and remote teams. The study, which was carried out in India, reveals that for global software development (GSD) projects, it is important to share knowledge, trust, commitment, and flow requirements. In turn, a study conducted in Sri Lanka [6] deals with the issue of verification of software quality by outsourced companies. 
In our study, we consider four aspects of technical leadership:

1. Technical leadership in autonomous Scrum teams. In this case teams that manage projects are associated with customers, and teams developing software are part of IT companies. Software development teams should be able to make their own technical decisions, so the role of a technical leader is important. It is necessary to identify competencies of technical leadership crucial for team members. Therefore, we decided to conduct surveys to determine team members' views on this role.

2. HR (Human Resources) management in an IT company which takes into consideration a technical leadership position. In this case it is important to develop skills corresponding to company's needs. This can be obtained by defining career paths and talent management to ensure retention of valuable staff members.

3. Software developers try to develop their own careers. It would be interesting to have knowledge regarding the orientation of their development in the context of the company. Usually they tend to choose a technology or management path. The survey conducted by the authors should show the developers' perception of the roles of a technical leader and a project manager.

4. Technical leadership requirement defined by IT companies depends on current needs. A good way to identify technical leadership requirements is to investigate companies' job offers. In the paper job offers investigated are limited to technical leadership in the data base domain.

Considering all four above mentioned aspects offers a full picture of the role of technical leadership in an IT company. On the basis of such information, sustainable development of human resources can be proposed.

\section{TECHNICAL LEADER ALLOCATION IN SOFTWARE DEVELOPMENT COMPANIES}

The quality of IT systems developed by a company depends on management of software development and the competency and availability of experts. Experts can specialize in various areas, e.g. connected to the domain, technology, or architecture. They can be in top management, in a specialized company division, or allocated to teams. Putting experts in a specialized division may cause bottlenecks and a lack of deep expert knowledge of the IT systems under development. Experts can be assigned to each team, but this can lead to a lack of specialists in the company. Assigning technical experts to the team is favorable for outsourced teams.

The problem to be solved is connected with how to best allocate experts and what type of competencies are needed. The allocation of technical experts depends on many factors. In case of an IT company that intends to outsource whole teams, having technical experts inside the teams is essential.
In a software development company, technical experts may be found in top management, in specialized divisions, or in teams. Fig. 1 shows possible allocations of technical experts with relation to software development and operation of companies.

Placing technical experts in top-level management can positively influence the outcome of an IT company. The study [7] examines how the inclusion of a technology leader (e.g., CIO or CTO) in the top-level management team influences the company's performance.

Experts can be gathered in a specialized division to support institutional solutions. TOGAF (The Open Group Architecture Framework) provides [8] a method of designing enterprise architectures. In TOGAF, the Architecture Board is a key element of architecture governance strategy. This body represents all key stakeholders in architecture. In project management solutions such as e.g. PMBOK [9], a PMO (Project Management Office) is proposed for leading and supporting projects. In the process of adapting enterprise architecture in a software development company [10], it is important to define the architect's role in software project implementation. Enterprise architects should be responsible for the coordination of work among units and the overall implementation of the company's strategy.

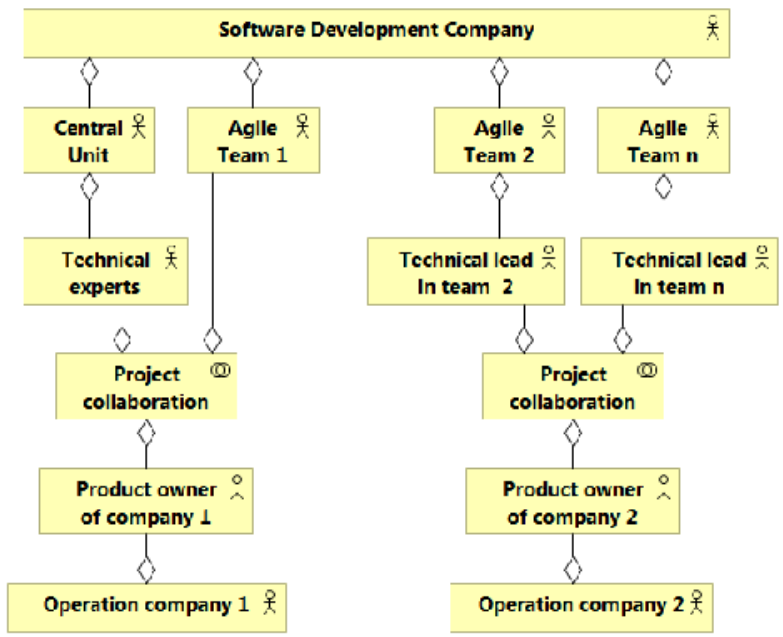

Fig. 1 Possible roles of a technical leader in software development collaboration

Placing experts in a team is important, as shown by the following case study. A solution architect is assigned to each project and is responsible for preparing and supervising the implementation of the entire solution architecture. Solution architects should cooperate closely with project managers and other persons responsible for collecting client's business requirements so that the developed solution best suits the client's needs. It is assumed [10] that a solution architect is not an active member of a project group. This solution proved to be incorrect because it leads to situations in which solution architecture is developed independently of implementation, and this might result in inconsistencies between them. 


\section{THE ROLE OF A TECHNICAL LEADER IN SOFTWARE DEVELOPMENT TEAMS}

There are many different definitions of leadership and leader in the literature. Leadership is defined here following [11] as the process of influencing others towards achieving some kind of a desired outcome. A leader is the owner of the process. A technical leader is a person who combines technical and soft skills in managing a group of people. This helps the project or the manager-especially if the manager does not have adequate technical knowledge - to lead the project to success.

A technical leader in a development team is a person who leads the team and acts as a guide for others thanks to his broad technical knowledge. He is an expert at technical issues, which allows him to organize and support team members in the implementation of tasks and resolve disputes in the team. He is the team representative and helps the team to overcome technical impediments and external stakeholders to understand technical issues.

There are other names used for a technical leader, such as a technical lead, a tech lead, and a tech leader. These names are considered synonymous. The term 'technical leader' seems more formal than 'technical lead'.

It is very important to choose the right person for the technical leader position. A technical leader must have above-average technical knowledge, engender respect and trust between team members, and be accepted by the team. The role is always played by a team member who is primarily a developer. There are various ways to identify this person. Most commonly, the leader is selected naturally in the team as the person with the broadest technical knowledge who has authority in technical issues. A team that chooses its own leader independently will never have a problem with accepting the role. It is very common that the person with the most experience is imposed on the team from outside as a leader. In selecting a leader, both hard and soft competences should be considered. A company can also ensure proper preparation of the candidate through training.

The technical leader role may cover different functions, e.g.:

1) Developing team building. The role and tasks of a leader are focused on team building. A team is defined [12] as "a small number of people with complementary skills who are committed to a common purpose, performance goals, and an approach for which they hold themselves mutually accountable".

2) Team learning. A technical leader plays an important role in the process of team learning. Clutterbuck says [13] that this process is cyclical and consists of five steps: (1) Determining development goals of the team; (2) Management of the learning process; (3) Learning process evaluation; (4) Knowledge sharing; (5) Determining relationship between effort put into the learning and understanding of the process.

3) Motivating the team. Motivating the team needs concrete actions that lead to the achievement of a predetermined purpose. Motivation is the result of unmet needs and occurs when a person becomes aware of the final outcome of his actions. In other words, need motivates action, which leads to achieving the goal [14]. A technical leader who wants motivation to be effective has to clearly define team goals. The role of a technical leader in motivating a team primarily consists of encouraging and supporting team members, giving feedback, and indicating the direction towards the goal. A leader should reward the team by offering his recognition and praise, entrusting greater responsibility, and showing trust by allowing more freedom in decision-making. The inner motivation of each member is also very important. It is created by ambition, responsibility, satisfaction, desire to develop, and the interests of each particular person. A technical leader should recognize the potential of each individual and best use it for the success of the whole team [14].

4) Communication with stakeholders. One of the major tasks of a technical leader is communication with the stakeholders on behalf of the team. He is the link between the developers and the outside world, for which technical issues are difficult to understand. A leader, who is, first of all, a developer, must have the ability to translate technical language for stakeholders [15]. He helps developers to understand and clarify the requirements of the customer. In [16].

The technical leader role should be beneficial for the software development company. A technical leader with inadequate technical and soft competences may result in the inefficient work of the team.

\section{COMPETENCY OF A TECHNICAL LEADER IN A SCRUM TEAM}

Scrum is the most popular agile software development methodology. According to the Scrum Guide [17], a Scrum team consists of a product owner, a scrum master, and the development team. The team is responsible for delivering the technical solution, which usually consists of working software. The team members are developers with different competencies, knowledge and experience. The team can include programmers, testers, analysts and solution architects. The proposed size of the team is $7 \pm 2$ members only, as this retains the ability to communicate and share knowledge.

The fact is that a technical leader in Scrum never appears as a formal role, but Rob Friesel [15] argues that the creation of a Scrum Tech Lead brings many benefits if it serves the leadership. In [16], a similar approach is presented which states that every Scrum team needs a Technical Lead to bridge the gap between technical perspectives. The author also emphasizes that responsibility for the code, making decisions autonomously, and imposing his opinions are not the Tech Lead's duties. According to [15], the Scrum Tech Lead's responsibilities fall into three categories: technical leadership, mentoring and support, and technical communication.

In order to distinguish the roles of a technical leader in Scrum teams, several surveys were conducted among 
employees of various teams in IT companies with the goal of gaining insight into these issues. Their primary objective was to obtain opinions on the desired characteristics of a technical leader, while the secondary theme was to find out how a technical team leader functions in real IT companies. In the first case, it was important to get important information about the respondents. Because only 25 people were interviewed, they were asked about the total number of years they had worked in IT teams and about the number of teams in which they had worked. The results are shown in Fig. 2.

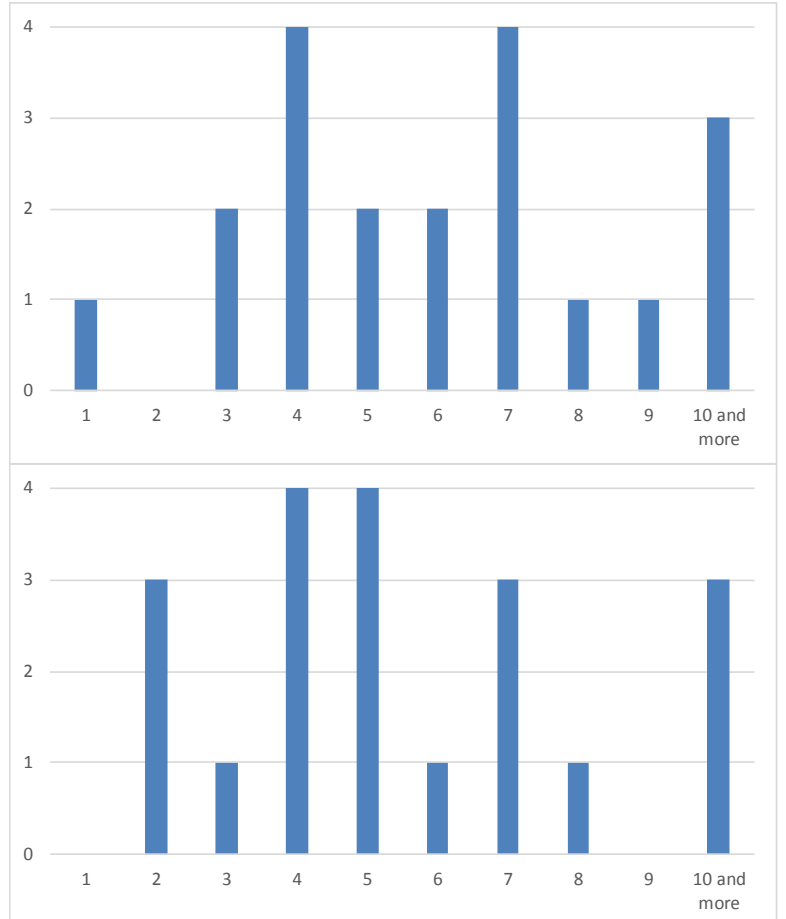

Fig. 2 Respondents' characteristics: years of work and the number of teams in which they have worked
The next question was connected with the presence of a leader in teams in which the respondents had worked. $80 \%$ of respondents answered this question positively. The second question clarified whether technical leaders were present in product development based on Scrum. In this case, $75 \%$ of respondents answered positively. From the above results, it is clear that having a technical leader is quite common in software teams.

The aim of the next question was to rank desirable features of a technical leader based on a group of 10 preselected features. The group of features was based on [18] and included: extensive knowledge and experience; communication ability; negotiation and mediation ability; building sustainable relationships in a team; openness to change; assertiveness; perseverance and patience in goal reaching; self-confidence; courage in taking decisions and responsibility; calmness.

For each characteristic respondents attributed a weight in the range 1-10. A weight of 1 meant the least important characteristic, and a weight of 10 meant the most desirable characteristic of a leader. The last column denoted as $\sum$ in Tab. 1 gives a total number of points collected for each characteristic. The obtained results reveal three characteristics - ability to communicate, extensive knowledge and experience, and the courage to make decisions - as the most important ones.

Another question was a supplementary list of other features of a technical leader that positively affect teamwork which were not included in the preselected group of 10 features. According to the respondents, who were specialists from the IT industry, a technical leader should also display good organization of work, charisma, honesty, reliability, knowledge transfer ability, vigor, understanding, positive attitude, ability to listen, and involvement.

TABLE I. MOST IMPORTANT CHARACTERISTICS OF TEAM LEADER AS SEEN FROM THE TEAM MEMBER'S PERSPRECTIVE

\begin{tabular}{|c|c|c|c|c|c|c|c|c|c|c|c|}
\hline & 1 & 2 & 3 & 4 & 5 & 6 & 7 & 8 & 9 & 10 & $\Sigma$ \\
\hline Communication ability & 0 & 0 & 0 & 0 & 0 & 0 & 0 & 24 & 63 & 100 & 187 \\
\hline Extensive knowledge and experience & 0 & 0 & 0 & 0 & 0 & 6 & 28 & 32 & 18 & 90 & 174 \\
\hline Assertiveness & 0 & 0 & 3 & 8 & 20 & 6 & 14 & 16 & 45 & 30 & 142 \\
\hline Openness to change & 0 & 2 & 0 & 8 & 0 & 24 & 35 & 8 & 54 & 10 & 141 \\
\hline Negotiation and mediation ability & 1 & 2 & 6 & 0 & 5 & 12 & 14 & 40 & 9 & 50 & 139 \\
\hline Self confidence & 0 & 0 & 12 & 0 & 10 & 6 & 28 & 40 & 9 & 30 & 135 \\
\hline Perseverance and patience in achieving goals & 2 & 0 & 0 & 12 & 0 & 12 & 21 & 48 & 9 & 30 & 134 \\
\hline Sustainable building of relationships in a team & 0 & 2 & 9 & 0 & 15 & 18 & 28 & 8 & 9 & 40 & 129 \\
\hline Calmness & 6 & 4 & 0 & 8 & 0 & 6 & 7 & 32 & 18 & 20 & 101 \\
\hline
\end{tabular}

Teams expect a leader to possess a certain level of soft competencies, e.g. the ability to transfer knowledge or listen, as well as appropriate character traits such as vigor, understanding, or a positive attitude. These qualities break down barriers between team members and a leader, making co-workers feel at ease. Commitment is also important for team members, as is reliability and good organizational skills, as these provide a role model for others to follow. 


\section{V.THE TECHNICAL LEADER'S CAREER PATH AND COMPETENCY DEVELOPMENT}

Generally, competency of a technical leader can be divided into domain and non-domain areas. Domain aspects are related to software engineering, while the non-domain aspects considered here mainly refer to social and management skills.

Basic domain requirements placed on a technical leader revolve around education and work experience. Technical leaders are required to possess skills related to programming language standards, encoding methods, company coding standards, and quality requirements. A software engineering competency model (SWECOM) may be used as a reference. This describes [19] competencies for software engineers. Additionally, it is also necessary to have knowledge of innovative solutions in related areas.

The non-domain characteristics of a technical leader cover appropriate predispositions and soft skills. By analyzing published job offers listing soft skills that IT companies require of software developers and project managers, the study [20] examines the issues of employees' competency definitions and career building in IT companies. In the conclusions, some proposals for HR departments and developers are given regarding the transition from software developer to project management positions. In [21], a study is conducted to find appropriate communication practices to be used in global outsourced agile software development projects.

For most IT companies defining a technical leader role for Scrum teams is followed by defining a technical leader position in the structure of the organization. After investigating career paths solutions in different IT companies, a typical example of career paths of technical leaders used in some IT companies is given in Fig. 3. Career paths depend largely on the size of the company, the level of the employees' technical knowledge, relationships with customers, and the type of products developed. Employees often play multiple roles in small companies. A classic path to leadership begins with a Junior Developer and then, when the employee is more independent, he is promoted to the position of a Developer. After gaining adequate experience, he becomes a Senior Developer. He then participates in the creation of products and supports less experienced team members, shares knowledge, and supervises work. Over time, a Senior Developer may decide to take on an additional role such as a Team Leader, which often necessitates the acquisition of extra soft skills. This process often requires the involvement of other people, for example, a special personal trainer. A Senior Developer can also remain in this position and become a Technical Leader. Companies frequently nominate unsuitable developers who do not have essential soft skills or competences needed for proper communication and human resources management.

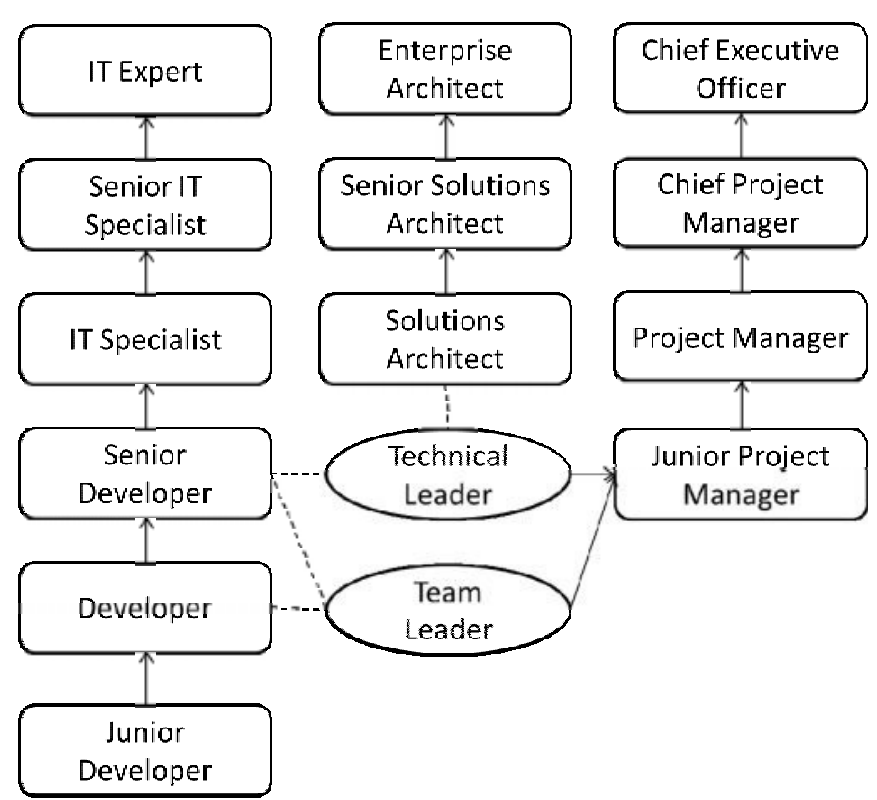

Fig. 3 Software engineer career paths, including a technical leader role

The role of a Technical Leader is usually offered to the person with the longest professional experience and the most technical knowledge, and little or no attention is paid to his soft skills. As shown in Fig. 3, fulfilling additional roles gives the developer the opportunity to an easier transition to being a Solution Architect or retraining as a Project Manager. Being a Solution Architect requires more technical competences than can be acquired as a Developer. A Project Manager takes a completely different career path that involves retraining. It is worth considering to what extent the competences an employee acquires as a Technical Leader overlap with the competences of a Project Manager. This raises the question whether being a Technical Leader is a good starting point for a career in project management.

Becoming a team leader may be an important step on the way to a project management position. Two surveys were conducted to compare the competences of technical leaders (Q1) and project managers (Q2). The results of the first survey are described in chapter 4 . Survey Q1 was conducted among software developers in various IT companies in order to assess the importance of project manager's soft skills from the point of view of developers. Survey Q2 was distributed in September 2015 to a group of selected 31 persons as a Google form.

As is evident from the surveys, project managers are expected to be able to manage themselves and their work, communicate with and understand other people, and negotiate. According to Q2 respondents, communication skills are the most important for this role. Negotiation skills also are useful in technical leadership, but to a lesser extent. Developers, however, say that one of the most important competencies of a technical leader is extensive knowledge and experience. In survey Q2 his most crucial skills include the ability to communicate his knowledge, the desire to share it with others, and a desire to be up to date with technical 
innovations; these skills appear at lower positions on the list of Project Manager competencies.

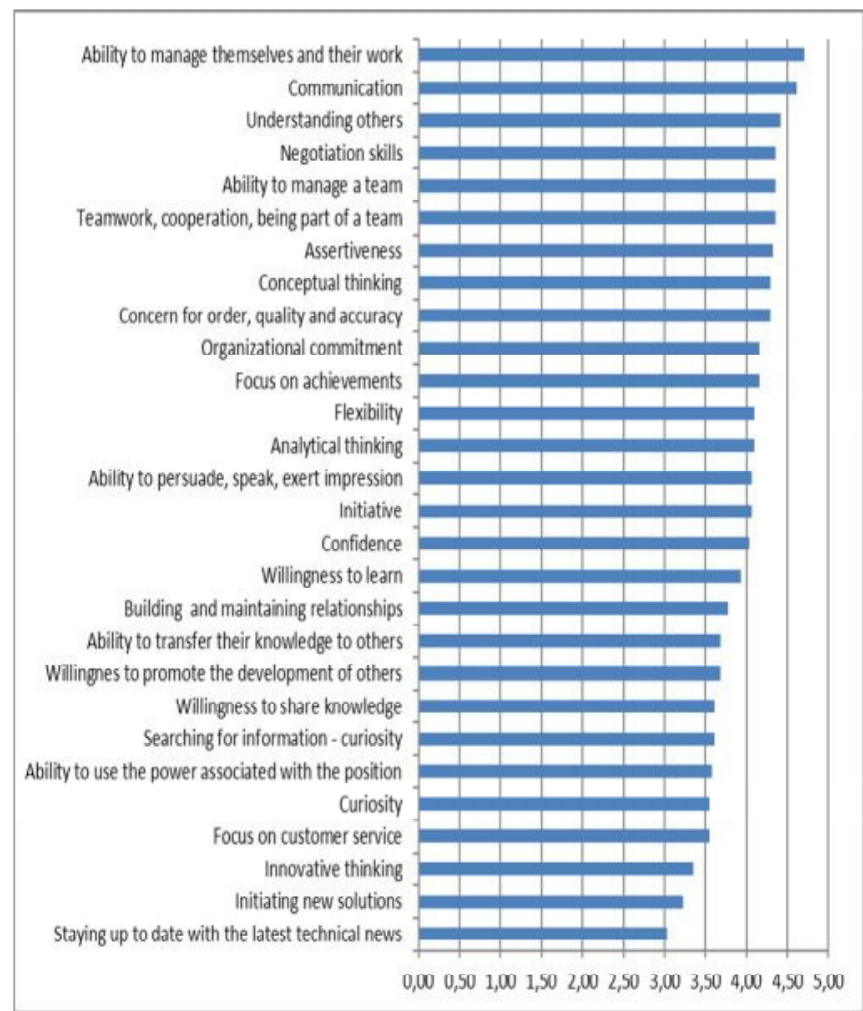

Fig. 4 Project manager competences - software developers' viewpoint

According to Q2 respondents, very important competencies of a project manager include team management skills, teamwork, cooperation, and behaving as a member of a team. However, they are not considered necessary for a technical leader. Both Q1 and Q2 respondents indicated the following competences as important: assertiveness, self-confidence, ability to build relationships, and flexibility. The last attribute in Q1 was openness to change. Other competences of a technical leader (Q1) cover courage in decision-making, perseverance, patience in the pursuit of goals, and calmness.

A technical leader who plans to be a project manager should also develop his soft skills such as conceptual and analytical thinking, organizational commitment, concern for order, quality and accuracy, the ability to speak to a group, persuasion and influence, and others. The soft competencies that are common to both lists mean that the role of a technical leader gives an employee a very good chance of becoming a Project Manager at a later stage. At the same time, we should be aware that a project manager needs more competencies and not only knowledge of project management, but also a strong commitment to personal development.

\section{DATABASE COMPETENCIES OF A TECHNICAL LEADER}

Outsourced teams may specialize in one or more areas of software engineering or technology. Almost every IT project deals with challenging problems in order to achieve scalability, security and integrity of data, and optimize database solutions.

Depending on such challenges, the competences of technical leaders may differ. As an example, technical leadership is discussed with relation to database competences. In building an optimal database solution, it is not sufficient to concentrate only on technology. There are many examples of disastrous failures of IT systems which occeured due to not only a lack of technological competence, but also to a lack of awareness of the environment in which the solution will be deployed. To overcome these problems in the future, a database practicum was introduced [22] as part of studies of database solutions at Regis University (Colorado, USA). The practicum fosters the development of soft skills by allowing students to volunteer in leadership roles, such as a Project Manager, a Technical Lead, and a Project Lead.

When considering technical leadership and database expertise, we can distinguish two approaches: the first concerns a technical leader specialized in databases, big data solutions etc., while the second is related to a general technical leader, who should have sufficient knowledge on databases. In order to determine the required competences of a technical leader specialized in databases, 100 job offers on indeed.com published in the US were analyzed.

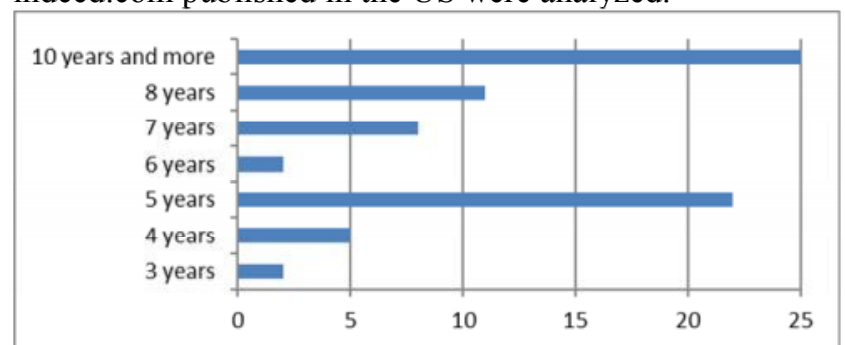

Fig. 5 Required experience of Database Technical Lead according to job offers

Most companies typically look for technical database leaders with five or ten years of experience (Fig. 5). Jobs offers for the position of a Database Technical Lead were analyzed and the required technical skills are considered below.

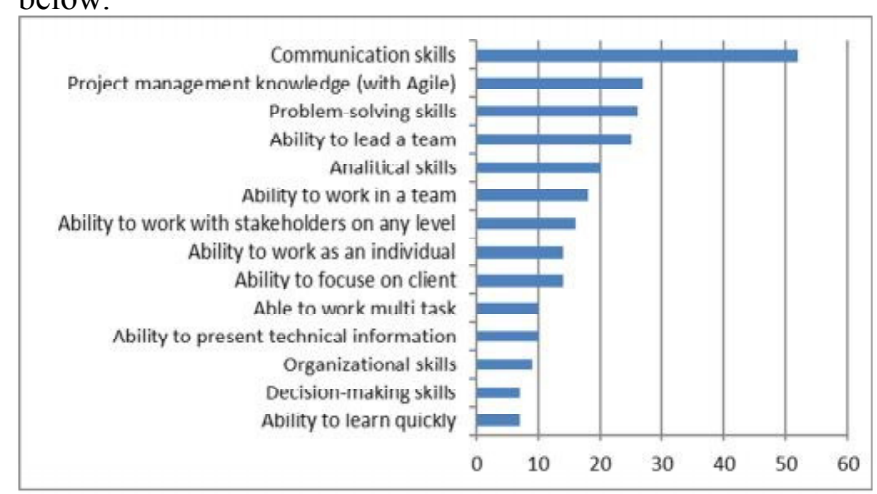

Fig. 6 Required non-domain skills of Database Technical Lead according to job offers

Supplementary to these requirements are skills and knowledge related to project management and experience as a technical leader. Job offers for the position of a Database 
Technical Lead require many non-domain competencies of leaders (Fig 6). The most common among them are communication skills (53), project management knowledge including agile approach (27), problem-solving skills (26), and ability to lead a team (25).

\section{CONCLUSIONS}

The quality of IT systems depends on the production process and the competence of the staff involved. Agile teams are now very popular. The quality of delivered software depends on technical experts who support development teams. For a Scrum team, it is important to have well prepared technical leadership that is represented by a technical leader. This paper shows that the technical lead role is well established in Scrum teams.

The role of a technical leader is not sufficiently described in the literature. In this paper, selected important characteristics of technical leaders and their career paths are described. The role's relation to project managers is clarified. It is shown that, when applying the best database solution, concentrating only on technology is not sufficient: the soft skills needed for cooperation with customers and the project team are also essential.

The study presented here is a pilot study and requires further in-depth analysis. Future studies investigating the role of a technical leader in Scrum teams should focus on the phenomenon of sustainable development. Technical leaders are required to continually develop and acquire new technical skills. Sustainable development should concentrate more precisely on different fields and the extent to which current needs are met. It is believed that acquiring knowledge and skills beyond the current demand is equivalent to wasting resources. In the rapidly changing IT sector, no one can guarantee that the knowledge gained today will be useful tomorrow. Sustainable development can also be applied in the area of soft skills development. The non-domain skills considered in the paper are a set of skills that may be useful to a technical leader, although not all of them will be used to the same extent. Further studies are needed to determine suitability of these competences, and thus to indicate the direction of the development of technical leaders.

\section{REFERENCES}

[1] R. Hoda, J. Noble, and S. Marshall, "Self-Organizing Roles on Agile Software Development Teams," IEEE Trans. Softw. Eng., vol. 39, no. 3, pp. 422-444, Mar. 2013.

[2] Tholons, "2015 Top 100 Outsourcing Destinations." www.THOLONS.com, 2015.

[3] L. D. Sienkiewicz and L. A. Maciaszek, "Adapting scrum for third party services and network organizations," in 2011 Federated Conference on Computer Science and Information Systems (FedCSIS), 2011, pp. 329-336.
[4] E. Hossain, P. L. Bannerman, and R. Jeffery, "Towards an Understanding of Tailoring Scrum in Global Software Development: A Multi-case Study," in Proceedings of the 2011 International Conference on Software and Systems Process, New York, NY, USA, 2011, pp. 110-119.

[5] S. A. Kumar and A. K. Thangavelu, "Factors affecting the outcome of Global Software Development projects: An empirical study," in 2013 International Conference on Computer Communication and Informatics (ICCCI), 2013, pp. $1-10$.

[6] D. Jayathilake, H. Yaggahavita, U. Senanayake, C. Elvitigala, and D. Sriyananda, "A scalable product quality verifier framework for a outsourcing supplier," in 2011 IEEE International Conference on Computer Applications and Industrial Electronics (ICCAIE), 2011, pp. 390-395.

[7] J. Taylor, A. Sahym, and J. Vithayathil, "Do Powerful Technology Leaders Make a Difference in Firm Performance?," 2015, pp. 4502-4512.

[8] The Open Group, TOGAF Version 9.1, 10th New edition edition. Zaltbommel: van Haren Publishing, 2011.

[9] PMI, A Guide to the Project Management Body of Knowledge: $P M B O K(R)$ Guide, 5 edition. Newtown Square, Pennsylvania: Project Management Institute, 2013.

[10] K. Jamróz, D. Pitulej, and J. Werewka, "Adapting Enterprise Architecture at a Software Development Company and the Resultant Benefits," in Software Architecture, vol. 8627, P. Avgeriou and U. Zdun, Eds. Cham: Springer International Publishing, 2014, pp. 170185.

[11] J. P. De Jong and D. N. Den Hartog, "How leaders influence employees' innovative behaviour," Eur. J. Innov. Manag., vol. 10, no. 1, pp. 41-64, 2007.

[12] J. R. Katzenbach and D. K. Smith, The Wisdom of Teams: Creating the High-Performance Organization, Reprint edition. Harvard Business Review Press, 2015.

[13] D. Clutterbuck, Coaching the Team at Work. London; Boston: Nicholas Brealey International, 2007.

[14] M. Armstrong, Managing People: A Practical Guide for Line Managers. Kogan Page Publishers, 1998.

[15] R. Friesel, "Defining the Scrum Tech Lead," Tech.Dealer.com, 23-Jul-2015. .

[16] Lemi Orhan Ergin, "Do we need Technical Leads in Scrum?," Flying to Moon, 12-Jun-2011. .

[17] K. Schwaber and K. Sutherland, Scrum Guide. 2016.

[18] M. Sieraczkiewicz, Technical leadership. Gliwice: Helion, 2016.

[19] IEEE Computer Society, "Software Engineering Competency Model Version 1.0 SWECOM." IEEE Computer Society, 2014.

[20] J. Werewka and M. Wietecha, "Impact analysis of soft skills in transition from software developer to project manager positions," Bus. Inform., vol. 38, no. 4, pp. 64-90, 2015.

[21] N. Schmidt and C. Meures, "'Mind the Gap': An Analysis of Communication in Agile Global Outsourced Software Development Projects," in 2016 49th Hawaii International Conference on System Sciences (HICSS), 2016, pp. 501510.

[22] R. T. Mason, "Database Practicum for Teaching Database Administration and Software Development at Regis University," J. Inf. Technol. Educ. Innov. Pract., vol. 12, 2013. 



\section{$4^{\text {th }}$ Conference on Multimedia, Interaction, Design and Innovation}

$\mathbf{M}$ IDI Conference provides an interdisciplinary forum for academics, designers and practitioners to discuss the challenges and opportunities for enriching human interaction with digital products and services.

The main focus of MIDI Conference is exploring design methods for creating novel human-system interaction, developing user interfaces and implementing innovations in usercentred development of advanced IT systems and on-line services.

\section{TOPICS}

Topics of interest include (but are not limited to) the following areas:

- interactive multimedia and multimodal interaction design

- novel interaction techniques, voice interfaces, interactive multimedia

- ubiquitous, multimodal, pervasive and mobile interaction, wearable computing

- novel information visualization and presentation techniques, Augmented/Virtual Reality

- design methods for usability, accessibility and outstanding user experience

- prototyping of user interfaces and interactive services

- human-centred design practices, methods and tools, user interface design

- unfolding trends in HCI research and practice, customer experience, Service Design

- advances in user-centred interaction design

- understanding people and interactions: theory, concepts, models and methods

- understanding people and interactions: contextual, ethnographical and field studies

- critique and evolution of methods, processes, theories and tools for human-computer interaction

- novel methodologies for conceptualization, design and evaluation of interactive products and services

\section{SteEring CommitTeE}

- Brocki, Lukasz, Polish-Japanese Academy of Information Technology

- Koržinek, Danijel, Polish-Japanese Academy of Information Technology, Poland

- Landowska, Agnieszka, Gdansk University of Technology, Poland

- Wichrowski, Marcin, Polish-Japanese Academy of Information Technology, Poland

- Wołk, Krzysztof, Polish-Japanese Academy of Information Technology, Poland
- Wróbel, Michat, Gdańsk University of Technology, Poland

\section{SECTION EDITORS}

- Marasek, Krzysztof, Polish-Japanese Academy of Information Technology, Poland

- Romanowski, Andrzej, Lodz University of Technology, Poland

- Sikorski, Marcin, Polish-Japanese Academy of Information Technology, Poland

\section{REVIEWERS}

- Ardito, Carmelo, Univeristy of Bari

- Brocki, Lukasz, Polish-Japanese Academy of Information Technology

- Fernández Iglesias, Manuel Jose, Vigo University, Spain

- Fjeld, Morten, Chalmers University of Technology, Sweden

- Forbrig, Peter, University of Rostock

- Guttormsen, Sissel, University of Bern, Institute of Medical Education, Switzerland

- Jaworski, Tomasz, Lodz University of Technology

- Kaptelinin, Victor, Umea University

- Koržinek, Danijel, Polish-Japanese Academy of Information Technology, Poland

- Kołakowska, Agata, Faculty of Electronics, Telecommunications And Informatics, Gdansk University of Technology, Poland

- Landowska, Agnieszka, Gdansk University of Technology, Poland

- Manzke, Robert

- Markopoulos, Panos, Eindhoven University of Technology

- Marti, Patrizia, University of Siena, Italy

- Masoodian, Masood, Aalto University

- Miler, Jakub, Faculty of Electronics, Telecommunications And Informatics, Gdansk University of Technology, Poland

- Obaid, Mohammad, Koç University

- Pribeanu, Costin, National Institute for Research and Development in Informatics - ICI Bucuresti

- Satalecka, Ewa, Polish-Japanese Academy of Information Technology

- Slavik, Pavel, Czech Technical University

- Toro, Carlos, Vicomtech 
- Unland, Rainer, Universität Duisburg-Essen, Germany

- Vanderdonckt, Jean, Université catholique de Louvain, Belgium

- Visciola, Michele, Experientia

- Wichrowski, Marcin, Polish-Japanese Academy of Information Technology, Poland

- Wieczorkowska, Alicja, Polish-Japanese Academy of Information Technology, Poland

- Windekilde, Iwona, Aalborg University

- Winkler, Marco, University Paul Sabatier
- Wojciechowski, Adam, Institute of Inf. Techn., Lodz Univ. of Techn.

- Woźniak, Paweł W., University of Stuttgart, Germany

- Wołk, Krzysztof, Polish-Japanese Academy of Information Technology, Poland

- Wróbel, Michał, Gdańsk University of Technology, Poland

- Zahiris, Panayiotis, Cyprus University of Technology

- Ziegler, Juergen, University of Duisburg-Essen 


\section{Affective Design Patterns in Computer Games. Scrollrunner Case Study}

\author{
Grzegorz J. Nalepa \\ Jagiellonian University, \\ ul. Gołębia 24, 31-007 Kraków, Poland \\ AGH University of Science and Technology, \\ al. Mickiewicza 30, 30-059 Kraków, Poland \\ e-mail: gjn@agh.edu.pl
}

\author{
Barbara Giżycka \\ Jagiellonian University, \\ ul. Gołębia 24, 31-007 Kraków, Poland \\ e-mail: barbarag.cka@gmail.com
}

\author{
Krzysztof Kutt \\ AGH University of Science and Technology, \\ al. Mickiewicza 30, 30-059 Kraków, Poland \\ e-mail: kkutt@agh.edu.pl
}

\author{
Jan K. Argasiński \\ Jagiellonian University, \\ ul. Gołębia 24, 31-007 Kraków, Poland \\ e-mail: jan.argasinski@uj.edu.pl
}

\begin{abstract}
The emotional state of the user is a new dimension in human-computer interaction, that can be used to improve the user experience. This is the domain of affective computing. In our work we focus on the applications of affective techniques in the design of video games. We assume that a change in the affective condition of a player can be detected based on the monitoring of physiological signals following the James-Lange theory of emotions. We propose the use of game design patterns introduced by Björk and Holopainen to build games. We identify a set of patterns that can be considered affective. Then we demonstrate how these patterns can be used in a design of a scroll-runner game. We address the problem of the calibration of measurements in order to reflect responses of individual users. We also provide results of practical experiments to verify our approach.
\end{abstract}

\section{INTRODUCTION}

$\mathbf{I}$ $\mathrm{N}$ ORDER to improve usability and provide superior user experience we seek new sources of information that can be used in human-computer interaction. A new dimension of such an information is related to the emotional state of the user. The affective condition can be determined based on number of data, e.g. heart rate, expression of face, etc. This is the domain of the Affective Computing (AfC) paradigm, that was originally introduced 20 years ago [1]. We believe, that information regarding the affective condition of a user, can lead to a better understanding of interactions of users with machines, and interfaces.

In our work we focus on the applications of AfC techniques in the area of the design of video games. We assert that these techniques can improve the gaming experience of the player. In fact, the affective component can, and should be incorporated into the design process of video games. In order to do so, we propose the use of game design patterns as introduced in [2].

There are number of models of human emotions considered in psychology and adopted in AfC. In our work, we assume that a change in the affective condition of a player can be detected and identified based on the monitoring of physiological signals, following the James-Lange theory of emotions [3]. The new generation of miniaturized computer sensors can be used to measure such signals and deliver sensor data in real time to a gaming system. In fact, our priority is to focus on sensors that can be worn by a user, e.g. wearable wristbands. While such devices do not offer high quality of measurement, they are non intrusive and can be easily used by a player during a video game. Furthermore, they are not very expensive which makes them accessible to many players.

The original contributions of this paper are as follows: First we identify a set of game design patterns that can be considered affective, which means that they elicit an emotional response from the player. Then we demonstrate how these patterns can be used in a design of a simple yet illustrative game. Next, we address the problem of the proper calibration of measurements in order to reflect differences in responses of individual users. Finally, we provide results of practical experiments supporting our claims. Our work follows our early experiments with virtual reality discussed in [4].

The rest of the paper is composed as follows. In Section II we briefly introduce the affective computing paradigm, and selected methods and models we use in our work. In Section III we discuss the concept of patterns in game design. We introduce our motivation in Section IV. Then in Section V we demonstrate a practical design example of a scroll-runner game with affective components. To verify our assertions, we planned and conducted practical experiments described in Section VI. We briefly discuss other works related to our research in Section VII. The paper ends with summary and plans for future works in Section VIII.

\section{AfFective COMPUTING}

Affective computing is a paradigm originally proposed in 1997 by Rosalind Picard from MIT Media Lab [1]. It uses results of biomedical engineering, psychology, and artificial intelligence. It aims at allowing computer systems to detect, 
use, and express emotions [5]. It is a constructive and practical approach oriented mainly at improving human-like decision support as well as human-computer interaction. AfC puts interest in design and description of systems that are able to collect, interpret, process (ultimately - simulate) emotional states (affects). Assuming that emotions are both physical and cognitive, they can be studied interdisciplinary by computer science, biomedical engineering and psychology. For affective computing there are two crucial elements to be considered: modes of data collection and ways of interpreting them in correlation with affective states corresponding to emotions.

The first is carried out by selection of methods for detecting information about emotions, using sensors capturing data about human physical states and behaviors. Today most often processed information is about: speech, body gestures and poses, facial expressions, physiological monitoring (blood pressure, blood volume pulse, galvanic skin response). In our research we focus on the last source of signals, and we assume a range of wearable physiological sensors.

The second crucial element of affective computing paradigm relies on application of selected algorithms on acquired data to develop models of interpretation for affective states. State of the art methodologies assume the use of the full range of available methods of data classification and interpretation [5].

Computational models of emotions derive from our interpretation of psychological states and cognitive processes and are in essence a way to describe relation between those two phenomena. Affective computing makes its own use of models by applying them to bio-physiological data obtained from sensors in such a way that they can be used in specific software. Defining "emotion" is a challenge. Modern theories of emotions have their origin in 19th century. William James theorized about affects in terms of reactions to stimuli. He was precursor to appraisal theory which is among most popular in the community of computational emotional modeling [6], [7], [8]. One of the most popular appraisal theories is OOC [9] which categorizes emotion on basis of appraisal of pleasure/displeasure (valence) and intensity (arousal). These are quantifiable values that can be measured and processed ascribing different kinds of emotions (i.e. positive self-attribution of intensive value might be interpret as "pride"). Another important set of theories of emotions is less about discrete states and more about relational affect states tracked on a number of continuous dimensions [10]. Dimensional models similarly to appraisal map emotion-evoking impulses and states triggered. Popular PAD theory [11] considers Pleasure, Arousal and Dominance dimensions. Different theories of emotion lead to various models which in turn lead to variety of emotion-oriented systems. Good example is EMA [10] - a system implemented on the Soar cognitive architecture which explains dynamic affects through a sequence of triggers. Another system is WASABI [12] believed to be one of most general models of emotion (mainly for simulation).

Nowadays most popular GUIs are based on windows, menus and forms. Natural User Interfaces (NUI) are meant to reduce the barriers for users even further while empowering them to perform more complicated tasks smoothly. In Adaptive User Interfaces (AUI) paradigm the assumption is that interfaces are subjects to modification as a result of interaction with the user. Adaptation can take place on many levels. Most often it means dynamically scalable quantity of displayed information depending on users demands and capabilities.

User interfaces based on emotion processing are in a way fusion of NUI and AUI. They are more "natural" than classic metaphor-based UIs because for humans "emotive" means natural. First, we have to keep in mind that in today's practice of UI design "emotive interface" means above all the use of techniques that cause emotions in receiver by using classical means of expression and communication. For us much more interesting will be the inclusion of affective processes at interfaces at the level of affective feedback for user.

In our work, we assume it is possible to identify a highlevel emotional state from low-level sensory data. We believe, that Jesse Prinz's Embodied Appraisal Theory [13] may be useful to reach this goal. According to it, emotions are build up by two parts: (a) form - bodily changes perception (as in the classical James-Lange theory [3]) and (b) content relationship between agent and environment. As an example, faster heart rate (form) and perception of a loud sudden noise (content) build up fear.

In the next section we will discuss the design patterns in video games. Extending them with affective information will lead to new design methods for computer games.

\section{GAMe Design PATterns}

One of the most important elements when it comes to game design are mechanics. They are basic building elements of the whole game structure. If we define the game as "the voluntary attempt to overcome unnecessary obstacles" [14], there are two main factors required to establish "gaming situation". These are the mental attitude of a player entering the play ("lusory attitude") and rules prohibiting the use of more efficient for less effectual means ("lusory means"). The role of the designer of the game is to create the frames (game design) that make this kind of activity (play) possible and pleasurable. It takes place mostly through creating constitutive rules (mechanics) which activated by the player (dynamics) result in his or her affects (user's experience). Another basis for the engagement are storytelling and interface design, leading to the interactivity of play that causes player's immersion (see: [15]), but these are less important from our point of view. In conclusion mechanics are main factor constructing gameplay resulting in player's affective experience.

We can express rules of play as the verbs describing what player can do inside designed game system: he can "jump" the avatar, "hit" the enemy, "collect" the coins, "solve" the puzzles. Usually, the player is aware of most of the rules. Mechanics are very similar "verbal" constructs, but widely they include invisible principles of the game system, those implemented deeper in the software, frequently not displayed to the user interface. For example when we play an arcade game we are aware of the rules that tell us to shoot the enemies, but we 
usually do not know about details of the algorithms governing enemy A.I. Both shooting and A.I.'s behaviors are mechanics.

Particular game mechanics are a repeating phenomena that strongly depend on the genre. An interesting fact is that the dynamics (groups of mechanics activated by player) tend to have emergent properties - it means that different gameplays cannot be directly derived from basic mechanics. This case seems to be the main source of diversity in the gaming domain.

Repetitive nature of the mechanics is also an origin of the idea of game design patterns. There are few formulations of the concept of modeling and aligning basic elements of lusory structures. One of the most interesting is that proposed by S. Björk and J. Holopainen [2]. It is an approach to creating a language for talking about gameplay. As the authors state: "Essential to the discussion of gameplay are the different aspects of gameplay that can exist. Understanding these aspects is important if one wants to go into details about a specific game one is playing [...] there is a lack of terminology associated with the elements of gameplay. We offer a solution to this [...]" [2]. In Björk's and Holopainen's framework besides the description of the structural elements of overall gaming situation, we find a semi-formal exposition of particular instances of gameplay.

For example components such as boundary (rules, modes, goals of play), temporal (actions, events, closures), holistic (game instance, session, play session), structural (interface, game elements, players, facilitator) are described in details.

Game design patterns rely, as authors state: "on general descriptions of particular areas of gameplay without using quantitative measures" [2]. That means characterizations are simple definitions with detailed descriptions of relationships to other patterns. There are three basic types of relations: instantiation (patterns tend to be present together with others, some naturally imply the presence of others), modulation (patterns change or are changed by the presence of others), conflict (patterns render the presence of others impossible).

Basic design pattern template has the following form: Name (arbitrary); Core definition; General Description; Clues for Using the Pattern; Consequences; Relations; References. In [2] authors identified and described in detail over 290 basic patterns. The Game Design Patterns framework is specially designed to facilitate the work of designers and analysts. They allow for easy diagnose of potential problems and identification of nodes where emergent properties can occur.

One of the goals of our proposal is to distinguish an arbitrary set of design patterns which, we believe, can cause affective reaction of the player. Our intention is also to test if embodied dynamics resulting from this emotion-invoking mechanics can be observed on a physiological level and included in the main game loop. In the case of success, such operation should allow for creating more complex feedback loops in order of suppressing or amplifying evoked emotions.

\section{Motivation}

In our work we aim at demonstrating, how methods of affective computing can improve design of video games. We base our work on the game design patterns [2]. Specifically, we identify certain patterns that in our opinion can be considered "affective". We expect an emotional response of a player of a video game that contains these patterns. We believe that a thoughtful application of these patterns can lead to more immersive games and improve the gaming experience. The motivation of our work is to provide a method for identification, personalization, and application of the affective design patterns in video games.

Our objective is to provide integration of a sensory framework with a gaming environment. The framework will use wearable physiological hardware sensors for detection of user affects. Ultimately we aim at extending the game design process by the use of affective patterns, and introduce an affective loop in the gameplay. Based on our experience with various hardware, we selected two most promising ones. Empatica E4 is an advanced sensory wristband based on the technologies previously developed in the Affective Computing division of MIT Media Lab. Blood volume pulse and galvanic skin response sensors, as well as infrared thermopile and accelerometer are on board [16]. Microsoft Band 2 was developed mainly for tracking fitness goals. Equipped with optical heart rate, skin temperature and galvanic skin response (GSR) sensors as well as accelerometer, available through well documented Software Development Kit.

To verify our hypothesis we provide a two phase experiment. We assume, that the emotional responses of a player can be detected by the analysis of his physiological responses, such as heart rate. We expect there are individual differences of the responses of different players. To address this challenge we provide an initial calibration phase. During it, the player is exposed to a series of pictures evoking emotional response. These pictures are selected from the NAPS [17] data base ${ }^{1}$.

The second phase of the experiment uses a simple video game. In this game a series of design patterns is identified. We consider two variants of the game. The first basic variant does not include the patterns that we consider affective. In the affective variant we augment the game with design patterns resulting in stronger emotional responses of the player.

In the next section we discuss a design of a video game to be used in our experiments, with the use of design patterns.

\section{Practical Design of an Affective Game}

In order to implement our ideas, we started with the design of a simple video game. In the game some basic design patterns selected from Björk's and Holopainen's collection can be observed. The selected design patterns, both affective and non-affective ones, that were implemented in the game design are described in part V-B.

\section{A. Bridge Scroll-runner}

For the purposes of the study, a simple video game was designed. As video games are already well known as a mul-

${ }^{1}$ NAPS stands for Nencki Affective Picture System (http://lobi.nencki.gov. $\mathrm{pl} / \mathrm{research} / 8$ ) and is a set of affective images. The dataset consists of standardized images, as well as normative ratings of valence and arousal. It is freely available for noncommercial use by request. 
timodal, interactive tool for providing entertainment [18], the game design was inspired by platformer games. In this subgenre of action games, the player's goal is to control the character in order to traverse through the game world and complete subsequent levels. The character usually moves by running and jumping on the platforms placed along the way, hence the name "platformer" game. The player is challenged by various obstacles that are placed in the game world in order to prevent him from completing the game, such as holes, gaps, enemies, etc. Some popular examples of platform games are Donkey Kong (1981) or Super Mario Bros (1985).

The reason we chose a platformer game and not some other genre is that platform games are relatively easy to design and to control all their parameters, which includes applying and subtracting design patterns. Some patterns, such as collectibles in case of platform games, are already an inherent feature of the genre [19]. What is more, the game sessions can be short, but nonetheless entertaining for the player.

The concept of the game that was designed for the purpose of this study refers to traditional English nursery rhyme, „London Bridge is Falling Down”. The player's avatar (the character that the player controls in the game), is an English gentleman that needs to rush past the bridge that is falling apart. To control the avatar, the player uses left and right arrow keys on keyboard to navigate (the character proceeds from left to right), and the spacebar to jump. Therefore, the goal of the game is to complete the level within the specific limited time, and completing all of the five designed levels results in completing the game. In the basic variant of the game, the player completes the level by collecting required number of points, which increases per level (1000 points for the first level, 2000 points for the second level, etc.). The player collects the points, thereby increasing his score, by picking up diamonds that are placed along the way through the bridge. Each diamond increases the player's score by 10 . Current score is showed in the top left corner of the screen.

To make the game more challenging, each subsequent level has its time limit narrowed (90 seconds the first level, 75 seconds for the second level, 60 seconds for the third, etc.). The remaining time is showed in the top center part of the screen. If the player fails to collect the objective of collecting the number of points required for the certain level, the game is over - the bridge falls down, and the player has to start the game from the first level. Besides the diamonds that are worth 10 points each, the player can pick up the little clocks that grant additional 5 seconds to the remaining time. To enable the new player to familiarize with the game mechanics, a tutorial level is provided at the beginning of each game session. The tutorial level serves as a teaching ground for the player to learn the controls and objectives of the game. The player is given 5 minutes, within which he explores the level and the game elements. The tutorial level is completed when the score reaches 1000 points. The tutorial level appears each time the game is started (after pressing Enter key), but it can be skipped by pressing $[\mathrm{P}]$ on the keyboard when it is entered.

In the affective variant of the game, the gameplay is en- hanced by several elements that instantiate some game design patterns, which will be specified in the following section. Firstly, the score and time indicators are replaced by narrative description (,bad”, „nice”, ,good”, and ,excellent”) and the remaining time is represented by a circular indicator. Secondly, once every few seconds (ranging from 5 to 15), an event will occur that will decrease the current score (a brick falling from the top of the screen) or the remaining time (a crow approaching the character from the right part of the screen).

The game was designed using Game Maker Studio version 1.4. software (YoYo Games). All of the game sprites and materials (background, music) were either created by us (some sprites, using GraphicsGale Free Edition) or acquired from free online resources (e.g. http://freepik.com). In Figure 1 an example of design session of the game is shown. Actual gameplay of the affective variant of the game is presented in Figure 2.

\section{B. Identification of Patterns}

In the basic variant of the game that is used in this study, a subset of game design patterns from those proposed by [2] was identified by us. These are:

1) Alarms: the notifications that appear as a red text in the top center part of the screen (below the remaining time indicator) that inform the player that half of the remaining time has passed.

2) Avatar: the character that is controlled by the player.

3) Collecting: the objectives of the game are reached by collecting the diamond that increase the player's score.

4) Deadly Traps: events that result in game over. In case of this game it is the falling off the platform.

5) Dexterity Based Actions, Evade and Maneuvering: these patterns refer to the mode of play that poses some challenge for the player's dexterity and eye-hand coordination (moving and jumping to collect the diamonds, evading falling bricks and incoming enemies).

6) Levels: spatial structures within the game world. The player traverses through the level in order to complete the objective of the game.

7) Movement: pattern refers to the action of moving within the game world.

8) Pick-Ups: items (clocks) that the player may optionally gather while playing, which grant him additional time to complete the level.

9) Rhythm-Based Actions: the collectibles in the game are placed in a manner (clusters) that resembles the rhythm of the tune played in the background.

10) Score: the numerical representation of the player's progress in the game.

11) Single-Player Games: this pattern refers to the general mode where there is only one player in a game instance.

12) Time Limits: some games, such as "London Bridge" that is used in this study, rely on the fact that the time within which the player can complete some action or reach a certain state in the game is somehow restricted. 


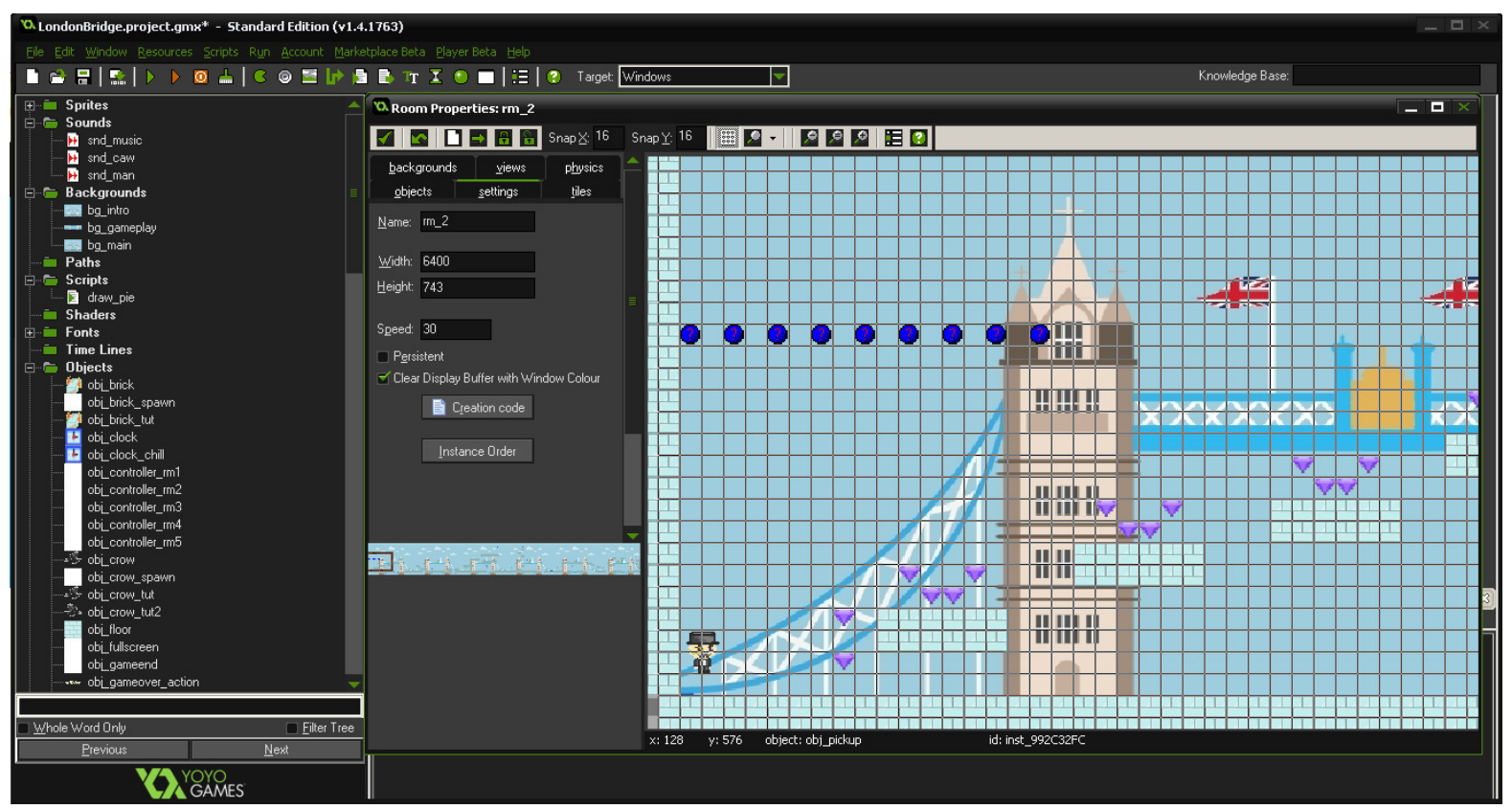

Fig. 1. Design of the scroll-runner game in the Game Makers Studio

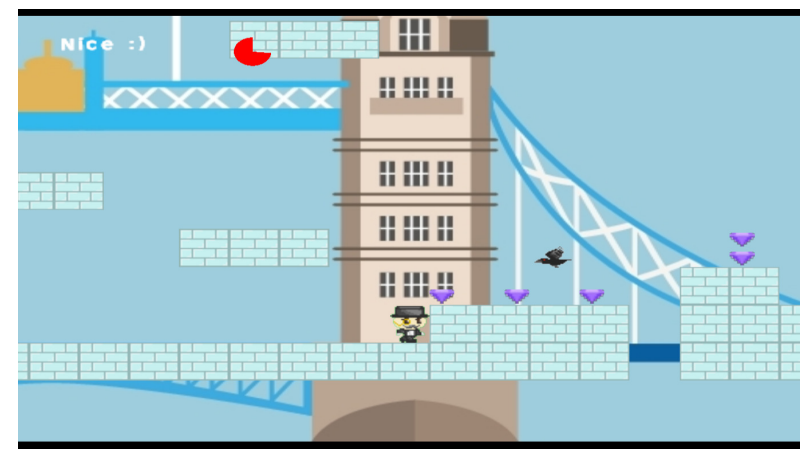

Fig. 2. Gameplay of the affective variant of the game. One can see the descriptive score (,nice”) and the crow approaching the character

\section{Affective Patterns}

In the affective variant of the game, some additional patterns are implemented:

1) Emotional Immersion: a higher level pattern that describes the player state that is hopefully reached when he becomes emotionally engaged in the gameplay.

2) Enemies and Obstacles: considered together, are elements that hinder the player trying to complete the game objective. Bricks and crows that consume score points and remaining time respectively.

3) Imperfect Information: occurs when some aspect of information about the game state is hidden from the player, for example - the exact score and time remaining.

In this study, player's affective responses - reflected by changes in his arousal, especially in heart rate and galvanic skin response - are anticipated in both variants of the game.
However, we hypothesize that the responses in the affective variant of the game will be stronger. We predict that exceptionally strong responses will appear when "Enemies and Obstacles" pattern occurs.

\section{EXPERIMENTS AND EVALUATION}

\section{A. Outline of the Calibration Phase}

Valence and arousal are two of several dimensions often used to characterize emotional experience (for overview of see [20]). Especially, valence differentiates states of pleasure and displeasure, and arousal contrasts states of low activation/relaxation and excitation [17], [21]. These dimensions are revealed in Autonomic Nervous System (ANS) activity, the part of nervous system responsible for unconscious autonomous functions like respiration and reflex actions. Research indicates that they can be measured by the use of ANS measures, inter alia, by the use of Heart Rate (HR) and Skin Conductance/Galvanic Skin Response (GSR) measures (for meta-analysis see [22]). Based on this research, the calibration phase was prepared. During it subjects are exposed to affective pictures from NAPS dataset. At the same time current levels of HR and GSR are collected by the wristband. Participants are also asked to evaluate the perceived arousal of each of the pictures. The goal of this phase is to combine physiological data with pictures' valence-arousal scores in order to prepare HR and GSR patterns as functions of them. These patterns will be used in the Gaming Phase as a reference to evaluate if affective design patters work as intended.

Experimental procedure was designed in the PsychoPyBuilder as shown in Figure 3. It was then detailed using the PsychoPy 2 (v 1.84.2) environment and executed on notebook with four cores processor $2.50 \mathrm{GHz}, 4 \mathrm{~GB}$ RAM working under 
the control of Windows 7 Professional OS $^{2}$. Stimuli were presented on 15,6" notebook screen with $1366 \times 768$ resolution. Physiological data was collected by MS Band 2 and Empatica E4 bands. Bands were paired over Bluetooth with Samsung Galaxy J3 smartphone, on which custom application for data acquisition, developed by the authors, was installed. Data from smartphone and notebook is synchronized using the Lab Stream Layer ${ }^{3}$, a protocol for time-synced data transmission over local network, developed at the Swartz Center for Computational Neuroscience, University of San Diego.

Affective stimuli was a subset of Nencki Affective Picture System. Pictures in this set have assigned valence and arousal scores on 7 point scale [1-7] [17]. It was arbitrarily divided into three same-length intervals: low $[1,3)$, neutral $[3,5]$ and high $(5,7]$. Then, based on these intervals, group of pictures were selected in order to cover the groups of interest: (a) neutral valence and neutral arousal -10 pictures $(+6$ pictures for training session), (b) low valence and high arousal - 15 pictures, (c) high valence and low arousal - 15 pictures, (d) high valence and high arousal - 15 pictures. NAPS has few low valence and low arousal pictures, therefore this group was not represented in the selected subset.

The experiment is done individually and takes about 22 minutes. At the beginning, subject seats comfortably in front of the notebook. The wrist band is then placed on the less used hand, to minimize muscle artifacts associated with user interaction with procedure. PsychoPy procedure starts with instruction and training session with 6 neutral images. Each stimuli is presented for 3 seconds, then it disappears and subject has 5 seconds to evaluate the arousal on 7-levels scale $[1,7]$. After that another image appears without any pause. Training session ends with time for questions to experimenter. Then main session starts with 30 seconds of blank screen to get baseline HR and GSR recordings during inactivity. After that subjects is exposed to 3 series of 18 pictures. Series are separated by the 30 seconds breaks.

\section{B. Outline of the Gaming Phase}

After the subject completes the task in the calibration phase, the experimenter immediately runs the „London Bridge” game on the same machine for the subject. The subject still has the wristband on while the synchronization between the smartphone and the notebook is maintained. Before the Gaming Phase, the subject is randomly ascribed either to control group, where subjects play the basic game variant, or experimental group, where subjects play the affective game variant. After pressing Enter key on the notebook to start the game, a 30 seconds of blank screen is presented to get the baseline HR and GSR recordings for the beginning of the Gaming Phase. After 30 seconds, tutorial level appears and the subject is allowed to freely explore the game mechanics, and then to play the game for the desired time, but not exceeding 20 minutes.

\footnotetext{
${ }^{2}$ PsychoPy (http://psychopy.org) is a standard software framework in Python to support a wide range of neuroscience, psychology and psychophysics experiments.

${ }^{3}$ See: https://github.com/sccn/labstreaminglayer.
}

\section{Practical Experiments}

The procedure of the Calibration Phase was applied in the first experiment that was conducted in late April 2017 (Eurokreator lab, Krakow, Poland). The subjects (6 persons) were two male and 4 female participants of the workshops which were held in the lab. The full procedure, consisting of Calibration Phase and Gaming Phase, has been conducted as the second experiment in mid-June 2017. The subjects ( 9 persons) were Polish students from AGH-UST.

At the time of experiments the Lab Stream Layer synchronization method was not running yet. Therefore, the synchronization between the phone and the notebook was acquired by the subject simultaneously pressing spacebar on the notebook and „START RECORDING” button on the phone. It should be noted that due to technical issues a total of 52 pictures was used in the first experiment instead of 54.

\section{Evaluation of Results}

During the calibration phase, the following data is acquired:

- NAPS Valence and arousal scores - shared with the NAPS set.

- Baseline HR and GSR - the average value of 30 seconds recordings of HR and GSR at the beginning of main session.

- Gamers arousal score - the rating given by the player after seeing each of the stimuli.

- HR and GSR reactions - the HR and GSR levels recorded from the appearance of the stimulus to the appearance of the next stimulus.

The ultimate goal of this phase is to prepare HR and GSR patterns as a function of valence-arousal scores. In order to achieve this, the following analyses are being conducted:

- Gamers arousal scores are compared with NAPS arousal scores as a simple verification of NAPS data. If these scores will be significantly different, further analyses will be conducted for both sets of values.

- Average absolute HR and GSR reactions are calculated for different valence, arousal and valence*arousal values (as described in Section VI-A). The statistical significance of the differences between them is checked.

- The same analysis are conducted for relative values: instead of taking HR and GSR values, the difference between actual reactions and baseline are considered.

- It is also checked "if change appears" in the moment of affective stimuli appearance: it is possible that band sometimes record growth and sometimes falls and there are no significant differences between reactions' levels, but there is always a change that can be used within game context.

Preliminary analyses were carried out. HR and GSR responses to the presented stimuli are depicted on the plots presented in Figure 4. Heart Rate varies as a function of arousal: higher arousal values are indicated by steeper HR changes than in the neutral condition and lower arousal values are associated with flatter HR changes. This is consistent 


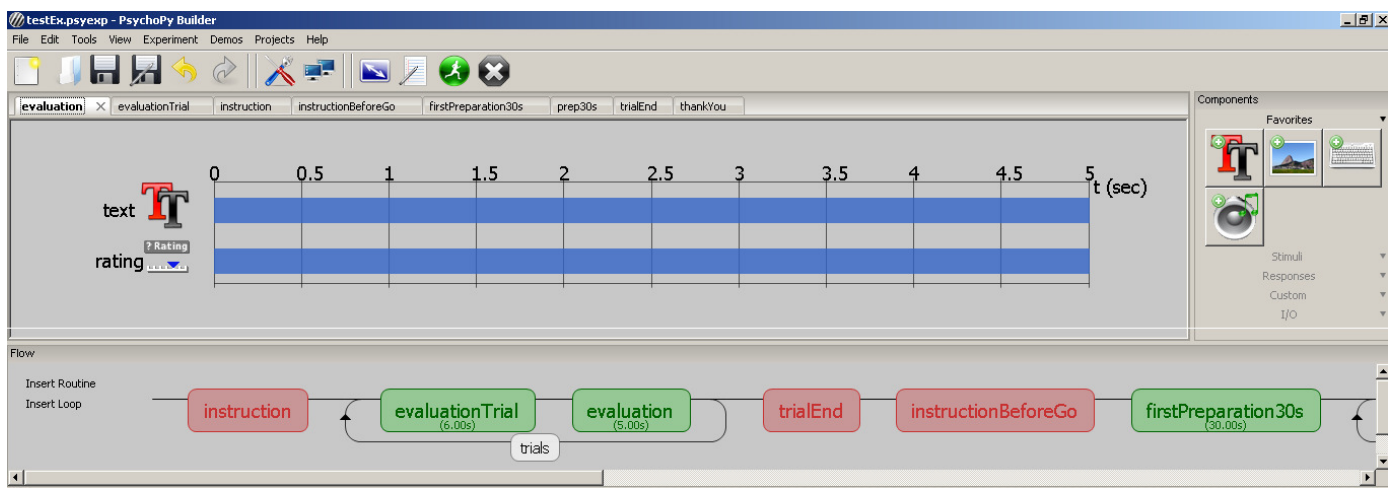

Fig. 3. PsychoPyBuilder Design of the experimental procedure for calibration
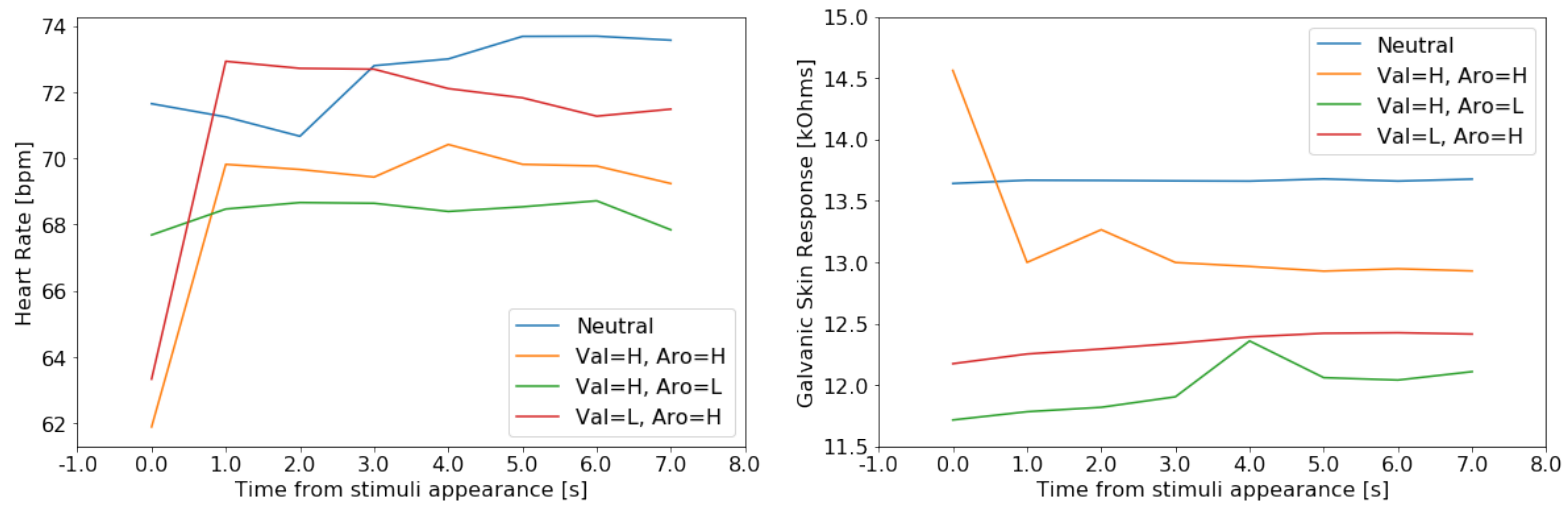

Fig. 4. HR and GSR responses with regard to the group to which the stimulus belonged $(\mathrm{Val}=$ Valence, $\mathrm{ArO}=\mathrm{Arousal}, \mathrm{H}=\mathrm{High}, \mathrm{L}=\mathrm{Low})$.

with both the general knowledge and the results of other research [20]: if a participant is more excited than his heart beats faster. On the other hand, Galvanic Skin Response varies as a function of valence. It shows changes to high valence values and no changes for others. These are important results because they point to the real utility of the budgetary wrist bands for differentiating emotional states.

As the data was collected just before the submission of the final version of this paper, a more exhaustive analyses leading to more accurate HR and GSR patterns identification are still being performed.

\section{RELATED RESEARCH}

In recent years there has been a lot of work related to the introduction of affective components into video games. Selected works are presented next.

In an early paper [23] an idea of enhancing biofeedback by placing it within a competitive virtual gaming environment was introduced. In fact, it was one of the first works to introduce affective feedback (loop). The virtual environment affects the players' level of relaxation while the levels of relaxation determine the outcome of the game. The most relaxed person wins the game. The study [24] investigates the hypothesis that the player's arousal will correspond with the pressure used to depress buttons on a gamepad. A practical video game was created to detect the force of each button press during play.
In [25] the authors examined phasic psychophysiological responses indexing emotional valence and arousal to different game events during the video game Monkey Bowling 2 . Event-related changes in skin conductance, cardiac interbeat intervals, and other signals were recorded. Furthermore, game events elicited reliable valence- and arousal-related phasic physiological responses. The authors proposed to use the information on emotion-related physiological responses to game events or event patterns, to guide choices in game design.

The paper [26] describes an investigation into how realtime yet low-cost biometric information can be interpreted by computer games to enhance gameplay. The primary benefit of incorporating this technique into computer games is that game developers can offer control over direction of game play and game events to the player.

The paper [27] discusses how the emerging discipline of affective computing contributes to important elements of affective game design, with emphasis on the importance of modeling, including sensing and recognition of the players' emotions, generating 'affective behaviors'.

The paper [28] presents two studies that aim to realize an emotionally adaptive game. It investigates the relations between game mechanics, a player's emotional state and his/her emotion-data. In an experiment, one game mechanic (speed) was manipulated. Emotional state was self-reported in 
terms of valence, arousal and boredom-frustration-enjoyment. A number of (mainly physiology-based) emotion-data features were measured. Significant correlations were found between the valence/arousal reports and the emotion-data features.

As our work is not directly comparable in detail to any of these works, certain objectives are similar. What seems novel in our approach wrt to the state of the art is addressing specific affective game design patterns.

\section{FUTURE WORKS}

In this paper we discussed applications of affective computing techniques to the design of video games. We employed Jesse Prinz's Embodied Appraisal Theory that follows the assumptions of James-Lange theory of emotions. In this approach a change in the affective condition of a player can be detected and identified based of the monitoring of physiological signals of the player. In fact, we focuses on heart rate and galvanic skin response. We used game design patterns introduced by Björk and Holopainen.

Our contribution consists in the identification of a set of affective game design patterns. We demonstrated how these patterns can be used in a design of a scroll-runner game. We addressed the problem of differences in responses of individual users by the introduction of a proper calibration phase using NAPS pictures. Our approach is novel wrt to the state of the art, by addressing and using specific affective game design patterns.

Our objective was to provide integration of a sensory framework with a specific gaming environment to detect emotions. In this paper we discuss the use of the Game Maker environment. In the future we plan to support the Unity environment. We aim at extending the game design process by the practical use of affective patterns. In this way, the designer could introduce and control the affective loop in the game. Moreover, new series of experiments are planned. Finally, we are aiming to incorporate this work with a platform [29] combining affective computing with context-aware systems for ambient intelligence applications [30].

\section{ACKNOWLEDGMENT}

This work is supported by the Jagiellonian University and AGH University grants.

\section{REFERENCES}

[1] R. W. Picard, Affective Computing. MIT Press, 1997.

[2] S. Björk and J. Holopainen, Patterns in Game Design. Charles River Media, 2005

[3] W. James, "What is an emotion?" Mind, no. 34, pp. 188-205, 1884.

[4] G. J. Nalepa, J. K. Argasinski, K. Kutt, P. Wegrzyn, S. Bobek, and M. Z. Lepicki, "Affective computing experiments in virtual reality with wearable sensors. methodological considerations and preliminary results," in Proceedings of the Workshop on Affective Computing and Context Awareness in Ambient Intelligence (AfCAI 2016), ser. CEUR Workshop Proceedings, M. T. H. Ezquerro, G. J. Nalepa, and J. T. P. Mendez, Eds., vol. 1794, 2016.

[5] R. A. Calvo, S. K. D'Mello, J. Gratch, and A. Kappas, Eds., The Oxford Handbook of Affective Computing, ser. Oxford Library of Psychology. Oxford University Press, 2015.

[6] M. B. Arnold, Emotion and personality. Columbia University Press, 1960.
[7] R. S. Lazarus, Psychological stress and the coping process. McGrawHill, 1966.

[8] S. K. R., "Apprasial theory," in Handbook of cognition and emotion, D. T. and P. M., Eds. John Wiley and Sons, 1999, pp. 637-663.

[9] A. Orthony, G. Clore, and A. Collins, The cognitive structure of emotions. Cambridge University Press, 1988.

[10] S. C. Marsella and J. Gratch, "Ema: A process model of appraisal dynamics," Cognitive Systems Research, vol. 10, pp. 70-90, 2009.

[11] A. Mehrabian and J. A. Russell, An approach to environmental psychology. MIT Press., 1974.

[12] C. Becker-Asano and I. Wachsmuth, "Affective computing with primary and secondary emotions in a virtual human," Autonomous Agents and Multi-Agent Systems, vol. 20, pp. 32-49, 2009.

[13] J. Prinz, "Which emotions are basic?" in Emotion, evolution, and rationality, D. Evans and P. Cruse, Eds. Oxford University Press New York, 2004, pp. 69-88.

[14] B. Suits, The Grasshopper: Games, Life and Utopia. Broadview Press, 2005.

[15] B. Winn, "The design, play and experience framework," in Handbook of Research on Effective Electronic Gaming in Education. Hershey, 2009, pp. 1010-1024.

[16] R. W. Picard, "Recognizing stress, engagement, and positive emotion," in Proceedings of the 20th International Conference on Intelligent User Interfaces. ACM, 2015, pp. 3-4

[17] A. Marchewka, Ł. Żurawski, K. Jednoróg, and A. Grabowska, "The Nencki Affective Picture System (NAPS): Introduction to a novel, standardized, wide-range, high-quality, realistic picture database," Behavior Research Methods, vol. 46, no. 2, pp. 596-610, 2014.

[18] S. Swink, Game Feel. A game designer's guide to virtual sensation. Elsevier, 2009.

[19] E. Andersen, Y.-E. Liu, R. Snider, R. Szeto, S. Cooper, and Z. Popović, "On the harmfulness of secondary game objectives," in Proceedings of the 6th International Conference on Foundations of Digital Games. ACM, 2011, pp. 30-37.

[20] I. B. Mauss and M. D. Robinson, "Measures of emotion: A review," Cognition and Emotion, vol. 23, no. 2, pp. 209-237, 2009.

[21] J. A. Russell, "A circumplex model of affect," Journal of Personality and Social Psychology, vol. 39, no. 6, pp. 1161-1178, 1980.

[22] J. T. Cacioppo, G. G. Berntson, J. T. Larsen, K. M. Poehlmann, and T. A. Ito, "The psychophysiology of emotion," in Handbook of emotions. Guildford Press, 2000, pp. 173-191.

[23] D. Bersak, G. McDarby, N. Augenblick, P. McDarby, D. McDonnell, B. McDonald, and R. Karkun, "Intelligent biofeedback using an immersive competitive environment," 2001, paper presented at the Designing Ubiquitous Computing Games Workshop at UbiComp 2001.

[24] J. Sykes and S. Brown, "Affective gaming: Measuring emotion through the gamepad," in CHI '03 Extended Abstracts on Human Factors in Computing Systems, ser. CHI EA '03. New York, NY, USA: ACM, 2003, pp. 732-733.

[25] R. Niklas, S. Timo, L. Jani, K. Kari, and S. Mikko, "The psychophysiology of video gaming: Phasic emotional responses to game events," in DiGRA \&\#3905 - Proceedings of the 2005 DiGRA International Conference: Changing Views: Worlds in Play, 2005.

[26] K. M. Gilleade and A. Dix, "Using frustration in the design of adaptive videogames," in Proceedings of the 2004 ACM SIGCHI International Conference on Advances in Computer Entertainment Technology, ser ACE '04. New York, NY, USA: ACM, 2004, pp. 228-232.

[27] E. Hudlicka, "Affective computing for game design," in Proceedings of the 4th International North American Conference on Intelligent Games and Simulation (GAMEON-NA), 2008.

[28] T. Tijs, D. Brokken, and W. Ijsselsteijn, "Creating an emotionally adaptive game," in Proceedings of the 7th International Conference on Entertainment Computing, ser. ICEC '08. Berlin, Heidelberg: SpringerVerlag, 2009, pp. 122-133.

[29] S. Bobek and G. J. Nalepa, "Uncertain context data management in dynamic mobile environments," Future Generation Computer Systems, vol. 66 , pp. $110-124,2017$.

[30] G. J. Nalepa, K. Kutt, S. Bobek, and M. Z. Lepicki, "AfCAI systems: Affective computing with context awareness for ambient intelligence. research proposal," in Proceedings of the Workshop on Affective Computing and Context Awareness in Ambient Intelligence (AfCAI 2016), ser. CEUR Workshop Proceedings, M. T. H. Ezquerro, G. J. Nalepa, and J. T. P. Mendez, Eds., vol. 1794, 2016. 


\title{
Proposal of an efficient rank-ordering method based on subjectivity
}

\author{
Taiki Nishijima*, Aoi Honda ${ }^{\dagger}$, Makoto Ohki ${ }^{\ddagger}$ \\ *Electronics and Information Systems Engineering Course \\ National Institute of Technology, Kumamoto College \\ 2659-2 Suya, Koshi, Kumamoto 861-1102, Japan \\ Email: ae17nishijima@g.kumamoto-nct.ac.jp \\ ${ }^{\dagger}$ Department of Systems Design and Informatics \\ Kyushu Institute of Technology \\ 680-4 Kawazu, Iizuka, Fukuoka, 820-8502, Japan \\ Email: aoi@ces.kyutech.ac.jp \\ $\ddagger$ Department of Information, Communication and Electronic Engineering \\ National Institute of Technology, Kumamoto College \\ 2659-2 Suya, Koshi, Kumamoto 861-1102, Japan \\ Email: ohki@kumamoto-nct.ac.jp
}

\begin{abstract}
An effective method to evaluate order relations based on subjectivity is proposed. This method is a kind of relative evaluation method and adopts "quicksort," which is to be the fastest sorting algorithm, to reduce the number of comparisons and the time of evaluation, so that the method has light burden on subjects. Therefore it is expected that the evaluation criteria of subjects do not fluctuate during evaluation.

We test the validity and effectiveness of the method through an experiment. In the experiment, subjects evaluate order relations of five landscape photographs about preference using the proposal method and pairwise comparison method. The experimental result shows that there is a strong correlation between order relations obtained by both of methods.

We conclude that the proposal method is effective to evaluate order relation about preference of photograph. Additionally, it is suggested that the same thing applies to other kinds of subjective criteria.
\end{abstract}

\section{INTRODUCTION}

$\mathbf{T}$ HIS paper discusses a new method to evaluate order relations based on individual subjectivity with a small number of comparisons and verify its effectiveness.

These days, there is necessity to evaluate order relations based on individual subjectivity such as comparing product designs, the usability of web sites or the quality of pictures. Additionally, as artificial intelligence develops, a larger number of teacher data of subjective evaluation is needed. In such situation, the order relation of stimuli has to be evaluated by many subjects in order to maintain generality, although it is difficult when the number of stimuli is large. Therefore methods to evaluate order relations are desirable to have little burden on subjects and a fewer number of comparisons.

To reflect individual subjectivity in order relations precisely, the evaluation criteria of subjects cannot fluctuate during the evaluation.

Subjective evaluation can be classified into absolute evaluation and relative evaluation. In absolute evaluation, stimuli are individually evaluated according to numeric rating scale.
When the number of stimuli is $N$, the number of evaluations is also $N$. Therefore even if the number of stimuli increases, the number of evaluations does not increase rapidly, but it gets difficult to keep evaluation criteria constant when the evaluation takes long. In relative evaluation, a stimulus is relatively evaluated with others. Pairwise comparison is frequently used relative evaluation method, which can reflect a tiny difference on the result. On the other hand, as the number of stimuli increases, the number of comparisons increases rapidly [1]. In this paper, pairwise comparison is assumed to be a method that compares all pairs of stimuli.

In this paper, we propose a type of relative rating method and test it through experiment. Using this method, order relation is evaluated with a small number of comparisons. This method is applied to order Japanese verbal expressions about pain and shown to have practicability [2]. Reference [2] reports that the method reduces burden on subjects and significant time without losing accuracy.

We extend this method to a subjective case other than pain.

\section{EXISTING METHODS}

In this section, well-known subjective evaluation methods are presented.

\section{A. Scoring method}

Scoring method is one of absolute evaluation methods. Every stimulus is evaluated according to discrete numeric rating scale one by one. Thus the number of evaluations is $N$ when the number of stimuli is $N$. A disadvantage of this method is difficulty in maintaining constant evaluation criteria of subjects when the number of stimuli is large.

\section{B. Whole ranking method}

In whole ranking method, all stimuli are ordered at once, so that subjects need to compare multiple stimuli at one 
evaluation. This might make evaluation criteria differ between subjects.

\section{Pairwise comparison}

Pairwise comparison [1] is one of the simplest method and frequently used for subjective evaluation such as preference test. In general, all pairs of stimuli are compared in this method. Thus precise results of order relations can be obtained. Nevertheless, the total number of comparisons $P_{N}$ is large, $P_{N}=N(N-1) / 2$.

\section{PROPOSAL METHOD, QUICK-PAIRWISE METHOD}

We propose a type of relative rating method and name it "quick-pairwise method." In this method, "quicksort [3]," which is to be the fastest sorting algorithm, is adopted to reduce the number of comparisons, so that it has a merit of simplicity of pairwise comparison with keeping the number of comparisons small.

\section{A. Procedure}

The procedure of quick-pairwise method follows below.

1) Choose a pivot from stimuli at random.

2) Compare the rest of stimuli with the pivot and divide them into a superior group, an equivalent group and an inferior group. If a stimulus is unable to compare, except it from the evaluation. If the pivot is excepted, choose a pivot again.

3) Choose a pivot from each of the superior group and the inferior group.

4) Divide the stimuli into the three groups in each groups.

5) Repeat this procedure until the numbers of stimuli in all groups are three or less.

6) Order stimuli in all groups

\section{B. An evaluation example}

How to order ten stimuli (A to J) by quick-pairwise method is shown below. In this example, stimuli are put in order of preference.

Firstly, choose a stimulus as a pivot. In this case, stimulus $\mathrm{A}$ is the pivot. Then, compare and divide the rest of stimuli whether it is preferred, not preferred or preferred equivalently to the pivot. At this process, except stimuli that are uncomparable for the subject. The result of the comparisons is shown in table I.

Next, choose a pivot in each of the preferred group and the not preferred group. In this case, B in the preferred group and $\mathrm{C}$ in the not preferred group are chosen as the pivots. Then, compare and divide the rest of the stimuli in each group likewise. The results of the comparisons are shown in table II and table III.

Because there is no group that has more than three stimuli now, compare each stimuli in all groups left. In this case, G is preferred to $\mathrm{F}$ and $\mathrm{I}$ is preferred to $\mathrm{H}$. Table IV shows the whole result of this evaluation.
TABLE I

THE RESUlT WHEN A IS THE PIVOT

\begin{tabular}{cc|c|cc}
\hline \multicolumn{2}{c|}{ preferred } & equivalent & \multicolumn{2}{c}{ not } \\
$\mathrm{B}$ & $\mathrm{F}$ & $\mathrm{E}$ & $\mathrm{C}$ & $\mathrm{D}$ \\
$\mathrm{G}$ & $\mathrm{J}$ & & $\mathrm{H}$ & $\mathrm{I}$ \\
\hline
\end{tabular}

TABLE II

THE RESULT WHEN B IS THE PIVOT

\begin{tabular}{c|c|lc}
\hline preferred & equivalent & \multicolumn{2}{|l}{ not preferred } \\
& $\mathrm{J}$ & $\mathrm{G}$ & $\mathrm{F}$ \\
\hline
\end{tabular}

TABLE III

THE RESULT WHEN C IS THE PIVOT

\begin{tabular}{c|l|lc}
\hline preferred & equivalent & \multicolumn{2}{|l}{ not preferred } \\
$\mathrm{D}$ & & $\mathrm{H}$ & $\mathrm{I}$ \\
\hline
\end{tabular}

TABLE IV

THE ORDER RELATION OF THE STIMULI

\begin{tabular}{llllllllll}
\hline $1 \mathrm{~B}$ & $1 \mathrm{~J}$ & $3 \mathrm{G}$ & $4 \mathrm{~F}$ & $5 \mathrm{~A}$ & $5 \mathrm{E}$ & $7 \mathrm{D}$ & $8 \mathrm{C}$ & $9 \mathrm{I}$ & $10 \mathrm{H}$ \\
\hline
\end{tabular}

\section{The number of comparisons}

Let $Q_{N}$ be the average number of comparisons when $N$ is the number of stimuli. When a pivot is randomly chosen from the stimuli, recurrence formula about $Q_{N}$ is

$$
\begin{aligned}
Q_{N} & =N-1+\frac{1}{N} \sum_{k=0}^{N-1}\left(Q_{k}+Q_{N-1-k}\right) \\
& =N-1+\frac{2}{N} \sum_{k=0}^{N-1} Q_{k} \\
Q_{0} & =0
\end{aligned}
$$

and using harmonic series $H_{N}, Q_{N}$ is

$$
\begin{aligned}
Q_{N} & =2(N+1) H_{N}-4 N \\
H_{N} & =1+\frac{1}{2}+\cdots+\frac{1}{N} \\
& =\sum_{k=1}^{N} \frac{1}{k} .
\end{aligned}
$$

Compared with the number of comparisons in pairwise comparison method, the ratio of $Q_{N}$ to $P_{N}$ is approximately $54 \%(N=10)$, and $29 \%(N=30)$.

Even if a subject makes the most inefficient judges, $Q_{N}=$ $P_{N}$. Therefore the number of comparisons is smaller in quickpairwise method than in pairwise comparison method.

\section{EXPERIMENT}

We tested the effectiveness of quick-pairwise method through the following experiment.

\section{A. Subjects}

A hundred subjects who were aged 17 to 22 participated in this experiment. 


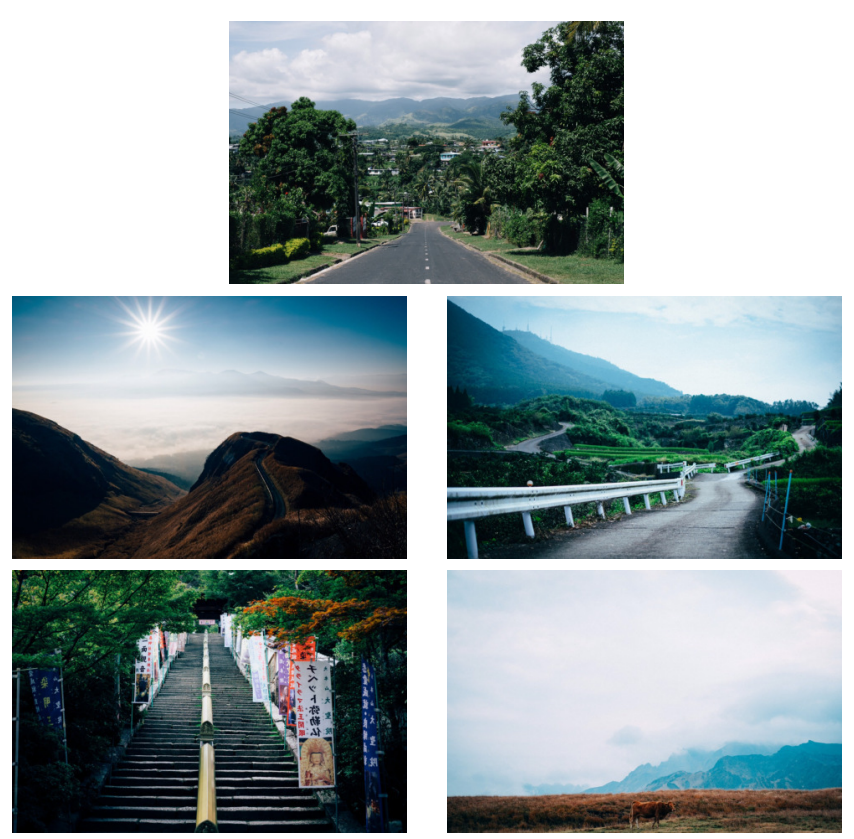

Fig. 1. Photographs for the stimuli

\section{B. Stimuli}

Five landscape photographs, taken by the same photographer, were used as stimuli. They are shown in Fig. 1.

\section{Process}

Because pairwise comparison method can evaluate the order relation of stimuli precisely, we compared quick-pairwise method with pairwise comparison method. The subjects were presented with five photographs of stimuli. They put the stimuli in order of their preferences in both ways of quickpairwise and pairwise comparison method using a web site which was made for this experiment. Firstly, they used quickpairwise method to order, then after five minutes, pairwise comparison method was used.

A week later, 57 out of all subjects did the same experiment again. This second experiment was held to test our hypothesis that there might be fluctuations in the evaluation criteria of subjects that would cause difference between order relations obtained by two kinds of method.

To compare the order relations obtained from the two kinds of evaluation, let the order of stimulus be the number of superior stimuli plus one. Correlation coefficients are calculated between order relations for each subjects.

\section{Web site for the experiment}

We made a web site shown in Fig. 2 for this experiment. Subjects compare photographs by clicking their preferred photograph or a button of "=." After clicking one of them, the pair of displayed photographs updates. Until the evaluation ends, subjects only repeat clicking.

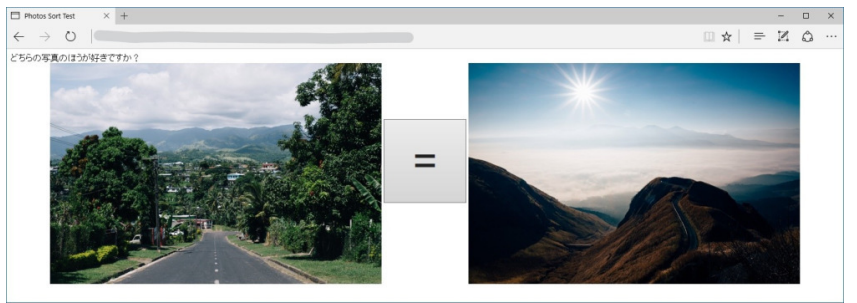

Fig. 2. Web site for the experiment

\section{E. Result}

We report the result of the experiment. Table $\mathrm{V}$ shows the average of the correlation coefficients of the obtained order relations. Table VI shows the correlation coefficients of the order relations evaluated by subjects who participated in the second experiment and had less than 0.75 correlation coefficient at the first experiment.

\section{Discussion}

We will discuss the effectiveness of quick-pairwise method compared with pairwise comparison method.

The first point to be discussed is the validity of quickpairwise method. The average of correlation coefficient between the order relations obtained at first evaluation with quick-pairwise and pairwise comparison method of table $\mathrm{V}$ is 0.854 . It shows that there is a strong correlation between the order relations. This result suggests that quick-pairwise method is as valid as pairwise comparison method to evaluate order relations about preference.

Even though it is supposed that quick-pairwise method is valid, it should be noted that there still are differences between the order relations obtained by two kinds of method. One plausible explanation for the differences is that there are fluctuations in the evaluation criteria of subjects. To examine this explanation, we will discuss correlation coefficients between the first and second pairwise comparison.

Table V shows that the average of correlation coefficient between the first and second pairwise comparison is 0.725 , which is less than the average of correlation coefficient between the first quick-pairwise and pairwise comparison, 0.874 .

Let us focus on low correlation coefficients shown in table VI. Correlation coefficients between quick-pairwise and pairwise comparison of subject A are 0.384 at the first evaluation and 0.354 at the second. Those are too low to say that quickpairwise method is strongly correlated with pairwise comparison method. On the other hand, his correlation coefficient between the first and the second pairwise comparison is 0.294 , which is smaller than 0.354 and 0.384 , that are correlation coefficients between quick-pairwise and pairwise comparison. The same thing applies to a half of the subjects in table VI. This result indicates that ordering stimuli even by pairwise comparison method, which can evaluate precisely, there are differences between the obtained order relations. Thus, it is quite possible that the differences between the order relations obtained by quick-pairwise and pairwise comparison method 
TABLE V

THE ORDER RELATION OF STIMULI

\begin{tabular}{|c|c|c|c|c|}
\hline \multirow{2}{*}{ number of subjects } & \multicolumn{3}{|c|}{ average of correlation coefficient } \\
\cline { 2 - 5 } & 1st quick-pairwise and pairwise & 2nd quick-pairwise and pairwise & 1st and 2nd quick-pairwise & 1st and 2nd pairwise \\
\hline $100^{*}$ & 0.854 & - & - & - \\
\hline $57^{\dagger}$ & 0.874 & 0.854 & 0.754 & 0.725 \\
\hline $12^{\ddagger}$ & 0.634 & 0.812 & 0.580 \\
\hline
\end{tabular}

* all the subjects

$\dagger$ subjects who participated in second evaluation

$\ddagger$ subjects who participated in second evaluation and had less than 0.75 correlation coefficient at first evaluation

TABLE VI

SUBJECTS WITH LOW CORRELATION COEFFICIENT AT THE FIRST EVALUATION

\begin{tabular}{|c|c|c|c|c|}
\hline \multirow{2}{*}{ subject } & \multicolumn{3}{|c|}{ correlation coefficient } \\
\cline { 2 - 5 } & 1st quick-pairwise and pairwise & 2nd quick-pairwise and pairwise & 1st and 2nd quick-pairwise & 1st and 2nd pairwise \\
\hline A & 0.384 & 0.354 & 0.853 & 0.294 \\
\hline B & 0.512 & 0.645 & -0.500 & 0.700 \\
\hline C & 0.530 & 0.900 & 0.700 & 0.707 \\
\hline D & 0.600 & 0.671 & 0.600 & -0.244 \\
\hline E & 0.671 & 0.900 & 0.700 & 0.400 \\
\hline F & 0.700 & 0.632 & 0.700 & 0.791 \\
\hline G & 0.700 & 0.900 & 0.700 & 0.700 \\
\hline H & 0.700 & 0.900 & 0.700 & 0.900 \\
\hline I & 0.700 & 0.945 & 0.700 & 1.000 \\
\hline J & 0.700 & 1.000 & 0.707 & 1.000 \\
\hline K & 0.700 & 1.000 & & 0.894 \\
\hline L & 0.707 & 0.894 & \\
\hline
\end{tabular}

were not caused by the small number of comparisons of quickpairwise method but by fluctuations in the evaluation criteria of subjects.

The next discussion deals with the effectiveness of quickpairwise method as a method to evaluate order relation. It is possible that order relation is cycled using pairwise comparison method. For example, stimuli $\mathrm{A}, \mathrm{B}$ and $\mathrm{C}$ may be judged that $\mathrm{A}$ is superior to $\mathrm{B}$, and $\mathrm{B}$ is superior to $\mathrm{C}$, but $\mathrm{C}$ is superior to $\mathrm{A}$. In this case, order relation cannot be evaluated, and when such evaluation happens in more than four stimuli, it may be a noise of the whole order relation. In fact, it happened in $20 \%$ of the order relations obtained by pairwise comparison method at the first evaluation of the experiment. However, it cannot happen with quick-pairwise method. From the abovedescribed point of view, it seems that quick-pairwise method is effective to evaluate order relations.

\section{CONCLUSION}

We conclude that quick-pairwise method evaluates precise order relations about preference of photograph with a small number of comparisons and light burden on subjects. Besides, using quick-pairwise method, cycle of order relations does not happen by fluctuations in the evaluation criteria of subjects.

\section{VIEW OF THE FUTURE}

In this paper, we tested the validity of quick-pairwise method about preference of photograph. More evaluations of order relation are needed to improve the credibility of the result and discussion.

Our future research will be evaluations of order relations of stimuli other than photograph. Furthermore, experiments about other subjective cases are required to prove the effectiveness of quick-pairwise method.

\section{ACKNOWLEDGMENT}

We extend our appreciation to all the participants in the experiment for their support. We are also thankful to $\mathrm{Mr}$. Kensho Murata for his great photographs.

\section{REFERENCES}

[1] Thomas L. Saaty, THE ANALYTIC HIERARCHY PROCESS, RWS 1996.

[2] Issei Shibao, Aoi Honda, Makoto Ohki, "The proposal of the method to check order relations onomatopoeias expressing pains using a relative rating method," Journal of Japan Society for Fuzzy Theory and Intelligent Informatics, Vol. 28, No. 4, 2016, pp. 734-743.

[3] C.A.R Hoare, "Quicksort," The Computer Journal, Vol. 5, No. 1, 1962, pp. 10-15. 


\section{A Method for Converting Colors for Color-impaired People, Considering Saturation and Contrast Ratio}

\author{
Junko Shirogane \\ Miho Saito
Waseda University
}

Tokyo Woman's Christian University

\author{
Yuko Iwase \\ Waseda University \\ Yoshiaki Fukazawa \\ Waseda University
}

\author{
Hajime Iwata \\ Kanagawa Institute of Technology
}

\begin{abstract}
Because color-impaired people have difficulty in perceiving specific colors, colors used in documents must be converted for them. The colors of documents include the intentions of their designers. That is, designers use different colors for parts of the document that they want to be attractive or distinguishable in some way. In existing conversion methods, however, colors are not converted based on a consideration of the designers' intentions. Hence, we propose a method for converting document colors for color-impaired people in a manner that takes the designers' intentions into account. Here, we define the designers' intentions as "attractiveness" and "distinguishability." In our method, the original colors are classified into three levels, low, middle, and high, based on their saturation. In each saturation level, some colors are defined as available for conversion. Next, the original colors are converted to colors in the corresponding saturation levels. According to the RGB (red-green-blue) values of the original colors, the target colors for conversion can be specified. Then, the contrast ratios of the pair of foreground and background colors are calculated. If the contrast ratios do not satisfy the specific thresholds, the colors are additionally converted to other colors in the corresponding saturation levels. Finally, when the contrast ratios satisfy the specific thresholds, the conversion is complete.
\end{abstract}

\section{INTRODUCTION}

V ARIOUS colors are used in artifacts such as computer displays, prints, and devices. The effectiveness of color in user interface aesthetics, attractiveness, and distinguishability is well known. Therefore, the usage of color is quite important in the design of artifacts. However, color-impaired people perceive color differently from people with full color vision. Specifically, they have difficultly distinguishing between red and green or blue and yellow. Additionally, some people have difficulty perceiving colors at all. Color impairments include both congenital cases and acquired cases due to advanced age and illnesses. Men have color impairments more frequently than women. Although the rates of color impairment differ among ethnicities, the frequencies of color impairment are about $2-10 \%$ for men and $0.2-0.5 \%$ for women [1]. Accordingly, approximately 200 million people worldwide have color impairments, nearly equal to the number of men with blood type AB. To make artifacts perceivable for color-impaired people, the colors of artifacts are often converted.

To convey information, attractiveness and distinguishability are important. Generally, colors with high saturation and luminance have high attractiveness. Designers often use colors with high attractiveness for artifact contents that they wish to emphasize. Meanwhile, background and foreground colors affect distinguishability. If the contrast ratio of relative luminance between the background and foreground colors of the contents of an artifact is low, the distinguishability of the contents will also be low. Regardless of color vision, distinguishability can be provided by ensuring that the contrast ratios between background and foreground colors are sufficiently high. Although many methods have been proposed for converting the original colors of artifacts to those that color-impaired people can easily perceive, in many cases the intentions of the artifact designers are not considered.

The purpose of our method is to convert original colors for color-impaired people in such a way that they can perceive the designers' intentions. Here, the designers' intentions are defined as "attractiveness" and "distinguishability." To convert colors, we focus on saturation and contrast ratios. In advance, we create a basic conversion table. For this purpose, colors that color-impaired people can easily perceive, selected by the Color Universal Design Organization (CUDO) of Japan [2], are classified into three saturation levels. For conversions of original colors, first, the saturations of the colors are calculated, and the saturation levels are identified. Second, the original colors are converted to the closest colors in the same saturation levels of the basic conversion table. Third, the contrast ratios of pairs of background and foreground colors are calculated. Finally, if the calculated values do not satisfy specific thresholds, the converted colors are additionally converted to other colors in the same saturation levels. Hereafter, the original colors that designers use are called "original colors," the converted colors based on the basic conversion table in the second step are called "provisional colors," and the ultimate converted colors based on the contrast ratios are called "final colors." In addition, the current targets of our method are limited to text, rather than the images in documents.

The rest of this paper is organized as follows. Section II describes related works. Section III summarizes the background of this research, while Section IV shows the features. Section $\mathrm{V}$ describes the details of strategies for converting colors. Section VI describes how to convert colors in documents. Section VII provides an evaluation of our method. Section VIII concludes this paper. 
TABLE I

TYPES OF COLOR VISION

\begin{tabular}{|l|l|l|l|}
\hline Types of color visions & Cone conditions & Descriptions \\
\hline Common & C-type & All three cones are normal & All colors can be perceived \\
\hline $\begin{array}{l}\text { Color } \\
\text { Impairments }\end{array}$ & P-type (Strong) & Lack of L-cones & \multirow{3}{*}{ Red and green colors are difficultly perceived } \\
\cline { 2 - 3 } & P-type (Weak) & Shift of spectral sensitivities of L-cones & \\
\cline { 2 - 3 } & D-type (Strong) & Lack of M-cones & \\
\cline { 2 - 3 } & D-type (Weak) & Shift of spectral sensitivities of M-cones & \\
\cline { 2 - 4 } & T-type & Lack of S-cones & Blue and yellow colors are difficultly perceived \\
\cline { 2 - 3 } & A-type & Lack of two cone types or all cones & Colors can not be perceived \\
\hline
\end{tabular}

\section{RELATED WORKS}

To make artifact colors perceivable to color-impaired people, the original colors are often converted for them, and many studies related to color conversion have been performed.

For converting document colors, Zhau et al. proposed a method for converting colors of web pages in a mobile environment [3]. Their purpose was to reduce the costs of selecting colors for conversion. To this end, a conversion method [4] was extended, pairs of background and foreground colors were extracted, and colors to be converted were limited by color pairs. In this manner, the costs of computational assessment of color conversion could be reduced. Then, colors were converted based on a model of users' subjective judgment of colors [5]. Although this method was targeted to text in documents, document structures were not considered.

To convert targets other than documents, Takimoto et al. proposed a method for converting image colors so that even people with full color vision would feel that the converted images were natural [6]. First, the number of colors was reduced using a median cut algorithm [7]. Next, saturation and lightness were modified using a modified Color2Gray algorithm [8]. In this case, because the converted colors sometimes became out of ranges of color gamut, color correlation was performed. A study by Wahab et al. aimed to convert image colors for people with protanope impairments [9]. First, colors in RGB (red-green-blue) color space were converted to LMS (long-medium-short) color space. Second, the LMS information that people with protanope impairments could not perceive were deleted from the LMS values. Third, the contrast between red and blue was increased. Meanwhile route maps and road signs were targeted using a multilayer neural network [10]. This network consisted of (1) layers of color conversion of images, (2) layers of perceptual models for color impairments, and (3) layers of color discrimination. Strategies of color conversion were determined by (1), perceptual models of color-impaired people were simulated by (2), and color edges of images for color-impaired people were simulated by (3). Color vision of color-impaired people was simulated by converting RGB color spaces to CIE XYZ [11][12] color spaces. $\mathrm{X}, \mathrm{Y}$, and $\mathrm{Z}$ values were calculated from red, green, and blue values [13], and reaction values of LMS were calculated from the $\mathrm{X}, \mathrm{Y}$, and $\mathrm{Z}$ values. Then, the $\mathrm{LMS}$ values of simulations for people with protanope and deuteranope impairments were calculated [14][15]. Layers (2) and (3) were fixed, and layers (1) were trained to determine color- conversion rules. In addition, Harwahyu et al. proposed a method for converting colors in embedded systems and smart phones so that color-impaired people can understand display colors [16]. First, colors on the display were represented by 16 color names. Users selected colors on the display, and the HSL (hue-saturation-lightness) values were calculated. Then, by changing hue and lightness values, colors were adjusted for the color-impaired users. In this manner, many color conversion methods for images were provided, but the designers' intentions were not focused on.

In study of color perception by color-impaired people, Tajima et al. quantified and visualized information that could be obtained by differences in color vision [17]. They then defined "visual salience" as "visual conspicuity predicted from image features." First, visual salience was modeled using images. Image colors were converted step by step, and loss/gain information was predicted by comparing models. Then, psychophysical experiments were performed, and subjective differences between the predictions and results of experiments were investigated. Correlations were observed. Meanwhile, color use guidelines for people with protanope and deuteranope impairments were provided based on scientific data [18]. Strategies for representing data were classified into five types, and guidelines were developed for individual classifications. These approaches can support determination of colors for color-impaired people; however, concrete strategies for converting colors of existing documents were not provided.

\section{PRELIMINARY}

\section{A. Types of color vision}

Due to differences in retinas among individual people, the colors that people see are different. Consequently, various types of color visions exist. There are two types of visual cells in human retinas, rod cells and cone cells. Rod cells work in the dark, whereas cone cells work in the light. Cone cells consist of L-cones, M-cones, and S-cones, which perceive red, green, and blue light, respectively. Color vision differs among individuals based on the presence or absence of these three types of cones, as well as whether their spectral sensitivities work normally. Accordingly, color vision can be classified into five types, as shown in Table I.

People that have all three types of cones are common type (C-type), whereas others with a subset of the three types of cones, or in whom the spectral sensitivities of cones are shifted, have color impairments. People who do not have 
L-cones or in whom the spectral sensitivities of L-cones are shifted toward those of M-cones are protanope (P-type), whereas people who do not have M-cones or in whom the spectral sensitivities of M-cones are shifted toward those of Lcones are deuteranope (D-type). People with P-type or D-type impairments have difficulty perceiving differences between red and green colors. Meanwhile, people who do not have Scones are tritanope (T-type), and have difficultly perceiving differences between blue and yellow colors. People who have only one or no cones are achromat (A-type), and perceive only light and darkness. The most common types are P-type and D-types, whereas T-type is rare [1].

\section{B. Color universal design}

Color-universal design refers to designs in which colors of artifacts are adjusted so that people can perceive colors regardless of their color vision status. Such designs are not only for color-impaired people, but also for people with normal color vision. Many studies have been conducted, such as [19][20][21], and many governments and companies have developed guidelines for color-universal design.

\section{FEATURES OF OUR METHOD}

\section{Representation of designers' intentions}

Generally, colors with high saturation have high attractiveness. Designers often use colors with high saturation for content that they want to emphasize. Meanwhile, when contrast ratios of relative luminance between background and foreground colors are high or low, the readability and distinguishability will be correspondingly high or low. When designers want to make contents readable or distinguishable, they use a pair of colors for which the contrast ratio is high. That is, designers represent intentions regarding the contents that they do or do not want to emphasize or make readable/distinguishable by the colors. In our method, colors can converted in a manner that reflects the designers' intentions.

\section{Conversion strategy for documents}

As mentioned in Section I, our method is targeted at text in documents. For text, background and foreground colors in documents do not always correspond in a one-to-one manner. In some cases, multiple foreground colors are used for one background color. If background and foreground colors are converted as pairs in a certain part of the document, the contrast ratios of relative luminance between background and foreground colors in other parts of the document may not satisfy the thresholds. Thus, in our method, the combinations of background and foreground colors are analyzed. Background and foreground colors are not converted as pairs. Instead, as necessary, background (foreground) colors are fixed, and only the foreground (background) colors are converted.

\section{STRATEGIES FOR COLOR CONVERSION}

Color conversions are performed in two steps. First, colors are converted based on a basic conversion table. After that, the contrast ratios of relative luminance between background and foreground colors are calculated. If the contrast ratios do not satisfy specific thresholds, additional conversions are performed. In this section, we describe the details of the basic conversion table and two conversion steps.

\section{A. Basic conversion table}

The basic conversion table is given by our method in advance. CUDO in Japan selects 20 colors that color-impaired people can easily perceive [2]. In this paper, these colors are called "recommended colors." Using the recommended colors, the basic conversion table is created. The purpose of creating the basic conversion table is to ensure that the color saturations are not significantly altered before and after conversion. Using this table, colors can be converted to other colors with almost the same saturation levels.

To create the basic conversion table, first, the HSV (huesaturation-value) values of the recommended colors are calculated using their RGB values by formulas (1)-(3) [22]. According to these formulas, because colors are represented by values of hue $(\mathrm{H})$, saturation $(\mathrm{S})$, and brightness $(\mathrm{V})$, the saturation values of the colors can be obtained. In formulas (1)-(3), R, G, and B indicate the red, green, and blue values of the RGB value for a color, whereas "Max" and "Min" indicate the maximum and minimum values among the $\mathrm{R}, \mathrm{G}$, and $\mathrm{B}$ values. These formulas operate on values as decimal numbers.

$$
\begin{aligned}
& H= \begin{cases}\text { Notdefined } & , \text { Max }=\text { Min } \\
60 \times \frac{G-B}{\text { Max }- \text { Min }} & , \text { Max }=R \\
60 \times \frac{B-R}{\text { Max-Min }}+120 & , \text { Max }=G \\
60 \times \frac{R-G}{\text { Max }- \text { Min }}+240 & , \text { Max }=B\end{cases} \\
& S= \begin{cases}\text { Notdefined }, \text { Max }=0 \\
\frac{\text { Max }- \text { Min }}{\text { Max }, \text { Max } \neq 0}\end{cases} \\
& V=\text { Max }
\end{aligned}
$$

Next, the recommended colors are classified into three saturation levels, low, middle, and high, according to their saturation values based on the Practical Color Coordinate System (PCCS) [23][24]. According to the PCCS, the calculated saturation values from 0 to 1 based on formula (2) are divided into 10 levels (0s-9s) at regular intervals. 0s indicates achromatic color, $1 \mathrm{~s}-3 \mathrm{~s}$ indicate low saturation level, $4 \mathrm{~s}-6 \mathrm{~s}$ indicate middle saturation level, and 7s-9s indicate high saturation level. As specified by the PCCS, Os is added to the low saturation level; thus, three saturation levels are defined in our method. That is, the saturation values of colors from 0 to 0.33 are classified into the low saturation level, colors from 0.34 to 0.67 into the middle saturation level, and colors from 0.68 to 1 into the high saturation level.

Next, recommended colors in each saturation level are sorted according to their color codes. Finally, all colors 
TABLE II

BASIC CONVERSION TABLE

\begin{tabular}{|c|c|c|c|}
\hline Saturation before conversion & Color codes before conversion & Conversion candidate 1 & Conversion candidate 2 \\
\hline \multirow{6}{*}{$\begin{array}{l}\text { Low } \\
\text { (Saturation value from } 0 \text { to } 0.33 \text { ) }\end{array}$} & (1) \#000000 - \#7F878F & $\# 000000$ & \#7F878F \\
\hline & (2) \#7F878F - \#B4EBFA & \#7F878F & \#B4EBFA \\
\hline & (3) \#B4EBFA - \#C7B2DE & \#B4EBFA & \#C7B2DE \\
\hline & (4) \#C7B2DE - \#C8C8CB & \#C7B2DE & \#C7B2DE \\
\hline & (5) \#C8C8CB - \#FFD1D1 & \#C8C8CB & \#FFD1D1 \\
\hline & (6) \#FFD1D1 - \#FFFFFF & \#FFD1D1 & \#FFFFFF \\
\hline \multirow{8}{*}{$\begin{array}{l}\text { Middle } \\
\text { (Saturation value from } 0.34 \text { to } 0.67 \text { ) }\end{array}$} & (7) $\# 000000$ - \#35A16B & $\# 35 \mathrm{~A} 16 \mathrm{~B}$ & $\mathrm{n} / \mathrm{a}$ \\
\hline & (8) \#35A16B - \#66CCFF & \#35A16B & \#66CCFF \\
\hline & (9) \#66CCFF - \#99E7B0 & \#66CCFF & \#99E7B0 \\
\hline & (10) \#99E7B0 - \#CBF266 & \#99E7B0 & \#CBF266 \\
\hline & (11) \#CBF266 - \#EDC58F & \#CBF266 & \#EDC58F \\
\hline & (12) \#EDC58F - \#FF99A0 & \#EDC58F & \#FF99A0 \\
\hline & (13) \#FF99A0 - \#FFFF99 & \#FF99A0 & \#FFFF99 \\
\hline & (14) \#FFFF99 - \#FFFFFF & \#FFFF99 & $\mathrm{n} / \mathrm{a}$ \\
\hline \multirow{7}{*}{$\begin{array}{l}\text { High } \\
\text { (Saturation value from } 0.68 \text { to } 1 \text { ) }\end{array}$} & (15) \#000000 - \#0041FF & $\# 0041 \mathrm{FF}$ & $\mathrm{n} / \mathrm{a}$ \\
\hline & (16) \#0041FF - \#663300 & $\# 0041 \mathrm{FF}$ & $\# 663300$ \\
\hline & (17) \#663300 - \#9A0079 & $\# 663300$ & \#9A0079 \\
\hline & (18) \#9A0079 - \#FAF500 & \#9A0079 & \#FAF500 \\
\hline & (19) \#FAF500 - \#FF2800 & \#FAF500 & \#FF2800 \\
\hline & (20) \#FF2800 - \#FF9900 & \#FF2800 & \#FF9900 \\
\hline & (21) \#FF9900 - \#FFFFFF & \#FF9900 & $\mathrm{n} / \mathrm{a}$ \\
\hline
\end{tabular}

from color code \#000000 to \#FFFFFF (hexadecimal notation) are divided by the color codes of the sorted recommended colors. That is, the color codes of the sorted recommended colors (\#7F878F, \#B4EBFA, etc.) are used as delimiters, and several divisions are created (divisions of \#000000 \#7F878F, \#7F878F - \#B4EBFA, etc.). This manipulation is performed in all three saturation levels. The first and last colors in each division are defined as conversion candidates 1 and 2, respectively. The reason for defining two conversion candidates is that the original colors of the document should be converted to the closest possible colors. However, for the first and last division of colors in the middle and high saturation levels, one of conversion candidates becomes either $\# 000000$ or \#FFFFFF. Because these color codes are achromatic, they are not appropriate conversion candidates for original colors in the middle and high saturation levels. Therefore, colors of \#000000 and \#FFFFFF are removed from the conversion candidates in the middle and high saturation levels. The resultant basic conversion table is shown in Table II.

\section{B. Conversion strategy}

First, original colors are converted using the basic conversion table to provisional colors. Next, the contrast ratios between background and foreground colors are calculated. Then, if the calculated contrast ratios do not satisfy specific thresholds, the provisional colors are additionally converted, and ultimately the final colors are determined.

1) Conversion by basic conversion table: First, the saturation value of an original color is calculated using formula (2). Next, the saturation level of the original color is determined using the calculated saturation value. Then, the corresponding divisions in the saturation level to the original color is determined. Finally, differences in R, G, and B values between the original color and two conversion candidates in the division are calculated using formula (4). In this formula, "Diff" indicates the difference. $R_{o}, G_{o}$, and $B_{o}$ indicate the $\mathrm{R}, \mathrm{G}$, and B values of the original color, whereas $R_{c}, G_{c}$, and $B_{c}$ indicate the $\mathrm{R}, \mathrm{G}$, and $\mathrm{B}$ values of the one of the candidate colors. After calculating the differences, the conversion candidate for which the difference is smaller is determined as the provisional color. Figure 1 shows the workflow for this determination.

$$
\operatorname{Diff}=\left|R_{o}-R_{c}\right|+\left|G_{o}-G_{c}\right|+\left|B_{o}-B_{c}\right|
$$

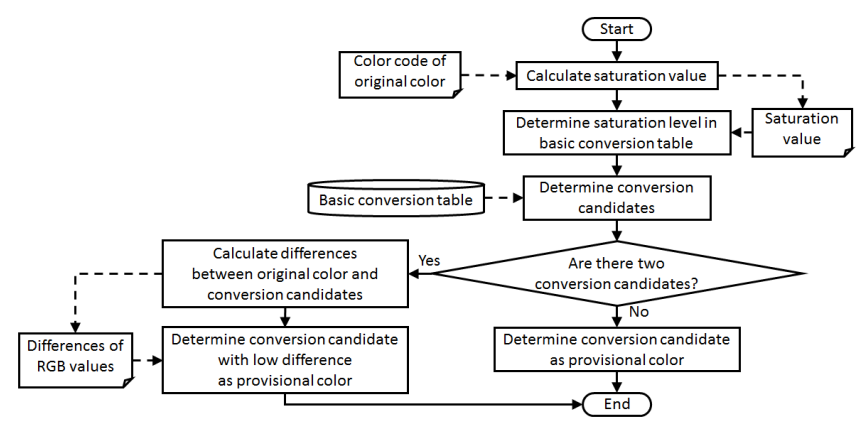

Fig. 1. Workflow of conversion of original color

As examples, original colors \#B6B7C6 (example 1-1), \#E8F2B0 (example 1-2), and \#004082 (example 1-3) are converted. For example 1-1, the calculated saturation value according to formula (2) is 0.08 . This saturation value is in the low range of saturation level in the basic conversion table (Table II). Accordingly, conversion candidates range from division (1) to division (6). According to the original color code, the original color is in the range of division (3). Thus, the conversion candidates are limited to \#B4EBFA and \#C7B2DE. Similarly, the saturation values of colors \#E8F2B0 
TABLE III

EXAMPLES OF DIFFERENCE CALCUATIONS

\begin{tabular}{|c|c|c|}
\hline & Example 1-1 & Example 1-2 \\
\hline \multirow[t]{3}{*}{ RGB value } & Original (\#B6B7C6) $\quad\left(R_{o}: 182, G_{o}: 183, B_{o}: 198\right)$ & $\left(R_{o}: 232, G_{o}: 242, B_{o}: 176\right)$ \\
\hline & \#B4EBFA $\quad\left(R_{c}: 180, G_{c}: 235, B_{c}: 250\right)$ & \#C8C8CB $\quad\left(R_{c}: 200, G_{c}: 200, B_{c}: 203\right)$ \\
\hline & $\left(R_{c}: 199, G_{c}: 178, B_{c}: 222\right)$ & $\left(R_{c}: 255, G_{c}: 209, B_{c}: 209\right)$ \\
\hline \multirow[t]{2}{*}{ Diff } & \#B4EBFA $\quad:|182-180|+|183-235|+|198-250|=106$ & $:|232-200|+|242-200|+|176-203|=101$ \\
\hline & \#C7B2DE $:|182-199|+|183-178|+|198-222|=46$ & $:|232-255|+|242-209|+|176-209|=89$ \\
\hline
\end{tabular}

(example 1-2) and \#004082 (example 1-3) are 0.27 and 1.00, respectively. According to their saturation values, \#E8F2B0 (example 1-2) and \#004082 (example 1-3) are in the low and high saturation levels, respectively. Next, the division of color \#E8F2B0 (example 1-2) is determined as (5) in Table II, yielding conversion candidates \#C8C8CB and \#FFD1D1. The division of the color \#004082 (example 1-3) is determined as (15); thus, the only conversion candidate is \#0041FF. The colors in examples 1-1 and 1-2 have two conversion candidates each, whereas the color in example 1-3 has only one. Thus, one of the candidates from each of examples 1-1 and 1-2 must be selected, whereas the conversion candidate of example 1-3 is determined as the provisional color.

TABLE IV

EXAMPLES OF COLOR CONVERSION TO PROVISIONAL COLOR

\begin{tabular}{|l|c|c|}
\hline & Original color & Provisional color \\
\hline Example & Color code: \#B6B7C6 & Color code: \#C7B2DE \\
$1-1$ & Saturation value: 0.08 & Saturation value: 0.20 \\
\hline Example & Color code: \#E8F2B0 & Color code: \#FFD1D1 \\
$1-2$ & Saturation value: 0.27 & Saturation value: 0.18 \\
\hline Example & Color code: \#004082 & Color code: \#0041FF \\
$1-3$ & Saturation value: 1.00 & Saturation value: 1.00 \\
\hline
\end{tabular}

For examples 1-1 and 1-2, the differences in R, G, and $\mathrm{B}$ values between the original and conversion colors are calculated using formula (4). The R, G, B values of examples 1-1 and 1-2 and the difference calculations are shown in Table III. For simplicity, the values are shown as decimal numbers. As a result, for example 1-1, the difference for color \#C7B2DE is smaller than that for \#B4EBFA; consequently, the color \#C7B2DE is determined as the provisional color for \#B6B7C6. For example 1-2, the difference for color \#FFD1D1 is smaller than that for $\# \mathrm{C} 8 \mathrm{C} 8 \mathrm{CB}$; consequently color \#FFD1D1 is determined as the provisional color of \#E8F2B0. Table IV shows the conversion results for these three examples.

2) Calculation of contrast ratio: After determination of provisional colors, the contrast ratios of relative luminance between background and foreground colors are calculated. If the contrast ratios do not satisfy specific thresholds, additional conversions are performed.

To calculate the contrast ratios, relative luminance values are calculated using formulas (5)-(7) [25]. The relative luminance values range from 0 to 1 . The colors \#000000 and \#FFFFFF are the darkest and the lightest, respectively, with relative luminance values are 0 and 1 , respectively. In this formula,
" $L_{R}$ ", " $L_{G}$ ", " $L_{B}$ " indicate the relative luminance for the red, green, and blue elements of a color, respectively. " $R$ " indicates the red value of the color, and " $L$ " indicates the calculated relative luminance. Although formulas (5) and (6) show the calculations for obtaining $L_{R}$ for the red element of the color, $L_{G}$ and $L_{B}$ for green and blue elements can be calculated similarly. These formulas calculate values as decimal numbers.

$$
\begin{gathered}
R s R G B=\frac{R}{255} \\
L_{R}= \begin{cases}\frac{R}{12.92}, & R s R G B \leq 0.03928 \\
\left(\frac{R s R G B+0.055}{1.055}\right)^{2.4}, & R s R G B>0.03928\end{cases} \\
L=0.2126 \times L_{R}+0.7152 \times L_{G}+0.0722 \times L_{B}
\end{gathered}
$$

After calculating the relative luminance of original colors, the contrast ratios between background and foreground colors are calculated using formula (8) [25]. For a pair of background and foreground colors, $L_{1}$ indicates the relative luminance value of the lighter color (the greater value), whereas $L_{2}$ indicates the relative luminance value of the darker color (the smaller value). $C R$ indicates the calculated contrast ratio.

$$
C R=\frac{L_{1}+0.05}{L_{2}+0.05}
$$

3) Additional conversion: When the contrast ratios of relative luminance between background and foreground colors are high, readability and distinguishability are also high. Thus, designers use a pair of background and foreground colors with a high contrast ratio to make contents readable and distinguishable, whereas they use a pair of background and foreground colors of contents with low contrast ratios when they do not want to make contents distinguishable. In addition, according to accessibility guidelines issued by the World Wide Web Consortium (W3C) [25], to ensure readability of documents, the contrast ratios between background and foreground colors should be greater than 4.5. Considering these factors, in our method, when the calculated contrast ratios in Section V-B2 are smaller than 4.5 and smaller than the contrast ratios of the pair of original background and foreground colors, the provisional colors must be additionally converted.

In the additional conversion, the colors in the saturation levels (Table II) of the background and foreground colors 
TABLE V

EXAMPLES OF DETERMINING FINAL COLORS

\begin{tabular}{|c|c|c|c|c|c|c|}
\hline & \multicolumn{2}{|c|}{ Original color } & \multicolumn{2}{|c|}{ Provisional color } & \multicolumn{2}{|c|}{ Final color } \\
\hline $\begin{array}{l}\text { Example } \\
2-1\end{array}$ & Sample & $\begin{array}{l}\text { Background color: \#FFE6E7 } \\
\text { Foreground color: \#CC0000 } \\
\text { Contrast ratio: } 4.97\end{array}$ & Sample & $\begin{array}{l}\text { Background color: \#FFD1D1 } \\
\text { Foreground color: \#9A0079 } \\
\text { Contrast ratio: } 5.77\end{array}$ & Sample & $\begin{array}{l}\text { Background color: \#FFD1D1 } \\
\text { Foreground color: \#9A0079 } \\
\text { Contrast ratio: } 5.77\end{array}$ \\
\hline $\begin{array}{l}\text { Example } \\
2-2\end{array}$ & Sample & $\begin{array}{l}\text { Background color: \#EAE6E7 } \\
\text { Foreground color: \#6E6C6C } \\
\text { Contrast ratio: } 4.22\end{array}$ & Sample & $\begin{array}{l}\text { Background color: \#FFD1D1 } \\
\text { Foreground color: \#7F878F } \\
\text { Contrast ratio: } 2.65\end{array}$ & Sample & $\begin{array}{l}\text { Background color: \#B4EBFA } \\
\text { Foreground color: \#000000 } \\
\text { Contrast ratio: } 16.2\end{array}$ \\
\hline $\begin{array}{l}\text { Example } \\
2-3\end{array}$ & Sample & $\begin{array}{l}\text { Background color: \#1E4611 } \\
\text { Foreground color: \#66FF33 } \\
\text { Contrast ratio: } 8.20\end{array}$ & & $\begin{array}{l}\text { Background color: \#663300 } \\
\text { Foreground color: \#663300 } \\
\text { Contrast ratio: } 1\end{array}$ & Sample & $\begin{array}{l}\text { Background color: \#663300 } \\
\text { Foreground color: \#FAF500 } \\
\text { Contrast ratio: } 8.89\end{array}$ \\
\hline
\end{tabular}

are sorted again according to relative luminance. Then, for a pair of background and foreground colors, the dark color (the color with the smaller value of relative luminance) and the light color (the color with the greater value of relative luminance) are shifted to the darker color and the lighter color in the corresponding saturation levels, respectively, one by one, and then the contrast ratio is calculated again. This process is repeated until the contrast ratio becomes greater than the threshold. The threshold is the contrast ratio of the pair of original background and foreground colors. However, if the shifted colors fall out of the ranges of the saturation levels in Table II determined in Section V-B1, the attractiveness of the original colors cannot be maintained, and the designers' intentions cannot be perceived by color-impaired people. Thus, even though the contrast ratio of a pair of background and foreground colors does not become greater than the threshold, the shifting of colors is stopped when both background and foreground colors become the first and the last colors in the corresponding saturation levels. When the shifting of colors is stopped, the shifted colors are considered to be the final colors.

Table V shows examples of conversion of original colors to final colors. In this table, the original colors are converted to provisional colors by the process of Section V-B1.

For example 2-1, the provisional colors of background and foreground are \#FFD1D1 and \#9A0079, respectively, and the contrast ratio of the colors are 5.77 and $>4.97$ (threshold for the contrast ratio of original colors). Thus, additional conversion of these colors is not performed, and the provisional colors are considered to be the final colors.

In example 2-2, the original background and foreground colors are \#EAE6E7 and \#6E6C6C, respectively, and the contrast ratio of the colors is 4.22 , whereas the provisional colors are \#FFD1D1 and \#7F878F, respectively, and the contrast ratio is 2.65. The contrast ratio of the provisional color is smaller than both 4.5 and the contrast ratio of the original colors (4.22). Therefore, the colors must be additionally converted. For the provisional colors, the relative luminance of background and foreground colors are calculated as 0.715 and 0.238 , respectively, by formulas (5)-(7). In addition, the saturation values of the background and foreground colors are 0.180 and 0.112 . Because the background color is lighter than the foreground color, and the saturation levels of both the background and foreground colors are low as determined in Section V-B1, the background and foreground colors are shifted to a lighter and darker color, respectively, in the low saturation level. Figure 2 shows the conversion process. The colors in this figure in the low saturation level are sorted by relative luminance. The additional conversion process is as follows:

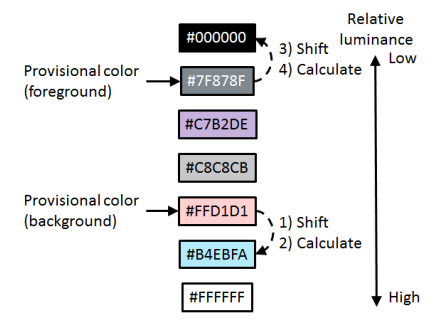

Fig. 2. Additional conversion process of example 2-2

1) Background color is shifted to the lighter color

2) Contrast ratio is calculated $\rightarrow 2.81$ (smaller than the threshold 4.22)

$\rightarrow$ Additional conversion is required $\rightarrow$ Go to 3 )

3) Foreground color is shifted to the darker color

4) Contrast ratio is calculated $\rightarrow 16.20$ (bigger than the threshold 4.22)

$\rightarrow$ Conversion is finished

When the background and foreground colors are shifted to \#B4EBFA and \#000000, respectively, the calculated contrast ratio is 16.20 , which is greater than 4.22 . Thus, the additional conversion is finished, and the shifted colors are considered to be the final colors.

In example 2-3, the provisional background and foreground colors become the same. Because the lighter and darker colors cannot be determined in this case, the relative luminance of the original background and foreground colors are used. The calculated relative luminance of the original background and foreground colors are 0.047 and 0.746 , respectively. Thus, because the background of the original color is darker than the foreground, the background and foreground of the provisional colors are shifted to the darker and lighter colors, respectively. In this example, the saturation level of both original colors 
were determined as 'high' in Section V-B1; accordingly, the provisional colors are converted to colors in the high saturation level. Figure 3 shows the conversion process. Colors in this figure in the high saturation level are sorted by relative luminance. The additional conversion process is as follows:

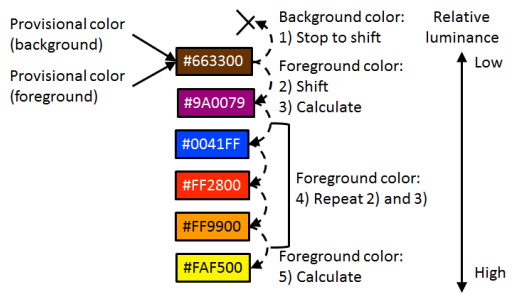

Fig. 3. Additional conversion process of example 2-3

1) Background of provisional color is the darkest color of this saturation level

$\rightarrow$ Background color cannot be shifted

2) Foreground of provisional color is shifted to the lighter color

3) Contrast ratio is calculated

$\rightarrow$ Smaller than threshold 8.20

$\rightarrow$ Additional conversion is required $\rightarrow$ Go to 4 )

4) 2) and 3) are repeated until the color \#FAF500 (until the contrast ratio become greater than 8.20)

5) Contrast ratio is calculated

$\rightarrow 8.89$ (bigger than threshold 8.20)

$\rightarrow$ Conversion is finished

When the foreground color is shifted to \#FAF500, the calculated contrast ratio is 8.89 and becomes greater than 8.20. Thus, the additional conversion is finished, and the shifted colors are considered to be the final colors.

\section{CONVERSION OF DOCUMENT COLORS}

\section{A. Conversion algorithm}

When background and foreground colors are converted, they do not always correspond one-to-one in the document. When document structures are represented by tree structures in which nodes represent blocks (parts of contents in documents) and edges represent inclusion of blocks, parent blocks often have some child blocks. This means that the size of parent blocks are bigger than child blocks. In this case, if the colors of a parent block are changed along with a child block, it may not be possible to convert colors of other child blocks appropriately. Thus, colors of parent blocks should be fixed when converting colors of child blocks. Document colors are converted by the algorithm shown in Fig. 4.

First, a tree is constructed from the target document. In the tree, nodes represent blocks, and edges represent the arrangement of child blocks on parent blocks. A block is extracted from the tree by depth-first search. If background or foreground colors are not assigned to the block, the colors are inherited from the parent block. If background (foreground) color is inherited, background (foreground) color is fixed, and foreground (background) color is converted by the conversion strategies described in Section V. Because colors of parent blocks are converted before conversion of the colors of child blocks, the fixed colors are the final colors of the parent blocks. If neither background nor foreground colors are inherited, both of them are converted.

\section{B. Example of converting document colors}

Figure 5 shows an example of the document structure at left and center. This example document is written by Hyper Text Markup Language (HTML). The source is shown at left, and the tree of the document is shown at center. In this document, blocks "h1," "h2," and "p" are arranged on the block "body." Background and foreground colors of the blocks are shown at right in Fig. 5. Because the background colors of blocks "h1" and "h2" are not given, the background color of the block "body" is applied to the background color of the block "h1" and "h2." In this case, if both the background and foreground colors of block "h1" are converted, the background color of the block "body" is also converted. This may result in an inappropriate combination between the background and foreground colors of block "h2." Thus, when the colors of block "h1" are converted, the background color is fixed.
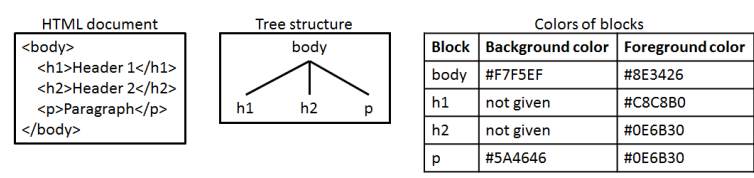

Fig. 5. Example of document structure

Applying the algorithm in Fig. 4, the colors of document are converted. First, the colors of block "body" is converted. Because block "body" does not have a parent block, both background and foreground colors are converted by the conversion strategies described in Section V. The final colors of the background and foreground colors of block "body" are \#FFD1D1 and \#663300, respectively.

Second, the colors of block "h1" are converted. Because the background color is not given, the background color of block "body" is applied. Thus, the additional conversion of the background color is not performed, according to the algorithm in Fig. 4, and only the foreground color of block "h1" is converted by the conversion strategies described in Section V. The final colors of the background and foreground of block "h1" are \#FFD1D1 (the final color of the background of the block "body") and \#C7B2DE, respectively.

Third, the colors of block "h2" are converted. As for block "h1," the background color is not given, but the foreground color is given. Thus, the conversion process is similar to that of block "h1." The final colors of the background and foreground of block "h2" are \#FFD1D1 (the final color of the background of the block "body") and \#663300, respectively.

Finally, the colors of block "p1" is converted. Because both the background and foreground colors are given, both of them are converted by the conversion strategies described in Section 


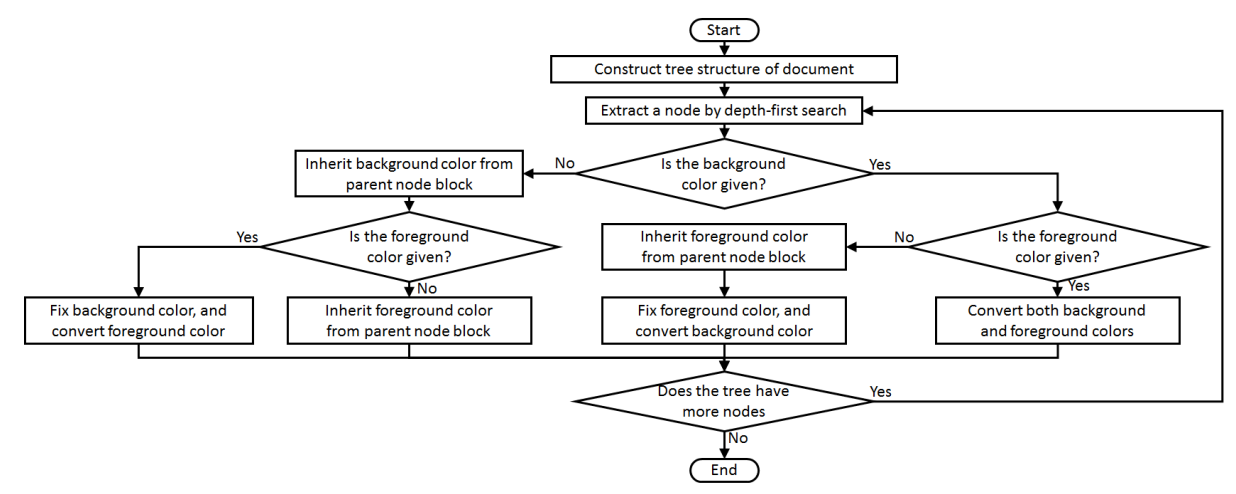

Fig. 4. Algorithm for converting document colors

$\mathrm{V}$. The final colors of the background and foreground of block "p" are \#7F878F and \#663300, respectively. Figure 6 shows the document before and after conversion.

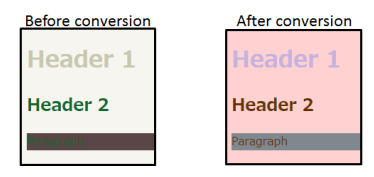

Fig. 6. Document colors before and after conversion

\section{EVALUATION}

To evaluate the validity of our method, we converted the colors of five web pages, and then our study subjects read them and answered questions.

\section{A. Procedures}

Five existing web pages were selected according to the following criteria:

1) To confirm distinguishability, more than one pair of background and foreground colors were used, with the exception of body colors.

2) To confirm attractiveness, more than one color with high saturation was used, with the exception of 1)

Although the selected web pages used image files to represent texts, our method is not designed to convert the colors of images, as mentioned in Section I. Therefore, we created web pages similar to the five selected using HTML and CSS files, and used the newly created web pages for this evaluation. As much as possible, image files were not used in the newly created web pages, and their appearances were almost the same as the original web pages. The existing web pages used for this evaluation were as follows (all are Japanese):

- Page A: http://www.ipa.go.jp/jinzai/asia/index.html (Referred on 19th December, 2016)

- Page B: https://www.nig.ac.jp/color/index.html (Referred on 19th December, 2016)

- Page C: http://www.dialoginthedark.com/today_ticket/ (Referred on 19th December, 2016)
- Page D: http://www-it.sci.waseda.ac.jp/TOP/ (Referred on 19th December, 2016)

- Page E: http://www.wul.waseda.ac.jp/imas/index.html (Referred on 19th December, 2016)

The colors of the created web pages were converted using our method, and screenshots of the color converted web pages were taken. Simulations of the appearances of these pages to color-impaired people with D-type impairments were generated using ImageJ [26] and Vischeck [27]. ImageJ is an image-processing application, and Vischeck is an application that can simulate files as they appear to color-impaired people. Vischeck can be used as plug-in for ImageJ. Then, subjects viewed the simulated screenshots and answered questions. Because the subjects saw the simulated screenshots, the contents of web pages did not change dynamically, and the web pages did not switch to other pages via hyperlinks.

A total of 12 subjects, all university students in their 20 s, took part in this evaluation. The evaluation conditions, including the order of presentation of the five web pages, the size of presentation, the computers used, and the display brightness, were the same for all subjects, and time limits were not set. Subjects answered four questions about each page by circling the appropriate text in response to the questions on the answer sheets. Answer sheets were printed for the five target web pages. Because the target web pages were printed in black and white on the answer sheets, subjects could not perceive the original attractive and distinguishable parts of the text from the answer sheets. The four questions were as follows:

Q1: Please circle the top three most attractive parts of the text, in terms of colors, and write their ranks in the blank spaces.

Q2: Please circle the top three least attractive parts of the text, in terms of colors, and write their ranks in the blank spaces.

Q3: Please circle the top three most distinguishable parts of the text, in terms of colors, and write their ranks in the blank spaces.

Q4: Please circle the top three least distinguishable parts of the test, in terms of colors, and write their ranks in the blank spaces. 


\section{B. Results}

Contrast ratios of each pair of background and foreground colors and saturation values of each color were calculated for the original five web pages, and the top three most attractive (high saturation values), least attractive (low saturation values), most distinguishable (high contrast ratio of relative luminance), and least distinguishable (low contrast ration of relative luminance) parts of the text were determined. These parts of the text were called "determined texts". The subjects' answers to questions Q1-Q4 were compared with the most attractive, least attractive, most distinguishable, and least distinguishable parts of the text, and the equivalence between the determined texts and the answers were confirmed.

Meanwhile, some of the original web pages contained images that could not be represented by HTML and CSS files, and these images remained in the newly created web pages. When the subjects answered the four questions, we explained that the images were not the target of this evaluation. However, there were answers in which the images were circled. Examples of these images were logo texts, which were difficult for the subjects to perceive them as images. Thus, we compared the determined texts with the answers in two cases: one in which answers including images were not counted ('no count' case), and another in which answers including images were evaluated as distinct from the determined texts ('distinct' case). The results are shown in Tables VI and VII.

TABLE VI

EQUIVALENCE OF ANSWERS ('NO COUNT' CASE)

\begin{tabular}{|l|l|l|l|l|}
\hline & \multicolumn{4}{|c|}{ Rates of identicalness (\%) } \\
\cline { 2 - 5 } & $\begin{array}{l}\text { Q1: Most } \\
\text { attractive }\end{array}$ & $\begin{array}{l}\text { Q2: Least. } \\
\text { attractive }\end{array}$ & $\begin{array}{l}\text { Q3: Most } \\
\text { distinguishable }\end{array}$ & $\begin{array}{l}\text { Q4: Least } \\
\text { distinguishable }\end{array}$ \\
\hline Page A & 65.0 & 44.1 & 56.3 & 25.0 \\
\hline Page B & 88.5 & 58.3 & 34.4 & 31.4 \\
\hline Page C & 76.7 & 51.6 & 46.7 & 71.0 \\
\hline Page D & 70.0 & 47.1 & 65.0 & 12.1 \\
\hline Page E & 48.0 & 75.0 & 66.7 & 37.1 \\
\hline Total & 70.3 & 55.6 & 52.1 & 34.9 \\
\hline
\end{tabular}

TABLE VII

EQUIVALENCE OF ANSWERS ('DISTINCT' CASE)

\begin{tabular}{|l|l|l|l|l|}
\hline & \multicolumn{4}{|c|}{ Rates of identicalness (\%) } \\
\cline { 2 - 5 } & $\begin{array}{l}\text { Q1: Most } \\
\text { attractive }\end{array}$ & $\begin{array}{l}\text { Q2: Least. } \\
\text { attractive }\end{array}$ & $\begin{array}{l}\text { Q3: Most } \\
\text { distinguishable }\end{array}$ & $\begin{array}{l}\text { Q4: Least } \\
\text { distinguishable }\end{array}$ \\
\hline Page A & 36.1 & 41.7 & 25.0 & 23.5 \\
\hline Page B & 65.7 & 58.3 & 27.8 & 31.4 \\
\hline Page C & 63.9 & 44.4 & 38.9 & 61.1 \\
\hline Page D & 19.4 & 44.4 & 36.1 & 11.1 \\
\hline Page E & 33.3 & 75.0 & 44.4 & 36.1 \\
\hline Total & 43.6 & 52.8 & 34.4 & 32.8 \\
\hline
\end{tabular}

The numbers in the tables are the rates at which the subjects' answers that were identical to the determined texts. Here, we only considered whether the answers were included in the top three determined texts; we did not evaluate the equivalence of individual rankings. Additionally, there were opinions that it was difficult to select answers because of few colors and factors except colors, such as text size, arrangement, text densities, and decorations.

\section{Discussion}

According to Tables VI and VII, the answer rates for Q1 and Q3 in the 'no count' case were clearly higher than those for Q2 and Q4 in the 'distinct' case. This means that the subjects selected images as the answers for Q1 and Q3 more often than for Q2 and Q4. Because images are not the targets of our method, this represents a problem of the evaluation procedure. To evaluate more appropriately, it is necessary to select web pages without images.

Although the rates of equivalence of answers for Q1 and Q3 in Table VI are higher than those in Table VII, the rates for Q2 and Q4 are similar in both tables. This indicates that it might be difficult for the subjects identify less distinguishable and less attractive parts of the text.

Meanwhile, the rates of equivalence for answers for Q1 and Q2 were higher than those for Q3 and Q4, respectively, in both the 'no count' and 'distinct' cases. Q1 and Q2 are questions related to attractiveness, whereas Q3 and Q4 are related to distinguishability. There were many common answers between Q1 (Q2) and Q3 (Q4). This indicates that attractiveness is related to distinguishability, and that the sizes and arrangements of text, in addition to color, affected attractiveness and distinguishability.

The answers that were not identical to the determined texts for Q1 and Q3 included headlines and itemized sentences. For Q2 and Q4, parts of the text that were small, or had narrow line spacing, were selected. These findings indicate that conditions other than colors were reflected in the answers.

In addition, the contrast ratios for some pairs of final background and foreground colors became significantly higher than the contrast ratios for their original colors. Due to this, the distinguishability of the parts of the text with the final colors might have been higher than those of the parts with the original colors. If the designers' intentions are considered, the conversion strategies should be improved in order to not make the contrast ratios too high.

However, the total rate of equivalence for answers for Q1 was over $70 \%$ for 'no count' case, indicating that the parts of the text with high attractiveness could be perceived. This result confirms the effectiveness of our method.

\section{Threats to validity}

In this evaluation, the converted colors of the target web pages were simulated for D-type color impairment. As noted in Section III-A, there are other types of color impairment, including P-type, T-type, and A-type. Even though certain colors are simulated, the results of simulations are often quite different among these types. Thus, if the converted colors of the target web pages were simulated for other types of vision, the results might be different.

Meanwhile, the subjects were all C-type people, and the converted colors of web pages in the evaluation were simulated for vision of color-impaired people. However, the conditions 
of color-impaired people are individually different, even within a given type of color impairment. This means that evaluation by color-impaired people might yield different results.

As described in Section I, the target of our method is text in documents, rather than images. These documents include images, which are not converted. In addition, our method assumes that the text in a target document is divided into blocks like HTML tags, and each block has one background color and one foreground color. If a block has multiple background or foreground colors, our method cannot be applied.

In addition, our method assumes that designers use highsaturation colors for parts of the text that they want to make attractive, and pairs of background and foreground colors with high contrast ratios for parts of the text that they want to make distinguishable. However, if designers do not follow these color-use strategies, our method will not work appropriately.

\section{CONCLUSION}

This paper describes our method for converting document colors so that color-impaired people can perceive the designers' intentions, here defined as attractiveness and distinguishability. High attractiveness is represented as colors with high saturations, whereas high distinguishability is represented as pairs of background and foreground colors with high contrast ratios. The conversion is performed in two steps. The first conversion is performed using the basic conversion table, whereas the second conversion is performed by calculating contrast ratios of pairs of background and foreground colors. In the second conversion, if the calculated contrast ratios do not satisfy certain thresholds, additional conversions are performed. In addition, we propose an algorithm for applying these conversion strategies to documents.

To evaluate the method, five web pages were selected and the colors were simulated to the appearance of D-type color impairment after color conversion. Twelve subjects viewed the simulated web pages and answered four questions. The results showed that although conditions other than colors affected the answers, attractive parts of the text could be perceived. These findings indicated that our method was effective.

Future work will focus on the following:

- Improvement of evaluation procedures

- Considerations of various types of color impairments

- Evaluation by color-impaired people

- Increasing the number of candidate colors for conversion

\section{REFERENCES}

[1] Facts About Color Blindness. [Online]. Available: https://nei.nih.gov/ health/color_blindness/facts_about (Referred in May 6, 2017).

[2] Color Universal Design Colorset Guidebook. [Online]. Available: http://www2.cudo.jp/wp/wp-content/uploads/2016/10/CUD_Colorset_ Guidebook.pdf (in Japanese) (Referred in May 6, 2017).

[3] L. Zhou, V. Bensal, and D. Zhang, "Color adaptation for improving mobile web accessibility", Procs. of 2014 IEEE/ACIS 13th International Conference on Computer and Information Science (ICIS), Taiyuan, China, 2014. http://dx.doi.org/10.1109/ICIS.2014.6912149

[4] D. R. Flatla, K. Reinecke, C. Gutwin, and K. Z. Gajos, "SPRWeb: preserving subjective responses to website colour schemes through automatic recolouring", Procs. of the SIGCHI Conference on Human Factors in Computing Systems, Paris, France, pp. 2069-2078, 2013. http://dx.doi.org/10.1145/2470654.2481283
[5] L. C. Ou, M. R. Luo, A. Woodcock, and A. Wright, "A study of colour emotion and colour preference. Part I: Colour emotions for single colours", Color Research \& Application, Volume 29, pp. 232240, 2004. http://dx.doi.org/10.1002/col.20010

[6] H. Takimoto, H. Yamauchi, M. Jindai, and A. Kanagawa, "Modification of indistinguishable colors for people with color vision deficiency", Journal of Signal Processing Volume 16, Number 6, pp. 587-592, 2012. http://dx.doi.org/10.2299/jsp.16.587

[7] A. Kruger, "Median-cut color quantization”, Dr. Dobb’s Jounal, 1994.

[8] A. A. Gooch, S. C. Olsen, J. Tumblin, and B. Gooch, "Color2Gray: salience-preserving color removal", Proc. SIGGRAPH, Los Angeles, California, pp. 634-639, 2005. http://dx.doi.org/10.1145/1073204.1073241

[9] N. H. N. A. Wahab, F. S. Ismail, and M. A. A. Nawawi, "Color Transformation Method for Protanopia Vision Deficiency using Artificial Neural Network", Journal of Telecommunication, Electronic and Computer Engineering, Volume 8, Number 11, pp. 29-33, 2016.

[10] H. Orii, H. Kawano, N. Suetake, and H. Maeda, "Color conversion for color blindness employing multilayer neural network with perceptual model", Proc. of the 7th Pacific-Rim Symposium on Image and Video Technology (PSIVT 2015), Volume 9431, pp. 3-14, Auckland, New Zealand, 2015. http://dx.doi.org/10.1007/978-3-319-29451-3_1

[11] CIE, "Commission internationale de l'Eclairage proceedings", Cambridge University Press, 1931.

[12] T. Smith and J. Guild, "The C.I.E. colorimetric standards and their use", Transactions of the Optical Society, Volume 33, Number 3, pp. 73-134, 1932. http://dx.doi.org/10.1088/1475-4878/33/3/301

[13] V. C. Smith and J. Pokorny, "Spectral sensitivity of the foveal cone photopigments between 400 and $500 \mathrm{~nm}$ ", Vision Research, Volume 15, Issue 2, pp. 161-171, 1975. http://dx.doi.org/10.1016/00426989(75)90203-5

[14] F. Vienot, H. Brettel, and J. D. Mollon, "Digital video colourmaps for checking the legibility of displays by dichromats", Color Research and Application Volume 24, Issue 4, pp. 243-252, 1999. http://dx.doi.org/10.1002/(SICI)1520-6378(199908)24:4<243::AIDCOL5 $>3.0 . \mathrm{CO} ; 2-3$

[15] N. Suetake and G. Tanaka, "Color transformation techniques for gamuts of lower dimension - color transformation for monochrome display/print and dichromacy -". IEICE ESS Fundamentals Review Volume 6, Number 2, pp. 102-113, 2012 http://dx.doi.org/10.1587/essfr.6.102

[16] R. Harwahyu, A. S. Manaf, B. S. Ananto, B. A. Wicaksana, and R. F. Sari, "Implementation of color-blind aid system", Journal of Computer Science, Volume 9, Number 6, pp. 794-810, 2013. http://dx.doi.org/10.3844/jcssp.2013.794.810

[17] S. Tajima and K. Komine, "Saliency-based color accessibility", IEEE Transactions on Image Processing, Volume 24, Number 3, pp. 1115 1126, 2015., http://dx.doi.org/10.1109/TIP.2015.2393056

[18] M. Geissbuehler and T. Lasser, "How to display data by color schemes compatible with red-green color perception deficiencies", Opt. Express 21, pp. 9862-9874, 2013. http://dx.doi.org/10.1364/OE.21.009862

[19] M. Tennekes and E. Jonge, "Tree colors: color schemes for treestructured data", IEEE Transactions on Visualization and Computer Graphics, Volume 20, Number 12, pp. 2072-2081, 2014. http://dx.doi.org/10.1109/TVCG.2014.2346277

[20] K. Tsuchida, C. Kato, and T. Nagshima, "Color barrier free displays in disaster situations", Open Journal of Social Sciences Volume 4, Number 2, pp. 25-29, 2016. http://dx.doi.org/10.4236/jss.2016.42005

[21] A. Frane, "A call for considering color vision deficiency when creating graphics for psychology reports", The Journal of General Psychology, Volume 142, Number 3, pp. 194-211, 2015. http://dx.doi.org/10.1080/00221309.2015.1063475

[22] A. R. Smith, "Color gamut transformation pairs", Computers Graphics vol.12, pp.12-19, 1978. http://dx.doi.org/10.1145/965139.807361.

[23] Japan Color Research Institute, "Practical color co-ordinate system", Japan Color Research Institute, 1964.

[24] Japan Color Research Institute, "PCCS harmonic color chart 201-L", Japan Color Research Institute, 1999.

[25] Web Content Accessibility Guidelines 2.0. [Online]. Available: https://www.w3.org/TR/WCAG20/ (Referred in May 6, 2017).

[26] ImageJ. [Online]. Available, https://imagej.nih.gov/ij/ (Referred in May $6,2017)$

[27] VischeckJ. [Online]. Available, http://www.vischeck.com/ (Referred in May 6, 2017) 


\title{
Distance-Profile Chart: a Novel Visual Representation of Mutual Location of 3D Objects
}

\author{
Magdalena A. Tkacz \\ Institute of Computer Science \\ University of Silesia in Katowice \\ ul. Będzińska 39, 41-200 Sosnowiec, Poland \\ Email: magdalena.tkacz@us.edu.pl
}

\begin{abstract}
This document presents a novel method for visual representation of mutual objects location (relative to each other) in 3D. The motivation and inspiration for such a work come from chromosome territory (CT) adjacency analysis. This paper describes: the idea of the cone of sight $(\mathrm{CoS})$, with an explanation of the origin of such approach; the way a mathematical model of $C o S$ was build and a process of a space segmentation with CoSes. Next, the way how distance-profile chart (DPC) is designed and created was described and finally, DPCs on the exemplary dataset was presented. Finally, some conclusions were formulated.
\end{abstract}

\section{INTRODUCTION}

$\mathbf{E}$ VERY DAY we assess where different objects are situated around us, and in which distance they are. In fact, this is a basis that allows us to live and move - by perpetual environment observation, we can notice any change in surrounding world. Then, in dependency on the situation, we can take a proper, adequate to a situation action: either we can come closer (e.g. to take something) or make a step back to avoid injury. For orientation in an environment (navigation) - determination of direction and distance in which any object is situated humans for ages used rhumb (marine navigation) [1], the wind rose or - so do now we use the clock-related method. It is the easiest way to instruct someone saying "It is on your four, in 2 meters distance" or during outdoor activity - "It is to the south-east of you, in 15 meters distance". This works, but only in 2D. We do not have either a 3D wind rose or $3 \mathrm{D}$ clock to determine direction when we want to orient in a $3 \mathrm{D}$ space.

\section{A. Motivation and background}

When we started to work on chromosome territory (CT) [2] modeling algorithm and application for model visualization [3] we did not assume that it will result in further methodological and algorithmic problems (others that concerns only model itself). But, when finishing Chromosome Territory Modeler (ChroTeMo) and Chromosome Territory Viewer (ChroTeVi) [3] subsequent question appears: $\mathrm{OK}$, so we have a probabilistic 3D model of chromosome territory for certain species. Such a model can be visually analyzed and compared by the human to the photos from FISH microscopy using chromosome paintings method, but ...how can we automate and objectify adjacency analysis of certain chromosomes, pairs of chromosomes or chromosome arms? Although at first sight it seems to be practically impossible, or at least difficult - an author decided to try to cope with that problem, and results of the first attempt are described and shown in this paper.

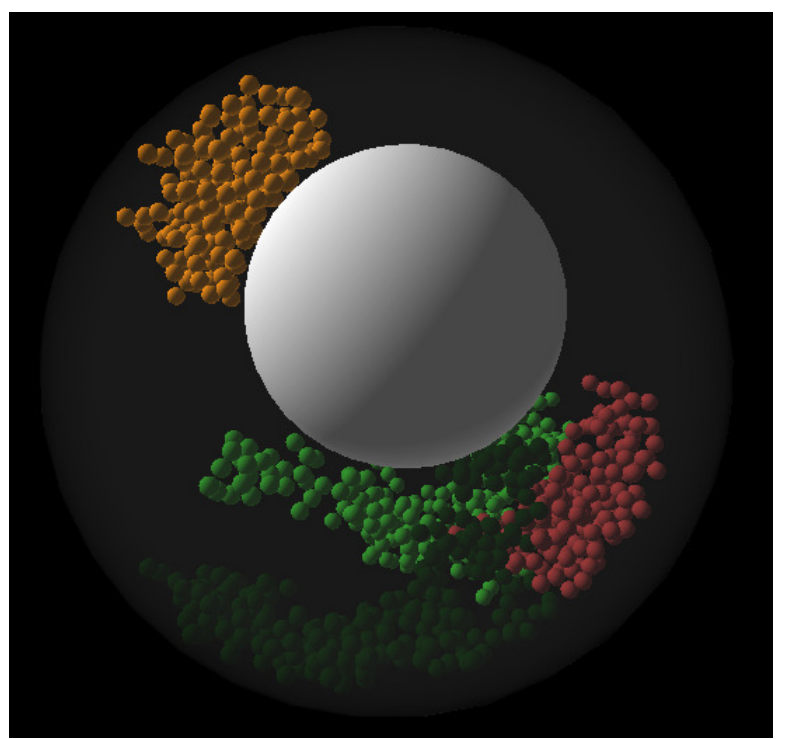

Fig. 1. Visualization of CT's with the use of ChroTeVi Model based on human species data.

This paper describes attempts of developing a method that allows automatically, in a reproducible and objective way get an answer to the question: which CT's (visualized as groups of spheres (see Fig. 1) are nearest to a given chromosome territory. How distant a surrounding objects are? Is a territory occupied by the cloud of a green spheres nearest to territory occupied by the red or rather by the orange ones (in 3D of course)? How near or how far they are? How they are situated in relation to each other? The first idea was to use some projection methods - but in that way, some information has been lost. There were also an attempt to make use of the Hausdorff distance to calculate distance among CT's. The 
Hausdorff distance (HD) was computed according Eq. 1 ([5], p.150) (for the two sets, $A$ and $B$, where $x \in A, y \in B$ ):

$H \operatorname{dist}(A, B)=\max (\limsup (\operatorname{dist}(x, B), \lim \sup \operatorname{dist}(y, A)))$

From my initial experiments, it looks that this method gives acceptable results with objects (approximated by the cloud of 3D points) with less complicated shape than CT's territories, so results of using $H D$ were not satisfactory. I also take a look at octree [4] (quadtree in 3D): this approach gives detailed and complete 3D space segmentation, but does not give intuitively imaginable description of direction where other objects are located in relation to a given object. Moreover, method itself is not easy to follow by non-tech/IT person - CT analysis are to be developed for life scientists as a target group. This led to the necessity of rethinking this problem in a different way.

\section{B. Shaft of Light and The Cone of Sight idea}

The idea of cone of sight was inspired by the shaft of light (a lighthouse or a torch). When you are in the dark and turn on the torch, pointing the light in a certain direction (for example to the left in relation to you), then you can see objects in the shaft of light. Then you can assess if at all, and how distant different objects are to the left of you. Directing the light in different directions (left, right, up, down, front, rear) allows you to explore entire space around you. This able you to make a kind of a "mental image" - the "map" of objects and their location in your surrounding.

To use this idea, developing a mathematical model of a shaft of light was necessary. The shaft of light has a shape that is similar to a cone (see Fig. 2): consists of a single point at the beginning and staying wider when far away.

The cone, as a solid block known from stereometry (solid geometry), cannot be described with the equation. The desirable solution for computational purposes would be the solution that allows writing conical-like shape in a form of equation. The solution came from analytical geometry: the conical surface [6] is one of the quadratic surfaces and can be described with a mathematical equation. Thus, the interior of space, limited by and located inside a conical surface could be considered as an appropriate model of the interior of the shaft of the light, suitable for computational purposes.

\section{Mathematical Model of the Cone of Sight}

Conical surface is known as a family of lines, crossing at a certain point and tangent to the sphere (see Fig. 2). The equation of conical surface can be derived as a set of lines that are tangent to a certain shape (base - often circular) and crossing one point (the cone vertex). For the purpose of this paper, the base is circular, i.e., it is a circle made by a cross-section of the sphere by the plane that passes through a diameter.

So, when there is a need to cover $3 \mathrm{D}$ space around fixed object(point) with a certain set of cones, it is enough to have a set of spheres basing on which a certain cone equation could be derived. The problem of covering space with spheres is

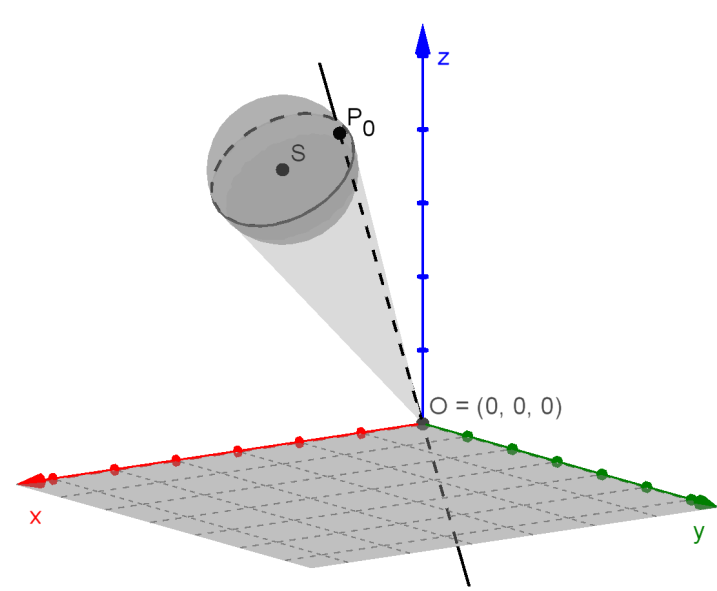

Fig. 2. Conical surface construction. Conical surface as a surface created by lines, crossing by a fixed point and tangent to the given sphere

known as "sphere packing" ([7], [8]) and will not be discussed here further.

To uniquely define a sphere in 3D you have to know the coordinates of the center of the sphere and the radius of this sphere. The coordinates of the additional point, that will become vertex should also be known. This point is also assumed to be the center of the given object, in relation to which we want to determine a relative position of other objects. For the purpose of this work, it is assumed that this point is a geometrical center of a given object - in general. In the case of $C T$ s, the centromere is regarded as center of chromosome. To simplify calculation it is also assumed that the center of object is the vertex of conical surface and is the center of the coordinate system $O(0,0,0)$. The equation of any sphere, with the center in $(a, b, c)$ and radius $R$ is shown on Eq. 2:

$$
(x-a)^{2}+(y-b)^{2}+(z-c)^{2}=R^{2}
$$

For a given sphere (or set of spheres) with a center in a point $(a, b, c)$ and a radius $R$, it is possible to derive the equation of a desirable conical surface (with a vertex in a given point).

A parametric equation of a line in $3 \mathrm{D}$ space, crossing a point $P_{0}\left(x_{0}, y_{0}, z_{0}\right)$ has the following form:

$$
\left\{\begin{array}{l}
x=x_{0} t \\
y=y_{0} t \\
z=z_{0} t
\end{array}\right.
$$

where $t$ is a parameter.

If point $P_{0}$ belongs to the line that is also tangent to the sphere, then both equations those of the sphere and the line have to been fulfilled. So:

$$
\left(x_{0}-a\right)^{2}+\left(y_{0}-b\right)^{2}+\left(z_{0}-c\right)^{2}=R^{2}
$$




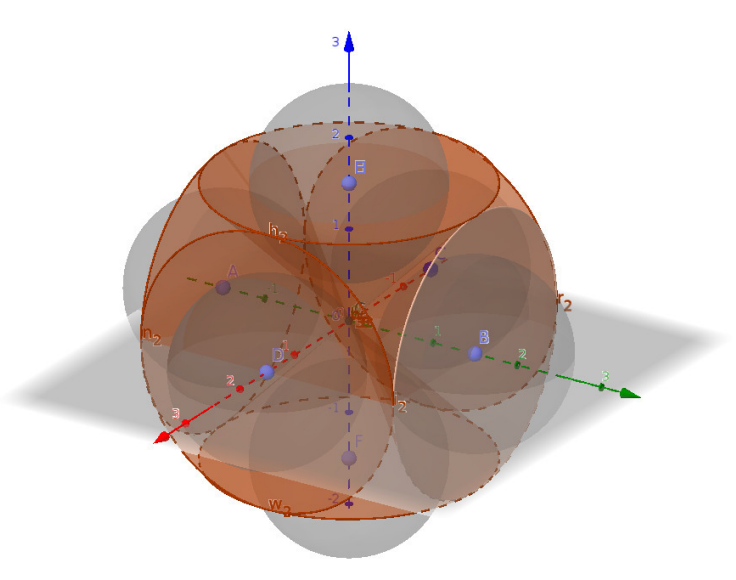

Fig. 3. Cones of sight that are located alongside axes.

Substituting equation 3 to the equation of the sphere 4 , after making some transformation (according [9], p. 227) we have a conical surface equation derived (Eq. 5):

$(-2 a x-2 b y-2 c z)^{2}-4\left(x^{2}+y^{2}+z^{2}\right)\left(a^{2}+b^{2}+c^{2}-R^{2}\right)=0$

With the inequality (Eq. 6) it is possible to determine which certain points in $3 \mathrm{D}$ space are outside or inside the conical surface. A certain point with coordinates $(x, y, z)$ will be inside conical surface if and only if its coordinates will conform (satisfy) Eq. 6.

$$
(-2 a x-2 b y-2 c z)^{2}-4\left(x^{2}+y^{2}+z^{2}\right)\left(a^{2}+b^{2}+c^{2}-R^{2}\right) \geq 0
$$

This is all we need to make $3 \mathrm{~d}$ space segmentation, and after segmentation we can check which objects are in which space segment represented by a $(C o S)$.

This approach was used by the author to create a set of cones, that allows to segment 3D space into precisely defined sectors (representing directions). Those sectors will be further refereed to as Cone of Sight $(\boldsymbol{C o S})$, and the process of dividing 3D space into cones will be refereed to as conification. Conification itself does not ideally cover the space - there are "gaps" between conical surfaces. Additional procedure that allows to assign yet unassigned points to the certain $\operatorname{CoS}$ is described later in this paper altogether with discussion of coverage during conification. In this paper the most intuitive (and thus relatively easy to understand) conification was used: CoSes were placed alongside axes in 3D Cartesian coordinate system (see Fig.3), with top of all cones placed at the beginning of this system, in the point $O(0,0,0)$.

Then, there will be six $C o S$ es (see also Fig. 3 and 5):

- $\mathrm{OX}+$ (relative direction: right),
- OX- (relative direction: left),

- $\mathrm{OY}+$ (relative direction: front),

- OY- (relative direction: rear),

- OZ+ (relative direction: up),

- OZ- (relative direction: down).

Each $\mathrm{CoS}$ represents direction in which someone can look at. To be able to uniquely determine to which $\operatorname{CoS}$ a certain point belongs the only thing is to substitute a given point coordinates to the equation of the conical surface (Eq. 6) and check whether it fulfills the inequality.

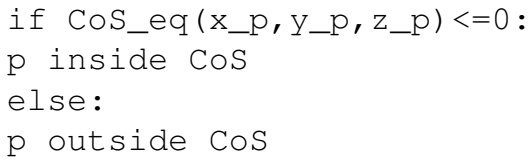

The number of required comparison, at these stage of segmentation, equals (number of points times number of CoSes).

After this procedure, some of the points may still reside outside any $\mathrm{CoS}$. As you can see on Fig. $3 \mathrm{CoS}$ s itself does not fulfill space entirely, there is a little space in between them. Anyway, we have to have all points (of all objects) being allocated to $C o S$. To solve this problem, the topological measure (known as the distance from the point $x$ to the set $A$ ([5], p.140)) was used (Eq. 7):

$$
\operatorname{dist}(x, A)=\liminf |x-a|, a \in A
$$

Points that remain unallocated after conification process are then allocated to the nearest $\operatorname{CoS}$ according mentioned measure (Eq. 7).

It was interesting how many points are conified and how many have to be assigned using additional, mentioned above measure. To check coverage of conification, a set of testing sets (with different number of points) were prepared. They were a spherical 3D sets generated with the normal or unified distribution. Percentage of points assigned in conification step and in the second (as distance between point and the set) step is shown in Tab. I.

It is easily to notice that during conification $76-81 \%$ of points are assigned to CoSes, so $19-24 \%$ have to be assigned using measure defined by the Eq. 7 to reach full conification. It can be also seen that most of the points were assigned during the first step. The assignment made during the second step assure us, that no point remains unallocated.

So, having uniquely segmented 3D space (or - thinking in categories of the shaft of light - everything visible inside a shaft) - we can go into checking what is inside this certain shaft $(C o S)$ (what can be "seen" inside). After full conification (conification and assignment of remaining points to the nearest $\operatorname{CoS}$ ) each point $P_{i}$ is characterized by the set of parameters $P_{i}\left(x_{i}, y_{i}, z_{i}, o b j_{i d}, C o S_{i d}\right)$. Therefore we can subset all points grouping them by $C o S_{i d}$.

Having sets of points located in a certain $C o S$ 's (segment of space) we are able to state which objects can be seen in a predefined part of a space $(C o S)$ around given object. When a set of points is limited only to points being inside $C o S$, 
TABLE I

NUMBER OF CONIFIED POINTS (SPHERICAL SET WITH UNIFORM AND NORMAL DISTRIBUTION WITH DIFFERENT NUMBERS OF POINTS). THE LOWEST IS IN italics, THE HIGHEST IN BOLD

\begin{tabular}{c|ccc} 
distribution & no of points & conified & remaining \\
\hline \hline & 1000 & $77 \%$ & $23 \%$ \\
& 2000 & $76 \%$ & $24 \%$ \\
& 5000 & $77 \%$ & $23 \%$ \\
& 7500 & $76 \%$ & $24 \%$ \\
& 10000 & $76 \%$ & $24 \%$ \\
\hline \multirow{3}{*}{} & 1000 & $\mathbf{8 1} \%$ & $19 \%$ \\
& 2000 & $\mathbf{8 1} \%$ & $19 \%$ \\
苛 & 5000 & $\mathbf{8 1} \%$ & $19 \%$ \\
& 7500 & $80 \%$ & $20 \%$ \\
& 10000 & $80 \%$ & $20 \%$
\end{tabular}

then it is easily to compute the less and the most distant points of a given object inside certain $C o S$. It is also possible to state (e.g. in percent), which part of points representing object are inside. Therefore, all the data necessary to make "a mental map" (mentioned in section I-B) of any object (with its surrounding) is present. Those (often numerous) data, although contains all information necessary to make adjacency analysis can be stored in a tabular form, but this is not easy to review format by humans. Humans have visual perception stronger than any other sense, so decision to present those relationships in visual form was taken. However, in some applications (e.g. computer vision) storing and processing this data in a form of a table or array can be more useful.

\section{Constructing Distance-PRofile Charts (DPCs)}

In this section the idea and details of distance-profile chart will be presented. After conification process, the following information concerning every given object is present: fraction of other objects that are located in the surrounding examined objects $C o S$ es and the distances of the nearest and the outermost point for every other object. Basing on these information, a kind of "mental map" (that allows us to imagine what object, to which extent, and in which ranges of distance it spreads) can be prepared. This is a situation similar to those, when in a darkness a torch is used: the one can turn and look around to determine the location and distance of objects that are visible in torch's shaft of light (as mentioned previously).

The assumption for the chart was that it should show all the information necessary to create "a mental map" of all objects surrounding a given one. That means, it should reflect the range of distances and the fraction of all objects located inside each $\operatorname{CoS}$ (direction), because this information is necessary to asses mutual location of objects in $3 \mathrm{D}$ space. Such set of charts were named the Distance-Profile Chart, (DPC). Such a name was given because this visualization shows the "profile of distances" of all other objects in relation to a given object.

Each DPC consists of a given number of sub-charts. Each sub-chart presents distance range, and a fraction of objects located in a certain $\operatorname{CoS}$ (so the number of sub-charts equals

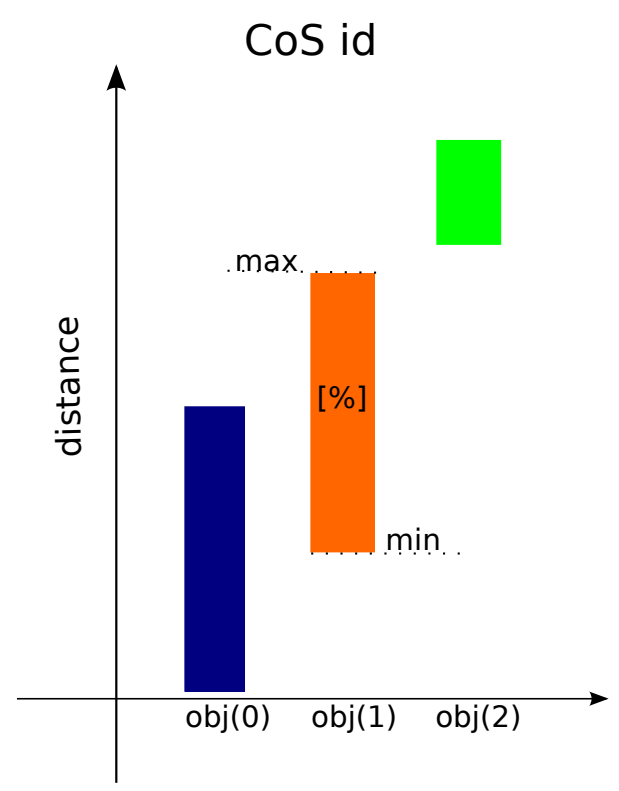

Fig. 4. Distance-profile chart construction schema.

to the number of $\operatorname{CoS}$ that divides the space, for every given object). The idea of creation each sub-chart and parameters is shown on Fig. 4.

It is easy to notice, that it is somehow inspired by a boxplot charts. The sub-charts are placed on a grid to present in a concise way all information about what is inside every $\mathrm{CoS}$ in relation to a given object. Each grid field represent one $C o S$, so this chart is "CoS-centered". The grid used to show arrangement of subcharts is presented in the Fig. 5.

The color of the bars corresponds to the scale that represents the fraction (percentage) of an object inside a given $C o S$ s. The scale used in this paper is shown in Fig. 6.

\section{A. DPC of the exemplary dataset}

To show how $C o S$ idea and DPC work in practice a special testing set (consists of three easy to show and imagine objects) was prepared. This set consists of three elliptical objects that are positioned in such a way that looks like the head of a mouse with ears (see Fig. 7).

This set was fully conified, and the DPCs were generated. Because this set of objects consists of three objects - also three DPCs were created (one for every object: head, left ear and right ear). All three DPCs are shown on Fig. 8-10.

Reading of DPCs needs some training. On the presented Mouse 3D exemplary dataset we can see three object: "the head", and two "ears". "Ears" are located above "head". Moreover, "ears" are located on opposite sideways. So, placing center of coordinate system in the center of the "head" we can see, that (refer to Mouse 3D image on Fig. 7):

- "head", as a center of coordinate system lie on every ax (in every direction), 


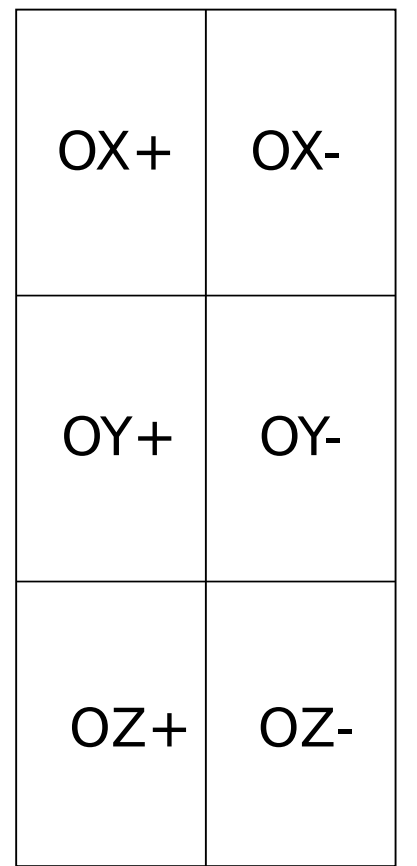

Fig. 5. Grid with fields for $D P C$ subcharts. $O X+$ means that this is a $C o S$ alongside $O X+$ axis, $\mathrm{OY}+$ alongside $O Y$ axis and so on.

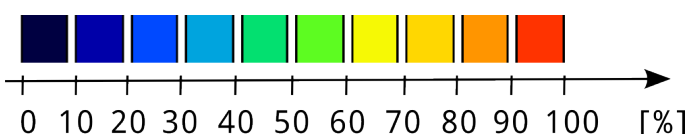

Fig. 6. Scale - percentage of object included in a certain $\operatorname{CoS}$

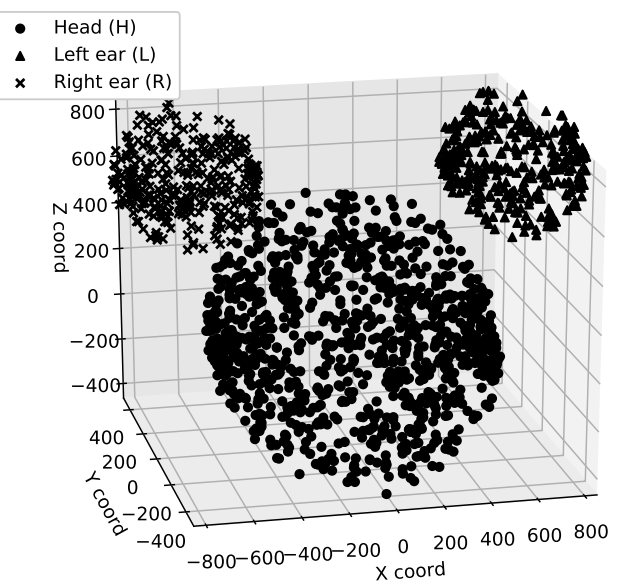

Fig. 7. Mouse 3D exemplary set.
- both ears lie in a "range" of OZ+ axis,

- one of the ear lies in a range of OX+ axis,

- the second ear lies in a range of OX-axis.

Let's take a look on the DPC "for" the "head" object (Fig. 8): it is the only object that has the smallest distance from the center of coordinate system (starting nearby zero) $A N D$ is in every CoS. It is depicted on a horizontal ax as second object. What does it means? The second object is around center of coordinate system, alongside $\mathrm{OY}$ and $\mathrm{OZ}$ axes the most distant point is in the same distance, but alongside OX ax distance is greater than alongside OY and OZ. That means that the object being a point of reference (object in relation to which we assess mutual position of other objects) is longer alongside OX. The dark blue color on fields OY+, OY-, OZ+, OZ- means, that there is $10-20 \%$ of the object. The light blue on fields that represents $\mathrm{OX}+$ and $\mathrm{OX}$ - means, that there is a $20-30 \%$ of the object. Putting together information about extent of the bar concerning the set and information about fraction of object coded as color, we can infer, that head of mouse is not symmetrical and is longer alongside OX axis.

Looking at length of bars, we can ascertain, that the part of the second object located in every $\operatorname{CoS}$ is more or less the same in size than entire objects one and three. On field that represent $\mathrm{OX}+, \mathrm{OX}-$ and $\mathrm{OZ}+$ we can see additional bars. They are related to the other objects. First object on horizontal axis of the DPC corresponds to the right ear, third object corresponds to the left ear

On OX+DPC field we can see an object that is farther than the head. The same object appears also in OZ+. This means that this object spans between $\mathrm{OX}+$ and $\mathrm{OZ}+$. We can see that it is located through diagonal to the upper right. Reading fraction from colors, we can say that this object is more in OZ+ (50-60\%) than in OX+, so is shifted more up than to the right. This is how the right ear is situated (this can be verified looking at Fig. 7)

Then, we can read information about third object. It appears on DPC in the field OX- and OZ+. Analyzing in the same way as in a case of the firs object, we can infer that this object is located in the left upper "corner". Opposite to the first object, it is more "shifted" towards OX- (what can be read from color - contains more points).

The next DPC to analyze is presented on Fig. 9. On this figure we can see the bars in all $\operatorname{CoS}$ for object one - so this is our object of reference. We can also see one additional bar (object) on field OZ-, and two additional bars on OX- field. This means, that, in relation to this object only one object is located below. But the same object is also located in OX- field in proportion $50-60 \%$ in OZ- and $40-50 \%$ in OX- field. This means that bigger part of object two is located below. Looking at length of bars, we can also ascertain that the second object is bigger (in size) than object one and two (spans more widely). Looking at third object presented as red bar in OX- field of $C o S$ we can see that it is about twice in size in comparison to the object one, begins somewhere in a middle of the object two, but ends further - the distance to its extreme point is 


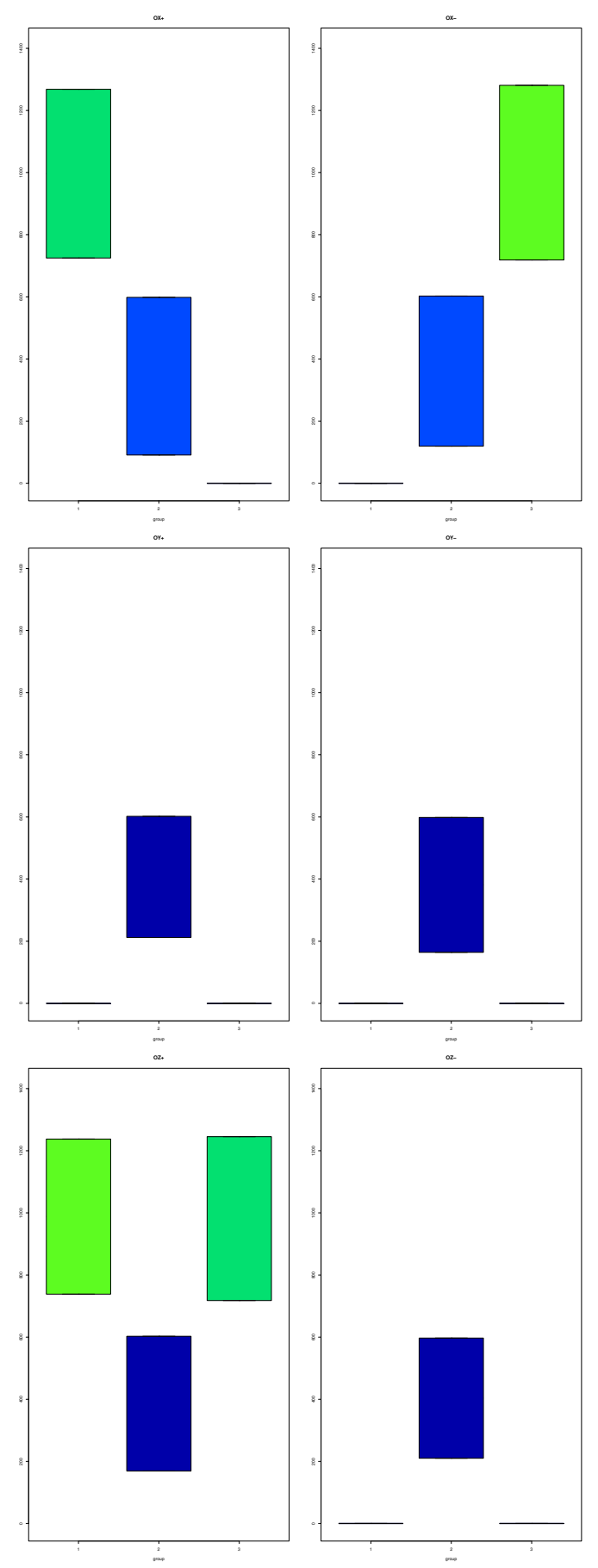

Fig. 8. Distance-Profile Chart for the Mouse 3D testing set. CoSes defined in relation to 3D Mouse's "head". greater than in a case of object two. The red color means that this third object is situated in OX- almost in full.

This description is adequate as description of a surrounding of the object being the left mouse "ear": the third object (right "ear") is entirely located to the right of the left "ear", on the bottom-right direction the "head" is located. Moreover, basing on bar length, we know that the "head" is bigger than the "ear".

The last DPC for the Mouse 3D set is presented on Fig. 10. This DPC is symmetric to those presented on Fig. 9 and can be read in a similar way as presented above.

As you can see, (comparing 3D set visualization (Fig. 7) and DPCs (Fig. 8 - 10), after a little training (to accustom to such charts and learn how to read them) you can recreate the most important features of objects positioning, their distance and distribution in relation to each other basing only on DPCs.

On Fig. 11 an exemplary DPC for the CT model is presented. This is DPC generated only for one object, as the number of pages in this paper is limited. This chart can be read in a similar way that charts for 3D Mouse exemplary set.

Data for this DPC charts was taken from Brachypodium Distachyon chromosome territory model, generated with ChroTeMo [3]. This certain DPC shows a mutual location of CTs of the other chromosome arms for the first arm of the fifth chromosome as an object of reference.

\section{CONCLUSION AND FUtURE WORK}

In this paper, a novel methodology for assessing mutual $3 \mathrm{D}$ objects location in a $3 \mathrm{D}$ space was presented with the use of the cone of sight idea. For the ease of perception for humans the novel method of visual presentation of 3D object adjacency - the distance-profile chart DPC was developed. The advantage of such approach is, that there is no need to have a special tool for visualization of $3 \mathrm{D}$ objects (which is computationally demanding and requires relatively powerful hardware). Also, the one examining the problem of the proximity of the objects in $3 \mathrm{D}$ does not have to spend a lot of time manually zooming in, zooming out and rotating scene to determine which objects are closer to the given object of interest, and how the objects are situated in relation to each other. The researcher can print $D P C$, share tis in such form and also analyze offline. Additionally, DPC approach allows to asses the mutual proximity of $3 \mathrm{D}$ objects by providing the exact and objective measure of proximity and thus made mutual proximity assessment of $3 \mathrm{D}$ objects reproducible, comparable and objective.

The future work is:

- refactor the code and provide it as a library for wide use,

- check different arrangement of spheres (basing on which conical surface equation is derived) in the context of space coverage, to maximize coverage during conification

- made benchmarks of the performance of this approach covering computational time, memory load in a context 

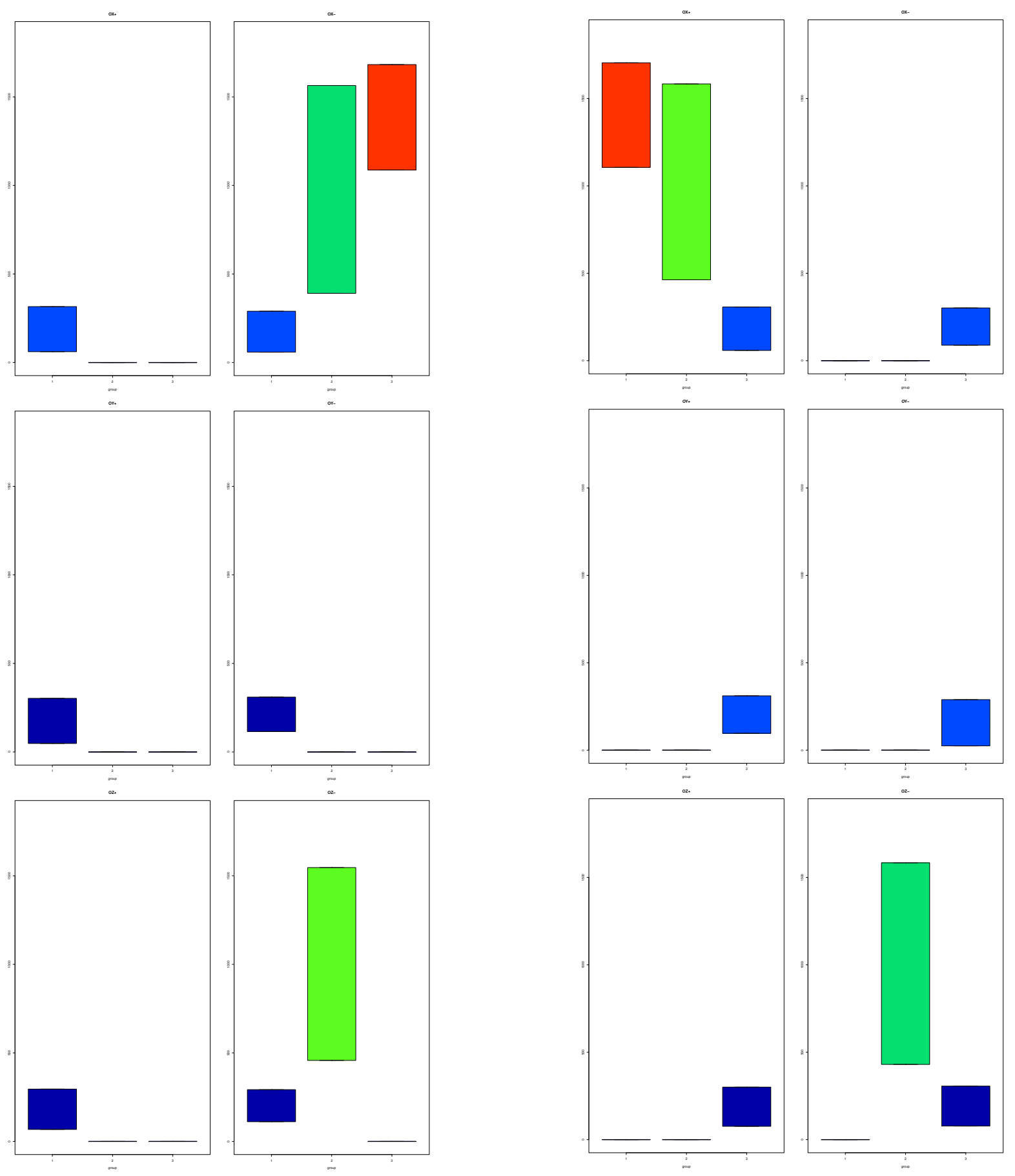

Fig. 9. Distance-profile chart for the Mouse 3D testing set. CoSes defined in relation to mouse's left ear.

Fig. 10. Distance-profile chart for the Mouse 3D testing set. CoS es defined in relation to mouse's right ear. 


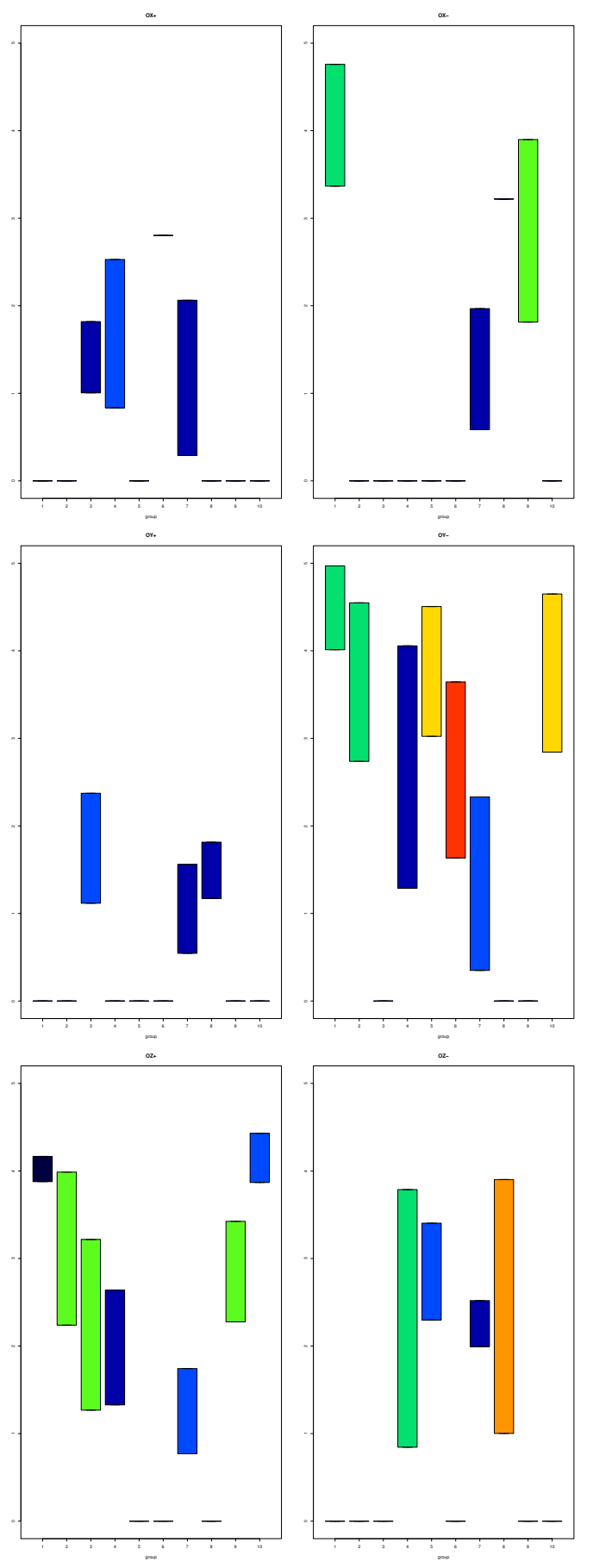

Fig. 11. Distance-profile chart for exemplary CTs model. Charts in relation to the 1 st arm of the 5th chromosome in Brachypodium Distachyon. of the type of sphere arrangement, the quantity of conified dataset and number of objects in a dataset.

\section{REFERENCES}

[1] Units in maritime navigation http://www.siranah.de/html/sail020e.htm

[2] Cremer T, Cremer C. Chromosome territories, nuclear architecture and gene regulation in mammalian cells. Nat Rev Genet. 2001;2(4):292âĂS301. Epub 2001/04/03. pmid:11283701.

[3] Magdalena Tkacz, Kornel Chromiński, Dominika Idziak-Helmcke, Ewa Robaszkiewicz, Robert Hasterok: Chromosome Territory Modeller and Viewer. PLoS ONE 11(8): e0160303. https://doi.org/10.1371/journal. pone. 0160303

[4] Mathworks: octree - partitioning 3D points into spatial subvolumes. https://www.mathworks.com/matlabcentral/fileexchange/ 40732-octree-partitioning-3d-points-into-spatial-subvolumes

[5] Kuratowski Kazimierz: Wstęp do teorii mnogości i topologii, PWN, Warszawa 1980 (Introduction to the Set Theory and Topology (in Polish))

[6] Conical surface. Encyclopedia of Mathematics. http://www. encyclopediaofmath.org/index.php?title=Conical_surface\&oldid=31530

[7] Sphere packing - Wolfram Alpha: http://mathworld.wolfram.com/ SpherePacking.html

[8] Conway, J. H. and Sloane, N. J. A. Sphere Packings, Lattices, and Groups, 2nd ed. New York: Springer-Verlag, 1993.

[9] Stark Marceli: Geometria analityczna z wstępem do geometrii wielowymiarowej, PWN, Warszawa 1974 (Analytical Geometry with introduction to multidimensional geometry (in Polish)) 


\section{The $37^{\text {th }}$ IEEE Software Engineering Workshop}

T HE IEEE Software Engineering Workshop (SEW) is the oldest Software Engineering event in the world, dating back to 1969 , and with the last $36^{\text {th }}$ workshop organized in Gdansk, Poland, 11-14 September 2016.

The workshop was original run as the NASA Software Engineering Workshop and focused on software engineering issues relevant to NASA and the space industry. After the 25th edition, it became the NASA/IEEE Software Engineering Workshop and expanded its remit to address many more areas of software engineering with emphasis on practical issues, industrial experience and case studies in addition to traditional technical papers. Since its 31st edition, it has been sponsored by IEEE and has continued to broaden its areas of interest.

\section{TOPICS}

The workshop aims to bring together all those with an interest in software engineering. Traditionally, the workshop attracts industrial and government practitioners and academics pursuing the advancement of software engineering principles, techniques and practice. The workshop provides a forum for reporting on past experiences, for describing new and emerging results and approaches, and for exchanging ideas on best practice and future directions.

Topics of interest include, but are not limited to:

- Experiments and experience reports

- Software quality assurance and Metrics

- Formal methods and formal approaches to software development

- Software engineering processes and process improvement

- Agile and Lean Methods

- Requirements engineering

- Software architectures

- Real-time Software Engineering

- Software maintenance, reuse, and legacy systems

- Agent-based software systems

- Self-managing systems

- New approaches to software engineering (e.g., search based software engineering)

- Software engineering issues Cyber-physical systems

- Software Engineering for social media

\section{SECTION EDITORS}

- Bowen, Jonathan, Museophile Ltd.

- Hinchey, Mike, Lero-the Irish Software Engineering Research Centre, Ireland

- Ryan, Kevin, Lero-the Irish Software Research Centre, Ireland

- Zalewski, Janusz, Florida Gulf Coast University, United States

\section{REVIEWERS}

- Ait Ameur, Yamine, LISI/ENSMA, France

- Banach, Richard, University of Manchester, United Kingdom

- Bensalem, Saddek, VERIMAG, France

- Bjorner, Nikolaj, Microsoft Research, United States

- Broy, Manfred, Technische Universitaet Muenchen, Germany

- Carter, John, University of Guelph, Canada

- Creissac Campos, José, Universidade do Minho, Portugal

- Denney, Ewen, SGT/NASA Ames, United States

- Derrick, John, Unversity of Sheffield

- Di Vito, Ben, NASA Langley Research Center, United States

- Eleftherakis, George, The University of Sheffield International Faculty, CITY College, Greece

- Fantechi, Alessandro, DSI - Universita' di Firenze, Italy

- Fidge, Colin, Queensland University of Technology, Australia

- Forbrig, Peter, University of Rostock

- Fortiers, Stephen, George Washington University

- Fuhrman, Christopher, ETS (École de technologie supérieure)

- Fujita, Masahiro, University of Tokyo, Japan

- Gracanin, Denis, Virginia Tech, United States

- Groce, Alex, Oregon State University, United States

- Grosu, Radu, Technische Universität Wien, Austria

- Havelund, Klaus, Jet Propulsion Laboratory, California Institute of Technology, United States

- Hsiao, Michael, Virginia Tech, United States

- Laplante, Phillip A., PennState University, United States

- Liu, Zhiming, United Nations University - International Institute for Software Technology, Macao

- Lopezo, Oscar Pastor, Valencia

- Malloy, Brian, Clemson University, United States

- Nesi, Paolo, DSI-DISIT, University of Florence, Italy

- Palanque, Philippe, ICS-IRIT, University Toulouse 3, France

- Pu, Geguang, East China Normal University

- Pullum, Laura, Oak Ridge National Laboratory, United States

- Qin, Shengchao, Teesside University

- Reeves, Steve, University of Waikato, New Zealand

- Rouff, Christopher, Lockheed Martin, United States

- Rozier, Kristin Yvonne, NASA Ames Research Center

- Seceleanu, Cristina, Mälardalen University, Västerås, Sweden 
- Sekerinski, Emil, McMaster University, Canada

- Sun, Jing, The University of Auckland, New Zealand

- Taguchi, Kenji, AIST, Japan

- Velev, Miroslav, Aries Design Automation, United States
- Vilkomir, Sergiy, East Carolina University, United States

- Yang, Hongli, Beijng University of Technology, China

- Zhu, Huibiao, Software Engineering Institute - East China Normal University 


\title{
Threefold Analysis of Distributed Systems: IMDS, Petri Net and Distributed Automata $\mathrm{DA}^{3}$
}

\author{
Wiktor B. Daszczuk \\ Institute of Computer Science, \\ Warsaw University of Technology \\ Nowowiejska Str. 15/19 \\ 00-665 Warsaw, Poland \\ Email: wbd@ii.pw.edu.pl
}

\begin{abstract}
Integrated Model of Distributed Systems is used for specification and verification of distributed systems. In the formalism, a system is modeled as a set of servers' states and agents' messages. The operation of a system is modeled as actions converting global system configuration (a set of states and messages) to a new configuration. The formalism is used in Dedan verification environment, in which specification and verification of distributed systems is performed. Equivalent Petri nets are used for structural analysis. For the graphical specification and simulation of distributed systems, Distributed Autonomous and Asynchronous Automata $\left(\mathbf{D A}^{3}\right)$ are invented. Such simulation does not require calculation of global configuration space of a system. Two forms of $D^{3}$ are shown: Server-DA ${ }^{3}\left(\mathrm{SDA}^{3}\right)$ for the server view and Agent-DA ${ }^{3}\left(\mathrm{ADA}^{3}\right)$ for the agent view.
\end{abstract}

\section{INTRODUCTION}

$\mathrm{I}^{\mathrm{N}}$ NTEGRATED Model of Distributed Systems (IMDS [1] [2]) is a formalism for specification of distributed systems. The formalism exploits the natural features of such systems:

- Communication duality: a model of a distributed system is uniform, it can be decomposed ("cut") to server processes communicating by messages or to agent processes communicating by shared resources (servers' states).

- Locality of actions: an action is executed entirely inside a server, on a basis of the server's state and a set of messages pending at this server.

- Autonomy of decisions: a server itself decides, which action will be executed (among enabled ones) and when.

- Asynchrony of actions: a server in given state awaits messages that enable some actions in it, or messages pend at a server, waiting for state that enables the server's actions.

- Asynchrony of communication: unidirectional cannels for message passing are assumed, without any acknowledgments (an acknowledgment may be sent, but using another asynchronous channel in opposite direction)

It is opposed to synchronous models, like CSP [3] or CCS [4], where distributed elements must agree on communication, which requires some kind of nonlocality.
The formal definition of IMDS can be found in [2], here we give an overview. IMDS is based on four sets: servers $S$, agents $A$, values $V$ and services $R$. We will use lowercase letters for elements of these sets. Any server has a set of states being pairs $p=(s, v)$. A server runs by accepting messages that invoke its services. The acceptance of a message at given state of a server causes the execution of an action in the context of this server. The action changes the state of the server and causes a next message to be sent (typically to some other server, but sometimes to the same server). In the system, distributed computations may be performed as sequences of actions. An agent is introduced to identify such a distributed computation.

The communication between servers is performed by means of messages. A message is an invocation of a server's service in a context of an agent, thus it is a triple: $m=(a, s, r)$. An action is defined for a pair $(m, p)$ and produces a new pair ( $m$ ', $\left.p^{\prime}\right)$. Therefore, the actions form a relation $(m, p) \lambda\left(m^{\prime}, p^{\prime}\right)$. We say that the pair $(m, p)$ matches if an action is defined for it. We impose the constraints on an action:

- The action is invoked by a message issued to a server and it is executed on this server, therefore server component $(s)$ of the message $m$ and the state $p$ must be the same.

- The action produces a new state of the server, therefore the server component $(s)$ of the input state $p$ and the output state $p$, must be the same.

- The action produces a new message of the agent (a distributed computation), therefore the agent component (a) of the input message $m$ and the output message $m$ ' must be the same.

A special kind of action terminates an agent, it is a relation between a pair $(m, p)$ and a singleton $\left(p^{\prime}\right)$ - the output message is missing: $(m, p) \lambda\left(p^{\prime}\right)$.

A global system configuration $T$ consists of current states of all servers and pending messages of agents (all but terminated ones). The actions are executed in interleaving way, therefore an action transforms its input configuration $T$, containing its input items $m$ and $p$, into an output 
configuration $T^{\prime}$, containing its output items $m^{\prime}$ and $p^{\prime}$ (or $p^{\text {' }}$ only). The initial configuration $T_{0}$ consists of initial states of all servers and initial messages of all agents.

The semantics of a distributed system is defined as Labeled Transition System (LTS), in which nodes are the configurations, initial node is initial configuration and transitions are actions.

A system may be decomposed into processes. If we group all actions to subsets on individual servers - we get server processes. Their carriers are servers' states and they communicate by messages. It is the server view of the system.

If we group all actions to subsets appointed to individual agents - we get agent processes. Their carriers are agents' messages and they communicate by servers' states (treated as resources). It is the agent view of the system.

A temporal logic is defined over IMDS, which allows to verify the correctness of modeled systems by model checking [5]. Especially, partial deadlock and termination (where not all processes participate) may be identified using universal temporal formulas. Universality of the formulas do not require from the designer any knowledge about temporal logic and model checking. Automatic partial deadlock and partial termination checking is a unique feature for systems without restriction on their structure (cycling, terminating, etc.).

The deadlocks identified using IMDS in the server view concern communication, while those detected in the agent view are resource deadlocks.

The formalism is incorporated in Dedan program for specification and verification of distributed systems [6]. Although automatic deadlock detection is useful, it has a typical drawback: model checking interrupts the verification if the evaluated formula is decided to be true or false. In deadlock detection, first deadlock found finishes the evaluation. Next deadlock may be identified after modification of the system. If many deadlocks are present, the procedure complicates the verification.

The situation is cured by static analysis of a Petri net corresponding to IMDS model [7]. In the network, subnets called siphons may be identified [8]: a siphon which is emptied from tokens, cannot restore them. If the emptying of a siphon is reachable, it may denote a deadlock. Also, some other properties of verified system may be identified using Petri net approach: unreachable actions ("dead code"), unrelated components, etc. A conversion of IMDS to a Petri net is described later in this paper.

In addition to static analysis (model checking and Petri net analysis), graphical tools for specification and simulation of the distributes systems are needed. The automata are widely used for such purpose, often called distributed automata. For example, automata on distributed alphabets, communicating on common symbols, based on Zielonka's automata [9]. These automata are called distributed automata in many papers concerning the behavior of concurrent systems (in some of them additionally equipped with real time clocks for temporal analysis with real-time constraints): [10] [11] [12]. These automata are called asynchronous for example in [13], although they perform actions (make the transitions) asynchronously only if the input symbols are distinct. They make synchronous moves on common input symbols (and it is the only common aspect of the automata). Similar are Timed Automata [10] and CSP processes [3], synchronizing on ! and ? operations rather than on symbols of input alphabet. Close to Zielonka's automata are Büchi automata. They differ in distinguishing some states as accepting. They are called distributed automata in [14].

Message Passing Automata (MPA, called distributed automata in [15]) are really distributed and asynchronous. They have ordered sets on symbols waiting for acceptance, called buffers or queues. Pushdown Distributed Automata (PDA) are equipped with local memories of input symbols (stacks) [16].

We developed a formalism of Distributed Autonomous and Asynchronous Automata (DAAA, $\mathrm{DA}^{3}$ in short) for the graphic specification and simulation.

The three modeling methods: IMDS, corresponding Petri nets and Distributed Automata are equivalent. The generate reachability spaces which have identical structures. Therefore, they all highlight the natural features of distributed systems: communication duality, locality of actions, autonomy of decisions, asynchrony of actions and communication. This paper describes the application of the three formalisms to the verification of distributed systems.

A Dedan program is presented in Section II. The example of a bounded buffer specification in IMDS is given in Section III. The conversion of IMDS to Petri net is described in Section IV. The notion of $\mathrm{DA}^{3}$ distributed automata is covered in detail in Section V. Two versions - Server $\mathrm{DA}^{3}$ $\left(\mathrm{SDA}^{3}\right)$ and Agent $\mathrm{DA}^{3}\left(\mathrm{ADA}^{3}\right)$ are defined. The operation of Dedan program on $\mathrm{DA}^{3}$ is described in Section VI. Conclusions and further work are covered in Section VII.

\section{THE DEDAN PROGRAM}

The IMDS formalism was used, together with model checking technique [5], to develop the Dedan program which finds various kinds of deadlock and termination in a verified system. A counterexample is generated if a deadlock is found, or it is a witness of distributed termination. Also, observation of global reachability graph and simulation over this graph are possible.

Dedan is built in such a way that the specification of temporal formulas and temporal verification are hidden to a user. The reason is that model checking techniques are seldom known by the engineers. Therefore, the program is constructed in such a way that a user specifies the system and simply "pushes the button" to check for the existence of deadlocks or to check distributed termination. 
Although the main target of Dedan is finding deadlocks and termination checking, a user may be interested in other properties of a verified system, for example:

- structural properties of a system: structural conflicts, dead code, pure cyclic system or not, etc.,

- temporal properties other than deadlock and termination: if a system is safe from some erroneous situation, if given situations are inevitable, etc.,

- graphical definition of concurrent components of a system (servers or agents),

- graphical simulation in terms of concurrent components rather than in terms of a global graph.

For the purpose of supporting the above possibilities, some additional facilities are included into Dedan:

- export to external model checkers for temporal analysis, for example Uppaal [17],

- export of a model to Petri net form for the analysis under Charlie Petri net analyzer [18] - to obtain a structural analysis,

- alternative formulation of a system as Distributed Automata, for facilitation of a system specification and simulation in terms of distributed components - automata representing server processes or agent processes.

\section{SIMPLE EXAMPLE - BUFFER}

To present the two views of IMDS model, a simple system containing a buffer with producer and consumer agents (each one originating from its own server) is included below. First the server view follows. The notation is intuitional: server types are defined (lines 2, 9, 16, (formal parameters specify agents and other servers used)). Every server includes states $(1.3,10,17)$, services $(1.4,11,18)$ and actions (1.6-7, 13-14, 20-21) (an action $((a, s, r),(s, v)) \lambda\left(\left(a, s^{\prime}, r^{\prime}\right),\left(s, v^{\prime}\right)\right)$ has the form \{a.s.r, $\left.\mathrm{S} \cdot \mathrm{V}\} \rightarrow\left\{\mathrm{a} \cdot \mathrm{s}^{\prime} \cdot \mathrm{r}^{\prime}, \mathrm{S} \cdot \mathrm{V}^{\prime}\right\}\right)$. Then, server and agent variables are declared (1.23-24). The variables have the same names as the types, they are distinguished by context. If a variable has the same identifier as its type, a declaration variable:type may be suppressed to a single identifier, as in the example. At the end, servers (1.26-28) and agents (1.29-30) are initialized (and variable names are bound with formal parameters of servers).

1. system BUF_server_view;

2. server: buf (agents Aprod,Acons; servers Sprod,Scons),

3. services \{put, get\},

4. states $\{$ no_elem,elem $\}$,

5. actions \{

6. $\{$ Aprod.buf.put, buf.no_elem\} -> \{Aprod.Sprod.ok_put, buf.elem\},

7. $\{$ Acons.buf.get, buf.elem\} -> \{Acons.Scons.ok_get, buf.no_elem\},
8. $\}$

9. server: Sprod (agents Aprod; servers buf),

10. services \{doSth,ok_put\}

11. states $\{$ neutral,prod $\}$

12. actions \{

13. \{Aprod.Sprod.doSth, Sprod.neutral\} -> \{Aprod.buf.put, Sprod.prod\}

14. \{Aprod.Sprod.ok_put, Sprod.prod\} ->

15. \}

16. server: Scons (agents Acons; servers buf),

17. services $\{$ doSth,ok_get\}

18. states \{neutral,cons\}

19. actions \{

20. \{Acons.Scons.doSth, Scons.neutral\} -> \{Acons.buf.get, Scons.cons\}

21. \{Acons.Scons.ok_get, Scons.cons\} ->

22. $\}$ \{Acons.Scons.doSth, Scons.neutral\}

23. servers buf,Sprod,Scons;

24. agents Aprod,Acons;

25. init $->\{$

26. Sprod(Aprod,buf).neutral,

27. Scons(Acons,buf).neutral,

28. buf(Aprod,Acons,Sprod,Scons).no_elem,

29. Aprod.Sprod.doSth,

30. Acons.Scons.doSth,

\} .

The Dedan program automatically converts the specification to the agent view. Now, the actions are grouped in agents rather than in servers (lines 13-15, 19-21).

1. system BUF_agent_view;

2. server: buf,

3. services \{put, get\}

4. states $\{$ no_elem, elem\};

5. server: Sprod,

6. services $\{$ doSth, ok_put\}

7. states $\{$ neutral, prod\};

8. server: Scons,

9. services $\{$ doSth, ok_get\}

10. states \{neutral, cons\};

11. agent: Aprod (servers buf:buf,Sprod:Sprod),

12. actions \{

13. $\{$ Aprod.buf.put, buf.no_elem $\}$-> \{Aprod.Sprod.ok_put, buf.elem\}, 


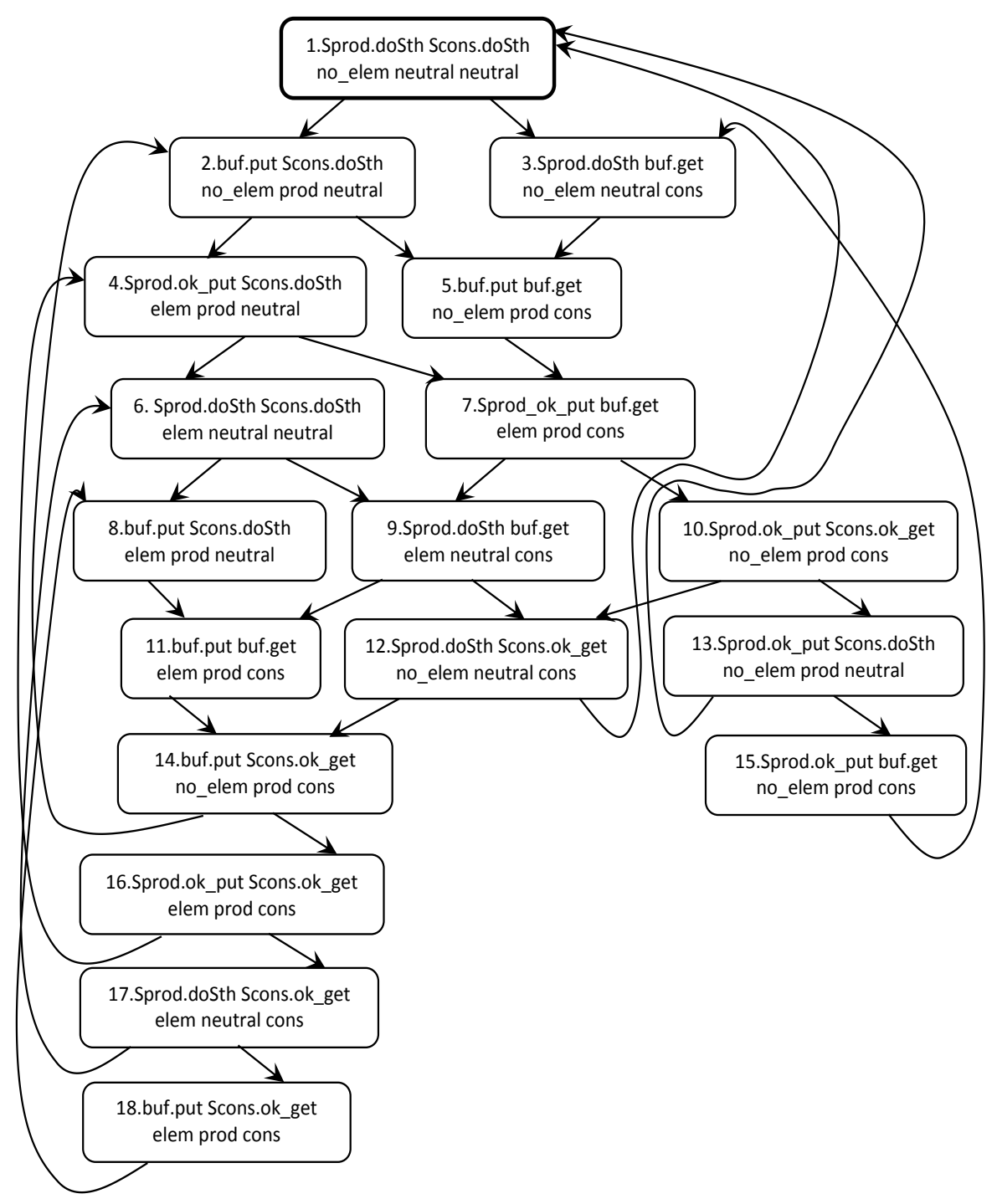

Fig. 1. LTS for the buffer system

14. $\{$ Aprod.Sprod.doSth, Sprod.neutral\} -> \{Aprod.buf.put, Sprod.prod\},

15. \{Aprod.Sprod.ok_put, Sprod.prod\} -> 16. \};

\{Aprod.Sprod.doSth, Sprod.neutral\},

17. agent: Acons (servers buf:buf,Scons:Scons),

18. actions \{

19. \{Acons.buf.get, buf.elem\} ->

\{Acons.Scons.ok_get, buf.no_elem\},

20. \{Acons.Scons.ok_get, Scons.cons\} ->

$\{$ Acons.Scons.doSth, Scons.neutral\},

21. \{Acons.Scons.doSth, Scons.neutral\} ->

22. \};

\{Acons.buf.get, Scons.cons\},

23. agents Aprod:Aprod,Acons:Acons;

24. servers buf:buf,Sprod:Sprod,Scons:Scons;
25. init $\rightarrow\{$

26. Aprod(buf,Sprod).Sprod.doSth,

27. Acons(buf,Scons).Scons.doSth,

28. buf.no_elem,

29. Sprod.neutral,

30. Scons.neutral,

31. $\}$

The LTS of the example system is presented in Fig. 1. In the nodes, messages of the agents Sprod and Scons are displayed (without agent identifiers) in the first line and the states of all servers (buf, Sprod, Scons) are displayed in the second line (without server identifiers). Of course, this LTS generated both from the server view and from the agent view is identical, as the views are projections onto servers and onto agents of a uniform system. 


\section{Petri Net Equivalent to IMDS}

A designer may be interested in some structural properties of a verified system, for example structural conflicts, dead code, pure cyclic system or not, etc. For this purpose, Petri nets equivalent to IMDS models were elaborated. The Dedan program exports IMDS models to Charlie Petri net analyzer [19] [18]. The export is in ANDL format (Abstract Net Description Language [20]).

An IMDS system is converted to an equivalent Petri net in such a way that every action is converted to a Petri net transition, as illustrated in Fig. 2. Input items (a message $m$ and a state $p$ ) are converted to the input places $m$ and $p$. In a regular action, output items (a message $m^{\prime}$ ' and a state $p^{\prime}$ ) are converted to the output places $m^{\prime}$ and $p^{\prime}$ (Fig. 2a). In an agent-terminating action, only one output place is present (corresponding to an output state $p^{\prime}$, Fig. 2b). The initial marking of the Petri net has tokens in all places of initial servers' states and all places of initial agents' messages. By construction of the described conversion of an IMDS system to a Petri net, the reachable markings graph has identical structure as LTS of IMDS (states $\leftrightarrow$ "state" places, messages $\leftrightarrow$ "message" places, actions $\leftrightarrow$ transitions, configuration $\leftrightarrow$ marking, initial configuration $\leftrightarrow$ initial marking).

The Petri net is not colored in the sense of [21], but we use read filling for states and green filling for messages in all figures, for readability.

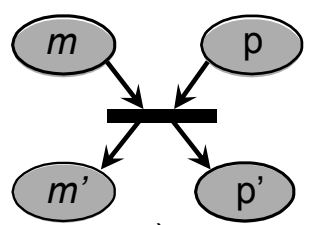

a)

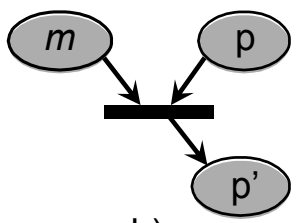

b)
Fig. 2 Petri net interpretation of a) regular action b) agent-terminating action

The Petri net of a buffer system is illustrated in Fig. 3. The states and messages in individual servers are grouped and separated by dashed lines. The states of servers are filled red while the messages are filled green. Also, Sprod states have dense grill while Scons states have rare grill. States of buf have chessboard filling. Messages of Aprod have diagonal hatching while messages of Acons have horizontal hatching. Initial states and initial messages are surrounded by bold ovals. All messages have identifiers in italics.

\section{V.DISTRIBUTED AUTONOMOUS AND ASYNCHRONOUS $\operatorname{AutOMATA}\left(D A^{3}\right)$}

In computer engineering practice, various forms of automata are used to express the behavior of concurrent components. There are two reasons: graphical representation and individual modeling of distinct components. UML state diagrams are the good example [22].

For a graphical representation of distributed systems, and for a simulation in terms of parallel components of a system, Distributed Autonomous and Asynchronous Automata $\left(\mathrm{DA}^{3}\right)$ were invented. We claim that our distributed automata are better to describe parallelism and cooperation in real distributed environment (with full asynchrony) than other formalisms in the literature known as distributed automata, mentioned is Section I.

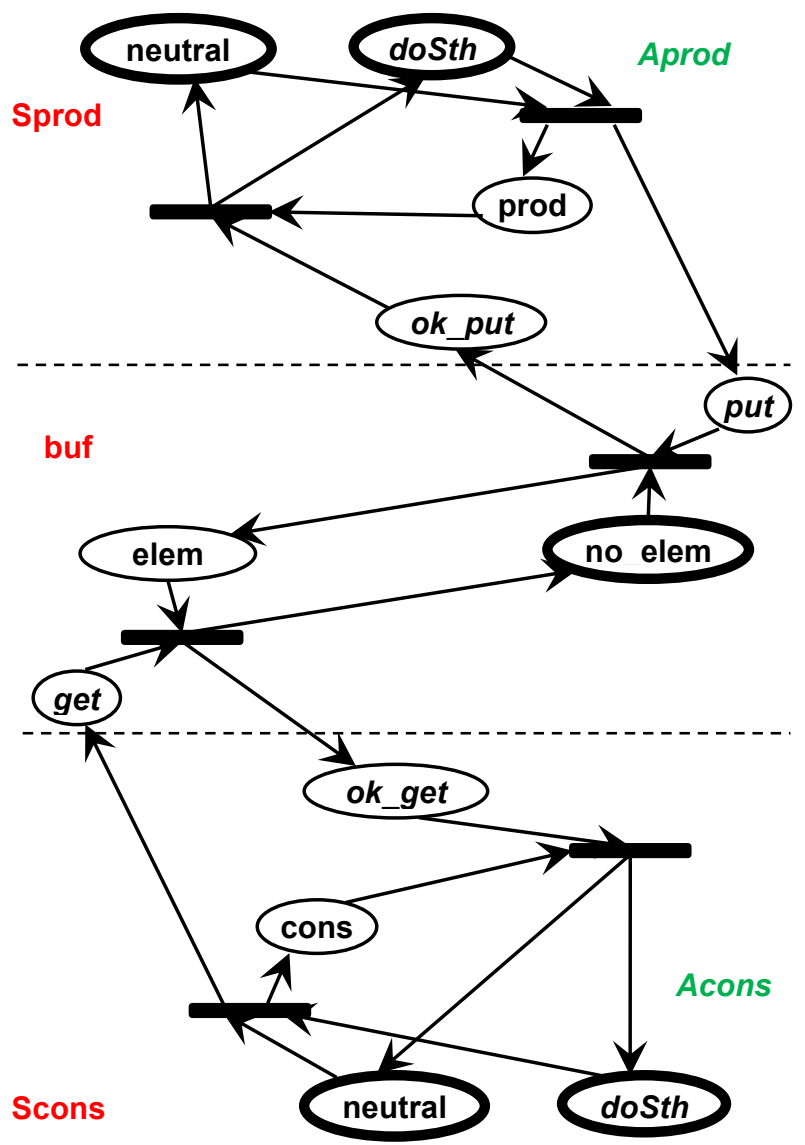

Fig. 3 Petri net representation of the buffer system: servers Sprod, Scons, buf, agents Aprod, Acons

We introduce a new version of distributed automata, equivalent to IMDS formalism. We call them Distributed Autonomous, Asynchronous Automata $-\mathrm{DA}^{3}$ (D-tripleA or DA-cubed) to distinguish them from all the previously mentioned formalisms, all called distributed automata. Our automata reflect the behavior of distributed components. The servers make decisions (perform actions) individually without any knowledge of other servers (autonomy) and messages are sent regardless of the states of target servers (asynchrony). As there are two views of a distributed system in IMDS, two forms of $\mathrm{DA}^{3}$ were developed - Server-DA ${ }^{3}$ and Agent-DA ${ }^{3}\left(\mathrm{SDA}^{3}\right.$ and $\left.\mathrm{ADA}^{3}\right)$.

\section{A. Server automata $\left(S D A^{3}\right)$}

An IMDS system in the server view may be shown as a set of communicating automata $\mathrm{SDA}^{3}$ (Distributed Server Automata), similar to MPA:

- States of a server are nodes (we use node instead of state to avoid ambiguity) of corresponding automaton. 
- An initial state of the server is an initial node of the automaton.

- Actions of the server process are transitions of the automaton.

- The automaton is Mealy-style [23], labels of the transitions in the automaton have the form extracted from actions; an IMDS action $(m, p) \lambda\left(m^{\prime}, p^{\prime}\right)$ is converted to a transition from $p$ to $p^{\prime}$ with a label $\mathrm{m} / \mathrm{m}^{\prime}$. It is a triple (node, transition label, node): ( $\left.p, m / m^{\prime}, p^{\prime}\right)$.

- The automaton is equipped with an input set - a set of input symbols pending, corresponding to a set of pending messages at the server. Firing a transition $\left(p, m / m^{\prime}, p^{\prime}\right)$ in the automaton of server $s$ retrieves the symbol $m$ from the input set of this automaton and inserts the symbol $m$ ' to the input set of an automaton of the server $s$ ' appointed by $m$ '. An initial input set consists of initial messages of agents directed to this server.

- The special agent-terminating action $(m, p) \lambda\left(p^{\prime}\right)$ is converted to a transition that does not produce an output symbol: $\left(p, m /, p^{\prime}\right)$.

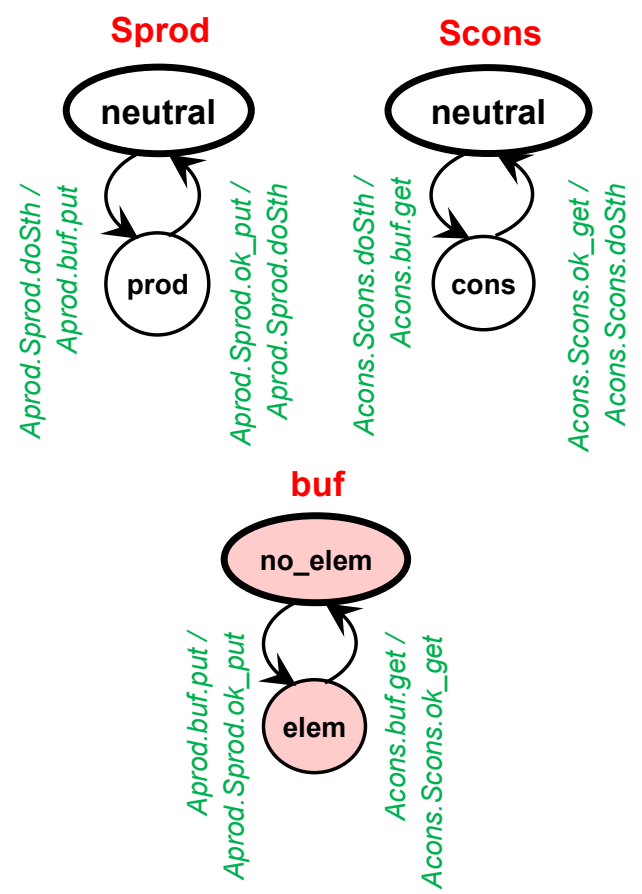

Fig. 4 Server automata of the buffer system

We denote automaton corresponding to a server $s$ as $z$ (reflected $s$ ). The set of all server automata we denote as $Z$. To define a behavior of server automata, we define a position of an automaton $(p, X)$ which consists of a node $p$ and given value (content) of the input set $X$. A global position of $Z$ is a set of nodes of all server automata (servers' states) and the union of their current input sets.

The execution of a transition in a server automaton moves its current node to the target node of the transition, extracts an input message from the input set of the automaton and inserts the output message to the input set of the automaton appointed by the output message. As in IMDS, the transitions are executed in interleaving manner, and in a case of many transitions possible, the choice is nondeterministic.

The server automata of the buffer system are presented in Fig. 4. A global graph of 2 cooperation may be elaborated in such a way that nodes are global positions, and edges are transitions in individual server automata. Of course, this graph is analogous to the LTS of IMDS system.

The initial sates of servers in Fig. 4 are in bold ovals. Server names are omitted in the state labels, because they are identical for all states in given server automaton.

Every automaton is equipped with the input set of pending messages, not shown in Fig. 4:

$$
\begin{aligned}
& X_{\text {buf }} \subseteq \exp (\{(\text { Aprod, buf, put }), \\
& \text { (Acons, buf, get) \}), } \\
& X_{\text {Sprod }} \subseteq \exp (\{(\text { Aprod, Sprod, doSth) } \text {, } \\
& \text { (Aprod, Sprod, ok put) \}), } \\
& X_{\text {Scons }} \subseteq \exp (\{(\text { Acons, Scons, doSth }), \\
& \text { (Acons, Scons, ok get) \}). }
\end{aligned}
$$

The initial input sets are:

$$
\begin{aligned}
& X_{\text {buf }}=\emptyset, \\
& X_{0 \text { Sprod }}=\{(\text { Aprod, Sprod, doSth })\}, \\
& X_{0 \text { Scons }}=\{(\text { Acons, Scons, doSth })\} .
\end{aligned}
$$

The $\mathrm{SDA}^{3}$ are similar to Message Passing Automata. The diff erence is in the ordering of messages on the input of the automaton: in MPA pending messages are ordered in the input queue (or input buffer) [15], while in $\mathrm{SDA}^{3}$ any message form the input set may cause a transition (no ordering). If the input buffers of MPA are bounded, a deadlock may occur because of all processes sending to full buff ers. Such a situation occurs when the size of buff ers is taken too small. IMDS helps to overcome this problem by posing an accurate limit for the input set maximum size (or the input buff er): it is simply the number of agents.

\section{B. Agent automata $\left(A D A^{3}\right)$}

An IMDS system in the agent view may be shown as a set of communicating automata $\mathrm{ADA}^{3}$ (Agent Distributed Autonomous and Asynchronous Automata). We use term node in these automata instead of state, because states ate attributed to servers in IMDS and it may be misleading. The $\mathrm{ADA}^{3}$ automata are similar to Timed Automata with variables used in Uppaal [17] (but we consider only timeless systems here):

- Messages of an agent are nodes of a corresponding automaton.

- An initial message of the agent is an initial node of the automaton.

- Actions of the agent process are transitions of the automaton.

- The automaton is Mealy-style [23]; the labels of the transitions in the automaton have the form extracted from actions; an IMDS action $(m, p) \lambda\left(m^{\prime}, p^{\prime}\right)$ is converted to a transition from $m$ to $m^{\prime}$ with a label $p / p^{\prime}$ ( $p$ is an input symbol conditioning the transition while $p$ ' is an output 
symbol produced on the transition; servers' states are $\left.p=(\mathrm{s}, v), p^{\prime}=\left(s, v^{\prime}\right)\right)$.

- For an agent-terminating action $(m, p) \lambda\left(p^{\prime}\right)$, a special node $t$ in the automaton is added as target node, and the transition is of the form $\left(m, p / p^{\prime}, t\right)$. For $t$ no outgoing transition is defined.

- The system is equipped with a global input vector (the vector of global current input symbols), corresponding to a vector of current states of the servers. Firing a transition $\left(m, p / p^{\prime}, m^{\prime}\right)$ in the automaton exchanges the symbol $p$ with the symbol $p$ ' in the vector. An initial global input vector consists of initial states of all servers.

We denote automaton corresponding to an agent $a$ with $e$ (reflected $a$ ). The set of all agent automata we denote as $U$ (reflected $A$, rounded to distinguish it from general quantifier). A global position of $U$ is a set of current nodes of agent automata (pending messages of non-terminated agents), and current value (content) of global input vector.

The execution of a transition in an agent automaton moves its current node to a target node of the transition (exchanges a message with the output message of the action), and replaces the state of server appointed by the transition to the output state of the action. As in IMDS, it is executed in interleaving manner, and in a case of many transitions possible, the choice is nondeterministic.
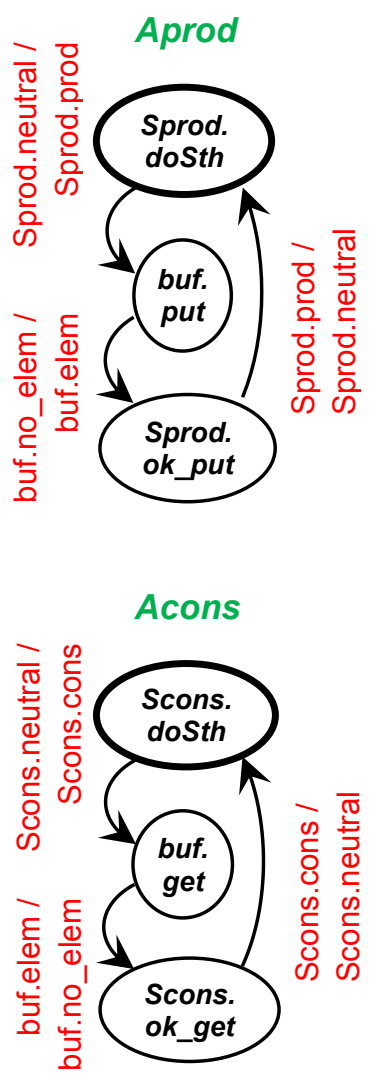

Fig. 5 Agent automata of the buffer system

Distributed agent automata for the buffer system are illustrated in Fig. 5. The initial messages of the agents are in bold ovals. Agent identifies are omitted in message labels (nodes of the automata), because they are identical for all messages in a given agent automaton. For completeness, a global input vector of current states of servers should be added.

A global graph of $U$ may be elaborated analogously to the global graph of 2 : nodes of the global graph are global positions of $U$, and edges are transitions in individual agent automata. This graph is analogous to the global graph of $\mathrm{SDA}^{3}$ and to the LTS of IMDS system (global positions contain messages of all non-terminated agents and a vector of states of all servers).

\section{Using Petri Net ANALYSIS AND DA ${ }^{3}$ IN DEDAN PROGRAM}

The basic form used in Dedan program is IMDS, because it allows for automatic conversion between the server view and the agent view of a system. Yet, the specification in the form of a relation between pairs $(m, p) \lambda\left(m^{\prime}, p^{\prime}\right)$ is exotic for the users. Therefore, an alternative input form of $\mathrm{DA}^{3}$ automata is provided as distributed automata.

A system may be simulated over the global space of configurations (LTS), but it is also possible to simulate it in terms of $\mathrm{SDA}^{3}$. This simulation does not require calculation of a global configuration space of verified system. All of the automata in the system are displayed, with input sets of pending messages under automata identifiers shown. The current states of the automata are distinguished by a separate color.

A user can choose an automaton, and then a list of transitions from the current node of the chosen automaton is displayed (with enabled ones distinguished; it is only one transition in this case, and it is enabled). Next, the user may choose a transition from the enabled ones. If the user clicks an enabled transition, it is "executed" and a destination automaton of the message becomes current.

The internal Dedan verifier, based on CBS evaluation algorithm [24], is limited to deadlock and termination detection formulas. However, the nature of model checking (evaluation of temporal formulas) allows to find only one deadlock in single verification (typically one which generates a shortest counterexample). A user may export a model to the Charlie program which finds many possible deadlocks as elementary siphons. Then, using an external verifier (Uppaal, for example) the reachability of siphon emptying may be examined. The configurations that terminate the counterexamples for every emptied siphon allow to reduce the results, as many siphons may denote the same deadlock [7].

\section{CONCLUSIONS AND FURTHER WORK}

The Dedan program supports an engineer in verification of distributed systems for deadlock freeness, without any knowledge on temporal logics and model checking 
TABLE I.

VERIFICATION FACILITIES IN THE THREE EQUIVALENT FORMALISMS

\begin{tabular}{|c|c|c|c|}
\hline Formalism: & IMDS & Petri net & $\mathbf{D A}^{3}$ \\
\hline $\begin{array}{l}\text { Main } \\
\text { features }\end{array}$ & $\begin{array}{l}\text { Specification, model checking, } \\
\text { simulation }\end{array}$ & Structural properties & Graphical input, simulation \\
\hline \multirow[t]{8}{*}{ Notions } & state & "red" place & $\begin{array}{l}\text { - } \text { node }\left(\mathrm{S}^{\left.-\mathrm{DA}^{3}\right)}\right. \\
\text { - element of global input } \\
\text { vector, input/output symbols } \\
\text { on transitions (A-DA })\end{array}$ \\
\hline & message & "green" place & 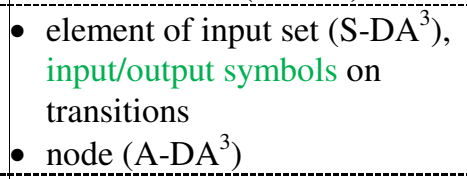 \\
\hline & configuration & marking & global position \\
\hline & action & transition & transition \\
\hline & initial state & $\begin{array}{l}\text { token in red place in initial } \\
\text { marking }\end{array}$ & $\begin{array}{l}\text { - initial node }\left(\mathrm{S}-\mathrm{DA}^{3}\right) \\
\text { initial element of global input } \\
\text { vector }\left(\mathrm{A}-\mathrm{DA}^{3}\right)\end{array}$ \\
\hline & initial message & $\begin{array}{l}\text { token in green place in initial } \\
\text { marking }\end{array}$ & $\begin{array}{l}\text { - initial element of input set } \\
\left(\mathrm{S}-\mathrm{DA}^{3}\right) \\
\text { - initial node }\left(\mathrm{A}-\mathrm{DA}^{3}\right)\end{array}$ \\
\hline & initial configuration & initial marking & $\begin{array}{l}\text { - initial nodes and initial input } \\
\text { sets of all automata }\left(\mathrm{S}-\mathrm{DA}^{3}\right) \\
\text { - initial nodes and initial global } \\
\text { input vector }\left(\mathrm{A}-\mathrm{DA}^{3}\right)\end{array}$ \\
\hline & Labeled Transition System & Marking reachability graph & $\begin{array}{l}\text { Global graph: } \\
\text { - all states and messages in } \\
\text { global positions, input and } \\
\text { output symbols (messages) on } \\
\text { transitions }\left(\mathrm{S}_{-} \mathrm{DA}^{3}\right) \\
\text { - all messages and global input } \\
\text { vector in global positions, } \\
\text { input and output symbols } \\
(\text { states) on transitions } \\
\left(\mathrm{A}-\mathrm{DA}^{3}\right)\end{array}$ \\
\hline Features & $\begin{array}{l}\text { - Resource deadlock } \\
\text { - Communication deadlock } \\
\text { - Partial deadlock } \\
\text { - Total deadlock } \\
\text { - Partial distributed termination } \\
\text { - Total distributed termination } \\
\text { - Counterexamples/ witnesses } \\
\text { - Configuration space inspection } \\
\text { - Simulation over configuration } \\
\text { space }\end{array}$ & $\begin{array}{l}\text { Structural properties } \\
\text { - Many deadlocks } \\
\text { - Existence of siphons } \\
\text { - Unreachable actions } \\
\text { - Separated components } \\
\text { - Invariants }\end{array}$ & $\begin{array}{l}\text { - Graphical definition of a } \\
\text { system } \\
\text { - Simulation over individual } \\
\text { automata } \\
\text { - Counterexample projected } \\
\text { onto individual automata } \\
\text { - Counterexample-guided } \\
\text { simulation }\end{array}$ \\
\hline
\end{tabular}

technique. If a communication deadlock occurs, a sequence diagram of messages is generated, leading from the initial configuration to the deadlock. In a case of resource deadlock, a sequence diagram of changes of servers' states and messages inside agents is generated. If the deadlock is not total, the servers/agents taking part in the deadlock are shown. With Petri net interpretation, some structural properties of a verified system are discovered using
Charlie[18]. Distributed automata (in $\mathrm{SDA}^{3}$ or in $\mathrm{ADA}^{3}$ version) allow to design the system in graphical form, and to simulate the components of the system and their cooperation instead of a simulation over the full configuration graph (LTS). Engineers are familiar with the notion of automata $\left(\mathrm{SDA}^{3}\right.$ are similar to Message Passing Automata [15] and $\mathrm{ADA}^{3}$ are like Timed Automata with global variables of Uppaal [17]) and they may be naturally used in distributed 
system design. It should be emphasized that simulation over $\mathrm{DA}^{3}$ does not require calculation of global reachability space of a verified system.

The three specification formalisms: IMDS, Petri nets with restricted structure and distributed automata $\mathrm{DA}^{3}$ (in two forms) are equivalent. The equivalence lays in mapping the basic structures of Petri net and $\mathrm{DA}^{3}$ onto structures of IMDS. All three formalisms generate analogous reachability graphs: LTS for IMDS, reachable markings graph for Petri net and global graph space for distributed automata of both forms. The construction elements and verification features, wider than deadlock and termination identification, are collected for every of the formalisms in the Table I. This variety of specification modes greatly facilitates analysis of the features of distributed systems.

For example, some models of transport cases were modeled. Observation of the server view is equivalent to exchange of messages between road marker controllers that automatically lead the vehicles on the roads. In the agent view, it is the observation of vehicles moving over the road, with interactions to other vehicles occupying some segments of the road. Possible deadlocks in communication may by easily identified, and the verifier shows the behavior of vehicles leading to a deadlock as transitions of $\mathrm{DA}^{3}$ automata. An example may be found in [25]. Server view and server automata allow to observe the system behavior from the point of view of road segment controllers, while agent view and agent automata from the point of view of guided vehicles. Petri net static analysis allows to locate the two possible deadlocks in specification. Such an approach of cooperation of distributed controllers by means of simple negotiation protocols follows the IoT paradigm [26].

The next steps of development of the Dedan environment are:

- Probabilistic automata allowing to identify a probability of a deadlock if the alternative actions in system processes are equipped with probabilities.

- Language-based input - elaboration of two languages for distributed systems specification: one for the server view (exploiting locality in servers and message passing) and the other one for the agent view (exploiting travelling of agents and resource sharing in distributed environment); a preliminary version of a declarative language-based preprocessor for a server view of verified systems is developed by the students of ICS, WUT under supervision of the author [27].

- Agent's own actions - equipping the agents with their own sets of actions, carried in their "backpacks", parametrizing their behavior; this will allow for modelling of mobile agents (agents carrying their own actions model code mobility) and to avoid many server types in specification, diff ering slightly.

The Dedan environment is successfully used in operating systems laboratory in ICS, WUT. The students verify their solutions of synchronization problems. More than 200 solutions were verified, ranging from several actions to over 5000 actions in a model. Also, Karlsruhe Production Cell benchmark [28] was modeled and successfully verified using the Dedan environment [29]. Several examples of IMDS specification in Dedan input form may found in [30].

In a design process, methods for its automatization are much needed to speed up and make it more dependable. Automatic verification in various forms and automatic code generation [31], which are subject of the research in ICS, WUT, are examples of such trends.

\section{REFERENCES}

[1] S. Chrobot and W. B. Daszczuk, "Communication Dualism in Distributed Systems with Petri Net Interpretation," Theor. Appl. Informatics, vol. 18, no. 4, pp. 261-278, 2006. https://taai.iitis.pl/taai/article/view/250/taai-vol.18-no.4-pp.261

[2] W. B. Daszczuk, "Communication and Resource Deadlock Analysis using IMDS Formalism and Model Checking," Comput. J., vol. 60, no.2, pp.729-750 (2017). doi: 10.1093/comjnl/bxw099

[3] C. A. R. Hoare, "Communicating sequential processes," Commun. $A C M$, vol. 21, no. 8, pp. 666-677, Aug. 1978. doi: $10.1145 / 359576.359585$

[4] R. Milner, A Calculus of Communicating Systems. Berlin Heidelberg: Springer-Verlag, 1984. ISBN: 0387102353

[5] E. M. Clarke, O. Grumberg, and D. Peled, Model Checking. Cambridge, MA: MIT Press, 1999. ISBN: 0-262-03270-8

[6] "Dedan" http://staff.ii.pw.edu.pl/dedan/files/DedAn.zip

[7] W. B. Daszczuk and W. M. Zuberek, "Deadlock detection in distributed systems using the IMDS formalism and Petri nets," in 12th International Conference on Dependability and Complex Systems, DepCoS-RELCOMEX 2017, Brunów, Poland, 2-6 July 2017, Advances in Intelligent Systems and Computing, vol 582, pp.118-130, Springer, Cham, 2018. doi: 10.1007/978-3-319-594156_12

[8] D. C. Craig and W. M. Zuberek, "Two-stage siphon-based deadlock detection in Petri nets," in Current Advances in Computing, Engineering and Information Technology, P. Petratos and P. Dandapami, Eds. Palermo, Italy: Int. Society for Advanced Research, 2008, pp. 317-330.

[9] W. Zielonka, "Notes on finite asynchronous automata," RAIRO Theor. Informatics Appl. - Inform. Théorique Appl., vol. 21, no. 2, pp. 99-135, 1987. http://archive.numdam.org/ARCHIVE/ITA/ITA_1987_21_2/ITA_1 987_21_2_99_0/ITA_1987_21_2_99_0.pdf

[10] P. Krishnan, "Distributed Timed Automata," Electron. Notes Theor. Comput. Sci., vol. 28, pp. 5-21, 2000. doi 10.1016/S15710661(05)80627-9

[11] D. Van Chieu and D. Van Hung, "An Extension of Mazukiewicz Traces and their Applications in Specification of Real-Time Systems," in 2010 Second International Conference on Knowledge and Systems Engineering, Hanoi, Vietnam, October 7-9, 2010, 2010, pp. 167-171. doi: 10.1109/KSE.2010.39

[12] V. Diekert and A. Muscholl, "On Distributed Monitoring of Asynchronous Systems," in 19th International Workshop on Logic, Language, Information and Computation, WoLLIC 2012, Buenos Aires, Argentina, September 3-6, 2012, 2012, pp. 70-84. doi: 10.1007/978-3-642-32621-9_5

[13] M. Mukund, "Automata on Distributed Alphabets," in Modern Applications of Automata Theory, Co-Published with Indian Institute of Science (IISc), Bangalore, India, 2012, pp. 257-288. doi: 10.1142/9789814271059_0009

[14] L. Brim, I. Černá, P. Moravec, and J. Šimša, "How to Order Vertices for Distributed LTL Model-Checking Based on Accepting Predecessors," Electron. Notes Theor. Comput. Sci., vol. 135, no. 2, pp. 3-18, Feb. 2006. doi: 10.1016/j.entcs.2005.10.015

[15] B. Bollig and M. Leucker, "A Hierarchy of Implementable MSC Languages," in Formal Techniques for Networked and Distributed 
Systems - FORTE 2005, Taipei, Taiwan, October 2-5, 2005, 2005, pp. 53-67. doi: 10.1007/11562436_6

[16] M. S. Balan, "Serializing the Parallelism in Parallel Communicating Pushdown Automata Systems," Electron. Proc. Theor. Comput. Sci., vol. 3, pp. 59-68, Jul. 2009. doi: 10.4204/EPTCS.3.5

[17] G. Behrmann, A. David, and K. G. Larsen, "A Tutorial on Uppaal 4.0," Report, Univ. Aalborg, Denmark, 2006. http://www.uppaal.com/admin/anvandarfiler/filer/uppaal-tutorial.pdf

[18] M. Heiner, M. Schwarick, and J.-T. Wegener, "Charlie - An Extensible Petri Net Analysis Tool," in 36th International Conference, PETRI NETS 2015, Brussels, Belgium, June 21-26, 2015, 2015, pp. 200-211. doi: 10.1007/978-3-319-19488-2_10

[19] Charlie, "Charlie Petri net analyzer.". http://www-dssz.informatik.tucottbus.de/DSSZ/Software/Charlie

[20] M. Schwarick, M. Heiner, and C. Rohr, "MARCIE - Model Checking and Reachability Analysis Done EffiCIEntly," in 2011 Eighth International Conference on Quantitative Evaluation of SysTems, Aachen, Germany, 5-8 Sept. 2011, 2011, pp. 91-100. doi: 10.1109/QEST.2011.19

[21] K. Jensen and L. M. Kristensen, Coloured Petri Nets. Berlin, Heidelberg: Springer-Verlag, 2009. doi: 10.1007/b95112

[22] "UML" http://www.uml.org/.

[23] G. Dick and X. Yao, "Model representation and cooperative coevolution for finite-state machine evolution," in 2014 IEEE Congress on Evolutionary Computation (CEC), Beijing, China, 6-11 July 2014, 2014, pp. 2700-2707. doi: 10.1109/CEC.2014.6900622

[24] W. B. Daszczuk, "Evaluation of temporal formulas based on "checking by spheres" in Proceedings Euromicro Symposium on Digital Systems Design, Warsaw, Poland, 4-6 September 2001, 2001, pp. 158-164. doi: 10.1109/DSD.2001.952267
[25] B. Czejdo, S. Bhattacharya, M. Baszun, and W. B. Daszczuk, "Improving Resilience of Autonomous Moving Platforms by realtime analysis of their Cooperation," Autobusy-TEST, vol. 17, no. 6, pp. 1294-1301, 2016. http://www.autobusytest.com.pl/images/stories/Do pobrania/2016/nr \%206/logistyka/10_1_czejdo_bhattacharya_baszun_daszczuk.pdf

[26] Z. Garofalaki, D. Kallergis, G. Katsikogiannis, I. Ellinas, and C. Douligeris, "Transport services within the IoT ecosystem using localisation parameters," in 2016 IEEE International Symposium on Signal Processing and Information Technology (ISSPIT), Limassol, Cyprus, 12-14 December 2016, 2016, pp. 87-92. doi: 10.1109/ISSPIT.2016.7886014

[27] W. B. Daszczuk, M. Bielecki, and J. Michalski, "Rybu: Imperativestyle Preprocessor for Verification of Distributed Systems in the Dedan Environment," in KKIO'17 - Software Engineering Conference, Rzeszów, Poland, 14-16 Sept 2017, 2017

[28] C. Lewerentz and T. Lindner, Eds., Formal Development of Reactive Systems, LNCS 891. Berlin, Heidelberg: Springer-Verlag, 1995. doi: $10.1007 / 3-540-58867-1$

[29] W. B. Daszczuk, "Asynchronous Specification of Production Cell Benchmark in Integrated Model of Distributed Systems," in 23rd International Symposium on Methodologies for Intelligent Systems, ISMIS 2017, Warsaw, Poland, 26-29 June 2017, 2017.

[30] "Dedan Examples" http://staff.ii.pw.edu.pl/dedan/files/examples.zip

[31] A. Derezińska, R. Pilitowski, Correctness issues of UML class and state machine models in the $\mathrm{C \#}$ code generation and execution framework, in: M. Ganzha, M. Paprzycki, T. Pełech-Pilichowski Eds., 2008 Int. Multiconference Comput. Sci. Inf. Technol. Wisła, Poland, 20-22 Oct. 2008, IEEE, Piscataway, NJ, 2008: pp. 517-524. doi:10.1109/IMCSIT.2008.4747293 


\title{
A Proof System for MDESL
}

\author{
Jianyu Lu, Wanling Xie, Huibiao Zhu, Yuan Fei \\ Shanghai Key Laboratory of Trustworthy Computing, \\ School of Computer and Software Engineering, \\ East China Normal University, Shanghai, China
}

\begin{abstract}
Hardware description language (HDL) Verilog has been standardized and widely used in industry. To describe the features such as event-driven computation, time and sharedvariable concurrency of hardware, a Verilog-like language MDESL (multithreaded discrete event simulation language), has been introduced. In this paper, we put forward a proof system for MDESL which is based on the classical Hoare Logic (precondition, program, postcondition). To deal with the guard statement, we add a new element trace to Hoare triples. We extend the primitives of assertion to express the global time of current program, and interpret the triples so that it can verify both terminating and nonterminating computations. To verify a concurrent program, we use a merger method of the trace to combine the traces in our parallel rule. Finally, there is an example about using our proof system to verify the correctness of a program written by MDESL.
\end{abstract}

\section{INTRODUCTION}

$\mathbf{W}$ ITH the increasing complexity of computer hardware, more and more modern hardware designs choose to use the hardware description language (HDL) to describe the designs at various levels of abstraction. As a high level programming language, HDL not only has the classical programming statements such as skip, assignments, conditionals, loops, but also has some extensions for real-time, concurrency, guard and new data structures appropriate for modelling hardware. The Verilog Hardware Description Language (Verilog HDL) became as IEEE standard in 1995 as IEEE std 1364-1995 [1], [2] due to its simple, intuitive and effective at multiple of abstraction. There are several important features in Verilog, including real-time [3], [4], event-driven computation, sharedvariable concurrency and simulator-based interpretation.

MDESL [5] (Multithreaded Discrete Simulation Language) is a Verilog-like language [6], [7], [8]. Parts of the statements and constructs in MDESL are similar to those in $\mathrm{C}$ programming language. However, as a hardware level programming language, it also has the statements and constructs which can describe the features of hardware, such as event-driven computation, real-time and shared-variable concurrency. In MDESL, the guard statement $(@(g))$ represents that a new state will be compared to its previous state, if the result satisfies $g$, then the program will continue to execute its rest statements, otherwise it will be in a state until the guard is trigged. This embodies the feature of the event-driven of MDESL. Time delay statement is introduced in MDESL, the synchronization of different parallel components can be based on time controls, and the parallel mechanism is an interleaving model.
In this paper, we put forward a proof system for MDESL in order to verify the correctness of the programs written by MDESL. Our proof system is based on classical Hoare Logic [9]. According to the sematic model of MDSEL, we have added a data structure trace in the front of the triple and extended the assertion languages to form a new triple which is convenient for the compositional verification of MDESL. Trace [10] is used to record the new time and values when an atomic action raises a data update. Thus it can help us to deal with the shared-variable feature. As usual, the precondition can express the set of initial or input states at the start of the execution, and the postcondition describes the set of final or output states at termination. To verify the property of time, we add a special variable time (similar to [11]) which represents the beginning time of the program in the precondition and the terminating time in the postcondition. We can specify the execution time of a program and merge the trace between different parallel components by the global clock time. In the classical Hoare Logic, we can only deal with the terminating program, but in our proof system, we can specify the terminating time by the use of time. If time $\in[0, \infty)$ we can deduce that the program will terminate or if time $=\infty$ this means that the process will run forever. We can use the rules for nonterminating in our proof system to deal with this situation.

The remainder of this paper is organized as follows. In Section II, we introduce the program language and the sematic model of MDESL, and give the specifications of the assertions and some definitions used in our proof system. In Section III, we provide the proof system for MDESL, including the rules for sequential programs, parallel composition and nontermination computations. In addition, we introduce some auxiliary axioms and rules which are useful for the verification of the programs. In Section IV, we apply our proof system to verify one example. Section $\mathrm{V}$ concludes the paper.

\section{BASIC FRAMEWORK}

In this section we introduce the basic framework. We first introduce the syntax of MDSEL in subsection A. Then the semantic model to describe the shared-variable concurrency and real-time computations is given in subsection $\mathrm{B}$. The formalism to specify a system which is described by MDESL is presented in subsection $\mathrm{C}$. 


\begin{tabular}{rlrl}
\hline$P:=$ & $P C$ & & primitive commands \\
& $\mid P ; Q$ & & sequential composition \\
& $\mid$ if $b$ then $P$ else $Q$ & & conditional construct \\
& $\mid$ while $b$ do $P$ & & iteration construction \\
& $P|| Q$ & & parallel composition \\
& $\mid M$ & & hybrid control \\
$M:=@(x:=e)|@(g)| \# n$ & hybrid control \\
$g:=\eta \mid g$ or $g \mid g$ and $g \mid g$ and $\neg g$ & & logic connection \\
$\eta:=v|\uparrow v| \downarrow v$ & TABLE I & event guard \\
\hline \multicolumn{2}{c}{ SYNTAX OF MDESL }
\end{tabular}

SYNTAX OF MDESL

\section{A. Programming Language}

In this subsection we introduce the syntax of Multithreaded Discrete Event Simulation Language (MDESL), first put forward by Zhu, a Verilog-like language, which not only has realtime feature but also supports the features of shared-variable concurrency and event-driven computation. The syntax is given in TABLE I.

We can explain the syntax (similar to [7], [8]) as follows :

- $P C$ consists of four primitive commands: Chaos, Stop, Skip, $x:=e$. Chaos represents the worst process, whose behaviour is totally unpredictable. Stop is the process that does nothing, in other word, idle process. $x:=e$ is the assignment statement, which executes instantaneously. Skip behaves the same as $x:=x$.

- $P ; Q$ is sequential composition. It executes process $P$ first. The process $Q$ starts to executes after $P$ terminates successfully.

- if $b$ then $P$ else $Q$ is the conditional construct.

- while $b$ do $P$ is the iteration construct.

- $P \| Q$ is parallel composition. In Verilog, parallel composition can occur only at the outmost level, here we allow it to occur anywhere.

- To accommodate the expansion laws of the parallel construct, we introduce the concept of guarded choice and extend Verilog's event category into the language.

(1) $@(x:=e)$ is an atomic assignment, whereas $x:=e$ is not.

(2) \#n is time delay which suspends the execution for $n$ time units, $n$ is an integer.

(3) An event guard $@(v)$ is triggered by the change of $v$, and $@(\downarrow v)$ is triggered by a decrease in $v$, however $@(\uparrow v)$ is triggered by an increase in $v$.

(4) $@\left(g_{1}\right.$ or $\left.g_{2}\right)$ is triggered if $@\left(g_{1}\right)$ or $@\left(g_{2}\right)$ is triggered.

(5) $@\left(g_{1}\right.$ and $\left.g_{2}\right)$ is triggered if $@\left(g_{1}\right)$ and $@\left(g_{2}\right)$ is triggered simultaneously.

(6) @ $\left(g_{1}\right.$ and $\left.\neg g_{2}\right)$ is triggered if @ $\left(g_{2}\right)$ remains untriggered and $@\left(g_{1}\right)$ are triggered.

We describe the execution of a statement is instantaneous if the execution of a statement lasts zero time. In MDESL, the following forms are instantaneous:

(1) $x:=e, S k i p, @(x:=e)$ are instantaneous.

(2) If $P$ and $Q$ are instantaneous, $P$; $Q$ is also instantaneous.
(3) The transition from if $b$ then $P$ else $Q$ to $P$ (or $Q$ ) is instantaneous.

(4) The transition from while $b$ do $P$ to $P$; while $b$ do $P$ (or to Skip) is instantaneous.

\section{B. The Semantical Model}

In this subsection, we will introduce the semantical model of MDESL. MDESL processes communicate with each other by shared variables. In order to record communications among them during execution, we use a trace of snapshots. When a process executes an atomic action, a snapshot will be added to the end of the trace. We use $t r$ to denote that trace.

Definition 2.1 (Snapshot) We use a triple $(t, \sigma, \mu)$ to denote a snapshot, which is used to specify the behaviour of an atomic action, where:

(1) $t$ represents the time when the atomic action happens.

(2) $\sigma$ represents the values of program variables when an atomic action is completed.

(3) $\mu$ denotes which process provides the status update. When $\mu=1$, it represents the process itself performs the atomic action, $\mu=0$ states the environment engages an atomic action.

We use the following projections to choose the components of a snapshot:

$$
\pi_{1}(t, \sigma, \mu)=_{d f} t, \pi_{2}(t, \sigma, \mu)=_{d f} \sigma, \pi_{3}(t, \sigma, \mu)=_{d f} \mu
$$

Definition 2.2 (Operators of Trace) We present the following main operators $O P$ among the trace. Let $t r_{1}$ and $t r_{2}$ be two traces, $s$ and $t$ be two snapshots.

$$
O P::=\uparrow \mid \text { last }|\preceq|-\mid \text { len }
$$

(1) $t r_{1} \widehat{t r_{2}}$ represents that $t r_{1}$ and $t r_{2}$ are connected.

(2) last $\left(t r_{1}\right)$ denotes the last snapshot of $t r_{1}$.

(3) $t r_{1} \preceq t r_{2}$ indicates that $t r_{1}$ is a prefix of $t r_{2}$, and we have $\forall t r, \emptyset \preceq t r$.

(4) $t r_{2}-t r_{1}$ indicates that the remain of removing all snapshots in $t r_{1}$ from $t r_{2}$ when $t r_{1} \preceq t r_{2}$, Combined with the definition of $t r_{1} \widehat{t} r_{2}$, we can conclude that

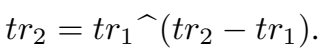

(5) len $\left(t r_{1}\right)$ stands for the length of $t r_{1}$, i.e., if $t r_{1}$ contains two snapshots, then $\operatorname{len}\left(\operatorname{tr}_{1}\right)=2$.

The Fig. 1. shows the trace behaviour of a process and its environment. Here, we use "•" to represent the process's atomic action and "०" to stand for the environment's atomic action. The numbers on the vertical line stand for the snapshots sequences in the process's trace, the numbers on the horizontal line indicate the time when the atomic action happens.

Example 2.1. Let $P={ }_{d f}(x:=1 ; \# 1 ; x:=2)$ and the initial trace of $P$ is $t r_{1}$. We assume that the initial time is time .

When $P$ completes its first atomic action $x:=1$, a snapshot $\left(\right.$ time $\left._{0}, \sigma(x:=1), 1\right)$ will be added to the end of $t r_{1}$. And we

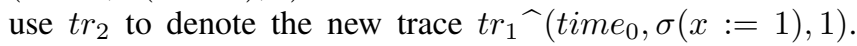




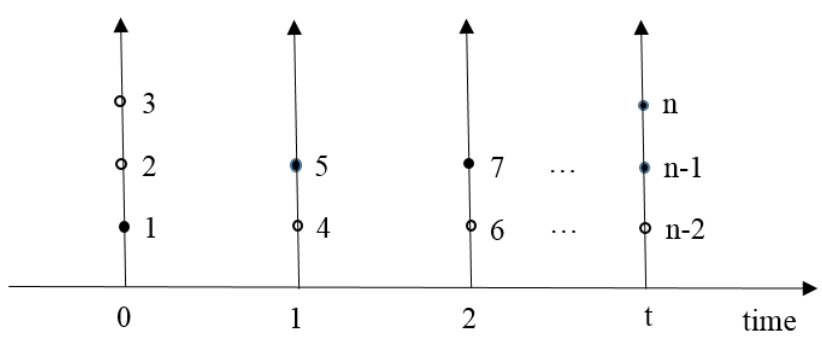

Fig. 1. The trace behaviour of a process

After one time unit, the atomic action $x:=2$ takes place, which generates a snapshot $\left(\right.$ time $\left._{0}+1, \sigma(x:=2), 1\right)$ attached to the end of the trace $t r_{2}$. And we use $t r_{3}$ to stand for the new trace $t r_{2} \widehat{ }\left(\right.$ time $\left._{0}+1, \sigma(x:=2), 1\right)$. According to Definition 2.2, we have

$$
\begin{aligned}
& t r_{1} \preceq t r_{2}, t r_{2} \preceq t r_{3} ; \\
& t r_{2}-t r_{1}=\left(\text { time }_{0}, \sigma(x:=1), 1\right) \\
& t r_{3}-t r_{2}=\left(\text { time }_{0}+1, \sigma(x:=2), 1\right) \text {; } \\
& t r_{3}-t r_{1}= \\
& \left(\text { time }_{0}, \sigma(x:=1), 1\right)^{\wedge}\left(\text { time }_{0}+1, \sigma(x:=2), 1\right) ; \\
& \text { last }\left(\mathrm{tr}_{2}\right)=\left(\text { time }_{0}, \sigma(x:=1), 1\right) \\
& \operatorname{last}\left(\operatorname{tr}_{3}\right)=\left(\text { time }_{0}+1, \sigma(x:=2), 1\right) \text {; } \\
& \operatorname{len}\left(\operatorname{tr}_{3}-t r_{2}\right)=1 ; \operatorname{len}\left(t r_{3}-t r_{1}\right)=2 ; \operatorname{len}\left(t r_{2}-t r_{1}\right)=1 \text {. }
\end{aligned}
$$

Now, we can describe the real-time behaviour of a process $P$ by the following aspects:

- the initial state, i.e., the values of the variables and the starting time at the start of the execution.

- the time and the values when the variables of $P$ are changed.

- if $P$ terminates, the final state, i.e., the values of the variables and the termination time at the end of the execution.

We can use $t r$ to record the second behaviour, as described above, it's a sequence of snapshots to record communications among them during execution. In order to record the global clock, we use a special variable time, ranging over $T I M E \bigcup\{\infty\}$, here TIME is a time domain which is discrete and TIME $=\{x \mid x \in \mathbb{N}\}$. We use $\sigma, \sigma_{0}, \sigma_{1} \ldots$ to represent states, assigns a value from $\mathbb{R}$ to a common variable and assigns a value from TIME $\{\infty\}$ to time variable.

A set of pairs of the form $(\sigma, t r)$ represents the semantics of a program $P$ starting in a state $\sigma_{0}$ denoted by $\mathscr{M}(P)\left(\sigma_{0}\right)$. Here $\sigma$ is a state and $t r$ is the trace of $P$, as we defined above, $\sigma_{0}(x)$ is the value of common variable $x$ at the start of the $P$ and $\sigma_{0}$ (time) represents the starting time, $t r_{0}$ denotes the initial trace when the program $P$ begins to execution. If $P$ terminates and a $\operatorname{pair}(\sigma, t r)$ is in $\mathscr{M}(P)\left(\sigma_{0}\right)$, then the value of $\sigma$ (time) represents the termination time. If $P$ does not terminate then $\sigma$ (time) $=\infty$ and $\sigma(x)$ is an arbitrary value, here $x$ is a common variable.

\section{Specifications}

Our specifications are based on classical Hoare triples $\{p\} S\{q\}$, it has the following meaning : if $S$ is executed in a state satisfying precondition $p$ and $S$ terminates then the final state satisfies postcondition $q$. In MDESL, @ $(g)$ statement needs to compare current state with the earlier state, the classical Hoare triples are not suitable for it. According to the semantic model of MDESL mentioned in subsection B, we can use the trace to help us to solve the problem. Thus the formula has the new form $\operatorname{tr}:\{p\} S\{q\}$ where $\operatorname{tr}$ represents the initial trace before $S$ executes its first statement, $p$ and $q$ are assertions and $S$ is a program.

Assertion $p$ expresses precondition described as below:

- the starting time of $S$,

- the initial values of the common variables of $S$.

Assertion $q$ expresses the postcondition described as follows:

- the terminating time of $S$ ( $\infty$ if $S$ does not terminate),

- the finial values of the common variables of $S$ if $S$ terminates.

Compared with classical Hoare triples, we add a special variable time in assertions, so our proof system can deal with total correctness as well as partial correctness. Then we will give some useful notations which will be used in our following proof system.

Definition 2.3 If a guard $g$ of a program $S$ is trigged and the current trace of $S$ is $t r$, we can denote it as $\operatorname{trig}(g)$ at $t r$.

$$
\begin{aligned}
& \operatorname{trig}(g) \text { at } \operatorname{tr}={ }_{d f} \exists t r_{1} \bullet \\
& \operatorname{tr}_{1} \preceq \operatorname{tr} \wedge \\
& \operatorname{len}\left(\operatorname{tr}-t r_{1}\right)=1 \wedge \\
& \text { fire }(g)\left(\pi_{2}\left(\text { last }\left(\operatorname{tr}_{1}\right)\right), \pi_{2}(\text { last }(t r))\right)
\end{aligned}
$$

where fire $(g)\left(\sigma_{0}, \sigma_{1}\right)$ represents the transition from state $\sigma_{0}$ to $\sigma_{1}$ can awake the guard $@ g$.

Definition 2.4 If a guard $g$ of a program $S$ is not trigged until the trace of $S$ is $t r_{1}$, and the beginning trace of $S$ is $t r_{0}$. During this period, denoted by await $(g)$ during $\left[\operatorname{tr}_{0}, t r_{1}\right)$.

$$
\begin{aligned}
& \operatorname{await}(g) \text { during }\left[\operatorname{tr}_{0}, t r_{1}\right)={ }_{d f} \forall t r_{2}, t r_{3} \bullet \\
& \operatorname{tr}_{0} \preceq t r_{2} \preceq t r_{3} \preceq t r_{1} \wedge \\
& \operatorname{len}\left(\operatorname{tr}_{3}-\operatorname{tr}_{2}\right)=1 \wedge \\
& \quad \neg \operatorname{fire}(g)\left(\pi_{2}\left(\operatorname{last}\left(\operatorname{tr}_{2}\right)\right), \pi_{2}\left(\operatorname{last}\left(\operatorname{tr}_{3}\right)\right)\right)
\end{aligned}
$$

Definition 2.5 If a guard $g$ of a program $S$ is trigged during $t r_{0}$ and $t r_{1}$, this period is represented by trig $(g)$ during $\left[t r_{0}, t r_{1}\right]$.

$$
\begin{gathered}
\operatorname{trig}(g) \text { during }\left[\operatorname{tr}_{0}, \operatorname{tr}_{1}\right]={ }_{d f} \exists t r_{2} \bullet \\
\operatorname{tr}_{0} \preceq \operatorname{tr}_{2} \preceq \operatorname{tr}_{1} \wedge \\
\text { await }(g) \text { during }\left[\operatorname{tr}_{0}, t r_{2}\right) \wedge \\
\operatorname{trig}(g) \text { at } \operatorname{tr}_{2}
\end{gathered}
$$

Definition 2.6 (Validity) For a program $S$, the beginning trace is $t r_{0}$, the program $S$ and assertions $p$ and $q$, if the correctness formula $\operatorname{tr}_{0}:\{p\} S\{q\}$ is true, we can write $=\operatorname{tr}_{0}:\{p\} S\{q\}$, iff for the initial state $\sigma_{0}$, and any $\sigma, t r$ with $(\sigma, t r) \in \mathscr{M}(P)\left(\sigma_{0}\right)$, we have that $\left(\sigma_{0}, t r_{0}\right) \models p$ implies $(\sigma, t r) \models q$. 


\section{The Proof System}

In this section, we will introduce a compositional proof system for MDESL. First we give the proof rules of the sequential program and some axioms that are generally applicable to each statement in subsection $\mathrm{A}$. Then in subsection $\mathrm{B}$, the rules for parallel composition are presented. Last, we will introduce some auxiliary axioms and rules in subsection $\mathrm{C}$.

\section{A. Axioms and Sequential Program Rules}

A skip statement means the program does nothing and terminates immediately, it will have no effect on itself and the environment.

Axioms 1. Skip

$$
\text { tr : }\{p\} \text { Skip }\{p\}
$$

The Chaos statement means that the behaviour of the program is totally unpredictable and the global clock will not stop, we use a notion time $=\infty$ to represent that the program is divergence.

\section{Axioms 2. Chaos}

$$
\operatorname{tr}:\{p\} \text { Chaos }\{q \wedge \text { time }=\infty\}
$$

The nontermination axiom represents that a program following a Chaos computation has no effect.

\section{Axioms 3. Nontermination}

$$
\operatorname{tr}:\{p \wedge \text { time }=\infty\} S\{p \wedge \text { time }=\infty\}
$$

The rule for an assignment $x:=e$ is same as the classical rule because the assignment statement takes 0 time unit to complete.

\section{Axioms 4. Assignment}

$$
\operatorname{tr}:\{q[x:=e]\} x:=e\{q\}
$$

About the rule for delay statement \#e, which means that the global clock time delays $e$ time units and no change takes place in common variables. We give the postcondition $q$, then the precondition $q[$ time $=$ time $+e]$ is required.

\section{Axioms 5. Delay}

$$
\text { tr }:\{q[\text { time }=\text { time }+e]\} \# e\{q\}
$$

About the rule for the $@(g)$ statement, there are two possibilities in sequential program. One possibility is that the guard is triggered by the execution of its prior atomic action (or it may be triggered by its environment and we will discuss it in subsection B). In this case, the notation $\operatorname{trig}(g)$ at $t r$ is true, due to no variables are updated, the postcondition and the precondition are same. The other possibility is that the execution of the program can not trig the guard $@(g)$, and the guard will be in a waiting state to be fired endlessly, which means the state of the program becomes Chaos. We use a notion $q_{\infty}$ to represent a nonterminating computation of infinite waiting.

\section{Rule 1. Guard -1}

$$
\begin{aligned}
& (p \wedge \text { time }<\infty) \wedge \operatorname{trig}(g) \text { at } t r \rightarrow p \\
& (p \wedge \text { time }<\infty) \wedge \neg \text { trig }(g) \text { at } t r \rightarrow q_{\infty} \\
& \hline \operatorname{tr}:\{p\} @(g)\left\{p \vee q_{\infty}\right\}
\end{aligned}
$$

\section{Rule 2. Conditional}

$$
\begin{aligned}
& t r:\{p \wedge b\} S_{1}\{q\}, t r:\{p \wedge \neg b\} S_{2}\{q\} \\
& t r:\{p\} \text { if } b \text { then } S_{1} \text { else } S_{2}\{q\}
\end{aligned}
$$

About the rule for the while construct, it has two parts. The first part is related to the classic rule of Hoare Logic. The second part is to handle the nonterminating statements.

\section{Rule 3. While}

$$
\begin{aligned}
& t r:\{I \wedge b \wedge t i m e<\infty\} S\{I\} \\
& \left(\forall t r_{1}, \exists t r_{2}, t r \preceq t r_{1} \preceq t r_{2}\right) \rightarrow q_{\infty} \\
& S:=S k i p \rightarrow q_{\infty} \\
& I \rightarrow I_{1} \\
& \left(\forall t_{1}, \exists t_{2}>t_{1}: I_{1}\left[t_{2} / t i m e\right]\right) \rightarrow q_{\infty} \\
& \hline t r:\{I\} \text { while } b \text { do } S \text { od }\left\{(I \wedge \neg b) \vee\left(q_{\infty} \wedge \text { time }=\infty\right)\right\}
\end{aligned}
$$

We will give an informal description of the soundness of the While rule, For a while program while $b$ do $S$ od, we assume that it starts in a state satisfying $p$. There are four cases.

The first is the same as the classic Hoare Logic. Program $S$ is a terminating computation, and the loop is terminated. Except the last one, for all these computations of $S, b$ is always true. So the condition $\operatorname{tr}:\{I \wedge b \wedge$ time $<\infty\} S\{I\}$ holds in the case, this means that the last computation $\neg b$ must be true, which leads to $(I \wedge \neg b)$.

In the second case, we assume that it starts in a state satisfying $I$ and nonterminating, i.e., for the initial state $\sigma_{0}$, $\sigma_{0}($ time $)=\infty$. Then model is the same as the nonterminating model (the property has been expressed in the Nontermination Axiom). time $=\infty$ and $I \rightarrow I_{1} \wedge$ time $=\infty$ hold in this model, so $\left(\forall t_{1}, \exists t_{2}>t_{1}: I_{1}\left[t_{2} /\right.\right.$ time $\left.]\right)$ is true. And it leads to $q_{\infty}$.

In the third case, we assume $S$ is a nonterminating computation. Then the computation becomes a nonterminating computation, and as in the first case, $I \wedge$ time $=\infty$ holds for this model. Thus, since $I \rightarrow I_{1}$, condition $\left(\forall t_{1}, \exists t_{2}>t_{1}\right.$ : $I_{1}\left[t_{2} /\right.$ time $\left.]\right)$ holds in the case, and it will lead to $q_{\infty}$.

The last case represents a nonterminating computation which program $S$ is a terminating computation and the loop is infinite. It means that the boolean condition $b$ will be true forever. In this case, program $S$ has three situations.

- If program $S$ is a Skip statement, then it will lead to $q_{\infty}$.

- If it doesn't contain any time delay, the trace of the while program will be infinite. Hence, we obtain $\left(\forall t r_{1}, \exists t r_{2}, t r \preceq t r_{1} \preceq t r_{2}\right) \rightarrow q_{\infty}$, and then it leads to $q_{\infty}$.

- If $S$ contains any time delay \#e, each computation of $S$ takes at least e time units, so we have $I \rightarrow I_{1}$, hence, $\left(\forall t_{1}, \exists t_{2}>t_{1}: I_{1}\left[t_{2} /\right.\right.$ time $\left.]\right) \rightarrow q_{\infty}$, and we obtain $q_{\infty}$. 


\section{B. Parallel Composition}

Before we give the rule of parallel composition, we will first introduce the merge of traces. Now consider the following example.

Example 3.1. Let $P={ }_{d f} x:=y+2 ; \# 1 ; y:=x+1$ and $Q={ }_{d f} \quad y:=y+2$. Assume that $P \| Q$ is activate with $x=y=0$ and time $=0$. If $P$ is scheduled to execute first, then the sequence of snapshots of $\mathrm{P}$ is :

$$
\begin{aligned}
\operatorname{seq}_{p}=< & (0,\{x=2, y=0\}, 1), \\
& (0,\{x=2, y=2\}, 0), \\
& (1,\{x=2, y=3\}, 1)>
\end{aligned}
$$

where the first and the third snapshots are produced by the atomic action $x=: y+2$ and $y:=x+1$ of $P$. And the second one is engaged by the environment of $P$. In this example, the environment of $P$ is $Q$ and the computation of $Q$ yields the following sequence :

$$
\begin{aligned}
\operatorname{seq}_{q}=< & (0,\{x=2, y=0\}, 0), \\
& (0,\{x=2, y=2\}, 1), \\
& (1,\{x=2, y=3\}, 0)>
\end{aligned}
$$

Due to $s e q_{p}$ and $s e q_{q}$ are built from the same initial state, they are comparable. In addition, all of their snapshots are made by both $P$ and $Q$. So their merge rises a trace of $P \| Q$ :

$$
\begin{aligned}
\operatorname{seq}_{p \| q}=< & (0,\{x=2, y=0\}, 1), \\
& (0,\{x=2, y=2\}, 1), \\
& (1,\{x=2, y=3\}, 1)>
\end{aligned}
$$

If $Q$ is executed first, then the traces of $P$ and $Q$ are :

$$
\begin{aligned}
\operatorname{seq}_{p}=< & (0,\{x=0, y=2\}, 0), \\
& (0,\{x=2, y=2\}, 1), \\
& (1,\{x=2, y=3\}, 1)> \\
\operatorname{seq}_{q}=< & (0,\{x=0, y=2\}, 1), \\
& (0,\{x=2, y=2\}, 0), \\
& (1,\{x=2, y=3\}, 0)>
\end{aligned}
$$

Their trace of $P \| Q$ is their merge. The trace is:

$$
\begin{aligned}
\operatorname{seq}_{p \| q}=< & (0,\{x=0, y=2\}, 1), \\
& (0,\{x=2, y=2\}, 1), \\
& (1,\{x=2, y=3\}, 1)>
\end{aligned}
$$

Definition 3.1 (Merge of Traces)) As we have seen in Example 3.1, two sequences seq1 and seq2 are said to be comparable if

(1) The time sequences from the two traces are the same $\pi_{1}(\operatorname{seq} 1)=\pi_{1}(\operatorname{seq} 2)$

(2) They are built from the same sequence of states $\pi_{2}(\operatorname{seq} 1)=\pi_{2}(\operatorname{seq} 2)$

(3) None of their snapshots is made by both components $2 \notin \pi_{1}(\operatorname{seq} 1)+\pi_{1}(\operatorname{seq} 2)$

We use the following predicate to present their merge:

$$
\begin{aligned}
& M\left(s e q, \operatorname{seq}_{1}, \text { seq }_{2}\right)=d f \\
& \left.\qquad \begin{array}{c}
\left(\pi_{1}\left(s e q_{1}\right)=\pi_{1}\left(s e q_{2}\right)=\pi_{1}(s e q)\right) \wedge \\
\left(\pi_{2}\left(s e q_{1}\right)=\pi_{2}\left(s e q_{2}\right)=\pi_{2}(s e q)\right) \wedge \\
\left(\pi_{3}(s e q)=\pi_{3}\left(s e q_{1}\right)+\pi_{3}\left(s e q_{2}\right)\right) \wedge \\
\left(2 \notin \pi_{3}\left(s e q_{1}\right)+\pi_{3}\left(s e q_{2}\right)\right)
\end{array}\right)
\end{aligned}
$$

In the sequential programs, the $@(g)$ can only be trigged by the execution of its prior atomic action, but in the parallel programs, it also can be trigged by its environment, and the Rule 1(Guard-1) will be replaced by the following rule.

\section{Rule 4. Guard -2}

$$
\begin{aligned}
& (p \wedge \text { time }<\infty) \wedge \operatorname{trig}(g) \text { at } t r \rightarrow p \\
& (p \wedge \text { time }<\infty) \wedge\left(\exists t r_{1} \bullet t r \leq t r_{1} \wedge\right. \\
& \left.\operatorname{await}(g) \text { during }\left[\operatorname{tr}, \operatorname{tr}_{1}\right) \wedge\left(\operatorname{trig}(g) \text { at } \operatorname{tr}_{1}\right)\right) \\
& \rightarrow q\left[\pi_{1}\left(\operatorname{last}\left(\operatorname{tr}_{1}\right)\right) / \text { time, } \pi_{2}\left(\operatorname{last}\left(\operatorname{tr}_{1}\right)\right) / \sigma\right] \\
& (p \wedge \text { time }<\infty) \wedge \text { await }(g) \text { during }\left[\text { tr, } \operatorname{tr}_{1}\right) \wedge \\
& \pi_{2}\left(\operatorname{last}\left(\operatorname{tr}_{1}\right)\right)=\infty \rightarrow q_{\infty} \\
& \operatorname{tr}:\{p\} @(g)\left\{p \vee q \vee q_{\infty}\right\}
\end{aligned}
$$

The second property of the rule notes that, in the guard statement, if the @ $(g)$ is trigged by itself, the atomic action @ $(g)$ will be scheduled immediately. This means that only if the guard cannot be trigged by itself, then it will be waiting to be trigged by it's environment. If the guard is fired, then the guard will complete it's computation, and the last snapshot of the trace records the terminating time and the finial values of the program, so the postcondition is $q\left[\pi_{1}\left(\operatorname{last}\left(\operatorname{tr}_{1}\right)\right) /\right.$ time,$\left.\pi_{2}\left(\operatorname{last}\left(\operatorname{tr}_{1}\right)\right) / \sigma\right]$.

The proof rule for parallel composition has the following form where we use a merge operator $M$ to combine the two traces, and use the trace which has been merged we can combine two assertions.

\section{Rule 5. Parallel}

$$
\begin{aligned}
& \operatorname{tr}:\left\{p_{1}\right\} S_{1}\left\{q_{1}\right\}, \operatorname{tr}:\left\{p_{2}\right\} S_{2}\left\{q_{2}\right\}, \\
& \forall t r_{1}, t r_{2} \bullet\left(M\left(\operatorname{tr}_{3}, \operatorname{tr}_{1}, \operatorname{tr}_{2}\right) \wedge\left(\operatorname{tr}_{1} \rightarrow q_{1}\right) \wedge\left(\operatorname{tr}_{2} \rightarrow q_{2}\right)\right) \\
& \quad \rightarrow q\left[\max T \operatorname{mime}\left(q_{1}, q_{2}\right) / \operatorname{time}, \pi_{2}\left(\operatorname{last}\left(\operatorname{tr}_{3}\right)\right) / \sigma\right] \\
& \operatorname{tr}:\left\{p_{1} \wedge p_{2}\right\} S_{1} \| S_{2}\{q\}
\end{aligned}
$$

where $t r_{1}$ is the trace of $S_{1}$ and $t r_{2}$ is the trace of $S_{2}$ when $S_{1} \| S_{2}$.

The $t r \rightarrow q$ represents that the assumption $q$ satisfies the state $\pi_{2}\left(\operatorname{last}\left(t r_{2}\right)\right)$, and the trace will not record the delay statement, so if a statement is followed by a time delay, it will not change the trace. For instance, consider a program $P: x=: x+1, \# 1, x=: x+2$ with the initial state $x=0$ and the initial time is 0 , and the sequence of the snapshots of $P:<(0, x=1,1),(1, x=3,1)>$. When we add a time delay to the end of $P$, the trace of $P$ will not change anything. So we define

$$
t r \rightarrow q={ }_{d f} \pi_{2}(\text { last }(\text { tr })) \wedge \text { time }<\infty \rightarrow q
$$

Due to the termination times of $S_{1}$ and $S_{2}$ will be different. To obtain a general rule, we use the notation $\operatorname{maxTime}\left(q_{1}, q_{2}\right)$ to denote the termination time of $S_{1} \| S_{2}$, the definition is given as below:

$\operatorname{maxTime}\left(q_{1}, q_{2}\right)={ }_{d f} \max \left(t_{1}, t_{2}\right)\left(t_{i}\right.$ is the value of time 
in $q_{i}$ )

\section{Auxiliary Axioms and Rules}

In this subsection, we will introduce some auxiliary axioms and rules which will be used in our proof system. Some of them have been presented in [9].

Axiom 6. Invariance

$$
\text { tr }:\{p\} S\{p\}
$$

where time does not occur in $p$, and $\operatorname{var}(S) \cap \operatorname{var}(p)=\phi$

\section{Rule 6. Disjunction}

$$
\frac{t r:\{p\} S\{q\}, t r:\{r\} S\{q\}}{t r:\{p \vee r\} S\{q\}}
$$

\section{Rule 7. Conjunction}

$$
\frac{t r:\left\{p_{1}\right\} S\left\{q_{1}\right\}, \operatorname{tr}:\left\{p_{2}\right\} S\left\{q_{2}\right\}}{\operatorname{tr}:\left\{p_{1} \wedge p_{2}\right\} S\left\{q_{1} \wedge q_{2}\right\}}
$$

The substitution rule means that if a variable does not occur in the program statement, we can use any arbitrary expression to replace it.

\section{Rule 8. Substitution}

$$
\frac{t r:\{p\} S\{q\}}{t r:\{p[z:=t]\} S\{q[z:=t]\}}
$$

where $(z \cup \operatorname{var}(t)) \cap$ changes $(S)=\phi$ and time does not occur in $t$.

\section{Rule 9. Consequence}

$$
\frac{t r:\{p\} S\{q\}, p_{1} \rightarrow p, q \rightarrow q_{1}}{t r:\left\{p_{1}\right\} S\left\{q_{1}\right\}}
$$

About the rule for sequential consequence, the construct is same as the classic rule of Hoare Logic. In our proof system, we use trace to help us record the state before a statement begins it's first statement, and as we described in the parallel rule, trace does not record the delay statement, so we assume that $S_{1}$ ends with a delay statement $\# e(0 \leq e \leq \infty)$.

\section{Rule 10. Sequential Consequence}

$$
\begin{aligned}
& t r:\{p\} S_{1}\{r\}, t r_{1}:\{r\} S_{2}\{q\} \\
& t r:\{p\} S_{1} ; S_{2}\{q\}
\end{aligned}
$$

where $t r \preceq t r_{1}, t r_{1} \rightarrow \mathrm{r}$ and $\pi_{1}($ last $(t r))+e=t_{r} .\left(t_{r}\right.$ is the value of time in $r$ ).

\section{Case Study}

In this section we give a parallel program written by MDESL, and show how to apply our method to prove the correctness of the program. Consider the program $P$ and $Q$ :

$$
\begin{gathered}
P:=\text { while } x>0 \text { do } \\
@(\uparrow y) ; \\
x:=x-1 ; \\
\text { od; } \\
z=1 ; \\
Q::=\# 1 ; \\
\text { while } z \neq 1 \text { do } \\
y:=y+1 ; \\
\# 1 ; \\
\text { od; }
\end{gathered}
$$

Program $P$ represents that the variable $x$ will decrease if $x>0$ and variable $y$ increases. If $x \leq 0$, it terminates. Program $Q$ denotes that $y$ increases by 1 per one time unit when $z \neq 1$ is true, so when $P$ terminates, it satisfies $z=1$. We have the following assumptions and notations:

- the initial trace $\operatorname{tr}$ is $\langle(0,\{x=2, y=0, z \neq 1\}, 1)>$.

- $x, y, z$ are all integers.

- the precondition $p_{1}$ is $x=2 \wedge y=0 \wedge z \neq 1 \wedge$ time $=0$.

- $S_{1}=(@(\uparrow y) ; x:=x-1)$ and $S_{2}=(y:=y+1 ; \# 1)$. For $P \| Q$, we want to prove the following correctness formulas:

$$
\begin{aligned}
& \operatorname{tr}:\left\{p_{1}\right\} P\{x \leq 0 \wedge \text { time }<\infty\} \\
& \operatorname{tr}:\left\{p_{1}\right\} Q\{z=1 \wedge \text { time }<\infty\} \\
& \operatorname{tr}:\left\{p_{1}\right\} P \| Q\{x \leq 0 \wedge z=1 \wedge \text { time }<\infty\}
\end{aligned}
$$

We denote $x \leq 0 \wedge$ time $<\infty$ as $q_{1}, z=1 \wedge$ time $<\infty$ as $q_{2}$ and $x \leq 0 \wedge z=1 \wedge$ time $<\infty$ as $q$.

\section{Proof :}

First, as same as the Example 3.1, we get the traces of $P$ and $Q$ when $P \| Q$.

$$
\begin{array}{rlr}
t r_{p}=< & (0,\{x=2, y=0, z \neq 1\}, 1), & \\
& (1,\{x=0, y=1, z \neq 1\}, 0), & \\
& (1,\{x=1, y=1, z \neq 1\}, 1), & \left(t r_{p 2}\right) \\
& (2,\{x=1, y=2, z \neq 1\}, 0), & \left(t r_{p 3}\right) \\
& (2,\{x=0, y=2, z \neq 1\}, 1), & \left(t r_{p 4}\right) \\
& (2,\{x=0, y=2, z=1\}, 1)> & \\
t r_{q}=< & (0,\{x=2, y=0, z \neq 1\}, 1), & \\
& (1,\{x=0, y=1, z \neq 1\}, 1), & \left(t r_{q 1}\right) \\
& (1,\{x=1, y=1, z \neq 1\}, 0), & \left(t r_{q 2}\right) \\
& (2,\{x=1, y=2, z \neq 1\}, 1), & \left(t r_{q 3}\right) \\
& (2,\{x=0, y=2, z \neq 1\}, 0), & \left(t r_{q 4}\right) \\
& (2,\{x=0, y=2, z=1\}, 0)> &
\end{array}
$$

According to the definition of Merge, we obtain the trace of $P \| Q$.

$$
\begin{aligned}
t r_{p \| q}=< & (0,\{x=2, y=0, z \neq 1\}, 1), \\
& (1,\{x=0, y=1, z \neq 1\}, 1), \\
& (1,\{x=1, y=1, z \neq 1\}, 1), \\
& (2,\{x=1, y=2, z \neq 1\}, 1), \\
& (2,\{x=0, y=2, z \neq 1\}, 1), \\
& (2,\{x=0, y=2, z=1\}, 1)>
\end{aligned}
$$

Then we prove the correctness of (4.1) and (4.2) by using their trace $t r_{p}$ and $t r_{q}$. 
To prove (4.1), we set a global invariant variable $I_{1}$ as : $I_{1}=z \neq 1 \wedge$ time $<\infty$.

And we need to prove the correctness of following formula :

and

$$
\text { tr }:\left\{I_{1} \wedge x>0\right\} @(\uparrow y)\{I \wedge x>0\}
$$

$$
\operatorname{tr}_{p 2}:\left\{I_{1} \wedge x>0\right\} @(\uparrow y)\{I \wedge x>0\}
$$

Note that the implications

$$
\begin{aligned}
I_{1} & \wedge\left(\exists t r_{p 1} \bullet t r \leq t r_{p 1} \wedge\right. \\
& \text { await } \left.(g) \text { during }\left[t r, t r_{p 1}\right) \wedge\left(\operatorname{trig}(g) \text { at } t r_{p 1}\right)\right) \\
& \rightarrow I_{1} \wedge x>0
\end{aligned}
$$

and

$$
\begin{aligned}
I_{1} & \wedge\left(\exists t r_{p 3} \bullet t r_{p 2} \leq t r_{p 3} \wedge\right. \\
& \left.\operatorname{await}(g) \text { during }\left[t r_{2}, t r_{p 3}\right) \wedge\left(\operatorname{trig}(g) \text { at } t r_{p 3}\right)\right) \\
& \rightarrow I_{1} \wedge x>0
\end{aligned}
$$

hold. Thus by the rule 4 (Guard -2), we prove (4.4) and (4.5). Further by the assignment axiom 4 , we prove

$$
\{I \wedge x>0\} x:=x-1\{I \wedge x \geq 0\}
$$

By (4.4), (4.5), (4.6) and the while rule 3, we obtain

$$
\text { tr }:\{I\} \text { while } x>0 \text { do } S_{1} \text { od }\{I \wedge x \leq 0\}
$$

By the consequence rule 9, we can prove that (4.1) is correct. We can use the same method to prove (4.2) is correct too.

At last we prove (4.3). Since

$$
\pi_{2}\left(\operatorname{last}\left(\operatorname{tr}_{p}\right)\right) \wedge \text { time }<\infty \rightarrow q_{1}
$$

and

$$
\pi_{2}\left(\operatorname{last}\left(\operatorname{tr}_{q}\right)\right) \wedge \text { time }<\infty \rightarrow q_{2} .
$$

$t r_{p} \rightarrow q_{1}$ and $t r_{q} \rightarrow q_{2}$ are hold in this model, and using the definition of maxtime and $\pi_{2}(t r)$, we get

$$
\operatorname{maxTime}\left(q_{1}, q_{2}\right)=_{d f} \text { time } \leq \infty
$$

and

$$
\pi_{2}\left(\operatorname{last}\left(\operatorname{tr}_{p \| q}\right)\right)=\{x=0, y=2, z=1\} .
$$

We obtain

$$
\begin{aligned}
& \forall t r_{p}, t r_{q} \bullet\left(M\left(t r_{p \| q}, t r_{p}, t r_{q}\right) \wedge\left(t r_{p} \rightarrow q_{1}\right) \wedge\left(t r_{q} \rightarrow q_{2}\right)\right) \\
& \quad \rightarrow q\left[\operatorname{maxTime}\left(q_{1}, q_{2}\right) / t i m e, \pi_{2}\left(\operatorname{last}\left(\operatorname{tr}_{3}\right)\right) / \sigma\right]
\end{aligned}
$$

Combine formula (4.1), (4.2) and (4.8) and by the Parallel rule, we can derive the correctness formula :

$$
\text { tr : }\left\{p_{1}\right\} P \| Q\{x \leq 0 \wedge z=1 \wedge \text { time }<\infty\}
$$

\section{CONCLUSION}

In this paper, we have presented a proof system for MDESL (Multithreaded Discrete Simulation Language) which aims to prove the correctness of MDESL. Compared to classical Hoare Logic, our proof system uses the global clock variable time to express the real-time feature. It represents the starting time in the precondition and the terminating time in the postcondition.
The value of time also can help us handle the nonterminating computations. And in order to verify the feature of event-driven we have extended the old triple $\{p\} s\{q\}$ to tr : $\{p\} s\{q\}$ by adding a data structure tr. To make the verification more straightforward, we have provided some axioms and rules for sequential. Then we give the composition rules which make our proof system become a complete system. Our key contribution is the guard rule. It can be applied in the verification of the property of event-driven in MDESL.

For the future, we want to explore our proof system for hardware description language (HDL). On one hand, the probability feature [12] has been proposed in a new Veriloglike language PTSC [13]. We can deduce some specific rules to describe the feature and verify the properties related to probability. On the other hand, we want to link our proof system with the semantics (operational, denotational, algebraic) [14], [15] respectively for MDESL. Furthermore, we want to implement the proof system in a tool so that it can verify the correctness of programs automatically.

\section{ACKNOWLEDGMENT}

This work was partly supported by Shanghai Collaborative Innovation Center of Trustworthy Software for Internet of Things (No. ZF1213).

\section{REFERENCES}

[1] IEEE, IEEE Standard Hardware Description Language based on the Verilog Hardware Description Laguage, Volume IEEE Standard 13641995. IEEE, 1995

[2] IEEE, IEEE Standard Hardware Description Language based on the Verilog Hardware Description Laguage, Volume IEEE Standard 13642001. IEEE, 2001.

[3] N. Nissanke, Realtime Systems, Prentice Hall International Series in computer Science, 1997.

[4] Haase, V., Real-time Behavior of Programs, IEEE Transactions on Software Engineering SE-7,5 (Sept. 1981), 497-501.

[5] Zhu, H., Linking the Semantics of a Multithreaded Discrete Event Simulation Language, London South Bank University, 2005.

[6] Golze, U., VLSI Chip Design with the Hardware Description Language Verilog: An Introduction Based on a Large RISC Processor Design, Springer-Verlag New York, Inc. 1996.

[7] Gordon, M.J.C., The semantic challenge of Verilog HDL, In Proc. Tenth Annual IEEE Symposium on Logic in Computer Science, page 136-145. IEEE Computer Society Press, June 1995.

[8] Gordon, M.J.C., Relating event and trace semantics of hardware description languages, The Computer Journal, 45(1): 27-36, 2002.

[9] Apt, K.R., de Boer, F.S., Olderog, E., Verification of sequential and concurrent programs, Texts in Computer Science, Springer, 2009.

[10] Hoare, C.A.R., Communicating Sequential Processes, Prentice Hall International Series in Computer Science, 1985.

[11] Hooman, J., Extending hoare logic to real-time, Formal Aspects of Computing, 1994, 6(1):801-825.

[12] Park, S., Pfenning, F., Thrun, S., A probabilistic language based upon sampling functions, Acm Transactions on Programming Languages, 2004, 40.1(2004):171-182.

[13] Zhu, H., Qin, S., He, J., Bowen, J.P., PTSC: probaility, time and sharedvariable concurrency, Innovations in Systems and Software Enginerring: A NASA Journal 5(4), 271-284(2009).

[14] Hoare, C.A.R., Algebra of concurrent programming, In: Meeting 52 of WG 2.3(2011).

[15] Hoare, C.A.R., He, J., Unifying Theories of programming, Prentice Hall International Series in Computer Science, 1998. 



\section{Author Index}

A jala, Oluwole I.

193

Angelova, Maria ....

...77

Argasiński, Jan K.

345

Artych, Rafał.

B abati, Bence

Barriviera, Rodolfo ............................ 199

Bisták, Pavol ................................ 315

Bocianiak, Krzysztof.......................... 127

Bodaniuk, Maksym ........................... 183

Bowzer, Christopher ............................65

Capiluppi, Andrea

Christo, Luiz Eduardo de......................... 207

Chujfi, Salim .................................... 303

Cohen, Kasey...................................65

Daszczuk, Wiktor

Deniziak, Stanisław

Dragg, Paweł

$\mathrm{F}_{\text {ei, Yuan }}$ 387

Fray, Imed El

Fukazawa, Yoshiaki

Fukuda, Munehiro

$\mathrm{G}_{\text {iżycka, Barbara }}$ 345

Grabska, Ewa

Grochowina, Marcin

Gryncewicz, Wieslawa .

$\mathrm{H}_{\text {affner, Oto }}$

Henriques, Pedro Rangel

Honda, Aoi

Hunka, Frantisek

Iliopoulos, Costas S

İşlek, İrem

Iwase, Yuko

Iwata, Hajime

$\mathbf{J}_{\text {ach, Tomasz }}$
Kaźmierczak, Adrian .............................249

Khan, Mujibur R............................ 193

Klasa, Tomasz...............................213

Kolesnik, Valerii ............................. 183

Kozák, Štefan............................. 3, 315

Krawiec, Łukasz.............................255

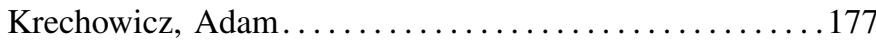

Kubus, Łukasz................................. 89

Kučera, Erik ............................ 3, 315

Kutera, Robert...............................265

Kutt, Krzysztof ................................... 345

$\mathrm{L}$

Łachwa, Andrzej..............................17

Leniowska, Lucyna ........................... 51

Ligęza, Antoni..................................295

Lu, Jianyu........................................

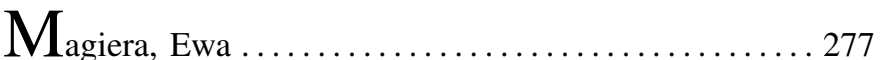

Matula, Jiri...............................241

Maziero, Carlos ............................... 199

Meinel, Christoph................................. 303

Morita, Naoya .................................285

Mozgovoy, Maxim .............................59

$\mathrm{N}_{\text {agao, Katashi } \ldots \ldots \ldots \ldots \ldots \ldots \ldots \ldots \ldots \ldots \ldots \ldots} 285$

Nakayama, Minoru ........................... 141

Nalepa, Grzegorz J. ................................ 345

Nekvapil, Viktor ............................... 9

Neuman, Clifford .................................. 323

Nishijima, Taiki ............................ 353

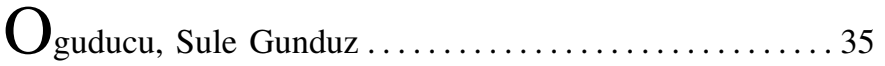

Ohira, Shigeki ...............................285

Ohki, Makoto ..................................... 353

Ośko, Tomasz ................................ 127

$P_{\text {alacz, wojciech } \ldots \ldots \ldots \ldots \ldots \ldots \ldots \ldots \ldots \ldots \ldots \ldots \ldots}$

Paszyńska, Anna ................................. 17

Pataki, Norbert ................................. 149

Patekha, Evgeny .......................... 45

Pencheva, Tania.............................. 77

Pereira, Maria João Varanda ....................... 157

Phan, Benjamin ...............................65

Poczęta, Katarzyna................................... 89

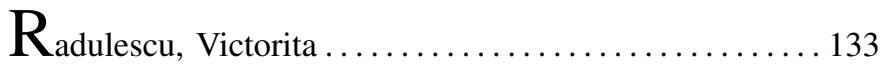

Ramos, Marcos ............................... 157

Raveendran, Nishanthi..........................95

Rolik, Oleksandr.............................183 
S

Šaikūnas, Audrius

Saito, Miho

Samotyy, Volodymyr.

Samuelsson, Christer.

Sendorek, Joanna

Shirogane, Junko

Skalniak, Tomasz . . .

Ślusarczyk, Grażyna

Sofronov, Georgy

Stark, Erich

Strug, Barbara.

Styczeń, Krystyn .

Suchenia (Mroczek), Anna

Szadkowska, Anna

Szadkowski, Zbigniew

Szydlo, Tomasz

$T_{\text {kacz, Magdalena }}$
Ur-Rehman, Obaid

W achnik, Bartosz .27

Wadhawan, Yatin ................................. 323

Wang, Zhenchen ................................ 277

Werewka, Jan................................... 335

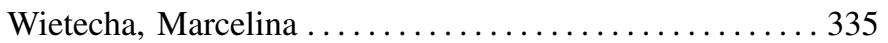

Wiśniewski, Piotr........................... 295

Wołczyk, Kamil .............................. 335

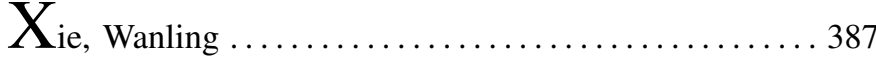

$Y_{\text {amaguchi, Hiroshi } \ldots \ldots \ldots \ldots \ldots \ldots \ldots \ldots \ldots \ldots}$

Yamazaki, Takashi ........................... 141

Yastrebov, Alexander........................... 89

Zacek, Jaroslav.........................241

Zhu, Huibiao .............................. 387

Zivic, Natasa .............................. 223 